Supporting Information for geometries of intermediates and transition states, a sample input, Cartesian coordinates and energies

\title{
A DFT Study of the Hydroxyl Radical Addition to 2'-Deoxyguanosine and to the Guanine Base in Four Double-Stranded B-Form Dimers
}

Shinichi Yamabe, ${ }^{a *}$ Noriko Tsuchida, ${ }^{\mathrm{b}}$ and Shoko Yamazaki ${ }^{\mathrm{a}}$

a Department of Chemistry, Nara University of Education, Takabatake-cho, Nara 630-8528, Japan

${ }^{b}$ Department of Liberal Arts, Faculty of Medicine, Saitama Medical University, 38 Morohongo, Moroyama-machi, Iruma-gun, Saitama 350-0495 Japan

email: yamabesh@nara-edu.ac.jp

phone: +81-742-27-9193

Figure S1. Reactions of dR-Gua and $\mathrm{HO} \bullet[n=0$, without water molecules $]$ for the $\mathrm{C} 4$-attack channel depicted in Scheme 4.

Page S3

Figure S2. Reactions of dR-Gua and $\mathrm{HO} \cdot[\mathrm{n}=1$, with a water molecule, "W1"] for the C4attack channel depicted in Scheme 4.

Page S4

Figure S3. Reactions of dR-Gua and $\mathrm{HO} \bullet[n=2$, with two water molecules, "W2"] for the C4attack channel depicted in Scheme 4.

Page S5

Figure S4. Reactions of dR-Gua and $\mathrm{HO} \bullet[n=0$, without water molecules $]$ for the C8-attack channel depicted in Scheme 4.

Page S6

Figure S5. Reactions of dR-Gua and $\mathrm{HO} \bullet[\mathrm{n}=1$, with a water molecule, "W1"] for the C8attack channel depicted in Scheme 4.

Page S7

Figure S6. Reactions of dR-Gua and $\mathrm{HO} \bullet[n=2$, with two water molecules, W2"] for the C8attack channel depicted in Scheme 4.

Page S8 
Figure S7. Reactions of dR-Gua and $\mathrm{HO} \bullet[n=0$, without water molecules $]$ for the C5-attack channel depicted in Scheme 4.

Page S9

Figure S8. Reactions of dR-Gua and $\mathrm{HO} \cdot[\mathrm{n}=1$, with a water molecule, "W1"] for the C5attack channel depicted in Scheme 4.

Page S10

Figure S9. Reactions of dR-Gua and $\mathrm{HO} \bullet[n=2$, with two water molecules, W2"] for the C5attack channel depicted in Scheme 4.

Page S11

Figure $\mathrm{S} 10$. The $\mathrm{HO} \bullet$ addition reaction to $\mathrm{C}(4)$ or $\mathrm{C}(8)$ of the guanine moiety of the (b) 5'-GG3' sequence.

Page S12

Figure $\mathrm{S} 11$. The $\mathrm{HO} \cdot$ addition reaction to $\mathrm{C}(4)$ or $\mathrm{C}(8)$ of the guanine moiety of the (c) 5'-GT3' sequence.

Page S13

Figure $\mathrm{S} 12$. The $\mathrm{HO} \bullet$ addition reaction to $\mathrm{C}(4)$ or $\mathrm{C}(8)$ of the guanine moiety of the (d) 5'-GC3' sequence.

Page S14

Figure $\mathrm{S} 13$. The $\mathrm{HO} \cdot\left(\mathrm{H}_{2} \mathrm{O}\right)_{2}$ addition reaction to $\mathrm{C}(8)$ of the guanine moiety of the (b) 5'-GG3 ' sequence and the subsequent isomerization.

Page S15

Figure $\mathrm{S} 14$. The $\mathrm{HO} \cdot\left(\mathrm{H}_{2} \mathrm{O}\right)_{2}$ addition reaction to $\mathrm{C}(8)$ of the guanine moiety of the (c) 5'-GT3 ' sequence and the subsequent isomerization.

Page S16

Figure $\mathrm{S} 15$. The $\mathrm{HO} \cdot\left(\mathrm{H}_{2} \mathrm{O}\right)_{2}$ addition reaction to $\mathrm{C}(8)$ of the guanine moiety of the (d) 5'-GC3 ' sequence and the subsequent isomerization.

Page S17

A sample input, Cartesian coordinates and energies

Pages S18-302 

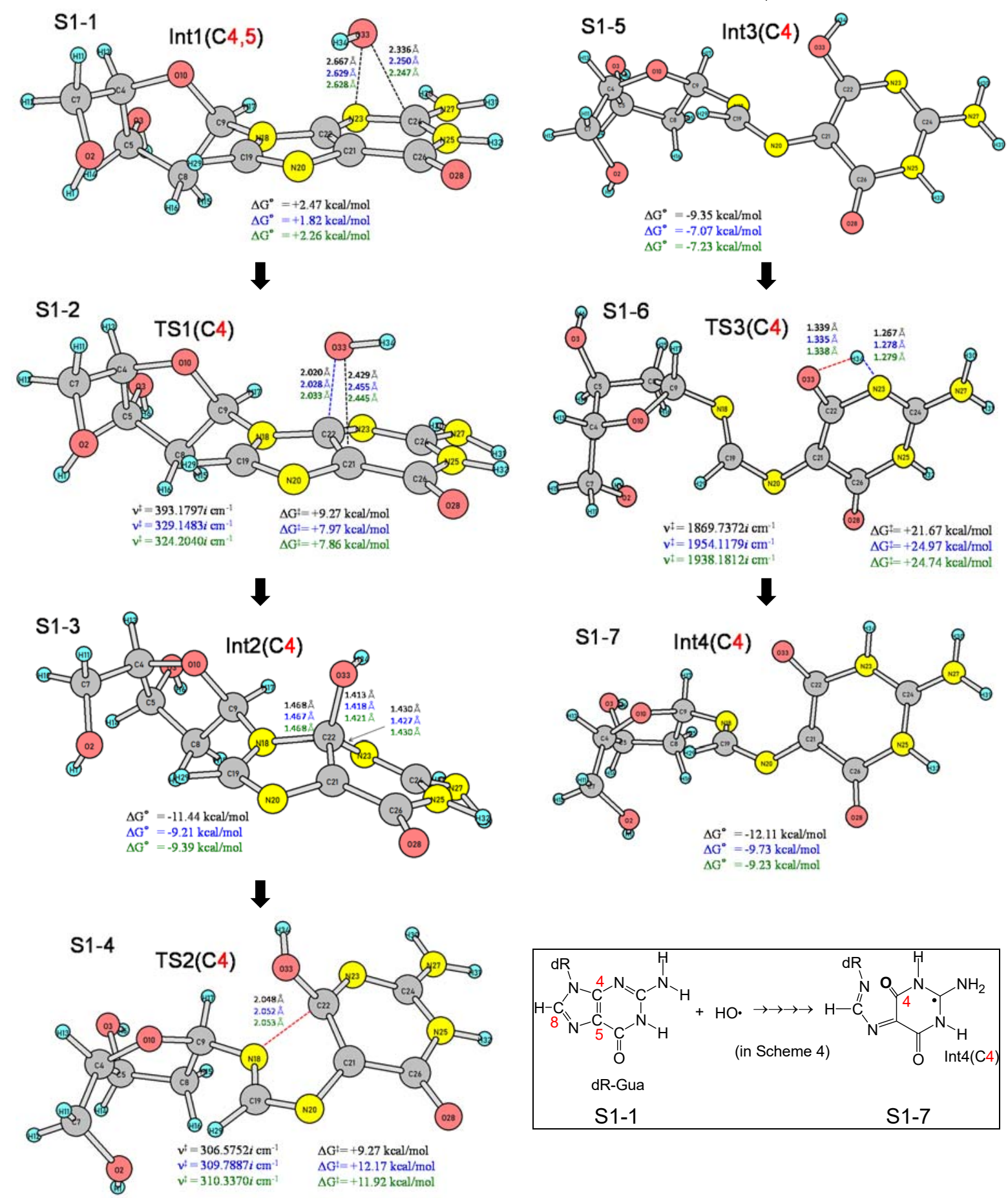

Figure S1. Reactions of dR-Gua and $\mathrm{HO} \cdot[\mathrm{n}=0$, without water molecules] for the $\mathrm{C} 4$-attack channel depicted in Scheme 4. Distances and $\Delta \mathrm{G}$ values of wB97X-D/6-31G** (black color), wB97X-D/6-311+G** (blue color) and wB97X-D/aug-cc-pVDZ (green color) with $\mathrm{SCRF}=(\mathrm{PCM}$,solvent=water $)$ are shown. $\operatorname{Int}(\mathrm{C} 4,5) "$ in Figure S1-1 means that the precursor for the $\mathrm{C} 4$ and $\mathrm{C} 5$ attack is common. That is to say, Figure S1-1 is the same as Figure S7-1(see Table 1). $\Delta \mathrm{G}$ values are relative to the sum of $\mathrm{G}^{\circ}(\mathrm{dR}-\mathrm{Gua})$ and $\mathrm{G}^{\circ}(\mathrm{HO} \bullet$ ). 

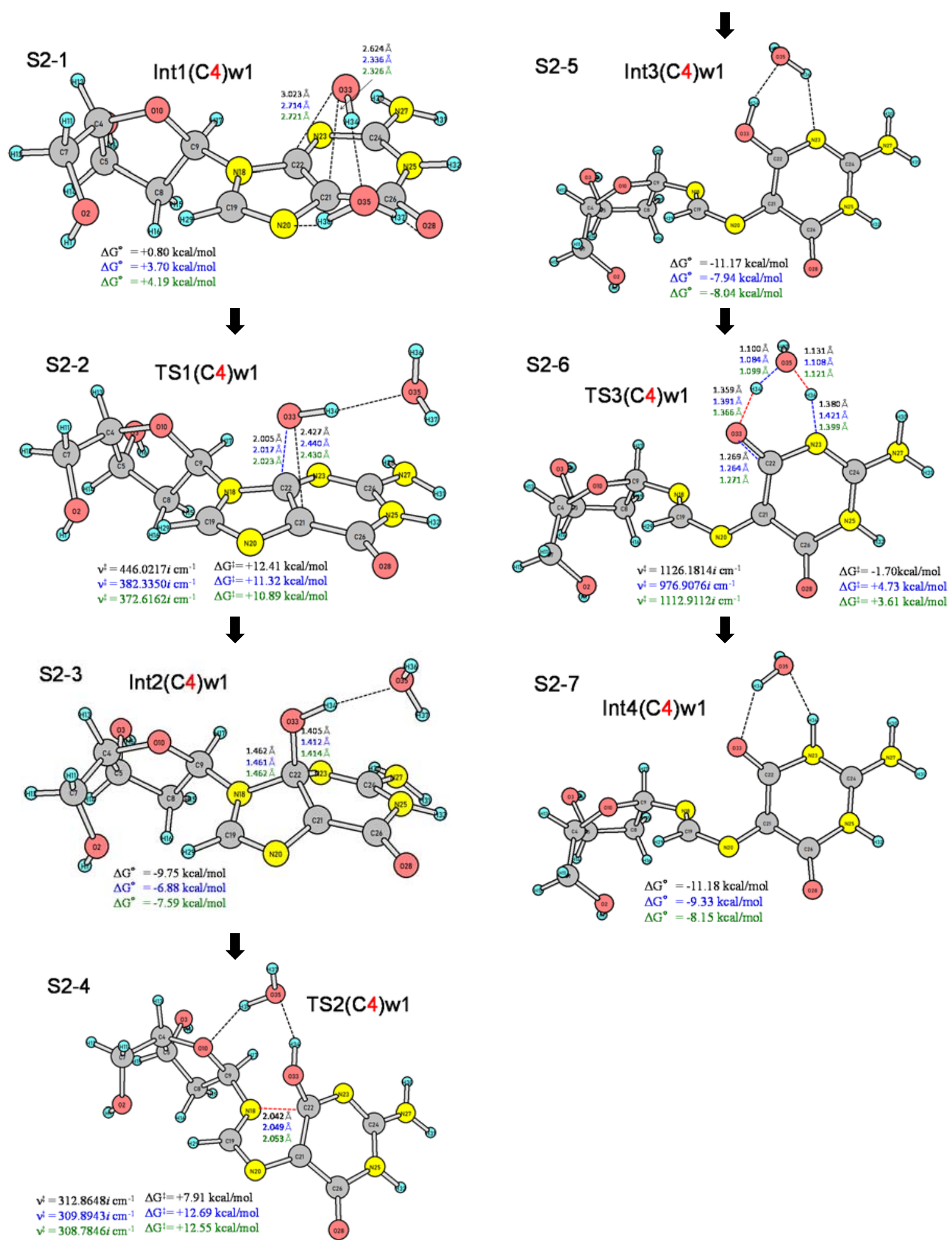

Figure S2. Reactions of dR-Gua and $\mathrm{HO} \cdot[\mathrm{n}=1$, with a water molecule, "W1"] for the C4attack channel depicted in Scheme $4 . \Delta \mathrm{G}$ values are relative to the sum of $\mathrm{G}^{\circ}$ (dR-Gua) and $\mathrm{G}^{\circ}$ $\left(\mathrm{HO} \cdot \mathrm{H}_{2} \mathrm{O}\right)$. 

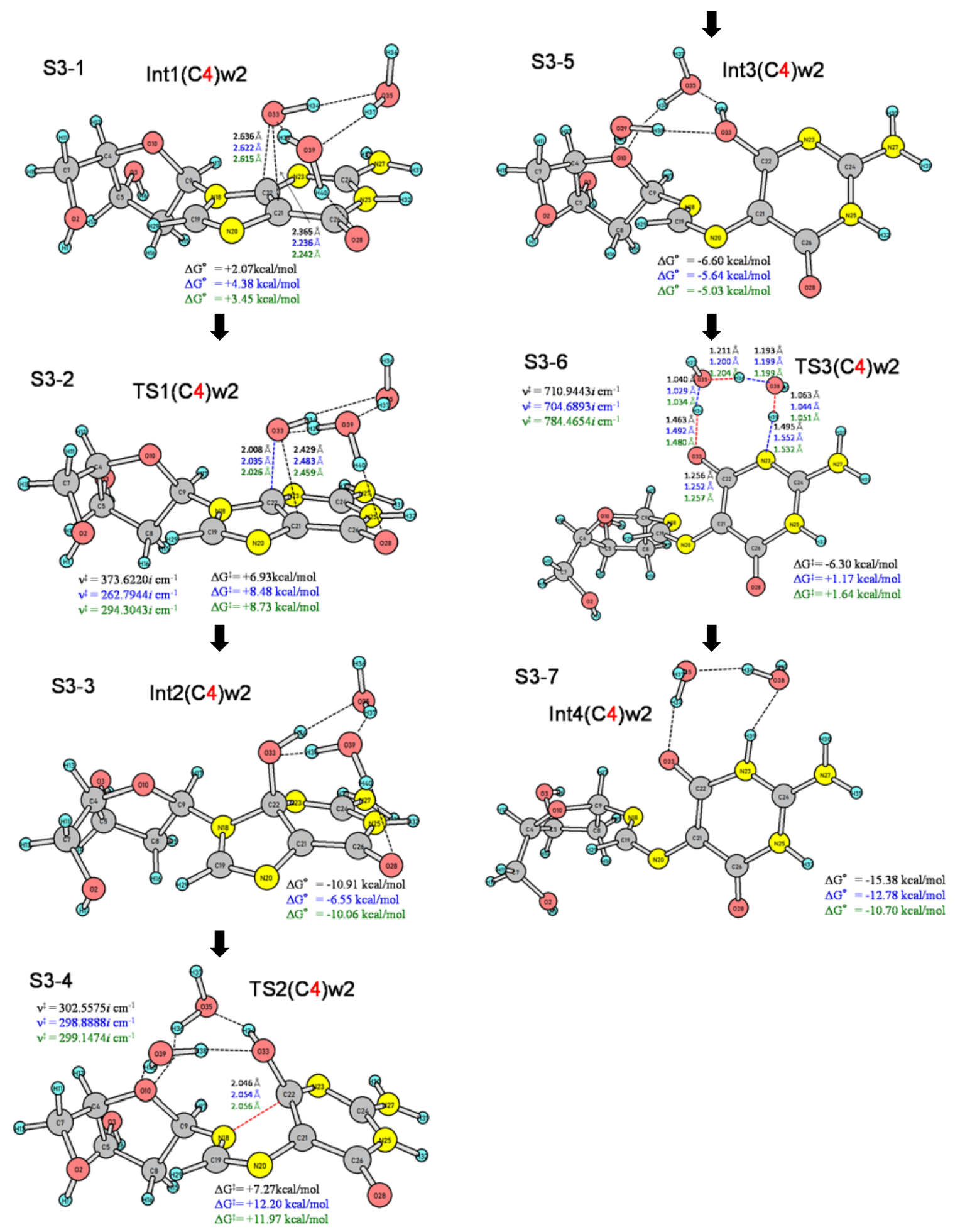

Figure S3. Reactions of dR-Gua and $\mathrm{HO} \bullet[\mathrm{n}=2$, with two water molecules, "W2"] for the C4attack channel depicted in Scheme 4. $\Delta \mathrm{G}$ values are relative to the sum of $\mathrm{G}^{\circ}$ (dR-Gua) and $\mathrm{G}^{\circ}$ $\left(\mathrm{HO} \cdot\left(\mathrm{H}_{2} \mathrm{O}\right)_{2}\right)$. 

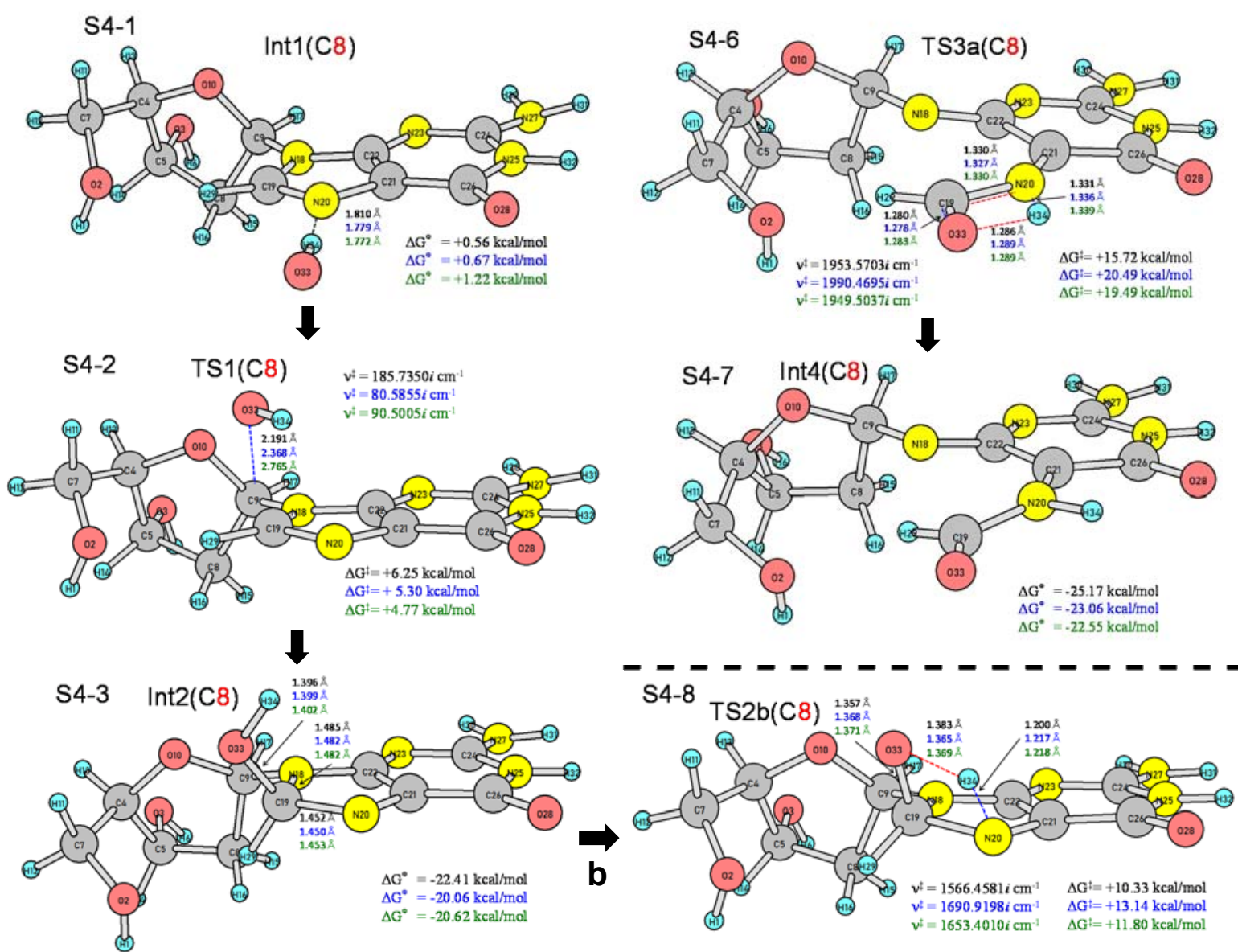

\section{a $\downarrow$}
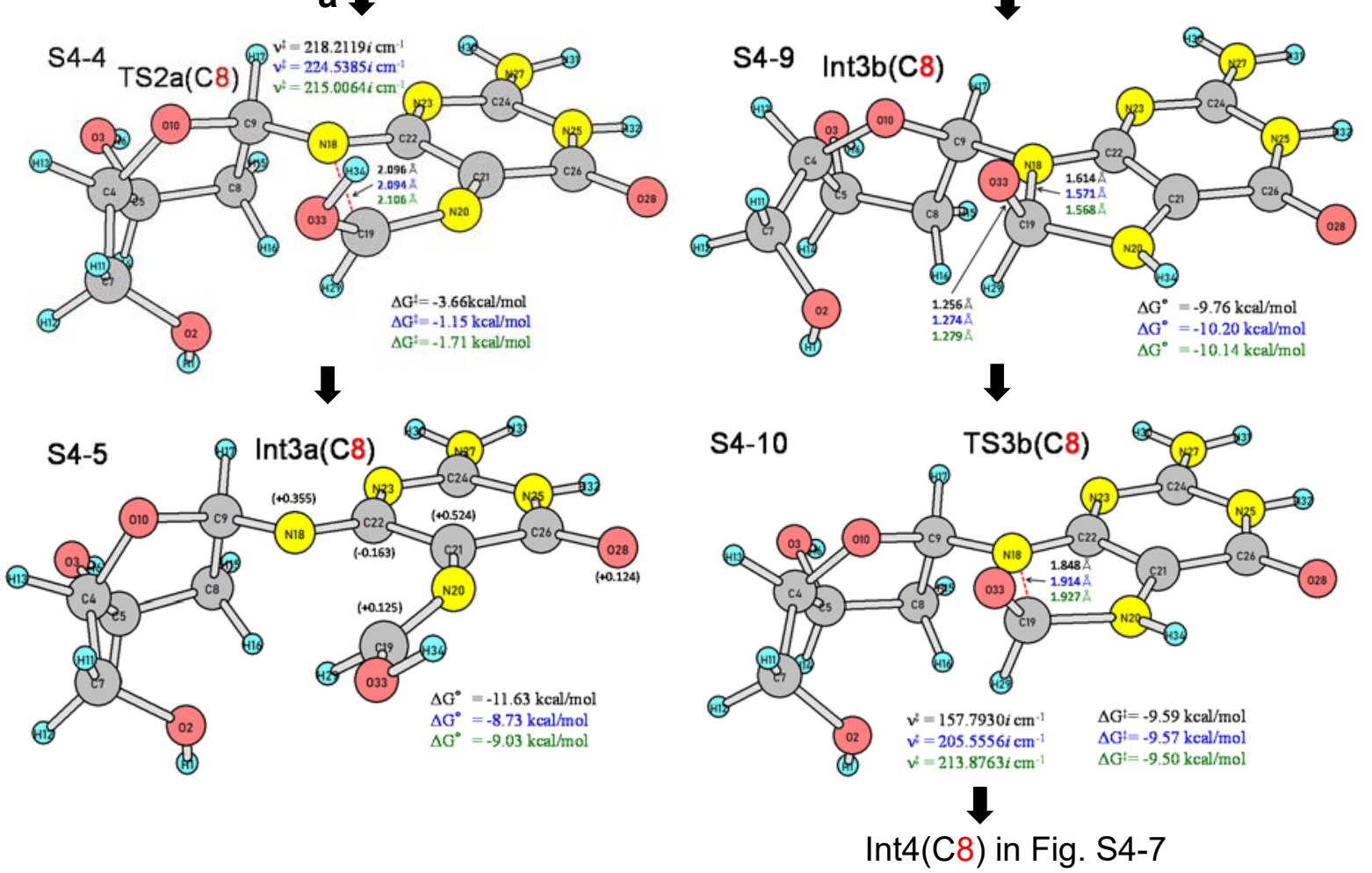

Figure S4. Reactions of dR-Gua and $\mathrm{HO} \cdot[\mathrm{n}=0$, without water molecules $]$ for the $\mathrm{C} 8$-attack channel depicted in Scheme 4. For Int3a(C8) in S4-5, Mulliken spin densities are shown in parentheses. 

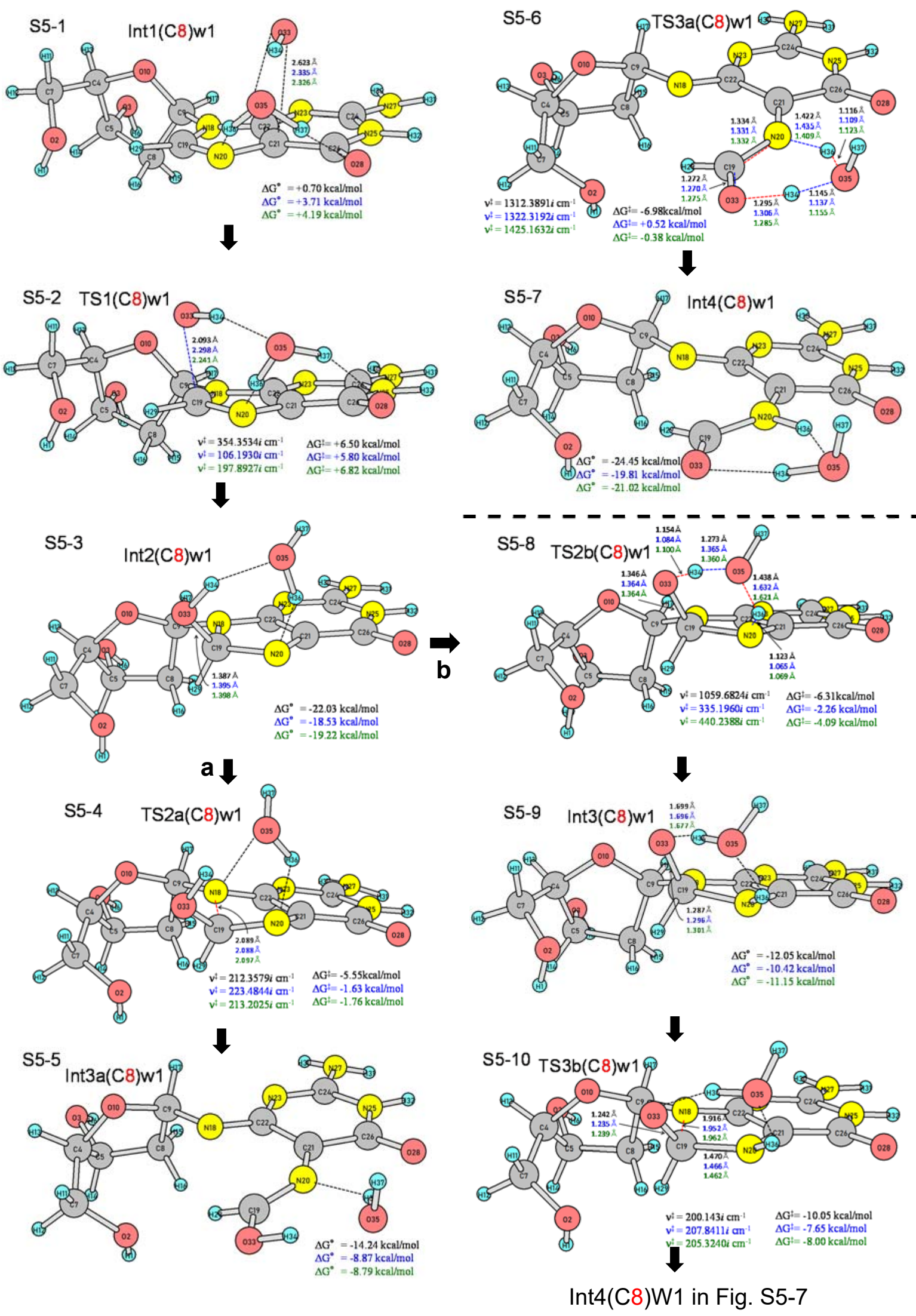

Figure S5. Reactions of dR-Gua and $\mathrm{HO} \bullet[n=1$, with a water molecule, "W1"] for the C8attack channel depicted in Scheme 4. 

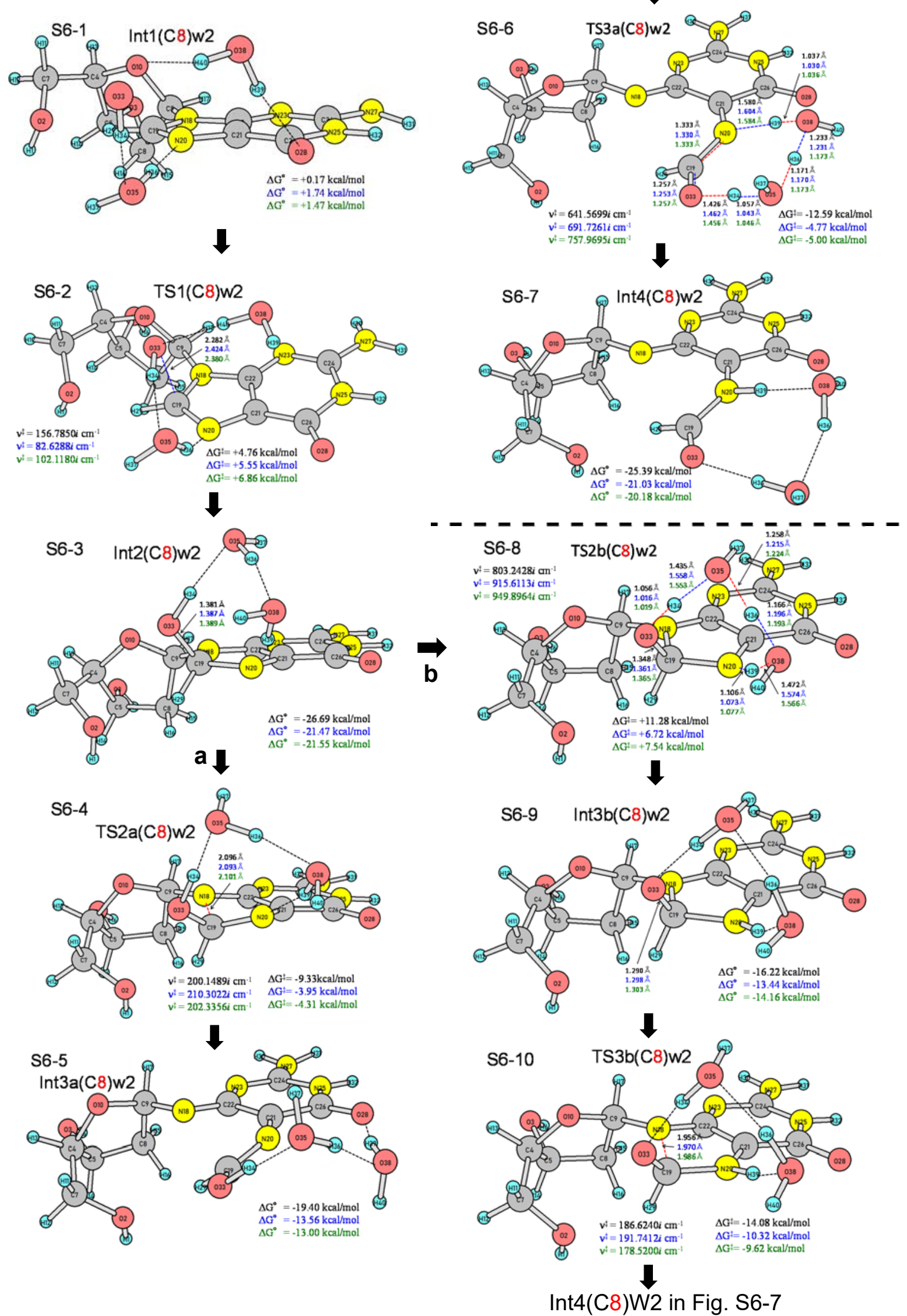

Figure S6. Reactions of dR-Gua and $\mathrm{HO} \bullet[n=2$, with two water molecules, W2"] for the C8attack channel depicted in Scheme 4. 
S7-1

Int1 $(\mathrm{C} 4,5)$ in Figure S1-1

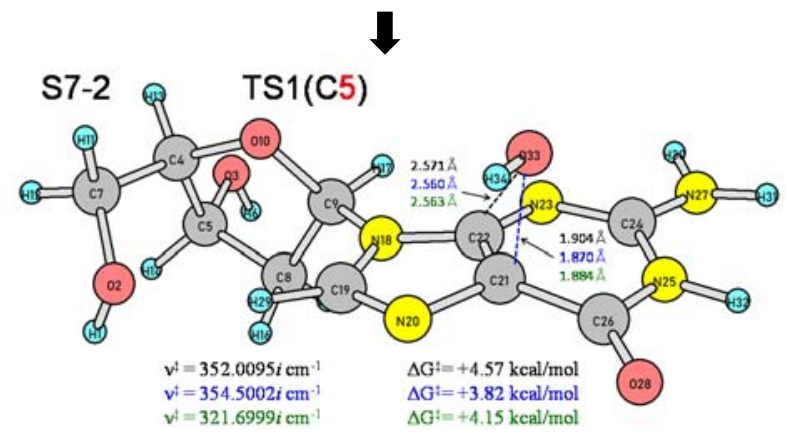

\section{$\downarrow$}

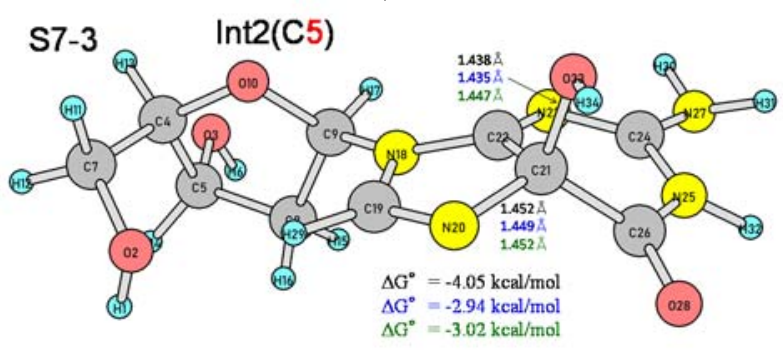

$\downarrow$

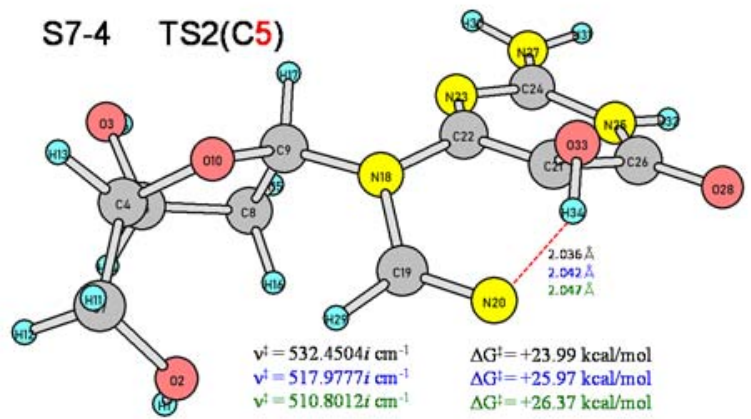

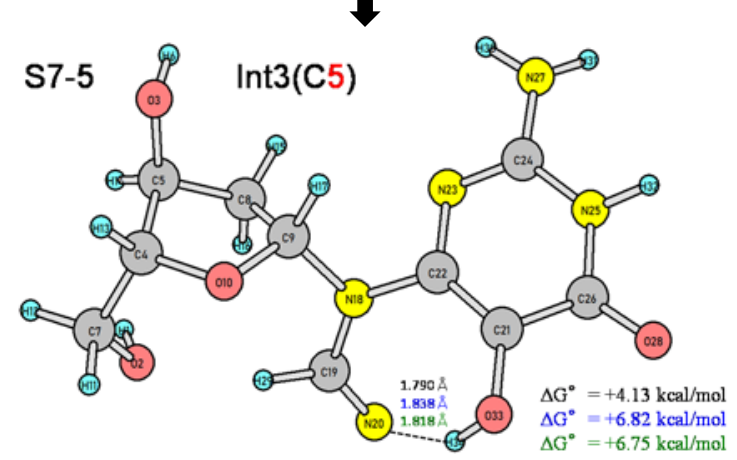
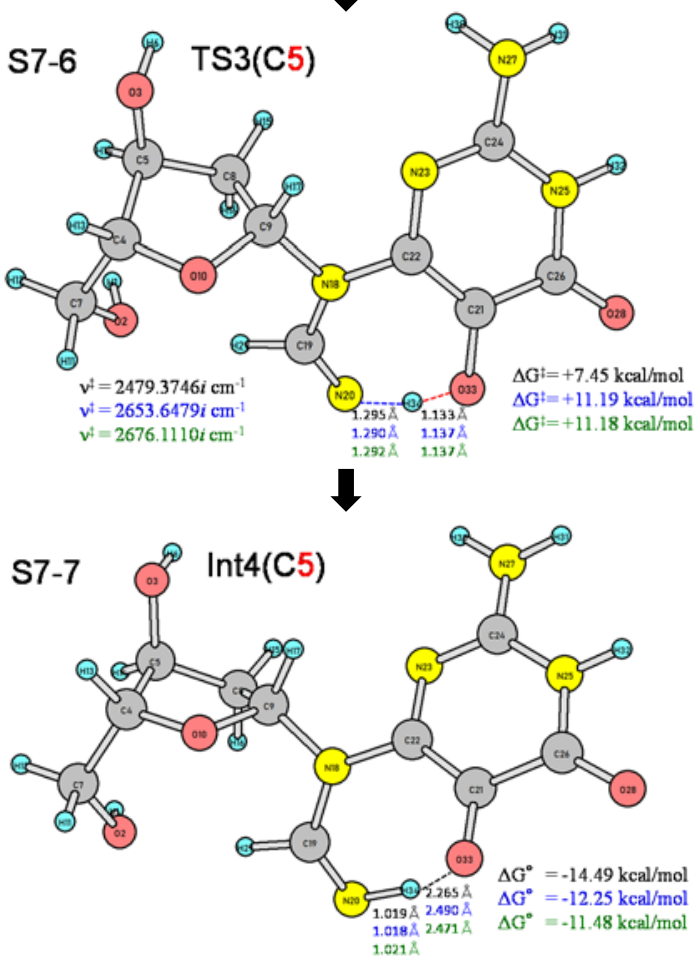

Figure S7. Reactions of dR-Gua and $\mathrm{HO} \bullet[n=0$, without water molecules $]$ for the C5-attack channel depicted in Scheme 4. 

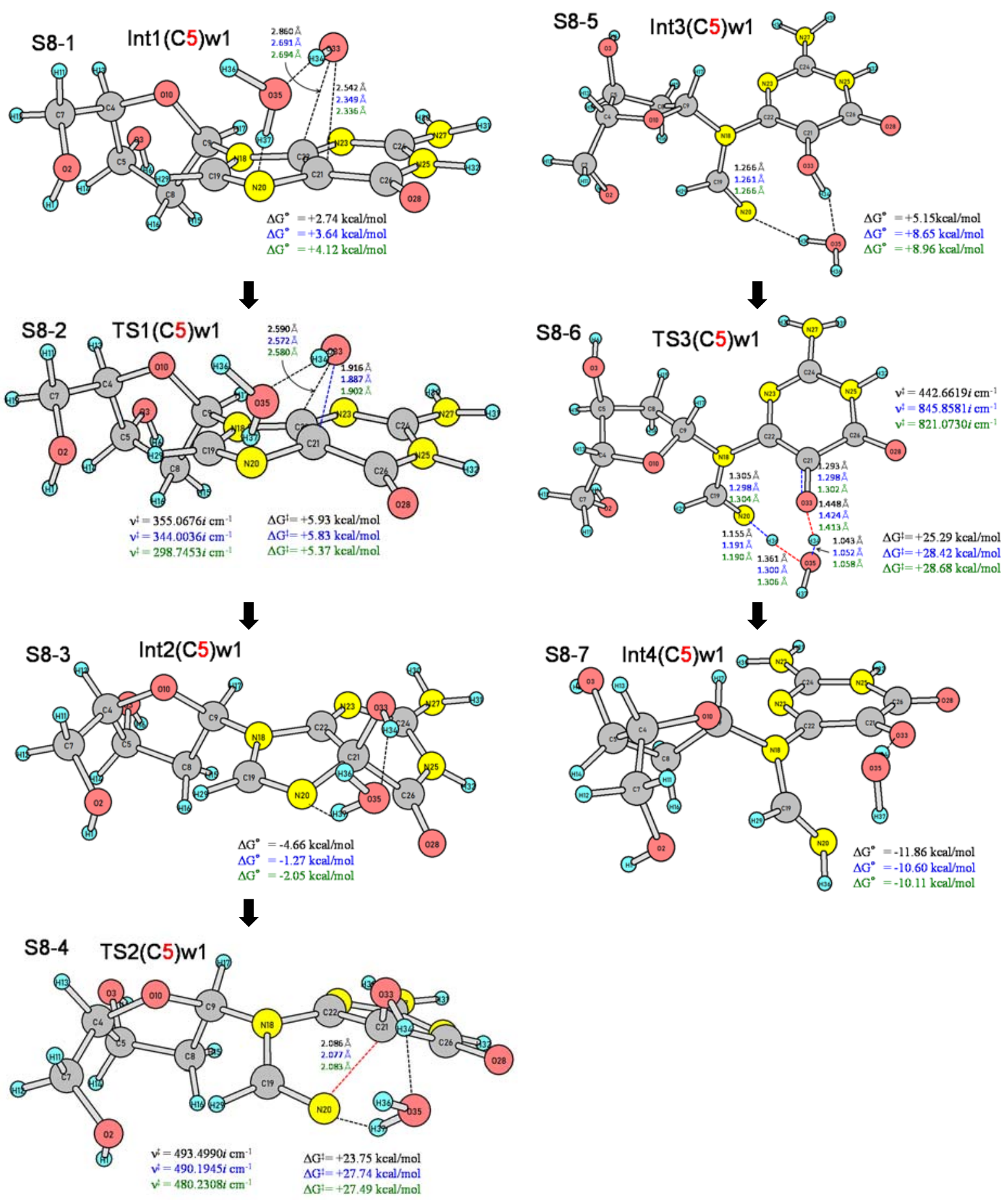

Figure S8. Reactions of dR-Gua and $\mathrm{HO} \cdot[\mathrm{n}=1$, with a water molecule, "W1"] for the C5attack channel depicted in Scheme 4. 

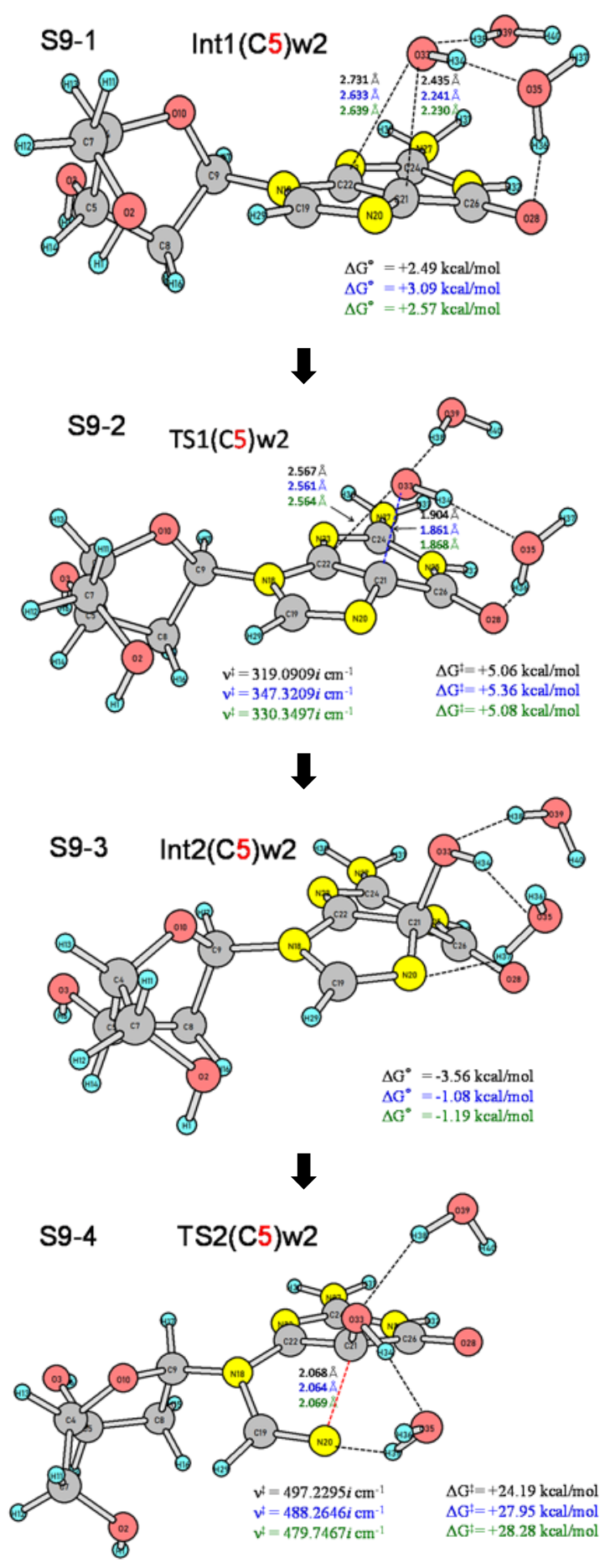
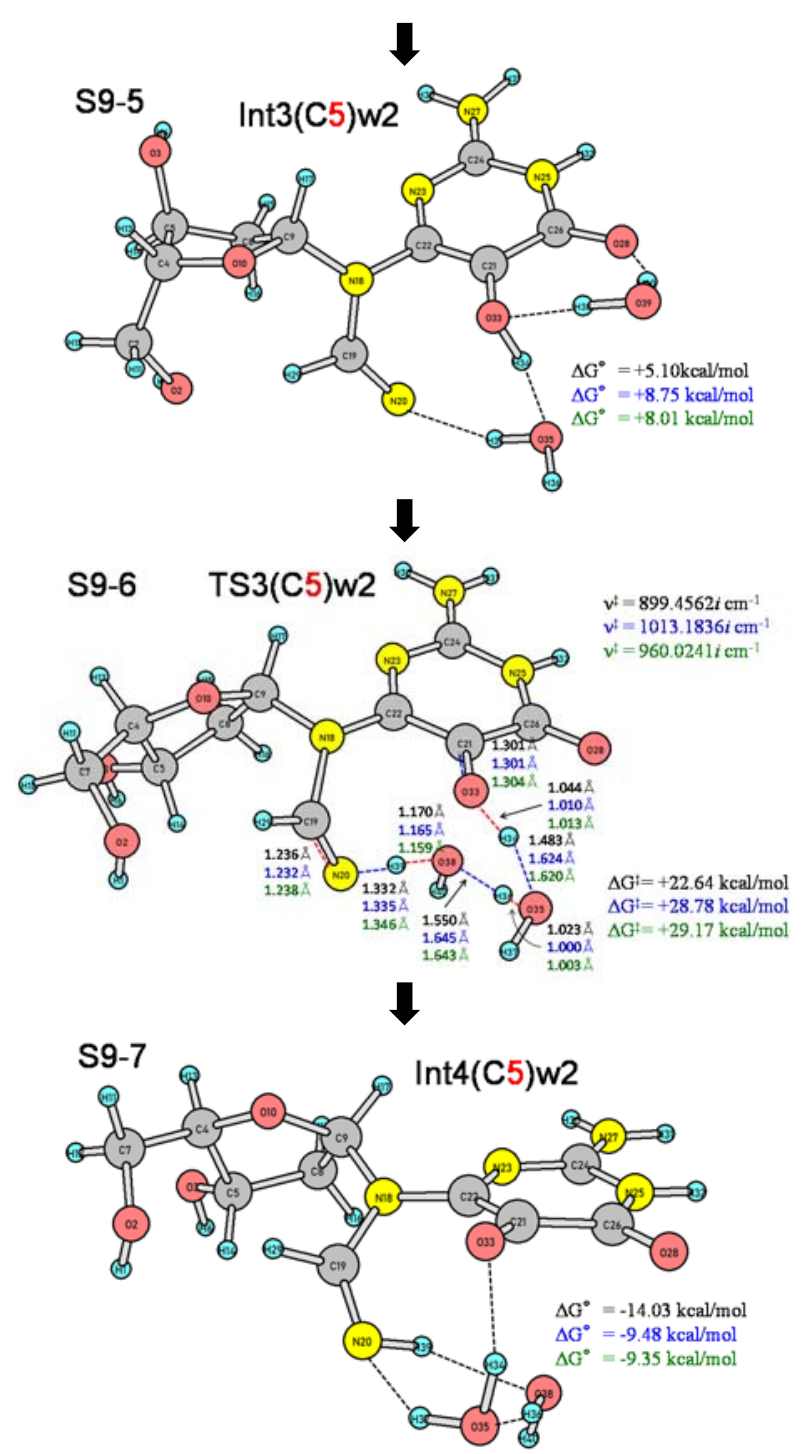

Figure S9. Reactions of dR-Gua and $\mathrm{HO} \cdot[\mathrm{n}=2$, with two water molecules, "W2"] for the C5attack channel depicted in Scheme 4. 


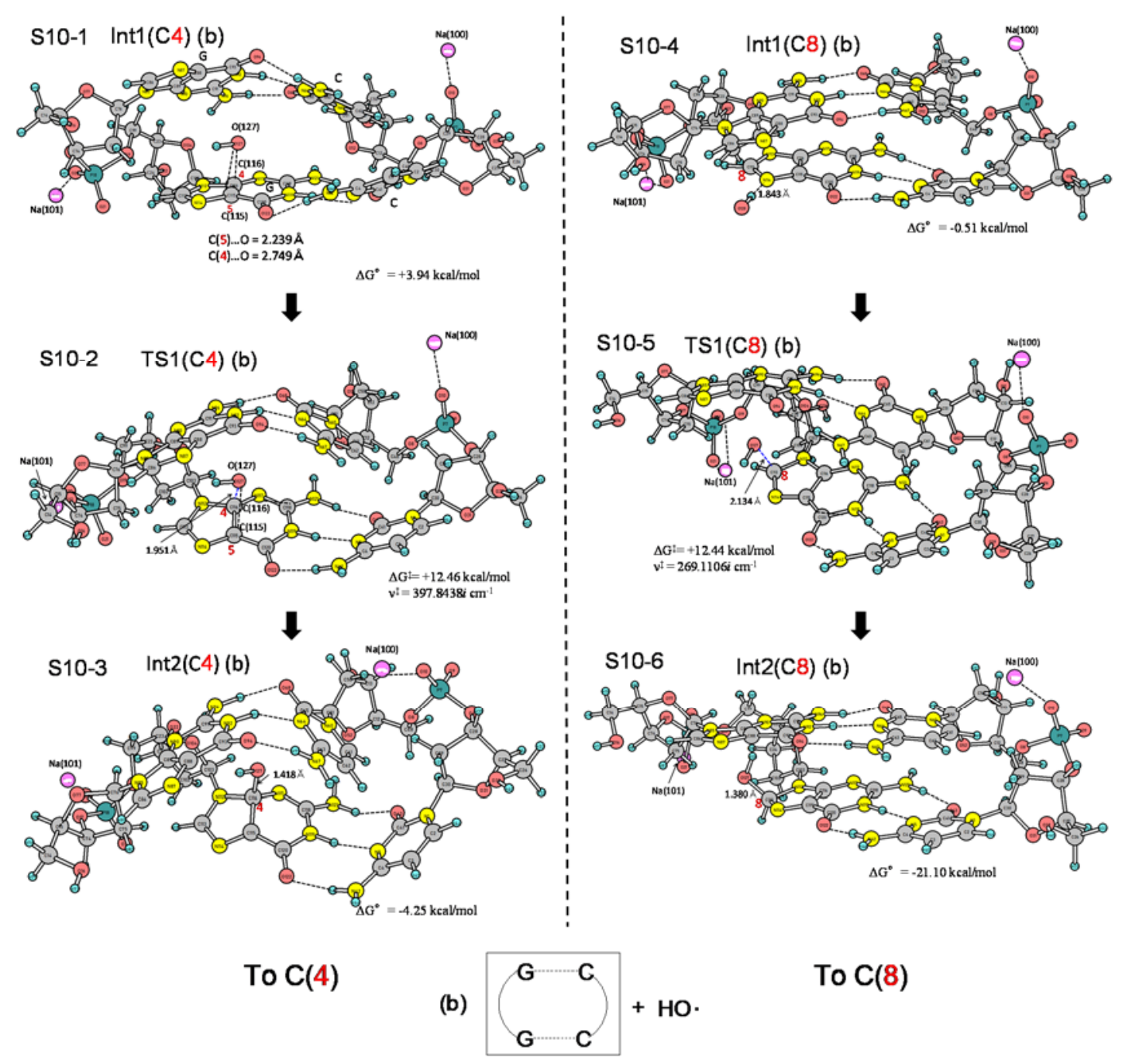

Figure $\mathrm{S} 10$. The $\mathrm{HO} \bullet$ addition reaction to $\mathrm{C}(4)$ or $\mathrm{C}(8)$ of the guanine moiety of the (b) 5'-GG3 ' sequence. Notation, Int1, TS1 or Int2, is the same as that used in Scheme 4 with the sequence classification, "(b)". 

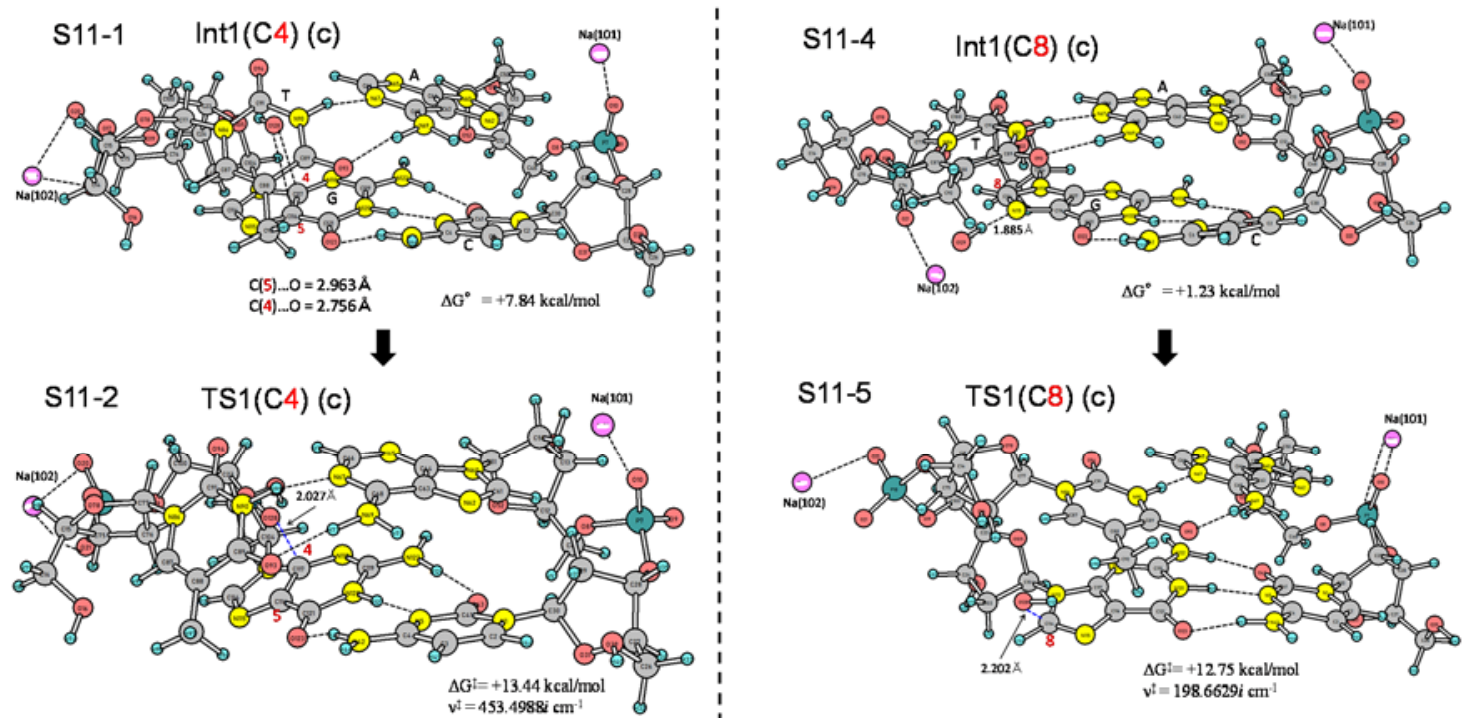

\section{$\downarrow$}
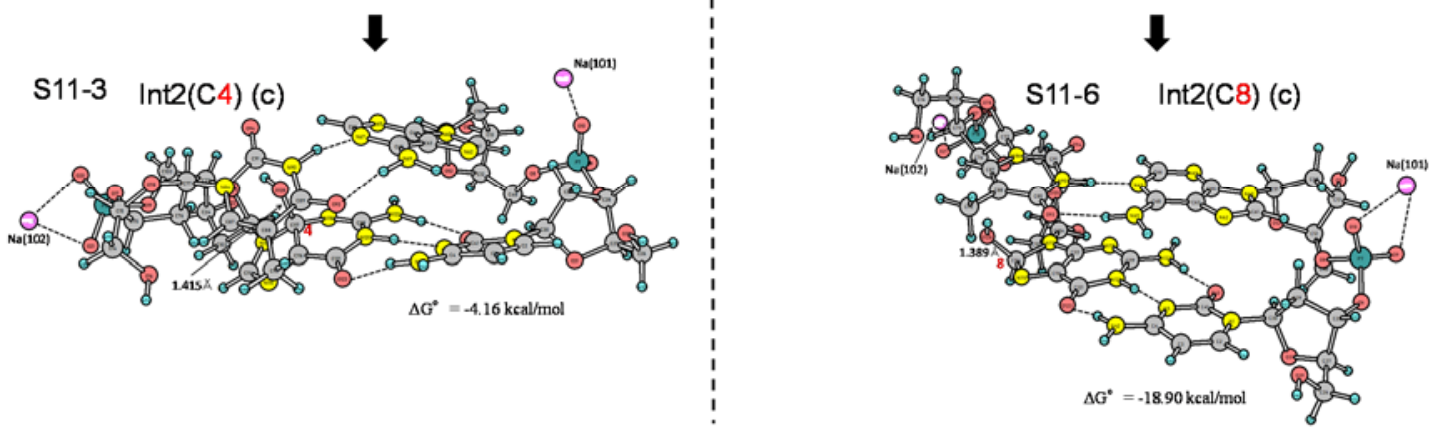

To C(4)

(c)

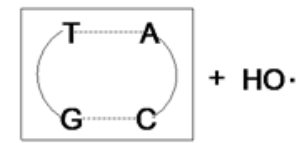

To $\mathrm{C}(8)$

Figure $\mathrm{S} 11$. The $\mathrm{HO} \bullet$ addition reaction to $\mathrm{C}(4)$ or $\mathrm{C}(8)$ of the adenine moiety of the (c) 5'-GT3' sequence. Notation, Int1, TS1 or Int2 is the same as that used in Scheme 4 with the sequence classification, "(c)". 


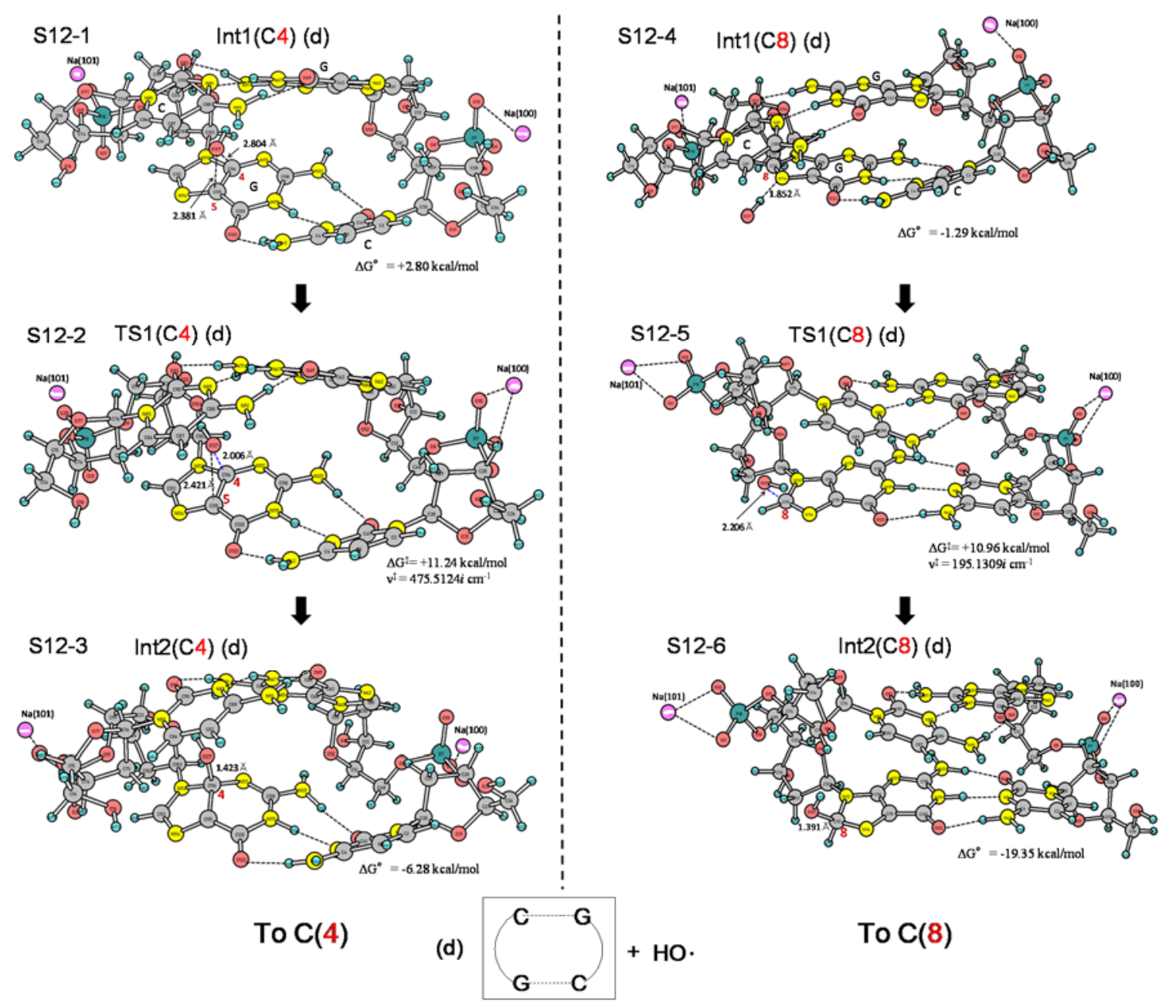

Figure S12. The HO• addition reaction to $\mathrm{C}(4)$ or $\mathrm{C}(8)$ of the guanine moiety of the (d)5'-GC3' sequence. Notation, Int1, TS1 or Int2, is the same as that used in Scheme 4 with the sequence classification, "(d)". 

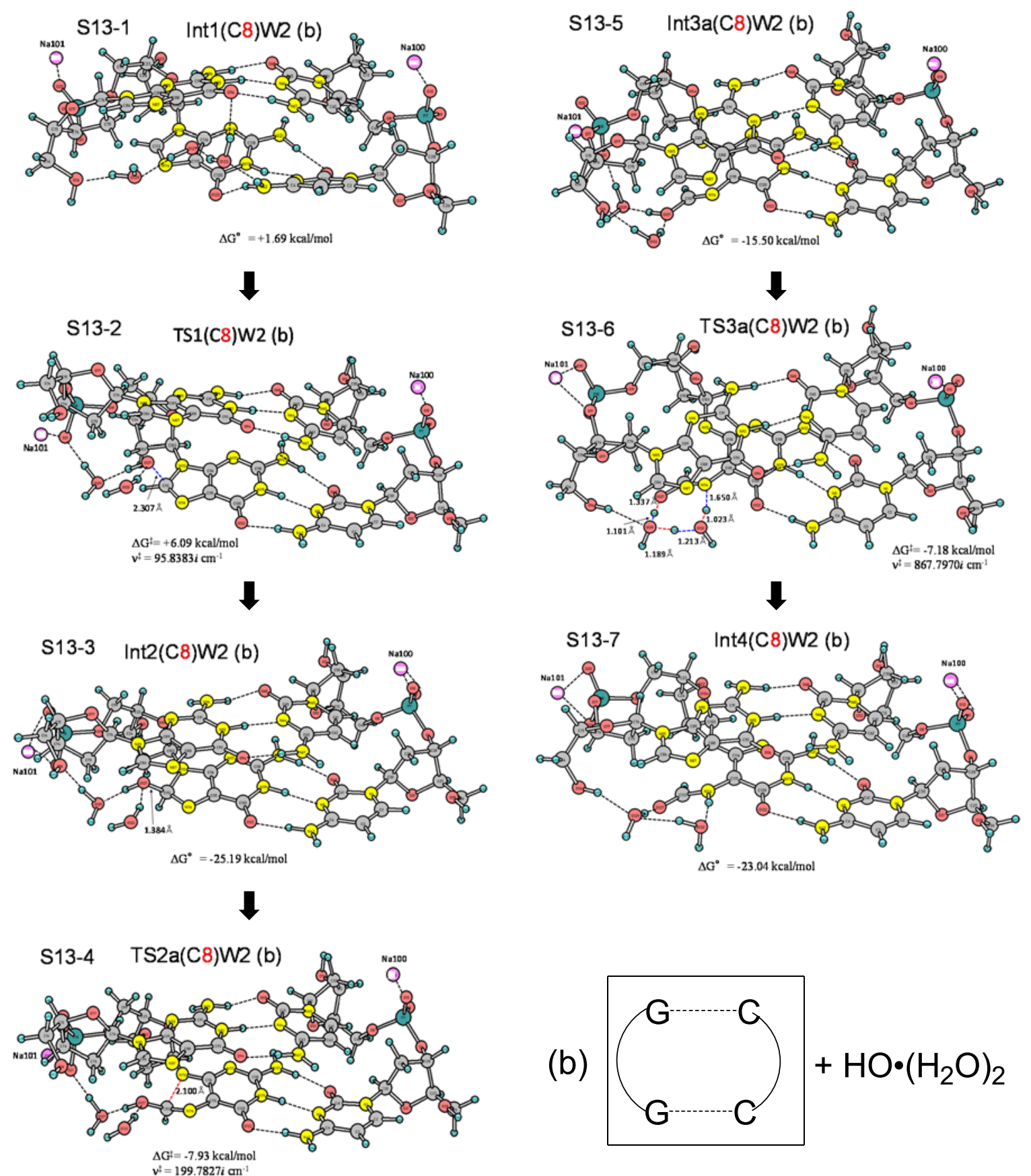

Figure $\mathrm{S} 13$. The $\mathrm{HO} \bullet\left(\mathrm{H}_{2} \mathrm{O}\right)_{2}$ addition reaction to $\mathrm{C}(8)$ of the guanine moiety of the (b) 5'-GG3' sequence and the subsequent isomerization. 

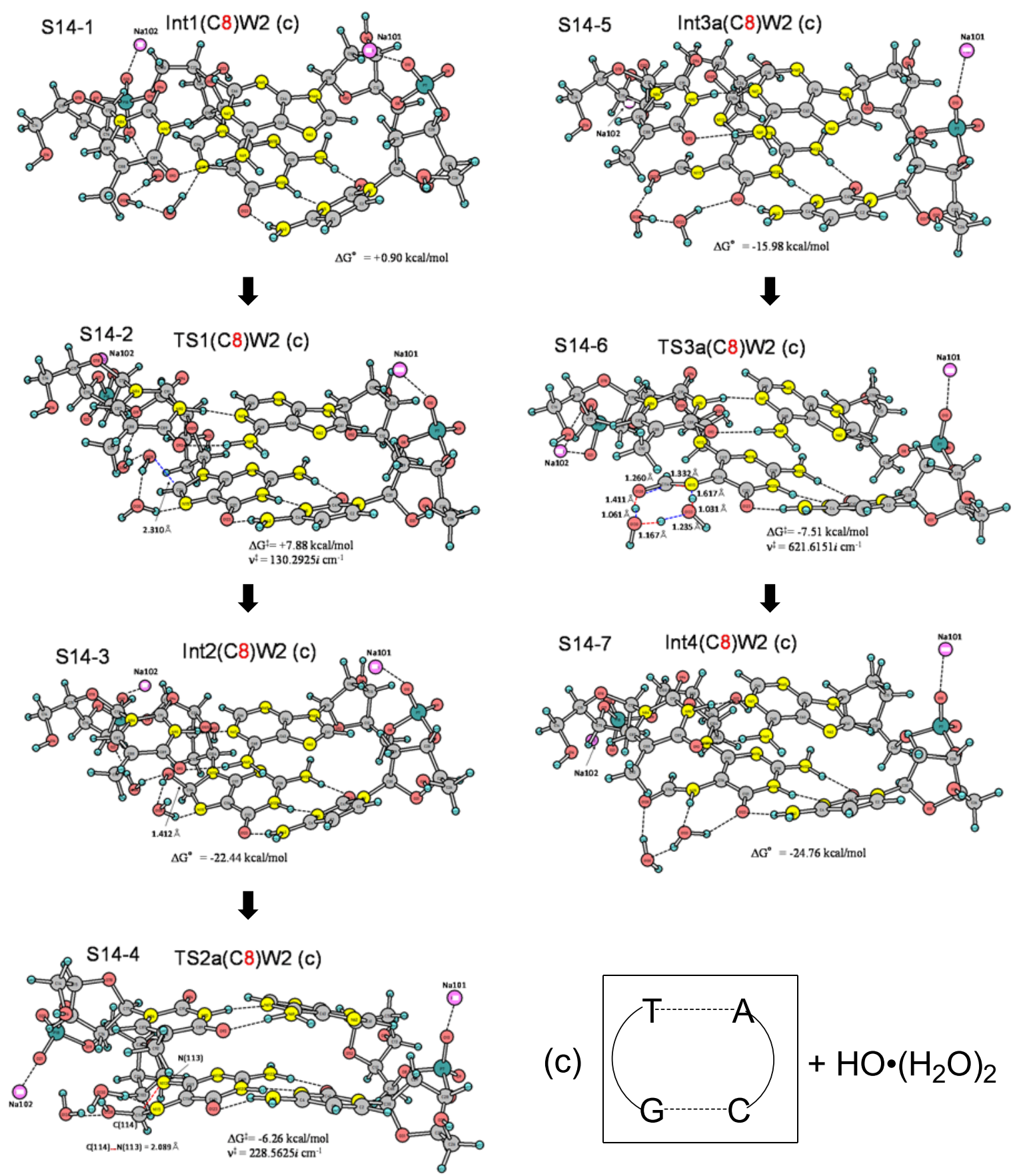

Figure $\mathrm{S} 14$. The $\mathrm{HO} \bullet\left(\mathrm{H}_{2} \mathrm{O}\right)_{2}$ addition reaction to $\mathrm{C}(8)$ of the guanine moiety of the (c) 5'-GT3' sequence and the subsequent isomerization. 

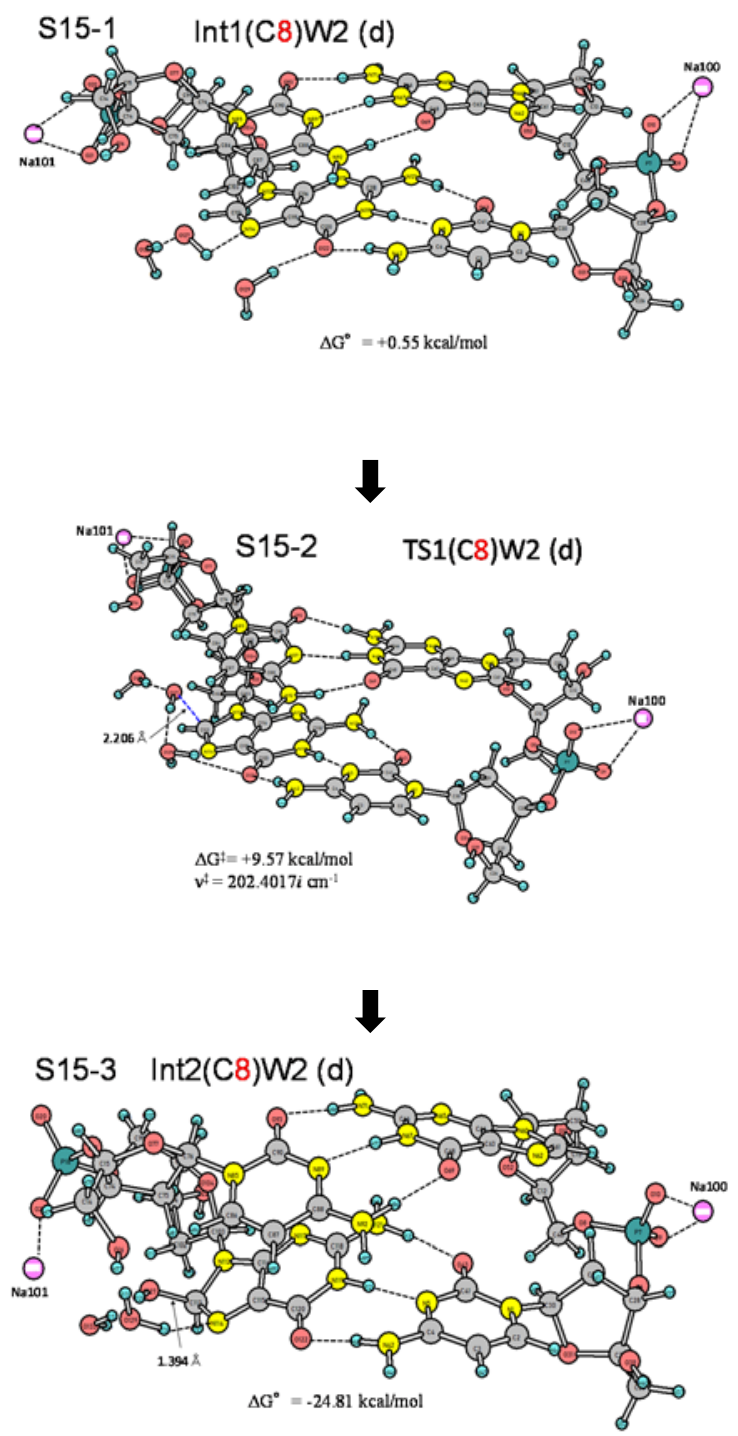

$\downarrow$

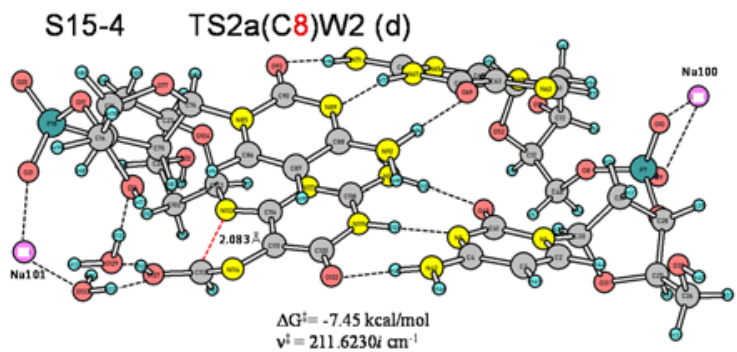

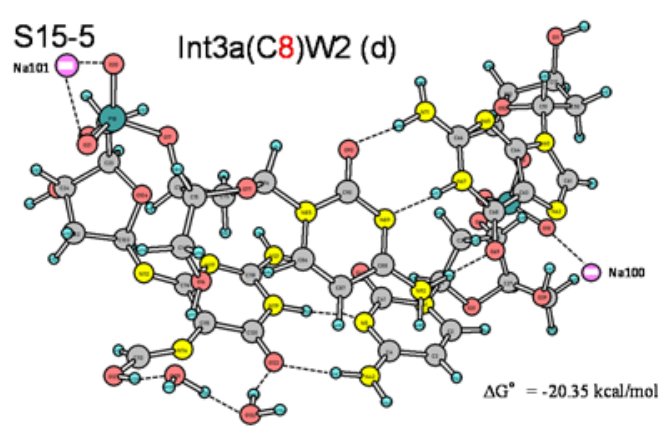

$\downarrow$

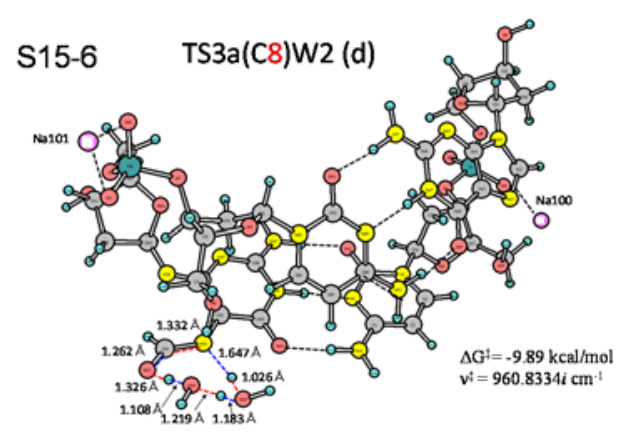

$\downarrow$

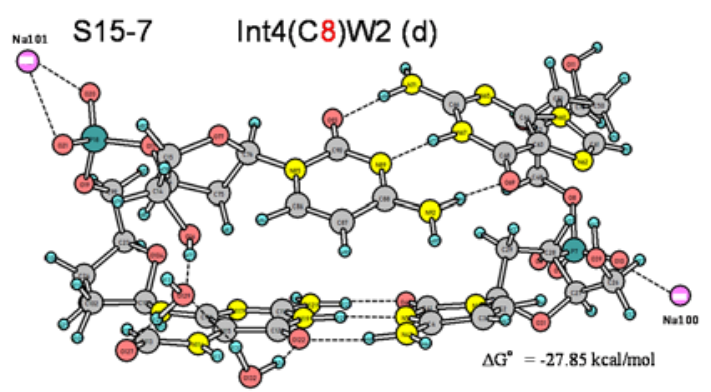

(d)

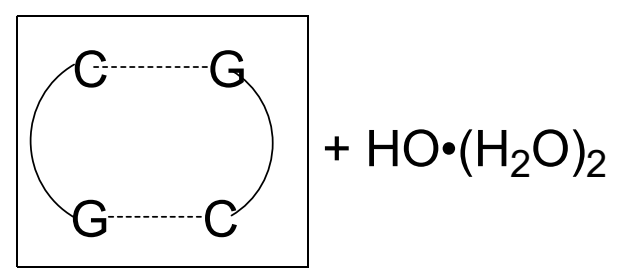

Figure $\mathrm{S} 15$. The $\mathrm{HO} \cdot\left(\mathrm{H}_{2} \mathrm{O}\right)_{2}$ addition reaction to $\mathrm{C}(8)$ of the guanine moiety of the (d) 5 '-GC3' sequence and the subsequent isomerization. 
A sample input, Cartesian coordinates and energies

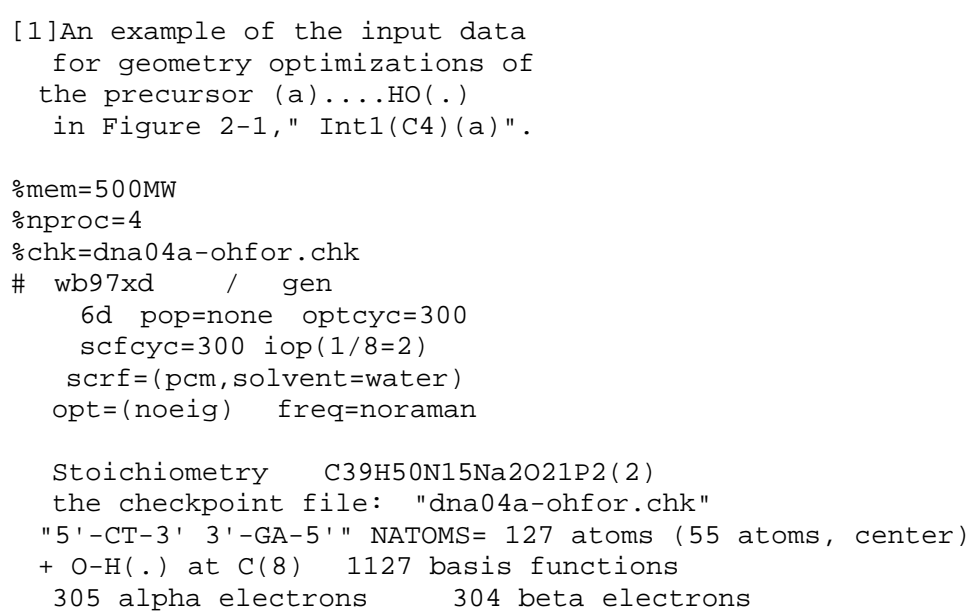

\begin{tabular}{|c|c|c|c|c|}
\hline \multicolumn{5}{|c|}{2} \\
\hline 7 & $\odot$ & 5.314848 & -1.986314 & -1.527377 \\
\hline 6 & 0 & 5.535606 & -3.332096 & -1.565323 \\
\hline 6 & $\odot$ & 4.506324 & -4.204136 & -1.469978 \\
\hline 6 & $\odot$ & 3.190387 & $-3.65 \odot 450$ & -1.318964 \\
\hline 7 & 0 & 2.984833 & -2.329995 & -1.304006 \\
\hline 8 & $\odot$ & 8.686087 & 0.797216 & -0.945263 \\
\hline 15 & 0 & 8.094323 & 1.797815 & 0.201194 \\
\hline 8 & 0 & 6.482422 & 1.763745 & -0.077097 \\
\hline 8 & $\odot$ & 8.638513 & 3.179150 & $-\odot .080067$ \\
\hline 8 & 0 & 8.299944 & 1.234602 & 1.589170 \\
\hline 8 & $\Theta$ & 4.683814 & 5.414638 & 1.209041 \\
\hline 6 & $\odot$ & 4.814827 & 3.538561 & $-\odot .254156$ \\
\hline 6 & $\odot$ & 5.355536 & 4.139549 & 1.047351 \\
\hline 6 & $\odot$ & -8.859404 & -2.610741 & 2.124093 \\
\hline 6 & $\odot$ & -8.483881 & -1.152360 & 1.959284 \\
\hline 8 & $\odot$ & -8.106679 & -3.401840 & 1.171991 \\
\hline 8 & $\odot$ & -8.911135 & 0.704149 & 0.491749 \\
\hline 15 & $\odot$ & -9.103341 & 1.534560 & -0.903582 \\
\hline 8 & $\odot$ & -7.586682 & 2.081209 & -1.191008 \\
\hline 8 & $\odot$ & -10.037032 & 2.685474 & -0.614011 \\
\hline 8 & $\odot$ & -9.463839 & 0.609312 & -2.041313 \\
\hline 8 & $\odot$ & -4.426171 & 4.688763 & -2.122831 \\
\hline 6 & $\odot$ & -5.507436 & 3.178997 & $-\odot .617473$ \\
\hline 6 & $\odot$ & -5.238253 & 3.487478 & -2.107060 \\
\hline 1 & $\odot$ & -4.284583 & 4.990341 & -3.036298 \\
\hline 6 & $\odot$ & 9.371855 & -2.516027 & -2.524719 \\
\hline 6 & $\odot$ & 8.688149 & -1.179982 & -2.318152 \\
\hline 6 & $\odot$ & 8.682692 & -0.670629 & $-\odot .869083$ \\
\hline 6 & $\odot$ & 7.367760 & -1.209155 & $-\odot .324709$ \\
\hline 6 & $\odot$ & 6.435798 & -1.060120 & -1.523945 \\
\hline 8 & $\odot$ & 7.272567 & -1.292608 & -2.692222 \\
\hline 1 & $\odot$ & 9.210190 & -2.836505 & -3.559077 \\
\hline 1 & $\odot$ & 10.448654 & -2.399957 & -2.355722 \\
\hline 1 & $\odot$ & 9.150770 & -0.437704 & -2.972414 \\
\hline 1 & $\odot$ & 9.554834 & -0.988852 & $-\odot .297185$ \\
\hline 1 & $\odot$ & 7.017333 & -0.661699 & 0.546444 \\
\hline 1 & $\odot$ & 7.505968 & -2.260698 & $-\odot .065374$ \\
\hline 1 & $\odot$ & 6.009280 & -0.063155 & -1.571263 \\
\hline 8 & 0 & 8.811863 & -3.479650 & -1.598739 \\
\hline 1 & 0 & 9.206080 & -4.357023 & -1.731025 \\
\hline 6 & 0 & 4. 015290 & -1.464254 & -1.445486 \\
\hline 7 & 0 & 2.123773 & -4.438113 & -1.189161 \\
\hline 8 & $\odot$ & 3.851704 & -0.239183 & -1.488607 \\
\hline 1 & 0 & 6.577849 & -3.627213 & -1.672853 \\
\hline 1 & 0 & 4.669599 & -5.272462 & -1.503880 \\
\hline 1 & 0 & 2.223760 & -5.438974 & -1.218699 \\
\hline 1 & 0 & 1.187607 & -4.023782 & -1.150447 \\
\hline 6 & 0 & 5.803283 & 2.665887 & -1.001755 \\
\hline 1 & $\odot$ & 4.958611 & 5.838381 & 2.040048 \\
\hline 6 & 0 & 4. 919611 & 3.135665 & 2.121169 \\
\hline 6 & 0 & 3.578351 & 2.618679 & 1.588612 \\
\hline
\end{tabular}




\begin{tabular}{|c|c|c|c|c|}
\hline 8 & $\odot$ & 3.642676 & 2.755539 & 0.137776 \\
\hline 1 & $\odot$ & 5.268591 & 2.047394 & -1.723762 \\
\hline 1 & $\odot$ & 6.540415 & 3.293058 & -1.508968 \\
\hline 1 & $\odot$ & 4.470376 & 4.336089 & -0.917075 \\
\hline 1 & $\odot$ & 6.441440 & 4.272579 & 1.012668 \\
\hline 1 & $\odot$ & 4.805117 & 3.604006 & 3.100905 \\
\hline 1 & $\odot$ & 5.669723 & 2.347453 & 2.198503 \\
\hline 1 & $\odot$ & 2.748969 & 3.223702 & 1.948146 \\
\hline 7 & $\odot$ & 3.237634 & 1.241295 & 1.914435 \\
\hline 6 & $\odot$ & 4.154820 & ๑. 219326 & 1.771433 \\
\hline 6 & $\odot$ & 3.845544 & -1.082411 & 1.948936 \\
\hline 6 & $\odot$ & 2.451128 & -1.424606 & 2.165606 \\
\hline 7 & $\odot$ & 1.587978 & -0.359029 & 2.285217 \\
\hline 6 & $\odot$ & 1.895535 & 0.971603 & 2.175093 \\
\hline 6 & $\odot$ & 4.836191 & -2.205321 & 1.920153 \\
\hline 8 & $\odot$ & 2.032274 & -2.583686 & 2.240258 \\
\hline 8 & $\odot$ & 1.049529 & 1.845505 & 2.307052 \\
\hline 1 & $\odot$ & 5.154640 & 0.538659 & 1.497492 \\
\hline 1 & $\odot$ & 4.729955 & -2.818472 & 2.819598 \\
\hline 1 & $\odot$ & 4.672996 & -2.868068 & 1.065458 \\
\hline 1 & $\odot$ & 5.858956 & -1.832257 & 1.885177 \\
\hline 1 & $\odot$ & 0.568586 & -0.576661 & 2.368636 \\
\hline 1 & $\odot$ & -8.355970 & -4.338751 & 1. 224221 \\
\hline 6 & $\odot$ & -8.436635 & -0.682818 & $\odot .503300$ \\
\hline 6 & $\odot$ & -6.961821 & -0.776408 & ๑. 120071 \\
\hline 6 & $\odot$ & -6.200920 & -0.595050 & 1.440812 \\
\hline 8 & $\odot$ & -7.147851 & -0.892525 & 2.501550 \\
\hline 1 & $\odot$ & -8.640511 & -2.922947 & 3.150383 \\
\hline 1 & $\odot$ & -9.934686 & -2.721266 & 1.942431 \\
\hline 1 & $\odot$ & -9.185826 & -0.539610 & 2.528831 \\
\hline 1 & $\odot$ & -9.075797 & -1.274083 & -0.152972 \\
\hline 1 & $\odot$ & -6.684980 & ๑. 001906 & -0.590799 \\
\hline 1 & $\odot$ & -6.754679 & -1.759335 & -0.309157 \\
\hline 1 & $\odot$ & -5.826119 & $\odot .421773$ & 1.566112 \\
\hline 7 & $\odot$ & -5.049342 & -1.468437 & 1.563460 \\
\hline 6 & $\odot$ & -5.036635 & -2.838849 & 1.440278 \\
\hline 7 & $\odot$ & -3.845229 & -3.364207 & 1.596685 \\
\hline 6 & $\odot$ & -3.031968 & -2.276850 & 1.842596 \\
\hline 6 & $\odot$ & -3.759037 & -1.091589 & 1.839287 \\
\hline 7 & $\odot$ & -3.283728 & 0.144322 & 2.055177 \\
\hline 6 & $\odot$ & -1.976822 & 0.130742 & 2.265130 \\
\hline 7 & $\odot$ & -1.152239 & -0.928546 & 2. 285134 \\
\hline 6 & $\odot$ & -1.645805 & -2.163876 & 2.069244 \\
\hline 7 & $\odot$ & -0.812412 & -3.213126 & 2.100467 \\
\hline 1 & $\odot$ & -5.964725 & -3.370299 & 1. 264523 \\
\hline 1 & $\odot$ & -1.487114 & 1.086416 & 2.435088 \\
\hline 1 & $\odot$ & $\odot .193948$ & -3.043750 & 2.135782 \\
\hline 1 & $\odot$ & -1.147551 & -4.098506 & 1.758191 \\
\hline 6 & $\odot$ & -6.953017 & 2.986464 & -0.241519 \\
\hline 11 & $\odot$ & 9.323799 & 3.408627 & 2.327848 \\
\hline 11 & $\odot$ & -11.527511 & 1.927871 & -2.504361 \\
\hline 6 & $\odot$ & -4.455127 & 2.265761 & -2.600233 \\
\hline 6 & $\odot$ & -3.733179 & 1.815450 & -1.329756 \\
\hline 8 & $\odot$ & -4.760922 & 1.956250 & $-\odot .308340$ \\
\hline 1 & $\odot$ & -7.023572 & 2.566913 & 0.766490 \\
\hline 1 & $\odot$ & -7.471688 & 3. 949161 & -0.265142 \\
\hline 1 & $\odot$ & -5.094914 & 4.001903 & -0.024091 \\
\hline 1 & $\odot$ & -6.163559 & 3.639749 & -2.667528 \\
\hline 1 & $\odot$ & -3.772875 & 2.502251 & -3.418058 \\
\hline 1 & $\odot$ & -5.149936 & 1.485790 & -2.924926 \\
\hline 1 & $\odot$ & -2.882659 & 2.464883 & -1.103235 \\
\hline 7 & $\odot$ & -3.237836 & 0.465064 & -1.329171 \\
\hline 6 & $\odot$ & -3.953672 & -0.688229 & -1.532862 \\
\hline 7 & $\odot$ & -3.212534 & -1.761511 & -1.485717 \\
\hline 6 & $\odot$ & -1.932909 & -1.296960 & -1.245805 \\
\hline 6 & $\odot$ & -1.929745 & $\bullet .085132$ & -1.139186 \\
\hline 7 & $\odot$ & -0.886914 & ๑. 911563 & -0.947670 \\
\hline 6 & $\odot$ & 0.270300 & ๑. 265775 & -0.899939 \\
\hline 7 & $\odot$ & 0.376237 & -1.102916 & -0.993675 \\
\hline 6 & $\odot$ & -0.686324 & -1.988502 & -1.164362 \\
\hline 7 & $\odot$ & 1.417579 & $\odot .937029$ & $-\odot .747749$ \\
\hline 8 & $\odot$ & -0.490724 & -3.206882 & -1.241216 \\
\hline 1 & $\odot$ & -5.016931 & -0.676547 & -1.709021 \\
\hline 1 & $\odot$ & 1.383769 & 1.940175 & $-\odot .671280$ \\
\hline 1 & $\odot$ & 2.328848 & 0.502376 & -0.923228 \\
\hline 1 & $\odot$ & 1.321743 & -1.530003 & -1.022509 \\
\hline 8 & $\odot$ & -5.124037 & -3.756861 & -1.179620 \\
\hline
\end{tabular}




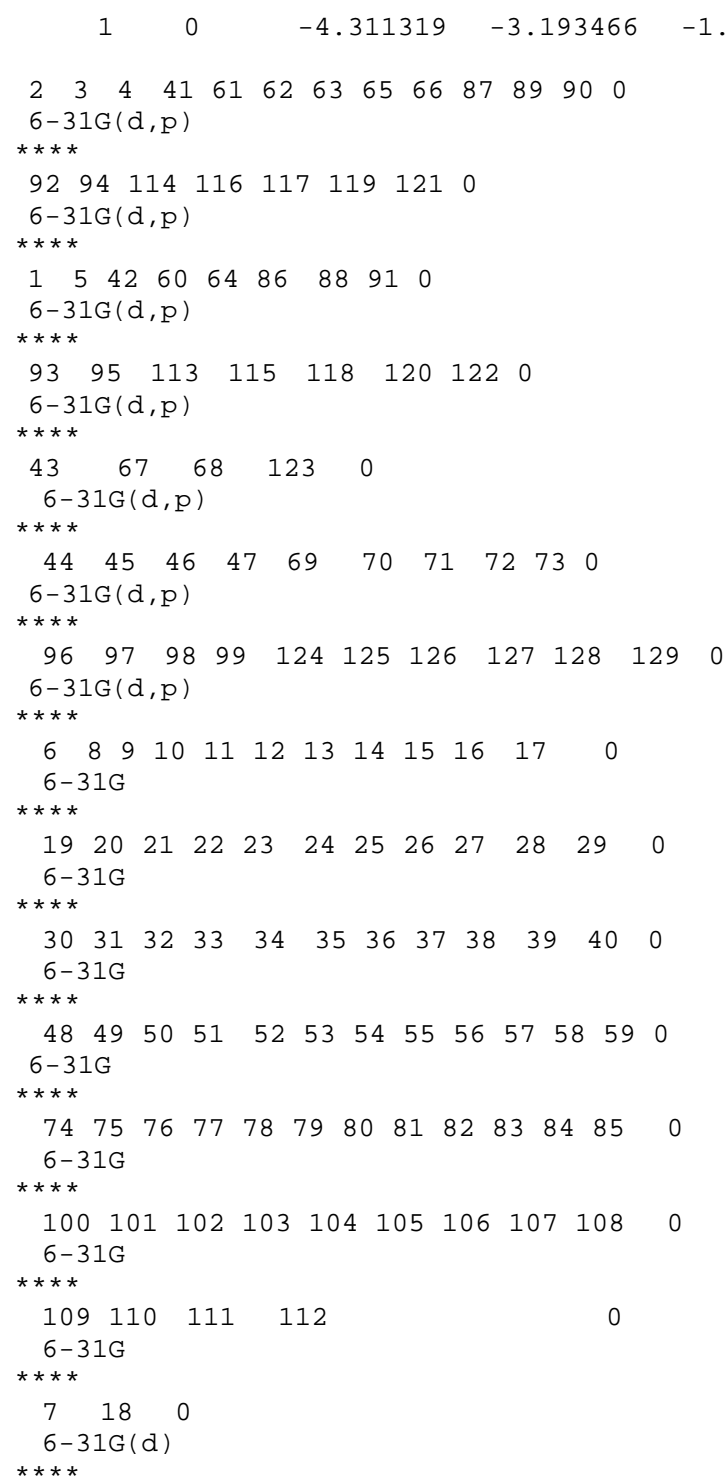

$\begin{array}{lllllllllllll}2 & 3 & 4 & 41 & 61 & 62 & 63 & 65 & 66 & 87 & 89 & 90 & 0\end{array}$

$6-31 G(d, p)$

****

$\begin{array}{llllllll}92 & 94 & 114 & 116 & 117 & 119 & 121 & 0\end{array}$

$6-31 \mathrm{G}(\mathrm{d}, \mathrm{p})$

$\begin{array}{lllllllll}1 & 5 & 42 & 60 & 64 & 86 & 88 & 91 & 0\end{array}$

$6-31 G(d, p)$

$\begin{array}{llllllll}93 & 95 & 113 & 115 & 118 & 120 & 122 & \odot\end{array}$

$6-31 G(d, p)$

****

$\begin{array}{lllll}43 & 67 & 68 & 123 & 0\end{array}$

$6-31 G(d, p)$

$\begin{array}{llllllllll}44 & 45 & 46 & 47 & 69 & 70 & 71 & 72 & 73 & 0\end{array}$

$6-31 G(d, p)$

$* * * *$

$\begin{array}{lllllllllll}96 & 97 & 98 & 99 & 124 & 125 & 126 & 127 & 128 & 129 & 0\end{array}$

$6-31 G(d, p)$

$* * * *$

$\begin{array}{llllllllllll}6 & 8 & 9 & 10 & 11 & 12 & 13 & 14 & 15 & 16 & 17 & 0\end{array}$

$6-31 \mathrm{G}$

****

$\begin{array}{llllllllllll}19 & 20 & 21 & 22 & 23 & 24 & 25 & 26 & 27 & 28 & 29 & \odot\end{array}$

$6-31 \mathrm{G}$

$\begin{array}{llllllllllll}30 & 31 & 32 & 33 & 34 & 35 & 36 & 37 & 38 & 39 & 40 & 0\end{array}$

$6-31 G$

****

$\begin{array}{lllllllllllll}48 & 49 & 50 & 51 & 52 & 53 & 54 & 55 & 56 & 57 & 58 & 59 & 0\end{array}$

$6-31 G$

$* \star * *$

$\begin{array}{lllllllllllll}74 & 75 & 76 & 77 & 78 & 79 & 80 & 81 & 82 & 83 & 84 & 85 & 0\end{array}$

$6-31 \mathrm{G}$

$100101102103 \quad 104105 \quad 106 \quad 107 \quad 108 \quad \odot$

$6-31 \mathrm{G}$

$\begin{array}{llll}109 & 110 & 111 & 112\end{array}$

$6-31 \mathrm{G}$

$\star \star * *$

$\begin{array}{lll}7 & 18 & 0\end{array}$

$6-31 G(d)$

$* * * *$

[2] Cartesian coordinates of geometries

optimized by wB97X-D/aug-CC-pVDZ SCRF=(PCM, solvent=water $)$

for reactions between 2'-deoxyguanosine

(dR-Gua) and hydroxy radical(HO.) with water molecules

$(\mathrm{H} 20) \mathrm{n}, \mathrm{n}=0,1$, and 2

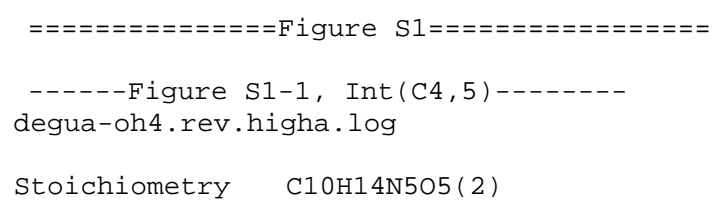

Standard orientation:

\begin{tabular}{|c|c|c|c|c|c|}
\hline \multirow{2}{*}{$\begin{array}{l}\text { Center } \\
\text { Number }\end{array}$} & \multirow{2}{*}{$\begin{array}{l}\text { Atomic } \\
\text { Number }\end{array}$} & \multirow{2}{*}{$\begin{array}{c}\text { Atomic } \\
\text { Type }\end{array}$} & \multicolumn{3}{|c|}{ Coordinates (Angstroms) } \\
\hline & & & $x$ & $\mathrm{Y}$ & Z \\
\hline$-\ldots-\ldots$ & & & $-\ldots$ & $-D_{-1}-\ldots$ &..----- \\
\hline 1 & 1 & $\odot$ & -3.900513 & -1.938947 & -1.145031 \\
\hline 2 & 8 & $\odot$ & -3.372760 & -2.053180 & -0.349989 \\
\hline 3 & 8 & $\odot$ & -3.850195 & 2.297043 & -0.193251 \\
\hline 4 & 6 & $\odot$ & -3.438155 & 0.154996 & 0.754911 \\
\hline 5 & 6 & $\odot$ & -3.574583 & $\odot .946746$ & -0.548697 \\
\hline 6 & 1 & $\odot$ & -3.901319 & 2.819091 & -0.999890 \\
\hline 7 & 6 & $\odot$ & -3.939320 & -1.282385 & 0.703852 \\
\hline 8 & 6 & $\odot$ & -2.198065 & $\odot .787078$ & -1.187503 \\
\hline 9 & 6 & $\odot$ & -1.271362 & ๑. 708142 & 0.026340 \\
\hline 10 & 8 & $\odot$ & -2.041684 & ๑. 184878 & 1.089807 \\
\hline 11 & 1 & $\odot$ & -3.656126 & -1.777323 & 1.640459 \\
\hline
\end{tabular}




\begin{tabular}{|c|c|c|c|c|c|}
\hline 12 & 1 & $\odot$ & -5.037235 & -1.274451 & 0.641081 \\
\hline 13 & 1 & $\odot$ & -3.985149 & 0.674111 & 1.553523 \\
\hline 14 & 1 & $\odot$ & -4.377001 & $\odot .551441$ & -1.186720 \\
\hline 15 & 1 & 0 & -1.936830 & 1.616644 & -1.851422 \\
\hline 16 & 1 & $\odot$ & -2.154184 & -0.146404 & -1.758607 \\
\hline 17 & 1 & $\odot$ & -0.866735 & 1.689151 & $\odot .301961$ \\
\hline 18 & 7 & $\odot$ & $-\odot .114886$ & -0.148349 & -0.174428 \\
\hline 19 & 6 & $\odot$ & -0.131307 & -1.503374 & -0.454011 \\
\hline 20 & 7 & $\odot$ & 1.061005 & -2.027602 & -0.559051 \\
\hline 21 & 6 & $\odot$ & 1.920175 & -0.978139 & -0.296458 \\
\hline 22 & 6 & $\odot$ & 1.193930 & 0.214447 & $-\odot .088545$ \\
\hline 23 & 7 & $\odot$ & 1.671025 & 1.443782 & $\odot .122188$ \\
\hline 24 & 6 & $\odot$ & 2.995558 & 1.504104 & 0.064786 \\
\hline 25 & 7 & $\odot$ & 3.792445 & $\odot .429827$ & -0.232250 \\
\hline 26 & 6 & $\odot$ & 3.351445 & $-\odot .885931$ & -0.451864 \\
\hline 27 & 7 & $\odot$ & 3. 600311 & 2.678544 & $\odot .286775$ \\
\hline 28 & 8 & $\odot$ & 4.147389 & -1.764748 & -0.745387 \\
\hline 29 & 1 & $\odot$ & -1.076346 & -2.029951 & -0.555767 \\
\hline 30 & 1 & $\odot$ & 3.026791 & 3.482640 & ๑. 488494 \\
\hline 31 & 1 & $\odot$ & 4.601506 & 2.789343 & $\odot .263448$ \\
\hline 32 & 1 & $\odot$ & 4.794360 & 0.563172 & -0.302650 \\
\hline 33 & 8 & $\odot$ & 2.238782 & -1.114249 & 1.924051 \\
\hline 34 & 1 & $\odot$ & 1.977331 & -2.043991 & 1.869778 \\
\hline
\end{tabular}

Standard basis: Aug-CC-pVDZ (6D, 7F)

626 basis functions

75 alpha electrons $\quad 74$ beta electrons

nuclear repulsion energy 1672.5966610066 Hartrees.

NAtoms $=34$ NActive $=34$

Nuclear repulsion after empirical dispersion term =

1672.5701950368 Hartrees.

Force inversion solution in PCM.

SCF Done: $E($ UWB97XD) $=-1039.19037501$ A.U. after 2 cycles

NFock $=2$ Conv $=0.25 \mathrm{D}-08 \quad-\mathrm{V} / \mathrm{T}=2.0071$

$\langle$ SX $>=0.0000<$ Sy $>=0.0000<$ Sz $>=0.5000$

$<S^{* *} 2>=0.7605 \mathrm{~S}=0.5052$

$<$ L.S $>=0.000000000000 \mathrm{E}+00$

Annihilation of the first spin contaminant:

$\mathrm{S}^{* * 2}$ before annihilation 0.7605 , after

$\odot .7501$

Zero-point correction=

Thermal correction to Energy=

Thermal correction to Enthalpy=

Thermal correction to Gibbs Free Energy=

Sum of electronic and zero-point Energies=

Sum of electronic and thermal Energies=

Sum of electronic and thermal Enthalpies=

$\odot .265267$ (a.u.)

0.284674

$\odot .285618$

0.216693

$-1038.925108$

$-1038.905701$

$-1038.904757$

Sum of electronic and thermal Free Energies=

$-1038.973682$

\begin{tabular}{|c|c|c|c|}
\hline${ }_{\mathrm{KC}}$ & $\begin{array}{l}\text { lermal) } \\
\text { /Mol } \\
78.635\end{array}$ & $\begin{array}{c}\text { CV } \\
\text { Cal/Mol-Kelvin } \\
72.516\end{array}$ & $\begin{array}{c}\mathrm{S} \\
\mathrm{Cal} / \mathrm{Mol}-\mathrm{Kel} \text { in } \\
145.064\end{array}$ \\
\hline Item & Value & \multicolumn{2}{|c|}{ Threshold Converged? } \\
\hline Maximum Force & 0.000063 & $\odot .0 \odot \odot 45 \odot$ & YES \\
\hline Force & 0.000006 & 0.000300 & YES \\
\hline Maximum Displacement & $\odot .002656$ & 0.001800 & NO \\
\hline RMS Displacement & $\odot .0 \odot \odot 6 \odot \odot$ & $\odot .0 \odot 120 \odot$ & YES \\
\hline
\end{tabular}

Normal termination of Gaussian 16 at Mon Mar 18 20:15:17 2019.

-...-Figure S1-2, TS1(C4) - .....

degua-oh4.higha. $\log$

Stoichiometry C10H14N505(2)

Standard orientation:

\begin{tabular}{|c|c|c|c|}
\hline Center & Atomic & Atomic & Coordinates (Angstroms) \\
\hline Number & Number & Type & Z \\
\hline
\end{tabular}




\begin{tabular}{|c|c|c|c|c|c|}
\hline 1 & 1 & 0 & -3.822023 & -1.863076 & -1.323191 \\
\hline 2 & 8 & 0 & -3.349038 & -2.023354 & -0.502136 \\
\hline 3 & 8 & $\odot$ & -3.741725 & 2.335337 & -0.188708 \\
\hline 4 & 6 & $\odot$ & -3.428590 & 0.144131 & 0.680303 \\
\hline 5 & 6 & $\odot$ & -3.486634 & $\odot .994198$ & $-\odot .591806$ \\
\hline 6 & 1 & 0 & -3.737852 & 2.894008 & -0.972041 \\
\hline 7 & 6 & $\odot$ & -3.959272 & -1.277274 & 0.545086 \\
\hline 8 & 6 & $\odot$ & -2.088022 & ๑.822581 & -1.175826 \\
\hline 9 & 6 & 0 & -1.217091 & 0.667333 & 0.071938 \\
\hline 10 & 8 & 0 & -2.049340 & 0.125451 & 1.079104 \\
\hline 11 & 1 & $\theta$ & -3.742424 & -1.813421 & 1.476680 \\
\hline 12 & 1 & 0 & -5.051221 & -1.240213 & 0.419396 \\
\hline 13 & 1 & $\odot$ & -4.000698 & $\odot .641816$ & 1.475021 \\
\hline 14 & 1 & $\odot$ & -4.270937 & 0.649613 & -1.279716 \\
\hline 15 & 1 & $\odot$ & -1.774984 & 1.669928 & -1.793452 \\
\hline 16 & 1 & 0 & -2.041842 & -0.088961 & -1.781727 \\
\hline 17 & 1 & 0 & -0.799004 & 1.624321 & 0.405294 \\
\hline 18 & 7 & 0 & -0.075116 & -0.207677 & -0.124546 \\
\hline 19 & 6 & $\odot$ & $-\odot .110159$ & -1.547357 & -0.384668 \\
\hline 20 & 7 & 0 & 1.083116 & -2.110818 & -0.458153 \\
\hline 21 & 6 & $\odot$ & 1.946835 & -1.091182 & -0.208507 \\
\hline 22 & 6 & $\odot$ & 1.249961 & 0.121571 & 0.077066 \\
\hline 23 & 7 & 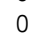 & 1.752300 & 1.376569 & 0.006428 \\
\hline 24 & 6 & 0 & 3.064976 & 1.403917 & -0.073988 \\
\hline 25 & 7 & $\odot$ & 3.853090 & $\odot .272367$ & -0.147432 \\
\hline 26 & 6 & 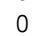 & 3.387643 & -1.042994 & -0.288700 \\
\hline 27 & 7 & $\odot$ & 3.707719 & 2.583375 & -0.070408 \\
\hline 28 & 8 & 0 & 4.165760 & -1.968190 & -0.467073 \\
\hline 29 & 1 & $\odot$ & -1.059753 & -2.061011 & -0.511166 \\
\hline 30 & 1 & $\odot$ & 3.155147 & 3.425893 & -0.038357 \\
\hline 31 & 1 & 0 & 4.695398 & 2.662626 & -0.253116 \\
\hline 32 & 1 & $\odot$ & 4.859766 & 0.376801 & -0.197429 \\
\hline 33 & 8 & 0 & 1.338837 & $-\odot .369579$ & 2.047536 \\
\hline 34 & 1 & $\theta$ & 2.268826 & -0.241885 & 2.277417 \\
\hline
\end{tabular}

Standard basis: Aug-CC-pVDZ (6D, 7F)

626 basis functions

75 alpha electrons

nuclear repulsion energy 1683.4552859297 Hartrees.

NAtoms $=34$ NActive $=34$

Force inversion solution in PCM.

SCF Done: $E($ UWB97XD $)=-1039.18223670 \quad$ A.U. after 1 cycles

$$
\text { NFock }=1 \text { Conv }=0.57 \mathrm{D}-08 \quad-\mathrm{V} / \mathrm{T}=2.0071
$$

$\langle\mathrm{S} X>=0.0000<\mathrm{S} y\rangle=0.0000\langle\mathrm{~S} z>=0.5000\langle\mathrm{~S} * * 2\rangle=0.7730 \mathrm{~S}=0.5115$

$<$ L.S $>=0.000000000000 \mathrm{E}+00$

Annihilation of the first spin contaminant:

$\mathrm{S}^{* * 2}$ before annihilation 0.7730 , after 0.7503

Harmonic frequencies $\left(\mathrm{cm}^{* *}-1\right)$, IR intensities (KM/Mole), Raman scattering activities ( $\left.A^{* *} 4 / \mathrm{AMU}\right)$, depolarization ratios for plane and unpolarized incident light, reduced masses (AMU), force constants (mDyne/A), and normal coordinates:

\begin{tabular}{|c|c|c|c|c|c|c|c|c|c|c|}
\hline & & & 1 & & 2 & & & 3 & & \\
\hline & & & A & & A & & & A & & \\
\hline Freque & ncies & - - & -324.20 & & & 31.390 & & & 13.7343 & \\
\hline Red. $n$ & asses & - - & 9.01 & & & 5.1939 & & & .7696 & \\
\hline $\mathrm{Frc} \mathrm{cc}$ & nsts & - - & 0.558 & & & ๑.๑०3० & & & .0076 & \\
\hline IR Int & & -- & 27.908 & & & 4.8488 & & & .0383 & \\
\hline Atom & AN & $x$ & $Y$ & Z & $x$ & $Y$ & Z & $x$ & Z & \\
\hline 1 & 1 & 0.01 & $-\odot . \odot \odot$ & $-\odot . \odot \odot$ & $-\odot . \odot 9$ & -0.10 & 0.24 & $-\odot . \odot 4$ & $\odot . \odot 8$ & $-\odot . \odot 8$ \\
\hline 2 & 8 & 0.01 & $\odot . \odot \odot$ & $\odot .0 \odot$ & $-\odot .02$ & -0.04 & 0.22 & $\odot . \odot 4$ & $\odot .02$ & -0.14 \\
\hline 3 & 8 & 0.00 & $-\odot . \odot \odot$ & $-\odot . \odot \odot$ & -0.04 & -0.03 & -0.10 & -0.05 & $-\odot .02$ & 0.25 \\
\hline 4 & 6 & $-\odot .01$ & $-\odot . \odot \odot$ & $\odot . \odot \odot$ & 0.05 & $\odot .05$ & $\odot .06$ & 0.06 & $-\odot .08$ & $\odot .06$ \\
\hline 5 & 6 & 0.01 & $-\odot . \odot \odot$ & $\odot . \odot \odot$ & $-\odot . \odot 4$ & -0.05 & $-\odot .01$ & $-\odot . \odot 6$ & $\odot .01$ & 0.12 \\
\hline 6 & 1 & 0.01 & $-\odot . \odot \odot$ & -0.01 & -0.11 & $-\odot .09$ & -0.15 & -0.10 & 0.04 & 0.30 \\
\hline 7 & 6 & $-\odot .01$ & $-\odot . \odot \odot$ & $-\odot . \odot \odot$ & 0.05 & $\odot .03$ & 0.21 & 0.11 & $-\odot . \odot 9$ & $-\odot . \odot 2$ \\
\hline 8 & 6 & 0.02 & -0.01 & $\odot . \odot 2$ & $-\odot .07$ & $-\odot .07$ & $-\odot . \odot 8$ & -0.10 & $\odot .08$ & $\odot .01$ \\
\hline 9 & 6 & 0.00 & $-\odot .02$ & $\odot . \odot 4$ & $\odot .01$ & 0.03 & -0.12 & $-\odot . \odot \odot$ & $\odot .03$ & $-\odot . \odot 6$ \\
\hline 10 & 8 & $-\odot .03$ & $\odot . \odot \odot$ & 0.03 & $\odot . \odot 8$ & $\odot . \odot 9$ & $-\odot .03$ & ๑.๑8 & $-\odot . \odot 4$ & -0.03 \\
\hline 11 & 1 & $-\odot .01$ & $-\odot . \odot \odot$ & $-\odot . \odot \odot$ & 0.13 & 0.11 & 0.23 & 0.22 & -0.16 & $-\odot .08$ \\
\hline 12 & 1 & $-\odot . \odot \odot$ & $-\odot .01$ & -0.01 & $\odot . \odot 4$ & 0.01 & 0.29 & $\odot . \odot 9$ & -0.13 & ๑.०9 \\
\hline 13 & 1 & $-\odot .03$ & $-\odot . \odot \odot$ & $-\odot .01$ & 0.10 & 0.11 & $\odot .06$ & $\odot . \odot 8$ & -0.17 & 0.13 \\
\hline 14 & 1 & 0.02 & $-\odot . \odot \odot$ & -0.01 & $-\odot . \odot 8$ & -0.12 & $\odot .07$ & -0.10 & 0.05 & 0.16 \\
\hline
\end{tabular}




$\begin{array}{rrrrr}15 & 1 & 0.03 & -0.01 & 0.03 \\ 16 & 1 & 0.02 & -0.01 & 0.01 \\ 17 & 1 & 0.00 & -0.02 & 0.03 \\ 18 & 7 & -0.00 & -0.02 & 0.08 \\ 19 & 6 & 0.02 & 0.03 & -0.05 \\ 20 & 7 & 0.04 & 0.00 & -0.11 \\ 21 & 6 & 0.03 & -0.07 & -0.02 \\ 22 & 6 & 0.03 & -0.09 & 0.56 \\ 23 & 7 & 0.01 & -0.04 & 0.09 \\ 24 & 6 & -0.01 & -0.02 & 0.01 \\ 25 & 7 & 0.02 & -0.02 & 0.02 \\ 26 & 6 & 0.03 & -0.02 & -0.02 \\ 27 & 7 & -0.01 & -0.02 & -0.04 \\ 28 & 8 & 0.03 & -0.02 & -0.03 \\ 29 & 1 & 0.03 & 0.03 & -0.16 \\ 30 & 1 & -0.01 & -0.02 & 0.00 \\ 31 & 1 & -0.04 & -0.02 & -0.20 \\ 32 & 1 & 0.02 & -0.01 & 0.03 \\ 33 & 8 & -0.14 & 0.26 & -0.41 \\ 34 & 1 & -0.18 & 0.39 & -0.32\end{array}$

$\begin{array}{ccc}-0.13 & -0.11 & -0.16 \\ -0.10 & -0.11 & -0.02 \\ 0.01 & 0.06 & -0.22 \\ 0.01 & 0.03 & -0.13 \\ 0.01 & 0.04 & -0.17 \\ 0.00 & 0.03 & -0.14 \\ 0.0 \odot & 0.01 & -0.07 \\ 0.00 & 0.01 & -0.06 \\ 0.02 & 0.01 & 0.02 \\ 0.02 & -0.01 & 0.10 \\ 0.01 & -0.01 & 0.09 \\ 0.01 & 0.00 & 0.00 \\ 0.04 & -0.01 & 0.20 \\ 0.00 & -0.0 \odot & 0.0 \odot \\ 0.01 & 0.05 & -0.20 \\ 0.05 & -0.01 & 0.21 \\ 0.05 & -0.02 & 0.26 \\ 0.02 & -0.02 & 0.15 \\ -0.10 & -0.07 & -0.08 \\ -0.11 & -0.09 & -0.02\end{array}$

$\begin{array}{ccc}-0.16 & 0.12 & 0.04 \\ -0.12 & 0.12 & -0.05 \\ 0.01 & 0.02 & -0.04 \\ 0.00 & 0.05 & -0.17 \\ -0.01 & 0.04 & -0.10 \\ -0.02 & 0.01 & -0.00 \\ -0.01 & 0.01 & -0.00 \\ -0.00 & 0.04 & -0.12 \\ 0.03 & 0.02 & -0.15 \\ 0.04 & -0.02 & -0.06 \\ 0.02 & -0.04 & 0.08 \\ -0.00 & -0.04 & 0.13 \\ 0.07 & -0.03 & -0.09 \\ -0.01 & -0.07 & 0.28 \\ -0.01 & 0.05 & -0.11 \\ 0.09 & -0.02 & -0.21 \\ 0.08 & -0.08 & -0.07 \\ 0.02 & -0.07 & 0.18 \\ -0.16 & 0.14 & -0.06 \\ -0.17 & 0.16 & -0.00\end{array}$

Zero-point correction=

Thermal correction to Energy=

Thermal correction to Enthalpy=

Thermal correction to Gibbs Free Energy=

Sum of electronic and zero-point Energies=

Sum of electronic and thermal Energies=

Sum of electronic and thermal Enthalpies=

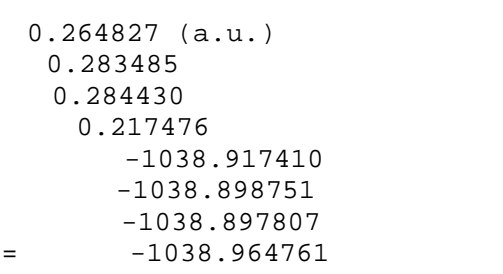

Sum of electronic and thermal Free Energies $=\quad-1038.964761$

\begin{tabular}{|c|c|c|c|}
\hline $\begin{array}{r}\mathrm{E}(\mathrm{T} \\
\mathrm{KCa}\end{array}$ & $\begin{array}{l}\text { lermal) } \\
\text { /Mol } \\
77.890\end{array}$ & $\begin{array}{c}\text { CV } \\
\text { Cal/Mol-Kelvin } \\
70.603\end{array}$ & $\begin{array}{c}\mathrm{S} \\
\mathrm{Cal} / \mathrm{Mol}-\mathrm{Kel} \text { in } \\
140.915\end{array}$ \\
\hline Item & value & Threshold Conv & verged? \\
\hline Maximum Force & 0.000004 & 0.000450 & YES \\
\hline Force & 0.000000 & 0.000300 & YES \\
\hline Maximum Displacement & 0.000345 & 0.001800 & YES \\
\hline RMS Displacement & 0.000043 & 0.001200 & YES \\
\hline \multicolumn{4}{|c|}{$\begin{array}{l}\text { Predicted change in Energy }=-7.209601 \mathrm{D}-10 \\
\text { Optimization completed. }\end{array}$} \\
\hline
\end{tabular}

Normal termination of Gaussian 16 at Sat Mar 9 20:37:12 2019.

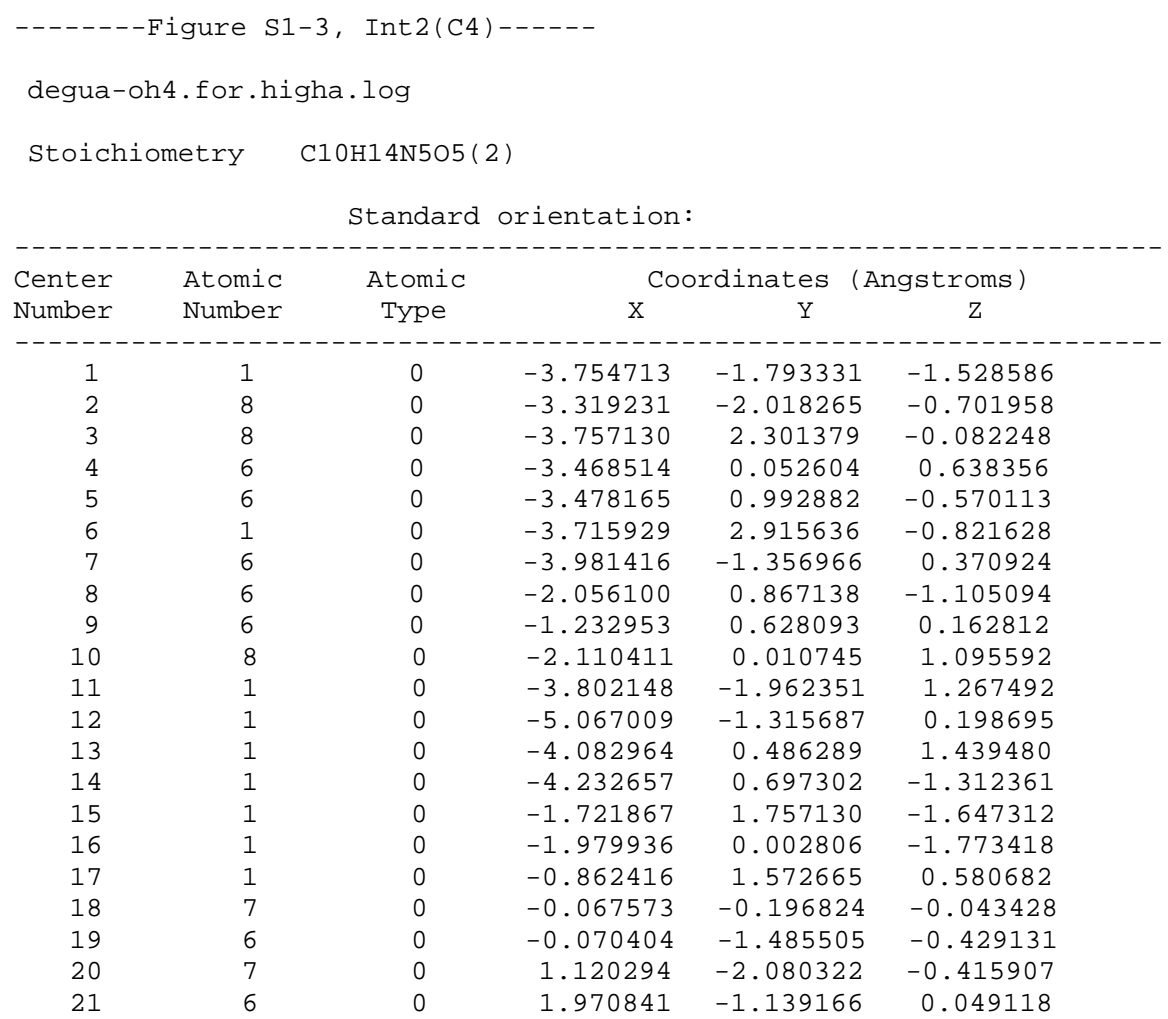




$\begin{array}{rrrrrr}22 & 6 & 0 & 1.267117 & 0.124163 & 0.476545 \\ 23 & 7 & 0 & 1.816314 & 1.324681 & -0.072639 \\ 24 & 6 & 0 & 3.107159 & 1.310930 & -0.209746 \\ 25 & 7 & 0 & 3.905250 & 0.185169 & -0.041785 \\ 26 & 6 & 0 & 3.404537 & -1.119736 & -0.082954 \\ 27 & 7 & 0 & 3.783843 & 2.454557 & -0.496668 \\ 28 & 8 & 0 & 4.154223 & -2.079520 & -0.237633 \\ 29 & 1 & 0 & -0.994005 & -1.976271 & -0.729384 \\ 30 & 1 & 0 & 3.212443 & 3.257295 & -0.717411 \\ 31 & 1 & 0 & 4.668738 & 2.397937 & -0.979954 \\ 32 & 1 & 0 & 4.909326 & 0.276513 & -0.132578 \\ 33 & 8 & 0 & 1.221767 & 0.219012 & 1.893219 \\ 34 & 1 & 0 & 1.229876 & 1.159828 & 2.109205 \\ ----\ldots-\ldots-\ldots-\ldots\end{array}$

Standard basis: Aug-CC-pVDZ (6D, 7F)

626 basis functions

75 alpha electrons

NAtoms $=34$ NActive $=34$

Nuclear repulsion after empirical dispersion term =

1690.2614882342 Hartrees.

Force inversion solution in PCM.

SCF Done: $E($ UWB97XD) $=-1039.21310590$ A.U. after 2 cycles

NFock $=2$ Conv $=0.94 \mathrm{D}-09-\mathrm{V} / \mathrm{T}=2.0071$

$<\mathrm{S} X>=0.0000<\mathrm{Sy}\rangle=0.0000<\mathrm{S} z>=0.5000$

$\left\langle S^{*} * 2>=0.7612 \mathrm{~S}=0.5056\right.$

$<$ L.S $>=0.000000000000 \mathrm{E}+\odot \odot$

Annihilation of the first spin contaminant:

$\mathrm{S}^{* *} 2$ before annihilation 0.7612 , after 0.7501

Zero-point correction=

Thermal correction to Energy=

Thermal correction to Enthalpy=

Thermal correction to Gibbs Free Energy=

Sum of electronic and zero-point Energies=

Sum of electronic and thermal Energies=

Sum of electronic and thermal Enthalpies=

$\odot .267716$ (a.u.)

$\odot .285921$

$\odot .286866$

0.220870

$-1038.945390$

$-1038.927184$

$-1038.926240$

Sum of electronic and thermal Free Energies=

$-1038.992236$

Total

$$
\begin{gathered}
\text { E (Thermal) } \\
\text { KCal/Mol }
\end{gathered}
$$

$\mathrm{CV}$

\section{$\mathrm{S}$}

Cal/Mol-Kelvin Cal/Mol-Kelvin

$70.200 \quad 138.900$

\begin{tabular}{lccc}
\multicolumn{1}{c}{ Item } & Value & Threshold Converged? \\
Maximum Force & 0.000033 & 0.000450 & YES \\
RMS Force & 0.000006 & 0.000300 & YES \\
Maximum Displacement & 0.001797 & 0.001800 & YES \\
RMS Displacement & 0.000454 & 0.001200 & YES \\
Predicted change in Energy=-3.319962D-08 & \\
Optimization completed. & & \\
\multicolumn{2}{l}{- - Stationary point found. }
\end{tabular}

Normal termination of Gaussian 16 at Mon Mar 18 19:31:41 2019.

-...-Figure S1-4, TS2(C4) - .....

degua-oh4.ts1.higha. $\log$

Stoichiometry C10H14N505(2)

Standard orientation:

\begin{tabular}{cccccc} 
Center & Atomic & Atomic & \multicolumn{3}{c}{ Coordinates } \\
Number & Number & $\begin{array}{c}\text { Type } \\
\text { Tyngstroms ) }\end{array}$ & X & Y & Z \\
\hline 1 & 1 & 0 & -3.827824 & -1.788815 & -1.554035 \\
2 & 8 & 0 & -3.490034 & -2.033148 & -0.688038 \\
3 & 8 & 0 & -3.865158 & 2.333647 & -0.321140 \\
4 & 6 & 0 & -3.615503 & 0.119877 & 0.521250 \\
5 & 6 & 0 & -3.566324 & 1.001710 & -0.731501 \\
6 & 1 & 0 & -3.778754 & 2.911813 & -1.085253 \\
7 & 6 & 0 & -4.172474 & -1.282511 & 0.308551 \\
8 & 6 & 0 & -2.123584 & 0.848363 & -1.197065
\end{tabular}




\begin{tabular}{|c|c|c|c|c|c|}
\hline 9 & 6 & $\odot$ & -1.354614 & ๑. 673511 & ๑. 118254 \\
\hline 10 & 8 & 0 & -2.275370 & 0.047423 & 1.014717 \\
\hline 11 & 1 & 0 & -4.059040 & -1.841824 & 1. 245059 \\
\hline 12 & 1 & $\odot$ & -5.247565 & -1.209692 & 0.083850 \\
\hline 13 & 1 & $\odot$ & -4.241020 & $\odot .613607$ & 1.279481 \\
\hline 14 & 1 & $\odot$ & -4.289985 & $\odot .673464$ & -1.491461 \\
\hline 15 & 1 & 0 & -1.767812 & 1.704399 & -1.779827 \\
\hline 16 & 1 & $\odot$ & -2.015887 & -0.059874 & -1.801320 \\
\hline 17 & 1 & $\odot$ & -1.070368 & 1.652201 & 0.535943 \\
\hline 18 & 7 & $\odot$ & -0.143278 & -0.077840 & -0.018137 \\
\hline 19 & 6 & 0 & -0.121304 & -1.381986 & ๑. . 006610 \\
\hline 20 & 7 & 0 & 1.075032 & -2.009104 & ๑. . 062401 \\
\hline 21 & 6 & 0 & 2.044221 & -1.089632 & ๑. 270516 \\
\hline 22 & 6 & $\odot$ & 1.694150 & $\odot .213792$ & $\odot .850319$ \\
\hline 23 & 7 & 0 & 2.335282 & 1.347316 & 0.498034 \\
\hline 24 & 6 & 0 & 3. 451911 & 1.224762 & -0.194058 \\
\hline 25 & 7 & $\odot$ & 4.015649 & 0.017041 & -0.485067 \\
\hline 26 & 6 & 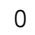 & 3.341370 & -1.210010 & -0.343532 \\
\hline 27 & 7 & $\odot$ & 4.098150 & 2.328127 & -0.591526 \\
\hline 28 & 8 & $\odot$ & 3.869304 & -2.233811 & -0.772653 \\
\hline 29 & 1 & 0 & -1.033703 & -1.981808 & -0.036834 \\
\hline 30 & 1 & $\odot$ & 3.730792 & 3.225827 & -0.316771 \\
\hline 31 & 1 & 0 & 4.977918 & 2.293179 & -1.081601 \\
\hline 32 & 1 & $\odot$ & 4.912027 & -0.026496 & -0.954111 \\
\hline 33 & 8 & $\odot$ & 1.181185 & ๑. 228996 & 2.096018 \\
\hline 34 & 1 & $\odot$ & 1.218872 & 1.143131 & 2.409652 \\
\hline
\end{tabular}

Standard basis: Aug-CC-pVDZ (6D, 7F)

626 basis functions

75 alpha electrons

nuclear repulsion energy 1655.6067924044 Hartrees.

NAtoms $=34$ NActive $=34$

Nuclear repulsion after empirical dispersion term =

1655.5814994453 Hartrees.

Force inversion solution in PCM.

SCF Done: $E($ UWB97XD) $=-1039.17575786 \mathrm{~A} . U$. after 1 cycles

$$
\text { NFock }=1 \text { Conv }=0.43 \mathrm{D}-08 \quad-\mathrm{V} / \mathrm{T}=2.0071
$$

$\left\langle\mathrm{S} X>=0.0000<\mathrm{S} y>=0.0000<\mathrm{S} z>=0.5000<\mathrm{S}{ }^{*} 2>=0.7619 \mathrm{~S}=0.5060\right.$

$<$ L.S $>=0.000000000000 \mathrm{E}+00$

Annihilation of the first spin contaminant:

$\mathrm{S}^{* *} 2$ before annihilation 0.7619 , after 0.7501

Harmonic frequencies $\left(\mathrm{cm}^{*}-1\right)$, IR intensities (KM/Mole), Raman activities $\left(A^{* *} 4 / A M U\right)$, depolarization ratios for plane and unpo incident light, reduced masses (AMU), force constants (mDyne/A), and normal coordinates:

\begin{tabular}{|c|c|c|c|c|c|c|c|c|c|c|}
\hline & & & $\begin{array}{l}1 \\
A\end{array}$ & & $\begin{array}{l}2 \\
A\end{array}$ & & & $\begin{array}{l}3 \\
A\end{array}$ & & \\
\hline Freque & ncies & - - & -310.33 & & & 20.248 & & & 5.4337 & \\
\hline Red. $\mathrm{n}$ & asses & - - & 8.06 & & & 5.3472 & & & .2982 & \\
\hline FrC $\mathrm{co}$ & nsts & -- & 0.45 & & & 0.0013 & & & .0047 & \\
\hline IR Int & en & -- & 136.121 & & & 5.1463 & & & .7710 & \\
\hline Atom & AN & $x$ & $Y$ & Z & $x$ & $Y$ & Z & $x$ & Z & \\
\hline 1 & 1 & -0.00 & $-\odot . \odot \odot$ & $-\odot .01$ & -0.10 & -0.14 & 0.23 & 0.35 & -0.09 & -0.04 \\
\hline 2 & 8 & -0.01 & $-0.0 \odot$ & $-0.0 \odot$ & -0.03 & -0.05 & 0.23 & 0.36 & -0.03 & -0.03 \\
\hline 3 & 8 & -0.03 & 0.01 & 0.01 & $-\odot .04$ & -0.02 & -0.12 & -0.18 & -0.06 & 0.04 \\
\hline 4 & 6 & -0.07 & $-\odot . \odot \odot$ & -0.01 & 0.04 & 0.05 & $\odot .06$ & $\odot .01$ & -0.05 & -0.01 \\
\hline 5 & 6 & -0.08 & 0.01 & -0.01 & $-\odot .04$ & -0.06 & -0.02 & -0.06 & -0.02 & 0.01 \\
\hline 6 & 1 & -0.01 & $\odot .02$ & $\odot .02$ & $-\odot .09$ & $-\odot . \odot 9$ & -0.18 & -0.21 & -0.03 & 0.06 \\
\hline 7 & 6 & -0.04 & -0.01 & -0.01 & 0.03 & 0.03 & 0.21 & 0.21 & -0.13 & -0.05 \\
\hline 8 & 6 & -0.11 & 0.00 & -0.04 & -0.06 & -0.07 & -0.09 & -0.04 & 0.11 & 0.02 \\
\hline 9 & 6 & -0.11 & -0.06 & $-\odot .07$ & 0.01 & 0.04 & -0.12 & -0.02 & 0.12 & 0.01 \\
\hline 10 & 8 & -0.09 & $\odot .00$ & $-\odot .02$ & 0.06 & 0.09 & -0.02 & -0.01 & 0.15 & 0.05 \\
\hline 11 & 1 & $-\odot .03$ & -0.01 & -0.01 & $\odot .09$ & 0.11 & 0.25 & 0.25 & -0.12 & -0.05 \\
\hline 12 & 1 & -0.04 & -0.05 & -0.02 & 0.01 & 0.01 & 0.27 & 0.21 & -0.28 & -0.10 \\
\hline 13 & 1 & -0.09 & $-\odot .02$ & -0.01 & $\odot .08$ & 0.11 & 0.05 & -0.10 & -0.16 & -0.03 \\
\hline 14 & 1 & -0.12 & 0.03 & 0.01 & -0.08 & -0.13 & 0.04 & -0.02 & -0.07 & -0.00 \\
\hline 15 & 1 & -0.11 & $\odot .02$ & -0.02 & -0.11 & -0.12 & -0.18 & -0.11 & 0.16 & $\odot .06$ \\
\hline 16 & 1 & -0.12 & 0.02 & -0.07 & -0.09 & -0.12 & -0.02 & 0.03 & 0.15 & -0.02 \\
\hline 17 & 1 & -0.05 & $-\odot .08$ & -0.06 & 0.01 & 0.07 & -0.20 & 0.01 & 0.11 & $-\odot . \odot \odot$ \\
\hline 18 & $\overline{7}$ & -0.21 & -0.07 & -0.20 & 0.01 & 0.04 & -0.12 & -0.03 & 0.09 & -0.01 \\
\hline 19 & 6 & -0.08 & -0.01 & 0.11 & -0.01 & 0.04 & -0.16 & -0.08 & $\odot .09$ & -0.01 \\
\hline 20 & 7 & 0.02 & 0.06 & 0.06 & -0.02 & $\odot . \odot 2$ & -0.14 & -0.10 & 0.05 & -0.01 \\
\hline
\end{tabular}




$\begin{array}{rrrrr}21 & 6 & 0.04 & 0.06 & 0.01 \\ 22 & 6 & 0.45 & -0.02 & 0.24 \\ 23 & 7 & 0.25 & -0.03 & 0.22 \\ 24 & 6 & 0.14 & 0.01 & -0.01 \\ 25 & 7 & 0.05 & 0.01 & -0.06 \\ 26 & 6 & 0.01 & 0.01 & -0.04 \\ 27 & 7 & 0.04 & 0.03 & -0.06 \\ 28 & 8 & -0.00 & 0.00 & -0.03 \\ 29 & 1 & 0.01 & -0.17 & 0.44 \\ 30 & 1 & 0.07 & 0.01 & 0.06 \\ 31 & 1 & -0.05 & 0.07 & -0.23 \\ 32 & 1 & 0.02 & -0.03 & -0.11 \\ 33 & 8 & -0.06 & 0.00 & -0.05 \\ 34 & 1 & -0.17 & -0.00 & -0.01\end{array}$

$\begin{array}{ccc}-0.01 & -0.00 & -0.07 \\ -0.02 & -0.03 & -0.02 \\ 0.02 & -0.02 & 0.07 \\ 0.04 & -0.00 & 0.11 \\ 0.03 & 0.01 & 0.06 \\ 0.01 & 0.01 & -0.03 \\ 0.08 & 0.01 & 0.20 \\ 0.00 & 0.02 & -0.06 \\ -0.02 & 0.06 & -0.21 \\ 0.08 & 0.00 & 0.23 \\ 0.10 & 0.02 & 0.23 \\ 0.05 & 0.02 & 0.10 \\ -0.07 & -0.08 & -0.04 \\ -0.08 & -0.10 & 0.01\end{array}$

$\begin{array}{ccc}-0.07 & 0.01 & -0.01 \\ -0.02 & 0.02 & -0.01 \\ 0.04 & -0.01 & -0.01 \\ 0.04 & -0.06 & -0.00 \\ -0.01 & -0.08 & 0.00 \\ -0.07 & -0.05 & -0.00 \\ 0.09 & -0.08 & 0.01 \\ -0.11 & -0.07 & 0.00 \\ -0.10 & 0.13 & -0.03 \\ 0.13 & -0.07 & 0.01 \\ 0.09 & -0.12 & 0.02 \\ -0.02 & -0.12 & 0.00 \\ -0.01 & 0.05 & -0.01 \\ 0.03 & 0.05 & -0.01\end{array}$

Zero-point correction=

Thermal correction to Energy=

$$
\begin{gathered}
0.265313(\mathrm{a} \cdot \mathrm{u} .) \\
0.283500 \\
0.284445 \\
0.217480 \\
-1038.910445 \\
-1038.892257 \\
-1038.891313 \\
-1038.958278
\end{gathered}
$$

Sum of electronic and thermal Enthalpies=

Sum of electronic and thermal Free Energies=

\section{$\mathrm{S}$}

$$
\begin{gathered}
\text { E (Thermal) } \\
\text { KCal/Mol } \\
177
\end{gathered}
$$

CV

Cal/Mol-Kelvin Cal/Mol-Kelvin

Total

$$
177.899
$$

140.939

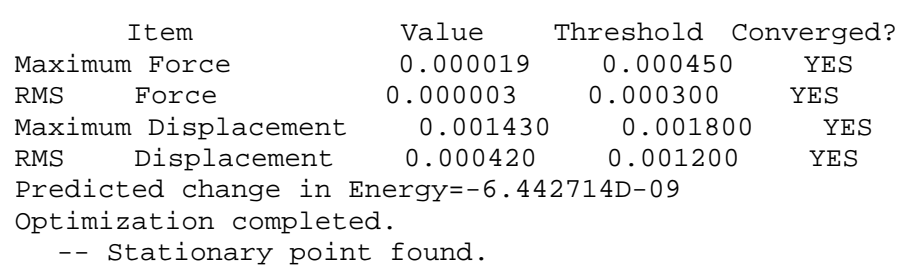

\begin{tabular}{|c|c|c|c|c|c|}
\hline \multirow{3}{*}{$\begin{array}{l}\text { Center } \\
\text { Number }\end{array}$} & \multirow{2}{*}{$\begin{array}{l}\text { Atomic } \\
\text { Number }\end{array}$} & \multirow{2}{*}{$\begin{array}{l}\text { Atomic } \\
\text { Type }\end{array}$} & \multicolumn{3}{|c|}{ Coordinates (Angstroms) } \\
\hline & & & $X$ & Y & Z \\
\hline & - & & - - - - - - - & $-\cdots----$ & - \\
\hline 1 & 1 & $\odot$ & -4.011660 & 2.293016 & 0.907110 \\
\hline 2 & 8 & $\odot$ & -3.585361 & 2.242873 & 0.047201 \\
\hline 3 & 8 & $\odot$ & -4.737619 & -1.930206 & $\odot .787890$ \\
\hline 4 & 6 & $\odot$ & -4.045840 & -0.108325 & -0.582487 \\
\hline 5 & 6 & $\odot$ & -4.205129 & -0.608657 & $\odot .857127$ \\
\hline 6 & 1 & $\odot$ & -4.772813 & -2.287673 & 1.680321 \\
\hline 7 & 6 & $\odot$ & -4.333948 & 1.373711 & -0.792164 \\
\hline 8 & 6 & $\odot$ & -2.775008 & -0.585169 & 1.381188 \\
\hline 9 & 6 & $\odot$ & -1.941843 & $-\odot .909778$ & $\odot .137163$ \\
\hline 10 & 8 & $\odot$ & -2.705446 & -0.419023 & -0.967079 \\
\hline 11 & 1 & $\odot$ & -4.061392 & 1.631927 & -1.822752 \\
\hline 12 & 1 & $\odot$ & -5.415254 & 1.544375 & -0.673876 \\
\hline 13 & 1 & $\odot$ & -4.731533 & -0.670661 & -1.233759 \\
\hline 14 & 1 & $\odot$ & -4.879519 & ๑. 032898 & 1.442742 \\
\hline 15 & 1 & $\odot$ & -2.608121 & -1.296121 & 2.197490 \\
\hline 16 & 1 & $\odot$ & -2.517606 & 0.421711 & 1.730469 \\
\hline 17 & 1 & $\odot$ & -1.837222 & -2.000162 & ๑. 015201 \\
\hline 18 & 7 & $\odot$ & -0.607996 & -0.381343 & ๑. 222718 \\
\hline 19 & 6 & $\odot$ & -0.261099 & 0.560571 & -0.572886 \\
\hline 20 & 7 & $\odot$ & ๑. 941750 & 1. 217241 & -0.482700 \\
\hline 21 & 6 & $\odot$ & 2.099961 & $\odot .589733$ & -0.261579 \\
\hline 22 & 6 & $\odot$ & 2.455838 & -0.735693 & -0.647370 \\
\hline 23 & 7 & $\odot$ & 3.625649 & -1.326046 & -0.391050 \\
\hline 24 & 6 & $\odot$ & 4.559148 & $-\odot .615609$ & ๑. 225986 \\
\hline 25 & 7 & $\odot$ & 4.374686 & 0.690439 & 0.544620 \\
\hline 26 & 6 & $\odot$ & 3.183853 & 1.388469 & ๑. 320698 \\
\hline 27 & 7 & $\odot$ & 5.723670 & -1.196376 & 0.530092 \\
\hline
\end{tabular}

Normal termination of Gaussian 16 at Sat Mar 9 20:08:09 2019.

-..--Figure S1-5, Int (C4)-.....

degua-oh4.for2.higha. $\log$

Stoichiometry C10H14N505(2)

Standard orientation: 


\begin{tabular}{cccrrr}
28 & 8 & 0 & 3.120901 & 2.569883 & 0.631862 \\
29 & 1 & $\odot$ & -0.956896 & 0.987370 & -1.303642 \\
30 & 1 & 0 & 5.857764 & -2.164077 & 0.280373 \\
31 & 1 & 0 & 6.465448 & -0.710619 & 1.009113 \\
32 & 1 & 0 & 5.120341 & 1.225491 & 0.975118 \\
33 & 8 & $\odot$ & 1.596127 & -1.443971 & -1.371949 \\
34 & 1 & 0 & 2.019739 & -2.286846 & -1.587113 \\
\hline
\end{tabular}

Standard basis: Aug-CC-pVDZ (6D, 7F)

626 basis functions

75 alpha electrons $\quad 74$ beta electrons

nuclear repulsion energy 1586.9442388778 Hartrees.

NAtoms $=34$ NActive $=34$

Nuclear repulsion after empirical dispersion term = 1586.9215327551 Hartrees.

Force inversion solution in PCM.

Polarizable Continuum Model (PCM)

=ニニニニニニニニニニニニニニニニニニニニニニニニニニニニニニニ=

Model : PCM.

Solvent : Water, Eps $=78.355300$ Eps $($ inf $)=1.777849$

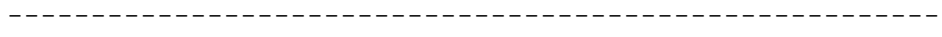

SCF Done: $E($ UWB97XD $)=-1039.20452372$ A.U. after 1 cycles $\mathrm{NFock}=1$ Conv $=0.46 \mathrm{D}-08 \quad-\mathrm{V} / \mathrm{T}=2.0072$

$\langle\mathrm{S} X\rangle=0.0000<\mathrm{Sy}\rangle=0.0000<\mathrm{S} z>=0.5000$

$<S^{* *} 2>=0.7661 \mathrm{~S}=0.5080$

$<$ L.S $>=0.000000000000 \mathrm{E}+00$

Annihilation of the first spin contaminant:

$\mathrm{S}^{* * 2}$ before annihilation 0.7661 , after 0.7502

Harmonic frequencies $\left(\mathrm{cm}^{*}-1\right)$, IR intensities (KM/Mole), Raman activities $\left(A^{* *} 4 / A M U\right)$, depolarization ratios for plane and unp incident light, reduced masses (AMU), force constants (mDyne/A), and normal coordinates:

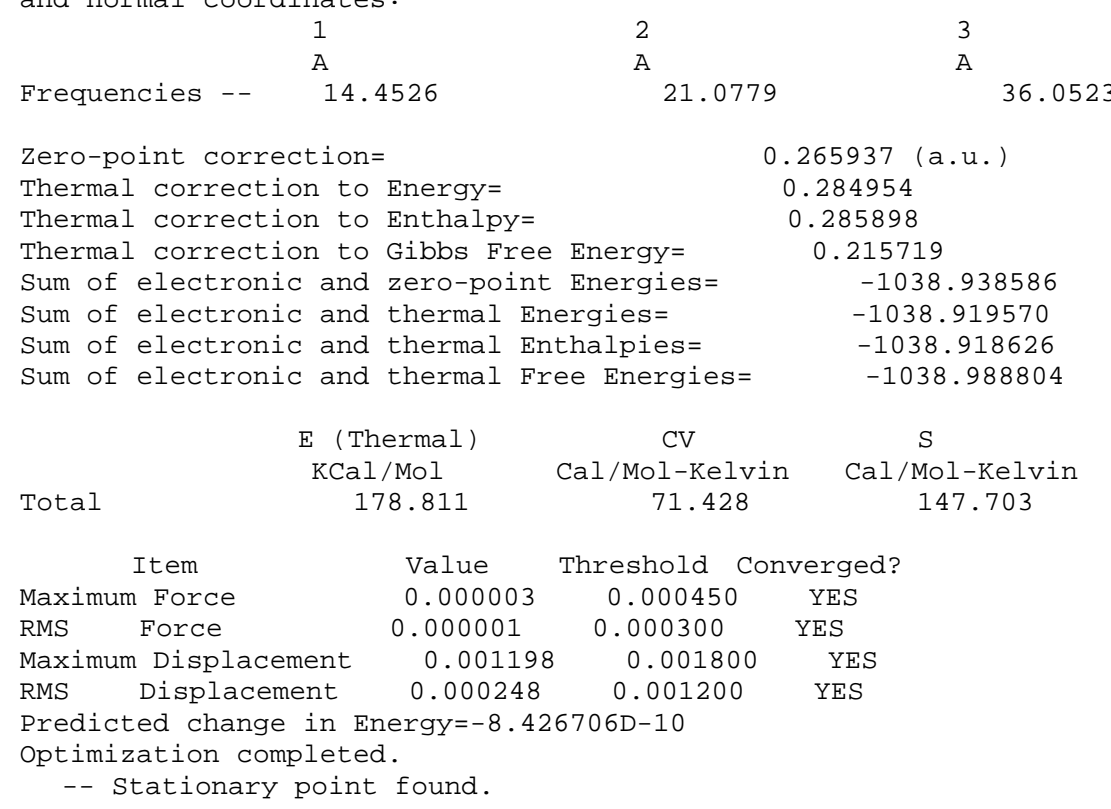

Normal termination of Gaussian 16 at Sat May 18 20:55:13 2019.

-...-.-Figure S1-6, TS3(C4)-....

degua-oh4.ts2.higha. $\log$

Stoichiometry C10H14N505(2)

Standard orientation:

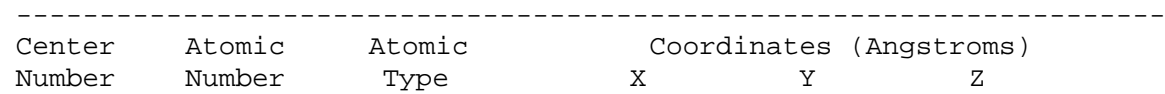




\begin{tabular}{|c|c|c|c|c|c|}
\hline 1 & 1 & $\odot$ & -4.041245 & 2.294186 & 0.856100 \\
\hline 2 & 8 & $\odot$ & -3.602012 & 2. 235976 & 0.003272 \\
\hline 3 & 8 & $\odot$ & -4.762767 & -1.933197 & 0.750624 \\
\hline 4 & 6 & $\odot$ & -4.054567 & -0.117798 & $-\odot .619924$ \\
\hline 5 & 6 & $\odot$ & -4.230458 & -0.611785 & ๑. 819985 \\
\hline 6 & 1 & $\odot$ & -4.812204 & -2.285081 & 1.644598 \\
\hline 7 & 6 & $\odot$ & -4.340756 & 1.362901 & -0.840819 \\
\hline 8 & 6 & $\odot$ & -2.806646 & -0.586370 & 1.361483 \\
\hline 9 & 6 & $\odot$ & -1.957753 & -0.913303 & ๑. 127847 \\
\hline 10 & 8 & $\odot$ & -2.709133 & -0.428524 & -0.986807 \\
\hline 11 & 1 & $\odot$ & -4.055535 & 1.615502 & -1.869413 \\
\hline 12 & 1 & $\odot$ & -5.423350 & 1.534323 & -0.736778 \\
\hline 13 & 1 & $\odot$ & -4.731512 & -0.684224 & -1.276717 \\
\hline 14 & 1 & $\odot$ & -4.910930 & 0.032800 & 1.395107 \\
\hline 15 & 1 & $\odot$ & -2.649492 & -1.294858 & 2.181740 \\
\hline 16 & 1 & $\odot$ & -2.554551 & $\odot .421790$ & 1.710994 \\
\hline 17 & 1 & $\odot$ & -1.846589 & -2.003272 & 0.010726 \\
\hline 18 & 7 & 0 & -0.627181 & -0.378794 & 0.226897 \\
\hline 19 & 6 & $\odot$ & -0.302930 & $\odot .615407$ & -0.515638 \\
\hline 20 & 7 & $\odot$ & $\odot .896060$ & 1.275601 & -0.413407 \\
\hline 21 & 6 & $\odot$ & 2.039573 & $\odot .614002$ & -0.191690 \\
\hline 22 & 6 & $\odot$ & 2.346408 & -0.723207 & -0.544366 \\
\hline 23 & 7 & $\odot$ & 3.581455 & -1.283618 & -0.317025 \\
\hline 24 & 6 & $\odot$ & 4.606773 & -0.599359 & 0.164056 \\
\hline 25 & 7 & $\odot$ & 4.395235 & ๑. 698099 & $\odot .490154$ \\
\hline 26 & 6 & $\odot$ & 3.173723 & 1.389743 & ๑. 331130 \\
\hline 27 & 7 & $\odot$ & 5.800804 & -1.161343 & 0. 325520 \\
\hline 28 & 8 & 0 & 3.142310 & 2.569584 & 0.644312 \\
\hline 29 & 1 & $\odot$ & -1.015987 & 1.075033 & -1.208667 \\
\hline 30 & 1 & $\odot$ & 5.928887 & -2.129553 & 0.072475 \\
\hline 31 & 1 & 0 & 6.597323 & -0.643643 & 0.663735 \\
\hline 32 & 1 & $\odot$ & 5.150637 & 1.254174 & $\odot .873589$ \\
\hline 33 & 8 & $\odot$ & 1.715613 & -1.649316 & -1.173207 \\
\hline 34 & 1 & $\odot$ & 2.927642 & -2.174363 & -0.960392 \\
\hline
\end{tabular}

Standard basis: Aug-CC-pVDZ (6D, 7F)

626 basis functions

75 alpha electrons nuclear repulsion energy 1585.7503168166 Hartrees.

NAtoms $=34$ NActive $=34$

Nuclear repulsion after empirical dispersion term =

1585.7273894343 Hartrees.

Force inversion solution in PCM.

Polarizable Continuum Model (PCM)

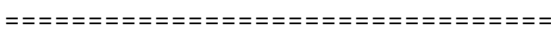

Model : PCM.

Solvent : Water, Eps $=78.355300$ Eps $($ inf $)=1.777849$

SCF Done: $E($ UWB97XD $)=-1039.14847416$ A.U. after 1 cycles

$$
\text { NFock }=1 \text { Conv }=0.71 \mathrm{D}-08 \quad-\mathrm{V} / \mathrm{T}=2.0072
$$

$<\mathrm{S} x>=0.0000<\mathrm{Sy}>=0.0000<\mathrm{S} z>=0.5000$

$<S * * 2>=0.7659 \mathrm{~S}=0.5079$

$<$ L.S $>=0.000000000000 \mathrm{E}+0 \odot$

Annihilation of the first spin contaminant:

$\mathrm{S}^{* * 2}$ before annihilation 0.7659 , after 0.7502

Harmonic frequencies $\left(\mathrm{cm}^{*}-1\right)$, IR intensities (KM/Mole), Raman activities $\left(A^{* *} 4 / A M U\right)$, depolarization ratios for plane and unpo incident light, reduced masses (AMU), force constants (mDyne/A), and normal coordinates:

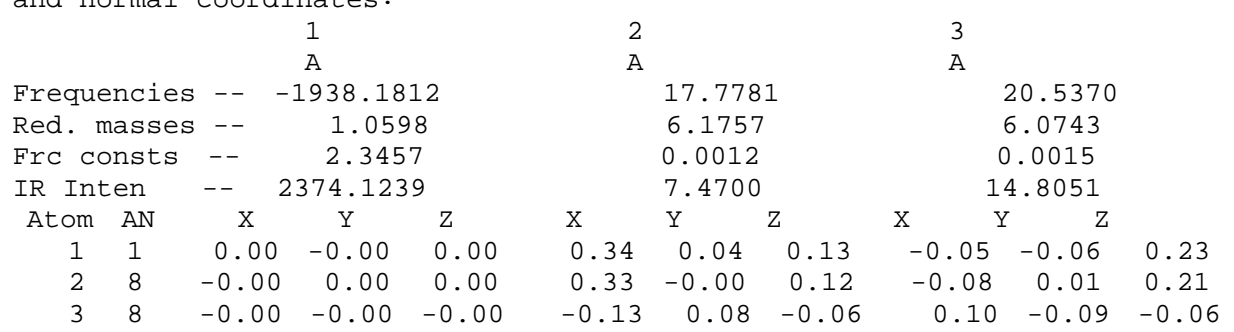




\begin{tabular}{|c|c|c|c|c|}
\hline 4 & 6 & $\odot . \odot \odot$ & $-\odot . \odot \odot$ & $-\odot . \odot \odot$ \\
\hline 5 & 6 & $\odot . \odot \odot$ & $\odot . \odot \odot$ & $\odot .0 \odot$ \\
\hline 6 & 1 & $\odot . \odot \odot$ & $\odot . \odot \odot$ & $-\odot . \odot \odot$ \\
\hline 7 & 6 & $-\odot . \odot \odot$ & $-\odot . \odot \odot$ & $\odot . \odot \odot$ \\
\hline 8 & 6 & $-\odot .00$ & -0.00 & -0.00 \\
\hline 9 & 6 & $\odot .0 \odot$ & $\odot . \odot \odot$ & $\odot . \odot \odot$ \\
\hline 10 & 8 & -0.00 & 0.00 & -0.00 \\
\hline 11 & 1 & $\odot .00$ & 0.00 & 0.00 \\
\hline 12 & 1 & $-\odot . \odot \odot$ & $\odot . \odot \odot$ & $\odot .0 \odot$ \\
\hline 13 & 1 & -0.00 & 0.00 & 0.00 \\
\hline 14 & 1 & -0.00 & $-0.0 \odot$ & -0.00 \\
\hline 15 & 1 & -0.00 & -0.00 & 0.00 \\
\hline 16 & 1 & $\odot .0 \odot$ & -0.00 & -0.00 \\
\hline 17 & 1 & $-0 . \odot \odot$ & $-0.0 \odot$ & -0.00 \\
\hline 18 & 7 & -0.00 & 0.00 & 0.00 \\
\hline 19 & 6 & $\odot . \odot \odot$ & -0.00 & $\odot .00$ \\
\hline 20 & 7 & $\odot . \odot \odot$ & $-0.0 \odot$ & -0.00 \\
\hline 21 & 6 & 0.00 & 0.00 & 0.00 \\
\hline 22 & 6 & -0.03 & -0.02 & -0.02 \\
\hline 23 & 7 & -0.02 & -0.01 & -0.01 \\
\hline 24 & 6 & 0.01 & -0.01 & -0.00 \\
\hline 25 & 7 & $-0 . \odot \odot$ & -0.01 & -0.00 \\
\hline 26 & 6 & -0.01 & 0.00 & $-\odot . \odot \odot$ \\
\hline 27 & 7 & -0.00 & $-\odot . \odot \odot$ & -0.00 \\
\hline 28 & 8 & $\odot . \odot \odot$ & $-\odot . \odot \odot$ & 0.00 \\
\hline 29 & 1 & $\odot . \odot \odot$ & -0.00 & -0.00 \\
\hline 30 & 1 & 0.01 & $-0.0 \odot$ & 0.00 \\
\hline 31 & 1 & -0.00 & 0.00 & -0.00 \\
\hline 32 & 1 & $\odot . \odot \odot$ & -0.02 & 0.00 \\
\hline 33 & 8 & -0.02 & 0.03 & $\odot .0 \odot$ \\
\hline 34 & 1 & 0.92 & $\odot .08$ & 0.37 \\
\hline
\end{tabular}

$\begin{array}{ccc}-0.00 & 0.08 & 0.02 \\ -0.03 & 0.04 & -0.0 \odot \\ -0.14 & 0.04 & -0.08 \\ 0.20 & 0.13 & 0.10 \\ -0.03 & -0.09 & -0.01 \\ -0.05 & -0.10 & -0.03 \\ -0.06 & -0.10 & -0.03 \\ 0.21 & 0.14 & 0.10 \\ 0.22 & 0.28 & 0.12 \\ -0.09 & 0.21 & 0.0 \odot \\ 0.02 & 0.06 & 0.03 \\ -0.07 & -0.14 & -0.05 \\ 0.05 & -0.13 & 0.03 \\ -0.05 & -0.10 & -0.04 \\ -0.04 & -0.10 & -0.04 \\ -0.05 & -0.11 & -0.05 \\ -0.06 & -0.08 & -0.05 \\ -0.04 & -0.04 & -0.02 \\ 0.01 & -0.04 & 0.02 \\ 0.04 & 0.02 & 0.03 \\ 0.01 & 0.08 & 0.01 \\ -0.05 & 0.08 & -0.01 \\ -0.08 & 0.02 & -0.03 \\ 0.04 & 0.14 & 0.02 \\ -0.13 & 0.02 & -0.05 \\ -0.05 & -0.12 & -0.05 \\ 0.08 & 0.14 & 0.04 \\ 0.01 & 0.19 & 0.00 \\ -0.08 & 0.12 & -0.02 \\ 0.05 & -0.08 & 0.05 \\ 0.07 & -0.02 & 0.06\end{array}$

$\begin{array}{lll}-0.02 & 0.04 & 0.05\end{array}$

$\begin{array}{llll}0.06 & -0.07 & 0.02\end{array}$

$0.15 \quad-0.16-0.08$

$\begin{array}{lll}-0.08 & 0.05 & 0.17\end{array}$

$\begin{array}{llll}0.08 & -0.07 & -0.05\end{array}$

$\begin{array}{llll}0.03 & 0.04 & -0.11\end{array}$

$\begin{array}{lll}-0.03 & 0.11 & -0.04\end{array}$

$\begin{array}{lll}-0.14 & 0.14 & 0.18\end{array}$

$\begin{array}{lll}-0.08 & 0.00 & 0.23\end{array}$

$\begin{array}{lll}-0.04 & 0.07 & 0.04\end{array}$

$0.06 \quad-0.14 \quad 0.10$

$0.14-0.12-0.10$

$\begin{array}{llll}0.07 & -0.09 & 0.01\end{array}$

$0.04 \quad 0.05 \quad-0.19$

$\begin{array}{llll}0.02 & 0.05 & -0.12\end{array}$

$\begin{array}{llll}0.02 & 0.04 & -0.14\end{array}$

$\begin{array}{lll}0.03 & 0.03 & -0.17\end{array}$

$0.01 \quad 0.01 \quad-0.09$

$\begin{array}{lll}-0.01 & -0.01 & 0.01\end{array}$

$\begin{array}{lll}-0.04 & -0.04 & 0.11\end{array}$

$\begin{array}{lll}-0.04 & -0.03 & 0.13\end{array}$

$\begin{array}{lll}-0.02 & -0.00 & 0.02\end{array}$

$\begin{array}{llll}0.01 & 0.02 & -0.09\end{array}$

$\begin{array}{lll}-0.07 & -0.07 & 0.24\end{array}$

$0.03 \quad 0.05 \quad-0.18$

$\begin{array}{lll}0.02 & 0.03 & -0.14\end{array}$

$-0.09-0.09 \quad 0.31$

$\begin{array}{lll}-0.07 & -0.07 & 0.24\end{array}$

$\begin{array}{lll}-0.02 & 0.00 & 0.02\end{array}$

$0.01 \quad-0.03 \quad 0.02$

Zero-point correction=

Thermal correction to Energy=

$-0.02 \quad-0.05 \quad 0.12$

Thermal correction to Enthalpy=

Thermal correction to Gibbs Free Energy=

Sum of electronic and zero-point Energies=

Sum of electronic and thermal Energies=

Sum of electronic and thermal Enthalpies=

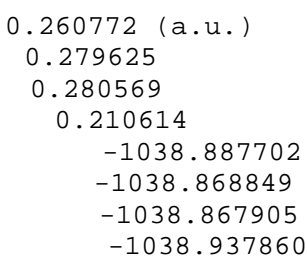

Total 175.467 70.261
Threshold Converged?

\begin{tabular}{lccc}
\multicolumn{1}{c}{ Item } & Value & Threshold & \multicolumn{2}{c}{ Converged? } \\
Maximum Force & 0.000008 & 0.000450 & YES \\
RMS Force & $0.0000 \odot 2$ & $0.00030 \odot$ & YES \\
Maximum Displacement & 0.001547 & $0.00180 \odot$ & YES \\
RMS Displacement & 0.000387 & 0.001200 & YES
\end{tabular}

Predicted change in Energy $=-4.879173 \mathrm{D}-09$

optimization completed.

- Stationary point found.

Normal termination of Gaussian 16 at Fri May 17 21:56:23 2019.

-...-Figure S1-7, Int4(C4)-...

degua-oh4.rev2.higha.log

Stoichiometry C10H14N505(2)

Standard orientation:

\begin{tabular}{|c|c|c|c|c|c|}
\hline \multirow{2}{*}{$\begin{array}{l}\text { Center } \\
\text { Number }\end{array}$} & \multirow{2}{*}{$\begin{array}{l}\text { Atomic } \\
\text { Number }\end{array}$} & \multirow{2}{*}{$\begin{array}{c}\text { Atomic } \\
\text { Type }\end{array}$} & \multicolumn{3}{|c|}{ Coordinates (Angstroms) } \\
\hline & & & $x$ & $\mathrm{Y}$ & Z \\
\hline & & & & 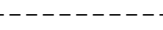 & \\
\hline 1 & 1 & $\odot$ & -4.278554 & 2.174773 & ๑. 978638 \\
\hline 2 & 8 & $\odot$ & -3.872584 & 2.219191 & 0.108597 \\
\hline 3 & 8 & $\odot$ & -4.673282 & -2.038572 & $\odot .753155$ \\
\hline 4 & 6 & $\odot$ & -4.089094 & -0.148451 & -0.580045 \\
\hline 5 & 6 & $\odot$ & -4.208129 & -0.693433 & 0.849755 \\
\hline 6 & 1 & $\odot$ & -4.690908 & -2.414773 & 1.638439 \\
\hline 7 & 6 & $\odot$ & -4.526794 & 1.300277 & -0.756163 \\
\hline 8 & 6 & $\odot$ & -2.776915 & -0.607850 & 1.363073 \\
\hline 9 & 6 & 0 & -1.944896 & -0.857827 & $\odot .103676$ \\
\hline 10 & 8 & 0 & -2.721454 & -0.301733 & -0.962182 \\
\hline
\end{tabular}




\begin{tabular}{|c|c|c|c|c|c|}
\hline 11 & 1 & $\Theta$ & -4.280892 & 1.613575 & -1.778013 \\
\hline 12 & 1 & $\odot$ & -5.620178 & 1.355707 & -0.637335 \\
\hline 13 & 1 & $\odot$ & -4.713248 & -0.764497 & -1.245290 \\
\hline 14 & 1 & 0 & -4.910257 & -0.102800 & 1.456430 \\
\hline 15 & 1 & $\odot$ & -2.565231 & -1.327775 & 2.160979 \\
\hline 16 & 1 & $\odot$ & -2.566990 & $\odot .403687$ & 1.731992 \\
\hline 17 & 1 & $\odot$ & -1.843649 & -1.941116 & $-\odot .078331$ \\
\hline 18 & 7 & $\odot$ & $-\odot .614567$ & -0.330590 & 0.210236 \\
\hline 19 & 6 & $\odot$ & -0.244657 & $\odot .601395$ & -0.588308 \\
\hline 20 & 7 & $\odot$ & $\odot .957459$ & 1.255505 & -0.486738 \\
\hline 21 & 6 & $\odot$ & 2.110185 & $\odot .612073$ & -0.270979 \\
\hline 22 & 6 & $\odot$ & 2.385961 & -0.735003 & -0.739776 \\
\hline 23 & 7 & $\odot$ & 3.654720 & -1.267113 & $-\odot .376860$ \\
\hline 24 & 6 & $\odot$ & 4.633054 & $-\odot .591870$ & $\odot .235961$ \\
\hline 25 & 7 & $\odot$ & $4.4 \odot 4616$ & ๑.689267 & 0.548839 \\
\hline 26 & 6 & $\odot$ & 3.187714 & 1.383493 & $\odot .321367$ \\
\hline 27 & 7 & $\Theta$ & 5.793843 & -1.171105 & 0.517494 \\
\hline 28 & 8 & $\odot$ & 3.147143 & 2.558501 & $\odot .662776$ \\
\hline 29 & 1 & $\odot$ & -0.926653 & 1.024716 & -1.334788 \\
\hline 30 & 1 & $\odot$ & 5.966243 & -2.136469 & 0.279349 \\
\hline 31 & 1 & $\odot$ & 6.530997 & -0.666040 & $\odot .986134$ \\
\hline 32 & 1 & $\odot$ & 5.130759 & 1.232659 & 1.000554 \\
\hline 33 & 8 & $\odot$ & 1.672363 & -1.442413 & -1.437568 \\
\hline 34 & 1 & $\odot$ & 3.820008 & -2.220004 & -0.679147 \\
\hline
\end{tabular}

Standard basis: Aug-CC-pVDZ (6D, 7F)

626 basis functions

75 alpha electrons $\quad 74$ beta electrons

nuclear repulsion energy 1577.8477729028 Hartrees.

NAtoms $=34$ NActive $=34$

Nuclear repulsion after empirical dispersion term $=$

1577.8251159358 Hartrees.

Force inversion solution in PCM.

Polarizable Continum Model (PCM)

Polarizable Continuum Model (PCM)

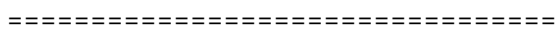

Model : PCM.

Solvent : Water, Eps $=78.355300$ Eps $($ inf $)=1.777849$

SCF Done: $E($ UWB97XD $)=-1039.20772171$ A.U. after 1 cycles

NFock $=1$ Conv $=0.56 \mathrm{D}-08 \quad-\mathrm{V} / \mathrm{T}=2.0072$

$\langle\mathrm{S} X>=0.0000<\mathrm{S} y>=0.0000\langle\mathrm{~S} z>=0.5000$

$\left\langle S^{* *} 2>=0.7618 \mathrm{~S}=0.5059\right.$

$<$ L.S $>=0.000000000000 \mathrm{E}+00$

Annihilation of the first spin contaminant:

$\mathrm{S}^{* * 2}$ before annihilation 0.7618 , after 0.7501

Harmonic frequencies $\left(\mathrm{cm}^{* *}-1\right)$, IR intensities (KM/Mole), Raman activities ( $\left.A^{* *} 4 / \mathrm{AMU}\right)$, depolarization ratios for plane and unpo incident light, reduced masses (AMU), force constants (mDyne/A), and normal coordinates:

$\begin{array}{cll}1 & 2 & 3 \\ \text { A } & \text { A } & \text { A }\end{array}$

$\begin{array}{lrrr}\text { Frequencies - } & 14.6549 & 25.4611 & 36.2648\end{array}$

Zero-point correction=

Thermal correction to Energy=

Thermal correction to Enthalpy=

Thermal correction to Gibbs Free Energy=

Sum of electronic and zero-point Energies=

Sum of electronic and thermal Energies=

Sum of electronic and thermal Enthalpies=

0.265975 (a.u.)

0.285042

0.285986

0.215736

$-1038.941746$

$-1038.922680$

$-1038.921736$

Sum of electronic and thermal Free Energies=

$-1038.991985$

\begin{tabular}{|c|c|c|c|}
\hline $\mathrm{E}$ & $\begin{array}{l}\text { ermal) } \\
\text { /Mol } \\
78.866\end{array}$ & $\begin{array}{c}\mathrm{CV} \\
\text { Cal/Mol-Kelvin } \\
71.407\end{array}$ & $\begin{array}{c}\mathrm{S} \\
\mathrm{Cal} / \mathrm{Mol}-\mathrm{Kelvin} \\
147.852\end{array}$ \\
\hline Item & Value & \multicolumn{2}{|c|}{ Threshold Converged? } \\
\hline Maximum Force & 0.000045 & 0.000450 & ES \\
\hline Force & $\odot . ๑ \odot \odot \odot \odot 6$ & 0.000300 & \\
\hline Maximum Displacement & $\odot .0 \odot 2690$ & 0.001800 & NO \\
\hline
\end{tabular}


$\begin{array}{llll}\text { RMS Displacement } & 0.000515 & 0.001200 & \text { YES }\end{array}$

Predicted change in Energy $=-1.436746 \mathrm{D}-\odot 8$

Normal termination of Gaussian 16 at Sun May 19 03:55:26 2019.

\begin{tabular}{|c|c|c|c|c|c|}
\hline \multicolumn{2}{|c|}{ Stoichiometry } & \multicolumn{4}{|c|}{ C10H16N506(2) } \\
\hline \multicolumn{6}{|c|}{ Standard orientation: } \\
\hline Center & Atomic & Atomic & $\operatorname{Coc}$ & dinates $(A$ & gstroms) \\
\hline Number & Number & Type & $x$ & $\mathrm{Y}$ & $\mathrm{Z}$ \\
\hline 1 & 1 & $\odot$ & -3.850934 & -2.067858 & -1.278951 \\
\hline 2 & 8 & 0 & -3.308361 & -2.183746 & -0.494151 \\
\hline 3 & 8 & $\odot$ & -4.305564 & 2.054597 & $-\odot .003955$ \\
\hline 4 & 6 & $\odot$ & -3.625605 & -0.087252 & 0.773006 \\
\hline 5 & 6 & $\odot$ & -3.880184 & 0.777055 & -0.464825 \\
\hline 6 & 1 & $\odot$ & -4.432708 & 2.626460 & -0.767167 \\
\hline 7 & 6 & $\odot$ & -3.950010 & -1.567077 & 0.616559 \\
\hline 8 & 6 & $\odot$ & -2.506913 & 0.830797 & -1.128527 \\
\hline 9 & 6 & $\odot$ & -1.554536 & $\odot .774189$ & 0.066697 \\
\hline 10 & 8 & 0 & -2.237160 & 0.088858 & 1.096849 \\
\hline 11 & 1 & $\odot$ & -3.596589 & -2.092124 & 1.511901 \\
\hline 12 & 1 & $\odot$ & -5.041591 & -1.687761 & $\odot .559005$ \\
\hline 13 & 1 & $\odot$ & -4.216771 & 0.299917 & 1.613779 \\
\hline 14 & 1 & $\odot$ & -4.643488 & 0.337210 & -1.121474 \\
\hline 15 & 1 & $\odot$ & -2.359071 & 1.732580 & -1.730431 \\
\hline 16 & 1 & 0 & -2.362768 & -0.045643 & -1.769291 \\
\hline 17 & 1 & $\odot$ & -1.261007 & 1.773365 & 0.409395 \\
\hline 18 & 7 & $\odot$ & $-\odot .309231$ & $\odot .075417$ & -0.207991 \\
\hline 19 & 6 & $\odot$ & -0.174374 & -1.250530 & -0.570565 \\
\hline 20 & 7 & $\odot$ & 1.068881 & -1.618069 & -0.726700 \\
\hline 21 & 6 & $\odot$ & 1.805968 & $-\odot .487486$ & -0.419354 \\
\hline 22 & 6 & $\odot$ & $\odot .950418$ & 0.587170 & -0.120749 \\
\hline 23 & 7 & $\odot$ & 1.281781 & 1.850937 & 0.162808 \\
\hline 24 & 6 & $\odot$ & 2.586440 & 2.074623 & $\odot .093181$ \\
\hline 25 & 7 & $\odot$ & 3.500950 & 1.117769 & -0.265180 \\
\hline 26 & 6 & $\odot$ & 3.212951 & -0.223826 & -0.542436 \\
\hline 27 & 7 & $\odot$ & 3.052455 & 3.300553 & $\odot .367512$ \\
\hline 28 & 8 & $\odot$ & 4.115997 & -0.999372 & -0.847427 \\
\hline 29 & 1 & $\odot$ & -1.050869 & -1.882013 & -0.685371 \\
\hline 30 & 1 & $\odot$ & 2.392603 & 4.017880 & $\odot .624549$ \\
\hline 31 & 1 & $\odot$ & 4.033415 & 3.528419 & $\odot .344986$ \\
\hline 32 & 1 & $\odot$ & 4.482237 & 1.366020 & -0.317033 \\
\hline 33 & 8 & $\odot$ & 2.208186 & $-\odot .805276$ & 1.849315 \\
\hline 34 & 1 & $\odot$ & 2.459181 & -1.718828 & 1.620697 \\
\hline 35 & 8 & $\odot$ & 3.052834 & -3.391077 & 0.393363 \\
\hline 36 & 1 & $\odot$ & 2.222040 & -3.172027 & -0.058915 \\
\hline 37 & 1 & $\odot$ & 3.670078 & -2.779711 & -0.038691 \\
\hline
\end{tabular}

Standard basis: Aug-CC-pVDZ (6D, 7F)

669 basis functions

80 alpha electrons

energy 1843.6897063335 Hartrees.

NAtoms $=37$ NActive $=37$

Force inversion solution in PCM.

Polarizable Continuum Model (PCM)

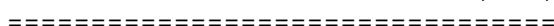

Model
: PCM.

Solvent: Water, Eps $=78.355300$ Eps $($ inf $)=1.777849$

SCF Done: $E($ UWB97XD) $=-1115.62997881$ A.U. after 1 cycles

NFock $=1$ Conv $=0.68 \mathrm{D}-08 \quad-\mathrm{V} / \mathrm{T}=2.0071$

$\langle\mathrm{S} x\rangle=0.0000<\mathrm{Sy}\rangle=0.0000<\mathrm{Sz}>=0.5000$

$<\mathrm{S}^{* *} 2>=0.7591 \mathrm{~S}=0.5045$ 
$<$ L.S $>=0.000000000000 \mathrm{E}+00$

Annihilation of the first spin contaminant:

$\mathrm{S}^{* * 2}$ before annihilation 0.7591 , after 0.7501

Harmonic frequencies $\left(\mathrm{cm}^{*}-1\right)$, IR intensities (KM/Mole), Raman

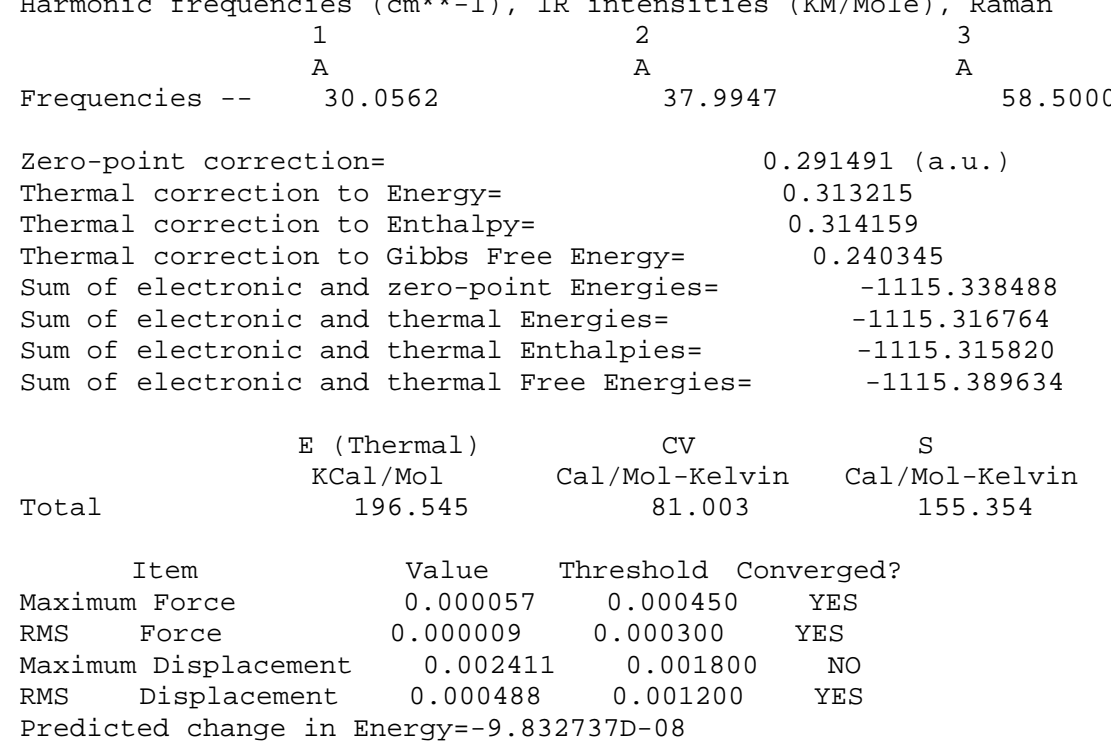

Normal termination of Gaussian 16 at Thu Jun 13 ๑4:30:51 2019.

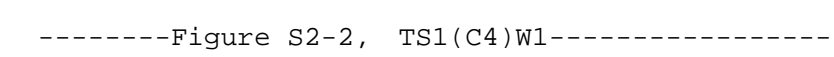

degua-oh4w1. higha. log

Stoichiometry C10H16N506(2)

Standard orientation:

\begin{tabular}{|c|c|c|c|c|c|}
\hline \multirow{2}{*}{$\begin{array}{l}\text { Center } \\
\text { Number }\end{array}$} & \multirow{2}{*}{$\begin{array}{l}\text { Atomic } \\
\text { Number }\end{array}$} & \multirow{2}{*}{$\begin{array}{c}\text { Atomic } \\
\text { Type }\end{array}$} & \multicolumn{3}{|c|}{ Coordinates (Angstroms) } \\
\hline & & & $\mathrm{X}$ & $\mathrm{Y}$ & Z \\
\hline & - & -- & & & \\
\hline 1 & 1 & $\odot$ & -4.239824 & -1.894036 & -0.976254 \\
\hline 2 & 8 & $\odot$ & -3.692426 & -2.013366 & -0.195405 \\
\hline 3 & 8 & $\odot$ & -3.986145 & 2.361841 & -0.169103 \\
\hline 4 & 6 & $\odot$ & -3.614669 & 0.234238 & 0.827044 \\
\hline 5 & 6 & $\odot$ & -3.787697 & 0.989925 & $-\odot .493344$ \\
\hline 6 & 1 & $\odot$ & -4.052478 & 2.860824 & $-\odot .989059$ \\
\hline 7 & 6 & $\odot$ & -4.179344 & -1.180322 & 0.850861 \\
\hline 8 & 6 & $\odot$ & -2.455900 & 0.749220 & -1.196805 \\
\hline 9 & 6 & $\odot$ & -1.466692 & 0.663892 & -0.033134 \\
\hline 10 & 8 & $\odot$ & -2.203529 & 0.212663 & 1.087955 \\
\hline 11 & 1 & $\odot$ & -3.876803 & -1.654135 & 1.792273 \\
\hline 12 & 1 & $\odot$ & -5.277781 & -1.126317 & 0.833779 \\
\hline 13 & 1 & $\odot$ & -4.097059 & ๑. 801575 & 1.634640 \\
\hline 14 & 1 & $\odot$ & -4.640299 & $\odot .611009$ & -1.073831 \\
\hline 15 & 1 & 0 & -2.194653 & 1.544501 & -1.901703 \\
\hline 16 & 1 & $\odot$ & -2.481395 & -0.203003 & -1.737645 \\
\hline 17 & 1 & $\odot$ & -1.005182 & 1.633386 & 0.187921 \\
\hline 18 & 7 & $\odot$ & -0.362774 & -0.244767 & -0.277654 \\
\hline 19 & 6 & $\odot$ & -0.442369 & -1.598420 & -0.441426 \\
\hline 20 & 7 & $\odot$ & 0.729345 & -2.190507 & -0.581631 \\
\hline 21 & 6 & $\odot$ & 1.629520 & -1.173576 & -0.478703 \\
\hline 22 & 6 & $\odot$ & ๑. 981138 & 0.066300 & -0.209181 \\
\hline 23 & 7 & $\odot$ & 1.497187 & 1.306168 & -0.400316 \\
\hline 24 & 6 & $\odot$ & 2.793261 & 1.298606 & -0.616967 \\
\hline 25 & 7 & $\odot$ & 3.548901 & 0.148188 & -0.717081 \\
\hline 26 & 6 & $\odot$ & 3.054307 & -1.161940 & -0.702932 \\
\hline 27 & 7 & $\odot$ & 3.454966 & 2.462053 & -0.747105 \\
\hline 28 & 8 & $\odot$ & 3.799569 & -2.115003 & -0.885569 \\
\hline 29 & 1 & $\odot$ & -1.407358 & -2.098551 & -0.447436 \\
\hline 30 & 1 & $\odot$ & 2.923979 & 3.317850 & $-\odot .711298$ \\
\hline 31 & 1 & $\odot$ & 4.419038 & 2.505697 & -1.035978 \\
\hline 32 & 1 & $\odot$ & 4.547273 & 0.227925 & -0.868670 \\
\hline 33 & 8 & $\odot$ & 1.241740 & -0.313960 & 1.761052 \\
\hline 34 & 1 & $\odot$ & 2.168987 & -0.057661 & 1.909281 \\
\hline 35 & 8 & $\odot$ & 4.010452 & 0.535589 & 2.386536 \\
\hline
\end{tabular}




\begin{tabular}{rrrrrr}
36 & 1 & 0 & 4.054959 & 0.642217 & 3.342038 \\
37 & 1 & 0 & 4.658607 & -0.148891 & 2.192229 \\
\hline
\end{tabular}

Standard basis: Aug-CC-pVDZ (6D, 7F)

669 basis functions

80 alpha electrons $\quad 79$ beta electrons

nuclear repulsion energy 1850.3209647280 Hartrees.

NAtoms $=37$ NActive $=37$

Force inversion solution in PCM.

Polarizable Continuum Model (PCM)

Polarizable Continuum Model (PCM)

$\begin{array}{ll}\text { Model } & \text { : PCM. }\end{array}$

Solvent : Water, Eps $=78.355300$ Eps $($ inf $)=1.777849$

SCF Done: $E($ UWB97XD) $=-1115.6156179$ A.U. after 2 cycles NFock $=2$ Conv $=0.66 \mathrm{D}-09 \quad-\mathrm{V} / \mathrm{T}=2.0071$

$<\mathrm{S} x>=0.0000<\mathrm{Sy}>=0.0000<\mathrm{Sz}>=0.5000$

$<S^{* *} 2>=0.7742 \mathrm{~S}=0.5120$

$<$ L.S $>=0.000000000000 \mathrm{E}+00$

Annihilation of the first spin contaminant:

$\mathrm{S}^{* *} 2$ before annihilation 0.7742 , after 0.7503

Harmonic frequencies $\left(\mathrm{cm}^{*}-1\right)$, IR intensities (KM/Mole), Raman activities ( $\left.A^{* *} 4 / A M U\right)$, depolarization ratios for plane and unp incident light, reduced masses (AMU), force constants (mDyne/A), and normal coordinates:

\begin{tabular}{|c|c|c|c|c|c|c|c|c|c|c|}
\hline & & & 1 & & 2 & & & 3 & & \\
\hline & & & A & & A & & & A & & \\
\hline Freque & ncies & - - & -372.61 & & & 26.372 & & & 3.7515 & \\
\hline Red. $n$ & lasses & - & 10.60 & & & 5.2004 & & & 5.9080 & \\
\hline Frc & nsts & - - & 0.867 & & & 0.0021 & & & .0040 & \\
\hline IR Int & en & - - & 52.359 & & & 7.2489 & & & .8371 & \\
\hline Atom & AN & $x$ & $Y$ & Z & $x$ & $Y$ & Z & $x$ & Z & \\
\hline 1 & 1 & 0.01 & $-0.0 \odot$ & $-\odot .0 \odot$ & $0.0 \odot$ & 0.12 & -0.24 & -0.05 & -0.01 & 0.14 \\
\hline 2 & 8 & 0.01 & 0.00 & 0.00 & -0.04 & 0.03 & -0.22 & 0.01 & 0.01 & 0.11 \\
\hline 3 & 8 & $\odot . \odot \odot$ & -0.00 & $-0.0 \odot$ & 0.06 & 0.04 & 0.16 & 0.03 & 0.01 & 0.04 \\
\hline 4 & 6 & -0.01 & 0.00 & $\odot . \odot \odot$ & -0.03 & -0.06 & -0.02 & 0.07 & 0.03 & 0.06 \\
\hline 5 & 6 & 0.01 & $-0.0 \odot$ & $-\odot . \odot \odot$ & 0.04 & 0.07 & 0.04 & -0.01 & $\odot .0 \odot$ & 0.06 \\
\hline 6 & 1 & 0.01 & -0.00 & $-\odot .0 \odot$ & 0.10 & 0.12 & 0.21 & -0.01 & $-0.0 \odot$ & 0.04 \\
\hline 7 & 6 & -0.01 & $-0.0 \odot$ & $-0.0 \odot$ & -0.06 & -0.05 & -0.17 & 0.07 & 0.03 & 0.12 \\
\hline 8 & 6 & $\odot . \odot 2$ & -0.01 & $\odot .02$ & 0.06 & 0.10 & 0.07 & -0.05 & -0.03 & -0.02 \\
\hline 9 & 6 & $\odot .01$ & -0.02 & 0.03 & 0.01 & -0.02 & 0.10 & 0.02 & $-0.0 \odot$ & -0.07 \\
\hline 10 & 8 & -0.02 & $0.0 \odot$ & 0.03 & -0.03 & -0.11 & $\odot . \odot 2$ & $\odot .08$ & 0.03 & -0.02 \\
\hline 11 & 1 & -0.01 & $-\odot . \odot \odot$ & $\odot .0 \odot$ & -0.11 & -0.14 & -0.20 & 0.12 & 0.05 & 0.11 \\
\hline 12 & 1 & -0.01 & -0.01 & $-\odot .01$ & -0.06 & -0.02 & -0.20 & 0.07 & $\odot .03$ & 0.19 \\
\hline 13 & 1 & -0.03 & $0.0 \odot$ & -0.01 & -0.04 & -0.12 & 0.01 & 0.11 & 0.04 & 0.08 \\
\hline 14 & 1 & 0.01 & -0.00 & -0.01 & 0.05 & 0.14 & -0.03 & -0.05 & $-0.0 \odot$ & 0.12 \\
\hline 15 & 1 & 0.03 & -0.01 & 0.02 & 0.10 & 0.16 & 0.15 & -0.09 & -0.05 & -0.06 \\
\hline 16 & 1 & 0.01 & -0.01 & 0.01 & 0.07 & 0.15 & -0.02 & -0.10 & -0.04 & 0.01 \\
\hline 17 & 1 & $0.0 \odot$ & -0.02 & 0.03 & 0.03 & -0.05 & 0.20 & 0.03 & 0.00 & -0.13 \\
\hline 18 & 7 & 0.00 & -0.01 & 0.08 & 0.01 & -0.02 & 0.06 & 0.00 & -0.01 & -0.11 \\
\hline 19 & 6 & 0.01 & 0.04 & -0.05 & $-0.0 \odot$ & -0.02 & 0.07 & -0.01 & $-0.0 \odot$ & -0.16 \\
\hline 20 & 7 & 0.03 & $-0.0 \odot$ & -0.10 & -0.01 & -0.03 & 0.06 & -0.01 & -0.01 & -0.15 \\
\hline 21 & 6 & 0.03 & -0.08 & -0.01 & -0.00 & -0.03 & 0.03 & $-\odot .0 \odot$ & -0.03 & -0.09 \\
\hline 22 & 6 & $\odot .08$ & -0.06 & 0.64 & 0.01 & -0.03 & $\odot .03$ & 0.01 & -0.03 & -0.06 \\
\hline 23 & 7 & 0.01 & -0.04 & 0.09 & 0.01 & -0.03 & $-\odot . \odot \odot$ & 0.03 & -0.03 & 0.01 \\
\hline 24 & 6 & -0.01 & -0.02 & 0.01 & $0.0 \odot$ & -0.04 & -0.04 & $\odot .04$ & -0.04 & 0.05 \\
\hline 25 & 7 & 0.02 & -0.02 & 0.01 & $-\odot .0 \odot$ & -0.04 & -0.03 & $\odot .03$ & -0.05 & 0.05 \\
\hline 26 & 6 & 0.03 & -0.02 & -0.02 & -0.01 & -0.04 & 0.01 & $\odot .01$ & -0.04 & -0.03 \\
\hline 27 & 7 & -0.02 & -0.03 & -0.03 & $-\odot .0 \odot$ & -0.05 & -0.10 & 0.05 & -0.04 & 0.09 \\
\hline 28 & 8 & $\odot .02$ & -0.02 & -0.03 & -0.01 & -0.05 & $\odot .02$ & 0.00 & -0.04 & -0.04 \\
\hline 29 & 1 & $\odot .02$ & 0.02 & -0.16 & -0.01 & $-\odot .02$ & 0.08 & -0.01 & 0.01 & -0.19 \\
\hline 30 & 1 & -0.01 & -0.02 & 0.03 & 0.01 & -0.04 & -0.06 & 0.07 & -0.04 & 0.13 \\
\hline 31 & 1 & -0.05 & -0.03 & -0.16 & 0.01 & -0.05 & -0.06 & 0.08 & -0.05 & 0.17 \\
\hline 32 & 1 & $\odot .02$ & -0.01 & 0.03 & -0.01 & -0.05 & -0.06 & 0.03 & -0.06 & 0.08 \\
\hline 33 & 8 & -0.14 & 0.23 & -0.47 & 0.04 & -0.00 & 0.04 & -0.05 & -0.14 & -0.07 \\
\hline 34 & 1 & -0.18 & 0.21 & -0.32 & 0.02 & 0.08 & $\odot .02$ & -0.09 & -0.01 & -0.02 \\
\hline 35 & 8 & $-0 . \odot \odot$ & 0.00 & $-\odot . \odot \odot$ & -0.03 & 0.29 & -0.07 & -0.25 & 0.29 & 0.11 \\
\hline 36 & 1 & -0.05 & -0.04 & 0.00 & 0.00 & 0.33 & -0.08 & -0.29 & 0.22 & 0.12 \\
\hline 37 & 1 & -0.02 & -0.01 & 0.00 & 0.02 & 0.34 & -0.08 & -0.11 & 0.43 & 0.06 \\
\hline
\end{tabular}


Zero-point correction=

Thermal correction to Energy=

Thermal correction to Enthalpy=

Thermal correction to Gibbs Free Energy=

Sum of electronic and zero-point Energies=

Sum of electronic and thermal Energies=

Sum of electronic and thermal Enthalpies=

Sum of electronic and thermal Free Energies=

$$
\text { E (Thermal) }
$$

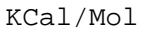

Total

$$
195.400
$$

CV
Cal/Mol-Kelvin
80.988

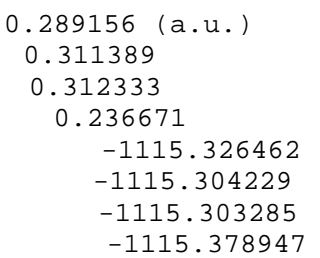

\section{$\mathrm{S}$}

Cal/Mol-Kelvin 159.245

Normal termination of Gaussian 16 at Sat Jun 8 03:32:41 2019 .

-...-Figure S2-3, Int2(C4)W1-.....

degua-oh4w1.rev . higha. $\log$

\begin{tabular}{|c|c|c|c|c|c|}
\hline \multirow{2}{*}{$\begin{array}{l}\text { Center } \\
\text { Number }\end{array}$} & \multirow{2}{*}{$\begin{array}{l}\text { Atomic } \\
\text { Number }\end{array}$} & \multirow{2}{*}{$\begin{array}{l}\text { Atomic } \\
\text { Type }\end{array}$} & \multicolumn{3}{|c|}{ Coordinates (Angstroms) } \\
\hline & & & $\mathrm{X}$ & $\mathrm{Y}$ & Z \\
\hline & & & & & \\
\hline 1 & 1 & $\odot$ & -4.169217 & -1.916031 & -1.062078 \\
\hline 2 & 8 & $\odot$ & -3.686870 & -2.019801 & -0.237308 \\
\hline 3 & 8 & $\odot$ & -3.898223 & 2.357928 & -0.382664 \\
\hline 4 & 6 & 0 & -3.644772 & 0.261249 & 0.713250 \\
\hline 5 & 6 & $\odot$ & -3.703773 & 0.971203 & -0.642457 \\
\hline 6 & 1 & $\odot$ & -3.880294 & 2.828119 & -1.221862 \\
\hline 7 & 6 & $\odot$ & -4.236552 & -1.142580 & 0.740133 \\
\hline 8 & 6 & $\odot$ & -2.328995 & 0.681282 & -1.233912 \\
\hline 9 & 6 & $\odot$ & -1.426395 & 0.623109 & $\odot .0 \odot \odot 951$ \\
\hline 10 & 8 & $\odot$ & -2.260504 & $\odot .225437$ & 1.082232 \\
\hline 11 & 1 & $\odot$ & -4.015548 & -1.588641 & 1.717159 \\
\hline 12 & 1 & $\odot$ & -5.329335 & -1.072278 & $\odot .636675$ \\
\hline 13 & 1 & $\odot$ & -4.179785 & ๑. 864011 & 1.460274 \\
\hline 14 & 1 & $\odot$ & -4.519317 & 0.587129 & -1.271328 \\
\hline 15 & 1 & $\odot$ & -1.999368 & 1.445131 & -1.945199 \\
\hline 16 & 1 & $\odot$ & -2.334836 & -0.288684 & -1.742624 \\
\hline 17 & 1 & $\odot$ & -0.981463 & 1.600255 & ๑. 223281 \\
\hline 18 & 7 & $\odot$ & -0.317486 & $-\odot .290489$ & -0.131313 \\
\hline 19 & 6 & $\odot$ & -0.404869 & -1.627123 & -0.261683 \\
\hline 20 & 7 & $\odot$ & ๑. 755854 & -2.275058 & -0.230204 \\
\hline 21 & 6 & $\odot$ & 1.682691 & -1.307873 & -0.038533 \\
\hline 22 & 6 & 0 & 1.063224 & 0.049964 & 0.207440 \\
\hline 23 & 7 & $\odot$ & 1.618067 & 1.090664 & -0.608146 \\
\hline 24 & 6 & $\odot$ & 2.881457 & 0.971763 & -0.863282 \\
\hline 25 & 7 & 0 & 3.653711 & -0.143202 & -0.540028 \\
\hline 26 & 6 & $\odot$ & 3.095374 & -1.402408 & -0.293551 \\
\hline 27 & 7 & $\odot$ & 3.573943 & 1.995431 & -1.438779 \\
\hline 28 & 8 & $\odot$ & 3.785768 & -2.418104 & -0.326684 \\
\hline 29 & 1 & $\odot$ & -1.370904 & -2.110855 & -0.392143 \\
\hline 30 & 1 & $\odot$ & 3.006087 & 2.763889 & -1.766258 \\
\hline 31 & 1 & $\odot$ & 4.376687 & 1.778711 & -2.012471 \\
\hline 32 & 1 & $\odot$ & 4.643951 & -0.133295 & -0.751346 \\
\hline 33 & 8 & $\odot$ & 1.053831 & 0.424148 & 1.572009 \\
\hline 34 & 1 & $\odot$ & 1.920059 & ๑. 819692 & 1.795539 \\
\hline 35 & 8 & $\odot$ & 3.523321 & 1.517473 & 2.258554 \\
\hline 36 & 1 & $\odot$ & 3.595847 & 1.704389 & 3.200106 \\
\hline 37 & 1 & $\odot$ & 4.245868 & ๑. 908879 & 2.072334 \\
\hline
\end{tabular}

Stoichiometry C10H16N506(2)

Standard basis: Aug-CC-pVDZ (6D, 7F)

669 basis functions 


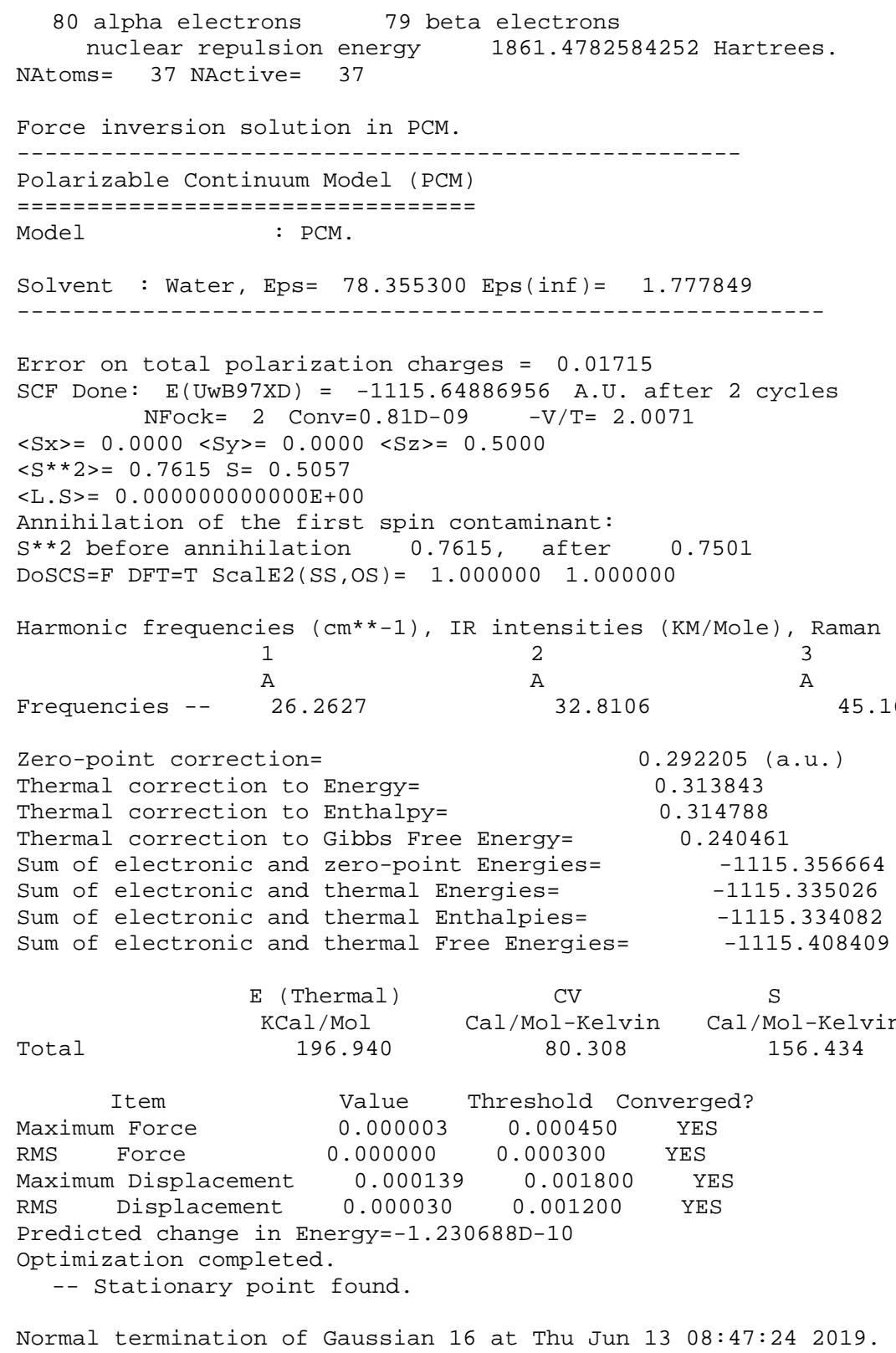

Normal termination of Gaussian 16 at Thu Jun 13 08:47:24 2019.

degua-oh4w1.ts1.higha.log

Stoichiometry C10H16N506(2)

\begin{tabular}{|c|c|c|c|c|c|}
\hline \multirow{2}{*}{$\begin{array}{l}\text { Center } \\
\text { Number }\end{array}$} & \multirow{2}{*}{$\begin{array}{l}\text { Atomic } \\
\text { Number }\end{array}$} & \multirow{2}{*}{$\begin{array}{c}\text { Atomic } \\
\text { Type }\end{array}$} & \multicolumn{3}{|c|}{ Coordinates (Angstroms) } \\
\hline & & & $\mathrm{x}$ & $\mathrm{Y}$ & Z \\
\hline$-x_{0}$ & - & --- & $-----\cdot--$ & ------ & $----\cdot--$ \\
\hline 1 & 1 & $\odot$ & -3.889902 & -2.469785 & -0.447924 \\
\hline 2 & 8 & $\odot$ & -3.396628 & -2.233328 & 0.342293 \\
\hline 3 & 8 & $\odot$ & -3.832225 & 1.736844 & -1.466244 \\
\hline 4 & 6 & $\odot$ & -3.452607 & 0.236186 & 0.339311 \\
\hline 5 & 6 & $\odot$ & -3.568613 & $\odot .366317$ & -1.181884 \\
\hline 6 & 1 & $\odot$ & -3.849663 & 1.850029 & -2.421517 \\
\hline 7 & 6 & $\odot$ & -3.973806 & -1.070680 & 0.923184 \\
\hline 8 & 6 & $\odot$ & -2.191784 & -0.071406 & -1.670984 \\
\hline 9 & 6 & $\odot$ & -1.258614 & 0.424734 & -0.563251 \\
\hline 10 & 8 & $\odot$ & -2.057574 & 0.386021 & ○. 642470 \\
\hline 11 & 1 & $\odot$ & -3.717804 & -1.095559 & 1.989158 \\
\hline 12 & 1 & $\odot$ & -5.070397 & -1.086304 & 0.836392 \\
\hline 13 & 1 & $\odot$ & -4.000495 & 1.060890 & ๑. 816143 \\
\hline 14 & 1 & $\odot$ & -4.375370 & -0.263462 & -1.583444 \\
\hline
\end{tabular}




\begin{tabular}{|c|c|c|c|c|c|}
\hline 15 & 1 & $\Theta$ & -1.932347 & $\odot .354538$ & -2.645490 \\
\hline 16 & 1 & $\odot$ & -2.148371 & -1.163472 & -1.742617 \\
\hline 17 & 1 & $\odot$ & -0.981542 & 1.473628 & -0.739859 \\
\hline 18 & 7 & 0 & -0.039130 & $-\odot .300206$ & -0.403423 \\
\hline 19 & 6 & $\odot$ & $-\odot .004983$ & -1.572830 & $-\odot .086965$ \\
\hline 20 & 7 & $\odot$ & 1.182056 & -2.126149 & $\odot .222434$ \\
\hline 21 & 6 & $\odot$ & 2.107070 & -1.142845 & $\odot .338543$ \\
\hline 22 & 6 & $\odot$ & 1.646754 & $\odot .219438$ & $\odot .646456$ \\
\hline 23 & 7 & $\odot$ & 2.267076 & 1.314208 & $\odot .148864$ \\
\hline 24 & 6 & $\odot$ & 3.445795 & 1.130525 & $-\odot .407498$ \\
\hline 25 & 7 & $\odot$ & 4.088353 & $-\odot .077859$ & -0.427880 \\
\hline 26 & 6 & $\odot$ & 3.458462 & -1.299596 & -0.133548 \\
\hline 27 & 7 & $\odot$ & 4.089973 & 2.177440 & $-\odot .943159$ \\
\hline 28 & 8 & $\odot$ & 4.062958 & -2.351585 & $-\odot .330185$ \\
\hline 29 & 1 & $\odot$ & $-\odot .898355$ & -2.203787 & -0.079005 \\
\hline 30 & 1 & $\odot$ & 3.668432 & $3.090 \odot 21$ & $-\odot .869055$ \\
\hline 31 & 1 & $\Theta$ & 5.014275 & $2.10 \odot 407$ & -1.336565 \\
\hline 32 & 1 & $\odot$ & 5.027299 & $-\odot .145668$ & -0.800811 \\
\hline 33 & 8 & $\odot$ & 1.017267 & $\odot .370777$ & 1.813064 \\
\hline 34 & 1 & $\odot$ & 0.584282 & 1.257167 & 1.875375 \\
\hline 35 & 8 & $\odot$ & $-\odot .719658$ & 2.411375 & 1.916280 \\
\hline 36 & 1 & $\odot$ & -1.396228 & 1.774645 & 1.609311 \\
\hline 37 & 1 & $\Theta$ & $-\Theta .991281$ & 2.674796 & 2.800440 \\
\hline
\end{tabular}

Standard basis: Aug-CC-pVDZ (6D, 7F)

669 basis functions

80 alpha electrons $\quad 79$ beta electrons

nuclear repulsion energy 1854.4300807365 Hartrees.

NAtoms $=37$ NActive $=37$

Force inversion solution in PCM.

Polarizable Continuum Model (PCM)

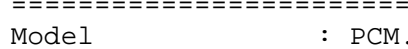

Solvent : Water, Eps $=78.355300$ Eps $($ inf $)=1.777849$

SCF Done: $E($ UWB97XD $)=-1115.61772463$ A.U. after 1 cycles NFock $=1$ Conv $=0.25 \mathrm{D}-08 \quad-\mathrm{V} / \mathrm{T}=2.0071$

$<\mathrm{S} x>=0.0000<\mathrm{Sy}\rangle=0.0000<\mathrm{Sz}>=0.5000$

$<S^{* *} 2>=0.7608 \mathrm{~S}=0.5054$

$<$ L.S $>=0.000000000000 \mathrm{E}+00$

Annihilation of the first spin contaminant:

$\mathrm{S}^{* *} 2$ before annihilation 0.7608 , after 0.7501

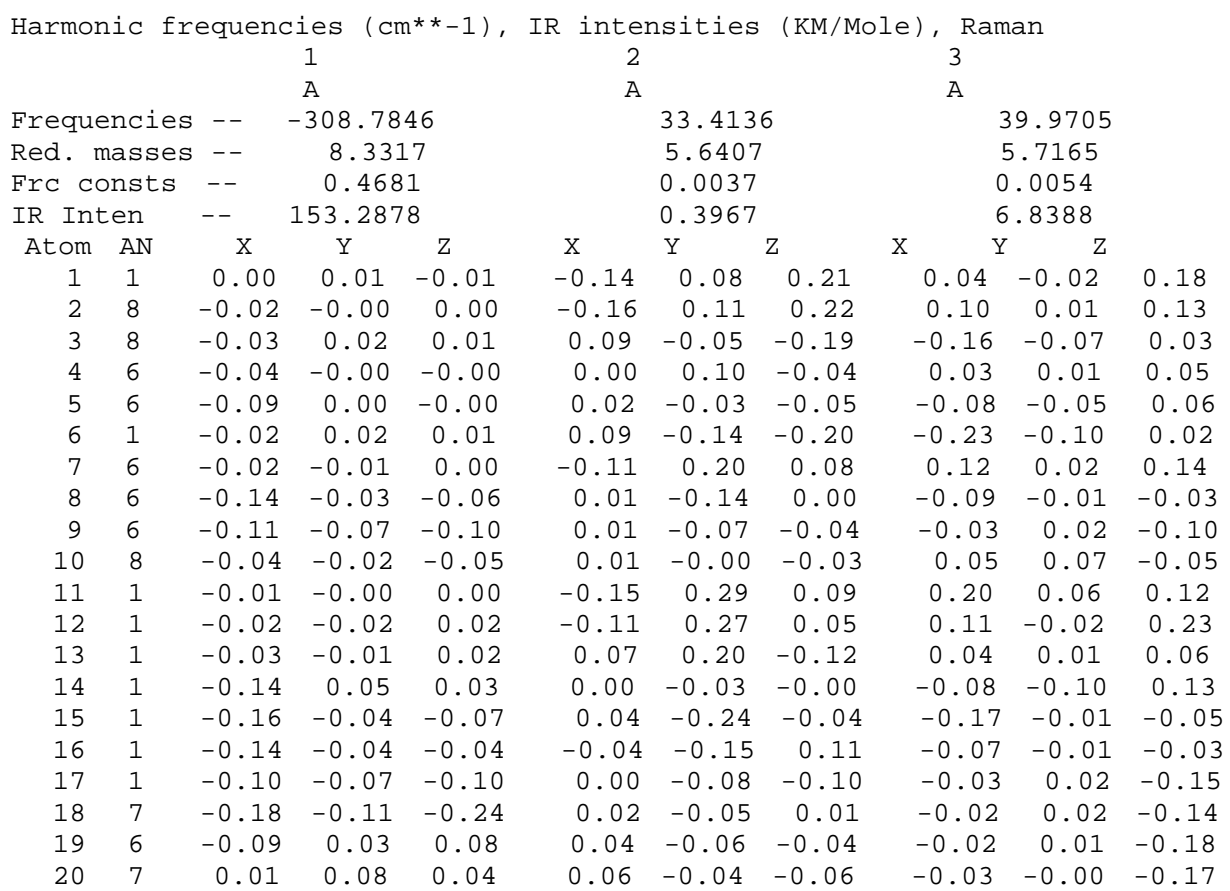




$\begin{array}{rrrrr}21 & 6 & 0.03 & 0.06 & 0.01 \\ 22 & 6 & 0.42 & 0.05 & 0.31 \\ 23 & 7 & 0.22 & 0.02 & 0.26 \\ 24 & 6 & 0.13 & 0.01 & 0.01 \\ 25 & 7 & 0.05 & 0.00 & -0.05 \\ 26 & 6 & 0.01 & 0.00 & -0.03 \\ 27 & 7 & 0.03 & 0.01 & -0.05 \\ 28 & 8 & 0.00 & -0.00 & -0.03 \\ 29 & 1 & -0.05 & -0.04 & 0.41 \\ 30 & 1 & 0.04 & 0.01 & 0.05 \\ 31 & 1 & -0.06 & 0.02 & -0.25 \\ 32 & 1 & 0.03 & -0.04 & -0.10 \\ 33 & 8 & -0.06 & -0.00 & -0.04 \\ 34 & 1 & 0.02 & 0.05 & -0.04 \\ 35 & 8 & 0.01 & -0.02 & -0.01 \\ 36 & 1 & 0.03 & -0.02 & -0.06 \\ 37 & 1 & -0.01 & -0.05 & -0.01\end{array}$

$\begin{array}{rrr}0.04 & -0.02 & -0.03 \\ -0.00 & -0.04 & 0.02 \\ -0.04 & -0.01 & 0.06 \\ -0.04 & 0.05 & 0.04 \\ -0.00 & 0.07 & -0.01 \\ 0.03 & 0.04 & -0.06 \\ -0.07 & 0.09 & 0.08 \\ 0.06 & 0.06 & -0.12 \\ 0.06 & -0.09 & -0.06 \\ -0.09 & 0.07 & 0.12 \\ -0.07 & 0.13 & 0.07 \\ -0.01 & 0.11 & -0.03 \\ 0.01 & -0.11 & 0.03 \\ 0.00 & -0.11 & 0.07 \\ 0.03 & -0.10 & 0.08 \\ 0.04 & -0.07 & 0.01 \\ -0.01 & -0.16 & 0.09\end{array}$

$\begin{array}{ccc}-0.04 & -0.01 & -0.09 \\ -0.04 & -0.02 & -0.06 \\ -0.00 & -0.01 & -0.00 \\ 0.05 & -0.01 & 0.11 \\ 0.05 & -0.01 & 0.14 \\ 0.00 & -0.01 & 0.03 \\ 0.12 & 0.01 & 0.21 \\ 0.01 & -0.01 & 0.05 \\ -0.02 & 0.01 & -0.23 \\ 0.12 & 0.01 & 0.19 \\ 0.15 & 0.00 & 0.28 \\ 0.08 & -0.00 & 0.23 \\ -0.09 & -0.04 & -0.09 \\ -0.03 & -0.02 & -0.07 \\ 0.06 & 0.08 & -0.10 \\ 0.04 & 0.09 & -0.10 \\ 0.05 & 0.10 & -0.11\end{array}$

Zero-point correction=

Thermal correction to Energy=

Thermal correction to Enthalpy=

$$
\begin{gathered}
0.291041 \text { (a.u. ) } \\
0.311621 \\
0.312565 \\
0.241424 \\
-1115.326683 \\
-1115.306103 \\
-1115.305159 \\
-1115.376300
\end{gathered}
$$

Sum of electronic and thermal Enthalpies=

Sum of electronic and thermal Free Energies=

\section{$\mathrm{S}$}

Total

$$
\begin{aligned}
& \text { E (Thermal) } \\
& \text { KCal/Mol }
\end{aligned}
$$

$\mathrm{CV}$

Cal/Mol-Kelvin

\begin{tabular}{|c|c|c|c|c|c|}
\hline \multirow{2}{*}{$\begin{array}{l}\text { Center } \\
\text { Number }\end{array}$} & \multirow{2}{*}{$\begin{array}{l}\text { Atomic } \\
\text { Number }\end{array}$} & \multirow{2}{*}{$\begin{array}{c}\text { Atomic } \\
\text { Type }\end{array}$} & \multicolumn{3}{|c|}{ Coordinates (Angstroms) } \\
\hline & & & $x$ & $\mathrm{Y}$ & Z \\
\hline$-\ldots-\ldots$ & & - & - & - & ---- \\
\hline 1 & 1 & $\odot$ & 4.390736 & -2.383747 & $\odot .059645$ \\
\hline 2 & 8 & $\odot$ & 3.886055 & -2.095665 & -0.705886 \\
\hline 3 & 8 & $\odot$ & 4.915475 & 1.695509 & 1.251218 \\
\hline 4 & 6 & $\odot$ & 4.184963 & 0.359848 & -0.581836 \\
\hline 5 & 6 & $\odot$ & 4.445325 & 0.388177 & 0.927745 \\
\hline 6 & 1 & $\odot$ & 5.003555 & 1.754501 & 2.207396 \\
\hline 7 & 6 & $\odot$ & 4.517275 & -0.956286 & -1.275035 \\
\hline 8 & 6 & $\odot$ & 3.067870 & 0.105274 & 1.513521 \\
\hline 9 & 6 & $\odot$ & 2.114128 & 0.740828 & 0.495884 \\
\hline 10 & 8 & 0 & 2.804628 & 0.684122 & -0.754818 \\
\hline 11 & 1 & $\odot$ & 4.163766 & $-\odot .894763$ & -2.311554 \\
\hline 12 & 1 & $\odot$ & 5.611153 & -1.079756 & -1.295969 \\
\hline 13 & 1 & $\odot$ & 4.787059 & 1.145017 & -1.063057 \\
\hline 14 & 1 & $\odot$ & 5.192730 & -0.360058 & 1. 229533 \\
\hline 15 & 1 & $\odot$ & 2.943288 & 0.514941 & 2.521650 \\
\hline 16 & 1 & $\odot$ & 2.886242 & -0.975406 & 1.545960 \\
\hline 17 & 1 & $\odot$ & 1.941588 & 1.802294 & 0.735016 \\
\hline 18 & 7 & $\odot$ & $\odot .821855$ & 0.108056 & $\odot .490813$ \\
\hline 19 & 6 & $\odot$ & 0.498376 & -0.620832 & -0.510146 \\
\hline 20 & 7 & $\odot$ & -0.647082 & -1.379407 & -0.549415 \\
\hline 21 & 6 & $\odot$ & -1.854281 & -0.903825 & -0.237467 \\
\hline 22 & 6 & $\odot$ & -2.305869 & $\odot .444169$ & -0.372747 \\
\hline 23 & 7 & $\odot$ & -3.535223 & $\odot .871344$ & -0.036875 \\
\hline 24 & 6 & $\odot$ & -4.411643 & -0.019702 & 0.401964 \\
\hline
\end{tabular}

Cal/Mol-Kelvin 78.262 149.729

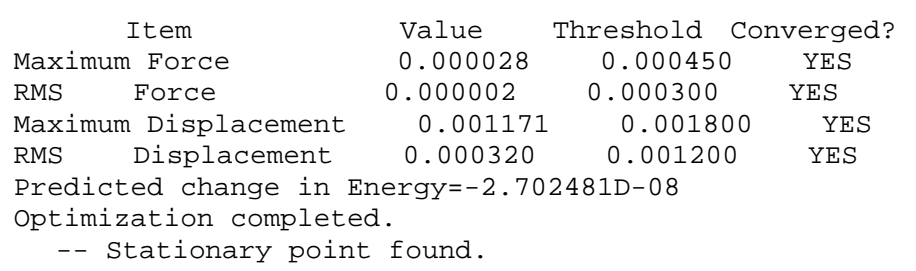

Normal termination of Gaussian 16 at Sat Jun 8 14:33:07 2019 .

Figure S2-5, Int3(C4)W1

degua-oh4w1 , rev2 . higha. log

Stoichiometry C10H16N506(2)

Standard orientation: 


\begin{tabular}{|c|c|c|c|c|c|}
\hline 25 & 7 & $\odot$ & -4.116444 & -1.341210 & $\odot .479340$ \\
\hline 26 & 6 & $\odot$ & -2.868614 & -1.884088 & $\odot .158175$ \\
\hline 27 & 7 & $\odot$ & -5.630774 & ๑. 387843 & $\odot .770002$ \\
\hline 28 & 8 & $\odot$ & -2.707961 & -3.094108 & 0.244813 \\
\hline 29 & 1 & $\odot$ & 1.180885 & -0.783922 & -1.352146 \\
\hline 30 & 1 & $\odot$ & -5.858359 & 1.367088 & $\odot .694782$ \\
\hline 31 & 1 & $\odot$ & -6.341608 & -0.250779 & 1.090406 \\
\hline 32 & 1 & $\odot$ & -4.815825 & -2.005550 & 0.790382 \\
\hline 33 & 8 & $\odot$ & -1.492567 & 1.331198 & -0.912322 \\
\hline 34 & 1 & $\odot$ & -1.948003 & 2.208409 & -0.966349 \\
\hline 35 & 8 & $\odot$ & -3.057680 & 3.526415 & -0.917408 \\
\hline 36 & 1 & $\odot$ & -3.644311 & 2.903988 & -0.455128 \\
\hline 37 & 1 & $\odot$ & -2.847992 & 4.210220 & -0.272740 \\
\hline
\end{tabular}

Standard basis: Aug-CC-pVDZ (6D, 7F)

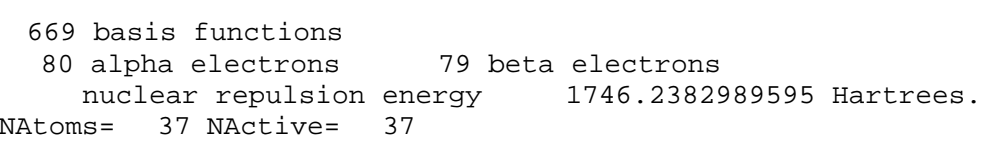

Nuclear repulsion after empirical dispersion term =

1746. 2132893481 Hartrees.

Force inversion solution in PCM.

Polarizable Continuum Model (PCM)

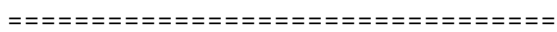

Model
Mod PCM.

Solvent : Water, Eps $=78.355300$ Eps $($ inf $)=1.777849$

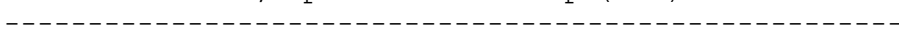

SCF Done: $E($ UWB97XD $)=-1115.64462360$ A.U. after 1 cycles NFock $=1$ Conv $=0.18 \mathrm{D}-08 \quad-\mathrm{V} / \mathrm{T}=2.0071$

$\langle\mathrm{S} x>=0.0000<\mathrm{S} y>=0.0000<\mathrm{S} z>=0.5000$

$<S^{* *} 2>=0.7648 \mathrm{~S}=0.5074$

$<$ L.S $>=0.000000000000 \mathrm{E}+00$

Annihilation of the first spin contaminant:

$\mathrm{S}^{* * 2}$ before annihilation 0.7648 , after 0.7501

Harmonic frequencies $\left(\mathrm{cm}^{*}-1\right)$, IR intensities (KM/Mole), Raman activities $\left(A^{* *} 4 / A M U\right)$, depolarization ratios for plane and unp incident light, reduced masses (AMU), force constants (mDyne/A), and normal coordinates:

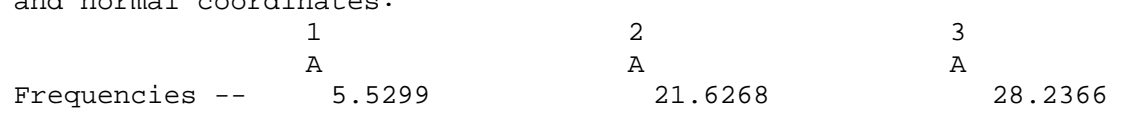

Zero-point correction=

Thermal correction to Energy=

Thermal correction to Enthalpy=

Thermal correction to Gibbs Free Energy=

Sum of electronic and zero-point Energies=

Sum of electronic and thermal Energies=

Sum of electronic and thermal Enthalpies=

๑. 290693 (a.u.)

$\odot .312726$

$\odot .313670$

$\odot .235510$

$-1115.353930$

$-1115.331898$

$-1115.330954$

Sum of electronic and thermal Free Energies $=\quad-1115.409113$

\begin{tabular}{lccc} 
& E (Thermal) & CV & \\
KCal/Mol & Cal/Mol-Kelvin & Cal/Mol \\
Total & 196.238 & 80.718 & \\
\multicolumn{1}{c}{ Item } & Value & Threshold & \multicolumn{2}{c}{ Converged? } \\
Maximum Force & 0.000040 & 0.000450 & YES \\
RMS Force & $0.00 \odot \odot \odot 7$ & $0.00030 \odot$ & YES \\
Maximum Displacement & 0.019327 & 0.001800 & NO \\
RMS Displacement & 0.005813 & 0.001200 & NO
\end{tabular}

Predicted change in Energy $=-1.265687 \mathrm{D}-07$

Normal termination of Gaussian 16 at Mon May 20 $03: 39: 242019$.

Figure S2-6, TS3(C4)W1 ........

degua-oh4w1.ts2.higha. log

Stoichiometry C10H16N506(2) 
Standard orientation:

\begin{tabular}{|c|c|c|c|c|c|}
\hline \multirow{2}{*}{$\begin{array}{l}\text { Center } \\
\text { Number }\end{array}$} & \multirow{2}{*}{$\begin{array}{l}\text { Atomic } \\
\text { Number }\end{array}$} & \multirow{2}{*}{$\begin{array}{l}\text { Atomic } \\
\text { Type }\end{array}$} & \multicolumn{3}{|c|}{ Coordinates (Angstroms) } \\
\hline & & & $\mathrm{x}$ & Y & Z \\
\hline & & & 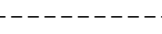 & & \\
\hline 1 & 1 & $\odot$ & 4.408589 & -2.378421 & 0.066463 \\
\hline 2 & 8 & $\odot$ & 3.895090 & -2.103343 & -0.697959 \\
\hline 3 & 8 & $\odot$ & 4.953010 & 1.714192 & 1.197567 \\
\hline 4 & 6 & $\odot$ & 4.192678 & $\odot .354749$ & -0.605645 \\
\hline 5 & 6 & $\odot$ & 4.481275 & 0.401389 & 0.898282 \\
\hline 6 & 1 & $\odot$ & 5.055342 & 1.785685 & 2.151444 \\
\hline 7 & 6 & $\odot$ & 4.515262 & -0.969120 & -1.289054 \\
\hline 8 & 6 & $\odot$ & 3.115906 & $\odot .121172$ & 1.512484 \\
\hline 9 & 6 & $\odot$ & 2.139937 & 0.741049 & 0.506297 \\
\hline 10 & 8 & $\odot$ & 2.809784 & $\odot .676066$ & -0.756723 \\
\hline 11 & 1 & $\odot$ & 4.145268 & -0.919801 & -2.320446 \\
\hline 12 & 1 & $\odot$ & 5.608952 & -1.091532 & -1.325861 \\
\hline 13 & 1 & $\odot$ & 4.785441 & 1.134332 & -1.107347 \\
\hline 14 & 1 & $\odot$ & 5.237372 & -0.340380 & 1.194505 \\
\hline 15 & 1 & $\odot$ & 3. 008996 & 0.542524 & 2.517965 \\
\hline 16 & 1 & $\odot$ & 2.938183 & -0.959554 & 1.561989 \\
\hline 17 & 1 & $\odot$ & 1.969023 & 1.804797 & $\odot .737195$ \\
\hline 18 & 7 & $\odot$ & 0.851176 & 0.102062 & 0.532558 \\
\hline 19 & 6 & $\odot$ & 0.495796 & -0.611016 & -0.468987 \\
\hline 20 & 7 & $\odot$ & -0.648292 & -1.372617 & -0.486169 \\
\hline 21 & 6 & 0 & -1.856803 & -0.876345 & -0.207847 \\
\hline 22 & 6 & $\odot$ & -2.260899 & ๑. 490925 & -0.415229 \\
\hline 23 & 7 & $\odot$ & -3.537413 & 0.906530 & -0.078808 \\
\hline 24 & 6 & $\odot$ & -4.438487 & ๑. .044761 & 0.362347 \\
\hline 25 & 7 & $\odot$ & -4.141249 & -1.268238 & 0.483237 \\
\hline 26 & 6 & 0 & -2.883512 & -1.826287 & 0.202022 \\
\hline 27 & 7 & $\odot$ & -5.664985 & 0.464155 & $\odot .688657$ \\
\hline 28 & 8 & $\odot$ & -2.743838 & -3.036728 & ○. 338212 \\
\hline 29 & 1 & $\odot$ & 1.155177 & -0.759811 & -1.332454 \\
\hline 30 & 1 & $\odot$ & -5.900347 & 1.439002 & $\odot .587665$ \\
\hline 31 & 1 & $\odot$ & -6.380733 & -0.171662 & 1. 004119 \\
\hline 32 & 1 & $\odot$ & -4.845641 & -1.920910 & 0.805428 \\
\hline 33 & 8 & $\odot$ & -1.501433 & 1. 360359 & -0.946859 \\
\hline 34 & 1 & $\odot$ & -2.205428 & 2.529668 & -0.994549 \\
\hline 35 & 8 & $\odot$ & -3.097768 & 3.158740 & -0.869885 \\
\hline 36 & 1 & $\odot$ & -3.590623 & 2.266013 & -0.404230 \\
\hline 37 & 1 & $\odot$ & -2.929898 & 3.834841 & -0.201050 \\
\hline
\end{tabular}

Standard basis: Aug-CC-pVDZ (6D, 7F)

669 basis functions

80 alpha electrons

nuclear repulsion energy 1754.7688022750 Hartrees

NAtoms $=37$ NActive $=37$

Nuclear repulsion after empirical dispersion term =

1754.7438749037 Hartrees.

Force inversion solution in PCM.

Polarizable Continuum Model (PCM)

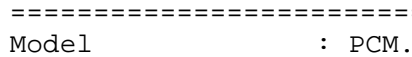

Solvent : Water, Eps $=78.355300$ Eps $($ inf $)=1.777849$

SCF Done: $E($ UWB97XD $)=-1115.62321535$ A.U. after 1 cycles

$$
\text { NFock }=1 \text { Conv }=0.46 \mathrm{D}-08 \quad-\mathrm{V} / \mathrm{T}=2.0071
$$

$<\mathrm{S} x>=0.0000<\mathrm{S} y>=0.0000<\mathrm{S} z>=0.5000$

$<S^{* *} 2>=0.7619 \mathrm{~S}=0.5059$

$<$ L.S $>=0.000000000000 \mathrm{E}+00$

Annihilation of the first spin contaminant:

$\mathrm{S}^{* * 2}$ before annihilation 0.7619 , after 0.7501

Harmonic frequencies $\left(\mathrm{cm}^{*}-1\right)$, IR intensities (KM/Mole), Raman activities $\left(A^{* *} 4 / A M U\right)$, depolarization ratios for plane and unp incident light, reduced masses (AMU), force constants (mDyne/A), and normal coordinates:

1

Frequencies - - -1112.9112
2

11.8897
3

25.9051 


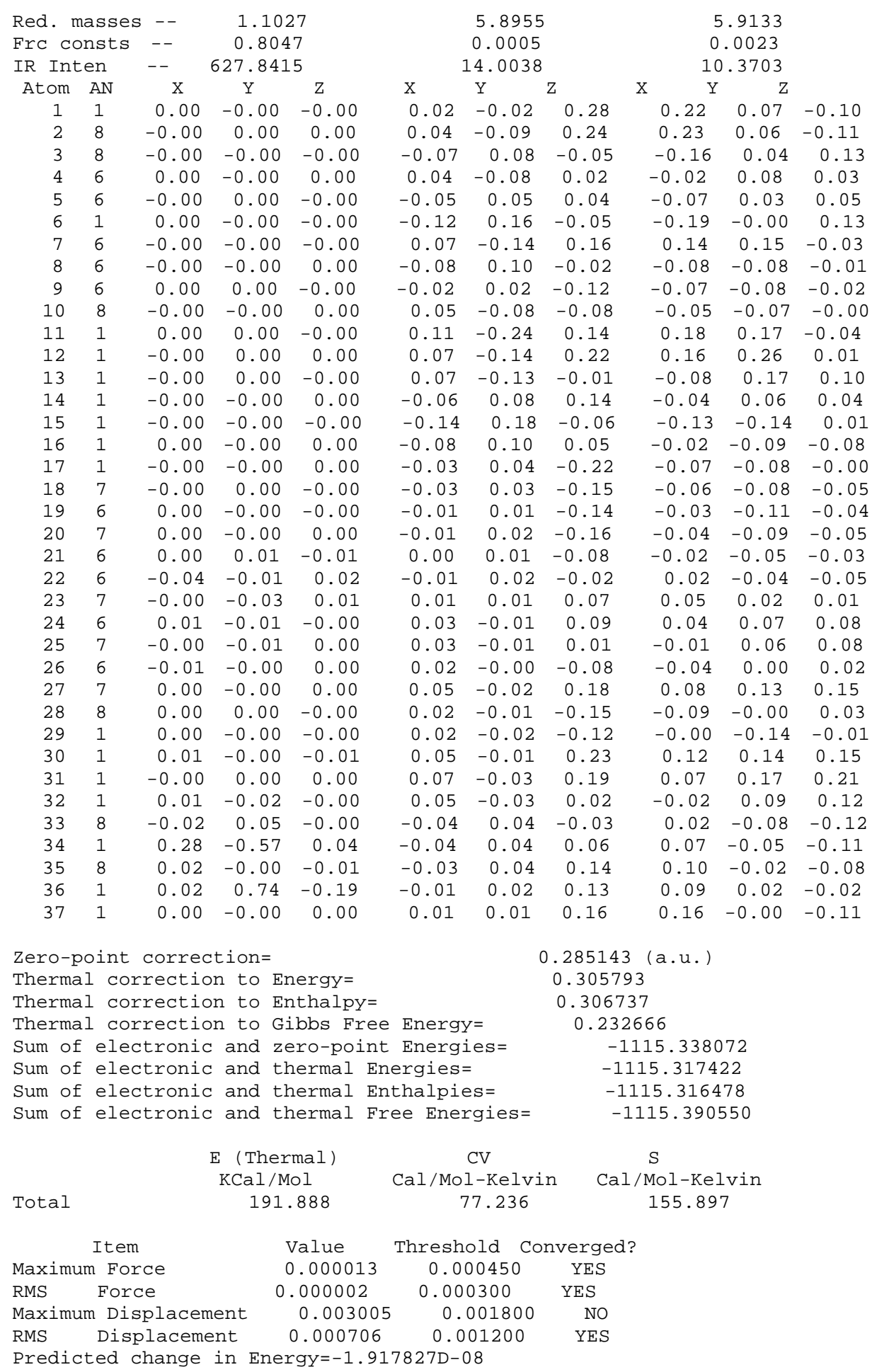

Normal termination of Gaussian 16 at Sat May 18 00:48:13 2019 .

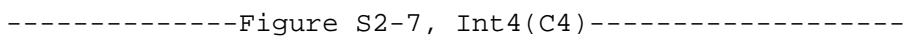

degua-oh4w1. for 2. higha . $\log$

Stoichiometry C10H16N506(2)

Standard orientation :

\begin{tabular}{|c|c|c|c|c|c|}
\hline Center & Atomic & Atomic & \multicolumn{3}{|c|}{ Coordinates (Angstroms) } \\
\hline Number & Number & Type & $\mathrm{x}$ & $\mathrm{Y}$ & Z \\
\hline & & & & & \\
\hline 1 & 1 & $\odot$ & 4.422331 & -2.367316 & 0.108670 \\
\hline 2 & 8 & $\odot$ & 3.909178 & -2.103655 & -0.659990 \\
\hline
\end{tabular}




\begin{tabular}{|c|c|c|c|c|c|}
\hline 3 & 8 & $\odot$ & 4.964069 & 1.746292 & 1.169557 \\
\hline 4 & 6 & 0 & 4.212132 & $\odot .355446$ & -0.612676 \\
\hline 5 & 6 & 0 & 4.495088 & 0.428029 & ๑.890972 \\
\hline 6 & 1 & $\odot$ & 5.063408 & 1.833990 & 2.122387 \\
\hline 7 & 6 & 0 & 4.532569 & -0.981752 & -1.270947 \\
\hline 8 & 6 & $\odot$ & 3.127674 & 0.156272 & $1.5 \odot 4703$ \\
\hline 9 & 6 & $\odot$ & 2.153903 & 0.756879 & 0.484438 \\
\hline 10 & 8 & 0 & 2.831140 & $\odot .680273$ & $-\odot .773920$ \\
\hline 11 & 1 & 0 & 4.164148 & $-\odot .950541$ & -2.303622 \\
\hline 12 & 1 & 0 & 5.626011 & -1.107299 & -1.303949 \\
\hline 13 & 1 & 0 & 4.809153 & 1.123625 & -1.126628 \\
\hline 14 & 1 & 0 & 5.250918 & $-\odot .307713$ & 1.202410 \\
\hline 15 & 1 & $\odot$ & 3.016345 & $\odot .594918$ & 2.502259 \\
\hline 16 & 1 & 0 & 2.951855 & -0.923749 & 1.572418 \\
\hline 17 & 1 & 0 & 1.974427 & 1.822367 & $\odot .699978$ \\
\hline 18 & 7 & 0 & $\odot .868950$ & $\odot .109607$ & $\odot .511964$ \\
\hline 19 & 6 & 0 & $\odot .524386$ & $-\odot .616771$ & -0.484029 \\
\hline 20 & 7 & 0 & $-\odot .612645$ & -1.388614 & $-\odot .501987$ \\
\hline 21 & 6 & $\odot$ & -1.824487 & -0.905808 & -0.212893 \\
\hline 22 & 6 & 0 & -2.219213 & $\odot .472007$ & -0.419384 \\
\hline 23 & 7 & 0 & -3.542970 & $\odot .812382$ & -0.051246 \\
\hline 24 & 6 & 0 & -4.467125 & -0.057311 & $\odot .362146$ \\
\hline 25 & 7 & $\odot$ & -4.120893 & -1.347195 & 0.466068 \\
\hline 26 & 6 & $\odot$ & -2.835499 & -1.871353 & $\odot .185114$ \\
\hline 27 & 7 & $\odot$ & -5.698749 & $\odot .343472$ & $\odot .656682$ \\
\hline 28 & 8 & 0 & -2.682422 & -3.078632 & ๑.316013 \\
\hline 29 & 1 & 0 & 1.190319 & $-\odot .769662$ & -1.341640 \\
\hline 30 & 1 & $\odot$ & -5.962459 & 1.312909 & $\odot .565181$ \\
\hline 31 & 1 & 0 & -6.405392 & -0.311602 & 0.955649 \\
\hline 32 & 1 & $\odot$ & -4.807291 & -2.026212 & 0.773028 \\
\hline 33 & 8 & 0 & -1.530518 & 1.364838 & -0.918792 \\
\hline 34 & 1 & 0 & -2.453233 & 3.036062 & -0.944268 \\
\hline 35 & 8 & 0 & -3.293085 & 3.509289 & -0.796957 \\
\hline 36 & 1 & 0 & -3.779982 & 1.805570 & -0.183359 \\
\hline 37 & 1 & 0 & -3.099874 & 4.166004 & -0.120830 \\
\hline
\end{tabular}

Standard basis: Aug-CC-pVDZ (6D, 7F)

669 basis functions

80 alpha electrons $\quad 79$ beta electrons

nuclear repulsion energy 1741.2794333681 Hartrees.

NAtoms $=37$ NActive $=37$

Nuclear repulsion after empirical dispersion term $=$ 1741.2543781715 Hartrees.

Force inversion solution in PCM.

Polarizable Continuum Model (PCM)

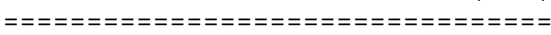

Model : PCM.

Solvent : Water, Eps $=78.355300$ Eps $($ inf $)=1.777849$

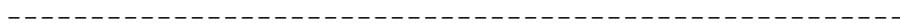

SCF Done: $E($ UWB97XD) $=-1115.64660950$ A.U. after 1 cycles NFock $=1$ Conv $=0.58 \mathrm{D}-08 \quad-\mathrm{V} / \mathrm{T}=2.0072$

$<\mathrm{S} x>=0.0000<\mathrm{Sy}>=0.0000<\mathrm{S} z>=0.5000$

$<S^{* *} 2>=0.7615 \mathrm{~S}=0.5057$

$<$ L.S $>=0.000000000000 \mathrm{E}+00$

Annihilation of the first spin contaminant:

$\mathrm{S}^{* * 2}$ before annihilation 0.7615 , after 0.7501

Harmonic frequencies $\left(\mathrm{cm}^{*}-1\right)$, IR intensities (KM/Mole), Raman activities $\left(A^{* *} 4 / A M U\right)$, depolarization ratios for plane and unp incident light, reduced masses (AMU), force constants (mDyne/A), and normal coordinates:

$\begin{array}{cccc}1 & 2 & 3 \\ \text { A } & \text { A } & \text { A }\end{array}$

Frequencies - - 14.5895

27.6946

30.3030

Zero-point correction=

Thermal correction to Energy=

Thermal correction to Enthalpy=

Thermal correction to Gibbs Free Energy=

Sum of electronic and zero-point Energies=

Sum of electronic and thermal Energies=

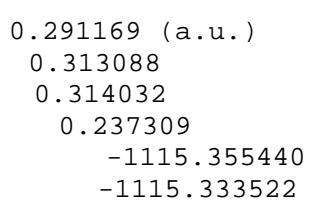


Sum of electronic and thermal Enthalpies= Sum of electronic and thermal Free Energies=

$-1115.332578$

$-1115.409301$

$$
\begin{gathered}
\text { E (Thermal) } \\
\text { KCal/Mol }
\end{gathered}
$$$$
196.466
$$

CV

Cal/Mol-Kelvin 80.574
$\mathrm{S}$

Cal/Mol-Kelvin 161.477

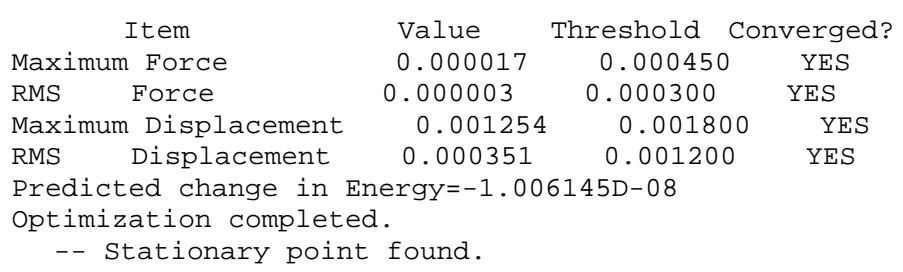

Normal termination of Gaussian 16 at Sun May 19 23:44:13 2019.

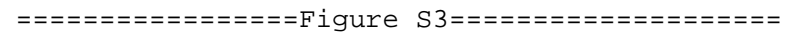

-...-Figure S3-1, Int1(C4)W2

degua-oh4w2 . for . higha. $\log$

\begin{tabular}{|c|c|c|c|c|c|}
\hline Center & Atomic & Atomic & $\mathrm{Co}$ & rdinates ( & igstroms) \\
\hline Number & Number & Type & $x$ & $Y$ & Z \\
\hline 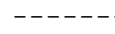 & - & - & - - - - - - - & --------- & ------- \\
\hline 1 & 1 & 0 & 4.283385 & 2.021058 & -1.289697 \\
\hline 2 & 8 & $\odot$ & 3.668402 & 2.204108 & -0.574334 \\
\hline 3 & 8 & $\odot$ & 4.665835 & -1.933401 & $\odot .468382$ \\
\hline 4 & 6 & $\odot$ & 3.890733 & ๑. 265031 & ๑. 938019 \\
\hline 5 & 6 & $\odot$ & 4.267730 & -0.724434 & -0.167986 \\
\hline 6 & 1 & $\odot$ & 4.875806 & -2.580510 & -0.211983 \\
\hline 7 & 6 & $\odot$ & 4.214222 & 1.724964 & ๑. 649607 \\
\hline 8 & 6 & $\odot$ & 2.961855 & - ๑. 881810 & -0.942266 \\
\hline 9 & 6 & $\odot$ & 1.901951 & -0.708637 & 0.146659 \\
\hline 10 & 8 & $\odot$ & 2.479436 & ๑. 099576 & 1.150797 \\
\hline 11 & 1 & $\odot$ & 3.776545 & 2.336928 & 1.447187 \\
\hline 12 & 1 & $\odot$ & 5.305291 & 1.860203 & ๑. 675924 \\
\hline 13 & 1 & $\odot$ & 4.403975 & -0.016926 & 1.867141 \\
\hline 14 & 1 & $\odot$ & 5.081617 & -0.341882 & -0.799273 \\
\hline 15 & 1 & $\odot$ & 2.878903 & -1.849160 & -1.446821 \\
\hline 16 & 1 & $\odot$ & 2.869321 & $-\odot .087815$ & -1.690845 \\
\hline 17 & 1 & $\odot$ & 1.584469 & -1.668293 & 0.571391 \\
\hline 18 & 7 & $\odot$ & 0.682838 & -0.067148 & -0.319837 \\
\hline 19 & 6 & $\odot$ & 0.570911 & 1. 208349 & -0.849348 \\
\hline 20 & 7 & $\odot$ & -0.650783 & 1.537172 & -1.173116 \\
\hline 21 & 6 & $\odot$ & -1.405045 & ○. 448899 & -0.794345 \\
\hline 22 & 6 & 0 & -0.571490 & -0.588211 & -0.301082 \\
\hline 23 & 7 & 0 & -0.922836 & -1.820935 & ๑. 061282 \\
\hline 24 & 6 & $\odot$ & -2.214153 & -2.074866 & -0.127393 \\
\hline 25 & 7 & $\odot$ & -3.087654 & -1.180679 & -0.690024 \\
\hline 26 & 6 & 0 & -2.776817 & 0.122423 & -1.098457 \\
\hline 27 & 7 & 0 & -2.699426 & -3.265446 & ๑. 235786 \\
\hline 28 & 8 & $\odot$ & -3.625488 & ๑. 826656 & -1.629396 \\
\hline 29 & 1 & 0 & 1.454001 & 1.832663 & -0.957681 \\
\hline 30 & 1 & $\odot$ & -2.068019 & -3.937190 & 0.644265 \\
\hline 31 & 1 & $\odot$ & -3.669773 & -3.514555 & $\odot .125698$ \\
\hline 32 & 1 & 0 & -4.056251 & -1.450589 & -0.816904 \\
\hline 33 & 8 & $\odot$ & -1.799824 & 1.045220 & 1.330048 \\
\hline 34 & 1 & $\odot$ & -2.447367 & ๑. 384196 & 1.623089 \\
\hline 35 & 8 & 0 & -4.803940 & ๑. 247138 & 1.668509 \\
\hline 36 & 1 & 0 & -5.168827 & ๑. 362506 & 2.550282 \\
\hline 37 & 1 & 0 & -4.635811 & 1.153651 & 1.349498 \\
\hline 38 & 1 & 0 & -2.984930 & 2.243205 & 0.874468 \\
\hline 39 & 8 & $\odot$ & -3.817081 & 2.711325 & ๑. 620594 \\
\hline 40 & 1 & $\odot$ & -3.915422 & 2.476542 & -0.311625 \\
\hline
\end{tabular}

Stoichiometry C10H18N507(2)

Standard basis: Aug-CC-pVDZ (6D, 7F)

712 basis functions

85 alpha electrons

84 beta electrons 
nuclear repulsion energy 2016.2388611210 Hartrees.

NAtoms $=40$ NActive $=40$

Force inversion solution in PCM.

Polarizable Continuum Model (PCM)

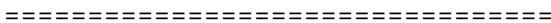

Model
: PCM.

Solvent : Water, Eps $=78.355300$ Eps $($ inf $)=1.777849$

SCF Done: $E($ UWB97XD $)=-1192.06572010$ A.U. after 2 cycles NFock $=2$ Conv $=0.65 \mathrm{D}-09 \quad-\mathrm{V} / \mathrm{T}=2.0071$

$<\mathrm{S} x>=0.0000<\mathrm{S} y>=0.0000<\mathrm{S} z>=0.5000$

$<\mathrm{S}^{* *} 2>=0.7612 \mathrm{~S}=0.5056$

$<$ L.S $>=0.000000000000 \mathrm{E}+00$

Annihilation of the first spin contaminant:

$\mathrm{S}^{* *} 2$ before annihilation 0.7612 , after 0.7501

Harmonic frequencies $\left(\mathrm{cm}^{*}{ }^{*}-1\right)$, IR intensities (KM/Mole), Raman

$\begin{array}{ccccc} & 1 & 2 & 3 & \\ \text { Frequencies - - } & 12.0868 & \text { A } & \text { A } & \\ \text { Fre.7072 } & & 20.5827 & & 30.7072\end{array}$

Zero-point correction=

Thermal correction to Energy=

0.315588 (a.u.)

Thermal correction to Enthalpy=

Thermal correction to Gibbs Free Energy=

Sum of electronic and zero-point Energies=

0.340614

0.341559

0.258087

$-1191.750132$

$-1191.725106$

$-1191.724162$

Sum of electronic and thermal Enthalpies=

$-1191.807633$

Sum of electronic and thermal Free Energies=

\section{$\mathrm{S}$}

$$
\begin{gathered}
\text { E (Thermal) } \\
\text { KCal/Mol }
\end{gathered}
$$

\begin{tabular}{lcccc} 
& KCal/Mol & Cal/Mol-Kelvin & Cal/Mol \\
Total & 213.739 & 90.773 & \\
\multicolumn{1}{c}{ Item } & Value & Threshold & Converged? \\
Maximum Force & 0.000012 & 0.000450 & YES \\
RMS Force & 0.000003 & 0.000300 & YES \\
Maximum Displacement & 0.005927 & 0.001800 & NO \\
RMS Displacement & 0.001021 & 0.001200 & YES
\end{tabular}

Predicted change in Energy $=-1.522051 \mathrm{D}-\odot 8$

Normal termination of Gaussian 16 at Fri Jun 14 05:34:42 2019.

$$
\text { -...-. Figure S3-2, TS1(C4)W2 -.... }
$$

degua-oh4w2. higha. $\log$

\begin{tabular}{|c|c|c|c|c|c|}
\hline \multirow{2}{*}{$\begin{array}{l}\text { Center } \\
\text { Number }\end{array}$} & \multirow{2}{*}{$\begin{array}{l}\text { Atomic } \\
\text { Number }\end{array}$} & \multirow{2}{*}{$\begin{array}{c}\text { Atomic } \\
\text { Type }\end{array}$} & \multicolumn{3}{|c|}{ Coordinates (Angstroms) } \\
\hline & & & $x$ & $\mathrm{Y}$ & Z \\
\hline & & & - & - & - - - - \\
\hline 1 & 1 & $\odot$ & 4.356987 & 1.706180 & -1.390470 \\
\hline 2 & 8 & $\odot$ & 3.767992 & 1.994457 & -0.687865 \\
\hline 3 & 8 & $\odot$ & 4.336483 & -2.174528 & $\odot .545594$ \\
\hline 4 & 6 & $\odot$ & 3.782227 & 0.109964 & 0.907537 \\
\hline 5 & 6 & $\odot$ & 4.068653 & -0.961277 & -0.148258 \\
\hline 6 & 1 & $\odot$ & 4.482497 & -2.869638 & -0.103459 \\
\hline 7 & 6 & $\odot$ & 4.255898 & 1.515188 & 0.560312 \\
\hline 8 & 6 & $\odot$ & 2.762110 & -1.019942 & -0.933555 \\
\hline 9 & 6 & $\odot$ & 1.711921 & $-\odot .689970$ & ๑. 128463 \\
\hline 10 & 8 & $\odot$ & 2.359845 & ๑. .098509 & 1.105844 \\
\hline 11 & 1 & $\odot$ & 3.879500 & 2.203253 & 1.326495 \\
\hline 12 & 1 & $\odot$ & 5.354991 & 1.538896 & 0.590593 \\
\hline 13 & 1 & $\odot$ & 4.254743 & $-\odot .179951$ & 1.855709 \\
\hline 14 & 1 & $\odot$ & 4.923001 & -0.690941 & -0.784024 \\
\hline 15 & 1 & $\odot$ & 2.585400 & -1.994233 & -1.399091 \\
\hline 16 & 1 & $\odot$ & 2.757863 & -0.252604 & -1.715188 \\
\hline 17 & 1 & $\odot$ & 1. 290862 & -1.590970 & 0.589679 \\
\hline 18 & 7 & $\odot$ & 0.572929 & 0.043715 & -0.398214 \\
\hline 19 & 6 & $\odot$ & 0.583130 & 1.311031 & -0.897158 \\
\hline
\end{tabular}

Stoichiometry C10H18N507(2)

Standard orientation: 


\begin{tabular}{|c|c|c|c|c|c|}
\hline 20 & 7 & 0 & -0.614334 & 1.762769 & -1.248204 \\
\hline 21 & 6 & 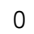 & -1.451403 & 0.744010 & -0.946722 \\
\hline 22 & 6 & 0 & -0.753179 & -0.335902 & -0.314102 \\
\hline 23 & 7 & $\odot$ & -1.189053 & -1.610544 & $-\odot .207196$ \\
\hline 24 & 6 & 0 & -2.473657 & -1.745606 & -0.475663 \\
\hline 25 & 7 & 0 & -3.283932 & -0.713833 & -0.903371 \\
\hline 26 & 6 & 0 & -2.866469 & $\odot .586187$ & -1.190492 \\
\hline 27 & 7 & 0 & -3.053504 & -2.944447 & -0.334017 \\
\hline 28 & 8 & 0 & -3.653761 & 1.432832 & -1.590509 \\
\hline 29 & 1 & 0 & 1.514663 & 1.864516 & -0.984775 \\
\hline 30 & 1 & $\odot$ & -2.489900 & -3.714106 & - ๑. . \\
\hline 31 & 1 & 0 & -4.016501 & -3.116295 & -0.574910 \\
\hline 32 & 1 & 0 & -4.273695 & -0.887076 & -1.033546 \\
\hline 33 & 8 & 0 & -1.181596 & 0.492953 & 1.484596 \\
\hline 34 & 1 & 0 & -1.837164 & -0.155632 & 1.781814 \\
\hline 35 & 8 & $\odot$ & -4.119412 & -0.306432 & 2.148992 \\
\hline 36 & 1 & 0 & -4.315028 & -0.350777 & 3.089100 \\
\hline 37 & 1 & $\odot$ & -3.980263 & $\odot .642437$ & 1.969839 \\
\hline 38 & 1 & $\odot$ & -2.354438 & 1.774096 & 1.448963 \\
\hline 39 & 8 & 0 & -3.191205 & 2.298490 & 1.520872 \\
\hline 40 & 1 & $\odot$ & -3.420426 & 2.536881 & 0.616221 \\
\hline
\end{tabular}

Standard basis: Aug-CC-pVDZ (6D, 7F)

712 basis functions

85 alpha electrons

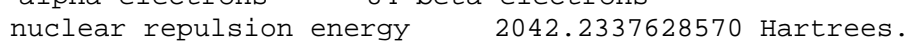
NAtoms $=40$

Force inversion solution in PCM.

Polarizable Continuum Model (PCM)

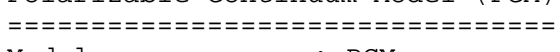

Model : PCM.

Atomic radii : UFF (Universal Force Field).

Solvent : Water, Eps $=78.355300$ Eps $($ inf $)=1.777849$

- - - - - - - - - - - - - - - - - - - - - - - - - - -

SCF Done: $E($ UWB97XD $)=-1192.05919107$ A.U. after 1 cycles NFock $=1$ Conv $=0.45 \mathrm{D}-08 \quad-\mathrm{V} / \mathrm{T}=2.0071$

$<\mathrm{S} X>=0.0000<\mathrm{Sy}>=0.0000<\mathrm{S} z>=0.5000$

$<S^{* *} 2>=0.7715 \mathrm{~S}=0.5107$

$<$ L.S $>=0.000000000000 \mathrm{E}+00$

Annihilation of the first spin contaminant:

$\mathrm{S}^{* * 2}$ before annihilation 0.7715 , after 0.7503

Harmonic frequencies $\left(\mathrm{cm}^{* *}-1\right)$, IR intensities (KM/Mole), Raman

\begin{tabular}{|c|c|c|c|c|c|c|c|c|c|c|}
\hline & & & $\begin{array}{l}1 \\
A\end{array}$ & & $\begin{array}{l}2 \\
A\end{array}$ & & & $\begin{array}{l}3 \\
A\end{array}$ & & \\
\hline Frequ & ncies & - & -294.30 & & & 20.027 & & & 30.2930 & \\
\hline Red. & asses & -- & 11.12 & & & 6.3185 & & & 5.4305 & \\
\hline Frc C & nsts & - - & 0.567 & & & 0.0015 & & & .0029 & \\
\hline IR In & en & -- & 76.061 & & & 1.0506 & & & 2974 & \\
\hline Atom & AN & $x$ & $Y$ & Z & $x$ & $Y$ & Z & $x$ & Z & \\
\hline 1 & 1 & -0.01 & 0.00 & $-\odot . \odot \odot$ & -0.05 & 0.16 & 0.13 & 0.05 & 0.15 & $\odot .21$ \\
\hline 2 & 8 & -0.00 & -0.00 & $\odot .0 \odot$ & -0.07 & $\odot .08$ & 0.14 & -0.01 & 0.07 & 0.20 \\
\hline 3 & 8 & -0.01 & 0.00 & -0.01 & 0.05 & $\odot .02$ & -0.12 & 0.01 & -0.01 & -0.06 \\
\hline 4 & 6 & $\odot .01$ & $\odot . \odot \odot$ & 0.01 & -0.02 & -0.02 & $\odot .02$ & -0.06 & -0.04 & 0.07 \\
\hline 5 & 6 & -0.01 & 0.00 & -0.00 & 0.02 & 0.06 & -0.04 & 0.02 & 0.03 & 0.01 \\
\hline 6 & 1 & -0.02 & $\odot .0 \odot$ & -0.01 & 0.07 & $\odot .07$ & -0.16 & 0.05 & 0.04 & -0.10 \\
\hline 7 & 6 & 0.01 & $\odot .00$ & $-\odot . \odot \odot$ & -0.06 & 0.02 & 0.11 & -0.07 & -0.01 & 0.19 \\
\hline 8 & 6 & -0.03 & 0.02 & 0.02 & 0.02 & 0.07 & -0.06 & 0.06 & 0.06 & -0.06 \\
\hline 9 & 6 & -0.02 & 0.04 & 0.04 & 0.01 & -0.03 & -0.04 & -0.00 & -0.04 & -0.10 \\
\hline 10 & 8 & 0.03 & 0.01 & $\odot .04$ & -0.03 & -0.07 & $\odot .01$ & -0.07 & -0.08 & -0.01 \\
\hline 11 & 1 & 0.01 & $\odot .0 \odot$ & $-\odot . \odot \odot$ & -0.09 & -0.04 & 0.15 & -0.13 & -0.07 & 0.22 \\
\hline 12 & 1 & 0.01 & 0.01 & -0.01 & -0.06 & 0.05 & 0.12 & -0.07 & 0.01 & 0.25 \\
\hline 13 & 1 & $\odot .04$ & 0.00 & -0.01 & $-\odot .02$ & -0.06 & 0.01 & -0.10 & -0.10 & 0.07 \\
\hline 14 & 1 & -0.02 & $-\odot .0 \odot$ & -0.02 & $\odot .01$ & 0.12 & -0.02 & 0.05 & 0.09 & 0.07 \\
\hline 15 & 1 & -0.05 & 0.02 & 0.03 & 0.06 & 0.09 & -0.12 & 0.11 & 0.09 & -0.14 \\
\hline 16 & 1 & -0.03 & 0.01 & 0.01 & 0.01 & 0.11 & -0.01 & 0.09 & 0.11 & -0.01 \\
\hline 17 & 1 & -0.01 & 0.03 & 0.04 & 0.03 & -0.07 & -0.10 & 0.00 & -0.08 & -0.18 \\
\hline 18 & 7 & -0.02 & 0.05 & 0.09 & -0.01 & -0.04 & -0.01 & 0.01 & -0.04 & -0.11 \\
\hline 19 & 6 & -0.01 & -0.04 & -0.07 & -0.04 & -0.02 & 0.02 & 0.02 & -0.05 & -0.15 \\
\hline 20 & 7 & -0.01 & -0.03 & -0.14 & -0.05 & -0.03 & 0.04 & 0.02 & -0.04 & -0.14 \\
\hline 21 & 6 & -0.04 & 0.06 & -0.04 & -0.03 & -0.06 & 0.02 & $0.0 \odot$ & -0.01 & -0.08 \\
\hline
\end{tabular}




$\begin{array}{rrrrr}22 & 6 & -0.14 & 0.23 & 0.58 \\ 23 & 7 & -0.04 & 0.07 & 0.11 \\ 24 & 6 & 0.01 & 0.03 & 0.02 \\ 25 & 7 & -0.02 & 0.03 & 0.00 \\ 26 & 6 & -0.02 & 0.02 & -0.04 \\ 27 & 7 & 0.01 & 0.02 & -0.04 \\ 28 & 8 & -0.03 & 0.01 & -0.05 \\ 29 & 1 & 0.00 & -0.07 & -0.19 \\ 30 & 1 & -0.00 & 0.02 & -0.00 \\ 31 & 1 & 0.07 & -0.02 & -0.24 \\ 32 & 1 & -0.02 & 0.02 & 0.03 \\ 33 & 8 & 0.17 & -0.37 & -0.36 \\ 34 & 1 & 0.12 & -0.19 & -0.10 \\ 35 & 8 & 0.01 & -0.01 & 0.01 \\ 36 & 1 & 0.00 & -0.00 & 0.01 \\ 37 & 1 & 0.03 & -0.01 & 0.01 \\ 38 & 1 & 0.05 & -0.00 & -0.12 \\ 39 & 8 & 0.07 & -0.04 & -0.01 \\ 40 & 1 & -0.01 & 0.01 & 0.03\end{array}$

$\begin{array}{rrr}-0.01 & -0.06 & -0.01 \\ 0.01 & -0.07 & -0.03 \\ 0.01 & -0.08 & -0.02 \\ -0.01 & -0.09 & 0.0 \odot \\ -0.03 & -0.07 & 0.03 \\ 0.03 & -0.09 & -0.02 \\ -0.04 & -0.07 & 0.06 \\ -0.05 & -0.0 \odot & 0.03 \\ 0.05 & -0.09 & -0.07 \\ 0.04 & -0.11 & -0.06 \\ -0.01 & -0.10 & 0.01 \\ 0.01 & -0.10 & 0.01 \\ -0.10 & -0.01 & -0.03 \\ -0.09 & 0.31 & -0.05 \\ -0.11 & 0.32 & -0.05 \\ 0.03 & 0.29 & -0.04 \\ 0.19 & 0.07 & -0.02 \\ 0.26 & 0.19 & -0.04 \\ 0.29 & 0.17 & -0.05\end{array}$

$\begin{array}{ccc}-0.00 & -0.00 & -0.06 \\ -0.03 & 0.01 & 0.01 \\ -0.04 & 0.03 & 0.06 \\ -0.03 & 0.03 & 0.05 \\ -0.01 & 0.01 & -0.03 \\ -0.06 & 0.05 & 0.12 \\ 0.00 & 0.01 & -0.05 \\ 0.02 & -0.06 & -0.17 \\ -0.07 & 0.05 & 0.14 \\ -0.08 & 0.06 & 0.17 \\ -0.04 & 0.05 & 0.08 \\ 0.05 & 0.08 & -0.09 \\ 0.10 & 0.07 & -0.03 \\ 0.19 & -0.05 & 0.19 \\ 0.30 & -0.01 & 0.21 \\ 0.10 & -0.05 & 0.11 \\ -0.02 & 0.02 & -0.08 \\ -0.04 & -0.02 & -0.04 \\ -0.11 & -0.06 & -0.04\end{array}$

Zero-point correction=

Thermal correction to Energy=

๑. 315170 (a.u.)

Thermal correction to Enthalpy=

Thermal correction to Gibbs Free Energy=

Sum of electronic and zero-point Energies=

Sum of electronic and thermal Energies=

Sum of electronic and thermal Enthalpies=

$\odot .339464$

$\odot .340408$

0.259969

$-1191.744021$

$-1191.719727$

$-1191.718783$

$-1191.799222$

Sum of electronic and thermal Free Energies=

$\mathrm{S}$

$\begin{array}{cccc} & \text { KCal/Mol } & \text { Cal/Mol-Kelvin } & \text { Cal/Mol-Kelvin } \\ \text { Total } & 213.017 & 88.978 & 169.298\end{array}$

\begin{tabular}{lccc}
\multicolumn{1}{c}{ Item } & Value & Threshold & Converged? \\
Maximum Force & $0.000 \odot 27$ & 0.000450 & YES \\
RMS Force & $0.0000 \odot 4$ & $0.00030 \odot$ & YES \\
Maximum Displacement & 0.001516 & $0.00180 \odot$ & YES \\
RMS Displacement & 0.000245 & 0.001200 & YES \\
Predicted change in Energy=-1.719620D-08 & \\
Optimization completed. & & \\
\multicolumn{2}{c}{- - Stationary point found. }
\end{tabular}

Normal termination of Gaussian 16 at Thu Jun 13 17:29:50 2019.

........ Figure S3-3, Int2(C4)W2

degua-oh4w2.rev. higha. log

Stoichiometry C10H18N507(2)

Standard orientation:

\begin{tabular}{|c|c|c|c|c|c|}
\hline \multirow{3}{*}{$\begin{array}{l}\text { Center } \\
\text { Number }\end{array}$} & \multirow{2}{*}{$\begin{array}{l}\text { Atomic } \\
\text { Number }\end{array}$} & \multirow{2}{*}{$\begin{array}{c}\text { Atomic } \\
\text { Type }\end{array}$} & \multicolumn{3}{|c|}{ Coordinates (Angstroms) } \\
\hline & & & $\mathrm{x}$ & $\mathrm{Y}$ & Z \\
\hline & 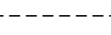 & - & ------ & ------ & ------- \\
\hline 1 & 1 & $\odot$ & -3.831523 & -1.442724 & -2.030869 \\
\hline 2 & 8 & $\odot$ & -3.303855 & -1.816751 & -1.320084 \\
\hline 3 & 8 & $\odot$ & -4.338410 & 2.009776 & 0.554084 \\
\hline 4 & 6 & $\odot$ & -3.660622 & $-\odot .270192$ & 0.574130 \\
\hline 5 & 6 & $\odot$ & -3.900854 & $\odot .961061$ & -0.303920 \\
\hline 6 & 1 & 0 & -4.439526 & 2.809122 & 0.028273 \\
\hline 7 & 6 & $\odot$ & -3.974005 & -1.609459 & -0.081043 \\
\hline 8 & 6 & $\odot$ & -2.519210 & 1.231833 & $-\odot .889690$ \\
\hline 9 & 6 & $\odot$ & -1.576890 & $\odot .776069$ & ๑. 226648 \\
\hline 10 & 8 & $\odot$ & -2.281739 & -0.215287 & 0.962425 \\
\hline 11 & 1 & $\odot$ & -3.637475 & -2.407104 & ๑. 591911 \\
\hline 12 & 1 & $\odot$ & -5.063172 & -1.702220 & - ๑ . 201937 \\
\hline 13 & 1 & $\odot$ & -4.271932 & -0.191414 & 1.483658 \\
\hline 14 & 1 & $\odot$ & -4.653795 & ๑. 769212 & -1.081159 \\
\hline 15 & 1 & $\odot$ & -2.364173 & 2.282923 & -1.152183 \\
\hline 16 & 1 & $\odot$ & -2.365137 & $\odot .622620$ & -1.786770 \\
\hline 17 & 1 & 0 & -1.308358 & 1.603264 & ○.894544 \\
\hline 18 & 7 & $\odot$ & -0.324591 & ๑. 236988 & -0.249008 \\
\hline 19 & 6 & $\odot$ & -0.177384 & -0.875643 & -0.984093 \\
\hline 20 & 7 & $\odot$ & 1.082600 & -1.272732 & -1.159276 \\
\hline 21 & 6 & $\odot$ & 1.822816 & -0.380693 & -0.462039 \\
\hline 22 & 6 & $\odot$ & ๑. 986027 & 0.596717 & ๑. 318941 \\
\hline
\end{tabular}




\begin{tabular}{|c|c|c|c|c|c|}
\hline 23 & 7 & $\odot$ & 1.346420 & 1.963296 & ๑. 086411 \\
\hline 24 & 6 & $\Theta$ & 2.618897 & 2.156241 & -0.045667 \\
\hline 25 & 7 & 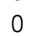 & 3.568693 & 1.137519 & -0.153349 \\
\hline 26 & 6 & $\odot$ & 3.242316 & -0.163094 & -0.515147 \\
\hline 27 & 7 & 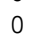 & 3.142094 & 3.414358 & -0.043997 \\
\hline 28 & 8 & $\theta$ & 4.112219 & -0.980408 & -0.831432 \\
\hline 29 & 1 & $\odot$ & -1.040761 & -1.396773 & -1.392147 \\
\hline 30 & 1 & 0 & 2. 461863 & 4.161021 & -0.057877 \\
\hline 31 & 1 & $\odot$ & 3.986603 & 3.590538 & -0.570219 \\
\hline 32 & 1 & $\odot$ & 4.552991 & 1.373839 & -0.147291 \\
\hline 33 & 8 & 0 & 1.006471 & 0.373404 & 1.708031 \\
\hline 34 & 1 & $\odot$ & 1. 051761 & -0.594886 & 1.870200 \\
\hline 35 & 8 & $\odot$ & 1.329830 & -2.337296 & 2.101022 \\
\hline 36 & 1 & 0 & 0.514190 & -2.846418 & 2.128820 \\
\hline 37 & 1 & $\odot$ & 1.859535 & -2.746147 & 1.385586 \\
\hline 38 & 1 & $\odot$ & 2.041791 & -3.118390 & -0.694381 \\
\hline 39 & 8 & $\Theta$ & 2.750395 & -3.398829 & -0.094883 \\
\hline 40 & 1 & $\theta$ & 3.434760 & -2.736380 & -0.295631 \\
\hline
\end{tabular}

Standard basis: Aug-CC-pVDZ (6D, 7F)

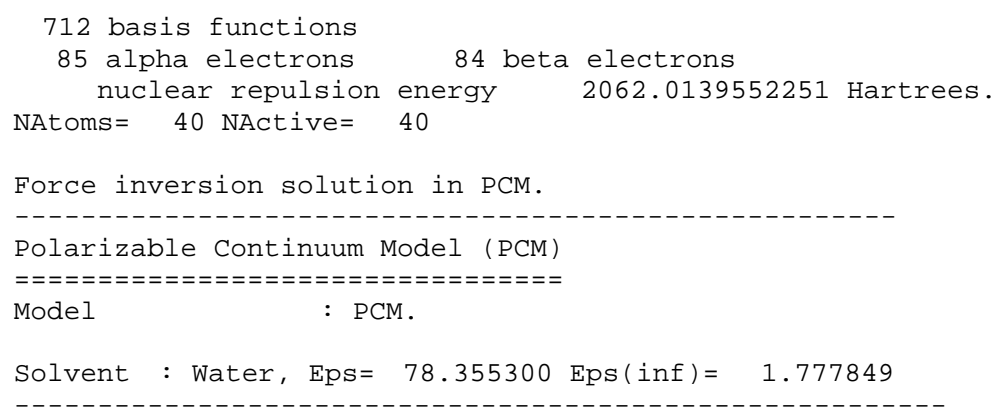

SCF Done: $E($ UWB97XD $)=-1192.09464340$ A.U. after 2 cycles NFock $=2$ Conv $=0.18 \mathrm{D}-08 \quad-\mathrm{V} / \mathrm{T}=2.0070$

$\langle S x>=0.0000\langle$ Sy $>=0.0000\langle$ Sz $>=0.5000$

$<S^{* *} 2>=0.7600 \mathrm{~S}=0.5050$

$<$ L.S $>=0.000000000000 \mathrm{E}+00$

Annihilation of the first spin contaminant:

$\mathrm{S}^{* * 2}$ before annihilation $\odot .7600$, after 0.7501

Harmonic frequencies $\left(\mathrm{cm}^{*}-1\right)$, IR intensities (KM/Mole), Raman

$\begin{array}{ccccc} & 1 & 2 & & \\ & \text { A } & \text { A } & \text { A } & \\ \text { Frequencies - - } & 19.9504 & \text { 31.9606 } & & 43.2283\end{array}$

Zero-point correction=

Thermal correction to Energy=

Thermal correction to Enthalpy=

Thermal correction to Gibbs Free Energy=

Sum of electronic and zero-point Energies=

Sum of electronic and thermal Energies=

Sum of electronic and thermal Enthalpies=

0.318953 (a.u.)

0.342387
0.343331

0.265479

$-1191.775690$

$-1191.752256$

$-1191.751312$

Sum of electronic and thermal Free Energies=

$-1191.829164$

\begin{tabular}{|c|c|c|c|}
\hline $\mathrm{E}(\mathrm{KC}$ & $\begin{array}{l}\text { ermal) } \\
\text { /Mol } \\
14.851\end{array}$ & $\begin{array}{c}\mathrm{CV} \\
\text { Cal/Mol-Kelvin } \\
87.879\end{array}$ & $\begin{array}{c}\mathrm{S} \\
\mathrm{Cal} / \mathrm{Mol}-\mathrm{Kelvin} \\
163.854\end{array}$ \\
\hline Item & Value & \multicolumn{2}{|c|}{ Threshold Converged? } \\
\hline Maximum Force & $\odot .00 \odot \odot 14$ & $\odot .00 \odot 45 \odot$ & \multirow{2}{*}{$\begin{array}{l}\text { YES } \\
\text { YES }\end{array}$} \\
\hline Force & 0.000003 & $\odot .000300$ & \\
\hline Maximum Displacement & 0.002668 & 0.001800 & NO \\
\hline RMS Displacement & 0.000539 & 0.001200 & YES \\
\hline Predicted change in & lergy $=-3.28$ & $32749 D-08$ & \\
\hline
\end{tabular}

Figure S3-4, TS2(C4)W2 ...........

degua-oh4w2.ts1.higha. log

Stoichiometry C10H18N507(2) 
Standard orientation:

\begin{tabular}{|c|c|c|c|c|c|}
\hline \multirow{2}{*}{$\begin{array}{l}\text { Center } \\
\text { Number }\end{array}$} & \multirow{2}{*}{$\begin{array}{l}\text { Atomic } \\
\text { Number }\end{array}$} & \multirow{2}{*}{$\begin{array}{l}\text { Atomic } \\
\text { Type }\end{array}$} & \multicolumn{3}{|c|}{ Coordinates (Angstroms) } \\
\hline & & & $x$ & Y & Z \\
\hline & & & 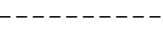 & 40 & \\
\hline 1 & 1 & $\odot$ & -3.896327 & -2.019841 & -1.402598 \\
\hline 2 & 8 & $\odot$ & -3.470664 & -2.031843 & -0.541056 \\
\hline 3 & 8 & $\odot$ & -3.623759 & 2.310540 & -1.166183 \\
\hline 4 & 6 & $\odot$ & -3.437062 & 0.335290 & 0.143314 \\
\hline 5 & 6 & $\odot$ & -3.432385 & 0.904388 & -1.278643 \\
\hline 6 & 1 & $\odot$ & -3.580748 & 2.694268 & -2.047470 \\
\hline 7 & 6 & $\odot$ & -4.066101 & -1.044020 & $\odot .288545$ \\
\hline 8 & 6 & $\odot$ & -2.040821 & 0.549231 & -1.791294 \\
\hline 9 & 6 & $\odot$ & -1.174284 & ๑. 668686 & -0.537943 \\
\hline 10 & 8 & $\odot$ & -2.056542 & ๑. 285322 & 0.553416 \\
\hline 11 & 1 & $\odot$ & -3.933718 & -1.382111 & 1.323759 \\
\hline 12 & 1 & $\odot$ & -5.146504 & -0.960136 & ๑. 101183 \\
\hline 13 & 1 & $\odot$ & -3.969738 & 1.027319 & $\odot .809247$ \\
\hline 14 & 1 & $\odot$ & -4.229484 & $\odot .462585$ & -1.893014 \\
\hline 15 & 1 & $\odot$ & -1.698479 & 1.216759 & -2.588173 \\
\hline 16 & 1 & $\odot$ & -2.027155 & -0.480812 & -2.163732 \\
\hline 17 & 1 & $\odot$ & -0.890208 & 1.716240 & -0.369442 \\
\hline 18 & 7 & $\odot$ & ๑ . 030121 & -0.091891 & -0.531655 \\
\hline 19 & 6 & $\odot$ & ๑ . 062819 & -1.398674 & -0.629106 \\
\hline 20 & 7 & $\odot$ & 1. 245611 & -2.024534 & -0.485353 \\
\hline 21 & 6 & 0 & 2.176861 & -1.129886 & -0.078200 \\
\hline 22 & 6 & $\odot$ & 1.735376 & ๑. 094449 & 0.602426 \\
\hline 23 & 7 & $\odot$ & 2.366100 & 1.275328 & 0.434812 \\
\hline 24 & 6 & $\odot$ & 3.540683 & 1.247140 & -0.162230 \\
\hline 25 & 7 & $\odot$ & 4.170929 & $\odot .087577$ & -0.522344 \\
\hline 26 & 6 & 0 & 3.530391 & -1.162191 & -0.575347 \\
\hline 27 & 7 & $\odot$ & 4.188375 & 2.397643 & -0.387891 \\
\hline 28 & 8 & $\odot$ & 4.123337 & -2.124423 & -1.054808 \\
\hline 29 & 1 & $\odot$ & -0.826184 & -1.999361 & -0.839989 \\
\hline 30 & 1 & $\odot$ & 3.773619 & 3.254861 & -0.056990 \\
\hline 31 & 1 & $\odot$ & 5.110878 & 2.429703 & -0.791817 \\
\hline 32 & 1 & $\odot$ & 5.107677 & 0.117717 & -0.905962 \\
\hline 33 & 8 & $\odot$ & 1.108431 & $-\odot .076870$ & 1.774408 \\
\hline 34 & 1 & $\odot$ & 0.657871 & $\odot .755616$ & 2.065488 \\
\hline 35 & 8 & $\odot$ & -0.647834 & 1.839222 & 2.391414 \\
\hline 36 & 1 & $\odot$ & -1.362464 & 1.355812 & 1.937068 \\
\hline 37 & 1 & $\odot$ & -0.912025 & 1.885536 & 3.315263 \\
\hline 38 & 1 & $\odot$ & -0.234740 & -1.546966 & 2.285568 \\
\hline 39 & 8 & $\odot$ & -1.151304 & -1.851011 & 2.303592 \\
\hline 40 & 1 & $\odot$ & -1.576384 & -1.230027 & 1.693230 \\
\hline
\end{tabular}

Standard basis: Aug-CC-pVDZ (6D, 7F)

712 basis functions

85 alpha electrons

nuclear repulsion energy 2060.6999771135 Hartrees.

NAtoms $=40$ NActive $=40$

Force inversion solution in PCM.

Polarizable Continuum Model (PCM)

=ニニニニニニニニニニニニニニニニニニニニニニニニニニニニニニニニ

Model : PCM.

Solvent: Water, Eps $=78.355300$ Eps $($ inf $)=1.777849$

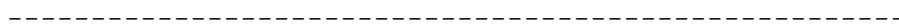

SCF Done: $E($ UWB97XD) $=-1192.05621352$ A.U. after 1 cycles

$$
\text { NFock }=1 \text { Conv }=0.24 \mathrm{D}-08 \quad-\mathrm{V} / \mathrm{T}=2.0071
$$

$<\mathrm{S} x>=0.0000<\mathrm{Sy}>=0.0000$

$\langle\mathrm{SZ}\rangle=0.5000<\mathrm{S} * * 2\rangle=0.7605 \mathrm{~S}=0.5052$

$<$ L.S $>=0.000000000 \odot \odot \odot E+\odot \odot$

Annihilation of the first spin contaminant:

$\mathrm{S}^{* * 2}$ before annihilation 0.7605 , after 0.7501

Harmonic frequencies $\left(\mathrm{cm}^{*}-1\right)$, IR intensities (KM/Mole), Raman activities $\left(A^{* *} 4 / A M U\right)$, depolarization ratios for plane and unp incident light, reduced masses (AMU), force constants (mDyne/A), and normal coordinates:

1 


\begin{tabular}{|c|c|c|c|c|}
\hline \multicolumn{2}{|c|}{ Frequencies } & - & \multicolumn{2}{|c|}{-299.1474} \\
\hline Red. $\mathrm{n}$ & asses & - & 8.402 & \\
\hline Frc $\mathrm{co}$ & nsts & -- & 0.443 & \\
\hline IR Int & en & -- & 138.905 & \\
\hline Atom & AN & $x$ & $\mathrm{Y}$ & Z \\
\hline 1 & 1 & 0.01 & 0.01 & -0.01 \\
\hline 2 & 8 & -0.01 & -0.01 & $\odot .0 \odot$ \\
\hline 3 & 8 & -0.03 & $\odot .02$ & 0.01 \\
\hline 4 & 6 & -0.05 & -0.00 & -0.00 \\
\hline 5 & 6 & -0.10 & 0.01 & -0.00 \\
\hline 6 & 1 & -0.02 & $\odot .02$ & $\odot .01$ \\
\hline 7 & 6 & -0.03 & -0.01 & $\odot .0 \odot$ \\
\hline 8 & 6 & -0.14 & -0.01 & -0.07 \\
\hline 9 & 6 & -0.12 & -0.04 & -0.12 \\
\hline 10 & 8 & -0.05 & -0.01 & -0.05 \\
\hline 11 & 1 & -0.01 & -0.01 & 0.00 \\
\hline 12 & 1 & -0.03 & -0.04 & 0.01 \\
\hline 13 & 1 & -0.04 & -0.02 & 0.02 \\
\hline 14 & 1 & -0.15 & $\odot . \odot 4$ & 0.04 \\
\hline 15 & 1 & -0.16 & -0.02 & -0.08 \\
\hline 16 & 1 & -0.15 & -0.02 & -0.06 \\
\hline 17 & 1 & -0.11 & -0.04 & -0.11 \\
\hline 18 & 7 & -0.17 & -0.03 & -0.24 \\
\hline 19 & 6 & -0.09 & 0.01 & 0.07 \\
\hline 20 & 7 & 0.01 & 0.07 & 0.07 \\
\hline 21 & 6 & $\odot .04$ & 0.05 & 0.03 \\
\hline 22 & 6 & $\odot .42$ & -0.04 & 0.31 \\
\hline 23 & 7 & 0.23 & -0.05 & 0.26 \\
\hline 24 & 6 & $\odot .14$ & $\odot .0 \odot$ & 0.01 \\
\hline 25 & 7 & $\odot .05$ & 0.01 & -0.06 \\
\hline 26 & 6 & 0.01 & 0.01 & -0.03 \\
\hline 27 & 7 & $\odot .03$ & $\odot .03$ & -0.05 \\
\hline 28 & 8 & $\odot . \odot \odot$ & 0.01 & -0.03 \\
\hline 29 & 1 & -0.04 & -0.17 & 0.36 \\
\hline 30 & 1 & 0.05 & -0.00 & 0.06 \\
\hline 31 & 1 & -0.05 & $\odot . \odot 9$ & -0.23 \\
\hline 32 & 1 & $\odot .03$ & -0.01 & -0.11 \\
\hline 33 & 8 & -0.06 & 0.01 & -0.03 \\
\hline 34 & 1 & $\odot .02$ & 0.06 & -0.04 \\
\hline 35 & 8 & $\odot .01$ & -0.01 & -0.02 \\
\hline 36 & 1 & $\odot .03$ & $\odot . \odot \odot$ & -0.06 \\
\hline 37 & 1 & -0.01 & -0.05 & -0.02 \\
\hline 38 & 1 & -0.00 & -0.05 & -0.01 \\
\hline 39 & 8 & -0.02 & 0.00 & $\odot .0 \odot$ \\
\hline 40 & 1 & 0.01 & $\odot . \odot \odot$ & -0.01 \\
\hline
\end{tabular}

\begin{tabular}{|c|c|c|}
\hline & $\begin{array}{r}6.773 \\
5.5118 \\
0.0001 \\
1.0449\end{array}$ & \\
\hline$x$ & $Y$ & Z \\
\hline-0.23 & $\odot .03$ & 0.24 \\
\hline-0.22 & 0.05 & 0.24 \\
\hline 0.06 & -0.00 & -0.14 \\
\hline 0.02 & 0.10 & 0.03 \\
\hline$\odot . \odot \odot$ & -0.02 & -0.02 \\
\hline 0.04 & -0.09 & -0.18 \\
\hline-0.10 & 0.17 & 0.17 \\
\hline-0.02 & -0.12 & -0.03 \\
\hline 0.01 & $-\odot .04$ & -0.06 \\
\hline 0.03 & $-\odot . \odot \odot$ & -0.02 \\
\hline-0.09 & 0.25 & 0.20 \\
\hline-0.10 & 0.25 & 0.21 \\
\hline 0.11 & 0.21 & -0.01 \\
\hline-0.03 & -0.04 & 0.04 \\
\hline-0.02 & -0.20 & -0.10 \\
\hline-0.08 & -0.15 & 0.06 \\
\hline-0.01 & -0.03 & -0.12 \\
\hline 0.02 & -0.02 & -0.03 \\
\hline 0.05 & -0.02 & -0.07 \\
\hline 0.06 & $-0.0 \odot$ & -0.06 \\
\hline 0.03 & 0.01 & -0.02 \\
\hline$-\odot . \odot \odot$ & -0.01 & -0.01 \\
\hline-0.02 & 0.00 & 0.02 \\
\hline-0.01 & $\odot .03$ & 0.05 \\
\hline 0.02 & 0.05 & 0.04 \\
\hline 0.05 & $\odot .04$ & 0.01 \\
\hline-0.02 & 0.05 & 0.08 \\
\hline 0.07 & 0.05 & 0.01 \\
\hline 0.07 & -0.04 & -0.11 \\
\hline-0.05 & 0.03 & 0.08 \\
\hline-0.02 & 0.07 & 0.09 \\
\hline$\odot .03$ & 0.07 & 0.07 \\
\hline-0.01 & -0.05 & -0.02 \\
\hline-0.01 & -0.05 & 0.00 \\
\hline$\odot . \odot \odot$ & -0.05 & $-0.0 \odot$ \\
\hline 0.03 & -0.05 & -0.05 \\
\hline-0.03 & $-\odot . \odot 9$ & -0.01 \\
\hline 0.01 & -0.08 & -0.12 \\
\hline 0.03 & -0.14 & -0.16 \\
\hline 0.01 & -0.14 & -0.13 \\
\hline
\end{tabular}

\begin{tabular}{|c|c|c|}
\hline \multicolumn{3}{|c|}{$\begin{array}{c}42.8556 \\
6.1011 \\
0.0066 \\
5.1560\end{array}$} \\
\hline$x$ & Z & \\
\hline 0.02 & -0.04 & 0.06 \\
\hline 0.07 & -0.04 & 0.04 \\
\hline-0.15 & -0.04 & 0.11 \\
\hline 0.00 & $-\odot .04$ & $\odot . \odot 7$ \\
\hline-0.10 & -0.03 & 0.08 \\
\hline-0.21 & -0.02 & 0.11 \\
\hline 0.07 & -0.07 & $\odot .08$ \\
\hline-0.11 & 0.04 & $-0.0 \odot$ \\
\hline-0.04 & 0.05 & -0.05 \\
\hline 0.03 & 0.02 & $-0.0 \odot$ \\
\hline 0.14 & -0.08 & 0.07 \\
\hline 0.06 & -0.12 & 0.15 \\
\hline 0.01 & -0.08 & 0.11 \\
\hline-0.11 & -0.05 & 0.12 \\
\hline-0.18 & 0.06 & -0.01 \\
\hline-0.09 & $\odot .04$ & -0.02 \\
\hline-0.06 & 0.05 & -0.06 \\
\hline-0.02 & 0.07 & -0.11 \\
\hline-0.03 & 0.07 & -0.13 \\
\hline-0.04 & 0.06 & -0.11 \\
\hline-0.04 & $\odot .03$ & -0.05 \\
\hline-0.03 & 0.04 & -0.07 \\
\hline 0.02 & 0.02 & -0.05 \\
\hline 0.07 & -0.04 & 0.06 \\
\hline 0.06 & -0.07 & 0.14 \\
\hline 0.01 & -0.04 & 0.08 \\
\hline 0.15 & -0.07 & 0.12 \\
\hline 0.01 & -0.08 & 0.15 \\
\hline-0.03 & $\odot . \odot 8$ & -0.16 \\
\hline 0.16 & -0.05 & 0.07 \\
\hline 0.19 & -0.12 & 0.20 \\
\hline 0.10 & -0.12 & 0.23 \\
\hline-0.07 & 0.06 & -0.09 \\
\hline-0.03 & $\odot .09$ & -0.09 \\
\hline 0.02 & 0.15 & -0.13 \\
\hline 0.01 & 0.11 & -0.07 \\
\hline 0.04 & 0.24 & -0.13 \\
\hline 0.07 & -0.05 & -0.09 \\
\hline 0.08 & -0.08 & -0.13 \\
\hline 0.07 & -0.05 & -0.09 \\
\hline
\end{tabular}

Zero-point correction=

Thermal correction to Energy=

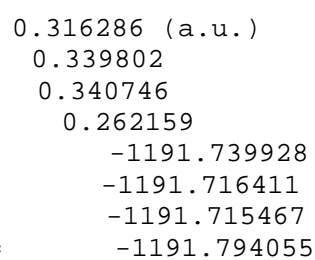




\begin{tabular}{|c|c|c|c|c|c|}
\hline \multirow{2}{*}{$\begin{array}{l}\text { Center } \\
\text { Number }\end{array}$} & \multirow{2}{*}{$\begin{array}{l}\text { Atomic } \\
\text { Number }\end{array}$} & \multirow{2}{*}{$\begin{array}{c}\text { Atomic } \\
\text { Type }\end{array}$} & \multicolumn{3}{|c|}{ Coordinates (Angstroms) } \\
\hline & & & $x$ & $\mathrm{Y}$ & Z \\
\hline-- & -- & --- & -------- & ------ & -------- \\
\hline 1 & 1 & $\odot$ & -4.056606 & -0.378261 & 2.119824 \\
\hline 2 & 8 & $\odot$ & -3.503391 & $\odot .403904$ & 2.038931 \\
\hline 3 & 8 & $\odot$ & -4.664368 & -0.770555 & -2.000605 \\
\hline 4 & 6 & $\odot$ & -3.519744 & $\odot .598357$ & -0.416154 \\
\hline 5 & 6 & $\odot$ & -4.061853 & -0.815394 & -0.711207 \\
\hline 6 & 1 & $\odot$ & -5.024068 & -1.641465 & -2.195280 \\
\hline 7 & 6 & $\odot$ & -3.917442 & 1.188026 & $\odot .926355$ \\
\hline 8 & 6 & $\odot$ & -2.813692 & -1.694959 & -0.670889 \\
\hline 9 & 6 & $\odot$ & -1.716770 & -0.730297 & -1.118143 \\
\hline 10 & 8 & $\odot$ & -2.092589 & 0.510603 & -0.478700 \\
\hline 11 & 1 & $\odot$ & -3.427589 & 2.161132 & 1.048382 \\
\hline 12 & 1 & $\odot$ & -5.005247 & 1.347045 & ๑. 940317 \\
\hline 13 & 1 & $\odot$ & -3.884626 & 1. 272801 & -1.205678 \\
\hline 14 & 1 & $\odot$ & -4.810230 & -1.128404 & 0.030817 \\
\hline 15 & 1 & $\odot$ & -2.895423 & -2.569225 & -1.324780 \\
\hline 16 & 1 & $\odot$ & -2.634002 & -2.039370 & ๑. 354471 \\
\hline 17 & 1 & $\odot$ & -1.782280 & -0.581092 & -2.205578 \\
\hline 18 & 7 & $\odot$ & -0.339209 & -1.029041 & -0.814114 \\
\hline 19 & 6 & $\odot$ & -0.052903 & -1.311899 & 0.407349 \\
\hline 20 & 7 & $\odot$ & 1.220507 & -1.601663 & ๑. 817384 \\
\hline 21 & 6 & $\odot$ & 2.237416 & -0.820882 & ○. 444071 \\
\hline 22 & 6 & $\odot$ & 2.139115 & 0.580970 & ๑. 182506 \\
\hline 23 & 7 & $\odot$ & 3. 099961 & 1.324497 & -0.363566 \\
\hline 24 & 6 & $\odot$ & 4.274661 & 0.740987 & -0.575711 \\
\hline 25 & 7 & $\odot$ & 4.534494 & $-\odot .533854$ & -0.183443 \\
\hline 26 & 6 & $\odot$ & 3.576416 & -1.400756 & ๑. 353976 \\
\hline 27 & 7 & $\odot$ & 5.246545 & 1.439607 & -1.168842 \\
\hline 28 & 8 & $\odot$ & 3.900371 & -2.538645 & 0.664349 \\
\hline 29 & 1 & $\odot$ & $-\odot .810089$ & -1.420194 & 1.195913 \\
\hline 30 & 1 & $\odot$ & 5.060860 & 2.397032 & -1.425496 \\
\hline 31 & 1 & $\odot$ & 6.162959 & 1.057967 & -1.344304 \\
\hline 32 & 1 & $\odot$ & 5. 461226 & -0.926802 & -0.303173 \\
\hline 33 & 8 & $\odot$ & 1.027011 & 1.186268 & $\odot .562701$ \\
\hline 34 & 1 & $\odot$ & $\odot .737708$ & 1.927194 & -0.034286 \\
\hline 35 & 8 & $\odot$ & -0.394321 & 2.634112 & -1.048571 \\
\hline 36 & 1 & $\odot$ & -1.092033 & 1. 961421 & -0.900837 \\
\hline 37 & 1 & $\odot$ & -0.775221 & 3.471347 & -0.766778 \\
\hline 38 & 1 & $\odot$ & -0.270059 & $\odot .925738$ & 2.043397 \\
\hline 39 & 8 & $\odot$ & -0.829104 & ๑. 865281 & 2.828590 \\
\hline 40 & 1 & $\odot$ & -1.711458 & 0.633659 & 2.494643 \\
\hline
\end{tabular}

Standard basis: Aug-CC-pVDZ (6D, 7F)

712 basis functions

85 alpha electrons 84 beta electrons

nuclear repulsion energy 2000.5409908094 Hartrees.

NAtoms $=40$ NActive $=40$

Force inversion solution in PCM.

Polarizable Continuum Model (PCM)

$\begin{array}{ll}\text { =ニ=ニニ=ニニ=ニニ=ニ=ニ=ニ=ニニニニ= } \\ \text { Model } & : \text { PCM. }\end{array}$

Solvent : Water, Eps $=78.355300$ Eps $($ inf $)=1.777849$

SCF Done: $E($ UWB97XD $)=-1192.08088600$ A.U. after 1 cycles NFock $=1$ Conv $=0.69 \mathrm{D}-08 \quad-\mathrm{V} / \mathrm{T}=2.0071$

$\langle\mathrm{S} x>=0.0000<\mathrm{Sy}>=0.0000<\mathrm{S} z>=0.5000$

$<S^{* *} 2>=0.7629 \mathrm{~S}=0.5064$

$<\mathrm{L} . \mathrm{S}>=0.000000000000 \mathrm{E}+00$

Annihilation of the first spin contaminant:

$\mathrm{S}^{* * 2}$ before annihilation 0.7629 , after 0.7501

Harmonic frequencies $\left(\mathrm{cm}^{*}-1\right)$, IR intensities (KM/Mole), Raman

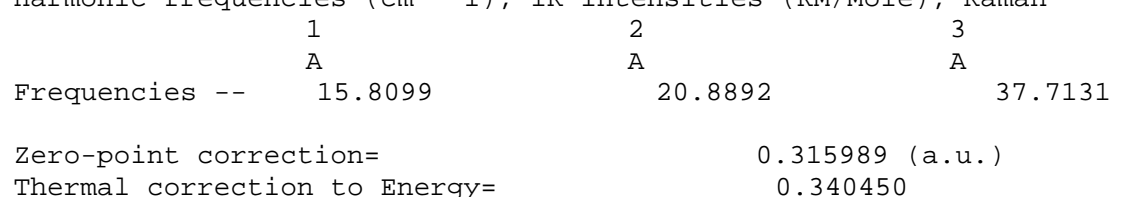


Thermal correction to Enthalpy=

Thermal correction to Gibbs Free Energy=

Sum of electronic and zero-point Energies=

Sum of electronic and thermal Energies=

Sum of electronic and thermal Enthalpies=

Sum of electronic and thermal Free Energies= $\odot .341394$

0.259747

$-1191.764897$

$-1191.740436$

$-1191.739492$

$-1191.821139$

$\begin{array}{lcccc} & & \text { KCal/Mol } & \text { Cal/Mol-Kelvin } & \text { Cal } \\ \text { Total } & \text { 213.635 } & 89.766 & \\ & & \text { Value } & \text { Threshold } & \text { Converged? } \\ \text { Maximum Force } & 0.000007 & 0.000450 & \text { YES } \\ \text { RMS Force } & 0.000002 & 0.00030 \odot & \text { YES } \\ \text { Maximum Displacement } & 0.002241 & 0.00180 \odot & \text { NO } \\ \text { RMS Displacement } & 0.000555 & 0.001200 & \text { YES }\end{array}$

Kelvin 171.840

Predicted change in Energy $=-7.506083 \mathrm{D}-\odot 9$

Normal termination of Gaussian 16 at Fri Jun 14 21:09:10 2019.

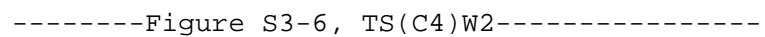

degua-oh4w2.ts2. higha. $\log$

Stoichiometry C10H18N507(2)

Standard orientation:

\begin{tabular}{|c|c|c|c|c|c|}
\hline \multirow{2}{*}{$\begin{array}{l}\text { Center } \\
\text { Number }\end{array}$} & \multirow{2}{*}{$\begin{array}{l}\text { Atomic } \\
\text { Number }\end{array}$} & \multirow{2}{*}{$\begin{array}{l}\text { Atomic } \\
\text { Type }\end{array}$} & \multicolumn{3}{|c|}{ Coordinates (Angstroms) } \\
\hline & & & $x$ & $\mathrm{Y}$ & Z \\
\hline & & & --------- & - & 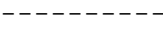 \\
\hline 1 & 1 & $\odot$ & 5.060524 & -2.143882 & 0.057694 \\
\hline 2 & 8 & $\odot$ & 4.588718 & -1.933219 & -0.752880 \\
\hline 3 & 8 & $\odot$ & 4.815079 & 1.830688 & 1.470318 \\
\hline 4 & 6 & $\odot$ & 4.369189 & $\odot .515300$ & $-\odot .471173$ \\
\hline 5 & 6 & $\odot$ & 4.549603 & 0.492132 & 1.053788 \\
\hline 6 & 1 & $\odot$ & 4.866449 & 1.841138 & 2.430952 \\
\hline 7 & 6 & $\odot$ & 5.004077 & -0.654126 & -1.212940 \\
\hline 8 & 6 & $\odot$ & 3.200014 & -0.013675 & 1.545290 \\
\hline 9 & 6 & $\odot$ & 2.225404 & $\odot .551821$ & 0.510663 \\
\hline 10 & 8 & $\odot$ & 2.962947 & $\odot .546726$ & -0.716789 \\
\hline 11 & 1 & $\odot$ & 4.707122 & -0.597436 & -2.267166 \\
\hline 12 & 1 & $\odot$ & 6.099413 & -0.552396 & -1.162166 \\
\hline 13 & 1 & $\odot$ & 4.820337 & 1.439567 & -0.863978 \\
\hline 14 & 1 & $\odot$ & 5.381540 & -0.159708 & 1.358381 \\
\hline 15 & 1 & $\odot$ & 2.969842 & $\odot .307125$ & 2.567055 \\
\hline 16 & 1 & $\odot$ & 3.167154 & -1.109489 & 1.500042 \\
\hline 17 & 1 & $\odot$ & 1.977450 & 1.598928 & $\odot .754110$ \\
\hline 18 & 7 & $\odot$ & ๑. 986297 & -0.169420 & $\odot .464410$ \\
\hline 19 & 6 & $\odot$ & $\odot .676516$ & -0.817300 & -0.597372 \\
\hline 20 & 7 & $\odot$ & -0.422258 & -1.633189 & -0.693225 \\
\hline 21 & 6 & $\odot$ & -1.645099 & -1.240805 & -0.320513 \\
\hline 22 & 6 & $\odot$ & -2.126736 & ๑. 130087 & -0.394039 \\
\hline 23 & 7 & $\odot$ & -3.381851 & ๑. 451835 & ๑. . 088166 \\
\hline 24 & 6 & $\odot$ & -4.202834 & -0.506979 & ๑. 482618 \\
\hline 25 & 7 & $\odot$ & -3.861508 & -1.814797 & ○. 421846 \\
\hline 26 & 6 & $\odot$ & -2.597624 & -2.279495 & ๑. . 028929 \\
\hline 27 & 7 & $\odot$ & -5.421298 & -0.194878 & $\odot .950075$ \\
\hline 28 & 8 & $\odot$ & -2.400830 & -3.493245 & ○. 029132 \\
\hline 29 & 1 & $\odot$ & 1.348488 & -0.856520 & -1.463214 \\
\hline 30 & 1 & $\odot$ & -5.722830 & $\odot .766496$ & ๑. 961887 \\
\hline 31 & 1 & $\odot$ & -6.068389 & $-\odot .900480$ & 1.264304 \\
\hline 32 & 1 & $\odot$ & -4.527138 & -2.528579 & $\odot .691718$ \\
\hline 33 & 8 & $\odot$ & -1.428587 & 1.032197 & -0.921798 \\
\hline 34 & 1 & $\odot$ & -1.815575 & 2.438110 & -1.172442 \\
\hline 35 & 8 & $\odot$ & -2.150209 & 3.414572 & -1.232654 \\
\hline 36 & 1 & $\odot$ & -3.130167 & 3.322050 & -0.539671 \\
\hline 37 & 1 & $\odot$ & -2.373553 & 3.602485 & -2.151666 \\
\hline 38 & 8 & $\odot$ & -4.018054 & 2.948126 & 0.173860 \\
\hline 39 & 1 & $\odot$ & -3.801657 & 1.920165 & ๑. 205881 \\
\hline 40 & 1 & $\odot$ & -3.922265 & 3.307051 & 1.064350 \\
\hline
\end{tabular}

Standard basis: Aug-CC-pVDZ (6D, 7F)

712 basis functions

85 alpha electrons

84 beta electrons 
nuclear repulsion energy 1913.6235269870 Hartrees. NAtoms $=40$ NActive $=40$

Nuclear repulsion after empirical dispersion term = 1913.5961643476 Hartrees.

Force inversion solution in PCM.

Polarizable Continuum Model (PCM)
$\begin{aligned} & \text { ========================== } \\ & \text { Model }\end{aligned}$
Solvent : Water, Eps= 78.355300 Eps(inf) $=1.777849$

SCF Done: $E($ UWB97XD) $=-1192.06633664$ A.U. after 1 cycles NFock $=1$ Conv $=0.30 \mathrm{D}-08 \quad-\mathrm{V} / \mathrm{T}=2.0071$

$\langle\mathrm{SX}>=0.0000<\mathrm{Sy}\rangle=0.0000<\mathrm{S} z>=0.5000$

$<S^{* *} 2>=0.7609 \mathrm{~S}=0.5055$

$<$ L.S $>=0.000000000000 \mathrm{E}+00$

Annihilation of the first spin contaminant:

$\mathrm{S}^{* *} 2$ before annihilation 0.7609 , after 0.7501

Harmonic frequencies $\left(\mathrm{cm}^{*}-1\right)$, IR intensities (KM/Mole), activities $\left(A^{* *} 4 / \mathrm{AMU}\right)$, depolarization ratios for plane and incident light, reduced masses (AMU), force constants and normal coordinates:

\begin{tabular}{|c|c|c|c|c|}
\hline & & \multicolumn{2}{|r|}{$\bar{A}$} & \\
\hline \multicolumn{2}{|c|}{ Frequencies } & -- & \multicolumn{2}{|c|}{$\begin{array}{l}\text { A } \\
784.4654\end{array}$} \\
\hline & -- & \multicolumn{2}{|c|}{1.1275} \\
\hline & Frc consts & -- & \multicolumn{2}{|c|}{$\odot .4088$} \\
\hline \multicolumn{2}{|c|}{ IR Inten } & -- & \multicolumn{2}{|c|}{2528.2850} \\
\hline Atom & AN & $X$ & $Y$ & Z \\
\hline 1 & 1 & $\odot . \odot \odot$ & $-\odot . \odot \odot$ & $-\odot .0 \odot$ \\
\hline 2 & 8 & $-\odot .0 \odot$ & 0.00 & 0.00 \\
\hline 3 & 8 & -0.00 & -0.00 & -0.00 \\
\hline 4 & 6 & 0.00 & -0.00 & 0.00 \\
\hline 5 & 6 & -0.00 & 0.00 & 0.00 \\
\hline 6 & 1 & $\odot . \odot \odot$ & $\odot . \odot \odot$ & $-\odot . \odot \odot$ \\
\hline 7 & 6 & -0.00 & -0.00 & 0.00 \\
\hline 8 & 6 & -0.00 & -0.00 & 0.00 \\
\hline 9 & 6 & $\odot . \odot \odot$ & $\odot . \odot \odot$ & -0.00 \\
\hline 10 & 8 & $-\odot . \odot \odot$ & $\odot .0 \odot$ & 0.00 \\
\hline 11 & 1 & 0.00 & -0.00 & $\odot .0 \odot$ \\
\hline 12 & 1 & -0.00 & $\odot .0 \odot$ & $\odot .0 \odot$ \\
\hline 13 & 1 & -0.00 & $\odot . \odot \odot$ & -0.00 \\
\hline 14 & 1 & $-0.0 \odot$ & $-\odot . \odot \odot$ & $\odot .0 \odot$ \\
\hline 15 & 1 & $-\odot . \odot \odot$ & $-\odot . \odot \odot$ & $-\odot . \odot \odot$ \\
\hline 16 & 1 & $-\odot . \odot \odot$ & $-\odot .0 \odot$ & $0.0 \odot$ \\
\hline 17 & 1 & -0.00 & -0.00 & 0.00 \\
\hline 18 & 7 & $-\odot . \odot \odot$ & $0.0 \odot$ & $-\odot .0 \odot$ \\
\hline 19 & 6 & $\odot . \odot \odot$ & $-\odot . \odot \odot$ & $-\odot . \odot \odot$ \\
\hline 20 & 7 & $\odot .00$ & -0.00 & -0.00 \\
\hline 21 & 6 & $\odot .0 \odot$ & 0.01 & -0.00 \\
\hline 22 & 6 & -0.02 & $-\odot . \odot \odot$ & 0.01 \\
\hline 23 & 7 & 0.01 & -0.03 & -0.01 \\
\hline 24 & 6 & $\odot .01$ & -0.01 & $-0.0 \odot$ \\
\hline 25 & 7 & $\odot .00$ & -0.01 & $\odot .00$ \\
\hline 26 & 6 & $-\odot . \odot \odot$ & $0.0 \odot$ & $\odot . \odot \odot$ \\
\hline 27 & 7 & $\odot . \odot \odot$ & $\odot . \odot \odot$ & $-\odot .0 \odot$ \\
\hline 28 & 8 & $\odot .0 \odot$ & $\odot . \odot \odot$ & -0.00 \\
\hline 29 & 1 & $\odot . \odot \odot$ & $-0.0 \odot$ & $\odot .0 \odot$ \\
\hline 30 & 1 & 0.03 & $\odot .01$ & -0.00 \\
\hline 31 & 1 & $-\odot . \odot \odot$ & 0.01 & $\odot .00$ \\
\hline 32 & 1 & $\odot .0 \odot$ & -0.01 & -0.00 \\
\hline 33 & 8 & -0.00 & 0.03 & -0.01 \\
\hline 34 & 1 & $\odot .01$ & -0.22 & 0.09 \\
\hline 35 & 8 & $-\odot .01$ & -0.05 & 0.02 \\
\hline 36 & 1 & 0.69 & 0.18 & -0.58 \\
\hline 37 & 1 & -0.07 & -0.01 & $\odot .03$ \\
\hline 38 & 8 & -0.03 & 0.04 & 0.02 \\
\hline 39 & 1 & -0.09 & 0.26 & 0.06 \\
\hline 40 & 1 & -0.05 & -0.03 & $\odot$ \\
\hline
\end{tabular}

Zero-point correction=

Thermal correction to Energy=

Thermal correction to Enthalpy=

\begin{tabular}{|c|c|c|c|c|c|}
\hline 2 & & \multicolumn{2}{|l|}{3} & \\
\hline A & & \multirow{2}{*}{\multicolumn{3}{|c|}{25.3265}} \\
\hline \multicolumn{3}{|c|}{18.5463} & & & \\
\hline \multicolumn{3}{|c|}{5.8726} & \multicolumn{3}{|c|}{6.1424} \\
\hline \multicolumn{3}{|c|}{$\odot .0012$} & \multicolumn{3}{|c|}{0.0023} \\
\hline \multicolumn{3}{|c|}{11.8706} & \multicolumn{3}{|c|}{12.4048} \\
\hline$x$ & $Y$ & Z & $X$ & Z & \\
\hline-0.15 & -0.09 & $\odot .23$ & 0.03 & $\odot .09$ & 0.17 \\
\hline-0.08 & -0.12 & 0.19 & 0.07 & 0.02 & 0.13 \\
\hline$\odot .00$ & -0.01 & -0.00 & -0.17 & 0.16 & -0.08 \\
\hline 0.06 & -0.09 & $\odot .04$ & -0.05 & 0.02 & -0.01 \\
\hline-0.03 & -0.02 & $\odot .05$ & $-\odot .09$ & 0.12 & -0.01 \\
\hline-0.05 & $\odot .03$ & $-\odot . \odot \odot$ & -0.19 & $\odot .22$ & -0.08 \\
\hline 0.03 & -0.17 & 0.14 & $\odot .03$ & 0.01 & $\odot .08$ \\
\hline-0.09 & 0.07 & -0.01 & -0.08 & 0.09 & -0.02 \\
\hline$-\odot .0 \odot$ & 0.06 & -0.09 & -0.07 & -0.01 & $-\odot . \odot 8$ \\
\hline 0.07 & -0.01 & -0.05 & -0.04 & -0.07 & -0.06 \\
\hline 0.08 & -0.21 & 0.12 & 0.07 & -0.07 & 0.06 \\
\hline 0.03 & -0.24 & 0.19 & 0.03 & 0.06 & 0.11 \\
\hline 0.14 & -0.14 & $\odot .01$ & -0.08 & 0.02 & -0.05 \\
\hline-0.08 & -0.05 & $\odot .13$ & -0.07 & 0.18 & 0.06 \\
\hline-0.13 & 0.13 & -0.04 & -0.13 & 0.13 & -0.04 \\
\hline-0.14 & 0.07 & $\odot .04$ & -0.03 & $\odot .08$ & 0.04 \\
\hline 0.02 & $\odot .08$ & -0.15 & -0.12 & -0.01 & -0.15 \\
\hline-0.02 & 0.10 & -0.13 & -0.05 & -0.05 & -0.07 \\
\hline$\odot .02$ & $\odot .06$ & -0.13 & -0.03 & $-\odot .09$ & -0.05 \\
\hline 0.01 & 0.07 & -0.15 & -0.03 & -0.09 & $-\odot . \odot 4$ \\
\hline 0.03 & 0.05 & $-\odot . \odot 8$ & -0.02 & -0.06 & -0.04 \\
\hline-0.01 & 0.04 & -0.03 & $\odot .02$ & -0.05 & -0.05 \\
\hline$\odot .01$ & 0.00 & 0.05 & 0.04 & -0.01 & -0.01 \\
\hline 0.06 & -0.02 & $\odot . \odot 8$ & $\odot .02$ & 0.02 & 0.01 \\
\hline 0.08 & $-\odot .01$ & 0.03 & -0.03 & $\odot . \odot \odot$ & -0.01 \\
\hline 0.07 & 0.02 & -0.05 & -0.04 & -0.03 & -0.03 \\
\hline 0.08 & -0.06 & 0.17 & 0.04 & 0.06 & 0.04 \\
\hline$\odot .09$ & $\odot .02$ & -0.09 & -0.08 & -0.04 & -0.03 \\
\hline 0.06 & 0.02 & -0.09 & -0.01 & -0.12 & -0.04 \\
\hline$\odot .06$ & $-\odot . \odot 6$ & $\odot .19$ & $\odot . \odot 7$ & $\odot .06$ & 0.03 \\
\hline 0.11 & -0.08 & 0.17 & $\odot .02$ & 0.07 & 0.04 \\
\hline 0.11 & -0.03 & $\odot .07$ & -0.04 & 0.03 & $-\odot .0 \odot$ \\
\hline-0.06 & 0.06 & -0.06 & $\odot .02$ & -0.08 & -0.09 \\
\hline-0.13 & 0.04 & -0.05 & 0.12 & -0.03 & 0.04 \\
\hline-0.17 & 0.03 & -0.04 & $\odot .20$ & $\odot . \odot \odot$ & 0.14 \\
\hline-0.12 & 0.00 & 0.03 & 0.22 & 0.02 & 0.17 \\
\hline-0.24 & 0.01 & -0.03 & 0.18 & 0.10 & 0.16 \\
\hline-0.06 & -0.02 & $\odot .08$ & 0.22 & 0.03 & 0.17 \\
\hline-0.02 & -0.01 & $\odot .08$ & 0.14 & $\odot .01$ & $\odot .09$ \\
\hline-0.02 & -0.01 & $\odot .07$ & $\odot .27$ & -0.04 & 0.19 \\
\hline & & $\begin{array}{l}0.33 \\
0.3\end{array}$ & $(\mathrm{a} \cdot \mathrm{u})$. & & \\
\hline
\end{tabular}


Thermal correction to Gibbs Free Energy= Sum of electronic and zero-point Energies= Sum of electronic and thermal Energies=

Sum of electronic and thermal Enthalpies=

Sum of electronic and thermal Free Energies=

$$
\text { E (Thermal) }
$$$$
\mathrm{KCal} / \mathrm{Mol}
$$

209.460
$\mathrm{CV}$

Cal/Mol-Kelvin 84.891
๑. 255819

$-1191.755403$

$-1191.732541$

$-1191.731597$

$-1191.810518$

\section{$\mathrm{S}$}

Cal/Mol-Kelvin

166.103

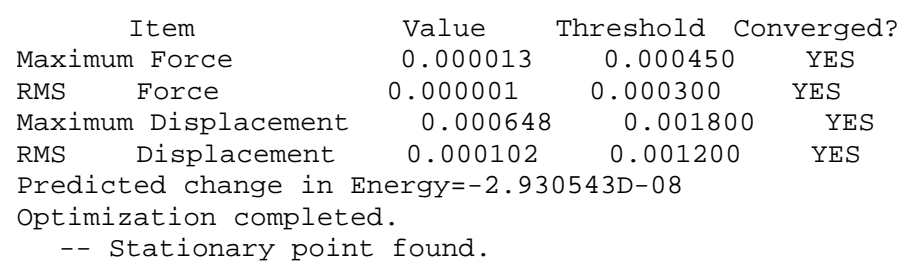

Normal termination of Gaussian 16 at Mon May 20 02:13:46 2019.

\begin{tabular}{|c|c|c|c|c|c|}
\hline \multicolumn{2}{|c|}{ Stoichiometry } & \multicolumn{4}{|c|}{ C10H18N507(2) } \\
\hline \multicolumn{6}{|c|}{ Standard orientation: } \\
\hline Center & Atomic & Atomic & $\mathrm{Coc}$ & rdinates ( & ggstroms) \\
\hline Number & Number & Type & $x$ & $\mathrm{Y}$ & Z \\
\hline 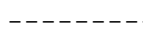 & ------ & ---- & $--_{-}--_{-}--$ & 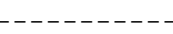 & ------ \\
\hline 1 & 1 & $\odot$ & 5.135535 & -2.061635 & ๑. . 888729 \\
\hline 2 & 8 & $\odot$ & 4.668867 & -1.860036 & -0.727081 \\
\hline 3 & 8 & 0 & 4.855617 & 1.929792 & 1.456686 \\
\hline 4 & 6 & $\odot$ & 4.441535 & 0.591177 & -0.475143 \\
\hline 5 & 6 & $\odot$ & 4.604911 & 0.585176 & 1.051603 \\
\hline 6 & 1 & $\odot$ & 4.897433 & 1.950824 & 2.417618 \\
\hline 7 & 6 & $\odot$ & 5.085634 & -0.585285 & -1.197656 \\
\hline 8 & 6 & $\odot$ & 3.253578 & 0.074643 & 1.533784 \\
\hline 9 & 6 & $\odot$ & 2.286536 & 0.622064 & 0.482203 \\
\hline 10 & 8 & $\odot$ & 3.037393 & 0.619023 & -0.735667 \\
\hline 11 & 1 & $\odot$ & 4.798201 & -0.540867 & -2.255098 \\
\hline 12 & 1 & $\odot$ & 6.180200 & -0.480448 & -1.138113 \\
\hline 13 & 1 & $\odot$ & 4.894763 & 1.511850 & -0.873548 \\
\hline 14 & 1 & $\odot$ & 5.437693 & $-\odot .057733$ & 1.372386 \\
\hline 15 & 1 & $\odot$ & 3.009567 & 0.405153 & 2.549146 \\
\hline 16 & 1 & $\odot$ & 3.229355 & -1.021726 & 1.500623 \\
\hline 17 & 1 & $\odot$ & 2.018803 & 1.666248 & $\odot .715980$ \\
\hline 18 & 7 & $\odot$ & 1.057739 & -0.116753 & 0.425708 \\
\hline 19 & 6 & $\odot$ & 0.774876 & -0.781703 & -0.632255 \\
\hline 20 & 7 & $\odot$ & -0.309478 & -1.618755 & -0.730609 \\
\hline 21 & 6 & $\odot$ & -1.531705 & -1.255176 & -0.326933 \\
\hline 22 & 6 & $\odot$ & -2.024058 & $\odot .107067$ & -0.362225 \\
\hline 23 & 7 & $\odot$ & -3.322120 & ๑. 315664 & 0.138038 \\
\hline 24 & 6 & $\odot$ & -4.166103 & -0.652373 & 0.503588 \\
\hline 25 & 7 & $\odot$ & -3.752513 & -1.923174 & 0.423528 \\
\hline 26 & 6 & $\odot$ & -2.457934 & -2.325356 & 0.010516 \\
\hline 27 & 7 & $\odot$ & -5.384688 & -0.335411 & 0.925641 \\
\hline 28 & 8 & 0 & -2.226481 & -3.526921 & -0.011696 \\
\hline 29 & 1 & $\odot$ & 1.455839 & -0.820844 & -1.490347 \\
\hline 30 & 1 & $\odot$ & -5.673274 & 0.634500 & 0.926301 \\
\hline 31 & 1 & $\odot$ & -6.045085 & -1.039958 & 1.216681 \\
\hline 32 & 1 & $\odot$ & -4.382615 & -2.676067 & 0.673490 \\
\hline 33 & 8 & $\odot$ & -1.427871 & 1.085539 & -0.822139 \\
\hline 34 & 1 & $\odot$ & -2.160498 & 2.661181 & -1.228765 \\
\hline 35 & 8 & $\odot$ & -2.653620 & 3.498883 & -1.355732 \\
\hline 36 & 1 & $\odot$ & -4.056638 & 3.212256 & -0.309277 \\
\hline 37 & 1 & 0 & -2.843849 & 3.542324 & -2.297498 \\
\hline 38 & 8 & $\odot$ & -4.662392 & 2.761012 & ๑. 319244 \\
\hline 39 & 1 & $\odot$ & -3.680365 & 1. 283001 & $\odot .193250$ \\
\hline 40 & 1 & $\odot$ & -4.573360 & 3. 241204 & 1.148475 \\
\hline
\end{tabular}

Standard basis: Aug-CC-pVDZ (6D, 7F)

712 basis functions 


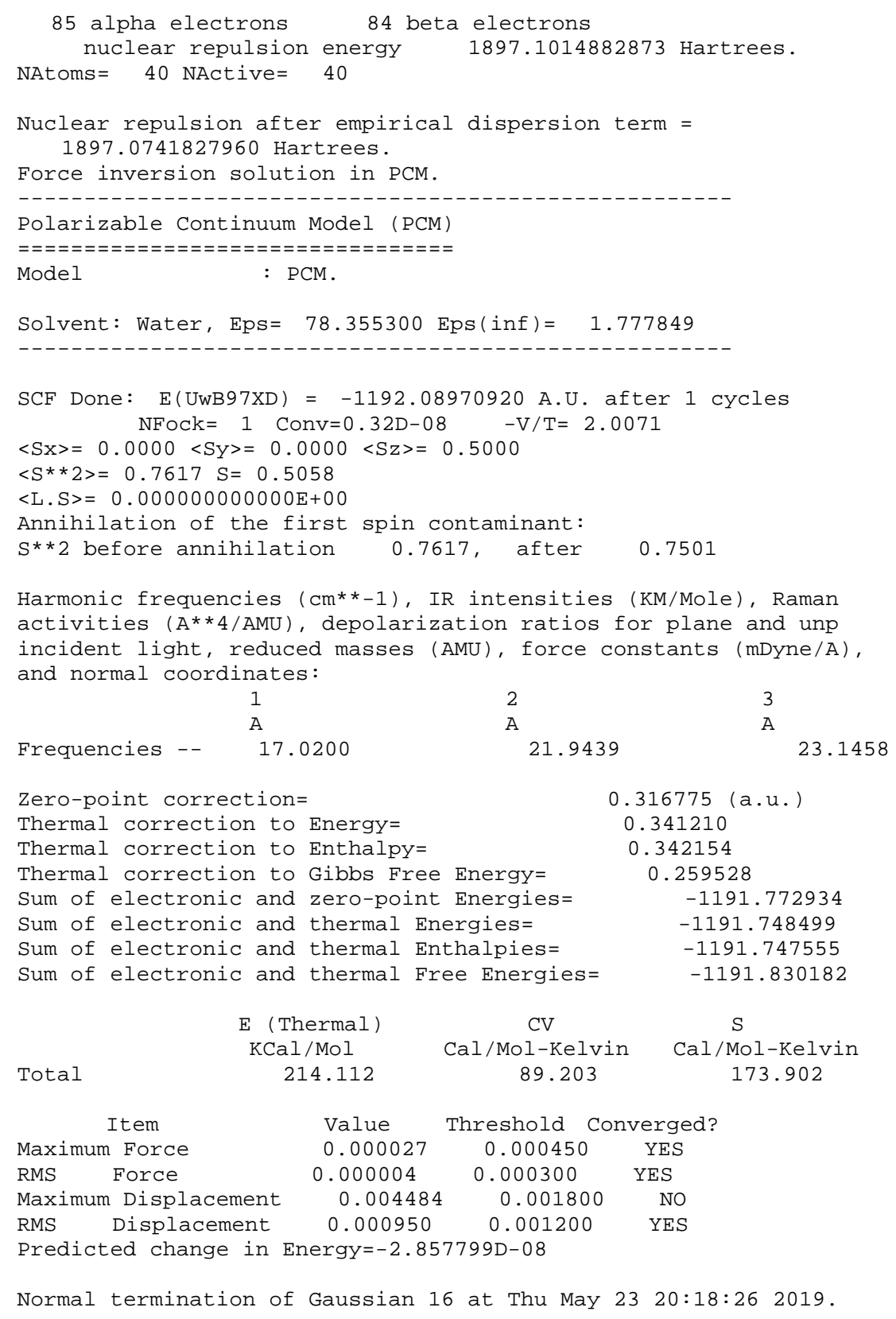

Harmonic frequencies $\left(\mathrm{cm}^{* *}-1\right)$, IR intensities (KM/Mole), Raman activities ( $\left.A^{* *} 4 / A M U\right)$, depolarization ratios for plane and unp incident light, reduced masses (AMU), force constants (mDyne/A), and normal coordinates:

$$
\begin{array}{ll} 
& 1 \\
\text { Frequencies - - } & \mathrm{A} \\
17.0200
\end{array}
$$

Zero-point correction= Thermal correction to Energy= Thermal correction to Enthalpy= Thermal correction to Gibbs Free Energy= Sum of electronic and zero-point Energies= Sum of electronic and thermal Energies= Sum of electronic and thermal Enthalpies= Sum of electronic and thermal Free Energies= $\begin{array}{ll}2 & 3 \\ \text { A } & \text { A }\end{array}$ 21.9439 23.1458

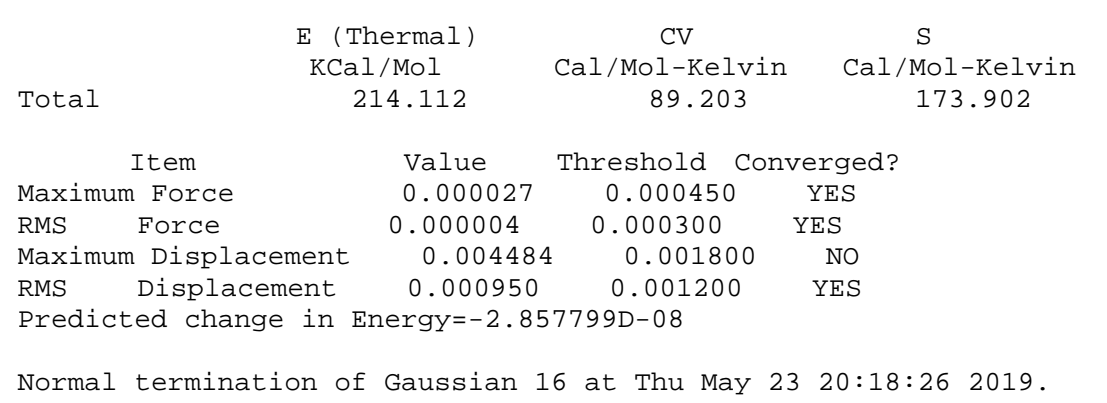

$$
\begin{gathered}
0.316775 \text { (a.u.) } \\
0.341210 \\
0.342154 \\
0.259528 \\
-1191.772934 \\
-1191.748499 \\
-1191.747555 \\
-1191.830182 \\
\text { S } \\
\text { Cal/Mol-Kelvin }
\end{gathered}
$$

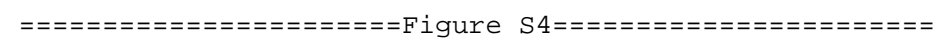

-..-Figure S4-1, Int1(C8)

Stoichiometry C10H14N505(2)

Standard orientation:

\begin{tabular}{cccccc} 
Center & Atomic & \multicolumn{2}{c}{ Atomic } & \multicolumn{3}{c}{ Coordinates } \\
Number & Number & Type & X & Y & Z \\
1 & 1 & 0 & -3.700996 & -2.089839 & -0.861424 \\
2 & 8 & 0 & -3.208634 & -2.071396 & -0.036349 \\
3 & 8 & 0 & -3.939688 & 2.230362 & -0.469909 \\
4 & 6 & 0 & -3.444419 & 0.255587 & 0.758618 \\
5 & 6 & 0 & -3.581628 & 0.863275 & -0.640046 \\
6 & 1 & 0 & -3.989209 & 2.642224 & -1.338012 \\
7 & 6 & 0 & -3.863180 & -1.203643 & 0.882144 \\
8 & 6 & 0 & -2.178981 & 0.700346 & -1.218047 \\
9 & 6 & 0 & -1.288666 & 0.835243 & 0.018103 \\
10 & 8 & 0 & -2.064084 & 0.411752 & 1.122428 \\
11 & 1 & 0 & -3.595051 & -1.553167 & 1.886281 \\
12 & 1 & 0 & -4.956087 & -1.270325 & 0.778676
\end{tabular}




\begin{tabular}{|c|c|c|c|c|c|}
\hline 13 & 1 & $\odot$ & -4.046613 & $\odot .839479$ & 1.467866 \\
\hline 14 & 1 & $\odot$ & -4.342425 & $\odot .343759$ & -1.238719 \\
\hline 15 & 1 & $\odot$ & -1.941365 & 1.448534 & -1.980276 \\
\hline 16 & 1 & $\odot$ & -2.066077 & -0.296420 & -1.657632 \\
\hline 17 & 1 & $\odot$ & -0.949135 & 1.866311 & 0.171402 \\
\hline 18 & 7 & $\odot$ & -0.079162 & $\odot .031913$ & -0.039500 \\
\hline 19 & 6 & $\odot$ & $-\odot . \odot \odot 8851$ & -1.337518 & $-\odot .122567$ \\
\hline 20 & 7 & $\odot$ & 1.224197 & -1.777620 & -0.130793 \\
\hline 21 & 6 & $\odot$ & 2.009623 & -0.641908 & -0.048991 \\
\hline 22 & 6 & $\odot$ & 1.210866 & $\odot .490329$ & $\odot .013720$ \\
\hline 23 & 7 & $\odot$ & 1.591554 & 1.783141 & $\odot .108046$ \\
\hline 24 & 6 & $\odot$ & 2.899273 & 1.929799 & $\odot .134652$ \\
\hline 25 & 7 & $\odot$ & 3.776269 & $\odot .875820$ & $\odot .075648$ \\
\hline 26 & 6 & $\odot$ & 3.429996 & -0.485988 & -0.018277 \\
\hline 27 & 7 & $\odot$ & 3.431402 & 3.172002 & 0.186407 \\
\hline 28 & 8 & $\odot$ & 4.309353 & -1.341146 & -0.062340 \\
\hline 29 & 1 & $\odot$ & -0.909131 & -1.943321 & -0.159006 \\
\hline 30 & 1 & $\odot$ & 2.795727 & 3.933031 & $\odot .371222$ \\
\hline 31 & 1 & $\odot$ & 4.400940 & 3.313851 & $\odot .423629$ \\
\hline 32 & 1 & $\odot$ & 4.772784 & 1.060220 & $\odot .087675$ \\
\hline 33 & 8 & $\odot$ & 1.918524 & -4.459381 & -0.265040 \\
\hline 34 & 1 & $\odot$ & 1.688838 & -3.485796 & -0.216522 \\
\hline
\end{tabular}

Standard basis: Aug-CC-pVDZ (6D, 7F)

626 basis functions

75 alpha electrons $\quad 74$ beta electrons

nuclear repulsion energy 1630.1320656568 Hartrees.

NAtoms $=34$ NActive $=34$

Nuclear repulsion after empirical dispersion term $=$

1630.1069428065 Hartrees.

Force inversion solution in PCM.

SCF Done: $E($ UWB97XD $)=-1039.19077816$

A.U. after

2 cycles

NFock $=2$ Conv $=0.86 \mathrm{D}-09 \quad-\mathrm{V} / \mathrm{T}=2.0071$

$\left\langle S x>=0.0000\left\langle\right.\right.$ Sy $>=0.0000\left\langle S z>=0.5000\left\langle S^{* *} 2>=0.7528 S=0.5014\right.\right.$

$<$ L.S $>=0.000000000000 \mathrm{E}+00$

Annihilation of the first spin contaminant:

$\mathrm{S}^{* \star} 2$ before annihilation 0.7528 , after 0.7500

Zero-point correction=

Thermal correction to Energy=

0.265264 (a.u.)

Thermal correction to Enthalpy=

Thermal correction to Gibbs Free Energy=

Sum of electronic and zero-point Energies=

Sum of electronic and thermal Energies=

$\odot .285375$

0.215446

$-1038.925514$

$-1038.906347$

Sum of electronic and thermal Enthalpies=

$-1038.906347$

Sum of electronic and thermal Free Energies=

$-1038.975333$

\begin{tabular}{|c|c|c|c|}
\hline $\mathrm{E}_{\mathrm{KC}}$ & $\begin{array}{l}\text { lermal) } \\
\text { l/Mol } \\
78.483\end{array}$ & $\begin{array}{c}\text { CV } \\
\text { Cal/Mol-Kelvin } \\
71.318\end{array}$ & $\begin{array}{c}\mathrm{S} \\
\mathrm{Cal} / \mathrm{Mol}-\mathrm{Kelvin} \\
147.179\end{array}$ \\
\hline Item & Value & \multicolumn{2}{|c|}{ Threshold Converged? } \\
\hline Maximum Force & 0.000016 & $\odot .000450$ & YES \\
\hline Force & 0.000003 & 0.000300 & YES \\
\hline Maximum Displacement & $\odot .0 \odot 2168$ & $\odot .001800$ & NO \\
\hline Displacement & $\odot .000370$ & 0.001200 & YES \\
\hline
\end{tabular}

Predicted change in Energy $=-2$.228911D-๑8

Normal termination of Gaussian 16 at Thu Mar 14 15:10:48 2019.

Figure S4-2, TS1(C8)

degua-oh8a.higha1.log

Stoichiometry C10H14N505(2)

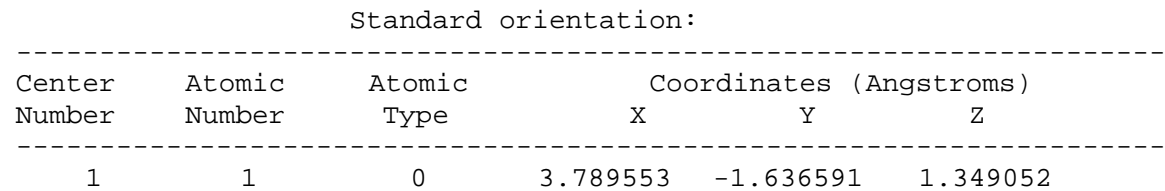




\begin{tabular}{|c|c|c|c|c|c|}
\hline 2 & 8 & 0 & 3.282175 & -1.833035 & $\odot .556825$ \\
\hline 3 & 8 & 0 & 3.677639 & $2.5 \odot 2574$ & $\odot .056625$ \\
\hline 4 & 6 & 0 & 3.321520 & $\odot .283100$ & $-\odot .719423$ \\
\hline 5 & 6 & 0 & 3.429481 & 1.178860 & $\odot .517515$ \\
\hline 6 & 1 & 0 & 3.697265 & 3.090587 & 0.817924 \\
\hline 7 & 6 & 0 & 3.847443 & -1.136276 & $-\odot .547794$ \\
\hline 8 & 6 & 0 & 2.051183 & 1.038832 & 1.156719 \\
\hline 9 & 6 & 0 & 1.136934 & ๑.847701 & -0.054409 \\
\hline 10 & 8 & $\odot$ & 1.929253 & $\odot .263061$ & -1.069809 \\
\hline 11 & 1 & $\odot$ & 3.584642 & -1.709407 & -1.444877 \\
\hline 12 & 1 & $\odot$ & 4.944321 & -1.102539 & -0.473869 \\
\hline 13 & 1 & $\odot$ & 3.869697 & $\odot .745714$ & -1.551341 \\
\hline 14 & 1 & $\odot$ & 4.235754 & $\odot .854087$ & 1.189565 \\
\hline 15 & 1 & 0 & 1.767193 & 1.910823 & 1.753690 \\
\hline 16 & 1 & $\odot$ & 2.021120 & $\odot .150306$ & 1.796347 \\
\hline 17 & 1 & $\odot$ & $\odot .716057$ & 1.796397 & -0.407452 \\
\hline 18 & 7 & $\Theta$ & -0.004253 & -0.013996 & 0.201732 \\
\hline 19 & 6 & $\odot$ & 0.035388 & -1.339031 & $\odot .566893$ \\
\hline 20 & 7 & $\odot$ & -1.161130 & -1.866486 & ๑.699251 \\
\hline 21 & 6 & 0 & -2.029493 & -0.837083 & ๑. 397013 \\
\hline 22 & 6 & $\odot$ & -1.326644 & ๑.319803 & $\odot .081877$ \\
\hline 23 & 7 & 0 & -1.812236 & 1.529715 & -0.267989 \\
\hline 24 & 6 & $\odot$ & -3.128993 & 1.562119 & -0.298484 \\
\hline 25 & 7 & $\odot$ & -3.916048 & $\odot .476465$ & $-\odot .007204$ \\
\hline 26 & 6 & $\odot$ & -3.458859 & -0.803909 & 0.360739 \\
\hline 27 & 7 & 0 & -3.760691 & 2.717036 & -0.598926 \\
\hline 28 & 8 & $\odot$ & -4.265967 & -1.697947 & $\odot .596589$ \\
\hline 29 & 1 & 0 & 0.982174 & -1.847506 & 0.719110 \\
\hline 30 & 1 & $\Theta$ & -3.194808 & 3.481952 & -0.932621 \\
\hline 31 & 1 & $\odot$ & -4.747425 & 2.739658 & -0.803222 \\
\hline 32 & 1 & $\Theta$ & -4.924059 & $\odot .573694$ & -0.044955 \\
\hline 33 & 8 & 0 & -0.187288 & -3.261436 & -1.408016 \\
\hline 34 & 1 & $\odot$ & -0.915247 & -3.416742 & -0.776994 \\
\hline
\end{tabular}

Standard basis: Aug-CC-pVDZ (6D, 7F)

626 basis functions

75 alpha electrons $\quad 74$ beta electrons

nuclear repulsion energy 1651.3166361317 Hartrees.

NAtoms $=34$ NActive $=34$

Nuclear repulsion after empirical dispersion term $=$

1651.2909853578 Hartrees.

Force inversion solution in PCM.

Polarizable Continuum Model (PCM)

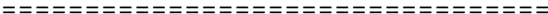

Model : PCM.

Cavity 2nd derivative terms included.

Solvent : Water, Eps $=78.355300$ Eps(inf $)=1.777849$

SCF Done: $E($ UWB97XD $)=-1039.18458211 \mathrm{~A} . \mathrm{U}$. after 1 cycles NFock $=1$ Conv $=0.60 \mathrm{D}-08 \quad-\mathrm{V} / \mathrm{T}=2.0071$

$\langle\mathrm{S} X\rangle=0.0000<\mathrm{S} y\rangle=0.0000<\mathrm{Sz}\rangle=0.5000$

$\left\langle S^{* *} 2>=0.7543 \mathrm{~S}=0.5022\right.$

$<$ L.S $>=0.000000000000 \mathrm{E}+00$

Annihilation of the first spin contaminant:

$\mathrm{S}^{* * 2}$ before annihilation 0.7543 , after 0.7500

Harmonic frequencies $\left(\mathrm{cm}^{* *}-1\right)$, IR intensities (KM/Mole), Raman activities $\left(A^{* *} 4 / A M U\right)$, depolarization ratios for plane and unpo incident light, reduced masses (AMU), force constants (mDyne/A), and normal coordinates:

\begin{tabular}{|c|c|c|c|c|c|c|c|c|c|c|}
\hline & & & 1 & & 2 & & & 3 & & \\
\hline & & & A & & A & & & A & & \\
\hline Frequ & ncies & ; - - & -90.50 & & & 24.628 & & & 8.2490 & \\
\hline Red. & asses & ; - - & 7.335 & & & 6.0071 & & & 7.7654 & \\
\hline Frc C & nsts & - - & $\odot .035$ & & & $\odot .0021$ & & & .0067 & \\
\hline IR In & & - - & 18.883 & & & 2. 0161 & & & .3987 & \\
\hline Atom & AN & $x$ & Y & Z & $x$ & Y & Z & $x$ & Z & \\
\hline 1 & 1 & $-\odot .01$ & $-\odot .01$ & $\odot .01$ & $\odot .04$ & -0.15 & -0.25 & -0.11 & $\odot .05$ & 0.13 \\
\hline 2 & 8 & 0.01 & -0.02 & 0.00 & -0.04 & -0.05 & -0.22 & -0.07 & $\odot .0 \odot$ & 0.11 \\
\hline 3 & 8 & -0.03 & $-\odot . \odot 2$ & -0.01 & $\odot . \odot 4$ & $-\odot .04$ & $\odot .08$ & $-\odot .01$ & $-\odot . \odot 2$ & $-\odot . \odot 8$ \\
\hline 4 & 6 & $\odot . \odot 1$ & $-\odot .03$ & $-\odot .01$ & $-\odot .07$ & 0.03 & $-\odot .07$ & $\odot . \odot \odot$ & -0.06 & $\odot .02$ \\
\hline 5 & 6 & -0.02 & $-\odot . \odot 2$ & -0.01 & 0.03 & $-\odot .06$ & -0.01 & -0.04 & $\odot . \odot \odot$ & $-\odot . \odot 2$ \\
\hline
\end{tabular}




$\begin{array}{rrrrr}6 & 1 & -0.04 & -0.02 & -0.01 \\ 7 & 6 & 0.02 & -0.02 & 0.01 \\ 8 & 6 & -0.03 & -0.04 & -0.03 \\ 9 & 6 & -0.01 & -0.06 & -0.04 \\ 10 & 8 & 0.01 & -0.05 & -0.03 \\ 11 & 1 & 0.04 & -0.03 & 0.01 \\ 12 & 1 & 0.02 & -0.01 & 0.03 \\ 13 & 1 & 0.02 & -0.03 & -0.0 \odot \\ 14 & 1 & -0.03 & -0.01 & 0.00 \\ 15 & 1 & -0.05 & -0.04 & -0.04 \\ 16 & 1 & -0.03 & -0.04 & -0.02 \\ 17 & 1 & -0.02 & -0.07 & -0.05 \\ 18 & 7 & -0.01 & -0.07 & -0.06 \\ 19 & 6 & -0.03 & -0.07 & -0.03 \\ 20 & 7 & -0.04 & -0.04 & 0.03 \\ 21 & 6 & -0.02 & -0.03 & 0.01 \\ 22 & 6 & -0.00 & -0.05 & -0.04 \\ 23 & 7 & 0.01 & -0.05 & -0.04 \\ 24 & 6 & 0.01 & -0.02 & -0.01 \\ 25 & 7 & -0.00 & -0.01 & 0.01 \\ 26 & 6 & -0.02 & -0.01 & 0.03 \\ 27 & 7 & 0.03 & -0.02 & -0.02 \\ 28 & 8 & -0.03 & 0.01 & 0.05 \\ 29 & 1 & -0.04 & -0.07 & 0.01 \\ 30 & 1 & 0.03 & -0.02 & -0.01 \\ 31 & 1 & 0.01 & 0.02 & 0.05 \\ 32 & 1 & -0.00 & 0.01 & 0.02 \\ 33 & 8 & 0.15 & 0.57 & 0.18 \\ 34 & 1 & -0.20 & -0.48 & -0.51\end{array}$

$\begin{array}{rrr}0.10 & -0.09 & 0.12 \\ -0.10 & 0.00 & -0.21 \\ 0.07 & -0.08 & 0.07 \\ -0.01 & 0.04 & 0.12 \\ -0.09 & 0.11 & 0.01 \\ -0.19 & 0.08 & -0.23 \\ -0.10 & -0.04 & -0.29 \\ -0.10 & 0.08 & -0.07 \\ 0.07 & -0.13 & -0.08 \\ 0.13 & -0.12 & 0.16 \\ 0.10 & -0.13 & 0.01 \\ -0.02 & 0.07 & 0.22 \\ -0.01 & 0.04 & 0.12 \\ -0.02 & 0.04 & 0.12 \\ -0.02 & 0.03 & 0.06 \\ -0.02 & 0.02 & 0.02 \\ -0.01 & 0.02 & 0.05 \\ -0.01 & 0.02 & 0.02 \\ -0.00 & 0.00 & -0.05 \\ -0.01 & -0.00 & -0.08 \\ -0.01 & 0.00 & -0.05 \\ -0.00 & -0.01 & -0.10 \\ -0.02 & -0.00 & -0.08 \\ -0.02 & 0.04 & 0.14 \\ 0.01 & -0.00 & -0.07 \\ 0.01 & -0.02 & -0.13 \\ -0.01 & -0.01 & -0.14 \\ 0.25 & -0.06 & 0.23 \\ 0.18 & -0.05 & 0.16\end{array}$

$\begin{array}{ccc}-0.04 & 0.01 & -0.10 \\ -0.02 & -0.05 & 0.10 \\ -0.07 & 0.04 & -0.07 \\ -0.02 & -0.01 & -0.10 \\ 0.01 & -0.05 & -0.04 \\ 0.01 & -0.09 & 0.12 \\ -0.02 & -0.06 & 0.14 \\ 0.04 & -0.10 & 0.02 \\ -0.07 & 0.02 & 0.02 \\ -0.08 & 0.08 & -0.12 \\ -0.10 & 0.07 & -0.03 \\ -0.01 & -0.02 & -0.15 \\ -0.03 & -0.00 & -0.10 \\ -0.03 & -0.01 & -0.14 \\ -0.03 & -0.01 & -0.14 \\ -0.03 & 0.01 & -0.09 \\ -0.03 & 0.02 & -0.05 \\ -0.03 & 0.05 & 0.04 \\ -0.03 & 0.07 & 0.11 \\ -0.03 & 0.06 & 0.08 \\ -0.03 & 0.03 & -0.02 \\ -0.03 & 0.10 & 0.23 \\ -0.04 & 0.03 & -0.04 \\ -0.03 & -0.02 & -0.16 \\ -0.03 & 0.11 & 0.24 \\ -0.03 & 0.12 & 0.25 \\ -0.03 & 0.08 & 0.15 \\ 0.46 & -0.17 & 0.10 \\ 0.34 & -0.11 & -0.02\end{array}$

Zero-point correction=

Thermal correction to Energy=

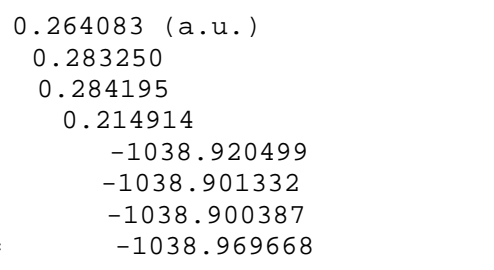

Thermal correction to Enthalpy=
Thermal correction to Gibbs Free Energy=

Sum of electronic and zero-point Energies=

Sum of electronic and thermal Energies=

Sum of electronic and thermal Enthalpies=

Sum of electronic and thermal Free Energies=
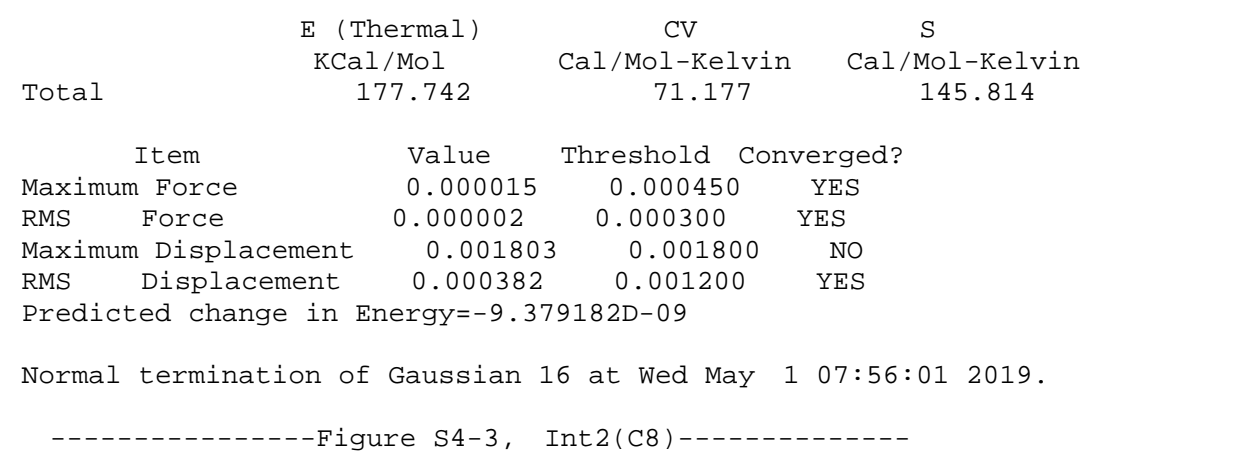

Stoichiometry C10H14N505(2)

Standard orientation:

\begin{tabular}{|c|c|c|c|c|c|}
\hline \multirow{2}{*}{$\begin{array}{l}\text { Center } \\
\text { Number }\end{array}$} & \multirow{2}{*}{$\begin{array}{l}\text { Atomic } \\
\text { Number }\end{array}$} & \multirow{2}{*}{$\begin{array}{c}\text { Atomic } \\
\text { Type }\end{array}$} & \multicolumn{3}{|c|}{ Coordinates (Angstroms) } \\
\hline & & & $x$ & $\mathrm{Y}$ & $\mathrm{Z}$ \\
\hline$\cdots$ & -- & $\cdots$ & 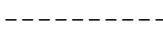 & $\ldots-\ldots$ & - - - - - \\
\hline 1 & 1 & $\odot$ & -3.646472 & -1.384817 & -1.724947 \\
\hline 2 & 8 & $\odot$ & -3.343786 & -1.686062 & -0.864025 \\
\hline 3 & 8 & $\odot$ & -3.296545 & 2.634503 & $-\odot .258667$ \\
\hline 4 & 6 & $\odot$ & -3.382814 & 0.378279 & 0.498582 \\
\hline 5 & 6 & $\odot$ & -3.100652 & 1.293283 & -0.696362 \\
\hline 6 & 1 & $\odot$ & -3.068526 & 3.225070 & -0.983182 \\
\hline 7 & 6 & $\odot$ & -4.027001 & -0.957820 & 0.148832 \\
\hline 8 & 6 & $\odot$ & -1.637717 & ๑. 993996 & -1.004631 \\
\hline 9 & 6 & $\odot$ & -1.058618 & $\odot .697092$ & ๑. 382869 \\
\hline 10 & 8 & $\odot$ & -2.122796 & 0.175391 & 1.151500 \\
\hline 11 & 1 & $\odot$ & - 4.018319 & -1.587875 & 1.046138 \\
\hline 12 & 1 & $\odot$ & -5.075818 & -0.780649 & -0.132973 \\
\hline 13 & 1 & $\odot$ & -4.047806 & $\odot .896635$ & 1.203399 \\
\hline 14 & 1 & $\odot$ & -3.763076 & 1.073969 & -1.545495 \\
\hline 15 & 1 & $\odot$ & -1.124159 & 1.828062 & -1.493869 \\
\hline 16 & 1 & $\odot$ & -1.562625 & $\odot .108817$ & -1.645088 \\
\hline
\end{tabular}




\begin{tabular}{|c|c|c|c|c|c|}
\hline 17 & 1 & $\odot$ & -0.671540 & 1.611564 & ๑.850827 \\
\hline 18 & 7 & $\odot$ & $\odot .043517$ & $-\odot .245008$ & ๑.380708 \\
\hline 19 & 6 & $\odot$ & -0.061933 & -1.687020 & ๑.053735 \\
\hline 20 & 7 & $\odot$ & 1.278847 & -2.108865 & -0.312518 \\
\hline 21 & 6 & $\odot$ & 2.055016 & -1.029124 & -0.181722 \\
\hline 22 & 6 & $\odot$ & 1.327499 & $\odot .128178$ & $\odot .220027$ \\
\hline 23 & 7 & $\odot$ & 1.807487 & 1.362077 & $\odot .396141$ \\
\hline 24 & 6 & $\odot$ & 3.116496 & 1.472831 & $\odot .187024$ \\
\hline 25 & 7 & $\odot$ & 3.908325 & $\odot .425503$ & -0.179575 \\
\hline 26 & 6 & $\odot$ & 3.467092 & $-\odot .895902$ & -0.401280 \\
\hline 27 & 7 & $\odot$ & 3.693185 & 2.673211 & $\odot .341337$ \\
\hline 28 & 8 & $\odot$ & 4.274106 & -1.756806 & -0.746611 \\
\hline 29 & 1 & $\odot$ & -0.784990 & -1.833074 & -0.758644 \\
\hline 30 & 1 & $\odot$ & 3.115789 & 3.449078 & $\odot .625301$ \\
\hline 31 & 1 & $\odot$ & 4.681837 & 2.822509 & $\odot .216808$ \\
\hline 32 & 1 & $\odot$ & 4.899222 & $\odot .577150$ & -0.327214 \\
\hline 33 & 8 & $\odot$ & -0.556942 & -2.446402 & 1.123366 \\
\hline 34 & 1 & $\odot$ & $\odot .130437$ & -2.482613 & 1.799338 \\
\hline
\end{tabular}

Standard basis: Aug-CC-pVDZ (6D, 7F)

626 basis functions

75 alpha electrons

nuclear repulsion energy 1683.6987648265 Hartrees.

NAtoms $=34$

Nuclear repulsion after empirical dispersion term $=$

1683.6722342179 Hartrees.

Force inversion solution in PCM.

Polarizable Continuum Model (PCM)

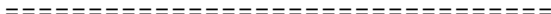

Model : PCM.

Atomic radii : UFF (Universal Force Field).

Solvent : Water, Eps $=78.355300$ Eps $($ inf $)=1.777849$

Solvent : W

SCF Done: $E($ UWB97XD $)=-1039.23093415$ A.U. after 2 cycles

NFock $=2$ Conv $=0.12 \mathrm{D}-08$

$-\mathrm{V} / \mathrm{T}=2.0071$

$\langle$ S $x>=0.0000\langle\mathrm{~S} y\rangle=0.0000\langle\mathrm{Sz}\rangle=0.5000$

$\left\langle S^{* *} 2>=0.7618 \mathrm{~S}=0.5059\right.$

$<$ L.S $>=0.000000000000 \mathrm{E}+00$

Annihilation of the first spin contaminant:

$\mathrm{S}^{* * 2}$ before annihilation 0.7618 , after 0.7501

DoSCS $=F$ DFT $=T$ ScalE2 $(S S, 0 S)=1.0000001 .000000$

Zero-point correction=

Thermal correction to Energy=

Thermal correction to Enthalpy=

Thermal correction to Gibbs Free Energy=

Sum of electronic and zero-point Energies=

Sum of electronic and thermal Energies=

Sum of electronic and thermal Enthalpies=

Sum of electronic and thermal Free Energies=

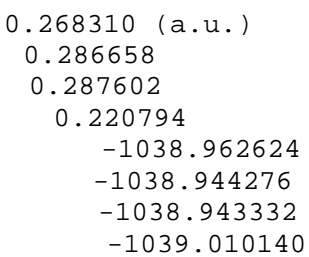

$$
\text { E (Thermal) }
$$$$
\mathrm{KCal} / \mathrm{Mol}
$$

Total 179.881

\section{Item}

Maximum Force

RMS Force

Maximum Displacement

RMS Displacement
$\mathrm{CV}$

Cal/Mol-Kelvin 69.923
$\mathrm{S}$

Cal/Mol-Kelvin 140.609

Predicted change in Energy $=-1.356568 \mathrm{D}-\odot 8$

Normal termination of Gaussian 16 at Thu Mar 14 15:09:๑7 2019.

$$
\text { -...-Figure S4-4, TS2a(C8) }
$$

Stoichiometry

C10H14N505(2)

Standard orientation:

Center Atomic Atomic Coordinates (Angstroms)




\begin{tabular}{|c|c|c|c|c|c|}
\hline Number & Number & Type & $x$ & $Y$ & $Z$ \\
\hline & - & & 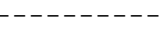 & - & - \\
\hline 1 & 1 & $\odot$ & 2.805231 & -1.015235 & 2.178799 \\
\hline 2 & 8 & $\odot$ & 2.649558 & -1.502491 & 1.365298 \\
\hline 3 & 8 & $\odot$ & 3.610364 & 2.497589 & -0.153025 \\
\hline 4 & 6 & $\odot$ & 3.392776 & $\odot .135492$ & -0.346393 \\
\hline 5 & 6 & $\odot$ & 3.098693 & 1.354293 & 0.527953 \\
\hline 6 & 1 & $\odot$ & 3.362045 & 3.281409 & ๑. 346324 \\
\hline 7 & 6 & $\odot$ & 3.655647 & -1.158011 & $\odot .419438$ \\
\hline 8 & 6 & 0 & 1.575107 & 1.353828 & 0.605076 \\
\hline 9 & 6 & 0 & 1.134936 & 0.700874 & -0.717829 \\
\hline 10 & 8 & $\odot$ & 2.273297 & ๑. 012936 & -1.224578 \\
\hline 11 & 1 & $\odot$ & 3.692665 & -1.981398 & -0.303651 \\
\hline 12 & 1 & $\odot$ & 4.640477 & -1.086371 & 0.906177 \\
\hline 13 & 1 & 0 & 4.278822 & 0.332432 & -0.966325 \\
\hline 14 & 1 & $\odot$ & 3.568030 & 1.269865 & 1.519012 \\
\hline 15 & 1 & $\odot$ & 1.159671 & 2.359683 & 0.720763 \\
\hline 16 & 1 & $\odot$ & 1.234990 & ๑. 739179 & 1.445338 \\
\hline 17 & 1 & $\odot$ & 0.845873 & 1.463533 & -1.457982 \\
\hline 18 & 7 & 0 & ๑. .057116 & -0.236716 & -0.569325 \\
\hline 19 & 6 & $\odot$ & -0.214858 & -2.247480 & -0.006334 \\
\hline 20 & 7 & $\odot$ & -1.541444 & -2.181921 & -0.100590 \\
\hline 21 & 6 & $\odot$ & -2.051862 & -0.944786 & ๑ . 004941 \\
\hline 22 & 6 & $\odot$ & -1.151875 & $\odot .159640$ & -0.294205 \\
\hline 23 & 7 & $\odot$ & -1.560810 & 1.465366 & -0.283106 \\
\hline 24 & 6 & $\odot$ & -2.829524 & 1.684196 & -0.015381 \\
\hline 25 & 7 & $\odot$ & -3.741438 & $\odot .695046$ & 0.240577 \\
\hline 26 & 6 & $\odot$ & -3.437829 & -0.673221 & 0.267009 \\
\hline 27 & 7 & $\odot$ & -3.281252 & 2.951160 & ๑. . 022708 \\
\hline 28 & 8 & $\odot$ & -4.326421 & -1.491206 & 0.506889 \\
\hline 29 & 1 & $\odot$ & 0.344316 & -1.973144 & 0.889236 \\
\hline 30 & 1 & $\odot$ & -2.637977 & 3.692107 & -0.206627 \\
\hline 31 & 1 & $\odot$ & -4.252658 & 3.178596 & 0.160020 \\
\hline 32 & 1 & $\odot$ & -4.705608 & $\odot .932096$ & 0.439780 \\
\hline 33 & 8 & $\odot$ & $\odot .447426$ & -3.121195 & -0.747974 \\
\hline 34 & 1 & $\odot$ & -0.122118 & -3.397320 & -1.481243 \\
\hline
\end{tabular}

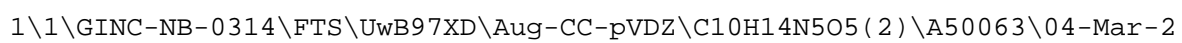

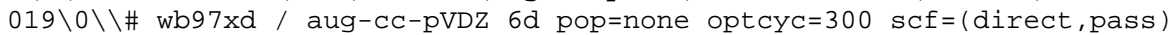
scfcyc $=300$ iop $(1 / 8=2)$ opt=(ts, readfc, nofreeze, noeig) IOP $(2 / 9=1)$ scrf= $(\mathrm{pcm}$, solvent=water $)$ iop $(1 / 18=120)$ geom=allcheck guess $=$ check $\backslash \backslash$ made from file: "degua-oh8a.rev.chk" 2'-deoxyguanosine + OH(.) at C8, C(19)-N(1 $H, 0.0204364673,-3.3094028996,-1.7420910925 \backslash \backslash$ Version=ES64L - G16RevB . $01 \backslash H$ $\mathrm{HF}=-1039.1981457 \backslash \mathrm{S} 2=$

File lengths (MBytes):

$\mathrm{RWF}=529 \mathrm{Int}=\quad \odot \mathrm{D} 2 \mathrm{E}=\quad 0 \mathrm{Chk}=48 \mathrm{Scr}=\quad 1$

Normal termination of Gaussian 16 at Mon Mar 4 12:58:33 2019.

Initial command:

Gaussian 16: ES64L-G16RevB.01 20-Dec-2017 $4-\operatorname{Mar}-2019$

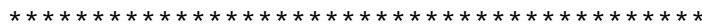

\%mem $=1500 \mathrm{MW}$

$\%$ nproc $=16$

Will use up to 16 processors via shared memory.

\%chk=degua - oh8c . higha. chk

\# wb97xd / aug-cc-pVDZ 6d pop=none optcyc $=300$

scf $=($ direct, pass) $s c f c y c=300$ iop $(1 / 8=2)$

freq=noraman $\operatorname{IOP}(2 / 9=1) \operatorname{scrf}=(\mathrm{pcm}$, solvent=water $)$ geom $=$ allcheck guess $=$ check

the checkpoint file: "degua-oh8c.higha.chk"

made from file: "degua-oh8a.rev.chk" 2'-deoxyguanosine

$+\mathrm{OH}($.$) at \mathrm{C} 8, \mathrm{C}(19)-\mathrm{N}(18)$ dissociation

Charge $=\odot$ Multiplicity $=2$

$\mathrm{H}, 0,2.9015462728,-1.0593895951,2.0363015274$

$0,0,2.753954292,-1.4967105892,1.1934471591$

$0,0,3.5276616818,2.6334276162,-0.0605210029$

C, $0,3.4041276247,0.2827380599,-0.4115721225$ 
C, $0,3.0733392033,1.42636559,0.5471440262$

$\mathrm{H}, 0,3.2548633281,3.370674808,0.493945662$

C, $0,3.7310167553,-1.0473717728,0.2612576946$

C, $0,1.552355409,1.356937706,0.6422732949$

C, $0,1.119794468,0.7769211183,-0.7166410874$

$0,0,2.2777561896,0.1729983809,-1.2826100356$

$\mathrm{H}, 0,3.7910295707,-1.8178013495,-0.5165799386$

$\mathrm{H}, 0,4.719169488,-0.9676003107,0.7399308602$

$\mathrm{H}, 0,4.272114985,0.5579790827,-1.0272171199$

$\mathrm{H}, 0,3.5603254603,1.2949492133,1.5244757941$

$\mathrm{H}, 0,1.0978418997,2.3344217914,0.8311034982$

$\mathrm{H}, 0,1.2501346958,0.6732804901,1.4427651616$

$\mathrm{H}, \Theta, 0.7888499725,1.5750334039,-1.3996670689$

$\mathrm{N}, 0,0.0835739905,-0.2128358168,-0.6194084209$

C, $0,-0.0975062885,-2.2666303354,-0.191348589$

$\mathrm{N}, 0,-1.4269111516,-2.2504305573,-0.265051593$

C, $0,-1.985935439,-1.0456862218,-0.0694777373$

C, $0,-1.1364348133,0.1131049998,-0.3035132109$

$\mathrm{N}, 0,-1.5982746099,1.3968451744,-0.198691822$

C $, 0,-2.8707912843,1.5438208417,0.0984594508$

$\mathrm{N}, 0,-3.7375647855,0.5023572112,0.2973965155$

$\mathrm{C}, 0,-3.3778365228,-0.8506678141,0.2270197037$

$\mathrm{N}, 0,-3.3733984525,2.7853075703,0.2280802848$

$0,0,-4.2285548837,-1.7194455762,0.4212872903$

$\mathrm{H}, 0,0.4631344436,-2.0302112292,0.7140630814$

$\mathrm{H}, 0,-2.7644542387,3.5663139177,0.0420238662$

$\mathrm{H}, 0,-4.3511663818,2.9621062172,0.3921587465$

$\mathrm{H}, \odot,-4.7075836351,0.6848639361,0.5237862597$

$0,0,0.5889662888,-3.0598110615,-0.9985950358$

$\mathrm{H}, 0,0.0204364673,-3.3094028996,-1.7420910925$

Recover connectivity data from disk.

Stoichiometry C10H14N505(2)

Standard orientation:

\begin{tabular}{|c|c|c|c|c|c|}
\hline \multirow{2}{*}{$\begin{array}{l}\text { Center } \\
\text { Number }\end{array}$} & \multirow{2}{*}{$\begin{array}{l}\text { Atomic } \\
\text { Number }\end{array}$} & \multirow{2}{*}{$\begin{array}{c}\text { Atomic } \\
\text { Type }\end{array}$} & \multicolumn{3}{|c|}{ Coordinates (Angstroms) } \\
\hline & & & $\mathrm{X}$ & $\mathrm{Y}$ & Z \\
\hline & & . & - - - - - - & ------ & $-\ldots-\ldots$ \\
\hline 1 & 1 & 0 & 2.805231 & -1.015235 & 2.178799 \\
\hline 2 & 8 & $\odot$ & 2.649558 & -1.502491 & 1.365298 \\
\hline 3 & 8 & $\odot$ & 3.610364 & 2.497589 & -0.153025 \\
\hline 4 & 6 & $\odot$ & 3.392776 & 0.135492 & -0.346393 \\
\hline 5 & 6 & $\odot$ & 3.098693 & 1.354293 & $\odot .527953$ \\
\hline 6 & 1 & $\odot$ & 3.362045 & 3.281409 & $\odot .346324$ \\
\hline 7 & 6 & $\odot$ & 3.655647 & -1.158011 & $\odot .419438$ \\
\hline 8 & 6 & $\odot$ & 1.575107 & 1.353828 & 0.605076 \\
\hline 9 & 6 & $\odot$ & 1.134936 & 0.700874 & -0.717829 \\
\hline 10 & 8 & $\odot$ & 2.273297 & $\odot .012936$ & -1.224578 \\
\hline 11 & 1 & $\odot$ & 3.692665 & -1.981398 & -0.303651 \\
\hline 12 & 1 & $\odot$ & 4.640477 & -1.086371 & 0.906177 \\
\hline 13 & 1 & $\odot$ & 4.278822 & ๑. 332432 & -0.966325 \\
\hline 14 & 1 & $\odot$ & 3.568030 & 1.269865 & 1.519012 \\
\hline 15 & 1 & $\odot$ & 1.159671 & 2.359683 & 0.720763 \\
\hline 16 & 1 & $\odot$ & 1.234990 & ๑. 739179 & 1.445338 \\
\hline 17 & 1 & $\odot$ & 0.845873 & 1.463533 & -1.457982 \\
\hline 18 & 7 & $\odot$ & 0.057116 & -0.236716 & -0.569325 \\
\hline 19 & 6 & $\odot$ & -0.214858 & -2.247480 & -0.006334 \\
\hline 20 & 7 & $\odot$ & -1.541444 & -2.181921 & -0.100590 \\
\hline 21 & 6 & $\odot$ & -2.051862 & -0.944786 & 0.004941 \\
\hline 22 & 6 & $\odot$ & -1.151875 & 0.159640 & -0.294205 \\
\hline 23 & 7 & $\odot$ & -1.560810 & 1.465366 & -0.283106 \\
\hline 24 & 6 & $\odot$ & -2.829524 & 1.684196 & -0.015381 \\
\hline 25 & 7 & $\odot$ & -3.741438 & $\odot .695046$ & 0.240577 \\
\hline 26 & 6 & $\odot$ & -3.437829 & -0.673221 & 0.267009 \\
\hline 27 & 7 & $\odot$ & -3.281252 & 2.951160 & 0.022708 \\
\hline 28 & 8 & $\odot$ & -4.326421 & -1.491206 & 0.506889 \\
\hline 29 & 1 & $\odot$ & ๑. 344316 & -1.973144 & ๑. 889236 \\
\hline 30 & 1 & $\odot$ & -2.637977 & 3.692107 & -0.206627 \\
\hline 31 & 1 & $\odot$ & -4.252658 & 3.178596 & 0.160020 \\
\hline 32 & 1 & $\odot$ & -4.705608 & $\odot .932096$ & ๑. 439780 \\
\hline 33 & 8 & $\odot$ & $\odot .447426$ & -3.121195 & -0.747974 \\
\hline 34 & 1 & 0 & -0.122118 & -3.397320 & -1.481243 \\
\hline
\end{tabular}

Standard basis: Aug-CC-pVDZ (6D, 7F) 


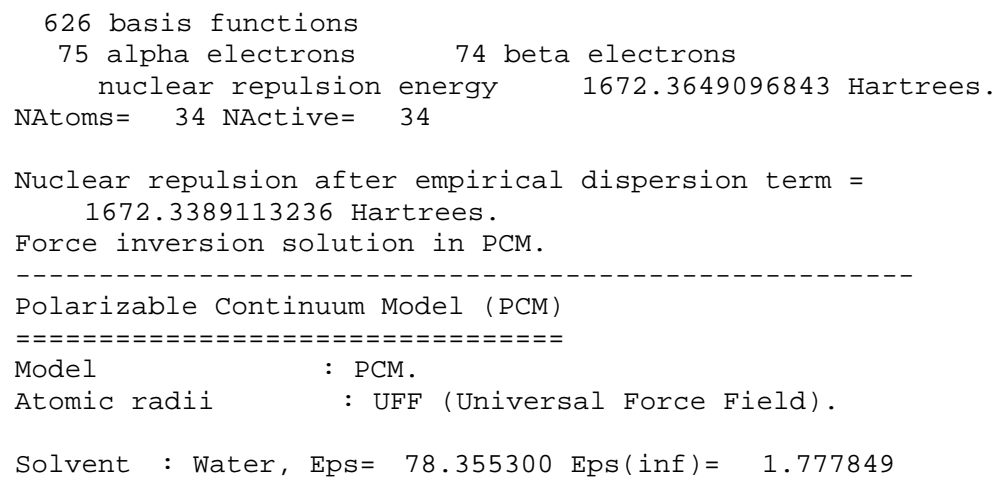


Principal axes and moments of inertia in atomic units:

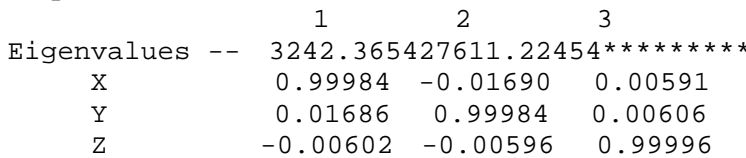

This molecule is an asymmetric top.

Rotational symmetry number 1 .

Warning - - assumption of classical behavior for rotation may cause significant error

$\begin{array}{llll}\text { Rotational temperatures (Kelvin) } & 0.02671 & 0.01138 & 0.00860\end{array}$

Rotational constants (GHZ): $\quad 0.55661 \quad 0.23712 \quad 0.17928$

1 imaginary frequencies ignored.

Zero-point vibrational energy 697900.9 (Joules/Mol) 166.80232 (Kcal/Mol)

Warning - - explicit consideration of 29 degrees of freedom as vibrations may cause significant error

Zero-point correction=

Thermal correction to Energy=

Thermal correction to Enthalpy=

Thermal correction to Gibbs Free Energy=

Sum of electronic and zero-point Energies=

Sum of electronic and thermal Energies=

Sum of electronic and thermal Enthalpies=

Sum of electronic and thermal Free Energies=

$\odot .265816$ ( a.u. )

0.284146

$\odot .285090$

$\odot .218145$

$-1038.932329$

$-1038.914000$

$-1038.913056$

$-1038.980001$

$$
\begin{gathered}
\text { E (Thermal) } \\
\text { KCal/Mol }
\end{gathered}
$$

CV

Total

178.304

Cal/Mol-Kelvin

140.897

\begin{tabular}{lccc}
\multicolumn{1}{c}{ Item } & Value & Threshold Converged? \\
Maximum Force & 0.000015 & 0.000450 & YES \\
RMS Force & 0.000002 & $0.00030 \odot$ & YES \\
Maximum Displacement & 0.001131 & $0.00180 \odot$ & YES \\
RMS Displacement & 0.000307 & 0.001200 & YES \\
Predicted change in Energy=-4.055340D-09 & \\
Optimization completed. & & \\
\multicolumn{2}{l}{- - Stationary point found. }
\end{tabular}

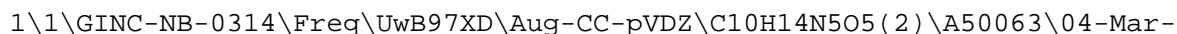

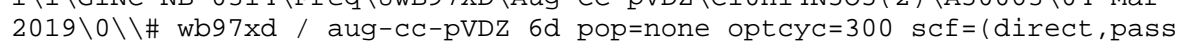
) $\mathrm{scfcyc}=300$ iop $(1 / 8=2)$ freq=noraman $\operatorname{IOP}(2 / 9=1)$ scrf= $(\mathrm{pcm}$, solvent=wate r) geom=allcheck guess=check \\made from file: "degua-oh8a.rev.chk" 2' -

File lengths (MBytes):

$\mathrm{RWF}=2403$ Int $=\quad \odot \mathrm{D} 2 \mathrm{E}=\quad \odot \mathrm{Chk}=62 \mathrm{Scr}=1$

Normal termination of Gaussian 16 at Mon Mar 4 16:55:11 2019.

Stoichiometry C10H14N505(2)

\begin{tabular}{|c|c|c|c|c|c|}
\hline \multirow{2}{*}{$\begin{array}{l}\text { Center } \\
\text { Number }\end{array}$} & \multirow{2}{*}{$\begin{array}{l}\text { Atomic } \\
\text { Number }\end{array}$} & \multirow{2}{*}{$\begin{array}{c}\text { Atomic } \\
\text { Type }\end{array}$} & \multicolumn{3}{|c|}{ Coordinates (Angstroms) } \\
\hline & & & $x$ & $\mathrm{Y}$ & $Z$ \\
\hline & & & $-\ldots-\ldots$ & - - - - - - - & 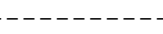 \\
\hline 1 & 1 & $\odot$ & 2.568753 & -1.298773 & 2.002099 \\
\hline 2 & 8 & $\odot$ & 2.401949 & -1.708201 & 1.148853 \\
\hline 3 & 8 & $\odot$ & 3.921722 & 2.255278 & $\odot .027555$ \\
\hline 4 & 6 & $\odot$ & 3.437275 & $-\odot .036926$ & -0.384726 \\
\hline 5 & 6 & $\odot$ & 3.233462 & 1.133121 & 0.576482 \\
\hline 6 & 1 & $\odot$ & 3.719699 & 3.024713 & 0.568246 \\
\hline 7 & 6 & $\odot$ & 3.484962 & -1.414831 & 0.273507 \\
\hline 8 & 6 & $\odot$ & 1.717178 & 1.318762 & 0.574727 \\
\hline 9 & 6 & $\odot$ & 1.255941 & $\odot .749770$ & -0.785791 \\
\hline 10 & 8 & $\odot$ & 2.387591 & 0.081747 & -1.342180 \\
\hline 11 & 1 & $\odot$ & 3. 451282 & -2.171191 & -0.519793 \\
\hline 12 & 1 & $\odot$ & 4.447063 & -1.516801 & ○. 799744 \\
\hline 13 & 1 & $\odot$ & 4.384870 & ๑. 089962 & $-\odot .926908$ \\
\hline 14 & 1 & $\odot$ & 3.625041 & ०. 915029 & 1.581010 \\
\hline 15 & 1 & $\odot$ & 1.430857 & 2.368316 & ๑. 691718 \\
\hline 16 & 1 & $\odot$ & 1.257803 & 0.745432 & 1.386874 \\
\hline 17 & 1 & $\odot$ & 0.984870 & 1.555114 & -1.484815 \\
\hline 18 & 7 & $\odot$ & 0.176030 & -0.187123 & -0.670278 \\
\hline 19 & 6 & $\odot$ & -0.762214 & -2.646456 & 0.096657 \\
\hline
\end{tabular}

Standard orientation: 


\begin{tabular}{|c|c|c|c|c|c|}
\hline 20 & 7 & $\odot$ & -1.868026 & -2.079396 & -0.223517 \\
\hline 21 & 6 & $\odot$ & -2.048677 & -0.733274 & -0.128540 \\
\hline 22 & 6 & $\odot$ & -1.004900 & $\odot .257461$ & -0.356340 \\
\hline 23 & 7 & $\odot$ & -1.277050 & 1.610137 & -0.229335 \\
\hline 24 & 6 & $\odot$ & -2.495941 & 1.979018 & 0.045940 \\
\hline 25 & 7 & $\odot$ & -3.540342 & 1.101973 & 0.219456 \\
\hline 26 & 6 & $\odot$ & -3.405635 & -0.277938 & 0.150067 \\
\hline 27 & 7 & $\odot$ & -2.786062 & 3. 288479 & $\odot .199959$ \\
\hline 28 & 8 & $\odot$ & -4.380744 & -1.000423 & ๑. 327279 \\
\hline 29 & 1 & $\odot$ & $\odot .071446$ & -2.184627 & 0.630903 \\
\hline 30 & 1 & $\odot$ & -2.064911 & 3.954458 & -0.029998 \\
\hline 31 & 1 & $\odot$ & -3.736362 & 3.620024 & ๑. 252944 \\
\hline 32 & 1 & 0 & -4.468004 & 1.445410 & $\odot .435693$ \\
\hline 33 & 8 & 0 & -0.550522 & -3.927013 & -0.181542 \\
\hline 34 & 1 & $\odot$ & -1.312925 & -4.268920 & -0.673320 \\
\hline
\end{tabular}

Standard basis: Aug-CC-pVDZ (6D, 7F)

626 basis functions

75 alpha electrons $\quad 74$ beta electrons

nuclear repulsion energy 1649.2482635123 Hartrees.

NAtoms $=34$ NActive $=34$

Force inversion solution in PCM.

SCF Done: $E(U W B 97 X D)=-1039.20811955$ A.U. after 1 cycles NFock $=1$ Conv $=0.56 \mathrm{D}-08 \quad-\mathrm{V} / \mathrm{T}=2.0071$

$\langle\mathrm{S} x\rangle=0.0000<\mathrm{S} y>=0.0000<\mathrm{S} z>=0.5000<\mathrm{S}^{* *} 2>=0.7759 \mathrm{~S}=0.5129$ $<$ L.S $>=0.000000000000 \mathrm{E}+0 \odot$

Annihilation of the first spin contaminant:

$\mathrm{S}^{* *} 2$ before annihilation 0.7759 , after

$\odot .7503$

Zero-point correction=

Thermal correction to Energy=

Thermal correction to Enthalpy=

Thermal correction to Gibbs Free Energy=

Sum of electronic and zero-point Energies=

Sum of electronic and thermal Energies=

Sum of electronic and thermal Enthalpies=

$\odot .265608$ (a.u.)

0.284865

$\odot .285809$

0.216444

$-1038.942512$

$-1038.923254$

$-1038.922310$

$-1038.991675$

Sum of electronic and thermal Free Energies=

$\mathrm{S}$

$$
\begin{gathered}
\text { E (Thermal) } \\
\text { KCal/Mol }
\end{gathered}
$$

$\mathrm{CV}$

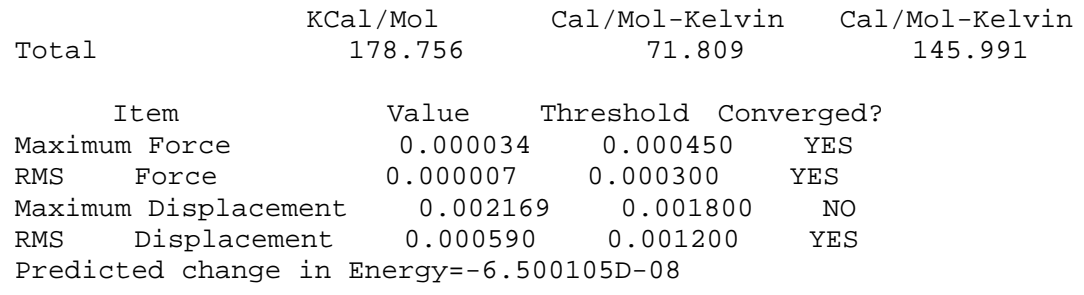

Predicted change in Energy $=-6.500105 \mathrm{D}-\odot 8$

Normal termination of Gaussian 16 at Thu Mar 14 15:38:20 2019.

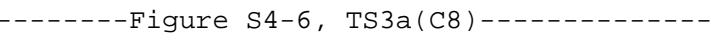

Stoichiometry C10H14N505(2)

\begin{tabular}{|c|c|c|c|c|c|}
\hline \multirow{2}{*}{$\begin{array}{l}\text { Center } \\
\text { Number }\end{array}$} & \multirow{2}{*}{$\begin{array}{l}\text { Atomic } \\
\text { Number }\end{array}$} & \multirow{2}{*}{$\begin{array}{l}\text { Atomic } \\
\text { Type }\end{array}$} & \multicolumn{3}{|c|}{ Coordinates (Angstroms) } \\
\hline & & & $x$ & $\mathrm{Y}$ & Z \\
\hline & & & & & \\
\hline 1 & 1 & $\odot$ & -2.649422 & 1.563992 & 1.729750 \\
\hline 2 & 8 & $\odot$ & -2.511795 & 1.908831 & 0.843318 \\
\hline 3 & 8 & $\odot$ & -4.025469 & -2.192488 & 0.160378 \\
\hline 4 & 6 & $\odot$ & -3.478991 & 0.031514 & $-\odot .481056$ \\
\hline 5 & 6 & $\odot$ & -3.349176 & -1.023360 & $\odot .618402$ \\
\hline 6 & 1 & $\odot$ & -3.878910 & -2.894421 & 0.801442 \\
\hline 7 & 6 & $\odot$ & -3.574040 & 1.476901 & $\odot .002219$ \\
\hline 8 & 6 & $\odot$ & -1.839034 & -1.226832 & 0.729393 \\
\hline 9 & 6 & $\odot$ & -1.285472 & -0.811447 & -0.650669 \\
\hline 10 & 8 & $\odot$ & -2.364845 & -0.184176 & -1.342938 \\
\hline 11 & 1 & $\odot$ & -3.547471 & 2.130725 & -0.877674 \\
\hline 12 & 1 & $\odot$ & -4.547052 & 1.615866 & ๑. 499510 \\
\hline 13 & 1 & $\odot$ & -4.388288 & -0.164687 & -1.067076 \\
\hline 14 & 1 & $\odot$ & -3.793852 & -0.686485 & 1.566422 \\
\hline
\end{tabular}

Standard orientation: 


\begin{tabular}{|c|c|c|c|c|c|}
\hline 15 & 1 & $\odot$ & -1.579444 & -2.261315 & $\odot .975803$ \\
\hline 16 & 1 & $\odot$ & -1.417916 & -0.574501 & 1.502558 \\
\hline 17 & 1 & $\odot$ & -0.990187 & -1.685113 & -1.248459 \\
\hline 18 & 7 & $\odot$ & $-\odot .202858$ & $\odot .122258$ & -0.533494 \\
\hline 19 & 6 & $\odot$ & ๑. 701382 & 2.735111 & $\odot .009114$ \\
\hline 20 & 7 & $\odot$ & 1.813332 & 2.007874 & -0.040905 \\
\hline 21 & 6 & $\odot$ & 2.032138 & 0.674769 & -0.080800 \\
\hline 22 & 6 & $\odot$ & ๑. 983831 & -0.322608 & -0.265210 \\
\hline 23 & 7 & $\odot$ & 1.280789 & -1.669218 & -0.138833 \\
\hline 24 & 6 & 0 & 2.524278 & -2.022186 & ๑ . 031713 \\
\hline 25 & 7 & $\odot$ & 3.573476 & -1.132173 & $\odot .106176$ \\
\hline 26 & 6 & $\odot$ & 3.415313 & ๑. 244427 & 0.077881 \\
\hline 27 & 7 & $\odot$ & 2.844391 & -3.324839 & 0.146804 \\
\hline 28 & 8 & $\odot$ & 4.383983 & ๑. 987565 & ๑. 185427 \\
\hline 29 & 1 & $\odot$ & -0.333951 & 2.397024 & 0.069665 \\
\hline 30 & 1 & $\odot$ & 2.107254 & -4.006606 & ๑. 062485 \\
\hline 31 & 1 & $\odot$ & 3.792440 & -3.646126 & ๑. 258183 \\
\hline 32 & 1 & $\odot$ & 4.521883 & -1.466262 & 0.224921 \\
\hline 33 & 8 & $\odot$ & 1. 089067 & 3.957879 & ๑. .005901 \\
\hline 34 & 1 & $\odot$ & 2.192096 & 3. 291970 & -0.038358 \\
\hline
\end{tabular}

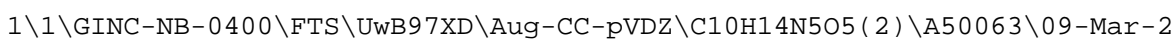

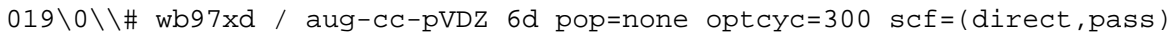
scfcyc $=300$ iop $(1 / 8=2)$ opt=(ts, readfc, nofreeze, noeig) $\operatorname{IOP}(2 / 9=1)$ scrf= $(\mathrm{pcm}$, solvent=water $)$ iop $(1 / 18=120)$ geom $=$ allcheck guess $=$ check $\backslash \backslash \mathrm{H} 34 \mathrm{migra}$ tion $\backslash \backslash 0,2 \backslash H, 2.8600618168,-1.1515710504,1.741612155 \backslash 0,2.7906326218,-1.4$ $829007713,0.8421589669 \backslash 0,3.6282431089,2.8303087175,0.3196041409 \backslash \mathrm{C}, 3.45$

$6,-3.5755465298,-\odot .1657374671 \backslash \backslash$ Version=ES64L - G16RevB . $01 \backslash H F=-1039.15736$ $61 \backslash S 2=0.773765 \backslash S 2-1=0 . \backslash S 2 A=0.750351 \backslash R M S D=6.944 e-09 \backslash R M S F=2.437 e-06 \backslash D i p o$ $l e=1.5344999,-3.6478871,2.079543 \backslash P G=C 01[X(C 10 H 14 N 505)] \backslash \backslash @$

File lengths (MBytes):

RWF $=529$ Int $=\quad \odot$ D2E $=\quad 0$ Chk $=50 \mathrm{Scr}=1$

Normal termination of Gaussian 16 at Sat Mar 9 19:18:00 2019.

Initial command:

*********************************************

Gaussian 16: ES64L-G16RevB.01 20-Dec-2017

$9-\operatorname{Mar}-2019$

$* * * * * * * * * * * * * * * * * * * * * * * * * * * * * * * * * * * * * * * * * *$

$\%$ mem $=1500 \mathrm{MW}$

$\%$ nproc $=16$

Will use up to 16 processors via shared memory.

\%chk=degua - oh8d. higha. chk

\# wb97xd / aug-cc-pVDZ 6d pop=none optcyc=300 scf=(direct, pass)

scfcyc $=300$ iop $(1 / 8=2)$ freq=noraman

$\operatorname{IOP}(2 / 9=1) \operatorname{scrf}=(\mathrm{pcm}$, solvent=water $)$ geom=allcheck guess=check

the checkpoint file: "degua-oh8d.higha.chk"

- - - - - - - - -

H34 migration

- - - - - - - - -

Charge $=0$ Multiplicity $=2$

$\mathrm{H}, 0,2.8600618168,-1.1515710504,1.741612155$

$0,0,2.7906326218,-1.4829007713,0.8421589669$

$0,0,3.6282431089,2.8303087175,0.3196041409$

C , $0,3.4567558949,0.5710615326,-0.4033428034$

C, $0,3.1447879824,1.5520558837,0.7274738786$

$\mathrm{H}, 0,3.3623511248,3.4765322699,0.9805509937$

C, $0,3.7788413446,-0.8560261069,0.0341684588$

C, $0,1.6204176291,1.5050776504,0.8187683829$

C $, 0,1.1578619456,1.0542857444,-0.5835058851$

$0,0,2.3327096254,0.6340687232,-1.2769761147$

$\mathrm{H}, 0,3.8689500797,-1.474398718,-0.86688649$

$\mathrm{H}, 0,4.755534171,-0.8533984757,0.5435060325$

$\mathrm{H}, 0,4.3291835954,0.9318376998,-0.9667656593$

$\mathrm{H}, 0,3.6268454693,1.2584472367,1.671602219$

$\mathrm{H}, 0,1.1938373181,2.4748345634,1.0941991424$

$\mathrm{H}, 0,1.3013253679,0.7666980958,1.5628351437$

$\mathrm{H}, 0,0.7321329984,1.889183706,-1.1575598028$

$\mathrm{N}, 0,0.2394072634,-0.0454528953,-0.5156470183$

$\mathrm{C}, 0,-\odot .2362032871,-2.7873558568,-\odot .0742450601$ 
$\mathrm{N}, 0,-1.4505617716,-2.2478558519,-\odot .1198672218$

C, $\odot,-1.8818501646,-0.9669859381,-0.1201093609$

C, $0,-1.0067593005,0.1923626827,-0.253680682$

$\mathrm{N}, 0,-1.5193253797,1.468123404,-0.0886825929$

C $, 0,-2.8054961393,1.609550868,0.0716312612$

$\mathrm{N}, 0,-3.6975446176,0.5597918568,0.0988124966$

C $, 0,-3.3182409362,-0.771381324,0.028440175$

$\mathrm{N}, 0,-3.3336584304,2.8386148729,0.2235164625$

$0,0,-4.1550064429,-1.6644397127,0.094866592$

$\mathrm{H}, 0,0.7299291582,-2.2889006365,0.0152744373$

$\mathrm{H}, \Theta,-2.715693672,3.6329585701,0.1742776305$

$\mathrm{H}, 0,-4.3224762752,2.998557005,0.3288076381$

$\mathrm{H}, 0,-4.68887638,0.7320441739,0.2118748017$

$0,0,-0.4207129834,-4.0558004895,-0.1240295528$

$\mathrm{H}, 0,-1.6164062286,-3.5755465298,-0.1657374671$

Recover connectivity data from disk.

Stoichiometry C10H14N505(2)

Standard orientation:

\begin{tabular}{|c|c|c|c|c|c|}
\hline \multirow{2}{*}{$\begin{array}{l}\text { Center } \\
\text { Number }\end{array}$} & \multirow{2}{*}{$\begin{array}{l}\text { Atomic } \\
\text { Number }\end{array}$} & \multirow{2}{*}{$\begin{array}{l}\text { Atomic } \\
\text { Type }\end{array}$} & \multicolumn{3}{|c|}{ Coordinates (Angstroms) } \\
\hline & & & $\mathrm{X}$ & Y & Z \\
\hline 7 & & & & & \\
\hline 1 & 1 & $\odot$ & -2.649422 & 1.563992 & 1.729750 \\
\hline 2 & 8 & 0 & -2.511795 & 1.908831 & 0.843318 \\
\hline 3 & 8 & $\odot$ & -4.025469 & -2.192488 & 0.160378 \\
\hline 4 & 6 & $\odot$ & -3.478991 & 0.031514 & -0.481056 \\
\hline 5 & 6 & $\odot$ & -3.349176 & -1.023360 & 0.618402 \\
\hline 6 & 1 & $\odot$ & -3.878910 & -2.894421 & $\odot .801442$ \\
\hline 7 & 6 & $\odot$ & -3.574040 & 1. 476901 & $\odot .0 \odot 2219$ \\
\hline 8 & 6 & $\odot$ & -1.839034 & -1.226832 & $\odot .729393$ \\
\hline 9 & 6 & $\odot$ & -1.285472 & -0.811447 & $-\odot .650669$ \\
\hline 10 & 8 & $\odot$ & -2.364845 & -0.184176 & -1.342938 \\
\hline 11 & 1 & $\odot$ & -3.547471 & 2.130725 & -0.877674 \\
\hline 12 & 1 & $\odot$ & -4.547052 & 1.615866 & ๑ . 499510 \\
\hline 13 & 1 & $\odot$ & -4.388288 & -0.164687 & -1.067076 \\
\hline 14 & 1 & $\odot$ & -3.793852 & -0.686485 & 1.566422 \\
\hline 15 & 1 & $\odot$ & -1.579444 & -2.261315 & 0.975803 \\
\hline 16 & 1 & $\odot$ & -1.417916 & -0.574501 & 1.502558 \\
\hline 17 & 1 & $\odot$ & -0.990187 & -1.685113 & -1.248459 \\
\hline 18 & 7 & $\odot$ & -0.202858 & ๑. 122258 & -0.533494 \\
\hline 19 & 6 & $\odot$ & ๑. 701382 & 2.735111 & $\odot .0 \odot 9114$ \\
\hline 20 & 7 & $\odot$ & 1.813332 & $2.0 \odot 7874$ & $-\odot .040905$ \\
\hline 21 & 6 & $\odot$ & 2.032138 & 0.674769 & -0.080800 \\
\hline 22 & 6 & $\odot$ & ๑. 983831 & -0.322608 & -0.265210 \\
\hline 23 & 7 & $\odot$ & 1. 280789 & -1.669218 & -0.138833 \\
\hline 24 & 6 & $\odot$ & 2.524278 & -2.022186 & $\odot .031713$ \\
\hline 25 & 7 & $\odot$ & 3.573476 & -1.132173 & $\odot .106176$ \\
\hline 26 & 6 & $\odot$ & 3.415313 & ๑. 244427 & ๑. . 077881 \\
\hline 27 & 7 & $\odot$ & 2.844391 & -3.324839 & $\odot .146804$ \\
\hline 28 & 8 & $\odot$ & 4.383983 & $\odot .987565$ & $\odot .185427$ \\
\hline 29 & 1 & $\odot$ & -0.333951 & 2.397024 & ๑.069665 \\
\hline 30 & 1 & $\odot$ & 2.107254 & -4.006606 & ๑. . 062485 \\
\hline 31 & 1 & $\odot$ & 3.792440 & -3.646126 & ๑. 258183 \\
\hline 32 & 1 & $\odot$ & 4.521883 & -1.466262 & ๑. 224921 \\
\hline 33 & 8 & $\odot$ & 1.089067 & 3.957879 & $\odot .0 \odot 5901$ \\
\hline 34 & 1 & $\odot$ & 2.192096 & 3.291970 & -0.038358 \\
\hline
\end{tabular}

Standard basis: Aug-CC-pVDZ (6D, 7F)

626 basis functions

75 alpha electrons

nuclear repulsion energy 1643.4281144727 Hartrees.

NAtoms $=34$ NActive $=34$

Nuclear repulsion after empirical dispersion term =

1643.4033071159 Hartrees.

Force inversion solution in PCM.

Polarizable Continuum Model (PCM)

Model : PCM.

Solvent : Water, Eps $=78.355300$ Eps $($ inf $)=1.777849$ 
SCF Done: $E($ UWB97XD) $=-1039.15736614$ A.U. after 1 cycles

$$
\text { NFock }=1 \text { Conv }=0.54 \mathrm{D}-08 \quad-\mathrm{V} / \mathrm{T}=2.0071
$$

$\langle\mathrm{S} X>=0.0000<\mathrm{Sy}\rangle=0.0000<\mathrm{S} z>=0.5000$

$<S^{*} 2>=0.7738 \mathrm{~S}=0.5118$

$<\mathrm{L} . \mathrm{S}>=0.000000000000 \mathrm{E}+00$

Annihilation of the first spin contaminant:

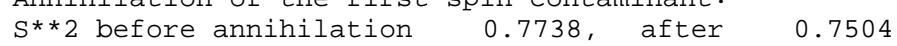

Harmonic frequencies $\left(\mathrm{cm}^{* *}-1\right)$, IR intensities (KM/Mole), Raman activities ( $\left.A^{* *} 4 / A M U\right)$, depolarization ratios for plane and unpo incident light, reduced masses (AMU), force constants (mDyne/A), and normal coordinates:

\begin{tabular}{|c|c|c|c|c|c|c|c|c|c|c|}
\hline & & & 1 & & 2 & & & 3 & & \\
\hline \multicolumn{2}{|c|}{ Frequencies } & - - & \multicolumn{2}{|c|}{-1949.5037} & \multicolumn{3}{|c|}{30.5691} & \multicolumn{3}{|c|}{34.4621} \\
\hline \multicolumn{2}{|c|}{ Red. masses } & - - & \multicolumn{2}{|c|}{1.0639} & \multicolumn{3}{|c|}{5.6381} & \multicolumn{3}{|c|}{5.8290} \\
\hline \multicolumn{2}{|c|}{ Frc consts } & -- & \multicolumn{2}{|c|}{2.3823} & \multicolumn{3}{|c|}{0.0031} & \multicolumn{3}{|c|}{0.0041} \\
\hline \multicolumn{2}{|c|}{ IR Inten } & - & \multicolumn{2}{|c|}{1020.7715} & \multicolumn{3}{|c|}{1.7384} & \multicolumn{3}{|c|}{0.4510} \\
\hline Atom & AN & $x$ & $Y$ & Z & $x$ & $Y$ & Z & $x$ & Z & \\
\hline 1 & 1 & $\odot . \odot \odot$ & -0.00 & -0.00 & 0.08 & -0.18 & 0.18 & 0.30 & -0.07 & 0.02 \\
\hline 2 & 8 & -0.00 & $\odot . \odot \odot$ & $\odot . \odot \odot$ & $\odot .03$ & -0.11 & 0.20 & 0.32 & -0.06 & 0.03 \\
\hline 3 & 8 & -0.00 & -0.00 & -0.00 & 0.08 & -0.07 & -0.12 & -0.13 & 0.13 & 0.06 \\
\hline 4 & 6 & $\odot .0 \odot$ & -0.00 & -0.00 & -0.01 & 0.01 & $\odot .08$ & 0.06 & 0.07 & 0.02 \\
\hline 5 & 6 & $\odot . \odot \odot$ & $\odot . \odot \odot$ & $\odot .0 \odot$ & $\odot .06$ & -0.10 & -0.03 & 0.03 & 0.05 & 0.01 \\
\hline 6 & 1 & $\odot . \odot \odot$ & $\odot .0 \odot$ & $-\odot . \odot \odot$ & 0.14 & -0.14 & -0.21 & -0.17 & 0.11 & 0.05 \\
\hline 7 & 6 & $-\odot . \odot \odot$ & $-\odot . \odot \odot$ & $\odot . \odot \odot$ & -0.01 & -0.04 & 0.22 & 0.26 & 0.07 & 0.04 \\
\hline 8 & 6 & $-0.0 \odot$ & $-0.0 \odot$ & $-\odot .0 \odot$ & 0.07 & -0.06 & -0.11 & $\odot .02$ & -0.11 & $-\odot .08$ \\
\hline 9 & 6 & $\odot .0 \odot$ & $\odot . \odot \odot$ & $\odot . \odot \odot$ & -0.00 & 0.06 & -0.10 & -0.01 & -0.07 & -0.09 \\
\hline 10 & 8 & $-\odot . \odot \odot$ & 0.00 & $-0.0 \odot$ & -0.05 & 0.11 & 0.00 & -0.03 & -0.07 & -0.06 \\
\hline 11 & 1 & -0.00 & $\odot . \odot \odot$ & $\odot . \odot \odot$ & -0.06 & $\odot .04$ & $\odot .28$ & 0.32 & 0.08 & 0.04 \\
\hline 12 & 1 & $\odot . \odot \odot$ & $\odot . \odot \odot$ & $\odot . \odot \odot$ & $\odot .02$ & -0.10 & $\odot .28$ & 0.28 & 0.19 & 0.05 \\
\hline 13 & 1 & $-0.0 \odot$ & 0.00 & 0.00 & -0.03 & 0.05 & 0.10 & $\odot . \odot \odot$ & 0.17 & 0.08 \\
\hline 14 & 1 & $-\odot . \odot \odot$ & $\odot .0 \odot$ & $-\odot . \odot \odot$ & 0.08 & -0.20 & 0.02 & 0.13 & 0.10 & 0.04 \\
\hline 15 & 1 & -0.00 & $-\odot .0 \odot$ & $\odot . \odot \odot$ & 0.12 & -0.07 & -0.20 & -0.08 & -0.15 & -0.17 \\
\hline 16 & 1 & 0.00 & 0.00 & -0.00 & $\odot .08$ & -0.10 & -0.08 & 0.12 & -0.21 & -0.06 \\
\hline 17 & 1 & $-\odot . \odot \odot$ & $-\odot .0 \odot$ & $\odot . \odot \odot$ & -0.03 & 0.11 & -0.19 & $-0.0 \odot$ & -0.05 & -0.11 \\
\hline 18 & 7 & $\odot .0 \odot$ & -0.00 & $\odot . \odot \odot$ & 0.00 & $\odot .06$ & -0.10 & -0.02 & -0.05 & -0.08 \\
\hline 19 & 6 & 0.01 & -0.05 & $-0.0 \odot$ & 0.04 & 0.05 & -0.12 & -0.12 & -0.05 & 0.02 \\
\hline 20 & 7 & -0.01 & -0.02 & $\odot .0 \odot$ & 0.03 & 0.03 & -0.08 & -0.09 & -0.01 & $-\odot . \odot \odot$ \\
\hline 21 & 6 & -0.01 & 0.00 & -0.00 & $-\odot .0 \odot$ & 0.02 & -0.03 & -0.05 & 0.00 & -0.00 \\
\hline 22 & 6 & $-0.0 \odot$ & $-\odot .0 \odot$ & -0.00 & -0.02 & 0.04 & -0.05 & -0.02 & -0.03 & -0.04 \\
\hline 23 & 7 & $-\odot . \odot \odot$ & -0.00 & -0.00 & -0.06 & 0.04 & -0.01 & 0.01 & -0.02 & -0.02 \\
\hline 24 & 6 & -0.00 & 0.00 & -0.00 & -0.07 & 0.01 & 0.07 & 0.01 & 0.01 & 0.01 \\
\hline 25 & 7 & $\odot . \odot \odot$ & $\odot . \odot \odot$ & $\odot . \odot \odot$ & -0.06 & -0.01 & 0.10 & -0.01 & $\odot .04$ & 0.03 \\
\hline 26 & 6 & 0.00 & 0.00 & 0.00 & -0.02 & -0.01 & $\odot .05$ & -0.04 & 0.04 & 0.03 \\
\hline 27 & 7 & $\odot .0 \odot$ & $-\odot . \odot \odot$ & $0.0 \odot$ & -0.12 & 0.01 & 0.13 & 0.04 & 0.02 & 0.04 \\
\hline 28 & 8 & $\odot .0 \odot$ & -0.00 & $\odot . \odot \odot$ & -0.01 & -0.03 & 0.07 & -0.07 & 0.06 & 0.06 \\
\hline 29 & 1 & -0.01 & 0.03 & $\odot . \odot \odot$ & 0.04 & 0.07 & -0.10 & -0.11 & -0.09 & 0.08 \\
\hline 30 & 1 & $0.0 \odot$ & $\odot .0 \odot$ & $-\odot .0 \odot$ & -0.13 & 0.02 & 0.08 & 0.06 & $\odot . \odot \odot$ & $-\odot . \odot \odot$ \\
\hline 31 & 1 & 0.00 & -0.00 & $\odot . \odot \odot$ & -0.12 & -0.02 & 0.13 & 0.05 & 0.05 & 0.02 \\
\hline 32 & 1 & $-0 . \odot \odot$ & -0.00 & 0.00 & -0.07 & -0.03 & 0.16 & -0.01 & 0.06 & 0.06 \\
\hline 33 & 8 & 0.04 & $0.0 \odot$ & $-\odot . \odot \odot$ & 0.06 & $\odot .04$ & -0.19 & -0.17 & -0.04 & -0.01 \\
\hline 34 & 1 & -0.43 & 0.90 & $\odot .02$ & 0.05 & 0.02 & -0.15 & -0.14 & 0.01 & -0.04 \\
\hline
\end{tabular}

- Thermochemistry

Temperature 298.150 Kelvin. Pressure 1.00000 Atm.

Molecular mass: 284.09949 amu.

Principal axes and moments of inertia in atomic units:

$$
123
$$

Eigenvalues - - 3696.355877617.02543**********

$\begin{array}{lrrr}X & 0.99982 & -0.01856 & -0.00435 \\ Y & 0.01854 & 0.99982 & -0.00455 \\ Z & 0.00443 & 0.00447 & 0.99998\end{array}$

This molecule is an asymmetric top.

Rotational symmetry number 1

Warning - - assumption of classical behavior for rotation may cause significant error

$\begin{array}{llll}\text { Rotational temperatures (Kelvin) } & 0.02343 & 0.01137 & 0.00806\end{array}$

Rotational constants (GHZ): $\quad 0.48825 \quad 0.23694 \quad 0.16788$

1 imaginary frequencies ignored.

Zero-point vibrational energy 684305.7 (Joules/Mol)

163.55299 (Kcal/Mol)

Warning - - explicit consideration of 29 degrees of freedom as 
vibrations may cause significant error

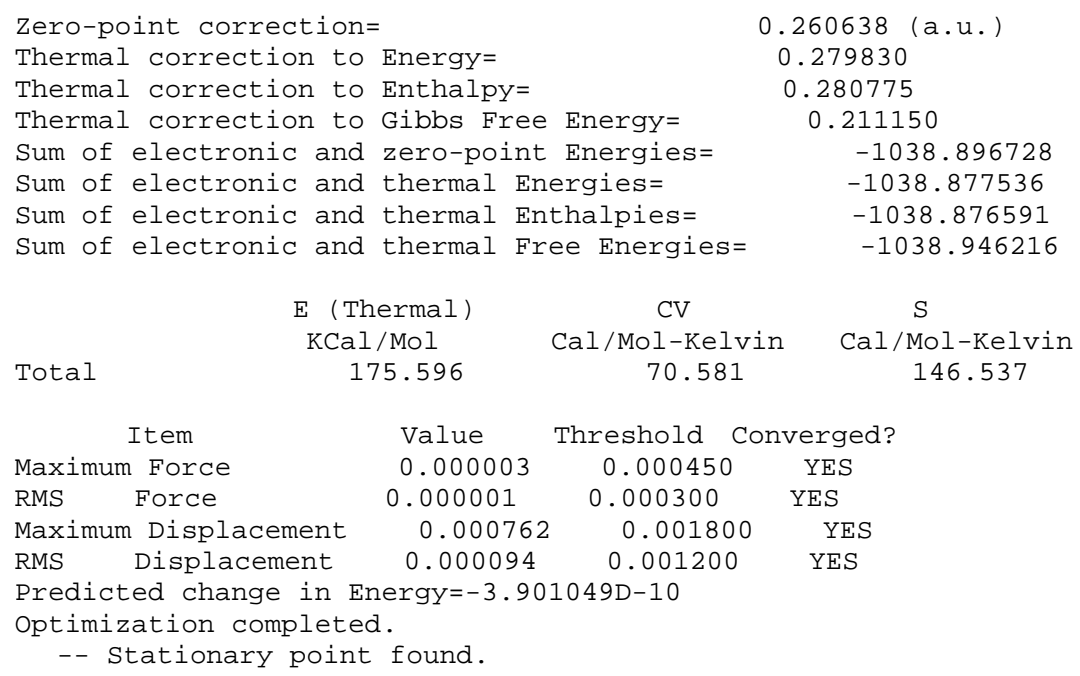
70.581 146.537

Total 175.596

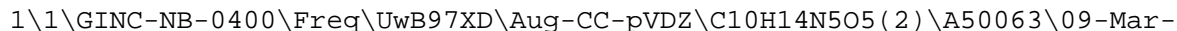
2019 $\ \backslash \backslash \#$ wb97xd / aug-cc-pVDZ 6d pop=none optcyc=300 scf=(direct, pass ) $\operatorname{scfcyc}=300$ iop $(1 / 8=2)$ freq=noraman $\operatorname{IOP}(2 / 9=1) \operatorname{scrf}=(\mathrm{pcm}$, solvent=wate r) geom=allcheck guess $=$ check $\backslash \backslash \mathrm{H} 34$ migration $\backslash \backslash 0,2 \backslash \mathrm{H}, 2.8600618168,-1.151$ $5710504,1.741612155 \backslash 0,2.7906326218,-1.4829007713,0.8421589669 \backslash 0,3.6282$

File lengths (MBytes):

RWF $=2403$ Int $=\quad \odot$ D2E $=\quad 0$ Chk $=05 \mathrm{Scr}=\quad 1$

Normal termination of Gaussian 16 at Sat Mar 9 23:05:34 2019.

-Figure S4-7, Int4(C8)

Stoichiometry C10H14N505(2)

Standard orientation:

\begin{tabular}{|c|c|c|c|c|c|}
\hline \multirow{2}{*}{$\begin{array}{l}\text { Center } \\
\text { Number }\end{array}$} & \multirow{2}{*}{$\begin{array}{l}\text { Atomic } \\
\text { Number }\end{array}$} & \multirow{2}{*}{$\begin{array}{c}\text { Atomic } \\
\text { Type }\end{array}$} & \multicolumn{3}{|c|}{ Coordinates (Angstroms) } \\
\hline & & & $x$ & Y & Z \\
\hline & & &.- & - - & 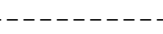 \\
\hline 1 & 1 & $\odot$ & -2.752697 & 1.518753 & 1.797559 \\
\hline 2 & 8 & 0 & -2.594509 & 1.869955 & $\odot .917199$ \\
\hline 3 & 8 & $\odot$ & -3.947118 & -2.247269 & $\odot .086975$ \\
\hline 4 & 6 & $\odot$ & -3.492483 & 0.017760 & -0.479875 \\
\hline 5 & 6 & $\odot$ & -3.326123 & -1.064764 & 0.586751 \\
\hline 6 & 1 & $\odot$ & -3.773723 & -2.962180 & 0.706673 \\
\hline 7 & 6 & $\odot$ & -3.637674 & 1.441574 & 0.051259 \\
\hline 8 & 6 & $\odot$ & -1.810211 & -1.205988 & 0.701823 \\
\hline 9 & 6 & $\odot$ & -1.276784 & -0.761568 & -0.676305 \\
\hline 10 & 8 & 0 & -2.365477 & -0.124575 & -1.341989 \\
\hline 11 & 1 & $\odot$ & -3.622890 & 2.125897 & -0.805643 \\
\hline 12 & 1 & $\odot$ & -4.619031 & 1.534438 & 0.542847 \\
\hline 13 & 1 & $\odot$ & -4.391006 & -0.193361 & -1.077140 \\
\hline 14 & 1 & $\odot$ & -3.790809 & -0.777299 & 1.541372 \\
\hline 15 & 1 & $\odot$ & -1.505230 & -2.228782 & 0.943672 \\
\hline 16 & 1 & $\odot$ & -1.419945 & -0.537570 & 1.477243 \\
\hline 17 & 1 & $\odot$ & -0.984521 & -1.625173 & -1.290969 \\
\hline 18 & 7 & $\odot$ & -0.190820 & ๑.170893 & -0.567086 \\
\hline 19 & 6 & $\odot$ & 0.812447 & 2.827337 & 0.046840 \\
\hline 20 & 7 & $\odot$ & 1.933857 & 2.036823 & -0.115214 \\
\hline 21 & 6 & $\odot$ & 2.061089 & 0.673682 & -0.106733 \\
\hline 22 & 6 & $\odot$ & ๑. 989952 & -0.284972 & -0.272604 \\
\hline 23 & 7 & $\odot$ & 1. 256651 & -1.635004 & -0.111726 \\
\hline 24 & 6 & 0 & 2.487609 & -2.023583 & 0.065778 \\
\hline 25 & 7 & 0 & 3.563101 & -1.161948 & ๑. 119691 \\
\hline 26 & 6 & $\odot$ & 3.427627 & ๑. 209017 & 0.057839 \\
\hline 27 & 7 & $\odot$ & 2.770126 & -3.331993 & ○. 204914 \\
\hline 28 & 8 & $\odot$ & 4.398137 & 0.959465 & $\odot .137105$ \\
\hline 29 & 1 & 0 & -0.128040 & 2.272898 & $\odot .167514$ \\
\hline 30 & 1 & 0 & 2.011215 & -3.992698 & 0.151126 \\
\hline 31 & 1 & 0 & 3.705455 & -3.676930 & 0.348474 \\
\hline 32 & 1 & 0 & 4.504197 & -1.516130 & ๑. 239027 \\
\hline 33 & 8 & $\odot$ & $\odot .909083$ & 4.042603 & $\odot .051751$ \\
\hline 34 & 1 & $\odot$ & 2.823004 & 2.534323 & -0.112791 \\
\hline
\end{tabular}


Standard basis: Aug-CC-pVDZ (6D, 7F)

626 basis functions

75 alpha electron

nuclear repulsion energy 1640.5295449838 Hartrees.

NAtoms $=34$ NActive $=34$

Nuclear repulsion after empirical dispersion term $=$

1640.5048006969 Hartrees.

Force inversion solution in PCM.

SCF Done: $E($ UWB97XD $)=-1039.22971033$ A.U. after 1 cycles

NFock $=1$ Conv $=0.48 \mathrm{D}-08$

$\langle$ SX $=0.0000<$ Sy $>=0.0000\langle$ Sz $>=0.5000$

$\left\langle\mathrm{S}^{* *} 2>=0.7741 \mathrm{~S}=0.5120\right.$

$<$ L.S $>=0.000000000000 \mathrm{E}+00$

Annihilation of the first spin contaminant:

$\mathrm{S}^{* * 2}$ before annihilation 0.7741 , after

0.7502

Zero-point correction=

Thermal correction to Energy=

Thermal correction to Enthalpy=

Thermal correction to Gibbs Free Energy=

Sum of electronic and zero-point Energies=

Sum of electronic and thermal Energies=

Sum of electronic and thermal Enthalpies=

0.265947 (a.u.)

$\odot .285304$

0.286248

0.216495

$-1038.963763$

$-1038.944406$

$-1038.943462$

Sum of electronic and thermal Free Energies=

$-1039.013215$

Total

$$
\text { E (Thermal) }
$$

$$
\mathrm{KCal} / \mathrm{Mol}
$$

$$
\mathrm{CV}
$$

$S$

Cal/Mol-Kelvin Cal/Mol-Kelvin

$$
71.532 \quad 146.808
$$

\begin{tabular}{lccc}
\multicolumn{1}{c}{ Item } & Value & Threshold & \multicolumn{2}{c}{ Converged? } \\
Maximum Force & 0.000047 & 0.000450 & YES \\
RMS Force & 0.000004 & 0.000300 & YES \\
Maximum Displacement & 0.000666 & 0.001800 & YES \\
RMS Displacement & 0.000113 & 0.001200 & YES \\
Predicted change in Energy=-4.503681D-09 & \\
Optimization completed. & & \\
\multicolumn{2}{l}{- - Stationary point found. } & &
\end{tabular}

Normal termination of Gaussian 16 at Thu Mar 14 18:23:23 2019.

\begin{tabular}{|c|c|c|c|c|c|}
\hline Center & Atomic & Atomic & & rdinates ( & gstroms) \\
\hline Number & Number & Type & $x$ & $\mathrm{Y}$ & Z \\
\hline 1 & 1 & $\odot$ & 3.820203 & -1.513678 & 1.501043 \\
\hline 2 & 8 & 0 & 3.432450 & -1.777046 & $\odot .662098$ \\
\hline 3 & 8 & $\odot$ & 3.440579 & 2.567047 & 0.213269 \\
\hline 4 & 6 & $\odot$ & 3.380235 & $\odot .336385$ & -0.617797 \\
\hline 5 & 6 & $\odot$ & 3.250327 & 1.218130 & 0.628031 \\
\hline 6 & 1 & $\odot$ & 3.312233 & 3.139206 & 0.976084 \\
\hline 7 & 6 & $\odot$ & 4.029885 & -1.021381 & -0.383674 \\
\hline 8 & 6 & 0 & 1.818899 & 0.949741 & 1.080535 \\
\hline 9 & 6 & $\odot$ & 1.092392 & $\odot .717895$ & -0.247607 \\
\hline 10 & 8 & $\odot$ & 2.050786 & $\odot .171606$ & -1.131318 \\
\hline 11 & 1 & 0 & 3.922244 & -1.615548 & -1.298996 \\
\hline 12 & 1 & $\odot$ & 5.104165 & $-\odot .871381$ & $-\odot .199097$ \\
\hline 13 & 1 & $\odot$ & 3.972766 & 0.867094 & -1.375955 \\
\hline 14 & 1 & $\odot$ & 3.989876 & $\odot .952061$ & $1.3960 \odot 9$ \\
\hline 15 & 1 & $\odot$ & 1.385521 & 1.780586 & 1.646392 \\
\hline 16 & 1 & $\odot$ & 1.782835 & $\odot .044759$ & 1.695858 \\
\hline 17 & 1 & $\odot$ & $\odot .695955$ & 1.657729 & $-\odot .652772$ \\
\hline 18 & 7 & $\odot$ & $-\odot .029507$ & -0.190625 & -0.161702 \\
\hline 19 & 6 & $\theta$ & 0.080897 & -1.652515 & 0.065255 \\
\hline 20 & 7 & $\odot$ & -1.302770 & -2.056721 & 0.438601 \\
\hline 21 & 6 & $\odot$ & -2.086430 & -0.969305 & 0.256455 \\
\hline 22 & 6 & $\theta$ & -1.310722 & 0.183694 & -0.064617 \\
\hline 23 & 7 & 0 & -1.781275 & 1.418447 & -0.257758 \\
\hline
\end{tabular}

Stoichiometry C10H14N505(2)

Stoichiometry C10H14N505(2)

Standard orientation: 


\begin{tabular}{|c|c|c|c|c|c|}
\hline 24 & 6 & 0 & -3.105520 & 1.523451 & -0.172955 \\
\hline 25 & 7 & $\odot$ & -3.935536 & $\odot .465479$ & $\odot .067446$ \\
\hline 26 & 6 & $\odot$ & -3.514580 & -0.860378 & $\odot .287958$ \\
\hline 27 & 7 & $\odot$ & -3.659860 & 2.727205 & -0.339528 \\
\hline 28 & 8 & $\odot$ & -4.337775 & -1.745948 & 0.486740 \\
\hline 29 & 1 & $\odot$ & ๑. 844302 & -1.838897 & 0.832202 \\
\hline 30 & 1 & $\odot$ & -3.053891 & 3.509488 & -0.533972 \\
\hline 31 & 1 & $\odot$ & -4.655391 & 2. 880901 & -0.306192 \\
\hline 32 & 1 & $\odot$ & -4.937875 & ๑. 612029 & 0.100436 \\
\hline 33 & 8 & $\odot$ & $\odot .166549$ & -2.456667 & -1.041939 \\
\hline 34 & 1 & $\odot$ & -1.053297 & -2.777088 & -0.510755 \\
\hline
\end{tabular}

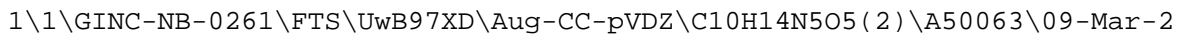
$019 \backslash 0 \backslash \backslash \#$ wb97xd / aug-cc-pVDZ 6d pop=none optcyc=300 scf=(direct, pass) scfcyc $=300$ iop $(1 / 8=2)$ opt $=(t s$, readfc, nofreeze, noeig) IOP $(2 / 9=1)$ scrf= $(\mathrm{pcm}$, solvent=water $)$ iop $(1 / 18=120)$ geom=allcheck guess $=$ check $\backslash \backslash$ made from file: "degua-oh8a.rev.chk" 2'-deoxyguanosine $+\mathrm{OH}($.$) at \mathrm{C} 8, \mathrm{O}(33) \ldots$

B. $01 \backslash H F=-1039.175155 \backslash S 2=0.757767 \backslash S 2-1=0 . \backslash S 2 A=0.750049 \backslash R M S D=9.087 \mathrm{e}-09 \backslash R$ $\mathrm{MSF}=4.354 \mathrm{e}-06 \backslash \mathrm{Dipole}=-0.4497999,4.9798051,3.2352415 \backslash \mathrm{PG}=\mathrm{CO} 1[\mathrm{X}(\mathrm{C} 10 \mathrm{H} 14 \mathrm{N5}$

Job cpu time: $\quad 1$ days 22 hours 6 minutes 12.0 seconds.

Elapsed time: $\odot$ days 2 hours 53 minutes 12.7 seconds.

File lengths (MBytes):

RWF $=528$ Int $=0$ D2E $=0$ Chk $=54 \mathrm{Scr}=\quad 1$

Normal termination of Gaussian 16 at Sat Mar 9 17:35:10 2019.

Initial command:

Gaussian 16: ES64L-G16RevB.01 20-Dec-2017 $9-\operatorname{Mar}-2019$

\section{$\%$ mem $=1500 \mathrm{MW}$}

$\%$ nproc $=16$

will use up to 16 processors via shared memory.

$\%$ chk $=$ degua - oh $8 x$. higha. chk

\# wb97xd / aug-cc-pVDZ 6d pop=none optcyc $=300$

scf $=($ direct, pass $)$ scfcyc $=300$ iop $(1 / 8=2)$

freq=noraman IOP $(2 / 9=1) \operatorname{scrf}=(p c m$, solvent=water $)$

geom=allcheck guess $=$ check

the checkpoint file: "degua-oh8x.higha.chk"

-

' 2'-deoxyguanosine

$+\mathrm{OH}($.$) at \mathrm{C} 8, \mathrm{O}(33) \ldots \mathrm{H}(34) \ldots \mathrm{N}(20)$

Charge $=\odot$ Multiplicity $=2$

$\mathrm{H}, 0,3.8528251911,-1.7163720853,1.286977412$

$0,0,3.4534656176,-1.8842344822,0.4291435632$

$0,0,3.4846616317,2.4827813087,0.4585104714$

C, $0,3.3999252145,0.3569271835,-0.6109423583$

C, $0,3.2905216037,1.0975537853,0.7254483905$

$\mathrm{H}, 0,3.369085713,2.9685937246,1.2809182683$

C, $0,4.0434156077,-1.0216731452,-0.5349849798$

C $, 0,1.8628216863,0.7886546806,1.1636657614$

C, $0,1.1191602877,0.7074739616,-0.1727121591$

$0,0,2.0634484558,0.2563056637,-1.12279754$

$\mathrm{H}, 0,3.9210797803,-1.5114273581,-1.5084378893$

$\mathrm{H}, 0,5.1207600624,-0.8983813887,-0.3485066084$

$\mathrm{H}, 0,3.9869027924,0.9644072891,-1.3137741602$

$\mathrm{H}, 0,4.0373465379,0.7451174184,1.4504008431$

$\mathrm{H}, 0,1.4416015095,1.5547532152,1.8224366341$

$\mathrm{H}, 0,1.8281261135,-0.1780875638,1.6766195283$

$\mathrm{H}, 0,0.7241077572,1.6880834492,-0.4675789683$

$\mathrm{N}, \odot,-\odot .0075549241,-0.1991452783,-0.1727993354$

C, $0,0.0959746525,-1.6776530595,-0.10863894$

$\mathrm{N}, \odot,-1.2857915363,-2.113127383,0.2354372424$

C, $0,-2.0644313211,-1.0082437952,0.1832166833$

C, $0,-1.2850598136,0.1689397598,-0.0193505972$

$\mathrm{N}, 0,-1.7497886293,1.4198488267,-0.0702746896$

C , $0,-3.0722238289,1.521812725,0.0420199115$

$\mathrm{N}, 0,-3.9062318768,0.4482017896,0.1754874318$

C, $0,-3.4913662713,-\odot .896002787,0.2442606421$ 
$\mathrm{N}, 0,-3.6206198333,2.7394348829,0.0151526005$

$0,0,-4.317920515,-1.7937277459,0.3551507943$

$\mathrm{H}, 0,0.8671623559,-1.9508942262,0.6237152476$

$\mathrm{H}, 0,-3.0118962073,3.5351500888,-0.1000242325$

$\mathrm{H}, 0,-4.6146620287,2.8937254638,0.0775259532$

$\mathrm{H}, 0,-4.9071321325,0.5954630366,0.2368186751$

$0,0,0.1632728083,-2.3561115929,-1.2981931443$

$\mathrm{H}, 0,-1.0522732878,-2.7264482757,-0.7901101003$

Recover connectivity data from disk.

Stoichiometry C10H14N505(2)

Standard orientation:

\begin{tabular}{|c|c|c|c|c|c|}
\hline \multirow{2}{*}{$\begin{array}{l}\text { Center } \\
\text { Number }\end{array}$} & \multirow{2}{*}{$\begin{array}{l}\text { Atomic } \\
\text { Number }\end{array}$} & \multirow{2}{*}{$\begin{array}{c}\text { Atomic } \\
\text { Type }\end{array}$} & \multicolumn{3}{|c|}{ Coordinates (Angstroms) } \\
\hline & & & $x$ & $\mathrm{Y}$ & Z \\
\hline & & -- & ------- & ------ & ------- \\
\hline 1 & 1 & $\odot$ & 3.820203 & -1.513678 & 1.501043 \\
\hline 2 & 8 & $\odot$ & 3.432450 & -1.777046 & 0.662098 \\
\hline 3 & 8 & $\odot$ & 3.440579 & 2.567047 & 0.213269 \\
\hline 4 & 6 & $\odot$ & 3.380235 & 0.336385 & -0.617797 \\
\hline 5 & 6 & $\odot$ & 3.250327 & 1.218130 & 0.628031 \\
\hline 6 & 1 & $\odot$ & 3.312233 & 3.139206 & 0.976084 \\
\hline 7 & 6 & $\odot$ & 4.029885 & -1.021381 & -0.383674 \\
\hline 8 & 6 & $\odot$ & 1.818899 & ๑. 949741 & 1.080535 \\
\hline 9 & 6 & $\odot$ & 1.092392 & ๑. 717895 & -0.247607 \\
\hline 10 & 8 & $\odot$ & 2.050786 & 0.171606 & -1.131318 \\
\hline 11 & 1 & $\odot$ & 3.922244 & -1.615548 & -1.298996 \\
\hline 12 & 1 & $\odot$ & 5.104165 & $-\odot .871381$ & -0.199097 \\
\hline 13 & 1 & $\odot$ & 3.972766 & ๑. 867094 & -1.375955 \\
\hline 14 & 1 & $\odot$ & 3.989876 & ๑. 952061 & 1.396009 \\
\hline 15 & 1 & $\odot$ & 1.385521 & 1.780586 & 1.646392 \\
\hline 16 & 1 & $\odot$ & 1.782835 & 0.044759 & 1.695858 \\
\hline 17 & 1 & $\odot$ & ๑. 695955 & 1.657729 & -0.652772 \\
\hline 18 & 7 & $\odot$ & -0.029507 & -0.190625 & -0.161702 \\
\hline 19 & 6 & $\odot$ & ๑. . 080897 & -1.652515 & 0.065255 \\
\hline 20 & 7 & $\odot$ & -1.302770 & -2.056721 & 0.438601 \\
\hline 21 & 6 & $\odot$ & -2.086430 & -0.969305 & 0.256455 \\
\hline 22 & 6 & $\odot$ & -1.310722 & ๑. 183694 & -0.064617 \\
\hline 23 & 7 & $\odot$ & -1.781275 & 1.418447 & -0.257758 \\
\hline 24 & 6 & $\odot$ & -3.105520 & 1.523451 & -0.172955 \\
\hline 25 & 7 & $\odot$ & -3.935536 & $\odot .465479$ & ๑. 067446 \\
\hline 26 & 6 & $\odot$ & -3.514580 & -0.860378 & ๑. 287958 \\
\hline 27 & 7 & $\odot$ & -3.659860 & 2.727205 & -0.339528 \\
\hline 28 & 8 & $\odot$ & -4.337775 & -1.745948 & 0.486740 \\
\hline 29 & 1 & $\odot$ & 0.844302 & -1.838897 & 0.832202 \\
\hline 30 & 1 & $\odot$ & -3.053891 & 3.509488 & -0.533972 \\
\hline 31 & 1 & $\odot$ & -4.655391 & 2.880901 & -0.306192 \\
\hline 32 & 1 & $\odot$ & -4.937875 & $\odot .612029$ & 0.100436 \\
\hline 33 & 8 & $\odot$ & 0.166549 & -2.456667 & -1.041939 \\
\hline 34 & 1 & $\odot$ & -1.053297 & -2.777088 & -0.510755 \\
\hline
\end{tabular}

Standard basis: Aug-CC-pVDZ (6D, 7F)

626 basis functions

75 alpha electrons

74 beta electrons

nuclear repulsion energy 1681.4094301898 Hartrees.

NAtoms $=34$ NActive $=34$

Nuclear repulsion after empirical dispersion term =

1681.3833096543 Hartrees.

Force inversion solution in PCM.

Polarizable Continuum Model (PCM)

=ニニニニニニニニニニニニニニニニニニニニニニニニニニニニニニニ=

Model : PCM.

Atomic radii : UFF (Universal Force Field).

Solvent : Water, Eps $=78.355300$ Eps $($ inf $)=1.777849$

SCF Done: E(UwB97XD) = -1039.17515504 A.U. after 1 cycles $\mathrm{NFock}=1$ Conv $=0.67 \mathrm{D}-08 \quad-\mathrm{V} / \mathrm{T}=2.0071$

$\langle\mathrm{S} x\rangle=0.0000<\mathrm{S} y>=0.0000<\mathrm{S} z>=0.5000$

$<S^{* *} 2>=0.7578 \mathrm{~S}=0.5039$

$<$ L.S $>=0.000000000000 \mathrm{E}+00$ 
Annihilation of the first spin contaminant:

$\mathrm{S}^{* * 2}$ before annihilation 0.7578 , after

0.7500

Harmonic frequencies $\left(\mathrm{cm}^{* *}-1\right)$, IR intensities (KM/Mole), Raman activities ( $\left.A^{* *} 4 / \mathrm{AMU}\right)$, depolarization ratios for plane and unp incident light, reduced masses (AMU), force constants (mDyne/A), and normal coordinates:

\begin{tabular}{|c|c|c|c|c|c|c|c|c|c|c|}
\hline \multicolumn{5}{|c|}{$\begin{array}{r}1 \\
\mathrm{~A}\end{array}$} & \multicolumn{3}{|l|}{$\begin{array}{l}2 \\
A\end{array}$} & \multicolumn{3}{|l|}{3} \\
\hline \multicolumn{2}{|c|}{ Frequencies } & 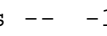 & \multicolumn{2}{|c|}{-1653.4010} & \multicolumn{3}{|c|}{19.2242} & \multicolumn{3}{|c|}{47.7120} \\
\hline \multicolumn{2}{|c|}{ Red. masses } & - - & \multicolumn{2}{|c|}{1.0702} & \multicolumn{3}{|c|}{5.2002} & \multicolumn{3}{|c|}{6.5920} \\
\hline \multirow{2}{*}{\multicolumn{2}{|c|}{$\begin{array}{l}\text { Frc consts } \\
\text { IR Inten }\end{array}$}} & - - & \multirow{2}{*}{\multicolumn{2}{|c|}{$\begin{array}{r}1.7237 \\
1984.9858\end{array}$}} & \multirow{2}{*}{\multicolumn{3}{|c|}{$\begin{array}{l}0.0011 \\
4.9110\end{array}$}} & \multirow{2}{*}{\multicolumn{3}{|c|}{$\begin{array}{l}0.0088 \\
1.6953\end{array}$}} \\
\hline & & -- & & & & & & & & \\
\hline Atom & AN & $\mathrm{x}$ & Y & Z & $x$ & Y & Z & $\mathrm{x}$ & Z & \\
\hline 1 & 1 & $-\odot .0 \odot$ & $-\odot . \odot \odot$ & $\odot .0 \odot$ & -0.12 & 0.11 & 0.24 & $\odot .06$ & $-\odot . \odot \odot$ & -0.06 \\
\hline 2 & 8 & $-\odot .0 \odot$ & -0.00 & $-\odot . \odot \odot$ & -0.05 & 0.04 & 0.22 & 0.17 & $-\odot . \odot \odot$ & -0.11 \\
\hline 3 & 8 & 0.00 & $\odot . \odot \odot$ & $-\odot . \odot \odot$ & -0.03 & $\odot .02$ & -0.11 & -0.14 & 0.03 & 0.20 \\
\hline 4 & 6 & $\odot . \odot \odot$ & $\odot . \odot \odot$ & -0.00 & 0.03 & -0.05 & 0.07 & $\odot .03$ & 0.08 & 0.05 \\
\hline 5 & 6 & $\odot . \odot \odot$ & $-\odot . \odot \odot$ & 0.00 & -0.06 & 0.05 & $-\odot . \odot 2$ & $-\odot .09$ & $-\odot . \odot \odot$ & 0.10 \\
\hline 6 & 1 & $-\odot . \odot \odot$ & $-\odot .0 \odot$ & $\odot . \odot \odot$ & $-\odot .08$ & 0.08 & -0.17 & -0.21 & -0.03 & 0.23 \\
\hline 7 & 6 & $\odot .0 \odot$ & 0.00 & -0.00 & 0.03 & -0.03 & 0.22 & 0.17 & 0.14 & -0.01 \\
\hline 8 & 6 & $\odot . \odot \odot$ & $\odot . \odot \odot$ & $-\odot . \odot \odot$ & -0.09 & $\odot . \odot 9$ & -0.10 & -0.10 & -0.11 & $\odot . \odot \odot$ \\
\hline 9 & 6 & $-\odot . \odot \odot$ & $-\odot .0 \odot$ & $\odot . \odot \odot$ & $\odot . \odot \odot$ & $-\odot .02$ & -0.13 & $-\odot .0 \odot$ & $-\odot .07$ & -0.05 \\
\hline 10 & 8 & $\odot .0 \odot$ & $-\odot . \odot \odot$ & $-\odot . \odot \odot$ & $\odot .06$ & -0.11 & -0.01 & 0.06 & -0.06 & 0.01 \\
\hline 11 & 1 & $\odot . \odot \odot$ & $-\odot . \odot \odot$ & $-\odot . \odot \odot$ & $\odot .10$ & $-\odot .09$ & 0.25 & 0.30 & 0.19 & $-\odot .06$ \\
\hline 12 & 1 & -0.00 & $-\odot .0 \odot$ & 0.00 & $\odot .01$ & 0.00 & 0.29 & $\odot .14$ & 0.24 & 0.07 \\
\hline 13 & 1 & $\odot . \odot \odot$ & $\odot .0 \odot$ & $\odot . \odot \odot$ & 0.07 & -0.11 & $\odot .06$ & -0.01 & $\odot .19$ & $\odot .09$ \\
\hline 14 & 1 & $\odot . \odot \odot$ & $\odot .0 \odot$ & $-\odot . \odot \odot$ & -0.11 & 0.11 & $\odot .06$ & -0.11 & -0.01 & 0.12 \\
\hline 15 & 1 & $\odot .0 \odot$ & $-\odot . \odot \odot$ & $\odot . \odot \odot$ & -0.13 & 0.13 & -0.19 & -0.17 & -0.17 & 0.03 \\
\hline 16 & 1 & $\odot . \odot \odot$ & $\odot . \odot \odot$ & $\odot . \odot \odot$ & -0.14 & 0.14 & $-\odot .03$ & -0.08 & $-\odot .15$ & $-\odot .06$ \\
\hline 17 & 1 & -0.00 & -0.00 & $\odot . \odot \odot$ & 0.04 & -0.05 & -0.24 & 0.03 & -0.06 & -0.06 \\
\hline 18 & 7 & $\odot .01$ & $-\odot . \odot \odot$ & $-\odot . \odot \odot$ & $-\odot .0 \odot$ & -0.01 & -0.13 & -0.01 & -0.06 & -0.15 \\
\hline 19 & 6 & $-\odot .02$ & $\odot .03$ & $\odot .03$ & $\odot .01$ & $-\odot .02$ & $-\odot .15$ & $-\odot . \odot 4$ & -0.05 & $-\odot . \odot 8$ \\
\hline 20 & 7 & -0.01 & $\odot .01$ & $\odot .02$ & $\odot .02$ & $-\odot . \odot 2$ & -0.11 & -0.03 & $-\odot . \odot \odot$ & 0.01 \\
\hline 21 & 6 & $\odot .01$ & $-\odot . \odot \odot$ & $\odot . \odot \odot$ & $\odot . \odot 2$ & $-\odot .01$ & $-\odot .04$ & -0.01 & $\odot .01$ & $-\odot . \odot \odot$ \\
\hline 22 & 6 & -0.01 & $-\odot . \odot \odot$ & $-\odot . \odot \odot$ & $\odot .01$ & -0.01 & -0.05 & $-\odot . \odot \odot$ & -0.03 & -0.10 \\
\hline 23 & 7 & $\odot . \odot \odot$ & $\odot . \odot \odot$ & $-\odot . \odot \odot$ & 0.01 & 0.01 & 0.02 & $\odot .02$ & -0.02 & -0.13 \\
\hline 24 & 6 & $\odot . \odot \odot$ & $-\odot . \odot \odot$ & $\odot . \odot \odot$ & $\odot . \odot 2$ & $\odot .01$ & $\odot . \odot 9$ & $\odot . \odot 3$ & $\odot .02$ & $-\odot . \odot 6$ \\
\hline 25 & 7 & $\odot . \odot \odot$ & $\odot . \odot \odot$ & $-\odot . \odot \odot$ & $\odot .02$ & $\odot .01$ & $\odot .10$ & $\odot .02$ & $\odot .06$ & $\odot . \odot 6$ \\
\hline 26 & 6 & $\odot . \odot \odot$ & $\odot . \odot \odot$ & 0.00 & $\odot .02$ & $-\odot . \odot \odot$ & $\odot . \odot 3$ & -0.01 & $\odot .06$ & $\odot .11$ \\
\hline 27 & 7 & $-\odot . \odot \odot$ & $\odot . \odot \odot$ & $-\odot . \odot \odot$ & $\odot .02$ & 0.03 & 0.18 & $\odot .06$ & $\odot . \odot 2$ & -0.11 \\
\hline 28 & 8 & $-\odot . \odot \odot$ & $-\odot . \odot \odot$ & $\odot . \odot \odot$ & $\odot . \odot 2$ & $-\odot . \odot \odot$ & $\odot . \odot 4$ & $-\odot . \odot 2$ & 0.10 & 0.25 \\
\hline 29 & 1 & 0.01 & -0.01 & -0.02 & $\odot .03$ & $-\odot .02$ & -0.18 & $-\odot .01$ & -0.03 & -0.10 \\
\hline 30 & 1 & $-\odot . \odot \odot$ & $\odot . \odot \odot$ & $-\odot . \odot \odot$ & $\odot . \odot 2$ & 0.03 & $\odot .17$ & $\odot . \odot 7$ & $-\odot .01$ & $-\odot .19$ \\
\hline 31 & 1 & $-\odot . \odot \odot$ & $\odot . \odot \odot$ & $-\odot . \odot \odot$ & $\odot . \odot 2$ & 0.03 & 0.23 & $\odot . \odot 6$ & $\odot .06$ & $-\odot . \odot 4$ \\
\hline 32 & 1 & $\odot . \odot \odot$ & $\odot . \odot \odot$ & $-\odot . \odot \odot$ & 0.02 & $\odot . \odot 2$ & $\odot .15$ & $\odot . \odot 2$ & $\odot .09$ & 0.13 \\
\hline 33 & 8 & -0.04 & $-\odot . \odot 2$ & $\odot . \odot \odot$ & $-\odot . \odot 3$ & $-\odot . \odot \odot$ & -0.16 & -0.10 & -0.11 & $-\odot .04$ \\
\hline 34 & 1 & 0.75 & -0.05 & -0.65 & -0.02 & $-\odot .0 \odot$ & -0.13 & -0.09 & -0.06 & 0.03 \\
\hline
\end{tabular}

- Thermochemistry

Temperature 298.150 Kelvin. Pressure 1.00000 Atm.

Molecular mass: 284.09949 amu

Principal axes and moments of inertia in atomic units:

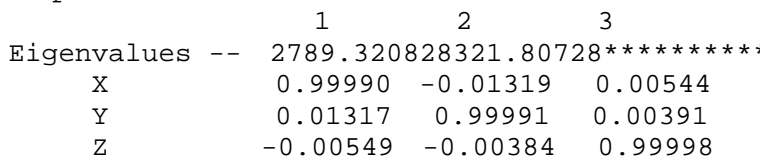

This molecule is an asymmetric top.

Rotational symmetry number 1 .

Warning - - assumption of classical behavior for rotation may cause significant error

$\begin{array}{llll}\text { Rotational temperatures (Kelvin) } & 0.03105 & 0.01041 & 0.00833\end{array}$

$\begin{array}{llll}\text { Rotational constants (GHZ): } & 0.64702 & 0.21687 & 0.17349\end{array}$

1 imaginary frequencies ignored.

Zero-point vibrational energy 692657.3 (Joules/Mol)

165.54906 (Kcal/Mol)

Warning - - explicit consideration of 27 degrees of freedom as vibrations may cause significant error

Zero-point correction=

Thermal correction to Energy=

Thermal correction to Enthalpy=

Thermal correction to Gibbs Free Energy=

$$
\begin{gathered}
0.263819 \text { (a.u.) } \\
0.281537 \\
0.282481 \\
0.216682
\end{gathered}
$$


Sum of electronic and zero-point Energies= Sum of electronic and thermal Energies=

Sum of electronic and thermal Enthalpies=

Sum of electronic and thermal Free Energies=
$-1038.911336$

$-1038.893618$

$-1038.892674$

$-1038.958473$

$\mathrm{S}$

CV

Cal/Mol-Kelvin Cal/Mol-Kelvin

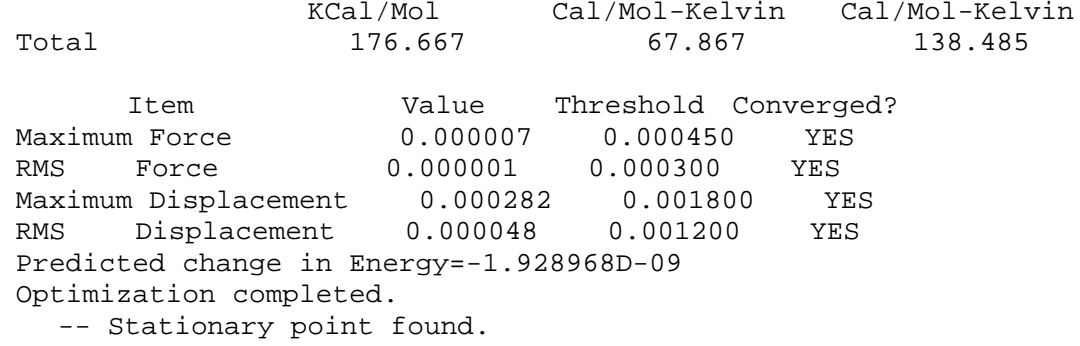

$67.867 \quad 138.485$

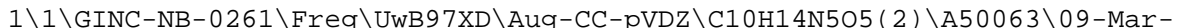
2019 \०\\\# wb97xd / aug-cc-pVDZ 6d pop=none optcyc=300 scf=(direct, pass ) $\operatorname{scfcyc}=300$ iop $(1 / 8=2)$ freq=noraman $\operatorname{IOP}(2 / 9=1) \operatorname{scrf}=(\mathrm{pcm}$, solvent=wate r) geom=allcheck guess=check \\made from file: "degua-oh8a.rev.chk" 2' deoxyguanosine $+\mathrm{OH}($.$) at \mathrm{C} 8, \mathrm{O}(33) \ldots \mathrm{H}(34) \ldots \mathrm{N}(20) \backslash \backslash 0,2 \backslash \mathrm{H}, 3.852825$ $1911,-1.7163720853,1.286977412 \backslash 0,3.4534656176,-1.8842344822,0.42914356$

File lengths (MBytes):

$\mathrm{RWF}=2403$ Int $=\quad \odot \mathrm{D} 2 \mathrm{E}=\quad \odot \mathrm{Chk}=60 \mathrm{Scr}=\quad 1$

Normal termination of Gaussian 16 at Sat Mar 9 21:40:42 2019.

Figure S4-9, Int3b(C8)

degua-oh8x. for . higha. $\log$

Stoichiometry C10H14N505(2)

Standard orientation:

\begin{tabular}{|c|c|c|c|c|c|}
\hline \multirow{2}{*}{$\begin{array}{l}\text { Center } \\
\text { Number }\end{array}$} & \multirow{2}{*}{$\begin{array}{l}\text { Atomic } \\
\text { Number }\end{array}$} & \multirow{2}{*}{$\begin{array}{c}\text { Atomic } \\
\text { Type }\end{array}$} & \multicolumn{3}{|c|}{ Coordinates (Angstroms) } \\
\hline & & & $\mathrm{X}$ & $\mathrm{Y}$ & Z \\
\hline & & 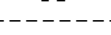 & -- & - - - & $--\ldots-2-$ \\
\hline 1 & 1 & $\odot$ & 3.872661 & -1.349617 & 1.626651 \\
\hline 2 & 8 & $\odot$ & 3.553243 & -1.680044 & $\odot .782572$ \\
\hline 3 & 8 & $\odot$ & 3.261674 & 2.634067 & 0.234155 \\
\hline 4 & 6 & $\odot$ & 3.385933 & 0.390008 & -0.559348 \\
\hline 5 & 6 & $\odot$ & 3.133064 & 1.281343 & 0.661461 \\
\hline 6 & 1 & $\odot$ & 3.056362 & 3.207200 & $\odot .979217$ \\
\hline 7 & 6 & $\odot$ & 4.126416 & -0.908387 & -0.264544 \\
\hline 8 & 6 & $\odot$ & 1.698933 & ๑. 927896 & 1.037741 \\
\hline 9 & 6 & $\odot$ & 1.068004 & 0.647718 & $-\odot .328997$ \\
\hline 10 & 8 & $\odot$ & 2.099875 & 0.112107 & -1.129400 \\
\hline 11 & 1 & $\odot$ & 4.095721 & -1.533427 & -1.165009 \\
\hline 12 & 1 & $\odot$ & 5.179682 & -0.671711 & -0.049951 \\
\hline 13 & 1 & $\odot$ & 3.973337 & ๑. 951700 & -1.299511 \\
\hline 14 & 1 & $\odot$ & 3.843881 & 1.074617 & 1.473678 \\
\hline 15 & 1 & $\odot$ & 1.183737 & 1.730502 & 1.575478 \\
\hline 16 & 1 & $\odot$ & 1.683721 & 0.018783 & 1.648448 \\
\hline 17 & 1 & $\odot$ & 0.683749 & 1.572839 & -0.779547 \\
\hline 18 & 7 & $\odot$ & -0.044364 & -0.277749 & -0.276695 \\
\hline 19 & 6 & $\odot$ & ๑. 095517 & -1.827965 & -0.089609 \\
\hline 20 & 7 & $\odot$ & -1.350360 & -2.065081 & 0.371634 \\
\hline 21 & 6 & $\odot$ & -2.121806 & -0.995354 & 0.186121 \\
\hline 22 & 6 & $\odot$ & -1.300935 & 0.125632 & -0.161331 \\
\hline 23 & 7 & $\odot$ & -1.753643 & 1.377271 & -0.314046 \\
\hline 24 & 6 & $\odot$ & -3.067628 & 1.525054 & -0.177924 \\
\hline 25 & 7 & $\odot$ & -3.926626 & $\odot .497733$ & 0.101358 \\
\hline 26 & 6 & $\odot$ & -3.537913 & -0.834060 & ๑. 315863 \\
\hline 27 & 7 & $\odot$ & -3.588993 & 2.746460 & -0.323650 \\
\hline 28 & 8 & $\odot$ & -4.362795 & -1.700518 & 0.585988 \\
\hline 29 & 1 & $\odot$ & $\odot .738852$ & -1.942567 & 0.807305 \\
\hline 30 & 1 & $\odot$ & -2.965538 & 3.509252 & -0.539084 \\
\hline 31 & 1 & $\odot$ & -4.575395 & 2.934853 & -0.238239 \\
\hline 32 & 1 & $\odot$ & -4.919818 & $\odot .680704$ & ๑.185599 \\
\hline 33 & 8 & $\odot$ & 0.401966 & -2.505036 & -1.131434 \\
\hline 34 & 1 & $\odot$ & -1.712268 & -3.009488 & 0.413110 \\
\hline
\end{tabular}

Standard basis: Aug-CC-pVDZ (6D, 7F) 


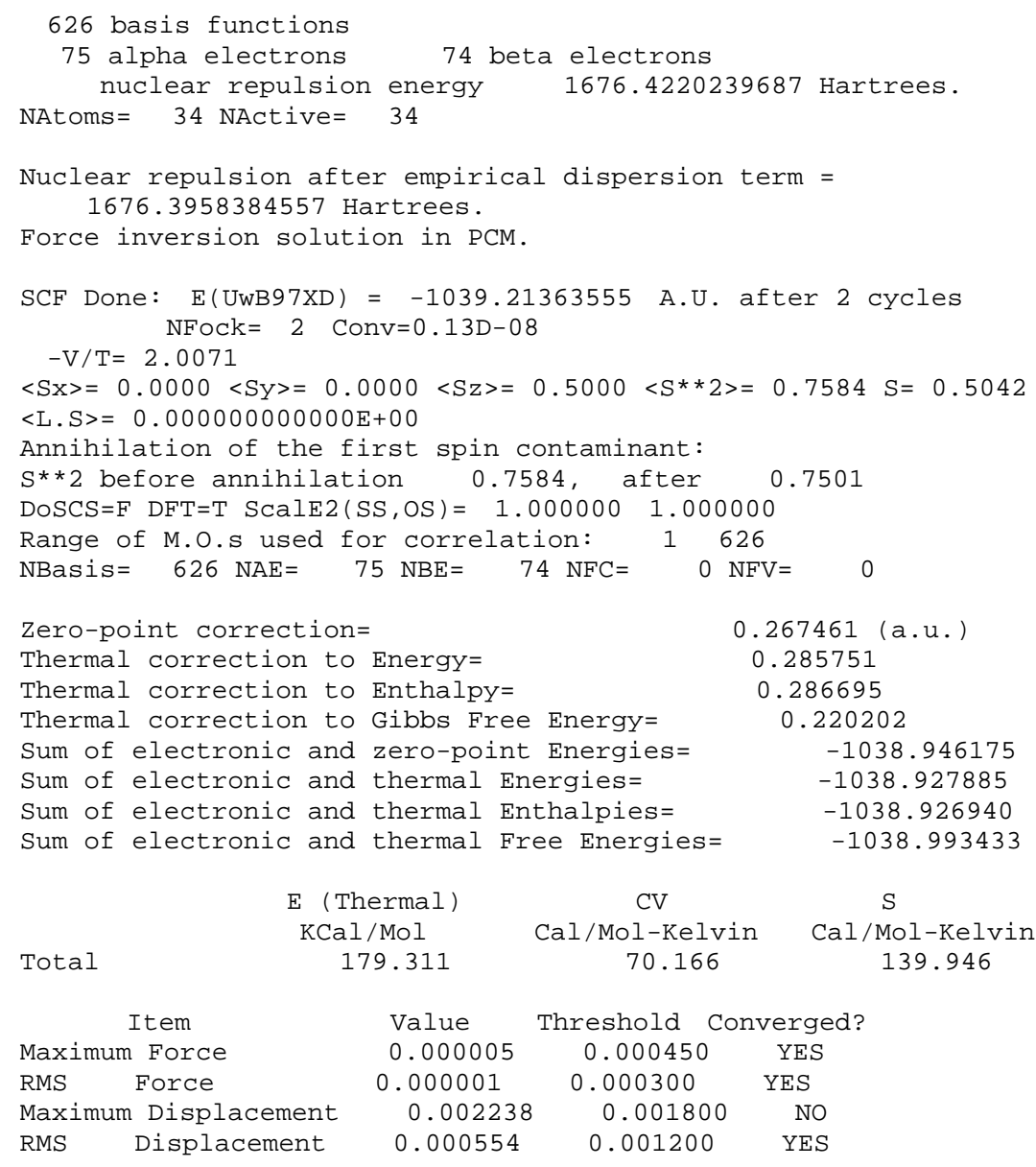

\begin{tabular}{lccc}
\multicolumn{1}{c}{ Item } & Value & Threshold Converged? \\
Maximum Force & $0.000 \odot \odot 5$ & $0.00 \odot 450$ & YES \\
RMS Force & $0.0000 \odot 1$ & $0.00030 \odot$ & YES \\
Maximum Displacement & 0.002238 & 0.001800 & NO \\
RMS Displacement & 0.000554 & 0.001200 & YES
\end{tabular}

Normal termination of Gaussian 16 at Thu Mar 14 19:10:51 2019.

Figure S4-10, TS3b(C8) - - - - -

Stoichiometry C10H14N505(2)

Standard orientation:

\begin{tabular}{|c|c|c|c|c|c|}
\hline \multirow{2}{*}{$\begin{array}{l}\text { Center } \\
\text { Number }\end{array}$} & \multirow{2}{*}{$\begin{array}{l}\text { Atomic } \\
\text { Number }\end{array}$} & \multirow{2}{*}{$\begin{array}{l}\text { Atomic } \\
\text { Type }\end{array}$} & \multicolumn{3}{|c|}{ Coordinates (Angstroms) } \\
\hline & & & $x$ & $\mathrm{Y}$ & Z \\
\hline & & 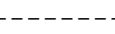 & $-\ldots$ & $-\ldots-\ldots$ & 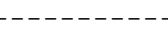 \\
\hline 1 & 1 & $\odot$ & 3.293560 & -1.086261 & 2.084323 \\
\hline 2 & 8 & $\odot$ & 3.084270 & -1.550208 & 1.269193 \\
\hline 3 & 8 & $\odot$ & 3.436301 & 2.580564 & -0.155680 \\
\hline 4 & 6 & $\odot$ & 3.413509 & $\odot .215509$ & -0.436342 \\
\hline 5 & 6 & $\odot$ & 3.110222 & 1.369720 & $\odot .521834$ \\
\hline 6 & 1 & $\odot$ & 3.183200 & 3.318503 & $\odot .407281$ \\
\hline 7 & 6 & $\odot$ & 3.920024 & -1.059965 & 0.228315 \\
\hline 8 & 6 & $\odot$ & 1.611631 & 1.222123 & $\odot .757172$ \\
\hline 9 & 6 & $\odot$ & 1.106102 & 0.660131 & -0.578304 \\
\hline 10 & 8 & $\odot$ & 2.201292 & -0.033272 & -1.153980 \\
\hline 11 & 1 & $\odot$ & 3.960300 & -1.848541 & -0.532586 \\
\hline 12 & 1 & $\odot$ & 4.943736 & -0.885793 & 0.593548 \\
\hline 13 & 1 & $\odot$ & 4.176611 & 0.536278 & -1.159967 \\
\hline 14 & 1 & $\odot$ & 3.691256 & 1.293160 & 1.452149 \\
\hline 15 & 1 & $\odot$ & 1.123284 & 2.166225 & 1.019035 \\
\hline 16 & 1 & $\odot$ & 1.423150 & ๑. 492298 & 1.552165 \\
\hline 17 & 1 & $\odot$ & ๑ . 801246 & 1.472267 & -1.256631 \\
\hline 18 & 7 & $\odot$ & 0.006390 & -0.255778 & -0.432567 \\
\hline 19 & 6 & $\odot$ & $\odot .014762$ & -2.159541 & -0.132076 \\
\hline 20 & 7 & $\odot$ & -1.446449 & -2.129009 & 0.112960 \\
\hline 21 & 6 & $\odot$ & -2.083764 & -0.952165 & 0.068540 \\
\hline 22 & 6 & $\odot$ & -1.211827 & 0.155889 & -0.222255 \\
\hline 23 & 7 & 0 & -1.648066 & 1.443241 & -0.246790 \\
\hline 24 & 6 & $\odot$ & -2.938880 & 1.625134 & -0.042477 \\
\hline 25 & 7 & $\odot$ & -3.832467 & 0.610544 & 0.193744 \\
\hline 26 & 6 & 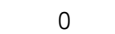 & $-3.4840 \odot 2$ & -0.741852 & 0.275647 \\
\hline
\end{tabular}




\begin{tabular}{|c|c|c|c|c|c|}
\hline 27 & 7 & $\odot$ & -3.429326 & 2.873163 & -0.060553 \\
\hline 28 & 8 & $\odot$ & -4.327948 & -1.606182 & $\odot .504158$ \\
\hline 29 & 1 & $\odot$ & 0.586462 & -2.051178 & 0.802745 \\
\hline 30 & 1 & 0 & -2.794797 & 3.632149 & -0.253634 \\
\hline 31 & 1 & $\odot$ & -4.407978 & 3. 078195 & ๑. 061328 \\
\hline 32 & 1 & $\odot$ & -4.811899 & ๑. 821849 & 0.342824 \\
\hline 33 & 8 & $\odot$ & 0.417344 & -2.858489 & -1.063144 \\
\hline 34 & 1 & $\odot$ & -1.955453 & -3.003490 & 0.075159 \\
\hline
\end{tabular}

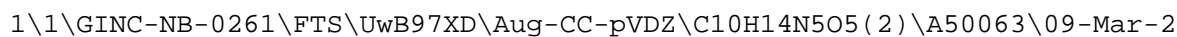
$019 \backslash 0 \backslash \backslash \#$ wb97xd / aug-cc-pVDZ 6d pop=none optcyc=300 scf=(direct, pass) scfcyc $=300$ iop $(1 / 8=2)$ opt $=(t s$, readfc, nofreeze, noeig) IOP $(2 / 9=1)$ scrf= ( $\mathrm{pcm}$, solvent=water ) iop $(1 / 18=120)$ geom=allcheck guess=check $\backslash \backslash$ made from

B. $01 \backslash H F=-1039.208732 \backslash S 2=0.76148 \backslash S 2-1=0 . \backslash S 2 A=0.750086 \backslash R M S D=5.307 e-09 \backslash R M$ $\mathrm{SF}=2.982 \mathrm{e}-06 \backslash \mathrm{Dipole}=-1.4477461,4.4644229,3.2988501 \backslash \mathrm{PG}=\mathrm{CO} 1 \quad[\mathrm{X}(\mathrm{C} 1 \mathrm{OH} 14 \mathrm{~N} 50$

Job cpu time: $\quad 2$ days 7 hours 57 minutes 32.1 seconds.

Elapsed time: $\odot$ days 3 hours 30 minutes 12.8 seconds.

File lengths (MBytes): RWF $=531$ Int $=0$ D2E $=0$

Chk $=49$ Scr=

Normal termination of Gaussian 16 at Sat Mar 9 18:14:49 2019.

Initial command:

$* * * * * * * * * * * * * * * * * * * * * * * * * * * * * * * * * * * * * * * * * *$

Gaussian 16: ES64L-G16RevB.01 20-Dec-2017 9 - Mar -2019

$* * * * * * * * * * * * * * * * * * * * * * * * * * * * * * * * * * * * * * * * * *$

$\%$ mem $=1500 \mathrm{MW}$

$\%$ nproc $=16$

Will use up to 16 processors via shared memory.

\%chk=degua - oh8y. higha. chk

\# wb97xd / aug-cc-pVDz 6d pop=none optcyc=300

scf $=($ direct, pass $)$ scfcyc $=300$ iop $(1 / 8=2)$

freq=noraman IOP $(2 / 9=1) \operatorname{scrf}=(\mathrm{pcm}$, solvent=water $)$

geom $=$ all check guess $=$ check

the checkpoint file: "degua-oh8y, higha,chk"

- . - - . -

made from file: "degua-oh8a.rev.chk" 2'-deoxyguanosine

$+\mathrm{OH}($.$) at \mathrm{C} 8, \mathrm{C}(19) \ldots \mathrm{N}(18)$ cleavage

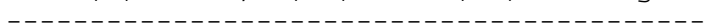

Charge $=\odot$ Multiplicity $=2$

$\mathrm{H}, 0,3.3635087331,-1.2518033612,1.9062382464$

$0,0,3.1355461441,-1.6630525742,1.0681498715$

$0,0,3.4815238194,2.5471569386,-0.1031312731$

C, $0,3.4403594554,0.2043669651,-0.5302357117$

C, $0,3.1624327268,1.2977237557,0.5039873884$

$\mathrm{H}, \odot, 3.2436627546,3.249535721,0.5097513753$

C, $0,3.9530805574,-1.1122289419,0.0432196192$

C, $0,1.6680401953,1.1419486313,0.7598573467$

C, $0,1.1330255081,0.6665819751,-0.5975948235$

$0,0,2.2127849625,0.0059431121,-1.2373821845$

$\mathrm{H}, 0,3.9739523026,-1.8518960528,-0.7660404947$

$\mathrm{H}, 0,4.9847791358,-\odot .9654715359,0.3978697144$

$\mathrm{H}, 0,4.1906474128,0.5665338972,-1.2476990312$

$\mathrm{H}, 0,3.7614268433,1.1607844714,1.4157994928$

$\mathrm{H}, 0,1.1901118327,2.0698726563,1.0898956552$

$\mathrm{H}, 0,1.4914575101,0.3646799335,1.5114256242$

$\mathrm{H}, 0,0.8191418123,1.5207641672,-1.2176584718$

$\mathrm{N}, \odot, 0.0314886124,-\odot .252059984,-\odot .4871008191$

C, $0,0.0355318206,-2.1708939649,-0.3061607799$

$\mathrm{N}, 0,-1.420331318,-2.1496498975,-0.0302586707$

C, $0,-2.0520262773,-0.9696890914,0.0116778884$

C, $0,-1.1800605934,0.1507295512,-0.2269807886$

$\mathrm{N}, 0,-1.609729019,1.4389025618,-0.1623684409$

C, $\odot,-2.8952230771,1.6130453577,0.078900378$

$\mathrm{N}, 0,-3.789413203,0.589406965,0.2693683357$

$\mathrm{C}, 0,-3.4467111407,-0.7669015135,0.2597152361$

$\mathrm{N}, 0,-3.3791707581,2.8617952998,0.1485995409$

$0,0,-4.2906114746,-1.6403003413,0.4508591734$

$\mathrm{H}, 0,0.6262833424,-2.1235141877,0.6218695323$

$\mathrm{H}, 0,-2.7445083879,3.6287148507,-0.0095351242$ 
$\mathrm{H}, 0,-4.3540820542,3.0628829844,0.3027567325$

$\mathrm{H}, 0,-4.7645299086,0.7950588734,0.4510733113$

$0,0,0.4157344034,-2.811985871,-1.2869436321$

$H, 0,-1.9347106731,-3.0179373511,-0.1122462166$

Recover connectivity data from disk.

Stoichiometry C10H14N505(2)

Standard orientation:

\begin{tabular}{|c|c|c|c|c|c|}
\hline \multirow{2}{*}{$\begin{array}{l}\text { Center } \\
\text { Number }\end{array}$} & \multirow{2}{*}{$\begin{array}{l}\text { Atomic } \\
\text { Number }\end{array}$} & \multirow{2}{*}{$\begin{array}{c}\text { Atomic } \\
\text { Type }\end{array}$} & \multicolumn{3}{|c|}{ Coordinates (Angstroms) } \\
\hline & & & $x$ & $\mathrm{Y}$ & $\mathrm{z}$ \\
\hline 1 & 1 & 0 & 3.293560 & -1.086261 & 2.084323 \\
\hline 2 & 8 & $\odot$ & 3.084270 & -1.550208 & 1.269193 \\
\hline 3 & 8 & 0 & 3.436301 & 2.580564 & $-\odot .15568 \odot$ \\
\hline 4 & 6 & $\odot$ & 3.413509 & $\odot .215509$ & -0.436342 \\
\hline 5 & 6 & $\odot$ & 3.110222 & 1.369720 & $\odot .521834$ \\
\hline 6 & 1 & 0 & 3.183200 & 3.318503 & $\odot .4 \odot 7281$ \\
\hline 7 & 6 & $\odot$ & 3.920024 & -1.059965 & $\odot .228315$ \\
\hline 8 & 6 & $\odot$ & 1.611631 & 1.222123 & $\odot .757172$ \\
\hline 9 & 6 & 0 & 1.106102 & $\odot .660131$ & -0.578304 \\
\hline 10 & 8 & $\odot$ & 2.201292 & $-\odot .033272$ & -1.153980 \\
\hline 11 & 1 & $\odot$ & 3.960300 & -1.848541 & -0.532586 \\
\hline 12 & 1 & 0 & 4.943736 & -0.885793 & $\odot .593548$ \\
\hline 13 & 1 & $\odot$ & 4.176611 & 0.536278 & -1.159967 \\
\hline 14 & 1 & $\odot$ & 3.691256 & 1.293160 & 1.452149 \\
\hline 15 & 1 & $\odot$ & 1.123284 & 2.166225 & 1.019035 \\
\hline 16 & 1 & $\Theta$ & 1.423150 & $\odot .492298$ & 1.552165 \\
\hline 17 & 1 & 0 & $\odot .801246$ & 1.472267 & -1.256631 \\
\hline 18 & 7 & 0 & $\odot .006390$ & -0.255778 & -0.432567 \\
\hline 19 & 6 & 0 & 0.014762 & -2.159541 & -0.132076 \\
\hline 20 & 7 & 0 & -1.446449 & -2.129009 & $\odot .112960$ \\
\hline 21 & 6 & $\odot$ & -2.083764 & -0.952165 & 0.068540 \\
\hline 22 & 6 & $\Theta$ & -1.211827 & 0.155889 & -0.222255 \\
\hline 23 & 7 & $\odot$ & -1.648066 & 1.443241 & -0.246790 \\
\hline 24 & 6 & $\odot$ & -2.938880 & 1.625134 & -0.042477 \\
\hline 25 & 7 & 0 & -3.832467 & 0.610544 & 0.193744 \\
\hline 26 & 6 & 0 & -3.484002 & -0.741852 & $\odot .275647$ \\
\hline 27 & 7 & $\odot$ & -3.429326 & 2.873163 & -0.060553 \\
\hline 28 & 8 & $\Theta$ & -4.327948 & -1.606182 & 0.504158 \\
\hline 29 & 1 & 0 & $\odot .586462$ & -2.051178 & $\odot .8 \odot 2745$ \\
\hline 30 & 1 & $\odot$ & -2.794797 & 3.632149 & -0.253634 \\
\hline 31 & 1 & $\odot$ & -4.407978 & 3.078195 & 0.061328 \\
\hline 32 & 1 & 0 & -4.811899 & $\odot .821849$ & $\odot .342824$ \\
\hline 33 & 8 & $\odot$ & 0.417344 & -2.858489 & -1.063144 \\
\hline 34 & 1 & $\odot$ & -1.955453 & -3.003490 & $\odot .075159$ \\
\hline
\end{tabular}

Standard basis: Aug-CC-pVDZ (6D, 7F)

626 basis functions

75 alpha electrons $\quad 74$ beta electrons

nuclear repulsion energy 1670.7585420298 Hartrees.

NAtoms $=34$ NActive $=34$

Nuclear repulsion after empirical dispersion term $=$ 1670.7323744834 Hartrees.

Force inversion solution in PCM.

Polarizable Continuum Model (PCM)

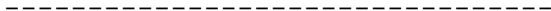

Model : PCM.

Atomic radii : UFF (Universal Force Field).

Solvent : Water, Eps $=78.355300$ Eps $($ inf $)=1.777849$

- - - - -

SCF Done: $E($ UWB97XD) $=-1039.20873197 \mathrm{~A} . \mathrm{U}$. after 1 cycles

NFock $=1$ Conv $=0.70 \mathrm{D}-08$

$\langle\mathrm{S} x>=0.0000<\mathrm{Sy}>=0.0000<\mathrm{S} z>=0.5000$

$\left\langle S^{* *} 2\right\rangle=0.7615 \mathrm{~S}=0.5057$

$<\mathrm{L} . \mathrm{S}\rangle=0.000000000000 \mathrm{E}+00$

Annihilation of the first spin contaminant:

$S^{* * 2}$ before annihilation 0.7615 , after

$\odot .7501$

DoSCS $=F$ DFT $=T$ ScalE2 $(S S, 0 S)=1.0000001 .000000$

Range of M.O.s used for correlation: 1626 
Harmonic frequencies $\left(\mathrm{cm}^{* *}-1\right)$, IR intensities (KM/Mole), Raman activities ( $\left.A^{* *} 4 / \mathrm{AMU}\right)$, depolarization ratios for plane and unp incident light, reduced masses (AMU), force constants (mDyne/A), and normal coordinates:

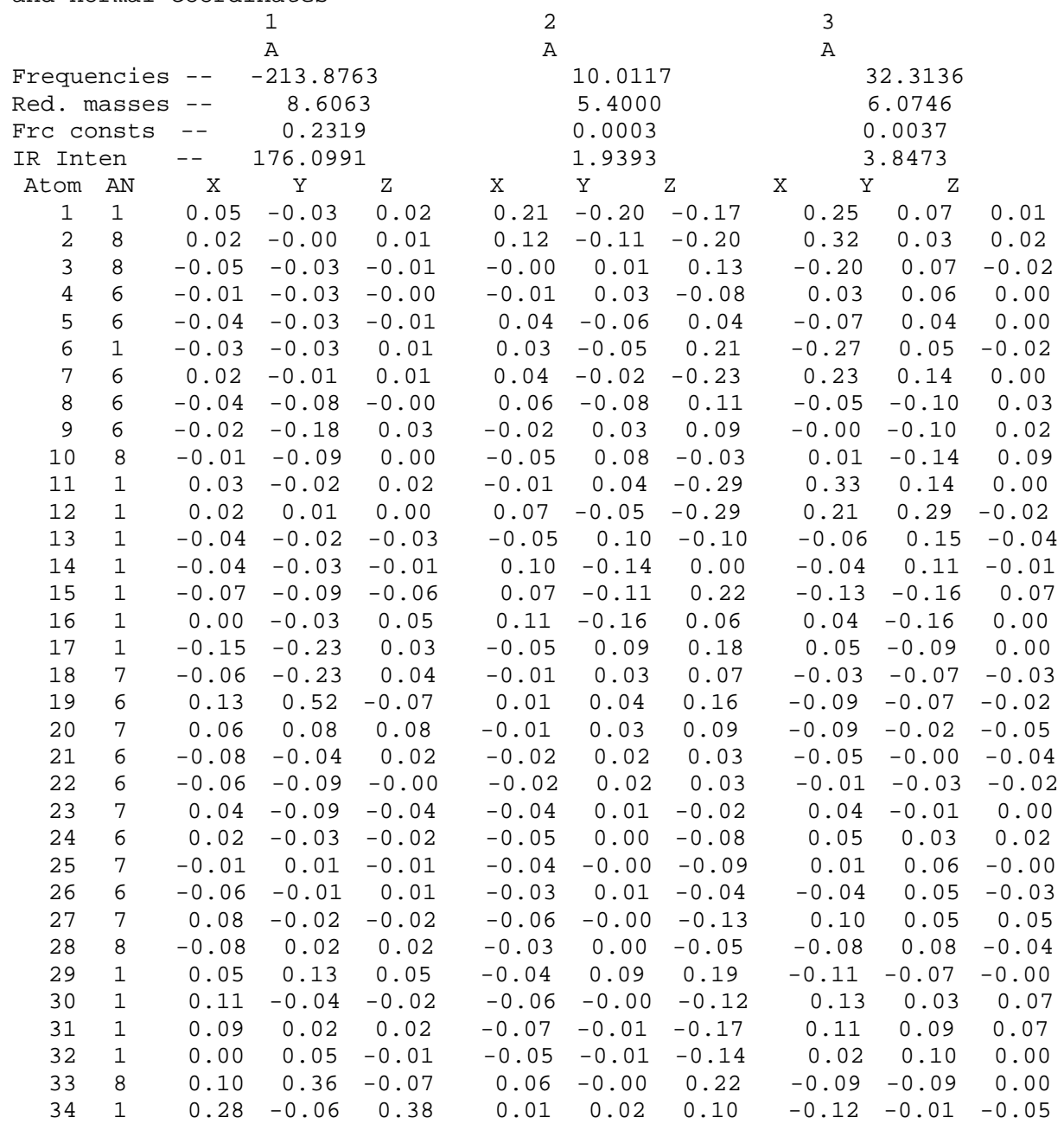

- Thermochemistry

\section{Thermochemistry -}

Temperature 298.150 Kelvin. Pressure 1.00000 Atm.

Molecular mass: 284.09949 amu.

Principal axes and moments of inertia in atomic units:

\begin{tabular}{cccr} 
& \multicolumn{1}{c}{1} & 2 & 3 \\
Eigenvalues & - & \multicolumn{3}{c}{$3091.325037936 .28797^{* * * * * * *}$} \\
X & 0.99992 & -0.01165 & 0.00554 \\
Y & 0.01166 & 0.99993 & -0.00052
\end{tabular}

$\begin{array}{lrrr}0.000553 & 0.00059 & 0.99998\end{array}$

This molecule is an asymmetric top.

Rotational symmetry number 1.

Warning - - assumption of classical behavior for rotation may cause significant error

$\begin{array}{lllll}\text { Rotational temperatures (Kelvin) } & 0.02802 & 0.01091 & 0.00844\end{array}$

$\begin{array}{llll}\text { Rotational constants (GHZ): } & 0.58381 & 0.22740 & 0.17586\end{array}$

1 imaginary frequencies ignored.

Zero-point vibrational energy 696937.1 (Joules/Mol) 166.57196 (Kcal/Mol)

Warning - - explicit consideration of 30 degrees of freedom as vibrations may cause significant error

Zero-point correction=

Thermal correction to Energy=

Thermal correction to Enthalpy=

Thermal correction to Gibbs Free Energy

Sum of electronic and zero-point Energies=

Sum of electronic and thermal Energies=

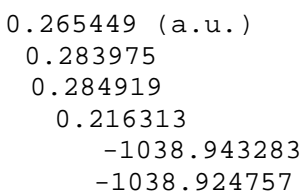


Sum of electronic and thermal Enthalpies= Sum of electronic and thermal Free Energies=

$$
\begin{gathered}
\text { E (Thermal) } \\
\text { KCal/Mol }
\end{gathered}
$$

Total

178.197
CV

Cal/Mol-Kelvin 69.763
$-1038.923813$

$-1038.992419$

Cal/Mol-Kelvin 144.395

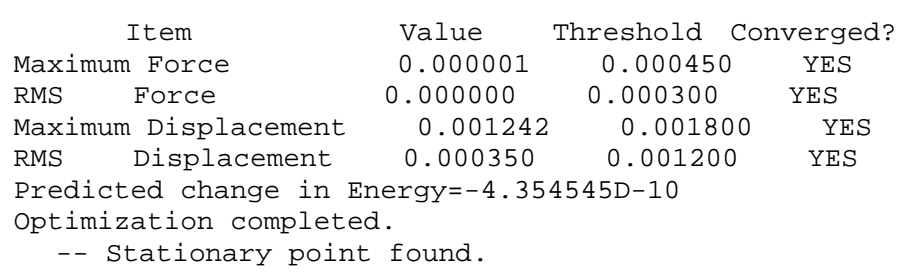

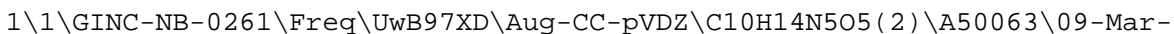

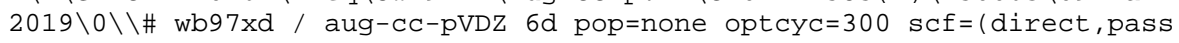
) $\operatorname{scfcyc}=300$ iop $(1 / 8=2)$ freq=noraman $\operatorname{IOP}(2 / 9=1)$ scrf $=(\mathrm{pcm}$, solvent=wate r) geom=allcheck guess=check \\made from file: "degua-oh8a.rev.chk" 2'deoxyguanosine $+\mathrm{OH}($.$) at \mathrm{C} 8, \mathrm{C}(19) \ldots . \mathrm{N}(18)$ cleavage $\backslash \backslash \odot, 2 \backslash \mathrm{H}, 3.363508$

File lengths (MBytes):

RWF $=2405$ Int $=\quad 0$ D2E $=\quad 0$ Chk $=62 \mathrm{Scr}=1$

Normal termination of Gaussian 16 at Sat Mar 9 22:14:51 2019.

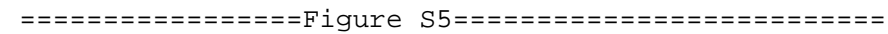

Figure S5-1, Int1(C8)W1

degua-oh8w1b.rev. higha. log

Stoichiometry C10H16N506(2)

\begin{tabular}{|c|c|c|c|c|c|}
\hline \multirow{2}{*}{$\begin{array}{l}\text { Center } \\
\text { Number }\end{array}$} & \multirow{2}{*}{$\begin{array}{l}\text { Atomic } \\
\text { Number }\end{array}$} & \multirow{2}{*}{$\begin{array}{c}\text { Atomic } \\
\text { Type }\end{array}$} & \multicolumn{3}{|c|}{ Coordinates (Angstroms) } \\
\hline & & & $x$ & $\mathrm{Y}$ & Z \\
\hline . & & -- & $---\cdot-\cdot--$ & -------- & ------- \\
\hline 1 & 1 & $\odot$ & -3.850854 & 2.068150 & 1.278471 \\
\hline 2 & 8 & $\odot$ & -3.308111 & 2.183803 & $\odot .493752$ \\
\hline 3 & 8 & $\odot$ & -4.305930 & -2.054368 & $\odot .004193$ \\
\hline 4 & 6 & $\odot$ & -3.625469 & 0.087164 & -0.773091 \\
\hline 5 & 6 & $\odot$ & -3.880258 & -0.776911 & 0.464880 \\
\hline 6 & 1 & $\odot$ & -4.433472 & -2.625980 & 0.767531 \\
\hline 7 & 6 & $\odot$ & -3.949713 & 1.567057 & $-\odot .616905$ \\
\hline 8 & 6 & $\odot$ & -2.506991 & -0.830905 & 1.128593 \\
\hline 9 & 6 & $\odot$ & -1.554546 & -0.774382 & -0.066586 \\
\hline 10 & 8 & $\odot$ & -2.237040 & - ๑. . 089071 & -1.096793 \\
\hline 11 & 1 & $\odot$ & -3.596140 & 2.091887 & -1.512292 \\
\hline 12 & 1 & $\odot$ & -5.041266 & 1.687896 & -0.559518 \\
\hline 13 & 1 & $\odot$ & -4.216617 & -0.300106 & -1.613808 \\
\hline 14 & 1 & $\odot$ & -4.643407 & $-\odot .336719$ & 1.121461 \\
\hline 15 & 1 & $\odot$ & -2.359304 & -1.732731 & 1.730434 \\
\hline 16 & 1 & $\odot$ & -2.362686 & ๑. . 045422 & 1.769431 \\
\hline 17 & 1 & $\odot$ & -1.261017 & -1.773577 & -0.409187 \\
\hline 18 & 7 & $\odot$ & -0.309253 & -0.075633 & 0.208218 \\
\hline 19 & 6 & $\odot$ & -0.174435 & 1.250314 & 0.570847 \\
\hline 20 & 7 & $\odot$ & 1.068780 & 1.617899 & $\odot .727064$ \\
\hline 21 & 6 & $\odot$ & 1.805901 & 0.487367 & 0.419583 \\
\hline 22 & 6 & $\odot$ & 0.950417 & -0.587323 & 0.120903 \\
\hline 23 & 7 & $\odot$ & 1.281855 & -1.851014 & -0.162864 \\
\hline 24 & 6 & $\odot$ & 2.586560 & -2.074592 & -0.093433 \\
\hline 25 & 7 & $\odot$ & 3.501014 & -1.117755 & 0. 265209 \\
\hline 26 & 6 & $\odot$ & 3. 212929 & 0.223792 & 0.542621 \\
\hline 27 & 7 & $\odot$ & 3.052712 & -3.300172 & -0.368780 \\
\hline 28 & 8 & $\odot$ & 4.115932 & ๑. 999342 & 0.847669 \\
\hline 29 & 1 & $\odot$ & -1.050947 & 1.881742 & $\odot .685648$ \\
\hline 30 & 1 & $\odot$ & 2.392734 & -4.018089 & -0.623762 \\
\hline 31 & 1 & $\odot$ & 4.033411 & -3.528752 & -0.343374 \\
\hline 32 & 1 & $\odot$ & 4.482330 & -1.365889 & 0.316906 \\
\hline 33 & 8 & $\odot$ & 2.208130 & ๑. 806311 & -1.849473 \\
\hline 34 & 1 & $\odot$ & 2.459664 & 1.719636 & -1.620362 \\
\hline 35 & 8 & $\odot$ & 3.052537 & 3.390617 & -0.393347 \\
\hline 36 & 1 & $\odot$ & 2.221635 & 3.171805 & 0.058783 \\
\hline 37 & 1 & $\odot$ & 3.669661 & 2.779476 & 0.039220 \\
\hline
\end{tabular}

Standard orientation: 
Standard basis: Aug-CC-pVDZ (6D, 7F)

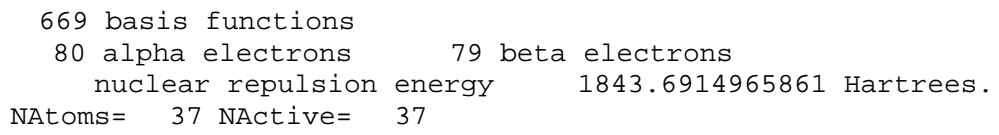

Force inversion solution in PCM.

SCF Done: $E($ UWB97XD $)=-1115.62997890$ A.U. after 2 cycles NFock $=2$ Conv $=0 \cdot 12 \mathrm{D}-08$

$<\mathrm{S} x>=0.0000<\mathrm{S} y>=0.0000<\mathrm{S} z>=0.5000$

$<S^{*} * 2>=0.7591 \mathrm{~S}=0.5045$

$<\mathrm{L} . \mathrm{S}>=0.000000000000 \mathrm{E}+00$

Annihilation of the first spin contaminant:

$\mathrm{S}^{* * 2}$ before annihilation 0.7591 , after 0.7501

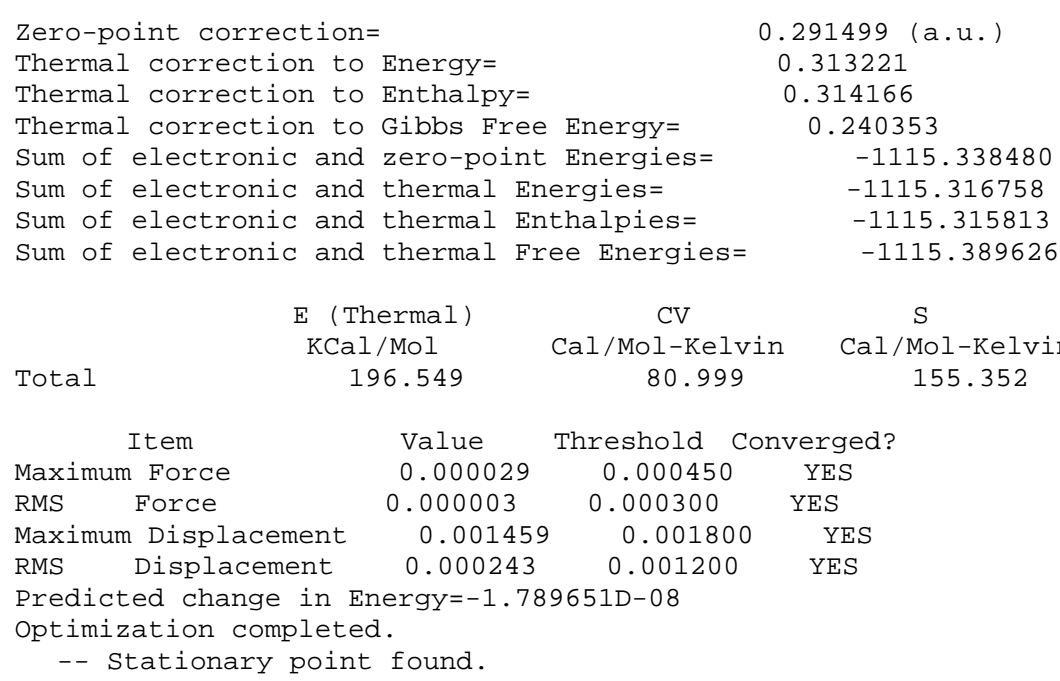

Total

$$
\text { E (Thermal) }
$$

Normal termination of Gaussian 16 at Fri Mar 15 22:48:50 2019.

Figure S5-2, TS1(C8)W1

degua-oh8w1b. higha. $\log$

Stoichiometry C10H16N506(2)

Standard orientation:

\begin{tabular}{|c|c|c|c|c|c|}
\hline \multirow{2}{*}{$\begin{array}{l}\text { Center } \\
\text { Number }\end{array}$} & \multirow{2}{*}{$\begin{array}{l}\text { Atomic } \\
\text { Number }\end{array}$} & \multirow{2}{*}{$\begin{array}{c}\text { Atomic } \\
\text { Type }\end{array}$} & \multicolumn{3}{|c|}{ Coordinates (Angstroms) } \\
\hline & & & $\mathrm{x}$ & $\mathrm{Y}$ & Z \\
\hline 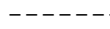 & & -- & ----- & - - - - - - & ------- \\
\hline 1 & 1 & $\odot$ & -3.666094 & 1.694437 & 1.489713 \\
\hline 2 & 8 & $\odot$ & -3.184319 & 1.861841 & $\odot .675194$ \\
\hline 3 & 8 & $\odot$ & -3.974074 & -2.409455 & 0.117639 \\
\hline 4 & 6 & $\odot$ & -3.482509 & -0.209952 & -0.641411 \\
\hline 5 & 6 & $\odot$ & -3.584326 & -1.122117 & 0.583361 \\
\hline 6 & 1 & $\odot$ & -3.996212 & -3.010093 & $\odot .868955$ \\
\hline 7 & 6 & $\odot$ & -3.869084 & 1.245396 & -0.409553 \\
\hline 8 & 6 & $\odot$ & -2.159800 & -1.111758 & 1.129799 \\
\hline 9 & 6 & 0 & -1.312315 & -0.954962 & -0.135502 \\
\hline 10 & 8 & $\odot$ & -2.121811 & -0.306385 & -1.089663 \\
\hline 11 & 1 & $\odot$ & -3.606805 & 1.815823 & -1.308396 \\
\hline 12 & 1 & $\odot$ & -4.958655 & 1.305853 & -0.272643 \\
\hline 13 & 1 & $\odot$ & -4.123506 & -0.602693 & -1.442128 \\
\hline 14 & 1 & $\odot$ & -4.313255 & -0.747209 & 1.314931 \\
\hline 15 & 1 & $\odot$ & -1.908638 & -2.022095 & 1.682600 \\
\hline 16 & 1 & $\odot$ & -2.014490 & -0.249605 & 1.789167 \\
\hline 17 & 1 & $\odot$ & -0.972316 & -1.921165 & -0.526447 \\
\hline 18 & 7 & $\odot$ & -0.098773 & -0.177796 & 0.077390 \\
\hline 19 & 6 & $\odot$ & - 0.019837 & 1.151115 & 0.430899 \\
\hline 20 & 7 & $\odot$ & 1.235031 & 1.527227 & $\odot .667648$ \\
\hline 21 & 6 & $\odot$ & 1.991149 & 0.426944 & ๑. 391515 \\
\hline 22 & 6 & $\odot$ & 1.179567 & -0.650322 & 0.023416 \\
\hline 23 & 7 & $\odot$ & 1.555219 & -1.897123 & -0.309491 \\
\hline 24 & 6 & $\odot$ & 2.865895 & -2.067771 & -0.276908 \\
\hline
\end{tabular}




\begin{tabular}{|c|c|c|c|c|c|}
\hline 25 & 7 & $\odot$ & 3.750304 & -1.073485 & ๑. . 060769 \\
\hline 26 & 6 & 0 & 3.413407 & 0.244847 & 0.417426 \\
\hline 27 & 7 & 0 & 3.380632 & -3.268889 & -0.584586 \\
\hline 28 & 8 & $\odot$ & 4.289650 & 1.053893 & ๑. 695966 \\
\hline 29 & 1 & 0 & -0.912066 & 1.721486 & $\odot .661032$ \\
\hline 30 & 1 & $\odot$ & 2.748756 & -4.009494 & -0.844908 \\
\hline 31 & 1 & 0 & 4.371088 & -3.451410 & -0.587162 \\
\hline 32 & 1 & 0 & 4.743346 & -1.276137 & ๑. 059177 \\
\hline 33 & 8 & $\odot$ & -0.038407 & 2.253770 & -1.519682 \\
\hline 34 & 1 & 0 & 0.577733 & 2.959681 & -1.246609 \\
\hline 35 & 8 & $\odot$ & 1.854960 & 4.153060 & -0.122848 \\
\hline 36 & 1 & $\odot$ & 1.792230 & 3.296533 & ๑. 345857 \\
\hline 37 & 1 & $\odot$ & 2.741107 & 4.164230 & -0.496344 \\
\hline
\end{tabular}

Standard basis: Aug-CC-pVDZ (6D, 7F)

669 basis functions

80 alpha electrons

nuclear repulsion energy 1833.9705132878 Hartrees.

NAtoms $=37$ NActive $=37$

Nuclear repulsion after empirical dispersion term =

1833.9418376259 Hartrees.

Force inversion solution in PCM.

SCF Done: $E($ UWB97XD $)=-1115.62346754$ A.U. after 2 cycles NFock $=2$ Conv $=0.59 \mathrm{D}-09 \quad-\mathrm{V} / \mathrm{T}=2.0071$

$\langle S x>=0.0000\langle$ Sy $>=0.0000\langle$ Sz $>=0.5000$

$<S^{* *} 2>=0.7690 \mathrm{~S}=0.5094$

$<$ L.S $>=0.000000000000 \mathrm{E}+00$

Annihilation of the first spin contaminant:

$\mathrm{S}^{* * 2}$ before annihilation 0.7690 , after 0.7501

Harmonic frequencies $\left(\mathrm{cm}^{* *}-1\right)$, IR intensities (KM/Mole), Raman activities ( $\left.A^{* *} 4 / \mathrm{AMU}\right)$, depolarization ratios for plane and unp incident light, reduced masses (AMU), force constants (mDyne/A), and normal coordinates:

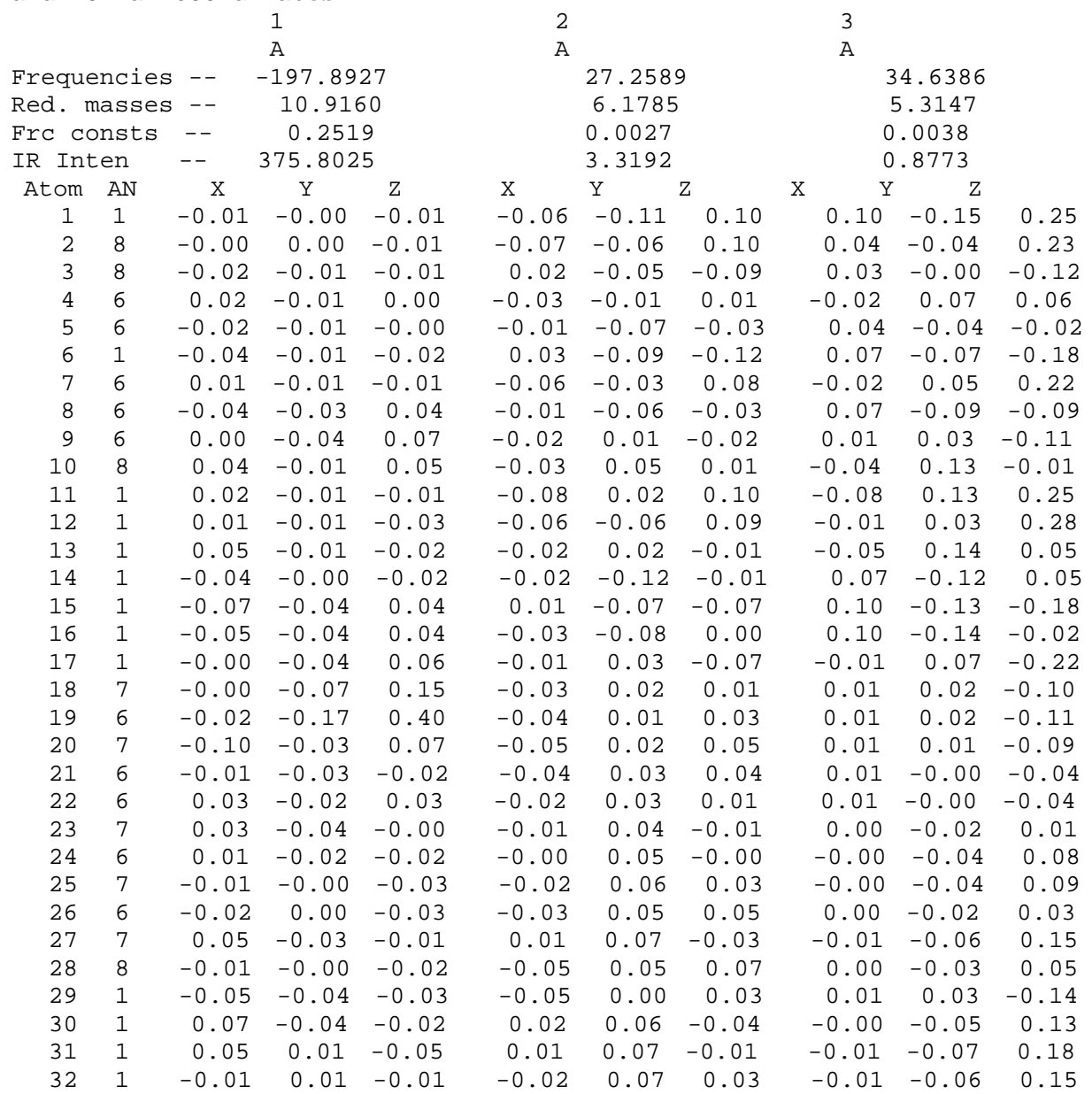




$\begin{array}{rrrrr}33 & 8 & 0.13 & 0.49 & -0.44 \\ 34 & 1 & 0.20 & 0.37 & -0.20 \\ 35 & 8 & -0.07 & -0.09 & -0.05 \\ 36 & 1 & -0.07 & -0.04 & 0.04 \\ 37 & 1 & -0.02 & -0.06 & 0.05\end{array}$

Zero-point correction=

Thermal correction to Energy=

Thermal correction to Enthalpy=

Thermal correction to Gibbs Free Energy=

Sum of electronic and zero-point Energies=

Sum of electronic and thermal Energies=

Sum of electronic and thermal Enthalpies=

Sum of electronic and thermal Free Energies=

$\begin{array}{ccc}-0.03 & 0.03 & 0.04 \\ 0.11 & -0.07 & -0.03 \\ 0.40 & -0.17 & -0.26 \\ 0.22 & -0.08 & -0.12 \\ 0.39 & -0.42 & -0.28\end{array}$

$\odot .03$

$0.00 \quad-0.15$

$-0.02$

$-0.15$

$-0.08$

$0.03-0.12$

$0.05-0.08$

$\begin{array}{lll}-0.15 & 0.13 & -0.07\end{array}$

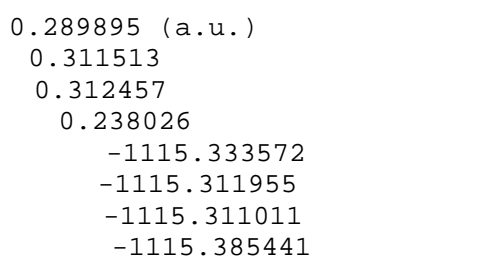

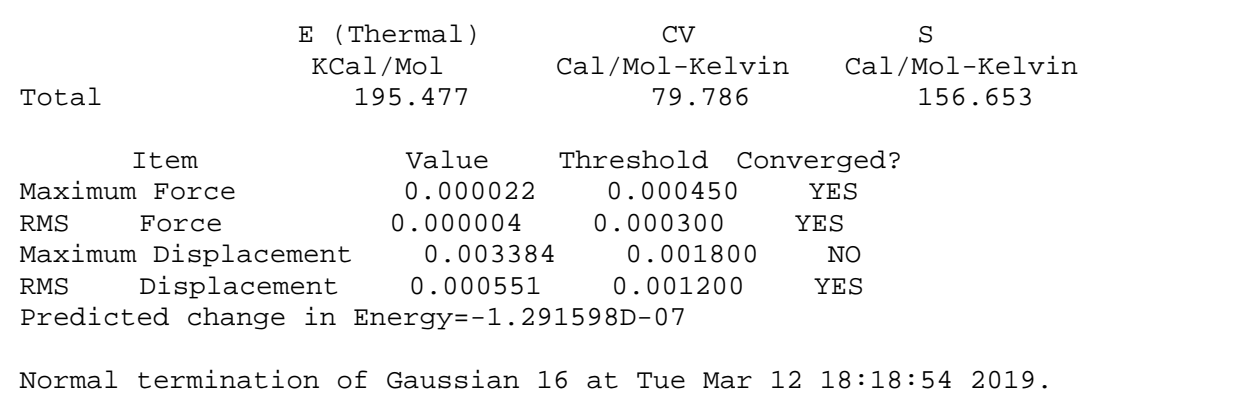

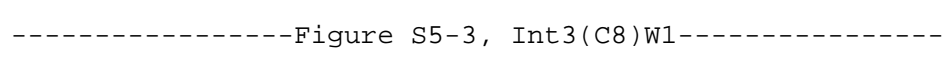

degua-oh8w1. for . higha . log

Stoichiometry C10H16N506(2)

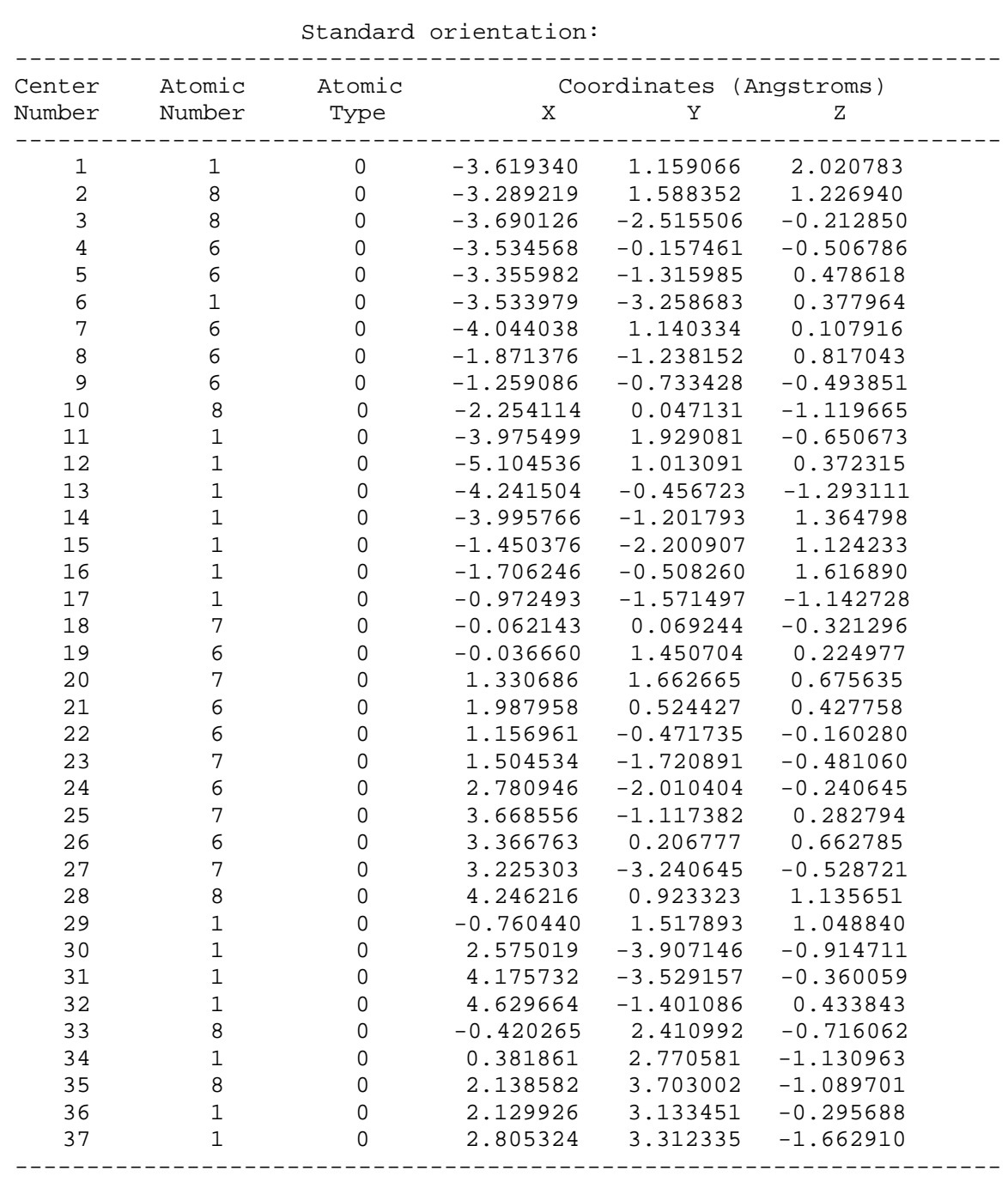


Standard basis: Aug-CC-pVDZ (6D, 7F)

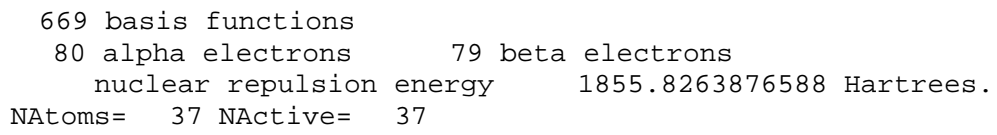

Force inversion solution in PCM.

SCF Done: $E($ UWB97XD) $=-1115.66832284$ A.U. after 2 cycles NFock $=2$ Conv $=0.82 \mathrm{D}-09 \quad-\mathrm{V} / \mathrm{T}=2.0071$

$<\mathrm{S} x>=0.0000<\mathrm{Sy}>=0.0000<\mathrm{S} z>=0.5000$

$<S^{*} * 2>=0.7607 \mathrm{~S}=0.5053$

$<\mathrm{L} . \mathrm{S}>=0.000000000000 \mathrm{E}+00$

Annihilation of the first spin contaminant:

$\mathrm{S}^{* * 2}$ before annihilation 0.7607 , after 0.7501

Harmonic frequencies $\left(\mathrm{cm}^{*}-1\right)$, IR intensities (KM/Mole), Raman activities $\left(A^{* *} 4 / A M U\right)$, depolarization ratios for plane and unpo incident light, reduced masses (AMU), force constants (mDyne/A), and normal coordinates:

$\begin{array}{cc}1 \\ \text { Frequencies - } & \mathrm{A} \\ & 21.6839\end{array}$

$\begin{array}{ll}2 & 3 \\ \text { A } & \text { A }\end{array}$

26.4923

Zero-point correction=

Thermal correction to Energy=

Thermal correction to Enthalpy=

Thermal correction to Gibbs Free Energy=

Sum of electronic and zero-point Energies=

Sum of electronic and thermal Energies=

Sum of electronic and thermal Enthalpies=

$$
\begin{gathered}
0.293260 \text { (a.u. ) } \\
0.314573 \\
0.315517 \\
0.241379 \\
-1115.375062 \\
-1115.353750 \\
-1115.352806 \\
-1115.426943
\end{gathered}
$$

Sum of electronic and thermal Free Energies=

\section{$\mathrm{S}$}

$$
\begin{gathered}
\text { E (Thermal) } \\
\text { KCal/Mol }
\end{gathered}
$$

$\mathrm{CV}$

Total

197.397

$$
\text { Cal/Mol-Kelvin }
$$

\begin{tabular}{|c|c|c|c|c|c|}
\hline \multirow{2}{*}{$\begin{array}{l}\text { Center } \\
\text { Number }\end{array}$} & \multirow{2}{*}{$\begin{array}{l}\text { Atomic } \\
\text { Number }\end{array}$} & \multirow{2}{*}{$\begin{array}{l}\text { Atomic } \\
\text { Type }\end{array}$} & \multicolumn{3}{|c|}{ Coordinates (Angstroms) } \\
\hline & & & $\mathrm{X}$ & $\mathrm{Y}$ & Z \\
\hline & & & - & - - - - - - - & \\
\hline 1 & 1 & $\odot$ & -2.935339 & ๑. 663691 & 2.381925 \\
\hline 2 & 8 & $\odot$ & -2.683888 & 1.267471 & 1.677788 \\
\hline 3 & 8 & $\odot$ & -3.963855 & -2.240088 & -0.624471 \\
\hline 4 & 6 & $\odot$ & -3.502861 & 0.077247 & -0.342019 \\
\hline 5 & 6 & $\odot$ & -3.377708 & -1.311127 & 0.284646 \\
\hline 6 & 1 & $\odot$ & -3.805193 & -3.128174 & -0.290543 \\
\hline 7 & 6 & $\odot$ & -3.670537 & 1.222129 & 0.652981 \\
\hline 8 & 6 & $\odot$ & -1.868119 & -1.489572 & 0.406520 \\
\hline 9 & 6 & $\odot$ & -1.302742 & -0.659170 & -0.759280 \\
\hline 10 & 8 & $\odot$ & -2.333817 & ๑. 242797 & -1.145837 \\
\hline 11 & 1 & $\odot$ & -3.586194 & 2.166698 & 0.102603 \\
\hline 12 & 1 & $\odot$ & -4.681264 & 1.168704 & 1.085863 \\
\hline 13 & 1 & $\odot$ & -4.372437 & 0.100490 & -1.014014 \\
\hline 14 & 1 & $\odot$ & -3.886546 & -1.370257 & 1.257818 \\
\hline 15 & 1 & $\odot$ & -1.562084 & -2.538542 & 0.346784 \\
\hline 16 & 1 & $\odot$ & -1.510115 & -1.075461 & 1.355112 \\
\hline 17 & 1 & $\odot$ & -1.073106 & -1.299951 & -1.625250 \\
\hline 18 & 7 & $\odot$ & -0.132210 & ๑. 099932 & -0.418241 \\
\hline
\end{tabular}

Cal/Mol-Kelvin

79.353

156.036

\begin{tabular}{lccc}
\multicolumn{1}{c}{ Item } & Value & Threshold Converged? \\
Maximum Force & $0.00 \odot \odot \odot 8$ & $0.00 \odot 450$ & YES \\
RMS Force & $0.000 \odot \odot 2$ & $0.00 \odot 30 \odot$ & YES \\
Maximum Displacement & 0.001919 & $0.00180 \odot$ & NO \\
RMS Displacement & 0.000515 & 0.001200 & YES \\
Predicted change in Energy=-7.467649D-09 &
\end{tabular}

Predicted change in Energy $=-7.467649 \mathrm{D}-09$

Normal termination of Gaussian 16 at Fri Mar 15 17:32:53 2019.

Figure S5-4, TS2a(C8)W1

degua-oh8w1c. higha. $\log$

Stoichiometry C10H16N506(2)

Standard orientation: 


\begin{tabular}{|c|c|c|c|c|c|}
\hline 19 & 6 & $\odot$ & 0.316643 & 1.932025 & $\odot .497092$ \\
\hline 20 & 7 & $\odot$ & 1.643657 & 1.729788 & $\odot .438801$ \\
\hline 21 & 6 & $\Theta$ & 2.007945 & $\odot .440955$ & $\odot .333986$ \\
\hline 22 & 6 & $\odot$ & 1.008396 & -0.481417 & -0.179889 \\
\hline 23 & 7 & $\Theta$ & 1.266743 & -1.809491 & -0.383446 \\
\hline 24 & 6 & $\Theta$ & 2.490967 & -2.217022 & -0.128109 \\
\hline 25 & 7 & $\odot$ & 3.495057 & -1.398072 & 0.315303 \\
\hline 26 & 6 & 0 & 3.343790 & -0.028779 & 0.572514 \\
\hline 27 & 7 & $\Theta$ & 2.800836 & -3.513688 & -0.307209 \\
\hline 28 & 8 & $\odot$ & 4.306923 & 0.629885 & $\odot .966249$ \\
\hline 29 & 1 & $\Theta$ & -0.296515 & 1.561246 & 1.320189 \\
\hline 30 & 1 & $\odot$ & 2.081098 & -4.134902 & -0.640757 \\
\hline 31 & 1 & $\odot$ & 3.721128 & -3.884351 & -0.133623 \\
\hline 32 & 1 & $\odot$ & 4.419045 & -1.775484 & $\odot .485042$ \\
\hline 33 & 8 & $\Theta$ & -0.210338 & 2.983832 & -0.089105 \\
\hline 34 & 1 & $\odot$ & 0.435056 & 3.345649 & -0.745301 \\
\hline 35 & 8 & $\Theta$ & 1.946466 & 3.748198 & -1.537806 \\
\hline 36 & 1 & $\odot$ & 2.331580 & 3.056614 & -0.970196 \\
\hline 37 & 1 & $\odot$ & 2.035722 & 3.416371 & -2.437304 \\
\hline
\end{tabular}

Standard basis: Aug-CC-pVDZ (6D, 7F)

669 basis functions

80 alpha electrons $\quad 79$ beta electrons

nuclear repulsion energy 1841.0418090280 Hartrees.

NAtoms $=37$ NActive $=37$

Force inversion solution in PCM.

SCF Done: $E($ UWB97XD $)=-1115.63837580$ A.U. after 1 cycles NFock $=1$ Conv $=0.58 \mathrm{D}-08 \quad-\mathrm{V} / \mathrm{T}=2.0071$

$\langle\mathrm{S} x\rangle=0.0000\langle\mathrm{Sy}\rangle=0.0000\langle\mathrm{Sz}\rangle=0.5000$

$\left\langle S^{* *} 2>=0.7632 \mathrm{~S}=0.5066\right.$

$<$ L.S $>=0.000000000000 \mathrm{E}+00$

Annihilation of the first spin contaminant:

$\mathrm{S}^{* * 2}$ before annihilation 0.7632 , after 0.7501

Harmonic frequencies $\left(\mathrm{cm}^{\star *}-1\right)$, IR intensities (KM/Mole), Raman activities ( $\left.A^{* *} 4 / \mathrm{AMU}\right)$, depolarization ratios for plane and unpo incident light, reduced masses (AMU), force constants (mDyne/A), and normal coordinates:

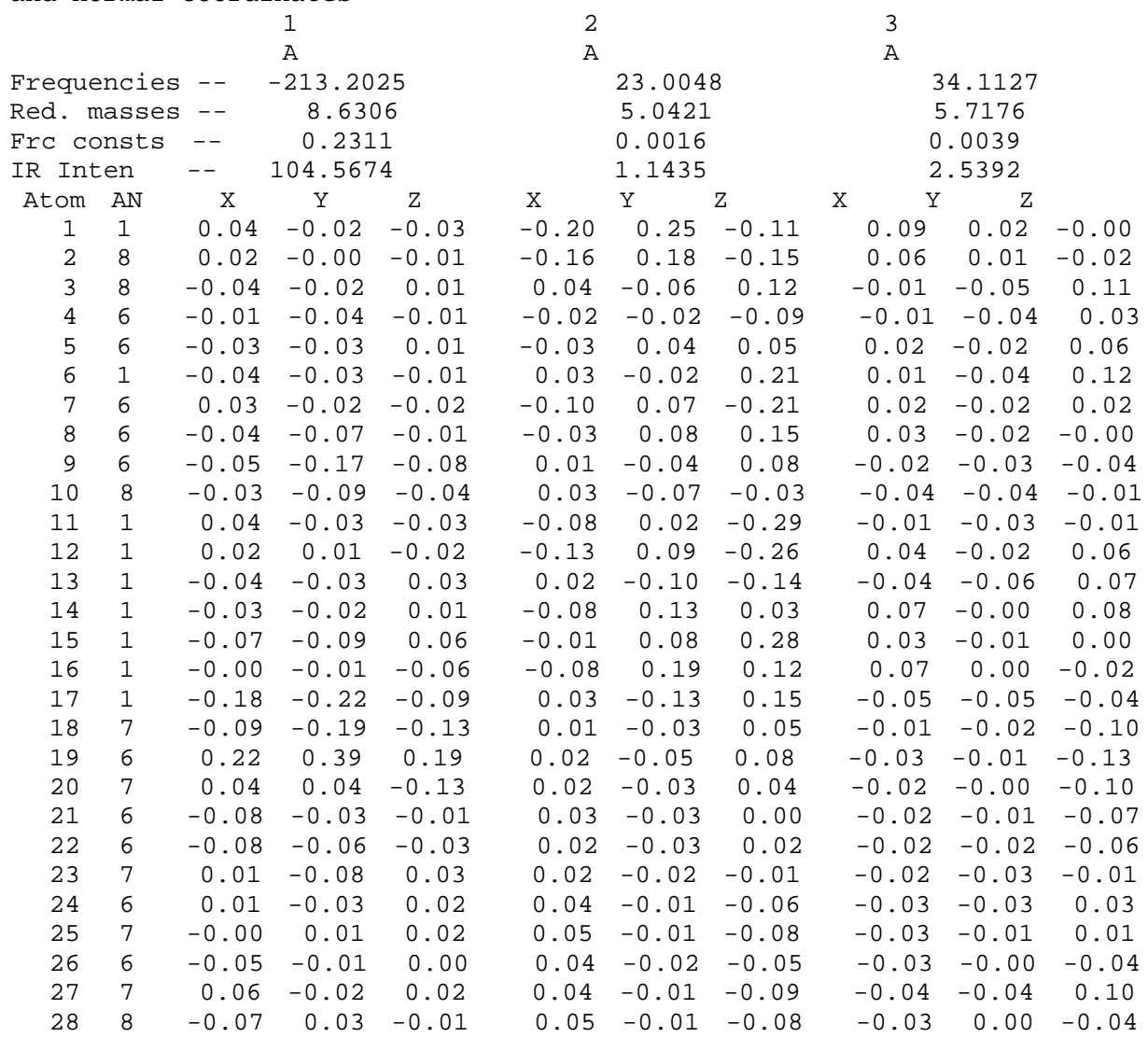




$\begin{array}{rrrrr}29 & 1 & 0.08 & 0.01 & -0.10 \\ 30 & 1 & 0.08 & -0.05 & 0.03 \\ 31 & 1 & 0.07 & 0.01 & 0.02 \\ 32 & 1 & 0.01 & 0.04 & 0.01 \\ 33 & 8 & 0.17 & 0.32 & 0.21 \\ 34 & 1 & 0.14 & 0.34 & 0.17 \\ 35 & 8 & 0.01 & 0.04 & -0.02 \\ 36 & 1 & 0.03 & -0.06 & -0.15 \\ 37 & 1 & -0.19 & 0.11 & -0.07\end{array}$

$\begin{array}{rrr}0.04 & -0.07 & 0.09 \\ 0.04 & -0.01 & -0.08 \\ 0.05 & -0.00 & -0.13 \\ 0.06 & -0.00 & -0.13 \\ -0.01 & -0.04 & 0.12 \\ -0.02 & -0.01 & 0.12 \\ -0.03 & 0.05 & 0.12 \\ -0.02 & 0.03 & 0.09 \\ -0.03 & 0.09 & 0.11\end{array}$

$\begin{array}{ccc}-0.05 & -0.00 & -0.14 \\ -0.04 & -0.04 & 0.09 \\ -0.04 & -0.03 & 0.10 \\ -0.04 & -0.01 & 0.05 \\ -0.01 & -0.01 & -0.15 \\ 0.04 & 0.05 & -0.06 \\ 0.18 & 0.29 & 0.29 \\ 0.08 & 0.19 & 0.24 \\ 0.40 & 0.47 & 0.25\end{array}$

Zero-point correction=

Thermal correction to Energy=

Thermal correction to Enthalpy=

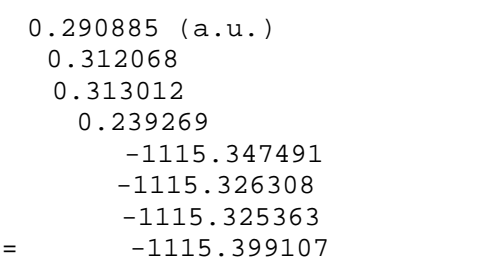

Sum of electronic and zero-point Energies=

Sum of electronic and thermal Energies=

Sum of electronic and thermal Enthalpies=

Sum of electronic and thermal Free Energies=

$\mathrm{S}$

Cal/Mol-Kelvin Cal/Mol-Kelvin 78.634

155.207

Total

$$
\begin{aligned}
& \text { E (Thermal) } \\
& \text { KCal/Mol }
\end{aligned}
$$$$
195.826
$$

\begin{tabular}{lccc}
\multicolumn{1}{c}{ Item } & Value & Threshold Converged? \\
Maximum Force & $0.00 \odot \odot \odot 9$ & $0.00 \odot 45 \odot$ & YES \\
RMS Force & $0.0000 \odot 2$ & $0.00030 \odot$ & YES \\
Maximum Displacement & $0.0040 \odot 1$ & $0.00180 \odot$ & NO \\
RMS Displacement & 0.000620 & 0.001200 & YES
\end{tabular}

Predicted change in Energy $=-5.596156 \mathrm{D}-\odot 8$

Normal termination of Gaussian 16 at Mon Mar 11 11:56:17 2019.

Figure S5-5, Int3a(C8)

degua-oh8w1c. rev. higha. $\log$

Stoichiometry C10H16N506(2)

Standard orientation:

\begin{tabular}{|c|c|c|c|c|c|}
\hline \multirow{2}{*}{$\begin{array}{l}\text { Center } \\
\text { Number }\end{array}$} & Atomic & Atomic & \multicolumn{3}{|c|}{ Coordinates (Angstroms) } \\
\hline & Number & Type & $x$ & $\mathrm{Y}$ & Z \\
\hline 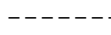 & - & - & --- & - - - & \\
\hline 1 & 1 & $\odot$ & -2.470423 & 1.621539 & 2.023853 \\
\hline 2 & 8 & $\odot$ & -2.193606 & 2.031976 & 1.200215 \\
\hline 3 & 8 & $\odot$ & -4.649273 & -1.361068 & -0.145601 \\
\hline 4 & 6 & $\odot$ & -3.572552 & 0.741405 & -0.426151 \\
\hline 5 & 6 & $\odot$ & -3.718457 & -0.480187 & 0.480204 \\
\hline 6 & 1 & $\odot$ & -4.678347 & -2.177079 & 0.362763 \\
\hline 7 & 6 & $\odot$ & -3.294442 & 2.056762 & 0.299064 \\
\hline 8 & 6 & $\odot$ & -2.303148 & -1.053614 & $\odot .514807$ \\
\hline 9 & 6 & $\odot$ & -1.654551 & -0.570963 & -0.801657 \\
\hline 10 & 8 & $\odot$ & -2.546294 & ○. 397171 & -1.353891 \\
\hline 11 & 1 & $\odot$ & -3.054208 & 2.815947 & -0.454858 \\
\hline 12 & 1 & $\odot$ & -4.214175 & 2.371010 & $\odot .817172$ \\
\hline 13 & 1 & 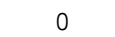 & -4.496970 & ๑. 885151 & -1.003246 \\
\hline 14 & 1 & $\odot$ & -4.082961 & -0.208790 & 1.482057 \\
\hline 15 & 1 & $\odot$ & -2.302491 & -2.145243 & 0.589305 \\
\hline 16 & 1 & $\odot$ & -1.744769 & -0.652053 & 1.367245 \\
\hline 17 & 1 & $\odot$ & -1.578203 & -1.389133 & -1.533345 \\
\hline 18 & 7 & $\odot$ & -0.371936 & ๑. . 040586 & -0.607044 \\
\hline 19 & 6 & $\odot$ & 1.168813 & 2.149995 & 0.310697 \\
\hline 20 & 7 & $\odot$ & 2.098151 & 1.297630 & 0.027483 \\
\hline 21 & 6 & $\odot$ & 1.898209 & -0.046990 & 0.010852 \\
\hline 22 & 6 & $\odot$ & ๑. 638524 & -0.713200 & -0.290335 \\
\hline 23 & 7 & $\odot$ & ๑. 542135 & -2.094503 & -0.226901 \\
\hline 24 & 6 & $\odot$ & 1.616099 & -2.785722 & 0.031165 \\
\hline 25 & 7 & $\odot$ & 2.854502 & -2.226509 & ๑. 245330 \\
\hline 26 & 6 & $\odot$ & 3. 086714 & -0.859397 & ๑. 249206 \\
\hline 27 & 7 & $\odot$ & 1.548750 & -4.130363 & 0.118080 \\
\hline 28 & 8 & $\odot$ & 4. 216198 & -0.424059 & $\odot .447982$ \\
\hline 29 & 1 & $\odot$ & ๑. 180259 & 1.901491 & $\odot .704117$ \\
\hline 30 & 1 & $\odot$ & ๑. 677871 & -4.573067 & -0.130214 \\
\hline 31 & 1 & $\odot$ & 2.374536 & -4.704586 & 0.180440 \\
\hline 32 & 1 & $\odot$ & 3.657002 & -2.814392 & 0.433828 \\
\hline 33 & 8 & $\odot$ & 1. 377972 & 3.444369 & 0.170487 \\
\hline 34 & 1 & 0 & 2. 295895 & 3.588328 & -0.172935 \\
\hline
\end{tabular}




$\begin{array}{rrrrrr}35 & 8 & 0 & 3.991168 & 3.277884 & -0.556560 \\ 36 & 1 & 0 & 3.742570 & 2.339587 & -0.449692 \\ 37 & 1 & 0 & 4.232629 & 3.374676 & -1.483289 \\ - & - & - & -\end{array}$

Standard basis: Aug-CC-pVDZ (6D, 7F)

669 basis functions

80 alpha electrons $\quad 79$ beta electrons

nuclear repulsion energy 1806.8917881200 Hartrees.

NAtoms $=37$ NActive $=37$

Force inversion solution in PCM.

SCF Done: $E(U W B 97 X D)=-1115.64892746$ A.U. after 2 cycles

$$
\text { NFock }=2 \text { Conv }=0.98 \mathrm{D}-09 \quad-\mathrm{V} / \mathrm{T}=2.0071
$$

$\langle\mathrm{S} x\rangle=0.0000\langle\mathrm{~S} y\rangle=0.0000\langle\mathrm{~S} z\rangle=0.5000\left\langle\mathrm{~S}^{* *} 2\right\rangle=0.7760 \mathrm{~S}=0.5129$

$<$ L.S $>=0.000000000000 \mathrm{E}+00$

Annihilation of the first spin contaminant:

$\mathrm{S}^{* * 2}$ before annihilation 0.7760 , after 0.7503

Harmonic frequencies $\left(\mathrm{cm}^{* *}-1\right)$, IR intensities (KM/Mole), Raman activities ( $\left.A^{* *} 4 / A M U\right)$, depolarization ratios for plane and unpo incident light, reduced masses (AMU), force constants (mDyne/A), and normal coordinates:

$\begin{array}{lllll} & 1 & 2 & 3 & \\ \text { Frequencies - } & \text { A } & 21.2549 & \text { A } & \text { A } \\ & & 38.9875 & & 44.3953\end{array}$

Zero-point correction=

Thermal correction to Energy=

Thermal correction to Enthalpy=

Thermal correction to Gibbs Free Energy=

Sum of electronic and zero-point Energies=

Sum of electronic and thermal Energies=

๑. 291056 (a.u.)

$\odot .312938$

0.313882

0.238607

$-1115.357872$

$-1115.335990$

$\begin{array}{ll}\text { Sum of electronic and thermal Enthalpies= } & -1115.335046 \\ \text { Sum of electronic and thermal Free Energies } & -1115.410321\end{array}$

\begin{tabular}{|c|c|c|c|}
\hline $\begin{array}{r}\mathrm{E}(\mathrm{T} \\
\mathrm{KCa} \\
1\end{array}$ & $\begin{array}{l}\text { lermal) } \\
\text { Mol } \\
96.371\end{array}$ & $\begin{array}{c}\text { CV } \\
\text { Cal/Mol-Kelvin } \\
80.682\end{array}$ & $\begin{array}{c}\mathrm{S} \\
\mathrm{Cal} / \mathrm{Mol}-\mathrm{Kel} \text { in } \\
158.429\end{array}$ \\
\hline Item & Value & Threshold Conv & erged? \\
\hline Maximum Force & $\odot .000007$ & 0.000450 & YES \\
\hline Force & 0.000001 & 0.000300 & YES \\
\hline Maximum Displacement & 0.000481 & 0.001800 & YES \\
\hline Displacement & $\odot .0 \odot \odot \odot 87$ & $\odot .001200$ & YES \\
\hline
\end{tabular}

Normal termination of Gaussian 16 at Sat Mar 16 03:00:09 2019.

$\ldots \ldots \ldots$ Figure S5-6, TS3a(C8)W1 $\ldots \ldots \ldots \ldots$

degua-oh8w1d. higha. $\log$

Stoichiometry C10H16N506(2)

\begin{tabular}{rccccc}
\multicolumn{7}{c}{ Standard orientation: } \\
Center & Atomic & Atomic & \multicolumn{3}{c}{ Coordinates } \\
Number & Number & Type & X & Y & Z \\
\hline 1 & 1 & 0 & -2.532105 & 1.726425 & 1.913608 \\
2 & 8 & 0 & -2.283198 & 2.095707 & 1.062062 \\
3 & 8 & 0 & -4.612152 & -1.481990 & -0.027883 \\
4 & 6 & 0 & -3.611870 & 0.633867 & -0.455564 \\
5 & 6 & 0 & -3.713613 & -0.526997 & 0.533563 \\
6 & 1 & 0 & -4.614159 & -2.260166 & 0.537454 \\
7 & 6 & 0 & -3.391216 & 2.007415 & 0.174869 \\
8 & 6 & 0 & -2.278852 & -1.045410 & 0.605248 \\
9 & 6 & 0 & -1.648145 & -0.635424 & -0.743339 \\
10 & 8 & 0 & -2.567357 & 0.270218 & -1.354663 \\
11 & 1 & 0 & -3.192849 & 2.723201 & -0.631962 \\
12 & 1 & 0 & -4.321111 & 2.313671 & 0.679793 \\
13 & 1 & 0 & -4.539329 & 0.698703 & -1.042178
\end{tabular}




\begin{tabular}{|c|c|c|c|c|c|}
\hline 14 & 1 & $\odot$ & -4.088178 & -0.200137 & 1.514916 \\
\hline 15 & 1 & $\odot$ & -2.237535 & -2.128109 & 0.758690 \\
\hline 16 & 1 & $\odot$ & -1.735212 & -0.562105 & 1.424382 \\
\hline 17 & 1 & $\odot$ & -1.552823 & -1.497814 & -1.419284 \\
\hline 18 & 7 & $\odot$ & -0.382935 & ๑. 023311 & -0.588708 \\
\hline 19 & 6 & $\odot$ & 1.142296 & 2.267700 & 0.180237 \\
\hline 20 & 7 & $\odot$ & 2.092786 & 1.354738 & -0.014286 \\
\hline 21 & 6 & 0 & 1.910831 & $\odot .008740$ & $-\odot .037669$ \\
\hline 22 & 6 & $\odot$ & $\odot .654990$ & $-\odot .690727$ & -0.273085 \\
\hline 23 & 7 & $\odot$ & $\odot .595287$ & -2.072109 & -0.157465 \\
\hline 24 & 6 & $\odot$ & 1.696087 & -2.734182 & 0.054647 \\
\hline 25 & 7 & $\odot$ & 2.932368 & -2.144282 & 0.179685 \\
\hline 26 & 6 & $\odot$ & 3.127433 & -0.772856 & 0.150202 \\
\hline 27 & 7 & $\odot$ & 1.663745 & -4.078187 & 0.185678 \\
\hline 28 & 8 & $\odot$ & 4.253629 & -0.301786 & $\odot .281846$ \\
\hline 29 & 1 & $\odot$ & 0.116040 & 1.981845 & 0.430705 \\
\hline 30 & 1 & $\odot$ & $\odot .794257$ & -4.543313 & $-\odot .024856$ \\
\hline 31 & 1 & $\odot$ & 2.507085 & -4.630382 & 0.176541 \\
\hline 32 & 1 & $\odot$ & 3.757400 & -2.708703 & 0.340619 \\
\hline 33 & 8 & $\odot$ & 1.437873 & 3.505414 & 0.103676 \\
\hline 34 & 1 & $\odot$ & 2.698134 & 3.538191 & -0.142617 \\
\hline 35 & 8 & 0 & 3.762610 & 3.106039 & $-\odot .257481$ \\
\hline 36 & 1 & $\odot$ & 3.278507 & 2.094281 & $-\odot .193074$ \\
\hline 37 & 1 & $\odot$ & 4.094227 & 3.248523 & -1.152175 \\
\hline
\end{tabular}

Standard basis: Aug-CC-pVDZ (6D, 7F)

669 basis functions

80 alpha electrons $\quad 79$ beta electrons

nuclear repulsion energy 1814.9519464941 Hartrees.

NAtoms $=37$ NActive $=37$

Force inversion solution in PCM.

SCF Done: $E($ UWB97XD) $=-1115.63053520$ A.U. after 1 cycles NFock $=1$ Conv $=0.49 \mathrm{D}-08 \quad-\mathrm{V} / \mathrm{T}=2.0071$

$\langle\mathrm{S} x\rangle=0.0000\langle\mathrm{~S} y\rangle=0.0000\langle\mathrm{Sz}\rangle=0.5000\left\langle\mathrm{~S}^{* *} 2\right\rangle=0.7734 \mathrm{~S}=0.5116$

$<$ L.S $>=0.000000000000 \mathrm{E}+00$

Annihilation of the first spin contaminant:

$\mathrm{S}^{* * 2}$ before annihilation 0.7734 , after 0.7503

Harmonic frequencies $\left(\mathrm{cm}^{* *}-1\right)$, IR intensities (KM/Mole), Raman activities ( $\left.A^{* *} 4 / A M U\right)$, depolarization ratios for plane and unp incident light, reduced masses (AMU), force constants (mDyne/A), and normal coordinates:

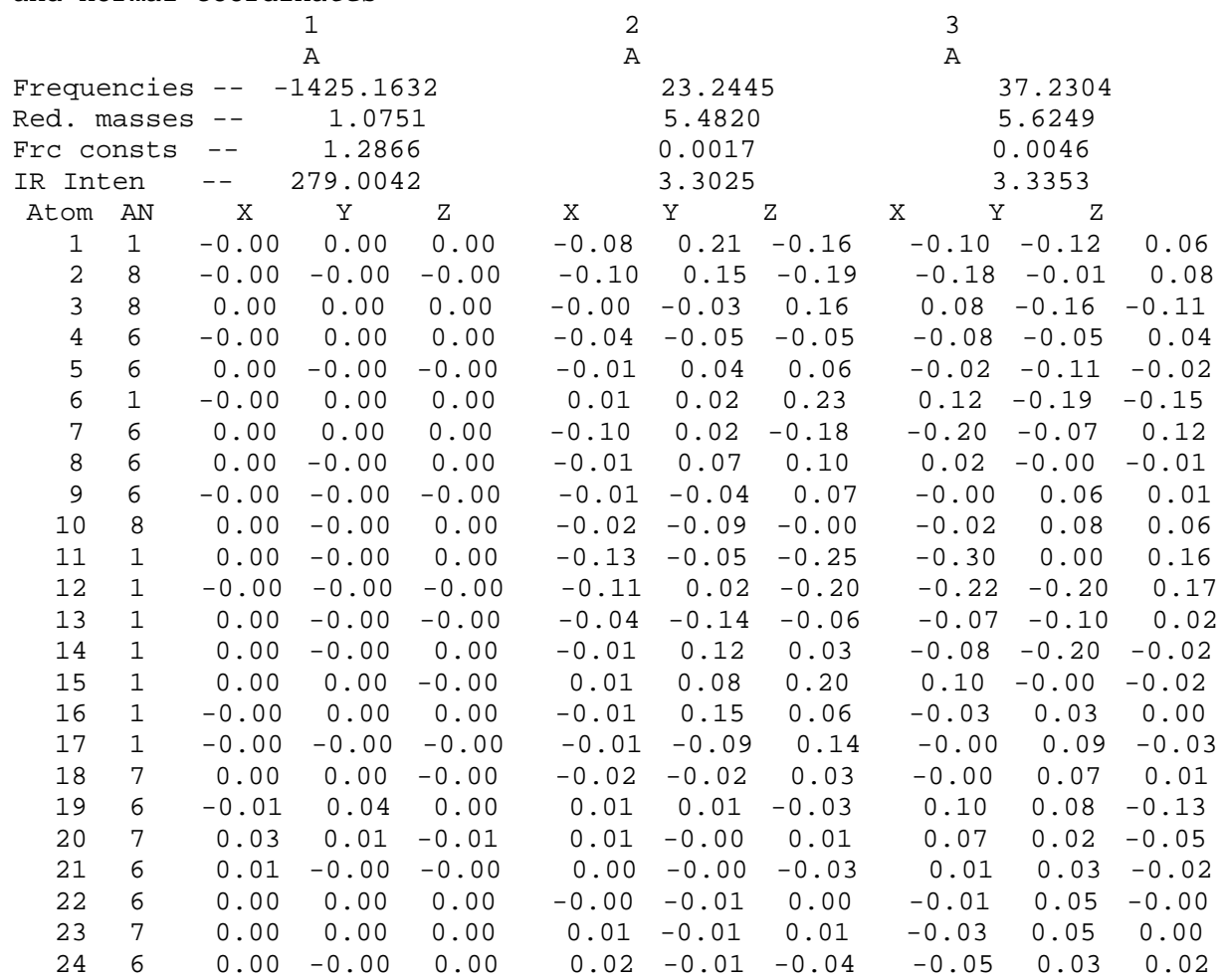




\begin{tabular}{|c|c|c|c|c|}
\hline 25 & 7 & -0.00 & -0.00 & -0.00 \\
\hline 26 & 6 & -0.00 & $-\odot . \odot \odot$ & $-\odot .0 \odot$ \\
\hline 27 & 7 & $-0.0 \odot$ & $\odot . \odot \odot$ & $-\odot .0 \odot$ \\
\hline 28 & 8 & 0.00 & 0.00 & -0.00 \\
\hline 29 & 1 & $\odot .02$ & -0.06 & -0.0 \\
\hline 30 & 1 & -0.00 & $-\odot . \odot \odot$ & $\odot .0$ \\
\hline 31 & 1 & $-\odot . \odot \odot$ & $-\odot . \odot \odot$ & $-0.0 \odot$ \\
\hline 32 & 1 & $\odot . \odot \odot$ & $\odot . \odot \odot$ & 0.00 \\
\hline 33 & 8 & -0.04 & -0.02 & 0.0 \\
\hline 34 & 1 & 0.74 & -0.03 & -0.1 \\
\hline 35 & 8 & -0.01 & 0.00 & 0.0 \\
\hline 36 & 1 & -0.49 & -0.42 & 0.0 \\
\hline 37 & 1 & -0.02 & 0.03 & -0.0 \\
\hline
\end{tabular}

Zero-point correction=

Thermal correction to Energy=

Thermal correction to Enthalpy=

Thermal correction to Gibbs Free Energy=

Sum of electronic and zero-point Energies=

Sum of electronic and thermal Energies=

Sum of electronic and thermal Enthalpies=

Sum of electronic and thermal Free Energies=

$\begin{array}{ccc}0.03 & -0.00 & -0.09 \\ 0.02 & 0.00 & -0.09 \\ 0.03 & -0.01 & -0.03 \\ 0.02 & 0.01 & -0.15 \\ -0.02 & 0.02 & -0.14 \\ 0.03 & -0.01 & 0.00 \\ 0.04 & -0.00 & -0.07 \\ 0.04 & 0.00 & -0.13 \\ 0.05 & 0.00 & 0.07 \\ 0.08 & -0.01 & 0.20 \\ 0.08 & -0.03 & 0.29 \\ 0.06 & -0.02 & 0.16 \\ 0.18 & 0.03 & 0.33\end{array}$

$-0.04 \quad 0.01$

$-0.01-0.00$

$-0.08 \quad 0.03$

$\odot .0 \odot-0.02$

$0.07 \quad 0.13 \quad-0.19$

$-0.09 \quad 0.05 \quad 0.03$

$\begin{array}{lll}-0.09 & 0.01 & 0.04\end{array}$

$\begin{array}{lll}-0.05 & -0.01 & 0.05\end{array}$

$0.18 \quad 0.06 \quad-0.14$

$\begin{array}{lll}0.21 & -0.02 & -0.04\end{array}$

$0.19-0.08 \quad 0.05$

$0.14-0.04 \quad 0.02$

$\begin{array}{lll}0.27 & -0.10 & 0.07\end{array}$

.284672 (a.u.)

$\odot .305253$

$\odot .306197$

$-1115.345863$

$-1115.325283$

$-1115.324338$

$-1115.396908$

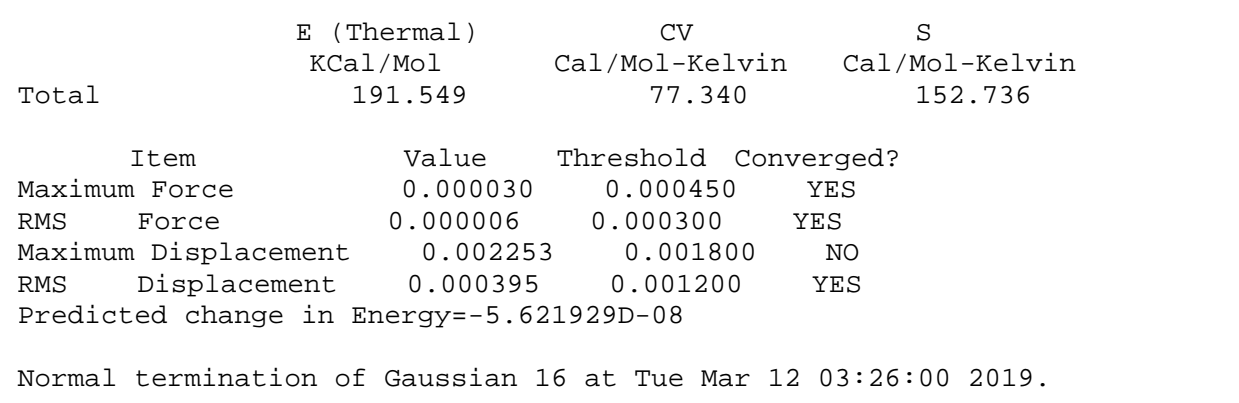

ol-Kelvin Cal/Mol-Kelvin

Cal/Mol

Value Threshold Converged?

Maximum Force

Maximum Displacement $0.002253 \quad 0.001800 \quad$ NO

Predicted change in Energy $=-5.621929 \mathrm{D}-\odot 8$

Normal termination of Gaussian 16 at Tue Mar 12 03:26:00 2019

degua-oh8w1a.rev . higha . log

Stoichiometry C10H16N506(2)

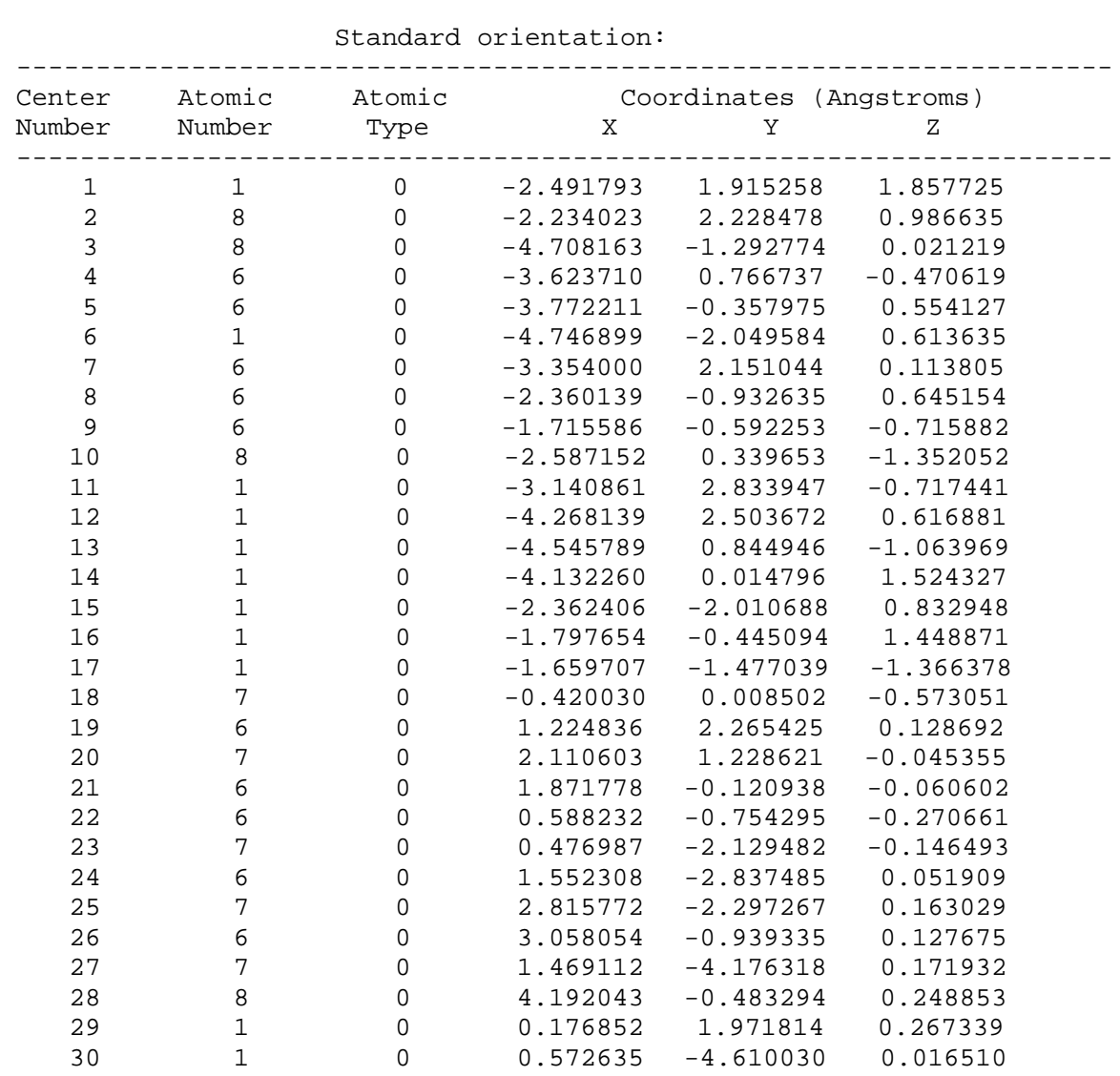




\begin{tabular}{rrrrrr}
31 & 1 & 0 & 2.285363 & -4.764903 & 0.221131 \\
32 & 1 & 0 & 3.620479 & -2.892943 & 0.315406 \\
33 & 8 & 0 & 1.611440 & 3.429328 & 0.128629 \\
34 & 1 & 0 & 3.473095 & 3.552428 & -0.115662 \\
35 & 8 & 0 & 4.382972 & 3.206400 & -0.152351 \\
36 & 1 & 0 & 3.101120 & 1.493800 & -0.056570 \\
37 & 1 & 0 & 4.674557 & 3.369162 & -1.054247 \\
\hline
\end{tabular}

Standard basis: Aug-CC-pVDZ (6D, 7F)

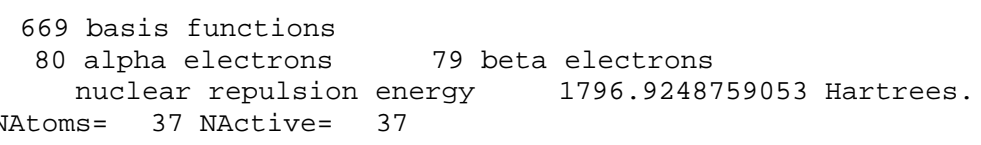

Force inversion solution in PCM.

SCF Done: $E($ UWB97XD $)=-1115.66571248$ A.U. after 1 cycles $\mathrm{NFOCk}=1$ Conv $=0.48 \mathrm{D}-08 \quad-\mathrm{V} / \mathrm{T}=2.0071$

$\langle\mathrm{S} X>=0.0000<\mathrm{Sy}\rangle=0.0000<\mathrm{S} z>=0.5000$

$\left\langle S^{*}{ }^{*} 2>=0.7750 \mathrm{~S}=0.5124\right.$

$<$ L.S $>=0.000000000000 \mathrm{E}+\odot \odot$

Annihilation of the first spin contaminant:

$\mathrm{S}^{* * 2}$ before annihilation 0.7750 , after 0.7503

Harmonic frequencies $\left(\mathrm{cm}^{*}-1\right)$, IR intensities (KM/Mole), Raman activities $\left(A^{* *} 4 / A M U\right)$, depolarization ratios for plane and unpo incident light, reduced masses (AMU), force constants (mDyne/A), and normal coordinates:

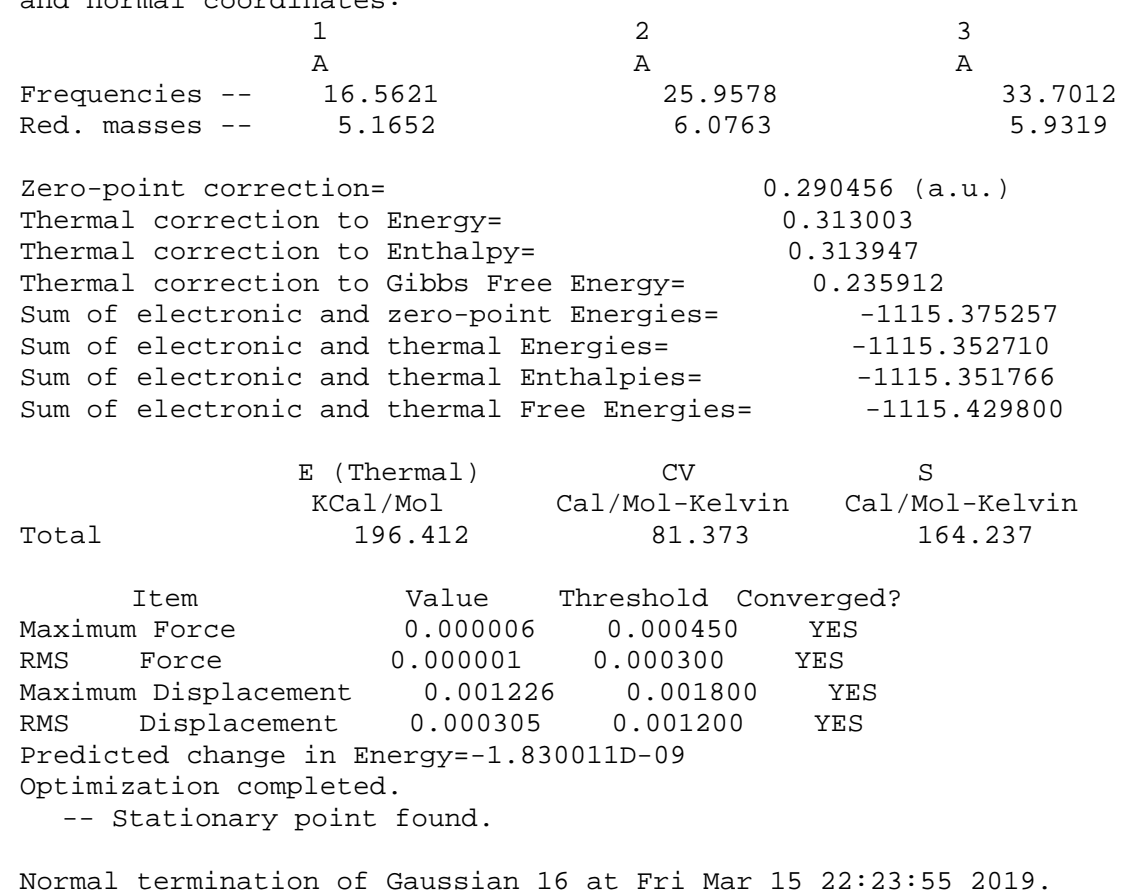

-Figure S5-8, TS2b(C8)W1

degua-oh8w1.higha. $\log$

Stoichiometry C10H16N506(2)

Standard orientation:

\begin{tabular}{|c|c|c|c|c|c|}
\hline \multirow{2}{*}{$\begin{array}{l}\text { Center } \\
\text { Number }\end{array}$} & Atomic & Atomic & \multicolumn{3}{|c|}{ Coordinates (Angstroms) } \\
\hline & Number & Type & $x$ & $\mathrm{Y}$ & Z \\
\hline 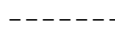 & & & & & \\
\hline 1 & 1 & $\odot$ & -3.615775 & 1.219687 & 1.901706 \\
\hline 2 & 8 & $\odot$ & -3.287448 & 1.575254 & 1.071572 \\
\hline 3 & 8 & $\odot$ & -3.595160 & -2.647443 & -0.023934 \\
\hline 4 & 6 & $\odot$ & -3.491248 & -0.318964 & -0.502268 \\
\hline 5 & 6 & $\odot$ & -3.294561 & -1.390718 & $\odot .573815$ \\
\hline 6 & 1 & $\odot$ & -3.431294 & -3.338994 & $\odot .624547$ \\
\hline 7 & 6 & $\odot$ & -4.031608 & 1.014627 & $-\odot .0 \odot 2646$ \\
\hline
\end{tabular}




\begin{tabular}{|c|c|c|c|c|c|}
\hline 8 & 6 & 0 & -1.814394 & -1.253372 & ๑.914851 \\
\hline 9 & 6 & 0 & -1.206814 & -0.843266 & -0.430344 \\
\hline 10 & 8 & 0 & -2.208511 & -0.137668 & -1.122233 \\
\hline 11 & 1 & $\odot$ & -3.980931 & 1.734992 & -0.827805 \\
\hline 12 & 1 & $\Theta$ & -5.088942 & $\odot .885848$ & $\odot .272711$ \\
\hline 13 & 1 & 0 & -4.183039 & -0.697162 & -1.267297 \\
\hline 14 & 1 & $\odot$ & -3.942306 & -1.221444 & 1.445089 \\
\hline 15 & 1 & $\odot$ & -1.374574 & -2.178096 & 1.301677 \\
\hline 16 & 1 & $\odot$ & -1.670130 & -0.456307 & 1.652022 \\
\hline 17 & 1 & $\odot$ & -0.887920 & -1.720093 & -1.008538 \\
\hline 18 & 7 & 0 & -0.030214 & 0.003238 & -0.306242 \\
\hline 19 & 6 & $\odot$ & -0.069279 & 1.441643 & 0.052996 \\
\hline 20 & 7 & $\odot$ & 1.313834 & 1.670538 & $\odot .528080$ \\
\hline 21 & 6 & $\odot$ & $2.0474 \odot 2$ & $\odot .574788$ & $\odot .299211$ \\
\hline 22 & 6 & 0 & 1.207310 & -0.481415 & -0.164290 \\
\hline 23 & 7 & $\odot$ & 1.591425 & -1.734997 & -0.394501 \\
\hline 24 & 6 & 0 & 2.893939 & -1.958523 & -0.211594 \\
\hline 25 & 7 & $\theta$ & 3.781476 & -0.996869 & 0.181318 \\
\hline 26 & 6 & $\odot$ & 3.449560 & $\odot .336764$ & $\odot .479106$ \\
\hline 27 & 7 & 0 & 3.360889 & -3.188143 & -0.426443 \\
\hline 28 & 8 & $\odot$ & 4.302648 & 1.133474 & 0.845969 \\
\hline 29 & 1 & $\odot$ & -0.783882 & 1.569281 & $\odot .881388$ \\
\hline 30 & 1 & $\odot$ & 2.713406 & -3.900535 & -0.727818 \\
\hline 31 & 1 & $\odot$ & 4.333998 & -3.428954 & -0.319676 \\
\hline 32 & 1 & $\odot$ & 4.760170 & -1.236276 & $\odot .294199$ \\
\hline 33 & 8 & 0 & -0.342776 & 2.265648 & -0.998540 \\
\hline 34 & 1 & $\odot$ & $\odot .328661$ & 3.129203 & $-\odot .885855$ \\
\hline 35 & 8 & $\odot$ & 1.261414 & 4.030399 & $-\odot .476276$ \\
\hline 36 & 1 & 0 & 1.620997 & 2.676753 & 0.340187 \\
\hline 37 & 1 & $\odot$ & 1.896500 & 4.199029 & -1.177492 \\
\hline
\end{tabular}

Standard basis: Aug-CC-pVDZ (6D, 7F)

669 basis functions

80 alpha electrons 79 beta electrons

nuclear repulsion energy 1862.3464764921 Hartrees.

NAtoms $=37$ NActive $=37$

Nuclear repulsion after empirical dispersion term = 1862.3174973320 Hartrees.

Force inversion solution in PCM.

SCF Done: $E($ UWB97XD $)=-1115.64331415$ A.U. after 2 cycles NFock $=2$ Conv $=0.64 \mathrm{D}-09 \quad-\mathrm{V} / \mathrm{T}=2.0071$

$\langle\mathrm{SX}\rangle=0.0000\langle\mathrm{~S} y\rangle=0.0000\langle\mathrm{Sz}\rangle=0.5000$

$\left\langle S^{* *} 2>=0.7580 \mathrm{~S}=0.5040\right.$

$<$ L.S $>=0.000000000000 \mathrm{E}+00$

Annihilation of the first spin contaminant:

$\mathrm{S}^{* *} 2$ before annihilation 0.7580 , after 0.7500

Harmonic frequencies $\left(\mathrm{cm}^{* *}-1\right)$, IR intensities (KM/Mole), Raman activities ( $\left.A^{* *} 4 / A M U\right)$, depolarization ratios for plane and unpolarized incident light, reduced masses (AMU), force constants (mDyne/A), and normal coordinates:

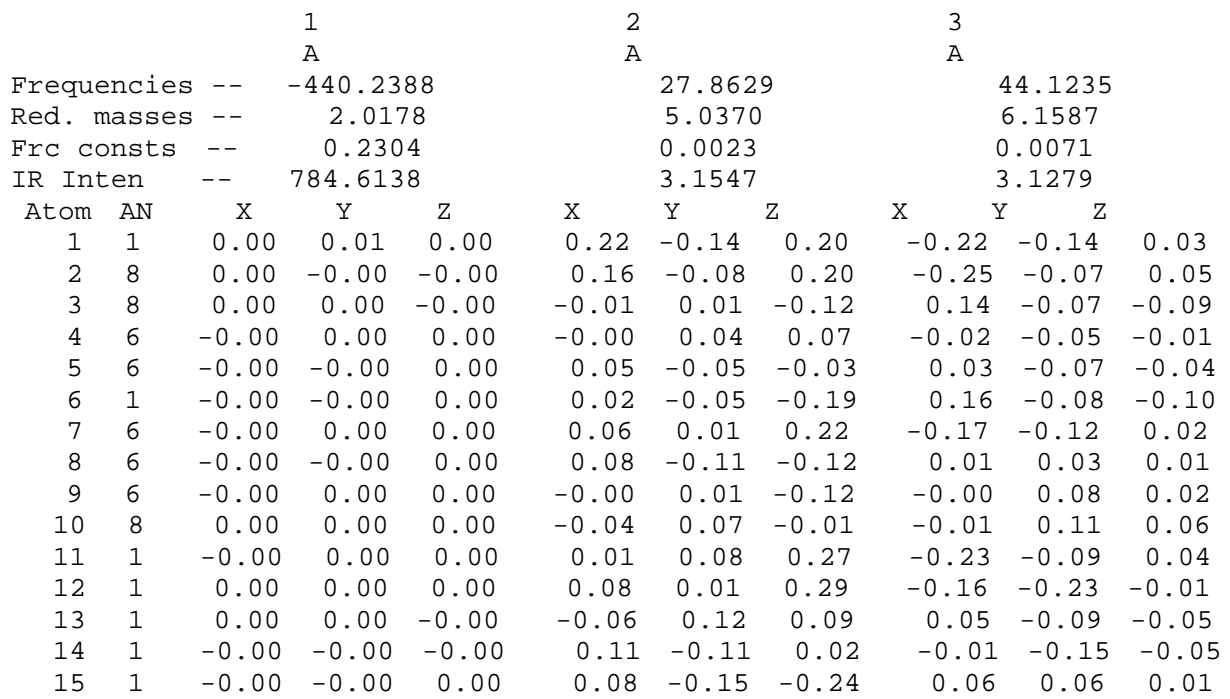




\begin{tabular}{|c|c|c|c|c|}
\hline 16 & 1 & $-\odot . \odot \odot$ & -0.00 & 0.01 \\
\hline 17 & 1 & 0.01 & 0.01 & $\odot . \odot \odot$ \\
\hline 18 & 7 & $-\odot . \odot \odot$ & 0.01 & $\odot . \odot \odot$ \\
\hline 19 & 6 & 0.02 & -0.06 & $\odot . \odot 4$ \\
\hline 20 & 7 & -0.01 & 0.06 & $-\odot .06$ \\
\hline 21 & 6 & -0.01 & 0.03 & -0.01 \\
\hline 22 & 6 & 0.01 & 0.01 & $\odot . \odot \odot$ \\
\hline 3 & 7 & $-0.0 \odot$ & 0.01 & $\odot . \odot \odot$ \\
\hline 24 & 6 & $\odot . \odot \odot$ & $\odot .0 \odot$ & $\odot .0 \odot$ \\
\hline 5 & 7 & -0.01 & 0.01 & $0.0 \odot$ \\
\hline 26 & 6 & -0.01 & 0.01 & $\odot . \odot \odot$ \\
\hline 27 & 7 & $-0.0 \odot$ & 0.01 & $\odot . \odot \odot$ \\
\hline 8 & 8 & $\odot . \odot \odot$ & $\odot . \odot \odot$ & $\odot . \odot \odot$ \\
\hline 29 & 1 & $\odot .0 \odot$ & 0.01 & 0.01 \\
\hline 30 & 1 & -0.00 & 0.01 & $\odot . \odot \odot$ \\
\hline 31 & 1 & $-\odot . \odot \odot$ & $\odot .0 \odot$ & $\odot . \odot \odot$ \\
\hline 32 & 1 & -0.01 & 0.01 & 0.01 \\
\hline 33 & 8 & -0.11 & $-\odot . \odot 9$ & -0.06 \\
\hline 34 & 1 & -0.57 & -0.50 & -0.24 \\
\hline 35 & 8 & 0.15 & 0.02 & 0.10 \\
\hline 36 & 1 & -0.15 & 0.22 & -0.36 \\
\hline & 1 & 0.22 & $\odot .09$ & 0.18 \\
\hline
\end{tabular}

$\begin{array}{ccc}0.14 & -0.18 & -0.05 \\ -0.03 & 0.07 & -0.21 \\ -0.01 & 0.02 & -0.10 \\ -0.02 & 0.02 & -0.10 \\ -0.03 & 0.02 & -0.07 \\ -0.03 & 0.02 & -0.02 \\ -0.02 & 0.01 & -0.04 \\ -0.02 & 0.00 & 0.01 \\ -0.03 & -0.00 & 0.08 \\ -0.04 & -0.00 & 0.10 \\ -0.04 & 0.01 & 0.05 \\ -0.03 & -0.01 & 0.13 \\ -0.05 & 0.01 & 0.08 \\ -0.04 & 0.00 & -0.11 \\ -0.02 & -0.01 & 0.11 \\ -0.04 & -0.02 & 0.18 \\ -0.05 & -0.01 & 0.15 \\ -0.01 & 0.02 & -0.10 \\ 0.01 & 0.01 & -0.11 \\ 0.02 & 0.00 & -0.12 \\ -0.02 & 0.02 & -0.08 \\ 0.03 & -0.03 & -0.12\end{array}$

$\begin{array}{ccc}-0.07 & 0.04 & 0.01 \\ -0.01 & 0.10 & -0.01 \\ 0.00 & 0.07 & 0.04 \\ 0.03 & 0.07 & 0.06 \\ 0.05 & 0.04 & 0.03 \\ 0.02 & 0.02 & 0.02 \\ -0.01 & 0.04 & 0.03 \\ -0.04 & 0.03 & 0.02 \\ -0.04 & 0.00 & 0.00 \\ -0.02 & -0.02 & -0.01 \\ 0.01 & -0.01 & 0.00 \\ -0.07 & -0.01 & -0.01 \\ 0.04 & -0.03 & -0.01 \\ 0.06 & 0.05 & 0.09 \\ -0.09 & 0.01 & -0.01 \\ -0.08 & -0.03 & -0.03 \\ -0.02 & -0.04 & -0.02 \\ -0.01 & 0.09 & 0.10 \\ 0.13 & 0.00 & -0.05 \\ 0.28 & -0.07 & -0.23 \\ 0.09 & 0.02 & -0.07 \\ 0.29 & -0.31 & -0.27\end{array}$

Zero-point correction=

Thermal correction to Energy=

$$
\begin{gathered}
0.289706 \text { (a.u.) } \\
0.309354 \\
0.310299 \\
0.240491 \\
-1115.353608 \\
-1115.333960 \\
-1115.333016 \\
-1115.402823
\end{gathered}
$$

Sum of electronic and thermal Enthalpies=

Sum of electronic and thermal Free Energies=

$\mathrm{S}$

Cal/Mol-Kelvin Cal/Mol-Kelvin 75.099 146.923$$
75.099
$$

Total

$$
\begin{gathered}
\text { E (Thermal) } \\
\text { KCal/Mol } \\
194.123
\end{gathered}
$$

\begin{tabular}{|c|c|c|c|c|c|}
\hline \multirow{2}{*}{$\begin{array}{l}\text { Center } \\
\text { Number }\end{array}$} & \multirow{2}{*}{$\begin{array}{l}\text { Atomic } \\
\text { Number }\end{array}$} & \multirow{2}{*}{$\begin{array}{c}\text { Atomic } \\
\text { Type }\end{array}$} & \multicolumn{3}{|c|}{ Coordinates (Angstroms) } \\
\hline & & & $\mathrm{x}$ & $\mathrm{Y}$ & Z \\
\hline & & -- & 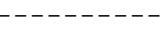 & ------ & ------- \\
\hline 1 & 1 & $\odot$ & -3.749747 & 1.181815 & 1.841511 \\
\hline 2 & 8 & $\odot$ & -3.411295 & 1.555677 & 1.023621 \\
\hline 3 & 8 & $\odot$ & -3.548684 & -2.685447 & -0.013666 \\
\hline 4 & 6 & $\odot$ & -3.486292 & -0.362930 & -0.535622 \\
\hline 5 & 6 & $\odot$ & -3.293706 & -1.409764 & $\odot .566193$ \\
\hline 6 & 1 & $\odot$ & -3.382106 & -3.359649 & $\odot .652139$ \\
\hline 7 & 6 & $\odot$ & -4.087724 & 0.958992 & -0.074949 \\
\hline 8 & 6 & $\odot$ & -1.827563 & -1.228778 & 0.942308 \\
\hline 9 & 6 & $\odot$ & -1.195464 & -0.840301 & -0.397763 \\
\hline 10 & 8 & $\odot$ & -2.191621 & -0.149197 & -1.116030 \\
\hline 11 & 1 & $\odot$ & -4.023620 & 1.672070 & -0.905568 \\
\hline 12 & 1 & $\odot$ & -5.151975 & 0. 799377 & ๑. 155304 \\
\hline 13 & 1 & $\odot$ & -4.142340 & -0.776076 & -1.314601 \\
\hline 14 & 1 & $\odot$ & -3.969180 & -1.240411 & 1.416271 \\
\hline 15 & 1 & $\odot$ & -1.376372 & -2.131567 & 1.366543 \\
\hline 16 & 1 & $\odot$ & -1.721774 & -0.406084 & 1.657520 \\
\hline 17 & 1 & $\odot$ & -0.875524 & -1.730587 & -0.955485 \\
\hline 18 & 7 & $\odot$ & $-\odot .020604$ & 0.000043 & -0.262795 \\
\hline 19 & 6 & $\odot$ & $-\odot .060996$ & 1.500508 & 0.067296 \\
\hline 20 & 7 & $\odot$ & 1.370811 & 1.635090 & $\odot .561687$ \\
\hline 21 & 6 & $\odot$ & 2.081712 & 0.535794 & 0.307836 \\
\hline
\end{tabular}

\begin{tabular}{lccc}
\multicolumn{1}{c}{ Item } & Value & Threshold & \multicolumn{2}{c}{ Converged? } \\
Maximum Force & $0.0 \odot \odot \odot 27$ & $\odot .0 \odot \odot 45 \odot$ & YES \\
RMS Force & $0.000 \odot \odot 4$ & $0.00 \odot 30 \odot$ & YES \\
Maximum Displacement & 0.002047 & $0.00180 \odot$ & NO \\
RMS Displacement & $0.00 \odot 259$ & 0.001200 & YES
\end{tabular}

Predicted change in Energy $=-1.344334 \mathrm{D}-08$

Normal termination of Gaussian 16 at Tue Mar 12 14:30:15 2019.

-Figure S5-9, Int3(C8)W1_........

degua-oh8w1.rev . higha. $\log$

Stoichiometry C10H16N506(2)

Standard orientation 


$\begin{array}{rrrrrr}22 & 6 & \odot & 1.204731 & -0.498088 & -0.148175 \\ 23 & 7 & 0 & 1.574087 & -1.760038 & -0.390815 \\ 24 & 6 & 0 & 2.872528 & -2.005922 & -0.234844 \\ 25 & 7 & 0 & 3.786770 & -1.063537 & 0.148025 \\ 26 & 6 & 0 & 3.480909 & 0.271576 & 0.458648 \\ 27 & 7 & 0 & 3.316942 & -3.243228 & -0.465971 \\ 28 & 8 & 0 & 4.351934 & 1.055879 & 0.816179 \\ 29 & 1 & 0 & -0.725720 & 1.591972 & 0.948663 \\ 30 & 1 & 0 & 2.652683 & -3.942601 & -0.760574 \\ 31 & 1 & 0 & 4.288034 & -3.499752 & -0.382426 \\ 32 & 1 & 0 & 4.762454 & -1.321172 & 0.243973 \\ 33 & 8 & 0 & -0.316074 & 2.298014 & -0.928349 \\ 34 & 1 & 0 & 0.601291 & 3.689415 & -0.744915 \\ 35 & 8 & 0 & 1.224977 & 4.411886 & -0.465142 \\ 36 & 1 & 0 & 1.768970 & 2.571239 & 0.604956 \\ 37 & 1 & 0 & 1.846191 & 4.502696 & -1.192710 \\ - & 1 & 0 & 0 & 0\end{array}$

Standard basis: Aug-CC-pVDZ (6D, 7F)

669 basis functions

80 alpha electrons $\quad 79$ beta electrons

nuclear repulsion energy 1845.2523934007 Hartrees.

NAtoms $=37$ NActive $=37$

Force inversion solution in PCM.

SCF Done: $E(U W B 97 X D)=-1115.65572313$ A.U. after 1 cycles NFock $=1$ Conv $=0.48 \mathrm{D}-08 \quad-\mathrm{V} / \mathrm{T}=2.0071$

$\left\langle\right.$ SX $>=0.0000\langle$ Sy $\rangle=0.0000\left\langle S z>=0.5000\left\langle S^{* *} 2\right\rangle=0.7583 S=0.5042\right.$

$<$ L.S $>=0.000000000000 \mathrm{E}+00$

Annihilation of the first spin contaminant:

$\mathrm{S}^{* * 2}$ before annihilation 0.7583 , after 0.7501

Harmonic frequencies $\left(\mathrm{cm}^{* *}-1\right)$, IR intensities (KM/Mole), Raman activities ( $\left.A^{* *} 4 / \mathrm{AMU}\right)$, depolarization ratios for plane and unpo incident light, reduced masses (AMU), force constants (mDyne/A), and normal coordinates:

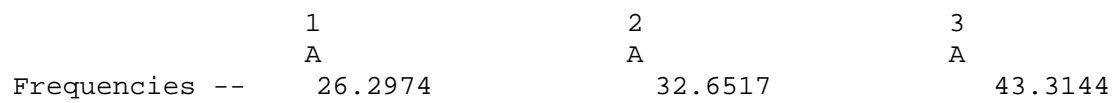

Zero-point correction=

Thermal correction to Energy=

Thermal correction to Enthalpy=

0.292894 (a.u.)

Thermal correction to Gibbs Free Energy=
Sum of electronic and zero-point Energie

Sum of electronic and zero-point Energies=

Sum of electronic and thermal Energies=

0.313891

0.314835

0.241647

$-1115.362829$

$-1115.341832$

$-1115.340888$

Sum of electronic and thermal Free Energies=

$-1115.414076$

Total

$$
\begin{gathered}
\text { E (Thermal) } \\
\text { KCal/Mol }
\end{gathered}
$$

$\mathrm{CV}$

\section{$\mathrm{S}$}

Cal/Mol-Kelvin Cal/Mol-Kelvin $78.681 \quad 154.037$

\begin{tabular}{lccc}
\multicolumn{1}{c}{ Item } & Value & Threshold Converged? \\
Maximum Force & 0.000321 & 0.000450 & YES \\
RMS Force & 0.000036 & $0.00030 \odot$ & YES \\
Maximum Displacement & 0.003280 & 0.001800 & NO \\
RMS Displacement & 0.000350 & 0.001200 & YES \\
Predicted change in Energy=-2.221902D-08 &
\end{tabular}

列

Normal termination of Gaussian 16 at Fri Mar 15 19:54:24 2019.

-....... Figure S5-10, TS3b(C8)W1

degua-oh8w1a.higha. log

Stoichiometry C10H16N506(2)

\begin{tabular}{|c|c|c|c|c|c|}
\hline \multirow{2}{*}{$\begin{array}{l}\text { Center } \\
\text { Number }\end{array}$} & \multirow{2}{*}{$\begin{array}{l}\text { Atomic } \\
\text { Number }\end{array}$} & \multirow{2}{*}{$\begin{array}{c}\text { Atomic } \\
\text { Type }\end{array}$} & \multicolumn{3}{|c|}{ Coordinates (Angstroms) } \\
\hline & & & $\mathrm{x}$ & $\mathrm{Y}$ & Z \\
\hline 1 & 1 & $\odot$ & -3.289487 & $\odot .900281$ & 2.249399 \\
\hline 2 & 8 & 0 & -2.985916 & 1.435833 & 1.511495 \\
\hline
\end{tabular}

Standard orientation: 


\begin{tabular}{|c|c|c|c|c|c|}
\hline 3 & 8 & 0 & -3.883737 & -2.336481 & -0.525900 \\
\hline 4 & 6 & $\odot$ & -3.530492 & 0.016487 & $-\odot .444567$ \\
\hline 5 & 6 & $\odot$ & -3.410202 & -1.298480 & $\odot .328184$ \\
\hline 6 & 1 & 0 & -3.740440 & -3.178837 & -0.083911 \\
\hline 7 & 6 & 0 & -3.858667 & 1.238976 & 0.405605 \\
\hline 8 & 6 & $\odot$ & -1.911092 & -1.399011 & $\odot .585986$ \\
\hline 9 & 6 & $\odot$ & -1.298999 & -0.709010 & -0.641754 \\
\hline 10 & 8 & 0 & -2.284632 & 0.188102 & -1.126360 \\
\hline 11 & 1 & $\odot$ & -3.762636 & 2.129621 & -0.226770 \\
\hline 12 & 1 & 0 & -4.905499 & 1.167991 & 0.738400 \\
\hline 13 & 1 & 0 & -4.319509 & $-\odot .078769$ & -1.204043 \\
\hline 14 & 1 & 0 & -3.995541 & -1.282425 & 1.258768 \\
\hline 15 & 1 & 0 & -1.564279 & -2.431737 & 0.692866 \\
\hline 16 & 1 & 0 & -1.643212 & -0.840341 & 1.489330 \\
\hline 17 & 1 & $\odot$ & -1.068005 & -1.441250 & -1.430986 \\
\hline 18 & 7 & 0 & -0.105230 & 0.036492 & -0.343703 \\
\hline 19 & 6 & 0 & $\odot .132671$ & 1.869872 & 0.314578 \\
\hline 20 & 7 & 0 & 1.559826 & 1.626646 & $\odot .517842$ \\
\hline 21 & 6 & 0 & 2.030743 & $\odot .38290 \odot$ & $\odot .316456$ \\
\hline 22 & 6 & 0 & 1.040709 & $-\odot .552658$ & -0.146328 \\
\hline 23 & 7 & $\odot$ & 1.313235 & -1.872582 & -0.334056 \\
\hline 24 & 6 & 0 & 2.563965 & -2.242721 & -0.139853 \\
\hline 25 & 7 & 0 & 3.571434 & -1.388549 & 0.236136 \\
\hline 26 & 6 & 0 & 3.391559 & $-\odot .027508$ & 0.497877 \\
\hline 27 & 7 & 0 & 2.897293 & -3.529109 & -0.317514 \\
\hline 28 & 8 & $\odot$ & 4.329873 & 0.686849 & $\odot .841152$ \\
\hline 29 & 1 & $\odot$ & -0.474376 & 1.631289 & 1.198377 \\
\hline 30 & 1 & 0 & 2.177962 & -4.172843 & -0.607699 \\
\hline 31 & 1 & $\odot$ & 3.836363 & -3.873005 & -0.195279 \\
\hline 32 & 1 & 0 & 4.513266 & -1.740029 & 0.359938 \\
\hline 33 & 8 & 0 & -0.188969 & 2.788240 & -0.452179 \\
\hline 34 & 1 & 0 & 1.231717 & 3.730498 & -1.025183 \\
\hline 35 & 8 & 0 & 2.108358 & 4.165894 & -1.062567 \\
\hline 36 & 1 & 0 & 2.174561 & 2.430651 & 0.404767 \\
\hline 37 & 1 & 0 & 2.512244 & 3.838044 & -1.871138 \\
\hline
\end{tabular}

Standard basis: Aug-CC-pVDZ (6D, 7F)

669 basis functions

80 alpha electrons $\quad 79$ beta electrons

nuclear repulsion energy 1833.3259255863 Hartrees.

NAtoms $=37$ NActive $=37$

Nuclear repulsion after empirical dispersion term $=$ 1833.2975760127 Hartrees.

Force inversion solution in PCM.

SCF Done: $E($ UWB97XD $)=-1115.64659505$ A.U. after 2 cycles NFock $=2$ Conv $=0.76 \mathrm{D}-09 \quad-\mathrm{V} / \mathrm{T}=2.0071$

$\langle\mathrm{S} X>=0.0000<\mathrm{Sy}\rangle=0.0000<\mathrm{Sz}\rangle=0.5000$

$<S^{* *} 2>=0.7631 \mathrm{~S}=0.5065$

$<$ L.S $>=0.000000000000 \mathrm{E}+00$

Annihilation of the first spin contaminant:

$\mathrm{S}^{* * 2}$ before annihilation 0.7631 , after 0.7501

Harmonic frequencies $\left(\mathrm{cm}^{* *}-1\right)$, IR intensities (KM/Mole), Raman activities $\left(A^{* *} 4 / A M U\right)$, depolarization ratios for plane and unp incident light, reduced masses (AMU), force constants (mDyne/A), and normal coordinates:

\begin{tabular}{|c|c|c|c|c|c|c|c|c|c|c|}
\hline \multicolumn{11}{|c|}{1} \\
\hline \multicolumn{2}{|c|}{ Frequencies } & - - & \multicolumn{2}{|c|}{-205.3240} & \multicolumn{3}{|c|}{9.5838} & \multicolumn{3}{|c|}{22.2850} \\
\hline \multicolumn{2}{|c|}{ Red. masses } & - - & \multicolumn{2}{|c|}{7.7311} & \multicolumn{3}{|c|}{6.4913} & \multicolumn{3}{|c|}{5.2171} \\
\hline \multirow{2}{*}{\multicolumn{2}{|c|}{$\begin{array}{l}\text { Frc consts } \\
\text { IR Inten }\end{array}$}} & - - & \multirow{2}{*}{\multicolumn{2}{|c|}{$\begin{array}{r}0.1920 \\
181.6732\end{array}$}} & \multirow{2}{*}{\multicolumn{3}{|c|}{$\begin{array}{l}\odot .0004 \\
8.2985\end{array}$}} & \multirow{2}{*}{\multicolumn{3}{|c|}{$\begin{array}{l}0.0015 \\
0.7027\end{array}$}} \\
\hline & & - - & & & & & & & & \\
\hline Atom & AN & $x$ & $\mathrm{Y}$ & Z & $\mathrm{x}$ & $\mathrm{Y}$ & Z & $\mathrm{x}$ & Z & \\
\hline 1 & 1 & $\odot . \odot 4$ & $-\odot .03$ & $-\odot .02$ & 0.03 & $\odot .07$ & $-\odot . \odot 3$ & 0.23 & -0.22 & 0.14 \\
\hline 2 & 8 & 0.03 & $-\odot .01$ & -0.02 & 0.01 & 0.05 & $-\odot .06$ & $\odot .20$ & -0.16 & 0.17 \\
\hline 3 & 8 & -0.05 & $-\odot .02$ & 0.01 & -0.00 & -0.03 & 0.10 & -0.05 & 0.05 & -0.10 \\
\hline 4 & 6 & -0.01 & -0.03 & $-0.0 \odot$ & -0.01 & -0.03 & 0.01 & 0.01 & 0.03 & 0.08 \\
\hline 5 & 6 & -0.03 & -0.03 & 0.01 & 0.01 & $\odot . \odot \odot$ & 0.06 & 0.02 & -0.03 & -0.04 \\
\hline 6 & 1 & $-\odot .04$ & -0.03 & -0.00 & 0.01 & $-\odot .01$ & 0.13 & -0.05 & $\odot .01$ & $-\odot .19$ \\
\hline 7 & 6 & 0.02 & -0.01 & -0.01 & $-0.0 \odot$ & 0.00 & -0.04 & 0.12 & -0.02 & 0.21 \\
\hline 8 & 6 & $-\odot . \odot 4$ & -0.07 & -0.01 & 0.01 & 0.02 & $\odot . \odot 4$ & $\odot .04$ & -0.10 & -0.14 \\
\hline 9 & 6 & -0.04 & -0.17 & -0.07 & -0.01 & -0.03 & $-\odot . \odot \odot$ & -0.01 & 0.02 & -0.10 \\
\hline 10 & 8 & -0.03 & -0.08 & -0.03 & $-\odot .02$ & -0.05 & $-\odot .02$ & -0.03 & 0.04 & -0.01 \\
\hline
\end{tabular}




$\begin{array}{rrrrr}11 & 1 & 0.04 & -0.02 & -0.02 \\ 12 & 1 & 0.02 & 0.01 & -0.01 \\ 13 & 1 & -0.04 & -0.02 & 0.02 \\ 14 & 1 & -0.03 & -0.02 & 0.01 \\ 15 & 1 & -0.07 & -0.08 & 0.04 \\ 16 & 1 & 0.01 & -0.02 & -0.06 \\ 17 & 1 & -0.17 & -0.20 & -0.08 \\ 18 & 7 & -0.08 & -0.20 & -0.10 \\ 19 & 6 & 0.16 & 0.40 & 0.17 \\ 20 & 7 & 0.06 & 0.09 & -0.10 \\ 21 & 6 & -0.09 & -0.02 & -0.02 \\ 22 & 6 & -0.07 & -0.08 & -0.02 \\ 23 & 7 & 0.02 & -0.09 & 0.04 \\ 24 & 6 & 0.01 & -0.03 & 0.02 \\ 25 & 7 & -0.01 & 0.01 & 0.01 \\ 26 & 6 & -0.06 & 0.00 & -0.01 \\ 27 & 7 & 0.07 & -0.03 & 0.02 \\ 28 & 8 & -0.08 & 0.03 & -0.02 \\ 29 & 1 & 0.08 & 0.01 & -0.01 \\ 30 & 1 & 0.10 & -0.05 & 0.03 \\ 31 & 1 & 0.09 & 0.01 & 0.01 \\ 32 & 1 & 0.01 & 0.05 & 0.01 \\ 33 & 8 & 0.06 & 0.29 & 0.19 \\ 34 & 1 & 0.04 & 0.20 & -0.07 \\ 35 & 8 & 0.11 & 0.03 & -0.02 \\ 36 & 1 & 0.18 & -0.04 & -0.46 \\ 37 & 1 & 0.01 & 0.14 & -0.11\end{array}$

$\begin{array}{ccc}-0.01 & -0.02 & -0.07 \\ 0.00 & 0.01 & -0.02 \\ -0.02 & -0.06 & 0.02 \\ 0.02 & 0.04 & 0.06 \\ 0.02 & 0.02 & 0.07 \\ 0.02 & 0.05 & 0.01 \\ -0.03 & -0.06 & 0.02 \\ -0.00 & -0.02 & -0.06 \\ 0.01 & -0.00 & -0.14 \\ 0.01 & 0.01 & -0.15 \\ 0.00 & -0.00 & -0.09 \\ -0.00 & -0.02 & -0.03 \\ -0.01 & -0.03 & 0.03 \\ -0.02 & -0.03 & 0.04 \\ -0.01 & -0.02 & -0.00 \\ 0.00 & -0.00 & -0.07 \\ -0.02 & -0.04 & 0.10 \\ 0.01 & 0.01 & -0.11 \\ 0.02 & 0.03 & -0.12 \\ -0.03 & -0.05 & 0.13 \\ -0.02 & -0.04 & 0.10 \\ -0.01 & -0.02 & 0.01 \\ -0.01 & -0.03 & -0.16 \\ 0.02 & 0.18 & 0.16 \\ 0.04 & 0.16 & 0.45 \\ -0.00 & 0.02 & -0.23 \\ 0.12 & 0.51 & 0.36\end{array}$

$\begin{array}{ccc}0.12 & 0.03 & 0.28 \\ 0.14 & -0.01 & 0.27 \\ -0.05 & 0.13 & 0.13 \\ 0.08 & -0.10 & -0.00 \\ 0.02 & -0.12 & -0.26 \\ 0.11 & -0.20 & -0.09 \\ -0.02 & 0.09 & -0.17 \\ -0.01 & 0.02 & -0.08 \\ -0.02 & 0.03 & -0.12 \\ -0.03 & 0.03 & -0.07 \\ -0.03 & 0.02 & -0.02 \\ -0.02 & 0.02 & -0.03 \\ -0.02 & 0.01 & 0.01 \\ -0.03 & 0.00 & 0.07 \\ -0.04 & 0.01 & 0.09 \\ -0.04 & 0.01 & 0.05 \\ -0.04 & -0.00 & 0.12 \\ -0.05 & 0.01 & 0.07 \\ -0.05 & 0.05 & -0.13 \\ -0.03 & -0.01 & 0.11 \\ -0.04 & -0.01 & 0.16 \\ -0.05 & 0.00 & 0.14 \\ -0.01 & 0.01 & -0.15 \\ 0.01 & 0.02 & -0.08 \\ 0.01 & 0.03 & -0.04 \\ -0.02 & 0.03 & -0.07 \\ 0.04 & 0.05 & -0.03\end{array}$

Zero-point correction=

Thermal correction to Energy=

Thermal correction to Enthalpy=

Thermal correction to Gibbs Free Energy=

Sum of electronic and zero-point Energies=

Sum of electronic and thermal Energies=

Sum of electronic and thermal Enthalpies=

Sum of electronic and thermal Free Energies=

$$
\begin{gathered}
0.290724 \text { (a.u. ) } \\
0.312129 \\
0.313073 \\
0.237540 \\
-1115.355871 \\
-1115.334466 \\
-1115.333522 \\
-1115.409055
\end{gathered}
$$

\begin{tabular}{|c|c|c|c|}
\hline${ }_{\mathrm{KCa}}$ & $\begin{array}{l}\text { lermal) } \\
\text { /Mol } \\
95.864\end{array}$ & $\begin{array}{c}\text { CV } \\
\text { Cal/Mol-Kelvin } \\
78.999\end{array}$ & $\begin{array}{c}\text { S } \\
\text { Cal/Mol-Kelvin } \\
158.972\end{array}$ \\
\hline Item & Value & \multicolumn{2}{|c|}{ Threshold Converged? } \\
\hline Maximum Force & $\odot .0 \odot \odot \odot 14$ & $\odot .000450$ & $\begin{array}{l}\text { erged? } \\
\text { YES }\end{array}$ \\
\hline Force & 0.000002 & 0.000300 & YES \\
\hline Maximum Displacement & 0.003118 & 0.001800 & NO \\
\hline RMS Displacement & $\odot .000488$ & 0.001200 & YES \\
\hline
\end{tabular}

\begin{tabular}{|c|c|c|c|c|c|}
\hline \multirow{2}{*}{$\begin{array}{l}\text { Center } \\
\text { Number }\end{array}$} & Atomic & Atomic & \multicolumn{3}{|c|}{ Coordinates (Angstroms) } \\
\hline & Number & Type & $X$ & $\mathrm{Y}$ & Z \\
\hline & & & ------- - & --- - - - - - & $------\cdot$ \\
\hline 1 & 1 & $\odot$ & 3.434087 & 2.822799 & $-\odot .989561$ \\
\hline 2 & 8 & $\odot$ & 2.897503 & 2.709428 & $-\odot .200389$ \\
\hline 3 & 8 & $\odot$ & 5.087754 & -1.084278 & -0.050140 \\
\hline 4 & 6 & $\odot$ & 3.833391 & 0.702114 & 0.893182 \\
\hline 5 & 6 & $\odot$ & 4.300923 & 0.046211 & $-\odot .408998$ \\
\hline 6 & 1 & $\odot$ & 5.363220 & -1.531558 & -0.856213 \\
\hline 7 & 6 & $\odot$ & 3.715313 & 2.220207 & 0.856587 \\
\hline 8 & 6 & $\odot$ & 2.987994 & -0.351256 & -1.077366 \\
\hline 9 & 6 & $\odot$ & 2.085935 & -0.666997 & ๑. 116520 \\
\hline 10 & 8 & $\odot$ & 2.560994 & ๑. 109726 & 1.198027 \\
\hline 11 & 1 & $\odot$ & 3.247422 & 2.550123 & 1.791783 \\
\hline 12 & 1 & $\odot$ & 4.724457 & 2.654560 & 0.808471 \\
\hline 13 & 1 & $\odot$ & 4.528391 & 0.438048 & 1.701705 \\
\hline 14 & 1 & 0 & 4.890044 & 0.737315 & -1.027325 \\
\hline
\end{tabular}

Normal termination of Gaussian 16 at Mon Mar 11 10:27:42 2019.

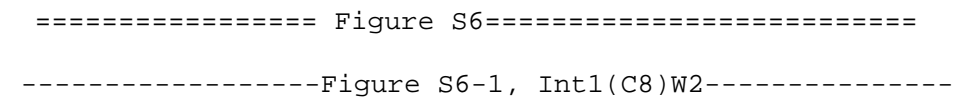

degua-oh8w2b.rev.higha . log

Stoichiometry C10H18N507(2)

Standard orientation: 


\begin{tabular}{|c|c|c|c|c|c|}
\hline 15 & 1 & $\odot$ & 3.093158 & -1.206094 & -1.752309 \\
\hline 16 & 1 & $\odot$ & 2.582369 & ๑. 494801 & -1.642422 \\
\hline 17 & 1 & $\odot$ & 2.106417 & -1.731030 & $\odot .379707$ \\
\hline 18 & 7 & $\odot$ & ๑. 685616 & -0.347393 & -0.106467 \\
\hline 19 & 6 & $\odot$ & 0.160960 & ๑. 898839 & -0.353148 \\
\hline 20 & 7 & $\odot$ & -1.140346 & $\odot .880258$ & -0.487406 \\
\hline 21 & 6 & $\odot$ & -1.496472 & -0.446293 & -0.320786 \\
\hline 22 & 6 & $\odot$ & -0.368315 & -1.220530 & -0.080957 \\
\hline 23 & 7 & $\odot$ & -0.294442 & -2.550838 & 0.130090 \\
\hline 24 & 6 & $\odot$ & -1.470555 & -3.142545 & 0.088825 \\
\hline 25 & 7 & $\odot$ & -2.643913 & -2.465670 & -0.137858 \\
\hline 26 & 6 & $\odot$ & -2.768439 & -1.085714 & -0.351682 \\
\hline 27 & 7 & $\odot$ & -1.552486 & -4.478896 & $\odot .248944$ \\
\hline 28 & 8 & $\odot$ & -3.885953 & -0.594779 & -0.533099 \\
\hline 29 & 1 & $\odot$ & 0.801590 & 1.773736 & -0.409291 \\
\hline 30 & 1 & $\odot$ & -0.714246 & -4.971540 & 0.514938 \\
\hline 31 & 1 & $\odot$ & -2.435920 & -4.949081 & 0.364734 \\
\hline 32 & 1 & $\odot$ & -3.517288 & -2.979902 & -0.153066 \\
\hline 33 & 8 & $\odot$ & -4.341069 & 3.890646 & 0.559640 \\
\hline 34 & 1 & $\odot$ & -3.668173 & 3.641460 & -0.140546 \\
\hline 35 & 8 & $\odot$ & -2.588293 & 3. 098682 & -1.290324 \\
\hline 36 & 1 & $\odot$ & -2.082428 & 2.300420 & -0.997513 \\
\hline 37 & 1 & $\odot$ & -1.924976 & 3.735067 & -1.572758 \\
\hline 38 & 8 & $\odot$ & -5.016261 & 1.183452 & 1.374649 \\
\hline 39 & 1 & $\odot$ & -4.606046 & 0.635329 & 0.685752 \\
\hline 40 & 1 & $\odot$ & -4.804885 & 2.096092 & 1.122830 \\
\hline
\end{tabular}

Standard basis: Aug-CC-pVDZ (6D, 7F)

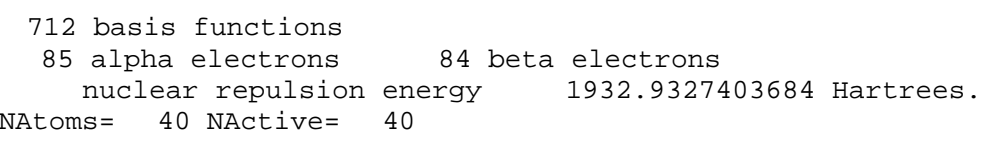

Force inversion solution in PCM.

SCF Done: $E(U W B 97 X D)=-1192.06638938$ A.U. after 2 cycles NFock $=2$ Conv $=0.57 \mathrm{D}-09 \quad-\mathrm{V} / \mathrm{T}=2.0070$

$\left\langle S x>=0.0000\left\langle\right.\right.$ Sy $>=0.0000\left\langle\right.$ Sz $>=0.5000\left\langle S^{* *} 2>=0.7528 \quad S=0.5014\right.$

$<$ L.S $>=0.000000000000 \mathrm{E}+00$

Annihilation of the first spin contaminant:

$\mathrm{S}^{* * 2}$ before annihilation 0.7528 , after 0.7500

DoSCS $=F$ DFT $=T$ ScalE2 $(S S, 0 S)=1.0000001 .000000$

Range of M.O.s used for correlation: 1712

Harmonic frequencies $\left(\mathrm{cm}^{* *}-1\right)$, IR intensities (KM/Mole), Raman activities ( $\left.A^{* *} 4 / \mathrm{AMU}\right)$, depolarization ratios for plane and unpo incident light, reduced masses (AMU), force constants (mDyne/A), and normal coordinates:

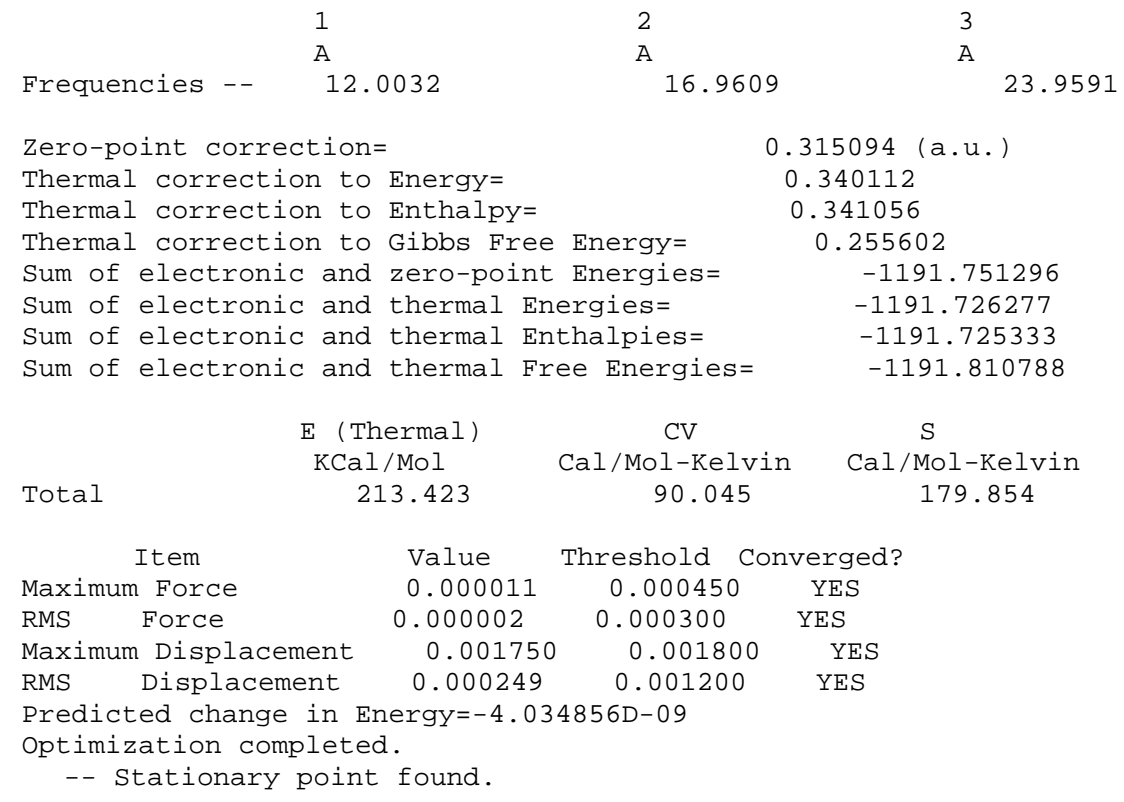

Normal termination of Gaussian 16 at Sun Mar 17 04:42:18 2019. 


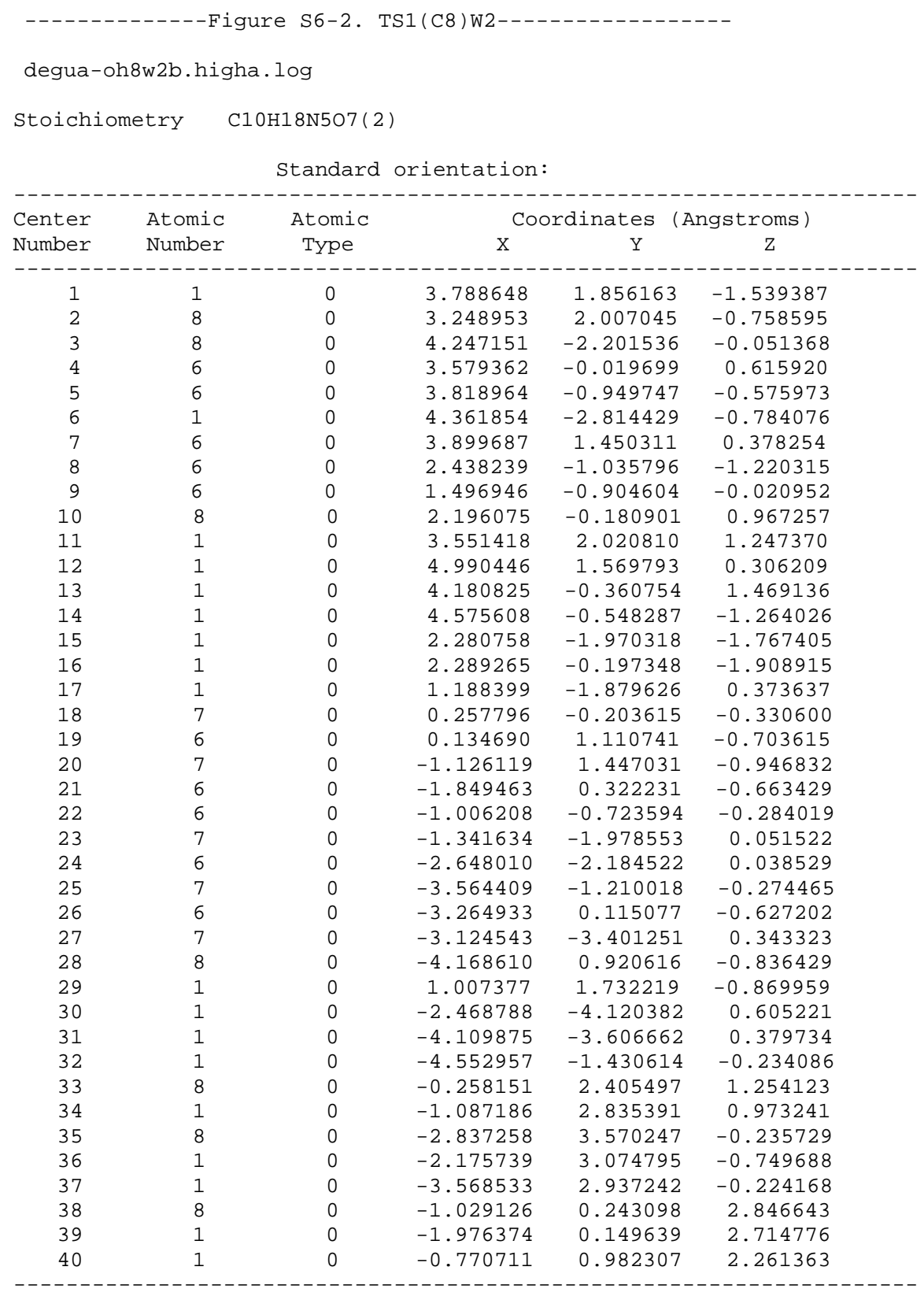

Standard basis: Aug-CC-pVDZ (6D, 7F)

712 basis functions

85 alpha electrons 84 beta electrons

nuclear repulsion energy 2039.8946115183 Hartrees.

NAtoms $=40$ NActive $=40$

Force inversion solution in PCM.

SCF Done: $E($ UWB97XD $)=-1192.06210838$ A.U. after 2 cycles

$$
\text { NFock }=2 \text { Conv }=0.73 \mathrm{D}-09 \quad-\mathrm{V} / \mathrm{T}=2.0070
$$

$\left\langle\mathrm{S} x>=0.0000<\mathrm{S} y>=0.0000<\mathrm{S} z>=0.5000<\mathrm{S} *{ }^{*} 2>=0.7612 \mathrm{~S}=0.5056\right.$

$<$ L.S $>=0.000000000000 \mathrm{E}+00$

Annihilation of the first spin contaminant:

$\mathrm{S}^{* *} 2$ before annihilation 0.7612 , after 0.7501

Harmonic frequencies $\left(\mathrm{cm}^{*}-1\right)$, IR intensities (KM/Mole), Raman activities ( $\left.A^{* *} 4 / A M U\right)$, depolarization ratios for plane and unp incident light, reduced masses (AMU), force constants (mDyne/A), and normal coordinates:

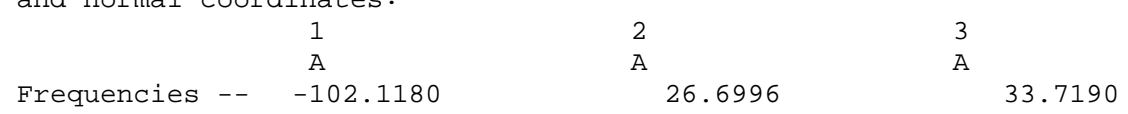




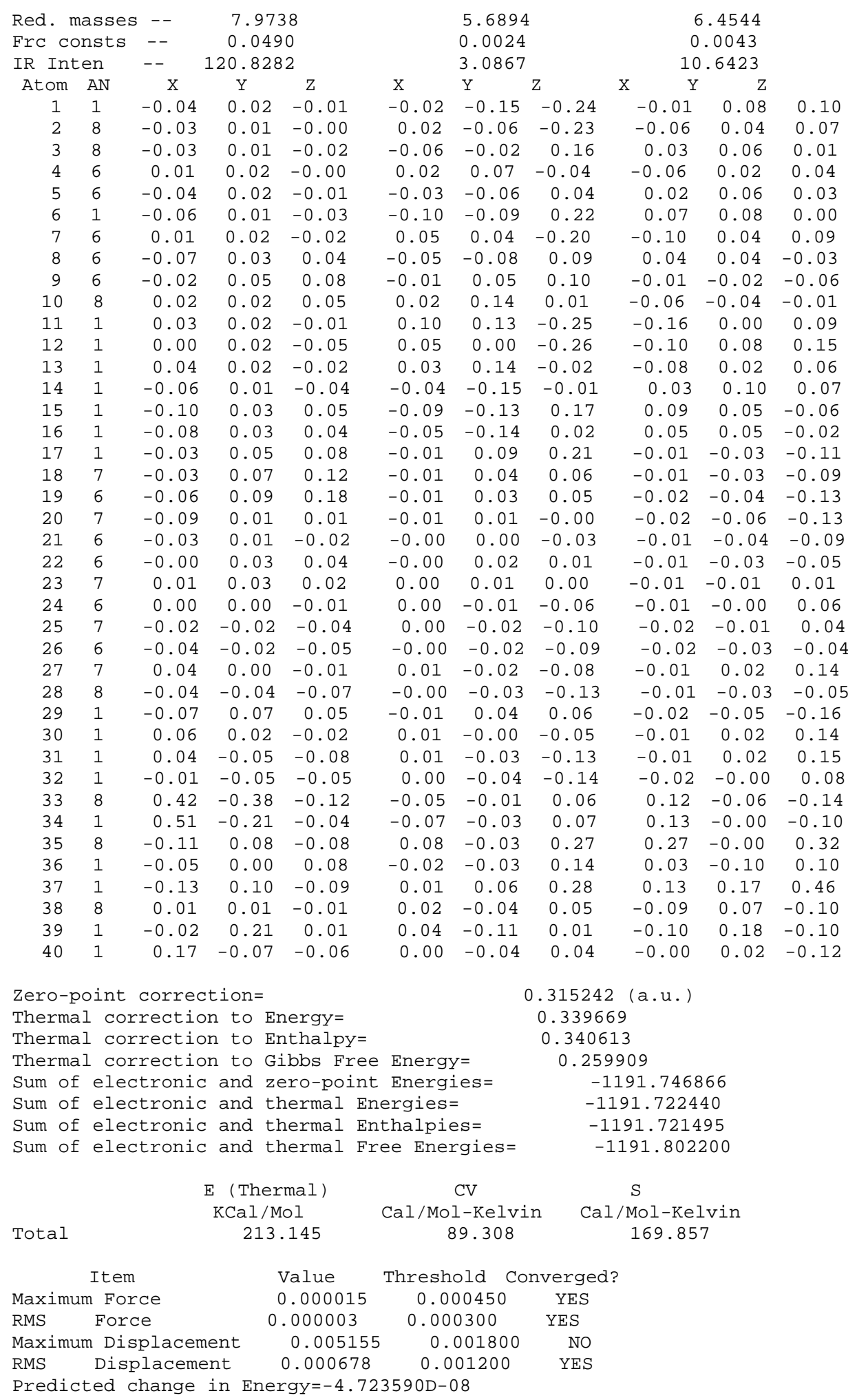

Normal termination of Gaussian 16 at Mon Mar 18 20:04:24 2019.

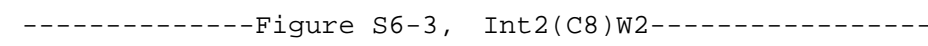

degua-oh8w2 . for . higha. $\log$

Stoichiometry C10H18N507(2)

Standard orientation:

\begin{tabular}{|c|c|c|c|}
\hline Center & Atomic & Atomic & Coordinates (Angstroms) \\
\hline Number & Number & Type & $\mathrm{x}$ \\
\hline
\end{tabular}




\begin{tabular}{|c|c|c|c|c|c|}
\hline 1 & 1 & $\odot$ & 3.680290 & -1.319373 & 2.043970 \\
\hline 2 & 8 & $\odot$ & 3.285082 & -1.737294 & 1. 274049 \\
\hline 3 & 8 & $\odot$ & 4.094786 & 2. 263018 & -0.266959 \\
\hline 4 & 6 & $\odot$ & 3.672046 & -0.068610 & -0.509466 \\
\hline 5 & 6 & $\odot$ & 3.648709 & 1.119877 & 0.456060 \\
\hline 6 & 1 & $\odot$ & 4.031989 & 3.029260 & 0.311515 \\
\hline 7 & 6 & $\odot$ & 4.049079 & -1.403124 & $\odot .122029$ \\
\hline 8 & 6 & $\odot$ & 2.174064 & 1.213238 & 0.831614 \\
\hline 9 & 6 & $\odot$ & 1.474958 & 0.756133 & $-\odot .452912$ \\
\hline 10 & 8 & 0 & 2.362367 & -0.138309 & -1.088930 \\
\hline 11 & 1 & $\odot$ & 3.866344 & -2.195018 & -0.613918 \\
\hline 12 & 1 & 0 & 5.125149 & -1.392993 & ๑. 351926 \\
\hline 13 & 1 & 0 & 4.388307 & 0.134660 & -1.317699 \\
\hline 14 & 1 & 0 & 4.294934 & 0.950789 & 1. 328811 \\
\hline 15 & 1 & 0 & 1.870854 & 2.222672 & 1.127927 \\
\hline 16 & $\overline{1}$ & $\odot$ & 1.949912 & 0.521547 & 1.650673 \\
\hline 17 & 1 & 0 & 1.264742 & 1.609346 & -1.111055 \\
\hline 18 & 7 & 0 & 0.200608 & 0.098114 & -0.233447 \\
\hline 19 & 6 & $\odot$ & $\odot .025171$ & -1.252610 & 0.362969 \\
\hline 20 & 7 & 0 & -1.352109 & -1.277453 & $\odot .842290$ \\
\hline 21 & 6 & 0 & -1.885021 & -0.090852 & 0.536316 \\
\hline 22 & 6 & 0 & -0.952080 & 0.775859 & -0.102381 \\
\hline 23 & 7 & 0 & -1.161505 & 2.037279 & -0.487808 \\
\hline 24 & 6 & $\odot$ & -2.395051 & 2.481016 & -0.260140 \\
\hline 25 & 7 & 0 & -3.370634 & 1.723101 & 0.316676 \\
\hline 26 & 6 & 0 & -3.213926 & $\odot .396452$ & 0.769699 \\
\hline 27 & 7 & 0 & -2.699546 & 3.736351 & -0.615078 \\
\hline 28 & 8 & 0 & -4.160202 & -0.183416 & 1. 297579 \\
\hline 29 & 1 & 0 & 0.745899 & -1.383590 & 1.180877 \\
\hline 30 & 1 & $\odot$ & -1.981398 & 4.299881 & -1.042635 \\
\hline 31 & 1 & 0 & -3.610523 & 4.140224 & -0.466514 \\
\hline 32 & 1 & 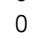 & -4.291628 & 2.119784 & 0.462642 \\
\hline 33 & 8 & 0 & 0.274283 & -2.280015 & -0.538481 \\
\hline 34 & 1 & $\odot$ & -0.465989 & -2.322678 & -1.178511 \\
\hline 35 & 8 & 0 & -1.959513 & -2.677361 & -2.163516 \\
\hline 36 & 1 & 0 & -2.432761 & -3.042009 & -1.386829 \\
\hline 37 & 1 & $\odot$ & -2.439064 & -1.875865 & -2.394059 \\
\hline 38 & 8 & 0 & -2.906932 & -3.518137 & ๑. 302098 \\
\hline 39 & 1 & 0 & -2.355839 & -2.784174 & 0.665301 \\
\hline 40 & 1 & 0 & -2.465585 & -4.326377 & $\odot .579699$ \\
\hline
\end{tabular}

Standard basis: Aug-CC-pVDZ (6D, 7F)

712 basis functions

85 alpha electrons 84 beta electrons

nuclear repulsion energy 2036.4312517326 Hartrees.

NAtoms $=40$ NActive $=40$

Force inversion solution in PCM.

SCF Done: $E($ UWB97XD) $=-1192.11175384$ A.U. after 2 cycles NFock $=2$ Conv $=0.54 \mathrm{D}-09 \quad-\mathrm{V} / \mathrm{T}=2.0071$

$\langle\mathrm{S} x\rangle=0.0000<\mathrm{S} y>=0.0000<\mathrm{S} z>=0.5000<\mathrm{S}^{* *} 2>=0.7609 \mathrm{~S}=0.5055$

$<$ L.S $>=0.000000000000 \mathrm{E}+00$

Annihilation of the first spin contaminant:

$\mathrm{S}^{* *} 2$ before annihilation 0.7609 , after 0.7501

Harmonic frequencies $\left(\mathrm{cm}^{*}-1\right)$, IR intensities (KM/Mole), Raman activities $\left(A^{* *} 4 / \mathrm{AMU}\right)$, depolarization ratios for plane and unpo incident light, reduced masses (AMU), force constants (mDyne/A), and normal coordinates:

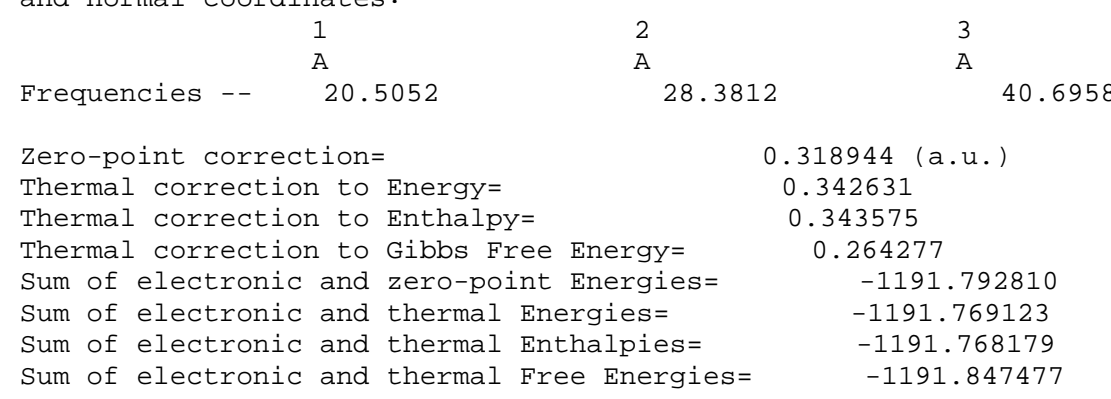

$$
\text { E (Thermal) CV S }
$$




\begin{tabular}{|c|c|c|c|}
\hline Total & $\begin{array}{l}/ \mathrm{Mol} \\
15.0 \odot 4\end{array}$ & $\begin{array}{c}\text { Cal/Mol-Kelvin } \\
87.787\end{array}$ & $\begin{array}{c}\text { Cal/Mol-Kelvin } \\
166.898\end{array}$ \\
\hline Item & Value & \multicolumn{2}{|c|}{ Threshold Converged? } \\
\hline Maximum Force & $\odot .000005$ & $\odot .000450$ & YES \\
\hline Force & $\odot .000001$ & $\odot .000300$ & YES \\
\hline Maximum Displacemen & $\odot .000990$ & $\odot .001800$ & \\
\hline Displacement & 0.000190 & 0.001200 & YES \\
\hline $\begin{array}{l}\text { Predicted change in } \\
\text { Optimization comple } \\
\text {-- Stationary poi }\end{array}$ & $\begin{array}{l}\text { ergy }=-5.14 \\
\text { d. } \\
\text { found. }\end{array}$ & $6124 D-\odot 9$ & \\
\hline
\end{tabular}

-...-Figure S6-4, TS2a(C8)W2 -.........

degua-oh8w2c. higha. log

Stoichiometry C10H18N507(2)

Standard orientation:

\begin{tabular}{|c|c|c|c|c|c|}
\hline \multirow{2}{*}{$\begin{array}{l}\text { Center } \\
\text { Number }\end{array}$} & \multirow{2}{*}{$\begin{array}{l}\text { Atomic } \\
\text { Number }\end{array}$} & \multirow{2}{*}{$\begin{array}{l}\text { Atomic } \\
\text { Type }\end{array}$} & \multicolumn{3}{|c|}{ Coordinates (Angstroms) } \\
\hline & & & $x$ & $Y$ & Z \\
\hline 1 & 1 & $\odot$ & -2.936094 & -0.868169 & -2.450416 \\
\hline 2 & 8 & $\odot$ & -2.559616 & -1.456861 & -1.790717 \\
\hline 3 & 8 & 0 & -4.552264 & 1.541441 & $\odot .708721$ \\
\hline 4 & 6 & 0 & -3.607519 & -0.602260 & $\odot .293324$ \\
\hline 5 & 6 & $\odot$ & -3.780902 & $\odot .816801$ & $-\odot .246599$ \\
\hline 6 & 1 & 0 & -4.589616 & 2.461148 & $\odot .429462$ \\
\hline 7 & 6 & 0 & -3.526025 & -1.691357 & -0.772734 \\
\hline 8 & 6 & $\odot$ & -2.343667 & 1.320452 & $-\odot .337405$ \\
\hline 9 & 6 & 0 & -1.607639 & $\odot .548363$ & $\odot .773133$ \\
\hline 10 & 8 & $\odot$ & -2.433043 & -0.562561 & 1.104978 \\
\hline 11 & 1 & $\odot$ & -3.234741 & -2.627564 & -0.282156 \\
\hline 12 & 1 & $\odot$ & -4.526627 & -1.831957 & -1.209781 \\
\hline 13 & 1 & 0 & -4.452782 & -0.852684 & 0.949759 \\
\hline 14 & 1 & $\odot$ & -4.290961 & $\odot .825779$ & -1.221114 \\
\hline 15 & 1 & $\odot$ & -2.270238 & 2.403605 & -0.198938 \\
\hline 16 & 1 & $\odot$ & -1.908381 & 1.064439 & -1.309094 \\
\hline 17 & 1 & $\odot$ & -1.498168 & 1.166983 & 1.677761 \\
\hline 18 & 7 & $\odot$ & -0.313476 & $\odot .061205$ & ๑.383928 \\
\hline 19 & 6 & $\odot$ & $\odot .485904$ & -1.588211 & -0.642016 \\
\hline 20 & 7 & $\odot$ & 1.748100 & -1.126064 & -0.558658 \\
\hline 21 & 6 & $\odot$ & 1.842902 & $\odot .203056$ & -0.382513 \\
\hline 22 & 6 & $\odot$ & ๑.684379 & $\odot .873027$ & 0.182954 \\
\hline 23 & 7 & $\odot$ & $\odot .673627$ & 2.211615 & $\odot .466466$ \\
\hline 24 & 6 & $\odot$ & 1.785186 & 2.872928 & $\odot .229047$ \\
\hline 25 & 7 & $\odot$ & 2.925027 & 2.303170 & -0.273222 \\
\hline 26 & 6 & $\odot$ & 3.047769 & $\odot .949142$ & $-\odot .612194$ \\
\hline 27 & 7 & $\odot$ & 1.829828 & 4.193426 & $\odot .481958$ \\
\hline 28 & 8 & $\odot$ & 4.111217 & $\odot .523764$ & -1.064462 \\
\hline 29 & 1 & $\odot$ & -0.180821 & -1.283294 & -1.450634 \\
\hline 30 & 1 & $\odot$ & 1.013962 & 4.631443 & 0.879067 \\
\hline 31 & 1 & $\odot$ & 2.664777 & 4.742713 & $\odot .357777$ \\
\hline 32 & 1 & $\odot$ & 3.745483 & 2.873054 & -0.438621 \\
\hline 33 & 8 & $\odot$ & $\odot .162923$ & -2.749035 & -0.137392 \\
\hline 34 & 1 & $\odot$ & $\odot .801126$ & -3.039384 & $\odot .574687$ \\
\hline 35 & 8 & $\odot$ & 1.826135 & -3.693597 & 1.692135 \\
\hline 36 & 1 & $\odot$ & 2.694491 & -3.457363 & 1.296425 \\
\hline 37 & 1 & $\odot$ & 1.801825 & -3.255347 & 2.548371 \\
\hline 38 & 8 & $\odot$ & 3.912798 & -2.753899 & $\odot .245875$ \\
\hline 39 & 1 & $\odot$ & 3.264954 & -2.145378 & -0.173832 \\
\hline 40 & 1 & $\odot$ & 4.232545 & -3.317160 & -0.465115 \\
\hline
\end{tabular}

Standard basis: Aug-CC-pVDZ (6D, 7F)

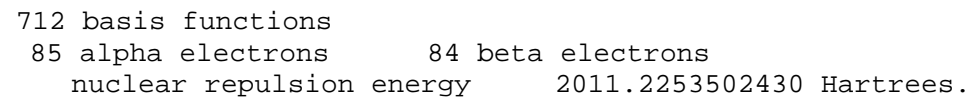

NAtoms $=40$ NActive $=40$

Force inversion solution in PCM.

SCF Done: $E($ UWB97XD $)=-1192.08115425$ A.U. after 1 cycles 
NFock $=1$ Conv $=0.37 \mathrm{D}-08 \quad-\mathrm{V} / \mathrm{T}=2.0071$

$\left\langle\mathrm{S} x>=0.0000<\mathrm{S} y>=0.0000<\mathrm{S} z>=0.5000<\mathrm{S}^{* *} 2>=0.7633 \mathrm{~S}=0.5066\right.$ $<$ L.S $>=0.000000000000 \mathrm{E}+00$

Annihilation of the first spin contaminant:

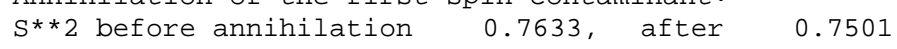

Harmonic frequencies $\left(\mathrm{cm}^{*}-1\right)$, IR intensities (KM/Mole), Raman activities ( $\left.A^{* *} 4 / A M U\right)$, depolarization ratios for plane and unpo incident light, reduced masses (AMU), force constants (mDyne/A), and normal coordinates:

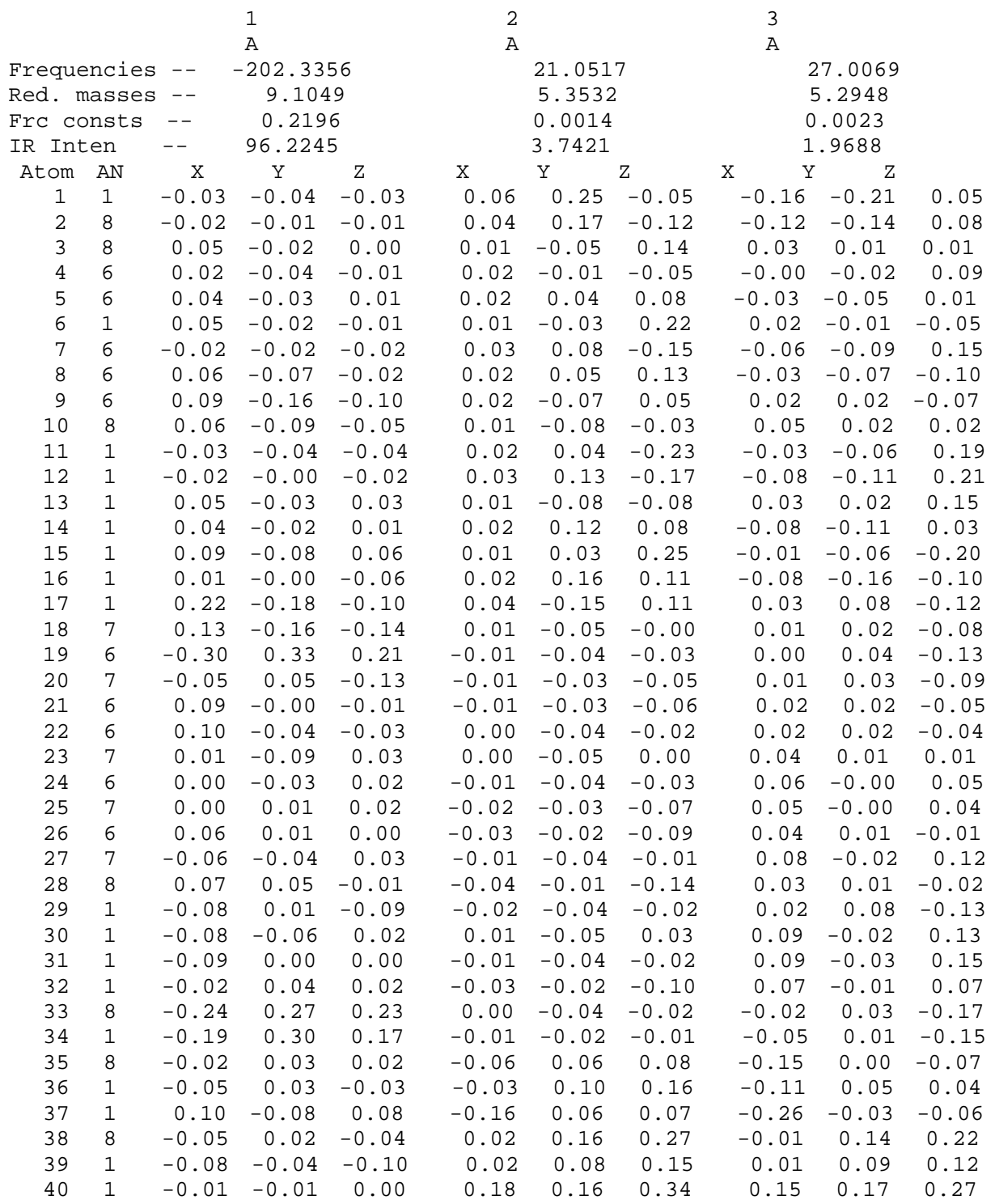

- . . . . . . . . . . . . .

Temperature 298.150 Kelvin. Pressure 1.00000 Atm.

Zero-point correction=

Thermal correction to Energy=

Thermal correction to Enthalpy=

Thermal correction to Gibbs Free Energy=

Sum of electronic and zero-point Energies=

Sum of electronic and thermal Energies=

Sum of electronic and thermal Enthalpies=

Sum of electronic and thermal Free Energies=
E (Thermal)
KCal/Mol

213.282
CV 87.210

$$
\begin{gathered}
\odot .316190 \text { (a.u. ) } \\
0.339887 \\
0.340831 \\
0.261163 \\
-1191.764964 \\
-1191.741267 \\
-1191.740323 \\
-1191.819991
\end{gathered}
$$

$\mathrm{S}$

Cal/Mol-Kelvin 167.675 


$\begin{array}{lccc}\text { Maximum Force } & \odot .0 \odot \odot \odot 28 & \odot .0 \odot \odot 45 \odot & \text { YES } \\ \text { RMS Force } & 0.0000 \odot 4 & 0.00 \odot 30 \odot & \text { YES } \\ \text { Maximum Displacement } & 0.003481 & 0.001800 & \text { NO } \\ \text { RMS Displacement } & 0.000682 & 0.001200 & \text { YES }\end{array}$

Predicted change in Energy $=-2.089734 \mathrm{D}-07$

Normal termination of Gaussian 16 at Tue Mar 12 19:24:23 2019.

-...-Figure S6-5, Int3a(C8)W2 ......

degua-oh8w2c. for . higha. $\log$

Stoichiometry C10H18N507(2)

\begin{tabular}{|c|c|c|c|c|c|}
\hline \multirow{2}{*}{$\begin{array}{l}\text { Center } \\
\text { Number }\end{array}$} & \multirow{2}{*}{$\begin{array}{l}\text { Atomic } \\
\text { Number }\end{array}$} & \multirow{2}{*}{$\begin{array}{c}\text { Atomic } \\
\text { Type }\end{array}$} & \multicolumn{3}{|c|}{ Coordinates (Angstroms) } \\
\hline & & & $X$ & $\mathrm{Y}$ & Z \\
\hline & & & & & \\
\hline 1 & 1 & $\odot$ & -2.581156 & -1.852970 & -2.085017 \\
\hline 2 & 8 & $\odot$ & -2.213901 & -2.216762 & -1.274963 \\
\hline 3 & 8 & $\odot$ & -5.110667 & $\odot .727389$ & $\odot .215578$ \\
\hline 4 & 6 & $\odot$ & -3.727101 & -1.197207 & 0.421646 \\
\hline 5 & 6 & $\odot$ & -4.070585 & 0.011284 & -0.447892 \\
\hline 6 & 1 & $\odot$ & -5.275725 & 1.538934 & $-\odot .273590$ \\
\hline 7 & 6 & $\odot$ & -3.269348 & -2.435802 & -0.346287 \\
\hline 8 & 6 & $\odot$ & -2.756927 & 0.789319 & -0.487307 \\
\hline 9 & 6 & $\odot$ & -2.025938 & 0.384411 & 0.811742 \\
\hline 10 & 8 & $\odot$ & -2.745737 & -0.728172 & 1.343213 \\
\hline 11 & 1 & $\odot$ & -2.891836 & -3.165532 & 0.380070 \\
\hline 12 & 1 & $\odot$ & -4.143995 & -2.877789 & -0.848937 \\
\hline 13 & 1 & $\odot$ & -4.608468 & -1.492846 & 1.008217 \\
\hline 14 & 1 & $\odot$ & -4.409168 & -0.286281 & -1.451392 \\
\hline 15 & 1 & $\odot$ & -2.918246 & 1.870299 & -0.540906 \\
\hline 16 & 1 & $\odot$ & -2.157494 & ๑. 487909 & -1.353094 \\
\hline 17 & 1 & $\odot$ & -2.075765 & 1.186489 & 1.563649 \\
\hline 18 & 7 & $\odot$ & -0.662904 & -0.008236 & 0.597783 \\
\hline 19 & 6 & $\odot$ & 1.183557 & -1.792351 & -0.422045 \\
\hline 20 & 7 & $\odot$ & 1.970805 & -0.830232 & -0.069894 \\
\hline 21 & 6 & $\odot$ & 1.559861 & ๑. 461584 & -0.029800 \\
\hline 22 & 6 & $\odot$ & ๑. 208519 & 0.906401 & ๑. 294412 \\
\hline 23 & 7 & $\odot$ & -0.113171 & 2.254220 & ๑. 258613 \\
\hline 24 & 6 & $\odot$ & ๑. 828812 & 3.117365 & ๑. .0०6077 \\
\hline 25 & 7 & 0 & 2.139521 & 2.770979 & -0.235984 \\
\hline 26 & 6 & $\odot$ & 2.584907 & 1.463639 & -0.263309 \\
\hline 27 & 7 & $\odot$ & ๑. 551548 & 4.435699 & -0.013163 \\
\hline 28 & 8 & $\odot$ & 3.777489 & 1.232002 & -0.483917 \\
\hline 29 & 1 & $\odot$ & $\odot .204358$ & -1.653382 & -0.888458 \\
\hline 30 & 1 & $\odot$ & -0.405308 & 4.722117 & $\odot .119603$ \\
\hline 31 & 1 & $\odot$ & 1.232128 & 5.129186 & -0.278190 \\
\hline 32 & 1 & $\odot$ & 2.837567 & 3.484944 & -0.405077 \\
\hline 33 & 8 & $\odot$ & 1.528507 & -3.049508 & -0.282651 \\
\hline 34 & 1 & $\odot$ & 2.435787 & -3.126992 & ๑. 139578 \\
\hline 35 & 8 & $\odot$ & 3.954063 & -3.335635 & $\odot .693952$ \\
\hline 36 & 1 & $\odot$ & 4.436443 & -2.499439 & 0.492228 \\
\hline 37 & 1 & $\odot$ & 4.004874 & -3.437546 & 1.649120 \\
\hline 38 & 8 & $\odot$ & 5.249589 & -1.035214 & 0.105688 \\
\hline 39 & 1 & $\odot$ & 4.622517 & -0.320927 & -0.131733 \\
\hline 40 & 1 & $\Theta$ & 5.883617 & -1.067851 & -0.616183 \\
\hline
\end{tabular}

Standard basis: Aug-CC-pVDZ (6D, 7F)

712 basis functions

85 alpha electrons 84 beta electrons

NAtoms $=40$ nuclear repulsion energy 1966.0343623941 Hartrees.

Force inversion solution in PCM.

SCF Done: $E($ UWB97XD $)=-1192.09292284 \quad$ A.U. after 1 cycles NFock $=1$ Conv $=0.34 \mathrm{D}-08 \quad-\mathrm{V} / \mathrm{T}=2.0071$

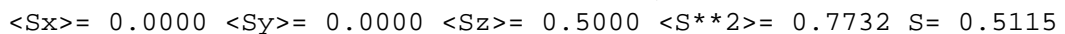

$<$ L.S $>=0.000000000000 \mathrm{E}+0 \odot$

Annihilation of the first spin contaminant:

$\mathrm{S}^{* * 2}$ before annihilation 0.7732 , after 0.7502 
Harmonic frequencies $\left(\mathrm{cm}^{* *}-1\right)$, IR intensities (KM/Mole), Raman activities ( $\left.A^{* *} 4 / \mathrm{AMU}\right)$, depolarization ratios for plane and unp incident light, reduced masses (AMU), force constants (mDyne/A), and normal coordinates:

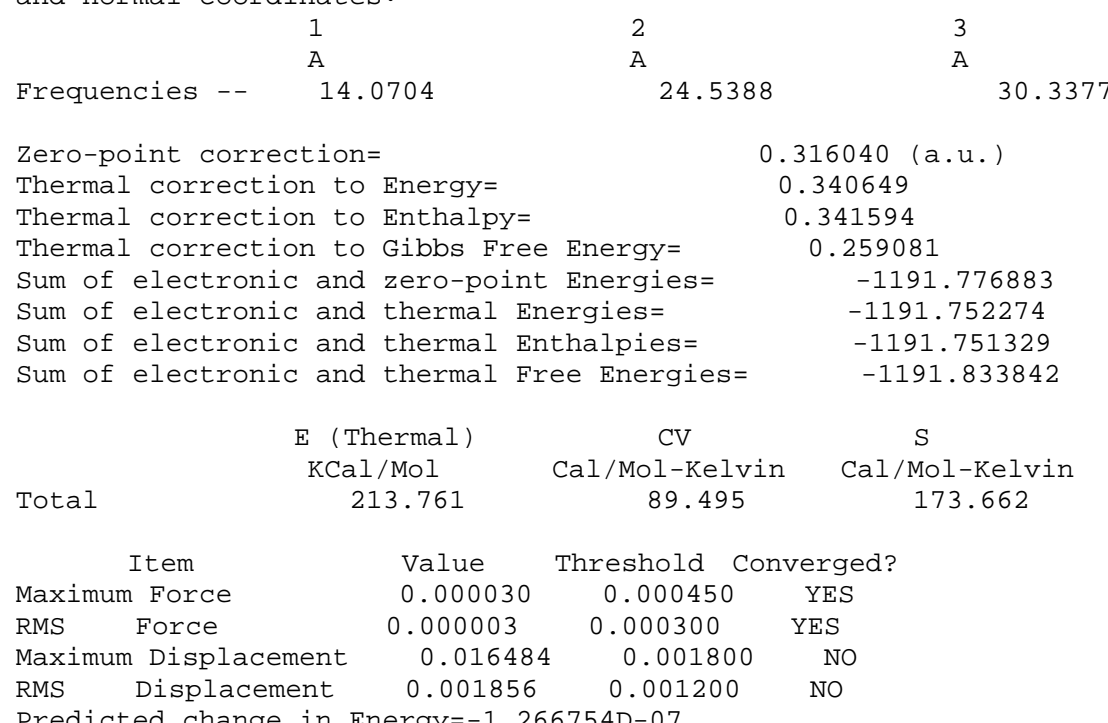

Predicted change in Energy $=-1.266754 \mathrm{D}-\Theta 7$

Normal termination of Gaussian 16 at Sun Mar 17 01:46:21 2019.

-

degua-oh8w2d.higha. log

Stoichiometry C10H18N507(2)

Standard orientation:

\begin{tabular}{|c|c|c|c|c|c|}
\hline \multirow{2}{*}{$\begin{array}{l}\text { Center } \\
\text { Number }\end{array}$} & \multirow{2}{*}{$\begin{array}{l}\text { Atomic } \\
\text { Number }\end{array}$} & \multirow{2}{*}{$\begin{array}{l}\text { Atomic } \\
\text { Type }\end{array}$} & \multicolumn{3}{|c|}{ Coordinates (Angstroms) } \\
\hline & & & $\mathrm{x}$ & $\mathrm{Y}$ & Z \\
\hline 1 & 1 & $\odot$ & 4.087786 & -2.206135 & 1.683315 \\
\hline 2 & 8 & 0 & 3.682937 & -2.639466 & $\odot .926810$ \\
\hline 3 & 8 & 0 & 4.653944 & 1.418959 & -0.306244 \\
\hline 4 & 6 & 0 & 3.873575 & $-\odot .804732$ & $-\odot .716087$ \\
\hline 5 & 6 & 0 & 4.033911 & 0.306995 & $\odot .337973$ \\
\hline 6 & 1 & 0 & 4.729885 & 2.132189 & 0.334964 \\
\hline 7 & 6 & 0 & 4.318124 & -2.187867 & -0.261855 \\
\hline 8 & 6 & $\odot$ & 2.597571 & $\odot .598089$ & 0.754117 \\
\hline 9 & 6 & 0 & 1.813928 & $\odot .287209$ & -0.523849 \\
\hline 10 & 8 & 0 & 2.494542 & -0.842139 & -1.076439 \\
\hline 11 & 1 & 0 & 4.057613 & -2.913545 & -1.041896 \\
\hline 12 & 1 & $\odot$ & 5.413522 & -2.187425 & -0.149179 \\
\hline 13 & 1 & $\theta$ & 4.479516 & $-\odot .531702$ & -1.595134 \\
\hline 14 & 1 & 0 & 4.657194 & -0.016477 & 1.184379 \\
\hline 15 & 1 & 0 & 2.448133 & 1.625734 & 1.098934 \\
\hline 16 & 1 & $\odot$ & 2.281526 & -0.097623 & 1.542045 \\
\hline 17 & 1 & 0 & 1.889381 & 1.131988 & -1.229620 \\
\hline 18 & 7 & $\odot$ & 0.451454 & $-\odot .088902$ & $-\odot .315859$ \\
\hline 19 & 6 & 0 & -1.549454 & -1.775883 & 0.759503 \\
\hline 20 & 7 & 0 & -2.273992 & $-\odot .797495$ & 0.216968 \\
\hline 21 & 6 & $\odot$ & -1.803677 & $\odot .475982$ & 0.140619 \\
\hline 22 & 6 & $\odot$ & $-\odot .414159$ & $\odot .857509$ & $-\odot .089779$ \\
\hline 23 & 7 & 0 & -0.050438 & 2.195985 & -0.078345 \\
\hline 24 & 6 & 0 & $-\odot .980065$ & 3.102720 & 0.017026 \\
\hline 25 & 7 & $\odot$ & -2.319189 & 2.815977 & 0.121848 \\
\hline 26 & 6 & 0 & -2.813507 & 1.522393 & $\odot .197778$ \\
\hline 27 & 7 & 0 & -0.651771 & 4.413180 & -0.018119 \\
\hline 28 & 8 & $\odot$ & -4.024189 & 1.332411 & 0.312572 \\
\hline 29 & 1 & 0 & $-\odot .648757$ & -1.502031 & 1.325830 \\
\hline 30 & 1 & 0 & $\odot .329000$ & 4.645488 & $\odot .005374$ \\
\hline 31 & 1 & 0 & -1.312875 & 5.130734 & 0.234657 \\
\hline 32 & 1 & 0 & -3.004303 & 3.560279 & $\odot .165287$ \\
\hline 33 & 8 & 0 & -1.867123 & -2.991238 & 0.714983 \\
\hline 34 & 1 & $\odot$ & -3.095853 & -3.446539 & 0.079318 \\
\hline 35 & 8 & $\odot$ & -4.029730 & -3.681561 & -0.329836 \\
\hline 36 & 1 & 0 & -4.440923 & -2.614852 & -0.596214 \\
\hline
\end{tabular}




$\begin{array}{cccccc}37 & 1 & 0 & -3.891505 & -4.209634 & -1.124989 \\ 38 & 8 & \odot & -4.597029 & -1.411164 & -0.793763 \\ 39 & 1 & \odot & -3.718971 & -1.047496 & -0.381911 \\ 40 & 1 & 0 & -5.317113 & -1.005074 & -0.297957 \\ - & - & - & -\end{array}$

Standard basis: Aug-CC-pVDZ (6D, 7F)

712 basis functions

85 alpha electrons 84 beta electrons

nuclear repulsion energy 1944.1594182991 Hartrees.

NAtoms $=40$ NActive $=40$

Force inversion solution in PCM.

SCF Done: $E($ UWB97XD $)=-1192.07617725$ A.U. after 2 cycles

NFock $=2$ Conv $=0.72 \mathrm{D}-09 \quad-\mathrm{V} / \mathrm{T}=2.0071$

$<\mathrm{S} X>=0.0000<\mathrm{S} y>=0.0000<\mathrm{S} z>=0.5000<\mathrm{S}^{* *} 2>=0.7703 \mathrm{~S}=0.5101$

$<$ L.S $>=0.0000000 \odot \odot \odot \odot \odot E+\odot \odot$

Annihilation of the first spin contaminant:

$\mathrm{S}^{* * 2}$ before annihilation 0.7703 , after 0.7502

Harmonic frequencies $\left(\mathrm{cm}^{* *}-1\right)$, IR intensities (KM/Mole)

activities $\left(A^{* *} 4 / \mathrm{AMU}\right)$, depolarization ratios for plane

incident light, reduced masses (AMU), force constants (mDyne/A), and normal coordinates:

\begin{tabular}{|c|c|c|c|c|c|c|c|c|c|c|}
\hline & & & $\begin{array}{l}1 \\
\mathrm{~A}\end{array}$ & & $\begin{array}{l}2 \\
A\end{array}$ & & & $\begin{array}{l}3 \\
A\end{array}$ & & \\
\hline Freque & ncies & - - & -757.96 & & & 17.249 & & & 24.1875 & \\
\hline Red. $n$ & lasses & ; - & 1.167 & & & 5.8526 & & & 6.3242 & \\
\hline Frc $\mathrm{cc}$ & nsts & -- & 0.395 & & & 0.0010 & & & $\odot .0 \odot 22$ & \\
\hline IR Int & en & -- & 804.906 & & & 0.8972 & & & 5.4023 & \\
\hline Atom & AN & $x$ & $Y$ & Z & $x$ & $Y$ & Z & $x$ & Z & \\
\hline 1 & 1 & $\odot . \odot \odot$ & $-\odot . \odot \odot$ & $\odot . \odot \odot$ & 0.27 & -0.13 & -0.18 & 0.27 & 0.19 & 0.12 \\
\hline 2 & 8 & $-0.0 \odot$ & $\odot .0 \odot$ & -0.00 & 0.29 & -0.11 & -0.20 & 0.24 & 0.12 & 0.18 \\
\hline 3 & 8 & $-0.0 \odot$ & -0.00 & $\odot .00$ & -0.01 & 0.05 & 0.20 & -0.07 & 0.07 & -0.21 \\
\hline 4 & 6 & $-0.0 \odot$ & $\odot . \odot \odot$ & 0.00 & 0.02 & 0.07 & -0.01 & $\odot .02$ & -0.00 & $\odot .02$ \\
\hline 5 & 6 & $-0.0 \odot$ & $\odot .0 \odot$ & -0.00 & -0.02 & -0.01 & 0.09 & 0.01 & 0.09 & -0.08 \\
\hline 6 & 1 & $\odot . \odot \odot$ & $-\odot . \odot \odot$ & $\odot . \odot \odot$ & 0.01 & -0.02 & 0.27 & -0.07 & 0.13 & -0.27 \\
\hline 7 & 6 & $-0.0 \odot$ & $\odot .0 \odot$ & 0.00 & 0.18 & 0.07 & -0.18 & 0.13 & 0.06 & 0.10 \\
\hline 8 & 6 & -0.00 & $\odot . \odot \odot$ & 0.00 & -0.04 & -0.05 & $\odot . \odot 8$ & $\odot .01$ & 0.07 & -0.05 \\
\hline 9 & 6 & $-\odot . \odot \odot$ & $\odot .0 \odot$ & $-\odot . \odot \odot$ & -0.01 & -0.03 & 0.06 & -0.01 & $-\odot .07$ & $\odot . \odot \odot$ \\
\hline 10 & 8 & -0.00 & 0.00 & -0.00 & -0.00 & -0.04 & 0.08 & -0.00 & -0.12 & 0.11 \\
\hline 11 & 1 & -0.00 & $\odot . \odot \odot$ & -0.00 & 0.22 & 0.13 & -0.24 & 0.13 & -0.02 & 0.18 \\
\hline 12 & 1 & -0.00 & $\odot . \odot \odot$ & -0.00 & $\odot .19$ & 0.18 & -0.24 & 0.14 & 0.14 & 0.03 \\
\hline 13 & 1 & $-\odot .0 \odot$ & $\odot . \odot \odot$ & $-\odot .0 \odot$ & -0.06 & 0.23 & -0.02 & -0.06 & -0.03 & -0.04 \\
\hline 14 & 1 & $-\odot . \odot \odot$ & $\odot . \odot \odot$ & 0.00 & -0.04 & -0.07 & 0.08 & 0.06 & 0.20 & -0.08 \\
\hline 15 & 1 & -0.00 & 0.00 & 0.00 & -0.06 & -0.06 & 0.11 & -0.01 & 0.09 & -0.13 \\
\hline 16 & 1 & $-\odot . \odot \odot$ & $\odot . \odot \odot$ & $-\odot . \odot \odot$ & -0.03 & -0.08 & 0.05 & 0.07 & 0.12 & 0.02 \\
\hline 17 & 1 & -0.00 & $-0.0 \odot$ & -0.00 & 0.01 & -0.03 & 0.06 & -0.04 & -0.14 & -0.08 \\
\hline 18 & 7 & -0.00 & $-0.0 \odot$ & -0.00 & -0.02 & -0.03 & 0.02 & -0.01 & -0.07 & 0.04 \\
\hline 19 & 6 & 0.01 & -0.01 & 0.01 & -0.06 & 0.01 & 0.02 & -0.07 & $-\odot .07$ & -0.05 \\
\hline 20 & 7 & -0.03 & $\odot .0 \odot$ & -0.02 & -0.04 & 0.01 & -0.01 & -0.04 & -0.02 & -0.01 \\
\hline 21 & 6 & -0.01 & $\odot . \odot \odot$ & $-\odot . \odot \odot$ & -0.02 & $-\odot .0 \odot$ & -0.02 & -0.01 & -0.03 & 0.03 \\
\hline 22 & 6 & $-\odot . \odot \odot$ & $-\odot . \odot \odot$ & 0.00 & $-\odot .02$ & -0.02 & $-\odot . \odot \odot$ & -0.00 & -0.06 & 0.02 \\
\hline 23 & 7 & -0.00 & $-\odot . \odot \odot$ & $\odot .00$ & $-\odot . \odot \odot$ & -0.02 & -0.02 & 0.02 & -0.06 & -0.01 \\
\hline 24 & 6 & -0.00 & $-0.0 \odot$ & $\odot .0 \odot$ & 0.00 & -0.01 & -0.05 & 0.04 & -0.04 & 0.02 \\
\hline 25 & 7 & -0.00 & $\odot . \odot \odot$ & $\odot .0 \odot$ & -0.00 & $\odot . \odot \odot$ & -0.07 & 0.04 & -0.02 & 0.07 \\
\hline 26 & 6 & $\odot . \odot \odot$ & $\odot .0 \odot$ & $-0.0 \odot$ & -0.01 & 0.01 & -0.06 & 0.01 & -0.01 & 0.06 \\
\hline 27 & 7 & $-\odot . \odot \odot$ & $-\odot . \odot \odot$ & $-0.0 \odot$ & 0.02 & -0.01 & -0.06 & 0.06 & -0.05 & $0.0 \odot$ \\
\hline 28 & 8 & -0.00 & -0.00 & -0.00 & -0.02 & 0.02 & -0.07 & 0.01 & 0.01 & 0.09 \\
\hline 29 & 1 & -0.01 & 0.07 & -0.01 & -0.06 & 0.01 & $\odot .02$ & -0.06 & -0.12 & -0.05 \\
\hline 30 & 1 & -0.00 & -0.00 & 0.00 & 0.02 & -0.02 & -0.05 & 0.06 & -0.07 & -0.04 \\
\hline 31 & 1 & -0.00 & $-\odot . \odot \odot$ & $-\odot . \odot \odot$ & 0.02 & $-\odot .0 \odot$ & -0.10 & 0.08 & -0.03 & -0.00 \\
\hline 32 & 1 & -0.00 & 0.00 & $\odot .00$ & 0.00 & 0.01 & $-\odot .09$ & 0.05 & -0.01 & $\odot .09$ \\
\hline 33 & 8 & 0.04 & 0.02 & 0.02 & -0.08 & 0.01 & 0.06 & -0.12 & -0.05 & -0.10 \\
\hline 34 & 1 & -0.21 & -0.16 & -0.12 & -0.10 & 0.02 & 0.09 & -0.14 & 0.01 & -0.12 \\
\hline 35 & 8 & -0.04 & -0.02 & -0.02 & -0.11 & 0.03 & 0.11 & -0.15 & 0.06 & -0.12 \\
\hline 36 & 1 & -0.21 & 0.87 & -0.13 & $-\odot . \odot 8$ & $\odot .02$ & $\odot .06$ & -0.11 & 0.08 & -0.08 \\
\hline 37 & 1 & $\odot . \odot \odot$ & -0.04 & 0.02 & -0.14 & -0.02 & 0.13 & -0.17 & 0.09 & -0.14 \\
\hline 38 & 8 & 0.05 & -0.03 & 0.02 & -0.05 & 0.02 & 0.01 & -0.07 & 0.10 & -0.03 \\
\hline 39 & 1 & 0.23 & -0.01 & $\odot .09$ & -0.04 & 0.01 & -0.01 & -0.05 & 0.05 & -0.02 \\
\hline 40 & 1 & $\odot .01$ & -0.13 & $\odot .06$ & -0.04 & $\odot . \odot 6$ & -0.01 & -0.05 & 0.11 & -0.02 \\
\hline zero & int & ש & $o n=$ & & & & .31 & (a.u & & \\
\hline Therma & $1 \mathrm{cor}$ & recti & on to & $g y=$ & & & 0.33 & & & \\
\hline Therma & $1 \mathrm{cor}$ & rectio & $n$ to $\mathrm{En}$ & halpy= & & & 0.3343 & & & \\
\hline
\end{tabular}


Thermal correction to Gibbs Free Energy= Sum of electronic and zero-point Energies= Sum of electronic and thermal Energies=

Sum of electronic and thermal Enthalpies=

Sum of electronic and thermal Free Energies=
E (Thermal) $\mathrm{KCal} / \mathrm{Mol}$
CV

Cal/Mol-Kelvin 85.369
0.255087

$-1191.765846$

$-1191.742751$

$-1191.741807$

$-1191.821091$

\section{$\mathrm{S}$}

Cal/Mol-Kelvin

166.866

Total

Value Threshold Converged?

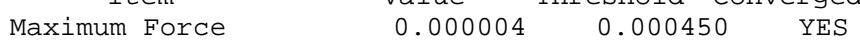

$\begin{array}{llll}\text { RMS Force } & 0.000001 & 0.000300 & \text { YES }\end{array}$

Maximum Displacement $0.000511 \quad 0.001800$ YES

$\begin{array}{llll}\text { RMS Displacement } & 0.000104 & 0.001200 & \text { YES }\end{array}$

Predicted change in Energy $=-1.075409 \mathrm{D}-09$

optimization completed.

- - Stationary point found.

Normal termination of Gaussian 16 at Thu Mar 14 16:45:47 2019.

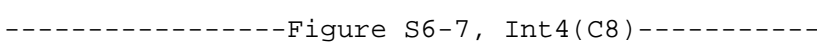

degua-oh8w2d.rev.higha. log

Stoichiometry C10H18N507(2)

Standard orientation:

\begin{tabular}{|c|c|c|c|c|c|}
\hline \multirow{2}{*}{$\begin{array}{l}\text { Center } \\
\text { Number }\end{array}$} & \multirow{2}{*}{$\begin{array}{l}\text { Atomic } \\
\text { Number }\end{array}$} & \multirow{2}{*}{$\begin{array}{c}\text { Atomic } \\
\text { Type }\end{array}$} & \multicolumn{3}{|c|}{ Coordinates (Angstroms) } \\
\hline & & & $x$ & $\mathrm{Y}$ & Z \\
\hline-- & - & -- & 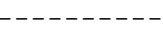 & ------- & 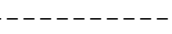 \\
\hline 1 & 1 & $\odot$ & 2.357213 & -2.243320 & 1.815471 \\
\hline 2 & 8 & $\odot$ & 2.034471 & -2.462615 & 0.937317 \\
\hline 3 & 8 & $\odot$ & 5.151603 & 0.509405 & 0.065434 \\
\hline 4 & 6 & $\odot$ & 3.699348 & -1.289703 & -0.493542 \\
\hline 5 & 6 & $\odot$ & 4.042196 & -0.237274 & $\odot .560161$ \\
\hline 6 & 1 & $\odot$ & 5.326390 & 1.229628 & 0.678559 \\
\hline 7 & 6 & $\odot$ & 3.143130 & -2.600936 & 0.056494 \\
\hline 8 & 6 & $\odot$ & 2.765219 & $\odot .597096$ & 0.642073 \\
\hline 9 & 6 & $\odot$ & 2.097667 & 0.417727 & -0.739291 \\
\hline 10 & 8 & $\odot$ & 2.793147 & -0.644619 & -1.386349 \\
\hline 11 & 1 & $\odot$ & 2.789020 & -3.201117 & -0.790248 \\
\hline 12 & 1 & $\odot$ & 3.962417 & -3.151687 & 0.544454 \\
\hline 13 & 1 & $\odot$ & 4.600348 & -1.540806 & -1.070676 \\
\hline 14 & 1 & $\odot$ & 4.303466 & -0.692588 & 1.526780 \\
\hline 15 & 1 & $\odot$ & 2.970982 & 1.649884 & 0.857366 \\
\hline 16 & 1 & $\odot$ & 2.102021 & ๑. 209398 & 1.422825 \\
\hline 17 & 1 & $\odot$ & 2.223533 & 1.313967 & -1.364493 \\
\hline 18 & 7 & $\odot$ & $\odot .707988$ & ๑. .070893 & -0.645113 \\
\hline 19 & 6 & $\odot$ & -1.292005 & -1.835546 & 0.112261 \\
\hline 20 & 7 & $\odot$ & -1.977660 & -0.693150 & -0.206965 \\
\hline 21 & 6 & $\odot$ & -1.522447 & ๑. 601897 & -0.133676 \\
\hline 22 & 6 & $\odot$ & -0.138352 & $\odot .993967$ & -0.290080 \\
\hline 23 & 7 & $\odot$ & 0.238533 & 2.306826 & -0.067744 \\
\hline 24 & 6 & $\odot$ & -0.685242 & 3. 195176 & 0.165346 \\
\hline 25 & 7 & $\odot$ & -2.030988 & 2.900590 & 0.219531 \\
\hline 26 & 6 & $\odot$ & -2.531560 & 1.620082 & $\odot .106522$ \\
\hline 27 & 7 & $\odot$ & -0.351334 & 4.481715 & 0.374239 \\
\hline 28 & 8 & $\odot$ & -3.738728 & 1.407048 & ๑. 216869 \\
\hline 29 & 1 & $\odot$ & -0.243448 & -1.697511 & 0.404845 \\
\hline 30 & 1 & $\odot$ & 0.619015 & 4.741039 & 0.292441 \\
\hline 31 & 1 & $\odot$ & -1.037122 & 5.207262 & $\odot .507433$ \\
\hline 32 & 1 & $\odot$ & -2.710564 & 3.629435 & 0.401491 \\
\hline 33 & 8 & $\odot$ & -1.833606 & -2.935700 & ०. 071617 \\
\hline 34 & 1 & $\odot$ & -3.597528 & -3.288504 & 0.404250 \\
\hline 35 & 8 & $\odot$ & -4.542270 & -3.310908 & 0.650066 \\
\hline 36 & 1 & $\odot$ & -4.945655 & -1.760315 & -0.268443 \\
\hline 37 & 1 & $\odot$ & -4.889584 & -4.111593 & 0.246063 \\
\hline 38 & 8 & $\odot$ & -4.809612 & -1.002006 & -0.866886 \\
\hline 39 & 1 & $\odot$ & -2.978869 & -0.818995 & -0.421270 \\
\hline 40 & 1 & $\odot$ & -4.865161 & -0.202054 & -0.326647 \\
\hline
\end{tabular}

Standard basis: Aug-CC-pVDZ (6D, 7F)

712 basis functions 
85 alpha electrons $\quad 84$ beta electrons NAtoms $=40$

nuclear repulsion energy 1963.8297941484 Hartrees.

Force inversion solution in PCM.

SCF Done: $E($ UWB97XD $)=-1192.10512192$ A.U. after 2 cycles NFock $=2$ Conv $=0.42 \mathrm{D}-09 \quad-\mathrm{V} / \mathrm{T}=2.0071$

$\langle\mathrm{S} X\rangle=0.0000<\mathrm{S} y\rangle=0.0000\langle\mathrm{Sz}\rangle=0.5000\langle\mathrm{~S} * * 2\rangle=0.7765 \mathrm{~S}=0.5132$

$<$ L.S $>=0.000000000000 \mathrm{E}+00$

Annihilation of the first spin contaminant:

$\mathrm{S}^{* * 2}$ before annihilation 0.7765 , after 0.7503

DoSCS $=$ F DFT $=$ T ScalE2 $(S S, 0 S)=1.0000001 .000000$

Harmonic frequencies $\left(\mathrm{cm}^{*}-1\right)$, IR intensities (KM/Mole), Raman activities $\left(A^{* *} 4 / \mathrm{AMU}\right)$, depolarization ratios for plane and unpo incident light, reduced masses (AMU), force constants (mDyne/A), and normal coordinates:

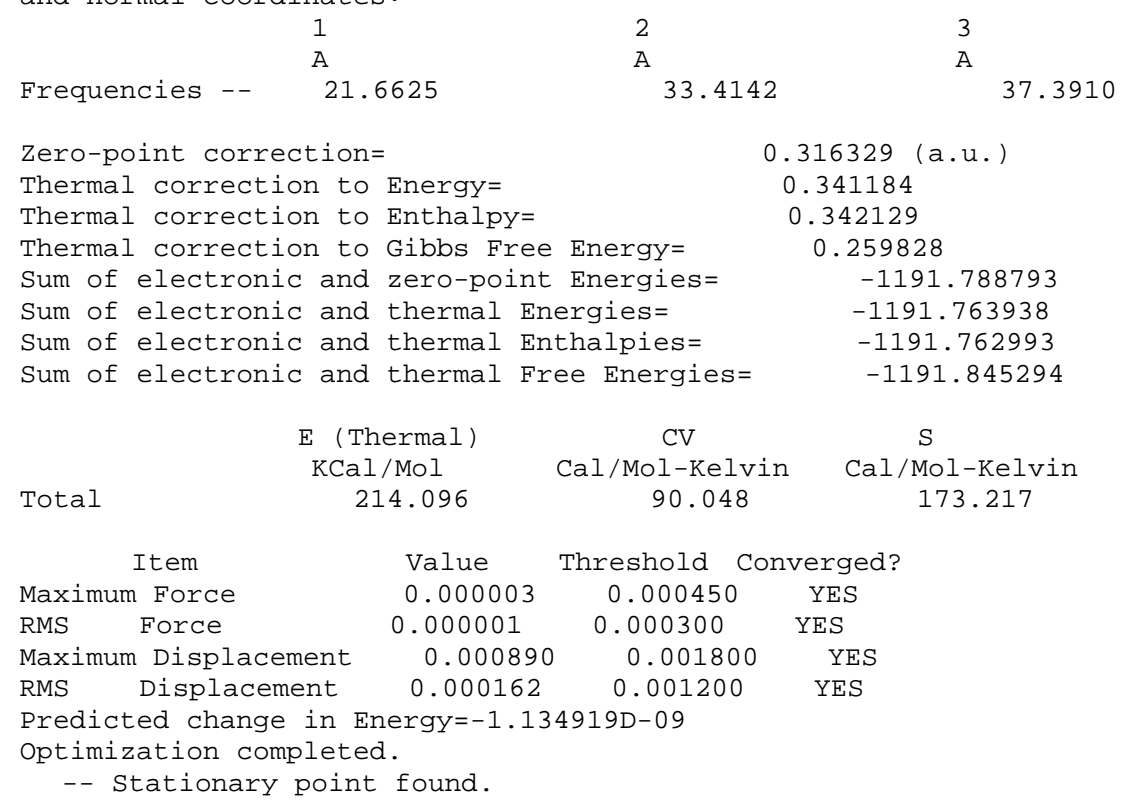

Normal termination of Gaussian 16 at Sat Mar 16 21:07:23 2019.

Figure S6-8, TS2b(C8)W2

degua-oh8w2.higha. $\log$

Stoichiometry $\mathrm{C} 10 \mathrm{H} 18 \mathrm{N507(2)}$

Standard orientation:

\begin{tabular}{|c|c|c|c|c|c|}
\hline \multirow{2}{*}{$\begin{array}{l}\text { Center } \\
\text { Number }\end{array}$} & \multirow{2}{*}{$\begin{array}{l}\text { Atomic } \\
\text { Number }\end{array}$} & \multirow{2}{*}{$\begin{array}{c}\text { Atomic } \\
\text { Type }\end{array}$} & \multicolumn{3}{|c|}{ Coordinates (Angstroms) } \\
\hline & & & $\mathrm{x}$ & $\mathrm{Y}$ & Z \\
\hline - & $-15+3$ & & ------ & ------ & ------ \\
\hline 1 & 1 & $\odot$ & 3.539039 & -1.303474 & 2.099642 \\
\hline 2 & 8 & $\odot$ & 3.150395 & -1.704079 & 1.317192 \\
\hline 3 & 8 & $\odot$ & 4.121967 & 2.250966 & -0.247873 \\
\hline 4 & 6 & $\odot$ & 3.655250 & -0.072580 & -0.470293 \\
\hline 5 & 6 & $\odot$ & 3.627499 & 1.129951 & 0.476431 \\
\hline 6 & 1 & $\odot$ & 4.063746 & 3.026640 & 0.318445 \\
\hline 7 & 6 & $\odot$ & 3.966669 & -1.409816 & $\odot .189880$ \\
\hline 8 & 6 & $\odot$ & 2.143582 & 1.262878 & 0.803559 \\
\hline 9 & 6 & 0 & 1.475180 & ๑. 793487 & -0.493218 \\
\hline 10 & 8 & $\odot$ & 2.365649 & -0.109156 & -1.101347 \\
\hline 11 & 1 & $\odot$ & 3.785837 & -2.204706 & -0.543164 \\
\hline 12 & 1 & $\odot$ & 5.033048 & -1.430153 & ๑. 459409 \\
\hline 13 & 1 & $\odot$ & 4.404355 & ๑. 098332 & -1.255133 \\
\hline 14 & 1 & $\odot$ & 4.238907 & ๑. 958949 & 1.373275 \\
\hline 15 & 1 & $\odot$ & 1.853817 & 2.284226 & 1.069637 \\
\hline 16 & 1 & $\odot$ & 1.877682 & $\odot .593587$ & 1.628587 \\
\hline 17 & 1 & $\odot$ & 1.270067 & 1.635949 & -1.166078 \\
\hline 18 & 7 & $\odot$ & 0.193459 & $\odot .133352$ & -0.280017 \\
\hline 19 & 6 & $\odot$ & 0.042181 & -1.262346 & 0.220941 \\
\hline 20 & 7 & $\odot$ & -1.345645 & -1.250953 & $\odot .705599$ \\
\hline
\end{tabular}




$\begin{array}{rrrrrr}21 & 6 & 0 & -1.915444 & -0.071673 & 0.434286 \\ 22 & 6 & 0 & -0.951266 & 0.808510 & -0.140646 \\ 23 & 7 & 0 & -1.151942 & 2.084794 & -0.457601 \\ 24 & 6 & 0 & -2.394235 & 2.521851 & -0.241560 \\ 25 & 7 & 0 & -3.391775 & 1.742007 & 0.269330 \\ 26 & 6 & 0 & -3.250904 & 0.399133 & 0.664490 \\ 27 & 7 & 0 & -2.679109 & 3.788642 & -0.541384 \\ 28 & 8 & 0 & -4.192994 & -0.215007 & 1.145306 \\ 29 & 1 & 0 & 0.752493 & -1.409624 & 1.045030 \\ 30 & 1 & 0 & -1.945626 & 4.365763 & -0.924247 \\ 31 & 1 & 0 & -3.594610 & 4.190121 & -0.410564 \\ 32 & 1 & 0 & -4.314789 & 2.137027 & 0.409555 \\ 33 & 8 & 0 & 0.258056 & -2.233080 & -0.713436 \\ 34 & 1 & 0 & -0.592332 & -2.452087 & -1.230718 \\ 35 & 8 & 0 & -1.894531 & -3.063285 & -1.816670 \\ 36 & 1 & 0 & -2.357004 & -3.328600 & -0.715180 \\ 37 & 1 & 0 & -2.473023 & -2.427584 & -2.245875 \\ 38 & 8 & 0 & -2.697261 & -3.470540 & 0.419845 \\ 39 & 1 & 0 & -1.889057 & -2.177758 & 0.777156 \\ 40 & 1 & 0 & -2.308799 & -4.284411 & 0.751379 \\ --------\ldots-\ldots-\ldots & --\ldots-\ldots\end{array}$

Standard basis: Aug-CC-pVDZ (6D, 7F)

712 basis functions

85 alpha electrons $\quad 84$ beta electrons

nuclear repulsion energy 2047.7751229593 Hartrees.

NAtoms $=40$ NActive $=40$

Nuclear repulsion after empirical dispersion term =

2047.7424405916 Hartrees.

Force inversion solution in PCM.

SCF Done: $E($ UWB97XD $)=-1192.08652605$ A.U. after 1 cycles

NFock $=1$ Conv $=0.83 \mathrm{D}-08 \quad-\mathrm{V} / \mathrm{T}=2.0070$

$\langle\mathrm{S} x\rangle=0.0000<\mathrm{S} y\rangle=0.0000\left\langle\mathrm{~S} z>=0.5000<\mathrm{S}^{* *} 2>=0.7580 \mathrm{~S}=0.5040\right.$

$<$ L.S $>=0.000000000000 \mathrm{E}+\odot \odot$

Annihilation of the first spin contaminant:

$\mathrm{S}^{* * 2}$ before annihilation 0.7580 , after 0.7500

Harmonic frequencies $\left(\mathrm{cm}^{*}-1\right)$, IR intensities (KM/Mole), Raman activities $\left(A^{* *} 4 / A M U\right)$, depolarization ratios for plane and unpo incident light, reduced masses (AMU), force constants (mDyne/A), and normal coordinates:

\begin{tabular}{|c|c|c|c|c|c|c|c|c|c|c|}
\hline & & & $\begin{array}{l}1 \\
A\end{array}$ & & $\begin{array}{l}2 \\
\text { A }\end{array}$ & & & $\begin{array}{l}3 \\
A\end{array}$ & & \\
\hline Frequ & ncies & $5-$ & -949.89 & & & 23.101 & & & 4.7250 & \\
\hline Red. & lasses & 5 & 1.05 & & & 5.395 & & & .0348 & \\
\hline Frc C & nsts & -- & 0.56 & & & $\odot .0017$ & & & $.0 \odot 43$ & \\
\hline IR In & en & - - & 078.20 & & & 2.6040 & & & .0985 & \\
\hline Atom & AN & $x$ & $Y$ & Z & $x$ & $Y$ & Z & $x$ & Z & \\
\hline 1 & 1 & $\odot .0 \odot$ & $-\odot .0 \odot$ & -0.00 & -0.12 & 0.19 & 0.18 & 0.03 & 0.14 & $\odot .09$ \\
\hline 2 & 8 & $\odot . \odot \odot$ & $\odot . \odot \odot$ & $\odot . \odot \odot$ & -0.07 & 0.12 & 0.19 & 0.01 & $\odot .09$ & 0.12 \\
\hline 3 & 8 & -0.00 & -0.00 & 0.00 & -0.03 & 0.01 & -0.08 & -0.02 & -0.02 & -0.22 \\
\hline 4 & 6 & $-\odot .0 \odot$ & $\odot .0 \odot$ & $\odot . \odot \odot$ & 0.01 & -0.01 & 0.09 & -0.01 & -0.04 & -0.01 \\
\hline 5 & 6 & -0.00 & 0.00 & -0.00 & -0.05 & 0.07 & -0.01 & 0.02 & 0.03 & -0.10 \\
\hline 6 & 1 & $0.0 \odot$ & 0.00 & $\odot .0 \odot$ & -0.08 & 0.06 & -0.16 & -0.01 & 0.03 & -0.28 \\
\hline 7 & 6 & $-\odot . \odot \odot$ & $-\odot . \odot \odot$ & $\odot . \odot \odot$ & $-\odot . \odot \odot$ & 0.05 & $\odot .22$ & $-\odot . \odot \odot$ & 0.01 & $\odot .08$ \\
\hline 8 & 6 & $-0.0 \odot$ & 0.00 & 0.00 & -0.07 & 0.07 & -0.10 & 0.03 & 0.05 & $-\odot .06$ \\
\hline 9 & 6 & $\odot .0 \odot$ & $-0.0 \odot$ & -0.00 & 0.01 & -0.03 & -0.10 & -0.01 & -0.04 & $-\odot . \odot \odot$ \\
\hline 10 & 8 & $-\odot . \odot \odot$ & $\odot . \odot \odot$ & $-\odot . \odot \odot$ & 0.04 & $-\odot .09$ & 0.03 & -0.02 & -0.07 & 0.02 \\
\hline 11 & 1 & $-0.0 \odot$ & $\odot . \odot \odot$ & $-0.0 \odot$ & 0.05 & -0.02 & $\odot .27$ & -0.02 & -0.05 & 0.15 \\
\hline 12 & 1 & $\odot . \odot \odot$ & $\odot .0 \odot$ & $-\odot . \odot \odot$ & -0.02 & 0.08 & $\odot .28$ & $\odot . \odot \odot$ & $\odot .02$ & 0.07 \\
\hline 13 & 1 & $-0.0 \odot$ & $\odot .0 \odot$ & $-\odot . \odot \odot$ & 0.05 & -0.07 & 0.11 & -0.02 & -0.11 & -0.04 \\
\hline 14 & 1 & $-0.0 \odot$ & $0.0 \odot$ & $\odot .00$ & -0.10 & 0.15 & 0.04 & 0.06 & 0.11 & -0.12 \\
\hline 15 & 1 & $-0.0 \odot$ & $\odot . \odot \odot$ & $-0.0 \odot$ & -0.10 & $\odot .09$ & -0.19 & $\odot .04$ & $\odot .06$ & -0.11 \\
\hline 16 & 1 & -0.00 & -0.00 & $-\odot . \odot \odot$ & -0.11 & 0.13 & -0.07 & $\odot .07$ & 0.10 & $-\odot . \odot \odot$ \\
\hline 17 & 1 & $-0.0 \odot$ & -0.00 & $-0.0 \odot$ & 0.04 & -0.09 & -0.19 & -0.04 & -0.09 & -0.05 \\
\hline 18 & 7 & -0.00 & 0.00 & 0.00 & 0.00 & -0.03 & -0.12 & 0.00 & -0.04 & 0.08 \\
\hline 19 & 6 & 0.01 & -0.01 & -0.01 & $\odot . \odot \odot$ & -0.04 & -0.15 & 0.01 & -0.03 & 0.12 \\
\hline 20 & 7 & 0.01 & 0.02 & $\odot . \odot \odot$ & 0.02 & $-\odot .03$ & -0.09 & -0.00 & -0.03 & 0.10 \\
\hline 21 & 6 & -0.00 & 0.01 & $-\odot . \odot \odot$ & 0.03 & -0.02 & -0.04 & -0.00 & -0.03 & 0.07 \\
\hline 22 & 6 & $\odot .0 \odot$ & 0.00 & -0.00 & $\odot .02$ & -0.02 & -0.05 & 0.00 & -0.04 & 0.06 \\
\hline 23 & 7 & -0.00 & 0.00 & $\odot . \odot \odot$ & 0.03 & -0.00 & 0.00 & $\odot .00$ & -0.04 & 0.03 \\
\hline 24 & 6 & 0.00 & 0.00 & -0.00 & 0.05 & 0.02 & 0.08 & -0.00 & -0.04 & 0.01 \\
\hline 25 & 7 & $-\odot . \odot \odot$ & $\odot . \odot \odot$ & $\odot .0 \odot$ & 0.06 & 0.02 & 0.09 & -0.01 & -0.04 & 0.01 \\
\hline 26 & 6 & -0.00 & $\odot .0 \odot$ & -0.00 & 0.05 & -0.00 & 0.04 & -0.01 & -0.03 & 0.04 \\
\hline
\end{tabular}




$\begin{array}{rrrrc}27 & 7 & 0.0 \odot & -0.0 \odot & 0.0 \odot \\ 28 & 8 & 0.0 \odot & -0.0 \odot & -0.0 \odot \\ 29 & 1 & 0.00 & 0.0 \odot & 0.0 \odot \\ 30 & 1 & 0.0 \odot & -0.0 \odot & 0.0 \odot \\ 31 & 1 & -0.0 \odot & -0.0 \odot & -0.0 \odot \\ 32 & 1 & -0.0 \odot & 0.0 \odot & -0.0 \odot \\ 33 & 8 & -0.02 & -0.0 \odot & -0.0 \odot \\ 34 & 1 & -0.10 & -0.06 & -0.02 \\ 35 & 8 & 0.03 & 0.02 & -0.02 \\ 36 & 1 & -0.29 & -0.22 & 0.90 \\ 37 & 1 & 0.07 & 0.03 & -0.05 \\ 38 & 8 & -0.01 & -0.02 & -0.02 \\ 39 & 1 & 0.07 & 0.13 & 0.08 \\ 40 & 1 & -0.01 & -0.04 & -0.07\end{array}$

$\begin{array}{ccc}0.06 & 0.03 & 0.14 \\ 0.06 & 0.00 & 0.06 \\ 0.03 & -0.06 & -0.18 \\ 0.05 & 0.03 & 0.13 \\ 0.07 & 0.05 & 0.19 \\ 0.07 & 0.03 & 0.15 \\ -0.05 & -0.02 & -0.18 \\ -0.08 & -0.04 & -0.12 \\ -0.10 & -0.07 & -0.04 \\ -0.05 & -0.05 & -0.01 \\ -0.13 & -0.08 & -0.02 \\ -0.00 & -0.03 & 0.01 \\ 0.02 & -0.03 & -0.06 \\ 0.01 & -0.03 & 0.00\end{array}$

$-0.00 \quad-0.05 \quad-0.02$

$\begin{array}{lll}-0.02 & -0.03 & 0.03\end{array}$

$\begin{array}{lll}-0.00 & 0.00 & 0.13\end{array}$

$-0.00-0.06 \quad-0.02$

$\begin{array}{lll}-0.01 & -0.05 & -0.04\end{array}$

$\begin{array}{lll}-0.01 & -0.04 & -0.02\end{array}$

$0.03 \quad-0.05 \quad 0.16$

$0.08 \quad 0.07 \quad 0.03$

$0.05 \quad 0.28-0.15$

$\begin{array}{lll}-0.01 & 0.18 & -0.20\end{array}$

$0.14 \quad 0.41 \quad-0.07$

$\begin{array}{lll}-0.07 & 0.05 & -0.23\end{array}$

$-0.02-0.02-0.01$

Zero-point correction=

Thermal correction to Energy=

Thermal correction to Enthalpy=

Thermal correction to Gibbs Free Energy=

Sum of electronic and zero-point Energies=

Sum of electronic and thermal Energies=

Sum of electronic and thermal Enthalpies=

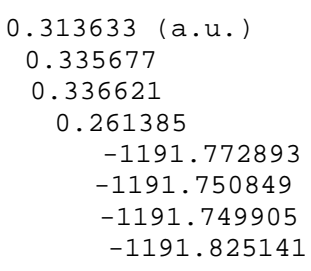

$\begin{array}{cccc} & \text { KCal/Mol } & \text { Cal/Mol-Kelvin } & \text { Cal/Mol-Kelvin } \\ \text { Total } & 210.641 & 83.753 & 158.348\end{array}$

CV

$\begin{array}{lccc} & \text { Value } & \text { Threshold } & \text { Converged? } \\ \text { Maximum Force } & 0.00 \odot \odot \odot 4 & 0.00 \odot 45 \odot & \text { YES } \\ \text { RMS Force } & 0.000 \odot \odot 1 & 0.00030 \odot & \text { YES } \\ \text { Maximum Displacement } & 0.000657 & 0.00180 \odot & \text { YES } \\ \text { RMS Displacement } & 0.000167 & 0.001200 & \text { YES }\end{array}$

Predicted change in Energy $=-3.235058 \mathrm{D}-09$

optimization completed.

- - Stationary point found.

Normal termination of Gaussian 16 at Tue Mar 12 23:14:29 2019.

Figure S6-9, Int3b(C8)W2-

degua-oh8w2 . rev . higha. $\log$

Stoichiometry C10H18N507(2)

Standard orientation:

\begin{tabular}{|c|c|c|c|c|c|}
\hline \multirow{2}{*}{$\begin{array}{l}\text { Center } \\
\text { Number }\end{array}$} & \multirow{2}{*}{$\begin{array}{l}\text { Atomic } \\
\text { Number }\end{array}$} & \multirow{2}{*}{$\begin{array}{c}\text { Atomic } \\
\text { Type }\end{array}$} & \multicolumn{3}{|c|}{ Coordinates (Angstroms) } \\
\hline & & & $\mathrm{x}$ & $\mathrm{Y}$ & Z \\
\hline & & 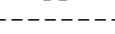 & $-\ldots$ & $---\ldots$ & 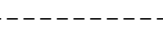 \\
\hline 1 & 1 & $\odot$ & 3.668835 & -1.473561 & 1.983575 \\
\hline 2 & 8 & $\odot$ & 3.273639 & -1.855811 & 1.195257 \\
\hline 3 & 8 & $\odot$ & 4.154094 & 2.201961 & -0.141485 \\
\hline 4 & 6 & $\odot$ & 3.683360 & -0.105488 & -0.503319 \\
\hline 5 & 6 & $\odot$ & 3.675870 & 1.034664 & 0.519562 \\
\hline 6 & 1 & $\odot$ & 4.106037 & 2.939149 & 0.474965 \\
\hline 7 & 6 & $\odot$ & 4.040796 & -1.475090 & 0.060667 \\
\hline 8 & 6 & $\odot$ & 2.200019 & 1.141674 & $\odot .885774$ \\
\hline 9 & 6 & $\odot$ & 1.509328 & 0.770096 & -0.430286 \\
\hline 10 & 8 & $\odot$ & 2.373073 & -0.129618 & -1.086675 \\
\hline 11 & 1 & $\odot$ & 3.845947 & -2.226550 & -0.713636 \\
\hline 12 & 1 & $\odot$ & 5.117456 & -1.491374 & 0. 288160 \\
\hline 13 & 1 & $\odot$ & 4.403675 & 0.128039 & -1.299787 \\
\hline 14 & 1 & $\odot$ & 4.308668 & 0.808642 & 1.389214 \\
\hline 15 & 1 & $\odot$ & 1.914705 & 2.139196 & 1.235283 \\
\hline 16 & 1 & $\odot$ & 1.949031 & 0.406804 & 1.658247 \\
\hline 17 & 1 & $\odot$ & 1.345232 & 1.658833 & -1.054215 \\
\hline 18 & 7 & $\odot$ & $\odot .207800$ & 0.155158 & -0.245087 \\
\hline 19 & 6 & $\odot$ & -0.015090 & -1.309578 & 0.176510 \\
\hline 20 & 7 & $\odot$ & -1.430499 & -1.164021 & 0.684903 \\
\hline 21 & 6 & $\odot$ & -1.936842 & ○. 034444 & 0.397443 \\
\hline 22 & 6 & $\odot$ & -0.905838 & 0.869632 & -0.137270 \\
\hline 23 & 7 & $\odot$ & -1.049808 & 2.163633 & -0.443889 \\
\hline 24 & 6 & $\odot$ & -2.275096 & 2.650120 & -0.265792 \\
\hline 25 & 7 & $\odot$ & -3.325576 & 1.913073 & 0.204696 \\
\hline 26 & 6 & $\odot$ & -3.254593 & 0.562265 & 0.585700 \\
\hline 27 & 7 & $\odot$ & -2.500358 & 3.932581 & -0.562662 \\
\hline
\end{tabular}




\begin{tabular}{|c|c|c|c|c|c|}
\hline 28 & 8 & $\odot$ & -4.238677 & -0.021333 & 1.025611 \\
\hline 29 & 1 & $\odot$ & 0.644727 & -1.467206 & 1.051191 \\
\hline 30 & 1 & $\odot$ & -1.733816 & 4.482967 & -0.918318 \\
\hline 31 & 1 & $\odot$ & -3.404794 & 4.366203 & -0.463056 \\
\hline 32 & 1 & $\odot$ & -4.232698 & 2.349714 & 0.320501 \\
\hline 33 & 8 & $\odot$ & $\odot .096420$ & -2.195642 & -0.772512 \\
\hline 34 & 1 & $\odot$ & -1.188414 & -2.831804 & -1.463849 \\
\hline 35 & 8 & $\odot$ & -1.988502 & -3.334153 & -1.811584 \\
\hline 36 & 1 & $\odot$ & -2.724500 & -3.609293 & -0.239897 \\
\hline 37 & 1 & $\odot$ & -2.473441 & -2.705753 & -2.353417 \\
\hline 38 & 8 & $\odot$ & -2.994145 & -3.562418 & 0.705507 \\
\hline 39 & 1 & $\odot$ & -1.997244 & -2.002844 & 0.871618 \\
\hline 40 & 1 & 0 & -2.599008 & -4.334453 & 1.120809 \\
\hline
\end{tabular}

Standard basis: Aug-CC-pVDZ (6D, 7F)
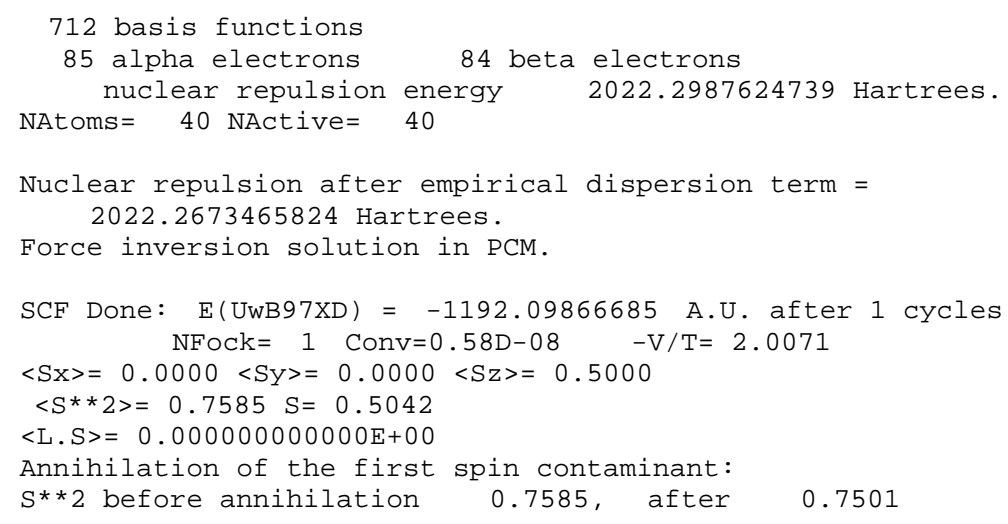

Harmonic frequencies $\left(\mathrm{cm}^{*}-1\right)$, IR intensities (KM/Mole), Raman activities ( $\left.A^{* *} 4 / A M U\right)$, depolarization ratios for plane and unp incident light, reduced masses (AMU), force constants (mDyne/A), and normal coordinates:

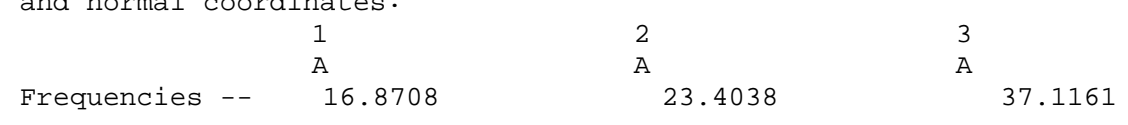

Zero-point correction=

Thermal correction to Energy=

Thermal correction to Enthalpy=
$\odot .317945$ (a.u.)
0.341508
$\odot .342452$
0.262976
$-1191.780722$
$-1191.757159$
$-1191.756214$
$-1191.835691$

Sum of electronic and thermal Energies=

Sum of electronic and thermal Enthalpies=

Sum of electronic and thermal Free Energies=

\section{$\mathrm{S}$}

$$
\begin{gathered}
\text { E (Thermal) } \\
\text { KCal/Mol }
\end{gathered}
$$

$\mathrm{CV}$

Cal/Mol-Kelvin Cal/Mol-Kelvin

Total

214.300

87.339

167.272

\begin{tabular}{lccc}
\multicolumn{1}{c}{ Item } & Value & Threshold Converged? \\
Maximum Force & $0.0000 \odot 4$ & 0.000450 & YES \\
RMS Force & $0.0000 \odot 1$ & $0.00030 \odot$ & YES \\
Maximum Displacement & 0.010490 & $0.00180 \odot$ & NO \\
RMS Displacement & 0.001636 & 0.001200 & NO
\end{tabular}

Predicted change in Energy $=-1.975347 \mathrm{D}-08$

optimization completed on the basis of negligible forces.

- Stationary point found.

Normal termination of Gaussian 16 at Sat Mar 16 20:40:15 2019.

-.............. Figure S6-10, TS3b(C8)W2 .......

degua-oh8w2a. higha. $\log$

Stoichiometry C10H18N507(2)

\begin{tabular}{|c|c|c|c|}
\hline Center & Atomic & Atomic & Coordinates (Angstroms) \\
\hline Number & Number & Type & $\mathrm{X}$ \\
\hline
\end{tabular}

Standard orientation: 


\begin{tabular}{|c|c|c|c|c|c|}
\hline 1 & 1 & 0 & -3.187434 & -1.096347 & -2.386133 \\
\hline 2 & 8 & 0 & -2.801161 & -1.633249 & -1.688892 \\
\hline 3 & 8 & 0 & -4.449051 & 1.701580 & $\odot .624048$ \\
\hline 4 & 6 & 0 & -3.639943 & -0.522401 & 0.360520 \\
\hline 5 & 6 & 0 & -3.768726 & $\odot .852704$ & -0.296986 \\
\hline 6 & 1 & 0 & -4.468987 & 2.590364 & 0.256368 \\
\hline 7 & 6 & 0 & -3.713043 & $-1.7 \odot 4825$ & -0.599471 \\
\hline 8 & 6 & $\odot$ & -2.315324 & 1.264603 & -0.501382 \\
\hline 9 & 6 & 0 & -1.589429 & $\odot .593694$ & 0.674450 \\
\hline 10 & 8 & 0 & -2.394719 & -0.508226 & 1.063341 \\
\hline 11 & 1 & 0 & -3.457528 & -2.613748 & -0.041816 \\
\hline 12 & 1 & 0 & -4.748660 & -1.804958 & -0.958969 \\
\hline 13 & 1 & 0 & -4.442785 & -0.648759 & 1.100783 \\
\hline 14 & 1 & 0 & -4.327061 & 0.802762 & -1.242938 \\
\hline 15 & 1 & 0 & -2.176230 & $2.35 \odot 266$ & -0.503864 \\
\hline 16 & 1 & 0 & -1.933436 & 0.857932 & -1.444058 \\
\hline 17 & 1 & 0 & -1.502112 & 1.283773 & 1.528289 \\
\hline 18 & 7 & 0 & -0.277730 & 0.108012 & 0.339047 \\
\hline 19 & 6 & 0 & $\odot .329023$ & -1.608197 & -0.454650 \\
\hline 20 & 7 & 0 & 1.677644 & -1.082250 & -0.578024 \\
\hline 21 & 6 & 0 & 1.880065 & $\odot .225613$ & -0.346961 \\
\hline 22 & 6 & 0 & $\odot .726251$ & $\odot .919961$ & 0.166139 \\
\hline 23 & 7 & $\odot$ & $\odot .726799$ & 2.258954 & $\odot .414835$ \\
\hline 24 & 6 & 0 & 1.866324 & 2.889879 & 0.214622 \\
\hline 25 & 7 & 0 & 3.014085 & 2.282899 & -0.229031 \\
\hline 26 & 6 & 0 & 3.113412 & $\odot .925910$ & -0.554304 \\
\hline 27 & 7 & 0 & 1.932953 & 4.208333 & $\odot .453281$ \\
\hline 28 & 8 & $\odot$ & 4.167582 & $\odot .451791$ & -0.971099 \\
\hline 29 & 1 & 0 & -0.300805 & -1.408276 & -1.332080 \\
\hline 30 & 1 & 0 & 1.105568 & 4.675840 & $\odot .788922$ \\
\hline 31 & 1 & 0 & 2.776547 & 4.743903 & $\odot .325922$ \\
\hline 32 & 1 & 0 & 3.854715 & 2.830458 & -0.368114 \\
\hline 33 & 8 & 0 & 0.153057 & -2.626874 & 0.226873 \\
\hline 34 & 1 & $\odot$ & 1.310451 & -3.188460 & 1.359121 \\
\hline 35 & 8 & 0 & 2.050761 & -3.596174 & 1.868607 \\
\hline 36 & 1 & 0 & 3.216530 & -3.417638 & $\odot .518057$ \\
\hline 37 & 1 & 0 & 2.219576 & -2.996072 & 2.600670 \\
\hline 38 & 8 & $\odot$ & 3.669980 & -3.135696 & -0.304198 \\
\hline 39 & 1 & 0 & 2.454564 & -1.758775 & -0.615469 \\
\hline 40 & 1 & $\odot$ & 3.587575 & -3.880756 & -0.906739 \\
\hline
\end{tabular}

Standard basis: Aug-CC-pVDZ (6D, 7F)

712 basis functions

85 alpha electrons

84 beta electrons

nuclear repulsion energy 2005.0740580009 Hartrees.

NAtoms $=40$ NActive $=40$

Nuclear repulsion after empirical dispersion term = 2005. 0431739963 Hartrees.

Force inversion solution in PCM.

Error on total polarization charges $=0.01714$

SCF Done: $\mathrm{E}($ UWB97XD) $=-1192.08878111 \quad$ A.U. a
NFock $=2$ Conv $=0.68 \mathrm{D}-09 \quad-\mathrm{V} / \mathrm{T}=2.0071$

$$
\text { NFock }=2 \text { Conv }=0.68 \mathrm{D}-09 \quad-\mathrm{V} / \mathrm{T}=2.0071
$$

2 cycles

$\langle\mathrm{S} X\rangle=0.0000\langle\mathrm{~S} y\rangle=0.0000\langle\mathrm{Sz}\rangle=0.5000\left\langle\mathrm{~S}^{* *} 2\right\rangle=0.7629 \mathrm{~S}=0.5064$

$<$ L.S $>=0.000000000000 \mathrm{E}+00$

Annihilation of the first spin contaminant:

$\mathrm{S}^{* * 2}$ before annihilation 0.7629 , after 0.7501

Harmonic frequencies $\left(\mathrm{cm}^{* *}-1\right)$, IR intensities (KM/Mole), Raman activities $\left(A^{* *} 4 / A M U\right)$, depolarization ratios for plane and unpo incident light, reduced masses (AMU), force constants (mDyne/A), and normal coordinates:

\begin{tabular}{|c|c|c|c|c|c|c|c|c|c|c|}
\hline & & & $\begin{array}{l}1 \\
A\end{array}$ & & $\begin{array}{l}2 \\
\text { A }\end{array}$ & & & $\begin{array}{l}3 \\
A\end{array}$ & & \\
\hline Frequ & encies & s.- & -178.52 & & & 19.321 & & & 24.6121 & \\
\hline Red. & nasses & S - - & 9.380 & & & 5.3783 & & & 5.4927 & \\
\hline Frc c & onsts & - - & 0.176 & & & 0.0012 & & & $\odot .0 \odot 2 \odot$ & \\
\hline IR In & zen & - - & 141.712 & & & 1.0939 & & & 1.4613 & \\
\hline Atom & AN & $x$ & $Y$ & z & $x$ & $\mathrm{Y}$ & Z & $x$ & z & \\
\hline 1 & 1 & -0.05 & -0.04 & -0.03 & 0.07 & 0.21 & $-\odot .09$ & -0.18 & -0.19 & 0.6 \\
\hline 2 & 8 & -0.03 & -0.01 & $-\odot .02$ & $\odot .07$ & 0.16 & -0.13 & -0.17 & -0.16 & 0.10 \\
\hline 3 & 8 & 0.07 & -0.01 & 0.00 & -0.02 & -0.06 & 0.13 & 0.04 & 0.04 & $-\odot . \odot 4$ \\
\hline 4 & 6 & $\odot . \odot 2$ & $-\odot .04$ & $-\odot .01$ & $\odot .01$ & $-\odot .03$ & $-\odot .05$ & $-\odot .02$ & $\odot .01$ & 0.0 \\
\hline 5 & 6 & $\odot .05$ & $-\odot .03$ & $\odot . \odot \odot$ & $-\odot . \odot \odot$ & $\odot .02$ & $\odot .06$ & $-\odot .02$ & -0.03 & $-\odot . \odot$ \\
\hline
\end{tabular}




$\begin{array}{rrrrr}6 & 1 & 0.06 & -0.02 & -0.01 \\ 7 & 6 & -0.02 & -0.02 & -0.02 \\ 8 & 6 & 0.06 & -0.08 & -0.03 \\ 9 & 6 & 0.08 & -0.17 & -0.09 \\ 10 & 8 & 0.05 & -0.08 & -0.04 \\ 11 & 1 & -0.03 & -0.03 & -0.03 \\ 12 & 1 & -0.03 & 0.00 & -0.01 \\ 13 & 1 & 0.05 & -0.02 & 0.02 \\ 14 & 1 & 0.04 & -0.02 & 0.01 \\ 15 & 1 & 0.10 & -0.08 & 0.03 \\ 16 & 1 & 0.01 & -0.03 & -0.07 \\ 17 & 1 & 0.22 & -0.17 & -0.10 \\ 18 & 7 & 0.13 & -0.18 & -0.12 \\ 19 & 6 & -0.26 & 0.36 & 0.23 \\ 20 & 7 & -0.07 & 0.06 & -0.07 \\ 21 & 6 & 0.10 & -0.01 & -0.02 \\ 22 & 6 & 0.09 & -0.06 & -0.03 \\ 23 & 7 & -0.00 & -0.10 & 0.03 \\ 24 & 6 & -0.01 & -0.04 & 0.02 \\ 25 & 7 & 0.0 \odot & 0.01 & 0.01 \\ 26 & 6 & 0.07 & 0.01 & -0.01 \\ 27 & 7 & -0.08 & -0.05 & 0.03 \\ 28 & 8 & 0.08 & 0.06 & -0.02 \\ 29 & 1 & -0.07 & -0.01 & -0.01 \\ 30 & 1 & -0.10 & -0.08 & 0.03 \\ 31 & 1 & -0.10 & -0.01 & 0.02 \\ 32 & 1 & -0.02 & 0.06 & 0.01 \\ 33 & 8 & -0.16 & 0.28 & 0.27 \\ 34 & 1 & -0.08 & 0.17 & -0.02 \\ 35 & 8 & -0.07 & 0.06 & -0.07 \\ 36 & 1 & -0.09 & 0.06 & -0.04 \\ 37 & 1 & -0.01 & -0.02 & -0.02 \\ 38 & 8 & -0.04 & 0.01 & -0.04 \\ 39 & 1 & -0.20 & -0.07 & -0.26 \\ 40 & 1 & 0.05 & -0.06 & 0.03 \\ & & & & \end{array}$

\begin{tabular}{|c|c|c|}
\hline-0.02 & -0.03 & 0.20 \\
\hline$\odot .05$ & 0.05 & $-0,15$ \\
\hline$\odot$. & 0.06 & 0.11 \\
\hline$\odot . \odot \odot$ & -0.04 & \\
\hline-0.01 & -0.06 & -0.02 \\
\hline 0.07 & 0.01 & -0.23 \\
\hline 0.06 & 0.05 & -0.18 \\
\hline-0.00 & -0.11 & -0.08 \\
\hline 0. & $\odot$. & 0.05 \\
\hline-0.02 & 0.06 & 0.21 \\
\hline 0.00 & 0. & 0.07 \\
\hline O. & -0. & 0.10 \\
\hline-0.01 & -0. & -0.0 \\
\hline-0.03 & -0.04 & -0.0 \\
\hline-0.03 & -0. & -0.0 \\
\hline-0.02 & -0.04 & -0.05 \\
\hline-0.01 & -0.04 & -0.0 \\
\hline$\odot .0 \odot$ & -0.05 & $\odot .02$ \\
\hline$\odot .0 \odot$ & -0.05 & 0.01 \\
\hline-0.01 & -0.05 & -0.03 \\
\hline-0.03 & -0.04 & -0.06 \\
\hline 0.02 & -0.06 & 0.0 \\
\hline-0.04 & -0.04 & -0.1 \\
\hline-0.06 & -0.05 & 0.0 \\
\hline 0.03 & -0.06 & 0.07 \\
\hline 0.02 & -0.06 & 0.03 \\
\hline-0.01 & -0.05 & -0.04 \\
\hline 0. & -0.04 & 0.06 \\
\hline$\odot .02$ & 0.18 & 0.10 \\
\hline 0.04 & 0.31 & 0.17 \\
\hline$\odot .02$ & 0.12 & 0.12 \\
\hline 0.02 & 0.47 & 0.05 \\
\hline-0.01 & -0.01 & $\odot .0$ \\
\hline$-\odot$ & -0.03 & $-\odot . \odot$ \\
\hline & & \\
\hline
\end{tabular}

$\begin{array}{ccc}0.03 & 0.02 & -0.10 \\ -0.12 & -0.05 & 0.15 \\ -0.02 & -0.07 & -0.11 \\ 0.01 & -0.00 & -0.08 \\ 0.03 & 0.01 & -0.02 \\ -0.13 & -0.03 & 0.19 \\ -0.14 & -0.03 & 0.20 \\ 0.02 & 0.08 & 0.12 \\ -0.06 & -0.08 & 0.01 \\ 0.00 & -0.07 & -0.19 \\ -0.07 & -0.15 & -0.09 \\ 0.01 & 0.05 & -0.12 \\ 0.00 & 0.00 & -0.09 \\ 0.01 & 0.01 & -0.14 \\ 0.01 & 0.02 & -0.11 \\ 0.02 & 0.00 & -0.05 \\ 0.02 & -0.00 & -0.03 \\ 0.03 & -0.02 & 0.03 \\ 0.05 & -0.03 & 0.09 \\ 0.05 & -0.02 & 0.08 \\ 0.03 & -0.00 & 0.01 \\ 0.07 & -0.04 & 0.17 \\ 0.03 & 0.0 \odot & 0.01 \\ 0.02 & 0.03 & -0.13 \\ 0.07 & -0.04 & 0.18 \\ 0.08 & -0.05 & 0.22 \\ 0.06 & -0.03 & 0.13 \\ 0.01 & -0.00 & -0.16 \\ -0.03 & 0.15 & -0.04 \\ -0.03 & 0.25 & 0.05 \\ -0.00 & 0.11 & 0.05 \\ -0.07 & 0.37 & -0.04 \\ 0.01 & 0.02 & 0.03 \\ 0.01 & 0.01 & -0.08 \\ -0.01 & -0.03 & 0.10\end{array}$

Zero-point correction=

Thermal correction to Energy=

Thermal correction to Enthalpy=

Thermal correction to Gibbs Free Energy=

Sum of electronic and zero-point Energies=

Sum of electronic and thermal Energies=

Sum of electronic and thermal Enthalpies=

Sum of electronic and thermal Free Energies=

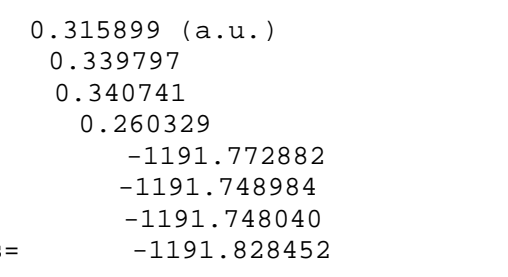

\begin{tabular}{|c|c|c|c|}
\hline $\begin{array}{r}\mathrm{E}(\mathrm{T} \\
\mathrm{KCa} \\
2\end{array}$ & $\begin{array}{l}\text { lermal) } \\
\text { /Mol } \\
13.226\end{array}$ & $\begin{array}{c}\text { CV } \\
\text { Cal/Mol-Kelvin } \\
87.733\end{array}$ & $\begin{array}{c}\mathrm{S} \\
\mathrm{Cal} / \mathrm{Mol}-\mathrm{Kel} \text { in } \\
169.240\end{array}$ \\
\hline Item & Value & \multicolumn{2}{|c|}{ Threshold Converged? } \\
\hline Maximum Force & $\odot .000011$ & 0.000450 & YES \\
\hline Force & 0.000002 & $\odot .000300$ & YES \\
\hline Maximum Displacement & 0.002030 & 0.001800 & NO \\
\hline Displacement & $\odot .000446$ & 0.001200 & YES \\
\hline
\end{tabular}

Normal termination of Gaussian 16 at Tue Mar 12 01:33:46 2019.

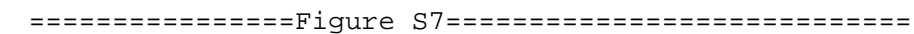

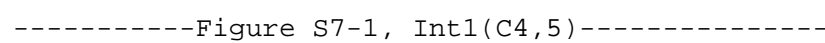

This geometry is the same as that of Int1(C4,5) in Figure S1-1.

-

degua-oh5.higha. $\log$

Stoichiometry C10H14N505(2)

Standard orientation:

\begin{tabular}{|c|c|c|c|c|c|}
\hline \multirow{2}{*}{$\begin{array}{l}\text { Center } \\
\text { Number }\end{array}$} & Atomic & Atomic & \multicolumn{3}{|c|}{ Coordinates (Angstroms) } \\
\hline & Number & Type & $x$ & $\mathrm{Y}$ & Z \\
\hline-1 & & & --- & 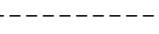 & ----- \\
\hline 1 & 1 & $\odot$ & -3.837280 & -1.908796 & -1.260377 \\
\hline 2 & 8 & $\odot$ & -3.370095 & -2.039497 & -0.430842 \\
\hline
\end{tabular}




\begin{tabular}{|c|c|c|c|c|c|}
\hline 3 & 8 & 0 & -3.822862 & 2.317142 & -0.231865 \\
\hline 4 & 6 & $\odot$ & -3.493322 & $\odot .153387$ & $\odot .698665$ \\
\hline 5 & 6 & $\odot$ & -3.540631 & $\odot .970323$ & -0.595586 \\
\hline 6 & 1 & 0 & -3.817189 & 2.854985 & -1.029665 \\
\hline 7 & 6 & $\odot$ & -4.004224 & -1.277412 & 0.590109 \\
\hline 8 & 6 & 0 & -2.129089 & $\odot .805752$ & -1.151016 \\
\hline 9 & 6 & 0 & -1.280652 & 0.698455 & 0.117075 \\
\hline 10 & 8 & $\odot$ & -2.120167 & 0.161120 & 1.119871 \\
\hline 11 & 1 & $\odot$ & -3.794365 & -1.788559 & 1.537258 \\
\hline 12 & 1 & 0 & -5.094657 & -1.256703 & 0.448435 \\
\hline 13 & 1 & $\odot$ & -4.084171 & $\odot .663943$ & 1.471173 \\
\hline 14 & 1 & $\odot$ & -4.306849 & $\odot .596353$ & -1.288419 \\
\hline 15 & 1 & $\odot$ & -1.818747 & 1.642481 & -1.784243 \\
\hline 16 & 1 & $\odot$ & -2.058932 & -0.119757 & -1.732702 \\
\hline 17 & 1 & $\odot$ & -0.890789 & 1.673010 & 0.434625 \\
\hline 18 & 7 & $\odot$ & -0.118069 & -0.160342 & $-\odot .023279$ \\
\hline 19 & 6 & $\odot$ & -0.121425 & -1.508674 & $-\odot .326583$ \\
\hline 20 & 7 & $\odot$ & 1.070334 & -2.034086 & -0.413911 \\
\hline 21 & 6 & $\odot$ & 1.938430 & -1.000205 & -0.058560 \\
\hline 22 & 6 & $\odot$ & 1.189278 & 0.210068 & ๑. .078068 \\
\hline 23 & 7 & $\odot$ & 1.665921 & 1.443136 & $\odot .256036$ \\
\hline 24 & 6 & $\odot$ & 2.978104 & 1.529977 & $\odot .090313$ \\
\hline 25 & 7 & $\odot$ & 3.759407 & $\odot .478426$ & -0.326744 \\
\hline 26 & 6 & $\odot$ & 3.321641 & -0.836739 & -0.510731 \\
\hline 27 & 7 & $\odot$ & 3.588141 & 2.705702 & $\odot .294808$ \\
\hline 28 & 8 & $\odot$ & 4.060107 & -1.682882 & -0.986473 \\
\hline 29 & 1 & $\odot$ & -1.063536 & -2.030573 & -0.473812 \\
\hline 30 & 1 & $\odot$ & 3.025319 & 3.499704 & $\odot .557526$ \\
\hline 31 & 1 & $\odot$ & 4.581903 & 2.830019 & $\odot .185192$ \\
\hline 32 & 1 & $\odot$ & 4.732727 & 0.646242 & $-\odot .552839$ \\
\hline 33 & 8 & $\odot$ & 2.399977 & -1.320594 & 1.739799 \\
\hline 34 & 1 & $\Theta$ & 2.342088 & -2.284093 & 1.705033 \\
\hline
\end{tabular}

Standard basis: Aug-CC-pVDZ (6D, 7F)

626 basis functions

75 alpha electrons

74 beta electrons

nuclear repulsion energy 1675.5380608757 Hartrees.

NAtoms $=34$ NActive $=34$

Nuclear repulsion after empirical dispersion term $=$

1675.5118091356 Hartrees.

Force inversion solution in PCM.

SCF Done: $E($ UWB97XD $)=-1039.18844641$ A.U. after 1 cycles NFock $=1$ Conv $=0.35 \mathrm{D}-08$

$\langle\mathrm{SX}\rangle=0.0000<\mathrm{Sy}>=0.0000<\mathrm{Sz}\rangle=0.5000$

$<S^{* *} 2>=0.7664 \mathrm{~S}=0.5081$

$<$ L.S $>=0.000000000000 \mathrm{E}+00$

Annihilation of the first spin contaminant:

$\mathrm{S}^{* *} 2$ before annihilation 0.7664 , after 0.7502

Harmonic frequencies $\left(\mathrm{cm}^{* *}-1\right)$, IR intensities (KM/Mole), Raman activities $\left(A^{* *} 4 / A M U\right)$, depolarization ratios for plane and unpo incident light, reduced masses (AMU), force constants (mDyne/A), and normal coordinates:

\begin{tabular}{|c|c|c|c|c|c|c|c|c|c|c|}
\hline & & & 1 & & 2 & & & 3 & & \\
\hline & & & A & & A & & & A & & \\
\hline Erequ & ncies & - - & -321.69 & & & 26.956 & & & 44.3873 & \\
\hline Red. & lasses & - - & 11.84 & & & 5.208 & & & 6.6976 & \\
\hline Frc c & nsts & - - & 0.722 & & & 0.0022 & & & 0.0078 & \\
\hline IR In & & - - & 97.574 & & & 2.7639 & & & 0.1710 & \\
\hline Atom & AN & $x$ & $Y$ & z & $x$ & $Y$ & Z & $x$ & $z$ & \\
\hline 1 & 1 & -0.00 & 0.00 & $-0.0 \odot$ & -0.08 & -0.10 & 0.24 & -0.01 & 0.07 & -0.1 \\
\hline 2 & 8 & -0.00 & $-0 . \odot \odot$ & -0. & -0.01 & -0.03 & 0.21 & 0.06 & 0.02 & -0.1 \\
\hline 3 & 8 & $-\odot . \odot \odot$ & $0.0 \odot$ & $0.0 \odot$ & -0.05 & -0.03 & -0.09 & -0.07 & -0.02 & 0.25 \\
\hline 4 & 6 & 0.00 & 0.6 & -0. & 0.05 & 0.05 & 0.06 & 0.05 & -0.08 & 0.05 \\
\hline 5 & 6 & -0.01 & $0.0 \odot$ & -0. & -0.05 & -0.06 & $-0.0 \odot$ & -0.06 & 0.01 & 0.12 \\
\hline 6 & 1 & $0.0 \odot$ & $\odot .0 \odot$ & 0.00 & -0.12 & -0.10 & -0.14 & -0.13 & 0.04 & 0.29 \\
\hline 7 & 6 & $\odot . \odot \odot$ & $\odot . \odot \odot$ & $-\odot$. & $\odot .06$ & & 0.21 & 0.12 & -0.10 & -0.03 \\
\hline 8 & 6 & -0.02 & -0. & -0 . & -0.08 & $-\odot$ & -0.08 & -0.10 & 0.09 & 0.0 \\
\hline 9 & 6 & 0.00 & -0.01 & -0.03 & $0.0 \odot$ & 0.03 & -0.13 & $-0 . \odot \odot$ & 0.04 & -0.0 \\
\hline 10 & 8 & $\odot .0 \odot$ & $\odot . \odot \odot$ & $-\odot$ & $\odot .07$ & 0.10 & -0.03 & $\odot .07$ & $-\odot . \odot 2$ & $-\odot . \odot$ \\
\hline 11 & 1 & $\odot .0 \odot$ & -0.0 & $-\Theta$ & 0.14 & & & 0.23 & -0.17 & -0.0 \\
\hline 12 & 1 & $\odot .0 \odot$ & $-\odot . \odot \odot$ & $0.0 \odot$ & 0.05 & 0.01 & 0.29 & 0.10 & -0.14 & $\odot . \odot$ \\
\hline 13 & 1 & $\odot . \odot \odot$ & $-\odot . \odot \odot$ & $\odot . \odot \odot$ & $\odot .09$ & 0.10 & 0.06 & 0.06 & -0.17 & 0.1 \\
\hline
\end{tabular}




$\begin{array}{rrrrr}14 & 1 & -0.02 & 0.0 \odot & 0.01 \\ 15 & 1 & -0.02 & -0.0 \odot & -0.01 \\ 16 & 1 & -0.03 & 0.00 & -0.02 \\ 17 & 1 & 0.01 & -0.01 & -0.04 \\ 18 & 7 & -0.00 & -0.01 & -0.03 \\ 19 & 6 & 0.05 & 0.03 & -0.04 \\ 20 & 7 & 0.05 & -0.03 & 0.17 \\ 21 & 6 & 0.11 & -0.12 & 0.66 \\ 22 & 6 & 0.02 & 0.0 \odot & 0.12 \\ 23 & 7 & 0.02 & 0.02 & -0.01 \\ 24 & 6 & -0.01 & 0.03 & -0.00 \\ 25 & 7 & -0.00 & 0.01 & -0.05 \\ 26 & 6 & 0.03 & 0.03 & 0.07 \\ 27 & 7 & 0.03 & 0.03 & 0.00 \\ 28 & 8 & -0.06 & 0.01 & -0.05 \\ 29 & 1 & 0.07 & 0.05 & -0.25 \\ 30 & 1 & 0.05 & 0.04 & -0.00 \\ 31 & 1 & 0.03 & 0.01 & -0.02 \\ 32 & 1 & -0.04 & 0.04 & -0.17 \\ 33 & 8 & -0.18 & -0.0 \odot & -0.54 \\ 34 & 1 & 0.13 & -0.03 & -0.08\end{array}$

$\begin{array}{ccc}-0.08 & -0.13 & 0.07 \\ -0.14 & -0.11 & -0.16 \\ -0.11 & -0.11 & -0.03 \\ 0.02 & 0.05 & -0.23 \\ 0.00 & 0.03 & -0.14 \\ 0.00 & 0.04 & -0.18 \\ 0.00 & 0.03 & -0.14 \\ -0.00 & 0.01 & -0.06 \\ 0.00 & 0.01 & -0.06 \\ 0.01 & -0.00 & 0.01 \\ 0.02 & -0.01 & 0.10 \\ 0.03 & -0.01 & 0.09 \\ 0.02 & 0.01 & 0.02 \\ 0.02 & -0.03 & 0.19 \\ 0.04 & 0.02 & 0.02 \\ 0.01 & 0.04 & -0.22 \\ 0.02 & -0.03 & 0.18 \\ 0.03 & -0.04 & 0.24 \\ 0.04 & -0.01 & 0.16 \\ -0.11 & -0.06 & -0.05 \\ -0.12 & -0.06 & -0.09\end{array}$

$\begin{array}{ccc}-0.10 & 0.05 & 0.14 \\ -0.16 & 0.14 & 0.04 \\ -0.11 & 0.12 & -0.05 \\ -0.00 & 0.03 & -0.03 \\ -0.00 & 0.06 & -0.16 \\ -0.01 & 0.05 & -0.10 \\ -0.02 & 0.02 & -0.01 \\ -0.02 & 0.01 & 0.01 \\ 0.00 & 0.04 & -0.10 \\ 0.02 & 0.04 & -0.15 \\ 0.03 & -0.01 & -0.07 \\ 0.04 & -0.05 & 0.06 \\ 0.02 & -0.05 & 0.11 \\ 0.05 & -0.00 & -0.12 \\ 0.04 & -0.11 & 0.24 \\ -0.02 & 0.06 & -0.13 \\ 0.05 & 0.03 & -0.22 \\ 0.06 & -0.04 & -0.07 \\ 0.06 & -0.09 & 0.12 \\ -0.20 & 0.09 & 0.05 \\ -0.21 & 0.09 & 0.10\end{array}$

Zero-point correction=

Thermal correction to Energy=

0.265221 (a.u. )
0.283812

Thermal correction to Enthalpy=

Thermal correction to Gibbs Free Energy=

Sum of electronic and zero-point Energies=

Sum of electronic and thermal Energies=

Sum of electronic and thermal Enthalpies=

Sum of electronic and thermal Free Energies=

$$
\begin{aligned}
& \odot .283812 \\
& 0.284757 \\
& 0.217772 \\
& -1038.923225 \\
& -1038.904634 \\
& -1038.903690 \\
& -1038.970674
\end{aligned}
$$

\begin{tabular}{|c|c|c|c|}
\hline $\begin{array}{l}\mathrm{E}(\mathrm{T} \\
\mathrm{KCa}\end{array}$ & $\begin{array}{l}\text { Iermal) } \\
\text { Mol } \\
78.095\end{array}$ & $\begin{array}{c}\text { CV } \\
\text { Cal/Mol-Kelvin } \\
70.390\end{array}$ & $\begin{array}{c}\text { S } \\
\text { Cal/Mol-Kelvin } \\
140.981\end{array}$ \\
\hline Item & Value & Threshold Conv & verged? \\
\hline Maximum Force & 0.000004 & 0.000450 & YES \\
\hline Force & 0.000000 & 0.000300 & YES \\
\hline Maximum Displacement & ๑ . 000154 & 0.001800 & YES \\
\hline Displacement & $\odot .0 \odot \odot \odot 32$ & 0.001200 & YES \\
\hline
\end{tabular}

\begin{tabular}{|c|c|c|c|c|c|}
\hline \multirow{2}{*}{$\begin{array}{l}\text { Center } \\
\text { Number }\end{array}$} & \multirow{2}{*}{$\begin{array}{l}\text { Atomic } \\
\text { Number }\end{array}$} & \multirow{2}{*}{$\begin{array}{c}\text { Atomic } \\
\text { Type }\end{array}$} & \multicolumn{3}{|c|}{ Coordinates (Angstroms) } \\
\hline & & & $\mathrm{X}$ & $\mathrm{Y}$ & Z \\
\hline-1 & & -- & -- & - & - \\
\hline 1 & 1 & $\odot$ & -3.710109 & -1.795167 & -1.477768 \\
\hline 2 & 8 & $\odot$ & -3.360735 & -1.992458 & -0.604487 \\
\hline 3 & 8 & $\odot$ & -3.715368 & 2.368924 & -0.255941 \\
\hline 4 & 6 & $\odot$ & -3.541252 & $\odot .156429$ & 0.600469 \\
\hline 5 & 6 & $\odot$ & -3.429255 & 1.031402 & -0.651689 \\
\hline 6 & 1 & $\odot$ & -3.600019 & 2.942796 & -1.019538 \\
\hline 7 & 6 & $\odot$ & -4.083362 & -1.248290 & 0.369697 \\
\hline 8 & 6 & $\odot$ & -1.971556 & 0.848970 & -1.061048 \\
\hline 9 & 6 & $\odot$ & -1.263787 & 0.660217 & ๑. 282714 \\
\hline 10 & 8 & $\odot$ & -2.221942 & $\odot .093140$ & 1.160345 \\
\hline 11 & 1 & $\odot$ & -4.001605 & -1.806259 & 1.310105 \\
\hline 12 & 1 & $\odot$ & -5.148987 & -1.180181 & 0.105415 \\
\hline 13 & 1 & $\odot$ & -4.195869 & 0.653170 & 1.329836 \\
\hline 14 & 1 & $\odot$ & -4.127751 & $\odot .711906$ & -1.437544 \\
\hline 15 & 1 & $\odot$ & -1.574014 & 1.702658 & -1.618949 \\
\hline 16 & 1 & $\odot$ & -1.862192 & -0.051806 & -1.674747 \\
\hline 17 & 1 & $\odot$ & -0.903413 & 1.614023 & 0.687346 \\
\hline 18 & 7 & $\odot$ & -0.107254 & -0.207469 & 0.235393 \\
\hline 19 & 6 & $\odot$ & $-\odot .093133$ & -1.529581 & -0.145739 \\
\hline 20 & 7 & $\odot$ & 1.078154 & -2.083636 & -0.205411 \\
\hline 21 & 6 & $\odot$ & 2.010774 & -1.081202 & 0.277287 \\
\hline 22 & 6 & $\odot$ & 1. 210874 & 0.170622 & 0.391497 \\
\hline
\end{tabular}

Normal termination of Gaussian 16 at Sat Mar 9 22:06:41 2019.

$$
\text { Figure S7-3, Int2(C5) }
$$

Stoichiometry C10H14N505(2)

Standard orientation: 


$\begin{array}{rrrrrr}23 & 7 & 0 & 1.678960 & 1.418686 & 0.443920 \\ 24 & 6 & 0 & 2.934610 & 1.550113 & 0.079369 \\ 25 & 7 & 0 & 3.651882 & 0.511924 & -0.493767 \\ 26 & 6 & 0 & 3.219175 & -0.797756 & -0.601851 \\ 27 & 7 & 0 & 3.563568 & 2.762438 & 0.136199 \\ 28 & 8 & 0 & 3.804106 & -1.610724 & -1.302624 \\ 29 & 1 & 0 & -1.034031 & -2.027207 & -0.374404 \\ 30 & 1 & 0 & 3.078270 & 3.462114 & 0.680675 \\ 31 & 1 & 0 & 4.567949 & 2.768900 & 0.253304 \\ 32 & 1 & 0 & 4.517298 & 0.729286 & -0.974164 \\ 33 & 8 & 0 & 2.513834 & -1.451205 & 1.582667 \\ 34 & 1 & 0 & 2.819622 & -2.364781 & 1.512183 \\ -\end{array}$

Standard basis: Aug-CC-pVDZ (6D, 7F)

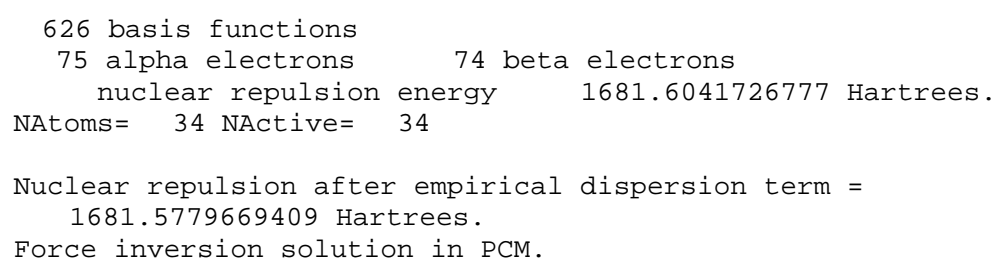

Normal termination of Gaussian 16 at Mon Mar 18 20:34:07 2019.

-....-Figure S7-4, TS2(C5) - . . .

degua-oh5.ts1.higha.log

Stoichiometry C10H14N505(2)

Standard orientation:

\begin{tabular}{|c|c|c|c|c|c|}
\hline \multirow{2}{*}{$\begin{array}{l}\text { Center } \\
\text { Number }\end{array}$} & \multirow{2}{*}{$\begin{array}{l}\text { Atomic } \\
\text { Number }\end{array}$} & \multirow{2}{*}{$\begin{array}{c}\text { Atomic } \\
\text { Type }\end{array}$} & \multicolumn{3}{|c|}{ Coordinates (Angstroms) } \\
\hline & & & $\mathrm{x}$ & $\mathrm{Y}$ & z \\
\hline 1 & 1 & $\odot$ & -3.661706 & -1.302193 & -1.929720 \\
\hline 2 & 8 & $\odot$ & -3.398275 & -1.761937 & -1.127833 \\
\hline 3 & 8 & $\odot$ & -3.564655 & 2.377782 & 0.318992 \\
\hline 4 & 6 & $\odot$ & -3.515919 & 0.012080 & $\odot .585056$ \\
\hline 5 & 6 & $\odot$ & -3.287956 & 1.173546 & $-\odot .389512$ \\
\hline 6 & 1 & $\odot$ & -3.389141 & 3.120581 & -0.266906 \\
\hline 7 & 6 & 0 & -4.130138 & -1.240884 & -0.024533 \\
\hline 8 & 6 & $\odot$ & -1.810925 & 1.036395 & -0.743166 \\
\hline 9 & 6 & 0 & -1.208545 & 0.465759 & 0.542148 \\
\hline 10 & 8 & 0 & -2.235236 & -0.297412 & 1.151209 \\
\hline
\end{tabular}




$\begin{array}{rrrrrr}11 & 1 & 0 & -4.138496 & -2.027296 & 0.739538 \\ 12 & 1 & 0 & -5.172132 & -1.027281 & -0.305370 \\ 13 & 1 & 0 & -4.176933 & 0.351023 & 1.394964 \\ 14 & 1 & 0 & -3.938781 & 1.099054 & -1.271895 \\ 15 & 1 & 0 & -1.347174 & 1.986045 & -1.027391 \\ 16 & 1 & 0 & -1.685544 & 0.325060 & -1.567441 \\ 17 & 1 & 0 & -0.879308 & 1.265133 & 1.216782 \\ 18 & 7 & 0 & -0.059753 & -0.404229 & 0.356684 \\ 19 & 6 & 0 & -0.127666 & -1.570040 & -0.421975 \\ 20 & 7 & 0 & 0.935639 & -2.160411 & -0.796481 \\ 21 & 6 & 0 & 2.191748 & -1.006234 & 0.335039 \\ 22 & 6 & 0 & 1.238686 & 0.049363 & 0.397515 \\ 23 & 7 & 0 & 1.484642 & 1.357584 & 0.227006 \\ 24 & 6 & 0 & 2.715463 & 1.686788 & -0.113010 \\ 25 & 7 & 0 & 3.659192 & 0.746265 & -0.412665 \\ 26 & 6 & 0 & 3.508583 & -0.621463 & -0.174645 \\ 27 & 7 & 0 & 3.062243 & 2.982677 & -0.200516 \\ 28 & 8 & 0 & 4.445174 & -1.384805 & -0.383871 \\ 29 & 1 & 0 & -1.138521 & -1.909667 & -0.674917 \\ 30 & 1 & 0 & 2.341282 & 3.677803 & -0.085695 \\ 31 & 1 & 0 & 3.961092 & 3.275679 & -0.549144 \\ 32 & 1 & 0 & 4.575259 & 1.034294 & -0.737642 \\ 33 & 8 & 0 & 2.223200 & -2.011168 & 1.292979 \\ 34 & 1 & 0 & 1.874720 & -2.812984 & 0.877324 \\ ---\ldots-\ldots-\ldots & -\ldots-\ldots\end{array}$

Standard basis: Aug-CC-pVDZ (6D, 7F)

626 basis functions

75 alpha electrons

nuclear repulsion energy 1676.6640398586 Hartrees.

NAtoms $=34$ NActive $=34$

Nuclear repulsion after empirical dispersion term = 1676.6376391677 Hartrees.

Force inversion solution in PCM.

SCF Done: $E($ UWB97XD $)=-1039.15349697$ A.U. after 1 cycles NFock $=1$ Conv $=0.37 \mathrm{D}-08$ $\mathrm{V} / \mathrm{T}=2.0071$

$\langle\mathrm{S} x\rangle=0.0000\langle\mathrm{~S} y\rangle=0.0000\langle\mathrm{Sz}\rangle=0.5000\left\langle\mathrm{~S}^{* *} 2\right\rangle=0.7801 \mathrm{~S}=0.5150$ $<$ L.S $>=0.000000000000 \mathrm{E}+00$

Annihilation of the first spin contaminant:

$\mathrm{S}^{* * 2}$ before annihilation 0.7801 , after 0.7504

Harmonic frequencies $\left(\mathrm{cm}^{* *}-1\right)$, IR intensities (KM/Mole), Raman activities ( $\left.A^{* *} 4 / A M U\right)$, depolarization ratios for plane and unpo incident light, reduced masses (AMU), force constants (mDyne/A), and normal coordinates:

\begin{tabular}{|c|c|c|c|c|c|c|c|c|c|c|}
\hline & & & 1 & & 2 & & & 3 & & \\
\hline & & & A & & A & & & A & & \\
\hline requ & ncies & - - & -510.80 & & & 31.734 & & & 4.1714 & \\
\hline Red. & asses & - - & $9.67 \mathrm{C}$ & & & 5.4071 & & & .2099 & \\
\hline Frc c & nsts & - - & 1.486 & & & $\odot .0032$ & & & $.0 \odot 71$ & \\
\hline IR In & & - - & 100.480 & & & 1.0896 & & & .0170 & \\
\hline Atom & AN & $x$ & $Y$ & Z & $x$ & $\mathrm{Y}$ & Z & $x$ & Z & \\
\hline 1 & 1 & $-\odot . \odot \odot$ & $-\odot . \odot \odot$ & $-\odot .0 \odot$ & -0.14 & -0.15 & 0.16 & $\odot .02$ & 0.14 & -0.10 \\
\hline 2 & 8 & $-0.0 \odot$ & $-\odot . \odot \odot$ & $0.0 \odot$ & -0.06 & -0.10 & 0.16 & 0.07 & 0.08 & -0.16 \\
\hline 3 & 8 & $\odot . \odot \odot$ & $-\odot . \odot \odot$ & $-\odot . \odot \odot$ & $-\odot .09$ & $-\odot .02$ & -0.06 & -0.09 & -0.07 & 0.25 \\
\hline 4 & 6 & 0.01 & $-\odot . \odot \odot$ & $\odot . \odot \odot$ & $\odot .01$ & $-\odot .01$ & $\odot .07$ & $-\odot . \odot \odot$ & $-\odot . \odot 9$ & $\odot .02$ \\
\hline 5 & 6 & 0.01 & $-\odot . \odot \odot$ & $\odot . \odot \odot$ & -0.10 & -0.06 & -0.01 & -0.08 & 0.01 & 0.12 \\
\hline 6 & 1 & $-\odot . \odot \odot$ & $-\odot . \odot \odot$ & $-\odot . \odot \odot$ & -0.16 & -0.05 & -0.12 & -0.14 & $-\odot .01$ & 0.32 \\
\hline 7 & 6 & $\odot . \odot \odot$ & $\odot . \odot \odot$ & $\odot . \odot \odot$ & 0.01 & $-\odot . \odot 6$ & $\odot .19$ & $\odot . \odot 7$ & $-\odot . \odot 7$ & $-\odot . \odot 9$ \\
\hline 8 & 6 & 0.01 & $-\odot . \odot \odot$ & $\odot . \odot \odot$ & -0.11 & -0.03 & -0.10 & -0.08 & 0.09 & 0.06 \\
\hline 9 & 6 & $\odot .02$ & $-\odot .01$ & $\odot . \odot \odot$ & $-\odot . \odot \odot$ & 0.05 & -0.12 & $-\odot .02$ & $\odot .02$ & $-\odot . \odot \odot$ \\
\hline 10 & 8 & $\odot .02$ & $-\odot . \odot \odot$ & $-\odot . \odot \odot$ & $\odot .06$ & 0.07 & $\odot . \odot \odot$ & $\odot .02$ & -0.05 & $-\odot .01$ \\
\hline 11 & 1 & $0.0 \odot$ & $\odot . \odot \odot$ & $\odot . \odot \odot$ & 0.09 & -0.02 & 0.23 & 0.14 & -0.15 & -0.16 \\
\hline 12 & 1 & $\odot .0 \odot$ & $\odot .01$ & $\odot . \odot \odot$ & $-\odot .02$ & -0.11 & 0.25 & 0.05 & -0.11 & -0.04 \\
\hline 13 & 1 & $\odot .02$ & $-\odot . \odot \odot$ & 0.01 & 0.06 & $\odot .02$ & 0.10 & $-\odot .02$ & $-\odot .20$ & 0.06 \\
\hline 14 & 1 & 0.01 & $-\odot . \odot \odot$ & $-0.0 \odot$ & -0.15 & -0.13 & 0.04 & -0.11 & 0.07 & 0.14 \\
\hline 15 & 1 & 0.01 & $-\odot . \odot \odot$ & $\odot .0 \odot$ & -0.17 & $-\odot .02$ & -0.18 & -0.13 & 0.13 & 0.12 \\
\hline 16 & 1 & 0.01 & $\odot . \odot \odot$ & $-\odot . \odot \odot$ & -0.15 & $-\odot .06$ & $-\odot .08$ & $-\odot .08$ & 0.16 & $\odot . \odot \odot$ \\
\hline 17 & 1 & 0.04 & -0.02 & $0 . \odot \odot$ & 0.03 & 0.10 & -0.19 & -0.02 & -0.01 & 0.04 \\
\hline 18 & 7 & 0.05 & $-\odot .03$ & $-\odot .02$ & $-\odot . \odot \odot$ & 0.07 & -0.16 & $-\odot . \odot \odot$ & 0.06 & $-\odot .08$ \\
\hline 19 & 6 & -0.12 & $-\odot .03$ & $\odot . \odot 2$ & $\odot .01$ & $\odot . \odot 7$ & -0.16 & $\odot .01$ & $\odot .06$ & $-\odot . \odot 7$ \\
\hline 20 & 7 & -0.37 & -0.26 & -0.23 & 0.01 & 0.06 & -0.12 & 0.02 & 0.03 & -0.01 \\
\hline 21 & 6 & 0.32 & 0.33 & 0.42 & $-\odot . \odot 2$ & 0.01 & $-\odot . \odot 5$ & $-\odot .03$ & $\odot . \odot 2$ & $\odot . \odot 4$ \\
\hline 22 & 6 & $\odot .08$ & -0.04 & $-\odot .04$ & $\odot .01$ & $\odot .04$ & $-\odot . \odot 8$ & $\odot . \odot \odot$ & $\odot .05$ & $-\odot .05$ \\
\hline
\end{tabular}




$\begin{array}{rrrrr}23 & 7 & -0.01 & -0.01 & -0.06 \\ 24 & 6 & 0.02 & 0.01 & -0.02 \\ 25 & 7 & 0.02 & 0.06 & -0.04 \\ 26 & 6 & 0.06 & 0.06 & 0.14 \\ 27 & 7 & -0.02 & 0.01 & 0.00 \\ 28 & 8 & 0.03 & 0.02 & 0.01 \\ 29 & 1 & -0.21 & 0.07 & 0.16 \\ 30 & 1 & -0.02 & 0.00 & -0.01 \\ 31 & 1 & 0.00 & 0.02 & 0.06 \\ 32 & 1 & -0.03 & 0.07 & -0.15 \\ 33 & 8 & -0.06 & -0.07 & -0.08 \\ 34 & 1 & -0.05 & 0.08 & -0.39\end{array}$

$\begin{array}{ccc}0.06 & 0.03 & -0.02 \\ 0.09 & 0.00 & 0.07 \\ 0.08 & -0.02 & 0.10 \\ 0.02 & -0.02 & 0.04 \\ 0.14 & -0.00 & 0.15 \\ 0.01 & -0.04 & 0.08 \\ 0.01 & 0.08 & -0.18 \\ 0.16 & 0.02 & 0.11 \\ 0.17 & -0.02 & 0.20 \\ 0.11 & -0.03 & 0.17 \\ -0.10 & -0.01 & -0.06 \\ -0.10 & 0.01 & -0.09\end{array}$

$0.03 \quad 0.03 \quad-0.13$

$0.05-0.02-0.12$

$0.05 \quad-0.06 \quad 0.00$

$0.01 \quad-0.04 \quad 0.09$

$0.08-0.03 \quad-0.21$

$0.01 \quad-0.07 \quad 0.21$

$0.02 \quad 0.07 \quad-0.11$

$0.09-0.01-0.31$

$0.09-0.08 \quad-0.23$

$0.07 \quad-0.10 \quad 0.03$

$\begin{array}{lll}-0.12 & 0.06 & 0.08\end{array}$

Zero-point correction=

Thermal correction to Energy=

Thermal correction to Enthalpy=

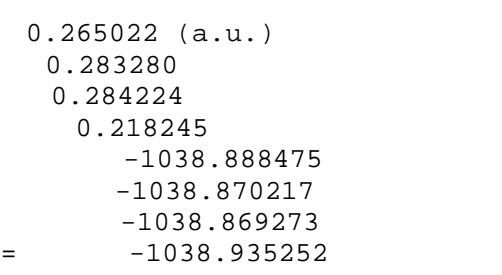

Thermal correction to Gibbs Free Energy=
Sum of electronic and zero-point Energies

Sum of electronic and thermal Energies=

Sum of electronic and thermal Enthalpies=

Sum of electronic and thermal Free Energies=

\section{$\mathrm{S}$}

$$
\begin{gathered}
\text { E (Thermal) } \\
\text { KCal/Mol }
\end{gathered}
$$

CV

$\mathrm{Cal} / \mathrm{Mol}-\mathrm{Kelvin}$

Cal/Mol-Kelvin

$$
70.109
$$

\begin{tabular}{|c|c|c|c|}
\hline Item & Value & Threshold C & Converged? \\
\hline Maximum Force & $\odot .000026$ & $\odot .000450$ & YES \\
\hline Force & 0.000002 & 0.000300 & YES \\
\hline Maximum Displacement & 0.000438 & 0.001800 & YES \\
\hline Displacement & $\odot .0 \odot \odot 109$ & 0.001200 & YES \\
\hline $\begin{array}{l}\text { Predicted change in } \\
\text { Optimization complet }\end{array}$ & ergy $=-1.471$ & 1966D - ๑8 & \\
\hline
\end{tabular}

138.864

Normal termination of Gaussian 16 at Sat Mar 9 20:29:57 2019.

Figure S7-5, Int3(C5)

degua-oh5.for1.higha.log

\begin{tabular}{|c|c|c|c|c|c|}
\hline \multirow{2}{*}{$\begin{array}{l}\text { Center } \\
\text { Number }\end{array}$} & \multirow{2}{*}{$\begin{array}{l}\text { Atomic } \\
\text { Number }\end{array}$} & \multirow{2}{*}{$\begin{array}{l}\text { Atomic } \\
\text { Type }\end{array}$} & \multicolumn{3}{|c|}{ Coordinates (Angstroms) } \\
\hline & & & $x$ & $\mathrm{Y}$ & Z \\
\hline & & & -----.-- & 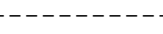 & ------- \\
\hline 1 & 1 & $\odot$ & -4.077092 & -1.125374 & -1.601930 \\
\hline 2 & 8 & $\odot$ & -3.719043 & -1.612496 & -0.854461 \\
\hline 3 & 8 & 0 & -3.274061 & 2.521144 & 0.560073 \\
\hline 4 & 6 & $\odot$ & -3.363919 & $\odot .157326$ & $\odot .825460$ \\
\hline 5 & 6 & $\odot$ & -3.228615 & 1.306361 & -0.181547 \\
\hline 6 & 1 & $\odot$ & -3.145680 & 3.253213 & -0.051031 \\
\hline 7 & 6 & $\odot$ & -4.189965 & -1.032402 & $\odot .357065$ \\
\hline 8 & 6 & $\odot$ & -1.857643 & 1.058838 & -0.801566 \\
\hline 9 & 6 & $\odot$ & -1.076120 & 0.424835 & 0.347389 \\
\hline 10 & 8 & $\odot$ & -2.028485 & -0.269789 & 1. 131651 \\
\hline 11 & 1 & $\odot$ & -4.127698 & -1.818270 & 1.119033 \\
\hline 12 & 1 & $\odot$ & -5.242436 & -0.726068 & ○. 267796 \\
\hline 13 & 1 & $\odot$ & -3.820615 & $\odot .543626$ & 1.747186 \\
\hline 14 & 1 & $\odot$ & -4.034807 & 1.291434 & -0.928144 \\
\hline 15 & 1 & $\odot$ & -1.379418 & 1.972716 & -1.166315 \\
\hline 16 & 1 & $\odot$ & -1.940870 & ๑. 352748 & -1.636216 \\
\hline 17 & 1 & $\odot$ & -0.569037 & 1.181502 & $\odot .954044$ \\
\hline 18 & 7 & $\odot$ & -0.038698 & -0.533492 & -0.050635 \\
\hline 19 & 6 & $\odot$ & -0.444425 & -1.688754 & -0.670290 \\
\hline 20 & 7 & $\odot$ & ๑. 297175 & -2.555447 & -1.232701 \\
\hline 21 & 6 & $\odot$ & 2.322086 & -1.011611 & 0. 299125 \\
\hline 22 & 6 & $\odot$ & 1.310926 & -0.129704 & -0.007587 \\
\hline 23 & 7 & $\odot$ & 1.501280 & 1.189488 & $-\odot .291499$ \\
\hline 24 & 6 & $\odot$ & 2.728928 & 1.642732 & -0.259099 \\
\hline 25 & 7 & $\odot$ & 3.792320 & 0.833828 & $\odot .0 \odot 2151$ \\
\hline 26 & 6 & $\odot$ & 3.683276 & -0.527164 & 0.286348 \\
\hline 27 & 7 & $\odot$ & 2.970929 & 2.961869 & -0.460838 \\
\hline 28 & 8 & $\odot$ & 4.694348 & -1.186168 & 0.522311 \\
\hline 29 & 1 & $\odot$ & -1.536423 & -1.845782 & -0.664571 \\
\hline
\end{tabular}

Stoichiometry C10H14N505(2)

Standard orientation:

S112 


$\begin{array}{cccccc}30 & 1 & 0 & 2.200046 & 3.503961 & -0.822155 \\ 31 & 1 & 0 & 3.896132 & 3.272821 & -0.717593 \\ 32 & 1 & 0 & 4.733197 & 1.210675 & 0.016929 \\ 33 & 8 & 0 & 2.142998 & -2.301073 & 0.700126 \\ 34 & 1 & 0 & 1.550422 & -2.756611 & 0.068223 \\ - & - & 0\end{array}$

Standard basis: Aug-CC-pVDZ (6D, 7F)

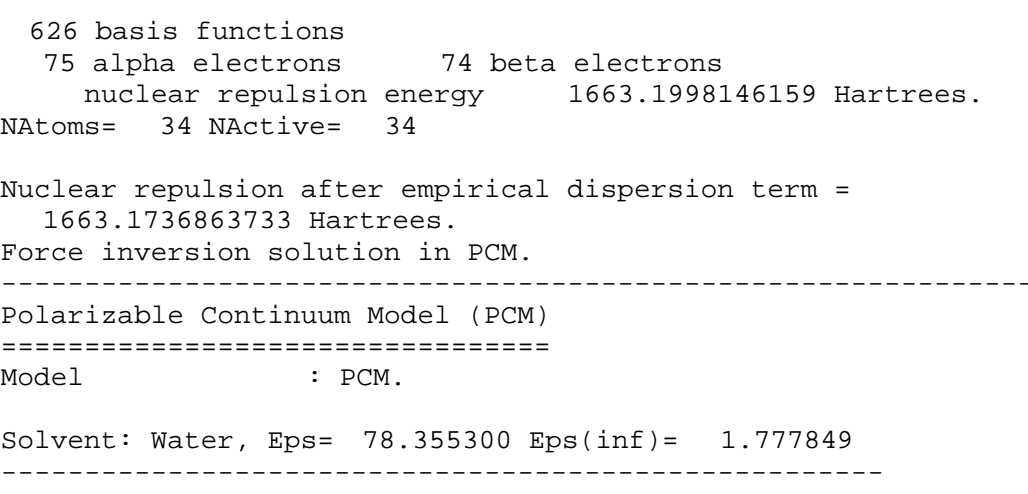

SCF Done: $E($ UWB97XD) $=-1039.18427798$ A.U. after 1 cycles NFock $=1$ Conv $=0.30 \mathrm{D}-08 \quad-\mathrm{V} / \mathrm{T}=2.0071$

$\langle\mathrm{S} x>=0.0000<\mathrm{S} y>=0.0000<\mathrm{Sz}>=0.5000$

$\left\langle\mathrm{S}^{* *} 2>=0.7597 \mathrm{~S}=0.5048\right.$

$<$ L.S $>=0.000000000000 \mathrm{E}+00$

Annihilation of the first spin contaminant:

$\mathrm{S}^{* * 2}$ before annihilation 0.7597 , after 0.7501

Harmonic frequencies $\left(\mathrm{cm}^{* *}-1\right)$, IR intensities (KM/Mole), Raman activities ( $\left.A^{* *} 4 / \mathrm{AMU}\right)$, depolarization ratios for plane and unpo incident light, reduced masses (AMU), force constants (mDyne/A), and normal coordinates:

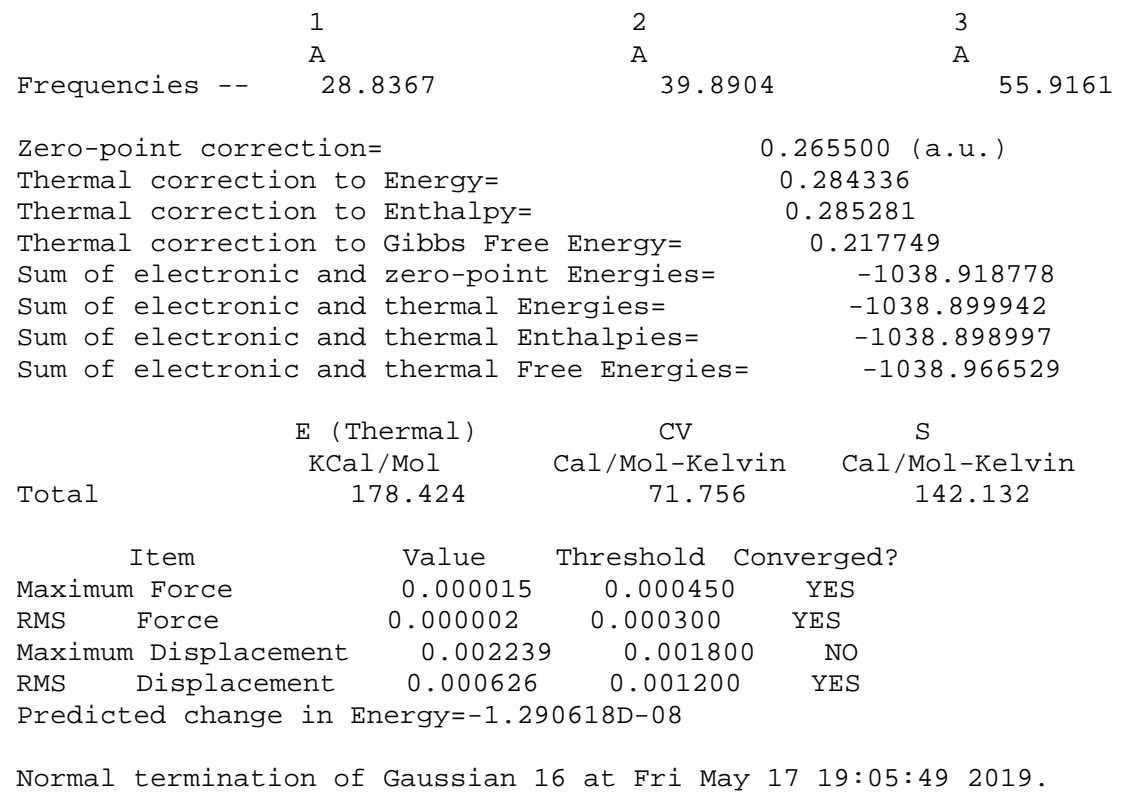

Figure S7-6, TS3(C5)

degua-oh5.ts2.higha. $\log$

Stoichiometry C10H14N505(2)

Standard orientation:

\begin{tabular}{|c|c|c|c|c|c|}
\hline Center & Atomic & Atomic & \multicolumn{3}{|c|}{ Coordinates (Angstroms) } \\
\hline Number & Number & Type & $x$ & $Y$ & Z \\
\hline 1 & 1 & $\odot$ & 3.943171 & -1.158569 & 1.719797 \\
\hline 2 & 8 & 0 & 3.678777 & -1.625452 & 0.922226 \\
\hline 3 & 8 & 0 & 3.221147 & 2.556152 & -0.330022 \\
\hline
\end{tabular}




\begin{tabular}{|c|c|c|c|c|c|}
\hline 4 & 6 & 0 & 3.405651 & $\odot .211921$ & -0.697331 \\
\hline 5 & 6 & 0 & 3.137657 & 1.305325 & $\odot .345125$ \\
\hline 6 & 1 & $\odot$ & 3.016152 & 3.254458 & $\odot .299360$ \\
\hline 7 & 6 & 0 & 4.232517 & -0.970130 & -0.212877 \\
\hline 8 & 6 & 0 & 1.719918 & 0.994657 & 0.813808 \\
\hline 9 & 6 & $\odot$ & 1.079325 & $\odot .4 \odot 2053$ & $-\odot .439299$ \\
\hline 10 & 8 & 0 & 2.118733 & -0.245967 & -1.142434 \\
\hline 11 & 1 & $\odot$ & 4.270022 & -1.718628 & -1.013092 \\
\hline 12 & 1 & $\odot$ & 5.259624 & -0.630512 & -0.014425 \\
\hline 13 & 1 & 0 & 3.925218 & $\odot .659184$ & -1.555882 \\
\hline 14 & 1 & 0 & 3.865342 & 1.272526 & 1.167870 \\
\hline 15 & 1 & 0 & 1.181272 & 1.876461 & 1.172119 \\
\hline 16 & 1 & 0 & 1.740880 & $\odot .247511$ & 1.616051 \\
\hline 17 & 1 & $\odot$ & $\odot .626087$ & 1.177499 & -1.063269 \\
\hline 18 & 7 & $\odot$ & ๑.017706 & -0.593203 & -0.203417 \\
\hline 19 & 6 & 0 & 0.438790 & -1.809221 & 0.335458 \\
\hline 20 & 7 & 0 & $-\odot .259980$ & -2.808518 & 0.684455 \\
\hline 21 & 6 & 0 & -2.371469 & -1.010365 & $-\odot .270901$ \\
\hline 22 & 6 & 0 & -1.300984 & -0.132203 & -0.157797 \\
\hline 23 & 7 & 0 & -1.430486 & 1.208243 & 0.039425 \\
\hline 24 & 6 & $\odot$ & -2.642411 & 1.692149 & 0.144501 \\
\hline 25 & 7 & 0 & -3.749755 & 0.896603 & 0.124878 \\
\hline 26 & 6 & 0 & -3.709208 & -0.484656 & $-\odot .051397$ \\
\hline 27 & 7 & 0 & -2.827511 & 3.026855 & 0.256929 \\
\hline 28 & 8 & 0 & -4.749388 & -1.136935 & -0.044210 \\
\hline 29 & 1 & $\odot$ & 1.531711 & -1.844812 & 0.464774 \\
\hline 30 & 1 & $\odot$ & -2.006855 & 3.589233 & $\odot .423299$ \\
\hline 31 & 1 & 0 & -3.714551 & 3.405106 & 0.551234 \\
\hline 32 & 1 & $\odot$ & -4.673040 & 1.298899 & 0.241035 \\
\hline 33 & 8 & 0 & -2.241775 & -2.298007 & -0.557203 \\
\hline 34 & 1 & 0 & -1.373514 & -2.733730 & 0.032647 \\
\hline
\end{tabular}

Standard basis: Aug-CC-pVDZ (6D, 7F)

626 basis functions

75 alpha electrons $\quad 74$ beta electrons

nuclear repulsion energy 1668.4193334332 Hartrees.

NAtoms $=34$ NActive $=34$

Force inversion solution in PCM.

Polarizable Continuum Model (PCM)

=ニニ=ニニ=ニニ=ニニ=ニニ=ニ=ニニ=ニ=
Model

Solvent : Water, Eps $=78.355300$ Eps $($ inf $)=1.777849$

SCF Done: $E($ UwB97XD) $=-1039.17350590$ A.U. after 2 cycles

NFock $=2$ Conv $=0.63 \mathrm{D}-09 \quad-\mathrm{V} / \mathrm{T}=2.0071$

$\langle\mathrm{S} x>=0.0000<\mathrm{Sy}\rangle=0.0000<\mathrm{S} z>=0.5000$

$<\mathrm{S}^{* *} 2>=0.7647 \mathrm{~S}=0.5073$

$<$ L.S $>=0.000000000000 \mathrm{E}+00$

Annihilation of the first spin contaminant:

$\mathrm{S}^{* *} 2$ before annihilation 0.7647 , after 0.7501

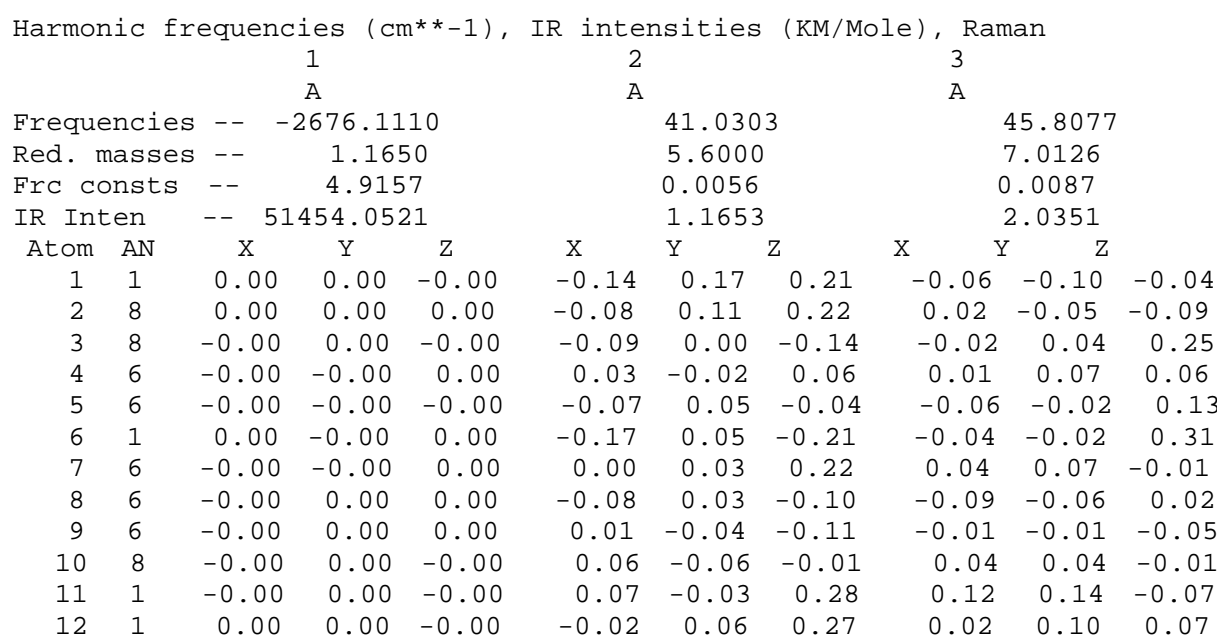




$\begin{array}{rrrrr}13 & 1 & -0.0 \odot & -0.0 \odot & -0.0 \odot \\ 14 & 1 & -0.0 \odot & -0.0 \odot & 0.0 \odot \\ 15 & 1 & 0.0 \odot & 0.0 \odot & -0.0 \odot \\ 16 & 1 & 0.0 \odot & -0.0 \odot & -0.0 \odot \\ 17 & 1 & 0.01 & 0.01 & -0.0 \odot \\ 18 & 7 & -0.02 & 0.02 & -0.01 \\ 19 & 6 & 0.0 \odot & -0.03 & 0.02 \\ 20 & 7 & -0.02 & -0.01 & -0.02 \\ 21 & 6 & -0.01 & -0.06 & -0.02 \\ 22 & 6 & 0.04 & 0.01 & 0.0 \odot \\ 23 & 7 & 0.0 \odot & -0.01 & -0.0 \odot \\ 24 & 6 & -0.01 & 0.01 & -0.0 \odot \\ 25 & 7 & -0.0 \odot & -0.0 \odot & 0.0 \odot \\ 26 & 6 & -0.02 & 0.0 \odot & 0.0 \odot \\ 27 & 7 & 0.0 \odot & -0.01 & 0.0 \odot \\ 28 & 8 & 0.01 & 0.0 \odot & 0.0 \odot \\ 29 & 1 & -0.03 & 0.10 & 0.04 \\ 30 & 1 & 0.0 \odot & 0.0 \odot & -0.01 \\ 31 & 1 & -0.0 \odot & 0.0 \odot & -0.01 \\ 32 & 1 & -0.0 \odot & 0.0 \odot & 0.0 \odot \\ 33 & 8 & -0.03 & 0.05 & -0.01 \\ 34 & 1 & 0.82 & -0.09 & 0.54\end{array}$

$\begin{array}{ccc}0.09 & -0.08 & 0.07 \\ -0.11 & 0.15 & -0.00 \\ -0.13 & 0.03 & -0.17 \\ -0.10 & 0.07 & -0.07 \\ 0.03 & -0.08 & -0.17 \\ 0.00 & -0.05 & -0.12 \\ -0.01 & -0.06 & -0.13 \\ -0.01 & -0.06 & -0.16 \\ -0.01 & 0.00 & -0.04 \\ 0.02 & -0.02 & -0.06 \\ 0.05 & -0.03 & -0.02 \\ 0.07 & -0.01 & 0.07 \\ 0.05 & 0.01 & 0.12 \\ 0.02 & 0.02 & 0.06 \\ 0.10 & -0.01 & 0.13 \\ 0.01 & 0.04 & 0.10 \\ -0.01 & -0.06 & -0.11 \\ 0.12 & -0.03 & 0.10 \\ 0.12 & 0.00 & 0.19 \\ 0.07 & 0.02 & 0.20 \\ -0.04 & 0.01 & -0.09 \\ -0.03 & -0.03 & -0.14\end{array}$

$0.01 \quad 0.16$

$\begin{array}{lll}-0.12 & -0.09 & 0.03\end{array}$

$\begin{array}{llll}-0.13 & -0.10 & -0.01\end{array}$

$\begin{array}{lll}0.02 & 0.01 & -0.04\end{array}$

$-0.01-0.03-0.12$

$\begin{array}{llll}0.01 & -0.03 & -0.13\end{array}$

$0.02-0.03-0.09$

$\begin{array}{lll}-0.03 & -0.02 & 0.04\end{array}$

$-0.00-0.02 \quad-0.09$

$0.02-0.01 \quad-0.17$

$\begin{array}{llll}0.03 & 0.02 & -0.13\end{array}$

$0.02 \quad 0.02 \quad 0.04$

$0.00 \quad 0.01 \quad 0.15$

$0.06 \quad 0.03 \quad-0.26$

$0.00 \quad 0.01 \quad 0.32$

$0.02-0.04-0.19$

$\begin{array}{llll}0.08 & 0.03 & -0.37\end{array}$

$0.09 \quad 0.07 \quad-0.20$

$0.04 \quad 0.04 \quad 0.09$

$\begin{array}{rrrr}-0.09 & -0.08 & -0.03 & 0.08 \\ -0.14 & -0.04 & -0.02 & -0.00\end{array}$

Zero-point correction=

Thermal correction to Energy=

Thermal correction to Enthalpy=

$\odot .260503$ (a.u.)

Thermal correction to Gibbs Free Energy=
Sum of electronic and zero-point Energie

Sum of electronic and thermal Energies=

Sum of electronic and thermal Enthalpies=

$\odot .278590$

$\odot .279534$

0.214045

$-1038.913003$

$-1038.894916$

$-1038.893972$

$-1038.959461$

Sum of electronic and thermal Free Energies=

\section{$\mathrm{S}$}

E (Thermal)

$$
\mathrm{KCal} / \mathrm{Mol}
$$

CV

Cal/Mol-Kelvin

Cal/Mol-Kelvin

Total

$$
174.818
$$
69.785

137.833

\begin{tabular}{lccc}
\multicolumn{1}{c}{ Item } & Value & Threshold & Converged? \\
Maximum Force & $0.000 \odot 26$ & 0.000450 & YES \\
RMS Force & 0.000003 & 0.000300 & YES \\
Maximum Displacement & 0.001251 & $0.00180 \odot$ & YES \\
RMS Displacement & 0.000269 & 0.001200 & YES \\
Predicted change in Energy=-1.332545D-08 & \\
Optimization completed. & & \\
\multicolumn{1}{l}{- - Stationary point found. }
\end{tabular}

Normal termination of Gaussian 16 at Mon Jun 10 18:39:29 2019.

Figure S7-7, Int4(C5)

degua-oh5.for2.higha.log

Stoichiometry C10H14N505(2)

\begin{tabular}{|c|c|c|c|c|c|}
\hline \multirow{2}{*}{$\begin{array}{l}\text { Center } \\
\text { Number }\end{array}$} & \multirow{2}{*}{$\begin{array}{l}\text { Atomic } \\
\text { Number }\end{array}$} & \multirow{2}{*}{$\begin{array}{c}\text { Atomic } \\
\text { Type }\end{array}$} & \multicolumn{3}{|c|}{ Coordinates (Angstroms) } \\
\hline & & & $x$ & $\mathrm{Y}$ & Z \\
\hline & & & -- & $-\cdots---$ & 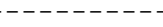 \\
\hline 1 & 1 & $\odot$ & 4.262738 & -1.155340 & 1.410290 \\
\hline 2 & 8 & $\odot$ & 3.869811 & -1.617717 & 0.664924 \\
\hline 3 & 8 & $\odot$ & 3.193896 & 2.569453 & -0.428047 \\
\hline 4 & 6 & $\odot$ & 3.324333 & $\odot .232732$ & -0.873157 \\
\hline 5 & 6 & $\odot$ & 3.209942 & 1.302066 & ๑. 222250 \\
\hline 6 & 1 & $\odot$ & 3.086787 & 3.251510 & $\odot .242099$ \\
\hline 7 & 6 & $\odot$ & 4.222243 & -0.950270 & -0.540391 \\
\hline 8 & 6 & $\odot$ & 1.875203 & $\odot .973938$ & ๑. 882397 \\
\hline 9 & 6 & $\odot$ & 1.058658 & $\odot .418190$ & $-\odot .282133$ \\
\hline 10 & 8 & $\odot$ & 1.990289 & -0.226125 & -1.134290 \\
\hline 11 & 1 & $\odot$ & 4.128367 & -1.691614 & -1.342941 \\
\hline 12 & 1 & $\odot$ & 5.267293 & -0.607233 & -0.514434 \\
\hline 13 & 1 & $\odot$ & 3.712443 & 0.703804 & -1.787335 \\
\hline 14 & 1 & $\odot$ & 4.050012 & 1.254371 & ๑. 929164 \\
\hline 15 & 1 & $\odot$ & 1.396923 & 1.845005 & 1.340899 \\
\hline 16 & 1 & $\odot$ & 2.014217 & 0.204285 & 1.650550 \\
\hline 17 & 1 & $\odot$ & 0.549713 & 1.220936 & $-\odot .824615$ \\
\hline 18 & 7 & $\odot$ & ๑. 022538 & -0.557363 & ๑. .076593 \\
\hline 19 & 6 & $\odot$ & 0.458250 & -1.730557 & 0.701078 \\
\hline
\end{tabular}

Standard orientation: 


\begin{tabular}{|c|c|c|c|c|c|}
\hline 20 & 7 & $\odot$ & -0.194387 & -2.561588 & 1.413187 \\
\hline 21 & 6 & $\odot$ & -2.337186 & -1.071983 & -0.376776 \\
\hline 22 & 6 & $\odot$ & -1.302646 & -0.148412 & 0.033130 \\
\hline 23 & 7 & $\odot$ & -1.522968 & 1.145739 & $\odot .292775$ \\
\hline 24 & 6 & $\odot$ & -2.764323 & 1.608049 & 0.243448 \\
\hline 25 & 7 & $\odot$ & -3.837508 & ๑. 810836 & -0.015237 \\
\hline 26 & 6 & $\odot$ & -3.732908 & -0.538466 & -0.332109 \\
\hline 27 & 7 & $\odot$ & -2.978509 & 2.905441 & 0.478626 \\
\hline 28 & 8 & $\odot$ & -4.730408 & -1.195933 & -0.574195 \\
\hline 29 & 1 & $\odot$ & 1.529300 & -1.884852 & $\odot .550353$ \\
\hline 30 & 1 & $\odot$ & -2.184235 & 3.493008 & $\odot .681535$ \\
\hline 31 & 1 & $\odot$ & -3.898540 & 3.317585 & 0.465688 \\
\hline 32 & 1 & $\odot$ & -4.775371 & 1.197610 & -0.012039 \\
\hline 33 & 8 & $\odot$ & -2.130014 & -2.230605 & -0.787220 \\
\hline 34 & 1 & $\odot$ & -1.181798 & -2.314838 & 1.493371 \\
\hline
\end{tabular}

Standard basis: Aug-CC-pVDZ (6D, 7F)

626 basis functions

75 alpha electrons $\quad 74$ beta electrons

nuclear repulsion energy 1658.0194508953 Hartrees.

NAtoms $=34$ NActive $=34$

Force inversion solution in PCM.

Polarizable Continuum Model (PCM)

=ニニニニニニニニニニニニニニニニニニニニニニニニニニ=ニニニニー

$\begin{array}{ll}\text { Model } & \text { : PCM. }\end{array}$

Solvent: Water, Eps $=78.355300$ Eps $($ inf $)=1.777849$

SCF Done: $E($ UWB97XD) $=-1039.21439603$ A.U. after 1 cycles $\mathrm{NFock}=1$ Conv $=0.47 \mathrm{D}-08 \quad-\mathrm{V} / \mathrm{T}=2.0071$

$<\mathrm{S} x>=0.0000<\mathrm{Sy}>=0.0000<\mathrm{S} z>=0.5000$

$<S^{* *} 2>=0.7612 \mathrm{~S}=0.5056$

$<$ L.S $>=0.000000000000 \mathrm{E}+00$

Annihilation of the first spin contaminant:

$\mathrm{S}^{* * 2}$ before annihilation 0.7612 , after 0.7501

Harmonic frequencies $\left(\mathrm{cm}^{*}-1\right)$, IR intensities (KM/Mole), Raman activities ( $\left.A^{* *} 4 / \mathrm{AMU}\right)$, depolarization ratios for plane and unp incident light, reduced masses (AMU), force constants (mDyne/A), and normal coordinates:

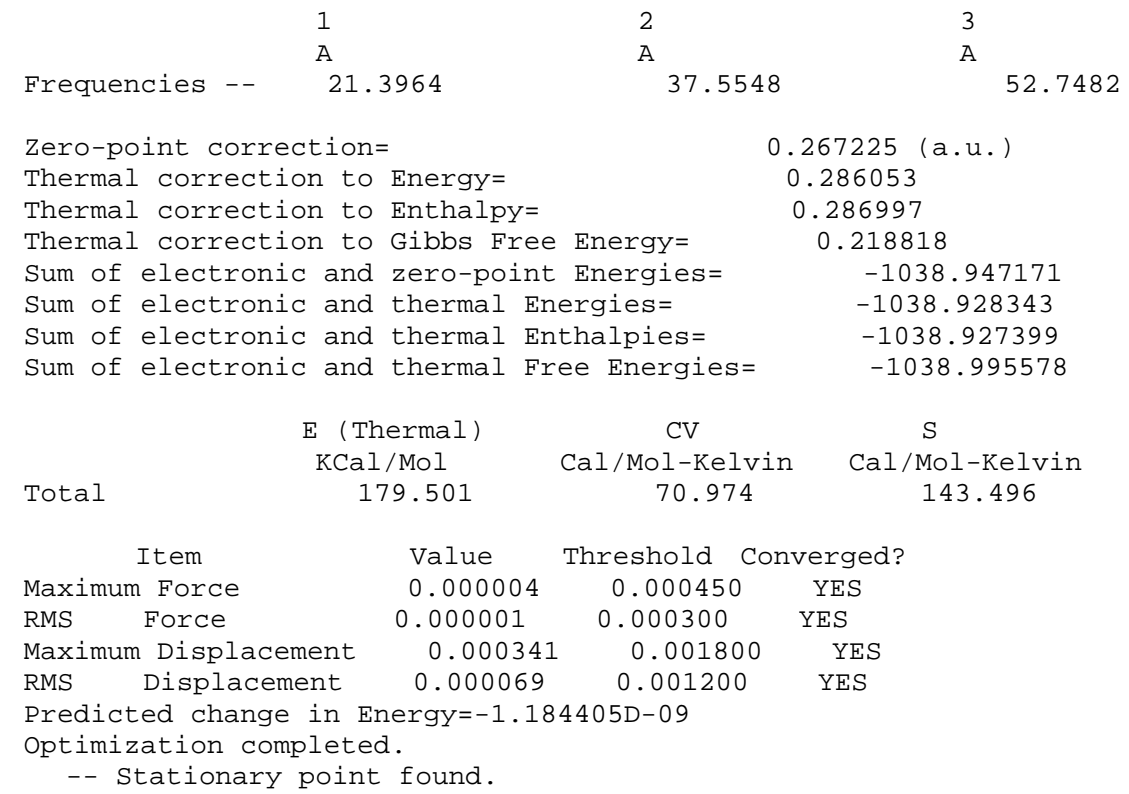

Normal termination of Gaussian 16 at Sun Jun 9 16:36:06 2019. 
degua-oh5w1. for . higha. $\log$

Stoichiometry C10H16N506(2)

Standard orientation:

\begin{tabular}{|c|c|c|c|c|c|}
\hline \multirow{2}{*}{$\begin{array}{l}\text { Center } \\
\text { Number }\end{array}$} & \multirow{2}{*}{$\begin{array}{l}\text { Atomic } \\
\text { Number }\end{array}$} & \multirow{2}{*}{$\begin{array}{l}\text { Atomic } \\
\text { Type }\end{array}$} & \multicolumn{3}{|c|}{ Coordinates (Angstroms) } \\
\hline & & & $\mathrm{x}$ & $\mathrm{Y}$ & Z \\
\hline 1 & 1 & $\odot$ & 3.862393 & 1.851336 & -1.413135 \\
\hline 2 & 8 & $\odot$ & 3.319270 & 2.027953 & -0.640134 \\
\hline 3 & 8 & $\odot$ & 4.174341 & -2.202984 & 0.122421 \\
\hline 4 & 6 & $\odot$ & 3.562650 & $\odot .005180$ & $\odot .756479$ \\
\hline 5 & 6 & 0 & 3.793094 & -0.944572 & -0.422077 \\
\hline 6 & 1 & $\odot$ & 4.281491 & -2.827154 & -0.601854 \\
\hline 7 & 6 & $\odot$ & 3.937774 & 1.460485 & 0.509256 \\
\hline 8 & 6 & $\odot$ & 2.421435 & $-\odot .996481$ & -1.088881 \\
\hline 9 & 6 & $\odot$ & 1.466693 & -0.831099 & 0.094277 \\
\hline 10 & 8 & $\odot$ & 2.167695 & -0.103082 & 1.081975 \\
\hline 11 & 1 & $\odot$ & 3.601038 & 2.051947 & 1.368926 \\
\hline 12 & 1 & $\odot$ & 5.032879 & 1.540328 & $\odot .448551$ \\
\hline 13 & 1 & 0 & 4.136840 & -0.347647 & 1.623713 \\
\hline 14 & 1 & $\odot$ & 4.572694 & -0.573247 & -1.101306 \\
\hline 15 & 1 & $\odot$ & 2.246621 & -1.930284 & -1.631907 \\
\hline 16 & 1 & $\odot$ & 2.307772 & -0.159341 & -1.785840 \\
\hline 17 & 1 & $\odot$ & 1.138197 & -1.795529 & 0.499072 \\
\hline 18 & 7 & $\odot$ & $\odot .246875$ & -0.110745 & -0.233694 \\
\hline 19 & 6 & 0 & $\odot .158600$ & 1.194394 & -0.669367 \\
\hline 20 & 7 & $\odot$ & -1.073163 & 1.590372 & -0.859974 \\
\hline 21 & 6 & $\odot$ & -1.848368 & 0.503115 & $-\odot .498970$ \\
\hline 22 & 6 & 0 & -1.030546 & -0.577064 & -0.129168 \\
\hline 23 & 7 & $\odot$ & -1.401791 & -1.812904 & 0.223935 \\
\hline 24 & 6 & $\odot$ & -2.712788 & -1.997731 & 0.158932 \\
\hline 25 & 7 & 0 & -3.593117 & -1.037976 & -0.266641 \\
\hline 26 & 6 & $\odot$ & -3.262325 & $\odot .276247$ & -0.639410 \\
\hline 27 & 7 & $\odot$ & -3.220148 & -3.188730 & 0.508107 \\
\hline 28 & 8 & 0 & -4.127986 & 1.044289 & -1.034076 \\
\hline 29 & 1 & $\odot$ & 1.054429 & 1.793410 & -0.806519 \\
\hline 30 & 1 & $\odot$ & -2.585010 & -3.911875 & 0.807185 \\
\hline 31 & 1 & 0 & -4.206848 & -3.389410 & 0.479927 \\
\hline 32 & 1 & $\odot$ & -4.579372 & -1.263334 & -0.323386 \\
\hline 33 & 8 & $\odot$ & -2.239078 & 0.905348 & 1.768241 \\
\hline 34 & 1 & 0 & -2.260342 & 1.857620 & 1.555507 \\
\hline 35 & 8 & $\odot$ & -2.172688 & 3.710330 & $\odot .677797$ \\
\hline 36 & 1 & $\odot$ & -1.459080 & 4.220050 & 1.072900 \\
\hline 37 & 1 & $\odot$ & -1.744055 & 3.193334 & -0.030185 \\
\hline
\end{tabular}

Standard basis: Aug-CC-pVDZ (6D, 7F)

669 basis functions

80 alpha electrons 79 beta electrons

nuclear repulsion energy 1841.2916445786 Hartrees.

NAtoms $=37$ NActive $=37$

Force inversion solution in PCM.

Polarizable Continuum Model (PCM)

Model
M PCM.

Solvent: Water, Eps $=78.355300$ Eps $($ inf $)=1.777849$

-

SCF Done: $E($ UWB97XD $)=-1115.62809542$ A.U. after 2 cycles NFock $=2$ Conv $=0.56 \mathrm{D}-09 \quad-\mathrm{V} / \mathrm{T}=2.0071$

$\langle\mathrm{SX}>=0.0000<\mathrm{Sy}\rangle=0.0000<\mathrm{Sz}>=0.5000$

$<S^{* *} 2>=0.7590 \mathrm{~S}=0.5045$

$<$ L.S $>=0.0000000000000 \mathrm{E}+00$

Annihilation of the first spin contaminant:

$\mathrm{S}^{* * 2}$ before annihilation 0.7590 , after 0.7501

Harmonic frequencies $\left(\mathrm{cm}^{* *}-1\right)$, IR intensities (KM/Mole), Raman

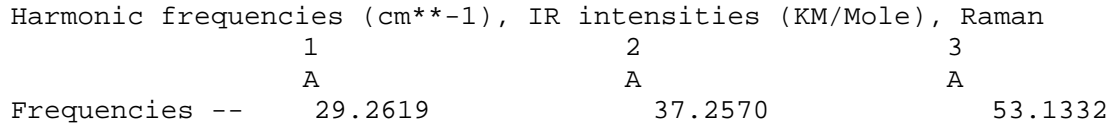


Zero-point correction=

Thermal correction to Energy=

Thermal correction to Enthalpy=

Thermal correction to Gibbs Free Energy=

Sum of electronic and zero-point Energies=

Sum of electronic and thermal Energies=

Sum of electronic and thermal Enthalpies=

Sum of electronic and thermal Free Energies=

$$
\text { E (Thermal) }
$$

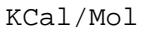

Total

$$
196.235
$$

CV
Cal/Mol-Kelvin
81.737

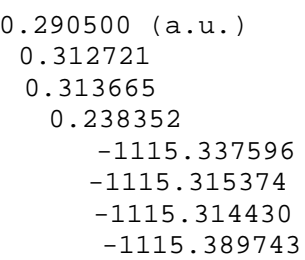

\section{$\mathrm{S}$}

Cal/Mol-Kelvin 158.510

$$
\begin{array}{llcc}
\multicolumn{1}{c}{\text { Item }} & \text { Value } & \text { Threshold Converged? } \\
\text { Maximum Force } & 0.000028 & 0.000450 & \text { YES } \\
\text { RMS Force } & 0.000003 & 0.000300 & \text { YES } \\
\text { Maximum Displacement } & 0.002133 & 0.001800 & \text { NO }
\end{array}
$$$$
\begin{array}{llll}
\text { RMS Force } & 0.000003 & 0.00030 \odot & \text { YES }
\end{array}
$$$$
\begin{array}{llll}
\text { RMS Displacement } & 0.000360 & 0.001200 & \text { YES }
\end{array}
$$

Predicted change in Energy $=-3.056010 \mathrm{D}-\odot 8$

Normal termination of Gaussian 16 at Sun Jun 9 20:07:21 2019.

\begin{tabular}{|c|c|c|c|c|c|}
\hline \multirow{2}{*}{$\begin{array}{l}\text { Center } \\
\text { Number }\end{array}$} & \multirow{2}{*}{$\begin{array}{l}\text { Atomic } \\
\text { Number }\end{array}$} & \multirow{2}{*}{$\begin{array}{c}\text { Atomic } \\
\text { Type }\end{array}$} & \multicolumn{3}{|c|}{ Coordinates (Angstroms) } \\
\hline & & & $\mathrm{x}$ & $\mathrm{Y}$ & Z \\
\hline & & & 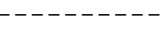 & , & 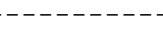 \\
\hline 1 & 1 & $\odot$ & 3.775616 & 1.917294 & -1.442009 \\
\hline 2 & 8 & $\odot$ & 3.290010 & 2.072320 & -0.627341 \\
\hline 3 & 8 & $\odot$ & 4.211454 & -2.172111 & -0.026967 \\
\hline 4 & 6 & $\odot$ & 3.638435 & 0.018731 & 0.698959 \\
\hline 5 & 6 & $\odot$ & 3.784605 & -0.904710 & -0.513775 \\
\hline 6 & 1 & $\odot$ & 4.273168 & -2.778295 & $-\odot .771623$ \\
\hline 7 & 6 & $\odot$ & 3.993363 & 1.480253 & 0.459020 \\
\hline 8 & 6 & $\odot$ & 2.367702 & -0.950221 & -1.078606 \\
\hline 9 & 6 & $\odot$ & 1.501297 & -0.810263 & 0.174126 \\
\hline 10 & 8 & $\odot$ & 2.270046 & -0.098136 & 1.121062 \\
\hline 11 & 1 & $\odot$ & 3.720876 & 2.050268 & 1.355195 \\
\hline 12 & 1 & $\odot$ & 5.080790 & 1.564767 & 0. 318992 \\
\hline 13 & 1 & $\odot$ & 4.273056 & -0.352218 & 1.515017 \\
\hline 14 & 1 & $\odot$ & 4.509635 & -0.514498 & -1.241136 \\
\hline 15 & 1 & $\odot$ & 2.155329 & -1.874570 & -1.624314 \\
\hline 16 & 1 & $\odot$ & 2.201114 & -0.100771 & -1.749575 \\
\hline 17 & 1 & $\odot$ & 1.205382 & -1.783862 & 0.582622 \\
\hline 18 & 7 & $\odot$ & ๑. 258995 & -0.088118 & -0.049888 \\
\hline 19 & 6 & $\odot$ & ๑. 136206 & 1.221669 & -0.458945 \\
\hline 20 & 7 & $\odot$ & -1.103316 & 1.607726 & -0.609937 \\
\hline 21 & 6 & $\odot$ & -1.872427 & 0.522326 & -0.188037 \\
\hline 22 & 6 & $\odot$ & -1.006776 & -0.583725 & ०. .061997 \\
\hline 23 & 7 & $\odot$ & -1.356983 & -1.841163 & 0.334542 \\
\hline 24 & 6 & $\odot$ & -2.649827 & -2.077417 & 0.158199 \\
\hline 25 & 7 & $\odot$ & -3.525135 & -1.151740 & -0.357282 \\
\hline 26 & 6 & $\odot$ & -3.220958 & 0.182873 & -0.642590 \\
\hline 27 & 7 & $\odot$ & -3.140223 & -3.288999 & 0.450501 \\
\hline 28 & 8 & $\odot$ & -4.028834 & 0.907415 & -1.198291 \\
\hline 29 & 1 & $\odot$ & 1.021069 & 1.829934 & -0.627132 \\
\hline 30 & 1 & $\odot$ & -2.507468 & -3.994170 & ๑.794895 \\
\hline 31 & 1 & $\odot$ & -4.116814 & -3.517329 & 0.354194 \\
\hline 32 & 1 & $\odot$ & -4.469281 & -1.440595 & -0.585054 \\
\hline 33 & 8 & $\odot$ & -2.427454 & 0.942345 & 1.581984 \\
\hline 34 & 1 & $\odot$ & -2.562343 & 1.892860 & 1.442738 \\
\hline 35 & 8 & $\odot$ & -2.422446 & 3.874096 & ๑. 449486 \\
\hline 36 & 1 & $\odot$ & -1.781860 & 4.386583 & 0.951116 \\
\hline 37 & 1 & $\odot$ & -1.883566 & 3. 246124 & -0.071023 \\
\hline
\end{tabular}

Figure S8-2, TS1(C5)W1

degua-oh5w1. higha. $\log$

Stoichiometry C10H16N506(2)

Standard orientation

Standard basis: Aug-CC-pVDZ (6D, 7F)

669 basis functions

80 alpha electrons 79 beta electrons nuclear repulsion energy 1841.6220745864 Hartrees. 
Force inversion solution in PCM.

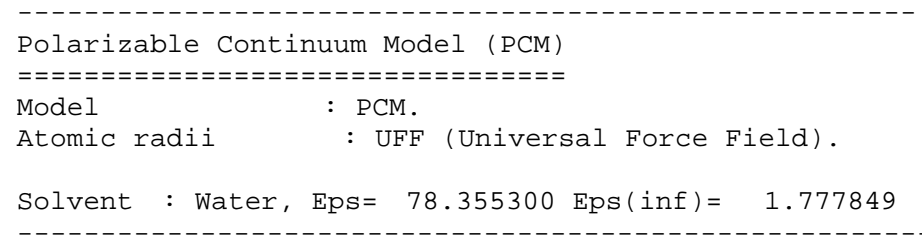

SCF Done: $E($ UWB97XD) $=-1115.62598489$ A.U. after 1 cycles NFock $=1$ Conv $=0.48 \mathrm{D}-08 \quad-\mathrm{V} / \mathrm{T}=2.0071$

$\langle S x>=0.0000\langle$ Sy $>=0.0000\langle S z\rangle=0.5000$

$<S^{* *} 2>=0.7672 \mathrm{~S}=0.5086$

$<$ L.S $>=0.000000000000 \mathrm{E}+00$

Annihilation of the first spin contaminant:

$\mathrm{S}^{* *} 2$ before annihilation 0.7672 , after 0.7502

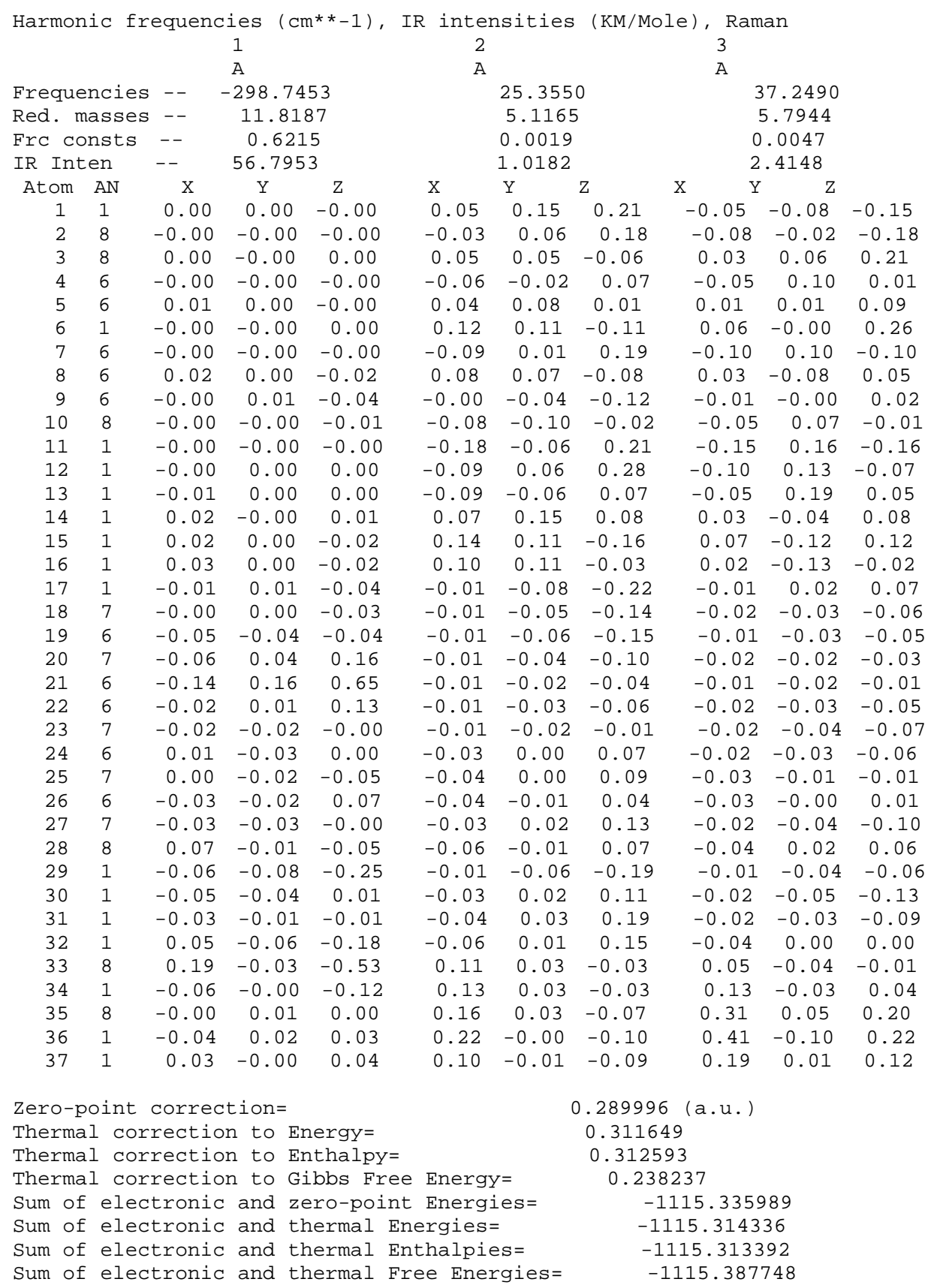




\begin{tabular}{lccc}
\multicolumn{1}{c}{ Item } & Value & Threshold & Converged? \\
Maximum Force & $0.00 \odot \odot 32$ & $0.00 \odot 45 \odot$ & YES \\
RMS Force & 0.000003 & 0.000300 & YES \\
Maximum Displacement & 0.001184 & 0.001800 & YES \\
RMS Displacement & 0.000212 & 0.001200 & YES \\
Predicted change in Energy=-6.475395D- -08 & \\
Optimization completed. & & \\
\multicolumn{2}{l}{- - Stationary point found. } & &
\end{tabular}

Normal termination of Gaussian 16 at Sat Jun 8 16:27:13 2019 .

Figure S8-3, Int2(C5)W1

degua-oh5w1.rev.higha.log

Stoichiometry C10H16N506(2)

Standard orientation:

\begin{tabular}{|c|c|c|c|c|c|}
\hline \multirow{2}{*}{$\begin{array}{l}\text { Center } \\
\text { Number }\end{array}$} & \multirow{2}{*}{$\begin{array}{l}\text { Atomic } \\
\text { Number }\end{array}$} & \multirow{2}{*}{$\begin{array}{l}\text { Atomic } \\
\text { Type }\end{array}$} & \multicolumn{3}{|c|}{ Coordinates (Angstroms) } \\
\hline & & & $X$ & $\mathrm{Y}$ & Z \\
\hline & & & & & \\
\hline 1 & 1 & $\odot$ & 3.642166 & 1.876728 & -1.621133 \\
\hline 2 & 8 & $\odot$ & 3.257065 & 2.091009 & -0.767088 \\
\hline 3 & 8 & $\odot$ & 4.182470 & -2.141750 & $-\odot .064322$ \\
\hline 4 & 6 & $\odot$ & 3.705708 & 0.089373 & 0.608785 \\
\hline 5 & 6 & $\odot$ & 3.725035 & -0.891056 & -0.567670 \\
\hline 6 & 1 & $\odot$ & 4.156464 & -2.784239 & -0.779983 \\
\hline 7 & 6 & $\odot$ & 4.060324 & 1.530363 & 0.265308 \\
\hline 8 & 6 & $\odot$ & 2.260725 & -0.938898 & -0.992197 \\
\hline 9 & 6 & $\odot$ & 1.516409 & -0.729156 & $\odot .328704$ \\
\hline 10 & 8 & $\odot$ & 2.382812 & ๑. . 021689 & 1.160515 \\
\hline 11 & 1 & $\odot$ & 3.894384 & 2.146334 & 1.157138 \\
\hline 12 & 1 & $\odot$ & 5.128499 & 1.583128 & $\odot .008396$ \\
\hline 13 & 1 & $\odot$ & 4.410705 & -0.255800 & 1.377438 \\
\hline 14 & 1 & $\odot$ & 4.383432 & -0.545384 & -1.376753 \\
\hline 15 & 1 & $\odot$ & 1.986230 & -1.882833 & -1.473418 \\
\hline 16 & 1 & $\odot$ & 2.040901 & -0.117414 & -1.682646 \\
\hline 17 & 1 & $\odot$ & 1.266937 & -1.682515 & ๑. 810222 \\
\hline 18 & 7 & $\odot$ & ๑. 262412 & $-\odot .015231$ & ○. 201592 \\
\hline 19 & 6 & $\odot$ & ๑. 101707 & 1.262015 & -0.269307 \\
\hline 20 & 7 & $\odot$ & -1.128796 & 1.661466 & -0.383420 \\
\hline 21 & 6 & $\odot$ & -1.952152 & $\odot .590331$ & 0.158394 \\
\hline 22 & 6 & $\odot$ & -1.004499 & -0.539175 & $\odot .375125$ \\
\hline 23 & 7 & $\odot$ & -1.318356 & -1.827993 & 0.516281 \\
\hline 24 & 6 & $\odot$ & -2.539471 & -2.138989 & 0.143031 \\
\hline 25 & 7 & $\odot$ & -3.359647 & -1.244859 & -0.527785 \\
\hline 26 & 6 & $\odot$ & -3.079450 & 0.094341 & -0.736326 \\
\hline 27 & 7 & $\odot$ & -3.020488 & -3.409437 & ๑. 291669 \\
\hline 28 & 8 & $\odot$ & -3.720751 & 0.770214 & -1.526493 \\
\hline 29 & 1 & $\odot$ & ๑. 979063 & 1.856154 & -0.518424 \\
\hline 30 & 1 & $\odot$ & -2.467521 & -3.998970 & 0.898450 \\
\hline 31 & 1 & $\odot$ & -4.019226 & -3.527367 & ๑. 396642 \\
\hline 32 & 1 & $\odot$ & -4.173363 & -1.605503 & -1.012655 \\
\hline 33 & 8 & $\odot$ & -2.547936 & 0.991296 & 1.406896 \\
\hline 34 & 1 & $\odot$ & -2.882495 & 1.897173 & 1.270587 \\
\hline 35 & 8 & 0 & -2.874666 & 3.686844 & $\odot .417019$ \\
\hline 36 & 1 & $\odot$ & -2.456550 & 4.327777 & $\odot .999771$ \\
\hline 37 & 1 & $\odot$ & -2.133468 & 3.235808 & -0.037286 \\
\hline
\end{tabular}

Standard basis: Aug-CC-pVDZ (6D, 7F)

669 basis functions

80 alpha electrons $\quad 79$ beta electrons

nuclear repulsion energy 1849.5994815736 Hartrees. NAtoms $=37$

Polarizable Continuum Model (PCM)

PO==I $=============$
Model

Solvent : Water, Eps $=78.355300$ Eps $($ inf $)=1.777849$ 
SCF Done: $E(U W B 97 X D)=-1115.6402608$ A.U. after 1 cycles

$$
\text { NFock }=1 \text { Conv }=0.21 \mathrm{D}-08 \quad-\mathrm{V} / \mathrm{T}=2.0071
$$

$\langle\mathrm{S} x\rangle=0.0000<\mathrm{Sy}\rangle=0.0000<\mathrm{S} z>=0.5000$

$<S^{* *} 2>=0.7661 \mathrm{~S}=0.5080$

$<$ L. S $>=0.000000000000 \mathrm{E}+00$

Annihilation of the first spin contaminant:

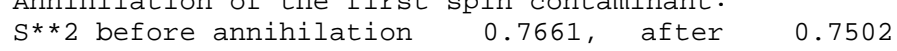

Harmonic frequencies $\left(\mathrm{cm}^{*}-1\right)$, IR intensities (KM/Mole), Raman activities ( $\left.A^{* *} 4 / A M U\right)$, depolarization ratios for plane and unp incident light, reduced masses (AMU), force constants (mDyne/A), and normal coordinates:

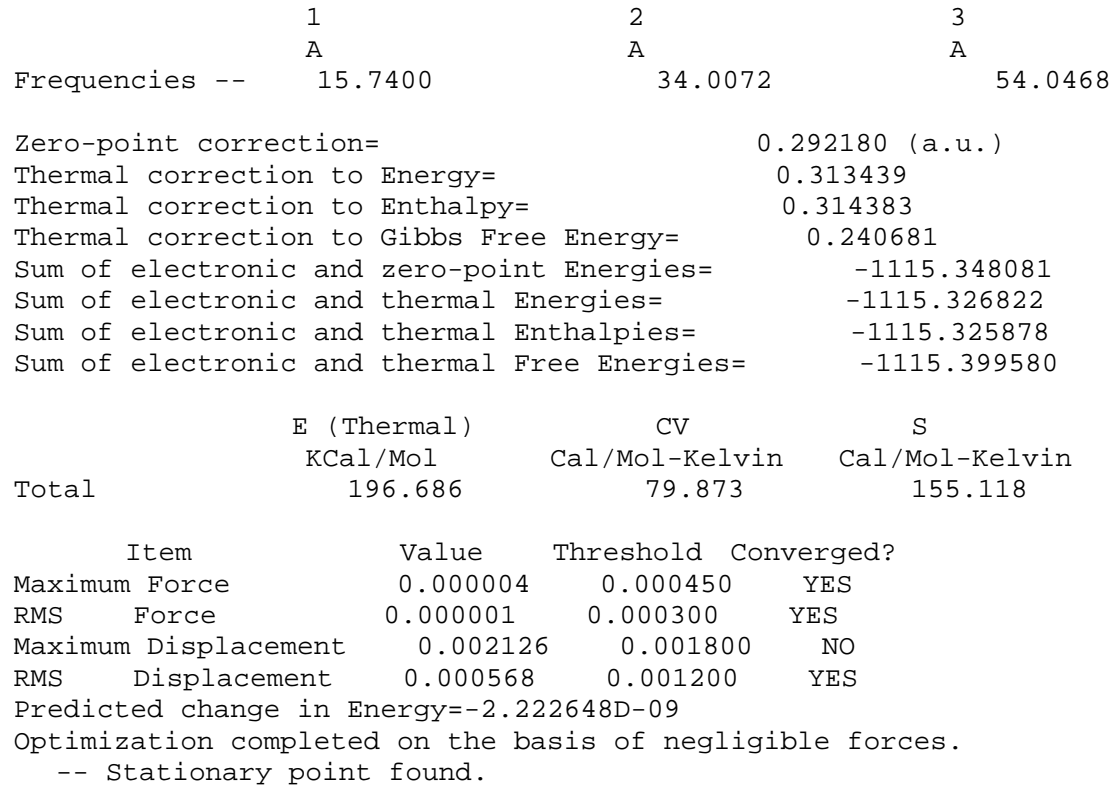

Normal termination of Gaussian 16 at Sun Jun 9 16:45:51 2019.

Figure S8-4, TS2(C5)W1

degua-oh5w1.ts1.higha. $\log$

Stoichiometry C10H16N506(2)

Standard orientation:

\begin{tabular}{|c|c|c|c|c|c|}
\hline \multirow{2}{*}{$\begin{array}{l}\text { Center } \\
\text { Number }\end{array}$} & \multirow{2}{*}{$\begin{array}{l}\text { Atomic } \\
\text { Number }\end{array}$} & \multirow{2}{*}{$\begin{array}{c}\text { Atomic } \\
\text { Type }\end{array}$} & \multicolumn{3}{|c|}{ Coordinates (Angstroms) } \\
\hline & & & $x$ & $\mathrm{Y}$ & Z \\
\hline & & & -- & - - & ------- \\
\hline 1 & 1 & $\odot$ & 3.639959 & 1.452343 & -1.965808 \\
\hline 2 & 8 & $\odot$ & 3.317307 & 1.905288 & $-1 \cdot 181681$ \\
\hline 3 & 8 & $\odot$ & 4.024898 & -2.129684 & 0.370424 \\
\hline 4 & 6 & $\odot$ & 3.669009 & 0.215210 & 0.584139 \\
\hline 5 & 6 & $\odot$ & 3.591432 & -0.987905 & -0.362530 \\
\hline 6 & 1 & $\odot$ & 3.934192 & -2.903134 & -0.194649 \\
\hline 7 & 6 & $\odot$ & 4.106619 & 1.523373 & $-\odot .061173$ \\
\hline 8 & 6 & $\odot$ & 2.107873 & -1.053041 & -0.708892 \\
\hline 9 & 6 & $\odot$ & 1.440709 & -0.540320 & $\odot .569071$ \\
\hline 10 & 8 & $\odot$ & 2.362122 & 0.363793 & 1.155057 \\
\hline 11 & 1 & $\odot$ & 4.000164 & 2.325313 & ๑. 678979 \\
\hline 12 & 1 & $\odot$ & 5.170211 & 1.449223 & -0.332543 \\
\hline 13 & 1 & $\odot$ & 4.374588 & -0.012579 & 1.395361 \\
\hline 14 & 1 & $\odot$ & 4.223887 & -0.849421 & -1.250588 \\
\hline 15 & 1 & $\odot$ & 1.773165 & -2.061611 & -0.970263 \\
\hline 16 & 1 & $\odot$ & 1.885915 & -0.382247 & -1.546672 \\
\hline 17 & 1 & $\odot$ & 1.225599 & -1.362980 & 1.261980 \\
\hline 18 & 7 & $\odot$ & ๑. 186297 & 0.167350 & ๑. 381956 \\
\hline 19 & 6 & $\odot$ & 0.092005 & 1.306460 & -0.431029 \\
\hline 20 & 7 & $\odot$ & -1.039658 & 1.730484 & -0.822420 \\
\hline 21 & 6 & $\odot$ & -2.130835 & 0.446324 & 0.401131 \\
\hline 22 & 6 & $\odot$ & -1.041947 & -0.463480 & 0.422307 \\
\hline 23 & 7 & $\odot$ & -1.107955 & -1.784751 & 0.191400 \\
\hline 24 & 6 & $\odot$ & -2.280652 & -2.269113 & -0.163633 \\
\hline 25 & 7 & 0 & -3.351710 & -1.457974 & -0.418308 \\
\hline
\end{tabular}




$\begin{array}{rrrrrr}26 & 6 & 0 & -3.389970 & -0.097850 & -0.117427 \\ 27 & 7 & 0 & -2.441890 & -3.597845 & -0.304823 \\ 28 & 8 & 0 & -4.423419 & 0.541439 & -0.276055 \\ 29 & 1 & 0 & 1.047239 & 1.774049 & -0.696402 \\ 30 & 1 & 0 & -1.620808 & -4.179484 & -0.239871 \\ 31 & 1 & 0 & -3.273459 & -3.995809 & -0.712007 \\ 32 & 1 & 0 & -4.226257 & -1.856183 & -0.742287 \\ 33 & 8 & 0 & -2.250525 & 1.406547 & 1.379215 \\ 34 & 1 & 0 & -2.564576 & 2.241629 & 0.978801 \\ 35 & 8 & 0 & -2.768279 & 3.753160 & -0.111293 \\ 36 & 1 & 0 & -2.305680 & 4.511956 & 0.257188 \\ 37 & 1 & 0 & -2.082015 & 3.245808 & -0.592976 \\ - & 1 & -\ldots-\ldots-\ldots-\ldots\end{array}$

Standard basis: Aug-CC-pVDZ (6D, 7F)

669 basis functions

80 alpha electrons $\quad 79$ beta electrons

nuclear repulsion energy 1843.8998464973 Hartrees.

NAtoms $=37$ NActive $=37$

Force inversion solution in PCM.

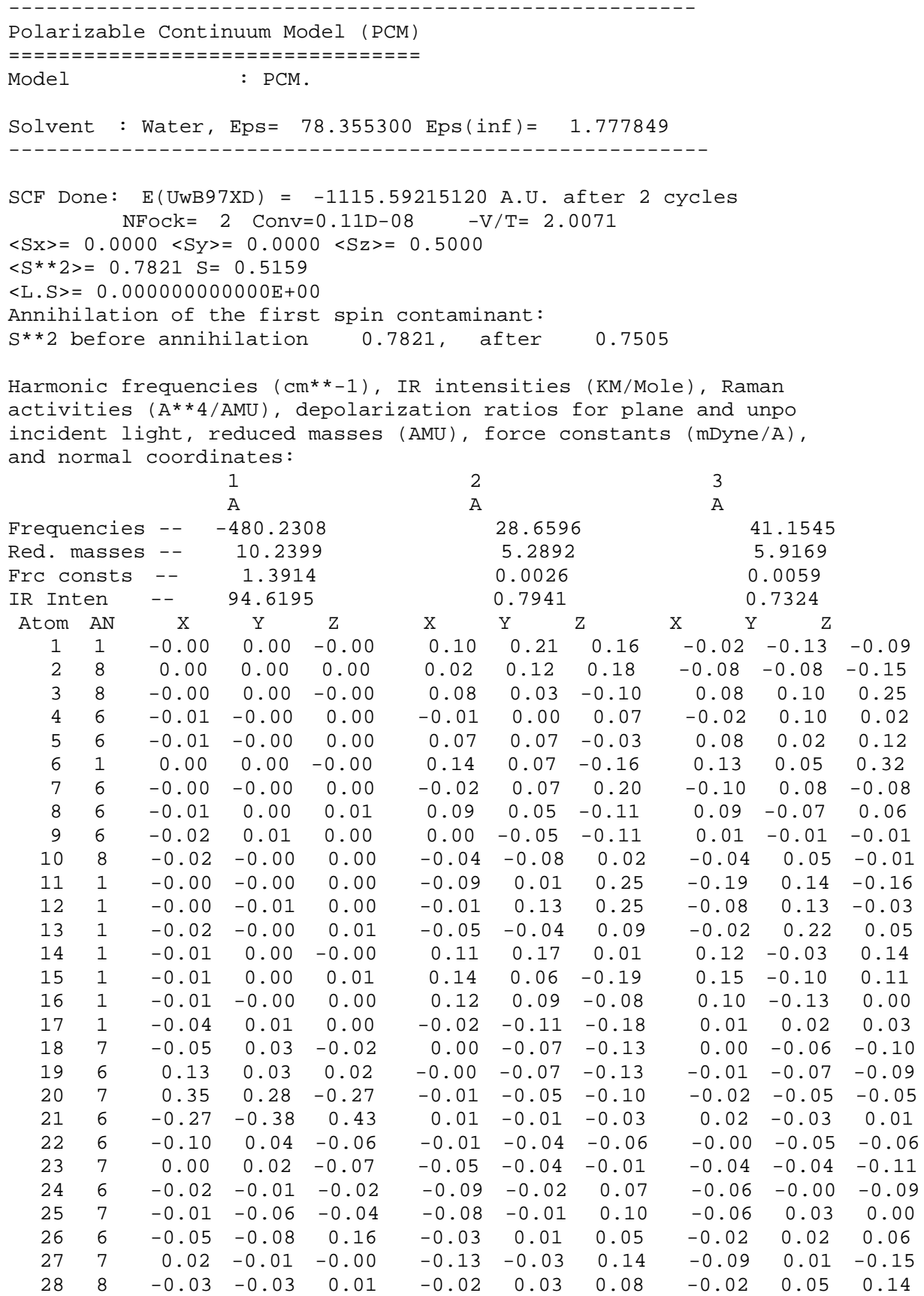




$\begin{array}{rrrrr}29 & 1 & 0.22 & -0.03 & 0.18 \\ 30 & 1 & 0.03 & -0.00 & -0.02 \\ 31 & 1 & -0.01 & -0.03 & 0.07 \\ 32 & 1 & 0.04 & -0.05 & -0.18 \\ 33 & 8 & 0.04 & 0.09 & -0.05 \\ 34 & 1 & 0.10 & 0.01 & -0.25 \\ 35 & 8 & -0.00 & 0.00 & -0.01 \\ 36 & 1 & -0.03 & 0.01 & 0.03 \\ 37 & 1 & 0.00 & 0.10 & -0.07\end{array}$

$\begin{array}{rrr}-0.01 & -0.07 & -0.16 \\ -0.14 & -0.04 & 0.10 \\ -0.16 & -0.01 & 0.17 \\ -0.11 & 0.00 & 0.16 \\ 0.08 & 0.02 & -0.05 \\ 0.09 & 0.02 & -0.05 \\ 0.08 & 0.01 & -0.07 \\ 0.12 & 0.00 & -0.10 \\ 0.05 & -0.02 & -0.09\end{array}$

$\begin{array}{rrr}-0.02 & -0.08 & -0.14 \\ -0.10 & -0.01 & -0.22 \\ -0.11 & 0.05 & -0.15 \\ -0.08 & 0.06 & 0.03 \\ 0.09 & -0.04 & 0.03 \\ 0.11 & -0.02 & 0.06 \\ 0.13 & 0.02 & 0.14 \\ 0.20 & -0.02 & 0.14 \\ 0.07 & 0.00 & 0.07\end{array}$

Zero-point correction=

Thermal correction to Energy=

$$
\begin{gathered}
\odot .289923 \text { (a.u. ) } \\
0.311017 \\
0.311961 \\
0.239646 \\
-1115.302228 \\
-1115.281134 \\
-1115.280190 \\
-1115.352505
\end{gathered}
$$$$
\text { Sum of electronic and thermal Energies= }
$$

Sum of electronic and thermal Free Energies=

\begin{tabular}{|c|c|c|c|}
\hline Item & Value & Threshold & Converged? \\
\hline Maximum Force & $\odot .0 \odot \odot \odot 44$ & $\odot .0 \odot \odot 45 \odot$ & YES \\
\hline Force & $\odot .0 \odot \odot \odot \odot 4$ & 0.000300 & YES \\
\hline Maximum Displacement & $\odot .000938$ & 0.001800 & YES \\
\hline Displacement & 0.000257 & 0.001200 & YES \\
\hline
\end{tabular}

\section{$\mathrm{S}$}

Total

$$
\begin{aligned}
& \text { E (Thermal) } \\
& \text { KCal/Mol }
\end{aligned}
$$

$\mathrm{CV}$

\begin{tabular}{|c|c|c|c|c|c|}
\hline \multirow{2}{*}{$\begin{array}{l}\text { Center } \\
\text { Number }\end{array}$} & \multirow{2}{*}{$\begin{array}{l}\text { Atomic } \\
\text { Number }\end{array}$} & \multirow{2}{*}{$\begin{array}{c}\text { Atomic } \\
\text { Type }\end{array}$} & \multicolumn{3}{|c|}{ Coordinates (Angstroms) } \\
\hline & & & $x$ & $\mathrm{Y}$ & Z \\
\hline & & & -------- & -- & 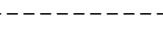 \\
\hline 1 & 1 & $\odot$ & 4.062592 & 1.456850 & -1.577996 \\
\hline 2 & 8 & $\odot$ & 3.661780 & 1.871454 & -0.808886 \\
\hline 3 & 8 & $\odot$ & 3.777619 & -2.331190 & 0.456627 \\
\hline 4 & 6 & $\odot$ & 3.575267 & 0.014114 & 0.812623 \\
\hline 5 & 6 & $\odot$ & 3.563812 & -1.105044 & -0.236142 \\
\hline 6 & 1 & $\odot$ & 3.717199 & -3.051072 & -0.178938 \\
\hline 7 & 6 & $\odot$ & 4.228498 & 1.317438 & 0.373463 \\
\hline 8 & 6 & $\odot$ & 2.159117 & -1.010579 & -0.820994 \\
\hline 9 & 6 & $\odot$ & 1.327592 & -0.525310 & 0.366310 \\
\hline 10 & 8 & $\odot$ & 2. 205183 & $\odot .251595$ & 1.163763 \\
\hline 11 & 1 & $\odot$ & 4.079897 & 2.062145 & 1.164249 \\
\hline 12 & 1 & $\odot$ & 5.309856 & 1.155081 & ๑. 255963 \\
\hline 13 & 1 & $\odot$ & 4.103357 & -0.342234 & 1.708024 \\
\hline 14 & 1 & $\odot$ & 4.345420 & $-\odot .961753$ & -0.995516 \\
\hline 15 & 1 & $\odot$ & 1.792754 & -1.963842 & -1.213493 \\
\hline 16 & 1 & $\odot$ & 2.133884 & $-\odot .270106$ & -1.628950 \\
\hline 17 & 1 & $\odot$ & ๑. 938148 & -1.367093 & 0.949220 \\
\hline 18 & 7 & $\odot$ & ๑. 167574 & 0.302163 & ๑. .036228 \\
\hline 19 & 6 & $\odot$ & ๑. 392024 & 1.501370 & -0.598727 \\
\hline 20 & 7 & $\odot$ & -0.457886 & 2.259118 & -1.152746 \\
\hline 21 & 6 & $\odot$ & -2.197265 & 0.415748 & 0.558769 \\
\hline 22 & 6 & $\odot$ & -1.115897 & -0.280930 & 0.077331 \\
\hline 23 & 7 & $\odot$ & -1.163472 & -1.568406 & -0.363832 \\
\hline 24 & 6 & $\odot$ & -2.336797 & -2.151633 & -0.388012 \\
\hline 25 & 7 & $\odot$ & -3.476646 & -1.503894 & -0.017738 \\
\hline 26 & 6 & $\odot$ & -3.498849 & -0.205884 & 0.491809 \\
\hline 27 & 7 & $\odot$ & -2.452547 & -3.421291 & -0.846330 \\
\hline 28 & 8 & $\odot$ & -4.563396 & ๑. 302094 & ๑.842327 \\
\hline 29 & 1 & $\odot$ & 1.454099 & 1.800035 & -0.624948 \\
\hline 30 & 1 & $\odot$ & -1.592040 & -3.932320 & -0.974437 \\
\hline 31 & 1 & $\odot$ & -3.277490 & -3.964811 & -0.642373 \\
\hline 32 & 1 & $\odot$ & -4.374904 & -1.969846 & -0.079334 \\
\hline
\end{tabular}

Cal/Mol-Kelvin Cal/Mol-Kelvin 79.438

152.200

Normal termination of Gaussian 16 at Sun Jun 9 0๑:46:45 2019 .

-Figure S8-5, Int3(C5)W1

degua-oh5w1. rev1.higha. $\log$

Stoichiometry C10H16N506(2)

Standard orientation: 


$\begin{array}{cccccc}33 & 8 & 0 & -2.076492 & 1.621794 & 1.176740 \\ 34 & 1 & 0 & -2.560961 & 2.301870 & 0.663470 \\ 35 & 8 & 0 & -2.997675 & 3.428608 & -0.678159 \\ 36 & 1 & 0 & -2.983140 & 4.354118 & -0.415872 \\ 37 & 1 & 0 & -2.109009 & 3.248546 & -1.036031 \\ -\end{array}$

Standard basis: Aug-CC-pVDZ (6D, 7F)

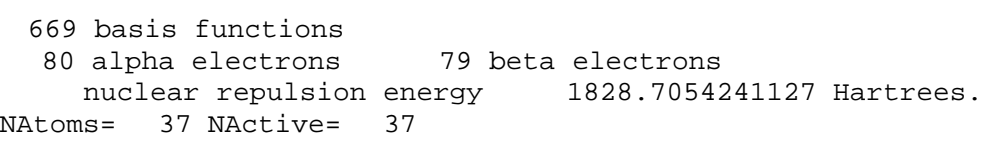

Force inversion solution in PCM.

Polarizable Continuum Model (PCM)

=ニ=ニ=ニ=ニニ=ニ=ニ=ニ=ニニニ=ニ=ニ=ニニ=ニ=ニ

Solvent : Water, Eps $=78.355300$ Eps $($ inf $)=1.777849$

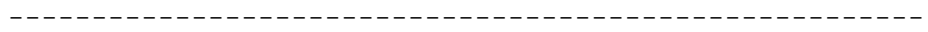

SCF Done: $E($ UWB97XD $)=-1115.62075476$ A.U. after 1 cycles NFock $=1$ Conv $=0.49 \mathrm{D}-08 \quad-\mathrm{V} / \mathrm{T}=2.0071$

$\langle\mathrm{S} x\rangle=0.0000\langle\mathrm{~S} y\rangle=0.0000\langle\mathrm{Sz}\rangle=0.5000$

$\left\langle S^{* *} 2>=0.7605 \mathrm{~S}=0.5052\right.$

$<$ L.S $>=0.000000000000 \mathrm{E}+00$

Annihilation of the first spin contaminant:

$\mathrm{S}^{* * 2}$ before annihilation 0.7605 , after 0.7501

Harmonic frequencies $\left(\mathrm{cm}^{* *}-1\right)$, IR intensities (KM/Mole), Raman activities ( $\left.A^{* *} 4 / A M U\right)$, depolarization ratios for plane and unp incident light, reduced masses (AMU), force constants (mDyne/A), and normal coordinates:

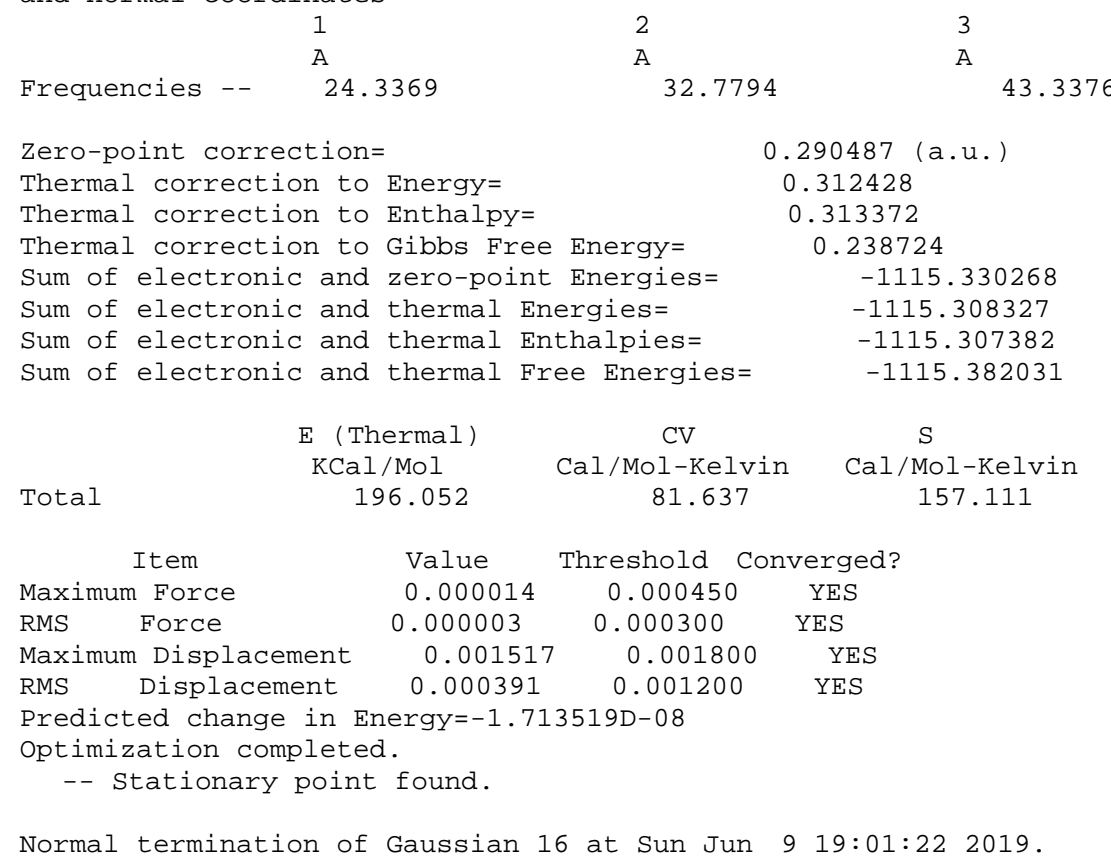

-Figure S8-6, TS3(C5)W1-......

degua-oh5w1.ts2.higha. $\log$

Stoichiometry C10H16N506(2)

Standard orientation:

\begin{tabular}{|c|c|c|c|c|c|}
\hline \multirow{2}{*}{$\begin{array}{l}\text { Center } \\
\text { Number }\end{array}$} & \multirow{2}{*}{$\begin{array}{l}\text { Atomic } \\
\text { Number }\end{array}$} & \multirow{2}{*}{$\begin{array}{l}\text { Atomic } \\
\text { Type }\end{array}$} & \multicolumn{3}{|c|}{ Coordinates (Angstroms) } \\
\hline & & & $\mathrm{x}$ & $\mathrm{Y}$ & z \\
\hline . & 1 & $\odot$ & -4.095047 & $-\odot .947764$ & -1.712817 \\
\hline 2 & 8 & 0 & -3.673517 & -1.484989 & -1.036071 \\
\hline$\checkmark$ & 8 & 0 & -3.548109 & 2.430409 & 0.966352 \\
\hline
\end{tabular}




\begin{tabular}{|c|c|c|c|c|c|}
\hline 4 & 6 & 0 & -3.461732 & $\odot .056025$ & 0.875284 \\
\hline 5 & 6 & 0 & -3.444425 & 1.346901 & ๑. 049584 \\
\hline 6 & 1 & $\odot$ & -3.496029 & 3.253607 & 0.470908 \\
\hline 7 & 6 & 0 & -4.185249 & -1.124303 & 0.242871 \\
\hline 8 & 6 & 0 & -2.082336 & 1.296640 & -0.635510 \\
\hline 9 & 6 & $\odot$ & -1.217195 & $\odot .556911$ & ๑.384064 \\
\hline 10 & 8 & $\odot$ & -2.085326 & -0.286688 & 1.106981 \\
\hline 11 & 1 & 0 & -4.047982 & -1.999801 & 0.888153 \\
\hline 12 & 1 & $\odot$ & -5.260754 & $-\odot .902297$ & 0.191502 \\
\hline 13 & 1 & $\odot$ & -3.928749 & 0.258338 & 1.848419 \\
\hline 14 & 1 & 0 & -4.272202 & 1.380491 & -0.672162 \\
\hline 15 & 1 & $\odot$ & -1.683098 & 2.286510 & -0.874558 \\
\hline 16 & 1 & $\odot$ & -2.146068 & $\odot .714692$ & -1.562078 \\
\hline 17 & 1 & 0 & -0.702300 & 1.244382 & 1.065448 \\
\hline 18 & 7 & $\odot$ & -0.147945 & $-\odot .266323$ & -0.207362 \\
\hline 19 & 6 & $\odot$ & -0.462481 & -1.351657 & -0.894613 \\
\hline 20 & 7 & 0 & 0.365769 & -2.082935 & -1.586351 \\
\hline 21 & 6 & 0 & 2.129425 & -0.570453 & 0.607119 \\
\hline 22 & 6 & $\odot$ & 1.206174 & $\odot .188564$ & $-\odot .086391$ \\
\hline 23 & 7 & 0 & 1.406923 & 1.421253 & $-\odot .654921$ \\
\hline 24 & 6 & $\odot$ & 2.595647 & 1.931613 & $-\odot .551453$ \\
\hline 25 & 7 & $\odot$ & 3.612893 & 1.265804 & $\odot .075321$ \\
\hline 26 & 6 & $\odot$ & 3.484329 & $\odot .020153$ & 0.674358 \\
\hline 27 & 7 & $\odot$ & 2.868616 & 3.195609 & -1.016833 \\
\hline 28 & 8 & $\odot$ & 4.455719 & -0.497727 & 1.221054 \\
\hline 29 & 1 & $\odot$ & -1.533886 & -1.615563 & -0.926387 \\
\hline 30 & 1 & $\odot$ & 2.167251 & 3.550049 & -1.653268 \\
\hline 31 & 1 & $\odot$ & 3.815232 & 3.380120 & -1.322245 \\
\hline 32 & 1 & $\odot$ & 4.529812 & 1.692923 & ๑.146923 \\
\hline 33 & 8 & $\odot$ & 1.905091 & -1.707188 & 1.201833 \\
\hline 34 & 1 & $\odot$ & 1.997156 & -2.848721 & 0.373860 \\
\hline 35 & 8 & $\odot$ & 1.938504 & -3.579742 & -0.388543 \\
\hline 36 & 1 & $\odot$ & 1.064739 & -2.908296 & -1.089294 \\
\hline 37 & 1 & $\odot$ & 1.536300 & -4.367483 & $-\odot .006099$ \\
\hline
\end{tabular}

Standard basis: Aug-CC-pVDZ (6D, 7F)

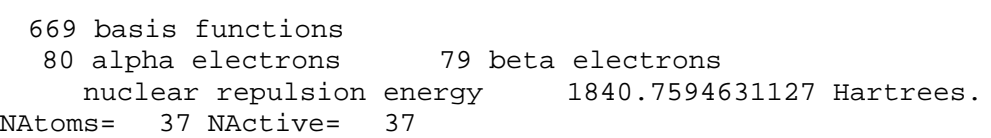

Nuclear repulsion after empirical dispersion term =

1840.7304412298 Hartrees.

Force inversion solution in PCM.

Polarizable Continuum Model (PCM)

$\begin{array}{ll}\text { Model } & \text { : PCM. }\end{array}$

Solvent : Water, Eps $=78.355300$ Eps $($ inf $)=1.777849$

Solvent : Water, Eps=

SCF Done: $E($ UWB97XD) $=-1115.58563996$ A.U. after 2 cycles NFock $=2$ Conv $=0.84 \mathrm{D}-09 \quad-\mathrm{V} / \mathrm{T}=2.0071$

$\langle\mathrm{S} x>=0.0000<\mathrm{Sy}\rangle=0.0000<\mathrm{Sz}>=0.5000$

$\left\langle\mathrm{S}^{* *} 2>=0.7569 \mathrm{~S}=0.5035\right.$

$<$ L.S $>=0.000000000000 \mathrm{E}+00$

Annihilation of the first spin contaminant:

$\mathrm{S}^{* * 2}$ before annihilation 0.7569 , after 0.7500

Harmonic frequencies $\left(\mathrm{cm}^{* *}-1\right)$, IR intensities (KM/Mole), Raman activities ( $\left.A^{* *} 4 / A M U\right)$, depolarization ratios for plane and unpo incident light, reduced masses (AMU), force constants (mDyne/A), and normal coordinates:

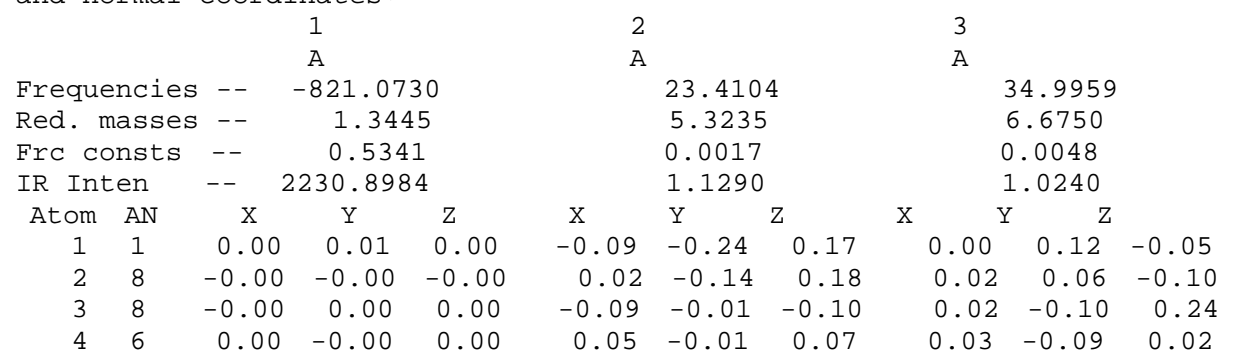




\begin{tabular}{|c|c|c|c|c|}
\hline 5 & 6 & $-\odot . \odot \odot$ & $-\odot . \odot \odot$ & $-0.0 \odot$ \\
\hline 6 & 1 & $\odot . \odot \odot$ & $-\odot . \odot \odot$ & $-\odot . \odot \odot$ \\
\hline 7 & 6 & $\odot . \odot \odot$ & 0.00 & $-0.0 \odot$ \\
\hline 8 & 6 & $-\odot . \odot \odot$ & $-\odot . \odot \odot$ & $\odot .0 \odot$ \\
\hline 9 & 6 & 0.01 & -0.00 & -0.01 \\
\hline 10 & 8 & -0.00 & $-\odot . \odot \odot$ & 0.00 \\
\hline 11 & 1 & $\odot .0 \odot$ & $\odot .0 \odot$ & -0.00 \\
\hline 12 & 1 & $-0.0 \odot$ & $\odot . \odot \odot$ & $\odot .00$ \\
\hline 13 & 1 & $-\odot . \odot \odot$ & $\odot . \odot \odot$ & $-\odot . \odot \odot$ \\
\hline 14 & 1 & -0.01 & $-0.0 \odot$ & 0.00 \\
\hline 15 & 1 & -0.00 & $-0.0 \odot$ & 0.00 \\
\hline 16 & 1 & -0.00 & -0.00 & 0.00 \\
\hline 17 & 1 & -0.02 & 0.01 & $-\odot .0 \odot$ \\
\hline 18 & 7 & 0.01 & 0.01 & 0.02 \\
\hline 19 & 6 & 0.02 & -0.03 & -0.05 \\
\hline 20 & 7 & 0.00 & -0.03 & $\odot .04$ \\
\hline 21 & 6 & 0.01 & 0.02 & -0.02 \\
\hline 22 & 6 & -0.01 & $\odot . \odot \odot$ & ๑. 01 \\
\hline 23 & 7 & $\odot . \odot \odot$ & -0.00 & 0.00 \\
\hline 24 & 6 & 0.01 & 0.00 & 0.00 \\
\hline 25 & 7 & $-0.0 \odot$ & $\odot . \odot \odot$ & $-0.0 \odot$ \\
\hline 26 & 6 & -0.01 & -0.01 & -0.00 \\
\hline 27 & 7 & -0.00 & -0.01 & -0.00 \\
\hline 28 & 8 & 0.00 & -0.00 & $\odot .0 \odot$ \\
\hline 29 & 1 & 0.02 & -0.01 & $-\odot .02$ \\
\hline 30 & 1 & -0.00 & $-\odot .0 \odot$ & $\odot .00$ \\
\hline 31 & 1 & 0.00 & 0.01 & $\odot .02$ \\
\hline 32 & 1 & 0.00 & -0.00 & 0.00 \\
\hline 33 & 8 & $\odot .00$ & -0.05 & -0.03 \\
\hline 34 & 1 & -0.03 & 0.33 & 0.16 \\
\hline 35 & 8 & -0.06 & $\odot . \odot 9$ & -0.02 \\
\hline 36 & 1 & 0.60 & -0.52 & 0.42 \\
\hline 37 & 1 & -0.10 & $\odot .07$ & -0.08 \\
\hline
\end{tabular}

$\begin{array}{ccc}-0.07 & -0.07 & -0.03 \\ -0.17 & -0.04 & -0.16 \\ 0.08 & -0.10 & 0.19 \\ -0.10 & -0.03 & -0.09 \\ -0.01 & 0.08 & -0.09 \\ 0.08 & 0.08 & 0.02 \\ 0.18 & -0.04 & 0.24 \\ 0.06 & -0.17 & 0.25 \\ 0.09 & 0.03 & 0.08 \\ -0.11 & -0.17 & 0.01 \\ -0.17 & -0.02 & -0.17 \\ -0.12 & -0.09 & -0.06 \\ -0.00 & 0.15 & -0.17 \\ -0.00 & 0.09 & -0.10 \\ 0.01 & 0.08 & -0.09 \\ 0.01 & 0.08 & -0.07 \\ -0.05 & 0.01 & -0.02 \\ 0.01 & 0.05 & -0.04 \\ 0.07 & 0.05 & -0.02 \\ 0.09 & 0.01 & 0.04 \\ 0.04 & -0.04 & 0.06 \\ -0.03 & -0.04 & 0.04 \\ 0.15 & 0.01 & 0.07 \\ -0.07 & -0.09 & 0.06 \\ 0.01 & 0.07 & -0.09 \\ 0.19 & 0.04 & 0.05 \\ 0.17 & -0.02 & 0.11 \\ 0.06 & -0.08 & 0.10 \\ -0.11 & 0.01 & -0.04 \\ -0.07 & 0.02 & -0.04 \\ -0.05 & 0.03 & -0.06 \\ -0.02 & 0.05 & -0.07 \\ -0.07 & 0.02 & -0.09 \\ 0.04 & 0.01\end{array}$

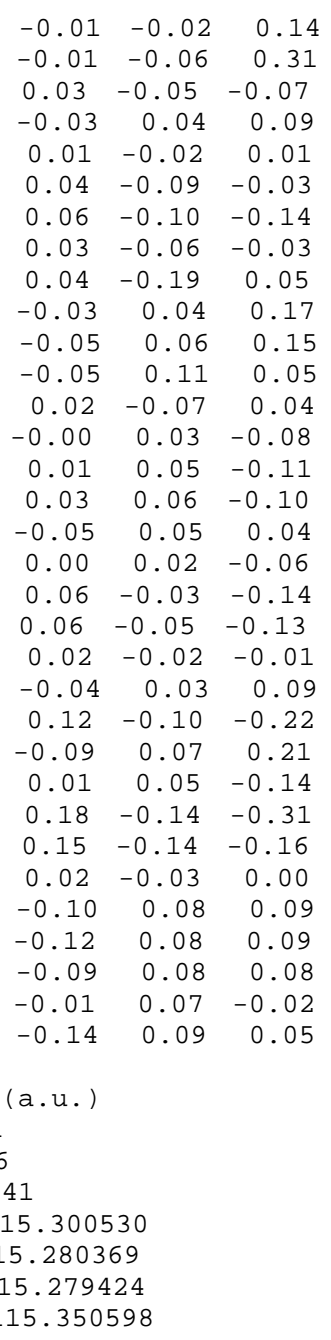

Zero-point correction=

Thermal correction to Energy=

Thermal correction to Enthalpy=

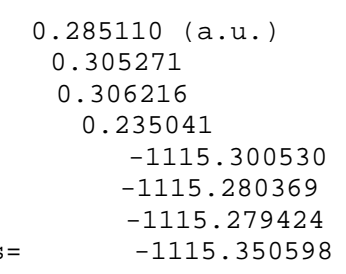

Sum of electronic and zero-point Energies=

Sum of electronic and thermal Energies=

Sum of electronic and thermal Enthalpies=

\section{$\mathrm{S}$}

E (Thermal) $\mathrm{KCal} / \mathrm{Mol}$

$\mathrm{CV}$

Cal/Mol-Kelvin Cal/Mol-Kelvin $\begin{array}{ll}76.903 & 149.798\end{array}$

Total

191.561

\begin{tabular}{lccc}
\multicolumn{1}{c}{ Item } & Value & Threshold & Converged? \\
Maximum Force & 0.000010 & 0.000450 & YES \\
RMS Force & 0.000001 & 0.000300 & YES \\
Maximum Displacement & 0.000700 & 0.001800 & YES \\
RMS Displacement & 0.000154 & 0.001200 & YES \\
Predicted change in Energy=-1.417243D-08 & \\
Optimization completed. & & \\
\multicolumn{2}{c}{- - Stationary point found. } & &
\end{tabular}

Normal termination of Gaussian 16 at Sun May 19 06:08:21 2019.

Figure S8-7, Int4(C5)W1

degua-oh5w1. for 2. higha.log

Stoichiometry C10H16N506(2)

Standard orientation:

\begin{tabular}{|c|c|c|c|c|c|}
\hline Center & Atomic & Atomic & \multicolumn{3}{|c|}{ Coordinates (Angstroms) } \\
\hline Number & Number & Type & $x$ & $\mathrm{Y}$ & Z \\
\hline \multicolumn{6}{|c|}{ - - - - - - - - - - - - - } \\
\hline 1 & 1 & $\odot$ & -4.197723 & 0.665953 & 1.680596 \\
\hline 2 & 8 & $\odot$ & -3.807223 & 1.240385 & 1.016285 \\
\hline 3 & 8 & $\odot$ & -3.207805 & -2.709859 & -0.796141 \\
\hline 4 & 6 & $\odot$ & -3.324727 & -0.332041 & -0.824977 \\
\hline 5 & 6 & $\odot$ & -3.215745 & -1.576185 & $\odot .066399$ \\
\hline 6 & 1 & $\odot$ & -3.090735 & -3.498585 & -0.257796 \\
\hline 7 & 6 & $\odot$ & -4.192934 & $\odot .792451$ & $-\odot .277552$ \\
\hline 8 & 6 & $\odot$ & -1.877146 & -1.375470 & $\odot .768492$ \\
\hline
\end{tabular}




\begin{tabular}{|c|c|c|c|c|c|}
\hline 9 & 6 & 0 & -1.056060 & -0.635995 & -0.286864 \\
\hline 10 & 8 & 0 & -1.985183 & 0.138569 & -1.027374 \\
\hline 11 & 1 & 0 & -4.097380 & 1.657467 & -0.944572 \\
\hline 12 & 1 & 0 & -5.244247 & 0.468377 & -0.288357 \\
\hline 13 & 1 & 0 & -3.736324 & -0.629957 & -1.799641 \\
\hline 14 & 1 & 0 & -4.054296 & -1.648207 & 0.773024 \\
\hline 15 & 1 & $\odot$ & -1.405444 & -2.315272 & 1.072060 \\
\hline 16 & 1 & $\odot$ & -2.009315 & -0.747175 & 1.657036 \\
\hline 17 & 1 & $\odot$ & -0.542843 & -1.338894 & -0.951216 \\
\hline 18 & 7 & 0 & -0.025751 & 0.267390 & 0.227824 \\
\hline 19 & 6 & 0 & $-\odot .435896$ & 1.292689 & 1.073346 \\
\hline 20 & 7 & 0 & 0.350411 & 1.859864 & 1.902727 \\
\hline 21 & 6 & $\odot$ & 2.307979 & $\odot .885506$ & -0.207270 \\
\hline 22 & 6 & $\odot$ & 1.307317 & $-\odot .104255$ & 0.115044 \\
\hline 23 & 7 & $\odot$ & 1.563042 & -1.411532 & 0.215436 \\
\hline 24 & 6 & 0 & 2.818651 & -1.829294 & 0.109048 \\
\hline 25 & 7 & 0 & 3.866028 & $-\odot .973325$ & $-\odot .039583$ \\
\hline 26 & 6 & 0 & 3.719208 & 0.399893 & -0.201879 \\
\hline 27 & 7 & $\odot$ & 3.068613 & -3.139083 & 0.175042 \\
\hline 28 & 8 & 0 & 4.696691 & 1.112519 & -0.352653 \\
\hline 29 & 1 & $\odot$ & -1.501241 & 1.518496 & 0.961041 \\
\hline 30 & 1 & $\odot$ & 2.291750 & -3.768081 & 0.310408 \\
\hline 31 & 1 & 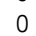 & 4.0000233 & -3.521116 & 0.120375 \\
\hline 32 & 1 & 0 & 4.816967 & -1.325663 & -0.063614 \\
\hline 33 & 8 & $\odot$ & 2.073951 & 2.069041 & -0.526426 \\
\hline 34 & 1 & 0 & 0.567847 & 3. 022648 & -0.882791 \\
\hline 35 & 8 & $\odot$ & -0.161098 & 3.606035 & -1.166069 \\
\hline 36 & 1 & 0 & -0.166599 & 2.580316 & 2.404912 \\
\hline 37 & 1 & $\odot$ & -0.565602 & 3.899159 & -0.345287 \\
\hline
\end{tabular}

Standard basis: Aug-CC-pVDZ (6D, 7F)

669 basis functions

80 alpha electrons

79 beta electrons

nuclear repulsion energy 1837.1661826923 Hartrees.

NAtoms $=37$ NActive $=37$

Force inversion solution in PCM.

Polarizable Continuum Model (PCM)

=ニニ=ニニニニニニニ=ニニ=ニニ=ニニニニニニニニニ=ニニニ=ニ

Model : PCM.

Solvent : Water, Eps $=78.355300$ Eps $($ inf $)=1.777849$

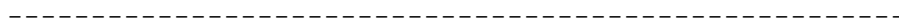

SCF Done: $E($ UWB97XD $)=-1115.65226004$ A.U. after 1 cycles

NFock $=1$ Conv $=0.36 \mathrm{D}-08$

$\langle\mathrm{S} x>=0.0000<\mathrm{Sy}>=0.0000<\mathrm{Sz}>=0.5000$

$<S^{* * 2}>=0.7605 \mathrm{~S}=0.5053$

$<$ L.S $>=0.000000000000 \mathrm{E}+00$

Annihilation of the first spin contaminant:

$\mathrm{S}^{* * 2}$ before annihilation 0.7605 , after 0.7501

Harmonic frequencies $\left(\mathrm{cm}^{*}-1\right)$, IR intensities (KM/Mole), Raman

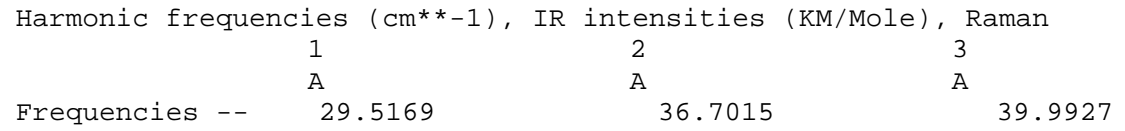

Zero-point correction=

Thermal correction to Energy=

Thermal correction to Enthalpy=

Thermal correction to Gibbs Free Energy=

Sum of electronic and zero-point Energies=

Sum of electronic and thermal Energies=

Sum of electronic and thermal Enthalpies=

0.291980 (a.u.)

$\odot .313942$

$\odot .314886$

0.239836

$-1115.360280$

$-1115.338318$

$-1115.337374$

Sum of electronic and thermal Free Energies=

$-1115.412424$

\begin{tabular}{|c|c|c|c|}
\hline $\begin{array}{l}\text { E ( T } \\
\mathrm{KCa}\end{array}$ & $\begin{array}{l}\text { ermal ) } \\
\text { /Mol }\end{array}$ & $\begin{array}{c}\mathrm{CV} \\
\mathrm{Cal} / \mathrm{Mol}-\mathrm{Kelvin}\end{array}$ & $\begin{array}{c}\mathrm{S} \\
\mathrm{Cal} / \mathrm{Mol}-\mathrm{Kel} \text { vin }\end{array}$ \\
\hline Item & Value & \multicolumn{2}{|c|}{ Threshold Converged? } \\
\hline Maximum Force & 0.000005 & 0.000450 & YES \\
\hline Force & 0.000001 & $\odot .000300$ & ES \\
\hline Maximum Displacement & 0.001432 & 0.001800 & YES \\
\hline
\end{tabular}


RMS Displacement $0.000283 \quad 0.001200 \quad$ YES

Predicted change in Energy $=-5.482495 \mathrm{D}-\odot 9$

optimization completed.

- Stationary point found.

Normal termination of Gaussian 16 at Sun Jun 16 18:52:23 2019.

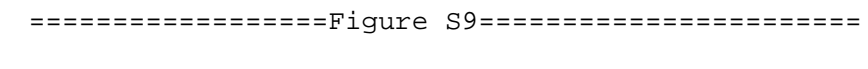

Figure S9-1, Int1(C5)W2

degua-oh5w2. for . higha. $\log$

Stoichiometry C10H18N507(2)

Standard orientation:

\begin{tabular}{|c|c|c|c|c|c|}
\hline \multirow{2}{*}{$\begin{array}{l}\text { Center } \\
\text { Number }\end{array}$} & \multirow{2}{*}{$\begin{array}{l}\text { Atomic } \\
\text { Number }\end{array}$} & \multirow{2}{*}{$\begin{array}{c}\text { Atomic } \\
\text { Type }\end{array}$} & \multicolumn{3}{|c|}{ Coordinates (Angstroms) } \\
\hline & & & $x$ & $\mathrm{Y}$ & Z \\
\hline & & & & & \\
\hline 1 & 1 & $\odot$ & 4.252562 & 2.080086 & -1.122023 \\
\hline 2 & 8 & $\odot$ & 3.657502 & 2.200823 & -0.377140 \\
\hline 3 & 8 & $\odot$ & 4.555180 & -2.052274 & $\odot .196785$ \\
\hline 4 & 6 & $\odot$ & 3.854901 & 0.103828 & 0.912507 \\
\hline 5 & 6 & $\odot$ & 4.185176 & -0.770989 & -0.299836 \\
\hline 6 & 1 & $\odot$ & 4.725292 & -2.631409 & -0.552433 \\
\hline 7 & 6 & $\odot$ & 4.212913 & 1.577719 & 0.775542 \\
\hline 8 & 6 & $\odot$ & 2.863281 & -0.807088 & -1.061534 \\
\hline 9 & 6 & $\odot$ & 1.828128 & -0.730621 & $\odot .061739$ \\
\hline 10 & 8 & $\odot$ & 2.443476 & -0.048400 & 1.134520 \\
\hline 11 & 1 & $\odot$ & 3.807956 & 2.110748 & 1.643942 \\
\hline 12 & 1 & $\odot$ & 5.307623 & 1.681013 & $\odot .792136$ \\
\hline 13 & 1 & $\odot$ & 4.376527 & $-\odot .288652$ & 1.795682 \\
\hline 14 & 1 & $\odot$ & 5.001090 & -0.346635 & -0.900968 \\
\hline 15 & 1 & $\odot$ & 2.745452 & -1.708869 & -1.669939 \\
\hline 16 & 1 & $\odot$ & 2.779781 & ๑. . 069118 & -1.713403 \\
\hline 17 & 1 & $\odot$ & 1.496259 & -1.723891 & ๑. 386529 \\
\hline 18 & 7 & $\odot$ & ๑. 616501 & -0.015172 & -0.305975 \\
\hline 19 & 6 & $\odot$ & $\odot .526289$ & 1.315975 & -0.680883 \\
\hline 20 & 7 & $\odot$ & -0.691380 & 1.702090 & -0.949171 \\
\hline 21 & 6 & $\odot$ & -1.464130 & $\odot .586673$ & -0.697899 \\
\hline 22 & 6 & $\odot$ & -0.646060 & -0.514130 & -0.343863 \\
\hline 23 & 7 & $\odot$ & -1.019050 & -1.773612 & -0.123463 \\
\hline 24 & 6 & $\odot$ & -2.309846 & -1.986909 & -0.355138 \\
\hline 25 & 7 & $\odot$ & -3.167833 & -1.019035 & -0.811669 \\
\hline 26 & 6 & $\odot$ & -2.844026 & 0.323617 & -1.014796 \\
\hline 27 & 7 & $\odot$ & -2.806895 & -3.213478 & -0.171192 \\
\hline 28 & 8 & $\odot$ & -3.695297 & 1.114490 & -1.414072 \\
\hline 29 & 1 & $\odot$ & 1.420620 & 1.931811 & $-\odot .724555$ \\
\hline 30 & 1 & $\odot$ & -2.191280 & -3.932406 & 0.176384 \\
\hline 31 & 1 & $\odot$ & -3.787389 & -3.422568 & -0.273650 \\
\hline 32 & 1 & $\odot$ & -4.135681 & -1.261776 & -0.988650 \\
\hline 33 & 8 & $\odot$ & -2.006163 & 0.866525 & 1.446700 \\
\hline 34 & 1 & $\odot$ & -2.383819 & 1.741521 & 1.243341 \\
\hline 35 & 8 & $\odot$ & -3.448934 & 3.265754 & $\odot .409957$ \\
\hline 36 & 1 & $\odot$ & -3.605230 & 2.669859 & -0.343561 \\
\hline 37 & 1 & $\odot$ & -4.301437 & 3.326984 & 0.851142 \\
\hline 38 & 1 & $\odot$ & -3.250849 & -0.207240 & 1.998388 \\
\hline 39 & 8 & $\odot$ & -3.914826 & -0.829018 & 2.378031 \\
\hline 40 & 1 & $\odot$ & -4.744135 & -0.584897 & 1.958657 \\
\hline
\end{tabular}

Standard basis: Aug-CC-pVDZ (6D, 7F)

712 basis functions

85 alpha electrons 84 beta electrons

nuclear repulsion energy 2017.1328062830 Hartrees.

NAtoms $=40$ NActive $=40$

Force inversion solution in PCM.

Polarizable Continuum Model (PCM)

=ニニニニニニニニニニニ=ニニ=ニニニニニ=
Model

Solvent : Water, Eps $=78.355300$ Eps $($ inf $)=1.777849$ 
SCF Done: $E($ UWB97XD) $=-1192.06686889$ A.U. after 2 cycles $\mathrm{NFock}=2$ Conv $=0.84 \mathrm{D}-09 \quad-\mathrm{V} / \mathrm{T}=2.0071$

$<\mathrm{S} x>=0.0000<\mathrm{Sy}>=0.0000<\mathrm{S} z>=0.5000$

$<S^{* *} 2>=0.7612 \mathrm{~S}=0.5056$

$<$ L.S $>=0.000000000000 \mathrm{E}+00$

Annihilation of the first spin contaminant:

$\mathrm{S}^{* *} 2$ before annihilation 0.7612 , after 0.7501

Harmonic frequencies $\left(\mathrm{cm}^{*}-1\right)$, IR intensities (KM/Mole), Raman

$\begin{array}{lcccc} & 1 & 2 & 3 & \\ \text { Frequencies - } & \mathrm{A} & \mathrm{A} & \mathrm{A} & \\ & 9.1467 & 21.8191 & & 36.9073\end{array}$

Zero-point correction=

Thermal correction to Energy=

Thermal correction to Enthalpy=

Thermal correction to Gibbs Free Energy=

Sum of electronic and zero-point Energies=

Sum of electronic and thermal Energies=

Sum of electronic and thermal Enthalpies=

Sum of electronic and thermal Free Energies=

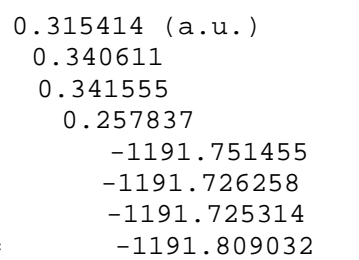

\begin{tabular}{|c|c|c|c|}
\hline E & $\begin{array}{l}\text { lermal) } \\
\text { /Mol } \\
13.736\end{array}$ & $\begin{array}{c}\text { CV } \\
\text { Cal/Mol-Kelvin } \\
91.009\end{array}$ & $\begin{array}{c}\mathrm{S} \\
\mathrm{Cal} / \mathrm{Mol}-\mathrm{Kel} \text { in } \\
176.198\end{array}$ \\
\hline Item & Value & Threshold Con & verged? \\
\hline Maximum Force & 0.000005 & 0.000450 & YES \\
\hline Force & 0.000001 & 0.000300 & YES \\
\hline Maximum Displacement & 0.001801 & 0.001800 & NO \\
\hline Displacement & 0.000466 & 0.001200 & YES \\
\hline
\end{tabular}

Predicted change in Energy $=-1.784221 \mathrm{D}-09$

Normal termination of Gaussian 16 at Wed Jun 19 ๑4:24: 09 2019.

degua-oh5w2.higha. log

Stoichiometry C10H18N507(2)

\begin{tabular}{|c|c|c|c|c|c|}
\hline \multirow{3}{*}{$\begin{array}{l}\text { Center } \\
\text { Number }\end{array}$} & \multirow{2}{*}{$\begin{array}{l}\text { Atomic } \\
\text { Number }\end{array}$} & \multirow{2}{*}{$\begin{array}{c}\text { Atomic } \\
\text { Type }\end{array}$} & \multicolumn{3}{|c|}{ Coordinates (Angstroms) } \\
\hline & & & $x$ & $\mathrm{Y}$ & Z \\
\hline & & & 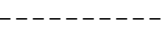 & 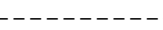 & ----- \\
\hline 1 & 1 & $\odot$ & 4.187570 & 2.114970 & -1.158779 \\
\hline 2 & 8 & $\odot$ & 3.644987 & 2.227080 & -0.373430 \\
\hline 3 & 8 & $\odot$ & 4.563409 & -2.037820 & ๑. 025195 \\
\hline 4 & 6 & $\odot$ & 3.916301 & 0.097579 & 0.847771 \\
\hline 5 & 6 & $\odot$ & 4.163893 & $-\odot .742487$ & -0.408035 \\
\hline 6 & 1 & $\odot$ & 4.684728 & -2.593983 & -0.750501 \\
\hline 7 & 6 & $\odot$ & 4.269086 & 1.574221 & 0.726437 \\
\hline 8 & 6 & $\odot$ & 2.794363 & $-\odot .755425$ & -1.081379 \\
\hline 9 & 6 & $\odot$ & 1.834989 & -0.710501 & ०. 109226 \\
\hline 10 & 8 & $\odot$ & 2.522519 & -0.059141 & 1.158040 \\
\hline 11 & 1 & $\odot$ & 3.920431 & 2.085943 & 1.631269 \\
\hline 12 & 1 & $\odot$ & 5.363000 & 1.675820 & 0.677100 \\
\hline 13 & 1 & $\odot$ & 4.494186 & -0.319522 & 1.683397 \\
\hline 14 & 1 & $\odot$ & 4.939301 & -0.300853 & -1.049113 \\
\hline 15 & 1 & $\odot$ & 2.633917 & -1.639430 & -1.706034 \\
\hline 16 & 1 & $\odot$ & 2.670309 & 0.139356 & -1.700815 \\
\hline 17 & 1 & $\odot$ & 1.525402 & -1.713488 & 0.426592 \\
\hline 18 & 7 & $\odot$ & ๑. 602894 & 0.014084 & -0.152880 \\
\hline 19 & 6 & 0 & 0.489645 & 1.350214 & -0.495147 \\
\hline 20 & 7 & $\odot$ & -0.735616 & 1.742050 & -0.706851 \\
\hline 21 & 6 & $\odot$ & -1.519018 & 0.627751 & -0.397792 \\
\hline 22 & 6 & $\odot$ & -0.658485 & -0.497116 & -0.172311 \\
\hline 23 & 7 & $\odot$ & -1.012076 & -1.774447 & -0.043270 \\
\hline 24 & 6 & $\odot$ & -2.275048 & -2.011618 & -0.366567 \\
\hline 25 & 7 & $\odot$ & -3.115058 & -1.048695 & -0.879260 \\
\hline 26 & 6 & $\odot$ & -2.816304 & 0.303219 & -0.991217 \\
\hline 27 & 7 & $\odot$ & -2.763701 & -3.252613 & -0.255216 \\
\hline 28 & 8 & $\odot$ & -3.606646 & 1.080267 & -1.517532 \\
\hline 29 & 1 & 0 & 1.381475 & 1.966951 & -0.573813 \\
\hline 30 & 1 & $\odot$ & -2.165984 & -3.969991 & 0.125153 \\
\hline
\end{tabular}




$\begin{array}{rrrrrr}31 & 1 & 0 & -3.735559 & -3.467145 & -0.413793 \\ 32 & 1 & 0 & -4.031748 & -1.322004 & -1.213948 \\ 33 & 8 & 0 & -2.149916 & 0.948265 & 1.331036 \\ 34 & 1 & 0 & -2.588883 & 1.801950 & 1.172674 \\ 35 & 8 & 0 & -3.671310 & 3.284291 & 0.262064 \\ 36 & 1 & 0 & -3.713234 & 2.668685 & -0.490350 \\ 37 & 1 & 0 & -4.567599 & 3.302540 & 0.610534 \\ 38 & 1 & 0 & -3.185337 & -0.268383 & 2.001499 \\ 39 & 8 & 0 & -3.715963 & -0.985481 & 2.419534 \\ 40 & 1 & 0 & -4.540249 & -0.998768 & 1.926077 \\ -\end{array}$

Standard basis: Aug-CC-pVDZ (6D, 7F)

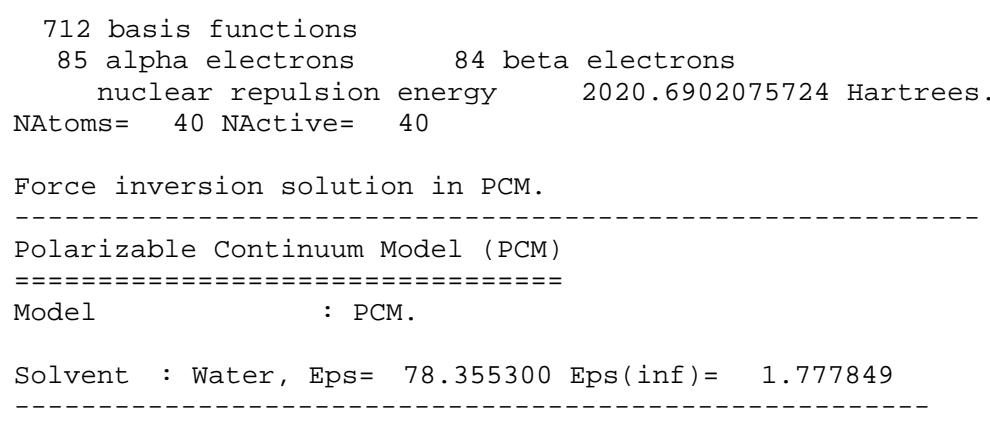

SCF Done: $E($ UWB97XD $)=-1192.06499696$ A.U. after 1 cycles NFock $=1$ Conv $=0.35 \mathrm{D}-08 \quad-\mathrm{V} / \mathrm{T}=2.0071$

$\langle$ SX $>=0.0000\langle$ Sy $>=0.0000\langle$ Sz $>=0.5000$

$<\mathrm{S}^{* *} 2>=0.7657 \mathrm{~S}=0.5078$

$<$ L.S $>=0.000000000000 \mathrm{E}+00$

Annihilation of the first spin contaminant:

$\mathrm{S}^{* * 2}$ before annihilation 0.7657 , after 0.7502

Harmonic frequencies $\left(\mathrm{cm}^{* *}-1\right)$, IR intensities (KM/Mole), Raman activities $\left(A^{* *} 4 / \mathrm{AMU}\right)$, depolarization ratios for plane and unp incident light, reduced masses (AMU), force constants (mDyne/A), and normal coordinates:

\begin{tabular}{|c|c|c|c|c|c|c|c|c|c|c|}
\hline & & & $\begin{array}{l}1 \\
\mathrm{~A}\end{array}$ & & $\begin{array}{l}2 \\
\text { A }\end{array}$ & & & $\begin{array}{l}3 \\
\text { A }\end{array}$ & & \\
\hline Freque & ncies & ; - - & -330.34 & & & 27.8741 & & & 9.5441 & \\
\hline Red. $n$ & asses & ; - & 11.41 & & & 6.6607 & & & 5.4891 & \\
\hline Frc cc & nsts & -- & 0.73 & & & 0.0030 & & & $.0 \odot 28$ & \\
\hline IR Int & & - - & 161.18 & & & 4.2503 & & & .6159 & \\
\hline Atom & AN & $x$ & $Y$ & Z & $x$ & $Y$ & Z & $x$ & Z & \\
\hline 1 & 1 & $\odot . \odot \odot$ & $-\odot . \odot \odot$ & $-\odot . \odot \odot$ & $\odot .02$ & $\odot .02$ & 0.12 & $-\odot . \odot \odot$ & 0.13 & 0.24 \\
\hline 2 & 8 & $\odot . \odot \odot$ & $\odot . \odot \odot$ & $-\odot . \odot \odot$ & $-\odot .02$ & $\odot . \odot \odot$ & 0.10 & -0.03 & 0.06 & 0.23 \\
\hline 3 & 8 & $\odot . \odot \odot$ & $-\odot . \odot \odot$ & $0.0 \odot$ & -0.03 & $-\odot . \odot \odot$ & $\odot .06$ & 0.08 & 0.05 & -0.16 \\
\hline 4 & 6 & $-\odot . \odot \odot$ & $-\odot . \odot \odot$ & $-\odot . \odot \odot$ & $-\odot . \odot 7$ & $-\odot . \odot 2$ & $\odot .07$ & $-\odot . \odot 2$ & $-\odot .05$ & 0.03 \\
\hline 5 & 6 & 0.01 & $\odot . \odot \odot$ & $-\odot . \odot \odot$ & $-\odot . \odot \odot$ & 0.01 & 0.07 & $\odot .04$ & ๑.๑8 & $-\odot .04$ \\
\hline 6 & 1 & $-\odot . \odot \odot$ & $-\odot . \odot \odot$ & $\odot . \odot \odot$ & 0.01 & $\odot .01$ & $\odot .06$ & 0.13 & 0.14 & -0.22 \\
\hline 7 & 6 & $-\odot . \odot \odot$ & $-\odot . \odot \odot$ & $-\odot . \odot \odot$ & $-\odot . \odot 7$ & $-\odot . \odot 1$ & 0.12 & $-\odot . \odot 6$ & $-\odot .03$ & 0.19 \\
\hline 8 & 6 & 0.02 & $\odot . \odot \odot$ & $-\odot .01$ & 0.03 & $\odot .02$ & $-\odot .01$ & $\odot .06$ & $\odot .10$ & $-\odot . \odot 9$ \\
\hline 9 & 6 & $-\odot . \odot \odot$ & ๑.01 & $-\odot .03$ & $-\odot .03$ & $-\odot .01$ & -0.06 & $\odot .02$ & $-\odot . \odot 3$ & -0.12 \\
\hline 10 & 8 & $0.0 \odot$ & $-\odot . \odot \odot$ & $-\odot .01$ & $-\odot . \odot 8$ & $-\odot .03$ & $-\odot . \odot \odot$ & $-\odot .03$ & -0.12 & -0.03 \\
\hline 11 & 1 & $-\odot . \odot \odot$ & $-0.0 \odot$ & $-\odot . \odot \odot$ & -0.12 & -0.03 & 0.11 & -0.10 & -0.13 & 0.23 \\
\hline 12 & 1 & $-\odot . \odot \odot$ & $\odot . \odot \odot$ & $\odot . \odot \odot$ & $-\odot . \odot 7$ & $-\odot .01$ & 0.17 & $-\odot .06$ & $\odot . \odot \odot$ & 0.24 \\
\hline 13 & 1 & $-\odot . \odot \odot$ & 0.00 & $\odot . \odot \odot$ & -0.11 & $-\odot .03$ & 0.09 & $-\odot . \odot 4$ & $-\odot .12$ & 0.01 \\
\hline 14 & 1 & $\odot .02$ & $-\odot . \odot \odot$ & $\odot .01$ & 0.03 & $\odot .02$ & 0.12 & $\odot .04$ & 0.18 & $\odot .02$ \\
\hline 15 & 1 & $\odot . \odot 2$ & $\odot . \odot \odot$ & $-\odot .01$ & $\odot . \odot 7$ & $\odot .03$ & -0.03 & 0.11 & 0.14 & $-\odot .16$ \\
\hline 16 & 1 & 0.03 & $-\odot . \odot \odot$ & $-\odot . \odot 2$ & $\odot .07$ & 0.03 & $\odot . \odot \odot$ & 0.06 & 0.14 & $-\odot .02$ \\
\hline 17 & 1 & -0.01 & 0.01 & $-\odot . \odot 4$ & -0.04 & $-\odot . \odot 2$ & -0.10 & 0.03 & $-\odot .07$ & -0.22 \\
\hline 18 & 7 & 0.01 & 0.01 & $-\odot .03$ & $-\odot . \odot 2$ & $-\odot .01$ & -0.10 & $\odot .01$ & $-\odot .03$ & $-\odot . \odot 9$ \\
\hline 19 & 6 & -0.04 & -0.04 & $-\odot .05$ & $-\odot .01$ & -0.01 & -0.11 & $\odot . \odot \odot$ & $-\odot .04$ & -0.10 \\
\hline 20 & 7 & -0.06 & 0.03 & 0.15 & -0.01 & -0.00 & -0.10 & $-\odot . \odot \odot$ & $-\odot .04$ & -0.07 \\
\hline 21 & 6 & -0.18 & 0.12 & 0.64 & $-\odot . \odot 2$ & 0.01 & $-\odot .07$ & 0.01 & $-\odot .03$ & -0.03 \\
\hline 22 & 6 & -0.03 & $\odot . \odot \odot$ & 0.14 & $-\odot . \odot 2$ & 0.00 & -0.07 & 0.01 & -0.03 & -0.04 \\
\hline 23 & 7 & -0.02 & $-\odot .02$ & -0.01 & $-\odot .04$ & 0.01 & -0.05 & 0.01 & $-\odot . \odot 2$ & 0.01 \\
\hline 24 & 6 & 0.01 & $-\odot .03$ & $\odot . \odot \odot$ & $-\odot .05$ & $\odot .02$ & $-\odot . \odot 2$ & $-\odot . \odot 1$ & $-\odot . \odot 2$ & $\odot .07$ \\
\hline 25 & 7 & $\odot .02$ & $-\odot . \odot \odot$ & $-\odot .05$ & -0.05 & 0.03 & $\odot . \odot \odot$ & $-\odot .02$ & $-\odot .03$ & 0.07 \\
\hline 26 & 6 & -0.03 & $-\odot . \odot 2$ & 0.07 & -0.04 & $\odot .02$ & -0.03 & -0.02 & $-\odot .04$ & $\odot .02$ \\
\hline 27 & 7 & -0.03 & $-\odot .04$ & $-\odot . \odot 2$ & $-\odot .06$ & 0.03 & 0.01 & -0.01 & -0.02 & 0.13 \\
\hline 28 & 8 & 0.06 & $-\odot . \odot \odot$ & $-\odot . \odot 4$ & -0.04 & $\odot .02$ & -0.02 & -0.03 & -0.05 & 0.03 \\
\hline 29 & 1 & -0.04 & -0.06 & -0.26 & -0.01 & -0.02 & -0.12 & 0.00 & -0.04 & -0.12 \\
\hline 30 & 1 & -0.07 & -0.03 & 0.05 & -0.05 & 0.02 & -0.00 & -0.00 & -0.01 & 0.13 \\
\hline
\end{tabular}




$\begin{array}{cccccc}32 & 1 & 0 & -3.891963 & -1.341608 & -1.537457 \\ 33 & 8 & 0 & -2.338058 & 1.084438 & 1.106056 \\ 34 & 1 & 0 & -2.617968 & 2.015200 & 0.994956 \\ 35 & 8 & 0 & -2.514195 & 3.786280 & 0.275285 \\ 36 & 1 & 0 & -2.131188 & 4.396979 & 0.912377 \\ 37 & 1 & 0 & -1.749268 & 3.374331 & -0.174978 \\ 38 & 1 & 0 & -3.525593 & -0.110564 & 1.869781 \\ 39 & 8 & 0 & -4.100324 & -0.781631 & 2.278683 \\ 40 & 1 & 0 & -4.822887 & -0.891750 & 1.654356 \\ -\end{array}$

Standard basis: Aug-CC-pVDZ (6D, 7F)

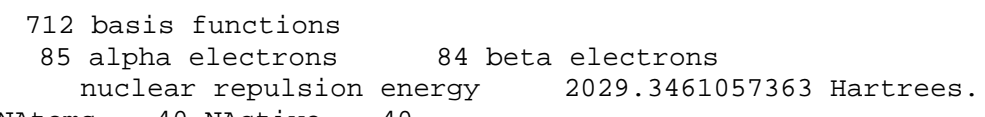

NAtoms $=40$ NActive $=40$

Force inversion solution in PCM.

Polarizable Continuum Model (PCM)

=ニニニニニニニニニニニニニニニニニニニニニニニニニニニニニニニニ

Model : PCM.

Solvent : Water, Eps $=78.355300$ Eps $($ inf $)=1.777849$

-.-. -

SCF Done: $E($ UWB97XD $)=-1192.07668353$ A.U. after NFock $=2$ Conv $=0.38 \mathrm{D}-09 \quad-\mathrm{V} / \mathrm{T}=2.0071$

$\langle\mathrm{SX}\rangle=0.0000\langle\mathrm{~S} y\rangle=0.0000\langle\mathrm{~S} z\rangle=0.5000\langle\mathrm{~S} * * 2\rangle=0.7658 \mathrm{~S}=0.5079$

$<$ L.S $>=0.000000000000 \mathrm{E}+00$

Annihilation of the first spin contaminant:

$\mathrm{S}^{* * 2}$ before annihilation 0.7658 , after 0.7502

Harmonic frequencies $\left(\mathrm{cm}^{*}-1\right)$, IR intensities (KM/Mole), Raman

$\begin{array}{ccccc} & 1 & 2 & & \\ \text { Frequencies - - } & \text { A } 23.5101 & \text { A } & \text { A } & \\ & 23.3158 & & 36.6854\end{array}$

Zero-point correction=

Thermal correction to Energy=

๑. 316855 (a.u.)

Thermal correction to Enthalpy=

$\odot .341253$

$\odot .342197$

$\odot .261659$

$-1191.759829$

$-1191.735431$

$-1191.734486$

Sum of electronic and thermal Energies=

Sum of electronic and thermal Enthalpies=

$-1191.815024$

Sum of electronic and thermal Free Energies=

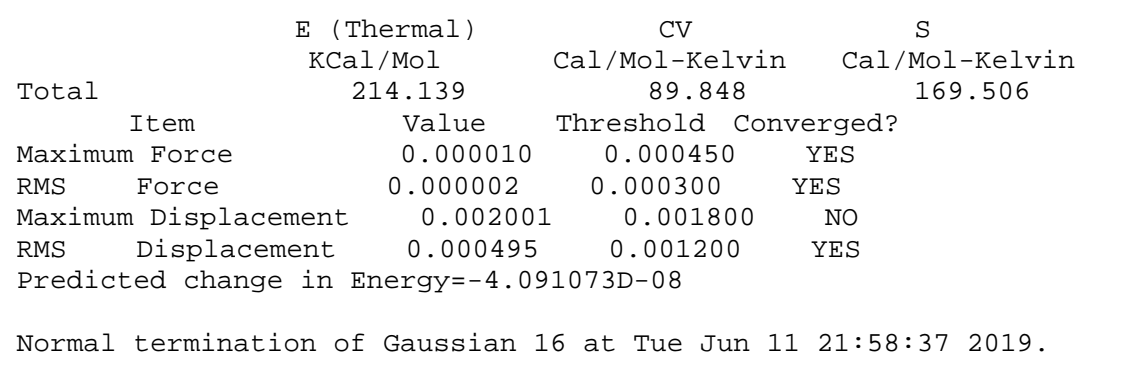

. Figure S9-4, TS2(C5)W2 $\ldots \ldots$

degua-oh5w2.ts1.higha. log

Stoichiometry C10H18N507(2)

Standard orientation:

\begin{tabular}{|c|c|c|c|c|c|}
\hline Center & Atomic & Atomic & \multicolumn{3}{|c|}{ Coordinates (Angstroms) } \\
\hline Number & Number & Type & $\mathrm{x}$ & $\mathrm{Y}$ & Z \\
\hline & - & & $-2-5-2-3$ & - & $--1--1$ \\
\hline 1 & 1 & $\odot$ & -4.004740 & -1.494960 & -1.847801 \\
\hline 2 & 8 & $\odot$ & -3.602114 & -1.957140 & -1.107469 \\
\hline 3 & 8 & $\odot$ & -4.345509 & 1.978750 & ๑. 682858 \\
\hline 4 & 6 & $\odot$ & -3.879875 & -0.354236 & $\odot .749871$ \\
\hline 5 & 6 & $\odot$ & -3.928982 & 0.893240 & $-\odot .139916$ \\
\hline 6 & 1 & 0 & -4.337263 & 2.781299 & 0.152264 \\
\hline
\end{tabular}




\begin{tabular}{|c|c|c|c|c|c|}
\hline 7 & 6 & 0 & -4.318674 & -1.650981 & ๑.082979 \\
\hline 8 & 6 & $\odot$ & -2.483144 & 1.040051 & $-\odot .601636$ \\
\hline 9 & 6 & 0 & -1.692780 & $\odot .496997$ & $\odot .590497$ \\
\hline 10 & 8 & 0 & -2.524404 & -0.472055 & 1.203688 \\
\hline 11 & 1 & $\odot$ & -4.129302 & -2.477140 & 0.778614 \\
\hline 12 & 1 & 0 & -5.401373 & -1.606412 & $-\odot .106548$ \\
\hline 13 & 1 & 0 & -4.522722 & -0.192604 & 1.626458 \\
\hline 14 & 1 & 0 & -4.626253 & 0.768921 & -0.980150 \\
\hline 15 & 1 & $\odot$ & -2.211157 & 2.073258 & -0.837915 \\
\hline 16 & 1 & 0 & -2.304967 & 0.420211 & -1.487727 \\
\hline 17 & 1 & $\odot$ & -1.450270 & 1.294299 & 1.303535 \\
\hline 18 & 7 & 0 & -0.432015 & -0.148536 & 0.263606 \\
\hline 19 & 6 & 0 & $-\odot .368560$ & -1.248850 & -0.605152 \\
\hline 20 & 7 & 0 & 0.738241 & -1.608653 & -1.113770 \\
\hline 21 & 6 & 0 & 1.882477 & -0.325009 & 0.037554 \\
\hline 22 & 6 & $\odot$ & 0.764734 & 0.534422 & $\odot .215832$ \\
\hline 23 & 7 & 0 & 0.755218 & 1.865547 & 0.059589 \\
\hline 24 & 6 & 0 & 1.868873 & 2.421600 & -0.377203 \\
\hline 25 & 7 & 0 & 2.942035 & 1.674500 & -0.775620 \\
\hline 26 & 6 & 0 & 3.065462 & $\odot .306770$ & -0.549467 \\
\hline 27 & 7 & $\odot$ & 1.957724 & 3.758215 & -0.469359 \\
\hline 28 & 8 & 0 & 4.116463 & $-\odot .270564$ & -0.816542 \\
\hline 29 & 1 & $\odot$ & -1.325684 & -1.742192 & -0.809395 \\
\hline 30 & 1 & 0 & 1.136061 & 4.306017 & -0.266822 \\
\hline 31 & 1 & 0 & 2.753066 & 4.218034 & -0.882871 \\
\hline 32 & 1 & $\odot$ & 3.766390 & 2.130348 & -1.151393 \\
\hline 33 & 8 & 0 & 2.143694 & -1.324451 & 0.961122 \\
\hline 34 & 1 & $\odot$ & 2.364730 & -2.158381 & 0.493674 \\
\hline 35 & 8 & $\odot$ & 2.563556 & -3.598372 & -0.605598 \\
\hline 36 & 1 & 0 & $2.17 \odot 261$ & -4.394446 & -0.234962 \\
\hline 37 & 1 & 0 & 1.826921 & -3.130140 & -1.048591 \\
\hline 38 & 1 & 0 & 3.771490 & $-\odot .949210$ & 1.938460 \\
\hline 39 & 8 & 0 & 4.682300 & $-\odot .704270$ & 2.168057 \\
\hline 40 & 1 & $\odot$ & 5.067329 & -0.533606 & 1. 300961 \\
\hline
\end{tabular}

Standard basis: Aug-CC-pVDZ (6D, 7F)

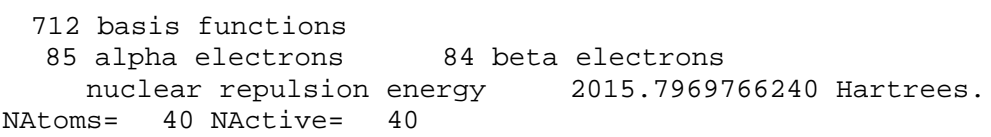

Force inversion solution in PCM.

Polarizable Continuum Model (PCM)

=ニニニニニニニニニニニニ=ニニニニニニニニニニニニニニニニーニ

Model : PCM.

Solvent : Water, Eps $=78.355300$ Eps $($ inf $)=1.777849$

SCF Done: $E($ UWB97XD $)=-1192.02781739$ A.U. after 2 cycles NFock $=2$ Conv $=0.85 \mathrm{D}-09 \quad-\mathrm{V} / \mathrm{T}=2.0071$

$\langle\mathrm{S} x\rangle=0.0000<\mathrm{Sy}\rangle=0.0000<\mathrm{Sz}>=0.5000$

$<S^{* *} 2>=0.7802 S=0.5150$

$<$ L.S $>=0.000000000000 \mathrm{E}+00$

Annihilation of the first spin contaminant:

$\mathrm{S}^{* * 2}$ before annihilation 0.7802 , after 0.7504

Harmonic frequencies $\left(\mathrm{cm}^{* *}-1\right)$, IR intensities (KM/Mole), Raman activities $\left(A^{* *} 4 / \mathrm{AMU}\right)$, depolarization ratios for plane and unpo incident light, reduced masses (AMU), force constants (mDyne/A), and normal coordinates:

\begin{tabular}{|c|c|c|c|c|c|c|c|c|c|c|}
\hline & & & 1 & & 2 & & & 3 & & \\
\hline & & & A & & A & & & A & & \\
\hline Freque & encies & s - - & -479.74 & & & 26.795 & & & 30.1009 & \\
\hline Red. $\mathrm{n}$ & nasses & S - - & 10.16 & & & 8.0255 & & & 5.4246 & \\
\hline FrC CO & onsts & - - & 1.378 & & & 0.0034 & & & 0.0029 & \\
\hline IR Int & Een & - - & 88.506 & & & 4.8479 & & & 0.5457 & \\
\hline Atom & AN & $x$ & $Y$ & Z & $x$ & $Y$ & Z & $x$ & Z & \\
\hline 1 & 1 & $-\odot . \odot \odot$ & $-\odot . \odot \odot$ & -0.00 & $-\odot . \odot 4$ & 0.11 & -0.03 & -0.09 & -0.22 & 0.18 \\
\hline 2 & 8 & $-\odot . \odot \odot$ & $-\odot . \odot \odot$ & 0.00 & -0.03 & $\odot .07$ & -0.06 & -0.02 & -0.14 & 0.19 \\
\hline 3 & 8 & $\odot . \odot \odot$ & $-\odot . \odot \odot$ & $-\odot . \odot \odot$ & $\odot .07$ & $-\odot . \odot \odot$ & 0.16 & $-\odot .07$ & -0.03 & $-\odot .08$ \\
\hline 4 & 6 & 0.01 & $0.0 \odot$ & $0.0 \odot$ & 0.02 & -0.01 & 0.03 & 0.03 & $-0.0 \odot$ & 0.08 \\
\hline 5 & 6 & $\odot .01$ & $\odot . \odot \odot$ & $\odot . \odot \odot$ & $\odot .02$ & 0.03 & $\odot .09$ & -0.06 & $-\odot . \odot 7$ & $-\odot .01$ \\
\hline 6 & 1 & -0.00 & $-\odot . \odot \odot$ & $-0.0 \odot$ & 0.08 & 0.03 & 0.21 & -0.15 & -0.07 & -0.16 \\
\hline
\end{tabular}




\begin{tabular}{|c|c|c|c|c|}
\hline 7 & 6 & $\odot .00$ & $\odot . \odot \odot$ & 0.00 \\
\hline 8 & 6 & 0.01 & -0.00 & $\odot . \odot \odot$ \\
\hline 9 & 6 & $\odot . \odot 2$ & $-\odot . \odot \odot$ & -0.00 \\
\hline 10 & 8 & 0.02 & 0.00 & -0.00 \\
\hline 11 & 1 & $\odot . \odot \odot$ & $\odot . \odot \odot$ & $\odot .0 \odot$ \\
\hline 12 & 1 & $\odot .0 \odot$ & $\odot .01$ & 0.01 \\
\hline 13 & 1 & $\odot .02$ & $\odot . \odot \odot$ & 0.01 \\
\hline 14 & 1 & 0.01 & $-\odot . \odot \odot$ & -0.00 \\
\hline 15 & 1 & 0.01 & -0.00 & 0.00 \\
\hline 16 & 1 & 0.01 & $\odot .0 \odot$ & -0.00 \\
\hline 17 & 1 & 0.04 & -0.01 & $-\odot . \odot \odot$ \\
\hline 18 & 7 & 0.05 & $-\odot .02$ & -0.03 \\
\hline 19 & 6 & -0.13 & -0.05 & 0.03 \\
\hline 20 & 7 & -0.36 & -0.30 & -0.25 \\
\hline 21 & 6 & 0.30 & 0.37 & 0.42 \\
\hline 22 & 6 & $\odot .09$ & -0.03 & -0.06 \\
\hline 23 & 7 & -0.01 & -0.02 & -0.07 \\
\hline 24 & 6 & 0.02 & $\odot .01$ & -0.02 \\
\hline 25 & 7 & 0.00 & $\odot .06$ & -0.04 \\
\hline 26 & 6 & 0.07 & $\odot .07$ & 0.15 \\
\hline 27 & 7 & -0.02 & 0.01 & 0.00 \\
\hline 28 & 8 & 0.04 & 0.03 & 0.02 \\
\hline 29 & 1 & -0.21 & 0.02 & 0.20 \\
\hline 30 & 1 & -0.03 & -0.00 & -0.01 \\
\hline 31 & 1 & 0.00 & 0.02 & 0.06 \\
\hline 32 & 1 & -0.06 & 0.06 & -0.17 \\
\hline 33 & 8 & -0.04 & -0.07 & -0.04 \\
\hline 34 & 1 & $-\odot .09$ & 0.02 & -0.23 \\
\hline 35 & 8 & 0.00 & -0.01 & -0.01 \\
\hline 36 & 1 & 0.04 & $-0.0 \odot$ & 0.04 \\
\hline 37 & 1 & -0.00 & -0.11 & -0.08 \\
\hline 38 & 1 & 0.01 & -0.09 & 0.04 \\
\hline 39 & 8 & -0.01 & $-\odot . \odot \odot$ & -0.01 \\
\hline 40 & 1 & -0.07 & 0.01 & -0.03 \\
\hline
\end{tabular}

$\begin{array}{ccc}-0.01 & 0.03 & -0.03 \\ 0.01 & 0.02 & 0.05 \\ 0.03 & -0.03 & 0.02 \\ 0.03 & -0.05 & -0.01 \\ -0.01 & -0.01 & -0.07 \\ -0.01 & 0.05 & -0.01 \\ 0.04 & -0.05 & 0.05 \\ -0.01 & 0.09 & 0.11 \\ 0.02 & 0.03 & 0.08 \\ -0.03 & 0.05 & 0.03 \\ 0.05 & -0.06 & 0.04 \\ 0.01 & -0.03 & -0.03 \\ -0.01 & -0.02 & -0.05 \\ -0.01 & -0.03 & -0.06 \\ 0.01 & -0.06 & -0.05 \\ 0.02 & -0.05 & -0.03 \\ 0.03 & -0.04 & -0.02 \\ 0.04 & -0.05 & -0.03 \\ 0.03 & -0.06 & -0.03 \\ 0.02 & -0.06 & -0.03 \\ 0.05 & -0.05 & -0.02 \\ 0.02 & -0.07 & -0.01 \\ -0.01 & -0.00 & -0.05 \\ 0.06 & -0.05 & -0.02 \\ 0.05 & -0.06 & -0.03 \\ 0.04 & -0.07 & -0.02 \\ -0.00 & -0.07 & -0.06 \\ 0.04 & -0.05 & -0.07 \\ 0.02 & -0.01 & -0.12 \\ 0.04 & -0.03 & -0.14 \\ 0.01 & -0.01 & -0.09 \\ -0.20 & 0.22 & 0.06 \\ -0.30 & 0.45 & 0.24 \\ -0.12 & 0.37 & 0.30\end{array}$

$\begin{array}{rrr}0.03 & -0.07 & 0.21 \\ -0.08 & -0.05 & -0.09\end{array}$

$\begin{array}{lll}-0.00 & 0.05 & -0.10\end{array}$

$0.06 \quad 0.07 \quad 0.02$

$\begin{array}{llll}0.09 & -0.01 & 0.26\end{array}$

$0.02 \quad-0.12 \quad 0.26$

$0.07 \quad 0.05 \quad 0.10$

$\begin{array}{llll}-0.09 & -0.17 & 0.03\end{array}$

$-0.13-0.05 \quad-0.16$

$-0.11-0.09-0.06$

$0.01 \quad 0.10 \quad-0.16$

$\begin{array}{lll}-0.01 & 0.07 & -0.12\end{array}$

$0.00 \quad 0.07 \quad-0.12$

$\begin{array}{llll}0.01 & 0.06 & -0.10\end{array}$

$\begin{array}{llll}-0.01 & 0.02 & -0.04\end{array}$

$\begin{array}{llll}0.01 & 0.04 & -0.06\end{array}$

$0.05 \quad 0.05 \quad-0.03$

$0.08 \quad 0.03 \quad 0.04$

$0.08 \quad 0.01 \quad 0.06$

$0.04 \quad 0.00 \quad 0.02$

$\begin{array}{lll}0.13 & 0.03 & 0.08\end{array}$

$0.03 \quad-0.02 \quad 0.06$

$0.00 \quad 0.07 \quad-0.14$

$0.13 \quad 0.04 \quad 0.06$

$\begin{array}{lll}0.15 & 0.02 & 0.12\end{array}$

$0.11 \quad 0.00 \quad 0.11$

$\begin{array}{lll}-0.07 & -0.01 & -0.05\end{array}$

$-0.06 \quad-0.00 \quad-0.06$

$\begin{array}{lll}-0.04 & 0.03 & -0.10\end{array}$

$\begin{array}{lll}-0.06 & 0.02 & -0.13\end{array}$

$\begin{array}{lll}-0.02 & 0.05 & -0.11\end{array}$

$-0.12-0.05 \quad 0.04$

$\begin{array}{lll}-0.13 & -0.08 & 0.10\end{array}$

Zero-point correction=

Thermal correction to Energy=

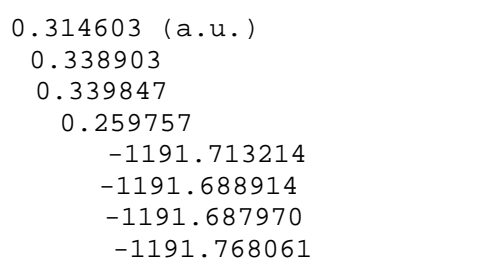

$-1191.768061$

Thermal correction to Enthalpy=

Thermal correction to Gibbs Free Energy=

Sum of electronic and zero-point Energies=

Sum of electronic and thermal Energies=

Sum of electronic and thermal Enthalpies=

Sum of electronic and thermal Free Energies=

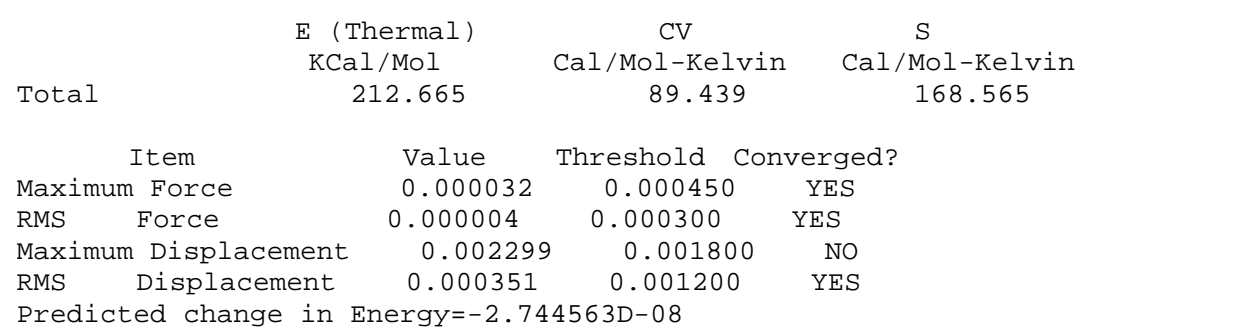

Normal termination of Gaussian 16 at Sun Jun 9 07:49:32 2019 .

.......... Figure S9-5, Int3(C5)W2

degua-oh5w2 . rev1.higha. log

Stoichiometry $\mathrm{C} 10 \mathrm{H} 18 \mathrm{~N} 507(2)$

\begin{tabular}{|c|c|c|c|c|c|}
\hline Center & Atomic & Atomic & & dinates ( & gstroms ) \\
\hline Number & Number & Type & $\mathrm{X}$ & $Y$ & Z \\
\hline--- & - & & -- & $--\cdots--$ & $--\cdots--$ \\
\hline 1 & 1 & 0 & -4.308390 & -1.650817 & -1.527240 \\
\hline 2 & 8 & $\odot$ & -3.785830 & -2.079668 & -0.843813 \\
\hline 3 & 8 & $\odot$ & -4.248699 & 1.926042 & 0.900741 \\
\hline 4 & 6 & 0 & -3.753767 & -0.400771 & ๑. 963859 \\
\hline 5 & 6 & $\odot$ & -3.969995 & 0.814938 & $\odot .054160$ \\
\hline 6 & 1 & $\odot$ & -4.343344 & 2.709336 & ๑. 350122 \\
\hline 7 & 6 & $\odot$ & -4.296168 & -1.721719 & 0.436038 \\
\hline 8 & 6 & $\odot$ & -2.627756 & ๑. 952683 & -0.656215 \\
\hline 9 & 6 & $\odot$ & -1.635462 & $\odot .451897$ & ๑. 392989 \\
\hline
\end{tabular}




\begin{tabular}{|c|c|c|c|c|c|}
\hline 10 & 8 & $\odot$ & -2.338005 & -0.504177 & 1.168416 \\
\hline 11 & 1 & $\Theta$ & -3.990890 & -2.519586 & 1.123294 \\
\hline 12 & 1 & $\odot$ & -5.395124 & -1.680620 & $\odot .427492$ \\
\hline 13 & 1 & $\odot$ & -4.228394 & -0.206325 & 1.935673 \\
\hline 14 & 1 & $\odot$ & -4.802275 & 0.658606 & -0.646269 \\
\hline 15 & 1 & $\odot$ & -2.409625 & 1.978696 & -0.967509 \\
\hline 16 & 1 & $\odot$ & -2.601120 & $\odot .307963$ & -1.542445 \\
\hline 17 & 1 & $\Theta$ & -1.286981 & 1.269886 & 1.032788 \\
\hline 18 & 7 & $\odot$ & -0.428280 & -0.191901 & -0.125916 \\
\hline 19 & 6 & $\odot$ & $-\odot .581912$ & -1.332238 & $-\odot .880792$ \\
\hline 20 & 7 & $\Theta$ & 0.286215 & -1.914226 & -1.594755 \\
\hline 21 & 6 & $\odot$ & 1.972102 & -0.095186 & 0.211285 \\
\hline 22 & 6 & $\odot$ & $\odot .784580$ & $\odot .524637$ & -0.106333 \\
\hline 23 & 7 & $\Theta$ & 0.660834 & 1.847362 & -0.389633 \\
\hline 24 & 6 & $\odot$ & 1.760594 & 2.563559 & -0.419470 \\
\hline 25 & 7 & $\odot$ & 2.987574 & 2.012615 & -0.200528 \\
\hline 26 & 6 & $\Theta$ & 3.180189 & $\odot .681725$ & 0.146452 \\
\hline 27 & 7 & $\odot$ & 1.702011 & 3.878142 & -0.716743 \\
\hline 28 & 8 & $\odot$ & 4.324946 & $\odot .272371$ & $\odot .377909$ \\
\hline 29 & 1 & $\odot$ & -1.602621 & -1.751320 & -0.852264 \\
\hline 30 & 1 & $\odot$ & $\odot .789189$ & 4.301222 & -0.780753 \\
\hline 31 & 1 & $\odot$ & 2.496923 & 4.485470 & $-\odot .593122$ \\
\hline 32 & 1 & $\odot$ & 3.824470 & 2.582108 & -0.254181 \\
\hline 33 & 8 & $\odot$ & 2.036722 & -1.373901 & $\odot .675530$ \\
\hline 34 & 1 & $\odot$ & 2.492810 & -1.939381 & $\odot .014126$ \\
\hline 35 & 8 & $\odot$ & 2.957304 & -2.823386 & -1.451202 \\
\hline 36 & 1 & $\odot$ & 3.078766 & -3.765539 & -1.298184 \\
\hline 37 & 1 & $\Theta$ & 2.032973 & -2.725278 & -1.743741 \\
\hline 38 & 1 & $\odot$ & 3.954223 & -2.333331 & 2.138752 \\
\hline 39 & 8 & $\odot$ & 4.829590 & -1.987398 & 1.942043 \\
\hline 40 & 1 & $\odot$ & 4.638286 & -1.221250 & 1.367648 \\
\hline
\end{tabular}

Standard basis: Aug-CC-pVDZ (6D, 7F)

712 basis functions

85 alpha electrons 84 beta electrons

nuclear repulsion energy 1991.9310144379 Hartrees.

NAtoms $=40$ NActive $=40$

Force inversion solution in PCM.

Polarizable Continuum Model (PCM)

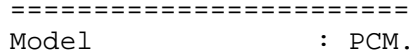

Solvent : Water, Eps $=78.355300$ Eps $($ inf $)=1.777849$

SCF Done: $E($ UWB97XD) $=-1192.05803028$ A.U. after 1 cycles NFock $=1$ Conv $=0.42 \mathrm{D}-08 \quad-\mathrm{V} / \mathrm{T}=2.0071$

$<\mathrm{S} x>=0.0000<\mathrm{Sy}>=0.0000<\mathrm{Sz}>=0.5000$

$<S^{* *} 2>=0.7605 \mathrm{~S}=0.5052$

$<$ L.S $>=0.000000000000 \mathrm{E}+0 \odot$

Annihilation of the first spin contaminant:

$\mathrm{S}^{* * 2}$ before annihilation 0.7605 , after 0.7501

Harmonic frequencies $\left(\mathrm{cm}^{*}{ }^{*}-1\right)$, IR intensities (KM/Mole), Raman

$\begin{array}{ccccc} & 1 & 2 & & \\ & \mathrm{~A} & \mathrm{~A} & \mathrm{~A} & \\ \text { Frequencies - - } & 18.3074 & 23.5509 & & 32.4698\end{array}$

Zero-point correction=

Thermal correction to Energy=

$\odot .314871$ (a.u. )

Thermal correction to Enthalpy=

0.340219

Thermal correction to Gibbs Free Energy=

Sum of electronic and zero-point Energies=

$\odot .341163$

0.257673

$-1191.743159$

$-1191.717811$

$-1191.716867$

Sum of electronic and thermal Enthalpies=

$-1191.800357$

Sum of electronic and thermal Free Energies=

\section{$\mathrm{S}$}

$$
\begin{aligned}
& \text { E (Thermal) } \\
& \text { KCal/Mol }
\end{aligned}
$$

$\begin{array}{ll}\text { Total } & \\ & \text { Item }\end{array}$

213. 491

$\mathrm{CV}$

Cal/Mol-Kelvin

91.598

Cal/Mol-Kelvin

175.720

Maximum Force

Value Threshold Converged?

$\odot .000003$

$\odot .000450$ YES 


$\begin{array}{llccr}\text { RMS } & \text { Force } & 0.000001 & 0.000300 & \text { YES } \\ \text { Maximum Displacement } & 0.002425 & 0.001800 & \text { NO } \\ \text { RMS } & \text { Displacement } & 0.000488 & 0.001200 & \text { YES }\end{array}$

Predicted change in Energy $=-4.579938 \mathrm{D}-09$

optimization completed on the basis of negligible forces.

- - Stationary point found.

Normal termination of Gaussian 16 at Tue Jun 11 05:34:36 2019.

Figure S9-6, TS3(C5)W2

degua-oh5w2.ts2.higha. $\log$

Stoichiometry C10H18N507(2)

Standard orientation:

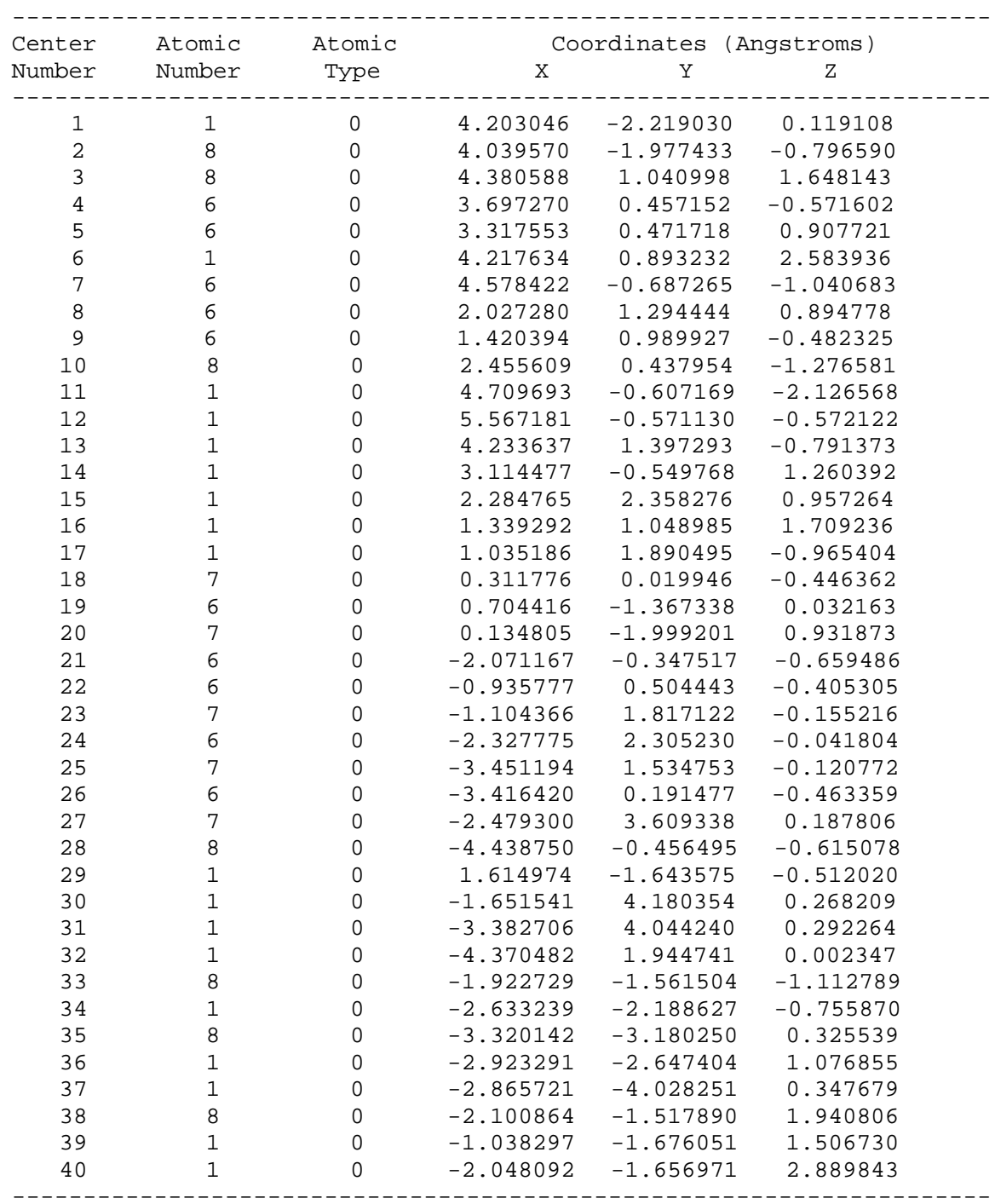

Standard basis: Aug-CC-pVDZ (6D, 7F)

712 basis functions

85 alpha electrons 84 beta electrons

nuclear repulsion energy 2033.8466976546 Hartrees.

NAtoms $=40$ NActive $=40$

Nuclear repulsion after empirical dispersion term =

2033.8137020515 Hartrees.

Force inversion solution in PCM.

Polarizable Continuum Model (PCM)

Model

: PCM. 
Solvent : Water, Eps $=78.355300$ Eps $($ inf $)=1.777849$

SCF Done: $E($ UWB97XD $)=-1192.02390166 \mathrm{~A} . \mathrm{U}$. after 1 cycles $\mathrm{NFock}=1$ Conv $=0.53 \mathrm{D}-08 \quad-\mathrm{V} / \mathrm{T}=2.0070$

$\langle\mathrm{S} X>=0.0000<\mathrm{S} y>=0.0000<\mathrm{Sz}>=0.5000$

$<S^{* *} 2>=0.7602 \mathrm{~S}=0.5051$

$<\mathrm{L} . \mathrm{S}\rangle=0.000000000000 \mathrm{E}+\odot \odot$

Annihilation of the first spin contaminant:

$\mathrm{S}^{* * 2}$ before annihilation 0.7602 , after 0.7501

Harmonic frequencies $\left(\mathrm{cm}^{*}-1\right)$, IR intensities (KM/Mole), Raman activities $\left(A^{* *} 4 / A M U\right)$, depolarization ratios for plane and unpo incident light, reduced masses (AMU), force constants (mDyne/A), and normal coordinates:

\begin{tabular}{|c|c|c|c|c|}
\hline \multicolumn{5}{|c|}{ 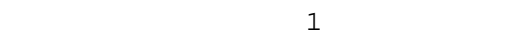 } \\
\hline \multicolumn{2}{|c|}{ Frequencies } & -- & \multicolumn{2}{|c|}{-960.0241} \\
\hline \multicolumn{2}{|c|}{ Red. masses } & -- & \multicolumn{2}{|c|}{1.1242} \\
\hline \multicolumn{2}{|c|}{ Frc consts } & -- & \multicolumn{2}{|c|}{$\odot .6105$} \\
\hline \multicolumn{2}{|c|}{ IR Inten } & -- & \multicolumn{2}{|c|}{4316.6062} \\
\hline Atom & AN & $x$ & $\mathrm{Y}$ & Z \\
\hline 1 & 1 & 0.01 & -0.01 & -0.00 \\
\hline 2 & 8 & $-\odot .0 \odot$ & $\odot .00$ & 0.00 \\
\hline 3 & 8 & $-\odot . \odot \odot$ & -0.00 & $-0.0 \odot$ \\
\hline 4 & 6 & 0.00 & 0.00 & $\odot .00$ \\
\hline 5 & 6 & $\odot . \odot \odot$ & $\odot .00$ & $\odot . \odot \odot$ \\
\hline 6 & 1 & $\odot .0 \odot$ & 0.00 & $\odot .0 \odot$ \\
\hline 7 & 6 & $\odot .0 \odot$ & $\odot .00$ & -0.00 \\
\hline 8 & 6 & $\odot . \odot \odot$ & $\odot .0 \odot$ & $-\odot . \odot \odot$ \\
\hline 9 & 6 & 0.01 & -0.00 & -0.00 \\
\hline 10 & 8 & $\odot . \odot \odot$ & -0.00 & 0.00 \\
\hline 11 & 1 & $\odot .0 \odot$ & -0.00 & -0.00 \\
\hline 12 & 1 & -0.00 & -0.00 & 0.00 \\
\hline 13 & 1 & -0.00 & $\odot .0 \odot$ & -0.00 \\
\hline 14 & 1 & $\odot . \odot \odot$ & -0.00 & 0.00 \\
\hline 15 & 1 & $-0.0 \odot$ & $\odot . \odot \odot$ & -0.00 \\
\hline 16 & 1 & -0.00 & $\odot . \odot \odot$ & -0.00 \\
\hline 17 & 1 & -0.02 & -0.02 & -0.00 \\
\hline 18 & 7 & 0.01 & -0.03 & 0.01 \\
\hline 19 & 6 & -0.04 & 0.06 & 0.00 \\
\hline 20 & 7 & -0.05 & $-\odot . \odot \odot$ & 0.01 \\
\hline 21 & 6 & $-\odot . \odot \odot$ & -0.01 & $\odot .0 \odot$ \\
\hline 22 & 6 & -0.01 & $\odot . \odot \odot$ & 0.01 \\
\hline 23 & 7 & 0.01 & -0.01 & -0.00 \\
\hline 24 & 6 & -0.01 & 0.00 & -0.00 \\
\hline 25 & 7 & $0.0 \odot$ & 0.00 & $\odot . \odot \odot$ \\
\hline 26 & 6 & $-\odot . \odot \odot$ & $-\odot . \odot \odot$ & $\odot . \odot \odot$ \\
\hline 27 & 7 & $\odot . \odot \odot$ & $-0.0 \odot$ & -0.00 \\
\hline 28 & 8 & $\odot . \odot \odot$ & $\odot . \odot \odot$ & -0.00 \\
\hline 29 & 1 & $-\odot .01$ & -0.04 & 0.11 \\
\hline 30 & 1 & $\odot .00$ & $-\odot . \odot \odot$ & $-\odot . \odot \odot$ \\
\hline 31 & 1 & $\odot . \odot \odot$ & $-\odot . \odot \odot$ & $\odot . \odot \odot$ \\
\hline 32 & 1 & $\odot .00$ & $\odot .0 \odot$ & $\odot .00$ \\
\hline 33 & 8 & -0.00 & $\odot . \odot \odot$ & $\odot .0 \odot$ \\
\hline 34 & 1 & 0.01 & -0.02 & 0.04 \\
\hline 35 & 8 & 0.01 & $\odot .01$ & $\odot .01$ \\
\hline 36 & 1 & 0.06 & 0.07 & 0.01 \\
\hline 37 & 1 & 0.01 & 0.01 & 0.00 \\
\hline 38 & 8 & $-0 . \odot \odot$ & -0.01 & -0.02 \\
\hline 39 & 1 & 0.86 & -0.11 & -0.46 \\
\hline 40 & 4 & $-\odot .03$ & -0.01 & -0 \\
\hline
\end{tabular}

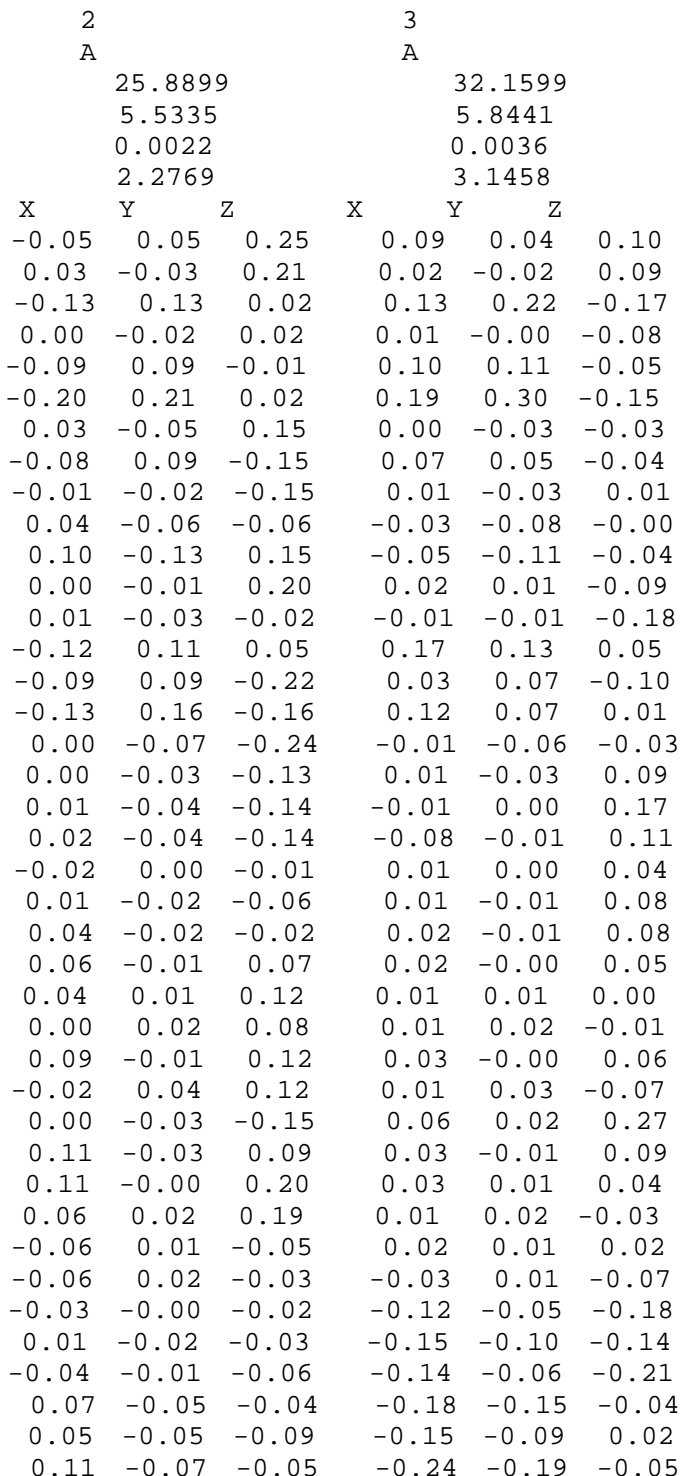

Zero-point correction=

Thermal correction to Energy=

Thermal correction to Enthalpy=

๑. 310464 ( a.u. )

$\odot .333887$

$\odot .334832$

0.257251

$-1191.713437$

$-1191.690014$

$-1191.689070$

Sum of electronic and thermal Energies=

$-1191.766651$

Sum of electronic and thermal Free Energies=

$\mathrm{S}$

E (Thermal) $\mathrm{KCal} / \mathrm{Mol}$

CV

Cal/Mol-Kelvin Cal/Mol-Kelvin

87.774

Total

209.517 


\begin{tabular}{lccc} 
Maximum Force & 0.000013 & 0.000450 & YES \\
RMS Force & 0.000001 & 0.000300 & YES \\
Maximum Displacement & 0.000588 & $0.00180 \odot$ & YES \\
RMS Displacement & 0.000139 & 0.001200 & YES \\
Predicted change in Energy=-1.522745D- & \\
Optimization completed. & & \\
\multicolumn{2}{l}{- - Stationary point found. } & &
\end{tabular}

Normal termination of Gaussian 16 at Sun May 19 13:50:36 2019 .

Figure s9-7, Int4(C5)W2

degua-oh5w2.rev2.higha.log

Stoichiometry C10H18N507(2)

Standard orientation:

\begin{tabular}{|c|c|c|c|c|c|}
\hline \multirow{2}{*}{$\begin{array}{l}\text { Center } \\
\text { Number }\end{array}$} & \multirow{2}{*}{$\begin{array}{l}\text { Atomic } \\
\text { Number }\end{array}$} & \multirow{2}{*}{$\begin{array}{c}\text { Atomic } \\
\text { Type }\end{array}$} & \multicolumn{3}{|c|}{ Coordinates (Angstroms) } \\
\hline & & & $x$ & $\mathrm{Y}$ & z \\
\hline 1 & 1 & $\odot$ & 4.208620 & -2.177934 & 0.408833 \\
\hline 2 & 8 & $\odot$ & 3.913821 & -2.029315 & -0.493495 \\
\hline 3 & 8 & 0 & 4.486313 & 1.312590 & 1.417890 \\
\hline 4 & 6 & $\odot$ & 3.690040 & 0.416562 & -0.655098 \\
\hline 5 & 6 & 0 & 3.380256 & $\odot .663773$ & $\odot .820396$ \\
\hline 6 & 1 & 0 & 4.364752 & 1.314074 & 2.371544 \\
\hline 7 & 6 & $\odot$ & 4.497020 & -0.828034 & -0.979624 \\
\hline 8 & 6 & 0 & 2.104381 & 1.503064 & 0.737866 \\
\hline 9 & 6 & $\odot$ & 1.418372 & $\odot .988698$ & -0.533020 \\
\hline 10 & 8 & $\odot$ & 2.416269 & $\odot .346154$ & -1.302041 \\
\hline 11 & 1 & $\Theta$ & 4.565191 & -0.929063 & -2.069586 \\
\hline 12 & 1 & $\odot$ & 5.515482 & -0.691594 & -0.588333 \\
\hline 13 & 1 & $\odot$ & 4.248435 & 1.288365 & -1.037228 \\
\hline 14 & 1 & $\odot$ & 3.181670 & -0.289120 & 1.332919 \\
\hline 15 & 1 & $\odot$ & 2.383050 & 2.555207 & $\odot .603695$ \\
\hline 16 & 1 & $\odot$ & 1.457079 & 1.419696 & 1.615392 \\
\hline 17 & 1 & $\odot$ & $\odot .972810$ & 1.800128 & -1.113986 \\
\hline 18 & 7 & $\odot$ & $\odot .328301$ & $\odot .020287$ & -0.294685 \\
\hline 19 & 6 & $\odot$ & $\odot .67 \odot 272$ & -1.229854 & $\odot .237240$ \\
\hline 20 & 7 & $\odot$ & -0.0000066 & -1.927853 & 1.066025 \\
\hline 21 & 6 & $\odot$ & -2.033176 & -0.369761 & -0.781599 \\
\hline 22 & 6 & $\odot$ & $-\odot .972446$ & $\odot .487981$ & -0.301026 \\
\hline 23 & 7 & 0 & -1.151027 & 1.765876 & 0.048640 \\
\hline 24 & 6 & $\odot$ & -2.381647 & 2.263765 & 0.035952 \\
\hline 25 & 7 & $\odot$ & -3.474992 & 1.518020 & $-\odot .280476$ \\
\hline 26 & 6 & $\odot$ & -3.408498 & $\odot .195310$ & $-0.70500 \odot$ \\
\hline 27 & 7 & $\odot$ & -2.558207 & 3.544579 & 0.368599 \\
\hline 28 & 8 & $\odot$ & -4.421739 & -0.408142 & -1.012584 \\
\hline 29 & 1 & $\odot$ & 1.645008 & -1.580460 & -0.109856 \\
\hline 30 & 1 & $\odot$ & -1.747035 & 4.090114 & 0.617290 \\
\hline 31 & 1 & $\odot$ & -3.465719 & 3.982927 & $\odot .394824$ \\
\hline 32 & 1 & $\odot$ & -4.401654 & 1.929830 & -0.251138 \\
\hline 33 & 8 & $\odot$ & -1.850367 & -1.503306 & -1.276700 \\
\hline 34 & 1 & $\odot$ & -2.290324 & -3.058624 & -0.363252 \\
\hline 35 & 8 & $\odot$ & -2.292231 & -3.679350 & 0.387019 \\
\hline 36 & 1 & $\Theta$ & -2.930153 & -2.326499 & 1.560348 \\
\hline 37 & 1 & $\odot$ & -1.374712 & -3.622691 & 0.691327 \\
\hline 38 & 8 & $\odot$ & -3.032851 & -1.478909 & 2.031305 \\
\hline 39 & 1 & $\Theta$ & -0.871073 & -1.466109 & 1.348952 \\
\hline 40 & 1 & $\odot$ & -3.010652 & -1.711417 & 2.963863 \\
\hline
\end{tabular}

Standard basis: Aug-CC-pVDZ (6D, 7F)

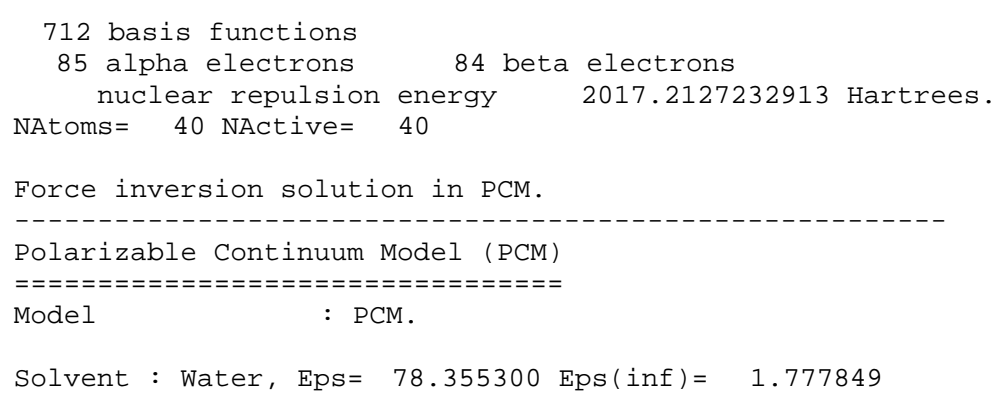


SCF Done: $E($ UWB97XD) $=-1192.0876172$ A.U. after 1 cycles NFock $=1$ Conv $=0.21 \mathrm{D}-08 \quad-\mathrm{V} / \mathrm{T}=2.0071$

$\langle\mathrm{S} X>=0.0000<\mathrm{Sy}>=0.0000<\mathrm{S} z>=0.5000$

$<S^{*} * 2>=0.7595 \mathrm{~S}=0.5048$

$<$ L.S $>=0.000000000000 \mathrm{E}+00$

Annihilation of the first spin contaminant:

$\mathrm{S}^{* *} 2$ before annihilation 0.7595 , after 0.7501

Harmonic frequencies $\left(\mathrm{cm}^{*}-1\right)$, IR intensities (KM/Mole), Raman

$\begin{array}{clll}1 & 2 & 3 \\ \text { A } & \text { A } & \text { A }\end{array}$

Frequencies - - 16.6183

26.3963

34.5189

Zero-point correction=

Thermal correction to Energy=

Thermal correction to Enthalpy=

Thermal correction to Gibbs Free Energy=

Sum of electronic and zero-point Energies=

Sum of electronic and thermal Energies=

Sum of electronic and thermal Enthalpies=

๑. 316592 (a.u.)

$\odot .341770$

$\odot .342714$

$\odot .259582$

$-1191.771025$

$-1191.745847$

$-1191.744903$

Sum of electronic and thermal Free Energies=

$-1191.828035$

$\begin{array}{cccc} & \text { E (Thermal) } & \text { CV } & \text { S } \\ \text { KCal/Mol } & \text { Cal/Mol-Kelvin } & \text { Cal/Mol-Kelvin } \\ \text { Total } & 214.464 & 90.751 & 174.967\end{array}$

\begin{tabular}{lccc}
\multicolumn{1}{c}{ Item } & Value & Threshold Converged? \\
Maximum Force & $0.0 \odot \odot \odot \odot 4$ & $0.0 \odot \odot 45 \odot$ & YES \\
RMS Force & $0.000 \odot \odot 1$ & $0.00030 \odot$ & YES \\
Maximum Displacement & 0.001930 & $0.00180 \odot$ & NO \\
RMS Displacement & 0.000280 & 0.001200 & YES
\end{tabular}

Predicted change in Energy $=-3.431739 \mathrm{D}-09$

optimization completed on the basis of negligible forces.

- - Stationary point found.

Normal termination of Gaussian 16 at Wed Jun 19 03:04:05 2019.

[3] Cartesian coordinates of geometries

optimized by WB97X-D/6-31G** and 6-31G SCRF=(PCM, solvent=water $)$ for reactions of the $\mathrm{HO}($.$) addition to \mathrm{C}(4)$ and $\mathrm{C}(8)$ sites of the guanine moiety of four dimer sequence models of B-form DNA with nucleotides moieties. Those for reactions of the $\mathrm{HO}().(\mathrm{H} 20) 2$ addition to the $\mathrm{C}(8)$ site and the subsequent steps, where (H20)2 is sbown by "W2". Single-point energy calculations, wb97X-D/6-311+G** with SCRF=PCM, were made on the

wb97X-D/6-31G** \& 6-31G with SCRF=PCM optimized geometries

Free energies composed of the wb97X-D/6-311+G** with SCRF=PCM electronic energeis and wb97X-D/6-31G** \& 6-31G with SCRF=PCM thermal corrections to Gibbs free energies are also shown by "G(high)" at the end of respective output data.

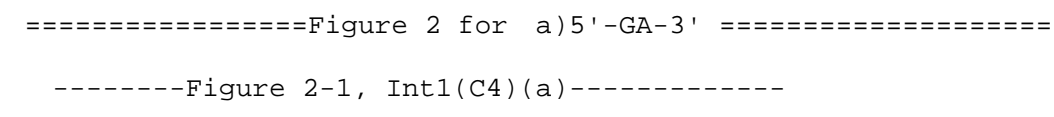

dna@4a-oh1afor. $\log$

Stoichiometry C39H50N15Na2021P2(2)

Standard orientation:

\begin{tabular}{|c|c|c|c|c|c|}
\hline Center & Atomic & Atomic & \multicolumn{3}{|c|}{ Coordinates (Angstroms) } \\
\hline Number & Number & Type & $x$ & $\mathrm{Y}$ & Z \\
\hline & & & & & \\
\hline 1 & 7 & 0 & 4.809296 & -2.009794 & -1.887384 \\
\hline 2 & 6 & $\odot$ & 4.922619 & -3.357999 & -2.056835 \\
\hline 3 & 6 & $\odot$ & 3.821943 & -4.143130 & -2.114935 \\
\hline 4 & 6 & $\odot$ & 2.548089 & -3.491807 & -2.013577 \\
\hline 5 & 7 & $\odot$ & 2.452330 & -2.174185 & -1.812108 \\
\hline 6 & 8 & $\odot$ & 8.307639 & 0.534136 & -1.269369 \\
\hline 7 & 15 & $\odot$ & 7.828005 & 1.582474 & -0.107391 \\
\hline 8 & 8 & $\odot$ & 6.189375 & 1.573384 & $-\odot .259029$ \\
\hline 9 & 8 & $\odot$ & 8.363096 & 2.932180 & -0.490414 \\
\hline 10 & 8 & $\odot$ & 8.099187 & 0.982293 & 1.256273 \\
\hline
\end{tabular}




\begin{tabular}{|c|c|c|c|c|c|}
\hline 11 & 8 & $\odot$ & $4.7 \odot 4922$ & $5.30 \odot 254$ & 1.193281 \\
\hline 12 & 6 & $\odot$ & 4.597255 & 3.436361 & -0.288403 \\
\hline 13 & 6 & $\odot$ & 5.282617 & 3.989341 & $\odot .964978$ \\
\hline 14 & 6 & 0 & -7.270506 & -3.601934 & 0.518377 \\
\hline 15 & 6 & $\odot$ & -7.715454 & -2.176718 & $\odot .769404$ \\
\hline 16 & 8 & $\odot$ & -5.828789 & -3.620175 & 0.424736 \\
\hline 17 & 8 & $\odot$ & -8.040083 & -0.075459 & -0.350487 \\
\hline 18 & 15 & $\odot$ & -8.067840 & 0.825560 & -1.709422 \\
\hline 19 & 8 & $\odot$ & -6.968519 & 1.987438 & -1.392439 \\
\hline 20 & 8 & 0 & -9.452648 & 1.404906 & -1.852582 \\
\hline 21 & 8 & $\odot$ & -7.510228 & $\odot .048917$ & -2.882911 \\
\hline 22 & 8 & $\odot$ & -4.569218 & 5.526229 & -0.889910 \\
\hline 23 & 6 & 0 & -5.716902 & 3.590837 & -0.122668 \\
\hline 24 & 6 & $\odot$ & -5.332885 & 4.391502 & -1.371396 \\
\hline 25 & 1 & $\odot$ & -4.260689 & 6.068936 & -1.635386 \\
\hline 26 & 6 & $\odot$ & 8.833768 & -2.826100 & -2.817066 \\
\hline 27 & 6 & $\odot$ & 8.243092 & -1.445594 & -2.615663 \\
\hline 28 & 6 & $\odot$ & 8.250266 & -0.927434 & -1.170365 \\
\hline 29 & 6 & $\odot$ & $6.9 \odot 4521$ & -1.397529 & -0.636172 \\
\hline 30 & 6 & $\odot$ & 5.998125 & -1.170801 & -1.843097 \\
\hline 31 & 8 & 0 & 6.826983 & -1.456126 & -3.005076 \\
\hline 32 & 1 & $\odot$ & 8.678894 & -3.124019 & -3.859292 \\
\hline 33 & 1 & $\odot$ & 9.911120 & -2.789295 & -2.617956 \\
\hline 34 & 1 & $\odot$ & 8.764105 & -0.738927 & -3.265353 \\
\hline 35 & 1 & $\odot$ & 9.101887 & -1.278331 & -0.586223 \\
\hline 36 & 1 & $\odot$ & 6.586488 & -0.837253 & 0.240283 \\
\hline 37 & 1 & $\odot$ & 6.976068 & -2.460794 & -0.397475 \\
\hline 38 & 1 & $\odot$ & 5.650555 & -0.143455 & -1.882585 \\
\hline 39 & 8 & 0 & 8.180846 & -3.757858 & -1.919896 \\
\hline 40 & 1 & $\odot$ & 8.512951 & -4.659971 & -2.056478 \\
\hline 41 & 6 & $\odot$ & 3.557837 & -1.397407 & -1.750488 \\
\hline 42 & 7 & $\odot$ & 1.408089 & -4.176757 & -2.112508 \\
\hline 43 & 8 & $\odot$ & 3.497894 & -0.174039 & -1.569107 \\
\hline 44 & 1 & $\odot$ & 5.942596 & -3.727585 & -2.142840 \\
\hline 45 & 1 & 0 & 3.901408 & -5.211954 & -2.257980 \\
\hline 46 & 1 & $\odot$ & 1.421115 & -5.165291 & -2.300244 \\
\hline 47 & 1 & $\odot$ & $\odot .519791$ & -3.671432 & -2.141133 \\
\hline 48 & 6 & $\odot$ & 5.460699 & 2.502827 & -1.115637 \\
\hline 49 & 1 & $\odot$ & 5.084603 & 5.702147 & 1.993113 \\
\hline 50 & 6 & $\odot$ & 4.869871 & 3. 001552 & 2.060735 \\
\hline 51 & 6 & 0 & 3.441246 & 2.628546 & 1.656080 \\
\hline 52 & 8 & $\odot$ & 3.406094 & 2.734121 & $\odot .203091$ \\
\hline 53 & 1 & $\odot$ & 4.824346 & 1.904864 & -1.768688 \\
\hline 54 & 1 & $\odot$ & 6.171601 & 3.089529 & -1.702698 \\
\hline 55 & 1 & $\odot$ & 4.252313 & 4.258792 & -0.919624 \\
\hline 56 & 1 & $\odot$ & 6.366988 & 4.056721 & 0.838171 \\
\hline 57 & 1 & 0 & 4.906782 & 3.443239 & 3.058557 \\
\hline 58 & 1 & $\odot$ & 5.530567 & 2.132771 & 2.030723 \\
\hline 59 & 1 & $\odot$ & 2.709948 & 3.316585 & 2.075009 \\
\hline 60 & 7 & $\odot$ & 3.010246 & 1.292676 & 2.035625 \\
\hline 61 & 6 & $\odot$ & 3.730783 & $\odot .187627$ & 1.623591 \\
\hline 62 & 6 & $\odot$ & 3.351499 & -1.077155 & 1.902555 \\
\hline 63 & 6 & $\odot$ & 2.114250 & -1.271649 & 2.639488 \\
\hline 64 & 7 & $\odot$ & 1.404892 & -0.125904 & 2.931002 \\
\hline 65 & 6 & $\odot$ & 1.752437 & 1.161256 & 2.614125 \\
\hline 66 & 6 & $\odot$ & 4.104880 & -2.303900 & 1.491091 \\
\hline 67 & 8 & $\odot$ & 1.687404 & -2.375971 & 2.986224 \\
\hline 68 & 8 & $\odot$ & 1.015107 & 2.109433 & 2.847644 \\
\hline 69 & 1 & 0 & 4.613894 & 0.417169 & 1.032274 \\
\hline 70 & 1 & $\odot$ & 4.319529 & -2.933075 & 2.359259 \\
\hline 71 & 1 & $\odot$ & 3.516071 & -2.908682 & $\odot .793760$ \\
\hline 72 & 1 & 0 & 5.047150 & -2.046099 & 1.012947 \\
\hline 73 & 1 & $\odot$ & 0.441304 & $-\odot .255794$ & 3.302284 \\
\hline 74 & 1 & $\odot$ & -5.487775 & -4.528730 & 0.414512 \\
\hline 75 & 6 & $\odot$ & -7.055241 & -1.142650 & -0.142601 \\
\hline 76 & 6 & $\odot$ & -5.847522 & -0.643497 & ๑.649995 \\
\hline 77 & 6 & $\odot$ & -6.220688 & $-\odot .887163$ & 2.123540 \\
\hline 78 & 8 & $\odot$ & -7.373815 & -1.746356 & 2.133233 \\
\hline 79 & 1 & $\odot$ & -7.623502 & -4.234883 & 1.339981 \\
\hline 80 & 1 & $\odot$ & -7.724717 & -3.956010 & $-\odot .415115$ \\
\hline 81 & 1 & $\odot$ & -8.801925 & -2.118709 & 0.691837 \\
\hline 82 & 1 & $\odot$ & -6.774640 & -1.553038 & -1.113734 \\
\hline 83 & 1 & 0 & -5.617798 & 0.404653 & 0.450298 \\
\hline 84 & 1 & $\odot$ & -4.974176 & -1.246096 & ๑.391033 \\
\hline 85 & 1 & $\odot$ & -6.469923 & $\odot .029634$ & 2.660737 \\
\hline 86 & 7 & 0 & -5.136892 & -1.557051 & 2.854648 \\
\hline 87 & 6 & $\odot$ & -5.087389 & -2.865267 & 3.261268 \\
\hline
\end{tabular}




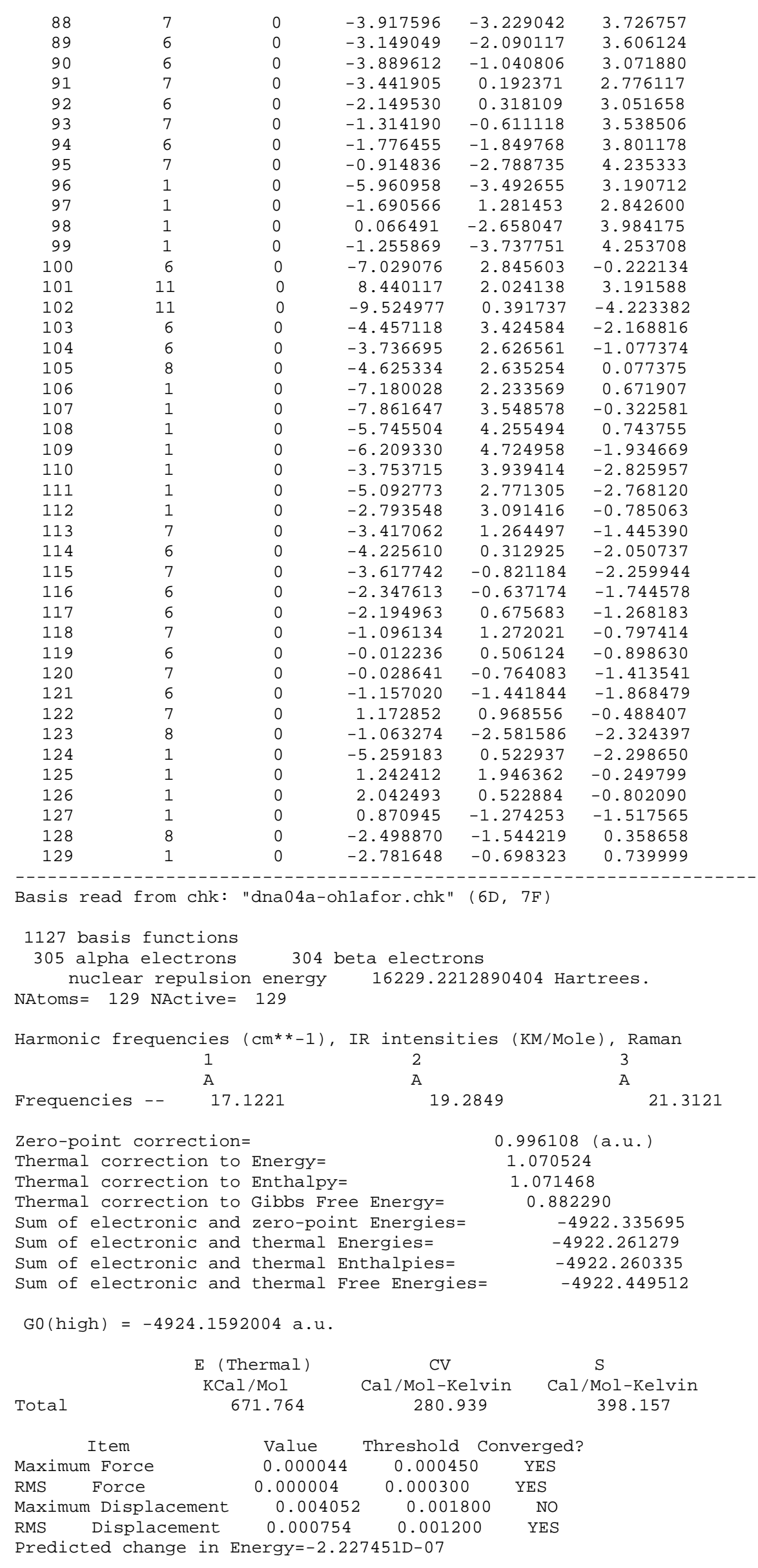


Normal termination of Gaussian 16 at Sun Feb 17 02:57:55 2019.

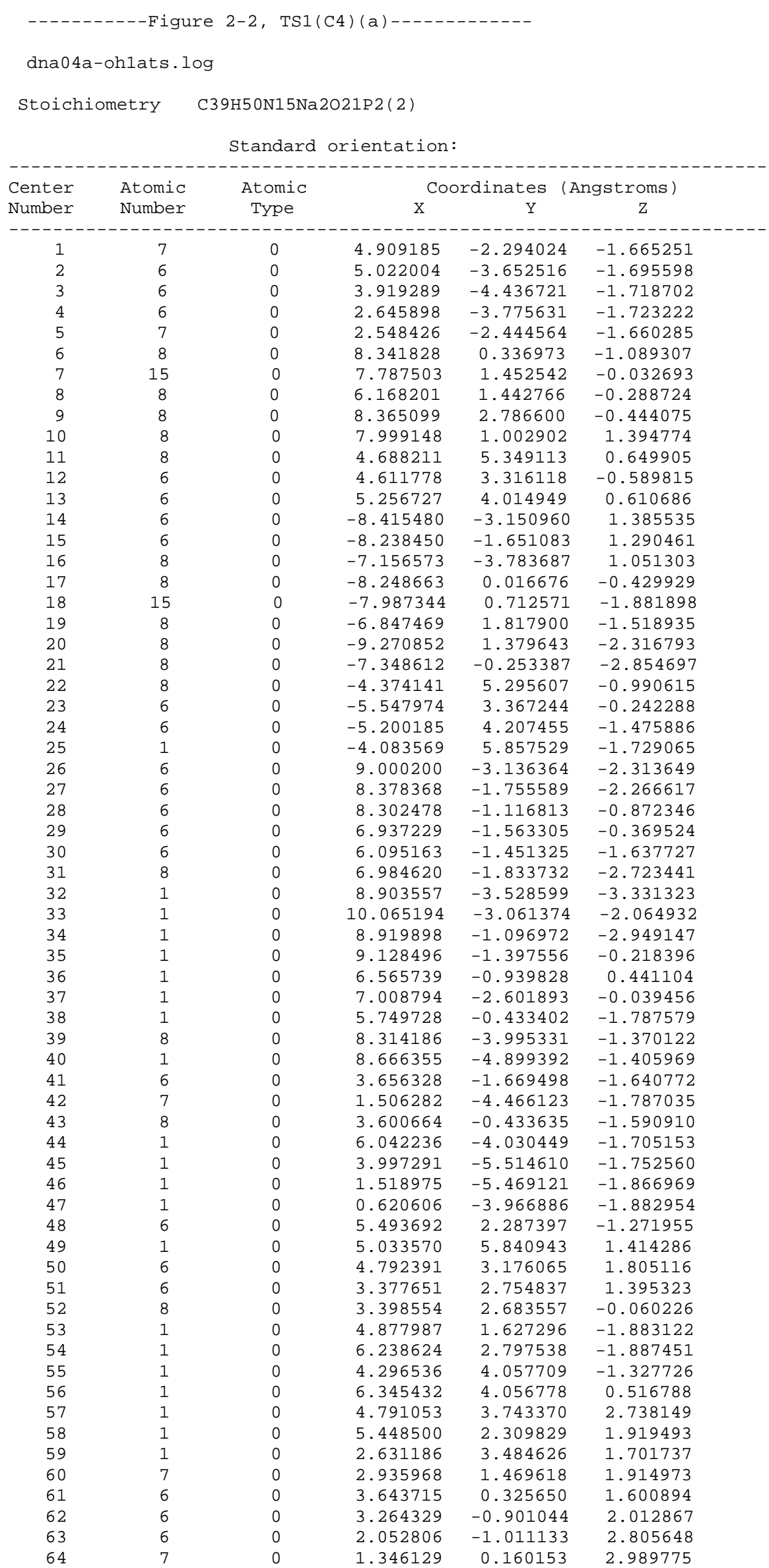




\begin{tabular}{|c|c|c|c|c|c|}
\hline 65 & 6 & 0 & 1.696793 & 1.410472 & 2.542476 \\
\hline 66 & 6 & 0 & 3.996182 & -2.169166 & 1.701066 \\
\hline 67 & 8 & 0 & 1.649241 & -2.072218 & 3.286593 \\
\hline 68 & 8 & 0 & $\odot .979134$ & 2.385395 & 2.713813 \\
\hline 69 & 1 & 0 & 4.517254 & $\odot .489717$ & $\odot .97440 \odot$ \\
\hline 70 & 1 & 0 & 4.264744 & -2.698865 & 2.619196 \\
\hline 71 & 1 & 0 & 3.368994 & -2.844489 & 1.110281 \\
\hline 72 & 1 & 0 & 4.906747 & -1.968227 & 1.141694 \\
\hline 73 & 1 & 0 & 0.412130 & 0.087635 & 3.434237 \\
\hline 74 & 1 & 0 & -7.249808 & -4.749097 & 1.003997 \\
\hline 75 & 6 & 0 & -7.535715 & -1.183416 & $\odot .011502$ \\
\hline 76 & 6 & 0 & -6.093074 & -0.894979 & 0.433254 \\
\hline 77 & 6 & 0 & -6.159412 & -0.629769 & 1.947607 \\
\hline 78 & 8 & 0 & -7.425513 & -1.153268 & 2.404916 \\
\hline 79 & 1 & 0 & -8.728028 & -3.416090 & 2.400754 \\
\hline 80 & 1 & 0 & -9.198030 & -3.458513 & 0.682451 \\
\hline 81 & 1 & 0 & -9.216290 & -1.171831 & 1.369019 \\
\hline 82 & 1 & 0 & -7.583489 & -1.924282 & -0.786676 \\
\hline 83 & 1 & 0 & -5.655046 & -0.049653 & -0.100034 \\
\hline 84 & 1 & 0 & -5.477975 & -1.776042 & 0.242317 \\
\hline 85 & 1 & 0 & -6.095944 & 0.433063 & 2.191698 \\
\hline 86 & 7 & 0 & -5.091100 & -1.289545 & 2.684620 \\
\hline 87 & 6 & 0 & -4.969910 & -2.645181 & 2.890972 \\
\hline 88 & 7 & 0 & -3.828237 & -2.990355 & 3.429614 \\
\hline 89 & 6 & 0 & -3.152129 & -1.793880 & 3.565013 \\
\hline 90 & 6 & 0 & -3.915772 & -0.726366 & 3.109486 \\
\hline 91 & 7 & 0 & -3.533433 & $\odot .558921$ & 3.052079 \\
\hline 92 & 6 & 0 & -2.266635 & $\odot .705556$ & 3.416271 \\
\hline 93 & 7 & 0 & -1.402544 & -0.234990 & 3.834507 \\
\hline 94 & 6 & 0 & -1.824223 & -1.511996 & 3.926227 \\
\hline 95 & 7 & 0 & $-\odot .971976$ & -2.465970 & 4.353925 \\
\hline 96 & 1 & 0 & -5.764908 & -3.310238 & 2.576328 \\
\hline 97 & 1 & 0 & -1.854480 & 1.709789 & 3.358708 \\
\hline 98 & 1 & 0 & $\odot .0219 \odot 2$ & -2.298216 & 4.188699 \\
\hline 99 & 1 & 0 & -1.283280 & -3.417365 & 4.224942 \\
\hline 100 & 6 & 0 & -6.876189 & 2.651640 & -0.331071 \\
\hline 101 & 11 & 0 & 9.082487 & 3.196692 & 1.964156 \\
\hline 102 & 11 & 0 & -9.225446 & $\odot .252801$ & -4.522923 \\
\hline 103 & 6 & 0 & -4.397553 & 3.246652 & -2.354413 \\
\hline 104 & 6 & 0 & -3.629188 & 2.419858 & -1.324894 \\
\hline 105 & 8 & 0 & -4.470339 & 2.380613 & -0.128721 \\
\hline 106 & 1 & 0 & -7.029276 & 2.022375 & 0.549236 \\
\hline 107 & 1 & 0 & -7.695812 & 3.373905 & $-\odot .4 \odot 0594$ \\
\hline 108 & 1 & 0 & -5.530710 & 3.995794 & $\odot .650691$ \\
\hline 109 & 1 & 0 & -6.092558 & 4.591640 & -1.978502 \\
\hline 110 & 1 & 0 & -3.726492 & 3.766435 & -3.040779 \\
\hline 111 & 1 & 0 & -5.082969 & 2.618763 & -2.924731 \\
\hline 112 & 1 & 0 & -2.682846 & 2.890915 & -1.053572 \\
\hline 113 & 7 & 0 & -3.304375 & 1.075947 & -1.727799 \\
\hline 114 & 6 & 0 & -4.083312 & 0.120053 & -2.324684 \\
\hline 115 & 7 & 0 & -3.465995 & -1.037534 & -2.475180 \\
\hline 116 & 6 & 0 & -2.236628 & -0.845170 & -1.924512 \\
\hline 117 & 6 & 0 & -2.119203 & 0.451665 & -1.359841 \\
\hline 118 & 7 & 0 & -0.981410 & 1.122682 & -1.073660 \\
\hline 119 & 6 & 0 & $\odot .074089$ & $\odot .337131$ & -1.057075 \\
\hline 120 & 7 & 0 & 0.035548 & -1.008572 & -1.380003 \\
\hline 121 & 6 & 0 & -1.070996 & -1.694226 & -1.862584 \\
\hline 122 & 7 & 0 & 1.272999 & $\odot .810439$ & -0.693338 \\
\hline 123 & 8 & 0 & -0.991177 & -2.878777 & -2.196684 \\
\hline 124 & 1 & $\odot$ & -5.113130 & 0.306376 & -2.611363 \\
\hline 125 & 1 & 0 & 1.369230 & 1.805612 & $-\odot .556966$ \\
\hline 126 & 1 & 0 & 2.132316 & $\odot .319757$ & -0.961226 \\
\hline 127 & 1 & 0 & 0.930156 & -1.531728 & -1.411535 \\
\hline 128 & 8 & 0 & -2.675238 & -0.324685 & 0.413923 \\
\hline 129 & 1 & 0 & -3.239668 & 0.431735 & 0.638512 \\
\hline
\end{tabular}

Harmonic frequencies $\left(\mathrm{cm}^{* *}-1\right)$, IR intensities (KM/Mole), Raman

\begin{tabular}{|c|c|c|c|c|c|c|c|c|c|}
\hline & & $\begin{array}{l}1 \\
A\end{array}$ & & A & . & & $\begin{array}{l}3 \\
A\end{array}$ & & \\
\hline Frequencies & - - & -479.9 & & & 14.94 & & & 17.3207 & \\
\hline Red. masses & - - & 12.80 & & & 8.544 & & & 7.3047 & \\
\hline Frc consts & - - & 1.73 & & & 0.001 & & & 0.0013 & \\
\hline IR Inten & - - & 63.19 & & & 0.236 & & & 3.6546 & \\
\hline Atom AN & $\mathrm{x}$ & $\mathrm{Y}$ & Z & $\mathrm{x}$ & $\mathrm{Y}$ & Z & $x$ & Z & \\
\hline 1 & 0.00 & 0.00 & 0.00 & 0.04 & 0.03 & -0.07 & -0.02 & $-0.00^{\circ}$ & 0.01 \\
\hline 6 & 0.00 & $0.0 \odot$ & $0.0 \odot$ & 0.05 & $\odot .03$ & -0.10 & -0.05 & $-\odot . \odot \odot$ & 0.02 \\
\hline
\end{tabular}




\begin{tabular}{|c|c|c|c|c|c|c|c|c|c|c|}
\hline 3 & 6 & $\odot . \odot \odot$ & $-\odot . \odot \odot$ & $\odot .0 \odot$ & $\odot .05$ & 0.03 & -0.14 & -0.06 & 0.01 & 0.02 \\
\hline 4 & 6 & $\odot . \odot \odot$ & $-\odot . \odot \odot$ & $\odot . \odot \odot$ & $\odot .05$ & 0.02 & -0.14 & $-\odot .05$ & 0.03 & $-\odot .01$ \\
\hline 5 & 7 & $\odot .00$ & -0.00 & $-0.0 \odot$ & 0.04 & 0.02 & -0.12 & -0.03 & 0.04 & -0.02 \\
\hline 6 & 8 & -0.00 & $-0.0 \odot$ & -0.00 & 0.05 & -0.01 & 0.09 & 0.02 & -0.06 & 0.04 \\
\hline 7 & 15 & 0.00 & $\odot .0 \odot$ & -0.00 & 0.02 & -0.03 & 0.10 & 0.02 & -0.05 & 0.03 \\
\hline 8 & 8 & $-\odot . \odot \odot$ & $-\odot .0 \odot$ & $-\odot . \odot \odot$ & $\odot .03$ & -0.01 & 0.04 & $\odot .02$ & -0.02 & $-\odot .0 \odot$ \\
\hline 9 & 8 & $-\odot . \odot \odot$ & $-\odot . \odot \odot$ & $-\odot . \odot \odot$ & 0.05 & -0.03 & 0.16 & 0.05 & -0.06 & 0.03 \\
\hline 10 & 8 & -0.00 & -0.00 & $-\odot .0 \odot$ & -0.04 & -0.07 & 0.10 & -0.02 & -0.05 & 0.03 \\
\hline 11 & 8 & -0.00 & $-\odot .0 \odot$ & -0.00 & 0.03 & -0.01 & 0.03 & 0.07 & 0.01 & -0.04 \\
\hline 12 & 6 & -0.00 & $-0.0 \odot$ & $-\odot . \odot \odot$ & 0.06 & 0.01 & 0.01 & 0.06 & $\odot .0 \odot$ & -0.03 \\
\hline 13 & 6 & -0.00 & -0.00 & -0.00 & 0.03 & -0.01 & 0.03 & 0.05 & -0.00 & -0.03 \\
\hline 14 & 6 & 0.00 & 0.00 & -0.00 & -0.04 & 0.04 & $\odot .09$ & 0.11 & -0.14 & -0.07 \\
\hline 15 & 6 & 0.00 & 0.00 & -0.00 & -0.04 & 0.04 & 0.06 & 0.05 & -0.13 & -0.04 \\
\hline 16 & 8 & $\odot . \odot \odot$ & $-\odot . \odot \odot$ & $-0.0 \odot$ & -0.03 & 0.03 & 0.12 & 0.14 & -0.08 & -0.06 \\
\hline 17 & 8 & 0.00 & $-0.0 \odot$ & -0.00 & -0.02 & 0.00 & 0.03 & 0.02 & -0.08 & 0.01 \\
\hline 18 & 15 & $0.0 \odot$ & $\odot . \odot \odot$ & $\odot . \odot \odot$ & -0.01 & -0.03 & 0.01 & $\odot . \odot \odot$ & -0.04 & 0.02 \\
\hline 19 & 8 & $\odot . \odot \odot$ & -0.00 & 0.00 & -0.02 & -0.02 & -0.01 & -0.01 & -0.03 & 0.04 \\
\hline 20 & 8 & $\odot . \odot \odot$ & $-\odot . \odot \odot$ & -0.00 & -0.01 & -0.04 & -0.01 & -0.01 & -0.04 & 0.05 \\
\hline 21 & 8 & 0.01 & $\odot . \odot \odot$ & $\odot . \odot \odot$ & $-\odot .01$ & -0.05 & 0.04 & 0.01 & $-\odot .0 \odot$ & -0.01 \\
\hline 22 & 8 & $\odot .00$ & $\odot .0 \odot$ & -0.00 & 0.02 & -0.04 & -0.02 & -0.11 & 0.04 & 0.07 \\
\hline 23 & 6 & -0.01 & 0.01 & $\odot . \odot \odot$ & -0.01 & -0.02 & -0.02 & -0.04 & -0.01 & 0.05 \\
\hline 24 & 6 & -0.00 & -0.00 & $-\odot .0 \odot$ & 0.01 & -0.03 & -0.02 & -0.08 & 0.02 & 0.06 \\
\hline 25 & 1 & $\odot . \odot \odot$ & $-\odot .0 \odot$ & $-\odot .0 \odot$ & $\odot .03$ & -0.05 & -0.02 & -0.14 & 0.06 & $\odot .07$ \\
\hline 26 & 6 & -0.00 & $-0.0 \odot$ & $-0.0 \odot$ & 0.05 & 0.03 & -0.01 & -0.03 & -0.08 & 0.06 \\
\hline 27 & 6 & $\odot .00$ & -0.00 & 0.00 & 0.06 & 0.03 & 0.02 & -0.01 & -0.07 & 0.05 \\
\hline 28 & 6 & $\odot . \odot \odot$ & $\odot .0 \odot$ & -0.00 & 0.03 & -0.01 & $\odot .04$ & -0.01 & -0.06 & 0.04 \\
\hline 29 & 6 & $\odot . \odot \odot$ & 0.00 & $\odot .0 \odot$ & 0.01 & -0.02 & $-\odot . \odot \odot$ & -0.03 & -0.03 & 0.03 \\
\hline 30 & 6 & $\odot .0 \odot$ & 0.00 & $-0.0 \odot$ & 0.04 & 0.03 & -0.02 & -0.01 & -0.02 & 0.02 \\
\hline 31 & 8 & -0.00 & -0.00 & 0.00 & 0.07 & 0.06 & -0.01 & -0.00 & -0.04 & 0.03 \\
\hline 32 & 1 & $\odot . \odot \odot$ & $-\odot . \odot \odot$ & $\odot . \odot \odot$ & $\odot .06$ & 0.07 & -0.03 & -0.03 & -0.08 & $\odot .06$ \\
\hline 33 & 1 & $-0.0 \odot$ & $-0.0 \odot$ & $\odot . \odot \odot$ & $\odot .04$ & $\odot .01$ & 0.01 & -0.04 & -0.10 & $\odot .08$ \\
\hline 34 & 1 & -0.00 & -0.00 & -0.00 & 0.08 & 0.05 & 0.06 & 0.01 & -0.08 & 0.05 \\
\hline 35 & 1 & $\odot .0 \odot$ & $\odot . \odot \odot$ & $0.0 \odot$ & 0.01 & -0.04 & 0.05 & -0.03 & -0.07 & 0.06 \\
\hline 36 & 1 & $\odot . \odot \odot$ & 0.00 & $\odot .00$ & $-0.0 \odot$ & -0.04 & 0.01 & -0.03 & -0.02 & 0.02 \\
\hline 37 & 1 & -0.00 & 0.00 & -0.00 & $-0.0 \odot$ & -0.03 & -0.03 & -0.05 & -0.03 & 0.03 \\
\hline 38 & 1 & 0.00 & $\odot .0 \odot$ & $-\odot . \odot \odot$ & 0.05 & 0.03 & 0.01 & 0.01 & -0.02 & $\odot .01$ \\
\hline 39 & 8 & $-\odot . \odot \odot$ & $-\odot . \odot \odot$ & $-\odot . \odot \odot$ & 0.02 & 0.00 & -0.05 & -0.06 & -0.06 & 0.06 \\
\hline 40 & 1 & $\odot .0 \odot$ & $\odot .0 \odot$ & $0.0 \odot$ & 0.01 & $\odot . \odot \odot$ & -0.07 & -0.08 & -0.07 & 0.07 \\
\hline 41 & 6 & $\odot .00$ & $-0.0 \odot$ & -0.00 & 0.04 & 0.03 & -0.09 & -0.02 & $\odot .02$ & -0.02 \\
\hline 42 & 7 & $\odot . \odot \odot$ & -0.00 & 0.00 & 0.05 & 0.02 & -0.17 & -0.06 & 0.05 & -0.01 \\
\hline 43 & 8 & -0.00 & $-0.0 \odot$ & $-0.0 \odot$ & $\odot .04$ & $\odot .02$ & -0.08 & 0.00 & 0.02 & -0.03 \\
\hline 44 & 1 & $\odot .00$ & $\odot .0 \odot$ & 0.00 & 0.05 & 0.03 & -0.09 & -0.05 & -0.02 & 0.04 \\
\hline 45 & 1 & $\odot . \odot \odot$ & $-\odot . \odot \odot$ & $\odot .0 \odot$ & 0.05 & 0.03 & -0.16 & -0.07 & 0.01 & 0.03 \\
\hline 46 & 1 & 0.00 & $-\odot . \odot \odot$ & -0.00 & 0.05 & 0.02 & -0.17 & -0.07 & 0.05 & 0.01 \\
\hline 47 & 1 & $\odot . \odot \odot$ & -0.01 & -0.00 & $\odot .05$ & 0.02 & -0.15 & -0.05 & 0.06 & -0.02 \\
\hline 48 & 6 & -0.00 & 0.00 & -0.00 & 0.08 & 0.01 & 0.02 & 0.05 & -0.01 & -0.02 \\
\hline 49 & 1 & $\odot . \odot \odot$ & $-0.0 \odot$ & $-0.0 \odot$ & $\odot .0 \odot$ & -0.02 & 0.05 & 0.07 & 0.00 & -0.03 \\
\hline 50 & 6 & $-\odot . \odot \odot$ & $-\odot . \odot \odot$ & $\odot . \odot \odot$ & -0.01 & -0.02 & 0.01 & $\odot .02$ & 0.01 & -0.03 \\
\hline 51 & 6 & $-\odot . \odot \odot$ & $\odot . \odot \odot$ & $-\odot . \odot \odot$ & $-\odot . \odot \odot$ & -0.01 & -0.04 & 0.02 & $\odot .03$ & -0.05 \\
\hline 52 & 8 & $-\odot . \odot \odot$ & $-\odot .0 \odot$ & $\odot .0 \odot$ & 0.04 & $0.0 \odot$ & -0.04 & 0.04 & 0.02 & -0.05 \\
\hline 53 & 1 & $\odot . \odot \odot$ & $\odot . \odot \odot$ & -0.00 & 0.10 & 0.03 & -0.02 & 0.06 & -0.00 & -0.03 \\
\hline 54 & 1 & $-0.0 \odot$ & $\odot .0 \odot$ & 0.00 & 0.11 & 0.02 & 0.06 & 0.07 & -0.02 & $-0 . \odot \odot$ \\
\hline 55 & 1 & -0.00 & $-0.0 \odot$ & -0.00 & 0.08 & 0.02 & 0.01 & 0.08 & 0.01 & -0.04 \\
\hline 56 & 1 & $-0 . \odot \odot$ & $-0.0 \odot$ & $-\odot . \odot \odot$ & 0.03 & -0.01 & $\odot .07$ & 0.05 & -0.02 & -0.01 \\
\hline 57 & 1 & $-\odot . \odot \odot$ & $-\odot .0 \odot$ & $-\odot . \odot \odot$ & -0.04 & -0.03 & $\odot .02$ & 0.01 & 0.01 & -0.03 \\
\hline 58 & 1 & -0.00 & -0.00 & $-\odot .0 \odot$ & -0.02 & -0.02 & 0.02 & -0.00 & $-\odot .0 \odot$ & -0.02 \\
\hline 59 & 1 & 0.00 & 0.00 & 0.00 & -0.01 & -0.01 & -0.06 & 0.02 & 0.04 & -0.07 \\
\hline 60 & 7 & -0.00 & -0.00 & -0.00 & -0.02 & -0.01 & -0.06 & -0.01 & $\odot .04$ & -0.05 \\
\hline 61 & 6 & $-0 . \odot \odot$ & $-\odot .0 \odot$ & $-\odot . \odot \odot$ & -0.03 & -0.01 & -0.06 & -0.02 & 0.03 & -0.04 \\
\hline 62 & 6 & $\odot . \odot \odot$ & $-\odot . \odot \odot$ & $-0.0 \odot$ & -0.05 & -0.01 & -0.08 & -0.03 & 0.04 & -0.02 \\
\hline 63 & 6 & $-0.0 \odot$ & -0.00 & $\odot . \odot \odot$ & -0.05 & -0.01 & -0.09 & -0.02 & $\odot .06$ & -0.00 \\
\hline 64 & 7 & -0.00 & -0.00 & 0.00 & -0.04 & 0.00 & -0.07 & -0.01 & 0.07 & -0.02 \\
\hline 65 & 6 & -0.00 & -0.00 & 0.00 & -0.02 & 0.00 & -0.06 & -0.00 & 0.06 & -0.05 \\
\hline 66 & 6 & $-\odot . \odot \odot$ & $-\odot .0 \odot$ & $\odot .0 \odot$ & $-\odot .06$ & -0.02 & -0.09 & -0.04 & 0.02 & -0.01 \\
\hline 67 & 8 & 0.00 & -0.00 & -0.00 & -0.07 & -0.01 & -0.10 & -0.01 & 0.07 & 0.03 \\
\hline 68 & 8 & -0.00 & 0.00 & -0.00 & -0.01 & 0.01 & -0.05 & 0.00 & 0.07 & -0.06 \\
\hline 69 & 1 & $-\odot . \odot \odot$ & $-\odot . \odot \odot$ & $-\odot . \odot \odot$ & -0.03 & -0.01 & -0.06 & -0.02 & 0.01 & -0.05 \\
\hline 70 & 1 & $-0.0 \odot$ & $\odot . \odot \odot$ & 0.00 & -0.07 & -0.03 & -0.10 & -0.05 & 0.03 & $-0.0 \odot$ \\
\hline 71 & 1 & -0.00 & -0.00 & 0.00 & -0.07 & -0.00 & -0.10 & -0.05 & 0.02 & 0.00 \\
\hline 72 & 1 & $-\odot . \odot \odot$ & $-\odot . \odot \odot$ & $\odot .0 \odot$ & -0.06 & -0.02 & -0.09 & -0.04 & $\odot .0 \odot$ & -0.01 \\
\hline 73 & 1 & $-\odot . \odot \odot$ & $-\odot . \odot \odot$ & $-\odot . \odot \odot$ & -0.04 & 0.01 & -0.07 & -0.00 & $\odot .08$ & -0.01 \\
\hline 74 & 1 & $\odot .0 \odot$ & $-\odot . \odot \odot$ & $\odot . \odot \odot$ & -0.03 & 0.03 & 0.13 & 0.18 & -0.08 & -0.08 \\
\hline 75 & 6 & 0.00 & $\odot .00$ & -0.00 & -0.02 & $\odot .01$ & 0.06 & 0.05 & -0.07 & -0.03 \\
\hline 76 & 6 & $\odot . \odot \odot$ & 0.00 & $-\odot . \odot \odot$ & -0.03 & $\odot .02$ & 0.07 & 0.04 & -0.05 & -0.01 \\
\hline 77 & 6 & $\odot . \odot \odot$ & 0.00 & -0.00 & -0.04 & 0.05 & 0.06 & 0.01 & -0.07 & -0.01 \\
\hline 78 & 8 & $\odot . \odot \odot$ & $\odot .0 \odot$ & $-\odot . \odot \odot$ & -0.05 & $\odot .06$ & 0.06 & 0.02 & -0.11 & $-\odot .03$ \\
\hline 79 & 1 & $\odot . \odot \odot$ & $\odot .0 \odot$ & $-\odot . \odot \odot$ & -0.05 & 0.06 & $\odot .09$ & 0.10 & -0.16 & $-\odot . \odot 8$ \\
\hline
\end{tabular}




\begin{tabular}{|c|c|c|c|c|}
\hline 80 & 1 & $\odot . \odot \odot$ & $\odot . \odot \odot$ & $-\odot .0 \odot$ \\
\hline 81 & 1 & $\odot .0 \odot$ & 0.00 & $-\odot . \odot \odot$ \\
\hline 82 & 1 & $\odot .0 \odot$ & $-\odot . \odot \odot$ & $-\odot .0 \odot$ \\
\hline 83 & 1 & 0.01 & $\odot .01$ & 0.01 \\
\hline 84 & 1 & 0.01 & 0.01 & -0.00 \\
\hline 85 & 1 & $\odot .0 \odot$ & $\odot .00$ & $-\odot . \odot \odot$ \\
\hline 86 & 7 & $\odot . \odot \odot$ & $-\odot . \odot \odot$ & $-\odot . \odot \odot$ \\
\hline 87 & 6 & $\odot .0 \odot$ & -0.00 & $\odot .0 \odot$ \\
\hline 88 & 7 & $\odot . \odot \odot$ & $\odot .0 \odot$ & $\odot . \odot \odot$ \\
\hline 89 & 6 & $\odot .0 \odot$ & 0.00 & $-\odot . \odot \odot$ \\
\hline 90 & 6 & $\odot .0 \odot$ & $\odot .00$ & $\odot .0 \odot$ \\
\hline 91 & 7 & $-\odot . \odot \odot$ & $-\odot . \odot \odot$ & 0.01 \\
\hline 92 & 6 & $-\odot . \odot \odot$ & $-0.0 \odot$ & $-0.0 \odot$ \\
\hline 93 & 7 & $\odot .00$ & $-\odot . \odot \odot$ & $-\odot .0 \odot$ \\
\hline 94 & 6 & $\odot .0 \odot$ & $\odot .0 \odot$ & $-\odot . \odot \odot$ \\
\hline 95 & 7 & $\odot .0 \odot$ & -0.00 & $\odot . \odot \odot$ \\
\hline 96 & 1 & $\odot . \odot \odot$ & $-\odot . \odot \odot$ & $\odot . \odot \odot$ \\
\hline 97 & 1 & $-\odot .0 \odot$ & $-0.0 \odot$ & $-0.0 \odot$ \\
\hline 98 & 1 & $\odot .0 \odot$ & $-\odot .0 \odot$ & $-\odot .0 \odot$ \\
\hline 99 & 1 & $\odot . \odot \odot$ & $\odot . \odot \odot$ & -0.00 \\
\hline 100 & 6 & $-0.0 \odot$ & $\odot .00$ & $-\odot . \odot \odot$ \\
\hline 101 & 11 & $\odot .00$ & $\odot .0 \odot$ & $-\odot . \odot \odot$ \\
\hline 102 & 11 & $-\odot . \odot \odot$ & -0.00 & $-\odot .0 \odot$ \\
\hline 103 & 6 & $\odot . \odot \odot$ & -0.01 & 0.00 \\
\hline 104 & 6 & -0.02 & -0.02 & 0.02 \\
\hline 105 & 8 & -0.02 & 0.02 & 0.02 \\
\hline 106 & 1 & $-0.0 \odot$ & $\odot . \odot \odot$ & $-\odot . \odot \odot$ \\
\hline 107 & 1 & $-\odot .01$ & $-\odot . \odot \odot$ & -0.01 \\
\hline 108 & 1 & -0.02 & $\odot .02$ & $-0.0 \odot$ \\
\hline 109 & 1 & $\odot . \odot \odot$ & $\odot .0 \odot$ & -0.01 \\
\hline 110 & 1 & 0.01 & -0.01 & 0.01 \\
\hline 111 & 1 & $-\odot . \odot \odot$ & $\odot . \odot \odot$ & $-\odot . \odot \odot$ \\
\hline 112 & 1 & -0.01 & $-\odot .02$ & 0.01 \\
\hline 113 & 7 & -0.02 & -0.01 & 0.04 \\
\hline 114 & 6 & $\odot . \odot 4$ & 0.03 & -0.01 \\
\hline 115 & 7 & 0.06 & -0.01 & -0.07 \\
\hline 116 & 6 & -0.03 & $-\odot . \odot 8$ & -0.01 \\
\hline 117 & 6 & -0.20 & -0.20 & 0.69 \\
\hline 118 & 7 & -0.03 & -0.03 & 0.03 \\
\hline 119 & 6 & -0.03 & 0.01 & -0.01 \\
\hline 120 & 7 & $-\odot . \odot \odot$ & $-\odot .03$ & $\odot . \odot 2$ \\
\hline 121 & 6 & $\odot .02$ & -0.03 & -0.01 \\
\hline 122 & 7 & -0.02 & $-\odot . \odot \odot$ & -0.04 \\
\hline 123 & 8 & 0.01 & -0.02 & -0.02 \\
\hline 124 & 1 & $\odot .06$ & 0.01 & -0.11 \\
\hline 125 & 1 & -0.02 & -0.01 & 0.03 \\
\hline 126 & 1 & -0.01 & $\odot .01$ & -0.06 \\
\hline 127 & 1 & $\odot . \odot \odot$ & -0.01 & 0.01 \\
\hline 128 & 8 & 0.16 & 0.31 & -0.48 \\
\hline 129 & 1 & 0.05 & 0.01 & 0.17 \\
\hline
\end{tabular}

\begin{tabular}{|c|c|c|c|c|c|}
\hline-0.03 & 0.02 & 0.08 & 0.13 & -0.15 & -0.09 \\
\hline$-\odot .04$ & 0.04 & $\odot .04$ & $\odot .03$ & -0.17 & -0.04 \\
\hline-0.01 & $-\odot .0 \odot$ & 0.07 & 0.07 & -0.05 & -0.05 \\
\hline-0.02 & $\odot .02$ & 0.05 & 0.03 & -0.03 & 0.00 \\
\hline$-\odot .02$ & 0.02 & 0.09 & 0.06 & -0.03 & -0.01 \\
\hline$-\odot . \odot 4$ & $\odot .05$ & 0.04 & -0.03 & -0.07 & 0.00 \\
\hline-0.05 & 0.06 & 0.08 & 0.02 & -0.04 & -0.00 \\
\hline-0.07 & 0.07 & 0.13 & 0.05 & -0.03 & 0.02 \\
\hline-0.06 & 0.07 & 0.13 & 0.05 & -0.00 & 0.04 \\
\hline-0.04 & 0.07 & 0.07 & $\odot .02$ & 0.02 & 0.03 \\
\hline-0.03 & 0.06 & 0.04 & 0.01 & -0.01 & $\odot . \odot \odot$ \\
\hline-0.02 & 0.05 & -0.02 & -0.02 & $-\odot . \odot \odot$ & -0.01 \\
\hline-0.01 & 0.05 & -0.04 & -0.02 & 0.03 & $-0.0 \odot$ \\
\hline-0.02 & 0.05 & -0.02 & $-\odot .01$ & $\odot .06$ & $\odot .02$ \\
\hline$-\odot .03$ & 0.06 & 0.03 & 0.01 & 0.05 & 0.04 \\
\hline-0.03 & $\odot .06$ & 0.04 & 0.02 & $\odot .08$ & 0.08 \\
\hline-0.08 & 0.07 & 0.16 & 0.07 & -0.05 & 0.02 \\
\hline 0.01 & 0.04 & -0.09 & -0.04 & 0.04 & -0.02 \\
\hline-0.04 & 0.04 & $-\odot . \odot \odot$ & 0.02 & 0.09 & $\odot .08$ \\
\hline$-\odot .05$ & 0.06 & 0.08 & 0.04 & 0.07 & 0.10 \\
\hline-0.02 & $-\odot . \odot \odot$ & -0.02 & -0.02 & -0.05 & 0.05 \\
\hline-0.02 & -0.01 & 0.11 & 0.01 & -0.10 & 0.08 \\
\hline-0.01 & -0.06 & 0.01 & -0.01 & -0.01 & 0.02 \\
\hline 0.01 & -0.05 & -0.00 & -0.06 & $\odot .06$ & $\odot .03$ \\
\hline-0.01 & -0.05 & 0.02 & -0.03 & 0.06 & 0.00 \\
\hline-0.03 & -0.03 & $\odot . \odot \odot$ & -0.01 & 0.02 & $\odot .02$ \\
\hline-0.04 & 0.02 & -0.01 & 0.01 & -0.07 & 0.04 \\
\hline-0.01 & ๑. 01 & -0.04 & -0.04 & -0.07 & 0.08 \\
\hline-0.01 & -0.01 & -0.02 & -0.05 & -0.03 & 0.06 \\
\hline 0.02 & -0.03 & -0.03 & -0.10 & $\odot . \odot \odot$ & 0.08 \\
\hline 0.02 & -0.07 & -0.00 & -0.09 & 0.09 & $\odot .03$ \\
\hline 0.01 & $-\odot .05$ & $0.0 \odot$ & -0.05 & 0.05 & 0.02 \\
\hline-0.01 & -0.05 & 0.03 & -0.03 & $\odot .07$ & -0.01 \\
\hline-0.01 & -0.05 & 0.03 & -0.01 & 0.07 & -0.02 \\
\hline-0.01 & -0.05 & 0.03 & -0.02 & 0.07 & -0.01 \\
\hline$\odot .0 \odot$ & -0.04 & 0.01 & -0.02 & 0.07 & -0.02 \\
\hline 0.01 & -0.03 & -0.01 & -0.01 & $\odot .07$ & -0.03 \\
\hline 0.00 & -0.04 & 0.01 & -0.01 & $\odot .07$ & -0.04 \\
\hline$\odot .0 \odot$ & -0.03 & -0.01 & $-0.0 \odot$ & $\odot .07$ & -0.05 \\
\hline 0.02 & $-\odot .01$ & -0.05 & $-\odot . \odot \odot$ & $\odot . \odot 7$ & $-\odot .06$ \\
\hline 0.03 & -0.01 & -0.07 & -0.01 & $\odot .07$ & -0.05 \\
\hline 0.03 & -0.02 & -0.05 & -0.02 & $\odot .07$ & -0.04 \\
\hline 0.02 & 0.01 & -0.09 & 0.00 & 0.07 & -0.08 \\
\hline 0.03 & -0.01 & -0.07 & -0.03 & 0.07 & -0.03 \\
\hline-0.01 & -0.06 & 0.05 & -0.02 & 0.07 & $-\odot . \odot \odot$ \\
\hline 0.00 & 0.01 & -0.07 & 0.01 & 0.07 & $-\odot .08$ \\
\hline 0.02 & 0.02 & $-\odot .09$ & $-0.0 \odot$ & 0.06 & $-\odot .07$ \\
\hline 0.04 & 0.01 & -0.10 & -0.01 & 0.06 & -0.05 \\
\hline 0.04 & -0.06 & 0.01 & $\odot .02$ & 0.08 & -0.02 \\
\hline 0.04 & $-\odot .07$ & $\odot .02$ & $-\odot .02$ & 0.05 & -0.03 \\
\hline \multicolumn{6}{|c|}{$\begin{array}{l}0.995121 \text { (a.u.) } \\
1.069263 \\
1.070207\end{array}$} \\
\hline \multicolumn{2}{|c|}{ Energy= } & \multicolumn{4}{|c|}{0.879482} \\
\hline \multicolumn{2}{|c|}{ Energies= } & \multicolumn{3}{|c|}{-4922.323089} & \\
\hline \multicolumn{2}{|c|}{ ergies= } & \multicolumn{3}{|c|}{-4922.248947} & \\
\hline \multicolumn{2}{|c|}{ thalpies= } & \multicolumn{3}{|c|}{-4922.248003} & \\
\hline \multicolumn{2}{|c|}{ ee Energies= } & \multicolumn{3}{|c|}{-4922.438728} & \\
\hline
\end{tabular}

Zero-point correction=

Thermal correction to Energy=

Thermal correction to Enthalpy=

Thermal correction to Gibbs Free Energy=

Sum of electronic and zero-point Energies=

Sum of electronic and thermal Energies=

Sum of electronic and thermal Enthalpies=

Sum of electronic and thermal Free Energies=

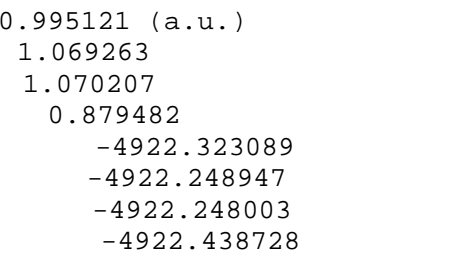

$\mathrm{G}(\mathrm{high})=-4924 \cdot 1464629 \mathrm{a} \cdot \mathrm{u}$.

\begin{tabular}{|c|c|c|c|}
\hline $\mathrm{E}_{\mathrm{KCa}}$ & $\begin{array}{l}\text { Iermal) } \\
\text { /Mol } \\
70.972\end{array}$ & $\begin{array}{c}\text { CV } \\
\text { Cal/Mol-Kelvin } \\
279.360\end{array}$ & $\begin{array}{c}\text { S } \\
\text { Cal/Mol-Kelvin } \\
401.415\end{array}$ \\
\hline Item & Value & Threshold Con & Converged? \\
\hline Maximum Force & 0.000008 & $\odot .000450$ & YES \\
\hline Force & 0.000001 & 0.000300 & YES \\
\hline Maximum Displacement & 0.001680 & 0.001800 & YES \\
\hline Displacement & 0.000253 & 0.001200 & YES \\
\hline
\end{tabular}

Predicted change in Energy $=-1.430526 \mathrm{D}-\odot 8$

Normal termination of Gaussian 16 at Tue Feb 5 19:00:24 2019. 
dna@4a-oh1a. log

Framework group C1[X(C39H50N15Na2021P2)]

Standard orientation

\begin{tabular}{|c|c|c|c|c|c|}
\hline \multirow{2}{*}{$\begin{array}{l}\text { Center } \\
\text { Number }\end{array}$} & \multirow{2}{*}{$\begin{array}{l}\text { Atomic } \\
\text { Number }\end{array}$} & \multirow{2}{*}{$\begin{array}{c}\text { Atomic } \\
\text { Type }\end{array}$} & \multicolumn{3}{|c|}{ Coordinates (Angstroms) } \\
\hline & & & $\mathrm{x}$ & $\mathrm{Y}$ & z \\
\hline 1 & 7 & 0 & 5.250537 & -2.002940 & -1.725278 \\
\hline 2 & 6 & $\odot$ & 5.501898 & -3.335725 & -1.865792 \\
\hline 3 & 6 & 0 & 4.486109 & -4.225001 & -1.955145 \\
\hline 4 & 6 & $\odot$ & 3.151838 & -3.700127 & -1.904071 \\
\hline 5 & 7 & $\odot$ & 2.917052 & -2.396281 & -1.734897 \\
\hline 6 & 8 & 0 & 8.447751 & 0.866937 & -0.894266 \\
\hline 7 & 15 & 0 & 7.790165 & 1.828858 & $\odot .249139$ \\
\hline 8 & 8 & $\odot$ & 6.180865 & 1.697601 & $-\odot .029566$ \\
\hline 9 & 8 & $\odot$ & 8.249581 & 3.239968 & -0.033582 \\
\hline 10 & 8 & $\odot$ & 8.032160 & 1.277754 & 1.636270 \\
\hline 11 & 8 & $\odot$ & 4.320978 & 5.321789 & 1.270007 \\
\hline 12 & 6 & 0 & 4.457547 & 3.436384 & -0.181206 \\
\hline 13 & 6 & $\odot$ & 5.013885 & 4.057947 & 1.103586 \\
\hline 14 & 6 & $\Theta$ & -8.758847 & -3.132680 & 1.087608 \\
\hline 15 & 6 & $\odot$ & -8.493375 & -1.659024 & 1.311516 \\
\hline 16 & 8 & $\odot$ & -7.626321 & -3.715398 & $\odot .397835$ \\
\hline 17 & 8 & 0 & -8.554679 & 0.448745 & 0.180924 \\
\hline 18 & 15 & $\odot$ & -8.636793 & 1.460387 & -1.097499 \\
\hline 19 & 8 & $\odot$ & -7.120325 & 2.060733 & -1.157784 \\
\hline 20 & 8 & 0 & -9.630791 & 2.541874 & -0.744954 \\
\hline 21 & 8 & 0 & -8.863401 & $\odot .700840$ & -2.382689 \\
\hline 22 & 8 & $\odot$ & -4.017241 & 4.881776 & -1.641880 \\
\hline 23 & 6 & 0 & -5.139965 & 3.184705 & -0.390275 \\
\hline 24 & 6 & 0 & -4.995951 & 3.821766 & -1.784060 \\
\hline 25 & 1 & 0 & -3.885724 & 5.339498 & -2.489718 \\
\hline 26 & 6 & 0 & 9.386709 & -2.397717 & -2.454853 \\
\hline 27 & 6 & $\odot$ & 8.646129 & -1.088773 & -2.272036 \\
\hline 28 & 6 & $\odot$ & 8.527355 & $-\odot .598947$ & -0.821745 \\
\hline 29 & 6 & $\odot$ & 7.208800 & -1.206469 & -0.365621 \\
\hline 30 & 6 & $\odot$ & 6.347779 & -1.054230 & -1.617166 \\
\hline 31 & 8 & $\odot$ & 7.261073 & -1.241769 & -2.735051 \\
\hline 32 & 1 & 0 & 9.311778 & -2.697741 & -3.505187 \\
\hline 33 & 1 & $\odot$ & 10.443999 & -2.251717 & -2.204949 \\
\hline 34 & 1 & 0 & 9.123109 & $-\odot .321491$ & -2.885822 \\
\hline 35 & 1 & $\odot$ & 9.378611 & -0.873441 & -0.198170 \\
\hline 36 & 1 & 0 & 6.790185 & -0.696648 & 0.499509 \\
\hline 37 & 1 & 0 & 7.374128 & -2.260318 & -0.131802 \\
\hline 38 & 1 & $\odot$ & 5.903248 & -0.065587 & -1.668645 \\
\hline 39 & 8 & $\odot$ & 8.792941 & -3.401282 & -1.594980 \\
\hline 40 & 1 & $\odot$ & 9.220775 & -4.263501 & -1.722733 \\
\hline 41 & 6 & 0 & 3.938362 & -1.511769 & -1.662061 \\
\hline 42 & 7 & $\odot$ & 2.088929 & -4.499038 & -2.018176 \\
\hline 43 & 8 & 0 & 3.754738 & -0.295314 & -1.531063 \\
\hline 44 & 1 & $\odot$ & 6.555363 & -3.605765 & -1.904724 \\
\hline 45 & 1 & $\odot$ & 4.674540 & -5.282486 & -2.078783 \\
\hline 46 & 1 & $\odot$ & 2.206074 & -5.484193 & -2.186628 \\
\hline 47 & 1 & 0 & 1.158679 & -4.082598 & -2.093497 \\
\hline 48 & 6 & 0 & 5.441714 & 2.567828 & -0.941491 \\
\hline 49 & 1 & 0 & 4.600198 & 5.753643 & 2.095361 \\
\hline 50 & 6 & $\odot$ & 4.610966 & 3.055178 & 2.189471 \\
\hline 51 & 6 & $\odot$ & 3.249950 & 2.552089 & 1.697854 \\
\hline 52 & 8 & 0 & 3.299348 & 2.644588 & $\odot .243989$ \\
\hline 53 & 1 & 0 & 4.897553 & 1.917527 & -1.626726 \\
\hline 54 & 1 & 0 & 6.143237 & 3.201990 & -1.488721 \\
\hline 55 & 1 & 0 & 4.089540 & 4.221398 & -0.846304 \\
\hline 56 & 1 & $\odot$ & 6.095172 & 4.211532 & 1.044942 \\
\hline 57 & 1 & 0 & 4.537510 & 3.517064 & 3.176153 \\
\hline 58 & 1 & 0 & 5.347677 & 2.249969 & 2.231846 \\
\hline 59 & 1 & $\odot$ & 2.435196 & 3.175297 & 2. 060137 \\
\hline 60 & 7 & 0 & 2.914528 & 1.185014 & 2.064292 \\
\hline 61 & 6 & 0 & 3.750387 & $\odot .148989$ & 1.697567 \\
\hline 62 & 6 & $\odot$ & 3.456511 & -1.145967 & 1.935858 \\
\hline 63 & 6 & $\odot$ & 2.180249 & -1.453838 & 2.554282 \\
\hline 64 & 7 & 0 & 1.368441 & -0.374190 & 2.827776 \\
\hline 65 & 6 & $\odot$ & 1.641287 & $\odot .945613$ & 2.570087 \\
\hline 66 & 6 & 0 & 4.344833 & -2.300707 & 1.591797 \\
\hline 67 & 8 & 0 & 1.812495 & -2.601584 & 2.820770 \\
\hline 68 & 8 & $\odot$ & $\odot .828591$ & 1.830915 & 2.797986 \\
\hline
\end{tabular}




\begin{tabular}{|c|c|c|c|c|c|}
\hline 69 & 1 & 0 & 4.654830 & $\odot .456975$ & 1.179328 \\
\hline 70 & 1 & $\odot$ & 4.578607 & -2.886846 & 2.485177 \\
\hline 71 & 1 & 0 & 3.852041 & -2.975056 & $\odot .884855$ \\
\hline 72 & 1 & 0 & 5.279587 & -1.960685 & 1.152069 \\
\hline 73 & 1 & $\odot$ & $\odot .4 \odot 3163$ & $-\odot .573138$ & 3.157025 \\
\hline 74 & 1 & $\odot$ & -7.792117 & -4.647142 & 0.180252 \\
\hline 75 & 6 & $\odot$ & -8.000449 & -0.901833 & 0.075019 \\
\hline 76 & 6 & $\odot$ & -6.479224 & $-\odot .897064$ & $\odot .217075$ \\
\hline 77 & 6 & 0 & -6.249509 & -0.910343 & 1.735983 \\
\hline 78 & 8 & $\odot$ & -7.450688 & -1.468483 & 2.324561 \\
\hline 79 & 1 & $\odot$ & -8.915898 & -3.619047 & 2.055950 \\
\hline 80 & 1 & 0 & -9.669285 & -3.240073 & 0.486643 \\
\hline 81 & 1 & $\odot$ & -9.402927 & -1.191151 & 1.693621 \\
\hline 82 & 1 & $\odot$ & -8.336435 & -1.349988 & $-\odot .861194$ \\
\hline 83 & 1 & 0 & $-6.0 \odot \odot 782$ & $-\odot .041723$ & $-\odot .263800$ \\
\hline 84 & 1 & 0 & -6.085193 & -1.817688 & -0.218868 \\
\hline 85 & 1 & $\odot$ & -6.077564 & $\odot .087919$ & 2.144670 \\
\hline 86 & 7 & 0 & -5.118268 & -1.724467 & 2.138948 \\
\hline 87 & 6 & 0 & -5.011695 & -3.090135 & 2.006432 \\
\hline 88 & 7 & $\odot$ & -3.845993 & -3.557803 & 2.374682 \\
\hline 89 & 6 & 0 & -3.144480 & -2.432277 & 2.759937 \\
\hline 90 & 6 & 0 & -3.915473 & -1.285065 & 2.624638 \\
\hline 91 & 7 & $\odot$ & -3.521700 & $-\odot .021418$ & 2.854512 \\
\hline 92 & 6 & 0 & -2.243039 & ๑. 029658 & 3.206289 \\
\hline 93 & 7 & $\odot$ & -1.379088 & -0.986521 & 3.355699 \\
\hline 94 & 6 & 0 & -1.800796 & -2.248467 & 3.132115 \\
\hline 95 & 7 & 0 & $-\odot .937664$ & -3.267593 & 3.273647 \\
\hline 96 & 1 & 0 & -5.847807 & -3.656665 & 1.612218 \\
\hline 97 & 1 & 0 & -1.819650 & 1.015772 & 3.379470 \\
\hline 98 & 1 & 0 & 0.062994 & -3.060855 & 3.249536 \\
\hline 99 & 1 & 0 & -1.237633 & -4.171225 & 2.942677 \\
\hline 100 & 6 & $\odot$ & -6.554803 & 2.798145 & $-\odot .038092$ \\
\hline 101 & 11 & $\odot$ & 8.915487 & 3.471912 & 2.405901 \\
\hline 102 & 11 & 0 & -10.919291 & 2.059558 & -2.816426 \\
\hline 103 & 6 & 0 & -4.461446 & 2.678762 & -2.654753 \\
\hline 104 & 6 & $\odot$ & -3.540336 & 1.969993 & -1.673490 \\
\hline 105 & 8 & $\odot$ & -4.299808 & 1.980827 & -0.410415 \\
\hline 106 & 1 & $\odot$ & -6.564809 & 2.170185 & 0.857485 \\
\hline 107 & 1 & $\odot$ & -7.154095 & 3.695081 & 0.146114 \\
\hline 108 & 1 & $\odot$ & -4.751959 & 3.874772 & 0.363714 \\
\hline 109 & 1 & 0 & -5.945026 & 4.217274 & -2.154532 \\
\hline 110 & 1 & $\odot$ & -3.942986 & 3.033744 & -3.546900 \\
\hline 111 & 1 & $\odot$ & -5.295828 & 2.033561 & -2.939225 \\
\hline 112 & 1 & $\odot$ & -2.623391 & 2.543419 & -1.508784 \\
\hline 113 & 7 & $\odot$ & -3.153369 & $\odot .630937$ & -1.973854 \\
\hline 114 & 6 & $\odot$ & -3.587958 & $-\odot .233990$ & -2.910917 \\
\hline 115 & 7 & 0 & -2.906355 & -1.371499 & -2.981048 \\
\hline 116 & 6 & $\odot$ & -1.972728 & -1.281946 & -2.006158 \\
\hline 117 & 6 & $\odot$ & -2.157581 & $-\odot .066497$ & -1.143173 \\
\hline 118 & 7 & $\odot$ & -0.994574 & 0.717912 & -0.922851 \\
\hline 119 & 6 & $\odot$ & $\odot .118825$ & $\odot .049173$ & -0.905636 \\
\hline 120 & 7 & $\odot$ & $\odot .239549$ & -1.309204 & -1.220300 \\
\hline 121 & 6 & 0 & -0.733247 & -2.013465 & -1.908324 \\
\hline 122 & 7 & $\odot$ & 1.278291 & $\odot .627594$ & -0.532186 \\
\hline 123 & 8 & $\odot$ & $-\odot .50566 \odot$ & -3.131329 & -2.384535 \\
\hline 124 & 1 & 0 & -4.399412 & 0.010574 & -3.583798 \\
\hline 125 & 1 & $\odot$ & 1.265795 & 1.622995 & $-\odot .369917$ \\
\hline 126 & 1 & $\odot$ & 2.175452 & $\odot .251951$ & -0.851853 \\
\hline 127 & 1 & 0 & 1.189316 & -1.710106 & -1.279598 \\
\hline 128 & 8 & 0 & -2.770326 & -0.467658 & 0.063513 \\
\hline 129 & 1 & $\odot$ & -3.112422 & 0.329072 & 0.499519 \\
\hline
\end{tabular}

Harmonic frequencies $\left(\mathrm{cm}^{* *}-1\right)$, IR intensities (KM/Mole), Raman

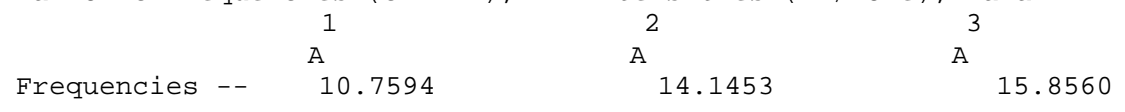

Zero-point correction=

Thermal correction to Energy=

Thermal correction to Enthalpy=

0.996983 (a.u.)

1.071157

1.072101

0.880960

Thermal correction to Gibbs Free Energy=

Sum of electronic and zero-point Energies=

Sum of electronic and thermal Energies=

$-4922.353669$

$-4922.279495$

$-4922.278550$

Sum of electronic and thermal Free Energies=

$-4922.469691$ 
$G($ high $)=-4924 \cdot 1761628 \mathrm{a} \cdot \mathrm{u}$.

\begin{tabular}{|c|c|c|c|}
\hline${ }_{\mathrm{KCa}}$ & $\begin{array}{l}\text { ermal) } \\
\text { /Mol } \\
72.161\end{array}$ & $\begin{array}{c}\text { CV } \\
\text { Cal/Mol-Kelvin } \\
279.793\end{array}$ & $\begin{array}{c}\mathrm{S} \\
\mathrm{Cal} / \mathrm{Mol}-\mathrm{Kel} \text { in } \\
402.290\end{array}$ \\
\hline Item & Value & \multicolumn{2}{|c|}{ Threshold Converged? } \\
\hline Maximum Force & 0.000062 & 0.000450 & YES \\
\hline Force & 0.000007 & 0.000300 & YES \\
\hline Maximum Displacement & $\odot .020252$ & 0.001800 & NO \\
\hline Displacement & $\odot .002730$ & 0.001200 & NO \\
\hline
\end{tabular}

-...-Figure 2-4, Int1(C8) (a) $\ldots$

dna@4a-ohfor. $\log$

Stoichiometry C39H50N15Na2021P2(2)

Standard orientation:

\begin{tabular}{|c|c|c|c|c|c|}
\hline \multirow{2}{*}{$\begin{array}{l}\text { Center } \\
\text { Number }\end{array}$} & \multirow{2}{*}{$\begin{array}{l}\text { Atomic } \\
\text { Number }\end{array}$} & \multirow{2}{*}{$\begin{array}{l}\text { Atomic } \\
\text { Type }\end{array}$} & \multicolumn{3}{|c|}{ Coordinates (Angstroms) } \\
\hline & & & $\mathrm{X}$ & $\mathrm{Y}$ & Z \\
\hline 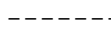 & 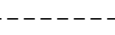 & 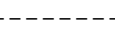 & ---- & --1 & -- \\
\hline 1 & 7 & $\odot$ & 5.314848 & -1.986314 & -1.527377 \\
\hline 2 & 6 & $\odot$ & 5.535606 & -3.332096 & -1.565323 \\
\hline 3 & 6 & $\odot$ & 4.506324 & -4.204136 & -1.469978 \\
\hline 4 & 6 & $\odot$ & 3.190387 & -3.650450 & -1.318964 \\
\hline 5 & 7 & $\odot$ & 2.984833 & -2.329995 & -1.304006 \\
\hline 6 & 8 & $\odot$ & 8.686087 & $\odot .797216$ & -0.945263 \\
\hline 7 & 15 & $\odot$ & 8.094323 & 1.797815 & $\odot .201194$ \\
\hline 8 & 8 & $\odot$ & 6.482422 & 1.763745 & -0.077097 \\
\hline 9 & 8 & 0 & 8.638513 & 3.179150 & -0.080067 \\
\hline 10 & 8 & $\odot$ & 8.299944 & 1.234602 & 1.589170 \\
\hline 11 & 8 & $\odot$ & 4.683814 & 5.414638 & 1.209041 \\
\hline 12 & 6 & $\odot$ & 4.814827 & 3.538561 & $-\odot .254156$ \\
\hline 13 & 6 & $\odot$ & 5.355536 & 4.139549 & 1.047351 \\
\hline 14 & 6 & $\odot$ & -8.859404 & -2.610741 & 2.124093 \\
\hline 15 & 6 & $\odot$ & -8.483881 & -1.152360 & 1.959284 \\
\hline 16 & 8 & $\odot$ & -8.106679 & -3.401840 & 1.171991 \\
\hline 17 & 8 & $\odot$ & -8.911135 & $\odot .704149$ & ๑. 491749 \\
\hline 18 & 15 & $\odot$ & -9.103341 & 1.534560 & -0.903582 \\
\hline 19 & 8 & $\odot$ & -7.586682 & 2.081209 & -1.191008 \\
\hline 20 & 8 & $\odot$ & -10.037032 & 2.685474 & -0.614011 \\
\hline 21 & 8 & $\odot$ & -9.463839 & 0.609312 & -2.041313 \\
\hline 22 & 8 & $\odot$ & -4.426171 & 4.688763 & -2.122831 \\
\hline 23 & 6 & $\odot$ & -5.507436 & 3.178997 & -0.617473 \\
\hline 24 & 6 & $\odot$ & -5.238253 & 3.487478 & -2.107060 \\
\hline 25 & 1 & $\odot$ & -4.284583 & 4.990341 & -3.036298 \\
\hline 26 & 6 & $\odot$ & 9.371855 & -2.516027 & -2.524719 \\
\hline 27 & 6 & $\odot$ & 8.688149 & -1.179982 & -2.318152 \\
\hline 28 & 6 & $\odot$ & 8.682692 & -0.670629 & -0.869083 \\
\hline 29 & 6 & $\odot$ & 7.367760 & -1.209155 & -0.324709 \\
\hline 30 & 6 & $\odot$ & 6.435798 & -1.060120 & -1.523945 \\
\hline 31 & 8 & $\odot$ & 7.272567 & -1.292608 & -2.692222 \\
\hline 32 & 1 & $\odot$ & 9.210190 & -2.836505 & -3.559077 \\
\hline 33 & 1 & $\odot$ & 10.448654 & -2.399957 & -2.355722 \\
\hline 34 & 1 & $\odot$ & 9.150770 & -0.437704 & -2.972414 \\
\hline 35 & 1 & $\odot$ & 9.554834 & -0.988852 & -0.297185 \\
\hline 36 & 1 & $\odot$ & 7.017333 & -0.661699 & 0.546444 \\
\hline 37 & 1 & $\odot$ & 7.505968 & -2.260698 & -0.065374 \\
\hline 38 & 1 & $\odot$ & 6.009280 & -0.063155 & -1.571263 \\
\hline 39 & 8 & $\odot$ & 8.811863 & -3.479650 & -1.598739 \\
\hline 40 & 1 & $\odot$ & 9.206080 & -4.357023 & -1.731025 \\
\hline 41 & 6 & $\odot$ & 4.015290 & -1.464254 & -1.445486 \\
\hline 42 & 7 & $\odot$ & 2.123773 & -4.438113 & -1.189161 \\
\hline 43 & 8 & $\odot$ & 3.851704 & -0.239183 & -1.488607 \\
\hline 44 & 1 & $\odot$ & 6.577849 & -3.627213 & -1.672853 \\
\hline 45 & 1 & $\odot$ & 4.669599 & -5.272462 & -1.503880 \\
\hline 46 & 1 & $\odot$ & 2.223760 & -5.438974 & -1.218699 \\
\hline 47 & 1 & $\odot$ & 1.187607 & -4.023782 & -1.150447 \\
\hline 48 & 6 & $\odot$ & 5.803283 & 2.665887 & -1.001755 \\
\hline 49 & 1 & $\odot$ & 4.958611 & 5.838381 & 2.040048 \\
\hline 50 & 6 & $\odot$ & 4.919611 & 3.135665 & 2.121169 \\
\hline 51 & 6 & $\odot$ & 3.578351 & 2.618679 & 1.588612 \\
\hline 52 & 8 & $\odot$ & 3.642676 & 2.755539 & 0.137776 \\
\hline 53 & 1 & $\odot$ & 5.268591 & 2.047394 & -1.723762 \\
\hline 54 & 1 & $\odot$ & 6.540415 & 3.293058 & -1.508968 \\
\hline
\end{tabular}

S148 


\begin{tabular}{|c|c|c|c|c|c|}
\hline 55 & 1 & 0 & 4.470376 & 4.336089 & -0.917075 \\
\hline 56 & 1 & 0 & 6.441440 & 4.272579 & 1.012668 \\
\hline 57 & 1 & 0 & 4.805117 & 3.604006 & 3.100905 \\
\hline 58 & 1 & 0 & 5.669723 & 2.347453 & 2.198503 \\
\hline 59 & 1 & 0 & 2.748969 & 3.223702 & 1.948146 \\
\hline 60 & 7 & 0 & 3.237634 & 1.241295 & 1.914435 \\
\hline 61 & 6 & 0 & 4.154820 & ๑.219326 & 1.771433 \\
\hline 62 & 6 & 0 & 3.845544 & -1.082411 & 1.948936 \\
\hline 63 & 6 & $\odot$ & 2.451128 & -1.424606 & 2.165606 \\
\hline 64 & 7 & 0 & 1.587978 & $-\odot .359029$ & 2.285217 \\
\hline 65 & 6 & 0 & 1.895535 & 0.971603 & 2.175093 \\
\hline 66 & 6 & 0 & 4.836191 & -2.205321 & 1.920153 \\
\hline 67 & 8 & 0 & 2.032274 & -2.583686 & $2.24 \odot 258$ \\
\hline 68 & 8 & 0 & 1.049529 & 1.845505 & 2.307052 \\
\hline 69 & 1 & 0 & 5.154640 & 0.538659 & 1.497492 \\
\hline 70 & 1 & 0 & 4.729955 & -2.818472 & 2.819598 \\
\hline 71 & 1 & 0 & 4.672996 & -2.868068 & 1.065458 \\
\hline 72 & 1 & 0 & 5.858956 & -1.832257 & 1.885177 \\
\hline 73 & 1 & 0 & 0.568586 & $-\odot .576661$ & 2.368636 \\
\hline 74 & 1 & 0 & -8.355970 & -4.338751 & 1.224221 \\
\hline 75 & 6 & 0 & -8.436635 & -0.682818 & $\odot .503300$ \\
\hline 76 & 6 & 0 & -6.961821 & -0.776408 & 0.120071 \\
\hline 77 & 6 & 0 & -6.200920 & -0.595050 & 1.440812 \\
\hline 78 & 8 & 0 & -7.147851 & -0.892525 & 2.501550 \\
\hline 79 & 1 & 0 & -8.640511 & -2.922947 & 3.150383 \\
\hline 80 & 1 & 0 & -9.934686 & -2.721266 & 1.942431 \\
\hline 81 & 1 & 0 & -9.185826 & -0.539610 & 2.528831 \\
\hline 82 & 1 & 0 & -9.075797 & -1.274083 & $-\odot .152972$ \\
\hline 83 & 1 & 0 & -6.684980 & 0.001906 & -0.590799 \\
\hline 84 & 1 & 0 & -6.754679 & -1.759335 & $-\odot .309157$ \\
\hline 85 & 1 & 0 & -5.826119 & 0.421773 & 1.566112 \\
\hline 86 & 7 & 0 & -5.049342 & -1.468437 & 1.563460 \\
\hline 87 & 6 & 0 & -5.036635 & -2.838849 & 1.440278 \\
\hline 88 & 7 & 0 & -3.845229 & -3.364207 & 1.596685 \\
\hline 89 & 6 & 0 & -3.031968 & -2.276850 & 1.842596 \\
\hline 90 & 6 & 0 & -3.759037 & -1.091589 & 1.839287 \\
\hline 91 & 7 & 0 & -3.283728 & $\odot .144322$ & 2.055177 \\
\hline 92 & 6 & $\odot$ & -1.976822 & 0.130742 & 2.265130 \\
\hline 93 & 7 & 0 & -1.152239 & -0.928546 & 2.285134 \\
\hline 94 & 6 & $\odot$ & -1.645805 & -2.163876 & 2.069244 \\
\hline 95 & 7 & 0 & -0.812412 & -3.213126 & 2.100467 \\
\hline 96 & 1 & 0 & -5.964725 & -3.370299 & 1.264523 \\
\hline 97 & 1 & 0 & -1.487114 & 1.086416 & 2.435088 \\
\hline 98 & 1 & 0 & 0.193948 & -3.043750 & 2.135782 \\
\hline 99 & 1 & 0 & -1.147551 & -4.098506 & 1.758191 \\
\hline 100 & 6 & $\odot$ & -6.953017 & 2.986464 & $-\odot .241519$ \\
\hline 101 & 11 & $\odot$ & 9.323799 & 3.408627 & 2.327848 \\
\hline 102 & 11 & 0 & -11.527511 & 1.927871 & -2.504361 \\
\hline 103 & 6 & $\odot$ & -4.455127 & 2.265761 & -2.600233 \\
\hline 104 & 6 & $\odot$ & -3.733179 & 1.815450 & -1.329756 \\
\hline 105 & 8 & 0 & -4.760922 & 1.956250 & $-\odot .308340$ \\
\hline 106 & 1 & $\odot$ & -7.023572 & 2.566913 & $\odot .766490$ \\
\hline 107 & 1 & $\odot$ & -7.471688 & 3.949161 & $-\odot .265142$ \\
\hline 108 & 1 & $\odot$ & -5.094914 & 4.001903 & $-\odot .024091$ \\
\hline 109 & 1 & $\odot$ & -6.163559 & 3.639749 & -2.667528 \\
\hline 110 & 1 & $\odot$ & -3.772875 & 2.502251 & -3.418058 \\
\hline 111 & 1 & $\odot$ & -5.149936 & 1.485790 & -2.924926 \\
\hline 112 & 1 & $\odot$ & -2.882659 & 2.464883 & -1.103235 \\
\hline 113 & 7 & 0 & -3.237836 & $\odot .465064$ & -1.329171 \\
\hline 114 & 6 & $\odot$ & -3.953672 & -0.688229 & -1.532862 \\
\hline 115 & 7 & $\odot$ & -3.212534 & -1.761511 & -1.485717 \\
\hline 116 & 6 & $\odot$ & -1.932909 & -1.296960 & -1.245805 \\
\hline 117 & 6 & $\odot$ & -1.929745 & 0.085132 & -1.139186 \\
\hline 118 & 7 & $\odot$ & -0.886914 & 0.911563 & $-\odot .947670$ \\
\hline 119 & 6 & 0 & 0.270300 & 0.265775 & -0.899939 \\
\hline 120 & 7 & $\odot$ & 0.376237 & -1.102916 & -0.993675 \\
\hline 121 & 6 & $\odot$ & -0.686324 & -1.988502 & -1.164362 \\
\hline 122 & 7 & $\odot$ & 1.417579 & $\odot .937029$ & $-\odot .747749$ \\
\hline 123 & 8 & 0 & -0.490724 & -3.206882 & -1.241216 \\
\hline 124 & 1 & $\odot$ & -5.016931 & -0.676547 & -1.709021 \\
\hline 125 & 1 & $\odot$ & 1.383769 & 1.940175 & -0.671280 \\
\hline 126 & 1 & $\odot$ & 2.328848 & 0.502376 & -0.923228 \\
\hline 127 & 1 & $\odot$ & 1.321743 & -1.530003 & -1.022509 \\
\hline 128 & 8 & $\odot$ & -5.124037 & -3.756861 & -1.179620 \\
\hline 129 & 1 & $\odot$ & -4.311319 & -3.193466 & -1.277903 \\
\hline
\end{tabular}




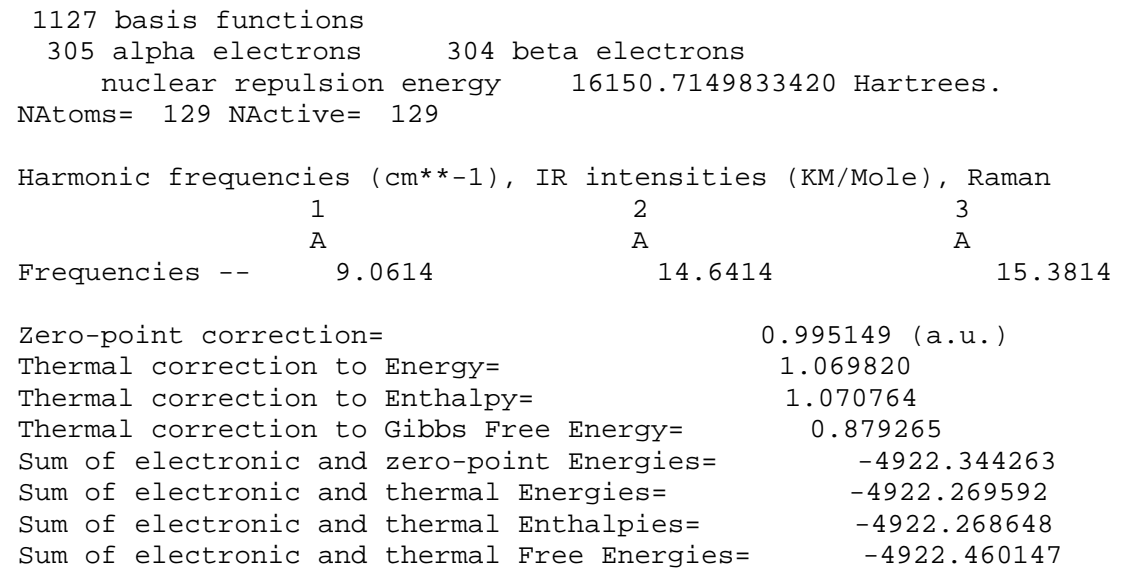

Normal termination of Gaussian 16 at Fri Jan 11 00:51:14 2019.

Figure 2-5, TS1(C8)(a)

dna@4a-ohts. $\log$

Stoichiometry C39H50N15Na2021P2(2)

Standard orientation:

\begin{tabular}{|c|c|c|c|c|c|}
\hline \multirow{2}{*}{$\begin{array}{l}\text { Center } \\
\text { Number }\end{array}$} & \multirow{2}{*}{$\begin{array}{l}\text { Atomic } \\
\text { Number }\end{array}$} & \multirow{2}{*}{$\begin{array}{l}\text { Atomic } \\
\text { Type }\end{array}$} & \multicolumn{3}{|c|}{ Coordinates (Angstroms) } \\
\hline & & & $X$ & $\mathrm{Y}$ & Z \\
\hline & 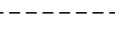 & - & 40 & & \\
\hline 1 & 7 & $\odot$ & 5.540137 & -1.928261 & -1.397158 \\
\hline 2 & 6 & $\odot$ & 5.855728 & -3.255284 & -1.429971 \\
\hline 3 & 6 & $\odot$ & 4.886245 & -4.197364 & -1.436950 \\
\hline 4 & 6 & $\odot$ & 3.527521 & -3.737722 & -1.405185 \\
\hline 5 & 7 & $\odot$ & 3.229809 & -2.434373 & -1.393223 \\
\hline 6 & 8 & $\odot$ & 8.743023 & 1.022313 & $-\odot .598743$ \\
\hline 7 & 15 & $\odot$ & 8.028932 & 1.999435 & $\odot .496225$ \\
\hline 8 & 8 & $\odot$ & 6.443457 & 1.883820 & 0.106313 \\
\hline 9 & 8 & $\odot$ & 8.520501 & 3.404579 & 0.233057 \\
\hline 10 & 8 & $\odot$ & 8.169216 & 1.459034 & 1.900076 \\
\hline 11 & 8 & $\odot$ & 4.341188 & 5.414007 & 1.253621 \\
\hline 12 & 6 & $\odot$ & 4.696282 & 3.557423 & -0.197613 \\
\hline 13 & 6 & $\odot$ & 5.101768 & 4.184038 & 1.141182 \\
\hline 14 & 6 & $\odot$ & -9.247614 & -2.277302 & 2.485631 \\
\hline 15 & 6 & $\odot$ & -8.712888 & -0.875524 & 2.275982 \\
\hline 16 & 8 & $\odot$ & -8.485798 & -3.198588 & 1.667413 \\
\hline 17 & 8 & $\odot$ & -8.827622 & $\odot .972228$ & $\odot .728890$ \\
\hline 18 & 15 & $\odot$ & -8.997103 & 1.727518 & -0.710808 \\
\hline 19 & 8 & $\odot$ & -7.449367 & 1.902222 & -1.215619 \\
\hline 20 & 8 & $\odot$ & -9.617028 & 3.076316 & -0.430271 \\
\hline 21 & 8 & $\odot$ & -9.687308 & $\odot .834597$ & -1.713666 \\
\hline 22 & 8 & $\odot$ & -4.389047 & 4.061300 & -3.042050 \\
\hline 23 & 6 & $\odot$ & -5.195227 & 2.791520 & -1.180776 \\
\hline 24 & 6 & $\odot$ & -5.222817 & 2.919462 & -2.722156 \\
\hline 25 & 1 & $\odot$ & -4.438086 & 4.261336 & -3.992185 \\
\hline 26 & 6 & $\odot$ & 9.652642 & -2.249713 & -2.139425 \\
\hline 27 & 6 & $\odot$ & 8.902125 & -0.944821 & -1.969798 \\
\hline 28 & 6 & $\odot$ & 8.786935 & -0.443921 & -0.523036 \\
\hline 29 & 6 & $\odot$ & 7.462953 & -1.036946 & -0.060244 \\
\hline 30 & 6 & $\odot$ & 6.598379 & $-\odot .933408$ & -1.314920 \\
\hline 31 & 8 & $\odot$ & 7.517775 & -1.107259 & -2.430809 \\
\hline 32 & 1 & $\odot$ & 9.559703 & -2.575642 & -3.180484 \\
\hline 33 & 1 & $\odot$ & 10.712845 & -2.086587 & -1.914137 \\
\hline
\end{tabular}




\begin{tabular}{|c|c|c|c|c|c|}
\hline 34 & 1 & 0 & 9.373900 & $-\odot .180615$ & -2.591328 \\
\hline 35 & 1 & $\odot$ & 9.632988 & $-\odot .731956$ & 0.101614 \\
\hline 36 & 1 & $\odot$ & 7.038010 & $-\odot .5 \odot 4212$ & $\odot .787464$ \\
\hline 37 & 1 & $\odot$ & 7.632086 & -2.080629 & 0.212219 \\
\hline 38 & 1 & $\odot$ & 6.114298 & $\odot .035488$ & -1.385270 \\
\hline 39 & 8 & $\odot$ & 9.089400 & -3.239229 & -1.242902 \\
\hline 40 & 1 & $\odot$ & 9.537279 & -4.094644 & -1.344848 \\
\hline 41 & 6 & $\odot$ & 4.205465 & -1.496303 & -1.427145 \\
\hline 42 & 7 & $\odot$ & 2.511964 & -4.599467 & -1.384611 \\
\hline 43 & 8 & $\odot$ & 3.958706 & -0.284833 & -1.467547 \\
\hline 44 & 1 & $\odot$ & 6.921341 & -3.478088 & -1.449813 \\
\hline 45 & 1 & 0 & 5.126677 & -5.251138 & -1.467120 \\
\hline 46 & 1 & $\odot$ & 2.684762 & -5.589997 & -1.424180 \\
\hline 47 & 1 & $\odot$ & 1.551555 & -4.249955 & -1.448724 \\
\hline 48 & 6 & $\odot$ & 5.790039 & 2.751731 & $-\odot .868045$ \\
\hline 49 & 1 & $\odot$ & 4.525970 & 5.849824 & 2.103092 \\
\hline 50 & 6 & $\odot$ & 4.648936 & 3.149124 & 2.177612 \\
\hline 51 & 6 & 0 & 3.372497 & 2.582765 & 1.549253 \\
\hline 52 & 8 & $\odot$ & 3.554076 & 2.694790 & $\odot .106394$ \\
\hline 53 & 1 & $\odot$ & 5.351288 & 2.110404 & -1.633653 \\
\hline 54 & 1 & $\odot$ & 6.527885 & 3.424055 & -1.312407 \\
\hline 55 & 1 & $\odot$ & 4.352168 & 4.335697 & $-\odot .883271$ \\
\hline 56 & 1 & $\odot$ & 6.176339 & 4.385619 & 1.187388 \\
\hline 57 & 1 & 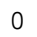 & 4.449500 & 3.599222 & 3.152236 \\
\hline 58 & 1 & $\odot$ & 5.427802 & 2.393871 & 2.293060 \\
\hline 59 & 1 & $\odot$ & 2.501125 & 3.169886 & 1.831220 \\
\hline 60 & 7 & $\odot$ & 3.042461 & 1.202187 & 1.864413 \\
\hline 61 & 6 & $\odot$ & 3.984187 & $\odot .196847$ & 1.768300 \\
\hline 62 & 6 & $\odot$ & 3.687220 & -1.111690 & 1.916737 \\
\hline 63 & 6 & 0 & 2.286958 & -1.478939 & 2.049868 \\
\hline 64 & 7 & $\odot$ & 1.403081 & -0.427171 & 2.130792 \\
\hline 65 & 6 & $\odot$ & 1.692531 & 0.906647 & 2.032863 \\
\hline 66 & 6 & $\odot$ & 4.699355 & -2.215151 & 1.929308 \\
\hline 67 & 8 & $\odot$ & 1.877729 & -2.643466 & 2.084103 \\
\hline 68 & 8 & 0 & $\odot .823247$ & 1.766599 & 2.094288 \\
\hline 69 & 1 & 0 & 4.991877 & $\odot .537273$ & 1.554096 \\
\hline 70 & 1 & $\odot$ & 4.619882 & -2.788548 & 2.857724 \\
\hline 71 & 1 & $\odot$ & 4.535843 & -2.918030 & 1.107979 \\
\hline 72 & 1 & $\odot$ & 5.713845 & -1.824421 & 1.859949 \\
\hline 73 & 1 & $\odot$ & $\odot .381075$ & -0.658352 & 2.117555 \\
\hline 74 & 1 & 0 & -8.817612 & -4.105623 & 1.767440 \\
\hline 75 & 6 & 0 & -8.561840 & $-\odot .468720$ & 0.806363 \\
\hline 76 & 6 & $\odot$ & -7.106574 & -0.777454 & 0.486058 \\
\hline 77 & 6 & $\odot$ & -6.381698 & $-\odot .554230$ & 1.817710 \\
\hline 78 & 8 & $\odot$ & -7.371693 & -0.760110 & 2.857422 \\
\hline 79 & 1 & $\odot$ & -9.158606 & -2.535957 & 3.545933 \\
\hline 80 & 1 & 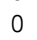 & -10.307072 & -2.300570 & 2.204613 \\
\hline 81 & 1 & $\odot$ & -9.359238 & $-\odot .166231$ & 2.797858 \\
\hline 82 & 1 & $\odot$ & -9.261263 & -0.988633 & $\odot .150018$ \\
\hline 83 & 1 & 0 & -6.693939 & $-\odot .154210$ & -0.306126 \\
\hline 84 & 1 & 0 & -7.019503 & -1.822020 & 0.188684 \\
\hline 85 & 1 & $\odot$ & -5.973039 & $\odot .454054$ & 1.910187 \\
\hline 86 & 7 & 0 & -5.268152 & -1.459828 & 2.017451 \\
\hline 87 & 6 & $\odot$ & -5.303166 & -2.832777 & 1.985407 \\
\hline 88 & 7 & $\odot$ & -4.113773 & -3.385173 & 1.979234 \\
\hline 89 & 6 & $\odot$ & -3.247340 & -2.307954 & 1.981588 \\
\hline 90 & 6 & $\odot$ & -3.946508 & -1.105663 & 2.006566 \\
\hline 91 & 7 & $\odot$ & -3.430318 & $\odot .132065$ & 2.019476 \\
\hline 92 & 6 & $\odot$ & -2.108099 & $\odot .102874$ & 1.987775 \\
\hline 93 & 7 & $\odot$ & -1.308880 & $-\odot .974663$ & 1.950045 \\
\hline 94 & 6 & $\odot$ & -1.841104 & -2.212574 & 1.942738 \\
\hline 95 & 7 & $\odot$ & -1.016903 & -3.266589 & 1.892339 \\
\hline 96 & 1 & $\odot$ & -6.258441 & -3.344883 & 1.971368 \\
\hline 97 & 1 & $\odot$ & -1.580279 & 1.053109 & 1.995807 \\
\hline 98 & 1 & 0 & -0.007972 & -3.121838 & 1.931588 \\
\hline 99 & 1 & $\odot$ & -1.397099 & -4.197221 & 1.925731 \\
\hline 100 & 6 & $\odot$ & -6.540560 & 2.788146 & -0.502172 \\
\hline 101 & 11 & 0 & 9.022781 & 3.794434 & 2.616936 \\
\hline 102 & 11 & 0 & -11.434372 & 2.635634 & -2.069733 \\
\hline 103 & 6 & $\odot$ & -4.599860 & 1.604149 & -3.211448 \\
\hline 104 & 6 & $\odot$ & -3.659775 & 1.270418 & -2.054985 \\
\hline 105 & 8 & $\odot$ & -4.489387 & 1.541972 & -0.887236 \\
\hline 106 & 1 & $\odot$ & -6.435213 & 2.449321 & 0.532859 \\
\hline 107 & 1 & $\odot$ & -6.955518 & 3.800212 & -0.505339 \\
\hline 108 & 1 & $\odot$ & -4.606222 & 3.628279 & -0.788340 \\
\hline 109 & 1 & $\odot$ & -6.236265 & 3.062464 & -3.103098 \\
\hline 110 & 1 & $\odot$ & -4.088011 & 1.710370 & -4.169015 \\
\hline
\end{tabular}

S151 


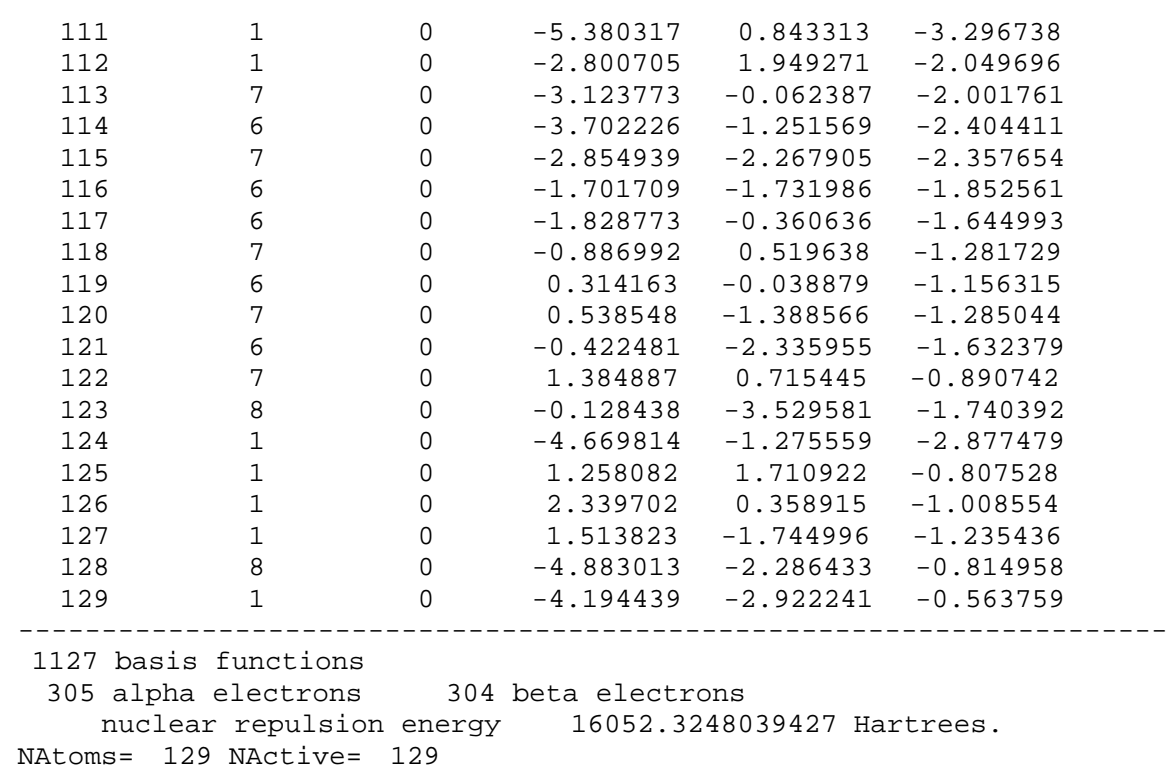

Harmonic frequencies $\left(\mathrm{cm}^{* *}-1\right)$, IR intensities (KM/Mole), Raman

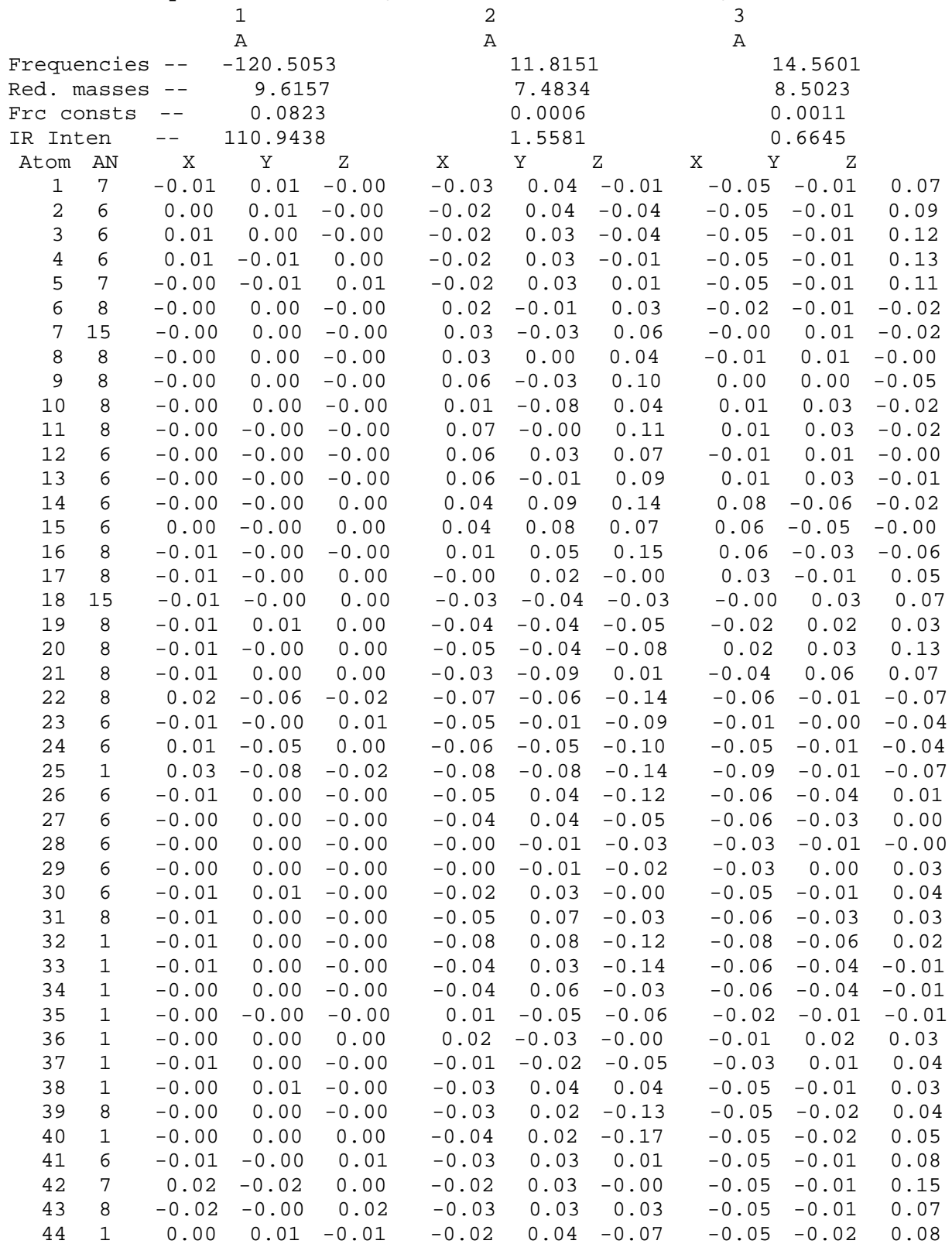




\begin{tabular}{|c|c|c|c|c|c|c|c|c|c|c|}
\hline 45 & 1 & 0.01 & $\odot . \odot \odot$ & $-0.0 \odot$ & -0.02 & 0.04 & -0.07 & -0.06 & -0.01 & 0.14 \\
\hline 46 & 1 & 0.03 & -0.01 & -0.00 & -0.01 & 0.03 & -0.03 & -0.06 & -0.01 & 0.15 \\
\hline 47 & 1 & 0.01 & -0.03 & 0.00 & -0.02 & 0.02 & 0.02 & -0.05 & -0.01 & 0.12 \\
\hline 48 & 6 & -0.00 & $-0.0 \odot$ & -0.00 & 0.06 & 0.04 & 0.06 & $-\odot .02$ & 0.00 & -0.01 \\
\hline 49 & 1 & $\odot . \odot \odot$ & -0.00 & -0.00 & $\odot .07$ & -0.02 & 0.12 & $\odot .02$ & $\odot .04$ & -0.02 \\
\hline 50 & 6 & $\odot . \odot \odot$ & -0.00 & -0.00 & 0.05 & -0.03 & 0.06 & 0.03 & 0.04 & 0.01 \\
\hline 51 & 6 & $\odot . \odot \odot$ & $-\odot .0 \odot$ & $0.0 \odot$ & 0.05 & -0.01 & 0.04 & $\odot .02$ & 0.02 & 0.03 \\
\hline 52 & 8 & $-\odot . \odot \odot$ & $-\odot . \odot \odot$ & $\odot .0 \odot$ & 0.05 & 0.03 & 0.05 & $-0.0 \odot$ & $\odot .02$ & 0.03 \\
\hline 53 & 1 & $-0.0 \odot$ & $-0.0 \odot$ & $-0.0 \odot$ & 0.06 & 0.06 & 0.04 & -0.03 & -0.01 & 0.01 \\
\hline 54 & 1 & $-0.0 \odot$ & $\odot .0 \odot$ & $-0.0 \odot$ & 0.07 & 0.04 & 0.08 & -0.02 & -0.01 & -0.02 \\
\hline 55 & 1 & -0.00 & -0.00 & $-0.0 \odot$ & 0.07 & 0.05 & 0.09 & -0.02 & 0.00 & -0.01 \\
\hline 56 & 1 & -0.00 & -0.00 & -0.00 & 0.06 & -0.02 & 0.10 & $\odot .01$ & 0.03 & -0.03 \\
\hline 57 & 1 & 0.00 & $-\odot . \odot \odot$ & $-\odot .0 \odot$ & $\odot .04$ & -0.04 & 0.07 & 0.04 & 0.05 & $\odot . \odot \odot$ \\
\hline 58 & 1 & $\odot .0 \odot$ & $-\odot .0 \odot$ & -0.00 & 0.04 & -0.03 & 0.05 & $\odot .04$ & 0.04 & 0.01 \\
\hline 59 & 1 & $\odot . \odot \odot$ & $-0.0 \odot$ & $\odot . \odot \odot$ & $\odot .05$ & -0.01 & 0.05 & $\odot .02$ & $\odot .02$ & $\odot .04$ \\
\hline 60 & 7 & $\odot . \odot \odot$ & -0.00 & -0.00 & 0.04 & -0.01 & 0.01 & 0.04 & $\odot .02$ & 0.04 \\
\hline 61 & 6 & $\odot . \odot \odot$ & $-0.0 \odot$ & 0.00 & 0.04 & -0.01 & 0.02 & 0.05 & 0.03 & 0.07 \\
\hline 62 & 6 & 0.00 & -0.00 & 0.00 & 0.03 & -0.01 & 0.00 & 0.05 & 0.03 & 0.08 \\
\hline 63 & 6 & $\odot .0 \odot$ & -0.00 & 0.00 & 0.03 & -0.01 & -0.02 & 0.05 & 0.02 & 0.05 \\
\hline 64 & 7 & $-\odot . \odot \odot$ & $-\odot . \odot \odot$ & -0.01 & 0.03 & -0.01 & -0.02 & 0.04 & 0.01 & 0.03 \\
\hline 65 & 6 & $\odot . \odot \odot$ & -0.00 & -0.00 & $\odot .04$ & -0.01 & $-\odot .0 \odot$ & 0.04 & 0.01 & 0.03 \\
\hline 66 & 6 & $\odot . \odot \odot$ & -0.00 & 0.01 & 0.03 & -0.02 & 0.01 & 0.06 & 0.04 & 0.11 \\
\hline 67 & 8 & $\odot . \odot \odot$ & $-\odot . \odot \odot$ & $0.0 \odot$ & 0.03 & -0.01 & -0.03 & 0.06 & 0.02 & 0.05 \\
\hline 68 & 8 & $-0.0 \odot$ & $-0.0 \odot$ & -0.01 & 0.04 & -0.01 & -0.00 & 0.03 & 0.01 & 0.02 \\
\hline 69 & 1 & $0.0 \odot$ & $-0.0 \odot$ & 0.00 & 0.04 & -0.02 & 0.04 & 0.05 & 0.03 & 0.09 \\
\hline 70 & 1 & $\odot . \odot \odot$ & $-\odot .0 \odot$ & 0.00 & 0.01 & -0.03 & $-\odot .0 \odot$ & $\odot .03$ & 0.03 & 0.11 \\
\hline 71 & 1 & $\odot . \odot \odot$ & $-\odot .0 \odot$ & $0.0 \odot$ & 0.05 & -0.00 & -0.01 & 0.10 & 0.04 & 0.10 \\
\hline 72 & 1 & 0.00 & $-0.0 \odot$ & 0.01 & 0.03 & -0.02 & 0.04 & 0.06 & 0.04 & 0.16 \\
\hline 73 & 1 & $-0.0 \odot$ & $-0.0 \odot$ & -0.00 & 0.03 & $-0.0 \odot$ & -0.04 & 0.05 & $\odot .0 \odot$ & -0.01 \\
\hline 74 & 1 & & $-\odot . \odot \odot$ & 0.01 & 0.01 & 0.06 & 0.19 & 0.07 & $-\odot .04$ & -0.08 \\
\hline 75 & 6 & $-\odot . \odot \odot$ & $-\odot . \odot \odot$ & $0.0 \odot$ & $\odot .0 \odot$ & 0.02 & 0.05 & 0.03 & -0.01 & $\odot . \odot \odot$ \\
\hline 76 & 6 & $\odot . \odot \odot$ & 0.01 & -0.00 & -0.00 & 0.01 & $\odot .02$ & $\odot .02$ & -0.01 & -0.04 \\
\hline 77 & 6 & 0.00 & 0.00 & -0.00 & 0.03 & 0.05 & -0.01 & 0.05 & -0.03 & -0.06 \\
\hline 78 & 8 & $\odot . \odot \odot$ & -0.00 & -0.00 & 0.06 & 0.10 & 0.03 & 0.08 & -0.06 & -0.04 \\
\hline 79 & 1 & $\odot . \odot \odot$ & $-\odot . \odot \odot$ & 0.00 & 0.07 & 0.13 & 0.14 & 0.10 & -0.08 & -0.03 \\
\hline 80 & 1 & $-\odot . \odot \odot$ & $\odot .0 \odot$ & 0.01 & 0.03 & 0.08 & 0.17 & 0.07 & -0.05 & 0.00 \\
\hline 81 & 1 & $\odot . \odot \odot$ & $-\odot .0 \odot$ & 0.00 & 0.06 & 0.10 & 0.06 & 0.07 & -0.06 & 0.03 \\
\hline 82 & 1 & $-\odot . \odot \odot$ & -0.01 & $\odot .0 \odot$ & -0.01 & -0.01 & 0.09 & $\odot .01$ & 0.01 & $\odot .01$ \\
\hline 83 & 1 & -0.01 & 0.02 & 0.01 & -0.02 & -0.01 & -0.01 & -0.00 & 0.01 & -0.04 \\
\hline 84 & 1 & 0.03 & 0.02 & -0.03 & -0.01 & 0.00 & 0.04 & 0.01 & $-0.0 \odot$ & -0.06 \\
\hline 85 & 1 & $\odot . \odot \odot$ & 0.00 & $\odot . \odot \odot$ & 0.05 & 0.05 & -0.05 & 0.05 & -0.03 & -0.05 \\
\hline 86 & 7 & $\odot . \odot \odot$ & $\odot .0 \odot$ & -0.01 & 0.03 & 0.04 & -0.01 & 0.06 & -0.03 & -0.10 \\
\hline 87 & 6 & & 0.00 & -0.01 & 0.02 & 0.04 & 0.03 & 0.07 & -0.03 & -0.13 \\
\hline 88 & 7 & $\odot .0 \odot$ & 0.00 & -0.02 & 0.01 & 0.03 & 0.02 & 0.07 & -0.02 & -0.15 \\
\hline 89 & 6 & $\odot . \odot \odot$ & $0.0 \odot$ & -0.01 & 0.02 & 0.03 & -0.01 & 0.06 & -0.02 & -0.13 \\
\hline 90 & 6 & $\odot . \odot \odot$ & $\odot .0 \odot$ & $0.0 \odot$ & 0.03 & 0.03 & -0.03 & 0.06 & -0.02 & -0.10 \\
\hline 91 & 7 & $\odot .0 \odot$ & 0.00 & $\odot .01$ & 0.04 & 0.03 & -0.06 & 0.05 & -0.02 & -0.07 \\
\hline 92 & 6 & 0.00 & 0.00 & 0.01 & 0.04 & 0.02 & -0.07 & 0.05 & -0.01 & -0.06 \\
\hline 93 & 7 & $\odot . \odot \odot$ & 0.00 & $-\odot .0 \odot$ & 0.03 & 0.01 & -0.06 & 0.06 & -0.01 & -0.08 \\
\hline 94 & 6 & & $\odot .0 \odot$ & $-0.0 \odot$ & & 0.02 & -0.03 & 0.06 & -0.01 & -0.11 \\
\hline 95 & 7 & -0.00 & $0.0 \odot$ & -0.01 & $\odot .02$ & 0.01 & -0.01 & 0.07 & -0.00 & -0.13 \\
\hline 96 & 1 & $\odot . \odot \odot$ & 0.00 & -0.02 & 0.01 & 0.05 & 0.06 & 0.07 & -0.04 & -0.14 \\
\hline 97 & 1 & $\odot .0 \odot$ & 0.00 & 0.01 & 0.05 & 0.01 & -0.09 & 0.04 & -0.01 & -0.03 \\
\hline 98 & 1 & $-0.0 \odot$ & $-0.0 \odot$ & $\odot .0 \odot$ & $\odot .02$ & 0.00 & -0.02 & 0.07 & 0.00 & -0.08 \\
\hline 99 & 1 & $-\odot . \odot \odot$ & $\odot .0 \odot$ & $-0.0 \odot$ & 0.01 & 0.01 & 0.01 & 0.08 & $-\odot . \odot \odot$ & -0.14 \\
\hline 100 & 6 & -0.02 & $\odot .02$ & -0.01 & -0.04 & $-\odot . \odot \odot$ & -0.09 & 0.01 & $\odot .01$ & 0.00 \\
\hline 101 & 11 & $\odot . \odot \odot$ & $-0.0 \odot$ & -0.00 & 0.03 & -0.08 & 0.11 & 0.01 & 0.02 & -0.05 \\
\hline 102 & 11 & $-\odot .0 \odot$ & 0.00 & $\odot .0 \odot$ & -0.06 & -0.10 & -0.02 & -0.01 & 0.09 & 0.12 \\
\hline 103 & 6 & -0.02 & -0.08 & 0.05 & -0.05 & -0.06 & -0.06 & -0.06 & -0.01 & -0.05 \\
\hline 104 & 6 & -0.04 & -0.08 & 0.07 & -0.04 & -0.02 & -0.06 & -0.03 & -0.01 & -0.07 \\
\hline 105 & 8 & -0.03 & -0.00 & 0.05 & -0.03 & 0.01 & -0.06 & -0.01 & -0.00 & -0.05 \\
\hline 106 & 1 & -0.03 & 0.04 & $\odot . \odot \odot$ & -0.03 & 0.03 & -0.08 & 0.04 & 0.01 & -0.00 \\
\hline 107 & 1 & -0.01 & 0.02 & -0.03 & -0.05 & -0.01 & -0.11 & 0.02 & 0.01 & 0.02 \\
\hline 108 & 1 & -0.01 & 0.00 & -0.01 & -0.05 & 0.01 & -0.12 & 0.01 & -0.00 & -0.06 \\
\hline 109 & 1 & 0.01 & -0.04 & -0.01 & -0.06 & -0.07 & -0.09 & -0.06 & -0.02 & -0.01 \\
\hline 110 & 1 & 0.00 & -0.11 & 0.05 & -0.06 & -0.09 & -0.07 & -0.09 & -0.02 & -0.06 \\
\hline 111 & 1 & -0.04 & -0.06 & 0.06 & -0.05 & -0.07 & -0.04 & -0.06 & -0.01 & -0.02 \\
\hline 112 & 1 & -0.02 & -0.10 & 0.05 & -0.04 & -0.02 & -0.09 & -0.03 & $-0.0 \odot$ & -0.09 \\
\hline 113 & 7 & -0.06 & -0.09 & 0.13 & -0.04 & -0.02 & -0.03 & -0.03 & $-\odot .0 \odot$ & -0.07 \\
\hline 114 & 6 & -0.07 & -0.13 & 0.17 & -0.03 & -0.03 & -0.01 & -0.01 & $-\odot . \odot \odot$ & -0.09 \\
\hline 115 & 7 & $\odot .09$ & -0.07 & -0.04 & -0.02 & -0.03 & 0.02 & -0.01 & -0.00 & -0.07 \\
\hline 116 & 6 & $\odot .02$ & -0.05 & -0.04 & -0.03 & -0.01 & 0.01 & -0.03 & $-0.0 \odot$ & -0.04 \\
\hline 117 & 6 & -0.03 & -0.04 & 0.05 & -0.04 & -0.01 & -0.01 & -0.04 & $-\odot . \odot \odot$ & -0.04 \\
\hline 118 & 7 & -0.05 & -0.05 & 0.05 & -0.04 & $\odot .0 \odot$ & -0.02 & -0.05 & $-0.0 \odot$ & -0.01 \\
\hline 119 & 6 & -0.02 & -0.02 & 0.00 & -0.04 & 0.01 & $-\odot . \odot \odot$ & -0.05 & $-\odot . \odot \odot$ & 0.02 \\
\hline 120 & 7 & 0.02 & -0.01 & -0.02 & -0.03 & 0.01 & 0.02 & -0.05 & -0.00 & 0.03 \\
\hline 121 & 6 & 0.04 & -0.02 & -0.02 & -0.03 & $-0.0 \odot$ & 0.03 & -0.04 & $-\odot .0 \odot$ & 0.00 \\
\hline
\end{tabular}




$\begin{array}{rrrrrrrrrrr}122 & 7 & -0.04 & 0.01 & -0.04 & -0.05 & 0.02 & -0.01 & -0.06 & -0.00 & 0.04 \\ 123 & 8 & 0.04 & -0.02 & -0.02 & -0.02 & -0.00 & 0.05 & -0.03 & -0.00 & 0.01 \\ 124 & 1 & 0.12 & -0.05 & -0.23 & -0.02 & -0.05 & -0.01 & -0.00 & -0.01 & -0.11 \\ 125 & 1 & -0.06 & -0.00 & 0.02 & -0.06 & 0.02 & -0.02 & -0.06 & -0.00 & 0.03 \\ 126 & 1 & -0.03 & 0.02 & -0.01 & -0.04 & 0.02 & 0.01 & -0.05 & -0.00 & 0.06 \\ 127 & 1 & 0.03 & 0.00 & -0.02 & -0.03 & 0.02 & 0.03 & -0.05 & -0.00 & 0.07 \\ 128 & 8 & 0.18 & 0.59 & -0.26 & -0.03 & -0.01 & 0.01 & -0.04 & -0.02 & -0.11 \\ 129 & 1 & 0.02 & 0.50 & -0.10 & -0.03 & -0.01 & 0.01 & -0.04 & -0.03 & -0.11\end{array}$

Zero-point correction=

Thermal correction to Energy=

Thermal correction to Enthalpy=

Thermal correction to Gibbs Free Energy=

Sum of electronic and zero-point Energies=

Sum of electronic and thermal Energies=

Sum of electronic and thermal Enthalpies=

Sum of electronic and thermal Free Energies=

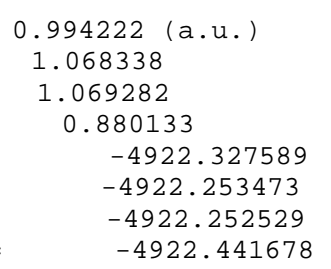

$G($ high $)=-4924 \cdot 1533896 \mathrm{a} \cdot \mathrm{u}$.

\begin{tabular}{|c|c|c|c|}
\hline $\begin{array}{r}\mathrm{E}(\mathrm{T} \\
\mathrm{KCa}\end{array}$ & $\begin{array}{l}\text { ermal) } \\
\text { /Mol } \\
0.392\end{array}$ & $\begin{array}{c}\text { CV } \\
\text { Cal/Mol-Kelvin } \\
280.179\end{array}$ & $\begin{array}{c}\text { S } \\
\text { Cal/Mol-Kelvin } \\
\text { 398.097 }\end{array}$ \\
\hline Item & Value & Threshold & verged? \\
\hline Maximum Force & $\odot .000 \odot 24$ & $\odot .000450$ & YES \\
\hline Force & 0.000003 & $\odot .00 \odot 30 \odot$ & YES \\
\hline Maximum Displacement & $\odot .003838$ & $\odot .0 \odot 180 \odot$ & NO \\
\hline Displacement & $\odot .000564$ & 0.001200 & YES \\
\hline
\end{tabular}

Normal termination of Gaussian 16 at Tue Jan 8 17:11:27 2019 .

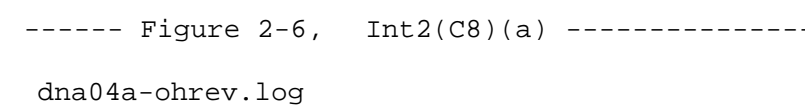

Stoichiometry C39H50N15Na2021P2(2)

Standard orientation:

\begin{tabular}{|c|c|c|c|c|c|}
\hline \multirow{2}{*}{$\begin{array}{l}\text { Center } \\
\text { Number }\end{array}$} & \multirow{2}{*}{$\begin{array}{l}\text { Atomic } \\
\text { Number }\end{array}$} & \multirow{2}{*}{$\begin{array}{l}\text { Atomic } \\
\text { Type }\end{array}$} & \multicolumn{3}{|c|}{ Coordinates (Angstroms) } \\
\hline & & & $x$ & $\mathrm{Y}$ & Z \\
\hline- & & & ( & --- & - - - - - - - \\
\hline 1 & 7 & $\odot$ & 5.616651 & -1.952815 & -1.323103 \\
\hline 2 & 6 & $\odot$ & 5.982985 & -3.267056 & -1.297500 \\
\hline 3 & 6 & $\odot$ & 5.051079 & -4.244719 & -1.252049 \\
\hline 4 & 6 & $\odot$ & 3.676259 & -3.836367 & -1.218171 \\
\hline 5 & 7 & $\odot$ & 3.328048 & -2.546199 & -1.276842 \\
\hline 6 & 8 & $\odot$ & 8.726480 & 1.135556 & -0.657911 \\
\hline 7 & 15 & $\odot$ & 7.984659 & 2.132653 & $\odot .399709$ \\
\hline 8 & 8 & $\odot$ & 6.403154 & 1.951779 & $\odot .017617$ \\
\hline 9 & 8 & $\odot$ & 8.431935 & 3.540969 & $\odot .079041$ \\
\hline 10 & 8 & $\odot$ & 8.144988 & 1.655088 & 1.823496 \\
\hline 11 & 8 & $\odot$ & 4.207325 & 5.475662 & $\odot .980451$ \\
\hline 12 & 6 & $\odot$ & 4.613371 & 3.559593 & -0.375149 \\
\hline 13 & 6 & $\odot$ & 4.998536 & 4.260927 & 0.932472 \\
\hline 14 & 6 & $\odot$ & -9.227754 & -2.068027 & 2.519339 \\
\hline 15 & 6 & $\odot$ & -8.697370 & -0.675451 & 2. 244134 \\
\hline 16 & 8 & $\odot$ & -8.523961 & -3.019523 & 1.682806 \\
\hline 17 & 8 & $\odot$ & -8.841388 & 1.135838 & ๑. 661648 \\
\hline 18 & 15 & $\odot$ & -9.027794 & 1.866211 & -0.789110 \\
\hline 19 & 8 & $\odot$ & -7.495946 & 1.940137 & -1.356990 \\
\hline 20 & 8 & $\odot$ & -9.564121 & 3.252344 & -0.518900 \\
\hline 21 & 8 & $\odot$ & -9.805626 & ๑. 988251 & -1.740252 \\
\hline 22 & 8 & $\odot$ & -4.237892 & 3.460952 & -3.358209 \\
\hline 23 & 6 & $\odot$ & -5.152000 & 2.563750 & -1.332817 \\
\hline 24 & 6 & 0 & -5.124231 & 2.419730 & -2.873819 \\
\hline 25 & 1 & $\odot$ & -4.249654 & 3.488433 & -4.329939 \\
\hline 26 & 6 & $\odot$ & 9.730520 & -2.163141 & -2.074277 \\
\hline 27 & 6 & $\odot$ & 8.938606 & $-\odot .877496$ & -1.952034 \\
\hline 28 & 6 & $\odot$ & 8.813437 & $-\odot .324616$ & -0.525170 \\
\hline 29 & 6 & $\odot$ & 7.510240 & -0.940297 & -0.032479 \\
\hline 30 & 6 & $\odot$ & 6.637668 & -0.917180 & -1.286233 \\
\hline 31 & 8 & $\odot$ & 7.558370 & -1.101561 & -2.399218 \\
\hline 32 & 1 & $\odot$ & 9.640775 & -2.535452 & -3.099919 \\
\hline 33 & 1 & $\odot$ & 10.786331 & -1.956316 & -1.864960 \\
\hline
\end{tabular}




\begin{tabular}{|c|c|c|c|c|c|}
\hline 34 & 1 & 0 & 9.382891 & -0.123328 & -2.605234 \\
\hline 35 & 1 & $\odot$ & 9.670259 & -0.563080 & 0.105651 \\
\hline 36 & 1 & $\odot$ & 7.070692 & $-\odot .388761$ & $\odot .795845$ \\
\hline 37 & 1 & $\odot$ & 7.715525 & -1.965877 & 0.281136 \\
\hline 38 & 1 & $\odot$ & 6.117808 & $\odot .02959 \odot$ & -1.392371 \\
\hline 39 & 8 & $\odot$ & 9.206773 & -3.131883 & -1.132249 \\
\hline 40 & 1 & $\odot$ & 9.684065 & -3.974729 & -1.199051 \\
\hline 41 & 6 & $\odot$ & 4.266331 & -1.574542 & -1.370155 \\
\hline 42 & 7 & $\odot$ & 2.697713 & -4.734023 & -1.124047 \\
\hline 43 & 8 & $\odot$ & 3.972916 & $-\odot .377150$ & -1.476084 \\
\hline 44 & 1 & $\odot$ & 7.056231 & -3.449674 & -1.316023 \\
\hline 45 & 1 & $\odot$ & 5.331954 & -5.288687 & -1.233746 \\
\hline 46 & 1 & $\odot$ & 2.911424 & -5.716531 & -1.083973 \\
\hline 47 & 1 & $\odot$ & 1.720487 & -4.425151 & -1.139886 \\
\hline 48 & 6 & $\odot$ & 5.729765 & 2.750394 & -1.001768 \\
\hline 49 & 1 & $\odot$ & 4.371832 & 5.955135 & 1.810168 \\
\hline 50 & 6 & $\odot$ & 4.565187 & 3.267597 & 2.016534 \\
\hline 51 & 6 & $\odot$ & 3.302052 & 2.647952 & 1.413031 \\
\hline 52 & 8 & $\odot$ & 3.492887 & 2.682433 & -0.032202 \\
\hline 53 & 1 & $\odot$ & 5.310868 & 2.059257 & -1.734524 \\
\hline 54 & 1 & $\odot$ & 6.450509 & 3.418564 & -1.479028 \\
\hline 55 & 1 & 0 & 4.251783 & 4.294639 & -1.098549 \\
\hline 56 & 1 & $\odot$ & 6.067798 & 4.489930 & $\odot .971699$ \\
\hline 57 & 1 & $\odot$ & 4.355461 & 3.758148 & 2.969284 \\
\hline 58 & 1 & $\odot$ & 5.357688 & 2.532471 & 2.166180 \\
\hline 59 & 1 & $\odot$ & 2.419852 & 3.235394 & 1.658311 \\
\hline 60 & 7 & $\odot$ & 2.993634 & 1.281544 & 1.801564 \\
\hline 61 & 6 & 0 & 3.945547 & $\odot .283888$ & 1.733292 \\
\hline 62 & 6 & $\odot$ & 3.667986 & -1.020459 & 1.944309 \\
\hline 63 & 6 & $\odot$ & 2.274348 & -1.397808 & 2.119462 \\
\hline 64 & 7 & 0 & 1.382967 & -0.350567 & 2.189513 \\
\hline 65 & 6 & $\odot$ & 1.653044 & $\odot .979696$ & 2.018334 \\
\hline 66 & 6 & 0 & 4.698929 & -2.105504 & 1.989586 \\
\hline 67 & 8 & 0 & 1.873769 & -2.563049 & 2.195643 \\
\hline 68 & 8 & $\odot$ & $\odot .774482$ & 1.831700 & 2.057120 \\
\hline 69 & 1 & $\odot$ & 4.944699 & 0.626460 & 1.484006 \\
\hline 70 & 1 & 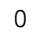 & 4.677261 & -2.607387 & 2.961779 \\
\hline 71 & 1 & $\odot$ & 4.508027 & -2.870528 & 1.233158 \\
\hline 72 & 1 & $\theta$ & 5.701385 & -1.706125 & 1.839225 \\
\hline 73 & 1 & 0 & $\odot .363813$ & -0.589469 & 2.228462 \\
\hline 74 & 1 & 0 & -8.850879 & -3.920909 & 1.835544 \\
\hline 75 & 6 & 0 & -8.617361 & -0.310599 & 0.758809 \\
\hline 76 & 6 & 0 & -7.190634 & -0.672290 & 0.370860 \\
\hline 77 & 6 & $\odot$ & -6.390420 & -0.411319 & 1.650337 \\
\hline 78 & 8 & 0 & -7.327747 & -0.545868 & 2.751063 \\
\hline 79 & 1 & $\theta$ & -9.078803 & -2.300269 & 3.578976 \\
\hline 80 & 1 & 0 & -10.301835 & -2.089454 & 2.301010 \\
\hline 81 & 1 & 0 & -9.313729 & 0.050655 & 2.778840 \\
\hline 82 & 1 & 0 & -9.367223 & $-\odot .823505$ & 0.154619 \\
\hline 83 & 1 & $\odot$ & -6.798661 & $-\odot .094362$ & -0.463239 \\
\hline 84 & 1 & 0 & -7.160419 & -1.730592 & 0.112981 \\
\hline 85 & 1 & $\odot$ & -5.952346 & $\odot .58749 \odot$ & 1.671963 \\
\hline 86 & 7 & $\odot$ & -5.288341 & -1.335424 & 1.826106 \\
\hline 87 & 6 & $\odot$ & -5.333282 & -2.706046 & 1.732189 \\
\hline 88 & 7 & $\odot$ & -4.146932 & -3.267301 & 1.750254 \\
\hline 89 & 6 & $\odot$ & -3.274349 & -2.201399 & 1.876890 \\
\hline $9 \odot$ & 6 & $\odot$ & -3.965347 & $-\odot .995528$ & 1.936046 \\
\hline 91 & 7 & $\odot$ & -3.444340 & 0.230678 & 2.086750 \\
\hline 92 & 6 & 0 & -2.125226 & 0.185509 & 2.160829 \\
\hline 93 & 7 & 0 & -1.332447 & $-\odot .897268$ & 2.112195 \\
\hline 94 & 6 & $\odot$ & -1.867735 & -2.123338 & 1.965698 \\
\hline 95 & 7 & 0 & -1.050697 & -3.182616 & 1.930901 \\
\hline 96 & 1 & $\odot$ & -6.289903 & -3.211883 & 1.664303 \\
\hline 97 & 1 & $\odot$ & -1.591891 & 1.125934 & 2.273406 \\
\hline 98 & 1 & 0 & -0.041735 & -3.042531 & 1.974520 \\
\hline 99 & 1 & 0 & -1.423106 & -4.093278 & 1.721587 \\
\hline 100 & 6 & 0 & -6.510419 & 2.807006 & -0.724980 \\
\hline 101 & 11 & 0 & 8.958017 & 4.055452 & 2.408920 \\
\hline 102 & 11 & 0 & -11.472867 & 2.849237 & -2.070677 \\
\hline 103 & 6 & 0 & -4.539595 & 1.018205 & -3.097299 \\
\hline 104 & 6 & 0 & -3.670637 & 0.847601 & -1.849118 \\
\hline 105 & 8 & 0 & -4.573589 & 1.332842 & -0.796411 \\
\hline 106 & 1 & 0 & -6.477233 & 2.607125 & 0.350311 \\
\hline 107 & 1 & 0 & -6.805139 & 3.847839 & -0.885063 \\
\hline 108 & 1 & 0 & -4.497349 & 3.404026 & -1.070806 \\
\hline 109 & 1 & 0 & -6.118232 & 2.527867 & -3.312504 \\
\hline 110 & 1 & 0 & -3.978267 & 0.942624 & -4.030561 \\
\hline
\end{tabular}




\begin{tabular}{|c|c|c|c|c|c|}
\hline 11 & 8 & $\odot$ & 4.896542 & 5.565133 & 1.106577 \\
\hline 12 & 6 & 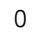 & 5.039724 & 3.660049 & -0.314679 \\
\hline 13 & 6 & $\odot$ & 5.542418 & 4.272475 & 0.994332 \\
\hline 14 & 6 & $\odot$ & -8.707343 & -3.319415 & 1.117251 \\
\hline 15 & 6 & 0 & -8.324832 & -1.858094 & 1.248648 \\
\hline 16 & 8 & 0 & -7.644681 & -4.090928 & 0.487685 \\
\hline 17 & 8 & $\odot$ & -8.328776 & $\odot .268172$ & $\odot .154543$ \\
\hline 18 & 15 & 0 & -7.725115 & 1.522631 & -0.706021 \\
\hline 19 & 8 & $\odot$ & -6.291352 & 1.804814 & ๑.029391 \\
\hline 20 & 8 & $\odot$ & -8.647567 & 2.687518 & -0.433149 \\
\hline 21 & 8 & 0 & -7.393449 & 1.088212 & -2.109321 \\
\hline 22 & 8 & $\odot$ & -5.042166 & 5.885029 & -0.785707 \\
\hline 23 & 6 & $\odot$ & -5.097756 & 3.878707 & 0.493488 \\
\hline 24 & 6 & 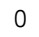 & -5.534237 & 4.521379 & -0.824789 \\
\hline 25 & 1 & $\odot$ & -5.249449 & 6.342894 & -1.618116 \\
\hline 26 & 6 & $\odot$ & 8.873003 & -2.936883 & -2.443419 \\
\hline 27 & 6 & 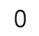 & 8.351746 & -1.518902 & -2.324994 \\
\hline 28 & 6 & $\odot$ & 8.449339 & -0.905652 & -0.919998 \\
\hline 29 & 6 & $\odot$ & 7.104895 & -1.262127 & -0.305228 \\
\hline 30 & 6 & $\Theta$ & 6.159549 & -1.079128 & -1.485411 \\
\hline 31 & 8 & $\odot$ & 6.920209 & -1.498439 & -2.654694 \\
\hline 32 & 1 & 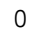 & 8.642504 & -3.312293 & -3.445810 \\
\hline 33 & 1 & 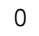 & 9.961123 & -2.933542 & -2.310150 \\
\hline 34 & 1 & $\odot$ & 8.872858 & -0.885605 & -3.046493 \\
\hline 35 & 1 & $\Theta$ & 9.302517 & -1.273544 & -0.348447 \\
\hline 36 & 1 & 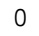 & 6.845515 & -0.624512 & 0.533695 \\
\hline 37 & 1 & $\odot$ & 7.135288 & -2.303938 & ๑.017265 \\
\hline 38 & 1 & 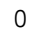 & 5.865593 & -0.039929 & -1.596566 \\
\hline 39 & 8 & $\Theta$ & 8.239507 & -3.761066 & -1.433395 \\
\hline 40 & 1 & $\odot$ & 8.516447 & -4.687498 & -1.520648 \\
\hline 41 & 6 & $\Theta$ & 3.710983 & -1.144413 & -1.387742 \\
\hline 42 & 7 & $\Theta$ & 1.413207 & -3.797888 & -0.992433 \\
\hline 43 & 8 & 0 & 3.722762 & ๑. 085866 & -1.510407 \\
\hline 44 & 1 & 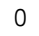 & 5.947923 & -3.650324 & -1.382900 \\
\hline 45 & 1 & $\Theta$ & 3.830875 & -4.994639 & -1.106021 \\
\hline 46 & 1 & $\odot$ & 1.369739 & -4.801263 & -0.931537 \\
\hline 47 & 1 & $\Theta$ & $\odot .544872$ & -3.263805 & -1.066716 \\
\hline 48 & 6 & 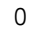 & 6.024876 & 2.737349 & -1.004425 \\
\hline 49 & 1 & 0 & 5.167441 & 6.008416 & 1.928565 \\
\hline 50 & 6 & 0 & 5.045139 & 3.297486 & 2.069483 \\
\hline 51 & 6 & $\odot$ & 3.727602 & 2.764215 & 1.489616 \\
\hline 52 & 8 & 0 & 3.818094 & 2.942142 & 0.046822 \\
\hline 53 & 1 & 0 & 5.491680 & 2.127336 & -1.735966 \\
\hline 54 & 1 & 0 & 6.797929 & 3.331644 & -1.498550 \\
\hline 55 & 1 & 0 & 4.751705 & 4.451754 & -1.010378 \\
\hline 56 & 1 & 0 & 6.631553 & 4.379086 & 1.003422 \\
\hline 57 & 1 & 0 & 4.884679 & 3.791716 & 3.029668 \\
\hline 58 & 1 & 0 & 5.793292 & 2.516676 & 2.202416 \\
\hline 59 & 1 & 0 & 2.875356 & 3.343340 & 1.838110 \\
\hline 60 & 7 & 0 & 3.400610 & 1.370850 & 1.780262 \\
\hline 61 & 6 & 0 & 4.359519 & 0.379573 & 1.812822 \\
\hline 62 & 6 & 0 & 4.070681 & -0.923926 & 2.011667 \\
\hline 63 & 6 & $\odot$ & 2.672489 & -1.312150 & 2.036448 \\
\hline 64 & 7 & $\odot$ & 1.764130 & -0.281266 & 1.950651 \\
\hline 65 & 6 & $\odot$ & 2.044358 & 1.060425 & 1.891449 \\
\hline 66 & 6 & 0 & 5.083716 & -2.008144 & 2.218501 \\
\hline 67 & 8 & 0 & 2.295998 & -2.484007 & 2.126973 \\
\hline 68 & 8 & 0 & 1.164344 & 1.907732 & 1.933375 \\
\hline 69 & 1 & 0 & 5.377733 & 0.720935 & 1.671459 \\
\hline 70 & 1 & 0 & 4.848131 & -2.560635 & 3.132545 \\
\hline 71 & 1 & 0 & 5.071948 & -2.732423 & 1.398582 \\
\hline 72 & 1 & 0 & 6.090109 & -1.602561 & 2.317247 \\
\hline 73 & 1 & 0 & $\odot .75 \odot 221$ & $-\odot .536159$ & 1.940617 \\
\hline 74 & 1 & 0 & -7.776764 & -5.038386 & 0.659510 \\
\hline 75 & 6 & 0 & -7.951429 & -1.133826 & -0.050869 \\
\hline 76 & 6 & 0 & -6.444729 & -1.316182 & -0.114105 \\
\hline 77 & 6 & $\odot$ & -6.027569 & -1.201425 & 1.348595 \\
\hline 78 & 8 & $\odot$ & -7.147474 & -1.717498 & 2.115128 \\
\hline 79 & 1 & $\odot$ & -8.896759 & -3.716653 & 2.118183 \\
\hline 80 & 1 & $\odot$ & -9.625760 & -3.397810 & $\odot .525655$ \\
\hline 81 & 1 & 0 & -9.154128 & -1.336497 & 1.732120 \\
\hline 82 & 1 & $\odot$ & -8.475239 & -1.516936 & $-\odot .927838$ \\
\hline 83 & 1 & 0 & -5.933080 & $-\odot .599427$ & -0.744854 \\
\hline 84 & 1 & 0 & -6.215910 & -2.314516 & -0.479955 \\
\hline 85 & 1 & 0 & -5.827553 & $-\odot .167288$ & 1.633931 \\
\hline 86 & 7 & 0 & -4.839106 & -1.968079 & 1.654789 \\
\hline 87 & 6 & 0 & -4.716507 & -3.330464 & 1.653301 \\
\hline
\end{tabular}




\begin{tabular}{|c|c|c|c|c|c|}
\hline 88 & 7 & $\odot$ & -3.474831 & -3.739101 & 1.756293 \\
\hline 89 & 6 & 0 & -2.739758 & -2.570528 & 1.790712 \\
\hline 90 & 6 & 0 & -3.568335 & $-1.46 \odot 261$ & 1.725050 \\
\hline 91 & 7 & 0 & -3.195793 & $-\odot .171589$ & 1.723068 \\
\hline 92 & 6 & 0 & -1.877737 & $-\odot .052334$ & 1.779324 \\
\hline 93 & 7 & 0 & -0.958527 & -1.029416 & 1.835605 \\
\hline 94 & 6 & $\odot$ & -1.354432 & -2.320560 & 1.833206 \\
\hline 95 & 7 & $\odot$ & -0.434241 & -3.288017 & 1.869531 \\
\hline 96 & 1 & $\odot$ & -5.593496 & -3.955585 & 1.553287 \\
\hline 97 & 1 & 0 & -1.469264 & 0.955633 & 1.771132 \\
\hline 98 & 1 & 0 & $\odot .558874$ & -3.051533 & 1.909512 \\
\hline 99 & 1 & 0 & -0.728099 & -4.249832 & 1.850270 \\
\hline 100 & 6 & $\odot$ & -6.039965 & 2.819974 & 1.034699 \\
\hline 101 & 11 & 0 & $8 . \odot \odot 7878$ & $\odot .603511$ & 3.500576 \\
\hline 102 & 11 & 0 & -9.779118 & 4.439339 & -1.095655 \\
\hline 103 & 6 & $\odot$ & -4.828723 & 3.688558 & -1.901869 \\
\hline 104 & 6 & $\odot$ & -3.558852 & 3.167505 & -1.211118 \\
\hline 105 & 8 & $\odot$ & -3.779815 & 3.308830 & 0.221297 \\
\hline 106 & 1 & $\odot$ & -5.575846 & 2.322213 & 1.887766 \\
\hline 107 & 1 & $\odot$ & -6.980969 & 3.283353 & 1.343658 \\
\hline 108 & 1 & 0 & -4.964008 & 4.651946 & 1.254173 \\
\hline 109 & 1 & $\odot$ & -6.622206 & 4.507596 & -0.943347 \\
\hline 110 & 1 & $\odot$ & -4.587614 & 4.285371 & -2.783888 \\
\hline 111 & 1 & $\Theta$ & -5.483743 & 2.873103 & -2.210613 \\
\hline 112 & 1 & $\odot$ & -2.675212 & 3.758316 & -1.455975 \\
\hline 113 & 7 & $\odot$ & -3.230534 & 1.790282 & -1.526959 \\
\hline 114 & 6 & $\Theta$ & -4.084088 & 0.736729 & -1.802910 \\
\hline 115 & 7 & $\odot$ & -3.467808 & $-\odot .4 \odot 9494$ & -1.872878 \\
\hline 116 & 6 & 0 & -2.143990 & $-\odot .113381$ & -1.618107 \\
\hline 117 & 6 & $\odot$ & -1.974316 & 1.246489 & -1.408322 \\
\hline 118 & 7 & $\odot$ & -0.832225 & 1.917863 & -1.181058 \\
\hline 119 & 6 & $\odot$ & $\odot .233998$ & 1.127898 & -1.191941 \\
\hline 120 & 7 & $\odot$ & 0.159054 & $-\odot .242749$ & -1.319299 \\
\hline 121 & 6 & $\odot$ & -1.011224 & $-\odot .960072$ & -1.509171 \\
\hline 122 & 7 & $\odot$ & 1.461735 & 1.646326 & -1.103460 \\
\hline 123 & 8 & $\odot$ & $-\odot .993579$ & -2.207233 & -1.561246 \\
\hline 124 & 1 & $\odot$ & -5.144120 & 0.886698 & -1.960739 \\
\hline 125 & 1 & $\odot$ & 1.566342 & 2.605154 & $-\odot .816342$ \\
\hline 126 & 1 & $\Theta$ & 2.306498 & 1.068694 & -1.145042 \\
\hline 127 & 1 & 0 & 1.031434 & $-\odot .802913$ & -1.255034 \\
\hline 128 & 8 & $\odot$ & -3.737762 & -2.843239 & -1.202856 \\
\hline 129 & 1 & $\odot$ & -2.777008 & -2.764317 & -1.404259 \\
\hline 130 & 8 & $\odot$ & -3.299337 & -5.508809 & $-\odot .597671$ \\
\hline 131 & 1 & $\odot$ & -3.241401 & -5.015408 & 0.235111 \\
\hline 132 & 1 & $\odot$ & -4.214037 & -5.349865 & $-\odot .884081$ \\
\hline 133 & 1 & 0 & -5.186074 & -3.831947 & -1.734369 \\
\hline 134 & 8 & $\odot$ & -5.814800 & -4.569340 & -1.649473 \\
\hline 135 & 1 & $\odot$ & -6.455661 & -4.288216 & $-\odot .975715$ \\
\hline
\end{tabular}

General basis read from cards: (6D, 7F)

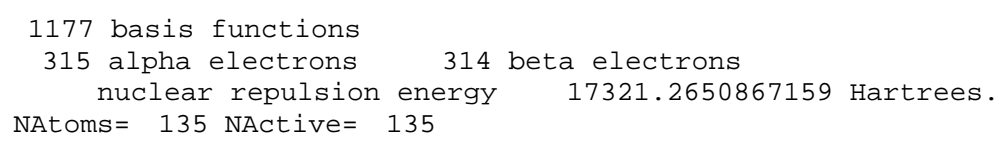

Force inversion solution in PCM.

Polarizable Continuum Model (PCM)

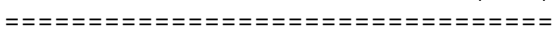

Model : PCM.

Atomic radii : UFF (Universal Force Field).

Solvent : Water, Eps $=78.355300$ Eps $($ inf $)=1.777849$

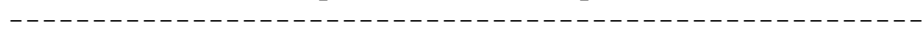

SCF Done: $E($ UwB97XD) $=-5076.18875567$ A.U. after 1 cycles NFock $=1$ Conv $=0.15 \mathrm{D}-09 \quad-\mathrm{V} / \mathrm{T}=2.0066$

$\langle\mathrm{S} x\rangle=0.0000<\mathrm{Sy}\rangle=0.0000\langle\mathrm{Sz}\rangle=0.5000$

$<S^{* *} 2>=0.7532 \mathrm{~S}=0.5016$

$<$ L.S $>=0.000000000000 \mathrm{E}+00$

Annihilation of the first spin contaminant:

$\mathrm{S}^{* * 2}$ before annihilation 0.7532 , after 0.7500

Harmonic frequencies $\left(\mathrm{cm}^{*}-1\right)$, IR intensities (KM/Mole), Raman 


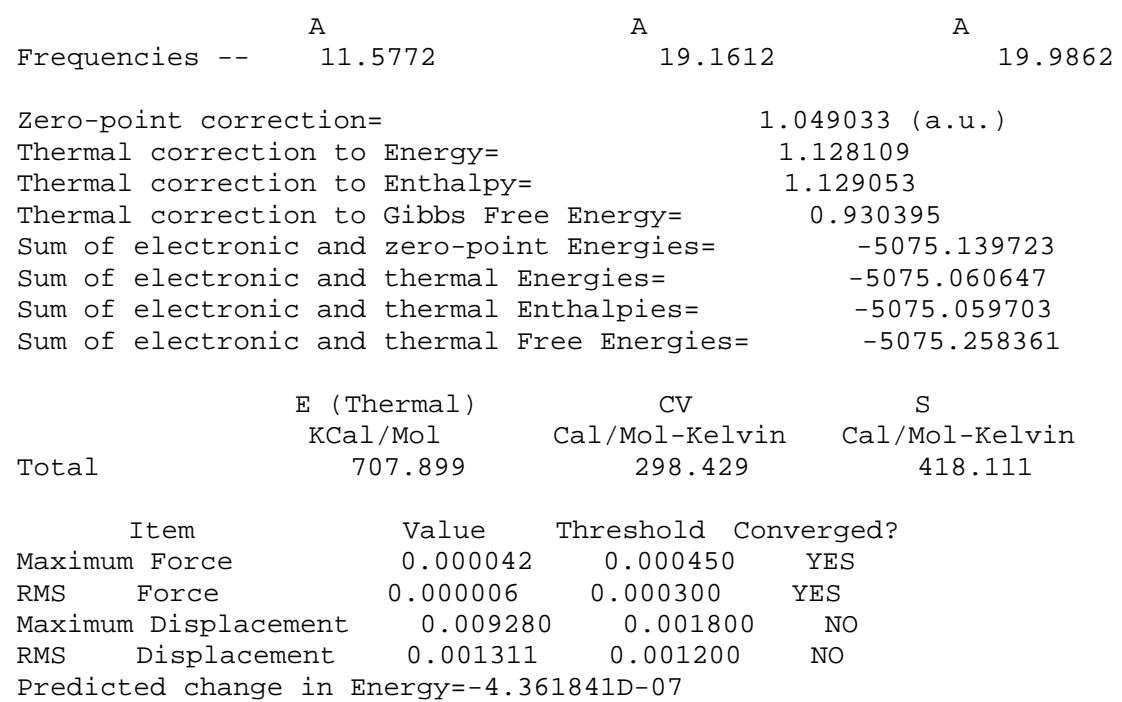

Predicted change in Energy $=-4.361841 \mathrm{D}-07$

Normal termination of Gaussian 16 at Sun Aug 18 01:58:47 2019.

$G($ high $)=-5077.023074 \mathrm{a} \cdot \mathrm{u}$.

(a) $3-2$ TS1(C8)W2(a)

dna.ts1aw2B. $\log$

Stoichiometry C39H54N15Na2023P2(2)

Standard orientation:

\begin{tabular}{|c|c|c|c|c|c|}
\hline \multirow{2}{*}{$\begin{array}{l}\text { Center } \\
\text { Number }\end{array}$} & \multirow{2}{*}{$\begin{array}{l}\text { Atomic } \\
\text { Number }\end{array}$} & \multirow{2}{*}{$\begin{array}{l}\text { Atomic } \\
\text { Type }\end{array}$} & \multicolumn{3}{|c|}{ Coordinates (Angstroms) } \\
\hline & & & $x$ & $Y$ & $z$ \\
\hline 1 & 7 & 0 & 5.673165 & -1.944063 & -1.559508 \\
\hline 2 & 6 & 0 & 5.910019 & -3.286452 & -1.609851 \\
\hline 3 & 6 & 0 & 4.886440 & -4.167531 & -1.681125 \\
\hline 4 & 6 & 0 & 3.557098 & -3.627359 & -1.693480 \\
\hline 5 & 7 & 0 & 3.337910 & -2.309164 & -1.652280 \\
\hline 6 & 8 & 0 & 8.930431 & 0.854751 & -0.588022 \\
\hline 7 & 15 & $\odot$ & 8.216224 & 1.855010 & $\odot .487152$ \\
\hline 8 & 8 & 0 & 6.634054 & 1.777371 & 0.066818 \\
\hline 9 & 8 & $\odot$ & 8.749112 & 3.243222 & $\odot .238695$ \\
\hline 10 & 8 & 0 & 8.304060 & 1.295377 & 1.891232 \\
\hline 11 & 8 & $\odot$ & 4.730911 & $5.4 \odot 4752$ & 1.269844 \\
\hline 12 & 6 & $\odot$ & 4.969484 & 3.548160 & $-\odot .205516$ \\
\hline 13 & 6 & $\odot$ & 5.412387 & 4.130822 & 1.141014 \\
\hline 14 & 6 & $\odot$ & -9.198880 & -2.285292 & 1.860123 \\
\hline 15 & 6 & $\odot$ & -8.684194 & -0.861282 & 1.869460 \\
\hline 16 & 8 & $\odot$ & -8.223655 & -3.182325 & 1.256723 \\
\hline 17 & 8 & $\odot$ & -8.644808 & 1.106829 & 0.483437 \\
\hline 18 & 15 & $\odot$ & -8.678337 & 1.952669 & -0.917532 \\
\hline 19 & 8 & $\odot$ & -7.094237 & 2.256750 & -1.188695 \\
\hline 20 & 8 & $\odot$ & -9.424503 & 3.235100 & -0.633968 \\
\hline 21 & 8 & 0 & -9.165160 & 1.092908 & -2.057557 \\
\hline 22 & 8 & $\odot$ & -3.925348 & 4.740033 & -2.404335 \\
\hline 23 & 6 & $\odot$ & -4.935613 & 3.272503 & -0.808392 \\
\hline 24 & 6 & $\Theta$ & -4.832928 & 3.612842 & -2.311437 \\
\hline 25 & 1 & $\odot$ & -3.865099 & 5.052465 & -3.323232 \\
\hline 26 & 6 & $\odot$ & 9.817250 & -2.451741 & -2.078139 \\
\hline 27 & 6 & 0 & 9.109476 & -1.118755 & -1.947288 \\
\hline 28 & 6 & $\odot$ & 8.934335 & -0.611962 & -0.508323 \\
\hline 29 & 6 & $\odot$ & 7.570123 & -1.162290 & -0.117658 \\
\hline 30 & 6 & $\odot$ & 6.779850 & -1.011079 & -1.415213 \\
\hline 31 & 8 & $\odot$ & 7.746479 & -1.232566 & -2.481275 \\
\hline 32 & 1 & $\odot$ & 9.778469 & -2.768176 & -3.125564 \\
\hline 33 & 1 & 0 & 10.866547 & -2.332608 & -1.784401 \\
\hline 34 & 1 & $\odot$ & 9.641886 & -0.373249 & -2.542043 \\
\hline 35 & 1 & $\odot$ & 9.738193 & -0.921878 & 0.160076 \\
\hline 36 & 1 & $\odot$ & 7.122405 & -0.620303 & $\odot .712260$ \\
\hline 37 & 1 & $\odot$ & 7.686247 & -2.215011 & 0.148365 \\
\hline 38 & 1 & $\odot$ & 6.355782 & $-\odot .016132$ & -1.505301 \\
\hline 39 & 8 & 0 & 9.157333 & -3.421895 & -1.227876 \\
\hline
\end{tabular}




\begin{tabular}{|c|c|c|c|c|c|}
\hline 40 & 1 & 0 & 9.571747 & -4.295809 & -1.312769 \\
\hline 41 & 6 & $\odot$ & 4.368851 & -1.432813 & -1.620495 \\
\hline 42 & 7 & $\odot$ & 2.490096 & -4.423628 & -1.742620 \\
\hline 43 & 8 & 0 & 4.197057 & -0.207620 & -1.627912 \\
\hline 44 & 1 & $\odot$ & 6.960214 & -3.572033 & -1.588764 \\
\hline 45 & 1 & $\odot$ & 5.064758 & -5.232905 & -1.727350 \\
\hline 46 & 1 & 0 & 2.600655 & -5.422771 & -1.788363 \\
\hline 47 & 1 & $\odot$ & 1.554109 & -4.013433 & -1.806221 \\
\hline 48 & 6 & $\odot$ & 6.010373 & 2.685028 & $-\odot .8909 \odot 4$ \\
\hline 49 & 1 & 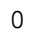 & 4.947554 & 5.819219 & 2.122432 \\
\hline 50 & 6 & $\odot$ & 4.893398 & 3.113578 & 2.163312 \\
\hline 51 & 6 & 0 & 3.582391 & 2.637010 & 1.530390 \\
\hline 52 & 8 & 0 & 3.772628 & 2.756908 & $\odot .089172$ \\
\hline 53 & 1 & $\odot$ & 5.527961 & 2.068828 & -1.650483 \\
\hline 54 & 1 & $\odot$ & 6.775558 & 3.320093 & -1.343785 \\
\hline 55 & 1 & 0 & 4.673357 & 4.355016 & -0.880418 \\
\hline 56 & 1 & $\odot$ & 6.497508 & 4.262844 & 1.189520 \\
\hline 57 & 1 & 0 & 4.725403 & 3.560781 & 3.145195 \\
\hline 58 & 1 & $\odot$ & 5.620133 & 2.305466 & 2.265709 \\
\hline 59 & 1 & $\odot$ & 2.746543 & 3.269456 & 1.822007 \\
\hline 60 & 7 & $\odot$ & 3.179406 & 1.272226 & 1.830322 \\
\hline 61 & 6 & $\odot$ & 4.058137 & $\odot .222483$ & 1.652769 \\
\hline 62 & 6 & $\odot$ & 3.701248 & -1.070853 & 1.799371 \\
\hline 63 & 6 & 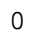 & 2.302171 & -1.365377 & 2.055607 \\
\hline 64 & 7 & $\odot$ & 1.477183 & $-\odot .271822$ & 2.192902 \\
\hline 65 & 6 & $\odot$ & 1.826190 & 1.046823 & 2.066914 \\
\hline 66 & 6 & $\odot$ & 4.645822 & -2.229471 & 1.714620 \\
\hline 67 & 8 & $\odot$ & 1.848104 & -2.510169 & 2.145257 \\
\hline 68 & 8 & $\odot$ & 1.004228 & 1.947776 & 2.169827 \\
\hline 69 & 1 & $\theta$ & 5.065374 & $\odot .515395$ & 1.373585 \\
\hline 70 & 1 & $\odot$ & 4.605329 & -2.817583 & 2.636162 \\
\hline 71 & 1 & $\odot$ & 4.378099 & -2.903119 & $\odot .896192$ \\
\hline 72 & 1 & 0 & 5.671778 & -1.895251 & 1.571111 \\
\hline 73 & 1 & $\odot$ & $\odot .455680$ & $-\odot .454984$ & 2.318101 \\
\hline 74 & 1 & 0 & -8.443399 & -4.106106 & 1.465064 \\
\hline 75 & 6 & 0 & -8.247794 & $-\odot .301802$ & 0.510171 \\
\hline 76 & 6 & $\odot$ & -6.733279 & -0.447642 & 0.514663 \\
\hline 77 & 6 & $\odot$ & -6.342792 & $-\odot .353826$ & 1.994269 \\
\hline 78 & 8 & $\odot$ & -7.512231 & -0.756096 & 2.744121 \\
\hline 79 & 1 & $\odot$ & -9.389410 & -2.596174 & 2.890733 \\
\hline 80 & 1 & 0 & -10.137224 & -2.323686 & 1.297065 \\
\hline 81 & 1 & $\odot$ & -9.466528 & $-\odot .223088$ & 2.287371 \\
\hline 82 & 1 & $\odot$ & -8.719864 & -0.814964 & -0.329816 \\
\hline 83 & 1 & $\odot$ & -6.221633 & $\odot .291754$ & $-\odot .101989$ \\
\hline 84 & 1 & 0 & -6.464429 & -1.433669 & 0.143132 \\
\hline 85 & 1 & $\odot$ & -6.040601 & $\odot .651357$ & 2.295271 \\
\hline 86 & 7 & 0 & -5.240766 & -1.242389 & 2.327523 \\
\hline 87 & 6 & $\odot$ & -5.274908 & -2.611378 & 2.307756 \\
\hline 88 & 7 & $\odot$ & -4.087323 & -3.161500 & 2.394791 \\
\hline 89 & 6 & 0 & -3.220771 & -2.086638 & 2.443837 \\
\hline 90 & 6 & $\odot$ & -3.918798 & $-\odot .8864 \odot 2$ & 2.400276 \\
\hline 91 & 7 & $\odot$ & -3.401622 & $\odot .350543$ & 2.397272 \\
\hline 92 & 6 & 0 & -2.077713 & $\odot .318209$ & 2.404880 \\
\hline 93 & 7 & 0 & -1.277759 & $-\odot .759026$ & 2.415577 \\
\hline 94 & 6 & $\odot$ & -1.814555 & -1.996503 & 2.430851 \\
\hline 95 & 7 & $\odot$ & -0.999006 & -3.056522 & 2.425783 \\
\hline 96 & 1 & $\odot$ & -6.218797 & -3.130602 & 2.206038 \\
\hline 97 & 1 & $\odot$ & -1.552259 & 1.270128 & 2.391260 \\
\hline 98 & 1 & $\theta$ & $\odot .009240$ & -2.919897 & 2.337231 \\
\hline 99 & 1 & $\odot$ & -1.388483 & -3.982957 & 2.380229 \\
\hline 100 & 6 & $\odot$ & -6.340161 & 3.115480 & -0.285828 \\
\hline 101 & 11 & 0 & 9.055261 & 3.273350 & 2.980890 \\
\hline 102 & 11 & 0 & -10.999227 & 2.878533 & -2.472448 \\
\hline 103 & 6 & $\odot$ & -4.227470 & 2.348751 & -2.931078 \\
\hline 104 & 6 & $\odot$ & -3.346765 & 1.846886 & -1.789295 \\
\hline 105 & 8 & $\odot$ & -4.199151 & 2.017924 & -0.619517 \\
\hline 106 & 1 & $\odot$ & -6.322292 & 2.670097 & 0.713562 \\
\hline 107 & 1 & $\odot$ & -6.825812 & 4.094344 & -0.231972 \\
\hline 108 & 1 & $\odot$ & -4.432856 & 4.064265 & -0.243573 \\
\hline 109 & 1 & $\odot$ & -5.805153 & 3.862320 & -2.742556 \\
\hline 110 & 1 & $\odot$ & -3.668433 & 2.549777 & -3.846053 \\
\hline 111 & 1 & $\odot$ & -5.023841 & 1.629720 & -3.140399 \\
\hline 112 & 1 & $\odot$ & -2.453979 & 2.470231 & -1.683718 \\
\hline 113 & 7 & 0 & -2.885489 & $\odot .487096$ & -1.876379 \\
\hline 114 & 6 & $\odot$ & -3.569567 & $-\odot .633266$ & -2.286176 \\
\hline 115 & 7 & $\odot$ & -2.809193 & -1.704616 & -2.348855 \\
\hline 116 & 6 & 0 & -1.570774 & -1.272517 & -1.928654 \\
\hline
\end{tabular}




\begin{tabular}{|c|c|c|c|c|c|}
\hline 117 & 6 & $\odot$ & -1.589767 & ๑. 084478 & -1.637004 \\
\hline 118 & 7 & $\odot$ & -0.575338 & $\odot .882651$ & -1.272422 \\
\hline 119 & 6 & $\odot$ & $\odot .586214$ & $\odot .240030$ & -1.228971 \\
\hline 120 & 7 & $\odot$ & $\odot .712645$ & -1.106851 & -1.471590 \\
\hline 121 & 6 & $\odot$ & -0.325239 & -1.968156 & -1.828051 \\
\hline 122 & 7 & $\odot$ & 1.711441 & $\odot .895007$ & -0.924112 \\
\hline 123 & 8 & $\odot$ & -0.109519 & -3.168564 & -2.023974 \\
\hline 124 & 1 & 0 & -4.614565 & $-\odot .595591$ & -2.551563 \\
\hline 125 & 1 & $\odot$ & 1.668451 & 1.892005 & -0.785625 \\
\hline 126 & 1 & $\odot$ & 2.634979 & $\odot .485337$ & -1.097985 \\
\hline 127 & 1 & $\odot$ & 1.661661 & -1.529428 & -1.473320 \\
\hline 128 & 8 & $\odot$ & -4.345180 & -2.119889 & -0.488606 \\
\hline 129 & 1 & $\odot$ & -3.831715 & -2.956148 & -0.525791 \\
\hline 130 & 8 & $\odot$ & $-4.0 \odot 7424$ & -4.838274 & $\odot .02474 \odot$ \\
\hline 131 & 1 & $\odot$ & -3.956208 & -4.538653 & $\odot .947986$ \\
\hline 132 & 1 & $\odot$ & -4.951632 & -4.732119 & -0.195191 \\
\hline 133 & 1 & $\odot$ & -5.876341 & -3.082448 & -0.996362 \\
\hline 134 & 8 & $\odot$ & -6.502863 & -3.815731 & -0.866293 \\
\hline 135 & 1 & $\odot$ & -7.135470 & -3.490326 & -0.202193 \\
\hline
\end{tabular}

General basis read from cards: (6D, 7F)

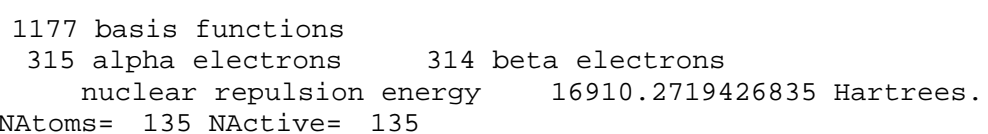

NAtoms $=135$ NActive $=135$

Force inversion solution in PCM.

Polarizable Continuum Model (PCM)

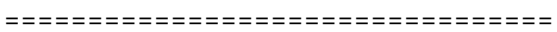

Model : PCM.

Solvent : Water, Eps $=78.355300$ Eps $($ inf $)=1.777849$

\begin{tabular}{|c|c|c|c|c|c|c|c|c|c|c|}
\hline \multicolumn{11}{|c|}{ 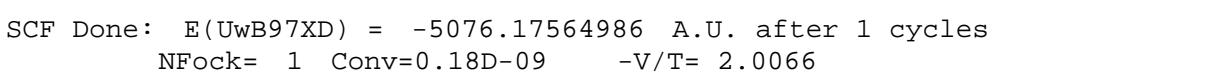 } \\
\hline \multicolumn{11}{|c|}{$\langle S x\rangle=0.0000<S y\rangle=0.0000<S z>=0.5000$} \\
\hline \multirow{2}{*}{\multicolumn{11}{|c|}{$\left\langle S^{* *} 2>=0.7553 \mathrm{~S}=0.5026\right.$}} \\
\hline & & & & \\
\hline \multirow{2}{*}{\multicolumn{11}{|c|}{ Annihilation of the first spin contaminant: }} \\
\hline$S^{* *} 2 \mathrm{~b}$ & & & & 0. & & fter & 0.750 & & & \\
\hline \multicolumn{11}{|c|}{ Harmonic frequencies $\left(\mathrm{cm}^{* *}-1\right)$, IR intensities (KM/Mole), Raman } \\
\hline & & & & & & & & 3 & & \\
\hline \multirow{2}{*}{\multicolumn{5}{|c|}{$\begin{array}{l}A \\
-29.0063\end{array}$}} & A & & & A & & \\
\hline & & & & & \multicolumn{3}{|c|}{12.2871} & \multicolumn{2}{|c|}{15.1451} & \\
\hline \multicolumn{3}{|c|}{ Red. masses - - } & \multicolumn{2}{|c|}{$\begin{array}{r}-29.0063 \\
8.2983\end{array}$} & \multicolumn{3}{|c|}{9.2937} & \multicolumn{3}{|c|}{$\begin{array}{l}15.1451 \\
7.2652\end{array}$} \\
\hline \multirow{2}{*}{\multicolumn{2}{|c|}{$\begin{array}{l}\text { Frc consts } \\
\text { IR Inten }\end{array}$}} & - - & \multicolumn{2}{|c|}{0.0041} & \multicolumn{3}{|c|}{0.0008} & \multicolumn{3}{|c|}{0.0010} \\
\hline & & - - & \multicolumn{2}{|c|}{6.4517} & \multicolumn{3}{|c|}{4.4130} & \multicolumn{3}{|c|}{0.8494} \\
\hline Atom & AN & $x$ & $\mathrm{Y}$ & Z & $\mathrm{x}$ & $Y$ & Z & $x$ & Z & \\
\hline 1 & 7 & 0.02 & $-\odot . \odot \odot$ & -0.00 & -0.01 & -0.01 & $\odot . \odot 8$ & -0.01 & -0.04 & 0.02 \\
\hline 2 & 6 & 0.04 & $\odot . \odot \odot$ & -0.01 & -0.01 & -0.01 & 0.11 & -0.02 & -0.04 & 0.05 \\
\hline 3 & 6 & 0.05 & -0.01 & -0.02 & -0.01 & -0.01 & 0.14 & -0.03 & -0.03 & 0.05 \\
\hline 4 & 6 & 0.05 & -0.03 & -0.03 & -0.01 & -0.01 & 0.13 & -0.03 & -0.02 & 0.02 \\
\hline 5 & 7 & 0.03 & -0.03 & -0.03 & -0.01 & -0.01 & 0.10 & -0.01 & -0.02 & 0.01 \\
\hline 6 & 8 & $\odot . \odot \odot$ & $\odot . \odot 2$ & 0.01 & $\odot .01$ & 0.01 & $\odot .01$ & -0.03 & $-\odot . \odot 2$ & $-\odot . \odot 4$ \\
\hline 7 & 15 & -0.01 & 0.01 & 0.01 & 0.01 & 0.03 & -0.01 & -0.03 & 0.01 & -0.06 \\
\hline 8 & 8 & $-\odot . \odot \odot$ & $\odot .01$ & $\odot .0 \odot$ & $\odot .01$ & $\odot .02$ & -0.00 & -0.03 & -0.02 & -0.03 \\
\hline 9 & 8 & -0.01 & $\odot . \odot 2$ & 0.01 & 0.01 & 0.02 & -0.03 & -0.04 & 0.01 & -0.11 \\
\hline 10 & 8 & -0.01 & 0.01 & 0.01 & 0.01 & 0.05 & 0.00 & 0.00 & 0.05 & -0.04 \\
\hline 11 & 8 & -0.02 & 0.00 & -0.00 & -0.02 & 0.02 & -0.05 & -0.02 & 0.01 & -0.11 \\
\hline 12 & 6 & -0.01 & $-\odot . \odot \odot$ & $-\odot . \odot \odot$ & $\odot . \odot \odot$ & 0.01 & $-\odot .03$ & $-\odot . \odot 4$ & $-\odot .03$ & -0.06 \\
\hline 13 & 6 & -0.01 & $0.0 \odot$ & $-\odot . \odot \odot$ & -0.01 & 0.02 & -0.03 & -0.02 & 0.01 & -0.09 \\
\hline 14 & 6 & -0.00 & 0.01 & -0.01 & 0.00 & -0.03 & -0.00 & 0.01 & -0.08 & -0.11 \\
\hline 15 & 6 & $-\odot . \odot 2$ & $\odot . \odot 2$ & $\odot . \odot 2$ & $\odot .01$ & $-\odot .03$ & $\odot .02$ & $-\odot .01$ & $-\odot . \odot 7$ & $-\odot .07$ \\
\hline 16 & 8 & 0.00 & 0.04 & -0.04 & -0.01 & -0.02 & -0.03 & 0.04 & -0.06 & -0.11 \\
\hline 17 & 8 & -0.03 & 0.04 & 0.04 & 0.02 & 0.01 & 0.08 & 0.00 & -0.04 & -0.02 \\
\hline 18 & 15 & -0.04 & 0.03 & $\odot .04$ & -0.01 & 0.06 & 0.11 & 0.02 & $-\odot . \odot \odot$ & $\odot . \odot \odot$ \\
\hline 19 & 8 & -0.04 & 0.01 & 0.01 & -0.01 & 0.01 & 0.05 & 0.02 & 0.01 & 0.04 \\
\hline 20 & 8 & -0.01 & 0.05 & 0.04 & 0.05 & $\odot .07$ & 0.17 & $\odot .01$ & -0.01 & 0.03 \\
\hline 21 & 8 & $-\odot .07$ & $\odot . \odot 4$ & 0.05 & $-\odot . \odot 8$ & 0.11 & 0.10 & 0.05 & $\odot .02$ & $-\odot .03$ \\
\hline 22 & 8 & 0.04 & -0.12 & 0.01 & -0.06 & -0.02 & -0.11 & 0.03 & 0.07 & 0.16 \\
\hline 23 & 6 & 0.01 & -0.10 & 0.01 & 0.02 & $-\odot . \odot \odot$ & -0.05 & $\odot .01$ & 0.01 & 0.10 \\
\hline 24 & & 0.03 & & 0.00 & -0.04 & -0.03 & $\theta$. & 0.03 & 0.07 & \\
\hline
\end{tabular}




\begin{tabular}{|c|c|c|c|c|c|c|c|c|c|c|}
\hline 25 & 1 & $\odot .04$ & -0.12 & 0.01 & -0.10 & -0.03 & -0.12 & $\odot .03$ & 0.11 & 0.18 \\
\hline 26 & 6 & 0.02 & $\odot .03$ & 0.01 & -0.01 & -0.02 & 0.07 & $\odot . \odot \odot$ & -0.07 & $\odot .09$ \\
\hline 27 & 6 & 0.01 & 0.02 & 0.01 & -0.01 & -0.02 & 0.04 & $\odot .0 \odot$ & -0.07 & 0.04 \\
\hline 28 & 6 & 0.01 & 0.02 & 0.01 & 0.00 & 0.01 & 0.04 & -0.02 & -0.02 & 0.02 \\
\hline 29 & 6 & $\odot .01$ & 0.01 & $\odot .01$ & $\odot . \odot \odot$ & $\odot .02$ & $\odot .05$ & -0.02 & -0.01 & $\odot .02$ \\
\hline 30 & 6 & 0.01 & 0.01 & 0.00 & -0.01 & -0.01 & $\odot .05$ & $-\odot . \odot \odot$ & -0.04 & 0.01 \\
\hline 31 & 8 & $\odot .02$ & 0.02 & 0.01 & -0.01 & -0.03 & $\odot .05$ & 0.01 & -0.09 & 0.03 \\
\hline 32 & 1 & 0.02 & 0.03 & 0.01 & -0.02 & -0.05 & 0.07 & 0.02 & -0.11 & 0.10 \\
\hline 33 & 1 & $\odot .02$ & 0.03 & 0.01 & -0.01 & -0.02 & $\odot .06$ & -0.00 & -0.06 & 0.11 \\
\hline 34 & 1 & 0.01 & 0.03 & 0.01 & -0.01 & -0.03 & 0.03 & 0.01 & -0.09 & 0.02 \\
\hline 35 & 1 & $\odot .01$ & 0.02 & $\odot .01$ & $\odot .0 \odot$ & 0.02 & 0.04 & -0.02 & 0.01 & 0.04 \\
\hline 36 & 1 & 0.01 & 0.01 & 0.01 & $\odot .01$ & 0.04 & 0.04 & -0.03 & 0.02 & -0.00 \\
\hline 37 & 1 & 0.02 & 0.01 & 0.01 & $-\odot . \odot \odot$ & 0.03 & $\odot . \odot 8$ & -0.02 & $\odot . \odot \odot$ & 0.05 \\
\hline 38 & 1 & 0.00 & 0.01 & 0.01 & -0.01 & -0.01 & 0.04 & 0.01 & -0.04 & -0.03 \\
\hline 39 & 8 & 0.02 & 0.02 & $\odot .0 \odot$ & -0.01 & -0.01 & $\odot .09$ & -0.01 & -0.04 & 0.11 \\
\hline 40 & 1 & 0.03 & 0.02 & $0.0 \odot$ & -0.01 & -0.01 & 0.11 & -0.01 & -0.05 & 0.15 \\
\hline 41 & 6 & $\odot .02$ & -0.02 & -0.01 & -0.01 & -0.01 & 0.07 & -0.01 & -0.03 & 0.01 \\
\hline 42 & 7 & 0.06 & -0.04 & -0.05 & -0.01 & -0.01 & 0.16 & -0.03 & -0.01 & 0.01 \\
\hline 43 & 8 & $-0.0 \odot$ & -0.02 & 0.00 & -0.01 & -0.01 & 0.03 & 0.01 & -0.03 & 0.01 \\
\hline 44 & 1 & 0.05 & 0.02 & $\odot .0 \odot$ & -0.01 & -0.01 & 0.11 & -0.03 & -0.05 & 0.07 \\
\hline 45 & 1 & 0.07 & -0.01 & $-\odot .02$ & -0.01 & -0.01 & 0.16 & $-\odot .04$ & -0.04 & 0.07 \\
\hline 46 & 1 & $\odot .08$ & -0.04 & -0.06 & -0.01 & -0.01 & 0.18 & -0.04 & -0.01 & 0.02 \\
\hline 47 & 1 & 0.06 & $-\odot .06$ & $-\odot .07$ & -0.01 & -0.01 & 0.14 & -0.03 & $\odot .0 \odot$ & -0.02 \\
\hline 48 & 6 & -0.01 & $\odot .0 \odot$ & 0.00 & 0.01 & 0.01 & -0.02 & -0.05 & -0.05 & -0.05 \\
\hline 49 & 1 & -0.02 & $\odot .0 \odot$ & $-\odot .0 \odot$ & -0.03 & 0.03 & -0.05 & -0.01 & 0.03 & -0.13 \\
\hline 50 & 6 & -0.01 & $\odot .0 \odot$ & $\odot .0 \odot$ & -0.01 & 0.03 & -0.03 & -0.01 & 0.04 & -0.05 \\
\hline 51 & 6 & -0.01 & $0.0 \odot$ & $\odot . \odot \odot$ & $-\odot .0 \odot$ & 0.01 & -0.03 & -0.02 & $\odot .02$ & -0.02 \\
\hline 52 & 8 & -0.01 & -0.01 & 0.00 & 0.01 & $-0.0 \odot$ & -0.03 & -0.04 & -0.01 & -0.03 \\
\hline 53 & 1 & $-0.0 \odot$ & $\odot .0 \odot$ & 0.00 & 0.02 & $\odot .0 \odot$ & -0.02 & -0.07 & -0.08 & -0.02 \\
\hline 54 & 1 & -0.01 & 0.01 & $\odot . \odot \odot$ & 0.01 & $\odot .01$ & -0.01 & -0.06 & -0.07 & $-\odot .09$ \\
\hline 55 & 1 & -0.01 & -0.00 & -0.00 & -0.00 & -0.00 & -0.04 & -0.04 & -0.05 & -0.09 \\
\hline 56 & 1 & -0.01 & $\odot .01$ & -0.00 & -0.01 & 0.03 & -0.02 & -0.02 & 0.01 & -0.10 \\
\hline 57 & 1 & -0.01 & $\odot .0 \odot$ & $\odot .00$ & -0.03 & 0.03 & -0.03 & -0.00 & 0.06 & -0.06 \\
\hline 58 & 1 & -0.01 & $\odot .0 \odot$ & $\odot . \odot \odot$ & $-0.0 \odot$ & 0.04 & -0.01 & -0.01 & 0.04 & -0.04 \\
\hline 59 & 1 & -0.01 & $\odot .0 \odot$ & $-\odot . \odot \odot$ & -0.01 & $\odot .0 \odot$ & -0.04 & -0.02 & 0.03 & -0.02 \\
\hline 60 & 7 & -0.01 & $\odot .0 \odot$ & 0.01 & 0.01 & 0.01 & -0.02 & -0.02 & 0.03 & 0.01 \\
\hline 61 & 6 & -0.01 & $\odot .0 \odot$ & $-\odot . \odot \odot$ & $\odot .02$ & 0.01 & 0.02 & -0.02 & $\odot .03$ & 0.01 \\
\hline 62 & 6 & -0.01 & $\odot .0 \odot$ & $\odot .0 \odot$ & 0.03 & $\odot .01$ & 0.03 & -0.02 & 0.03 & $\odot .03$ \\
\hline 63 & 6 & -0.01 & $\odot .0 \odot$ & 0.01 & 0.03 & -0.00 & -0.02 & -0.01 & 0.03 & 0.07 \\
\hline 64 & 7 & -0.01 & 0.01 & 0.02 & 0.01 & -0.01 & -0.06 & -0.01 & 0.03 & 0.07 \\
\hline 65 & 6 & -0.01 & 0.01 & 0.01 & 0.00 & -0.01 & -0.05 & $-\odot .01$ & 0.03 & $\odot .04$ \\
\hline 66 & 6 & -0.02 & $\odot . \odot \odot$ & -0.01 & $\odot .05$ & 0.02 & 0.10 & -0.02 & 0.03 & 0.03 \\
\hline 67 & 8 & -0.01 & 0.01 & 0.02 & 0.04 & -0.01 & -0.03 & -0.01 & 0.03 & 0.10 \\
\hline 68 & 8 & -0.01 & 0.01 & $\odot .01$ & -0.01 & -0.01 & -0.08 & -0.01 & 0.03 & 0.03 \\
\hline 69 & 1 & -0.01 & $\odot .0 \odot$ & -0.01 & 0.03 & 0.02 & 0.05 & -0.03 & $\odot .02$ & -0.02 \\
\hline 70 & 1 & $-0.0 \odot$ & $0.0 \odot$ & -0.01 & $\odot .02$ & 0.04 & 0.10 & -0.00 & 0.03 & 0.03 \\
\hline 71 & 1 & -0.03 & $\odot .0 \odot$ & -0.01 & 0.10 & $\odot .0 \odot$ & 0.10 & -0.04 & 0.02 & 0.04 \\
\hline 72 & 1 & -0.02 & $\odot .0 \odot$ & -0.03 & 0.06 & 0.03 & 0.13 & -0.02 & 0.02 & 0.00 \\
\hline 73 & 1 & -0.01 & 0.01 & $\odot .02$ & 0.01 & -0.02 & -0.09 & -0.01 & 0.03 & 0.08 \\
\hline 74 & 1 & $\odot .0 \odot$ & 0.03 & -0.08 & -0.01 & -0.02 & -0.03 & 0.05 & -0.06 & -0.13 \\
\hline 75 & 6 & -0.01 & 0.04 & 0.03 & -0.01 & $\odot .0 \odot$ & $\odot .03$ & 0.01 & -0.04 & -0.05 \\
\hline 76 & 6 & -0.01 & 0.05 & 0.04 & -0.01 & -0.02 & -0.01 & 0.01 & -0.03 & -0.03 \\
\hline 77 & 6 & -0.02 & 0.03 & 0.04 & $\odot .01$ & -0.05 & -0.01 & -0.01 & -0.05 & -0.02 \\
\hline 78 & 8 & -0.02 & 0.02 & 0.03 & 0.03 & -0.06 & $0.0 \odot$ & -0.02 & -0.08 & -0.05 \\
\hline 79 & 1 & 0.01 & -0.02 & -0.01 & 0.01 & -0.04 & -0.01 & $-\odot . \odot \odot$ & -0.11 & -0.12 \\
\hline 80 & 1 & $-\odot . \odot \odot$ & $\odot .02$ & $-\odot . \odot \odot$ & $-0.0 \odot$ & -0.01 & 0.00 & 0.03 & -0.08 & -0.13 \\
\hline 81 & 1 & -0.02 & $\odot . \odot \odot$ & $\odot .03$ & 0.02 & -0.03 & 0.05 & -0.02 & -0.10 & -0.07 \\
\hline 82 & 1 & -0.00 & $\odot .04$ & 0.02 & -0.04 & 0.04 & $\odot .02$ & 0.03 & -0.02 & -0.07 \\
\hline 83 & 1 & -0.01 & 0.05 & 0.05 & -0.01 & -0.02 & -0.01 & 0.02 & -0.01 & $-0 . \odot \odot$ \\
\hline 84 & 1 & -0.01 & 0.06 & 0.03 & $-\odot .04$ & -0.02 & -0.03 & 0.03 & -0.02 & -0.04 \\
\hline 85 & 1 & -0.03 & 0.03 & 0.06 & 0.02 & -0.05 & $-\odot . \odot \odot$ & $-\odot .03$ & -0.05 & 0.01 \\
\hline 86 & 7 & -0.02 & 0.03 & $\odot .03$ & 0.02 & -0.05 & -0.05 & -0.01 & -0.04 & -0.01 \\
\hline 87 & 6 & -0.01 & $\odot .03$ & $\odot .0 \odot$ & 0.02 & -0.05 & -0.06 & 0.01 & -0.04 & -0.04 \\
\hline 88 & 7 & -0.01 & 0.03 & -0.02 & 0.03 & -0.05 & -0.09 & 0.01 & -0.03 & -0.02 \\
\hline 89 & 6 & -0.02 & 0.03 & 0.00 & 0.02 & -0.05 & -0.10 & $-\odot . \odot \odot$ & -0.02 & 0.02 \\
\hline 90 & 6 & -0.02 & 0.03 & $\odot .03$ & 0.02 & -0.05 & -0.07 & -0.01 & -0.02 & 0.03 \\
\hline 91 & 7 & -0.01 & 0.03 & 0.05 & 0.01 & -0.05 & -0.06 & -0.03 & -0.02 & 0.06 \\
\hline 92 & 6 & -0.01 & $\odot .03$ & 0.04 & 0.01 & -0.04 & -0.09 & -0.03 & -0.00 & $\odot .09$ \\
\hline 93 & 7 & -0.01 & 0.03 & 0.02 & 0.02 & -0.04 & -0.11 & -0.01 & 0.01 & 0.09 \\
\hline 94 & 6 & -0.02 & 0.03 & 0.00 & 0.02 & -0.04 & -0.12 & $-\odot . \odot \odot$ & $\odot . \odot \odot$ & 0.06 \\
\hline 95 & 7 & -0.02 & 0.03 & -0.02 & 0.03 & -0.04 & -0.16 & 0.01 & 0.01 & 0.06 \\
\hline 96 & 1 & -0.01 & $\odot .03$ & -0.01 & $\odot .02$ & -0.05 & -0.05 & 0.02 & -0.05 & -0.07 \\
\hline 97 & 1 & -0.01 & 0.03 & 0.05 & 0.01 & -0.04 & -0.08 & -0.04 & 0.00 & 0.11 \\
\hline 98 & 1 & -0.02 & 0.03 & -0.01 & 0.03 & -0.03 & -0.13 & 0.01 & 0.02 & 0.08 \\
\hline 99 & 1 & -0.02 & 0.03 & -0.02 & 0.04 & -0.04 & -0.13 & 0.02 & 0.01 & 0.03 \\
\hline 100 & 6 & 0.00 & -0.03 & 0.01 & 0.04 & 0.00 & 0.02 & 0.01 & -0.01 & $\odot .08$ \\
\hline 101 & 11 & -0.01 & 0.02 & 0.01 & 0.01 & 0.05 & -0.01 & 0.03 & 0.07 & -0.13 \\
\hline
\end{tabular}




$\begin{array}{rrrrr}102 & 11 & -0.03 & 0.04 & 0.03 \\ 103 & 6 & 0.01 & -0.12 & 0.01 \\ 104 & 6 & -0.02 & -0.14 & 0.03 \\ 105 & 8 & -0.05 & -0.14 & 0.01 \\ 106 & 1 & -0.02 & -0.02 & 0.01 \\ 107 & 1 & 0.05 & -0.0 \odot & -0.00 \\ 108 & 1 & 0.06 & -0.13 & 0.00 \\ 109 & 1 & 0.03 & -0.10 & -0.00 \\ 110 & 1 & 0.03 & -0.13 & 0.02 \\ 111 & 1 & -0.00 & -0.10 & -0.01 \\ 112 & 1 & -0.02 & -0.14 & 0.06 \\ 113 & 7 & -0.01 & -0.14 & 0.02 \\ 114 & 6 & 0.02 & -0.14 & -0.03 \\ 115 & 7 & 0.06 & -0.11 & -0.10 \\ 116 & 6 & 0.04 & -0.10 & -0.07 \\ 117 & 6 & -0.00 & -0.11 & -0.00 \\ 118 & 7 & -0.02 & -0.10 & 0.03 \\ 119 & 6 & -0.0 \odot & -0.08 & 0.00 \\ 120 & 7 & 0.03 & -0.07 & -0.04 \\ 121 & 6 & 0.05 & -0.08 & -0.08 \\ 122 & 7 & -0.02 & -0.06 & 0.01 \\ 123 & 8 & 0.07 & -0.07 & -0.12 \\ 124 & 1 & 0.03 & -0.14 & -0.09 \\ 125 & 1 & -0.05 & -0.06 & 0.03 \\ 126 & 1 & -0.01 & -0.03 & 0.00 \\ 127 & 1 & 0.04 & -0.05 & -0.05 \\ 128 & 8 & -0.02 & 0.28 & -0.02 \\ 129 & 1 & -0.0 \odot & 0.29 & -0.02 \\ 130 & 8 & 0.07 & 0.20 & -0.13 \\ 131 & 1 & 0.04 & 0.15 & -0.11 \\ 132 & 1 & 0.07 & 0.19 & -0.13 \\ 133 & 1 & 0.03 & 0.20 & -0.02 \\ 134 & 8 & 0.05 & 0.18 & -0.07 \\ 135 & 1 & 0.04 & 0.12 & -0.05 \\ & & & & \end{array}$

$\begin{array}{ccc}-0.03 & 0.17 & 0.20 \\ -0.06 & -0.03 & -0.07 \\ -0.01 & -0.01 & -0.09 \\ 0.03 & 0.00 & -0.06 \\ 0.09 & -0.00 & 0.01 \\ 0.05 & 0.00 & 0.04 \\ 0.04 & 0.01 & -0.08 \\ -0.06 & -0.04 & -0.02 \\ -0.09 & -0.04 & -0.09 \\ -0.06 & -0.04 & -0.03 \\ -0.01 & 0.00 & -0.13 \\ -0.00 & -0.00 & -0.08 \\ 0.01 & -0.01 & -0.07 \\ 0.00 & -0.01 & -0.03 \\ -0.00 & -0.01 & -0.02 \\ -0.00 & 0.00 & -0.05 \\ -0.01 & 0.01 & -0.06 \\ -0.01 & 0.01 & -0.03 \\ -0.01 & 0.00 & 0.02 \\ -0.00 & -0.01 & 0.03 \\ -0.01 & 0.02 & -0.03 \\ -0.00 & -0.01 & 0.07 \\ 0.01 & -0.02 & -0.08 \\ -0.01 & 0.02 & -0.05 \\ -0.01 & 0.01 & -0.01 \\ -0.01 & -0.00 & 0.05 \\ -0.04 & -0.03 & -0.07 \\ -0.04 & -0.03 & -0.08 \\ -0.03 & -0.04 & -0.10 \\ -0.01 & -0.04 & -0.10 \\ -0.03 & -0.03 & -0.08 \\ -0.04 & -0.02 & -0.06 \\ -0.04 & -0.02 & -0.05 \\ -0.03 & -0.02 & -0.04\end{array}$

$\begin{array}{ccc}0.03 & 0.03 & 0.01 \\ 0.03 & 0.09 & 0.07 \\ 0.02 & 0.05 & 0.06 \\ 0.02 & 0.01 & 0.06 \\ -0.00 & -0.05 & 0.06 \\ 0.00 & -0.02 & 0.11 \\ 0.00 & -0.01 & 0.13 \\ 0.03 & 0.09 & 0.11 \\ 0.03 & 0.12 & 0.08 \\ 0.03 & 0.10 & 0.04 \\ 0.02 & 0.04 & 0.08 \\ 0.01 & 0.05 & 0.01 \\ 0.00 & 0.06 & -0.02 \\ -0.00 & 0.06 & -0.05 \\ 0.00 & 0.04 & -0.05 \\ 0.01 & 0.03 & -0.01 \\ 0.02 & 0.01 & 0.02 \\ 0.01 & 0.00 & 0.00 \\ -0.00 & 0.01 & -0.03 \\ -0.01 & 0.02 & -0.06 \\ 0.01 & -0.02 & 0.02 \\ -0.02 & 0.03 & -0.08 \\ 0.00 & 0.07 & -0.01 \\ 0.03 & -0.02 & 0.03 \\ 0.01 & -0.02 & 0.01 \\ -0.01 & -0.00 & -0.03 \\ 0.05 & 0.04 & -0.01 \\ 0.06 & 0.05 & -0.03 \\ 0.09 & 0.04 & -0.07 \\ 0.07 & 0.01 & -0.06 \\ 0.09 & 0.02 & -0.08 \\ 0.08 & 0.02 & -0.06 \\ 0.09 & 0.01 & -0.09 \\ 0.07 & -0.02 & -0.10 \\ & & \end{array}$

Zero-point correction=

Thermal correction to Energy=

Thermal correction to Enthalpy=

Thermal correction to Gibbs Free Energy=

Sum of electronic and zero-point Energies=

Sum of electronic and thermal Energies=

Sum of electronic and thermal Enthalpies=

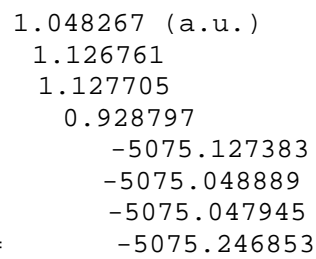

\begin{tabular}{|c|c|c|c|c|c|}
\hline \multirow{2}{*}{$\begin{array}{l}\text { Center } \\
\text { Number }\end{array}$} & \multirow{2}{*}{$\begin{array}{l}\text { Atomic } \\
\text { Number }\end{array}$} & \multirow{2}{*}{$\begin{array}{c}\text { Atomic } \\
\text { Type }\end{array}$} & \multicolumn{3}{|c|}{ Coordinates (Angstroms) } \\
\hline & & & $x$ & $Y$ & Z \\
\hline & & - & & & \\
\hline 1 & 7 & $\odot$ & 5.889142 & -1.895749 & -1.398369 \\
\hline 2 & 6 & $\odot$ & 6.235515 & -3.214476 & -1.353702 \\
\hline 3 & 6 & $\odot$ & 5.289542 & -4.179687 & -1.371482 \\
\hline 4 & 6 & $\odot$ & 3.921155 & -3.752758 & -1.418445 \\
\hline 5 & 7 & $\odot$ & 3.594051 & -2.457236 & -1.485544 \\
\hline 6 & 8 & $\odot$ & 8.961561 & 1.166529 & -0.590911 \\
\hline 7 & 15 & $\odot$ & 8.201790 & 2.219831 & 0.404241 \\
\hline
\end{tabular}




\begin{tabular}{|c|c|c|c|c|c|}
\hline 8 & 8 & $\odot$ & 6.621169 & 2.008980 & $\odot .001007$ \\
\hline 9 & 8 & 0 & 8.646630 & 3.597612 & 0.006483 \\
\hline 10 & 8 & $\odot$ & 8.311073 & 1.734032 & 1.833971 \\
\hline 11 & 8 & $\theta$ & 4.395499 & 5.521969 & $\odot .896775$ \\
\hline 12 & 6 & 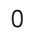 & 4.831967 & 3.607616 & -0.453550 \\
\hline 13 & 6 & $\theta$ & 5.180283 & 4.302580 & $\odot .867185$ \\
\hline 14 & 6 & 0 & -9.293516 & -1.863806 & 2.100821 \\
\hline 15 & 6 & 0 & -8.686942 & -0.477087 & 2.076126 \\
\hline 16 & 8 & $\Theta$ & -8.415290 & -2.804735 & 1.419894 \\
\hline 17 & 8 & $\Theta$ & -8.560211 & 1.458649 & 0.634170 \\
\hline 18 & 15 & 0 & -8.646036 & 2.229511 & -0.807732 \\
\hline 19 & 8 & 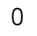 & -7.092838 & 2.222312 & -1.314492 \\
\hline 20 & 8 & 0 & -9.120579 & 3.634707 & -0.533888 \\
\hline 21 & 8 & $\Theta$ & -9.428407 & $1.4 \odot 4865$ & -1.803849 \\
\hline 22 & 8 & $\Theta$ & -3.835085 & 3.585104 & -3.449732 \\
\hline 23 & 6 & 0 & -4.755167 & 2.845936 & -1.363661 \\
\hline 24 & 6 & $\Theta$ & -4.785643 & 2.638503 & -2.896682 \\
\hline 25 & 1 & 0 & -3.859373 & 3.558490 & -4.421314 \\
\hline 26 & 6 & 0 & 10.049517 & -2.184515 & -1.805125 \\
\hline 27 & 6 & $\Theta$ & 9.268517 & $-\odot .886908$ & -1.785171 \\
\hline 28 & 6 & $\odot$ & 9.034010 & -0.282934 & -0.392962 \\
\hline 29 & 6 & $\odot$ & 7.690600 & -0.875122 & 0.013427 \\
\hline 30 & 6 & $\Theta$ & 6.922396 & -0.874397 & -1.306524 \\
\hline 31 & 8 & $\Theta$ & 7.925166 & -1.108826 & -2.335710 \\
\hline 32 & 1 & $\theta$ & 10.045005 & -2.582418 & -2.825150 \\
\hline 33 & 1 & $\Theta$ & 11.085791 & -1.984468 & -1.509261 \\
\hline 34 & 1 & 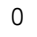 & 9.774305 & -0.159216 & -2.423570 \\
\hline 35 & 1 & $\Theta$ & 9.835637 & -0.502661 & 0.313488 \\
\hline 36 & 1 & $\odot$ & 7.193222 & $-\odot .295653$ & 0.788145 \\
\hline 37 & 1 & 0 & 7.857994 & -1.895584 & $\odot .364968$ \\
\hline 38 & 1 & $\Theta$ & 6.431726 & 0.077974 & -1.480616 \\
\hline 39 & 8 & $\Theta$ & 9.432104 & -3.123123 & -0.889784 \\
\hline 40 & 1 & 0 & 9.900185 & -3.973740 & -0.896612 \\
\hline 41 & 6 & $\Theta$ & 4.549254 & -1.498176 & -1.510456 \\
\hline 42 & 7 & $\odot$ & 2.926060 & -4.636734 & -1.390655 \\
\hline 43 & 8 & 0 & 4.277637 & -0.294692 & -1.609266 \\
\hline 44 & 1 & $\Theta$ & 7.305109 & -3.410472 & -1.300477 \\
\hline 45 & 1 & $\Theta$ & 5.554680 & -5.227424 & -1.337994 \\
\hline 46 & 1 & $\theta$ & 3.121997 & -5.621754 & -1.329006 \\
\hline 47 & 1 & $\Theta$ & 1.954780 & -4.310474 & -1.421651 \\
\hline 48 & 6 & $\Theta$ & 5.961007 & 2.785139 & -1.041663 \\
\hline 49 & 1 & 0 & 4.553522 & 6.006901 & 1.724550 \\
\hline 50 & 6 & $\Theta$ & 4.703874 & 3.307359 & 1.930993 \\
\hline 51 & 6 & $\Theta$ & 3.445994 & 2.717407 & 1.290082 \\
\hline 52 & 8 & $\theta$ & 3.686683 & 2.745545 & -0.147763 \\
\hline 53 & 1 & $\Theta$ & 5.553824 & 2.080219 & -1.768172 \\
\hline 54 & 1 & 0 & 6.688263 & 3.446352 & -1.518948 \\
\hline 55 & 1 & $\Theta$ & 4.502076 & 4.346250 & -1.188203 \\
\hline 56 & 1 & $\Theta$ & 6.249169 & 4.523661 & 0.941369 \\
\hline 57 & 1 & 0 & 4.483958 & 3.788974 & 2.885900 \\
\hline 58 & 1 & 0 & 5.475892 & 2.551323 & 2.082045 \\
\hline 59 & 1 & $\Theta$ & 2.569825 & 3.324264 & 1.507988 \\
\hline 60 & 7 & 0 & 3.098047 & 1.357649 & 1.668903 \\
\hline 61 & 6 & $\Theta$ & 4.024643 & 0.337057 & 1.591460 \\
\hline 62 & 6 & $\Theta$ & 3.714478 & $-\odot .961596$ & 1.793125 \\
\hline 63 & 6 & 0 & 2.312885 & -1.304597 & 1.978582 \\
\hline 64 & 7 & $\Theta$ & 1.446183 & -0.236042 & 2.040465 \\
\hline 65 & 6 & $\Theta$ & 1.749197 & 1.087705 & 1.874756 \\
\hline 66 & 6 & $\theta$ & 4.718760 & -2.071816 & 1.829032 \\
\hline 67 & 8 & $\odot$ & 1.885143 & -2.459041 & 2.068596 \\
\hline 68 & 8 & $\theta$ & $\odot .890992$ & 1.960718 & 1.909618 \\
\hline 69 & 1 & 0 & 5.031912 & 0.656793 & 1.343718 \\
\hline 70 & 1 & $\Theta$ & 4.728332 & -2.544004 & 2.816322 \\
\hline 71 & 1 & $\Theta$ & 4.473097 & -2.852242 & 1.105377 \\
\hline 72 & 1 & 0 & 5.723054 & -1.704484 & 1.620923 \\
\hline 73 & 1 & 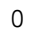 & $\odot .423869$ & -0.452986 & 2.111197 \\
\hline 74 & 1 & $\Theta$ & -8.650941 & -3.719981 & 1.646267 \\
\hline 75 & 6 & 0 & -8.271685 & 0.024289 & 0.687239 \\
\hline 76 & 6 & $\Theta$ & -6.774254 & -0.230767 & 0.633774 \\
\hline 77 & 6 & $\Theta$ & -6.317918 & -0.123345 & 2.090160 \\
\hline 78 & 8 & 0 & -7.470939 & -0.452435 & 2.896862 \\
\hline 79 & 1 & 0 & -9.426659 & -2.174959 & 3.139941 \\
\hline 80 & 1 & 0 & -10.269555 & -1.841407 & 1.605391 \\
\hline 81 & 1 & 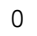 & -9.401343 & $\odot .220267$ & 2.519630 \\
\hline 82 & 1 & $\theta$ & -8.817894 & -0.473106 & -0.117120 \\
\hline 83 & 1 & 0 & -6.231943 & $\odot .441727$ & -0.028790 \\
\hline 84 & 1 & $\Theta$ & -6.588092 & -1.246244 & 0.292273 \\
\hline
\end{tabular}




\begin{tabular}{|c|c|c|c|c|c|}
\hline 85 & 1 & 0 & -5.949658 & $\odot .869330$ & 2.355889 \\
\hline 86 & 7 & 0 & -5.246306 & -1.064608 & 2.373691 \\
\hline 87 & 6 & 0 & -5.342081 & -2.429205 & 2.328547 \\
\hline 88 & 7 & $\odot$ & -4.178118 & -3.031368 & 2.283174 \\
\hline 89 & 6 & $\odot$ & -3.261079 & -1.995817 & 2.265301 \\
\hline 90 & 6 & 0 & -3.909160 & $-\odot .766781$ & 2.326125 \\
\hline 91 & 7 & $\odot$ & -3.350008 & 0.450615 & 2.329288 \\
\hline 92 & 6 & 0 & -2.032433 & 0.371478 & 2.236069 \\
\hline 93 & 7 & 0 & -1.280119 & -0.735509 & 2.149653 \\
\hline 94 & 6 & 0 & -1.854622 & -1.954071 & 2.158184 \\
\hline 95 & 7 & 0 & -1.068174 & -3.031664 & 2.056153 \\
\hline 96 & 1 & 0 & -6.313119 & -2.906271 & 2.304174 \\
\hline 97 & 1 & 0 & -1.468012 & 1.300489 & 2.221785 \\
\hline 98 & 1 & 0 & -0.055796 & -2.914784 & 2.025829 \\
\hline 99 & 1 & 0 & -1.470907 & -3.953215 & 2.069760 \\
\hline 100 & 6 & 0 & -6.097071 & 3.107519 & -0.724387 \\
\hline 101 & 11 & 0 & 8.085726 & 2.797206 & 3.772292 \\
\hline 102 & 11 & 0 & -10.963479 & 3.239108 & -2.299678 \\
\hline 103 & 6 & 0 & -4.312062 & 1.190154 & -3.073975 \\
\hline 104 & 6 & $\odot$ & -3.362892 & 1.042350 & -1.884255 \\
\hline 105 & 8 & 0 & -4.160407 & 1.633101 & -0.798814 \\
\hline 106 & 1 & 0 & -6.036116 & 2.931089 & 0.354289 \\
\hline 107 & 1 & $\odot$ & -6.398770 & 4.143475 & -0.901026 \\
\hline 108 & 1 & 0 & -4.092875 & 3.693504 & -1.153341 \\
\hline 109 & 1 & $\odot$ & -5.780819 & 2.805125 & -3.313432 \\
\hline 110 & 1 & $\odot$ & -3.829766 & 1.020158 & -4.038443 \\
\hline 111 & 1 & 0 & -5.162236 & 0.510721 & -2.963220 \\
\hline 112 & 1 & 0 & -2.480237 & 1.673777 & -2.033783 \\
\hline 113 & 7 & 0 & -2.891128 & $-\odot .265890$ & -1.555709 \\
\hline 114 & 6 & 0 & -3.557375 & -1.548982 & -1.887641 \\
\hline 115 & 7 & 0 & -2.529713 & -2.564167 & -1.853490 \\
\hline 116 & 6 & $\odot$ & -1.396555 & -1.925861 & -1.552902 \\
\hline 117 & 6 & $\odot$ & -1.562484 & $-0.5276 \odot 2$ & -1.397828 \\
\hline 118 & 7 & 0 & -0.608591 & 0.378197 & -1.209389 \\
\hline 119 & 6 & 0 & 0.629934 & -0.130881 & -1.193351 \\
\hline 120 & 7 & 0 & 0.893503 & -1.469516 & -1.264631 \\
\hline 121 & 6 & 0 & -0.071868 & -2.460775 & -1.441073 \\
\hline 122 & 7 & 0 & 1.679063 & 0.685290 & -1.097869 \\
\hline 123 & 8 & 0 & $\odot .250562$ & -3.653299 & -1.513485 \\
\hline 124 & 1 & 0 & -3.981270 & -1.462482 & -2.898691 \\
\hline 125 & 1 & 0 & 1.518259 & 1.676989 & -1.026977 \\
\hline 126 & 1 & 0 & 2.643490 & 0.352441 & -1.209857 \\
\hline 127 & 1 & 0 & 1.880607 & -1.796806 & -1.291804 \\
\hline 128 & 8 & 0 & -4.583939 & -1.859385 & -0.989372 \\
\hline 129 & 1 & $\odot$ & -4.411039 & -2.777525 & -0.672826 \\
\hline 130 & 8 & 0 & -4.691188 & -4.484404 & -0.149467 \\
\hline 131 & 1 & 0 & -4.495806 & -4.300322 & 0.783586 \\
\hline 132 & 1 & $\odot$ & -5.645909 & -4.309113 & -0.261468 \\
\hline 133 & 1 & 0 & -6.535100 & -2.651873 & -1.237218 \\
\hline 134 & 8 & 0 & -7.101375 & -3.374334 & -0.931531 \\
\hline 135 & 1 & $\odot$ & -7.628626 & -3.014642 & -0.193334 \\
\hline
\end{tabular}

General basis read from cards: (6D, 7F)

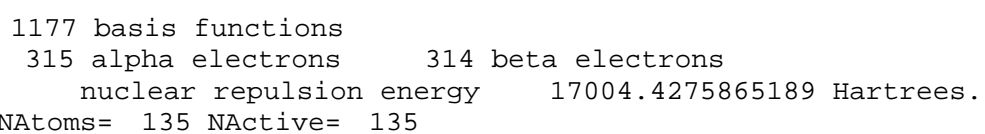

NAtoms $=135$ NActive $=135$

Force inversion solution in PCM.

Polarizable Continuum Model (PCM)

==ニ=ニ=ニ=ニ=ニ=ニ=ニ=ニ=ニ=ニ=ニ=ニ=ニ=ニ=

Model : PCM.

Solvent : Water, Eps $=78.355300$ Eps $($ inf $)=1.777849$

SCF Done: E(UWB97XD) = -5076.22929106 A.U. after 1 cycles

SCF Done: $E(U W B 97 X D)=-5076.22929106$ A.U. after 1 cycles
NFock $=1$ Conv $=0.35 \mathrm{D}-08 \quad-\mathrm{V} / \mathrm{T}=2.0066$

$\langle\mathrm{S} x\rangle=0.0000<\mathrm{S} y>=0.0000<\mathrm{Sz}>=0.5000$

$<S^{* *} 2>=0.7620 \mathrm{~S}=0.5060$

$<$ L.S $>=0.0000000000000 \mathrm{E}+00$

Annihilation of the first spin contaminant:

$\mathrm{S}^{* * 2}$ before annihilation 0.7620 , after 0.7501 
Harmonic frequencies $\left(\mathrm{cm}^{*}-1\right)$, IR intensities (KM/Mole)

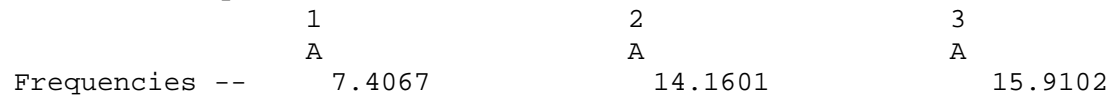

Zero-point correction=

Thermal correction to Energy=

Thermal correction to Enthalpy=

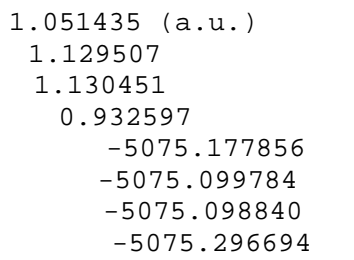

Thermal correction to Gibbs Free Energy=
Sum of electronic and zero-point Energies

Sum of electronic and thermal Energies=

Sum of electronic and thermal Enthalpies=

Sum of electronic and thermal Free Energies=

$$
\mathrm{S}
$$

$$
\begin{gathered}
\text { E (Thermal) } \\
\text { KCal/Mol }
\end{gathered}
$$

$\mathrm{CV}$

Cal/Mol-Kelvin

Cal/Mol-Kelvin

$\begin{array}{ll}295.068 & 416.420\end{array}$

Normal termination of Gaussian 16 at Wed Aug 14 19:58:34 2019.

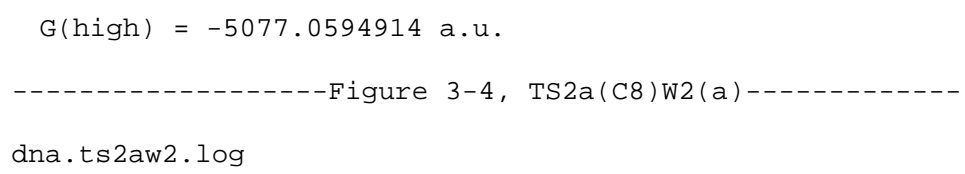

\begin{tabular}{|c|c|c|c|c|c|}
\hline \multirow{2}{*}{$\begin{array}{l}\text { Center } \\
\text { Number }\end{array}$} & \multirow{2}{*}{$\begin{array}{l}\text { Atomic } \\
\text { Number }\end{array}$} & \multirow{2}{*}{$\begin{array}{l}\text { Atomic } \\
\text { Type }\end{array}$} & \multicolumn{3}{|c|}{ Coordinates (Angstroms) } \\
\hline & & & $x$ & $\mathrm{Y}$ & Z \\
\hline$-1-5$ - & -1. & & - - - - & 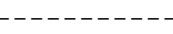 & -------6 \\
\hline 1 & 7 & $\odot$ & 5.576525 & -2.051402 & -1.402517 \\
\hline 2 & 6 & $\odot$ & 5.833792 & -3.391146 & -1.382767 \\
\hline 3 & 6 & $\odot$ & 4.825202 & -4.288532 & -1.307996 \\
\hline 4 & 6 & $\odot$ & 3.490049 & -3.767181 & -1.238331 \\
\hline 5 & 7 & $\odot$ & 3.249523 & -2.452975 & -1.279414 \\
\hline 6 & 8 & $\odot$ & 8.885995 & 0.815957 & -0.824073 \\
\hline 7 & 15 & $\odot$ & 8.236592 & 1.871446 & ๑. 237995 \\
\hline 8 & 8 & $\odot$ & 6.633825 & 1.770546 & -0.080320 \\
\hline 9 & 8 & $\odot$ & 8.749087 & 3.246594 & -0.115024 \\
\hline 10 & 8 & $\odot$ & 8.417611 & 1.399000 & 1.664194 \\
\hline 11 & 8 & $\odot$ & 4.713169 & 5.419107 & 1.005545 \\
\hline 12 & 6 & $\odot$ & 4.926201 & 3.490451 & -0.377893 \\
\hline 13 & 6 & $\odot$ & 5.417334 & 4.154575 & ๑. 912901 \\
\hline 14 & 6 & $\odot$ & -8.975041 & -2.331330 & 1.799002 \\
\hline 15 & 6 & $\odot$ & -8.528535 & -0.895840 & 1.968493 \\
\hline 16 & 8 & $\odot$ & -7.984616 & -3.114678 & 1.068178 \\
\hline 17 & 8 & $\odot$ & -8.551619 & 1.208939 & 0.795745 \\
\hline 18 & 15 & $\odot$ & -8.858827 & 2.113885 & -0.535755 \\
\hline 19 & 8 & $\odot$ & -7.330829 & 2.393628 & -1.110924 \\
\hline 20 & 8 & $\odot$ & -9.469474 & 3.397067 & -0.067746 \\
\hline 21 & 8 & $\odot$ & -9.503471 & 1.241838 & -1.586092 \\
\hline 22 & 8 & $\odot$ & -4.202824 & 4.618039 & -2.656939 \\
\hline 23 & 6 & $\odot$ & -5.049762 & 3.208655 & -0.920423 \\
\hline 24 & 6 & $\odot$ & -4.798013 & 3.315622 & -2.440281 \\
\hline 25 & 1 & $\odot$ & -4.158136 & 4.814818 & -3.607556 \\
\hline 26 & 6 & $\odot$ & 9.669521 & -2.565406 & -2.194547 \\
\hline 27 & 6 & $\odot$ & 8.964656 & -1.228837 & -2.086199 \\
\hline 28 & 6 & $\odot$ & 8.891573 & -0.644931 & -0.667700 \\
\hline 29 & 6 & $\odot$ & 7.559256 & -1.170255 & -0.151232 \\
\hline 30 & 6 & $\odot$ & 6.676794 & -1.100187 & -1.395357 \\
\hline 31 & 8 & $\odot$ & 7.567533 & -1.374214 & -2.513995 \\
\hline 32 & 1 & $\odot$ & 9.551619 & -2.943070 & -3.215414 \\
\hline 33 & 1 & $\odot$ & 10.737569 & -2.427054 & -1.990674 \\
\hline 34 & 1 & $\odot$ & 9.448306 & -0.515864 & -2.757519 \\
\hline 35 & 1 & $\odot$ & 9.740758 & -0.922613 & -0.042594 \\
\hline 36 & 1 & $\odot$ & 7.165955 & -0.581776 & 0.674406 \\
\hline 37 & 1 & $\odot$ & 7.699288 & -2.204602 & ๑. 169322 \\
\hline 38 & 1 & $\odot$ & 6.233263 & -0.116685 & -1.512180 \\
\hline 39 & 8 & $\odot$ & 9.084124 & -3.485247 & -1.240051 \\
\hline 40 & 1 & 0 & 9.502615 & -4.359355 & -1.298444 \\
\hline 41 & 6 & $\odot$ & 4.261304 & -1.561798 & -1.398766 \\
\hline 42 & 7 & $\odot$ & 2. 440141 & -4.579482 & -1.127889 \\
\hline 43 & 8 & $\odot$ & 4.065096 & -0.343617 & -1.486633 \\
\hline 44 & 1 & $\odot$ & 6.887584 & -3.660896 & -1.428475 \\
\hline
\end{tabular}

Stoichiometry C39H54N15Na2023P2(2)

Standard orientation: 


\begin{tabular}{|c|c|c|c|c|c|}
\hline 45 & 1 & $\odot$ & 5.018880 & -5.352287 & -1.295713 \\
\hline 46 & 1 & 0 & 2.568899 & -5.577219 & -1.106866 \\
\hline 47 & 1 & 0 & 1.492278 & -4.189394 & -1.129135 \\
\hline 48 & 6 & 0 & 5.953809 & 2.610695 & -1.061130 \\
\hline 49 & 1 & $\odot$ & 4.956182 & 5.882264 & 1.825298 \\
\hline 50 & 6 & 0 & 4.971424 & 3.183480 & 2.012587 \\
\hline 51 & 6 & 0 & 3.646680 & 2.641956 & 1.466927 \\
\hline 52 & 8 & $\odot$ & 3.763042 & 2.693693 & 0.013906 \\
\hline 53 & 1 & 0 & 5.451141 & 1.945864 & -1.764724 \\
\hline 54 & 1 & 0 & 6.687088 & 3.232174 & -1.580602 \\
\hline 55 & 1 & $\odot$ & 4.583190 & 4.253173 & -1.081350 \\
\hline 56 & 1 & 0 & 6.500008 & 4.311690 & 0.901846 \\
\hline 57 & 1 & 0 & 4.834041 & 3.682222 & 2.974233 \\
\hline 58 & 1 & 0 & 5.725553 & 2.403391 & 2.127225 \\
\hline 59 & 1 & 0 & 2.810461 & 3.270480 & 1.765510 \\
\hline 60 & 7 & 0 & 3.284797 & 1.286370 & 1.850937 \\
\hline 61 & 6 & $\odot$ & 4.188164 & $\odot .247024$ & 1.753655 \\
\hline 62 & 6 & 0 & 3.855993 & -1.045095 & 1.959323 \\
\hline 63 & 6 & 0 & 2.451514 & -1.360711 & 2.158404 \\
\hline 64 & 7 & 0 & 1.605334 & -0.277925 & 2.236446 \\
\hline 65 & 6 & 0 & 1.933843 & 1.042508 & 2.084198 \\
\hline 66 & 6 & $\odot$ & 4.833627 & -2.179182 & 1.975963 \\
\hline 67 & 8 & $\odot$ & 2.008363 & -2.509747 & 2.249333 \\
\hline 68 & 8 & 0 & 1.096094 & 1.931983 & 2.154628 \\
\hline 69 & 1 & 0 & 5.196860 & $\odot .544532$ & 1.487530 \\
\hline 70 & 1 & 0 & 4.768188 & -2.718318 & 2.925747 \\
\hline 71 & 1 & 0 & 4.621730 & -2.903852 & 1.185495 \\
\hline 72 & 1 & $\odot$ & 5.856588 & -1.823269 & 1.860182 \\
\hline 73 & 1 & 0 & 0.578492 & $-\odot .475306$ & 2.278406 \\
\hline 74 & 1 & 0 & -8.284399 & -4.036933 & 1.001726 \\
\hline 75 & 6 & 0 & -8.095674 & $-\odot .1754 \odot 2$ & $\odot .686092$ \\
\hline 76 & 6 & 0 & -6.575020 & $-\odot .250563$ & 0.707874 \\
\hline 77 & 6 & 0 & -6.219380 & -0.296282 & 2.199522 \\
\hline 78 & 8 & $\odot$ & -7.379071 & $-\odot .824585$ & 2.877301 \\
\hline 79 & 1 & 0 & -9.126089 & -2.773737 & 2.787507 \\
\hline 80 & 1 & 0 & -9.923536 & -2.343260 & 1.252573 \\
\hline 81 & 1 & 0 & -9.347407 & -0.343916 & 2.435551 \\
\hline 82 & 1 & 0 & -8.531442 & $-\odot .624475$ & -0.208660 \\
\hline 83 & 1 & $\odot$ & -6.081256 & 0.572680 & 0.189390 \\
\hline 84 & 1 & $\odot$ & -6.253469 & -1.183267 & 0.244886 \\
\hline 85 & 1 & 0 & -5.963207 & $\odot .683584$ & 2.606535 \\
\hline 86 & 7 & 0 & -5.096837 & -1.189888 & 2.461436 \\
\hline 87 & 6 & 0 & -5.147556 & -2.557206 & 2.529322 \\
\hline 88 & 7 & 0 & -3.967393 & -3.129212 & 2.474873 \\
\hline 89 & 6 & 0 & -3.089962 & -2.066813 & 2.364865 \\
\hline 90 & 6 & 0 & -3.770885 & $-\odot .854637$ & 2.360685 \\
\hline 91 & 7 & 0 & -3.240861 & $\odot .375627$ & 2.291716 \\
\hline 92 & 6 & $\odot$ & -1.921053 & $\odot .324324$ & 2.213904 \\
\hline 93 & 7 & 0 & -1.136842 & -0.765102 & 2.200948 \\
\hline 94 & 6 & 0 & -1.683290 & -1.994356 & 2.271837 \\
\hline 95 & 7 & 0 & -0.879354 & -3.061662 & 2.254651 \\
\hline 96 & 1 & 0 & -6.097983 & -3.066737 & 2.599602 \\
\hline 97 & 1 & 0 & -1.382452 & 1.266529 & 2.156907 \\
\hline 98 & 1 & $\odot$ & $\odot .132810$ & -2.935134 & 2.217116 \\
\hline 99 & 1 & 0 & -1.275083 & -3.985156 & 2.302933 \\
\hline 100 & 6 & 0 & -6.470381 & 3.360864 & -0.439635 \\
\hline 101 & 11 & $\odot$ & 9.270830 & 3.540177 & 2.434159 \\
\hline 102 & 11 & $\odot$ & -8.921030 & $-\odot .115104$ & -3.247094 \\
\hline 103 & 6 & 0 & -3.854620 & 2.137749 & -2.751067 \\
\hline 104 & 6 & 0 & -3.381031 & 1.641622 & -1.371101 \\
\hline 105 & 8 & 0 & -4.550934 & 1.907256 & -0.525861 \\
\hline 106 & 1 & 0 & -6.510686 & 3.189239 & 0.640161 \\
\hline 107 & 1 & 0 & -6.832377 & 4.368563 & -0.657636 \\
\hline 108 & 1 & 0 & -4.446852 & 3.995408 & -0.445373 \\
\hline 109 & 1 & 0 & -5.730210 & 3.233466 & -3.007516 \\
\hline 110 & 1 & 0 & -3.022494 & 2.437589 & -3.391279 \\
\hline 111 & 1 & 0 & -4.419646 & 1.339554 & -3.237737 \\
\hline 112 & 1 & 0 & -2.543999 & 2.248883 & -1.001704 \\
\hline 113 & 7 & 0 & -3.064001 & $\odot .255667$ & -1.303411 \\
\hline 114 & 6 & 0 & -3.747218 & -1.449206 & -2.212212 \\
\hline 115 & 7 & 0 & -2.835108 & -2.252317 & -1.650483 \\
\hline 116 & 6 & 0 & -1.701344 & -1.586417 & -1.364154 \\
\hline 117 & 6 & 0 & -1.816890 & -0.152907 & -1.185858 \\
\hline 118 & 7 & 0 & -0.744729 & $\odot .649803$ & -0.971809 \\
\hline 119 & 6 & 0 & $\odot .437557$ & $\odot .054154$ & -0.937327 \\
\hline 120 & 7 & 0 & 0.614050 & -1.303893 & -0.991851 \\
\hline 121 & 6 & 0 & -0.419436 & -2.208121 & -1.198665 \\
\hline
\end{tabular}




$\begin{array}{rrrrrr}122 & 7 & 0 & 1.552027 & 0.783824 & -0.833294 \\ 123 & 8 & 0 & -0.189514 & -3.423990 & -1.264530 \\ 124 & 1 & 0 & -3.531227 & -0.942353 & -3.153412 \\ 125 & 1 & 0 & 1.467520 & 1.785140 & -0.772246 \\ 126 & 1 & 0 & 2.484600 & 0.383907 & -0.976972 \\ 127 & 1 & 0 & 1.573902 & -1.696848 & -1.021738 \\ 128 & 8 & 0 & -5.033620 & -1.691746 & -2.067680 \\ 129 & 1 & 0 & -5.213712 & -2.359969 & -1.333441 \\ 130 & 8 & 0 & -5.660132 & -3.467160 & -0.313785 \\ 131 & 1 & 0 & -4.980446 & -3.618908 & 0.355807 \\ 132 & 1 & 0 & -6.479037 & -3.250181 & 0.179452 \\ 133 & 1 & 0 & -6.154575 & -0.262409 & -2.515573 \\ 134 & 8 & 0 & -6.688704 & 0.441124 & -2.912774 \\ 135 & 1 & 0 & -6.773089 & 1.135576 & -2.231207 \\ -\end{array}$

General basis read from cards: (6D, 7F)

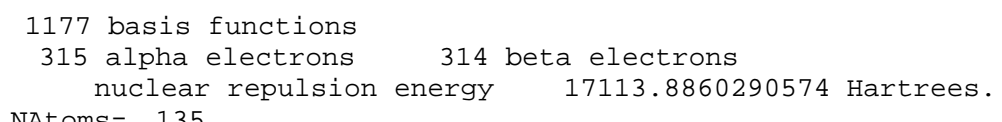

NAtoms $=135$

Force inversion solution in PCM.

Polarizable Continuum Model (PCM)

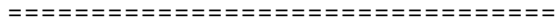

Model : PCM.

Atomic radii : UFF (Universal Force Field).

Solvent : Water, Eps $=78.355300$ Eps $($ inf $)=1.777849$

SCF Done: $E($ UWB97XD $)=-5076.21672841$ A.U. after 1 cycles NFock $=1$ Conv $=0.10 \mathrm{D}-08 \quad-\mathrm{V} / \mathrm{T}=2.0066$

$\langle\mathrm{S} x>=0.0000<\mathrm{S} y>=0.0000<\mathrm{Sz}>=0.5000$

$\left\langle S^{* *} 2>=0.7618 \quad S=0.5059\right.$

$<$ L.S $>=0.000000000000 \mathrm{E}+00$

Annihilation of the first spin contaminant:

\begin{tabular}{|c|c|c|c|c|c|c|c|c|c|c|}
\hline \multicolumn{3}{|c|}{ Harmonic frequenci } & $\begin{array}{l}\text { ies } \\
1 \\
\text { A }\end{array}$ & & $\begin{array}{l}2 \\
\mathrm{~A}\end{array}$ & & & $\begin{array}{l}3 \\
\mathrm{~A}\end{array}$ & & \\
\hline \multicolumn{3}{|c|}{ Frequencies - - } & \multicolumn{2}{|c|}{-286.3233} & \multirow{2}{*}{\multicolumn{3}{|c|}{10.3903}} & \multicolumn{3}{|c|}{14.0857} \\
\hline \multirow{2}{*}{\multicolumn{2}{|c|}{$\begin{array}{l}\text { Red. masses } \\
\text { Frc consts }\end{array}$}} & - - & \multicolumn{2}{|c|}{9.2513} & \multicolumn{2}{|c|}{8.1823} & & \multicolumn{3}{|c|}{8.0608} \\
\hline & & - - & 0.446 & & & $\odot .0005$ & & \multirow{2}{*}{\multicolumn{3}{|c|}{$\begin{array}{l}0.0009 \\
4.4871\end{array}$}} \\
\hline \multicolumn{2}{|c|}{ IR Inten } & -- & \multicolumn{2}{|c|}{131.4608} & \multicolumn{3}{|c|}{1.1395} & & & \\
\hline Atom & AN & $x$ & Y & Z & $x$ & $Y$ & Z & $x$ & Z & \\
\hline 1 & 7 & $-\odot . \odot \odot$ & $\odot . \odot \odot$ & $-\odot . \odot \odot$ & 0.03 & $\odot . \odot 3$ & -0.05 & -0.03 & 0.03 & $-\odot . \odot \odot$ \\
\hline 2 & 6 & $-\odot . \odot \odot$ & $\odot . \odot \odot$ & $-\odot . \odot \odot$ & 0.03 & $\odot .03$ & $-\odot . \odot 8$ & $-\odot . \odot 3$ & $\odot . \odot 3$ & -0.04 \\
\hline 3 & 6 & $\odot . \odot \odot$ & $\odot . \odot \odot$ & $-\odot . \odot \odot$ & $\odot .03$ & $\odot . \odot 3$ & -0.11 & $-\odot . \odot 2$ & 0.03 & -0.03 \\
\hline 4 & 6 & $-\odot . \odot \odot$ & $-0.0 \odot$ & $\odot . \odot \odot$ & 0.03 & $\odot .03$ & -0.11 & -0.02 & $\odot . \odot 2$ & 0.01 \\
\hline 5 & 7 & $-\odot . \odot \odot$ & $\odot . \odot \odot$ & $\odot . \odot \odot$ & $\odot .03$ & $\odot .03$ & $-\odot . \odot 8$ & $-\odot .02$ & $\odot .02$ & 0.05 \\
\hline 6 & 8 & $-\odot . \odot \odot$ & $\odot .0 \odot$ & $\odot .00$ & $\odot .05$ & $\odot . \odot \odot$ & 0.08 & $-\odot .01$ & $\odot .02$ & -0.07 \\
\hline 7 & 15 & $-0.0 \odot$ & $\odot .0 \odot$ & $-\odot . \odot \odot$ & 0.04 & -0.03 & 0.10 & $\odot . \odot 4$ & $\odot .0 \odot$ & -0.03 \\
\hline 8 & 8 & $-\odot . \odot \odot$ & $\odot . \odot \odot$ & $\odot . \odot \odot$ & 0.05 & $\odot . \odot \odot$ & $\odot .06$ & $\odot . \odot 3$ & $\odot .02$ & $\odot .03$ \\
\hline 9 & 8 & $-\odot . \odot \odot$ & $\odot . \odot \odot$ & $-\odot . \odot \odot$ & $\odot .06$ & -0.02 & 0.16 & 0.03 & 0.01 & $-\odot . \odot 4$ \\
\hline 10 & 8 & $-\odot . \odot \odot$ & $\odot .0 \odot$ & $-\odot . \odot \odot$ & $\odot . \odot \odot$ & -0.07 & 0.09 & 0.10 & -0.01 & -0.05 \\
\hline 11 & 8 & $-\odot . \odot \odot$ & $-\odot . \odot \odot$ & $-\odot . \odot \odot$ & $\odot .04$ & $-\odot .01$ & $\odot . \odot 6$ & 0.03 & $-\odot .03$ & 0.18 \\
\hline 12 & 6 & $-\odot . \odot \odot$ & $-\odot . \odot \odot$ & $-\odot . \odot \odot$ & $\odot .07$ & $\odot .01$ & 0.03 & $\odot .01$ & $\odot .01$ & 0.11 \\
\hline 13 & 6 & $-0.0 \odot$ & $-0.0 \odot$ & $-\odot . \odot \odot$ & 0.03 & -0.01 & $\odot .06$ & 0.03 & -0.03 & 0.13 \\
\hline 14 & 6 & $-\odot . \odot \odot$ & $-\odot . \odot \odot$ & $0.0 \odot$ & -0.03 & 0.05 & $\odot .07$ & $\odot .01$ & 0.03 & $\odot .01$ \\
\hline 15 & 6 & $-\odot .0 \odot$ & 0.00 & $\odot . \odot \odot$ & $-\odot .03$ & 0.05 & 0.05 & $\odot .01$ & 0.03 & -0.01 \\
\hline 16 & 8 & $-\odot . \odot \odot$ & $\odot .0 \odot$ & $\odot . \odot \odot$ & -0.02 & $\odot .03$ & 0.10 & $-\odot . \odot \odot$ & $\odot .02$ & 0.01 \\
\hline 17 & 8 & $\odot . \odot \odot$ & $\odot . \odot \odot$ & $\odot . \odot \odot$ & -0.01 & $\odot . \odot 3$ & $\odot .01$ & $\odot . \odot \odot$ & 0.01 & -0.04 \\
\hline 18 & 15 & $\odot . \odot \odot$ & $0.0 \odot$ & $\odot . \odot \odot$ & -0.01 & -0.01 & $-\odot .02$ & -0.01 & -0.01 & $-\odot .06$ \\
\hline 19 & 8 & $\odot . \odot \odot$ & $\odot . \odot \odot$ & $\odot .0 \odot$ & -0.01 & -0.03 & -0.03 & $-\odot . \odot 2$ & -0.01 & -0.07 \\
\hline 20 & 8 & $\odot . \odot \odot$ & $\odot . \odot \odot$ & $\odot . \odot \odot$ & $-\odot .01$ & $\odot . \odot 1$ & $-\odot .05$ & $-\odot . \odot 2$ & $-\odot . \odot 1$ & $-\odot . \odot 8$ \\
\hline 21 & 8 & $\odot . \odot \odot$ & $\odot .0 \odot$ & $\odot .0 \odot$ & $-\odot .02$ & -0.04 & $\odot . \odot \odot$ & $-\odot .02$ & $-\odot . \odot 4$ & -0.04 \\
\hline 22 & 8 & $\odot .01$ & -0.03 & $\odot .01$ & -0.04 & $-\odot .06$ & -0.10 & $-\odot .02$ & -0.04 & -0.13 \\
\hline 23 & 6 & $\odot . \odot 2$ & $-\odot .05$ & $-\odot . \odot \odot$ & -0.01 & $-\odot . \odot 2$ & $-\odot .06$ & -0.03 & $\odot .01$ & $-\odot .09$ \\
\hline 24 & 6 & $-\odot .0 \odot$ & $-\odot .02$ & $-\odot . \odot \odot$ & $-\odot .02$ & $-\odot .06$ & $-\odot . \odot 6$ & $-\odot .03$ & -0.03 & $-\odot .09$ \\
\hline 25 & 1 & $-\odot .03$ & $-0.0 \odot$ & 0.01 & -0.04 & $-\odot . \odot 8$ & -0.11 & $-\odot .01$ & -0.07 & -0.13 \\
\hline 26 & 6 & $-\odot . \odot \odot$ & 0.00 & -0.00 & 0.03 & 0.05 & -0.04 & -0.06 & 0.03 & -0.14 \\
\hline 27 & 6 & $-\odot . \odot \odot$ & $\odot .0 \odot$ & $\odot . \odot \odot$ & $\odot .04$ & $\odot .05$ & 0.00 & -0.05 & $\odot .03$ & -0.10 \\
\hline 28 & 6 & $-\odot . \odot \odot$ & $\odot . \odot \odot$ & $\odot . \odot \odot$ & $\odot . \odot 3$ & $-\odot . \odot \odot$ & $\odot . \odot 2$ & $-\odot . \odot \odot$ & $\odot .01$ & $-\odot .09$ \\
\hline 29 & 6 & $-0.0 \odot$ & $\odot .0 \odot$ & $-\odot . \odot \odot$ & 0.02 & -0.01 & -0.00 & 0.01 & 0.01 & -0.05 \\
\hline
\end{tabular}




\begin{tabular}{|c|c|c|c|c|c|c|c|c|c|c|}
\hline 30 & 6 & $-\odot . \odot \odot$ & $\odot . \odot \odot$ & $-\odot .0 \odot$ & $\odot .03$ & 0.03 & -0.01 & -0.03 & 0.03 & -0.02 \\
\hline 31 & 8 & -0.00 & $\odot . \odot \odot$ & 0.00 & $\odot .04$ & $\odot .07$ & -0.01 & -0.07 & 0.05 & -0.06 \\
\hline 32 & 1 & -0.00 & 0.00 & 0.00 & 0.03 & 0.08 & -0.05 & -0.10 & 0.05 & -0.14 \\
\hline 33 & 1 & $-0.0 \odot$ & $0.0 \odot$ & -0.00 & $\odot .03$ & 0.03 & -0.03 & -0.05 & 0.03 & -0.18 \\
\hline 34 & 1 & $-\odot . \odot \odot$ & $\odot . \odot \odot$ & $\odot .0 \odot$ & 0.05 & 0.07 & $\odot .03$ & -0.07 & 0.04 & -0.10 \\
\hline 35 & 1 & -0.00 & $-\odot . \odot \odot$ & $\odot . \odot \odot$ & 0.02 & -0.04 & 0.02 & 0.02 & $\odot .01$ & -0.12 \\
\hline 36 & 1 & $-\odot . \odot \odot$ & $\odot . \odot \odot$ & $\odot .0 \odot$ & 0.02 & -0.03 & 0.01 & 0.04 & $-0.0 \odot$ & -0.03 \\
\hline 37 & 1 & $-\odot . \odot \odot$ & $\odot . \odot \odot$ & $-\odot . \odot \odot$ & 0.01 & -0.02 & -0.03 & 0.02 & $\odot .0 \odot$ & -0.07 \\
\hline 38 & 1 & $\odot . \odot \odot$ & $\odot .0 \odot$ & -0.00 & 0.03 & 0.04 & 0.02 & -0.03 & 0.03 & 0.01 \\
\hline 39 & 8 & $-\odot .0 \odot$ & $\odot .0 \odot$ & $0.0 \odot$ & 0.02 & $\odot .02$ & -0.07 & -0.03 & $\odot .02$ & -0.13 \\
\hline 40 & 1 & -0.00 & 0.00 & -0.00 & 0.02 & 0.02 & -0.10 & -0.03 & 0.02 & -0.16 \\
\hline 41 & 6 & -0.00 & $\odot . \odot \odot$ & $\odot .00$ & 0.03 & 0.03 & -0.06 & -0.03 & 0.03 & 0.04 \\
\hline 42 & 7 & $\odot .0 \odot$ & $-\odot . \odot \odot$ & $\odot . \odot \odot$ & 0.03 & $\odot .02$ & -0.13 & -0.02 & $\odot .02$ & $\odot .02$ \\
\hline 43 & 8 & $-\odot .01$ & $\odot .0 \odot$ & $\odot .01$ & 0.03 & 0.03 & -0.03 & -0.03 & 0.03 & 0.07 \\
\hline 44 & 1 & $-\odot . \odot \odot$ & $\odot . \odot \odot$ & $-0.0 \odot$ & $\odot .03$ & $\odot . \odot 4$ & -0.08 & -0.03 & $\odot .03$ & -0.07 \\
\hline 45 & 1 & $\odot . \odot \odot$ & $\odot .0 \odot$ & -0.00 & $\odot .03$ & 0.03 & -0.14 & -0.02 & 0.03 & -0.06 \\
\hline 46 & 1 & $\odot .01$ & $\odot .0 \odot$ & $\odot .0 \odot$ & 0.04 & $\odot .02$ & -0.13 & -0.01 & $\odot .02$ & $-0.0 \odot$ \\
\hline 47 & 1 & -0.00 & $\odot .0 \odot$ & 0.00 & 0.03 & 0.02 & -0.10 & -0.02 & 0.02 & 0.05 \\
\hline 48 & 6 & $-0.0 \odot$ & -0.00 & -0.00 & 0.09 & 0.02 & 0.05 & -0.00 & 0.03 & 0.07 \\
\hline 49 & 1 & $\odot .0 \odot$ & 0.00 & -0.00 & 0.02 & -0.03 & $\odot .08$ & 0.04 & -0.06 & 0.19 \\
\hline 50 & 6 & -0.00 & -0.00 & -0.00 & -0.02 & -0.03 & 0.03 & 0.04 & -0.06 & 0.10 \\
\hline 51 & 6 & -0.00 & $\odot .0 \odot$ & 0.00 & -0.00 & -0.01 & -0.03 & 0.03 & -0.04 & 0.11 \\
\hline 52 & 8 & $-\odot . \odot \odot$ & $-0.0 \odot$ & $\odot . \odot \odot$ & 0.05 & $\odot .01$ & -0.03 & 0.02 & $-\odot . \odot \odot$ & 0.11 \\
\hline 53 & 1 & -0.00 & -0.00 & $-0.0 \odot$ & 0.11 & 0.04 & 0.01 & -0.02 & 0.05 & 0.06 \\
\hline 54 & 1 & $-\odot .0 \odot$ & $\odot .0 \odot$ & 0.00 & 0.11 & 0.03 & 0.09 & -0.02 & 0.05 & 0.07 \\
\hline 55 & 1 & $-\odot .0 \odot$ & -0.00 & $-0.0 \odot$ & 0.10 & 0.03 & 0.03 & -0.00 & 0.03 & 0.14 \\
\hline 56 & 1 & $-\odot .0 \odot$ & -0.00 & $-0.0 \odot$ & 0.03 & -0.03 & 0.10 & 0.03 & -0.03 & 0.12 \\
\hline 57 & 1 & 0.00 & 0.00 & -0.00 & -0.05 & -0.05 & 0.03 & 0.06 & -0.09 & 0.12 \\
\hline 58 & 1 & -0.00 & $-\odot . \odot \odot$ & -0.00 & -0.03 & -0.04 & 0.04 & $\odot .04$ & -0.07 & 0.06 \\
\hline 59 & 1 & $\odot . \odot \odot$ & 0.00 & 0.00 & $-\odot .01$ & -0.01 & -0.05 & 0.04 & -0.04 & 0.13 \\
\hline 60 & 7 & $-\odot .0 \odot$ & -0.00 & -0.00 & -0.02 & -0.02 & -0.07 & 0.03 & -0.04 & 0.08 \\
\hline 61 & 6 & $\odot .0 \odot$ & -0.00 & $\odot .00$ & $-\odot .03$ & -0.02 & -0.07 & 0.02 & -0.05 & 0.05 \\
\hline 62 & 6 & $\odot .0 \odot$ & $-\odot . \odot \odot$ & $\odot .0 \odot$ & -0.04 & -0.02 & -0.08 & $\odot .02$ & -0.05 & 0.03 \\
\hline 63 & 6 & $\odot .0 \odot$ & $-\odot .0 \odot$ & $\odot .00$ & -0.04 & -0.01 & -0.08 & $\odot .02$ & -0.04 & 0.05 \\
\hline 64 & 7 & $-\odot . \odot \odot$ & $-0.0 \odot$ & $-\odot . \odot \odot$ & $-\odot .04$ & -0.01 & -0.09 & 0.03 & $-\odot .04$ & 0.07 \\
\hline 65 & 6 & 0.00 & $-\odot .0 \odot$ & 0.00 & -0.03 & -0.01 & -0.08 & 0.03 & -0.04 & 0.09 \\
\hline 66 & 6 & $\odot . \odot \odot$ & $-\odot . \odot \odot$ & $\odot .0 \odot$ & -0.05 & -0.03 & -0.08 & $\odot .02$ & -0.05 & -0.01 \\
\hline 67 & 8 & $\odot . \odot \odot$ & $-\odot . \odot \odot$ & $\odot .0 \odot$ & -0.05 & -0.01 & $-\odot . \odot 9$ & 0.02 & $-\odot .04$ & 0.04 \\
\hline 68 & 8 & -0.00 & -0.00 & $-0.0 \odot$ & -0.02 & -0.00 & -0.07 & $\odot .04$ & -0.04 & 0.12 \\
\hline 69 & 1 & $-\odot .0 \odot$ & $\odot .0 \odot$ & $-\odot .0 \odot$ & -0.03 & -0.02 & -0.07 & 0.02 & -0.05 & 0.03 \\
\hline 70 & 1 & $\odot .0 \odot$ & $-\odot .0 \odot$ & 0.00 & -0.05 & -0.02 & -0.08 & 0.06 & -0.04 & $-\odot .0 \odot$ \\
\hline 71 & 1 & $\odot .0 \odot$ & $\odot .00$ & $\odot .0 \odot$ & -0.05 & -0.03 & -0.08 & -0.02 & -0.05 & $\odot .01$ \\
\hline 72 & 1 & 0.00 & $-\odot . \odot \odot$ & $\odot .0 \odot$ & -0.05 & -0.03 & -0.09 & 0.01 & -0.05 & -0.06 \\
\hline 73 & 1 & $-\odot .00$ & -0.00 & 0.00 & -0.04 & -0.00 & -0.08 & 0.03 & -0.03 & 0.08 \\
\hline 74 & 1 & $\odot .0 \odot$ & 0.00 & $\odot .0 \odot$ & -0.02 & 0.03 & 0.11 & -0.01 & 0.02 & $\odot .02$ \\
\hline 75 & $\overline{6}$ & $-\odot . \odot \odot$ & $-\odot .0 \odot$ & $\odot .0 \odot$ & -0.01 & 0.02 & 0.04 & 0.01 & 0.01 & -0.02 \\
\hline 76 & 6 & $-\odot .0 \odot$ & -0.01 & $\odot .0 \odot$ & -0.01 & 0.02 & 0.06 & 0.01 & 0.01 & -0.03 \\
\hline 77 & 6 & -0.00 & $-0.0 \odot$ & 0.00 & -0.03 & 0.05 & 0.06 & 0.02 & 0.03 & -0.03 \\
\hline 78 & 8 & $-\odot .0 \odot$ & $\odot .0 \odot$ & $\odot .0 \odot$ & -0.03 & 0.07 & 0.06 & 0.02 & 0.04 & -0.02 \\
\hline 79 & 1 & $-\odot . \odot \odot$ & $0.0 \odot$ & $\odot . \odot \odot$ & -0.03 & & $\odot .08$ & & & $\odot .02$ \\
\hline 80 & 1 & $-0.0 \odot$ & 0.00 & $\odot .00$ & -0.02 & 0.04 & 0.07 & 0.00 & 0.03 & $\odot .02$ \\
\hline 81 & 1 & -0.00 & 0.00 & 0.00 & -0.03 & 0.06 & 0.03 & 0.02 & 0.04 & -0.01 \\
\hline 82 & 1 & $-\odot . \odot \odot$ & $\odot .0 \odot$ & 0.00 & -0.00 & $\odot .0 \odot$ & 0.04 & $\odot .0 \odot$ & -0.00 & -0.01 \\
\hline 83 & 1 & -0.00 & -0.01 & -0.01 & -0.01 & 0.01 & 0.04 & 0.00 & 0.01 & -0.04 \\
\hline 84 & 1 & -0.01 & -0.01 & $\odot .01$ & -0.01 & $\odot .02$ & $\odot .08$ & 0.01 & 0.01 & -0.03 \\
\hline 85 & 1 & $-0.0 \odot$ & $-0.0 \odot$ & $-0.0 \odot$ & -0.03 & 0.06 & 0.05 & 0.03 & 0.03 & -0.04 \\
\hline 86 & 7 & $-\odot . \odot \odot$ & -0.00 & $-0.0 \odot$ & -0.03 & 0.05 & $\odot .08$ & 0.01 & 0.02 & -0.03 \\
\hline 87 & 6 & $-\odot . \odot \odot$ & $-0.0 \odot$ & $-0.0 \odot$ & -0.04 & 0.05 & 0.12 & 0.00 & 0.02 & -0.01 \\
\hline 88 & 7 & $\odot .0 \odot$ & $-\odot .0 \odot$ & 0.00 & -0.05 & 0.04 & 0.10 & $-0.0 \odot$ & 0.01 & 0.01 \\
\hline 89 & 6 & $-\odot . \odot \odot$ & $-\odot . \odot \odot$ & $\odot .0 \odot$ & $-\odot .04$ & & 0.05 & 0.01 & 0.01 & 0.02 \\
\hline 90 & 6 & $-\odot . \odot \odot$ & -0.00 & $-0.0 \odot$ & -0.03 & 0.04 & $\odot .04$ & $\odot .02$ & 0.01 & -0.01 \\
\hline 91 & 7 & -0.00 & -0.00 & -0.00 & -0.03 & 0.03 & -0.01 & 0.03 & 0.01 & -0.00 \\
\hline 92 & 6 & $-\odot . \odot \odot$ & 0.00 & $\odot .00$ & -0.03 & 0.02 & -0.04 & 0.03 & $-0.0 \odot$ & 0.03 \\
\hline 93 & 7 & $\odot .0 \odot$ & $\odot .0 \odot$ & $\odot .0 \odot$ & -0.04 & $\odot .01$ & -0.04 & 0.02 & -0.01 & 0.06 \\
\hline 94 & 6 & $-\odot . \odot \odot$ & $-0.0 \odot$ & $\odot .0 \odot$ & -0.04 & 0.02 & 0.01 & 0.01 & $-0.0 \odot$ & 0.05 \\
\hline 95 & 7 & $-0.0 \odot$ & 0.00 & 0.00 & -0.05 & 0.01 & 0.01 & 0.00 & -0.01 & $\odot .08$ \\
\hline 96 & 1 & $-\odot . \odot \odot$ & $-0.0 \odot$ & $-\odot . \odot \odot$ & -0.04 & 0.06 & 0.15 & -0.00 & 0.03 & -0.02 \\
\hline 97 & 1 & $-\odot .0 \odot$ & $\odot .0 \odot$ & 0.00 & -0.02 & 0.01 & -0.08 & 0.04 & -0.01 & 0.04 \\
\hline 98 & 1 & -0.00 & -0.00 & $-0.0 \odot$ & -0.05 & $\odot .0 \odot$ & -0.03 & $\odot .0 \odot$ & -0.02 & 0.07 \\
\hline 99 & 1 & $-\odot . \odot \odot$ & $\odot .0 \odot$ & $-\odot . \odot \odot$ & $-\odot .06$ & 0.02 & 0.05 & -0.01 & -0.01 & 0.05 \\
\hline 100 & 6 & 0.02 & -0.02 & 0.01 & -0.01 & -0.01 & -0.05 & -0.03 & 0.01 & -0.10 \\
\hline 101 & 11 & $\odot . \odot \odot$ & $\odot . \odot \odot$ & $-\odot . \odot \odot$ & $\odot .02$ & -0.04 & 0.12 & $\odot .15$ & 0.04 & -0.13 \\
\hline 102 & 11 & $-0.0 \odot$ & $\odot .0 \odot$ & $\odot .0 \odot$ & -0.01 & -0.07 & 0.04 & -0.03 & -0.06 & -0.02 \\
\hline 103 & 6 & -0.06 & -0.05 & -0.06 & -0.02 & -0.06 & $-\odot .04$ & -0.03 & -0.04 & -0.06 \\
\hline 104 & 6 & -0.11 & -0.20 & -0.11 & -0.01 & -0.02 & -0.03 & -0.02 & $\odot . \odot \odot$ & -0.05 \\
\hline 105 & 8 & -0.01 & -0.08 & -0.03 & -0.01 & -0.01 & -0.03 & -0.01 & 0.03 & -0.04 \\
\hline 106 & 1 & $\odot .02$ & -0.02 & $\odot .01$ & -0.00 & 0.01 & -0.05 & -0.03 & 0.04 & -0.09 \\
\hline
\end{tabular}




$\begin{array}{rrrrr}107 & 1 & 0.05 & -0.01 & 0.01 \\ 108 & 1 & 0.05 & -0.08 & 0.01 \\ 109 & 1 & -0.02 & 0.01 & 0.02 \\ 110 & 1 & -0.02 & -0.04 & -0.01 \\ 111 & 1 & -0.09 & 0.03 & -0.17 \\ 112 & 1 & 0.00 & -0.36 & -0.11 \\ 113 & 7 & -0.07 & -0.25 & -0.13 \\ 114 & 6 & 0.08 & 0.53 & 0.19 \\ 115 & 7 & 0.05 & 0.04 & -0.11 \\ 116 & 6 & 0.04 & -0.06 & 0.03 \\ 117 & 6 & 0.03 & -0.11 & -0.01 \\ 118 & 7 & -0.07 & -0.04 & 0.03 \\ 119 & 6 & -0.02 & -0.02 & 0.02 \\ 120 & 7 & 0.01 & 0.01 & 0.01 \\ 121 & 6 & 0.02 & -0.03 & 0.02 \\ 122 & 7 & -0.05 & 0.02 & -0.01 \\ 123 & 8 & 0.05 & -0.03 & 0.01 \\ 124 & 1 & 0.00 & 0.02 & -0.12 \\ 125 & 1 & -0.06 & 0.02 & 0.01 \\ 126 & 1 & -0.04 & 0.04 & -0.00 \\ 127 & 1 & 0.02 & 0.02 & 0.00 \\ 128 & 8 & 0.06 & 0.32 & 0.15 \\ 129 & 1 & 0.14 & 0.27 & 0.09 \\ 130 & 8 & 0.02 & 0.02 & -0.01 \\ 131 & 1 & -0.01 & -0.04 & 0.00 \\ 132 & 1 & 0.01 & 0.00 & -0.02 \\ 133 & 1 & -0.03 & -0.01 & 0.01 \\ 134 & 8 & -0.01 & 0.0 \odot & -0.01 \\ 135 & 1 & -0.02 & 0.00 & -0.01 \\ & & & & \end{array}$

$\begin{array}{ccc}-0.01 & -0.02 & -0.07 \\ -0.01 & -0.01 & -0.08 \\ -0.03 & -0.08 & -0.06 \\ -0.02 & -0.07 & -0.05 \\ -0.02 & -0.07 & -0.02 \\ -0.01 & -0.01 & -0.05 \\ -0.00 & -0.02 & -0.00 \\ -0.01 & -0.05 & 0.05 \\ 0.01 & -0.03 & 0.05 \\ 0.01 & -0.01 & 0.01 \\ 0.00 & -0.01 & -0.02 \\ 0.00 & 0.00 & -0.05 \\ 0.01 & 0.01 & -0.06 \\ 0.02 & 0.01 & -0.05 \\ 0.02 & -0.00 & -0.01 \\ 0.00 & 0.02 & -0.09 \\ 0.03 & 0.00 & -0.00 \\ -0.04 & -0.07 & 0.04 \\ -0.01 & 0.02 & -0.09 \\ 0.01 & 0.03 & -0.08 \\ 0.02 & 0.02 & -0.06 \\ -0.01 & -0.05 & 0.09 \\ 0.01 & -0.03 & 0.11 \\ 0.00 & -0.00 & 0.14 \\ -0.01 & 0.01 & 0.15 \\ -0.01 & 0.01 & 0.12 \\ -0.01 & -0.07 & 0.05 \\ -0.01 & -0.08 & 0.03 \\ -0.01 & -0.06 & 0.01\end{array}$

$\begin{array}{ccc}-0.04 & 0.00 & -0.12 \\ -0.04 & 0.03 & -0.11 \\ -0.03 & -0.04 & -0.09 \\ -0.04 & -0.07 & -0.08 \\ -0.04 & -0.06 & -0.03 \\ -0.02 & 0.01 & -0.07 \\ -0.02 & 0.00 & -0.01 \\ -0.00 & -0.01 & 0.00 \\ -0.01 & -0.00 & 0.03 \\ -0.02 & 0.00 & 0.05 \\ -0.03 & 0.01 & 0.03 \\ -0.03 & 0.01 & 0.05 \\ -0.03 & 0.01 & 0.07 \\ -0.03 & 0.01 & 0.08 \\ -0.02 & 0.01 & 0.07 \\ -0.03 & 0.01 & 0.09 \\ -0.02 & 0.01 & 0.09 \\ 0.02 & -0.02 & -0.00 \\ -0.04 & 0.01 & 0.07 \\ -0.03 & 0.02 & 0.09 \\ -0.02 & 0.01 & 0.09 \\ -0.00 & -0.01 & -0.02 \\ -0.02 & -0.01 & -0.02 \\ -0.02 & 0.01 & -0.01 \\ -0.02 & 0.01 & -0.02 \\ -0.02 & 0.01 & -0.01 \\ -0.02 & -0.03 & -0.04 \\ -0.03 & -0.04 & -0.05 \\ -0.03 & -0.03 & -0.06\end{array}$

Zero-point correction=

Thermal correction to Energy=

$$
\begin{gathered}
1.049726(\mathrm{a} . \mathrm{u} .) \\
1.127306 \\
1.128251 \\
0.932733 \\
-5075.167002 \\
-5075.089422 \\
-5075.088478 \\
-5075.283996
\end{gathered}
$$

Sum of electronic and thermal Free Energies= $\mathrm{S}$

$$
\begin{aligned}
& \text { Thermal) } \\
& \text { al/Mol } \\
& 707.395
\end{aligned}
$$

$$
\begin{array}{cc}
\text { CV } & \text { S } \\
\text { Cal/Mol-Kelvin } & \text { Cal/Mol-Kelvin }
\end{array}
$$$$
294.205
$$

411.502

\begin{tabular}{lccc}
\multicolumn{1}{c}{ Item } & Value & Threshold Converged? \\
Maximum Force & 0.000007 & 0.000450 & YES \\
RMS Force & 0.000001 & 0.000300 & YES \\
Maximum Displacement & 0.001315 & $0.00180 \odot$ & YES \\
RMS Displacement & 0.000225 & 0.001200 & YES
\end{tabular}

Predicted change in Energy $=-7.178188 \mathrm{D}-09$

optimization completed.

Normal termination of Gaussian 16 at Fri Jul 12 22:58:45 2019.

$\mathrm{G}(\mathrm{high})=-5077.040741 \mathrm{a} \cdot \mathrm{u}$.

3-5, Int3a(C8)W2(a)

dna. for $3 a w 2 x \cdot \log$

Stoichiometry C39H54N15Na2023P2(2)

Standard orientation:

\begin{tabular}{rccccc} 
Center & Atomic & Atomic & \multicolumn{3}{c}{ Coordinates } \\
Number & Number & Type & $X$ & $Y$ & (Angstroms ) \\
1 & 7 & 0 & 5.497952 & -1.922694 & -1.579924 \\
2 & 6 & 0 & 5.781036 & -3.256671 & -1.613276 \\
3 & 6 & 0 & 4.789779 & -4.175628 & -1.573282 \\
4 & 6 & 0 & 3.445622 & -3.683536 & -1.477382 \\
5 & 7 & 0 & 3.178516 & -2.373941 & -1.472444 \\
6 & 8 & 0 & 8.728801 & 1.001218 & -0.921443 \\
7 & 15 & 0 & 8.073702 & 2.001925 & 0.188899 \\
8 & 8 & 0 & 6.467202 & 1.877019 & -0.105871 \\
9 & 8 & 0 & 8.547448 & 3.400586 & -0.125941 \\
10 & 8 & 0 & 8.293776 & 1.486869 & 1.593916 \\
11 & 8 & 0 & 4.522828 & 5.477035 & 1.105951
\end{tabular}




\begin{tabular}{|c|c|c|c|c|c|}
\hline 12 & 6 & $\odot$ & 4.730761 & 3.584496 & -0.328060 \\
\hline 13 & 6 & 0 & 5.234973 & 4.220395 & $\odot .971965$ \\
\hline 14 & 6 & $\odot$ & -9.076093 & -1.959183 & 1.745020 \\
\hline 15 & 6 & 0 & -8.567488 & -0.534511 & 1.652082 \\
\hline 16 & 8 & 0 & -8.230210 & -2.820121 & 0.942566 \\
\hline 17 & 8 & 0 & -8.609405 & 1.336383 & 0.138137 \\
\hline 18 & 15 & 0 & -8.658254 & 2.117345 & -1.295689 \\
\hline 19 & 8 & 0 & -7.100994 & 2.547363 & -1.528512 \\
\hline 20 & 8 & $\odot$ & -9.524092 & 3.342056 & -1.103766 \\
\hline 21 & 8 & 0 & -9.030391 & 1.172810 & -2.414904 \\
\hline 22 & 8 & 0 & -4.215260 & 4.626867 & -2.589960 \\
\hline 23 & 6 & 0 & -4.968649 & 3.353051 & -0.693415 \\
\hline 24 & 6 & 0 & -4.323005 & 3.284803 & -2.074007 \\
\hline 25 & 1 & 0 & -3.987763 & 4.611955 & -3.533748 \\
\hline 26 & 6 & $\odot$ & 9.599646 & -2.308869 & -2.411811 \\
\hline 27 & 6 & $\odot$ & 8.862457 & -0.995210 & -2.251514 \\
\hline 28 & 6 & 0 & 8.783358 & -0.462954 & -0.813448 \\
\hline 29 & 6 & $\odot$ & 7.470995 & -1.044492 & -0.306665 \\
\hline 30 & 6 & 0 & 6.577899 & -0.948612 & -1.541794 \\
\hline 31 & 8 & $\odot$ & 7.466276 & -1.160876 & -2.675306 \\
\hline 32 & 1 & $\odot$ & 9.487146 & -2.650154 & -3.446030 \\
\hline 33 & 1 & 0 & 10.664766 & -2.151647 & -2.206311 \\
\hline 34 & 1 & 0 & 9.323252 & -0.247122 & -2.900319 \\
\hline 35 & 1 & $\odot$ & 9.645449 & -0.734461 & -0.203497 \\
\hline 36 & 1 & 0 & 7.071487 & -0.498325 & 0.545106 \\
\hline 37 & 1 & 0 & 7.641511 & -2.086606 & -0.028609 \\
\hline 38 & 1 & $\odot$ & 6.112197 & 0.028751 & -1.618609 \\
\hline 39 & 8 & 0 & 9.041345 & -3.278834 & -1.491371 \\
\hline 40 & 1 & 0 & 9.479151 & -4.140101 & -1.587443 \\
\hline 41 & 6 & $\odot$ & 4.174114 & -1.460048 & -1.556285 \\
\hline 42 & 7 & $\odot$ & 2.412279 & -4.519901 & -1.386230 \\
\hline 43 & 8 & 0 & 3.954893 & -0.243529 & -1.594341 \\
\hline 44 & 1 & 0 & 6.839715 & -3.503816 & -1.671046 \\
\hline 45 & 1 & 0 & 5.004247 & -5.235050 & -1.601036 \\
\hline 46 & 1 & 0 & 2.558923 & -5.515234 & -1.373227 \\
\hline 47 & 1 & $\odot$ & 1.463234 & -4.146796 & -1.314225 \\
\hline 48 & 6 & $\odot$ & 5.755914 & 2.733606 & -1.051141 \\
\hline 49 & 1 & 0 & 4.771825 & 5.919774 & 1.935221 \\
\hline 50 & 6 & 0 & 4.809151 & 3.219680 & 2.051886 \\
\hline 51 & 6 & $\odot$ & 3.476820 & 2.689310 & 1.513430 \\
\hline 52 & 8 & 0 & 3.580607 & 2.765687 & $\odot .060344$ \\
\hline 53 & 1 & $\odot$ & 5.248266 & 2.078977 & -1.760622 \\
\hline 54 & 1 & 0 & 6.470779 & 3.377090 & -1.569280 \\
\hline 55 & 1 & 0 & 4.369012 & 4.361460 & -1.006037 \\
\hline 56 & 1 & 0 & 6.316090 & 4.386416 & 0.949727 \\
\hline 57 & 1 & 0 & 4.688194 & 3.690462 & 3.029632 \\
\hline 58 & 1 & 0 & 5.562682 & 2.433931 & 2.131480 \\
\hline 59 & 1 & 0 & 2.642694 & 3.309595 & 1.834545 \\
\hline 60 & 7 & 0 & 3.129813 & 1.325504 & 1.878823 \\
\hline 61 & 6 & $\odot$ & 4.019146 & $\odot .292076$ & 1.663967 \\
\hline 62 & 6 & 0 & 3.704327 & -1.004608 & 1.865281 \\
\hline 63 & 6 & 0 & 2.328676 & -1.325664 & 2.207262 \\
\hline 64 & 7 & $\odot$ & 1.492330 & -0.246286 & 2.393450 \\
\hline 65 & 6 & $\odot$ & 1.805374 & 1.077457 & 2.229486 \\
\hline 66 & 6 & $\odot$ & 4.674288 & -2.139821 & 1.755263 \\
\hline 67 & 8 & $\odot$ & 1.901244 & -2.477252 & 2.324660 \\
\hline 68 & 8 & 0 & $\odot .979310$ & 1.965728 & 2.392107 \\
\hline 69 & 1 & 0 & 4.996395 & 0.601646 & 1.306191 \\
\hline 70 & 1 & 0 & 4.735566 & -2.677989 & 2.706038 \\
\hline 71 & 1 & 0 & 4.357989 & -2.864288 & 1.000861 \\
\hline 72 & 1 & 0 & 5.672624 & -1.785199 & 1.504130 \\
\hline 73 & 1 & $\odot$ & 0.483585 & -0.449445 & 2.583867 \\
\hline 74 & 1 & $\odot$ & -8.551457 & -3.736264 & $\odot .961390$ \\
\hline 75 & 6 & $\odot$ & -8.240392 & $-\odot .080822$ & 0.226338 \\
\hline 76 & 6 & 0 & -6.735053 & -0.290076 & 0.097466 \\
\hline 77 & 6 & $\odot$ & -6.198797 & -0.140962 & 1.527035 \\
\hline 78 & 8 & 0 & -7.328539 & -0.373862 & 2.414664 \\
\hline 79 & 1 & $\odot$ & -9.067175 & -2.273571 & 2.793857 \\
\hline 80 & 1 & $\odot$ & -10.107665 & -1.991137 & 1.375126 \\
\hline 81 & 1 & $\odot$ & -9.309797 & 0.132680 & 2.096266 \\
\hline 82 & 1 & 0 & -8.804844 & -0.629991 & -0.528424 \\
\hline 83 & 1 & 0 & -6.253154 & 0.432513 & -0.560354 \\
\hline 84 & 1 & 0 & -6.550432 & -1.299758 & -0.273349 \\
\hline 85 & 1 & 0 & -5.790182 & 0.854123 & 1.703591 \\
\hline 86 & 7 & $\odot$ & -5.142370 & -1.084311 & 1.839223 \\
\hline 87 & 6 & $\odot$ & -5.194891 & -2.450427 & 1.685815 \\
\hline 88 & 7 & $\odot$ & -4.049899 & -3.044354 & 1.900553 \\
\hline
\end{tabular}




\begin{tabular}{|c|c|c|c|c|c|}
\hline 89 & 6 & 0 & -3.188791 & -2.007674 & 2.207187 \\
\hline 90 & 6 & 0 & -3.853496 & $-\odot .785806$ & 2.197626 \\
\hline 91 & 7 & 0 & -3.327165 & 0.415396 & 2.483142 \\
\hline 92 & 6 & 0 & -2.028498 & 0.330767 & 2.719646 \\
\hline 93 & 7 & 0 & -1.249296 & $-\odot .763149$ & 2.693266 \\
\hline 94 & 6 & 0 & -1.796345 & -1.965867 & 2.419968 \\
\hline 95 & 7 & $\odot$ & -1.005239 & -3.050835 & 2.365524 \\
\hline 96 & 1 & 0 & -6.131350 & -2.924916 & 1.415228 \\
\hline 97 & 1 & $\odot$ & -1.500785 & 1.255126 & 2.941758 \\
\hline 98 & 1 & 0 & 0.002732 & -2.894821 & 2.370219 \\
\hline 99 & 1 & $\theta$ & -1.363591 & -3.863080 & 1.867618 \\
\hline 100 & 6 & $\odot$ & -6.465138 & 3.514347 & -0.642606 \\
\hline 101 & 11 & $\Theta$ & 9.135028 & 3.662596 & 2.369413 \\
\hline 102 & 11 & 0 & -10.933710 & 2.697443 & -3.040121 \\
\hline 103 & 6 & 0 & -2.976242 & 2.603672 & -1.783514 \\
\hline 104 & 6 & $\odot$ & -3.218343 & 1.773543 & -0.489823 \\
\hline 105 & 8 & 0 & -4.591140 & 2.097479 & -0.079755 \\
\hline 106 & 1 & $\odot$ & -6.812175 & 3.360510 & 0.383464 \\
\hline 107 & 1 & $\odot$ & -6.735935 & 4.521777 & -0.968714 \\
\hline 108 & 1 & 0 & -4.520443 & 4.195934 & -0.145060 \\
\hline 109 & 1 & 0 & -4.943429 & 2.668093 & -2.731399 \\
\hline 110 & 1 & 0 & -2.221042 & 3.373588 & -1.604347 \\
\hline 111 & 1 & 0 & -2.637740 & 1.966782 & -2.602564 \\
\hline 112 & 1 & $\Theta$ & -2.545623 & 2.098702 & 0.311322 \\
\hline 113 & 7 & 0 & -3.140824 & 0.355024 & -0.670560 \\
\hline 114 & 6 & 0 & -3.998135 & -2.172354 & -1.499830 \\
\hline 115 & 7 & $\Theta$ & -2.839332 & -2.479370 & -1.016272 \\
\hline 116 & 6 & 0 & -1.812503 & -1.581044 & -0.965696 \\
\hline 117 & 6 & 0 & -1.937739 & -0.145600 & -0.767019 \\
\hline 118 & 7 & $\odot$ & -0.812882 & 0.649841 & -0.697206 \\
\hline 119 & 6 & 0 & 0.365627 & 0.095460 & -0.821517 \\
\hline 120 & 7 & $\Theta$ & 0.559513 & -1.259897 & -0.989749 \\
\hline 121 & 6 & 0 & -0.474203 & -2.156422 & -1.053190 \\
\hline 122 & 7 & $\Theta$ & 1.481766 & 0.830711 & -0.781004 \\
\hline 123 & 8 & 0 & -0.252652 & -3.365637 & -1.204000 \\
\hline 124 & 1 & 0 & -4.197298 & -1.236965 & -2.016280 \\
\hline 125 & 1 & 0 & 1.403416 & 1.825539 & -0.647788 \\
\hline 126 & 1 & 0 & 2.403410 & 0.444688 & -1.004510 \\
\hline 127 & 1 & 0 & 1.515412 & -1.642175 & -1.130182 \\
\hline 128 & 8 & 0 & -5.028262 & -2.971393 & -1.443413 \\
\hline 129 & 1 & 0 & -4.842727 & -3.840559 & -0.961722 \\
\hline 130 & 8 & 0 & -4.729187 & -5.306854 & -0.331346 \\
\hline 131 & 1 & 0 & -3.813703 & -5.342883 & 0.023760 \\
\hline 132 & 1 & 0 & -4.747165 & -5.949960 & -1.047574 \\
\hline 133 & 8 & 0 & -2.149363 & -4.872981 & 0.347355 \\
\hline 134 & 1 & 0 & -2.387965 & -4.009975 & -0.063400 \\
\hline 135 & 1 & 0 & -1.421513 & -5.160381 & -0.216038 \\
\hline
\end{tabular}

General basis read from cards: (6D, 7F)

1177 basis functions

315 alpha electrons 314 beta electrons

nuclear repulsion energy 17102.6192243012 Hartrees.

NAtoms $=135$ NActive $=135$

Force inversion solution in PCM.

Polarizable Continuum Model (PCM)

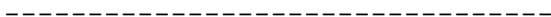

Model : PCM.

Solvent : Water, Eps= 78.355300 Eps $($ inf $)=1.777849$

SCF Done: $E($ UWB97XD) $=-5076.2237524$ A.U. after 1 cycles

NFock $=1$ Conv $=0.10 \mathrm{D}-08 \quad-\mathrm{V} / \mathrm{T}=2.0066$

$\langle\mathrm{S} X>=0.0000<\mathrm{S} y\rangle=0.0000\langle\mathrm{~S} z\rangle=0.5000$

$\left\langle S^{* *} 2>=0.7764 \mathrm{~S}=0.5131\right.$

$<$ L.S $>=0.000000000000 \mathrm{E}+00$

Annihilation of the first spin contaminant:

$\mathrm{S}^{* * 2}$ before annihilation 0.7764 , after 0.7503

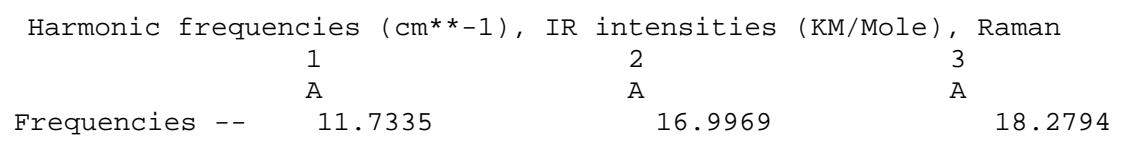


Zero-point correction=

Thermal correction to Energy=

Thermal correction to Enthalpy=

Thermal correction to Gibbs Free Energy=

Sum of electronic and zero-point Energies=

Sum of electronic and thermal Energies=

Sum of electronic and thermal Enthalpies=

Sum of electronic and thermal Free Energies=

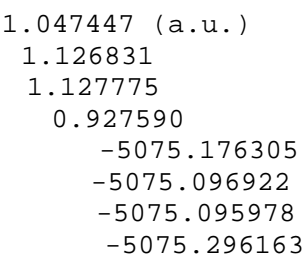

Cal/Mol-Kelvin 421.325

Normal termination of Gaussian 16 at Mon Aug 12 05:57:20 2019.

$G($ high $)=-5077.0550825 \mathrm{a} \cdot \mathrm{u}$.

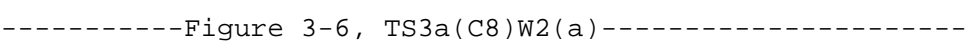

dna.ts3aw2x. log

Stoichiometry C39H54N15Na2023P2(2)

Standard orientation

\begin{tabular}{|c|c|c|c|c|c|}
\hline \multirow{3}{*}{$\begin{array}{l}\text { Center } \\
\text { Number }\end{array}$} & \multirow{2}{*}{$\begin{array}{l}\text { Atomic } \\
\text { Number }\end{array}$} & \multirow{2}{*}{$\begin{array}{c}\text { Atomic } \\
\text { Type }\end{array}$} & \multicolumn{3}{|c|}{ Coordinates (Angstroms) } \\
\hline & & & $x$ & $\mathrm{Y}$ & Z \\
\hline & & & -8 & - & ---- \\
\hline 1 & 7 & $\odot$ & 5.500292 & -1.910189 & -1.596437 \\
\hline 2 & 6 & $\odot$ & 5.776189 & -3.245415 & -1.640904 \\
\hline 3 & 6 & $\odot$ & 4.780544 & -4.159523 & -1.601961 \\
\hline 4 & 6 & $\odot$ & 3.439771 & -3.660953 & -1.489543 \\
\hline 5 & 7 & $\odot$ & 3.179192 & -2.350226 & -1.481086 \\
\hline 6 & 8 & $\odot$ & 8.755450 & 0.982625 & -0.891735 \\
\hline 7 & 15 & $\odot$ & 8.101286 & 1.969358 & 0.231729 \\
\hline 8 & 8 & $\odot$ & 6.496889 & 1.867386 & -0.080536 \\
\hline 9 & 8 & $\odot$ & 8.592657 & 3.368845 & -0.054056 \\
\hline 10 & 8 & $\odot$ & 8.303628 & 1.428150 & 1.628975 \\
\hline 11 & 8 & $\odot$ & 4.563988 & 5.460466 & 1.162489 \\
\hline 12 & 6 & $\odot$ & 4.775649 & 3.588435 & -0.298134 \\
\hline 13 & 6 & $\odot$ & 5.267583 & 4.200178 & 1.018167 \\
\hline 14 & 6 & $\odot$ & -9.103856 & -1.920597 & 1.784097 \\
\hline 15 & 6 & $\odot$ & -8.581709 & -0.502500 & 1.670645 \\
\hline 16 & 8 & $\odot$ & -8.261927 & -2.801768 & 1.000012 \\
\hline 17 & 8 & $\odot$ & -8.615541 & 1. 339891 & 0.123151 \\
\hline 18 & 15 & $\odot$ & -8.657565 & 2.095984 & -1.323737 \\
\hline 19 & 8 & $\odot$ & -7.101586 & 2.532804 & -1.551129 \\
\hline 20 & 8 & $\odot$ & -9.532117 & 3.318822 & -1.158779 \\
\hline 21 & 8 & $\odot$ & -9.015844 & 1.131527 & -2.430380 \\
\hline 22 & 8 & $\odot$ & -4.235654 & 4.623384 & -2.621844 \\
\hline 23 & 6 & $\odot$ & -4.980759 & 3.365286 & -0.710797 \\
\hline 24 & 6 & $\odot$ & -4.328776 & 3.286857 & -2.087551 \\
\hline 25 & 1 & $\odot$ & -3.993893 & 4.597745 & -3.561872 \\
\hline 26 & 6 & $\odot$ & 9.599821 & -2.308443 & -2.436543 \\
\hline 27 & 6 & $\odot$ & 8.870374 & -0.993211 & -2.255323 \\
\hline 28 & 6 & $\odot$ & 8.795077 & -0.483696 & -0.808747 \\
\hline 29 & 6 & $\odot$ & 7.477455 & -1.061809 & -0.311879 \\
\hline 30 & 6 & $\odot$ & 6.585491 & -0.942958 & -1.545857 \\
\hline 31 & 8 & $\odot$ & 7.473427 & -1.143787 & -2.681951 \\
\hline 32 & 1 & $\odot$ & 9.484189 & -2.633202 & -3.475726 \\
\hline 33 & 1 & $\odot$ & 10.666072 & -2.160404 & -2.230008 \\
\hline 34 & 1 & $\odot$ & 9.335891 & -0.237631 & -2.892000 \\
\hline 35 & 1 & $\odot$ & 9.654253 & -0.773529 & -0.203105 \\
\hline 36 & 1 & $\odot$ & 7.081295 & -0.524786 & 0.547262 \\
\hline 37 & 1 & $\odot$ & 7.639646 & -2.108848 & $-\odot .047702$ \\
\hline 38 & 1 & $\odot$ & 6.124897 & 0.037756 & -1.609238 \\
\hline 39 & 8 & $\odot$ & 9.037153 & -3.289104 & -1.530229 \\
\hline 40 & 1 & $\odot$ & 9.467618 & -4.152128 & -1.642501 \\
\hline 41 & 6 & $\odot$ & 4.178180 & -1.440554 & -1.568776 \\
\hline
\end{tabular}




\begin{tabular}{|c|c|c|c|c|c|}
\hline 42 & 7 & 0 & 2.403632 & -4.492250 & -1.385703 \\
\hline 43 & 8 & $\odot$ & 3.964770 & $-\odot .223206$ & -1.605419 \\
\hline 44 & 1 & 0 & 6.833269 & -3.497692 & -1.705387 \\
\hline 45 & 1 & 0 & 4.989419 & -5.219830 & -1.637695 \\
\hline 46 & 1 & $\odot$ & 2.547533 & -5.487940 & -1.367957 \\
\hline 47 & 1 & $\odot$ & 1.457616 & -4.113844 & -1.287960 \\
\hline 48 & 6 & 0 & 5.805662 & 2.744340 & -1.021783 \\
\hline 49 & 1 & 0 & 4.805412 & 5.887982 & 2.001940 \\
\hline 50 & 6 & $\odot$ & 4.820498 & 3.186130 & 2.076711 \\
\hline 51 & 6 & 0 & 3.493428 & 2.669978 & 1.511436 \\
\hline 52 & 8 & 0 & 3.617309 & 2.768667 & $\odot .061625$ \\
\hline 53 & 1 & 0 & 5.304306 & 2.105651 & -1.750094 \\
\hline 54 & 1 & 0 & 6.532191 & 3.391965 & -1.518152 \\
\hline 55 & 1 & 0 & 4.426483 & 4.378544 & -0.967522 \\
\hline 56 & 1 & 0 & 6.350346 & 4.357708 & 1.012478 \\
\hline 57 & 1 & 0 & 4.687513 & 3.643070 & 3.059445 \\
\hline 58 & 1 & $\odot$ & 5.568928 & 2.395519 & 2.155741 \\
\hline 59 & 1 & 0 & 2.657594 & 3.288884 & 1.830916 \\
\hline 60 & 7 & 0 & 3.135316 & 1.302699 & 1.852723 \\
\hline 61 & 6 & $\odot$ & 4.024098 & 0.267677 & 1.644452 \\
\hline 62 & 6 & $\odot$ & 3.701261 & -1.029785 & 1.828283 \\
\hline 63 & 6 & 0 & 2.317581 & -1.348929 & 2.138470 \\
\hline 64 & 7 & 0 & 1.484587 & $-\odot .267557$ & 2.326497 \\
\hline 65 & 6 & 0 & 1.806340 & 1.056694 & 2.186887 \\
\hline 66 & 6 & 0 & 4.671606 & -2.165951 & 1.732708 \\
\hline 67 & 8 & 0 & 1.881103 & -2.499601 & 2.230564 \\
\hline 68 & 8 & 0 & 0.983899 & 1.947151 & 2.356461 \\
\hline 69 & 1 & 0 & 5.009419 & 0.576532 & 1.309243 \\
\hline 70 & 1 & 0 & 4.716119 & -2.705346 & 2.683801 \\
\hline 71 & 1 & 0 & 4.367994 & -2.889189 & 0.972247 \\
\hline 72 & 1 & $\odot$ & 5.674654 & -1.812130 & 1.499409 \\
\hline 73 & 1 & 0 & 0.474359 & -0.469177 & 2.512203 \\
\hline 74 & 1 & 0 & -8.592739 & -3.714205 & 1.030288 \\
\hline 75 & 6 & 0 & -8.242633 & -0.074791 & 0.239503 \\
\hline 76 & 6 & 0 & -6.736157 & -0.284220 & 0.124589 \\
\hline 77 & 6 & 0 & -6.209718 & $-\odot .124053$ & 1.556850 \\
\hline 78 & 8 & 0 & -7.346885 & -0.339684 & 2.439043 \\
\hline 79 & 1 & $\odot$ & -9.103422 & -2.218224 & 2.837895 \\
\hline 80 & 1 & $\odot$ & -10.133823 & -1.948876 & 1.409333 \\
\hline 81 & 1 & 0 & -9.321261 & $\odot .178326$ & 2.098509 \\
\hline 82 & 1 & 0 & -8.800568 & -0.638379 & $-\odot .509452$ \\
\hline 83 & 1 & 0 & -6.249287 & 0.435214 & -0.533204 \\
\hline 84 & 1 & $\odot$ & -6.542406 & -1.295038 & $-\odot .239780$ \\
\hline 85 & 1 & 0 & -5.794578 & $\odot .869662$ & 1.726559 \\
\hline 86 & 7 & $\odot$ & -5.162785 & -1.072577 & 1.885630 \\
\hline 87 & 6 & 0 & -5.228758 & -2.439756 & 1.749266 \\
\hline 88 & 7 & 0 & -4.086126 & -3.041524 & 1.953630 \\
\hline 89 & 6 & 0 & -3.211826 & -2.007892 & 2.230398 \\
\hline 90 & 6 & $\odot$ & -3.865607 & -0.780427 & 2.218589 \\
\hline 91 & 7 & 0 & -3.323532 & 0.417823 & 2.485358 \\
\hline 92 & 6 & 0 & -2.021232 & 0.324311 & 2.695224 \\
\hline 93 & 7 & $\odot$ & -1.251089 & -0.776335 & 2.653205 \\
\hline 94 & 6 & 0 & -1.815439 & -1.973547 & 2.398801 \\
\hline 95 & 7 & 0 & -1.035252 & -3.067809 & 2.317032 \\
\hline 96 & 1 & $\odot$ & -6.172308 & -2.907613 & 1.493131 \\
\hline 97 & 1 & 0 & -1.481175 & 1.244734 & 2.903401 \\
\hline 98 & 1 & 0 & $-\odot .026488$ & -2.921871 & 2.278840 \\
\hline 99 & 1 & 0 & -1.420452 & -3.864246 & 1.822032 \\
\hline 100 & 6 & 0 & -6.479066 & 3.513474 & -0.670253 \\
\hline 101 & 11 & 0 & 9.188939 & 3.628452 & 2.400018 \\
\hline 102 & 11 & $\odot$ & -10.924396 & 2.652032 & -3.090589 \\
\hline 103 & 6 & 0 & -2.976366 & 2.626237 & -1.781620 \\
\hline 104 & 6 & 0 & -3.218642 & 1.794976 & $-\odot .488954$ \\
\hline 105 & 8 & 0 & -4.594356 & 2.121889 & $-\odot .079478$ \\
\hline 106 & 1 & 0 & -6.831037 & 3.365370 & 0.354977 \\
\hline 107 & 1 & 0 & -6.757407 & 4.515340 & -1.007140 \\
\hline 108 & 1 & 0 & -4.543896 & 4.220195 & -0.171644 \\
\hline 109 & 1 & 0 & -4.939059 & 2.653845 & -2.739048 \\
\hline 110 & 1 & 0 & -2.234990 & 3.408546 & -1.596994 \\
\hline 111 & 1 & 0 & -2.619037 & 1.992492 & -2.594941 \\
\hline 112 & 1 & 0 & -2.549148 & 2.123012 & 0.314003 \\
\hline 113 & 7 & 0 & -3.144712 & $\odot .376282$ & -0.666523 \\
\hline 114 & 6 & 0 & -4.041436 & -2.206457 & -1.407297 \\
\hline 115 & 7 & 0 & -2.817128 & -2.483680 & -0.961185 \\
\hline 116 & 6 & 0 & -1.811002 & -1.567818 & $-\odot .940139$ \\
\hline 117 & 6 & 0 & -1.945301 & $-\odot .125900$ & $-\odot .774328$ \\
\hline 118 & 7 & 0 & -0.819807 & 0.676378 & -0.733624 \\
\hline
\end{tabular}




\begin{tabular}{|c|c|c|c|c|c|}
\hline 119 & 6 & $\odot$ & $\odot .361085$ & $\odot .126236$ & $-\odot .844967$ \\
\hline 120 & 7 & 0 & $\odot .562652$ & -1.230106 & -0.977406 \\
\hline 121 & 6 & $\odot$ & -0.470138 & -2.130983 & -1.014257 \\
\hline 122 & 7 & 0 & 1.474776 & ๑. 869861 & -0.826020 \\
\hline 123 & 8 & $\odot$ & -0.239202 & -3.345030 & -1.132518 \\
\hline 124 & 1 & $\odot$ & -4.204749 & -1.246902 & -1.904801 \\
\hline 125 & 1 & 0 & 1.391159 & 1.864704 & -0.699580 \\
\hline 126 & 1 & $\odot$ & 2.399494 & 0.482179 & -1.029486 \\
\hline 127 & 1 & 0 & 1.517446 & -1.611262 & -1.118081 \\
\hline 128 & 8 & 0 & -5.011482 & -3.002210 & -1.319974 \\
\hline 129 & 1 & $\odot$ & -4.853857 & -4.291351 & -0.685068 \\
\hline 130 & 8 & $\odot$ & -4.662129 & -5.232198 & -0.256420 \\
\hline 131 & 1 & 0 & -3.513012 & -5.146684 & -0.021297 \\
\hline 132 & 1 & $\odot$ & -4.817988 & -5.898882 & -0.935837 \\
\hline 133 & 8 & $\odot$ & -2.357857 & -4.751161 & 0.139326 \\
\hline 134 & 1 & $\odot$ & -2.477722 & -3.794199 & -0.313182 \\
\hline 135 & 1 & $\odot$ & -1.697856 & -5.187253 & -0.413799 \\
\hline
\end{tabular}

General basis read from cards: (6D, 7F)

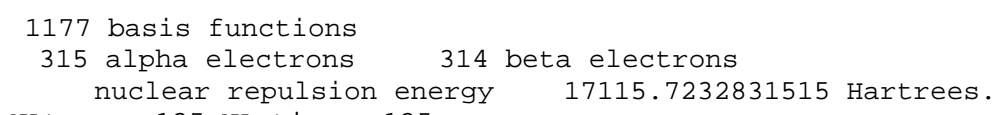

NAtoms $=135$ NActive $=135$

Nuclear repulsion after empirical dispersion term $=$

17115.5486398556 Hartrees.

Force inversion solution in PCM.

Polarizable Continuum Model (PCM)

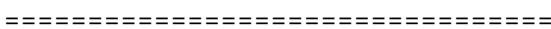

Model : PCM.

Solvent : Water, Eps $=78.355300$ Eps $($ inf $)=1.777849$

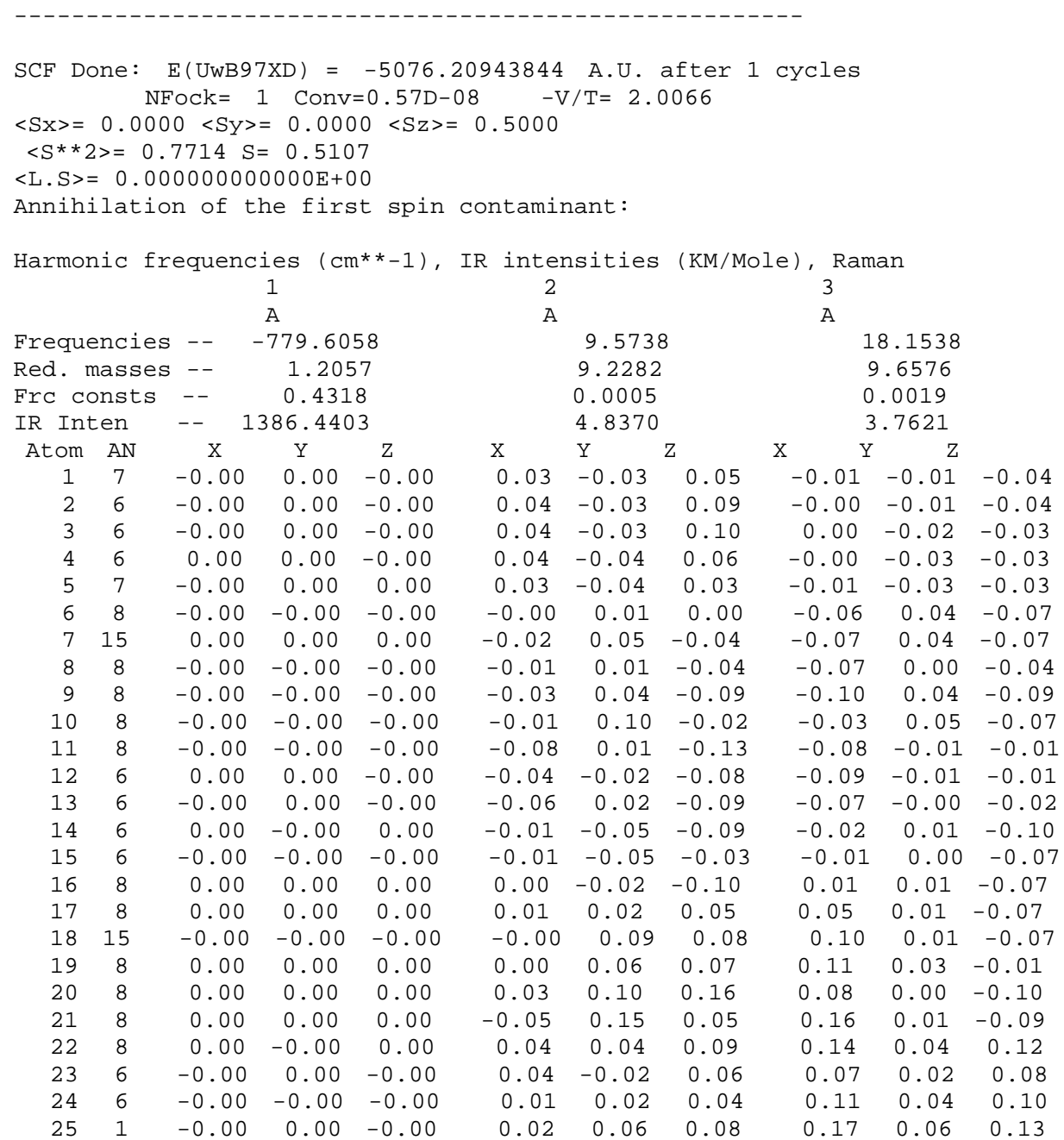




\begin{tabular}{|c|c|c|c|c|c|c|c|c|c|c|}
\hline 26 & 6 & $-\odot . \odot \odot$ & $-\odot . \odot \odot$ & $-\odot . \odot \odot$ & $\odot .04$ & -0.04 & 0.13 & $-\odot . \odot \odot$ & $\odot .04$ & $-\odot . \odot 4$ \\
\hline 27 & 6 & 0.00 & -0.00 & 0.00 & 0.04 & -0.04 & $\odot .08$ & -0.02 & 0.03 & -0.05 \\
\hline 28 & 6 & $-\odot . \odot \odot$ & $\odot . \odot \odot$ & $-0.0 \odot$ & 0.01 & 0.01 & 0.06 & -0.03 & 0.04 & -0.06 \\
\hline 29 & 6 & $-\odot . \odot \odot$ & $-\odot . \odot \odot$ & $\odot . \odot \odot$ & $\odot .01$ & $\odot .02$ & $\odot .06$ & -0.02 & 0.02 & -0.05 \\
\hline 30 & 6 & $\odot . \odot \odot$ & $\odot . \odot \odot$ & -0.00 & 0.03 & -0.02 & 0.04 & -0.02 & $-\odot . \odot \odot$ & -0.05 \\
\hline 31 & 8 & $-\odot . \odot \odot$ & $-0.0 \odot$ & $\odot .0 \odot$ & 0.04 & -0.06 & 0.06 & -0.02 & $-0.0 \odot$ & -0.05 \\
\hline 32 & 1 & 0.00 & $-\odot . \odot \odot$ & -0.00 & 0.06 & -0.08 & 0.14 & 0.01 & 0.03 & -0.04 \\
\hline 33 & 1 & $-\odot . \odot \odot$ & $-\odot . \odot \odot$ & $\odot .0 \odot$ & $\odot .04$ & -0.03 & 0.14 & $-\odot .01$ & $\odot .06$ & -0.04 \\
\hline 34 & 1 & $-\odot . \odot \odot$ & $-\odot . \odot \odot$ & $-\odot . \odot \odot$ & $\odot .04$ & -0.06 & $\odot .06$ & -0.04 & $\odot .03$ & -0.06 \\
\hline 35 & 1 & $-0.0 \odot$ & $-0.0 \odot$ & $\odot . \odot \odot$ & $\odot .01$ & 0.04 & 0.08 & -0.02 & 0.06 & -0.05 \\
\hline 36 & 1 & $\odot . \odot \odot$ & $-\odot .0 \odot$ & 0.00 & -0.01 & 0.04 & 0.03 & -0.03 & 0.01 & -0.05 \\
\hline 37 & 1 & 0.00 & -0.00 & -0.00 & 0.01 & 0.03 & 0.09 & -0.00 & 0.02 & -0.05 \\
\hline 38 & 1 & $\odot .00$ & 0.00 & $-\odot .0 \odot$ & 0.02 & -0.03 & $\odot .00$ & -0.03 & -0.01 & -0.05 \\
\hline 39 & 8 & $-0.0 \odot$ & -0.00 & -0.00 & 0.03 & -0.01 & 0.16 & 0.01 & 0.03 & -0.04 \\
\hline 40 & 1 & $-0.0 \odot$ & 0.00 & 0.00 & $\odot .04$ & -0.01 & 0.20 & 0.02 & 0.04 & -0.03 \\
\hline 41 & 6 & $\odot . \odot \odot$ & 0.00 & $\odot .00$ & 0.03 & -0.03 & $\odot .02$ & -0.01 & -0.02 & -0.03 \\
\hline 42 & 7 & $\odot .0 \odot$ & $-\odot . \odot \odot$ & $-\odot . \odot \odot$ & 0.04 & -0.04 & 0.07 & $\odot .0 \odot$ & -0.03 & -0.02 \\
\hline 43 & 8 & $-\odot . \odot \odot$ & 0.00 & 0.00 & 0.02 & -0.04 & -0.01 & -0.02 & -0.02 & $-\odot .03$ \\
\hline 44 & 1 & $-0 . \odot \odot$ & $\odot . \odot \odot$ & $-\odot . \odot \odot$ & $\odot . \odot 4$ & -0.03 & 0.11 & $-\odot . \odot \odot$ & $-0.0 \odot$ & -0.04 \\
\hline 45 & 1 & -0.00 & 0.00 & 0.00 & 0.04 & -0.03 & 0.13 & 0.01 & -0.02 & -0.03 \\
\hline 46 & 1 & $\odot .00$ & -0.00 & $-0.0 \odot$ & 0.04 & -0.04 & 0.09 & 0.01 & -0.03 & -0.01 \\
\hline 47 & 1 & $\odot . \odot \odot$ & $-0.0 \odot$ & $-\odot .0 \odot$ & $\odot .03$ & -0.04 & $\odot .04$ & $\odot . \odot \odot$ & $-\odot .03$ & -0.00 \\
\hline 48 & 6 & $-0 . \odot \odot$ & $-\odot . \odot \odot$ & $-\odot . \odot \odot$ & -0.03 & -0.02 & -0.06 & -0.10 & -0.01 & $-\odot .03$ \\
\hline 49 & 1 & $\odot . \odot \odot$ & 0.00 & $-\odot .0 \odot$ & -0.09 & 0.03 & -0.13 & -0.07 & -0.00 & -0.02 \\
\hline 50 & 6 & $\odot .0 \odot$ & $-\odot . \odot \odot$ & 0.00 & -0.05 & 0.04 & -0.07 & -0.03 & -0.00 & -0.01 \\
\hline 51 & 6 & 0.00 & 0.00 & 0.00 & -0.04 & 0.01 & -0.07 & -0.05 & -0.01 & 0.03 \\
\hline 52 & 8 & $-\odot . \odot \odot$ & $-0.0 \odot$ & $\odot . \odot \odot$ & -0.03 & -0.02 & -0.07 & -0.08 & -0.02 & 0.02 \\
\hline 53 & 1 & 0.00 & $\odot .0 \odot$ & $-\odot .0 \odot$ & $-\odot .02$ & -0.05 & -0.04 & -0.12 & -0.02 & -0.01 \\
\hline 54 & 1 & $-\odot . \odot \odot$ & $0.0 \odot$ & $\odot . \odot \odot$ & -0.04 & -0.02 & -0.07 & -0.12 & -0.01 & -0.05 \\
\hline 55 & 1 & $-0.0 \odot$ & $-0.0 \odot$ & -0.00 & -0.05 & -0.04 & -0.10 & -0.11 & -0.02 & -0.01 \\
\hline 56 & 1 & $-\odot . \odot \odot$ & $-\odot . \odot \odot$ & $-\odot . \odot \odot$ & -0.06 & 0.04 & $-\odot . \odot 9$ & -0.07 & 0.01 & -0.05 \\
\hline 57 & 1 & $\odot . \odot \odot$ & $-0.0 \odot$ & $\odot . \odot \odot$ & -0.05 & 0.05 & -0.08 & -0.01 & $-0.0 \odot$ & -0.01 \\
\hline 58 & 1 & 0.00 & -0.00 & 0.00 & -0.04 & 0.05 & -0.06 & -0.03 & 0.00 & -0.03 \\
\hline 59 & 1 & $\odot .0 \odot$ & 0.00 & 0.00 & -0.05 & 0.01 & -0.08 & -0.04 & -0.01 & 0.04 \\
\hline 60 & 7 & $-0.0 \odot$ & -0.00 & $-0.0 \odot$ & -0.03 & 0.01 & -0.04 & -0.03 & -0.01 & 0.04 \\
\hline 61 & 6 & -0.00 & $-0.0 \odot$ & 0.00 & -0.02 & 0.02 & -0.01 & -0.04 & -0.01 & 0.00 \\
\hline 62 & 6 & $-\odot . \odot \odot$ & $-0.0 \odot$ & $\odot .0 \odot$ & -0.01 & 0.02 & 0.01 & -0.03 & -0.01 & -0.00 \\
\hline 63 & 6 & $\odot .0 \odot$ & $-\odot . \odot \odot$ & 0.00 & -0.01 & 0.01 & $\odot .0 \odot$ & -0.02 & -0.02 & 0.03 \\
\hline 64 & 7 & $\odot .0 \odot$ & $-0.0 \odot$ & -0.00 & -0.02 & 0.01 & -0.02 & -0.02 & -0.02 & 0.07 \\
\hline 65 & 6 & $\odot . \odot \odot$ & $\odot .0 \odot$ & 0.00 & $-\odot .03$ & 0.01 & -0.04 & -0.02 & -0.02 & 0.06 \\
\hline 66 & 6 & -0.00 & -0.00 & 0.00 & $\odot .0 \odot$ & 0.02 & 0.04 & -0.03 & -0.01 & -0.04 \\
\hline 67 & 8 & -0.00 & $-0.0 \odot$ & $-\odot . \odot \odot$ & $\odot . \odot \odot$ & 0.01 & 0.02 & -0.02 & -0.02 & 0.03 \\
\hline 68 & 8 & $-0.0 \odot$ & -0.00 & -0.00 & -0.04 & 0.00 & -0.06 & -0.02 & -0.02 & 0.09 \\
\hline 69 & 1 & $-\odot .0 \odot$ & -0.00 & -0.00 & -0.02 & 0.02 & -0.01 & -0.05 & -0.00 & -0.02 \\
\hline 70 & 1 & $\odot . \odot \odot$ & $0.0 \odot$ & $\odot . \odot \odot$ & $-\odot . \odot \odot$ & $\odot .04$ & $\odot .05$ & $\odot .01$ & $-0.0 \odot$ & -0.04 \\
\hline 71 & 1 & 0.00 & $-\odot . \odot \odot$ & 0.00 & 0.02 & 0.01 & 0.05 & -0.06 & -0.01 & -0.02 \\
\hline 72 & 1 & $-0.0 \odot$ & $\odot . \odot \odot$ & 0.00 & 0.00 & 0.03 & 0.05 & -0.04 & $-0.0 \odot$ & -0.08 \\
\hline 73 & 1 & 0.00 & 0.00 & 0.00 & -0.02 & -0.00 & -0.02 & -0.01 & -0.02 & 0.08 \\
\hline 74 & 1 & $\odot .0 \odot$ & $-\odot . \odot \odot$ & $-\odot . \odot \odot$ & 0.01 & -0.03 & -0.13 & $-\odot . \odot \odot$ & 0.01 & $-\odot . \odot 9$ \\
\hline 75 & 6 & $\odot .0 \odot$ & $-0.0 \odot$ & $\odot . \odot \odot$ & -0.01 & 0.01 & -0.02 & 0.05 & 0.01 & -0.06 \\
\hline 76 & 6 & $0.0 \odot$ & -0.00 & -0.00 & -0.01 & 0.00 & -0.02 & 0.05 & 0.01 & -0.01 \\
\hline 77 & 6 & $-0.0 \odot$ & $-0.0 \odot$ & 0.00 & -0.02 & -0.03 & -0.02 & 0.00 & -0.01 & 0.01 \\
\hline 78 & 8 & $\odot .0 \odot$ & $-\odot . \odot \odot$ & $-\odot . \odot \odot$ & -0.02 & -0.07 & -0.02 & $-\odot .03$ & -0.01 & -0.03 \\
\hline 79 & 1 & -0.00 & 0.00 & 0.00 & -0.02 & -0.08 & -0.09 & -0.06 & 0.00 & -0.10 \\
\hline 80 & 1 & $-0.0 \odot$ & 0.00 & 0.00 & -0.00 & -0.04 & -0.10 & -0.01 & 0.02 & -0.14 \\
\hline 81 & 1 & 0.00 & 0.00 & 0.00 & -0.02 & -0.06 & -0.01 & -0.02 & 0.01 & -0.09 \\
\hline 82 & 1 & $\odot .0 \odot$ & $-\odot . \odot \odot$ & $-0.0 \odot$ & -0.02 & 0.05 & -0.04 & 0.07 & 0.01 & -0.08 \\
\hline 83 & 1 & $-\odot . \odot \odot$ & $\odot . \odot \odot$ & $-\odot .0 \odot$ & $-\odot . \odot \odot$ & 0.01 & $-\odot . \odot \odot$ & 0.07 & 0.01 & $\odot . \odot 2$ \\
\hline 84 & 1 & $\odot .0 \odot$ & 0.00 & $-0.0 \odot$ & -0.02 & 0.01 & -0.04 & 0.06 & 0.01 & -0.01 \\
\hline 85 & 1 & $\odot .0 \odot$ & $-\odot .0 \odot$ & 0.00 & -0.03 & -0.03 & 0.01 & -0.00 & -0.01 & 0.03 \\
\hline 86 & 7 & $\odot . \odot \odot$ & $-0.0 \odot$ & $\odot . \odot \odot$ & -0.01 & -0.03 & -0.03 & -0.01 & -0.01 & $\odot .04$ \\
\hline 87 & 6 & $\odot .0 \odot$ & $-\odot . \odot \odot$ & $-\odot . \odot \odot$ & $-\odot . \odot \odot$ & -0.03 & -0.05 & -0.01 & -0.01 & 0.03 \\
\hline 88 & 7 & $\odot .0 \odot$ & $-\odot . \odot \odot$ & $-\odot .0 \odot$ & $\odot .0 \odot$ & -0.03 & -0.06 & -0.02 & -0.02 & 0.04 \\
\hline 89 & 6 & $-0.0 \odot$ & 0.00 & 0.00 & -0.00 & -0.03 & -0.04 & -0.02 & -0.02 & 0.06 \\
\hline 90 & 6 & $-\odot . \odot \odot$ & 0.00 & 0.00 & -0.01 & -0.03 & -0.03 & -0.02 & -0.02 & $\odot .06$ \\
\hline 91 & 7 & $\odot .0 \odot$ & $-\odot . \odot \odot$ & $-0.0 \odot$ & -0.02 & -0.03 & -0.01 & $-\odot .02$ & -0.03 & 0.08 \\
\hline 92 & 6 & $\odot .0 \odot$ & $\odot .0 \odot$ & $-\odot .0 \odot$ & $-\odot .02$ & -0.02 & -0.01 & -0.02 & $-\odot .03$ & 0.09 \\
\hline 93 & 7 & $-\odot . \odot \odot$ & $-0.0 \odot$ & $-\odot . \odot \odot$ & -0.01 & -0.02 & -0.02 & -0.02 & -0.03 & 0.09 \\
\hline 94 & 6 & -0.00 & 0.00 & 0.00 & -0.01 & -0.02 & -0.03 & -0.02 & -0.03 & 0.07 \\
\hline 95 & 7 & $\odot . \odot \odot$ & 0.00 & $\odot .0 \odot$ & $\odot .00$ & -0.01 & -0.04 & -0.02 & -0.03 & 0.06 \\
\hline 96 & 1 & 0.00 & $-0.0 \odot$ & -0.00 & 0.00 & -0.03 & -0.07 & -0.01 & -0.01 & 0.00 \\
\hline 97 & 1 & 0.00 & -0.00 & -0.00 & -0.02 & -0.02 & 0.00 & -0.02 & -0.03 & 0.11 \\
\hline 98 & 1 & $\odot .0 \odot$ & -0.01 & $\odot .0 \odot$ & 0.00 & -0.01 & -0.03 & -0.02 & -0.02 & 0.06 \\
\hline 99 & 1 & -0.00 & -0.02 & 0.06 & 0.01 & -0.01 & -0.05 & -0.02 & -0.02 & 0.05 \\
\hline 100 & 6 & $-0.0 \odot$ & $-\odot .0 \odot$ & $\odot . \odot \odot$ & 0.04 & 0.02 & $\odot . \odot 9$ & 0.07 & 0.02 & 0.03 \\
\hline 101 & 11 & 0.00 & -0.00 & $\odot .00$ & -0.10 & 0.09 & -0.02 & -0.09 & 0.05 & -0.07 \\
\hline$\odot 2$ & 11 & $-0.0 \odot$ & $\odot . \odot \odot$ & -0.00 & -0.02 & 0.21 & 0.16 & 0.18 & 0.03 & -0.16 \\
\hline
\end{tabular}




\begin{tabular}{|c|c|c|c|c|}
\hline 103 & 6 & $\odot .00$ & -0.00 & -0.00 \\
\hline 104 & 6 & $-\odot .0 \odot$ & $\odot . \odot \odot$ & 0.00 \\
\hline 105 & 8 & 0.00 & $-\odot . \odot \odot$ & 0.00 \\
\hline 106 & 1 & $\odot .0 \odot$ & -0.00 & 0.00 \\
\hline 107 & 1 & 0.00 & -0.00 & 0.00 \\
\hline 108 & 1 & $\odot . \odot \odot$ & -0.00 & $\odot .0 \odot$ \\
\hline 109 & 1 & $\odot .00$ & $-0.0 \odot$ & -0.00 \\
\hline 110 & 1 & $\odot . \odot \odot$ & $-\odot .0 \odot$ & 0.00 \\
\hline 111 & 1 & $\odot .00$ & -0.00 & -0.00 \\
\hline 112 & 1 & $\odot . \odot \odot$ & $-0.0 \odot$ & $\odot .0 \odot$ \\
\hline 113 & 7 & $-\odot . \odot \odot$ & $-0.0 \odot$ & $\odot . \odot \odot$ \\
\hline 114 & 6 & -0.01 & $-\odot . \odot \odot$ & $-\odot . \odot \odot$ \\
\hline 115 & 7 & 0.02 & -0.04 & 0.03 \\
\hline 116 & 6 & 0.01 & -0.01 & 0.01 \\
\hline 117 & 6 & $-\odot . \odot \odot$ & $-\odot . \odot \odot$ & $-\odot . \odot \odot$ \\
\hline 118 & 7 & $-\odot . \odot \odot$ & $-\odot . \odot \odot$ & $-\odot . \odot \odot$ \\
\hline 119 & 6 & $\odot .00$ & $\odot .00$ & -0.00 \\
\hline 120 & 7 & $\odot . \odot \odot$ & $-\odot .0 \odot$ & -0.00 \\
\hline 121 & 6 & $\odot . \odot \odot$ & $\odot . \odot \odot$ & 0.00 \\
\hline 122 & 7 & -0.00 & -0.00 & 0.00 \\
\hline 123 & 8 & -0.00 & $-0.0 \odot$ & 0.00 \\
\hline 124 & 1 & $\odot .08$ & 0.02 & 0.01 \\
\hline 125 & 1 & $\odot . \odot \odot$ & 0.00 & -0.00 \\
\hline 126 & 1 & $\odot . \odot \odot$ & -0.00 & -0.00 \\
\hline 127 & 1 & $\odot .0 \odot$ & -0.00 & -0.00 \\
\hline 128 & 8 & $\odot . \odot \odot$ & $\odot .05$ & -0.02 \\
\hline 129 & 1 & -0.04 & -0.28 & 0.13 \\
\hline 130 & 8 & $\odot . \odot \odot$ & -0.05 & 0.02 \\
\hline 131 & 1 & 0.82 & 0.18 & 0.11 \\
\hline 132 & 1 & -0.03 & 0.01 & -0.02 \\
\hline 133 & 8 & -0.05 & $\odot . \odot 4$ & -0.03 \\
\hline 134 & 1 & -0.12 & 0.29 & -0.18 \\
\hline 135 & 1 & -0.12 & -0.05 & -0.05 \\
\hline
\end{tabular}

$0.00 \quad-0.04 \quad-0.01$

$0.02-0.04 \quad-0.01$

$0.02 \quad-0.05 \quad 0.00$

$\begin{array}{llll}0.06 & -0.00 & 0.09\end{array}$

$0.06 \quad 0.04 \quad 0.12$

$0.07 \quad-0.05 \quad 0.08$

$\begin{array}{lll}-0.02 & 0.06 & 0.03\end{array}$

$\begin{array}{llll}0.04 & -0.07 & -0.03\end{array}$

$-0.05-0.04-0.03$

$0.02-0.04-0.02$

$0.02-0.04-0.02$

$0.02-0.03 \quad-0.05$

$0.02 \quad-0.04 \quad-0.04$

$0.02-0.04-0.03$

$0.02-0.04-0.03$

$0.02-0.04-0.04$

$0.02-0.03-0.03$

$0.02-0.04-0.02$

$0.02-0.04-0.02$

$0.02 \quad-0.03 \quad-0.05$

$0.02-0.04-0.01$

$0.03-0.04-0.05$

$0.02-0.03 \quad-0.05$

$0.02-0.03 \quad-0.03$

$0.02-0.04-0.01$

$0.02-0.03-0.05$

$0.02-0.03-0.05$

$0.01-0.03 \quad-0.04$

$0.01-0.03 \quad-0.04$

$0.01-0.03 \quad-0.04$

$0.01-0.04-0.03$

$0.01-0.04-0.04$

$0.01-0.04-0.03$

$\begin{array}{ccc}0.09 & 0.02 & 0.13 \\ 0.05 & -0.00 & 0.10 \\ 0.05 & 0.02 & 0.08 \\ 0.03 & 0.01 & 0.01 \\ 0.08 & 0.02 & 0.03 \\ 0.05 & 0.02 & 0.10 \\ 0.12 & 0.05 & 0.07 \\ 0.10 & 0.01 & 0.17 \\ 0.11 & 0.03 & 0.13 \\ 0.04 & -0.03 & 0.12 \\ 0.02 & -0.00 & 0.08 \\ 0.00 & 0.00 & 0.06 \\ 0.00 & -0.01 & 0.05 \\ 0.01 & -0.01 & 0.06 \\ 0.02 & -0.02 & 0.08 \\ 0.02 & -0.02 & 0.08 \\ 0.02 & -0.03 & 0.06 \\ 0.01 & -0.03 & 0.04 \\ 0.00 & -0.02 & 0.04 \\ 0.02 & -0.04 & 0.06 \\ -0.00 & -0.02 & 0.03 \\ 0.00 & 0.01 & 0.07 \\ 0.03 & -0.03 & 0.06 \\ 0.01 & -0.04 & 0.03 \\ 0.00 & -0.03 & 0.02 \\ -0.00 & 0.00 & 0.04 \\ -0.00 & -0.00 & 0.02 \\ -0.01 & -0.01 & 0.02 \\ -0.01 & -0.01 & 0.02 \\ -0.00 & -0.00 & 0.01 \\ -0.01 & -0.01 & 0.04 \\ -0.01 & -0.01 & 0.04 \\ -0.00 & -0.01 & 0.04 \\ & & \end{array}$

$$
\begin{array}{r}
1.041788 \text { (a.u. ) } \\
1.119626 \\
1.120570 \\
0.923935 \\
-5075.167651 \\
-5075.089813 \\
-5075.088868 \\
-5075.285504
\end{array}
$$

Thermal correction to Gibbs Free Energy=

Sum of electronic and zero-point Energies=

Sum of electronic and thermal Enthalpies=

Sum of electronic and thermal Free Energies=
E (Thermal)

702.576
$\mathrm{CV}$

$\begin{array}{cr}\mathrm{CV} & \mathrm{S} \\ \mathrm{Cal} / \mathrm{Mol}-\mathrm{Kelvin} & \mathrm{Cal} / \mathrm{Mol}\end{array}$ 293.694 413.854

Total

Item

Maximum Force

Value

Threshold Converged?

RMS Force

Maximum Displacement

RMS Displacement

$0.000010 \quad 0.000450$ YES

0.0001 YES

$0.004266 \quad 0.001800 \quad$ NO
0.000643

\begin{tabular}{|c|c|c|c|c|c|}
\hline \multirow{2}{*}{$\begin{array}{l}\text { Center } \\
\text { Number }\end{array}$} & \multirow{2}{*}{$\begin{array}{l}\text { Atomic } \\
\text { Number }\end{array}$} & \multirow{2}{*}{$\begin{array}{c}\text { Atomic } \\
\text { Type }\end{array}$} & \multicolumn{3}{|c|}{ Coordinates (Angstroms) } \\
\hline & & & $\mathrm{x}$ & Y & Z \\
\hline & & & & & \\
\hline 1 & 7 & ○ & 5.507943 & -1.914563 & -1.563242 \\
\hline 2 & 6 & $\odot$ & 5.793575 & -3.248048 & -1.585774 \\
\hline 3 & 6 & $\odot$ & 4.803291 & -4.167822 & -1.543874 \\
\hline 4 & 6 & $\odot$ & 3.458591 & -3.677276 & -1.454305 \\
\hline 5 & 7 & $\odot$ & 3.188283 & -2.367898 & -1.462100 \\
\hline 6 & 8 & $\odot$ & 8.730274 & 1.016310 & $-\odot .892760$ \\
\hline 7 & 15 & $\odot$ & 8.064638 & 2.023671 & 0.205477 \\
\hline 8 & 8 & 0 & 6.460989 & 1.896918 & -0.104189 \\
\hline
\end{tabular}

Predicted change in Energy $=-6.384641 \mathrm{D}-\odot 8$

Normal termination of Gaussian 16 at wed Aug 7 04:25:32 2019.

$G($ high $)=-5077.041869 \mathrm{a} \cdot \mathrm{u}$.

-Figure 3-7, Int4(C8)W2(a)

dna. rev3aw2x. $\log$

Stoichiometry C39H54N15Na2023P2(2)

Standard orientation: 


\begin{tabular}{|c|c|c|c|c|c|}
\hline 9 & 8 & $\odot$ & 8.541432 & 3.420445 & -0.112393 \\
\hline 10 & 8 & 0 & 8.270844 & 1.515650 & 1.615201 \\
\hline 11 & 8 & $\odot$ & 4.493727 & 5.494301 & 1.076248 \\
\hline 12 & 6 & 0 & 4.723418 & 3.599423 & -0.351009 \\
\hline 13 & 6 & 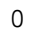 & 5.210354 & 4.239124 & $\odot .953756$ \\
\hline 14 & 6 & $\theta$ & -9.133898 & -1.932524 & 1.768150 \\
\hline 15 & 6 & 0 & -8.614520 & -0.512659 & 1.665868 \\
\hline 16 & 8 & 0 & -8.283938 & -2.806478 & 0.984628 \\
\hline 17 & 8 & $\Theta$ & -8.635497 & 1.343392 & 0.133783 \\
\hline 18 & 15 & 0 & -8.671236 & 2.110503 & -1.308230 \\
\hline 19 & 8 & 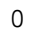 & -7.109444 & 2.529121 & -1.533413 \\
\hline 20 & 8 & 0 & -9.530469 & 3.342171 & -1.133416 \\
\hline 21 & 8 & 0 & -9.041001 & 1.156921 & -2.420201 \\
\hline 22 & 8 & 0 & -4.233029 & 4.617406 & -2.606914 \\
\hline 23 & 6 & $\Theta$ & -4.979701 & 3.347227 & -0.706258 \\
\hline 24 & 6 & 0 & -4.338927 & 3.276269 & -2.089214 \\
\hline 25 & 1 & $\Theta$ & -4.020381 & 4.601900 & -3.554135 \\
\hline 26 & 6 & 0 & 9.616991 & -2.304723 & -2.349524 \\
\hline 27 & 6 & $\odot$ & 8.878338 & $-\odot .989816$ & -2.207035 \\
\hline 28 & 6 & $\Theta$ & 8.783904 & -0.446982 & -0.773807 \\
\hline 29 & 6 & 0 & 7.466245 & -1.024965 & -0.276749 \\
\hline 30 & 6 & 0 & 6.586584 & -0.938512 & -1.522081 \\
\hline 31 & 8 & 0 & 7.486547 & -1.158856 & -2.644355 \\
\hline 32 & 1 & $\odot$ & 9.516675 & -2.653467 & -3.382488 \\
\hline 33 & 1 & 0 & 10.679612 & -2.146390 & -2.132376 \\
\hline 34 & 1 & 0 & 9.345779 & -0.246533 & -2.856566 \\
\hline 35 & 1 & 0 & 9.639442 & -0.714164 & -0.152851 \\
\hline 36 & 1 & 0 & 7.057316 & -0.472582 & $\odot .566500$ \\
\hline 37 & 1 & 0 & 7.633973 & -2.065017 & 0.010651 \\
\hline 38 & 1 & 0 & 6.120795 & 0.037678 & -1.611295 \\
\hline 39 & 8 & 0 & 9.047148 & -3.267791 & -1.428891 \\
\hline 40 & 1 & 0 & 9.487893 & -4.129016 & -1.510674 \\
\hline 41 & 6 & 0 & 4.184093 & -1.453869 & -1.550924 \\
\hline 42 & 7 & 0 & 2.428422 & -4.516631 & -1.356425 \\
\hline 43 & 8 & 0 & 3.964154 & $-\odot .237936$ & -1.602068 \\
\hline 44 & 1 & 0 & 6.853031 & -3.493794 & -1.635569 \\
\hline 45 & 1 & 0 & 5.018921 & -5.227162 & -1.562598 \\
\hline 46 & 1 & 0 & 2.578118 & -5.511168 & -1.327481 \\
\hline 47 & 1 & 0 & 1.480415 & -4.147198 & -1.279831 \\
\hline 48 & 6 & 0 & 5.759036 & 2.749354 & -1.059889 \\
\hline 49 & 1 & 0 & 4.732617 & 5.940359 & 1.906706 \\
\hline 50 & 6 & 0 & 4.774084 & 3.239583 & 2.030641 \\
\hline 51 & 6 & 0 & 3.449549 & 2.705064 & 1.477456 \\
\hline 52 & 8 & 0 & 3.570615 & 2.778650 & ๑. 025369 \\
\hline 53 & 1 & 0 & 5.261491 & 2.092229 & -1.774237 \\
\hline 54 & 1 & 0 & 6.478507 & 3.393419 & -1.570912 \\
\hline 55 & 1 & 0 & 4.367886 & 4.374100 & -1.034811 \\
\hline 56 & 1 & 0 & 6.291284 & 4.407552 & 0.944622 \\
\hline 57 & 1 & 0 & 4.640172 & 3.712137 & 3.005819 \\
\hline 58 & 1 & 0 & 5.528975 & 2.456340 & 2.120842 \\
\hline 59 & 1 & 0 & 2.610543 & 3.324684 & 1.786937 \\
\hline 60 & 7 & 0 & 3.099706 & 1.341475 & 1.841415 \\
\hline 61 & 6 & 0 & 3.994683 & $\odot .308679$ & 1.647863 \\
\hline 62 & 6 & 0 & 3.678607 & -0.988039 & 1.847382 \\
\hline 63 & 6 & 0 & 2.296415 & -1.311075 & 2.160512 \\
\hline 64 & 7 & 0 & 1.455364 & -0.231950 & 2.328583 \\
\hline 65 & 6 & 0 & 1.770495 & 1.092364 & 2.173122 \\
\hline 66 & 6 & $\odot$ & 4.654490 & -2.120516 & 1.765055 \\
\hline 67 & 8 & 0 & 1.867020 & -2.462657 & 2.268437 \\
\hline 68 & 8 & 0 & 0.941571 & 1.979950 & 2.324089 \\
\hline 69 & 1 & 0 & 4.978495 & 0.618423 & 1.308969 \\
\hline 70 & 1 & 0 & 4.700968 & -2.649152 & 2.722010 \\
\hline 71 & 1 & 0 & 4.354348 & -2.853698 & 1.012663 \\
\hline 72 & 1 & $\odot$ & 5.655946 & -1.764547 & 1.528349 \\
\hline 73 & 1 & 0 & 0.446270 & -0.436437 & 2.516795 \\
\hline 74 & 1 & 0 & -8.612777 & -3.719861 & 1.005636 \\
\hline 75 & 6 & 0 & -8.273335 & $-\odot .073947$ & 0.238507 \\
\hline 76 & 6 & 0 & -6.767460 & -0.290475 & 0.124327 \\
\hline 77 & 6 & 0 & -6.243318 & $-\odot .131519$ & 1.558003 \\
\hline 78 & 8 & $\odot$ & -7.380090 & -0.355124 & 2.436971 \\
\hline 79 & 1 & 0 & -9.139145 & -2.236001 & 2.820201 \\
\hline 80 & 1 & 0 & -10.161317 & -1.961545 & 1.386707 \\
\hline 81 & 1 & 0 & -9.354900 & 0.164344 & 2.098088 \\
\hline 82 & 1 & 0 & -8.833081 & -0.628544 & -0.515772 \\
\hline 83 & 1 & 0 & -6.277907 & 0.424589 & -0.536595 \\
\hline 84 & 1 & 0 & -6.585007 & -1.304981 & -0.235614 \\
\hline 85 & 1 & 0 & -5.833167 & $\odot .863774$ & 1.731041 \\
\hline
\end{tabular}




\begin{tabular}{|c|c|c|c|c|c|}
\hline 86 & 7 & 0 & -5.191093 & -1.075704 & 1.883724 \\
\hline 87 & 6 & $\theta$ & -5.248317 & -2.443296 & 1.740903 \\
\hline 88 & 7 & 0 & -4.100370 & -3.037866 & 1.939348 \\
\hline 89 & 6 & $\theta$ & -3.233929 & -1.999359 & 2.226515 \\
\hline 90 & 6 & 0 & -3.896408 & -0.776772 & 2.221747 \\
\hline 91 & 7 & 0 & -3.363627 & 0.425585 & 2.491557 \\
\hline 92 & 6 & 0 & -2.060739 & $\odot .340361$ & $2.7 \odot 4 \odot 56$ \\
\hline 93 & 7 & $\theta$ & -1.281800 & -0.753059 & 2.656357 \\
\hline 94 & 6 & $\odot$ & -1.835221 & -1.955841 & 2.392646 \\
\hline 95 & 7 & $\theta$ & -1.048042 & -3.037403 & 2.288889 \\
\hline 96 & 1 & 0 & -6.190025 & -2.915431 & 1.485088 \\
\hline 97 & 1 & $\theta$ & -1.528897 & 1.264389 & 2.917670 \\
\hline 98 & 1 & 0 & -0.039677 & -2.885751 & 2.289082 \\
\hline 99 & 1 & 0 & -1.417427 & -3.848092 & 1.791995 \\
\hline 100 & 6 & $\odot$ & -6.476535 & 3.501115 & -0.651070 \\
\hline 101 & 11 & 0 & 9.104663 & 3.687390 & 2.396064 \\
\hline 102 & 11 & $\odot$ & -10.940090 & 2.688362 & -3.065364 \\
\hline 103 & 6 & 0 & -2.989633 & 2.596099 & -1.804811 \\
\hline 104 & 6 & $\odot$ & -3.218967 & 1.786504 & -0.495189 \\
\hline 105 & 8 & 0 & -4.593515 & 2.095137 & -0.088624 \\
\hline 106 & 1 & $\odot$ & -6.820111 & 3.347115 & 0.376145 \\
\hline 107 & 1 & $\odot$ & -6.753024 & 4.506697 & -0.978011 \\
\hline 108 & 1 & $\odot$ & -4.532967 & 4.193378 & -0.162203 \\
\hline 109 & 1 & 0 & -4.961660 & 2.658627 & -2.743307 \\
\hline 110 & 1 & $\odot$ & -2.229498 & 3.365172 & -1.645933 \\
\hline 111 & 1 & 0 & -2.663591 & 1.946246 & -2.618680 \\
\hline 112 & 1 & 0 & -2.546815 & 2.133312 & $\odot .297654$ \\
\hline 113 & 7 & $\odot$ & -3.123272 & 0.366301 & -0.651532 \\
\hline 114 & 6 & $\odot$ & -4.085003 & -2.241941 & -1.369961 \\
\hline 115 & 7 & $\odot$ & -2.819300 & -2.450257 & -0.908072 \\
\hline 116 & 6 & $\odot$ & -1.775639 & -1.552185 & $-\odot .897700$ \\
\hline 117 & 6 & 0 & -1.913848 & -0.121959 & -0.760638 \\
\hline 118 & 7 & $\odot$ & -0.794382 & $\odot .678503$ & -0.744263 \\
\hline 119 & 6 & $\odot$ & $\odot .386640$ & $\odot .125929$ & -0.858413 \\
\hline 120 & 7 & $\odot$ & $\odot .583343$ & -1.237195 & -0.985022 \\
\hline 121 & 6 & $\odot$ & -0.446416 & -2.130786 & -1.008842 \\
\hline 122 & 7 & $\odot$ & 1.498987 & ๑.862695 & $-\odot .852912$ \\
\hline 123 & 8 & 0 & -0.240231 & -3.346385 & -1.147170 \\
\hline 124 & 1 & $\odot$ & -4.261147 & -1.244441 & -1.777646 \\
\hline 125 & 1 & $\odot$ & 1.424866 & 1.859158 & $-\odot .727976$ \\
\hline 126 & 1 & $\odot$ & 2.423389 & $\odot .467389$ & -1.051201 \\
\hline 127 & 1 & $\odot$ & 1.539128 & -1.625165 & -1.132640 \\
\hline 128 & 8 & 0 & -4.951072 & -3.109735 & -1.330567 \\
\hline 129 & 1 & $\odot$ & -4.597606 & -4.808730 & -0.817416 \\
\hline 130 & 8 & $\odot$ & -4.281980 & -5.719419 & -0.651653 \\
\hline 131 & 1 & $\odot$ & -2.685016 & -5.428977 & 0.003690 \\
\hline 132 & 1 & $\odot$ & -4.208661 & -6.109976 & -1.527661 \\
\hline 133 & 8 & $\odot$ & -1.862161 & -5.049919 & 0.380624 \\
\hline 134 & 1 & 0 & -2.609073 & -3.380763 & -0.535089 \\
\hline 135 & 1 & $\odot$ & -1.256983 & -4.864214 & -0.349380 \\
\hline
\end{tabular}

General basis read from cards: (6D, 7F)

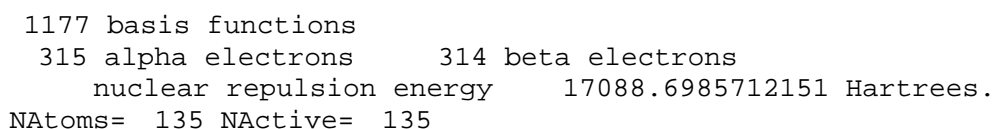

Force inversion solution in PCM.

Polarizable Continuum Model (PCM)

ニニニニニニニニニニニニニニニニニニニニニニニニニニニニニニ=
Model
:PCM.

Solvent : Water, Eps $=78.355300$ Eps $($ inf $)=1.777849$

SCF Done: $E($ UwB97XD $)=-5076.23573344$ A.U. after 1 cycles

NFock $=1$ Conv $=0.32 \mathrm{D}-08 \quad-\mathrm{V} / \mathrm{T}=2.0066$

$<\mathrm{S} x>=0.0000<\mathrm{Sy}\rangle=0.0000<\mathrm{Sz}>=0.5000$

$\left\langle S^{* *} 2\right\rangle=0.7783 \mathrm{~S}=0.5141$

$<$ L.S $>=0.000000000000 \mathrm{E}+\odot \odot$

Annihilation of the first spin contaminant:

$\mathrm{S}^{* * 2}$ before annihilation 0.7783 , after 0.7503

Harmonic frequencies $\left(\mathrm{cm}^{*}-1\right)$, IR intensities (KM/Mole), Raman 


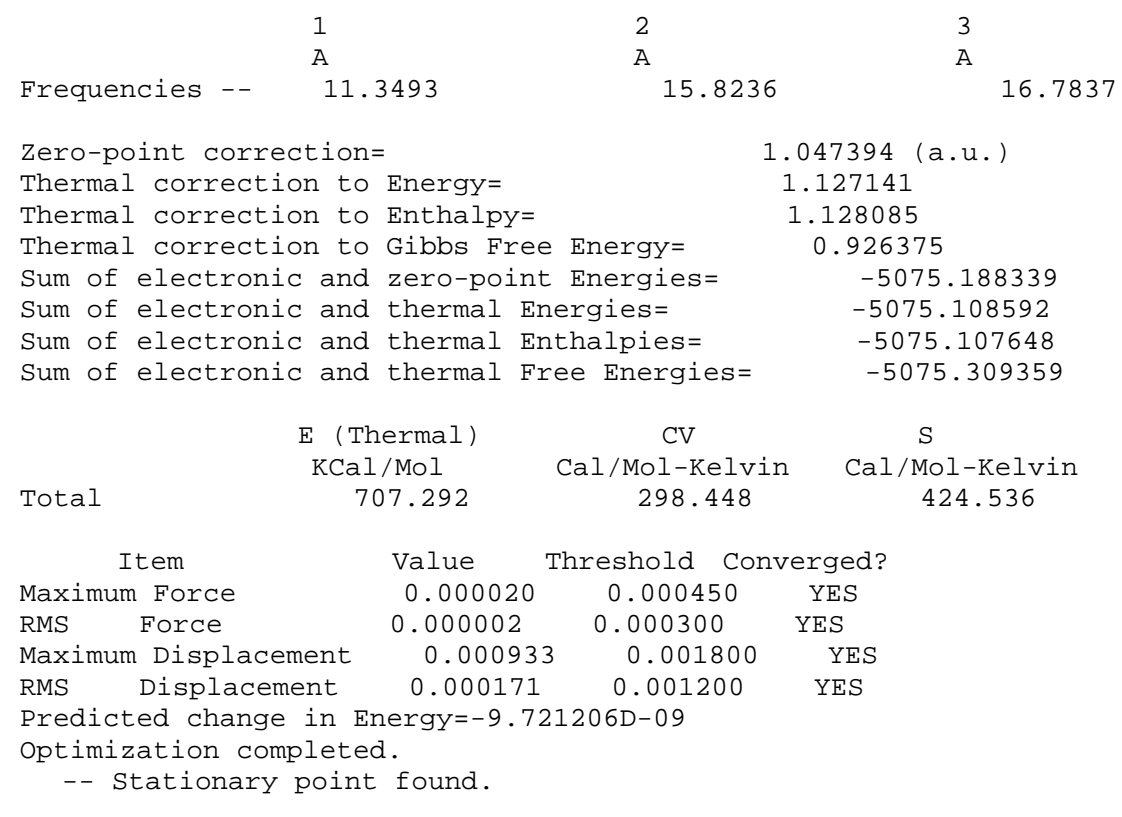

Normal termination of Gaussian 16 at Mon Aug 12 04:01:38 2019.

$\mathrm{G}(\mathrm{high})=-5077.0709425 \mathrm{a} \cdot \mathrm{u}$.

============= Figure S10 for (b) $5^{\prime}$ - GG - $3^{\prime}$ ======================

S10-1, Int1(C4)(b) (b)

dna@4b-oh1afor . log

Stoichiometry C38H49N16Na2021P2(2)

Standard orientation:

\begin{tabular}{|c|c|c|c|c|c|}
\hline \multirow{2}{*}{$\begin{array}{l}\text { Center } \\
\text { Number }\end{array}$} & \multirow{2}{*}{$\begin{array}{l}\text { Atomic } \\
\text { Number }\end{array}$} & \multirow{2}{*}{$\begin{array}{c}\text { Atomic } \\
\text { Type }\end{array}$} & \multicolumn{3}{|c|}{ Coordinates (Angstroms) } \\
\hline & & & $x$ & $Y$ & Z \\
\hline 1 & 7 & $\odot$ & 5.155178 & -2.113657 & -1.653417 \\
\hline 2 & 6 & $\odot$ & 5.173998 & -3.468300 & -1.478108 \\
\hline 3 & 6 & 0 & 4.055957 & -4.205574 & -1.662206 \\
\hline 4 & 6 & $\odot$ & 2.865556 & -3.502307 & -2.045783 \\
\hline 5 & 7 & $\odot$ & 2.870757 & -2.182188 & -2.252847 \\
\hline 6 & 8 & 0 & 8.499378 & 0.350788 & -0.331230 \\
\hline 7 & 15 & $\odot$ & 7.812405 & 1.568660 & 0.514929 \\
\hline 8 & 8 & $\odot$ & 6.292510 & 1.621104 & -0.117183 \\
\hline 9 & 8 & 0 & 8.541578 & 2.833560 & 0.167989 \\
\hline 10 & 8 & $\odot$ & 7.632034 & 1.125319 & 1.952073 \\
\hline 11 & 8 & $\odot$ & 4.620434 & 5.389888 & 1.091748 \\
\hline 12 & 6 & $\odot$ & 4.812394 & 3.553918 & -0.411059 \\
\hline 13 & 6 & $\odot$ & 5.224842 & 4.078102 & 0.967210 \\
\hline 14 & 6 & $\odot$ & -9.041714 & -3.513257 & 1.047198 \\
\hline 15 & 6 & $\odot$ & -8.620512 & -2.057998 & 1.073664 \\
\hline 16 & 8 & $\odot$ & -7.936530 & -4.325426 & 0.585429 \\
\hline 17 & 8 & $\odot$ & -8.333961 & $-\odot .070012$ & -0.218227 \\
\hline 18 & 15 & $\odot$ & -7.562224 & 1.064704 & -1.106282 \\
\hline 19 & 8 & $\odot$ & -6.160707 & 1.287435 & -0.294773 \\
\hline 20 & 8 & $\odot$ & -8.401533 & 2.314653 & -0.974670 \\
\hline 21 & 8 & $\odot$ & -7.163021 & $\odot .515071$ & -2.449857 \\
\hline 22 & 8 & $\odot$ & -4.687748 & 5.320002 & -0.869437 \\
\hline 23 & 6 & $\odot$ & -4.925798 & 3.281554 & 0.332660 \\
\hline 24 & 6 & $\odot$ & -5.251867 & 3.989554 & -0.982875 \\
\hline 25 & 1 & $\odot$ & -4.826666 & 5.818205 & -1.692763 \\
\hline 26 & 6 & $\odot$ & 9.189270 & -3.220594 & -1.187842 \\
\hline 27 & 6 & $\odot$ & 8.683949 & -1.802471 & -1.358959 \\
\hline 28 & 6 & $\odot$ & 8.299019 & -1.077384 & $-\odot .061740$ \\
\hline 29 & 6 & $\odot$ & 6.821582 & -1.416415 & $\odot .086399$ \\
\hline 30 & 6 & $\odot$ & 6.341382 & -1.324506 & -1.361046 \\
\hline 31 & 8 & $\odot$ & 7.455323 & -1.810787 & -2.163619 \\
\hline 32 & 1 & $\odot$ & 9.317467 & -3.667580 & -2.179108 \\
\hline 33 & 1 & $\odot$ & 10.162256 & -3.197046 & -0.683572 \\
\hline
\end{tabular}




\begin{tabular}{|c|c|c|c|c|c|}
\hline 34 & 1 & 0 & 9.434852 & -1.222261 & -1.899681 \\
\hline 35 & 1 & $\odot$ & 8.905339 & -1.366884 & 0.797263 \\
\hline 36 & 1 & $\odot$ & 6.298595 & $-\odot .740506$ & 0.758536 \\
\hline 37 & 1 & 0 & 6.738843 & -2.438593 & 0.461126 \\
\hline 38 & 1 & $\odot$ & 6.108274 & $-\odot .299772$ & -1.638157 \\
\hline 39 & 8 & $\odot$ & 8.231244 & -3.979070 & $-\odot .408681$ \\
\hline 40 & 1 & 0 & 8.510859 & -4.904937 & $-\odot .323275$ \\
\hline 41 & 6 & $\odot$ & 3.997401 & -1.453496 & -2.085213 \\
\hline 42 & 7 & $\odot$ & 1.710887 & -4.144924 & -2.216452 \\
\hline 43 & 8 & 0 & 4.026624 & $-\odot .229580$ & -2.275618 \\
\hline 44 & 1 & $\odot$ & 6.137083 & -3.883707 & -1.186767 \\
\hline 45 & 1 & 0 & 4.061129 & -5.278252 & -1.526325 \\
\hline 46 & 1 & 0 & 1.651610 & -5.137602 & -2.062475 \\
\hline 47 & 1 & 0 & 0.873385 & -3.628199 & -2.496530 \\
\hline 48 & 6 & $\odot$ & 5.818789 & 2.621376 & -1.063414 \\
\hline 49 & 1 & 0 & 4.826259 & 5.776886 & 1.959679 \\
\hline 50 & 6 & $\odot$ & 4.594839 & 3.074917 & 1.938995 \\
\hline 51 & 6 & 0 & 3.291839 & 2.671012 & 1.239370 \\
\hline 52 & 8 & 0 & 3.534734 & 2.865871 & -0.185566 \\
\hline 53 & 1 & $\odot$ & 5.343221 & 2.079328 & -1.882153 \\
\hline 54 & 1 & 0 & 6.668146 & 3.200549 & -1.433995 \\
\hline 55 & 1 & 0 & 4.612951 & 4.389598 & -1.085630 \\
\hline 56 & 1 & $\odot$ & 6.312793 & 4.139302 & 1.069717 \\
\hline 57 & 1 & 0 & 4.407852 & 3.509911 & 2.922998 \\
\hline 58 & 1 & 0 & 5.260207 & 2.216200 & 2.055617 \\
\hline 59 & 1 & 0 & 2.447225 & 3.286201 & 1.538894 \\
\hline 60 & 7 & 0 & 2.889243 & 1.284482 & 1.451894 \\
\hline 61 & 6 & 0 & 3.707890 & $\odot .281550$ & 1.021255 \\
\hline 62 & 6 & 0 & 3.338325 & -1.012753 & 1.124782 \\
\hline 63 & 6 & 0 & 2.048313 & -1.281020 & 1.687221 \\
\hline 64 & 7 & 0 & 1.234337 & -0.293562 & 2.068000 \\
\hline 65 & 6 & 0 & 1.596049 & 1.004692 & 1.924424 \\
\hline 66 & 1 & 0 & 3.986991 & -1.814781 & 0.799546 \\
\hline 67 & 7 & 0 & 1.627073 & -2.535583 & 1.843276 \\
\hline 68 & 8 & 0 & $\odot .835458$ & 1.938611 & 2.206642 \\
\hline 69 & 1 & 0 & 4.649535 & 0.603298 & $\odot .583711$ \\
\hline 70 & 1 & 0 & 2.221830 & -3.302657 & 1.577016 \\
\hline 71 & 1 & 0 & $\odot .734412$ & -2.729116 & 2.309804 \\
\hline 72 & 1 & 0 & -0.537118 & -0.644958 & 2.641632 \\
\hline 73 & 1 & 0 & -8.161276 & -5.268907 & ๑. 629831 \\
\hline 74 & 6 & 0 & -8.038366 & -1.507792 & $-\odot .237171$ \\
\hline 75 & 6 & 0 & -6.549271 & -1.786656 & -0.081216 \\
\hline 76 & 6 & 0 & -6.329665 & -1.470273 & 1.395617 \\
\hline 77 & 8 & 0 & -7.554702 & -1.868908 & 2.066734 \\
\hline 78 & 1 & 0 & -9.338614 & -3.806008 & 2.060025 \\
\hline 79 & 1 & 0 & -9.907854 & -3.622788 & $\odot .383337$ \\
\hline 80 & 1 & 0 & -9.473883 & -1.450197 & 1.382518 \\
\hline 81 & 1 & $\odot$ & -8.480260 & -1.950353 & -1.130774 \\
\hline 82 & 1 & 0 & -5.911122 & -1.191569 & $-\odot .730052$ \\
\hline 83 & 1 & 0 & -6.376655 & -2.849332 & $-\odot .262419$ \\
\hline 84 & 1 & 0 & -6.160987 & -0.404248 & 1.546596 \\
\hline 85 & 7 & 0 & -5.201892 & -2.150973 & 1.982159 \\
\hline 86 & 6 & 0 & -5.035478 & -3.499191 & 2.221937 \\
\hline 87 & 7 & $\odot$ & -3.828000 & -3.798241 & 2.622358 \\
\hline 88 & 6 & 0 & -3.157383 & -2.583702 & 2.632810 \\
\hline 89 & 6 & 0 & -3.996819 & -1.554599 & 2.229664 \\
\hline $9 \odot$ & 7 & 0 & -3.721144 & $-\odot .253605$ & 2.043964 \\
\hline 91 & 6 & 0 & -2.444043 & $\odot .028686$ & 2.252071 \\
\hline 92 & 7 & 0 & -1.537971 & -0.895978 & 2.712696 \\
\hline 93 & 6 & 0 & -1.786663 & -2.261891 & 2.862357 \\
\hline 94 & 8 & 0 & $-\odot .860019$ & -3.035224 & 3.146407 \\
\hline 95 & 1 & 0 & -5.860099 & -4.182453 & 2.067251 \\
\hline 96 & 7 & 0 & -2.002664 & 1.280338 & 2.051734 \\
\hline 97 & 1 & 0 & -2.584501 & 1.851746 & 1.437338 \\
\hline 98 & 1 & 0 & -1.002752 & 1.480213 & 2.094575 \\
\hline 99 & 6 & 0 & -5.936399 & 2.242852 & $\odot .773949$ \\
\hline 100 & 11 & 0 & 7.764584 & 2.217900 & 3.875813 \\
\hline 101 & 11 & 0 & -9.402373 & 4.032417 & -1.895340 \\
\hline 102 & 6 & 0 & -4.533773 & 3.154869 & -2.049154 \\
\hline 103 & 6 & $\odot$ & -3.322902 & 2.557441 & -1.317767 \\
\hline 104 & 8 & 0 & -3.620786 & 2.655251 & 0.104358 \\
\hline 105 & 1 & $\odot$ & -5.530303 & 1.686921 & 1.620694 \\
\hline 106 & 1 & $\odot$ & -6.874967 & 2.731087 & 1.050225 \\
\hline 107 & 1 & $\odot$ & -4.792424 & 4.016469 & 1.129498 \\
\hline 108 & 1 & $\odot$ & -6.330567 & 4.036414 & -1.160099 \\
\hline 109 & 1 & 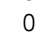 & -4.223129 & 3.763214 & -2.900903 \\
\hline 110 & 1 & 0 & -5.204014 & 2.375126 & -2.412217 \\
\hline
\end{tabular}

S181 


\begin{tabular}{|c|c|c|c|c|c|}
\hline 111 & 1 & $\odot$ & -2.407904 & 3.125769 & -1.487964 \\
\hline 112 & 7 & $\odot$ & -3.020960 & 1.188660 & -1.688182 \\
\hline 113 & 6 & $\odot$ & -3.875877 & $\odot .171958$ & -2.092088 \\
\hline 114 & 7 & $\odot$ & -3.253608 & -0.933102 & -2.397404 \\
\hline 115 & 6 & $\odot$ & -1.918957 & -0.664163 & -2.147308 \\
\hline 116 & 6 & $\odot$ & -1.756017 & 0.667564 & -1.729334 \\
\hline 117 & 7 & $\odot$ & -0.627219 & 1.325157 & -1.463694 \\
\hline 118 & 6 & $\odot$ & $\odot .460454$ & 0.590639 & -1.700118 \\
\hline 119 & 7 & $\odot$ & 0.418709 & -0.692671 & -2.182899 \\
\hline 120 & 6 & $\odot$ & -0.734864 & -1.424703 & -2.451807 \\
\hline 121 & 7 & $\odot$ & 1.670883 & 1.101773 & -1.487164 \\
\hline 122 & 8 & $\odot$ & -0.660711 & -2.567069 & -2.908174 \\
\hline 123 & 1 & $\odot$ & -4.947607 & 0.320417 & -2.154567 \\
\hline 124 & 1 & $\odot$ & 1.778743 & $2 . \odot 4 \odot 803$ & -1.132737 \\
\hline 125 & 1 & $\odot$ & 2.524192 & $\odot .605844$ & -1.760051 \\
\hline 126 & 1 & $\odot$ & 1.313725 & -1.204759 & -2.301322 \\
\hline 127 & 8 & 0 & -1.623223 & -1.552555 & -0.113659 \\
\hline 128 & 1 & $\odot$ & -2.501282 & -1.942690 & -0.236706 \\
\hline
\end{tabular}

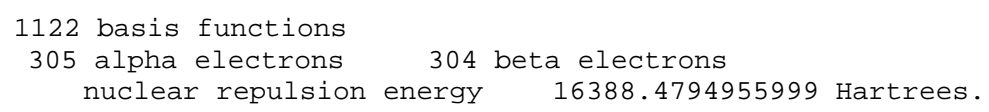

$\mathrm{CV}$

Cal/Mol-Kelvin Cal/Mol-Kelvin

Total 664.678 280.004 393.358

\begin{tabular}{lccc}
\multicolumn{1}{c}{ Item } & Value & Threshold Converged? \\
Maximum Force & 0.000027 & 0.000450 & YES \\
RMS Force & 0.000004 & 0.000300 & YES \\
Maximum Displacement & 0.003072 & 0.001800 & NO \\
RMS Displacement & 0.000456 & 0.001200 & YES
\end{tabular}

Normal termination of Gaussian 16 at Mon Feb 18 21:50:19 2019.

-.... Figure S10-2, TS1(C4)(b)

dna@4b-oh1ats. log

Stoichiometry C38H49N16Na2021P2(2)

Standard orientation:

\begin{tabular}{|c|c|c|c|c|c|}
\hline \multirow{2}{*}{$\begin{array}{l}\text { Center } \\
\text { Number }\end{array}$} & \multirow{2}{*}{$\begin{array}{l}\text { Atomic } \\
\text { Number }\end{array}$} & \multirow{2}{*}{$\begin{array}{c}\text { Atomic } \\
\text { Type }\end{array}$} & \multicolumn{3}{|c|}{ Coordinates (Angstroms) } \\
\hline & & & $x$ & $\mathrm{Y}$ & Z \\
\hline-- & --- & -- & ------- & ----- & - - - - - - \\
\hline 1 & 7 & $\odot$ & 5.173784 & -2.155417 & -1.723206 \\
\hline 2 & 6 & $\odot$ & 5.159033 & -3.500998 & -1.488154 \\
\hline 3 & 6 & $\odot$ & 4.047214 & -4.232084 & -1.727197 \\
\hline 4 & 6 & $\odot$ & 2.897646 & -3.529533 & -2.221912 \\
\hline 5 & 7 & $\odot$ & 2.941464 & -2.223042 & -2.498541 \\
\hline 6 & 8 & $\odot$ & 8.355069 & 0.365610 & -0.172198 \\
\hline 7 & 15 & $\odot$ & 7.599479 & 1.601685 & 0.583992 \\
\hline 8 & 8 & $\odot$ & 6.127269 & 1.616939 & -0.156875 \\
\hline 9 & 8 & $\odot$ & 8.337878 & 2.864493 & ๑. 248572 \\
\hline 10 & 8 & $\odot$ & 7.329543 & 1. 200312 & 2.019815 \\
\hline 11 & 8 & $\odot$ & 4.360948 & 5.366756 & 1.026285 \\
\hline 12 & 6 & $\odot$ & 4.638087 & 3.541382 & -0.475831 \\
\hline 13 & 6 & $\odot$ & 4.991726 & 4.066281 & ๑. 918077 \\
\hline 14 & 6 & $\odot$ & -8.952774 & -3.569799 & ๑. 792484 \\
\hline
\end{tabular}




\begin{tabular}{|c|c|c|c|c|c|}
\hline 15 & 6 & $\odot$ & -8.550783 & -2.114055 & $\odot .913536$ \\
\hline 16 & 8 & 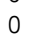 & -7.809963 & -4.349136 & 0.367399 \\
\hline 17 & 8 & $\odot$ & -8.204015 & $-\odot .066828$ & -0.269133 \\
\hline 18 & 15 & $\odot$ & -7.415939 & 1.093508 & -1.108929 \\
\hline 19 & 8 & $\odot$ & -6.030067 & 1.291734 & $-\odot .267416$ \\
\hline 20 & 8 & $\odot$ & -8.259077 & 2.340722 & $-\odot .967157$ \\
\hline 21 & 8 & $\odot$ & -6.988352 & 0.580217 & -2.458719 \\
\hline 22 & 8 & $\odot$ & -4.392046 & 5.312204 & $-\odot .502816$ \\
\hline 23 & 6 & $\odot$ & -4.760942 & 3.197035 & 0.521086 \\
\hline 24 & 6 & $\odot$ & -5.023047 & 4.027993 & -0.735627 \\
\hline 25 & 1 & $\odot$ & -4.491534 & 5.885150 & -1.282155 \\
\hline 26 & 6 & $\odot$ & 9.151799 & -3.231616 & $-\odot .808597$ \\
\hline 27 & 6 & $\odot$ & 8.659892 & -1.825892 & -1.087034 \\
\hline 28 & 6 & $\odot$ & 8.142214 & -1.053649 & 0.134779 \\
\hline 29 & 6 & $\odot$ & 6.659431 & -1.398235 & 0.146772 \\
\hline 30 & 6 & $\odot$ & 6.331472 & -1.360685 & -1.344619 \\
\hline 31 & 8 & $\odot$ & 7.517919 & -1.879865 & -2.009585 \\
\hline 32 & 1 & $\odot$ & 9.385984 & -3.714775 & -1.762850 \\
\hline 33 & 1 & $\odot$ & 10.066282 & -3.180296 & -0.206341 \\
\hline 34 & 1 & $\odot$ & 9.458014 & -1.260619 & -1.573219 \\
\hline 35 & 1 & $\odot$ & 8.661282 & -1.301358 & 1.061232 \\
\hline 36 & 1 & $\odot$ & 6.065981 & $-\odot .700593$ & $\odot .732674$ \\
\hline 37 & 1 & $\odot$ & 6.541442 & -2.406832 & $\odot .548069$ \\
\hline 38 & 1 & $\odot$ & 6.136315 & $-\odot .345952$ & -1.681712 \\
\hline 39 & 8 & $\odot$ & 8.120245 & -3.967726 & -0.105748 \\
\hline 40 & 1 & $\odot$ & 8.390449 & -4.889165 & ๑.037986 \\
\hline 41 & 6 & $\odot$ & 4.067218 & -1.504427 & -2.284712 \\
\hline 42 & 7 & $\odot$ & 1.742714 & -4.161996 & -2.429078 \\
\hline 43 & 8 & $\odot$ & 4.138118 & $-\odot .294057$ & -2.539488 \\
\hline 44 & 1 & $\odot$ & 6.088871 & -3.912992 & -1.100582 \\
\hline 45 & 1 & $\odot$ & 4.024633 & -5.297176 & -1.542471 \\
\hline 46 & 1 & $\odot$ & 1.647871 & -5.138562 & -2.205154 \\
\hline 47 & 1 & 0 & $\odot .921576$ & -3.638478 & -2.738740 \\
\hline 48 & 6 & $\odot$ & 5.681960 & 2.631543 & -1.100890 \\
\hline 49 & 1 & $\odot$ & 4.530103 & 5.753719 & 1.902139 \\
\hline 50 & 6 & $\odot$ & 4.345372 & 3.046551 & 1.860338 \\
\hline 51 & 6 & $\odot$ & 3.070847 & 2.630161 & 1.117838 \\
\hline 52 & 8 & $\odot$ & 3.366348 & 2.826072 & -0.293418 \\
\hline 53 & 1 & 0 & 5.249454 & 2.098743 & -1.949272 \\
\hline 54 & 1 & $\odot$ & 6.538101 & 3.227881 & -1.426023 \\
\hline 55 & 1 & $\odot$ & 4.441345 & 4.375558 & -1.152675 \\
\hline 56 & 1 & $\odot$ & 6.074152 & 4.145548 & 1.059473 \\
\hline 57 & 1 & $\odot$ & 4.125855 & 3.465663 & 2.844510 \\
\hline 58 & 1 & $\odot$ & 5.014233 & 2.190595 & 1.980852 \\
\hline 59 & 1 & 0 & 2.207457 & 3.232290 & 1.389935 \\
\hline 60 & 7 & $\odot$ & 2.686276 & 1.233324 & 1.320799 \\
\hline 61 & 6 & $\odot$ & 3.454368 & $\odot .241787$ & $\odot .786318$ \\
\hline 62 & 6 & $\odot$ & 3.120512 & -1.057072 & $\odot .944999$ \\
\hline 63 & 6 & $\odot$ & 1.934132 & -1.340257 & 1.696580 \\
\hline 64 & 7 & $\odot$ & 1.187141 & -0.364587 & 2.218081 \\
\hline 65 & 6 & 0 & 1.508482 & 0.938276 & 2.026395 \\
\hline 66 & 1 & $\odot$ & 3.725783 & -1.851831 & 0.531230 \\
\hline 67 & 7 & $\odot$ & 1.552962 & -2.600743 & 1.903260 \\
\hline 68 & 8 & $\odot$ & 0.813147 & 1.864787 & 2.460595 \\
\hline 69 & 1 & $\odot$ & 4.331365 & $\odot .572792$ & $\odot .238214$ \\
\hline 70 & 1 & $\odot$ & 2.082638 & -3.359487 & 1.507471 \\
\hline 71 & 1 & $\odot$ & $\odot .706992$ & -2.804987 & 2.443824 \\
\hline 72 & 1 & $\odot$ & -0.560812 & -0.714231 & 2.868721 \\
\hline 73 & 1 & $\odot$ & -8.025806 & -5.295622 & 0.352360 \\
\hline 74 & 6 & $\odot$ & -7.890105 & -1.499907 & -0.329659 \\
\hline 75 & 6 & $\odot$ & -6.410242 & -1.766636 & -0.086426 \\
\hline 76 & 6 & $\odot$ & -6.295924 & -1.517674 & 1.415555 \\
\hline 77 & 8 & $\odot$ & -7.554260 & -1.964501 & 1.983119 \\
\hline 78 & 1 & $\odot$ & -9.311966 & -3.912523 & 1.768765 \\
\hline 79 & 1 & $\odot$ & -9.772154 & -3.655170 & 0.068511 \\
\hline 80 & 1 & $\odot$ & -9.428559 & -1.527397 & 1.193387 \\
\hline 81 & 1 & $\odot$ & -8.264247 & -1.908009 & -1.269387 \\
\hline 82 & 1 & $\odot$ & -5.740333 & -1.131457 & -0.661721 \\
\hline 83 & 1 & $\odot$ & -6.208240 & -2.817270 & -0.303410 \\
\hline 84 & 1 & $\odot$ & -6.155870 & -0.458252 & 1.630057 \\
\hline 85 & 7 & $\odot$ & -5.196589 & -2.212504 & 2. 041784 \\
\hline 86 & 6 & $\odot$ & -5.027215 & -3.568832 & 2.226824 \\
\hline 87 & 7 & 0 & -3.834728 & -3.877353 & 2.663536 \\
\hline 88 & 6 & $\odot$ & -3.176195 & -2.660073 & 2.755807 \\
\hline 89 & 6 & $\odot$ & -4.008675 & -1.620766 & 2.366957 \\
\hline 90 & 7 & $\odot$ & -3.736329 & -0.307899 & 2.255329 \\
\hline 91 & 6 & $\odot$ & -2.472106 & $-\odot .021384$ & 2.524881 \\
\hline
\end{tabular}

S183 


\begin{tabular}{|c|c|c|c|c|c|}
\hline 92 & 7 & 0 & -1.562090 & -0.971366 & 2.920254 \\
\hline 93 & 6 & $\odot$ & -1.811394 & -2.343430 & 3.025380 \\
\hline 94 & 8 & $\odot$ & -0.886563 & -3.123640 & 3.291653 \\
\hline 95 & 1 & 0 & -5.837478 & -4.250764 & 2.005342 \\
\hline 96 & 7 & 0 & -2.051415 & $1.2556 \odot 2$ & 2.443054 \\
\hline 97 & 1 & $\odot$ & -2.568317 & 1.800086 & 1.749324 \\
\hline 98 & 1 & 0 & -1.045341 & 1.428477 & 2.472535 \\
\hline 99 & 6 & $\odot$ & -5.831999 & 2.182055 & 0.861087 \\
\hline 100 & 11 & 0 & 7.293565 & 2.285686 & 3.951221 \\
\hline 101 & 11 & 0 & -8.918529 & 3.951528 & -2.330057 \\
\hline 102 & 6 & $\odot$ & -4.327672 & 3.247196 & -1.854304 \\
\hline 103 & 6 & 0 & -3.156578 & 2.539378 & -1.157549 \\
\hline 104 & 8 & 0 & -3.490399 & 2.515221 & 0.263759 \\
\hline 105 & 1 & 0 & -5.489092 & 1.572606 & 1.698984 \\
\hline 106 & 1 & $\odot$ & -6.765862 & 2.691615 & 1.113945 \\
\hline 107 & 1 & 0 & -4.606201 & 3.857408 & 1.377472 \\
\hline 108 & 1 & $\Theta$ & -6.094183 & 4.148942 & -0.924138 \\
\hline 109 & 1 & 0 & -3.976997 & 3.902448 & -2.654143 \\
\hline 110 & 1 & 0 & -5.026247 & 2.527532 & -2.282510 \\
\hline 111 & 1 & 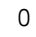 & -2.219564 & 3.089938 & -1.250343 \\
\hline 112 & 7 & $\odot$ & -2.888703 & 1.212207 & -1.664994 \\
\hline 113 & 6 & 0 & -3.758088 & 0.273725 & -2.176687 \\
\hline 114 & 7 & $\odot$ & -3.160502 & -0.817342 & -2.611660 \\
\hline 115 & 6 & 0 & -1.834743 & $-\odot .59989 \odot$ & -2.375201 \\
\hline 116 & 6 & 0 & -1.624629 & 0.636922 & -1.695756 \\
\hline 117 & 7 & 0 & $-\odot .475860$ & 1.348388 & -1.643642 \\
\hline 118 & 6 & $\odot$ & 0.575009 & 0.630031 & -1.994849 \\
\hline 119 & 7 & 0 & 0.499910 & $-\odot .679877$ & -2.439392 \\
\hline 120 & 6 & $\odot$ & -0.666526 & -1.389306 & -2.673446 \\
\hline 121 & 7 & 0 & 1.802038 & 1.153430 & -1.968036 \\
\hline 122 & 8 & $\odot$ & $-\odot .637681$ & -2.549877 & -3.090579 \\
\hline 123 & 1 & $\odot$ & -4.829888 & $\odot .439370$ & -2.219891 \\
\hline 124 & 1 & 0 & 1.985635 & 2.007098 & -1.453954 \\
\hline 125 & 1 & $\odot$ & 2.621638 & $\odot .598927$ & -2.226224 \\
\hline 126 & 1 & $\odot$ & 1.382144 & -1.203918 & -2.577937 \\
\hline 127 & 8 & $\odot$ & -1.505961 & -0.475824 & $-\odot .097124$ \\
\hline 128 & 1 & 0 & -2.431369 & -0.721309 & 0.045282 \\
\hline
\end{tabular}

1122 basis functions

305 alpha electrons

304 beta electrons

nuclear repulsion energy 16423.9103215697 Hartrees.

NAtoms $=128$

\begin{tabular}{|c|c|c|c|c|c|c|c|c|c|c|}
\hline \multicolumn{11}{|c|}{$\begin{array}{c}\text { Harmonic frequencies (c } \\
1\end{array}$} \\
\hline \multicolumn{2}{|c|}{ Frequencies } & - & \multirow{2}{*}{\multicolumn{2}{|c|}{$\begin{array}{r}-391.8438 \\
10.7815\end{array}$}} & \multicolumn{3}{|c|}{15.3215} & \multicolumn{3}{|c|}{16.4516} \\
\hline \multirow{2}{*}{\multicolumn{2}{|c|}{$\begin{array}{l}\text { Red. masses } \\
\text { Frc consts }\end{array}$}} & - - & & & & 8.449 & & \multicolumn{3}{|c|}{6.4844} \\
\hline & & -- & 1.005 & & \multirow{2}{*}{\multicolumn{3}{|c|}{$\begin{array}{r}0.0012 \\
10.0373\end{array}$}} & \multirow{2}{*}{\multicolumn{3}{|c|}{$\begin{array}{l}0.0010 \\
1.0864\end{array}$}} \\
\hline \multicolumn{2}{|c|}{ IR Inten } & - - & \multicolumn{2}{|c|}{32.4107} & & & & & & \\
\hline Atom & AN & $\mathrm{x}$ & $Y$ & Z & $\mathrm{x}$ & $\mathrm{Y}$ & Z & $x$ & Z & \\
\hline 1 & 7 & 0.00 & $\odot .0 \odot$ & 0.00 & -0.00 & -0.02 & $\odot .02$ & 0.04 & $\odot .02$ & -0.03 \\
\hline 2 & 6 & 0.00 & 0.00 & 0.00 & -0.01 & -0.01 & 0.08 & 0.06 & 0.02 & -0.05 \\
\hline 3 & 6 & 0.00 & -0.00 & 0.00 & -0.01 & -0.02 & 0.10 & 0.07 & 0.01 & -0.07 \\
\hline 4 & 6 & 0.00 & $-0.0 \odot$ & 0.00 & -0.00 & -0.03 & 0.05 & 0.06 & 0.01 & -0.05 \\
\hline 5 & 7 & 0.00 & -0.00 & -0.00 & 0.01 & -0.05 & -0.01 & 0.05 & 0.01 & -0.03 \\
\hline 6 & 8 & 0.00 & $0.0 \odot$ & -0.00 & -0.04 & 0.04 & $-\odot . \odot \odot$ & 0.01 & 0.03 & 0.03 \\
\hline 7 & 15 & -0.00 & 0.00 & $-\odot . \odot \odot$ & -0.05 & 0.06 & -0.05 & $-0.0 \odot$ & 0.01 & 0.04 \\
\hline 8 & 8 & 0.00 & $\odot .0 \odot$ & 0.00 & -0.05 & 0.03 & -0.06 & 0.00 & 0.01 & 0.03 \\
\hline 9 & 8 & 0.00 & $\odot .0 \odot$ & $-0.0 \odot$ & -0.06 & 0.05 & -0.09 & -0.01 & 0.02 & 0.07 \\
\hline 10 & 8 & 0.00 & $0.0 \odot$ & -0.00 & -0.05 & 0.11 & -0.03 & -0.01 & -0.01 & 0.03 \\
\hline 11 & 8 & -0.00 & -0.00 & $-0.0 \odot$ & -0.10 & 0.01 & -0.06 & -0.03 & -0.01 & 0.04 \\
\hline 12 & 6 & -0.00 & $-\odot . \odot \odot$ & $-\odot . \odot \odot$ & -0.08 & 0.01 & -0.06 & -0.00 & 0.00 & 0.03 \\
\hline 13 & 6 & -0.00 & $-\odot . \odot \odot$ & $-\odot . \odot \odot$ & -0.08 & 0.02 & -0.06 & -0.02 & $-\odot . \odot \odot$ & 0.03 \\
\hline 14 & 6 & 0.00 & 0.00 & 0.00 & -0.02 & $\odot .04$ & -0.02 & -0.12 & 0.12 & 0.21 \\
\hline 15 & 6 & $\odot .0 \odot$ & $\odot .0 \odot$ & 0.00 & -0.00 & 0.03 & -0.01 & -0.07 & 0.11 & 0.13 \\
\hline 16 & 8 & $\odot . \odot \odot$ & $\odot . \odot \odot$ & $\odot . \odot \odot$ & $-\odot . \odot 4$ & 0.03 & -0.04 & -0.16 & $\odot . \odot 6$ & $\odot .22$ \\
\hline 17 & 8 & $\odot .0 \odot$ & $-\odot . \odot \odot$ & $-\odot . \odot \odot$ & 0.01 & 0.04 & 0.01 & -0.05 & 0.05 & 0.02 \\
\hline 18 & 15 & $\odot .0 \odot$ & $-\odot . \odot \odot$ & $-\odot . \odot \odot$ & $\odot .02$ & 0.05 & 0.02 & -0.01 & $\odot . \odot \odot$ & $0.0 \odot$ \\
\hline 19 & 8 & $\odot . \odot \odot$ & $-\odot . \odot \odot$ & $-\odot . \odot \odot$ & 0.03 & 0.02 & $\odot .02$ & -0.01 & $-\odot . \odot 2$ & 0.01 \\
\hline 20 & 8 & 0.00 & $-\odot . \odot \odot$ & -0.00 & $\odot .04$ & 0.05 & 0.04 & 0.03 & 0.03 & -0.04 \\
\hline 21 & 8 & $\odot .0 \odot$ & $-\odot . \odot \odot$ & $-\odot . \odot \odot$ & 0.02 & 0.06 & 0.02 & $-\odot . \odot \odot$ & $-\odot .04$ & 0.02 \\
\hline 22 & 8 & $\odot . \odot \odot$ & $-\odot . \odot \odot$ & $-\odot . \odot \odot$ & 0.12 & -0.01 & $\odot . \odot 9$ & $\odot .01$ & $-\odot . \odot 3$ & $-\odot . \odot 6$ \\
\hline 23 & 6 & -0.00 & 0.00 & 0.01 & 0.06 & -0.01 & 0.06 & $0.0 \odot$ & -0.01 & -0.02 \\
\hline 24 & 6 & 0.01 & -0.01 & $-\odot . \odot \odot$ & 0.09 & 0.01 & 0.07 & 0.01 & -0.03 & -0.04 \\
\hline 25 & 1 & 0.00 & -0.01 & -0.01 & 0.13 & 0.00 & 0.09 & 0.02 & -0.04 & -0.08 \\
\hline
\end{tabular}




\begin{tabular}{|c|c|c|c|c|c|c|c|c|c|c|}
\hline 26 & 6 & $\odot . \odot \odot$ & $\odot . \odot \odot$ & $\odot . \odot \odot$ & -0.01 & $\odot . \odot 2$ & 0.14 & 0.05 & 0.05 & -0.03 \\
\hline 27 & 6 & $\odot . \odot \odot$ & $\odot . \odot \odot$ & $\odot . \odot \odot$ & $-\odot .01$ & $\odot .01$ & $\odot . \odot 8$ & $\odot . \odot 4$ & 0.05 & $-\odot .01$ \\
\hline 28 & 6 & $\odot . \odot \odot$ & $\odot . \odot \odot$ & $\odot .0 \odot$ & $-\odot .04$ & $\odot .05$ & $\odot . \odot 4$ & $\odot .02$ & 0.02 & $\odot .0 \odot$ \\
\hline 29 & 6 & $\odot . \odot \odot$ & $\odot . \odot \odot$ & $\odot .00$ & -0.04 & 0.04 & $\odot .03$ & .03 & 0.01 & $-\odot .01$ \\
\hline 30 & 6 & $\odot . \odot \odot$ & $\odot . \odot \odot$ & $\odot . \odot \odot$ & $-\odot .01$ & $-\odot . \odot 1$ & $\odot . \odot 2$ & $\odot .03$ & $\odot .03$ & -0.01 \\
\hline 31 & 8 & $\odot . \odot \odot$ & $\odot . \odot \odot$ & $\odot . \odot \odot$ & $\odot .01$ & $-\odot .03$ & $\odot .06$ & $\odot .04$ & 0.05 & $-\odot .01$ \\
\hline 32 & 1 & $\odot . \odot \odot$ & $0.0 \odot$ & $\odot . \odot \odot$ & 0.01 & -0.02 & $\odot .17$ & $\odot .06$ & 0.07 & -0.03 \\
\hline 33 & 1 & $\odot . \odot \odot$ & $\odot . \odot \odot$ & $\odot . \odot \odot$ & $-\odot .03$ & $\odot . \odot 4$ & 0.16 & ๑.05 & 0.05 & $-\odot . \odot 2$ \\
\hline 34 & 1 & $\odot . \odot \odot$ & $\odot .0 \odot$ & $\odot .0 \odot$ & $-\odot . \odot \odot$ & $-\odot .01$ & & $\odot .03$ & 0.06 & 0.01 \\
\hline 35 & 1 & $\odot . \odot \odot$ & $\odot .0 \odot$ & $\odot . \odot \odot$ & -0.05 & $\odot . \odot 8$ & $\odot .06$ & $\odot .02$ & 0.01 & $\odot .0 \odot$ \\
\hline 36 & 1 & $\odot . \odot \odot$ & $\odot . \odot \odot$ & $\odot . \odot \odot$ & -0.05 & $\odot . \odot 6$ & $-\odot . \odot 1$ & $\odot .01$ & $-\odot . \odot \odot$ & -0.01 \\
\hline 37 & 1 & $\odot . \odot \odot$ & $\odot .0 \odot$ & $\odot .0 \odot$ & $-\odot .04$ & 0.05 & $\odot .06$ & 0.03 & $\odot .0 \odot$ & -0.03 \\
\hline 38 & 1 & $-\odot . \odot \odot$ & $\odot . \odot \odot$ &.$\odot \odot$ & $-\odot . \odot \odot$ & $-\odot .02$ & $-\odot . \odot 2$ & .02 & $\odot .03$ & $-\odot . \odot \odot$ \\
\hline 39 & 8 & $\odot . \odot \odot$ & $\odot . \odot \odot$ & $\odot . \odot \odot$ & $-\odot . \odot 2$ & $\odot . \odot 4$ & 0.15 & $\odot .05$ & $\odot .03$ & $-\odot . \odot 4$ \\
\hline 40 & 1 & $\odot . \odot \odot$ & $-\odot . \odot \odot$ & $-\odot . \odot \odot$ & $-\odot .03$ & 0.04 & 0.18 & 0.06 & $\odot .03$ & $-\odot .05$ \\
\hline 41 & 6 & $\odot . \odot \odot$ & $\odot . \odot \odot$ & $-\odot . \odot \odot$ & $\odot .01$ & $-\odot . \odot 4$ & -0.03 & 0.04 & $\odot . \odot 2$ & $-\odot .02$ \\
\hline 42 & 7 & $\odot . \odot \odot$ & $-\odot . \odot \odot$ & $\odot . \odot \odot$ & $-\odot . \odot \odot$ & $-\odot .03$ & $\odot .07$ & $\odot .07$ & $-\odot . \odot \odot$ & $-\odot .06$ \\
\hline 43 & 8 & $\odot . \odot \odot$ & $-\odot . \odot \odot$ & $-\odot . \odot \odot$ & 0.02 & -0.05 & $-\odot . \odot 8$ & 0.03 & $\odot .02$ & -0.01 \\
\hline 44 & 1 & $\odot . \odot \odot$ & $\odot . \odot \odot$ & $\odot . \odot \odot$ & -0.02 & $-\odot . \odot \odot$ & 0.11 & $\odot .06$ & $\odot .02$ & -0.06 \\
\hline 45 & 1 & $\odot . \odot \odot$ & $-\odot . \odot \odot$ & $\odot . \odot \odot$ & $-\odot . \odot 2$ & $-\odot .01$ & & .08 & $\odot .01$ & $-\odot .08$ \\
\hline 46 & 1 & $\odot . \odot \odot$ & $-\odot . \odot \odot$ & $-\odot . \odot \odot$ & $-\odot . \odot 2$ & -0.02 & $\odot .12$ & .08 & $-\odot .01$ & $-\odot . \odot 8$ \\
\hline 47 & 1 & $\odot .01$ & $-\odot .01$ & $-\odot .01$ & $\odot . \odot \odot$ & $-\odot .04$ & $\odot .04$ & 0.06 & $-\odot .01$ & $-\odot .05$ \\
\hline 48 & 6 & $-\odot . \odot \odot$ & $-\odot . \odot \odot$ & $-\odot . \odot \odot$ & $-\odot .06$ & $\odot .02$ & $-\odot . \odot 6$ & $\odot .01$ & $\odot . \odot 2$ & $\odot .03$ \\
\hline 49 & 1 & $-\odot . \odot \odot$ & $-\odot . \odot \odot$ & $-\odot . \odot \odot$ & -0.11 & 0.01 & $-\Theta$ & -0.04 & -0.01 & $\odot .04$ \\
\hline 50 & 6 & $-\odot . \odot \odot$ & $-\odot . \odot \odot$ & $-\odot . \odot \odot$ & $-\odot .07$ & 0.01 & $-\odot . \odot 6$ & $-\odot . \odot 2$ & $-\odot .01$ & $\odot .02$ \\
\hline 51 & 6 & $-\odot . \odot \odot$ & $-\odot$. & $-\odot$. & $-\odot .06$ & $-\odot .01$ & & $-\odot .01$ & $-\odot .02$ & 0.01 \\
\hline 52 & 8 & $-\odot . \odot \odot$ & $-\odot . \odot \odot$ & $-\odot . \odot \odot$ & -0.06 & -0.01 & -0.05 & 0.00 & -0.01 & $\odot .01$ \\
\hline 53 & 1 & $\odot . \odot \odot$ & $-\odot . \odot \odot$ & $\odot . \odot \odot$ & $-\odot .06$ & $\odot .01$ & -0.05 & 0.02 & $\odot .02$ & $\odot . \odot 2$ \\
\hline 54 & 1 & $-\odot . \odot \odot$ & 0.00 & -0. & -0.07 & 0.03 & -0.06 & 0.01 & 0.02 & 0.04 \\
\hline 55 & 1 & $-\odot . \odot \odot$ & $-\odot . \odot \odot$ & $-\odot . \odot \odot$ & -0.09 & $\odot .0 \odot$ & $-\odot . \odot 6$ & $-\odot . \odot \odot$ & 0.01 & 0.03 \\
\hline 56 & 1 & $-\odot . \odot \odot$ & $-\odot . \odot \odot$ & $-\odot . \odot \odot$ & $-\odot . \odot 8$ & 0.03 & $-\odot . \odot 6$ & $-\odot . \odot 2$ & $\odot . \odot \odot$ & $\odot . \odot 4$ \\
\hline 57 & 1 & $-\odot . \odot \odot$ & $-\odot . \odot \odot$ & $-\odot . \odot \odot$ & $-\odot .07$ & 0.01 & $-\odot .06$ & $-\odot . \odot 3$ & $-\odot .02$ & 0.02 \\
\hline 58 & 1 & $-\odot . \odot \odot$ & $-\odot . \odot \odot$ & $-\odot . \odot \odot$ & $-\odot . \odot 6$ & 0.02 & -0.05 & -0.02 & $-\odot .01$ & 0.02 \\
\hline 59 & 1 & $-\odot . \odot \odot$ & $-\odot . \odot \odot$ & $-\odot . \odot \odot$ & $-\odot . \odot 7$ & $-\odot . \odot 2$ & $-\odot .05$ & $-\odot . \odot 2$ & $-\odot . \odot 2$ & $\odot .0 \odot$ \\
\hline 60 & 7 & $-\odot . \odot \odot$ & $-\odot . \odot \odot$ & $-\odot . \odot \odot$ & $-\odot .04$ & $-\odot . \odot 2$ & -0.05 & $-\odot . \odot \odot$ & $-\odot .02$ & -0.01 \\
\hline 61 & 6 & $-\odot . \odot \odot$ & $-\odot . \odot \odot$ & $-\odot . \odot \odot$ & -0.05 & -0.01 & $-\odot .07$ & $\odot . \odot \odot$ & $-\odot .01$ & -0.01 \\
\hline 62 & 6 & $\odot . \odot \odot$ & $-\odot . \odot \odot$ & $-\odot . \odot \odot$ & $-\odot .03$ & -0.01 & $-\odot .05$ & $\odot . \odot \odot$ & $-\odot . \odot 2$ & $-\odot .02$ \\
\hline 63 & 6 & $\odot . \odot \odot$ & $-\odot . \odot \odot$ & $\odot . \odot \odot$ & $-\odot . \odot \odot$ & $-\odot . \odot 2$ & $-\odot .02$ & $\odot . \odot \odot$ & -0.02 & $-\odot .03$ \\
\hline 64 & 7 & $\odot .00$ & $-\odot$. & $\odot . \odot \odot$ & $\odot .01$ & -0.03 & 0.01 & $-\odot . \odot \odot$ & -0.03 & $-\odot . \odot 2$ \\
\hline 65 & 6 & $\odot . \odot \odot$ & $-\odot . \odot \odot$ & $\odot . \odot \odot$ & $-\odot .01$ & -0.03 & $\odot . \odot \odot$ & $-\odot . \odot \odot$ & $-\odot .03$ & $-\odot .01$ \\
\hline 66 & 1 & -0.00 & $-\odot . \odot \odot$ & $-\odot . \odot \odot$ & -0.03 & $-\odot .0 \odot$ & $-\odot .07$ & 0.01 & -0.01 & -0.03 \\
\hline 67 & 7 & 000 & $-\odot . \odot \odot$ & $-\odot . \odot \odot$ & 0.01 & $-\odot .03$ & $-\odot . \odot 2$ & $-\odot . \odot \odot$ & -0.03 & -0.05 \\
\hline 68 & 8 & $\odot . \odot \odot$ & $-\odot . \odot \odot$ & $\odot . \odot \odot$ & $\odot . \odot \odot$ & $-\odot . \odot 4$ & $\odot .04$ & $-\odot . \odot \odot$ & -0.03 & 0.01 \\
\hline 69 & 1 & $-\odot . \odot \odot$ & $-\odot . \odot \odot$ & $-\odot . \odot \odot$ & $-\odot .07$ & $\odot . \odot \odot$ & $-\odot . \odot 9$ & $\odot . \odot \odot$ & -0.01 & -0.01 \\
\hline 70 & 1 & $\odot . \odot \odot$ & $-\odot . \odot \odot$ & $\odot . \odot \odot$ & $-\odot . \odot \odot$ & $-\odot . \odot 2$ & $-\odot$ & $-\odot . \odot \odot$ & -0.02 & -0.06 \\
\hline 71 & 1 & $\odot . \odot \odot$ & $-\odot . \odot \odot$ & $\odot .0 \odot$ & 0.02 & $-\odot . \odot 4$ & $-\odot .02$ & $-\odot .01$ & -0.03 & $-\odot .06$ \\
\hline 72 & 1 & $\odot .0 \odot$ & $\odot . \odot \odot$ & $-\odot . \odot \odot$ & 0.01 & -0.07 & 0.01 & $-\odot .01$ & -0.03 & $-\odot .04$ \\
\hline 73 & 1 & $\odot . \odot \odot$ & $\odot$ & 0. & -0.05 & $\odot .03$ & $-\odot$. & -0.19 & $\odot .07$ & 0.26 \\
\hline 74 & 6 & $-0 . \odot \odot$ & $-\odot . \odot \odot$ & $-\odot . \odot \odot$ & -0.01 & 0.04 & -0.01 & -0.11 & 0.03 & 0.07 \\
\hline 75 & 6 & $-\odot . \odot \odot$ & $-\odot . \odot \odot$ & $-\odot . \odot \odot$ & $-\odot .01$ & 0.01 & -0.04 & -0.10 & -0.01 & 0.01 \\
\hline 76 & 6 & 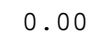 & $-\odot . \odot \odot$ & $-\odot . \odot \odot$ & ค 01 & $-\odot .01$ & $-\odot . \odot 4$ & $-\odot . \odot 2$ & 0.04 & $-\odot .01$ \\
\hline 7 & 8 & $\odot \odot$ & & -0. & & 0.01 & -0. & -0.02 & 0.13 & 0.08 \\
\hline 78 & 1 & $\odot . \odot \odot$ & $\odot .0 \odot$ & $\odot$. & $-\odot .02$ & $\odot .04$ & -0. & -0.10 & 0.18 & 0.24 \\
\hline 79 & 1 & $0 \cap 00$ & & & & $\odot .06$ & $-\odot$ & -0 & 0.10 & 0.24 \\
\hline 80 & 1 & 0.0 & $\odot$. & $\theta$ & 0 & $\odot .04$ & $\odot$. & $-\odot .04$ & 0.15 & 0.14 \\
\hline 81 & 1 & $-\odot . \odot \odot$ & $-\odot . \odot \odot$ & $\Theta$. & 04 & 0.06 & $-\odot$ & -0.17 & 0.01 & 0.10 \\
\hline 82 & 1 & $\circ ค ค$ & & $-\odot . \odot \odot$ & & $\odot .01$ & & -0.11 & $-\odot .05$ & -0.05 \\
\hline 83 & 1 & 0.0 & $\odot$. & $-\Theta$. & $-\odot$. & 1 & $-\Theta$ & -0.15 & -0.03 & 0.03 \\
\hline 84 & 1 & $\odot . \odot \odot$ & $-\odot$. & $-\Theta$. & 04 & $-\odot .01$ & $-\odot$ & 0.04 & $\odot .04$ & -0.05 \\
\hline 85 & 7 & ? & & $-\odot$. & & $-\odot$ & & $-\odot . \odot 2$ & $\odot .01$ & $-\odot .03$ \\
\hline 86 & 6 & 0 & -0 & & & $-\Theta$ & & -0 & 0.01 & -0 \\
\hline 87 & 7 & $-\odot . \odot \odot$ & $-\Theta$. & & $\odot \odot$ & -0 & & -0.03 & -0.01 & -0.07 \\
\hline مo & 0 & & $-\odot$. & & & $-\odot$ & & & $-\odot .01$ & \\
\hline 89 & 6 & A & $-\Theta$. & -0. & 1 & -0 & -0 & -0 & $-\odot . \odot \odot$ & -0.03 \\
\hline 90 & 7 & $\odot .0 \odot$ & $-\odot$. & -0. & 01 & -0 & $\odot$. & $-\odot .01$ & $-\odot . \odot \odot$ & -0.01 \\
\hline 91 & 0 & & & & & $-\odot$. & & & & \\
\hline 9 & 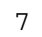 & & $-\Theta$. & -0 & & -0 & -0 & 1 & -0.03 & $-\Theta$ \\
\hline 93 & 6 & $\odot . \odot \odot$ & $-\odot$. & & 0 & $-\Theta$. & $-\odot$. & $-\odot . \odot 2$ & $-\odot .03$ & -0.06 \\
\hline 94 & 8 & & & & & $-\odot$. & & & $-\odot . \odot 4$ & \\
\hline 95 & 1 & คค & $-\Theta$. & & 1 & -0.03 & -0 & -0.04 & 0.02 & -0.06 \\
\hline 96 & 7 &.$\odot \odot$ & $-\odot . \odot \odot$ & $\odot$. & 0.01 & -0.05 & 0.05 & $\odot . \odot \odot$ & -0.02 & $\odot . \odot \odot$ \\
\hline 97 & 1 & & $-\odot$. & $-\odot$ & & $-\odot . \odot 4$ & & & $-\odot .01$ & 0.01 \\
\hline 98 & 1 & $-0 . \odot \odot$ & -0. & $-\Theta$. & & -0.06 & 0.06 & $\odot .0 \odot$ & $-\odot . \odot 2$ & 0.00 \\
\hline 99 & 6 & $-\odot$. & $\odot . \odot \odot$ & $\odot$. & $\odot .04$ & $\odot . \odot \odot$ & $\odot . \odot 4$ & $-\odot . \odot \odot$ & $\odot .01$ & $-\odot .01$ \\
\hline & + & & 0.00 & & -0.07 & 0.11 & -0.0 & -0.02 & -0.01 & 0.03 \\
\hline & 11 & $-\odot .0 \odot$ & $-\odot . \odot \odot$ & $-\odot$ & 0.10 & 0.14 & 0.11 & 0.01 & -0.01 & -0.08 \\
\hline 10 & 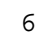 & $\odot .01$ & $-\odot .02$ & $\odot .01$ & $\odot . \odot 8$ & $\odot .01$ & $\odot . \odot 6$ & $\odot .01$ & -0.05 & $-\odot . \odot$ \\
\hline
\end{tabular}




\begin{tabular}{|c|c|c|c|c|}
\hline 103 & 6 & 0.00 & -0.04 & $\odot .02$ \\
\hline 104 & 8 & $-0.0 \odot$ & 0.02 & 0.03 \\
\hline 105 & 1 & -0.00 & $\odot .0 \odot$ & $\odot .0 \odot$ \\
\hline 106 & 1 & -0.01 & -0.00 & $-\odot . \odot \odot$ \\
\hline 107 & 1 & -0.02 & 0.01 & 0.00 \\
\hline 108 & 1 & 0.01 & -0.01 & -0.01 \\
\hline 109 & 1 & 0.03 & -0.02 & 0.01 \\
\hline 110 & 1 & ๑. . 01 & -0.00 & -0.00 \\
\hline 111 & 1 & 0.01 & -0.06 & 0.02 \\
\hline 112 & 7 & -0.01 & -0.06 & 0.09 \\
\hline 113 & 6 & 0.03 & 0.02 & -0.03 \\
\hline 114 & 7 & 0.02 & 0.00 & -0.11 \\
\hline 115 & 6 & -0.02 & -0.06 & -0.10 \\
\hline 116 & 6 & 0.01 & -0.30 & 0.57 \\
\hline 117 & 7 & -0.01 & -0.05 & 0.05 \\
\hline 118 & 6 & -0.03 & $-0.0 \odot$ & 0.01 \\
\hline 119 & 7 & 0.00 & -0.03 & -0.00 \\
\hline 120 & 6 & $\odot .01$ & -0.03 & -0.04 \\
\hline 121 & 7 & -0.02 & $-\odot . \odot \odot$ & -0.03 \\
\hline 122 & 8 & 0.01 & -0.03 & -0.03 \\
\hline 123 & 1 & $\odot .03$ & 0.03 & -0.12 \\
\hline 124 & 1 & $-\odot . \odot \odot$ & $-\odot .03$ & 0.01 \\
\hline 125 & 1 & -0.03 & 0.01 & -0.08 \\
\hline 126 & 1 & $\odot .00$ & -0.02 & 0.02 \\
\hline 127 & 8 & $-\odot .03$ & 0.47 & -0.29 \\
\hline 128 & 1 & 0.02 & 0.44 & -0.05 \\
\hline
\end{tabular}

$\begin{array}{ccc}0.06 & -0.02 & 0.06 \\ 0.05 & -0.03 & 0.05 \\ 0.02 & -0.01 & 0.03 \\ 0.05 & 0.02 & 0.04 \\ 0.07 & -0.03 & 0.07 \\ 0.09 & 0.04 & 0.06 \\ 0.09 & 0.01 & 0.07 \\ 0.06 & 0.02 & 0.05 \\ 0.07 & -0.03 & 0.07 \\ 0.05 & -0.01 & 0.04 \\ 0.03 & -0.01 & 0.06 \\ 0.01 & -0.01 & 0.05 \\ 0.02 & -0.02 & 0.02 \\ 0.04 & -0.02 & 0.01 \\ 0.05 & -0.03 & -0.01 \\ 0.03 & -0.03 & -0.04 \\ 0.02 & -0.04 & -0.03 \\ 0.01 & -0.03 & 0.00 \\ 0.04 & -0.03 & -0.09 \\ -0.01 & -0.04 & 0.02 \\ 0.03 & 0.00 & 0.08 \\ 0.05 & -0.05 & -0.07 \\ 0.02 & -0.04 & -0.10 \\ 0.01 & -0.04 & -0.04 \\ 0.07 & -0.02 & 0.02 \\ 0.08 & -0.04 & 0.02\end{array}$

$0.01 \quad-0.04-0.00$

$\begin{array}{lll}-0.00 & -0.02 & -0.01\end{array}$

$\begin{array}{lll}-0.01 & 0.02 & 0.01\end{array}$

$\begin{array}{lll}-0.00 & 0.02 & -0.02\end{array}$

$\begin{array}{ccc}-0.00 & 0.02 & -0.02 \\ 0.00 & 0.01 & -0.04\end{array}$

$\begin{array}{llll}0.01 & -0.03 & -0.05\end{array}$

$0.01 \quad-0.07 \quad-0.04$

$0.01-0.06 \quad-0.01$

$0.01 \quad-0.04 \quad-0.01$

$0.01 \quad-0.05 \quad 0.02$

$0.02 \quad-0.06 \quad 0.02$

$0.02 \quad-0.05 \quad 0.02$

$\begin{array}{lll}0.02 & -0.04 & 0.01\end{array}$

$0.02 \quad-0.04 \quad 0.01$

$0.01 \quad-0.03 \quad 0.01$

$\begin{array}{llll}0.01 & -0.02 & -0.00\end{array}$

$0.02-0.02-0.01$

$0.03-0.03-0.00$

$0.01-0.01-0.01$

$0.04 \quad-0.02 \quad-0.01$

$\begin{array}{lll}0.01 & -0.06 & 0.03\end{array}$

$\begin{array}{llll}0.00 & -0.01 & -0.00\end{array}$

$\begin{array}{llll}0.01 & 0.00 & -0.01\end{array}$

$0.03-0.01-0.02$

$0.04 \quad-0.04 \quad 0.01$

$0.05-0.08-0.00$

Zero-point correction=

Thermal correction to Energy=

Thermal correction to Enthalpy=

Thermal correction to Gibbs Free Energy=

Sum of electronic and zero-point Energies=

Sum of electronic and thermal Energies=

Sum of electronic and thermal Enthalpies=

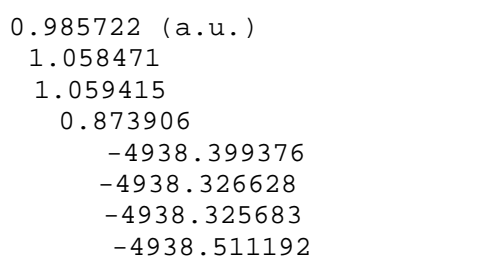

\begin{tabular}{|c|c|c|c|}
\hline $\begin{array}{r}\mathrm{E}(\mathrm{T} \\
\mathrm{KCa}\end{array}$ & $\begin{array}{l}\text { lermal) } \\
\text { /Mol } \\
64.200\end{array}$ & $\begin{array}{c}\text { CV } \\
\text { Cal/Mol-Kelvin } \\
277.606\end{array}$ & $\begin{array}{c}\text { S } \\
\text { Cal/Mol-Kelvin } \\
390.436\end{array}$ \\
\hline Item & Value & Threshold Conv & erged? \\
\hline Maximum Force & 0.000016 & $\odot .000450$ & YES \\
\hline Force & $\odot .0 \odot \odot \odot \odot 3$ & $\odot .0 \odot \odot 3 \odot \odot$ & YES \\
\hline Maximum Displacement & 0.003028 & 0.001800 & NO \\
\hline Displacement & $\odot .000697$ & 0.001200 & YES \\
\hline
\end{tabular}

Normal termination of Gaussian 16 at Sun Feb 10 08:49:01 2019.

Figure S10-3, Int2(C4)(b)

dna@4b-oh1arev. $\log$

Stoichiometry C38H49N16Na2021P2(2)

Standard orientation:

\begin{tabular}{|c|c|c|c|c|c|}
\hline \multirow{2}{*}{$\begin{array}{l}\text { Center } \\
\text { Number }\end{array}$} & \multirow{2}{*}{$\begin{array}{l}\text { Atomic } \\
\text { Number }\end{array}$} & \multirow{2}{*}{$\begin{array}{l}\text { Atomic } \\
\text { Type }\end{array}$} & \multicolumn{3}{|c|}{ Coordinates (Angstroms) } \\
\hline & & & $\mathrm{X}$ & Y & Z \\
\hline . & & & --- - - - - & - & $-----\cdot--$ \\
\hline 1 & 7 & $\odot$ & 5.388298 & -1.865780 & -1.969405 \\
\hline 2 & 6 & $\odot$ & 5.420425 & -3.225795 & -1.853064 \\
\hline 3 & 6 & $\odot$ & 4.428258 & -3.987647 & -2.366416 \\
\hline 4 & 6 & $\odot$ & 3.324098 & -3.295944 & -2.964786 \\
\hline 5 & 7 & $\odot$ & 3.339841 & -1.972177 & -3.143236 \\
\hline 6 & 8 & $\odot$ & 7.806828 & $\odot .593160$ & $\odot .740091$ \\
\hline 7 & 15 & $\odot$ & 6.796252 & 1.679687 & 1.436998 \\
\hline 8 & 8 & $\odot$ & 5.565453 & 1.769296 & $\odot .352378$ \\
\hline 9 & 8 & $\odot$ & 7.523340 & 2.991155 & 1.490620 \\
\hline 10 & 8 & $\odot$ & 6.180374 & 1.053736 & 2.669224 \\
\hline 11 & 8 & $\odot$ & 3.597181 & 5.597735 & ๑. 674791 \\
\hline 12 & 6 & $\odot$ & 4.311761 & 3.665226 & -0.510346 \\
\hline 13 & 6 & $\odot$ & 4.243372 & 4.314714 & $\odot .870664$ \\
\hline 14 & 6 & $\odot$ & -8.598837 & -3.792950 & 0.331783 \\
\hline 15 & 6 & $\odot$ & -8.237815 & -2.342298 & 0.577783 \\
\hline 16 & 8 & $\odot$ & -7.414578 & -4.519215 & -0.072870 \\
\hline
\end{tabular}




\begin{tabular}{|c|c|c|c|c|c|}
\hline 17 & 8 & $\odot$ & -7.864898 & -0.210486 & -0.435590 \\
\hline 18 & 15 & $\odot$ & -7.069052 & 1. 016441 & -1.169211 \\
\hline 19 & 8 & $\odot$ & -5.729410 & 1.196000 & -0.252129 \\
\hline 20 & 8 & $\odot$ & -7.948712 & 2.230321 & -0.988561 \\
\hline 21 & 8 & $\odot$ & -6.567548 & $\odot .591473$ & -2.523223 \\
\hline 22 & 8 & $\odot$ & -4.126378 & 5.233082 & -0.153177 \\
\hline 23 & 6 & $\odot$ & -4.521549 & 3.054442 & $\odot .717661$ \\
\hline 24 & 6 & $\odot$ & -4.715519 & 3.955281 & -0.503038 \\
\hline 25 & 1 & $\odot$ & -4.178350 & 5.847438 & -0.905133 \\
\hline 26 & 6 & $\odot$ & 8.985512 & -2.868408 & -0.009926 \\
\hline 27 & 6 & $\odot$ & 8.499070 & -1.461775 & $-\odot .290729$ \\
\hline 28 & 6 & $\odot$ & 7.587151 & -0.855415 & 0.786596 \\
\hline 29 & 6 & $\odot$ & 6.193480 & -1.221168 & $\odot .297734$ \\
\hline 30 & 6 & $\odot$ & 6.334573 & -1.054895 & -1.213722 \\
\hline 31 & 8 & $\odot$ & 7.694002 & -1.461785 & -1.521496 \\
\hline 32 & 1 & $\odot$ & 9.522724 & -3.236299 & $-\odot .890183$ \\
\hline 33 & 1 & $\odot$ & 9.676168 & -2.847742 & $\odot .841048$ \\
\hline 34 & 1 & $\odot$ & 9.361938 & -0.812723 & -0.455468 \\
\hline 35 & 1 & $\odot$ & 7.806910 & -1.217786 & 1.791844 \\
\hline 36 & 1 & $\odot$ & 5.406999 & $-\odot .591136$ & $\odot .703174$ \\
\hline 37 & 1 & $\odot$ & 5.996675 & -2.264556 & $\odot .552278$ \\
\hline 38 & 1 & $\odot$ & 6.180872 & $-\odot .024687$ & -1.524358 \\
\hline 39 & 8 & $\odot$ & 7.844777 & -3.713934 & $\odot .280124$ \\
\hline 40 & 1 & $\odot$ & 8.122187 & -4.634414 & 0.416233 \\
\hline 41 & 6 & $\odot$ & 4.371487 & -1.223257 & -2.687596 \\
\hline 42 & 7 & $\odot$ & 2.231833 & -3.956857 & -3.360464 \\
\hline 43 & 8 & $\odot$ & 4.415888 & $\odot .006009$ & -2.843273 \\
\hline 44 & 1 & $\odot$ & 6.274971 & -3.621994 & -1.307601 \\
\hline 45 & 1 & $\odot$ & 4.440285 & -5.064559 & -2.271669 \\
\hline 46 & 1 & $\odot$ & 2.140469 & -4.936447 & -3.145129 \\
\hline 47 & 1 & $\odot$ & 1.379125 & -3.427586 & -3.550087 \\
\hline 48 & 6 & $\odot$ & 5.491548 & 2.738995 & -0.726855 \\
\hline 49 & 1 & $\odot$ & 3.503687 & 6.061412 & 1.524227 \\
\hline 50 & 6 & $\odot$ & 3.370226 & 3.357465 & 1.690237 \\
\hline 51 & 6 & $\odot$ & 2.440942 & 2.700637 & $\odot .655973$ \\
\hline 52 & 8 & $\odot$ & 3.046391 & 2.922661 & -0.630863 \\
\hline 53 & 1 & $\odot$ & 5.357267 & 2.180939 & -1.656364 \\
\hline 54 & 1 & $\odot$ & 6.413737 & 3.324636 & -0.772205 \\
\hline 55 & 1 & $\odot$ & 4.298830 & 4.427272 & -1.291445 \\
\hline 56 & 1 & $\odot$ & 5.239625 & 4.440034 & 1.306610 \\
\hline 57 & 1 & $\odot$ & 2.807098 & 3.874693 & 2.467459 \\
\hline 58 & 1 & 0 & 3.997407 & 2.594167 & 2.154815 \\
\hline 59 & 1 & $\odot$ & 1.439863 & 3.129648 & $\odot .645509$ \\
\hline 60 & 7 & $\odot$ & 2.280078 & 1.246980 & ๑.885511 \\
\hline 61 & 6 & $\odot$ & 2.864852 & 0.316468 & $\odot .094151$ \\
\hline 62 & 6 & $\odot$ & 2.660583 & -1.005146 & $\odot .314159$ \\
\hline 63 & 6 & $\odot$ & 1.814260 & -1.365397 & 1.404939 \\
\hline 64 & 7 & $\odot$ & 1.253942 & $-\odot .442610$ & 2.190765 \\
\hline 65 & 6 & $\odot$ & 1.434164 & $\odot .875547$ & 1.933749 \\
\hline 66 & 1 & 0 & 3.107456 & -1.756672 & -0.321287 \\
\hline 67 & 7 & $\odot$ & 1.574960 & -2.653316 & 1.682702 \\
\hline 68 & 8 & $\odot$ & $\odot .896969$ & 1.768623 & 2.606099 \\
\hline 69 & 1 & 0 & 3.482041 & $\odot .693479$ & -0.709395 \\
\hline 70 & 1 & $\odot$ & 1.911559 & -3.368504 & 1.059500 \\
\hline 71 & 1 & $\odot$ & $\odot .872684$ & -2.906521 & 2.379975 \\
\hline 72 & 1 & 0 & -0.412363 & -0.900558 & 3.055060 \\
\hline 73 & 1 & $\odot$ & -7.608564 & -5.465335 & -0.171939 \\
\hline 74 & 6 & $\odot$ & -7.519245 & -1.629819 & -0.577913 \\
\hline 75 & 6 & $\odot$ & -6.050759 & -1.886811 & -0.265102 \\
\hline 76 & 6 & $\odot$ & -6.031947 & -1.738018 & 1.254060 \\
\hline 77 & 8 & $\odot$ & -7.308287 & -2.250995 & 1.713022 \\
\hline 78 & 1 & 0 & -9.010076 & -4.210071 & 1.257256 \\
\hline 79 & 1 & $\Theta$ & -9.369779 & -3.841721 & -0.446732 \\
\hline 80 & 1 & $\odot$ & -9.143301 & -1.793205 & 0.845230 \\
\hline 81 & 1 & $\odot$ & -7.828425 & -1.977079 & -1.564547 \\
\hline 82 & 1 & $\odot$ & -5.362407 & -1.202385 & -0.755324 \\
\hline 83 & 1 & 0 & -5.813046 & -2.916452 & -0.538820 \\
\hline 84 & 1 & $\odot$ & -5.932825 & $-\odot .692227$ & 1.545966 \\
\hline 85 & 7 & $\Theta$ & -4.952903 & -2.448977 & 1.899315 \\
\hline 86 & 6 & $\odot$ & -4.765508 & -3.809232 & 2.026559 \\
\hline 87 & 7 & $\odot$ & -3.594063 & -4.117205 & 2.518114 \\
\hline 88 & 6 & $\Theta$ & -2.968464 & -2.894873 & 2.710414 \\
\hline 89 & 6 & 0 & -3.800093 & -1.853365 & 2.323626 \\
\hline 90 & 7 & $\odot$ & -3.555253 & -0.530215 & 2.295132 \\
\hline 91 & 6 & $\odot$ & -2.319856 & -0.229869 & 2.656523 \\
\hline 92 & 7 & 0 & -1.404498 & -1.183667 & 3.036931 \\
\hline 93 & 6 & $\Theta$ & -1.627042 & -2.567936 & 3.072695 \\
\hline
\end{tabular}




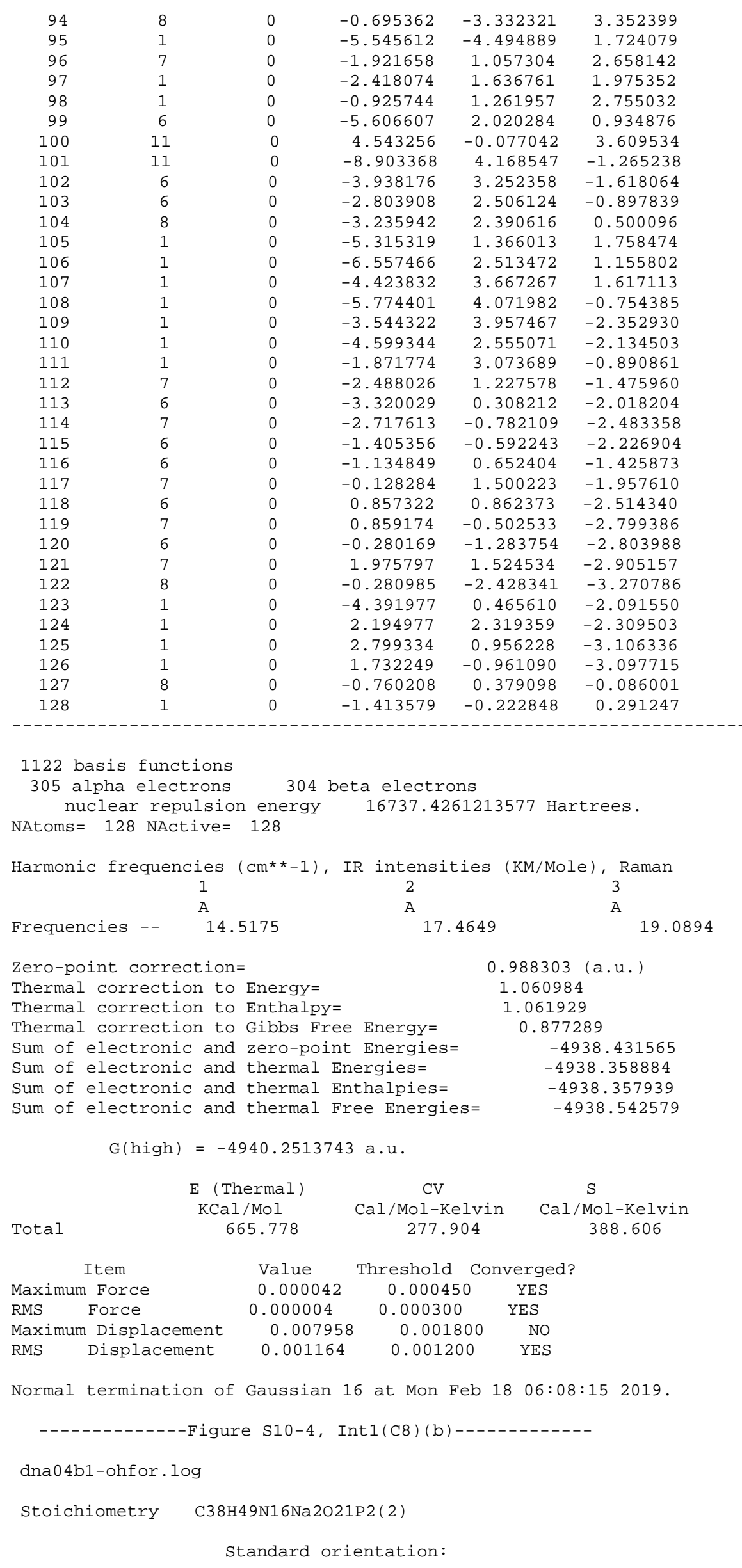




\begin{tabular}{|c|c|c|c|c|c|}
\hline \multirow{2}{*}{$\begin{array}{l}\text { Center } \\
\text { Number }\end{array}$} & \multirow{2}{*}{$\begin{array}{l}\text { Atomic } \\
\text { Number }\end{array}$} & \multirow{2}{*}{$\begin{array}{l}\text { Atomic } \\
\text { Type }\end{array}$} & \multicolumn{3}{|c|}{ Coordinates (Angstroms) } \\
\hline & & & $x$ & $\mathrm{Y}$ & Z \\
\hline 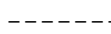 & & - & ------- & --- & -- \\
\hline 1 & 7 & $\odot$ & 5.139602 & -2.058058 & -1.451559 \\
\hline 2 & 6 & $\odot$ & 5.206062 & -3.403345 & -1.228701 \\
\hline 3 & 6 & $\odot$ & 4.082379 & -4.154222 & -1.180384 \\
\hline 4 & 6 & $\odot$ & 2.831573 & -3.470587 & -1.352917 \\
\hline 5 & 7 & $\odot$ & 2.782422 & -2.162071 & -1.617914 \\
\hline 6 & 8 & $\odot$ & 8.606177 & 0.493745 & -0.612323 \\
\hline 7 & 15 & $\odot$ & 8.058894 & 1.673170 & 0.385478 \\
\hline 8 & 8 & $\odot$ & 6.474978 & 1.792220 & -0.044380 \\
\hline 9 & 8 & $\odot$ & 8.776361 & 2.938316 & $\odot .022157$ \\
\hline 10 & 8 & $\odot$ & 8.027776 & 1.146577 & 1.802654 \\
\hline 11 & 8 & $\odot$ & 4.670509 & 5.566520 & 0.886354 \\
\hline 12 & 6 & $\odot$ & 4.919905 & 3.629093 & -0.472778 \\
\hline 13 & 6 & $\odot$ & 5.309723 & 4.265853 & 0.863360 \\
\hline 14 & 6 & $\odot$ & -9.032965 & -3.273667 & 1.477427 \\
\hline 15 & 6 & $\odot$ & -8.546491 & -1.839455 & 1.404568 \\
\hline 16 & 8 & $\odot$ & -8.179493 & -4.116272 & 0.666057 \\
\hline 17 & 8 & $\odot$ & -8.576668 & 0.163521 & $\odot .081136$ \\
\hline 18 & 15 & $\Theta$ & -7.842698 & 1.350751 & -0.751972 \\
\hline 19 & 8 & $\odot$ & -6.409192 & 1.515716 & $\odot .013154$ \\
\hline 20 & 8 & $\odot$ & -8.660805 & 2.609468 & -0.590002 \\
\hline 21 & 8 & $\odot$ & -7.503370 & $\odot .917704$ & -2.163627 \\
\hline 22 & 8 & $\odot$ & -4.757375 & 5.439440 & -0.638225 \\
\hline 23 & 6 & $\odot$ & -5.062480 & 3.452572 & 0.632682 \\
\hline 24 & 6 & $\odot$ & -5.355039 & 4.121306 & -0.711762 \\
\hline 25 & 1 & $\odot$ & -4.879149 & 5.914377 & -1.477847 \\
\hline 26 & 6 & $\odot$ & 9.235249 & -3.042924 & -1.634101 \\
\hline 27 & 6 & $\odot$ & 8.666078 & -1.640959 & -1.711073 \\
\hline 28 & 6 & $\odot$ & 8.467763 & -0.942741 & -0.357507 \\
\hline 29 & 6 & $\odot$ & 7.036884 & -1.317198 & 0.003263 \\
\hline 30 & 6 & $\odot$ & 6.337346 & -1.239057 & -1.351538 \\
\hline 31 & 8 & $\odot$ & 7.328277 & -1.689715 & -2.317151 \\
\hline 32 & 1 & $\odot$ & 9.213310 & -3.485372 & -2.635286 \\
\hline 33 & 1 & $\odot$ & 10.276149 & -2.990941 & -1.294429 \\
\hline 34 & 1 & $\odot$ & 9.304237 & -1.031700 & -2.354791 \\
\hline 35 & 1 & $\odot$ & 9.199391 & -1.243145 & 0.393660 \\
\hline 36 & 1 & $\odot$ & 6.602383 & -0.650025 & 0.740775 \\
\hline 37 & 1 & $\odot$ & 7.035023 & -2.339004 & 0.387637 \\
\hline 38 & 1 & 0 & 6.034176 & -0.222077 & -1.586083 \\
\hline 39 & 8 & $\odot$ & 8.438779 & -3.828842 & -0.712665 \\
\hline 40 & 1 & $\odot$ & 8.748942 & -4.748451 & -0.685328 \\
\hline 41 & $\overline{6}$ & $\odot$ & 3.914307 & -1.426244 & -1.711080 \\
\hline 42 & 7 & $\odot$ & 1.673394 & -4.120699 & -1.249648 \\
\hline 43 & 8 & $\odot$ & 3. 900201 & -0.221285 & -1.990750 \\
\hline 44 & 1 & $\odot$ & 6.210399 & -3.802732 & -1.100496 \\
\hline 45 & 1 & $\odot$ & 4.126097 & -5.221040 & -1.009119 \\
\hline 46 & 1 & $\odot$ & 1.659117 & -5.105745 & -1.044320 \\
\hline 47 & 1 & $\odot$ & ๑. 788382 & -3.620198 & -1.378506 \\
\hline 48 & 6 & $\odot$ & 5.963104 & 2.697532 & -1.062982 \\
\hline 49 & 1 & $\odot$ & 4.874087 & 6.029349 & 1.716924 \\
\hline 50 & 6 & $\odot$ & 4.706890 & 3. 322701 & 1.911355 \\
\hline 51 & 6 & $\odot$ & 3.442858 & 2.775651 & 1. 232272 \\
\hline 52 & 8 & $\odot$ & 3.672003 & 2.910272 & -0.202267 \\
\hline 53 & 1 & $\odot$ & 5.507466 & 2.089699 & -1.846891 \\
\hline 54 & 1 & $\odot$ & 6.789135 & 3.284652 & -1.472242 \\
\hline 55 & 1 & 0 & 4.689447 & 4.408167 & -1.203052 \\
\hline 56 & 1 & $\odot$ & 6.395169 & 4.363563 & 0.965864 \\
\hline 57 & 1 & $\odot$ & 4.468096 & 3.841783 & 2.841643 \\
\hline 58 & 1 & $\odot$ & 5.422523 & 2.529157 & 2.133134 \\
\hline 59 & 1 & $\odot$ & 2.553008 & 3.343205 & 1.491788 \\
\hline 60 & 7 & $\odot$ & 3.134398 & 1.379022 & 1.526279 \\
\hline 61 & 6 & $\odot$ & 4.085644 & 0.425871 & 1.316045 \\
\hline 62 & 6 & $\odot$ & 3.805433 & -0.887010 & 1. 455562 \\
\hline 63 & 6 & $\odot$ & 2.445185 & -1.239175 & 1.730086 \\
\hline 64 & 7 & $\odot$ & 1.502887 & -0.307273 & 1.895094 \\
\hline 65 & 6 & $\odot$ & 1.801705 & 1.013170 & 1.799110 \\
\hline 66 & 1 & $\odot$ & 4.561553 & -1.645333 & 1.305980 \\
\hline 67 & 7 & $\odot$ & 2.088816 & -2.519724 & 1.822617 \\
\hline 68 & 8 & $\odot$ & ๑. 951836 & 1.897186 & 1.950698 \\
\hline 69 & 1 & $\odot$ & 5.058554 & $\odot .795436$ & 1.004797 \\
\hline 70 & 1 & $\odot$ & 2.768259 & -3.239420 & 1.638409 \\
\hline 71 & 1 & $\odot$ & 1.097291 & -2.782245 & 1.886184 \\
\hline 72 & 1 & $\odot$ & -0.309281 & -0.797316 & 1.962191 \\
\hline 73 & 1 & $\odot$ & -8.430380 & -5.049631 & 0.758355 \\
\hline
\end{tabular}




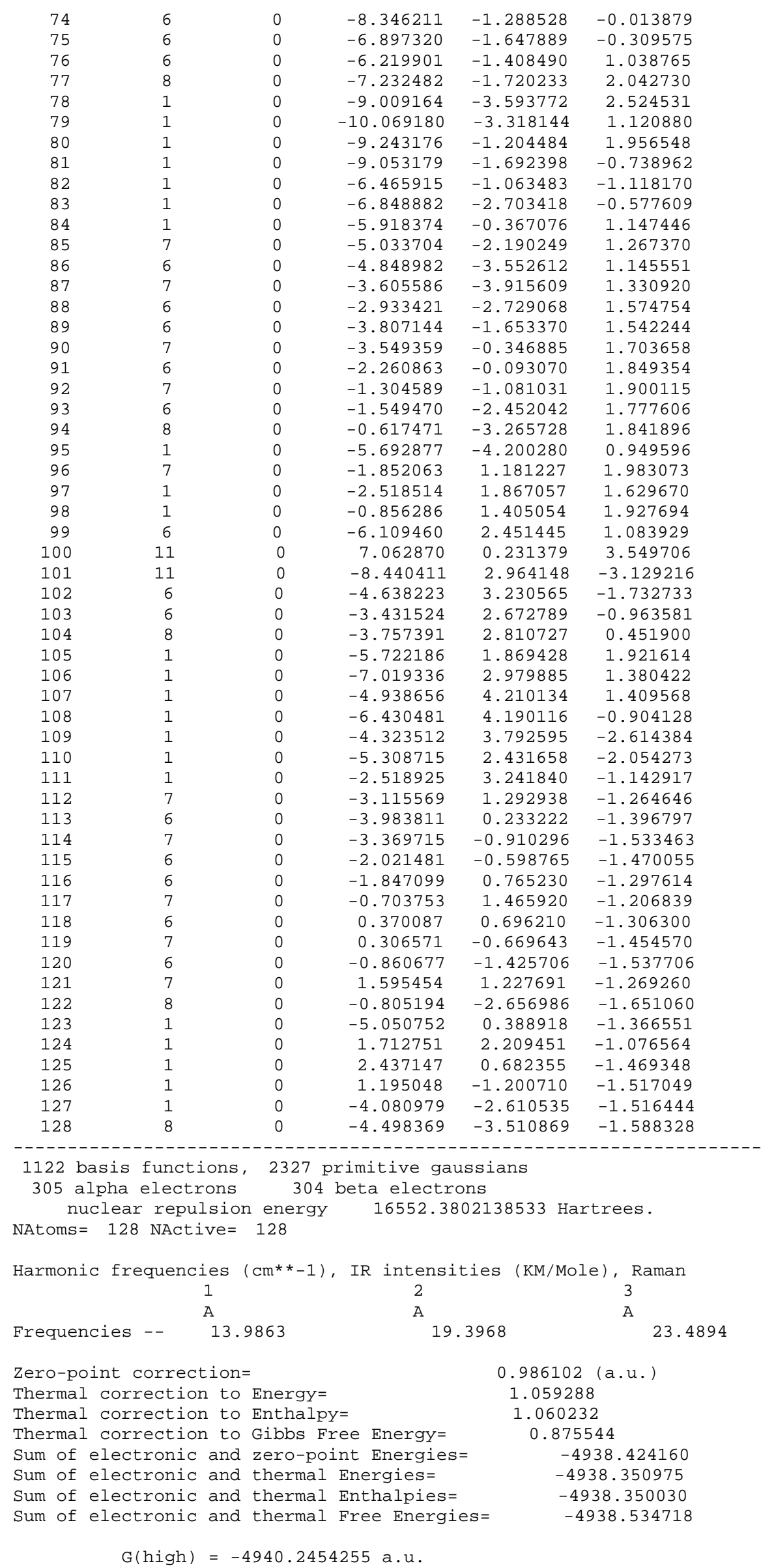




\begin{tabular}{|c|c|c|c|}
\hline $\begin{array}{c}\mathrm{E}(\mathrm{T} \\
\mathrm{KCa}\end{array}$ & $\begin{array}{l}\text { ermal) } \\
\text { /Mol } \\
44.713\end{array}$ & $\begin{array}{c}\text { CV } \\
\text { Cal/Mol-Kelvin } \\
279.113\end{array}$ & $\begin{array}{c}\mathrm{S} \\
\mathrm{Cal} / \mathrm{Mol}-\mathrm{Kelvin} \\
388.708\end{array}$ \\
\hline Item & Value & \multicolumn{2}{|c|}{ Threshold Converged? } \\
\hline Maximum Force & 0.000028 & 0.000450 & YES \\
\hline Force & ๑.00०००5 & $\odot .000300$ & ES \\
\hline Maximum Displacement & 0.010661 & 0.001800 & NO \\
\hline Displacement & 0.001828 & 0.001200 & NO \\
\hline
\end{tabular}

Normal termination of Gaussian 16 at Sun Feb 10 13:47:๑3 2019.

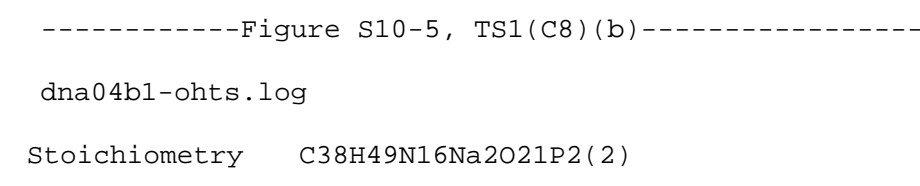

Standard orientation:

\begin{tabular}{|c|c|c|c|c|c|}
\hline \multirow{2}{*}{$\begin{array}{l}\text { Center } \\
\text { Number }\end{array}$} & \multirow{2}{*}{$\begin{array}{l}\text { Atomic } \\
\text { Number }\end{array}$} & \multirow{2}{*}{$\begin{array}{l}\text { Atomic } \\
\text { Type }\end{array}$} & \multicolumn{3}{|c|}{ Coordinates (Angstroms) } \\
\hline & & & $\mathrm{x}$ & $\mathrm{Y}$ & Z \\
\hline 1 & 7 & 0 & 5.398493 & -2.031852 & -1.755883 \\
\hline 2 & 6 & 0 & 5.432685 & -3.394159 & -1.659305 \\
\hline 3 & 6 & 0 & 4.345890 & -4.135595 & -1.967238 \\
\hline 4 & 6 & 0 & 3.167252 & -3.427415 & -2.376613 \\
\hline 5 & 7 & 0 & 3.163662 & -2.097920 & -2.520153 \\
\hline 6 & 8 & 0 & 8.565118 & $\odot .366623$ & $\odot .061328$ \\
\hline 7 & 15 & 0 & 7.776319 & 1.514197 & $\odot .917084$ \\
\hline 8 & 8 & $\odot$ & 6.334016 & 1.608239 & 0.125960 \\
\hline 9 & 8 & $\odot$ & 8.525047 & 2.803297 & $\odot .746520$ \\
\hline 10 & 8 & 0 & 7.446411 & 0.958808 & 2.285651 \\
\hline 11 & 8 & $\odot$ & 4.537390 & 5.297113 & 1.396169 \\
\hline 12 & 6 & 0 & 4.893989 & 3.563991 & $-\odot .197735$ \\
\hline 13 & 6 & 0 & 5.160287 & 3.996754 & 1.246546 \\
\hline 14 & 6 & 0 & -9.468957 & -2.989934 & 1.486771 \\
\hline 15 & 6 & $\odot$ & -8.894086 & -1.590581 & 1. 401811 \\
\hline 16 & 8 & 0 & -8.460127 & -3.944998 & 1.079788 \\
\hline 17 & 8 & $\odot$ & -8.431670 & 0.210271 & -0.126949 \\
\hline 18 & 15 & 0 & -7.543594 & 1.142852 & -1.126074 \\
\hline 19 & 8 & 0 & -6.155355 & 1.369537 & -0.309065 \\
\hline 20 & 8 & 0 & -8.277083 & 2.454094 & -1.293296 \\
\hline 21 & 8 & 0 & -7.152558 & ๑.398807 & -2.386805 \\
\hline 22 & 8 & $\odot$ & -4.383477 & 5.233773 & -1.118685 \\
\hline 23 & 6 & $\odot$ & -4.811051 & 3.311214 & 0.213775 \\
\hline 24 & 6 & 0 & -5.038233 & 3.940864 & -1.162544 \\
\hline 25 & 1 & $\odot$ & -4.477520 & 5.691377 & -1.971365 \\
\hline 26 & 6 & $\odot$ & 9.380807 & -3.127728 & $-\odot .972635$ \\
\hline 27 & 6 & 0 & 8.873010 & -1.705764 & -1.094340 \\
\hline 28 & 6 & $\odot$ & 8.349771 & -1.076997 & 0.204813 \\
\hline 29 & 6 & 0 & 6.868105 & -1.428480 & 0.177162 \\
\hline 30 & 6 & 0 & 6.535795 & -1.246466 & -1.302899 \\
\hline 31 & 8 & 0 & 7.731745 & -1.667650 & -2.018095 \\
\hline 32 & 1 & $\odot$ & 9.612650 & -3.503025 & -1.974777 \\
\hline 33 & 1 & 0 & 10.298939 & -3.131723 & -0.373942 \\
\hline 34 & 1 & 0 & 9.665008 & -1.082226 & -1.514892 \\
\hline 35 & 1 & 0 & 8.867657 & -1.427560 & 1.098310 \\
\hline 36 & 1 & 0 & 6.269656 & -0.798994 & $\odot .831860$ \\
\hline 37 & 1 & 0 & 6.757007 & -2.473083 & 0.475030 \\
\hline 38 & 1 & 0 & 6.313460 & $-\odot .209012$ & -1.537857 \\
\hline 39 & 8 & 0 & 8.362251 & -3.946558 & -0.346372 \\
\hline 40 & 1 & 0 & 8.648043 & -4.872947 & $-\odot .291935$ \\
\hline 41 & 6 & 0 & 4.266970 & -1.365636 & -2.244242 \\
\hline 42 & 7 & 0 & 2.033122 & -4.076611 & -2.633669 \\
\hline 43 & 8 & 0 & 4.295700 & -0.134363 & -2.379379 \\
\hline 44 & 1 & 0 & 6.379425 & -3.810420 & -1.319187 \\
\hline 45 & 1 & 0 & 4.361229 & -5.214158 & -1.892794 \\
\hline 46 & 1 & 0 & 1.978072 & -5.073466 & -2.507119 \\
\hline 47 & 1 & 0 & 1.188724 & -3.551247 & -2.877506 \\
\hline 48 & 6 & 0 & 5.966472 & 2.671865 & -0.798057 \\
\hline 49 & 1 & 0 & 4.663644 & 5.630669 & 2.300796 \\
\hline 50 & 6 & 0 & 4.440540 & 2.930499 & 2.078552 \\
\hline 51 & 6 & 0 & 3.212754 & 2.590599 & 1.227888 \\
\hline 52 & 8 & 0 & 3.604199 & 2.862200 & -0.149088 \\
\hline 53 & 1 & 0 & 5.585038 & 2.189150 & -1.699122 \\
\hline 54 & 1 & $\odot$ & 6.849941 & 3.270219 & -1.034106 \\
\hline
\end{tabular}




\begin{tabular}{|c|c|c|c|c|c|}
\hline 55 & 1 & $\odot$ & 4.759771 & 4.440971 & -0.834683 \\
\hline 56 & 1 & $\odot$ & 6.231562 & 4.050619 & 1.463024 \\
\hline 57 & 1 & $\odot$ & 4.160522 & 3.292633 & 3.069857 \\
\hline 58 & 1 & $\odot$ & 5.089563 & 2.058937 & 2.189010 \\
\hline 59 & 1 & $\odot$ & 2.349686 & 3.202125 & 1.478369 \\
\hline 60 & 7 & $\odot$ & 2.772597 & 1.200588 & 1.313903 \\
\hline 61 & 6 & $\odot$ & 3.589219 & ๑. 207019 & $\odot .859477$ \\
\hline 62 & 6 & $\odot$ & 3.186382 & -1.082593 & ๑. 867196 \\
\hline 63 & 6 & $\odot$ & 1.865377 & -1.352858 & 1.353463 \\
\hline 64 & 7 & $\odot$ & 1.057798 & -0.370824 & 1.761791 \\
\hline 65 & 6 & $\odot$ & 1.465006 & ๑. 922032 & 1.741931 \\
\hline 66 & 1 & $\odot$ & 3.832874 & -1.879098 & 0.524177 \\
\hline 67 & 7 & $\odot$ & 1.401256 & -2.601873 & 1.405629 \\
\hline 68 & 8 & 0 & 0.732904 & 1.853372 & 2.096651 \\
\hline 69 & 1 & $\odot$ & 4.558717 & ๑. 531912 & $\odot .490745$ \\
\hline 70 & 1 & $\odot$ & 1.993277 & -3.369923 & 1.135774 \\
\hline 71 & 1 & $\odot$ & $\odot .502481$ & -2.797978 & 1.858451 \\
\hline 72 & 1 & $\odot$ & -0.692190 & -0.709021 & 2.395673 \\
\hline 73 & 1 & $\odot$ & -8.784604 & -4.853913 & 1.185992 \\
\hline 74 & 6 & $\odot$ & -8.241635 & -1.237712 & 0.055351 \\
\hline 75 & 6 & $\odot$ & -6.783983 & -1.600088 & ๑. 280464 \\
\hline 76 & 6 & $\odot$ & -6.565613 & -1.186692 & 1.734669 \\
\hline 77 & 8 & $\odot$ & -7.830306 & -1.436869 & 2.403553 \\
\hline 78 & 1 & $\odot$ & -9.783515 & -3.175224 & 2.519379 \\
\hline 79 & 1 & $\odot$ & -10.348837 & -3.055498 & 0.835321 \\
\hline 80 & 1 & $\odot$ & -9.680863 & -0.869283 & 1.635383 \\
\hline 81 & 1 & $\odot$ & -8.697504 & -1.754764 & -0.789869 \\
\hline 82 & $\overline{1}$ & $\odot$ & -6.069118 & -1.116326 & -0.380036 \\
\hline 83 & 1 & 0 & -6.687088 & -2.684035 & ๑.190569 \\
\hline 84 & 1 & $\odot$ & -6.314705 & -0.129286 & 1.815692 \\
\hline 85 & 7 & $\odot$ & -5.493298 & -1.912256 & 2.376152 \\
\hline 86 & 6 & $\odot$ & -5.365555 & -3.284933 & 2.470543 \\
\hline 87 & 7 & $\odot$ & -4.140668 & -3.672448 & 2.700388 \\
\hline 88 & 6 & $\odot$ & -3.408306 & -2.493781 & 2.732756 \\
\hline 89 & 6 & $\odot$ & -4.233568 & -1.395566 & 2.530033 \\
\hline 90 & 7 & $\odot$ & -3.905340 & -0.095132 & 2.443867 \\
\hline 91 & 6 & $\odot$ & -2.599470 & ๑. 095821 & 2.412810 \\
\hline 92 & 7 & $\odot$ & -1.688452 & -0.919527 & 2.580997 \\
\hline 93 & 6 & $\odot$ & -1.999025 & -2.273435 & 2.718020 \\
\hline 94 & 8 & $\odot$ & -1.091455 & -3.117342 & 2.765039 \\
\hline 95 & 1 & $\odot$ & -6.235421 & -3.918067 & 2.350236 \\
\hline 96 & 7 & $\odot$ & -2.107492 & 1.338393 & 2.227740 \\
\hline 97 & 1 & $\odot$ & -2.680286 & 1.920884 & 1.616335 \\
\hline 98 & 1 & 0 & -1.096680 & 1.458832 & 2.143592 \\
\hline 99 & 6 & 0 & -5.913800 & 2.391979 & $\odot .692922$ \\
\hline 100 & 11 & $\odot$ & 7.077538 & 1. 329187 & 4.413435 \\
\hline 101 & 11 & $\odot$ & -7.926580 & 2.231385 & -3.826554 \\
\hline 102 & 6 & 0 & -4.333157 & 2.984767 & -2.126813 \\
\hline 103 & 6 & $\odot$ & -3.161922 & 2.426623 & -1.306357 \\
\hline 104 & 8 & $\odot$ & -3.564239 & 2.551094 & ๑. . 086672 \\
\hline 105 & 1 & $\odot$ & -5.585720 & 1.882917 & 1.601072 \\
\hline 106 & 1 & 0 & -6.824780 & 2.965535 & ๑. 884658 \\
\hline 107 & 1 & 0 & -4.642796 & 4.094604 & 0.956303 \\
\hline 108 & 1 & 0 & -6.103675 & 4.045107 & -1.389752 \\
\hline 109 & 1 & 0 & -3.984319 & 3.489125 & -3.030082 \\
\hline 110 & 1 & $\odot$ & -5.019602 & 2.188027 & -2.416837 \\
\hline 111 & 1 & $\odot$ & -2.252034 & 3.014624 & -1.437537 \\
\hline 112 & 7 & $\odot$ & -2.801502 & 1.064402 & -1.647343 \\
\hline 113 & 6 & $\odot$ & -3.624201 & -0.002184 & -1.978906 \\
\hline 114 & 7 & 0 & -2.942282 & -1.008387 & -2.528490 \\
\hline 115 & 6 & $\odot$ & -1.634894 & $-\odot .635568$ & -2.420330 \\
\hline 116 & 6 & 0 & -1.514508 & 0.643599 & -1.873492 \\
\hline 117 & 7 & 0 & -0.400801 & 1. 345101 & -1.632066 \\
\hline 118 & 6 & $\odot$ & 0.706363 & $\odot .675926$ & -1.948706 \\
\hline 119 & 7 & 0 & ๑. 698210 & -0.607479 & -2.442064 \\
\hline 120 & 6 & 0 & -0.439368 & -1.363860 & -2.712376 \\
\hline 121 & 7 & 0 & 1.904335 & 1.240792 & -1.804671 \\
\hline 122 & 8 & 0 & -0.343588 & -2.519979 & -3.137473 \\
\hline 123 & 1 & 0 & -4.701057 & 0.105676 & -2.000161 \\
\hline 124 & 1 & 0 & 2.005750 & 2.125616 & -1.327996 \\
\hline 125 & 1 & 0 & 2.765578 & 0.733511 & -2.026999 \\
\hline 126 & 1 & $\odot$ & 1.603283 & -1.100190 & -2.569075 \\
\hline 127 & 8 & 0 & -3.675879 & -1.208462 & -0.219371 \\
\hline 128 & 1 & $\odot$ & -3.632506 & -2.070652 & -0.659923 \\
\hline
\end{tabular}


nuclear repulsion energy 16345.0649382981 Hartrees. NAtoms $=128$ NActive $=128$

\begin{tabular}{|c|c|c|c|c|c|c|c|c|c|c|}
\hline & 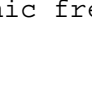 & . & $\begin{array}{l}1 \\
A\end{array}$ & & $\begin{array}{l}2 \\
\mathrm{~A}\end{array}$ & & & $\begin{array}{l}3 \\
A\end{array}$ & & \\
\hline Freque & ncies & -- & -269.11 & & & 14.138 & & & 7.8008 & \\
\hline Red. $n$ & lasses & -- & 10.00 & & & 7.6771 & & & .3559 & \\
\hline Frc co & insts & -- & 0.426 & & & $\odot .00 \odot 9$ & & & .0017 & \\
\hline IR Int & en & -- & 215.618 & & & 3.5226 & & & .2370 & \\
\hline Atom & AN & $x$ & $Y$ & Z & $x$ & $\mathrm{Y}$ & Z & $x$ & Z & \\
\hline 1 & 7 & -0.00 & $\odot .0 \odot$ & $-\odot .0 \odot$ & -0.02 & -0.03 & 0.01 & -0.01 & -0.01 & $\odot .08$ \\
\hline 2 & 6 & $\odot .0 \odot$ & $\odot . \odot \odot$ & $-\odot . \odot \odot$ & -0.04 & -0.03 & $\odot .06$ & -0.01 & -0.01 & 0.12 \\
\hline 3 & 6 & $\odot . \odot \odot$ & $\odot . \odot \odot$ & $-\odot . \odot \odot$ & -0.04 & -0.02 & $\odot .06$ & -0.02 & -0.02 & 0.17 \\
\hline 4 & 6 & $\odot .0 \odot$ & -0.00 & 0.00 & -0.02 & -0.02 & 0.01 & -0.02 & -0.03 & 0.16 \\
\hline 5 & 7 & $-\odot . \odot \odot$ & -0.00 & $\odot . \odot \odot$ & 0.00 & -0.02 & $-\odot . \odot 4$ & -0.02 & -0.03 & 0.12 \\
\hline 6 & 8 & $-\odot . \odot \odot$ & $-\odot . \odot \odot$ & -0.00 & -0.05 & $\odot .0 \odot$ & $-\odot . \odot \odot$ & $\odot . \odot 4$ & 0.05 & -0.08 \\
\hline 7 & 15 & 0.00 & 0.00 & $-\odot .0 \odot$ & -0.05 & 0.03 & -0.05 & $\odot .06$ & 0.06 & -0.07 \\
\hline 8 & 8 & $-0.0 \odot$ & 0.00 & $-0.0 \odot$ & -0.05 & 0.01 & -0.06 & 0.02 & 0.02 & -0.02 \\
\hline 9 & 8 & -0.00 & -0.00 & -0.00 & -0.05 & 0.03 & -0.09 & 0.03 & 0.06 & -0.13 \\
\hline 10 & 8 & $-\odot . \odot \odot$ & -0.00 & $-0.0 \odot$ & -0.06 & 0.08 & -0.03 & 0.12 & 0.08 & -0.05 \\
\hline 11 & 8 & $-\odot . \odot \odot$ & $-\odot . \odot \odot$ & $-\odot . \odot \odot$ & -0.07 & 0.02 & -0.10 & $-\odot . \odot \odot$ & $-\odot . \odot \odot$ & $-0.0 \odot$ \\
\hline 12 & 6 & -0.00 & $-0.0 \odot$ & -0.00 & -0.06 & -0.00 & -0.08 & -0.02 & -0.01 & -0.00 \\
\hline 13 & 6 & -0.00 & $-0.0 \odot$ & $-0.0 \odot$ & -0.06 & ๑. 02 & -0.08 & 0.01 & $\odot .0 \odot$ & -0.01 \\
\hline 14 & 6 & -0.00 & $\odot .00$ & $-\odot .0 \odot$ & 0.03 & -0.04 & -0.11 & -0.04 & 0.02 & -0.03 \\
\hline 15 & 6 & $\odot .00$ & -0.00 & $-\odot . \odot \odot$ & $\odot .02$ & -0.04 & -0.06 & -0.02 & 0.02 & -0.01 \\
\hline 16 & 8 & -0.00 & $-\odot . \odot \odot$ & $-0.0 \odot$ & 0.04 & -0.03 & -0.12 & -0.06 & 0.02 & -0.07 \\
\hline 17 & 8 & -0.00 & 0.00 & -0.00 & 0.04 & 0.01 & -0.00 & -0.04 & 0.04 & 0.01 \\
\hline 18 & 15 & $-0.0 \odot$ & $0.0 \odot$ & $-0.0 \odot$ & 0.05 & 0.04 & $\odot .03$ & -0.04 & 0.06 & 0.03 \\
\hline 19 & 8 & -0.01 & 0.01 & -0.01 & 0.05 & 0.01 & 0.05 & -0.03 & 0.02 & $\odot .03$ \\
\hline 20 & 8 & -0.01 & -0.00 & -0.01 & 0.05 & 0.04 & 0.07 & -0.03 & 0.07 & 0.07 \\
\hline 21 & 8 & $-\odot . \odot \odot$ & 0.00 & $-0.0 \odot$ & 0.05 & 0.07 & 0.01 & -0.06 & 0.09 & $\odot .01$ \\
\hline 22 & 8 & 0.00 & -0.02 & -0.02 & 0.07 & 0.01 & 0.11 & 0.03 & -0.01 & -0.00 \\
\hline 23 & 6 & -0.01 & $\odot .01$ & 0.01 & 0.06 & -0.01 & 0.07 & 0.01 & $-0.0 \odot$ & 0.01 \\
\hline 24 & 6 & 0.00 & -0.02 & -0.01 & 0.06 & 0.02 & 0.09 & $-0.0 \odot$ & -0.00 & 0.01 \\
\hline 25 & 1 & 0.01 & -0.03 & -0.02 & 0.08 & 0.03 & 0.11 & 0.02 & -0.02 & -0.00 \\
\hline 26 & 6 & $-\odot . \odot \odot$ & $\odot .0 \odot$ & $-\odot . \odot \odot$ & -0.04 & -0.04 & 0.15 & 0.03 & 0.03 & -0.04 \\
\hline 27 & 6 & -0.00 & $\odot .0 \odot$ & $-\odot .0 \odot$ & -0.03 & -0.04 & $\odot .08$ & $\odot .01$ & 0.02 & -0.05 \\
\hline 28 & 6 & -0.00 & $\odot .0 \odot$ & $-\odot . \odot \odot$ & -0.05 & 0.01 & 0.05 & $\odot .06$ & 0.05 & -0.04 \\
\hline 29 & 6 & $-\odot . \odot \odot$ & $\odot .0 \odot$ & $-\odot . \odot \odot$ & -0.05 & 0.02 & 0.03 & 0.06 & 0.03 & 0.02 \\
\hline 30 & 6 & -0.00 & 0.00 & $-\odot .00$ & -0.02 & -0.03 & 0.02 & $\odot .00$ & 0.00 & 0.03 \\
\hline 31 & 8 & -0.00 & $\odot .0 \odot$ & $-\odot . \odot \odot$ & -0.01 & -0.07 & 0.06 & -0.02 & -0.01 & -0.01 \\
\hline 32 & 1 & -0.00 & $\odot .0 \odot$ & -0.00 & -0.02 & -0.08 & 0.17 & -0.01 & $\odot .01$ & -0.04 \\
\hline 33 & 1 & -0.00 & 0.00 & -0.00 & -0.06 & -0.03 & 0.17 & 0.05 & 0.05 & -0.07 \\
\hline 34 & 1 & -0.00 & 0.00 & $-\odot . \odot \odot$ & -0.01 & -0.06 & 0.08 & -0.01 & 0.02 & -0.09 \\
\hline 35 & 1 & -0.00 & -0.00 & -0.00 & -0.07 & 0.04 & 0.07 & $\odot .09$ & 0.07 & -0.05 \\
\hline 36 & 1 & -0.00 & 0.00 & $-\odot .0 \odot$ & -0.06 & 0.04 & $-\odot .0 \odot$ & $\odot .08$ & 0.04 & 0.03 \\
\hline 37 & 1 & -0.00 & 0.00 & -0.00 & -0.07 & 0.03 & 0.06 & 0.08 & 0.04 & 0.05 \\
\hline 38 & 1 & 0.00 & $\odot .00$ & -0.00 & 0.00 & -0.03 & -0.02 & -0.01 & -0.00 & $\odot .02$ \\
\hline 39 & 8 & $-\odot . \odot \odot$ & $\odot .0 \odot$ & $-\odot . \odot \odot$ & -0.06 & -0.01 & 0.15 & 0.06 & 0.04 & 0.02 \\
\hline 40 & 1 & -0.00 & $0.0 \odot$ & $-\odot .00$ & -0.07 & -0.01 & 0.20 & $\odot .07$ & 0.04 & 0.03 \\
\hline 41 & 6 & -0.00 & 0.00 & 0.00 & 0.00 & -0.03 & -0.03 & -0.02 & -0.03 & $\odot .09$ \\
\hline 42 & 7 & $\odot .01$ & -0.01 & $-\odot . \odot \odot$ & $-\odot .03$ & -0.01 & $\odot . \odot \odot$ & -0.02 & -0.04 & 0.19 \\
\hline 43 & 8 & -0.01 & 0.00 & & 0.02 & -0.03 & -0.07 & -0.02 & -0.03 & $\odot .06$ \\
\hline 44 & 1 & $0.0 \odot$ & 0.01 & -0.00 & -0.05 & -0.03 & 0.10 & $-\odot .0 \odot$ & -0.00 & 0.11 \\
\hline 45 & 1 & 0.00 & $0.0 \odot$ & -0.00 & -0.06 & -0.02 & 0.10 & -0.01 & -0.02 & 0.20 \\
\hline 46 & 1 & 0.01 & -0.01 & $-0.0 \odot$ & -0.05 & $-0.0 \odot$ & 0.04 & -0.02 & -0.04 & 0.21 \\
\hline 47 & 1 & 0.01 & -0.01 & -0.01 & -0.01 & $-\odot . \odot \odot$ & -0.03 & -0.02 & -0.05 & 0.16 \\
\hline 48 & 6 & -0.00 & $-0.0 \odot$ & -0.00 & -0.06 & -0.00 & -0.07 & -0.03 & 0.00 & -0.02 \\
\hline 49 & 1 & $\odot . \odot \odot$ & $-\odot .0 \odot$ & $-0.0 \odot$ & -0.06 & 0.04 & -0.11 & $\odot .02$ & 0.01 & -0.01 \\
\hline 50 & 6 & $-\odot . \odot \odot$ & $-\odot . \odot \odot$ & $-\odot . \odot \odot$ & -0.05 & 0.03 & -0.07 & 0.04 & $-\odot . \odot \odot$ & 0.01 \\
\hline 51 & 6 & $-\odot . \odot \odot$ & -0.00 & -0.00 & -0.05 & 0.01 & -0.06 & 0.02 & -0.02 & 0.05 \\
\hline 52 & 8 & $-0.0 \odot$ & $-\odot . \odot \odot$ & $-\odot . \odot \odot$ & -0.06 & -0.01 & -0.06 & -0.01 & -0.02 & 0.03 \\
\hline 53 & 1 & 0.00 & $-\odot . \odot \odot$ & $-\odot . \odot \odot$ & -0.06 & -0.02 & -0.06 & -0.05 & -0.02 & $\odot .00$ \\
\hline 54 & 1 & -0.00 & 0.00 & 0.00 & -0.06 & $-\odot . \odot \odot$ & -0.08 & -0.04 & 0.01 & -0.06 \\
\hline 55 & 1 & -0.00 & $-\odot . \odot \odot$ & $-\odot . \odot \odot$ & -0.07 & -0.01 & -0.09 & $-\odot .04$ & -0.01 & $-0.0 \odot$ \\
\hline 56 & 1 & $-\odot . \odot \odot$ & $-0.0 \odot$ & $-\odot . \odot \odot$ & -0.06 & 0.03 & $-\odot .09$ & 0.01 & 0.02 & -0.03 \\
\hline 57 & 1 & $-0.0 \odot$ & $-0.0 \odot$ & $-0.0 \odot$ & -0.05 & 0.04 & -0.07 & 0.06 & 0.00 & $\odot .02$ \\
\hline 58 & 1 & 0.00 & -0.00 & -0.00 & -0.05 & 0.03 & -0.06 & 0.06 & 0.01 & 0.00 \\
\hline 59 & 1 & $-\odot . \odot \odot$ & $-\odot . \odot \odot$ & $-\odot . \odot \odot$ & -0.06 & 0.01 & $-\odot .07$ & 0.03 & $-\odot .02$ & 0.06 \\
\hline 60 & 7 & 0.00 & $-\odot . \odot \odot$ & $-\odot . \odot \odot$ & -0.04 & 0.01 & -0.04 & $\odot .04$ & -0.02 & 0.06 \\
\hline 61 & 6 & 0.00 & $-\odot .00$ & $\odot . \odot \odot$ & $-\odot .04$ & 0.01 & -0.05 & 0.06 & -0.02 & 0.08 \\
\hline 62 & 6 & 0.00 & $-\odot . \odot \odot$ & $\odot . \odot \odot$ & -0.03 & $\odot .01$ & -0.03 & 0.07 & -0.02 & 0.09 \\
\hline 63 & 6 & -0.00 & $-0.0 \odot$ & 0.00 & -0.02 & -0.00 & 0.01 & 0.05 & -0.03 & 0.05 \\
\hline 64 & 7 & $\odot .0 \odot$ & 0.00 & -0.00 & -0.01 & -0.01 & 0.03 & 0.03 & $-\odot .03$ & 0.02 \\
\hline 65 & 6 & $\odot .0 \odot$ & $-\odot . \odot \odot$ & $\odot . \odot \odot$ & -0.02 & $-\odot .0 \odot$ & $\odot .02$ & 0.03 & -0.03 & 0.02 \\
\hline 66 & 1 & 0.00 & $-\odot .0 \odot$ & $\odot . \odot \odot$ & -0.04 & 0.01 & -0.04 & $\odot .08$ & -0.02 & 0.11 \\
\hline
\end{tabular}




\begin{tabular}{|c|c|c|c|c|}
\hline 67 & 7 & -0.00 & 0.00 & 0.00 \\
\hline 68 & 8 & $\odot . \odot \odot$ & $\odot . \odot \odot$ & -0.00 \\
\hline 69 & 1 & $\odot . \odot \odot$ & $-\odot .0 \odot$ & $\odot . \odot \odot$ \\
\hline 70 & 1 & -0.00 & -0.00 & 0.00 \\
\hline 71 & 1 & -0.00 & 0.00 & 0.00 \\
\hline 72 & 1 & -0.00 & $-0.0 \odot$ & $-\odot . \odot \odot$ \\
\hline 73 & 1 & $-\odot . \odot \odot$ & $-\odot . \odot \odot$ & $\odot .0 \odot$ \\
\hline 74 & 6 & 0.01 & $\odot .0 \odot$ & $-\odot . \odot \odot$ \\
\hline 75 & 6 & $\odot .01$ & 0.00 & 0.00 \\
\hline 76 & 6 & -0.00 & $-0.0 \odot$ & 0.01 \\
\hline 77 & 8 & -0.00 & $-0.0 \odot$ & $\odot .0 \odot$ \\
\hline 78 & 1 & $-\odot . \odot \odot$ & 0.00 & $-\odot . \odot \odot$ \\
\hline 79 & 1 & $\odot .0 \odot$ & $\odot . \odot \odot$ & $-\odot . \odot \odot$ \\
\hline 80 & 1 & $\odot . \odot \odot$ & $-\odot . \odot \odot$ & $-0.0 \odot$ \\
\hline 81 & 1 & 0.01 & $-0.0 \odot$ & $-\odot . \odot \odot$ \\
\hline 82 & 1 & 0.03 & $\odot . \odot \odot$ & $\odot .03$ \\
\hline 83 & 1 & 0.01 & $\odot . \odot \odot$ & 0.01 \\
\hline 84 & 1 & $-\odot . \odot \odot$ & $-\odot . \odot \odot$ & 0.01 \\
\hline 85 & 7 & $\odot .0 \odot$ & $\odot .0 \odot$ & $\odot . \odot \odot$ \\
\hline 86 & 6 & $\odot . \odot \odot$ & $-\odot . \odot \odot$ & -0.00 \\
\hline 87 & 7 & $\odot . \odot \odot$ & $-\odot . \odot \odot$ & $-\odot . \odot \odot$ \\
\hline 88 & 6 & $-0.0 \odot$ & 0.00 & 0.01 \\
\hline 89 & 6 & $\odot .0 \odot$ & $-0.0 \odot$ & 0.01 \\
\hline 90 & 7 & -0.00 & -0.00 & 0.02 \\
\hline 91 & 6 & -0.00 & -0.00 & 0.01 \\
\hline 92 & 7 & $-\odot . \odot \odot$ & $-\odot . \odot \odot$ & -0.00 \\
\hline 93 & 6 & -0.00 & -0.00 & 0.00 \\
\hline 94 & 8 & $\odot .00$ & $-\odot . \odot \odot$ & $-\odot .0 \odot$ \\
\hline 95 & 1 & 0.00 & 0.00 & -0.01 \\
\hline 96 & 7 & $-\odot . \odot \odot$ & $\odot . \odot \odot$ & 0.01 \\
\hline 97 & 1 & $-\odot . \odot \odot$ & -0.01 & -0.00 \\
\hline 98 & 1 & $-0.0 \odot$ & -0.00 & -0.00 \\
\hline 99 & 6 & -0.01 & $\odot . \odot \odot$ & $\odot .0 \odot$ \\
\hline 100 & 11 & $\odot .0 \odot$ & $-0.0 \odot$ & $-\odot . \odot \odot$ \\
\hline 101 & 11 & -0.00 & 0.00 & 0.00 \\
\hline 102 & 6 & 0.01 & -0.05 & 0.02 \\
\hline 103 & 6 & -0.01 & -0.06 & 0.05 \\
\hline 104 & 8 & $\odot .0 \odot$ & 0.03 & 0.04 \\
\hline 105 & 1 & -0.01 & $-\odot . \odot \odot$ & $-\odot . \odot \odot$ \\
\hline 106 & 1 & -0.01 & $\odot .0 \odot$ & 0.01 \\
\hline 107 & 1 & -0.03 & 0.02 & -0.00 \\
\hline 108 & 1 & 0.01 & -0.02 & -0.02 \\
\hline 109 & 1 & 0.03 & -0.07 & 0.02 \\
\hline 110 & 1 & 0.00 & -0.05 & 0.02 \\
\hline 111 & 1 & 0.00 & -0.09 & 0.01 \\
\hline 112 & 7 & -0.00 & -0.09 & 0.14 \\
\hline 113 & 6 & $\odot .02$ & -0.27 & 0.45 \\
\hline 114 & 7 & 0.10 & -0.08 & -0.07 \\
\hline 115 & 6 & 0.01 & -0.01 & -0.08 \\
\hline 116 & 6 & -0.01 & -0.01 & 0.02 \\
\hline 117 & 7 & -0.02 & -0.02 & $\odot .0 \odot$ \\
\hline 118 & 6 & $0.0 \odot$ & -0.01 & -0.02 \\
\hline 119 & 7 & 0.02 & -0.01 & -0.02 \\
\hline 120 & 6 & 0.03 & 0.00 & -0.05 \\
\hline 121 & 7 & -0.02 & $-0.0 \odot$ & 0.01 \\
\hline 122 & 8 & $\odot .02$ & $-\odot .0 \odot$ & -0.02 \\
\hline 123 & 1 & $\odot .04$ & -0.12 & 0.05 \\
\hline 124 & 1 & -0.03 & 0.01 & -0.02 \\
\hline 125 & 1 & -0.01 & 0.02 & -0.01 \\
\hline 126 & 1 & 0.03 & -0.01 & 0.01 \\
\hline 127 & 8 & -0.05 & 0.46 & -0.35 \\
\hline 128 & 1 & -0.12 & 0.43 & -0.27 \\
\hline
\end{tabular}

\begin{tabular}{|c|c|c|}
\hline ๑.๑९ & $-\odot .01$ & \\
\hline 0.01 & 01 & \\
\hline & 1 & \\
\hline & $-\odot .0 \odot$ & -0.00 \\
\hline & -0.01 & \\
\hline$\odot . \odot \odot$ & -0 & $\odot .06$ \\
\hline & $-\odot$. & -0.16 \\
\hline$\odot .04$ & $\odot$. & -0.04 \\
\hline .04 & $\odot . \odot \odot$ & -0.03 \\
\hline & -0.03 & -0.01 \\
\hline$\odot .0 \odot$ & -0. & -0.04 \\
\hline 0.02 & -0.08 & -0.12 \\
\hline & $-\odot$. & -0.13 \\
\hline 0.01 & -0. & -0.05 \\
\hline 0.06 & 0.03 & -0.06 \\
\hline 0.0 & 0.02 & $-\cap \cap \Omega$ \\
\hline 0.05 & 0.01 & -0.05 \\
\hline$\odot .00$ & -0.03 & 0.02 \\
\hline 0.01 & -0.04 & -0.01 \\
\hline & -0 . & -0 \\
\hline ○. & -0. & -0.02 \\
\hline U. & $-\odot$ & $\odot .01$ \\
\hline & $-\odot$. & \\
\hline$\odot$. & -0. & 0.04 \\
\hline$\odot .0 \odot$ & -0 & $\odot$ \\
\hline & $-\odot$ & \\
\hline 0.00 & -0. & 0.03 \\
\hline 0.01 & -0.03 & 0.03 \\
\hline & $-\odot$ & -0. \\
\hline$\odot$. & -0. & ○. \\
\hline & -0 & 0.07 \\
\hline & -0 & \\
\hline 0 . & $-\odot$ & $\theta$. \\
\hline-0.07 & 0.11 & -0.04 \\
\hline & 0.09 & 0. \\
\hline$\odot$. & $\odot$. & .07 \\
\hline 0.05 & 0 . & .06 \\
\hline 0.05 & -0.02 & 0.06 \\
\hline & -0.03 & 0.05 \\
\hline 0.06 & -0.00 & 0.07 \\
\hline & -0.02 & 09 \\
\hline 6 & 3 & 9 \\
\hline & ○. & 8 \\
\hline 0.05 & 0.04 & 6 \\
\hline & $\Theta$. & 7 \\
\hline$\odot$ & 0 & \\
\hline & O. & 3 \\
\hline & J & 1 \\
\hline$\odot$ & 0. & 2 \\
\hline & $\odot$. & -0.01 \\
\hline & $\odot$. & -0 \\
\hline & $\odot$. & -0.06 \\
\hline 0.01 & $\odot . \odot \odot$ & $-\odot .07$ \\
\hline & 0.01 & -0.05 \\
\hline 0.04 & $-0.0 \odot$ & -0.10 \\
\hline-0.01 & 0.01 & $-\odot . \odot 6$ \\
\hline & 0.03 & 0.05 \\
\hline & -0.02 & $-\odot . \odot 7$ \\
\hline 0.03 & -0.02 & -0.11 \\
\hline & -0.00 & $-\odot .08$ \\
\hline & $-\odot . \odot \odot$ & 0.02 \\
\hline & $0.0 \odot$ & \\
\hline
\end{tabular}

\begin{tabular}{|c|c|c|}
\hline 0.06 & -0.03 & \\
\hline 01 & -0.03 & -0.01 \\
\hline & & \\
\hline & 3 & \\
\hline & -0. & -0.00 \\
\hline$\odot \odot$ & & $-\odot$ \\
\hline & & $-\odot$ \\
\hline & & -0.02 \\
\hline & & -0.06 \\
\hline & & -0.05 \\
\hline & & -0.03 \\
\hline & & -0.02 \\
\hline (.05 & & -0.00 \\
\hline & & 0.02 \\
\hline & & $-\odot .02$ \\
\hline 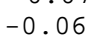 & 0.03 & -0.06 \\
\hline & & -0. \\
\hline & & -0 \\
\hline-0.0 & -0 & -0.09 \\
\hline & & -0.12 \\
\hline & & -0.13 \\
\hline - & $-\odot$ & -0.11 \\
\hline & & $-\odot .08$ \\
\hline$\odot$ & & $-\odot$. \\
\hline$\odot$. & -0 & $-\odot .05$ \\
\hline$-\mathrm{C}$ & & -0.08 \\
\hline & & -0.10 \\
\hline & & -0.11 \\
\hline-0. & -0 & -0.13 \\
\hline & & -0.03 \\
\hline 0. & & -0.02 \\
\hline 0. & -0 & $-\odot$ \\
\hline 0.0 & & 0. \\
\hline$\odot$. & & -0.05 \\
\hline-0.0 & & $\odot$. \\
\hline 4 & & \\
\hline-0 & -0 & -0.02 \\
\hline-0.0 & & $-\odot .01$ \\
\hline$\odot$. & & $\odot$. \\
\hline 0. & $\odot$. & 0.05 \\
\hline & -0 & 0.01 \\
\hline-0. & $\odot$ & 0.03 \\
\hline t & -0 & -0.01 \\
\hline-0.05 & -0.01 & 0.03 \\
\hline 03 & -0. & -0.03 \\
\hline & -0 & 93 \\
\hline-0. & -0 & -0.05 \\
\hline$-\odot .02$ & & $-\odot .04$ \\
\hline$-\odot$. & & $-\odot$ \\
\hline-0 & -0 & $\odot . \odot \odot$ \\
\hline$-\odot$. & $-\odot$. & $\odot .03$ \\
\hline$-\odot$. & $-\odot$ & 0.06 \\
\hline-0 & $-\odot$ & 0.06 \\
\hline-0.02 & -0.04 & $\odot .03$ \\
\hline & & 0.10 \\
\hline-0 & -0.04 & 0.04 \\
\hline-0.03 & -0.01 & $-\odot .07$ \\
\hline & -0.04 & $\odot .08$ \\
\hline & & 0.09 \\
\hline$-\odot$ & -0.05 & $\odot .09$ \\
\hline & & -0.07 \\
\hline & & $-0 \Omega 8$ \\
\hline
\end{tabular}

Zero-point correction=

Thermal correction to Energy=

Thermal correction to Enthalpy=

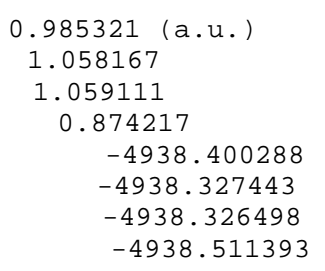

Sum of electronic and zero-point Energies=
Sum of electronic and thermal Energies=

Sum of electronic and thermal Enthalpies=

Sum of electronic and thermal Free Energies=

$\mathrm{G}(\mathrm{high})=-4940.2247781 \mathrm{a} \cdot \mathrm{u}$.

$\begin{array}{cccc} & \text { E (Thermal) } & \text { CV } & \text { S } \\ \text { KCal/Mol } & \text { Cal/Mol-Kelvin } & \text { Cal/Mol-Kelvin } \\ \text { Total } & 664.010 & 277.882 & 389.143\end{array}$




\begin{tabular}{lccc}
\multicolumn{1}{c}{ Item } & Value & Threshold Converged? \\
Maximum Force & 0.000014 & 0.000450 & YES \\
RMS Force & 0.000001 & 0.000300 & YES \\
Maximum Displacement & 0.001779 & 0.001800 & YES \\
RMS Displacement & 0.000130 & 0.001200 & YES
\end{tabular}

Normal termination of Gaussian 16 at Mon Jan 28 20:37:๑7 2019.

dna@4b1-ohrev.log

Stoichiometry C38H49N16Na2021P2(2)

Standard orientation:

\begin{tabular}{|c|c|c|c|c|c|}
\hline \multirow{2}{*}{$\begin{array}{l}\text { Center } \\
\text { Number }\end{array}$} & \multirow{2}{*}{$\begin{array}{l}\text { Atomic } \\
\text { Number }\end{array}$} & \multirow{2}{*}{$\begin{array}{c}\text { Atomic } \\
\text { Type }\end{array}$} & \multicolumn{3}{|c|}{ Coordinates (Angstroms) } \\
\hline & & & $x$ & $\mathrm{Y}$ & Z \\
\hline 1 & 7 & $\odot$ & 5.554768 & -1.779613 & -1.560147 \\
\hline 2 & 6 & 0 & 5.710176 & -3.136398 & -1.545307 \\
\hline 3 & 6 & $\odot$ & 4.647506 & -3.955735 & -1.699112 \\
\hline 4 & 6 & $\odot$ & 3.361860 & -3.335320 & -1.844118 \\
\hline 5 & 7 & 0 & 3.229314 & -2.006587 & -1.915645 \\
\hline 6 & 8 & $\odot$ & 8.810626 & 0.743111 & 0.014662 \\
\hline 7 & 15 & $\odot$ & 8.056292 & 1.746435 & 1.066978 \\
\hline 8 & 8 & 0 & 6.547906 & 1.852921 & $\odot .419915$ \\
\hline 9 & 8 & 0 & 8.739463 & 3.078292 & $\odot .985119$ \\
\hline 10 & 8 & $\odot$ & 7.854591 & 1.029185 & 2.382813 \\
\hline 11 & 8 & 0 & 4.419164 & 5.413156 & 1.465837 \\
\hline 12 & 6 & $\odot$ & 4.982011 & 3.660161 & -0.041622 \\
\hline 13 & 6 & $\odot$ & 5.143116 & 4.159030 & 1.397235 \\
\hline 14 & 6 & 0 & -9.265020 & -3.284023 & 1.907867 \\
\hline 15 & 6 & $\odot$ & -8.686617 & -1.883063 & 1.892212 \\
\hline 16 & 8 & $\odot$ & -8.570748 & -4.105748 & 0.939354 \\
\hline 17 & 8 & 0 & -8.697426 & 0.244488 & 0.805788 \\
\hline 18 & 15 & $\odot$ & -8.207559 & 1.442601 & $-\odot .187268$ \\
\hline 19 & 8 & $\odot$ & -6.596048 & 1.494835 & $\odot .063714$ \\
\hline 20 & 8 & $\odot$ & -8.836754 & 2.729024 & $\odot .301903$ \\
\hline 21 & 8 & $\odot$ & -8.451102 & 1.071370 & -1.633473 \\
\hline 22 & 8 & $\odot$ & -4.731805 & 5.034275 & -1.489738 \\
\hline 23 & 6 & 0 & -4.869867 & 3.238221 & 0.071592 \\
\hline 24 & 6 & $\odot$ & -5.335641 & 3.726997 & -1.305680 \\
\hline 25 & 1 & $\odot$ & -4.957051 & 5.390314 & -2.366196 \\
\hline 26 & 6 & 0 & 9.681441 & -2.560165 & -1.479278 \\
\hline 27 & 6 & $\odot$ & 9.053833 & -1.184053 & -1.394783 \\
\hline 28 & 6 & $\odot$ & 8.694204 & -0.717205 & 0.023646 \\
\hline 29 & 6 & 0 & 7.250773 & -1.180425 & 0.171851 \\
\hline 30 & 6 & $\odot$ & 6.680951 & -0.923832 & -1.222112 \\
\hline 31 & 8 & $\odot$ & 7.785072 & -1.174465 & -2.136125 \\
\hline 32 & 1 & $\Theta$ & 9.773821 & -2.840674 & -2.533496 \\
\hline 33 & 1 & $\odot$ & 10.682488 & -2.526268 & -1.034176 \\
\hline 34 & 1 & $\odot$ & 9.721321 & $-\odot .45937 \odot$ & -1.865855 \\
\hline 35 & 1 & 0 & 9.358634 & -1.119157 & 0.789791 \\
\hline 36 & 1 & $\odot$ & 6.713661 & -0.648339 & $\odot .951462$ \\
\hline 37 & 1 & $\odot$ & 7.257201 & -2.248273 & ๑.398977 \\
\hline 38 & 1 & $\odot$ & 6.335771 & 0.100138 & -1.334241 \\
\hline 39 & 8 & 0 & 8.843308 & -3.507896 & -0.771750 \\
\hline 40 & 1 & $\odot$ & 9.204599 & -4.406733 & -0.837287 \\
\hline 41 & 6 & 0 & 4.306071 & -1.192482 & -1.815652 \\
\hline 42 & 7 & $\odot$ & 2.253670 & -4.069696 & -1.906904 \\
\hline 43 & 8 & $\odot$ & 4.219421 & $\odot .038111$ & -1.910086 \\
\hline 44 & 1 & $\odot$ & 6.731426 & -3.484252 & -1.398917 \\
\hline 45 & 1 & 0 & 4.758768 & -5.031189 & -1.688387 \\
\hline 46 & 1 & $\odot$ & 2.305060 & -5.072624 & -1.842444 \\
\hline 47 & 1 & $\odot$ & 1.340071 & -3.607941 & -1.976575 \\
\hline 48 & 6 & $\odot$ & 6.153380 & 2.851715 & -0.562862 \\
\hline 49 & 1 & $\odot$ & 4.468200 & 5.785183 & 2.362744 \\
\hline 50 & 6 & $\odot$ & 4.465152 & 3.071663 & 2.238238 \\
\hline 51 & 6 & 0 & 3.331236 & 2.576381 & 1.331799 \\
\hline 52 & 8 & 0 & 3.764008 & 2.845777 & -0.033113 \\
\hline 53 & 1 & $\odot$ & 5.856797 & 2.324991 & -1.471682 \\
\hline 54 & 1 & $\odot$ & 6.998640 & 3.513682 & -0.767667 \\
\hline 55 & 1 & $\odot$ & 4.804315 & 4.505011 & -0.711184 \\
\hline 56 & 1 & $\odot$ & 6.195285 & 4.299837 & 1.663840 \\
\hline 57 & 1 & 0 & 4.082729 & 3.458404 & 3.185048 \\
\hline
\end{tabular}




\begin{tabular}{|c|c|c|c|c|c|}
\hline 58 & 1 & $\odot$ & 5.185474 & 2.279292 & 2.450751 \\
\hline 59 & 1 & $\odot$ & 2.400061 & 3.107149 & 1.511641 \\
\hline 60 & 7 & $\odot$ & 3.016710 & 1.155750 & 1.449432 \\
\hline 61 & 6 & $\odot$ & 3.996085 & ๑. 226123 & 1.265731 \\
\hline 62 & 6 & $\odot$ & 3.715629 & -1.095870 & 1.268224 \\
\hline 63 & 6 & $\odot$ & 2.336322 & -1.470931 & 1.373171 \\
\hline 64 & 7 & $\odot$ & 1.374231 & -0.555511 & 1.516201 \\
\hline 65 & 6 & $\odot$ & 1.671204 & $\odot .766678$ & 1.558140 \\
\hline 66 & 1 & $\odot$ & 4.492112 & -1.837334 & 1.134039 \\
\hline 67 & 7 & $\odot$ & 1.973663 & -2.752293 & 1.319508 \\
\hline 68 & 8 & $\odot$ & ๑. 802613 & 1.637016 & 1.688718 \\
\hline 69 & 1 & $\odot$ & 4.991083 & $\odot .627359$ & 1.093714 \\
\hline 70 & 1 & $\odot$ & 2.666954 & -3.467449 & 1.175996 \\
\hline 71 & 1 & $\odot$ & $\odot .986786$ & -3.023058 & 1.376664 \\
\hline 72 & 1 & $\odot$ & $-\odot .433495$ & -1.086831 & 1.492126 \\
\hline 73 & 1 & 0 & -8.873602 & -5.026702 & ๑. 991931 \\
\hline 74 & 6 & 0 & -8.638843 & -1.197359 & ๑. 519485 \\
\hline 75 & 6 & $\odot$ & -7.277423 & -1.615706 & -0.018088 \\
\hline 76 & 6 & $\odot$ & -6.417680 & -1.531383 & 1.238489 \\
\hline 77 & 8 & $\odot$ & -7.289457 & -1.917583 & 2.339444 \\
\hline 78 & 1 & $\odot$ & -9.147030 & -3.694831 & 2.916360 \\
\hline 79 & 1 & $\odot$ & -10.335595 & -3.231566 & 1.674937 \\
\hline 80 & 1 & $\odot$ & -9.246650 & -1.264406 & 2.597180 \\
\hline 81 & 1 & $\odot$ & -9.473472 & -1.461311 & -0.131139 \\
\hline 82 & 1 & $\odot$ & -6.893947 & -0.980246 & -0.813078 \\
\hline 83 & 1 & $\odot$ & -7.342486 & -2.649964 & -0.361751 \\
\hline 84 & 1 & $\odot$ & -6.071025 & -0.508053 & 1.396574 \\
\hline 85 & 7 & $\odot$ & -5.235595 & -2.360700 & 1.244820 \\
\hline 86 & 6 & $\odot$ & -5.118440 & -3.734595 & 1. 298814 \\
\hline 87 & 7 & $\odot$ & -3.876225 & -4.135885 & 1.376213 \\
\hline 88 & 6 & $\odot$ & -3.130980 & -2.964451 & 1.374779 \\
\hline 89 & 6 & $\odot$ & -3.966475 & -1.863435 & 1.291793 \\
\hline 90 & 7 & $\odot$ & -3.657059 & $-\odot .555549$ & 1.243313 \\
\hline 91 & 6 & $\odot$ & -2.351864 & -0.331881 & 1. 341022 \\
\hline 92 & 7 & $\odot$ & -1.435675 & -1.348227 & 1.453494 \\
\hline 93 & 6 & $\odot$ & -1.723542 & -2.718404 & 1.450316 \\
\hline 94 & 8 & $\odot$ & -0.802515 & -3.542083 & 1.514535 \\
\hline 95 & 1 & $\odot$ & $-6.0 \odot 4 \odot 75$ & -4.356075 & 1. 284814 \\
\hline 96 & 7 & $\odot$ & -1.902407 & $\odot .924256$ & 1.347528 \\
\hline 97 & 1 & $\odot$ & -2.549246 & 1.614710 & 0.960460 \\
\hline 98 & 1 & $\odot$ & -0.901028 & 1.129477 & 1.378473 \\
\hline 99 & 6 & $\odot$ & -5.904212 & 2.476869 & $\odot .879889$ \\
\hline 100 & 11 & $\odot$ & 6.694984 & $-\odot .183949$ & 3.792923 \\
\hline 101 & 11 & $\odot$ & -9.627169 & 3.264244 & -1.980768 \\
\hline 102 & 6 & $\odot$ & -4.761057 & 2.695343 & -2.278368 \\
\hline 103 & 6 & 0 & -3.420335 & 2.318605 & -1.641735 \\
\hline 104 & 8 & $\odot$ & -3.702161 & 2.395837 & -0.191641 \\
\hline 105 & 1 & $\odot$ & -5.404551 & 1.939197 & 1.688468 \\
\hline 106 & 1 & 0 & -6.630699 & 3.181919 & 1. 293021 \\
\hline 107 & 1 & 0 & -4.536317 & 4.097437 & ๑. 660942 \\
\hline 108 & 1 & $\odot$ & -6.426252 & 3.797128 & -1.368319 \\
\hline 109 & 1 & 0 & -4.645347 & 3. 088751 & -3.290176 \\
\hline 110 & 1 & $\odot$ & -5.422067 & 1.826926 & -2.289087 \\
\hline 111 & 1 & 0 & -2.637227 & 3.047403 & -1.862762 \\
\hline 112 & 7 & 0 & -2.927740 & 1.017574 & -1.969660 \\
\hline 113 & 6 & 0 & -3.775556 & -0.171910 & -2.237873 \\
\hline 114 & 7 & 0 & -2.883148 & -1.313071 & -2.215206 \\
\hline 115 & 6 & $\odot$ & -1.658708 & -0.809685 & -2.036577 \\
\hline 116 & 6 & $\odot$ & -1.638949 & 0.600793 & -1.866092 \\
\hline 117 & 7 & $\odot$ & $-\odot .574249$ & 1.370194 & -1.659498 \\
\hline 118 & 6 & 0 & 0.586128 & 0.700733 & -1.657990 \\
\hline 119 & 7 & 0 & $\odot .676552$ & -0.653382 & -1.817134 \\
\hline 120 & 6 & 0 & -0.410191 & -1.507586 & -1.987347 \\
\hline 121 & 7 & 0 & 1.732705 & 1.361435 & -1.506244 \\
\hline 122 & 8 & 0 & -0.245375 & -2.731410 & -2.091603 \\
\hline 123 & 1 & 0 & -4.217364 & -0.077428 & -3.240859 \\
\hline 124 & 1 & 0 & 1.711678 & 2.342629 & -1.281730 \\
\hline 125 & 1 & 0 & 2.644660 & ๑. 897814 & -1.561390 \\
\hline 126 & 1 & 0 & 1.611233 & -1.107163 & -1.827380 \\
\hline 127 & 8 & 0 & -4.839909 & -0.263451 & -1.364787 \\
\hline 128 & 1 & 0 & -4.520877 & -0.085756 & -0.458100 \\
\hline
\end{tabular}

\section{2 basis functions}

305 alpha electrons

304 beta electrons

nuclear repulsion energy 16420.4926950550 Hartrees.

NAtoms $=128$ NActive $=128$ 
Harmonic frequencies $\left(\mathrm{cm}^{*}-1\right)$, IR intensities (KM/Mole), Raman

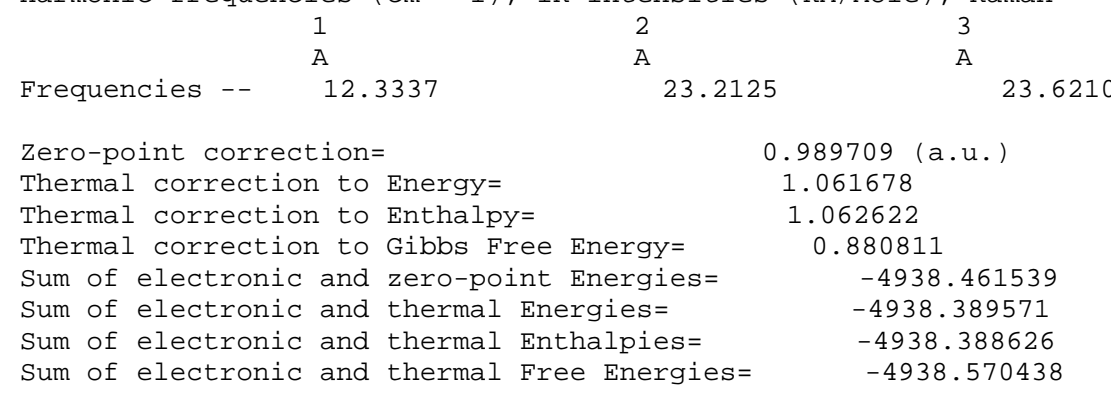

$$
\begin{gathered}
G(\text { high })=-4940 \\
\text { E (Thermal) } \\
\text { KCal/Mol }
\end{gathered}
$$

Total
666.213
CV
Cal/Mol-Kelvin Cal/Mol-Kelvin

$\begin{array}{ll}276.276 & 382.654\end{array}$

\begin{tabular}{lccc}
\multicolumn{1}{c}{ Item } & Value & Threshold Converged? \\
Maximum Force & $0.00 \odot \odot 29$ & $0.00045 \odot$ & YES \\
RMS Force & $0.000 \odot \odot 3$ & $0.00030 \odot$ & YES \\
Maximum Displacement & 0.002371 & $0.00180 \odot$ & NO \\
RMS Displacement & $0.00 \odot 40 \odot$ & $0.00120 \odot$ & YES
\end{tabular}

Normal termination of Gaussian 16 at Tue Feb 5 19:23:34 2019.

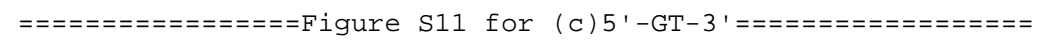

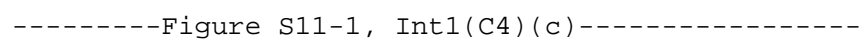

dna@4c-oh1afor. $\log$

Stoichiometry C39H50N15Na2021P2(2)

Standard orientation:

\begin{tabular}{|c|c|c|c|c|c|}
\hline \multirow{2}{*}{$\begin{array}{l}\text { Center } \\
\text { Number }\end{array}$} & \multirow{2}{*}{$\begin{array}{l}\text { Atomic } \\
\text { Number }\end{array}$} & \multirow{2}{*}{$\begin{array}{l}\text { Atomic } \\
\text { Type }\end{array}$} & \multicolumn{3}{|c|}{ Coordinates (Angstroms) } \\
\hline & & & $x$ & $\mathrm{Y}$ & Z \\
\hline & . & 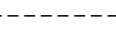 & 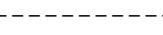 & & \\
\hline 1 & 7 & $\odot$ & 4.909711 & -1.920141 & -1.279296 \\
\hline 2 & 6 & $\odot$ & 4.949362 & -3.262419 & -1.047072 \\
\hline 3 & 6 & $\odot$ & 3.811134 & -3.990083 & -0.972983 \\
\hline 4 & 6 & $\odot$ & 2.573388 & -3.289325 & -1.165761 \\
\hline 5 & 7 & $\odot$ & 2.548130 & -1.976931 & -1.412550 \\
\hline 6 & 8 & $\odot$ & 8.470112 & 0.562062 & -0.839069 \\
\hline 7 & 15 & $\odot$ & 8.045170 & 1.783876 & $\odot .164810$ \\
\hline 8 & 8 & $\odot$ & 6.434781 & 1.942087 & -0.116224 \\
\hline 9 & 8 & $\odot$ & 8.761084 & 3.016431 & -0.300694 \\
\hline 10 & 8 & $\odot$ & 8.129339 & 1.299285 & 1.594828 \\
\hline 11 & 8 & $\odot$ & 4.648947 & 5.730440 & $\odot .777054$ \\
\hline 12 & 6 & $\odot$ & 4.827379 & 3.744209 & -0.521340 \\
\hline 13 & 6 & $\odot$ & 5.296524 & 4.434254 & $\odot .760031$ \\
\hline 14 & 6 & $\odot$ & -8.039052 & -3.579096 & 0.159493 \\
\hline 15 & 6 & $\odot$ & -7.793898 & -2.331442 & 0.981612 \\
\hline 16 & 8 & $\odot$ & -6.947463 & -3.725720 & -0.782371 \\
\hline 17 & 8 & 0 & -8.111513 & $\odot .057822$ & 0.717303 \\
\hline 18 & 15 & $\odot$ & -8.384248 & 1.389027 & -0.191016 \\
\hline 19 & 8 & $\odot$ & -6.911621 & 2.101652 & -0.248365 \\
\hline 20 & 8 & $\odot$ & -9.366139 & 2.251994 & $\odot .558248$ \\
\hline 21 & 8 & $\odot$ & -8.716772 & $\odot .999067$ & -1.614018 \\
\hline 22 & 8 & $\odot$ & -4.562495 & 5.621479 & -0.656905 \\
\hline 23 & 6 & $\odot$ & -5.157355 & 3.600752 & 0.451167 \\
\hline 24 & 6 & $\odot$ & -5.373309 & 4.431255 & -0.820660 \\
\hline 25 & 1 & $\odot$ & -4.654232 & 6.205619 & -1.428909 \\
\hline 26 & 6 & $\odot$ & 8.946319 & -2.993515 & -1.882777 \\
\hline 27 & 6 & $\odot$ & 8.398015 & -1.580715 & -1.902380 \\
\hline 28 & 6 & $\odot$ & 8.359315 & $-\odot .870218$ & -0.541651 \\
\hline 29 & 6 & $\odot$ & 6.978802 & -1.241012 & -0.015680 \\
\hline 30 & 6 & $\odot$ & 6.131574 & -1.136612 & -1.280555 \\
\hline 31 & 8 & $\odot$ & 7.002466 & -1.599664 & -2.356752 \\
\hline 32 & 1 & $\odot$ & 8.807260 & -3.434428 & -2.875381 \\
\hline 33 & 1 & $\odot$ & 10.019299 & -2.959432 & -1.660199 \\
\hline 34 & 1 & $\odot$ & 8.975625 & $-\odot .987776$ & -2.615053 \\
\hline 35 & 1 & $\odot$ & 9.174112 & -1.159410 & 0.123498 \\
\hline
\end{tabular}

S197 


\begin{tabular}{|c|c|c|c|c|c|}
\hline 36 & 1 & 0 & 6.636572 & $-\odot .592297$ & $\odot .782756$ \\
\hline 37 & 1 & $\odot$ & 7.016151 & -2.268331 & 0.350011 \\
\hline 38 & 1 & $\odot$ & 5.833796 & $-\odot .10929 \odot$ & -1.471572 \\
\hline 39 & 8 & 0 & 8.244726 & -3.769189 & -0.879419 \\
\hline 40 & 1 & $\odot$ & 8.531016 & -4.696687 & $-\odot .898976$ \\
\hline 41 & 6 & $\odot$ & 3.691528 & -1.258390 & -1.490350 \\
\hline 42 & 7 & 0 & 1.405784 & -3.928293 & -1.120857 \\
\hline 43 & 8 & $\odot$ & 3.692810 & $-\odot . \odot 46 \odot 29$ & -1.739121 \\
\hline 44 & 1 & $\odot$ & 5.945522 & -3.688816 & $-\odot .950977$ \\
\hline 45 & 1 & 0 & 3.836413 & -5.057596 & $-\odot .802071$ \\
\hline 46 & 1 & $\odot$ & 1.358094 & -4.893051 & $-\odot .840262$ \\
\hline 47 & 1 & 0 & $\odot .533445$ & -3.413303 & -1.262429 \\
\hline 48 & 6 & 0 & 5.836193 & 2.788284 & -1.135902 \\
\hline 49 & 1 & 0 & 4.900698 & 6.223613 & 1.576312 \\
\hline 50 & 6 & $\odot$ & 4.776088 & 3.525420 & 1.880281 \\
\hline 51 & 6 & 0 & 3.500888 & 2.889156 & 1.298660 \\
\hline 52 & 8 & $\odot$ & 3.586662 & 3.059091 & -0.144329 \\
\hline 53 & 1 & 0 & 5.330243 & 2.139072 & -1.853085 \\
\hline 54 & 1 & 0 & 6.622379 & 3.362602 & -1.633657 \\
\hline 55 & 1 & $\odot$ & 4.553592 & 4.490480 & -1.270384 \\
\hline 56 & 1 & 0 & 6.385393 & 4.542942 & 0.788353 \\
\hline 57 & 1 & 0 & 4.564472 & 4.083842 & 2.794183 \\
\hline 58 & 1 & $\odot$ & 5.525746 & 2.764904 & 2.105571 \\
\hline 59 & 1 & 0 & 2.587983 & 3.379330 & 1.638128 \\
\hline 60 & 7 & 0 & 3.356490 & 1.480652 & 1.612481 \\
\hline 61 & 6 & 0 & 4.316049 & $\odot .514059$ & 1.429859 \\
\hline 62 & 7 & 0 & 3.879285 & $-\odot .705908$ & 1.598149 \\
\hline 63 & 6 & 0 & 2.538118 & -0.535845 & 1.884117 \\
\hline 64 & 6 & 0 & 2.188566 & $\odot .809576$ & 1.890143 \\
\hline 65 & 7 & 0 & 0.968308 & 1.317679 & 2.109065 \\
\hline 66 & 6 & 0 & 0.071677 & 0.359603 & 2.307889 \\
\hline 67 & 7 & 0 & 0.266459 & $-\odot .969856$ & 2.309448 \\
\hline 68 & 6 & 0 & 1.498000 & -1.459938 & 2.078571 \\
\hline 69 & 7 & 0 & 1.681549 & -2.795739 & 2.056103 \\
\hline 70 & 1 & 0 & 5.309947 & 0.806526 & 1.124451 \\
\hline 71 & 1 & 0 & -0.958294 & $\odot .666873$ & 2.461960 \\
\hline 72 & 1 & 0 & 2.569818 & -3.118069 & 1.702058 \\
\hline 73 & 1 & 0 & $\odot .862150$ & -3.362380 & 1.826546 \\
\hline 74 & 1 & 0 & -7.061769 & -4.517649 & -1.332239 \\
\hline 75 & 6 & 0 & -7.421452 & -1.101017 & 0.143574 \\
\hline 76 & 6 & 0 & -5.910592 & -0.974250 & 0.291740 \\
\hline 77 & 6 & 0 & -5.584868 & -1.639171 & 1.633136 \\
\hline 78 & 8 & 0 & -6.676216 & -2.557087 & 1.901056 \\
\hline 79 & 1 & 0 & -8.097430 & -4.445626 & 0.825856 \\
\hline 80 & 1 & 0 & -8.991426 & -3.475669 & -0.373103 \\
\hline 81 & 1 & 0 & -8.681447 & -2.116174 & 1.581366 \\
\hline 82 & 1 & 0 & -7.731222 & -1.204413 & -0.897279 \\
\hline 83 & 1 & $\odot$ & -5.569503 & 0.059478 & 0.256572 \\
\hline 84 & 1 & 0 & -5.420750 & -1.513942 & -0.521306 \\
\hline 85 & 1 & 0 & -5.499897 & $-\odot .926774$ & 2.451472 \\
\hline 86 & 7 & 0 & -4.331043 & -2.385965 & 1.599680 \\
\hline 87 & 6 & 0 & -4.197545 & -3.419837 & 0.697581 \\
\hline 88 & 6 & 0 & -3.030338 & -4.054904 & 0.469625 \\
\hline 89 & 6 & $\odot$ & -1.859848 & -3.601882 & 1.198861 \\
\hline 90 & 7 & 0 & -2.071656 & -2.557366 & 2.078461 \\
\hline 91 & 6 & 0 & -3.257982 & -1.910770 & 2.332262 \\
\hline 92 & 6 & 0 & -2.871237 & -5.152729 & -0.536572 \\
\hline 93 & 8 & 0 & -0.734242 & -4.094919 & 1.077569 \\
\hline 94 & 8 & 0 & -3.342524 & -0.999025 & 3.145301 \\
\hline 95 & 1 & 0 & -5.118711 & -3.682132 & 0.178480 \\
\hline 96 & 1 & 0 & -2.211472 & -4.822243 & -1.344561 \\
\hline 97 & 1 & 0 & -2.422593 & -6.042108 & -0.084707 \\
\hline 98 & 1 & 0 & -3.836948 & -5.428783 & -0.965928 \\
\hline 99 & 1 & 0 & -1.222833 & -2.059432 & 2.406161 \\
\hline 100 & 6 & 0 & -6.361857 & 2.806345 & 0.898993 \\
\hline 101 & 11 & 0 & 7.376704 & $\odot .552979$ & 3.510578 \\
\hline 102 & 11 & $\odot$ & -10.811125 & 2.150160 & -1.725095 \\
\hline 103 & 6 & $\odot$ & -4.834290 & 3.537403 & -1.942081 \\
\hline 104 & 6 & $\odot$ & -3.646503 & 2.849849 & -1.276161 \\
\hline 105 & 8 & $\odot$ & -4.053110 & 2.691421 & 0.128023 \\
\hline 106 & 1 & 0 & -6.070536 & 2.082916 & 1.667142 \\
\hline 107 & 1 & 0 & -7.120617 & 3.479285 & 1.308911 \\
\hline 108 & 1 & $\odot$ & -4.830490 & 4.251981 & 1.264790 \\
\hline 109 & 1 & $\odot$ & -6.425056 & 4.692048 & -0.966427 \\
\hline 110 & 1 & $\odot$ & -4.537595 & 4.104421 & -2.825908 \\
\hline 111 & 1 & $\odot$ & -5.603534 & 2.814174 & -2.216844 \\
\hline 112 & 1 & 0 & -2.746452 & 3.465693 & -1.301454 \\
\hline
\end{tabular}




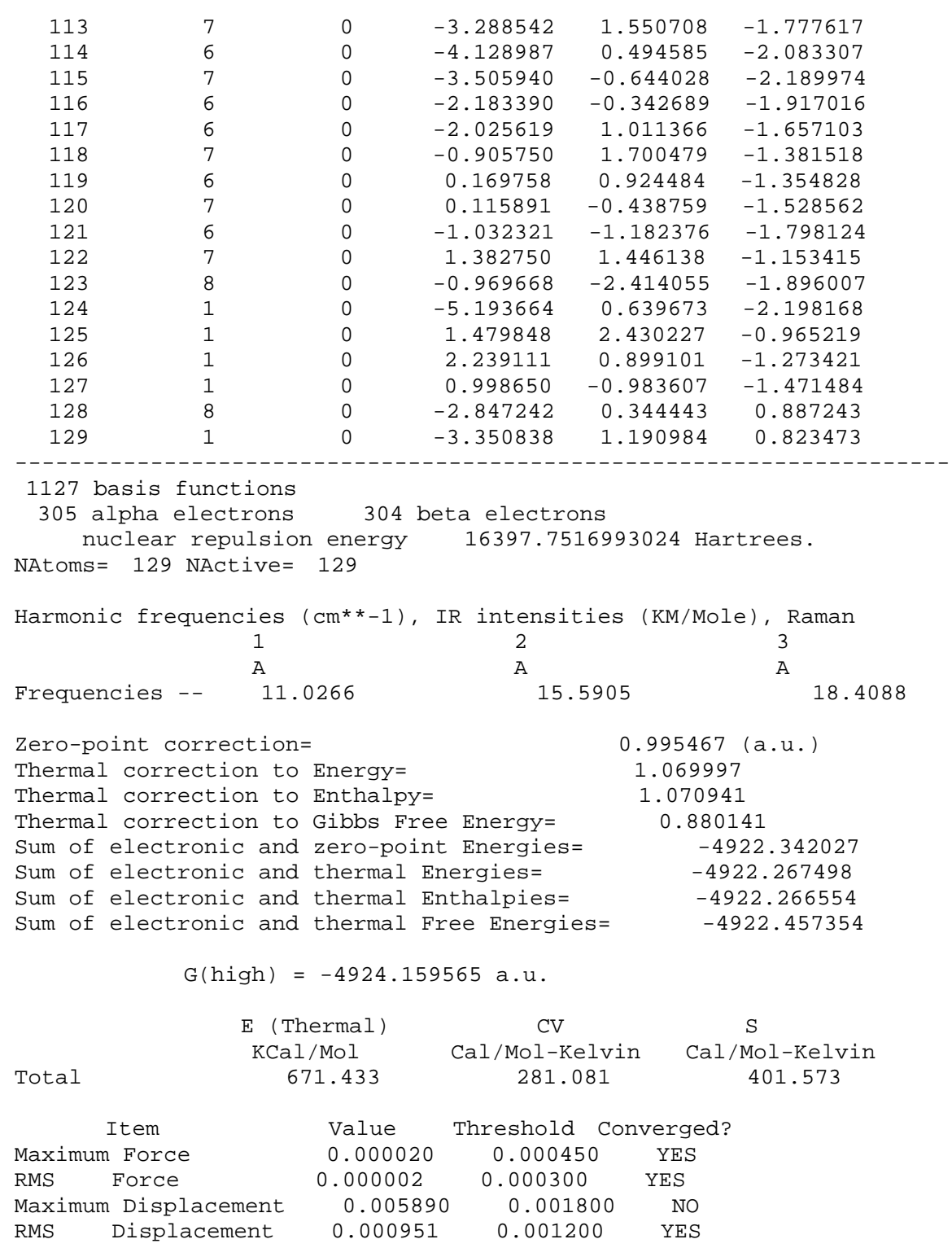

Normal termination of Gaussian 16 at wed Feb 13 19:46:12 2019.

Figure S11-2, TS1(C4)(c)

dna@4c-oh1ats. $\log$

Stoichiometry C39H50N15Na2021P2(2)

Standard orientation:

\begin{tabular}{rrrrrr} 
Center & $\begin{array}{c}\text { Atomic } \\
\text { Number }\end{array}$ & $\begin{array}{c}\text { Nomber } \\
\text { Type }\end{array}$ & \multicolumn{2}{c}{$\begin{array}{c}\text { Coordinates } \\
\text { X Angstroms) }\end{array}$} \\
\hline 1 & 7 & 0 & 5.011221 & -1.975550 & -1.185849 \\
2 & 6 & 0 & 5.108639 & -3.306977 & -0.911482 \\
3 & 6 & 0 & 4.002181 & -4.079579 & -0.813350 \\
4 & 6 & 0 & 2.735453 & -3.435167 & -1.012065 \\
5 & 7 & 0 & 2.653385 & -2.133974 & -1.299690 \\
6 & 8 & 0 & 8.467429 & 0.668932 & -0.801716 \\
7 & 15 & 0 & 7.979711 & 1.917221 & 0.138470 \\
8 & 8 & 0 & 6.372353 & 2.006749 & -0.184997 \\
9 & 8 & 0 & 8.663420 & 3.153498 & -0.364140 \\
10 & 8 & 0 & 8.047760 & 1.497299 & 1.590043 \\
11 & 8 & 0 & 4.545900 & 5.807799 & 0.605362 \\
12 & 6 & 0 & 4.743383 & 3.782864 & -0.627112 \\
13 & 6 & 0 & 5.218579 & 4.524580 & 0.622671 \\
14 & 6 & 0 & -7.972266 & -3.503082 & 0.073292 \\
15 & 6 & 0 & -7.760499 & -2.262350 & 0.915269
\end{tabular}




\begin{tabular}{|c|c|c|c|c|c|}
\hline 16 & 8 & $\odot$ & -6.893468 & -3.597925 & -0.889553 \\
\hline 17 & 8 & $\odot$ & -8.100802 & $\odot .124478$ & $\odot .77589 \odot$ \\
\hline 18 & 15 & 0 & -8.578547 & 1.439668 & -0.068789 \\
\hline 19 & 8 & 0 & -7.165186 & 2.208580 & -0.361646 \\
\hline 20 & 8 & $\odot$ & -9.449406 & 2.260649 & $\odot .852812$ \\
\hline 21 & 8 & 0 & -9.150555 & 1.037065 & -1.406168 \\
\hline 22 & 8 & $\odot$ & -4.514248 & 5.392849 & -1.140187 \\
\hline 23 & 6 & $\odot$ & -5.199590 & 3.509622 & $\odot .153466$ \\
\hline 24 & 6 & $\odot$ & -5.365128 & 4.220930 & -1.200580 \\
\hline 25 & 1 & 0 & -4.586945 & 5.908966 & -1.961066 \\
\hline 26 & 6 & $\odot$ & 9.094662 & -2.905325 & -1.685781 \\
\hline 27 & 6 & 0 & 8.491233 & -1.517974 & -1.773964 \\
\hline 28 & 6 & 0 & 8.401750 & -0.752535 & $-\odot .445558$ \\
\hline 29 & 6 & $\odot$ & 7.026647 & -1.152284 & $\odot .073027$ \\
\hline 30 & 6 & $\odot$ & 6.198505 & -1.139315 & -1.208489 \\
\hline 31 & 8 & 0 & 7.105329 & -1.612832 & -2.248731 \\
\hline 32 & 1 & $\odot$ & 8.992077 & -3.392123 & -2.661209 \\
\hline 33 & 1 & 0 & 10.160979 & -2.818805 & -1.446195 \\
\hline 34 & 1 & $\odot$ & 9.056649 & -0.932636 & -2.502423 \\
\hline 35 & 1 & $\odot$ & 9.214270 & $-\odot .983808$ & $\odot .244547$ \\
\hline 36 & 1 & 0 & 6.643441 & $-\odot .482392$ & $\odot .834440$ \\
\hline 37 & 1 & $\odot$ & 7.096057 & -2.160066 & $\odot .485834$ \\
\hline 38 & 1 & $\odot$ & 5.860584 & $-\odot .135149$ & -1.450087 \\
\hline 39 & 8 & $\odot$ & 8.405004 & -3.666255 & -0.663029 \\
\hline 40 & 1 & $\odot$ & 8.729649 & -4.580921 & $-\odot .63646 \odot$ \\
\hline 41 & 6 & $\odot$ & 3.766951 & -1.375295 & -1.420384 \\
\hline 42 & 7 & 0 & 1.594746 & -4.118533 & -0.925182 \\
\hline 43 & 8 & $\odot$ & 3.717967 & $-\odot .177794$ & -1.728352 \\
\hline 44 & 1 & 0 & 6.122657 & -3.685740 & $-\odot .801098$ \\
\hline 45 & 1 & $\odot$ & 4.072573 & -5.138964 & $-\odot .608159$ \\
\hline 46 & 1 & $\odot$ & 1.591850 & -5.082770 & $-\odot .639041$ \\
\hline 47 & 1 & $\odot$ & $\odot .702673$ & -3.646994 & -1.074231 \\
\hline 48 & 6 & $\odot$ & 5.760738 & 2.822172 & -1.221097 \\
\hline 49 & 1 & $\odot$ & 4.797377 & 6.332141 & 1.384524 \\
\hline 50 & 6 & 0 & 4.732516 & 3.644806 & 1.780738 \\
\hline 51 & 6 & $\odot$ & 3.457373 & 2.972210 & 1.242581 \\
\hline 52 & 8 & $\odot$ & 3.517921 & 3.093383 & $-\odot .207895$ \\
\hline 53 & 1 & $\odot$ & 5.262301 & 2.148551 & -1.920692 \\
\hline 54 & 1 & $\odot$ & 6.537853 & 3.393472 & -1.736360 \\
\hline 55 & 1 & $\odot$ & 4.448249 & 4.498022 & -1.398052 \\
\hline 56 & 1 & 0 & 6.305543 & 4.654409 & $\odot .629885$ \\
\hline 57 & 1 & $\odot$ & 4.528728 & 4.228983 & 2.680248 \\
\hline 58 & 1 & 0 & 5.498504 & 2.903783 & 2.015864 \\
\hline 59 & 1 & $\odot$ & 2.543611 & 3.463087 & 1.578576 \\
\hline 60 & 7 & $\odot$ & 3.334757 & 1.573698 & 1.605225 \\
\hline 61 & 6 & $\odot$ & 4.304077 & 0.612085 & 1.444955 \\
\hline 62 & 7 & 0 & 3.879987 & $-\odot .607368$ & 1.644112 \\
\hline 63 & 6 & $\odot$ & 2.537086 & $-\odot .444162$ & 1.927887 \\
\hline 64 & 6 & 0 & 2.173810 & $\odot .897631$ & 1.901090 \\
\hline 65 & 7 & 0 & 0.948314 & 1.400381 & 2.101267 \\
\hline 66 & 6 & $\odot$ & $\odot .059430$ & $\odot .439172$ & 2.319493 \\
\hline 67 & 7 & $\odot$ & $\odot .270190$ & $-\odot .886166$ & 2.360272 \\
\hline 68 & 6 & 0 & 1.504502 & -1.372052 & 2.141792 \\
\hline 69 & 7 & $\odot$ & 1.693821 & -2.708003 & 2.148177 \\
\hline 70 & 1 & $\odot$ & 5.294801 & $\odot .905698$ & 1.131660 \\
\hline 71 & 1 & 0 & -0.975061 & $\odot .736856$ & 2.458257 \\
\hline 72 & 1 & 0 & 2.584062 & -3.032531 & 1.801794 \\
\hline 73 & 1 & $\odot$ & $\odot .877626$ & -3.278717 & 1.916181 \\
\hline 74 & 1 & $\odot$ & -6.988120 & -4.388349 & -1.445412 \\
\hline 75 & 6 & $\odot$ & -7.443905 & $-\odot .999416$ & 0.104237 \\
\hline 76 & 6 & 0 & -5.927624 & $-\odot .867623$ & $\odot .181931$ \\
\hline 77 & 6 & 0 & -5.558924 & -1.509523 & 1.524025 \\
\hline 78 & 8 & 0 & -6.624070 & -2.454583 & 1.817999 \\
\hline 79 & 1 & $\odot$ & -7.989508 & -4.381819 & $\odot .726011$ \\
\hline 80 & 1 & $\odot$ & -8.936779 & -3.424526 & -0.441600 \\
\hline 81 & 1 & $\odot$ & -8.647933 & -2.093889 & 1.529800 \\
\hline 82 & 1 & $\odot$ & -7.813043 & -1.061627 & -0.920758 \\
\hline 83 & 1 & $\odot$ & -5.589401 & $\odot .167283$ & $\odot .109821$ \\
\hline 84 & 1 & $\odot$ & -5.479470 & -1.437588 & -0.634847 \\
\hline 85 & 1 & $\odot$ & -5.484620 & -0.784106 & 2.332186 \\
\hline 86 & 7 & $\odot$ & -4.293075 & -2.229485 & 1.491408 \\
\hline 87 & 6 & $\odot$ & -4.151489 & -3.291952 & $\odot .626064$ \\
\hline 88 & 6 & 0 & -2.989252 & -3.953187 & $\odot .452537$ \\
\hline 89 & 6 & $\odot$ & -1.834792 & -3.497817 & 1.205723 \\
\hline 90 & 7 & 0 & -2.057696 & -2.436976 & 2.058760 \\
\hline 91 & 6 & 0 & -3.231968 & -1.744608 & 2.236606 \\
\hline 92 & 6 & $\odot$ & -2.823024 & -5.088254 & -0.510222 \\
\hline
\end{tabular}




\begin{tabular}{|c|c|c|c|c|c|}
\hline 93 & 8 & $\odot$ & -0.711597 & -4.008492 & 1.127832 \\
\hline 94 & 8 & $\odot$ & -3.326985 & $-\odot .807491$ & 3.017453 \\
\hline 95 & 1 & $\odot$ & -5.061679 & -3.558424 & ๑. 091033 \\
\hline 96 & 1 & $\odot$ & -2.130019 & -4.799689 & -1.305890 \\
\hline 97 & 1 & $\odot$ & -2.410764 & -5.971226 & -0.012903 \\
\hline 98 & 1 & $\odot$ & -3.781138 & -5.358168 & $-\odot .960513$ \\
\hline 99 & 1 & $\odot$ & -1.209394 & -1.941364 & 2.394444 \\
\hline 100 & 6 & 0 & -6.465872 & 2.905877 & 0.708939 \\
\hline 101 & 11 & 0 & 7.345365 & 0.735358 & 3.520167 \\
\hline 102 & 11 & $\odot$ & -11.385626 & 2.116563 & $-\odot .678851$ \\
\hline 103 & 6 & 0 & -4.838309 & 3.201850 & -2.218081 \\
\hline 104 & 6 & 0 & -3.690504 & 2.565762 & -1.444865 \\
\hline 105 & 8 & 0 & -4.213836 & 2.446793 & -0.078640 \\
\hline 106 & 1 & 0 & -6.221924 & 2.201955 & 1.510912 \\
\hline 107 & 1 & 0 & -7.113111 & 3.692820 & 1.105987 \\
\hline 108 & 1 & 0 & -4.784460 & 4.212386 & 0.880408 \\
\hline 109 & 1 & 0 & -6.403257 & 4.501734 & -1.393471 \\
\hline 110 & 1 & 0 & -4.513818 & 3.664116 & -3.151305 \\
\hline 111 & 1 & 0 & -5.624428 & 2.472168 & -2.423313 \\
\hline 112 & 1 & $\odot$ & -2.809841 & 3.211718 & -1.427888 \\
\hline 113 & 7 & 0 & -3.251550 & 1.263708 & -1.859845 \\
\hline 114 & 6 & 0 & -4.000747 & 0.206870 & -2.306411 \\
\hline 115 & 7 & 0 & -3.345278 & -0.932128 & -2.344210 \\
\hline 116 & 6 & 0 & -2.108761 & $-\odot .625100$ & -1.860810 \\
\hline 117 & 6 & 0 & -2.016228 & 0.737491 & -1.481740 \\
\hline 118 & 7 & $\odot$ & $-\odot .892950$ & 1.474975 & -1.347661 \\
\hline 119 & 6 & $\odot$ & $\odot .194390$ & 0.728655 & -1.292101 \\
\hline 120 & 7 & 0 & 0.182682 & -0.652610 & -1.395163 \\
\hline 121 & 6 & 0 & -0.919768 & -1.428652 & -1.715176 \\
\hline 122 & 7 & 0 & 1.399889 & 1.279775 & -1.139607 \\
\hline 123 & 8 & $\odot$ & -0.821941 & -2.649369 & -1.855424 \\
\hline 124 & 1 & 0 & -5.033516 & $\odot .327801$ & -2.599868 \\
\hline 125 & 1 & 0 & 1.483475 & 2.270815 & -0.979557 \\
\hline 126 & 1 & 0 & 2.262712 & 0.744786 & -1.270947 \\
\hline 127 & 1 & 0 & 1.083055 & -1.167524 & -1.334395 \\
\hline 128 & 8 & 0 & -2.464534 & 0.177773 & 0.413838 \\
\hline 129 & 1 & $\odot$ & -3.167979 & $\odot .840733$ & 0.523185 \\
\hline
\end{tabular}

1127 basis functions

305 alpha electrons

304 beta electrons

nuclear repulsion energy 16418.7923681671 Hartrees.

NAtoms $=129$ NActive $=129$

Harmonic frequencies $\left(\mathrm{cm}^{* *}-1\right)$, IR intensities (KM/Mole),

\begin{tabular}{|c|c|c|c|c|c|c|c|c|c|c|}
\hline & & & 1 & & 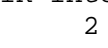 & & & 3 & & \\
\hline & & & A & & A & & & A & & \\
\hline Frequ & encies & - - & -453.49 & & & 12.768 & & & 16.2834 & \\
\hline Red. & nasses & - - & 13.02 & & & 10.2086 & & & 7.0320 & \\
\hline Frc c & nsts & -- & 1.577 & & & $\odot .0010$ & & & 0.0011 & \\
\hline IR In & en & -- & 73.274 & & & 0.9188 & & & 6.6561 & \\
\hline Atom & AN & $x$ & $\mathrm{Y}$ & Z & $x$ & $\mathrm{Y}$ & Z & $x$ & Z & \\
\hline 1 & 7 & $\odot . \odot \odot$ & $\odot . \odot \odot$ & $-\odot . \odot \odot$ & 0.03 & $\odot . \odot \odot$ & $\odot . \odot 2$ & -0.01 & $-\odot .01$ & $\odot .05$ \\
\hline 2 & 6 & $\odot . \odot \odot$ & $\odot . \odot \odot$ & $-\odot . \odot \odot$ & $\odot .04$ & $\odot .0 \odot$ & 0.01 & -0.03 & -0.01 & $\odot . \odot 8$ \\
\hline 3 & 6 & $\odot . \odot \odot$ & $-\odot .0 \odot$ & $\odot . \odot \odot$ & 0.04 & -0.01 & -0.01 & -0.04 & 0.01 & 0.06 \\
\hline 4 & 6 & $\odot . \odot \odot$ & $-\odot . \odot \odot$ & $\odot .00$ & $\odot . \odot 4$ & $-\odot .02$ & -0.01 & -0.03 & 0.01 & 0.01 \\
\hline 5 & 7 & $\odot . \odot \odot$ & -0.00 & $-\odot .0 \odot$ & 0.03 & -0.02 & $-\odot . \odot \odot$ & $-\odot .01$ & 0.01 & $-\odot .02$ \\
\hline 6 & 8 & $\odot . \odot \odot$ & -0.00 & $\odot . \odot \odot$ & $\odot .01$ & 0.03 & 0.05 & 0.01 & -0.05 & 0.05 \\
\hline 7 & 15 & $-\odot . \odot \odot$ & $\odot .0 \odot$ & $\odot . \odot \odot$ & $-\odot .02$ & 0.02 & 0.05 & $\odot .01$ & -0.01 & $\odot . \odot \odot$ \\
\hline 8 & 8 & $\odot . \odot \odot$ & $\odot . \odot \odot$ & $-\odot . \odot \odot$ & -0.01 & $\odot .01$ & $\odot .03$ & $\odot . \odot 2$ & -0.01 & $-\odot .02$ \\
\hline 9 & 8 & $\odot . \odot \odot$ & -0.00 & $\odot . \odot \odot$ & -0.02 & $\odot .03$ & $\odot .06$ & 0.03 & -0.04 & -0.03 \\
\hline 10 & 8 & $\odot . \odot \odot$ & $-\odot .00$ & $\odot . \odot \odot$ & -0.03 & 0.02 & 0.05 & -0.01 & 0.03 & 0.02 \\
\hline 11 & 8 & $\odot . \odot \odot$ & $-\odot . \odot \odot$ & $-\odot .0 \odot$ & -0.05 & $-\odot . \odot \odot$ & 0.01 & 0.01 & 0.02 & -0.14 \\
\hline 12 & 6 & $-\odot . \odot \odot$ & $-\odot . \odot \odot$ & $-\odot . \odot \odot$ & $-\odot . \odot 2$ & $-\odot . \odot \odot$ & $\odot .01$ & $\odot . \odot 2$ & -0.02 & -0.08 \\
\hline 13 & 6 & $-\odot . \odot \odot$ & $-\odot . \odot \odot$ & $-\odot . \odot \odot$ & $-\odot .03$ & $\odot . \odot \odot$ & 0.01 & 0.01 & 0.02 & -0.10 \\
\hline 14 & 6 & $\odot .0 \odot$ & $\odot . \odot \odot$ & $-\odot . \odot \odot$ & $-\odot .02$ & $\odot .06$ & -0.04 & 0.02 & -0.03 & -0.09 \\
\hline 15 & 6 & $\odot . \odot \odot$ & $\odot . \odot \odot$ & $-\odot . \odot \odot$ & $-\odot .01$ & $\odot . \odot 3$ & $-\odot .01$ & 0.01 & -0.05 & $-\odot .06$ \\
\hline 16 & 8 & $\odot . \odot \odot$ & $\odot .0 \odot$ & $-\odot . \odot \odot$ & $-\odot .03$ & $\odot .06$ & $-\odot .04$ & 0.03 & $-\odot .01$ & $-\odot .08$ \\
\hline 17 & 8 & $\odot . \odot \odot$ & $-\odot . \odot \odot$ & $-\odot . \odot \odot$ & 0.04 & $\odot . \odot 4$ & $\odot . \odot 8$ & $-\odot . \odot \odot$ & -0.05 & 0.01 \\
\hline 18 & 15 & $\odot .0 \odot$ & $\odot . \odot \odot$ & $-\odot . \odot \odot$ & $-\odot . \odot \odot$ & $\odot .06$ & 0.14 & $\odot . \odot \odot$ & $-\odot . \odot 2$ & $\odot .06$ \\
\hline 19 & 8 & $-\odot . \odot \odot$ & $-\odot . \odot \odot$ & $\odot . \odot \odot$ & -0.01 & $\odot .04$ & $\odot .03$ & $\odot . \odot \odot$ & $-\odot .02$ & $\odot .07$ \\
\hline 20 & 8 & $-\odot . \odot \odot$ & $-\odot . \odot \odot$ & -0.00 & 0.09 & $\odot .07$ & 0.22 & $\odot .01$ & -0.05 & $\odot .09$ \\
\hline 21 & 8 & $-\odot . \odot \odot$ & $-\odot . \odot \odot$ & $\odot . \odot \odot$ & -0.12 & $\odot . \odot 9$ & 0.18 & $-\odot .01$ & $\odot .02$ & 0.05 \\
\hline 22 & 8 & -0.00 & $\odot . \odot \odot$ & $-\odot .0 \odot$ & -0.01 & -0.05 & -0.24 & $-\odot .01$ & 0.01 & 0.15 \\
\hline 23 & 6 & -0.01 & $\odot .01$ & $\odot . \odot \odot$ & 0.05 & $\odot .01$ & $-\odot .12$ & $\odot . \odot \odot$ & -0.03 & $\odot . \odot 9$ \\
\hline 24 & 6 & $-\odot . \odot \odot$ & $\odot . \odot \odot$ & $-\odot . \odot \odot$ & $-\odot . \odot \odot$ & $-\odot .06$ & -0.15 & $-\odot .01$ & $\odot .01$ & 0.12 \\
\hline 25 & 1 & $\odot .0 \odot$ & $-\odot . \odot \odot$ & $-\odot .0 \odot$ & -0.05 & $-\odot . \odot 8$ & -0.26 & -0.01 & 0.04 & 0.17 \\
\hline 26 & 6 & $-\odot . \odot \odot$ & $-\odot . \odot \odot$ & $\odot . \odot \odot$ & $\odot .04$ & 0.04 & $\odot .04$ & $-\odot .02$ & $-\odot . \odot 8$ & 0.16 \\
\hline
\end{tabular}




\begin{tabular}{|c|c|c|c|c|c|c|c|c|c|c|}
\hline 27 & 6 & $\odot . \odot \odot$ & $-\odot . \odot \odot$ & $\odot .0 \odot$ & $\odot .03$ & $\odot .04$ & $\odot .04$ & $\odot . \odot \odot$ & $-\odot .08$ & 0.11 \\
\hline 28 & 6 & 0.00 & 0.00 & 0.00 & 0.01 & 0.03 & 0.04 & -0.01 & -0.04 & 0.09 \\
\hline 29 & 6 & $-0.0 \odot$ & 0.00 & $\odot .0 \odot$ & 0.01 & 0.02 & 0.03 & $-\odot .03$ & -0.01 & 0.07 \\
\hline 30 & 6 & $\odot . \odot \odot$ & $\odot . \odot \odot$ & $-\odot .0 \odot$ & $\odot .02$ & 0.01 & $\odot .03$ & $-\odot . \odot \odot$ & -0.03 & 0.05 \\
\hline 31 & 8 & $-\odot . \odot \odot$ & 0.00 & $\odot .00$ & 0.03 & 0.03 & 0.03 & 0.01 & -0.07 & $\odot .08$ \\
\hline 32 & 1 & $-\odot . \odot \odot$ & $-0.0 \odot$ & 0.00 & 0.05 & 0.04 & 0.03 & -0.01 & -0.11 & 0.17 \\
\hline 33 & 1 & $-0.0 \odot$ & $-0.0 \odot$ & $\odot .00$ & 0.04 & 0.05 & 0.04 & -0.02 & -0.09 & 0.18 \\
\hline 34 & 1 & $-\odot . \odot \odot$ & $-\odot . \odot \odot$ & $\odot . \odot \odot$ & 0.03 & 0.04 & 0.05 & 0.03 & -0.11 & 0.11 \\
\hline 35 & 1 & $-\odot . \odot \odot$ & $-\odot . \odot \odot$ & $\odot .0 \odot$ & 0.01 & 0.03 & $\odot .05$ & $-\odot .03$ & $-\odot .03$ & 0.11 \\
\hline 36 & 1 & $-0.0 \odot$ & $\odot . \odot \odot$ & 0.00 & $\odot .00$ & $\odot .01$ & 0.03 & -0.03 & 0.02 & 0.05 \\
\hline 37 & 1 & $-\odot .0 \odot$ & $\odot .0 \odot$ & 0.00 & $\odot .02$ & 0.01 & 0.03 & -0.05 & 0.00 & 0.10 \\
\hline 38 & 1 & 0.00 & 0.00 & -0.00 & 0.01 & 0.01 & 0.03 & 0.02 & -0.03 & 0.02 \\
\hline 39 & 8 & $-\odot . \odot \odot$ & $-0.0 \odot$ & 0.00 & 0.04 & 0.03 & 0.03 & -0.05 & -0.05 & 0.16 \\
\hline 40 & 1 & $\odot .0 \odot$ & $\odot . \odot \odot$ & 0.00 & 0.05 & 0.03 & $\odot .03$ & -0.07 & -0.05 & 0.19 \\
\hline 41 & 6 & $0.0 \odot$ & $-0.0 \odot$ & -0.00 & 0.02 & -0.01 & 0.01 & -0.00 & $-0.0 \odot$ & 0.01 \\
\hline 42 & 7 & $\odot .0 \odot$ & $-\odot . \odot \odot$ & 0.00 & $\odot .05$ & -0.03 & -0.02 & -0.04 & 0.02 & -0.03 \\
\hline 43 & 8 & $-\odot . \odot \odot$ & $-0.0 \odot$ & $-\odot . \odot \odot$ & $\odot .02$ & $-\odot . \odot \odot$ & 0.02 & $\odot .02$ & -0.01 & -0.01 \\
\hline 44 & 1 & $\odot . \odot \odot$ & $\odot . \odot \odot$ & $-\odot . \odot \odot$ & $\odot .04$ & $\odot .01$ & ๑. . 01 & $-\odot . \odot 4$ & -0.01 & 0.12 \\
\hline 45 & 1 & $\odot . \odot \odot$ & $-0.0 \odot$ & $\odot . \odot \odot$ & $\odot .05$ & -0.01 & -0.01 & -0.06 & 0.01 & $\odot . \odot 9$ \\
\hline 46 & 1 & 0.01 & -0.00 & 0.00 & 0.05 & -0.03 & -0.03 & -0.06 & 0.03 & 0.01 \\
\hline 47 & 1 & $\odot .00$ & -0.01 & 0.01 & 0.04 & -0.04 & -0.02 & -0.03 & 0.03 & -0.05 \\
\hline 48 & 6 & $-\odot . \odot \odot$ & $-\odot . \odot \odot$ & $-\odot . \odot \odot$ & -0.01 & 0.01 & $\odot .02$ & $\odot . \odot 3$ & -0.03 & -0.05 \\
\hline 49 & 1 & $-0.0 \odot$ & $-0.0 \odot$ & $\odot .00$ & -0.06 & $-0.0 \odot$ & 0.01 & $\odot . \odot \odot$ & 0.04 & -0.15 \\
\hline 50 & 6 & $-\odot . \odot \odot$ & $-\odot . \odot \odot$ & $-\odot . \odot \odot$ & $-\odot .04$ & $-\odot .0 \odot$ & $\odot .01$ & -0.00 & $\odot .05$ & -0.08 \\
\hline 51 & 6 & $-0.0 \odot$ & $-0.0 \odot$ & $\odot . \odot \odot$ & -0.03 & -0.01 & -0.00 & $\odot .00$ & 0.03 & -0.07 \\
\hline 52 & 8 & -0.00 & -0.00 & $-\odot .0 \odot$ & -0.02 & -0.01 & 0.00 & $\odot .02$ & -0.00 & -0.08 \\
\hline 53 & 1 & $\odot . \odot \odot$ & $-\odot . \odot \odot$ & $-\odot . \odot \odot$ & 0.01 & 0.00 & 0.02 & 0.04 & -0.05 & -0.04 \\
\hline 54 & 1 & $-0.0 \odot$ & $\odot . \odot \odot$ & $\odot . \odot \odot$ & $-\odot . \odot \odot$ & $\odot .01$ & $\odot .03$ & 0.04 & -0.04 & -0.05 \\
\hline 55 & 1 & $-\odot . \odot \odot$ & $-0.0 \odot$ & $-\odot .0 \odot$ & $-\odot .02$ & -0.00 & 0.01 & 0.03 & -0.03 & -0.10 \\
\hline 56 & 1 & $-0.0 \odot$ & $-0.0 \odot$ & $-\odot . \odot \odot$ & -0.04 & 0.01 & $\odot .02$ & $\odot .01$ & $\odot .02$ & -0.09 \\
\hline 57 & 1 & $-\odot . \odot \odot$ & $-\odot . \odot \odot$ & $-\odot . \odot \odot$ & -0.05 & $-0.0 \odot$ & 0.01 & -0.01 & 0.07 & -0.10 \\
\hline 58 & 1 & -0.00 & $-\odot . \odot \odot$ & $-\odot . \odot \odot$ & -0.03 & 0.01 & $\odot . \odot 2$ & -0.00 & 0.05 & -0.05 \\
\hline 59 & 1 & $-0.0 \odot$ & $-0.0 \odot$ & 0.00 & -0.03 & -0.02 & -0.01 & 0.00 & 0.04 & -0.10 \\
\hline 60 & 7 & $-\odot .0 \odot$ & $-0.0 \odot$ & $-\odot . \odot \odot$ & $-\odot .02$ & -0.01 & -0.00 & $\odot .0 \odot$ & 0.04 & -0.04 \\
\hline 61 & 6 & $\odot . \odot \odot$ & $\odot .0 \odot$ & -0.00 & -0.01 & -0.01 & 0.01 & 0.01 & $\odot .04$ & $\odot .01$ \\
\hline 62 & 7 & $\odot . \odot \odot$ & $\odot .0 \odot$ & $-\odot .0 \odot$ & $-\odot .01$ & -0.01 & 0.01 & 0.01 & $\odot .04$ & 0.02 \\
\hline 63 & 6 & $\odot .0 \odot$ & $-\odot . \odot \odot$ & $\odot .00$ & $-\odot .01$ & -0.02 & -0.01 & 0.00 & 0.04 & -0.02 \\
\hline 64 & 6 & $-\odot . \odot \odot$ & $-0.0 \odot$ & $\odot .0 \odot$ & -0.02 & -0.02 & -0.02 & $-0.0 \odot$ & $\odot . \odot 4$ & -0.06 \\
\hline 65 & 7 & -0.00 & -0.00 & $-\odot . \odot \odot$ & -0.02 & -0.03 & -0.03 & -0.01 & 0.04 & -0.10 \\
\hline 66 & 6 & $-\odot . \odot \odot$ & $-0.0 \odot$ & $-\odot .01$ & $-\odot .02$ & -0.03 & -0.04 & -0.01 & 0.04 & -0.11 \\
\hline 67 & 7 & 0.00 & $-\odot .0 \odot$ & 0.00 & -0.01 & -0.03 & -0.04 & -0.01 & 0.04 & -0.08 \\
\hline 68 & 6 & $\odot .0 \odot$ & 0.00 & $\odot .00$ & -0.01 & -0.02 & -0.02 & $-0.0 \odot$ & 0.05 & -0.03 \\
\hline 69 & 7 & 0.00 & $-\odot . \odot \odot$ & 0.00 & -0.01 & -0.02 & -0.03 & 0.00 & 0.05 & -0.00 \\
\hline 70 & 1 & $-\odot .0 \odot$ & 0.00 & -0.00 & -0.01 & -0.00 & 0.03 & $\odot .02$ & 0.04 & 0.03 \\
\hline 71 & 1 & -0.01 & $-\odot .01$ & -0.04 & $-\odot .02$ & -0.04 & -0.05 & -0.02 & $\odot .04$ & -0.15 \\
\hline 72 & 1 & 0.00 & -0.00 & -0.00 & -0.00 & -0.02 & -0.02 & 0.02 & 0.04 & 0.04 \\
\hline 73 & 1 & $\odot .0 \odot$ & 0.00 & $-0.0 \odot$ & $-\odot .0 \odot$ & -0.02 & -0.04 & 0.01 & 0.04 & -0.01 \\
\hline 74 & 1 & 0.00 & 0.00 & -0.00 & -0.04 & 0.07 & -0.06 & 0.04 & 0.00 & -0.10 \\
\hline 75 & 6 & $\odot .0 \odot$ & $\odot .0 \odot$ & $-\odot . \odot \odot$ & $-\odot . \odot \odot$ & 0.05 & 0.02 & $-\odot . \odot \odot$ & -0.03 & -0.03 \\
\hline 76 & 6 & $\odot .0 \odot$ & $\odot .0 \odot$ & $-0.0 \odot$ & $-\odot . \odot \odot$ & $\odot .02$ & $-0.0 \odot$ & -0.00 & -0.03 & -0.02 \\
\hline 77 & 6 & $0.0 \odot$ & $\odot .0 \odot$ & -0.00 & 0.00 & 0.00 & -0.02 & -0.01 & -0.05 & -0.03 \\
\hline 78 & 8 & $\odot .00$ & 0.00 & -0.00 & $-0.0 \odot$ & $\odot .0 \odot$ & -0.02 & 0.00 & -0.07 & -0.06 \\
\hline 79 & 1 & $\odot .0 \odot$ & $\odot .0 \odot$ & 0.00 & -0.03 & 0.04 & -0.06 & 0.02 & -0.05 & -0.11 \\
\hline 80 & 1 & 0.00 & 0.00 & -0.00 & $-\odot .02$ & 0.08 & -0.03 & 0.02 & -0.03 & -0.10 \\
\hline 81 & 1 & $\odot .0 \odot$ & $\odot .0 \odot$ & -0.00 & $-\odot . \odot \odot$ & $\odot .02$ & $\odot .0 \odot$ & 0.00 & -0.08 & -0.06 \\
\hline 82 & 1 & -0.00 & 0.00 & -0.00 & -0.03 & 0.10 & 0.03 & -0.00 & 0.00 & -0.03 \\
\hline 83 & 1 & 0.01 & $\odot .0 \odot$ & $-0.0 \odot$ & $\odot .02$ & 0.02 & $\odot .0 \odot$ & $-\odot .0 \odot$ & -0.02 & -0.01 \\
\hline 84 & 1 & $-\odot . \odot \odot$ & -0.01 & $-\odot . \odot \odot$ & -0.03 & 0.02 & $-\odot .02$ & $\odot . \odot \odot$ & -0.01 & -0.03 \\
\hline 85 & 1 & $\odot .0 \odot$ & 0.00 & $-0.0 \odot$ & 0.01 & -0.01 & -0.01 & $-\odot .02$ & -0.06 & -0.02 \\
\hline 86 & 7 & $\odot .0 \odot$ & 0.00 & $-0.0 \odot$ & $-0.0 \odot$ & -0.00 & -0.03 & $\odot .0 \odot$ & -0.03 & -0.04 \\
\hline 87 & 6 & $\odot . \odot \odot$ & $\odot .0 \odot$ & -0.00 & -0.01 & $\odot .0 \odot$ & -0.04 & 0.02 & -0.02 & -0.04 \\
\hline 88 & 6 & $\odot .0 \odot$ & 0.00 & -0.00 & -0.02 & -0.00 & -0.05 & $\odot .03$ & -0.01 & -0.04 \\
\hline 89 & 6 & $\odot .0 \odot$ & $\odot .0 \odot$ & $-0.0 \odot$ & -0.01 & -0.02 & -0.04 & 0.02 & 0.01 & -0.03 \\
\hline 90 & 7 & 0.00 & 0.01 & -0.01 & -0.00 & -0.02 & -0.04 & 0.01 & 0.00 & -0.04 \\
\hline 91 & 6 & $\odot .0 \odot$ & 0.01 & -0.00 & 0.00 & -0.01 & -0.03 & $-0.0 \odot$ & -0.01 & -0.04 \\
\hline 92 & 6 & $-\odot . \odot \odot$ & $-0.0 \odot$ & $\odot .0 \odot$ & -0.02 & 0.00 & -0.06 & 0.04 & -0.01 & -0.04 \\
\hline 93 & 8 & $\odot .0 \odot$ & $\odot .00$ & $-0.0 \odot$ & -0.01 & -0.02 & -0.05 & $\odot .03$ & $\odot .02$ & -0.03 \\
\hline 94 & 8 & $-\odot . \odot \odot$ & $\odot .0 \odot$ & -0.00 & 0.01 & -0.02 & -0.03 & -0.01 & -0.01 & -0.04 \\
\hline 95 & 1 & 0.00 & -0.00 & 0.00 & -0.01 & 0.01 & -0.04 & 0.02 & -0.03 & -0.04 \\
\hline 96 & 1 & $\odot .0 \odot$ & -0.01 & $-\odot .0 \odot$ & -0.03 & 0.01 & -0.06 & 0.04 & 0.00 & -0.04 \\
\hline 97 & 1 & -0.00 & $-\odot . \odot \odot$ & $\odot . \odot \odot$ & $-\odot .03$ & $-\odot . \odot \odot$ & -0.07 & 0.05 & $-0.0 \odot$ & -0.04 \\
\hline 98 & 1 & -0.00 & 0.00 & 0.00 & -0.03 & 0.01 & -0.05 & 0.05 & -0.02 & -0.04 \\
\hline 99 & 1 & 0.01 & 0.01 & -0.02 & 0.00 & -0.03 & -0.04 & 0.00 & 0.02 & -0.05 \\
\hline 100 & 6 & -0.01 & 0.01 & 0.00 & 0.06 & 0.06 & -0.03 & 0.01 & -0.05 & $\odot .09$ \\
\hline 101 & 11 & -0.00 & $-\odot .00$ & $-0.0 \odot$ & -0.03 & 0.04 & 0.05 & -0.01 & 0.06 & 0.03 \\
\hline 102 & 11 & 0.00 & $\odot .0 \odot$ & -0.00 & -0.03 & 0.09 & 0.29 & -0.00 & 0.01 & $\odot .09$ \\
\hline 103 & 6 & -0.00 & -0.00 & $\odot . \odot \odot$ & -0.02 & -0.10 & -0.12 & -0.01 & 0.05 & 0.08 \\
\hline
\end{tabular}




\begin{tabular}{|c|c|c|c|c|c|c|c|c|c|c|}
\hline 104 & 6 & -0.02 & -0.01 & 0.02 & $-\odot . \odot \odot$ & -0.07 & -0.12 & -0.01 & 0.02 & 0.05 \\
\hline 105 & 8 & -0.02 & 0.02 & $\odot .02$ & 0.02 & -0.02 & -0.10 & $\odot .0 \odot$ & -0.02 & 0.05 \\
\hline 106 & 1 & -0.00 & 0.01 & 0.00 & 0.09 & $\odot .09$ & -0.01 & 0.02 & -0.07 & 0.06 \\
\hline 107 & 1 & -0.02 & $-\odot .0 \odot$ & $\odot .00$ & 0.10 & 0.09 & -0.03 & 0.01 & -0.06 & $\odot .12$ \\
\hline 108 & 1 & -0.02 & 0.02 & 0.00 & 0.09 & 0.05 & -0.18 & 0.01 & -0.05 & 0.11 \\
\hline 109 & 1 & $-\odot . \odot \odot$ & 0.01 & -0.01 & -0.01 & -0.08 & -0.14 & -0.01 & 0.02 & 0.14 \\
\hline 110 & 1 & 0.01 & -0.01 & 0.01 & -0.05 & -0.14 & -0.15 & $-\odot .02$ & $\odot . \odot 8$ & $\odot .09$ \\
\hline 111 & 1 & -0.01 & 0.01 & $-0.0 \odot$ & -0.03 & -0.11 & -0.07 & -0.01 & 0.05 & 0.0 \\
\hline 112 & 1 & -0.01 & -0.02 & 0.02 & -0.01 & -0.06 & -0.15 & -0.01 & 0.03 & 0.06 \\
\hline 113 & 7 & -0.02 & $-\odot . \odot \odot$ & 0.06 & $\odot . \odot \odot$ & -0.08 & -0.07 & $-\odot .01$ & $\odot . \odot 4$ & $\odot .01$ \\
\hline 114 & 6 & 0.05 & 0.03 & -0.02 & 0.01 & -0.09 & -0.07 & -0.02 & 0.04 & 0.00 \\
\hline 115 & 7 & 0.06 & -0.02 & -0.07 & 0.03 & -0.08 & -0.05 & -0.02 & $\odot .04$ & -0.02 \\
\hline 116 & 6 & -0.03 & -0.09 & 0.02 & $\odot .02$ & -0.06 & -0.04 & -0.01 & 0.03 & $-0.0<$ \\
\hline 117 & 6 & -0.16 & -0.12 & 0.73 & $\odot .0 \odot$ & -0.06 & -0.06 & -0.00 & 0.03 & -0.0 \\
\hline 118 & 7 & -0.03 & -0.03 & 0.03 & -0.01 & -0.05 & -0.06 & 0.00 & $\odot .02$ & -0.0 \\
\hline 119 & 6 & -0.03 & 0.00 & -0.01 & 0.00 & -0.04 & -0.04 & 0.00 & 0.02 & -0.05 \\
\hline 120 & 7 & 0.00 & -0.03 & 0.02 & 0.02 & -0.04 & -0.03 & -0.01 & 0.02 & -0.06 \\
\hline 121 & 6 & $\odot .02$ & -0.03 & 0.00 & 0.03 & -0.05 & -0.03 & -0.01 & 0.03 & -0.05 \\
\hline 122 & 7 & $-\odot .02$ & $-\odot .01$ & $-\odot .03$ & -0.01 & -0.02 & $-\odot .03$ & 0.01 & 0.01 & -0.0 \\
\hline 123 & 8 & $\odot .0 \odot$ & -0.03 & -0.02 & 0.04 & -0.05 & -0.03 & -0.02 & $\odot .03$ & -0.0 \\
\hline 124 & 1 & $\odot .08$ & 0.01 & -0.14 & 0.02 & -0.10 & -0.08 & -0.02 & 0.05 & 0.02 \\
\hline 125 & 1 & -0.02 & -0.02 & 0.01 & -0.02 & -0.02 & -0.03 & 0.01 & ๑. 01 & -0.0 \\
\hline 126 & 1 & -0.02 & -0.01 & -0.06 & $\odot . \odot \odot$ & -0.01 & -0.01 & $0.0 \odot$ & ๑. . 01 & -0.06 \\
\hline 127 & 1 & 0.01 & -0.02 & 0.01 & $\odot .02$ & -0.03 & $-\odot . \odot 2$ & $-\odot .01$ & $\odot .02$ & -0.0 \\
\hline 128 & 8 & 0.15 & 0.23 & -0.53 & -0.01 & -0.03 & -0.05 & 0.02 & -0.01 & -0.0 \\
\hline 129 & 1 & 0.04 & -0.01 & 0.05 & 0.01 & -0.01 & -0.06 & 0.01 & -0.02 & -0.0 \\
\hline
\end{tabular}

Zero-point correction=

Thermal correction to Energy=

Thermal correction to Enthalpy=

Thermal correction to Gibbs Free Energy=

Sum of electronic and zero-point Energies=

Sum of electronic and thermal Energies=

Sum of electronic and thermal Enthalpies=

Sum of electronic and thermal Free Energies=

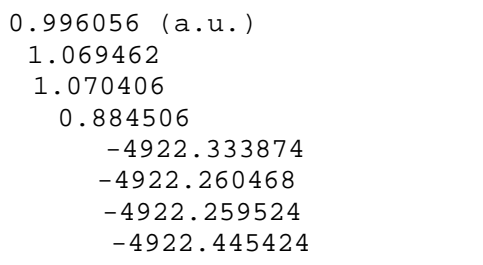

$G($ high $)=-4924 \cdot 150635 \mathrm{a} \cdot \mathrm{u}$.

Total

Electronic

Translational

Rotational

Vibrational

Item
Maximum Force
RMS Force
Maximum Displacement
RMS Displacement

CV

Cal/Mol-Kelvin Cal/Mol-Kelvin $279.143 \quad 391.259$

$0.000 \quad 1.377$

$2.981 \quad 47.055$

$2.981 \quad 41.910$

$273.181-300.916$

Normal termination of Gaussian 16 at Tue Feb 5 19:13:07 2019.

Figure S11-3, Int2(C4)(c)

dna@4c-oh1a. log

Stoichiometry C39H50N15Na2021P2(2)

Standard orientation:

\begin{tabular}{|c|c|c|c|c|c|}
\hline \multirow{2}{*}{$\begin{array}{l}\text { Center } \\
\text { Number }\end{array}$} & \multirow{2}{*}{$\begin{array}{l}\text { Atomic } \\
\text { Number }\end{array}$} & \multirow{2}{*}{$\begin{array}{c}\text { Atomic } \\
\text { Type }\end{array}$} & \multicolumn{3}{|c|}{ Coordinates (Angstroms) } \\
\hline & & & $\mathrm{X}$ & $\mathrm{Y}$ & Z \\
\hline 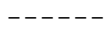 & 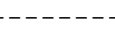 & $\ldots$ & 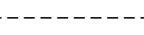 & & \\
\hline 1 & 7 & $\odot$ & 5.095224 & -1.974226 & -1.197374 \\
\hline 2 & 6 & $\odot$ & 5.242494 & -3.302302 & -0.928276 \\
\hline 3 & 6 & $\odot$ & 4.165240 & -4.113408 & -0.816738 \\
\hline 4 & 6 & $\odot$ & 2.874822 & -3.509622 & -0.986683 \\
\hline 5 & 7 & $\odot$ & 2.743892 & -2.213276 & -1.272905 \\
\hline 6 & 8 & $\odot$ & 8.486873 & 0.769683 & -0.828456 \\
\hline 7 & 15 & $\odot$ & 7.958572 & 2.002438 & 0.108862 \\
\hline 8 & 8 & $\odot$ & 6.349731 & 2.037874 & -0.218134 \\
\hline 9 & 8 & $\odot$ & 8.601785 & 3.259274 & -0.396438 \\
\hline 10 & 8 & $\odot$ & 8.038312 & 1.588665 & 1.562002 \\
\hline 11 & 8 & $\odot$ & 4.459616 & 5.812772 & 0.571847 \\
\hline
\end{tabular}




\begin{tabular}{|c|c|c|c|c|c|}
\hline 12 & 6 & $\odot$ & 4.689887 & 3.788187 & -0.655181 \\
\hline 13 & 6 & 0 & 5.164448 & 4.546784 & 0.584907 \\
\hline 14 & 6 & $\odot$ & -7.973791 & -3.434209 & 0.096422 \\
\hline 15 & 6 & $\odot$ & -7.768873 & -2.176988 & 0.915581 \\
\hline 16 & 8 & 0 & -6.909784 & -3.529589 & -0.882978 \\
\hline 17 & 8 & $\odot$ & -8.127852 & 0.202326 & 0.756611 \\
\hline 18 & 15 & $\odot$ & -8.645777 & 1.498039 & -0.092076 \\
\hline 19 & 8 & 0 & -7.250482 & 2.266729 & -0.452700 \\
\hline 20 & 8 & $\odot$ & -9.489284 & 2.331782 & 0.842922 \\
\hline 21 & 8 & $\odot$ & -9.263858 & 1.066201 & -1.400822 \\
\hline 22 & 8 & 0 & -4.466805 & 5.195065 & -1.548144 \\
\hline 23 & 6 & $\odot$ & -5.227410 & 3.511404 & -0.036687 \\
\hline 24 & 6 & $\odot$ & -5.350854 & 4.048918 & -1.473134 \\
\hline 25 & 1 & 0 & -4.512055 & 5.602634 & -2.429824 \\
\hline 26 & 6 & $\odot$ & 9.191495 & -2.787803 & -1.718884 \\
\hline 27 & 6 & $\odot$ & 8.554386 & -1.415441 & -1.804652 \\
\hline 28 & 6 & 0 & 8.452226 & -0.653298 & -0.475485 \\
\hline 29 & 6 & $\odot$ & 7.088270 & -1.084831 & 0.048214 \\
\hline 30 & 6 & $\odot$ & 6.253440 & -1.098714 & -1.229475 \\
\hline 31 & 8 & 0 & 7.169565 & -1.542594 & -2.274616 \\
\hline 32 & 1 & $\odot$ & 9.090816 & -3.279433 & -2.692051 \\
\hline 33 & 1 & 0 & 10.257656 & -2.675069 & -1.490177 \\
\hline 34 & 1 & 0 & 9.103059 & -0.816543 & -2.534822 \\
\hline 35 & 1 & $\odot$ & 9.271294 & -0.868367 & $\odot .212144$ \\
\hline 36 & 1 & 0 & 6.691949 & -0.420328 & 0.808278 \\
\hline 37 & 1 & 0 & 7.184969 & -2.088616 & 0.465465 \\
\hline 38 & 1 & $\odot$ & 5.879783 & -0.106724 & -1.469545 \\
\hline 39 & 8 & 0 & 8.530073 & -3.562517 & -0.687687 \\
\hline 40 & 1 & 0 & 8.878057 & -4.468646 & -0.661290 \\
\hline 41 & 6 & $\odot$ & 3.827795 & -1.416693 & -1.419386 \\
\hline 42 & 7 & $\odot$ & 1.755391 & -4.225354 & -0.863429 \\
\hline 43 & 8 & 0 & 3.732952 & -0.225611 & -1.738564 \\
\hline 44 & 1 & 0 & 6.270733 & -3.644985 & -0.831235 \\
\hline 45 & 1 & 0 & 4.276465 & -5.169960 & -0.615423 \\
\hline 46 & 1 & 0 & 1.792537 & -5.203451 & -0.631396 \\
\hline 47 & 1 & $\odot$ & $\odot .849500$ & -3.787655 & -1.038507 \\
\hline 48 & 6 & $\odot$ & 5.716543 & 2.839612 & -1.252510 \\
\hline 49 & 1 & 0 & 4.707931 & 6.346804 & 1.345388 \\
\hline 50 & 6 & 0 & 4.713870 & 3.658569 & 1.750321 \\
\hline 51 & 6 & $\odot$ & 3.436391 & 2.975039 & 1.234073 \\
\hline 52 & 8 & 0 & 3.482015 & 3.078835 & -0.218171 \\
\hline 53 & 1 & $\odot$ & 5.221803 & 2.153801 & -1.942800 \\
\hline 54 & 1 & 0 & 6.480192 & 3.418834 & -1.778763 \\
\hline 55 & 1 & 0 & 4.374537 & 4.492696 & -1.427896 \\
\hline 56 & 1 & 0 & 6.247904 & 4.703120 & 0.576963 \\
\hline 57 & 1 & 0 & 4.523754 & 4.234408 & 2.658142 \\
\hline 58 & 1 & $\odot$ & 5.491966 & 2.923192 & 1.961484 \\
\hline 59 & 1 & 0 & 2.525120 & 3.466213 & 1.576533 \\
\hline 60 & 7 & 0 & 3.321599 & 1.579638 & 1.610200 \\
\hline 61 & 6 & $\odot$ & 4.273729 & $\odot .609364$ & 1.401150 \\
\hline 62 & 7 & 0 & 3.845489 & -0.606846 & 1.611202 \\
\hline 63 & 6 & 0 & 2.517675 & -0.431911 & 1.953567 \\
\hline 64 & 6 & $\odot$ & 2.168326 & 0.913530 & 1.951488 \\
\hline 65 & 7 & $\odot$ & ๑. 957828 & 1.427687 & 2.206710 \\
\hline 66 & 6 & $\odot$ & ๑.068982 & 0.473679 & 2.453122 \\
\hline 67 & 7 & $\odot$ & $\odot .263537$ & -0.854399 & 2.468341 \\
\hline 68 & 6 & $\odot$ & 1.483002 & -1.351073 & 2.191665 \\
\hline 69 & 7 & 0 & 1.657918 & -2.688076 & 2.162878 \\
\hline 70 & 1 & 0 & 5.253327 & ๑.896348 & 1.047417 \\
\hline 71 & 1 & $\odot$ & -0.952881 & 0.783315 & 2.651386 \\
\hline 72 & 1 & 0 & 2.530326 & -3.011649 & 1.773471 \\
\hline 73 & 1 & $\odot$ & 0.830037 & -3.249793 & 1.950566 \\
\hline 74 & 1 & $\odot$ & -7.002631 & -4.330241 & -1.424331 \\
\hline 75 & 6 & $\odot$ & -7.475148 & -0.923508 & 0.082523 \\
\hline 76 & 6 & 0 & -5.958317 & -0.778907 & 0.131392 \\
\hline 77 & 6 & $\odot$ & -5.565967 & -1.392438 & 1.480386 \\
\hline 78 & 8 & $\odot$ & -6.622922 & -2.336005 & 1.812181 \\
\hline 79 & 1 & $\odot$ & -7.967668 & -4.302245 & 0.763561 \\
\hline 80 & 1 & $\odot$ & -8.947245 & -3.378043 & -0.404234 \\
\hline 81 & 1 & $\odot$ & -8.653179 & -2.009829 & 1.534790 \\
\hline 82 & 1 & 0 & -7.864015 & -1.000792 & -0.934114 \\
\hline 83 & 1 & 0 & -5.626826 & 0.256525 & $\odot .036905$ \\
\hline 84 & 1 & $\odot$ & -5.520546 & -1.362023 & -0.682425 \\
\hline 85 & 1 & $\odot$ & -5.484536 & -0.648925 & 2.271158 \\
\hline 86 & 7 & $\odot$ & -4.299434 & -2.110003 & 1.449237 \\
\hline 87 & 6 & $\odot$ & -4.166799 & -3.194646 & 0.610609 \\
\hline 88 & 6 & $\odot$ & -3.019378 & -3.889881 & 0.476511 \\
\hline
\end{tabular}




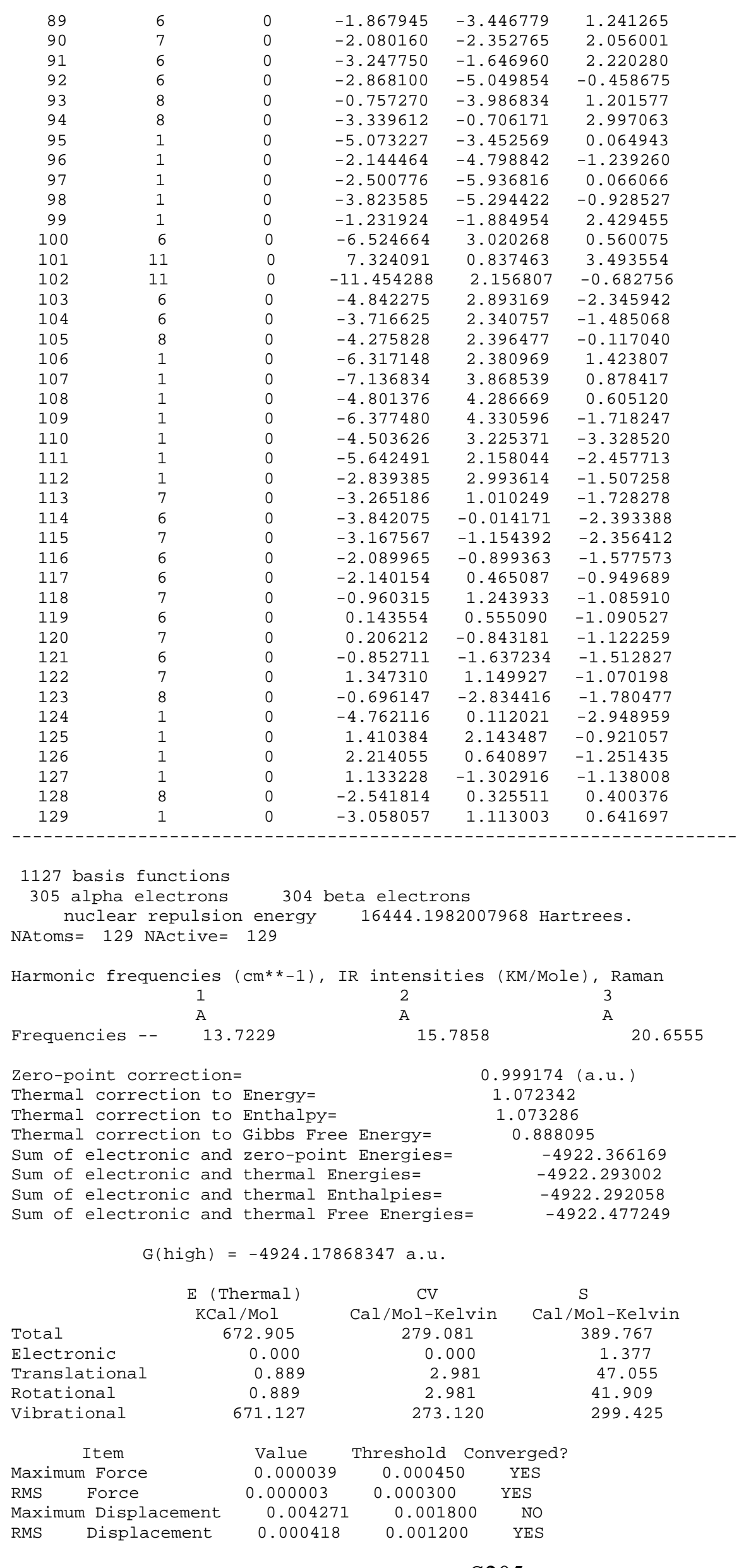


Normal termination of Gaussian $\odot 9$ at Fri Jan 25 23:05:06 2019.

dna๑4c-ohreva. log

Stoichiometry C39H50N15Na2021P2(2)

Standard orientation:

\begin{tabular}{|c|c|c|c|c|c|}
\hline \multirow{2}{*}{$\begin{array}{l}\text { Center } \\
\text { Number }\end{array}$} & \multirow{2}{*}{$\begin{array}{l}\text { Atomic } \\
\text { Number }\end{array}$} & \multirow{2}{*}{$\begin{array}{c}\text { Atomic } \\
\text { Type }\end{array}$} & \multicolumn{3}{|c|}{ Coordinates (Angstroms) } \\
\hline & & & $\mathrm{x}$ & $\mathrm{Y}$ & Z \\
\hline & & & - & -1 & -- \\
\hline 1 & 7 & $\odot$ & 5.109122 & -2.081851 & -1.351150 \\
\hline 2 & 6 & $\odot$ & 5.109777 & -3.426699 & -1.125305 \\
\hline 3 & 6 & $\odot$ & 3.957403 & -4.133026 & -1.152808 \\
\hline 4 & 6 & $\odot$ & 2.748511 & -3.400092 & -1.399768 \\
\hline 5 & 7 & $\odot$ & 2.765998 & -2.094523 & -1.678441 \\
\hline 6 & 8 & $\odot$ & 8.591971 & $\odot .355146$ & -0.342008 \\
\hline 7 & 15 & $\odot$ & 8.007248 & 1.570022 & $\odot .585786$ \\
\hline 8 & 8 & $\odot$ & 6.471879 & 1.740407 & $\odot .024699$ \\
\hline 9 & 8 & 0 & 8.799854 & 2.802662 & 0.266967 \\
\hline 10 & 8 & $\odot$ & 7.839953 & 1.071303 & 2.004255 \\
\hline 11 & 8 & $\odot$ & 4.828691 & 5.612929 & $\odot .892524$ \\
\hline 12 & 6 & $\odot$ & 5.048529 & 3.676550 & -0.472837 \\
\hline 13 & 6 & 0 & 5.411407 & 4.286289 & ๑. 881536 \\
\hline 14 & 6 & 0 & -8.994672 & -2.881568 & 1.472144 \\
\hline 15 & 6 & 0 & -8.449121 & -1.469341 & 1.530252 \\
\hline 16 & 8 & $\odot$ & -8.186222 & -3.670253 & 0.563742 \\
\hline 17 & 8 & $\odot$ & -8.429522 & 0.634420 & $\odot .379553$ \\
\hline 18 & 15 & $\odot$ & -7.842994 & 1.827147 & -0.574002 \\
\hline 19 & 8 & $\odot$ & -6.320002 & 2.024405 & ๑. 012649 \\
\hline 20 & 8 & $\odot$ & -8.651427 & 3.060728 & -0.302326 \\
\hline 21 & 8 & $\odot$ & -7.647069 & 1. 294252 & -1.975952 \\
\hline 22 & 8 & $\odot$ & -4.507176 & 5.839554 & -0.775114 \\
\hline 23 & 6 & $\odot$ & -4.881184 & 3. 917029 & 0.576571 \\
\hline 24 & 6 & 0 & -5.138618 & 4.535015 & -0.799242 \\
\hline 25 & 1 & $\odot$ & -4.618212 & 6.285599 & -1.631923 \\
\hline 26 & 6 & $\odot$ & 9.183414 & -3.199741 & -1.324021 \\
\hline 27 & 6 & $\odot$ & 8.657164 & -1.782300 & -1.426107 \\
\hline 28 & 6 & $\odot$ & 8.409944 & -1.077433 & -0.083729 \\
\hline 29 & 6 & 0 & 6.956994 & -1.424539 & 0.208260 \\
\hline 30 & 6 & $\odot$ & 6.326747 & -1.310429 & -1.176964 \\
\hline 31 & 8 & 0 & 7.350780 & -1.790234 & -2.097513 \\
\hline 32 & 1 & $\odot$ & 9.210322 & -3.636848 & -2.327461 \\
\hline 33 & 1 & $\odot$ & 10.203412 & -3.175774 & -0.922929 \\
\hline 34 & 1 & $\odot$ & 9.344687 & -1.191266 & -2.035197 \\
\hline 35 & 1 & $\odot$ & 9.101447 & -1.386666 & $\odot .701138$ \\
\hline 36 & 1 & $\odot$ & 6.499471 & -0.766836 & $\odot .939312$ \\
\hline 37 & 1 & $\odot$ & 6.915610 & -2.451543 & 0.574778 \\
\hline 38 & 1 & $\odot$ & 6.076841 & -0.280005 & -1.415522 \\
\hline 39 & 8 & 0 & 8.315381 & -3.971566 & -0.456802 \\
\hline 40 & 1 & $\odot$ & 8.596818 & -4.900257 & -0.425718 \\
\hline 41 & 6 & 0 & 3.930231 & -1.404974 & -1.690879 \\
\hline 42 & 7 & $\odot$ & 1.560401 & -4.005677 & -1.362505 \\
\hline 43 & 8 & $\odot$ & 3.985460 & -0.204128 & -1.985397 \\
\hline 44 & 1 & 0 & 6.087774 & -3.865520 & -0.937740 \\
\hline 45 & 1 & $\odot$ & 3.946999 & -5.200243 & -0.978661 \\
\hline 46 & 1 & 0 & 1.494147 & -4.981082 & -1.124058 \\
\hline 47 & 1 & 0 & 0.704424 & -3.454047 & -1.478814 \\
\hline 48 & 6 & 0 & 6.056644 & 2.673871 & -1.010916 \\
\hline 49 & 1 & $\odot$ & 5.018779 & 6.059069 & 1.735321 \\
\hline 50 & 6 & $\odot$ & 4.727133 & 3.359344 & 1.893184 \\
\hline 51 & 6 & $\odot$ & 3.475709 & 2.853998 & 1.156579 \\
\hline 52 & 8 & $\odot$ & 3. 731821 & 3.063339 & -0.261968 \\
\hline 53 & 1 & $\odot$ & 5.600419 & 2.089982 & -1.812312 \\
\hline 54 & 1 & $\odot$ & 6.932485 & 3.209362 & -1.386765 \\
\hline 55 & 1 & $\odot$ & 4.908587 & 4.464411 & -1.216083 \\
\hline 56 & 1 & $\odot$ & 6.494915 & 4.334836 & 1.029116 \\
\hline 57 & 1 & 0 & 4.467574 & 3.879588 & 2.817077 \\
\hline 58 & 1 & $\odot$ & 5.399448 & 2.534689 & 2.135471 \\
\hline 59 & 1 & 0 & 2.575736 & 3.410722 & 1.419643 \\
\hline 60 & $\overline{7}$ & 0 & 3.177405 & 1.454388 & 1.389779 \\
\hline 61 & 6 & 0 & 4.047428 & ๑. 399953 & 1.241062 \\
\hline 62 & 7 & 0 & 3.476482 & -0.772605 & 1.329925 \\
\hline 63 & 6 & $\odot$ & 2.139721 & -0.474653 & 1.518378 \\
\hline
\end{tabular}




\begin{tabular}{|c|c|c|c|c|c|}
\hline 64 & 6 & 0 & 1.930431 & $\odot .899007$ & 1.549815 \\
\hline 65 & 7 & $\odot$ & $\odot .757742$ & 1.531875 & 1.697254 \\
\hline 66 & 6 & 0 & $-\odot .247365$ & 0.672215 & 1.793966 \\
\hline 67 & 7 & 0 & $-\odot .192403$ & $-\odot .668461$ & 1.771543 \\
\hline 68 & 6 & 0 & $\odot .995851$ & -1.288173 & 1.627011 \\
\hline 69 & 7 & 0 & 1.033528 & -2.628069 & 1.606713 \\
\hline 70 & 1 & 0 & 5.087475 & 0.598303 & 1.024812 \\
\hline 71 & 1 & 0 & -1.249112 & 1.083643 & 1.893022 \\
\hline 72 & 1 & 0 & 1.899139 & -3.077925 & 1.357851 \\
\hline 73 & 1 & 0 & 0.162140 & -3.150930 & 1.505274 \\
\hline 74 & 1 & 0 & -8.479230 & -4.595881 & $\odot .555597$ \\
\hline 75 & 6 & 0 & -8.226524 & -0.802245 & 0.165517 \\
\hline 76 & 6 & $\odot$ & -6.777262 & -1.144507 & -0.144808 \\
\hline 77 & 6 & 0 & -6.111801 & -1.038807 & 1.226487 \\
\hline 78 & 8 & 0 & -7.130143 & -1.470716 & 2.175905 \\
\hline 79 & 1 & 0 & -8.970761 & -3.309672 & 2.479574 \\
\hline 80 & 1 & 0 & -10.035038 & -2.848604 & 1.127887 \\
\hline 81 & 1 & 0 & -9.117322 & $-\odot .856388$ & 2.139221 \\
\hline 82 & 1 & 0 & -8.925331 & -1.144624 & -0.598989 \\
\hline 83 & 1 & 0 & -6.336613 & $-\odot .483645$ & -0.882970 \\
\hline 84 & 1 & 0 & -6.725426 & -2.164250 & -0.525824 \\
\hline 85 & 1 & $\odot$ & -5.813255 & -0.017247 & 1.453301 \\
\hline 86 & 7 & 0 & -4.925455 & -1.863337 & 1.376151 \\
\hline 87 & 6 & 0 & -4.995038 & -3.207065 & 1.089104 \\
\hline 88 & 6 & 0 & -3.905438 & -4.010795 & 1.049852 \\
\hline 89 & 6 & 0 & -2.606560 & -3.410469 & 1.322170 \\
\hline 90 & 7 & $\odot$ & -2.629757 & $-2.070 \odot 27$ & 1.641979 \\
\hline 91 & 6 & $\odot$ & -3.724949 & -1.247309 & 1.701947 \\
\hline 92 & 6 & 0 & -3.975900 & -5.476528 & 0.757413 \\
\hline 93 & 8 & 0 & -1.541413 & -4.032103 & 1.301690 \\
\hline 94 & 8 & 0 & -3.635903 & $-\odot .069103$ & 2.017673 \\
\hline 95 & 1 & 0 & -6.003906 & -3.578807 & 0.924709 \\
\hline 96 & 1 & 0 & -3.372808 & -5.719934 & -0.121346 \\
\hline 97 & 1 & 0 & -3.587238 & -6.062294 & 1.595783 \\
\hline 98 & 1 & 0 & -5.005940 & -5.783232 & $\odot .565672$ \\
\hline 99 & 1 & 0 & -1.715309 & -1.582824 & 1.766652 \\
\hline 100 & 6 & 0 & -5.963294 & 2.968992 & 1.059709 \\
\hline 101 & 11 & $\odot$ & 6.800143 & 0.229115 & 3.738533 \\
\hline 102 & 11 & $\odot$ & -6.557603 & $\odot .490034$ & -3.697036 \\
\hline 103 & 6 & 0 & -4.428419 & 3.580297 & -1.764187 \\
\hline 104 & 6 & 0 & -3.241977 & 3.041248 & -0.947819 \\
\hline 105 & 8 & 0 & -3.594165 & 3.229178 & 0.449393 \\
\hline 106 & 1 & 0 & -5.594924 & 2.398163 & 1.914340 \\
\hline 107 & 1 & 0 & -6.849146 & 3.541303 & 1.348030 \\
\hline 108 & 1 & 0 & -4.747953 & 4.705433 & 1.321193 \\
\hline 109 & 1 & 0 & -6.208679 & 4.623889 & -1.012648 \\
\hline 110 & 1 & 0 & -4.097349 & 4.082314 & -2.675294 \\
\hline 111 & 1 & 0 & -5.112264 & 2.774921 & -2.037827 \\
\hline 112 & 1 & 0 & -2.316461 & 3.584619 & -1.142162 \\
\hline 113 & 7 & 0 & -2.951404 & 1.640755 & -1.188369 \\
\hline 114 & 6 & $\odot$ & -3.848897 & $\odot .601932$ & -1.141925 \\
\hline 115 & 7 & 0 & -3.283187 & -0.567232 & -1.284782 \\
\hline 116 & 6 & 0 & -1.932954 & $-\odot .288158$ & -1.411862 \\
\hline 117 & 6 & 0 & -1.707548 & 1.078245 & -1.341501 \\
\hline 118 & 7 & 0 & -0.542887 & 1.744500 & -1.418974 \\
\hline 119 & 6 & 0 & 0.497127 & 0.934779 & -1.558137 \\
\hline 120 & 7 & 0 & 0.381718 & $-\odot .434896$ & -1.634422 \\
\hline 121 & 6 & 0 & -0.810939 & -1.153734 & -1.567016 \\
\hline 122 & 7 & 0 & 1.737174 & 1.430162 & -1.651878 \\
\hline 123 & 8 & 0 & $-\odot .809797$ & -2.388598 & -1.638298 \\
\hline 124 & 1 & 0 & -4.893111 & 0.804817 & -0.956522 \\
\hline 125 & 1 & 0 & 1.913716 & 2.378907 & -1.356796 \\
\hline 126 & 1 & 0 & 2.551067 & $\odot .812114$ & -1.716563 \\
\hline 127 & 1 & 0 & 1.244144 & -1.008265 & -1.698630 \\
\hline 128 & 1 & 0 & -4.022065 & -2.301161 & -1.322336 \\
\hline 129 & 8 & 0 & -4.455881 & -3.183019 & -1.454099 \\
\hline
\end{tabular}

Basis read from chk: "/LARGE@/a50063/Gau-17101.chk" (6D, 7F) 1127 basis functions

305 alpha electrons 304 beta electrons nuclear repulsion energy 16498.7414489903 Hartrees.

NAtoms $=129$

Harmonic frequencies $\left(\mathrm{cm}^{* *}-1\right)$, IR intensities (KM/Mole), Raman

$\begin{array}{lll}1 & 2 & 3 \\ \mathrm{~A} & \mathrm{~A} & \mathrm{~A}\end{array}$


Zero-point correction=

Thermal correction to Energy=

Thermal correction to Enthalpy=

Thermal correction to Gibbs Free Energy=

Sum of electronic and zero-point Energies=

Sum of electronic and thermal Energies=

Sum of electronic and thermal Enthalpies=

Sum of electronic and thermal Free Energies=

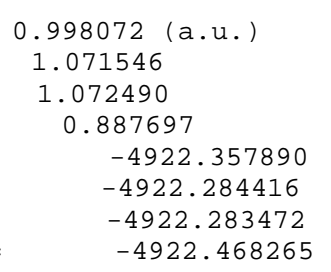

$\mathrm{G}(\mathrm{high})=-4924 \cdot 1700992 \mathrm{a} \cdot \mathrm{u}$.

\begin{tabular}{|c|c|c|c|}
\hline $\begin{array}{r}\mathrm{E}(\mathrm{T} \\
\mathrm{KCa}\end{array}$ & $\begin{array}{l}\text { ermal) } \\
\text { /Mol } \\
2.405\end{array}$ & $\begin{array}{c}\text { CV } \\
\text { Cal/Mol-Kelvin } \\
279.777\end{array}$ & $\begin{array}{c}\mathrm{S} \\
\mathrm{Cal} / \mathrm{Mol}-\mathrm{Kel} \text { in } \\
388.930\end{array}$ \\
\hline Item & Value & \multicolumn{2}{|c|}{ Threshold Converged? } \\
\hline Maximum Force & $\odot .000019$ & 0.000450 & YES \\
\hline Force & $\odot .000003$ & 0.000300 & YES \\
\hline Maximum Displacement & 0.001814 & 0.001800 & NO \\
\hline Displacement & 0.000342 & 0.001200 & YES \\
\hline
\end{tabular}

Normal termination of Gaussian $\odot 9$ at Mon Jan 28 02:47:33 2019.

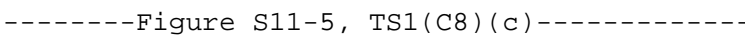

dna๑4c-ohts. $\log$

Standard orientation:

\begin{tabular}{|c|c|c|c|c|c|}
\hline \multirow{2}{*}{$\begin{array}{l}\text { Center } \\
\text { Number }\end{array}$} & \multirow{2}{*}{$\begin{array}{l}\text { Atomic } \\
\text { Number }\end{array}$} & \multirow{2}{*}{$\begin{array}{c}\text { Atomic } \\
\text { Type }\end{array}$} & \multicolumn{3}{|c|}{ Coordinates (Angstroms) } \\
\hline & & & $x$ & $\mathrm{Y}$ & Z \\
\hline & & & & $\ldots$ & - - - \\
\hline 1 & 7 & $\odot$ & 5.018408 & -2.206349 & -0.809525 \\
\hline 2 & 6 & $\odot$ & 5.287407 & -3.538585 & -0.684874 \\
\hline 3 & 6 & $\odot$ & 4.292734 & -4.442813 & -0.556160 \\
\hline 4 & 6 & $\odot$ & 2.951779 & -3.943856 & -0.586856 \\
\hline 5 & 7 & $\odot$ & 2.693466 & -2.641984 & -0.744293 \\
\hline 6 & 8 & $\odot$ & 8.745458 & 0.411882 & -0.713244 \\
\hline 7 & 15 & $\odot$ & 8.204296 & 1.682091 & 0.138258 \\
\hline 8 & 8 & $\odot$ & 6.664804 & 1.830683 & -0.383625 \\
\hline 9 & 8 & $\odot$ & 9.017283 & 2.888614 & -0.257746 \\
\hline 10 & 8 & $\odot$ & 8.106226 & 1.359964 & 1.618770 \\
\hline 11 & 8 & $\odot$ & 5.480838 & 5.941994 & 0.321536 \\
\hline 12 & 6 & $\odot$ & 5.367412 & 3.848111 & -0.802771 \\
\hline 13 & 6 & $\odot$ & 6.012992 & 4.593770 & ๑. 368891 \\
\hline 14 & 6 & $\odot$ & -8.186779 & -1.991795 & 2.870653 \\
\hline 15 & 6 & $\odot$ & -7.717594 & -0.573157 & 2.621163 \\
\hline 16 & 8 & $\odot$ & -7.606055 & -2.862422 & 1.868737 \\
\hline 17 & 8 & $\odot$ & -8.174567 & 1.327001 & 1. 203732 \\
\hline 18 & 15 & $\odot$ & -8.767029 & 2.091394 & $-\odot .111185$ \\
\hline 19 & 8 & $\odot$ & -7.483260 & 2.130350 & -1.126175 \\
\hline 20 & 8 & $\odot$ & -9.144113 & 3.487697 & 0.322356 \\
\hline 21 & 8 & $\odot$ & -9.830939 & 1.259043 & -0.788400 \\
\hline 22 & 8 & $\odot$ & -4.895710 & 3. 260628 & -4.145632 \\
\hline 23 & 6 & $\odot$ & -5.245366 & 2.711343 & -1.842671 \\
\hline 24 & 6 & $\odot$ & -5.684736 & 2.396213 & -3.290781 \\
\hline 25 & 1 & $\odot$ & -5.193094 & 3.189728 & -5.068561 \\
\hline 26 & 6 & $\odot$ & 8.864026 & -3.038888 & -2.032313 \\
\hline 27 & 6 & $\odot$ & 8.314934 & -1.633682 & -1.894937 \\
\hline 28 & 6 & $\odot$ & 8.479926 & -1.015099 & -0.499624 \\
\hline 29 & 6 & $\odot$ & 7.148929 & -1.322572 & ๑. 183154 \\
\hline 30 & 6 & $\odot$ & 6.127229 & -1.269433 & -0.955361 \\
\hline 31 & 8 & $\odot$ & 6.873464 & -1.614199 & -2.160977 \\
\hline 32 & 1 & $\odot$ & 8.526108 & -3.461470 & -2.983968 \\
\hline 33 & 1 & $\odot$ & 9.959042 & -2.994154 & -2.033623 \\
\hline 34 & 1 & $\odot$ & 8.794134 & $-\odot .995589$ & -2.640377 \\
\hline 35 & 1 & $\odot$ & 9.331046 & -1.423871 & 0.045335 \\
\hline 36 & 1 & $\odot$ & 6.914450 & -0.637803 & $\odot .993251$ \\
\hline 37 & 1 & $\odot$ & 7.219201 & -2.331372 & $\odot .594808$ \\
\hline 38 & 1 & $\odot$ & 5.691241 & -0.279609 & -1.065452 \\
\hline 39 & 8 & $\odot$ & 8.396789 & -3.842290 & -0.919741 \\
\hline 40 & 1 & $\odot$ & 8.732526 & -4.751251 & -0.980671 \\
\hline 41 & 6 & $\odot$ & 3. 698168 & -1.739693 & -0.888916 \\
\hline 42 & 7 & $\odot$ & 1.914982 & -4.774701 & -0.453312 \\
\hline 43 & 8 & $\odot$ & 3.481869 & -0.537500 & -1.085861 \\
\hline
\end{tabular}




\begin{tabular}{|c|c|c|c|c|c|}
\hline 44 & 1 & $\odot$ & 6.341774 & -3.809815 & -0.717835 \\
\hline 45 & 1 & $\odot$ & 4.502710 & -5.499250 & -0.460533 \\
\hline 46 & 1 & $\odot$ & 2.080571 & -5.767097 & $-\odot .415560$ \\
\hline 47 & 1 & 0 & $\odot .969631$ & -4.448934 & -0.674033 \\
\hline 48 & 6 & $\odot$ & 6.220603 & 2.754151 & -1.415271 \\
\hline 49 & 1 & $\odot$ & 5.842317 & 6.473945 & 1.050730 \\
\hline 50 & 6 & 0 & 5.523690 & 3.836270 & 1.609438 \\
\hline 51 & 6 & $\odot$ & 4.156459 & 3.279339 & 1.192091 \\
\hline 52 & 8 & $\odot$ & 4.130956 & 3.284381 & $-\odot .263339$ \\
\hline 53 & 1 & 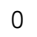 & 5.626818 & 2.176332 & -2.124843 \\
\hline 54 & 1 & $\odot$ & 7.083046 & 3.192691 & -1.923937 \\
\hline 55 & 1 & 0 & 5.088080 & 4.559087 & -1.584137 \\
\hline 56 & 1 & 0 & 7.105031 & 4.602246 & $\odot .294528$ \\
\hline 57 & 1 & $\odot$ & 5.441857 & 4.490605 & 2.479657 \\
\hline 58 & 1 & $\odot$ & 6.222102 & 3.033537 & 1.848415 \\
\hline 59 & 1 & 0 & 3.328714 & 3.902472 & 1.532961 \\
\hline 60 & 7 & $\odot$ & 3.880445 & 1.938632 & 1.670484 \\
\hline 61 & 6 & 0 & 4.735366 & $\odot .863843$ & 1.789235 \\
\hline 62 & 7 & 0 & 4.124840 & $-\odot .268459$ & 2.023667 \\
\hline 63 & 6 & $\odot$ & 2.784581 & $\odot .070085$ & 2.040059 \\
\hline 64 & 6 & $\odot$ & 2.613394 & 1.431964 & 1.826154 \\
\hline 65 & 7 & 0 & 1.455755 & 2.108478 & 1.785507 \\
\hline 66 & 6 & $\odot$ & $\odot .421958$ & 1.301566 & 1.972436 \\
\hline 67 & 7 & 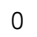 & $\odot .438183$ & -0.026591 & 2.155649 \\
\hline 68 & 6 & 0 & 1.607990 & $-\odot .693102$ & 2.167048 \\
\hline 69 & 7 & $\odot$ & 1.589461 & -2.029767 & 2.312188 \\
\hline 70 & 1 & $\odot$ & 5.805502 & $\odot .993080$ & 1.703578 \\
\hline 71 & 1 & $\odot$ & -0.571938 & 1.742471 & 1.958577 \\
\hline 72 & 1 & $\odot$ & 2.440172 & -2.528418 & 2.105728 \\
\hline 73 & 1 & $\odot$ & $\odot .7 \odot 4818$ & -2.513923 & 2.149545 \\
\hline 74 & 1 & $\odot$ & -7.857715 & -3.787137 & 2.023952 \\
\hline 75 & 6 & $\odot$ & -7.855229 & $-\odot .107722$ & 1.166930 \\
\hline 76 & 6 & $\odot$ & -6.477579 & -0.339097 & 0.568033 \\
\hline 77 & 6 & $\odot$ & -5.527997 & $-\odot .127194$ & 1.744973 \\
\hline 78 & 8 & 0 & -6.293428 & -0.454134 & 2.941357 \\
\hline 79 & 1 & $\odot$ & -7.877524 & -2.296376 & 3.875957 \\
\hline 80 & 1 & $\odot$ & -9.281206 & -2.021912 & 2.813551 \\
\hline 81 & 1 & $\odot$ & -8.265209 & $\odot .102496$ & 3.282709 \\
\hline 82 & 1 & $\odot$ & -8.651317 & -0.628651 & 0.632333 \\
\hline 83 & 1 & $\odot$ & -6.246772 & $\odot .336278$ & $-\odot .253739$ \\
\hline 84 & 1 & 0 & -6.404875 & -1.358265 & 0.186267 \\
\hline 85 & 1 & $\odot$ & -5.173556 & 0.899143 & 1.821072 \\
\hline 86 & 7 & $\odot$ & -4.336615 & -0.966733 & 1.704664 \\
\hline 87 & 6 & $\odot$ & -4.472484 & -2.336162 & 1.630599 \\
\hline 88 & 6 & $\odot$ & -3.421112 & -3.183188 & 1.610926 \\
\hline 89 & 6 & $\odot$ & -2.089489 & -2.615578 & 1.732201 \\
\hline 90 & 7 & 0 & -2.037778 & -1.239675 & 1.811006 \\
\hline 91 & 6 & $\odot$ & -3.094411 & $-\odot .366049$ & 1.835735 \\
\hline 92 & 6 & $\odot$ & -3.559495 & -4.670932 & 1.492416 \\
\hline 93 & 8 & 0 & -1.050231 & -3.280905 & 1.781881 \\
\hline 94 & 8 & 0 & -2.937735 & 0.841974 & 1.963798 \\
\hline 95 & 1 & $\odot$ & -5.504496 & -2.682160 & 1.611106 \\
\hline 96 & 1 & 0 & -3.056958 & -5.030750 & 0.588652 \\
\hline 97 & 1 & $\odot$ & -3.096657 & -5.179585 & 2.343085 \\
\hline 98 & 1 & $\odot$ & -4.611132 & -4.962661 & 1.444658 \\
\hline 99 & 1 & $\odot$ & -1.096934 & -0.803770 & 1.951264 \\
\hline 100 & 6 & $\odot$ & -6.363667 & 3.028911 & -0.880353 \\
\hline 101 & 11 & 0 & 9.275665 & 3.300434 & 2.410555 \\
\hline 102 & 11 & 0 & -11.460374 & 3.138012 & -0.616792 \\
\hline 103 & 6 & $\odot$ & -5.305442 & $\odot .918734$ & -3.476388 \\
\hline 104 & 6 & $\odot$ & -4.079508 & $\odot .82160 \odot$ & -2.576237 \\
\hline 105 & 8 & $\odot$ & -4.515148 & 1.525240 & -1.375128 \\
\hline 106 & 1 & $\odot$ & -6.009053 & 2.912722 & 0.148517 \\
\hline 107 & 1 & $\odot$ & -6.699649 & 4.058644 & -1.028596 \\
\hline 108 & 1 & $\odot$ & -4.550910 & 3.557368 & -1.874992 \\
\hline 109 & 1 & $\odot$ & -6.751731 & 2.568877 & -3.442139 \\
\hline 110 & 1 & $\odot$ & -5.109398 & $\odot .664507$ & -4.519085 \\
\hline 111 & 1 & $\odot$ & -6.107226 & $\odot .286126$ & -3.084168 \\
\hline 112 & 1 & $\odot$ & -3.235723 & 1.369290 & -3.009966 \\
\hline 113 & 7 & $\odot$ & -3.608047 & -0.498724 & -2.244497 \\
\hline 114 & 6 & $\odot$ & -4.145388 & -1.728473 & -2.591089 \\
\hline 115 & 7 & $\odot$ & -3.295451 & -2.726563 & -2.367925 \\
\hline 116 & 6 & $\odot$ & -2.197632 & -2.133562 & -1.818091 \\
\hline 117 & 6 & $\odot$ & -2.341844 & -0.746412 & -1.771131 \\
\hline 118 & 7 & $\odot$ & -1.426804 & $\odot .176562$ & -1.458484 \\
\hline 119 & 6 & $\odot$ & -0.232590 & -0.356271 & -1.201046 \\
\hline 120 & 7 & $\odot$ & -0.004071 & -1.707469 & -1.129568 \\
\hline
\end{tabular}




$\begin{array}{rrrrrr}121 & 6 & 0 & -0.934879 & -2.697628 & -1.439079 \\ 122 & 7 & 0 & 0.821545 & 0.437309 & -1.008019 \\ 123 & 8 & 0 & -0.633312 & -3.891803 & -1.394779 \\ 124 & 1 & 0 & -5.025506 & -1.797154 & -3.207843 \\ 125 & 1 & 0 & 0.667858 & 1.431130 & -1.049676 \\ 126 & 1 & 0 & 1.781145 & 0.075033 & -0.955292 \\ 127 & 1 & 0 & 0.963827 & -2.034183 & -0.927306 \\ 128 & 8 & 0 & -5.589083 & -2.716129 & -1.252913 \\ 129 & 1 & 0 & -4.921550 & -3.305858 & -0.870125 \\ - & - & - & -\end{array}$

1127 basis functions

305 alpha electrons 304 beta electrons

nuclear repulsion energy 16098.0711716913 Hartrees.

NAtoms $=129$

Harmonic frequencies $\left(\mathrm{cm}^{*}-1\right)$, IR intensities (KM/Mole), Raman

\begin{tabular}{|c|c|c|c|c|c|c|c|c|c|c|}
\hline & & & A & & A & & & $\begin{array}{l}3 \\
A\end{array}$ & & \\
\hline Freque & encie & ; - - & 198.66 & & & 13.6961 & & & 15.8629 & \\
\hline Atom & AN & $x$ & Y & Z & $x$ & $\mathrm{Y}$ & Z & $x$ & Z & \\
\hline 1 & 7 & $-\odot .01$ & $\odot .01$ & $-\odot . \odot \odot$ & $-\odot . \odot 4$ & $-\odot .01$ & $\odot .07$ & -0.03 & $\odot .02$ & $-\odot .01$ \\
\hline 2 & 6 & $-\odot . \odot \odot$ & $\odot .01$ & $-\odot . \odot \odot$ & $-\odot .06$ & $-\odot .01$ & 0.10 & $-\odot .03$ & 0.02 & $-\odot .04$ \\
\hline 3 & 6 & $\odot .0 \odot$ & $\odot . \odot \odot$ & $-\odot . \odot \odot$ & $-\odot .07$ & $-\odot .0 \odot$ & 0.11 & -0.03 & 0.01 & -0.06 \\
\hline 4 & 6 & $\odot . \odot \odot$ & $-\odot . \odot \odot$ & $\odot . \odot \odot$ & $-\odot .07$ & 0.01 & $\odot .07$ & $-\odot .03$ & $\odot .01$ & -0.05 \\
\hline 5 & 7 & -0.01 & $-\odot . \odot \odot$ & $\odot .0 \odot$ & $-\odot .05$ & 0.01 & $\odot . \odot 4$ & $-\odot .03$ & $\odot .01$ & -0.03 \\
\hline 6 & 8 & $-0.0 \odot$ & $-\odot . \odot \odot$ & $\odot . \odot \odot$ & -0.02 & -0.05 & 0.01 & 0.03 & -0.03 & 0.05 \\
\hline 7 & 15 & $-\odot . \odot \odot$ & $\odot .0 \odot$ & $-\odot . \odot \odot$ & $-0.0 \odot$ & $-\odot .02$ & -0.02 & 0.04 & $-\odot .03$ & 0.07 \\
\hline 8 & 8 & $-\odot . \odot \odot$ & $\odot . \odot \odot$ & $-\odot . \odot \odot$ & $-\odot .0 \odot$ & $-\odot . \odot 1$ & $-\odot .01$ & 0.05 & $\odot . \odot \odot$ & 0.06 \\
\hline 9 & 8 & $-\odot . \odot \odot$ & $\odot . \odot \odot$ & $-\odot . \odot \odot$ & $\odot .01$ & $-\odot . \odot 4$ & $-\odot .05$ & 0.07 & $-\odot . \odot 4$ & 0.10 \\
\hline 10 & 8 & $-\odot . \odot \odot$ & $\odot . \odot \odot$ & $-\odot . \odot \odot$ & $\odot . \odot \odot$ & 0.01 & $-\odot .01$ & 0.02 & -0.05 & 0.06 \\
\hline 11 & 8 & $-\odot . \odot \odot$ & $\odot . \odot \odot$ & -0.00 & $\odot .04$ & 0.01 & -0.10 & 0.13 & 0.02 & 0.07 \\
\hline 12 & 6 & $\odot . \odot \odot$ & $\odot . \odot \odot$ & $-\odot . \odot \odot$ & 0.01 & $-\odot .01$ & -0.05 & 0.10 & 0.03 & $\odot .06$ \\
\hline 13 & 6 & 0.00 & $\odot .0 \odot$ & $-0 . \odot \odot$ & 0.03 & 0.01 & -0.07 & 0.10 & 0.01 & 0.07 \\
\hline 14 & 6 & $\odot . \odot \odot$ & $-0 . \odot \odot$ & $-0.0 \odot$ & 0.07 & -0.09 & -0.04 & 0.07 & 0.05 & 0.10 \\
\hline 15 & 6 & $-\odot . \odot \odot$ & $-\odot . \odot \odot$ & $-\odot . \odot \odot$ & 0.05 & $-\odot .08$ & -0.01 & 0.06 & 0.05 & 0.05 \\
\hline 16 & 8 & 0.00 & -0.00 & $-0.0 \odot$ & $\odot .07$ & -0.06 & -0.06 & 0.05 & 0.03 & 0.11 \\
\hline 17 & 8 & -0.01 & $-\odot .0 \odot$ & $\odot . \odot \odot$ & 0.01 & $-\odot .06$ & 0.02 & 0.00 & $\odot .01$ & 0.01 \\
\hline 18 & 15 & $-\odot . \odot \odot$ & $\odot . \odot \odot$ & $\odot . \odot \odot$ & $-\odot .01$ & $-\odot .04$ & 0.04 & -0.04 & $-\odot .04$ & $\odot . \odot \odot$ \\
\hline 19 & 8 & -0.00 & $0.0 \odot$ & $0.0 \odot$ & -0.02 & $-\odot . \odot \odot$ & 0.03 & -0.06 & -0.04 & -0.02 \\
\hline 20 & 8 & -0.00 & $0.0 \odot$ & 0.00 & -0.03 & -0.05 & 0.07 & -0.05 & -0.03 & -0.03 \\
\hline 21 & 8 & $-\odot . \odot \odot$ & $\odot . \odot \odot$ & $-\odot . \odot \odot$ & $-\odot . \odot \odot$ & $-\odot . \odot 4$ & $\odot .03$ & $-\odot .04$ & $-\odot .07$ & 0.04 \\
\hline 22 & 8 & 0.01 & -0.04 & -0.00 & -0.04 & 0.08 & 0.04 & -0.12 & -0.08 & -0.09 \\
\hline 23 & 6 & -0.00 & -0.01 & $0.0 \odot$ & -0.03 & 0.03 & 0.03 & -0.07 & -0.04 & -0.08 \\
\hline 24 & 6 & $\odot . \odot \odot$ & $-\odot .03$ & 0.01 & $-\odot .03$ & 0.06 & $\odot . \odot 3$ & $-\odot . \odot 9$ & $-\odot .07$ & $-\odot .06$ \\
\hline 25 & 1 & $0.0 \odot$ & -0.04 & $0.0 \odot$ & -0.04 & 0.09 & 0.04 & -0.14 & -0.10 & -0.09 \\
\hline 26 & 6 & $-\odot . \odot \odot$ & $\odot . \odot \odot$ & $-\odot .0 \odot$ & -0.05 & $-\odot .09$ & 0.12 & -0.06 & 0.03 & $-\odot .07$ \\
\hline 27 & 6 & $-\odot . \odot \odot$ & $\odot . \odot \odot$ & $\odot . \odot \odot$ & $-\odot .03$ & $-\odot . \odot 8$ & $\odot . \odot 7$ & -0.04 & $\odot .03$ & $-\odot . \odot 2$ \\
\hline 28 & 6 & -0.00 & $0.0 \odot$ & 0.00 & -0.03 & -0.04 & 0.05 & -0.02 & -0.02 & 0.00 \\
\hline 29 & 6 & $-0.0 \odot$ & $\odot .0 \odot$ & $-0.0 \odot$ & -0.04 & -0.01 & $\odot .06$ & $-\odot .02$ & -0.01 & $\odot .0 \odot$ \\
\hline 30 & 6 & $-\odot . \odot \odot$ & 0.01 & $-\odot . \odot \odot$ & -0.03 & $-\odot .03$ & $\odot .06$ & -0.03 & $\odot .02$ & 0.01 \\
\hline 31 & 8 & $-0.0 \odot$ & $0.0 \odot$ & $-0.0 \odot$ & -0.03 & -0.07 & 0.07 & -0.04 & 0.06 & -0.01 \\
\hline 32 & 1 & -0.00 & $\odot . \odot \odot$ & $-\odot . \odot \odot$ & -0.05 & -0.11 & 0.13 & $-\odot . \odot 8$ & 0.06 & $-\odot . \odot 8$ \\
\hline 33 & 1 & $-\odot . \odot \odot$ & $\odot . \odot \odot$ & $-\odot . \odot \odot$ & -0.05 & -0.10 & 0.12 & -0.06 & 0.01 & $-\odot . \odot 9$ \\
\hline 34 & 1 & -0.00 & $0.0 \odot$ & $\odot . \odot \odot$ & -0.02 & -0.11 & 0.06 & -0.04 & 0.05 & 0.00 \\
\hline 35 & 1 & $-\odot .01$ & $-\odot . \odot \odot$ & $\odot . \odot \odot$ & -0.04 & $-\odot .03$ & $\odot .07$ & -0.03 & $-\odot .07$ & -0.02 \\
\hline 36 & 1 & $-\odot . \odot \odot$ & $\odot . \odot \odot$ & $-\odot .0 \odot$ & -0.03 & $\odot .01$ & 0.04 & $-\odot .01$ & $-\odot .02$ & $\odot .01$ \\
\hline 37 & 1 & -0.01 & $\odot .0 \odot$ & -0.00 & -0.05 & $-\odot . \odot \odot$ & $\odot . \odot 8$ & -0.04 & -0.02 & -0.01 \\
\hline 38 & 1 & $-\odot . \odot \odot$ & 0.01 & $-\odot . \odot \odot$ & $-\odot . \odot 2$ & -0.03 & 0.03 & $-\odot . \odot 3$ & 0.03 & $\odot . \odot 4$ \\
\hline 39 & 8 & $-\odot . \odot \odot$ & $\odot . \odot \odot$ & $\odot . \odot \odot$ & $-\odot . \odot 6$ & -0.05 & $\odot .14$ & -0.05 & $-\odot . \odot \odot$ & $-\odot .08$ \\
\hline 40 & 1 & $-0.0 \odot$ & $\odot .0 \odot$ & $\odot .0 \odot$ & -0.08 & -0.06 & 0.16 & -0.05 & $-\odot . \odot \odot$ & -0.11 \\
\hline 41 & 6 & $-\odot .01$ & $\odot . \odot \odot$ & $\odot . \odot \odot$ & $-\odot . \odot 4$ & $-\odot . \odot \odot$ & $\odot .05$ & $-\odot .03$ & $\odot .02$ & $-\odot . \odot \odot$ \\
\hline 42 & 7 & $\odot .01$ & $-\odot .01$ & $\odot .01$ & $-\odot . \odot 8$ & $\odot .02$ & $\odot .06$ & $-\odot . \odot 2$ & $-\odot . \odot \odot$ & $-\odot . \odot 7$ \\
\hline 43 & 8 & -0.02 & $\odot .0 \odot$ & $\odot . \odot \odot$ & $-\odot . \odot 2$ & $\odot . \odot \odot$ & $\odot .03$ & $-\odot .04$ & 0.02 & 0.02 \\
\hline 44 & 1 & $-\odot . \odot \odot$ & $\odot .01$ & $-\odot . \odot \odot$ & $-\odot .06$ & -0.03 & 0.13 & $-\odot .03$ & $\odot .02$ & -0.05 \\
\hline 45 & 1 & $\odot .01$ & $\odot . \odot \odot$ & $\odot . \odot \odot$ & $-\odot .08$ & $-\odot . \odot \odot$ & 0.13 & $-\odot .02$ & $\odot . \odot 1$ & $-\odot . \odot 9$ \\
\hline 46 & 1 & $\odot .02$ & -0.01 & 0.01 & $-\odot . \odot 9$ & 0.02 & $\odot . \odot 8$ & -0.02 & $-\odot . \odot \odot$ & -0.09 \\
\hline 47 & 1 & 0.01 & $-\odot . \odot 2$ & $\odot . \odot 2$ & $-\odot . \odot 7$ & $\odot . \odot 3$ & $\odot . \odot 2$ & $-\odot .03$ & $-\odot . \odot \odot$ & $-\odot .05$ \\
\hline 48 & 6 & $\odot .0 \odot$ & 0.00 & $-\odot . \odot \odot$ & $-\odot . \odot \odot$ & -0.04 & -0.03 & 0.08 & $\odot .02$ & 0.06 \\
\hline 49 & 1 & $\odot .0 \odot$ & $\odot . \odot \odot$ & $-\odot . \odot \odot$ & 0.05 & 0.03 & -0.11 & 0.14 & 0.01 & 0.08 \\
\hline 50 & 6 & $-\odot . \odot \odot$ & $\odot . \odot \odot$ & $-\odot . \odot \odot$ & $\odot .03$ & $\odot .04$ & $-\odot .05$ & $\odot . \odot 7$ & $\odot .01$ & $\odot .06$ \\
\hline 51 & 6 & $\odot . \odot \odot$ & $\odot . \odot \odot$ & $-\odot .0 \odot$ & $\odot .02$ & $\odot .04$ & -0.03 & $\odot . \odot 7$ & 0.03 & 0.04 \\
\hline 52 & 8 & $\odot . \odot \odot$ & $\odot . \odot \odot$ & $-\odot . \odot \odot$ & $\odot .01$ & 0.02 & $-\odot . \odot 3$ & $\odot . \odot 8$ & $\odot . \odot 6$ & $\odot . \odot 4$ \\
\hline 53 & 1 & $\odot . \odot \odot$ & $\odot . \odot \odot$ & $-\odot . \odot \odot$ & $-\odot .01$ & $-\odot .05$ & $-\odot . \odot 1$ & $\odot . \odot 8$ & $\odot .04$ & 0.05 \\
\hline 54 & 1 & $\odot . \odot \odot$ & $\odot . \odot \odot$ & $\odot .0 \odot$ & -0.00 & -0.06 & -0.04 & 0.10 & $0.0 \odot$ & 0.07 \\
\hline 55 & 1 & $-\odot . \odot \odot$ & $\odot . \odot \odot$ & $-\odot .0 \odot$ & $\odot .02$ & $-\odot .03$ & -0.06 & 0.12 & $\odot .04$ & 0.06 \\
\hline
\end{tabular}




\begin{tabular}{|c|c|c|c|c|c|c|c|c|c|c|}
\hline 56 & 1 & $\odot . \odot \odot$ & $\odot . \odot \odot$ & $-\odot .0 \odot$ & 0.03 & $-\odot . \odot \odot$ & -0.08 & 0.10 & -0.02 & $\odot . \odot 8$ \\
\hline 57 & 1 & $-0.0 \odot$ & $\odot .00$ & -0.00 & $\odot . \odot 4$ & 0.06 & $-\odot .07$ & $\odot .07$ & 0.01 & 0.06 \\
\hline 58 & 1 & $-\odot . \odot \odot$ & $\odot .0 \odot$ & $-\odot .0 \odot$ & 0.03 & 0.04 & -0.03 & 0.06 & $-0.0 \odot$ & 0.07 \\
\hline 59 & 1 & $-\odot . \odot \odot$ & $\odot . \odot \odot$ & $-\odot .0 \odot$ & $\odot .03$ & 0.05 & -0.03 & $\odot .07$ & $\odot .03$ & 0.04 \\
\hline 60 & 7 & $-\odot . \odot \odot$ & $-\odot . \odot \odot$ & $-\odot . \odot \odot$ & $\odot .02$ & 0.05 & $-\odot . \odot \odot$ & $\odot .05$ & $\odot .02$ & $\odot .02$ \\
\hline 61 & 6 & $\odot . \odot \odot$ & $\odot . \odot \odot$ & $\odot . \odot \odot$ & $\odot .03$ & $\odot .05$ & 0.02 & 0.04 & 0.02 & $\odot .04$ \\
\hline 62 & 7 & $\odot . \odot \odot$ & $\odot .0 \odot$ & 0.00 & 0.03 & 0.05 & $\odot .04$ & 0.03 & 0.02 & $\odot .03$ \\
\hline 63 & 6 & $\odot . \odot \odot$ & -0.00 & $\odot .0 \odot$ & 0.03 & 0.05 & $\odot .03$ & 0.04 & 0.03 & $-\odot . \odot \odot$ \\
\hline 64 & 6 & $\odot .00$ & -0.00 & -0.00 & 0.03 & 0.05 & $-\odot . \odot \odot$ & 0.05 & 0.03 & -0.01 \\
\hline 65 & 7 & $-\odot . \odot \odot$ & $-\odot . \odot \odot$ & $-\odot . \odot \odot$ & $\odot .02$ & $\odot .04$ & -0.02 & $\odot .05$ & $\odot .03$ & -0.05 \\
\hline 66 & 6 & -0.00 & $-\odot . \odot \odot$ & $-\odot . \odot \odot$ & $\odot .02$ & 0.04 & -0.01 & $\odot .04$ & 0.04 & -0.06 \\
\hline 67 & 7 & $-\odot . \odot \odot$ & -0.00 & -0.01 & 0.03 & 0.04 & 0.01 & 0.03 & 0.04 & -0.05 \\
\hline 68 & 6 & $\odot . \odot \odot$ & $-\odot . \odot \odot$ & $-0.0 \odot$ & 0.03 & 0.05 & 0.03 & $\odot .03$ & 0.04 & -0.02 \\
\hline 69 & 7 & $-0.0 \odot$ & $\odot .0 \odot$ & -0.00 & $\odot .04$ & $\odot .05$ & 0.06 & 0.02 & 0.04 & $-\odot .0 \odot$ \\
\hline 70 & 1 & $\odot . \odot \odot$ & $\odot .0 \odot$ & $\odot .0 \odot$ & 0.03 & 0.05 & 0.03 & 0.05 & 0.01 & 0.07 \\
\hline 71 & 1 & $-\odot . \odot \odot$ & $-\odot . \odot \odot$ & $-\odot . \odot \odot$ & 0.02 & 0.03 & -0.03 & 0.04 & 0.04 & -0.09 \\
\hline 72 & 1 & $\odot . \odot \odot$ & $-\odot . \odot \odot$ & $\odot .02$ & $\odot .05$ & 0.05 & $\odot .08$ & $\odot .03$ & $\odot .03$ & $\odot . \odot 3$ \\
\hline 73 & 1 & $-\odot . \odot \odot$ & $-\odot . \odot \odot$ & $\odot .01$ & $\odot .05$ & $\odot .04$ & $\odot .03$ & 0.03 & $\odot .04$ & -0.01 \\
\hline 74 & 1 & $\odot .0 \odot$ & -0.00 & 0.00 & 0.09 & -0.07 & $-\odot .08$ & 0.06 & 0.03 & 0.14 \\
\hline 75 & 6 & $\odot .0 \odot$ & -0.00 & $\odot .0 \odot$ & 0.03 & -0.05 & -0.01 & $\odot .01$ & 0.01 & $\odot . \odot 4$ \\
\hline 76 & 6 & $\odot . \odot \odot$ & 0.01 & -0.00 & 0.03 & -0.02 & $-\odot .02$ & $\odot . \odot \odot$ & $\odot .01$ & $\odot .01$ \\
\hline 77 & 6 & $-\odot . \odot \odot$ & $\odot .0 \odot$ & 0.00 & $\odot .03$ & -0.03 & -0.02 & 0.03 & $\odot .04$ & -0.02 \\
\hline 78 & 8 & $-\odot . \odot \odot$ & $-\odot . \odot \odot$ & $\odot .0 \odot$ & 0.05 & -0.06 & -0.02 & 0.06 & 0.07 & $\odot .01$ \\
\hline 79 & 1 & $\odot .0 \odot$ & -0.00 & -0.00 & $\odot .08$ & -0.10 & -0.05 & 0.11 & $\odot .08$ & 0.10 \\
\hline 80 & 1 & $\odot . \odot \odot$ & -0.00 & $-0.0 \odot$ & $\odot .07$ & -0.10 & -0.03 & 0.07 & 0.05 & 0.14 \\
\hline 81 & 1 & -0.00 & $-\odot . \odot \odot$ & $-0.0 \odot$ & 0.04 & -0.10 & 0.00 & 0.07 & 0.07 & 0.05 \\
\hline 82 & 1 & $\odot .0 \odot$ & -0.00 & $-\odot .0 \odot$ & 0.03 & -0.05 & -0.01 & 0.01 & -0.01 & 0.08 \\
\hline 83 & 1 & $-0.0 \odot$ & 0.04 & 0.02 & $\odot .01$ & $-0.0 \odot$ & -0.01 & -0.02 & -0.01 & -0.01 \\
\hline 84 & 1 & 0.03 & 0.03 & -0.06 & 0.04 & -0.01 & -0.04 & -0.00 & $-0.0 \odot$ & 0.03 \\
\hline 85 & 1 & $\odot .0 \odot$ & $\odot . \odot \odot$ & $\odot .01$ & $\odot . \odot 2$ & -0.03 & -0.01 & 0.03 & 0.04 & -0.06 \\
\hline 86 & 7 & -0.00 & 0.00 & 0.00 & 0.04 & -0.01 & -0.05 & 0.03 & 0.04 & -0.03 \\
\hline 87 & 6 & $-0.0 \odot$ & $\odot . \odot \odot$ & -0.01 & $\odot .06$ & -0.01 & -0.08 & 0.03 & 0.04 & 0.01 \\
\hline 88 & 6 & -0.00 & 0.00 & -0.00 & 0.07 & 0.00 & -0.09 & 0.03 & 0.03 & 0.01 \\
\hline 89 & 6 & $-\odot .0 \odot$ & $-\odot . \odot \odot$ & $\odot .0 \odot$ & 0.06 & 0.02 & -0.06 & 0.03 & 0.04 & -0.01 \\
\hline 90 & 7 & -0.00 & $-\odot . \odot \odot$ & $-0.0 \odot$ & 0.05 & 0.02 & -0.03 & 0.03 & 0.04 & -0.05 \\
\hline 91 & 6 & $-\odot . \odot \odot$ & $-\odot . \odot \odot$ & $\odot . \odot \odot$ & 0.03 & 0.00 & $-\odot .03$ & 0.03 & $\odot .04$ & -0.05 \\
\hline 92 & 6 & $-\odot . \odot \odot$ & $\odot .0 \odot$ & -0.01 & $\odot .09$ & 0.00 & -0.13 & $\odot .02$ & 0.03 & 0.04 \\
\hline 93 & 8 & $-\odot . \odot \odot$ & $-\odot . \odot \odot$ & -0.01 & $\odot .07$ & 0.03 & -0.05 & $\odot .03$ & 0.03 & $-\odot .0 \odot$ \\
\hline 94 & 8 & $\odot . \odot \odot$ & -0.00 & $\odot .0 \odot$ & $\odot .02$ & $\odot .0 \odot$ & -0.01 & 0.04 & 0.04 & -0.08 \\
\hline 95 & 1 & -0.00 & 0.00 & -0.03 & $\odot .07$ & -0.03 & -0.09 & 0.03 & 0.03 & 0.03 \\
\hline 96 & 1 & -0.01 & $\odot .0 \odot$ & -0.01 & 0.09 & 0.04 & -0.14 & 0.01 & 0.01 & 0.04 \\
\hline 97 & 1 & -0.00 & $-\odot . \odot \odot$ & -0.01 & 0.11 & -0.01 & -0.15 & 0.04 & $\odot .05$ & $\odot .05$ \\
\hline 98 & 1 & $-\odot .0 \odot$ & 0.00 & -0.01 & 0.10 & -0.01 & -0.13 & 0.02 & 0.03 & 0.06 \\
\hline 99 & 1 & $-\odot . \odot \odot$ & $-\odot . \odot \odot$ & $\odot .0 \odot$ & 0.04 & $\odot .03$ & -0.01 & 0.03 & 0.04 & -0.05 \\
\hline 100 & 6 & $-0.0 \odot$ & $\odot .0 \odot$ & -0.00 & -0.03 & 0.00 & 0.05 & -0.06 & -0.03 & -0.06 \\
\hline 101 & 11 & $0.0 \odot$ & $\odot .0 \odot$ & $\odot .0 \odot$ & $\odot .02$ & -0.01 & -0.02 & 0.04 & -0.08 & 0.11 \\
\hline 102 & 11 & 0.00 & $-0 . \odot \odot$ & $\odot . \odot \odot$ & -0.03 & -0.06 & 0.07 & -0.05 & -0.08 & 0.02 \\
\hline 103 & 6 & $-\odot .02$ & $-\odot .04$ & 0.03 & $-\odot .03$ & 0.06 & $\odot .0 \odot$ & -0.08 & -0.07 & -0.04 \\
\hline 104 & 6 & -0.04 & -0.07 & 0.05 & -0.02 & $\odot .05$ & -0.01 & -0.07 & -0.04 & -0.05 \\
\hline 105 & 8 & -0.01 & -0.00 & 0.02 & -0.01 & 0.03 & 0.00 & -0.06 & -0.02 & -0.06 \\
\hline 106 & 1 & -0.01 & 0.01 & $-0.0 \odot$ & -0.02 & $-\odot .01$ & 0.04 & -0.04 & $\odot .0 \odot$ & -0.07 \\
\hline 107 & 1 & -0.00 & $\odot .0 \odot$ & -0.02 & -0.04 & 0.00 & 0.07 & -0.07 & -0.03 & -0.08 \\
\hline 108 & 1 & $\odot . \odot \odot$ & $-\odot .01$ & -0.01 & $-\odot .04$ & 0.04 & 0.05 & $-\odot . \odot 8$ & -0.03 & -0.11 \\
\hline 109 & 1 & 0.00 & $-\odot .02$ & $\odot .0 \odot$ & -0.04 & 0.06 & $\odot .04$ & -0.10 & -0.09 & -0.05 \\
\hline 110 & 1 & -0.00 & -0.05 & 0.03 & -0.04 & 0.08 & -0.01 & -0.09 & -0.09 & -0.04 \\
\hline 111 & 1 & -0.04 & -0.02 & 0.01 & -0.03 & $\odot .06$ & -0.00 & -0.07 & -0.07 & -0.01 \\
\hline 112 & 1 & -0.01 & -0.13 & 0.04 & -0.03 & 0.06 & -0.01 & -0.08 & -0.05 & -0.07 \\
\hline 113 & 7 & -0.09 & -0.08 & 0.12 & -0.02 & 0.05 & -0.04 & -0.05 & -0.03 & -0.03 \\
\hline 114 & 6 & -0.13 & -0.13 & 0.18 & -0.02 & 0.05 & -0.06 & -0.05 & -0.04 & -0.01 \\
\hline 115 & 7 & 0.10 & $-\odot .08$ & -0.03 & $-\odot .02$ & 0.05 & -0.08 & -0.05 & -0.03 & $\odot .0 \odot$ \\
\hline 116 & 6 & 0.01 & -0.05 & -0.04 & -0.02 & 0.04 & -0.07 & -0.04 & -0.02 & -0.02 \\
\hline 117 & 6 & -0.05 & -0.03 & 0.03 & -0.02 & 0.04 & -0.04 & -0.05 & -0.02 & -0.04 \\
\hline 118 & 7 & -0.06 & -0.04 & $\odot .02$ & -0.02 & $\odot .03$ & -0.02 & -0.05 & -0.01 & -0.05 \\
\hline 119 & 6 & -0.02 & -0.02 & -0.01 & -0.02 & 0.03 & -0.02 & -0.05 & -0.01 & -0.05 \\
\hline 120 & 7 & 0.01 & -0.02 & -0.01 & -0.03 & 0.03 & -0.04 & -0.04 & -0.00 & -0.03 \\
\hline 121 & 6 & $\odot .03$ & -0.02 & -0.01 & $-\odot .02$ & 0.03 & -0.06 & -0.04 & -0.01 & -0.02 \\
\hline 122 & 7 & -0.05 & -0.01 & -0.03 & -0.02 & 0.02 & $-\odot . \odot \odot$ & -0.05 & 0.00 & -0.05 \\
\hline 123 & 8 & 0.02 & -0.02 & $-0.0 \odot$ & -0.03 & 0.03 & -0.08 & -0.04 & -0.01 & -0.01 \\
\hline 124 & 1 & 0.21 & -0.05 & -0.32 & -0.02 & 0.06 & -0.07 & -0.06 & -0.06 & 0.00 \\
\hline 125 & 1 & -0.07 & -0.01 & $\odot . \odot \odot$ & -0.02 & 0.02 & 0.02 & -0.06 & -0.00 & -0.06 \\
\hline 126 & 1 & -0.04 & 0.01 & -0.02 & -0.03 & 0.02 & 0.01 & -0.05 & 0.01 & -0.03 \\
\hline 127 & 1 & $\odot .02$ & $-\odot . \odot \odot$ & $-\odot . \odot \odot$ & $-\odot .03$ & $\odot .02$ & -0.02 & -0.04 & $\odot . \odot \odot$ & $-\odot .03$ \\
\hline 128 & 8 & $\odot .30$ & 0.52 & -0.23 & $-\odot . \odot \odot$ & $\odot .02$ & $-\odot . \odot 8$ & -0.03 & -0.02 & $\odot .03$ \\
\hline 129 & 1 & 0.10 & 0.48 & $\odot .04$ & $-\odot . \odot \odot$ & 0.01 & -0.10 & -0.03 & -0.02 & 0.03 \\
\hline
\end{tabular}


Thermal correction to Enthalpy=

Thermal correction to Gibbs Free Energy=

Sum of electronic and zero-point Energies=

Sum of electronic and thermal Energies=

Sum of electronic and thermal Enthalpies=

Sum of electronic and thermal Free Energies=

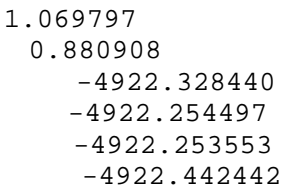

$$
\begin{aligned}
& \text { E (Thermal) } \\
& \text { KCal/Mol }
\end{aligned}
$$$$
G(\text { high })=-4924 \cdot 1700992 \mathrm{a} \cdot \mathrm{u}
$$

Total

$$
670.715
$$
CV
Cal/Mol-Kelvin 279.646

\begin{tabular}{lccc}
\multicolumn{1}{c}{ Item } & Value & Threshold Converged? \\
Maximum Force & 0.000120 & 0.000450 & YES \\
RMS Force & 0.000013 & $0.00030 \odot$ & YES \\
Maximum Displacement & 0.008657 & 0.001800 & NO \\
RMS Displacement & 0.001151 & 0.001200 & YES
\end{tabular}

Normal termination of Gaussian 16 at Wed Jan 9 01:51:24 2019.

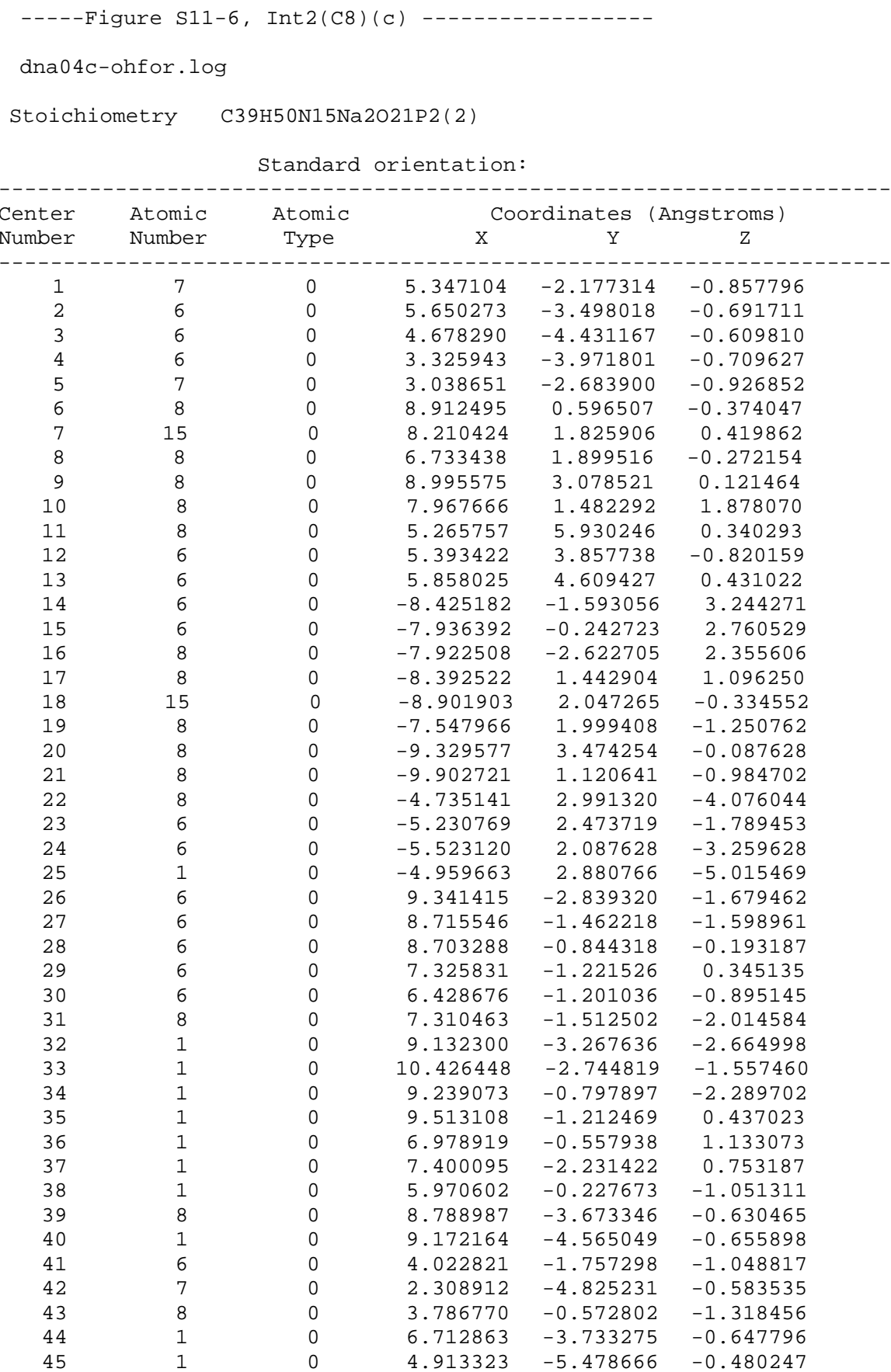




\begin{tabular}{|c|c|c|c|c|c|}
\hline 46 & 1 & 0 & 2.495570 & -5.808789 & -0.478357 \\
\hline 47 & 1 & 0 & 1.351656 & -4.522861 & -0.794483 \\
\hline 48 & 6 & 0 & 6.365351 & 2.816518 & -1.339732 \\
\hline 49 & 1 & 0 & 5.502487 & 6.461192 & 1.119652 \\
\hline 50 & 6 & $\odot$ & 5.262660 & 3.803890 & 1.591644 \\
\hline 51 & 6 & 0 & 3.972215 & 3.217031 & 1.006054 \\
\hline 52 & 8 & 0 & 4.131836 & 3.222998 & -0.440820 \\
\hline 53 & 1 & $\odot$ & 5.887941 & 2.221609 & -2.119375 \\
\hline 54 & 1 & 0 & 7.258354 & 3.304112 & -1.739072 \\
\hline 55 & 1 & 0 & 5.174926 & 4.570597 & -1.619048 \\
\hline 56 & 1 & $\odot$ & 6.949098 & 4.673898 & 0.487218 \\
\hline 57 & 1 & 0 & 5.058501 & 4.431872 & 2.461297 \\
\hline 58 & 1 & 0 & 5.959523 & 3.019131 & 1.890420 \\
\hline 59 & 1 & 0 & 3.096234 & 3.822674 & 1.242538 \\
\hline 60 & 7 & 0 & 3.663756 & 1.871102 & 1.445221 \\
\hline 61 & 6 & 0 & 4.511161 & $\odot .798929$ & 1.620981 \\
\hline 62 & 7 & $\odot$ & 3.889109 & $-\odot .334659$ & 1.815552 \\
\hline 63 & 6 & 0 & 2.549358 & -0.000114 & 1.739022 \\
\hline 64 & 6 & 0 & 2.390861 & 1.361967 & 1.513679 \\
\hline 65 & 7 & 0 & 1.238925 & 2.039732 & 1.396951 \\
\hline 66 & 6 & 0 & 0.194178 & 1.235655 & 1.523723 \\
\hline 67 & 7 & $\odot$ & $\odot .198123$ & $-\odot .092081$ & 1.710540 \\
\hline 68 & 6 & $\odot$ & 1.364283 & $-\odot .761520$ & 1.798308 \\
\hline 69 & 7 & 0 & 1.329417 & -2.095186 & 1.945255 \\
\hline 70 & 1 & 0 & 5.583105 & 0.935615 & 1.597524 \\
\hline 71 & 1 & 0 & -0.796686 & 1.678462 & 1.456528 \\
\hline 72 & 1 & 0 & 2.181869 & -2.613368 & 1.808195 \\
\hline 73 & 1 & $\odot$ & $\odot .437629$ & -2.573937 & 1.819828 \\
\hline 74 & 1 & 0 & -8.193792 & -3.504761 & 2.657208 \\
\hline 75 & 6 & 0 & -8.151328 & $\odot .005252$ & 1.263460 \\
\hline 76 & 6 & 0 & -6.828491 & -0.404825 & 0.631276 \\
\hline 77 & 6 & 0 & -5.795602 & $-\odot .035007$ & 1.696848 \\
\hline 78 & 8 & 0 & -6.491973 & -0.128390 & 2.974483 \\
\hline 79 & 1 & $\odot$ & -8.066460 & -1.755419 & 4.265806 \\
\hline 80 & 1 & 0 & -9.521308 & -1.597097 & 3.248938 \\
\hline 81 & 1 & 0 & -8.423674 & 0.541717 & 3.344307 \\
\hline 82 & 1 & 0 & -9.005542 & -0.542120 & $\odot .862298$ \\
\hline 83 & 1 & 0 & -6.615825 & $\odot .093449$ & -0.312044 \\
\hline 84 & 1 & $\odot$ & -6.856000 & -1.480892 & 0.463158 \\
\hline 85 & 1 & $\odot$ & -5.414758 & $\odot .975779$ & 1.567298 \\
\hline 86 & 7 & 0 & -4.630375 & -0.905546 & 1.723486 \\
\hline 87 & 6 & 0 & -4.803356 & -2.271567 & 1.813179 \\
\hline 88 & 6 & 0 & -3.780477 & -3.151654 & 1.761328 \\
\hline 89 & 6 & 0 & -2.427072 & -2.615742 & 1.680078 \\
\hline 90 & 7 & 0 & -2.337688 & -1.241928 & 1.637783 \\
\hline 91 & 6 & 0 & -3.365911 & $-\odot .333548$ & 1.702674 \\
\hline 92 & 6 & 0 & -3.961034 & -4.637159 & 1.820349 \\
\hline 93 & 8 & $\odot$ & -1.407314 & -3.308878 & 1.672447 \\
\hline 94 & 8 & 0 & -3.166481 & $\odot .871313$ & 1.742530 \\
\hline 95 & 1 & 0 & -5.838740 & -2.584600 & 1.947410 \\
\hline 96 & 1 & 0 & -3.567747 & -5.102705 & $\odot .911525$ \\
\hline 97 & 1 & 0 & -3.416531 & -5.064930 & 2.666964 \\
\hline 98 & 1 & 0 & -5.016918 & -4.898418 & 1.915215 \\
\hline 99 & 1 & $\odot$ & -1.375458 & -0.830536 & 1.646968 \\
\hline 100 & 6 & 0 & -6.428552 & 2.889717 & $-\odot .972320$ \\
\hline 101 & 11 & $\odot$ & 8.971543 & 3.467765 & 2.790576 \\
\hline 102 & 11 & $\odot$ & -11.551156 & 2.973182 & -1.158984 \\
\hline 103 & 6 & 0 & -5.027067 & $\odot .637838$ & -3.364139 \\
\hline 104 & 6 & 0 & -3.932558 & 0.609865 & -2.297494 \\
\hline 105 & 8 & 0 & -4.589126 & 1.308298 & -1.182570 \\
\hline 106 & 1 & 0 & -6.180423 & 2.853494 & 0.092753 \\
\hline 107 & 1 & 0 & -6.719215 & 3.910108 & -1.236430 \\
\hline 108 & 1 & 0 & -4.513504 & 3.303534 & -1.804823 \\
\hline 109 & 1 & 0 & -6.583707 & 2.178290 & -3.502379 \\
\hline 110 & 1 & 0 & -4.666500 & 0.393777 & -4.365264 \\
\hline 111 & 1 & 0 & -5.829716 & -0.050755 & -3.085152 \\
\hline 112 & 1 & 0 & -3.077388 & 1.218534 & -2.614150 \\
\hline 113 & 7 & 0 & -3.432612 & $-\odot .665987$ & -1.897070 \\
\hline 114 & 6 & 0 & -4.083767 & -1.979528 & -2.152559 \\
\hline 115 & 7 & 0 & -3.079677 & -2.982019 & -1.875335 \\
\hline 116 & 6 & 0 & -1.943689 & -2.309335 & -1.659665 \\
\hline 117 & 6 & 0 & -2.110481 & -0.900984 & -1.689280 \\
\hline 118 & 7 & 0 & -1.166857 & 0.026095 & -1.577872 \\
\hline 119 & 6 & 0 & $\odot .067872$ & $-\odot .474929$ & -1.438260 \\
\hline 120 & 7 & 0 & $\odot .329789$ & -1.807426 & -1.318949 \\
\hline 121 & 6 & 0 & -0.625985 & -2.819346 & -1.421682 \\
\hline 122 & 7 & 0 & 1.111896 & $\odot .352404$ & -1.427081 \\
\hline
\end{tabular}

S213 


$\begin{array}{rrrrrr}123 & 8 & 0 & -0.296770 & -4.008714 & -1.338420 \\ 124 & 1 & 0 & -4.391446 & -2.014271 & -3.207060 \\ 125 & 1 & 0 & 0.922364 & 1.341161 & -1.432611 \\ 126 & 1 & 0 & 2.079775 & 0.017595 & -1.342228 \\ 127 & 1 & 0 & 1.314749 & -2.113501 & -1.173301 \\ 128 & 8 & 0 & -5.244906 & -2.175302 & -1.416472 \\ 129 & 1 & 0 & -4.964717 & -2.455172 & -0.533874 \\ -\end{array}$

\section{7 basis functions}

305 alpha electrons

304 beta electrons

nuclear repulsion energy 16094.6591872030 Hartrees.

NAtoms $=129$ NActive $=129$

Harmonic frequencies $\left(\mathrm{cm}^{*}-1\right)$, IR intensities (KM/Mole), Raman

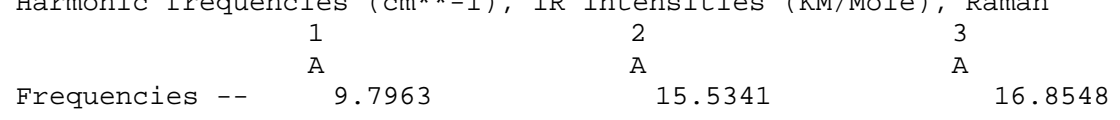

Zero-point correction=

Thermal correction to Energy=

Thermal correction to Enthalpy=

Thermal correction to Gibbs Free Energy=

Sum of electronic and zero-point Energies=

Sum of electronic and thermal Energies=

Sum of electronic and thermal Enthalpies=

๑. 998517 (a.u.)

1.072044

1.072988

0.885445

$-4922.382938$

$-4922.309410$

$-4922.308466$

Sum of electronic and thermal Free Energies=

$-4922.496009$

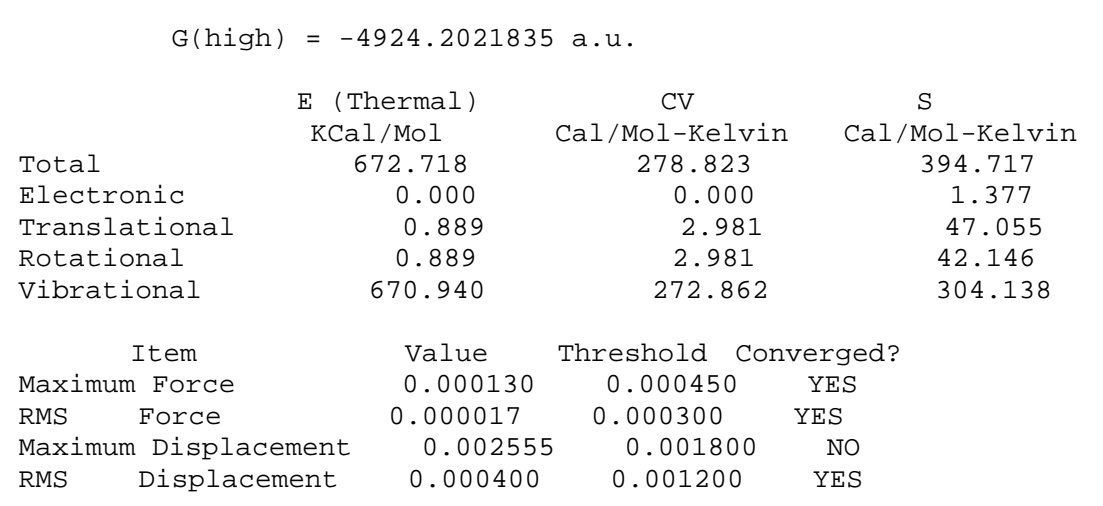

Normal termination of Gaussian 16 at Mon Jan 14 15:56:47 2019.

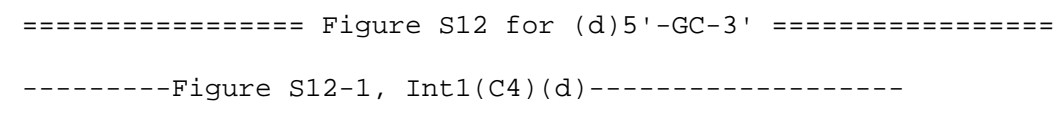

dna@4d-oh1afor. $\log$

Stoichiometry C38H49N16Na2021P2(2)

Standard orientation:

\begin{tabular}{|c|c|c|c|c|c|}
\hline \multirow{2}{*}{$\begin{array}{l}\text { Center } \\
\text { Number }\end{array}$} & Atomic & Atomic & \multicolumn{3}{|c|}{ Coordinates (Angstroms) } \\
\hline & Number & Type & $x$ & $\mathrm{Y}$ & Z \\
\hline & 1 & & & & \\
\hline 1 & 7 & $\odot$ & -4.632054 & 2.472656 & -1.669698 \\
\hline 2 & 6 & $\odot$ & -4.484621 & 3.790122 & -1.349147 \\
\hline 3 & 6 & $\odot$ & -3.322393 & 4.437895 & -1.597212 \\
\hline 4 & 6 & $\odot$ & -2.255553 & 3.667118 & -2.165455 \\
\hline 5 & 7 & $\odot$ & -2.418650 & 2.379918 & -2.484766 \\
\hline 6 & 8 & $\odot$ & -7.902940 & 0.073268 & -0.098030 \\
\hline 7 & 15 & $\odot$ & -7.387394 & -1.333494 & 0.560026 \\
\hline 8 & 8 & $\odot$ & -5.857677 & -1.469352 & $\odot .006022$ \\
\hline 9 & 8 & $\odot$ & -8.233170 & -2.412141 & -0.074255 \\
\hline 10 & 8 & $\odot$ & -7.270129 & -1.195774 & 2.055351 \\
\hline 11 & 8 & $\odot$ & -4.469979 & -5.557302 & ๑. . 030381 \\
\hline 12 & 6 & $\odot$ & -4.499256 & -3.319240 & -0.775370 \\
\hline 13 & 6 & $\odot$ & -5.061381 & -4.260042 & ๑. 291370 \\
\hline 14 & 6 & $\odot$ & 8.181615 & 3.795619 & ๑. 032499 \\
\hline 15 & 6 & $\odot$ & 7.926960 & 2.409488 & $\odot .586629$ \\
\hline 16 & 8 & $\odot$ & 6.965097 & 4.295230 & -0.573710 \\
\hline 17 & 8 & $\odot$ & 7.822491 & ๑. 082791 & 0.041368 \\
\hline 18 & 15 & $\odot$ & 7.328090 & -1.325867 & -0.625257 \\
\hline
\end{tabular}




\begin{tabular}{|c|c|c|c|c|c|}
\hline 19 & 8 & 0 & 5.806292 & -1.491760 & $-\odot .058704$ \\
\hline 20 & 8 & $\Theta$ & 8.198397 & -2.395306 & -0.009156 \\
\hline 21 & 8 & 0 & 7.193152 & -1.179016 & -2.118396 \\
\hline 22 & 8 & 0 & 4.436687 & -5.565987 & $-\odot .097392$ \\
\hline 23 & 6 & $\Theta$ & 4.473359 & -3.340346 & 0.744432 \\
\hline 24 & 6 & $\odot$ & 5.020175 & -4.263087 & -0.345780 \\
\hline 25 & 1 & 0 & 4.716349 & -6.198592 & $-\odot .780848$ \\
\hline 26 & 6 & 0 & -8.329302 & 3.784009 & $-\odot .087278$ \\
\hline 27 & 6 & 0 & -8.036396 & 2.401787 & -0.632806 \\
\hline 28 & 6 & 0 & -7.475360 & 1.393384 & ๑.381196 \\
\hline 29 & 6 & $\Theta$ & -5.969336 & 1.565636 & $\odot .233532$ \\
\hline 30 & 6 & 0 & -5.834092 & 1.749235 & -1.274701 \\
\hline 31 & 8 & 0 & $-7.0104 \odot 7$ & 2.498106 & -1.681060 \\
\hline 32 & 1 & 0 & -8.632377 & 4.431003 & $-\odot .917226$ \\
\hline 33 & 1 & 0 & -9.157440 & 3.719241 & 0.628209 \\
\hline 34 & 1 & $\odot$ & -8.944501 & 2.000652 & -1.088603 \\
\hline 35 & 1 & $\Theta$ & -7.842012 & 1.544802 & 1.397343 \\
\hline 36 & 1 & 0 & -5.386753 & $\odot .722377$ & 0.595815 \\
\hline 37 & 1 & 0 & -5.669686 & 2.477021 & $\odot .755141$ \\
\hline 38 & 1 & $\odot$ & -5.809650 & 0.795582 & -1.796553 \\
\hline 39 & 8 & 0 & -7.138883 & 4.303588 & 0.553284 \\
\hline 40 & 1 & 0 & -7.289263 & 5.204510 & 0.882948 \\
\hline 41 & 6 & 0 & -3.589891 & 1.747633 & -2.253013 \\
\hline 42 & 7 & 0 & -1.054703 & 4.200689 & -2.396886 \\
\hline 43 & 8 & 0 & -3.750935 & $\odot .545926$ & -2.518271 \\
\hline 44 & 1 & 0 & -5.352341 & 4.249080 & -0.880626 \\
\hline 45 & 1 & 0 & -3.198829 & 5.482989 & -1.348885 \\
\hline 46 & 1 & 0 & $-\odot .855899$ & 5.150894 & -2.131915 \\
\hline 47 & 1 & 0 & -0.299140 & 3.606826 & -2.746533 \\
\hline 48 & 6 & 0 & -5.425337 & -2.187493 & -1.177494 \\
\hline 49 & 1 & 0 & -4.751453 & -6.200074 & $\odot .703486$ \\
\hline 50 & 6 & 0 & -4.574417 & -3.664475 & 1.619979 \\
\hline 51 & 6 & 0 & -3.268255 & -2.939311 & 1.263683 \\
\hline 52 & 8 & 0 & -3.262701 & -2.798888 & -0.187924 \\
\hline 53 & 1 & 0 & -4.894161 & -1.475780 & -1.812378 \\
\hline 54 & 1 & 0 & -6.291529 & -2.591500 & -1.709495 \\
\hline 55 & 1 & 0 & -4.214768 & -3.885645 & -1.664774 \\
\hline 56 & 1 & 0 & -6.153790 & -4.316600 & $\odot .246259$ \\
\hline 57 & 1 & $\odot$ & -4.406027 & -4.438907 & 2.370977 \\
\hline 58 & 1 & $\odot$ & -5.326273 & -2.974705 & 2.002613 \\
\hline 59 & 1 & $\odot$ & -2.381175 & -3.520369 & 1.516978 \\
\hline 60 & 7 & $\odot$ & -3.105991 & -1.642067 & 1.890237 \\
\hline 61 & 6 & 0 & -4.059892 & -0.752293 & 2.356850 \\
\hline 62 & 7 & $\odot$ & -3.545482 & $\odot .371471$ & 2.776731 \\
\hline 63 & 6 & $\odot$ & -2.185317 & $\odot .23004 \odot$ & 2.571800 \\
\hline 64 & 6 & 0 & -1.894501 & -1.007206 & 2.017978 \\
\hline 65 & 7 & $\odot$ & $-\odot .693968$ & -1.518792 & 1.673638 \\
\hline 66 & 6 & $\odot$ & 0.296595 & -0.680388 & 1.914641 \\
\hline 67 & 7 & 0 & 0.115856 & $\odot .562965$ & 2.469396 \\
\hline 68 & 6 & $\odot$ & -1.107485 & 1.132060 & 2.816244 \\
\hline 69 & 8 & $\odot$ & -1.157199 & 2.287102 & 3.260725 \\
\hline 70 & 1 & 0 & -5.118665 & -0.981609 & 2.351720 \\
\hline 71 & 7 & $\odot$ & 1.570490 & $-\odot .984289$ & 1.587589 \\
\hline 72 & 1 & $\odot$ & $\odot .943335$ & 1.179753 & 2.584100 \\
\hline 73 & 1 & 0 & 7.090630 & 5.198787 & -0.906530 \\
\hline 74 & 6 & $\odot$ & 7.342099 & 1.395494 & $-\odot .408048$ \\
\hline 75 & 6 & 0 & 5.840714 & 1.532608 & -0.194342 \\
\hline 76 & 6 & $\odot$ & 5.761264 & 1.722077 & 1.316777 \\
\hline 77 & 8 & $\odot$ & 6.942246 & 2.489746 & 1.674354 \\
\hline 78 & 1 & $\odot$ & 8.498759 & 4.448386 & $\odot .852657$ \\
\hline 79 & 1 & 0 & 8.989282 & 3.742958 & -0.707045 \\
\hline 80 & 1 & $\odot$ & 8.857586 & 2.019550 & 1.005422 \\
\hline 81 & 1 & 0 & 7.659950 & 1.561515 & -1.438364 \\
\hline 82 & 1 & $\odot$ & 5.255890 & $\odot .679408$ & -0.527098 \\
\hline 83 & 1 & $\odot$ & 5.496400 & 2.433264 & $-\odot .706590$ \\
\hline 84 & 1 & $\odot$ & 5.768054 & 0.773236 & 1.848095 \\
\hline 85 & 7 & $\odot$ & 4.565711 & 2.434923 & 1.745174 \\
\hline 86 & 6 & $\odot$ & 4.389128 & 3.737846 & 1.385723 \\
\hline 87 & 6 & 0 & 3.218258 & 4.370901 & 1.625455 \\
\hline 88 & 6 & $\odot$ & 2.170734 & 3.595041 & 2.219299 \\
\hline 89 & 7 & $\odot$ & 2.361915 & 2.323054 & 2.581352 \\
\hline 90 & 6 & $\odot$ & 3.541034 & 1.703645 & 2.349683 \\
\hline 91 & 1 & $\odot$ & 3.072080 & 5.405847 & 1.348411 \\
\hline 92 & 7 & $\odot$ & $\odot .961692$ & 4.115417 & 2.437807 \\
\hline 93 & 8 & 0 & 3.728081 & $\odot .513050$ & 2.648821 \\
\hline 94 & 1 & $\odot$ & 5.243156 & 4.199894 & $\odot .895568$ \\
\hline 95 & 1 & $\odot$ & $\odot .748269$ & 5.051140 & 2.135299 \\
\hline
\end{tabular}




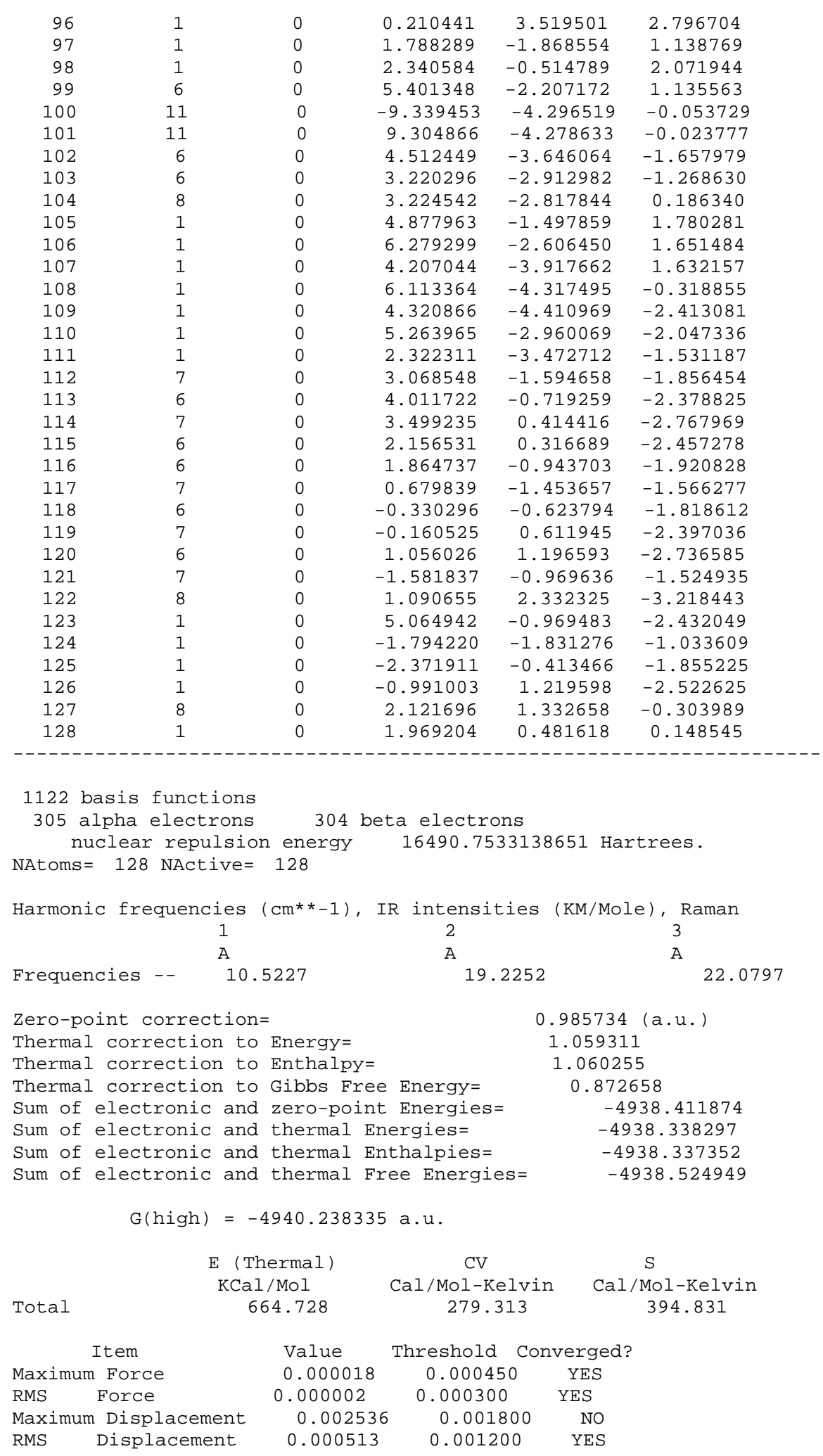

Normal termination of Gaussian 16 at Mon Feb 25 05:25:37 2019.

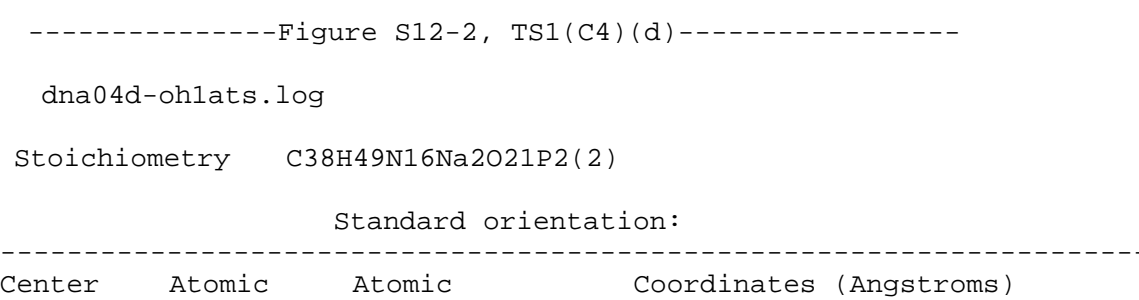




\begin{tabular}{|c|c|c|c|c|c|}
\hline Number & Number & Type & $x$ & $Y$ & Z \\
\hline & & & & & -- \\
\hline 1 & 7 & $\odot$ & 4.579133 & -2.638187 & -1.669905 \\
\hline 2 & 6 & $\odot$ & 4.465386 & -3.925188 & -1.233073 \\
\hline 3 & 6 & $\odot$ & 3.311697 & -4.614331 & -1.398054 \\
\hline 4 & 6 & $\odot$ & 2.217383 & -3.914050 & -2.002957 \\
\hline 5 & 7 & $\odot$ & 2.346708 & -2.657287 & -2.435928 \\
\hline 6 & 8 & $\odot$ & 7.823751 & $-\odot .035579$ & -0.399000 \\
\hline 7 & 15 & $\odot$ & 7.295099 & 1.410177 & ๑. 139171 \\
\hline 8 & 8 & $\odot$ & 5.742539 & 1.457587 & -0.352146 \\
\hline 9 & 8 & $\odot$ & 8.095800 & 2.479929 & -0.569784 \\
\hline 10 & 8 & $\odot$ & 7.254905 & 1.433284 & 1.650789 \\
\hline 11 & 8 & $\odot$ & 4.330509 & 5.548899 & -0.660164 \\
\hline 12 & 6 & $\odot$ & 4.324712 & 3.235462 & -1.208256 \\
\hline 13 & 6 & $\odot$ & 4.957836 & 4.292714 & -0.301208 \\
\hline 14 & 6 & $\odot$ & -7.620262 & -4.081954 & -0.036031 \\
\hline 15 & 6 & $\odot$ & -7.583229 & -2.662887 & $\odot .490401$ \\
\hline 16 & 8 & $\odot$ & -6.299657 & -4.446299 & -0.504607 \\
\hline 17 & 8 & $\odot$ & -7.709496 & -0.358167 & -0.134242 \\
\hline 18 & 15 & $\odot$ & -7.396765 & 1.061631 & -0.881603 \\
\hline 19 & 8 & $\odot$ & -5.923108 & 1.463655 & -0.311055 \\
\hline 20 & 8 & $\odot$ & -8.419843 & 2.033748 & -0.343056 \\
\hline 21 & 8 & $\odot$ & -7.201738 & 0.853825 & -2.360102 \\
\hline 22 & 8 & $\odot$ & -4.280337 & 5.409554 & -0.108699 \\
\hline 23 & 6 & $\odot$ & -4.587077 & 3.184653 & $\odot .671843$ \\
\hline 24 & 6 & $\odot$ & -4.967249 & 4.175351 & -0.428878 \\
\hline 25 & 1 & $\odot$ & -4.446745 & 6.078989 & -0.794024 \\
\hline 26 & 6 & $\odot$ & 8.343168 & -3.722570 & -0.070665 \\
\hline 27 & 6 & $\odot$ & $8.0 \odot 4224$ & -2.400331 & -0.727357 \\
\hline 28 & 6 & $\odot$ & 7.441783 & -1.320376 & ๑. 208752 \\
\hline 29 & 6 & $\odot$ & 5.937419 & -1.541871 & 0.118234 \\
\hline 30 & 6 & $\odot$ & 5.771535 & -1.857742 & -1.365903 \\
\hline 31 & 8 & $\odot$ & 6.956331 & -2.611863 & -1.735647 \\
\hline 32 & 1 & $\odot$ & 8.642904 & -4.432002 & -0.849141 \\
\hline 33 & 1 & $\odot$ & 9.185359 & -3.578040 & ๑. 616084 \\
\hline 34 & 1 & $\odot$ & 8.891114 & -2.020534 & -1.239470 \\
\hline 35 & 1 & $\odot$ & 7.839517 & -1.369204 & 1.222957 \\
\hline 36 & 1 & $\odot$ & 5.339665 & -0.686140 & 0.424198 \\
\hline 37 & 1 & $\odot$ & 5.679473 & -2.413807 & 0.723279 \\
\hline 38 & 1 & $\odot$ & 5.712387 & -0.953026 & -1.966427 \\
\hline 39 & 8 & $\odot$ & 7.179992 & -4.209968 & 0.640863 \\
\hline 40 & 1 & $\odot$ & 7.355844 & -5.079213 & 1.036309 \\
\hline 41 & 6 & $\odot$ & 3.512301 & -1.988335 & -2.295788 \\
\hline 42 & 7 & $\odot$ & 1.019663 & -4.483673 & -2.156468 \\
\hline 43 & 8 & $\odot$ & 3.645327 & -0.814989 & -2.677013 \\
\hline 44 & 1 & $\odot$ & 5.352298 & -4.325168 & -0.746796 \\
\hline 45 & 1 & $\odot$ & 3.214741 & -5.635853 & -1.057331 \\
\hline 46 & 1 & $\odot$ & ๑. 839551 & -5.409238 & -1.805217 \\
\hline 47 & 1 & $\odot$ & ๑. 249581 & -3.933741 & -2.537273 \\
\hline 48 & 6 & $\odot$ & 5.228947 & 2.070588 & -1.564500 \\
\hline 49 & 1 & $\odot$ & 4.667042 & 6.268894 & $-0.100 \odot 46$ \\
\hline 50 & 6 & $\odot$ & 4.578010 & 3.851851 & 1.117835 \\
\hline 51 & 6 & $\odot$ & 3.238737 & 3.120729 & $\odot .946663$ \\
\hline 52 & 8 & $\odot$ & 3.147660 & 2.773028 & -0.469506 \\
\hline 53 & 1 & $\odot$ & 4.665127 & 1. 301402 & -2.095347 \\
\hline 54 & 1 & $\odot$ & 6.055359 & 2.423423 & -2.187868 \\
\hline 55 & 1 & $\odot$ & 3.964422 & 3.701701 & -2.128018 \\
\hline 56 & 1 & $\odot$ & 6.042660 & 4.346162 & -0.438645 \\
\hline 57 & 1 & $\odot$ & 4.482568 & 4.703418 & 1.794579 \\
\hline 58 & 1 & $\odot$ & 5.348716 & 3.187695 & 1.510535 \\
\hline 59 & 1 & $\odot$ & 2.380979 & 3.756853 & 1.166532 \\
\hline 60 & 7 & $\odot$ & 3.093424 & 1.932193 & 1.758929 \\
\hline 61 & 6 & $\odot$ & 4.062967 & 1.034390 & 2.178710 \\
\hline 62 & 7 & $\odot$ & 3.565277 & -0.003937 & 2.791114 \\
\hline 63 & 6 & $\odot$ & 2.197561 & ๑. 203885 & 2.770783 \\
\hline 64 & 6 & $\odot$ & 1.884456 & 1.393646 & 2.131703 \\
\hline 65 & 7 & $\odot$ & 0.672068 & 1.942576 & 1.922052 \\
\hline 66 & 6 & $\odot$ & -0.314467 & 1.191577 & 2.385189 \\
\hline 67 & 7 & $\odot$ & -0.111337 & -0.020937 & 3. 000952 \\
\hline 68 & 6 & $\odot$ & 1.129548 & -0.611219 & 3.243404 \\
\hline 69 & 8 & $\odot$ & 1.203759 & -1.718259 & 3.794202 \\
\hline 70 & 1 & $\odot$ & 5.118049 & 1. 203604 & 2.004365 \\
\hline 71 & 7 & $\odot$ & -1.590545 & 1.620790 & 2.321048 \\
\hline 72 & 1 & $\odot$ & -0.929364 & -0.629823 & 3.179125 \\
\hline 73 & 1 & $\odot$ & -6.286672 & -5.362854 & -0.825050 \\
\hline 74 & 6 & $\odot$ & -7.026621 & -1.610412 & -0.479688 \\
\hline 75 & 6 & $\odot$ & -5.549798 & -1.547715 & -0.113568 \\
\hline
\end{tabular}




\begin{tabular}{|c|c|c|c|c|c|}
\hline 76 & 6 & $\odot$ & -5.593529 & -1.705960 & 1.402885 \\
\hline 77 & 8 & $\odot$ & -6.707780 & -2.598962 & 1.668865 \\
\hline 78 & 1 & $\odot$ & -7.939415 & -4.748716 & 0.772051 \\
\hline 79 & 1 & $\odot$ & -8.351109 & -4.142527 & -0.850974 \\
\hline 80 & 1 & $\odot$ & -8.589080 & -2.376347 & 0.805881 \\
\hline 81 & 1 & $\odot$ & -7.211236 & -1.843682 & -1.529310 \\
\hline 82 & 1 & $\odot$ & -5.046295 & -0.632276 & -0.412630 \\
\hline 83 & 1 & 0 & -5.040642 & -2.402853 & -0.562996 \\
\hline 84 & 1 & $\odot$ & -5.758890 & -0.760457 & 1.913694 \\
\hline 85 & 7 & $\odot$ & -4.372636 & -2.278408 & 1.956690 \\
\hline 86 & 6 & $\odot$ & -4.076316 & -3.586485 & 1.717620 \\
\hline 87 & 6 & $\odot$ & -2.900836 & -4.112951 & 2.127835 \\
\hline 88 & 6 & $\odot$ & -1.984158 & -3.227115 & 2.781591 \\
\hline 89 & 7 & 0 & -2.283479 & -1.944024 & 3.005754 \\
\hline $9 \odot$ & 6 & $\odot$ & -3.460011 & -1.425500 & 2.584322 \\
\hline 91 & 1 & $\odot$ & -2.654597 & -5.149959 & 1.945085 \\
\hline 92 & 7 & $\odot$ & -0.792123 & -3.663964 & 3.195369 \\
\hline 93 & 8 & $\odot$ & -3.751366 & -0.229359 & 2.741400 \\
\hline 94 & 1 & $\odot$ & -4.837683 & -4.139471 & 1.172955 \\
\hline 95 & 1 & $\odot$ & -0.496874 & -4.599625 & 2.971105 \\
\hline 96 & 1 & $\odot$ & -0.095836 & -2.989163 & 3.526398 \\
\hline 97 & 1 & 0 & -1.823717 & 2.259988 & 1.568432 \\
\hline 98 & 1 & $\odot$ & -2.333239 & $\odot .949405$ & 2.528749 \\
\hline 99 & 6 & $\odot$ & -5.623580 & 2.116761 & $\odot .946148$ \\
\hline 100 & 11 & 0 & 8.673383 & 3.819823 & 1.301934 \\
\hline 101 & 11 & $\odot$ & -9.216966 & 4.072624 & -0.512163 \\
\hline 102 & 6 & $\odot$ & -4.429498 & 3.539394 & -1.718697 \\
\hline 103 & 6 & 0 & -3.219632 & 2.717739 & -1.259286 \\
\hline 104 & 8 & 0 & -3.348941 & 2.562076 & 0.191703 \\
\hline 105 & 1 & 0 & -5.208795 & 1.376280 & 1.634869 \\
\hline 106 & 1 & 0 & -6.530310 & 2.560969 & 1.367806 \\
\hline 107 & 1 & 0 & -4.342759 & 3.713539 & 1.594937 \\
\hline 108 & 1 & 0 & -6.049019 & 4.334991 & -0.475406 \\
\hline 109 & 1 & 0 & -4.142315 & 4.294674 & -2.452715 \\
\hline 110 & 1 & 0 & -5.200848 & 2.905243 & -2.153287 \\
\hline 111 & 1 & 0 & -2.271965 & 3.231449 & -1.421740 \\
\hline 112 & 7 & $\odot$ & -3.090140 & 1.415646 & -1.877589 \\
\hline 113 & 6 & 0 & -4.008869 & $\odot .607419$ & -2.496509 \\
\hline 114 & 7 & 0 & -3.512334 & $-\odot .554239$ & -2.880531 \\
\hline 115 & 6 & 0 & -2.218165 & -0.527556 & -2.467142 \\
\hline 116 & 6 & 0 & -1.921541 & $\odot .669528$ & -1.759558 \\
\hline 117 & 7 & 0 & -0.703943 & 1.225065 & -1.567473 \\
\hline 118 & 6 & $\odot$ & $\odot .275156$ & $\odot .369459$ & -1.781192 \\
\hline 119 & 7 & 0 & 0.074107 & -0.919525 & -2.249331 \\
\hline 120 & 6 & 0 & -1.137811 & -1.467942 & -2.637976 \\
\hline 121 & 7 & 0 & 1.546513 & $\odot .699491$ & -1.537777 \\
\hline 122 & 8 & $\odot$ & -1.205741 & -2.622267 & -3.068758 \\
\hline 123 & 1 & 0 & -5.048184 & 0.888812 & -2.626756 \\
\hline 124 & 1 & $\odot$ & 1.785606 & 1.610027 & -1.154829 \\
\hline 125 & 1 & 0 & 2.308577 & 0.153154 & -1.940734 \\
\hline 126 & 1 & 0 & 0.898516 & -1.534223 & -2.376787 \\
\hline 127 & 8 & 0 & -2.292005 & -0.323046 & -0.056241 \\
\hline 128 & 1 & 0 & -2.848108 & $\odot .391652$ & 0.291345 \\
\hline
\end{tabular}

1122 basis functions

305 alpha electrons

304 beta electrons

nuclear repulsion energy 16508.7816314739 Hartrees.

NAtoms $=128$ NActive $=128$

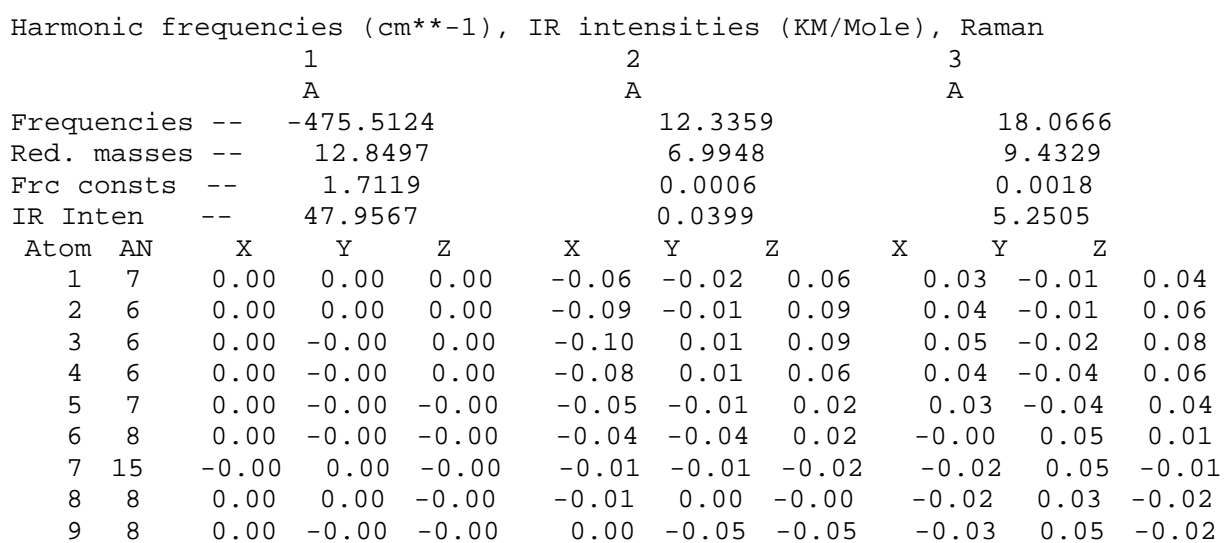




\begin{tabular}{|c|c|c|c|c|c|c|c|c|c|c|}
\hline 10 & 8 & $\odot . \odot \odot$ & $\odot . \odot \odot$ & $-\odot . \odot \odot$ & 0.01 & 0.03 & $-\odot .02$ & -0.02 & $\odot .06$ & -0.01 \\
\hline 11 & 8 & -0.00 & -0.00 & -0.00 & -0.00 & -0.01 & -0.07 & -0.07 & $\odot .01$ & -0.02 \\
\hline 12 & 6 & $-\odot .0 \odot$ & $-\odot . \odot \odot$ & $-0.0 \odot$ & -0.01 & -0.02 & -0.03 & -0.04 & 0.01 & -0.02 \\
\hline 13 & 6 & $-\odot . \odot \odot$ & $-\odot . \odot \odot$ & $-\odot . \odot \odot$ & $-\odot . \odot \odot$ & 0.00 & -0.05 & -0.05 & 0.01 & -0.02 \\
\hline 14 & 6 & $\odot .0 \odot$ & $\odot .00$ & -0.00 & 0.10 & -0.03 & -0.10 & $-\odot . \odot 9$ & 0.05 & -0.01 \\
\hline 5 & 6 & $\odot . \odot \odot$ & $\odot . \odot \odot$ & $-\odot . \odot \odot$ & 0.07 & -0.04 & -0.06 & -0.05 & 0.05 & $\odot . \odot \odot$ \\
\hline 16 & 8 & 0.00 & 0.00 & 0.00 & 0.10 & 0.01 & -0.11 & -0.11 & 0.03 & -0.04 \\
\hline 17 & 8 & $\odot . \odot \odot$ & 0.00 & -0.00 & 0.03 & -0.03 & $-0.0 \odot$ & -0.02 & $\odot .05$ & 0.02 \\
\hline 18 & 15 & $\odot . \odot \odot$ & $\odot .0 \odot$ & $-\odot . \odot \odot$ & $-\odot . \odot \odot$ & -0.01 & $\odot .02$ & 0.02 & 0.06 & 0.07 \\
\hline 19 & 8 & $\odot . \odot \odot$ & $-0.0 \odot$ & $-\odot . \odot \odot$ & $\odot . \odot \odot$ & 0.01 & 0.01 & 0.04 & $-0.0 \odot$ & 0.07 \\
\hline 20 & 8 & -0.00 & $-\odot . \odot \odot$ & $-\odot .0 \odot$ & -0.01 & -0.04 & 0.06 & 0.06 & 0.08 & 0.11 \\
\hline 21 & 8 & 0.01 & -0.00 & -0.00 & -0.02 & 0.02 & 0.02 & 0.00 & 0.10 & $\odot .06$ \\
\hline 22 & 8 & $-\odot .0 \odot$ & $\odot .00$ & $-\odot .0 \odot$ & -0.01 & $\odot .00$ & 0.09 & 0.08 & 0.00 & -0.13 \\
\hline 23 & 6 & -0.01 & 0.01 & $\odot . \odot \odot$ & $\odot .0 \odot$ & -0.02 & $\odot .04$ & 0.07 & 0.04 & -0.04 \\
\hline 24 & 6 & -0.00 & $\odot .0 \odot$ & -0.00 & -0.01 & 0.01 & 0.07 & 0.05 & -0.00 & -0.07 \\
\hline 25 & 1 & $\odot . \odot \odot$ & $-\odot . \odot \odot$ & $-\odot . \odot \odot$ & -0.02 & 0.02 & 0.11 & 0.08 & -0.03 & -0.16 \\
\hline 26 & 6 & $\odot .0 \odot$ & 0.00 & $\odot . \odot \odot$ & -0.10 & -0.03 & 0.14 & $\odot .04$ & 0.06 & $\odot .08$ \\
\hline 27 & 6 & $\odot .0 \odot$ & -0.00 & $\odot .0 \odot$ & -0.07 & -0.05 & 0.10 & 0.03 & 0.04 & 0.05 \\
\hline 28 & 6 & $\odot .0 \odot$ & $\odot . \odot \odot$ & $\odot .00$ & -0.07 & -0.01 & 0.06 & 0.01 & 0.05 & 0.03 \\
\hline 29 & 6 & 0.00 & 0.00 & 0.00 & -0.07 & 0.01 & 0.04 & 0.01 & 0.03 & 0.02 \\
\hline 30 & 6 & $\odot .0 \odot$ & 0.00 & 0.00 & -0.05 & -0.03 & 0.05 & 0.02 & 0.00 & 0.03 \\
\hline 31 & 8 & $\odot .0 \odot$ & $-\odot . \odot \odot$ & 0.00 & -0.06 & -0.06 & 0.09 & 0.03 & 0.01 & 0.05 \\
\hline 32 & 1 & $\odot .0 \odot$ & -0.00 & 0.00 & -0.10 & -0.06 & 0.16 & 0.05 & 0.05 & 0.09 \\
\hline 33 & 1 & $\odot .0 \odot$ & $\odot .0 \odot$ & $\odot .0 \odot$ & -0.11 & -0.03 & 0.15 & 0.04 & 0.08 & 0.08 \\
\hline 34 & 1 & $\odot . \odot \odot$ & $-\odot .0 \odot$ & $\odot . \odot \odot$ & -0.06 & -0.08 & 0.10 & 0.02 & 0.04 & 0.05 \\
\hline 35 & 1 & 0.00 & 0.00 & 0.00 & -0.08 & 0.01 & 0.06 & 0.00 & 0.08 & 0.03 \\
\hline 36 & 1 & $\odot .0 \odot$ & $\odot . \odot \odot$ & $\odot .0 \odot$ & -0.06 & $\odot .02$ & 0.01 & $-0.0 \odot$ & 0.03 & 0.00 \\
\hline 37 & 1 & $\odot .0 \odot$ & 0.00 & 0.00 & -0.09 & 0.03 & 0.07 & $\odot .02$ & $\odot .04$ & $\odot .04$ \\
\hline 38 & 1 & $\odot .0 \odot$ & $\odot .00$ & $\odot .00$ & -0.03 & -0.05 & 0.03 & 0.02 & -0.01 & $\odot .01$ \\
\hline 39 & 8 & $\odot . \odot \odot$ & 0.00 & 0.00 & -0.12 & 0.01 & 0.14 & 0.04 & 0.06 & 0.08 \\
\hline 40 & 1 & $\odot .0 \odot$ & 0.00 & $\odot .00$ & -0.14 & 0.01 & 0.17 & 0.05 & 0.07 & 0.10 \\
\hline 41 & 6 & $\odot .0 \odot$ & $-\odot . \odot \odot$ & $-\odot . \odot \odot$ & -0.04 & -0.02 & 0.03 & $\odot .03$ & -0.03 & 0.02 \\
\hline 42 & 7 & $\odot .00$ & -0.00 & $\odot . \odot \odot$ & -0.08 & 0.02 & 0.05 & 0.04 & -0.05 & $\odot .07$ \\
\hline 43 & 8 & $\odot .0 \odot$ & $-0.0 \odot$ & $-\odot . \odot \odot$ & -0.02 & -0.03 & $-\odot . \odot \odot$ & 0.02 & -0.04 & $\odot . \odot \odot$ \\
\hline 44 & 1 & $\odot . \odot \odot$ & $\odot .0 \odot$ & $\odot .0 \odot$ & -0.10 & -0.01 & 0.11 & $\odot .04$ & 0.01 & 0.07 \\
\hline 45 & 1 & $\odot .0 \odot$ & $-\odot . \odot \odot$ & $\odot .0 \odot$ & -0.12 & 0.02 & 0.12 & 0.05 & -0.01 & 0.10 \\
\hline 46 & 1 & $\odot .0 \odot$ & $-\odot . \odot \odot$ & $-\odot . \odot \odot$ & -0.10 & 0.03 & 0.08 & 0.05 & -0.04 & $\odot .09$ \\
\hline 47 & 1 & $\odot .01$ & -0.01 & -0.01 & -0.07 & $\odot .02$ & 0.03 & 0.04 & -0.06 & $\odot .06$ \\
\hline 48 & 6 & -0.00 & $-0.0 \odot$ & -0.00 & -0.01 & -0.02 & -0.01 & -0.03 & 0.02 & -0.02 \\
\hline 49 & 1 & $-\odot . \odot \odot$ & $-\odot . \odot \odot$ & $-\odot . \odot \odot$ & $0.0 \odot$ & 0.00 & $-\odot .09$ & $-\odot .08$ & 0.01 & -0.01 \\
\hline 50 & 6 & -0.00 & -0.00 & -0.00 & $\odot .00$ & 0.03 & -0.04 & -0.05 & 0.01 & -0.02 \\
\hline 51 & 6 & -0.00 & $-\odot . \odot \odot$ & -0.00 & $\odot . \odot \odot$ & 0.02 & -0.02 & -0.04 & $-\odot .0 \odot$ & -0.02 \\
\hline 52 & 8 & $-0.0 \odot$ & $-0.0 \odot$ & -0.00 & -0.01 & $-0.0 \odot$ & -0.02 & -0.04 & -0.00 & -0.02 \\
\hline 53 & 1 & 0.00 & -0.00 & $\odot . \odot \odot$ & -0.02 & -0.03 & 0.00 & -0.02 & 0.01 & -0.01 \\
\hline 54 & 1 & $-\odot . \odot \odot$ & $-\odot . \odot \odot$ & $-\odot . \odot \odot$ & $-\odot . \odot 2$ & -0.04 & -0.02 & $-\odot .03$ & $\odot .03$ & -0.02 \\
\hline 55 & 1 & -0.00 & -0.00 & -0.00 & -0.02 & -0.03 & -0.04 & -0.05 & 0.00 & -0.02 \\
\hline 56 & 1 & -0.00 & $-0.0 \odot$ & -0.00 & -0.01 & $-0.0 \odot$ & -0.06 & -0.05 & 0.03 & -0.02 \\
\hline 57 & 1 & -0.00 & $-0 . \odot \odot$ & -0.00 & 0.01 & 0.04 & -0.06 & -0.05 & 0.01 & -0.02 \\
\hline 58 & 1 & $-\odot . \odot \odot$ & $\odot . \odot \odot$ & $-\odot . \odot \odot$ & $\odot .01$ & $\odot .04$ & -0.04 & $-\odot .04$ & 0.02 & -0.02 \\
\hline 59 & 1 & $-\odot . \odot \odot$ & $-\odot . \odot \odot$ & $-\odot . \odot \odot$ & $\odot .0 \odot$ & 0.02 & -0.02 & -0.04 & -0.01 & -0.01 \\
\hline 60 & 7 & $\odot . \odot \odot$ & 0.00 & 0.00 & 0.01 & 0.03 & $-0.0 \odot$ & -0.03 & -0.01 & -0.02 \\
\hline 61 & 6 & 0.00 & -0.00 & 0.00 & 0.02 & 0.05 & 0.01 & -0.02 & $-0.0 \odot$ & -0.03 \\
\hline 62 & 7 & $\odot .0 \odot$ & $-\odot . \odot \odot$ & $-\odot . \odot \odot$ & 0.03 & 0.05 & 0.02 & -0.01 & -0.02 & -0.04 \\
\hline 63 & 6 & 0.00 & -0.00 & $-\odot . \odot \odot$ & 0.03 & 0.04 & 0.02 & -0.01 & -0.03 & -0.04 \\
\hline 64 & 6 & $-0.0 \odot$ & $0.0 \odot$ & $\odot .0 \odot$ & 0.02 & 0.03 & 0.00 & -0.02 & -0.02 & -0.03 \\
\hline 65 & 7 & $\odot .0 \odot$ & $\odot .0 \odot$ & 0.00 & 0.01 & 0.01 & -0.01 & -0.02 & -0.02 & -0.02 \\
\hline 66 & 6 & $-0.0 \odot$ & 0.00 & 0.00 & 0.02 & 0.01 & $-0.0 \odot$ & -0.02 & -0.03 & -0.03 \\
\hline 67 & 7 & $\odot . \odot \odot$ & $\odot .0 \odot$ & $\odot .0 \odot$ & 0.03 & 0.01 & 0.01 & -0.01 & -0.04 & -0.05 \\
\hline 68 & 6 & 0.00 & $0.0 \odot$ & $-\odot . \odot \odot$ & 0.03 & 0.03 & $\odot .02$ & -0.01 & -0.04 & -0.06 \\
\hline 69 & 8 & $-0.0 \odot$ & $-\odot . \odot \odot$ & $\odot .0 \odot$ & 0.04 & 0.03 & $\odot .02$ & -0.01 & -0.05 & -0.08 \\
\hline 70 & 1 & $\odot . \odot \odot$ & $-\odot . \odot \odot$ & $-\odot . \odot \odot$ & 0.02 & 0.05 & $\odot .02$ & $-\odot .02$ & 0.01 & -0.02 \\
\hline 71 & 7 & $\odot .0 \odot$ & 0.01 & 0.01 & 0.01 & -0.01 & -0.01 & -0.02 & -0.04 & -0.02 \\
\hline 72 & 1 & $\odot .0 \odot$ & 0.00 & 0.01 & 0.04 & $0.0 \odot$ & 0.00 & -0.01 & -0.05 & -0.06 \\
\hline 73 & 1 & $\odot .0 \odot$ & 0.00 & 0.00 & 0.12 & 0.02 & -0.14 & -0.13 & 0.03 & -0.04 \\
\hline 74 & $\overline{6}$ & $\odot .0 \odot$ & 0.00 & $-0.0 \odot$ & 0.05 & -0.01 & -0.03 & -0.05 & 0.04 & $-\odot . \odot \odot$ \\
\hline 75 & 6 & $\odot .0 \odot$ & $\odot .0 \odot$ & $-0.0 \odot$ & 0.05 & 0.01 & -0.04 & -0.04 & 0.01 & -0.04 \\
\hline 76 & 6 & $\odot .0 \odot$ & 0.00 & 0.00 & 0.06 & -0.03 & -0.04 & -0.02 & 0.01 & -0.04 \\
\hline 77 & 8 & $\odot .0 \odot$ & 0.00 & -0.00 & 0.07 & -0.06 & -0.06 & -0.03 & 0.02 & -0.02 \\
\hline 78 & 1 & $\odot .0 \odot$ & 0.00 & 0.00 & 0.11 & -0.06 & -0.12 & -0.08 & 0.05 & -0.00 \\
\hline 79 & 1 & $\odot .0 \odot$ & 0.00 & -0.00 & 0.10 & -0.02 & -0.10 & -0.11 & 0.07 & 0.01 \\
\hline 80 & 1 & 0.00 & 0.00 & -0.00 & 0.07 & -0.07 & -0.05 & -0.04 & 0.06 & 0.02 \\
\hline 81 & 1 & 0.00 & 0.00 & -0.00 & 0.05 & 0.02 & -0.04 & -0.08 & 0.05 & -0.00 \\
\hline 82 & 1 & 0.02 & $0.0 \odot$ & 0.01 & 0.04 & $\odot .02$ & -0.01 & -0.03 & -0.00 & -0.04 \\
\hline 83 & 1 & -0.00 & -0.00 & 0.00 & 0.07 & 0.03 & -0.06 & -0.07 & $-0.0 \odot$ & -0.05 \\
\hline 84 & 1 & $\odot . \odot \odot$ & 0.00 & $-\odot . \odot \odot$ & 0.04 & -0.05 & -0.01 & 0.01 & 0.01 & -0.03 \\
\hline 85 & 7 & $0.0 \odot$ & 0.00 & -0.00 & 0.07 & -0.02 & -0.06 & -0.02 & -0.01 & -0.06 \\
\hline 86 & 6 & $\odot . \odot \odot$ & $-\odot . \odot \odot$ & 0.00 & 0.10 & -0.01 & -0.09 & -0.02 & -0.01 & -0.10 \\
\hline
\end{tabular}




\begin{tabular}{|c|c|c|c|c|c|c|c|c|c|c|}
\hline 87 & 6 & $-\odot . \odot \odot$ & $-\odot .0 \odot$ & $\odot . \odot \odot$ & 0.11 & -0.00 & -0.11 & -0.01 & -0.02 & -0.14 \\
\hline 88 & 6 & $\odot .0 \odot$ & $-0.0 \odot$ & 0.00 & $\odot .08$ & $-0.0 \odot$ & $-\odot .08$ & -0.01 & -0.04 & -0.12 \\
\hline 89 & 7 & $-0 . \odot \odot$ & -0.00 & $-\odot . \odot \odot$ & 0.06 & -0.02 & -0.04 & -0.01 & -0.05 & -0.07 \\
\hline 90 & 6 & $\odot .00$ & $\odot .00$ & 0.00 & 0.05 & -0.02 & -0.03 & -0.01 & -0.03 & -0.04 \\
\hline 91 & 1 & $-\odot . \odot \odot$ & -0.00 & $\odot .00$ & 0.13 & 0.01 & -0.14 & -0.02 & -0.01 & -0.17 \\
\hline 92 & 7 & $-\odot . \odot \odot$ & 0.00 & $\odot .00$ & $\odot .09$ & 0.01 & -0.08 & $\odot .0 \odot$ & -0.05 & -0.16 \\
\hline 93 & 8 & $\odot .0 \odot$ & $-\odot .0 \odot$ & $-\odot . \odot \odot$ & 0.03 & -0.03 & $-\odot . \odot \odot$ & -0.01 & -0.04 & 0.01 \\
\hline 94 & 1 & $-\odot . \odot \odot$ & -0.00 & $\odot .00$ & 0.11 & -0.01 & -0.11 & -0.03 & 0.01 & -0.11 \\
\hline 95 & 1 & $\odot . \odot \odot$ & $\odot . \odot \odot$ & $\odot . \odot \odot$ & 0.11 & $\odot . \odot 2$ & -0.10 & $\odot . \odot \odot$ & -0.04 & -0.19 \\
\hline 96 & 1 & $-\odot . \odot \odot$ & $\odot . \odot \odot$ & $-\odot .0 \odot$ & 0.07 & 0.01 & -0.05 & $\odot .00$ & -0.06 & -0.14 \\
\hline 97 & 1 & $-\odot .01$ & -0.01 & $-\odot . \odot \odot$ & 0.01 & -0.01 & -0.01 & -0.03 & -0.04 & -0.02 \\
\hline 98 & 1 & 0.01 & $-\odot . \odot \odot$ & -0.00 & 0.02 & -0.01 & $-\odot .0 \odot$ & -0.02 & -0.04 & -0.01 \\
\hline 99 & 6 & $-\odot . \odot \odot$ & $\odot .0 \odot$ & -0.00 & 0.01 & -0.02 & 0.03 & 0.07 & 0.06 & 0.03 \\
\hline 100 & 11 & -0.00 & $\odot . \odot \odot$ & -0.00 & 0.03 & $\odot . \odot \odot$ & -0.09 & -0.07 & $\odot .06$ & 0.01 \\
\hline 101 & 11 & $-\odot .0 \odot$ & $\odot . \odot \odot$ & $-\odot . \odot \odot$ & -0.01 & $-\odot .03$ & $\odot .06$ & $\odot . \odot 9$ & 0.11 & 0.30 \\
\hline 102 & 6 & $\odot .0 \odot$ & -0.00 & $\odot .00$ & -0.01 & 0.04 & 0.05 & $\odot .02$ & -0.06 & -0.05 \\
\hline 103 & 6 & -0.01 & -0.02 & $\odot .02$ & -0.01 & $\odot .03$ & $\odot .02$ & $\odot . \odot 2$ & -0.04 & $-\odot .05$ \\
\hline 104 & 8 & -0.00 & 0.02 & 0.02 & 0.00 & -0.00 & $\odot .02$ & 0.05 & 0.00 & -0.04 \\
\hline 105 & 1 & $\odot . \odot \odot$ & 0.00 & $-0.0 \odot$ & 0.01 & -0.04 & 0.01 & 0.07 & $\odot .08$ & 0.06 \\
\hline 106 & 1 & -0.01 & -0.01 & -0.00 & 0.01 & -0.04 & 0.04 & $\odot . \odot 8$ & $\odot . \odot 8$ & 0.03 \\
\hline 107 & 1 & -0.02 & 0.01 & $\odot .01$ & 0.01 & -0.03 & 0.05 & 0.10 & $\odot .07$ & -0.06 \\
\hline 108 & 1 & $-\odot . \odot \odot$ & $\odot . \odot \odot$ & -0.01 & -0.01 & $\odot . \odot \odot$ & $\odot .07$ & $\odot . \odot 6$ & 0.01 & -0.05 \\
\hline 109 & 1 & $\odot .01$ & -0.01 & $\odot . \odot \odot$ & -0.01 & $\odot .06$ & $\odot .07$ & $-\odot . \odot \odot$ & -0.09 & $-\odot .09$ \\
\hline 110 & 1 & -0.01 & $\odot .01$ & $-0.0 \odot$ & -0.01 & 0.05 & 0.04 & $-0.0 \odot$ & -0.08 & -0.00 \\
\hline 111 & 1 & $-\odot . \odot \odot$ & $-\odot . \odot 4$ & $\odot .01$ & -0.01 & $\odot .03$ & $\odot .03$ & $\odot . \odot 2$ & -0.05 & $-\odot . \odot 8$ \\
\hline 112 & 7 & -0.03 & -0.03 & 0.06 & -0.01 & 0.04 & -0.00 & $\odot .01$ & -0.06 & -0.01 \\
\hline 113 & 6 & $\odot . \odot 4$ & 0.02 & -0.01 & -0.02 & $\odot .05$ & -0.00 & $\odot .01$ & -0.06 & -0.01 \\
\hline 114 & 7 & 0.05 & -0.01 & -0.08 & -0.02 & 0.05 & -0.02 & 0.01 & -0.07 & $\odot . \odot \odot$ \\
\hline 115 & 6 & -0.03 & -0.08 & -0.04 & -0.02 & 0.04 & -0.02 & 0.01 & -0.07 & 0.01 \\
\hline 116 & 6 & -0.12 & -0.29 & 0.67 & -0.01 & 0.04 & -0.02 & 0.01 & -0.06 & -0.00 \\
\hline 117 & 7 & -0.03 & -0.04 & 0.03 & -0.01 & 0.03 & -0.02 & 0.01 & -0.06 & -0.00 \\
\hline 118 & 6 & -0.03 & 0.01 & $-0.0 \odot$ & -0.01 & $\odot .02$ & -0.03 & 0.01 & -0.06 & $\odot . \odot \odot$ \\
\hline 119 & 7 & -0.00 & -0.03 & 0.01 & -0.02 & 0.03 & -0.03 & 0.01 & -0.06 & $\odot .02$ \\
\hline 120 & 6 & 0.01 & -0.03 & -0.02 & -0.03 & 0.03 & -0.03 & 0.01 & -0.07 & $\odot . \odot 2$ \\
\hline 121 & 7 & -0.02 & $\odot . \odot \odot$ & -0.04 & -0.01 & 0.01 & -0.03 & $\odot .01$ & -0.05 & $\odot .01$ \\
\hline 122 & 8 & $\odot .0 \odot$ & -0.02 & -0.02 & -0.04 & 0.03 & -0.02 & $\odot .02$ & -0.07 & 0.03 \\
\hline 123 & 1 & 0.05 & 0.03 & -0.11 & -0.02 & 0.06 & $\odot .0 \odot$ & 0.01 & -0.06 & -0.01 \\
\hline 124 & 1 & -0.02 & -0.01 & $\odot . \odot \odot$ & $-\odot . \odot \odot$ & 0.01 & -0.02 & $\odot . \odot \odot$ & -0.05 & $-\odot .01$ \\
\hline 125 & 1 & -0.03 & $\odot .02$ & -0.07 & -0.02 & 0.00 & -0.02 & 0.01 & -0.05 & $\odot .00$ \\
\hline 126 & 1 & $\odot .0 \odot$ & -0.02 & 0.01 & -0.03 & 0.01 & -0.02 & 0.01 & -0.06 & $\odot .02$ \\
\hline 127 & 8 & 0.11 & 0.37 & -0.45 & 0.00 & $\odot .02$ & -0.02 & -0.00 & -0.05 & -0.00 \\
\hline 128 & 1 & $\odot .03$ & -0.02 & 0.18 & $\odot .0 \odot$ & $\odot .01$ & -0.01 & $-\odot . \odot \odot$ & -0.05 & -0.01 \\
\hline
\end{tabular}

Zero-point correction=

Thermal correction to Energy=

Thermal correction to Enthalpy=

Thermal correction to Gibbs Free Energy=

Sum of electronic and zero-point Energies=

Sum of electronic and thermal Energies=

Sum of electronic and thermal Enthalpies=

Sum of electronic and thermal Free Energies=

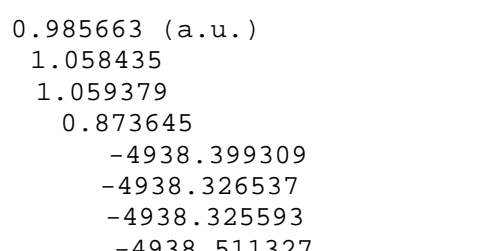

$G($ high $)=-4940.224893 \mathrm{a} \cdot \mathrm{u}$.

E (Thermal)
KCal/Mol

664.178
CV

Cal/Mol-Kelvin Cal/Mol-Kelvin $277.550 \quad 390.910$

$\begin{aligned} \text { Total } & \\ & \text { Item }\end{aligned}$

Maximum Force

RMS Force

Maximum Displacement

RMS Displacement

Value Threshold Converged?

$0.000010 \quad 0.000450$ YES

$0.000001 \quad 0.000300 \quad$ YES

$0.001576 \quad 0.001800 \quad$ YES

Normal termination of Gaussian 16 at Sun Feb 24 23:27:11 2019.

Figure 12-3, Int2(C4)(d)

dna@4d-oh1a. $\log$

Stoichiometry C38H49N16Na2021P2(2)

Standard orientation

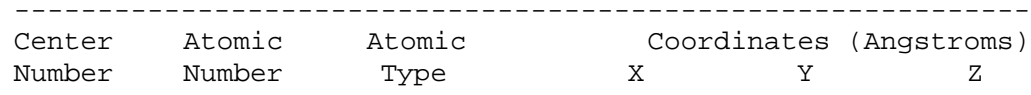




\begin{tabular}{|c|c|c|c|c|c|}
\hline 1 & 7 & 0 & 4.617003 & -2.715180 & -1.685108 \\
\hline 2 & 6 & $\odot$ & 4.535312 & $-4.0 \odot 4565$ & -1.249809 \\
\hline 3 & 6 & 0 & 3.402145 & -4.725820 & -1.423675 \\
\hline 4 & 6 & $\odot$ & 2.292422 & -4.051786 & -2.029146 \\
\hline 5 & 7 & 0 & 2.387520 & -2.790239 & -2.455048 \\
\hline 6 & 8 & $\odot$ & 7.762679 & -0.019012 & -0.361316 \\
\hline 7 & 15 & 0 & 7.251981 & 1.433539 & ๑. 192049 \\
\hline 8 & 8 & $\odot$ & 5.687059 & 1.483174 & -0.268307 \\
\hline 9 & 8 & $\odot$ & 8.028299 & 2.465789 & $-\odot .593172$ \\
\hline 10 & 8 & $\odot$ & 7.230650 & 1.436135 & 1.698034 \\
\hline 11 & 8 & 0 & 4.239683 & 5.533184 & -0.654829 \\
\hline 12 & 6 & $\odot$ & 4.238453 & 3.207885 & -1.160699 \\
\hline 13 & 6 & $\odot$ & 4.870923 & 4.284444 & -0.275911 \\
\hline 14 & 6 & $\odot$ & -7.442322 & -4.035375 & -0.276739 \\
\hline 15 & 6 & 0 & -7.497599 & -2.623615 & 0.266357 \\
\hline 16 & 8 & 0 & -6.076030 & -4.338706 & -0.647406 \\
\hline 17 & 8 & 0 & -7.673516 & -0.325750 & -0.390011 \\
\hline 18 & 15 & $\odot$ & -7.405954 & 1.066681 & -1.207506 \\
\hline 19 & 8 & $\odot$ & $-6.0 \odot 3099$ & 1.599996 & -0.568605 \\
\hline 20 & 8 & $\odot$ & -8.523418 & 1.993784 & -0.790646 \\
\hline 21 & 8 & $\odot$ & -7.099434 & 0.790330 & -2.653658 \\
\hline 22 & 8 & 0 & -4.114136 & 5.379431 & -0.109320 \\
\hline 23 & 6 & $\odot$ & -4.690156 & 3.194267 & $\odot .625633$ \\
\hline 24 & 6 & $\odot$ & -4.847016 & 4.198528 & -0.516606 \\
\hline 25 & 1 & $\odot$ & -4.138181 & 6.053650 & -0.809404 \\
\hline 26 & 6 & 0 & 8.337864 & -3.685032 & ๑. .069025 \\
\hline 27 & 6 & $\odot$ & 7.998549 & -2.384349 & -0.628721 \\
\hline 28 & 6 & 0 & 7.382374 & -1.293580 & $\odot .26000 \odot$ \\
\hline 29 & 6 & 0 & 5.885645 & -1.539526 & 0.115496 \\
\hline 30 & 6 & 0 & 5.782422 & -1.899411 & -1.364774 \\
\hline 31 & 8 & 0 & 6.993620 & -2.637970 & -1.670826 \\
\hline 32 & 1 & $\odot$ & 8.683704 & -4.404479 & -0.680728 \\
\hline 33 & 1 & 0 & 9.148535 & -3.507834 & 0.785564 \\
\hline 34 & 1 & $\odot$ & 8.896686 & -1.998549 & -1.116304 \\
\hline 35 & 1 & 0 & 7.738941 & -1.314899 & 1.290703 \\
\hline 36 & 1 & $\odot$ & 5.268669 & $-\odot .680598$ & $\odot .371828$ \\
\hline 37 & 1 & 0 & 5.610720 & -2.398029 & $\odot .732182$ \\
\hline 38 & 1 & $\odot$ & 5.725390 & -1.013714 & -1.993551 \\
\hline 39 & 8 & 0 & 7.157050 & -4.182196 & 0.743500 \\
\hline 40 & 1 & $\odot$ & 7.335807 & -5.037024 & 1.168041 \\
\hline 41 & 6 & 0 & 3.532595 & -2.087687 & -2.305824 \\
\hline 42 & 7 & 0 & 1.107435 & -4.649399 & -2.186866 \\
\hline 43 & 8 & $\odot$ & 3.631424 & -0.905950 & -2.669000 \\
\hline 44 & 1 & $\odot$ & 5.427015 & -4.378147 & -0.751966 \\
\hline 45 & 1 & 0 & 3.330607 & -5.749530 & -1.083102 \\
\hline 46 & 1 & 0 & 0.944824 & -5.574632 & -1.826312 \\
\hline 47 & 1 & 0 & ๑. 320698 & -4.105511 & -2.541974 \\
\hline 48 & 6 & 0 & 5.154046 & 2.044799 & -1.493656 \\
\hline 49 & 1 & 0 & 4.568101 & 6.261292 & -0.100333 \\
\hline 50 & 6 & 0 & 4.500219 & 3.868056 & 1.152883 \\
\hline 51 & 6 & 0 & 3.163908 & 3.129109 & 1.000667 \\
\hline 52 & 8 & 0 & 3.068111 & 2.750908 & -0.407134 \\
\hline 53 & 1 & $\odot$ & 4.596007 & 1. 253032 & -1.997866 \\
\hline 54 & 1 & 0 & 5.969388 & 2.391194 & -2.135521 \\
\hline 55 & 1 & 0 & 3.870083 & 3.653992 & -2.087184 \\
\hline 56 & 1 & 0 & 5.954886 & 4.338714 & -0.418581 \\
\hline 57 & 1 & $\odot$ & 4.404676 & 4.730832 & 1.815288 \\
\hline 58 & 1 & 0 & 5.274285 & 3.212421 & 1.552923 \\
\hline 59 & 1 & $\odot$ & 2.304546 & 3.765671 & 1.213156 \\
\hline 60 & 7 & $\odot$ & 3.024675 & 1.955509 & 1.835309 \\
\hline 61 & 6 & $\odot$ & 3.989310 & 1.039668 & 2.226876 \\
\hline 62 & 7 & 0 & 3.489615 & $\odot .009363$ & 2.851300 \\
\hline 63 & 6 & 0 & 2.125439 & ๑. 240818 & 2.866662 \\
\hline 64 & 6 & 0 & 1.817486 & 1.437688 & 2.239193 \\
\hline 65 & 7 & 0 & ๑. 610515 & 2.010966 & 2.065882 \\
\hline 66 & 6 & 0 & -0.376032 & 1.271581 & 2.541791 \\
\hline 67 & 7 & 0 & -0.182115 & 0.049765 & 3.140549 \\
\hline 68 & 6 & 0 & 1.055361 & -0.557805 & 3.361671 \\
\hline 69 & 8 & 0 & 1.124032 & -1.663741 & 3.914568 \\
\hline 70 & 1 & $\odot$ & 5.041693 & 1.193017 & 2.024782 \\
\hline 71 & 7 & $\odot$ & -1.646672 & 1.724276 & 2.512040 \\
\hline 72 & 1 & 0 & -1.006844 & -0.545884 & 3.328329 \\
\hline 73 & 1 & $\odot$ & -6.002739 & -5.242463 & -0.994983 \\
\hline 74 & 6 & 0 & -6.901496 & -1.545040 & -0.648546 \\
\hline 75 & 6 & $\odot$ & -5.470825 & -1.400323 & -0.146438 \\
\hline 76 & 6 & 0 & -5.620724 & -1.618439 & 1.357448 \\
\hline
\end{tabular}




\begin{tabular}{|c|c|c|c|c|c|}
\hline 77 & 8 & 0 & -6.724146 & -2.546078 & 1.513370 \\
\hline 78 & 1 & $\odot$ & -7.794709 & -4.726859 & 0.495893 \\
\hline 79 & 1 & $\odot$ & -8.106217 & -4.110742 & -1.145829 \\
\hline 80 & 1 & $\odot$ & -8.534403 & -2.373782 & 0.502525 \\
\hline 81 & 1 & $\odot$ & -6.967018 & -1.792685 & -1.709135 \\
\hline 82 & 1 & $\odot$ & -5.018227 & -0.438680 & -0.374579 \\
\hline 83 & 1 & $\odot$ & -4.859364 & -2.195295 & -0.578038 \\
\hline 84 & 1 & $\odot$ & -5.844120 & -0.700004 & 1.895404 \\
\hline 85 & 7 & $\odot$ & -4.423282 & -2.187120 & 1.972009 \\
\hline 86 & 6 & $\odot$ & -4.113922 & -3.495242 & 1.752592 \\
\hline 87 & 6 & $\odot$ & -2.962812 & -4.019637 & 2.228995 \\
\hline 88 & 6 & $\odot$ & -2.082163 & -3.131772 & 2.925826 \\
\hline 89 & 7 & $\odot$ & -2.387608 & -1.844428 & 3.120310 \\
\hline 90 & 6 & $\odot$ & -3.540570 & -1.328842 & 2.633681 \\
\hline 91 & 1 & $\odot$ & -2.705528 & -5.056373 & 2.060779 \\
\hline 92 & 7 & 0 & -0.916780 & -3.572095 & 3.408071 \\
\hline 93 & 8 & $\odot$ & -3.834322 & -0.127597 & 2.749867 \\
\hline 94 & 1 & $\odot$ & -4.838796 & -4.048222 & 1.160851 \\
\hline 95 & 1 & $\odot$ & -0.614891 & -4.508170 & 3.193346 \\
\hline 96 & 1 & $\odot$ & -0.218938 & -2.895652 & 3. 732691 \\
\hline 97 & 1 & $\odot$ & -1.875568 & 2.403096 & 1.795763 \\
\hline 98 & 1 & $\odot$ & -2.404623 & 1.074360 & 2.733190 \\
\hline 99 & 6 & 0 & -5.819277 & 2.193308 & ๑. 737991 \\
\hline 100 & 11 & $\odot$ & 9.137187 & 4.349160 & -0.285380 \\
\hline 101 & 11 & $\odot$ & -9.085076 & 4.101563 & -0.514793 \\
\hline 102 & 6 & $\odot$ & -4.198755 & 3.507444 & -1.726795 \\
\hline 103 & 6 & $\odot$ & -3.132556 & 2.590003 & -1.117926 \\
\hline 104 & 8 & $\odot$ & -3.433715 & 2.504348 & 0.324610 \\
\hline 105 & 1 & $\odot$ & -5.538702 & 1.415378 & 1.453125 \\
\hline 106 & 1 & $\odot$ & -6.740594 & 2.686148 & 1. 063712 \\
\hline 107 & 1 & $\odot$ & -4.551550 & 3.710168 & 1.577189 \\
\hline 108 & 1 & $\odot$ & -5.897689 & 4.441557 & -0.705037 \\
\hline 109 & 1 & $\odot$ & -3.754704 & 4.230948 & -2.413283 \\
\hline 110 & 1 & $\odot$ & -4.959731 & 2.937972 & -2.257030 \\
\hline 111 & 1 & $\odot$ & -2.128158 & 3.012275 & -1.168121 \\
\hline 112 & 7 & $\odot$ & -3.049380 & 1.263731 & -1.667880 \\
\hline 113 & 6 & $\odot$ & -3.846784 & ๑. 568182 & -2.509110 \\
\hline 114 & 7 & $\odot$ & -3.373668 & -0.625501 & -2.858232 \\
\hline 115 & 6 & 0 & -2.215121 & -0.760701 & -2.174893 \\
\hline 116 & 6 & $\odot$ & -2.010336 & 0.346753 & -1.185025 \\
\hline 117 & 7 & $\odot$ & -0.722490 & 0.940777 & -1.161728 \\
\hline 118 & 6 & $\odot$ & $\odot .247130$ & 0.122602 & -1.438773 \\
\hline 119 & 7 & $\odot$ & 0.067431 & -1.185021 & -1.902690 \\
\hline 120 & 6 & $\odot$ & -1.125750 & -1.675102 & -2.402098 \\
\hline 121 & 7 & $\odot$ & 1.538822 & $\odot .465910$ & -1.258356 \\
\hline 122 & 8 & $\odot$ & -1.178918 & -2.774505 & -2.968506 \\
\hline 123 & 1 & $\odot$ & -4.801169 & 0.943151 & -2.861691 \\
\hline 124 & 1 & $\odot$ & 1.762279 & 1.403653 & -0.938506 \\
\hline 125 & 1 & $\odot$ & 2.263855 & $-\odot .0 \odot 2978$ & -1.799384 \\
\hline 126 & 1 & $\odot$ & 0.902805 & -1.747159 & -2.127262 \\
\hline 127 & 8 & $\odot$ & -2.391250 & -0.149475 & ๑. 093566 \\
\hline 128 & 1 & $\odot$ & -2.761816 & ๑. 597211 & $\odot .591305$ \\
\hline
\end{tabular}

1122 basis functions

305 alpha electrons

304 beta electrons

nuclear repulsion energy 16521.7593466216 Hartrees.

NAtoms $=128$

Harmonic frequencies $\left(\mathrm{cm}^{*}-1\right)$, IR intensities (KM/Mole), Raman

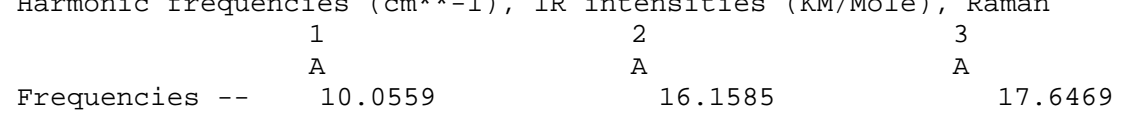

Zero-point correction=

Thermal correction to Energy=

Thermal correction to Enthalpy=

Thermal correction to Gibbs Free Energy=

Sum of electronic and zero-point Energies=

Sum of electronic and thermal Energies=

Sum of electronic and thermal Enthalpies=

๑. 987822 (a.u.)

1.060709

1.061654

0.875025

$-4938.429118$

$-4938.356231$

$-4938.355286$

$-4938.541915$

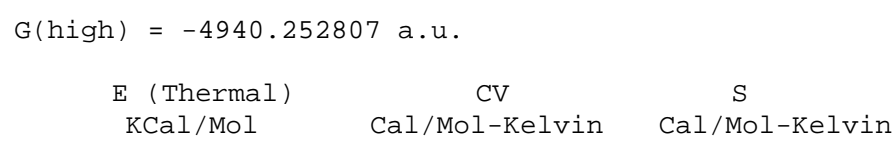




\begin{tabular}{lccc}
\multicolumn{1}{c}{ Item } & Value & Threshold Converged? \\
Maximum Force & 0.000040 & 0.000450 & YES \\
RMS Force & 0.000005 & 0.000300 & YES \\
Maximum Displacement & 0.011586 & $0.00180 \odot$ & NO \\
RMS Displacement & 0.001445 & 0.001200 & NO
\end{tabular}

Normal termination of Gaussian 16 at Mon Feb 11 09:47:41 2019.

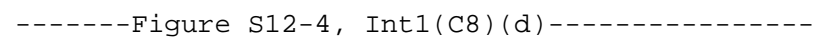

dna@4d1-ohfor. $\log$

Stoichiometry C38H49N16Na2021P2(2)

Standard orientation:

\begin{tabular}{|c|c|c|c|c|c|}
\hline \multirow{2}{*}{$\begin{array}{l}\text { Center } \\
\text { Number }\end{array}$} & \multirow{2}{*}{$\begin{array}{l}\text { Atomic } \\
\text { Number }\end{array}$} & \multirow{2}{*}{$\begin{array}{c}\text { Atomic } \\
\text { Type }\end{array}$} & \multicolumn{3}{|c|}{ Coordinates (Angstroms) } \\
\hline & & & $\mathrm{x}$ & $Y$ & Z \\
\hline & - & 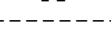 & $-\ldots-\ldots$ & ------- & $-\ldots$ \\
\hline 1 & 7 & $\odot$ & 5.259748 & -2.162237 & -1.172847 \\
\hline 2 & 6 & $\odot$ & 5.299656 & -3.477304 & -0.812432 \\
\hline 3 & 6 & $\odot$ & 4.179497 & -4.233506 & -0.830951 \\
\hline 4 & 6 & 0 & 2.959255 & -3.583918 & -1.217589 \\
\hline 5 & 7 & $\odot$ & 2.944806 & -2.316087 & -1.636109 \\
\hline 6 & 8 & $\odot$ & 8.589104 & 0.514205 & -0.217885 \\
\hline 7 & 15 & $\odot$ & 7.903876 & 1.780551 & 0.560841 \\
\hline 8 & 8 & 0 & 6.397687 & 1.827274 & -0.092201 \\
\hline 9 & 8 & $\odot$ & 8.662115 & 3.014431 & $\odot .170823$ \\
\hline 10 & 8 & $\odot$ & 7.679942 & 1.406057 & 2.009695 \\
\hline 11 & 8 & $\odot$ & 4.582229 & 5.692287 & 0.369975 \\
\hline 12 & 6 & $\odot$ & 4.925937 & 3.655326 & -0.809228 \\
\hline 13 & 6 & $\odot$ & 5.212518 & 4.393188 & $\odot .498936$ \\
\hline 14 & 6 & $\odot$ & -8.962650 & -3.474441 & 0.978512 \\
\hline 15 & 6 & $\odot$ & -8.474874 & -2.049722 & 1.148106 \\
\hline 16 & 8 & $\odot$ & -8.051617 & -4.196158 & 0.113417 \\
\hline 17 & 8 & $\odot$ & -8.408342 & 0.132192 & 0.168305 \\
\hline 18 & 15 & $\odot$ & -7.828691 & 1.381334 & -0.716338 \\
\hline 19 & 8 & $\odot$ & -6.277910 & 1.512152 & -0.181087 \\
\hline 20 & 8 & 0 & -8.587178 & 2.600276 & -0.244581 \\
\hline 21 & 8 & $\odot$ & -7.746656 & 1.008439 & -2.168089 \\
\hline 22 & 8 & $\odot$ & -4.687876 & 5.376245 & $-\odot .965666$ \\
\hline 23 & 6 & $\odot$ & -4.857427 & 3.430808 & ๑. 396931 \\
\hline 24 & 6 & $\odot$ & -5.203063 & 4.021632 & -0.969931 \\
\hline 25 & 1 & $\odot$ & -4.831351 & 5.795015 & -1.831461 \\
\hline 26 & 6 & $\odot$ & 9.371438 & -3.081580 & -0.850220 \\
\hline 27 & 6 & $\odot$ & 8.791028 & -1.704882 & -1.102244 \\
\hline 28 & 6 & $\odot$ & 8.444894 & $-\odot .896529$ & 0.157733 \\
\hline 29 & 6 & $\odot$ & 6.992548 & -1.278553 & ○. 406951 \\
\hline 30 & 6 & $\odot$ & 6.432495 & -1.322277 & -1.011852 \\
\hline 31 & 8 & $\odot$ & 7.524039 & -1.833496 & -1.832758 \\
\hline 32 & 1 & $\odot$ & 9.463809 & -3.602090 & -1.809109 \\
\hline 33 & 1 & $\odot$ & 10.369643 & -2.975767 & -0.409474 \\
\hline 34 & 1 & $\odot$ & 9.483934 & -1.137114 & -1.727224 \\
\hline 35 & 1 & $\odot$ & 9.103849 & -1.107193 & 1. 001231 \\
\hline 36 & 1 & $\odot$ & 6.467816 & -0.576696 & 1.046029 \\
\hline 37 & 1 & $\odot$ & 6.971639 & -2.267428 & 0.867784 \\
\hline 38 & 1 & 0 & 6.150006 & -0.331503 & -1.358059 \\
\hline 39 & 8 & $\odot$ & 8.496938 & -3.813541 & 0.044313 \\
\hline 40 & 1 & $\odot$ & 8.812419 & -4.723731 & 0.166042 \\
\hline 41 & 6 & $\odot$ & 4.079179 & -1.579346 & -1.652557 \\
\hline 42 & 7 & $\odot$ & 1.797817 & -4.239288 & -1.197739 \\
\hline 43 & 8 & $\odot$ & 4.107009 & -0.414899 & -2.072410 \\
\hline 44 & 1 & $\odot$ & 6.281103 & -3.851393 & -0.528238 \\
\hline 45 & 1 & $\odot$ & 4.200813 & -5.277641 & -0.550322 \\
\hline 46 & 1 & $\odot$ & 1.751640 & -5.170083 & -0.818067 \\
\hline 47 & 1 & $\odot$ & ๑. 927798 & -3.730181 & -1.380713 \\
\hline 48 & 6 & $\odot$ & 5. 991042 & 2.650662 & -1.219587 \\
\hline 49 & 1 & $\odot$ & 4.710091 & 6.211737 & 1.181861 \\
\hline 50 & 6 & $\odot$ & 4.520392 & 3.532966 & 1.561762 \\
\hline 51 & 6 & $\odot$ & 3.315006 & 2.920879 & 0.828892 \\
\hline 52 & 8 & 0 & 3.626160 & 3.009648 & -0.594201 \\
\hline 53 & 1 & $\odot$ & 5.589240 & 1.980602 & -1.981805 \\
\hline 54 & 1 & $\odot$ & 6.861082 & 3.185126 & -1.610031 \\
\hline 55 & 1 & $\odot$ & 4.788333 & 4.371550 & -1.622243 \\
\hline 56 & 1 & $\odot$ & 6.286776 & 4.497820 & 0.680876 \\
\hline
\end{tabular}




\begin{tabular}{|c|c|c|c|c|c|}
\hline 57 & 1 & $\odot$ & 4.208686 & 4.121394 & 2.427105 \\
\hline 58 & 1 & $\odot$ & 5.207466 & 2.754560 & 1.899056 \\
\hline 59 & 1 & $\odot$ & 2.389520 & 3.470537 & 1.002822 \\
\hline 60 & 7 & $\odot$ & 3. 051782 & 1.541782 & 1.177693 \\
\hline 61 & 6 & $\odot$ & 3. 963812 & 0.507198 & 1.145086 \\
\hline 62 & 7 & $\odot$ & 3.425726 & -0.663375 & 1.345061 \\
\hline 63 & 6 & $\odot$ & 2.074956 & -0.394356 & 1.492948 \\
\hline 64 & 6 & $\odot$ & 1.822332 & 0.966287 & 1.377714 \\
\hline 65 & 7 & $\odot$ & $\odot .645775$ & 1.614445 & 1.449969 \\
\hline 66 & 6 & $\odot$ & -0.377679 & 0.790769 & 1.619125 \\
\hline 67 & 7 & $\odot$ & -0.237988 & -0.573837 & 1.725265 \\
\hline 68 & 6 & $\odot$ & $\odot .968490$ & -1.272405 & 1.678383 \\
\hline 69 & 8 & $\odot$ & $\odot .984834$ & -2.506510 & 1.781957 \\
\hline 70 & 1 & $\odot$ & 4.999013 & 0.720172 & ๑. 922244 \\
\hline 71 & 7 & $\odot$ & -1.627219 & 1.266498 & 1.721216 \\
\hline 72 & 1 & $\odot$ & -1.092554 & -1.158590 & 1.784859 \\
\hline 73 & 1 & $\odot$ & -8.307852 & -5.130286 & 0.046689 \\
\hline 74 & 6 & $\odot$ & -8.202748 & -1.286506 & -0.155015 \\
\hline 75 & 6 & $\odot$ & -6.736480 & -1.593027 & -0.423196 \\
\hline 76 & 6 & $\odot$ & -6.149771 & -1.514381 & 0.985555 \\
\hline 77 & 8 & $\odot$ & -7.190752 & -2.047783 & 1.858810 \\
\hline 78 & 1 & $\odot$ & -9.010576 & -3.946391 & 1.965453 \\
\hline 79 & 1 & $\odot$ & -9.970473 & -3.459044 & 0.547070 \\
\hline 80 & 1 & $\odot$ & -9.197016 & -1.500280 & 1.756379 \\
\hline 81 & 1 & $\odot$ & -8.868913 & -1.565624 & -0.972182 \\
\hline 82 & 1 & $\odot$ & -6.282517 & -0.897875 & -1.123456 \\
\hline 83 & 1 & $\odot$ & -6.648307 & -2.599847 & -0.831547 \\
\hline 84 & 1 & $\odot$ & -5.932282 & -0.488338 & 1.267958 \\
\hline 85 & 7 & $\odot$ & -4.923012 & -2.263975 & 1.183336 \\
\hline 86 & 6 & $\odot$ & -4.876994 & -3.588527 & 0.867457 \\
\hline 87 & 6 & $\odot$ & -3.706157 & -4.269397 & 0.914844 \\
\hline 88 & 6 & $\odot$ & -2.536136 & -3.535361 & 1.305747 \\
\hline 89 & 7 & $\odot$ & -2.606430 & -2.253880 & 1.675030 \\
\hline 90 & 6 & $\odot$ & -3.782692 & -1.586639 & 1.639391 \\
\hline 91 & 1 & $\odot$ & -3.659465 & -5.321724 & $\odot .672567$ \\
\hline 92 & 7 & $\odot$ & -1.343153 & -4.127792 & 1.349372 \\
\hline 93 & 8 & $\odot$ & -3.881990 & -0.403259 & 1.990694 \\
\hline 94 & 1 & $\odot$ & -5.830208 & -4.039549 & 0.605099 \\
\hline 95 & 1 & $\odot$ & -1.234679 & -5.060353 & 0.986785 \\
\hline 96 & 1 & $\odot$ & -0.505388 & -3.564346 & 1.528473 \\
\hline 97 & 1 & $\odot$ & -1.830685 & 2.190817 & 1.367715 \\
\hline 98 & 1 & $\odot$ & -2.425234 & ๑. 628224 & 1.776767 \\
\hline 99 & 6 & $\odot$ & -5.835503 & 2.379736 & ๑. 899964 \\
\hline 100 & 11 & $\odot$ & 6.616484 & 0.581325 & 3.739376 \\
\hline 101 & 11 & $\odot$ & -8.952898 & 4.669569 & 0.360743 \\
\hline 102 & 6 & $\odot$ & -4.447933 & 3.123046 & -1.957774 \\
\hline 103 & 6 & $\odot$ & -3.210298 & 2.647214 & -1.178022 \\
\hline 104 & 8 & $\odot$ & -3.507871 & 2.880187 & ๑. 228082 \\
\hline 105 & 1 & $\odot$ & -5.342582 & 1.750286 & 1.643260 \\
\hline 106 & 1 & $\odot$ & -6.705122 & 2.871923 & 1.342258 \\
\hline 107 & 1 & $\odot$ & -4.772175 & 4.221585 & 1.144882 \\
\hline 108 & 1 & $\odot$ & -6.280976 & 4.018509 & -1.156445 \\
\hline 109 & 1 & $\Theta$ & -4.167064 & 3. 659108 & -2.866385 \\
\hline 110 & 1 & 0 & -5.084149 & 2.280784 & -2.236045 \\
\hline 111 & 1 & $\odot$ & -2.309717 & 3.210009 & -1.423840 \\
\hline 112 & 7 & 0 & -2.888337 & 1.248076 & -1.380243 \\
\hline 113 & 6 & $\odot$ & -3.751366 & 0.185256 & -1.255595 \\
\hline 114 & 7 & $\odot$ & -3.150803 & -0.970115 & -1.350057 \\
\hline 115 & 6 & $\odot$ & -1.812903 & -0.658141 & -1.520843 \\
\hline 116 & 6 & $\odot$ & -1.630177 & 0.715953 & -1.527663 \\
\hline 117 & 7 & $\odot$ & -0.488587 & 1.412307 & -1.655383 \\
\hline 118 & 6 & $\odot$ & 0.576470 & 0.629313 & -1.753444 \\
\hline 119 & 7 & $\odot$ & 0.504332 & -0.745449 & -1.742403 \\
\hline 120 & 6 & $\odot$ & -0.665841 & -1.496538 & -1.635018 \\
\hline 121 & 7 & $\odot$ & 1.798221 & 1.157882 & -1.892657 \\
\hline 122 & 8 & $\odot$ & -0.625749 & -2.733012 & -1.637894 \\
\hline 123 & 1 & $\Theta$ & -4.797813 & 0.363372 & -1.053618 \\
\hline 124 & 1 & 0 & 1.948489 & 2.125356 & -1.645103 \\
\hline 125 & 1 & 0 & 2.630802 & $\odot .561994$ & -1.907348 \\
\hline 126 & 1 & 0 & 1.385485 & -1.293140 & -1.767693 \\
\hline 127 & 8 & $\odot$ & -4.285058 & -3.554953 & -1.680111 \\
\hline 128 & 1 & $\odot$ & -3.862320 & -2.672675 & -1.504993 \\
\hline
\end{tabular}

1122 basis functions

305 alpha electrons

304 beta electrons

nuclear repulsion energy 16547.2413510584 Hartrees. 
NAtoms $=128$ NActive $=128$ NUniq $=128$ SFac $=1.00 \mathrm{D}+00$

Harmonic frequencies $\left(\mathrm{cm}^{*}-1\right)$, IR intensities (KM/Mole), Raman

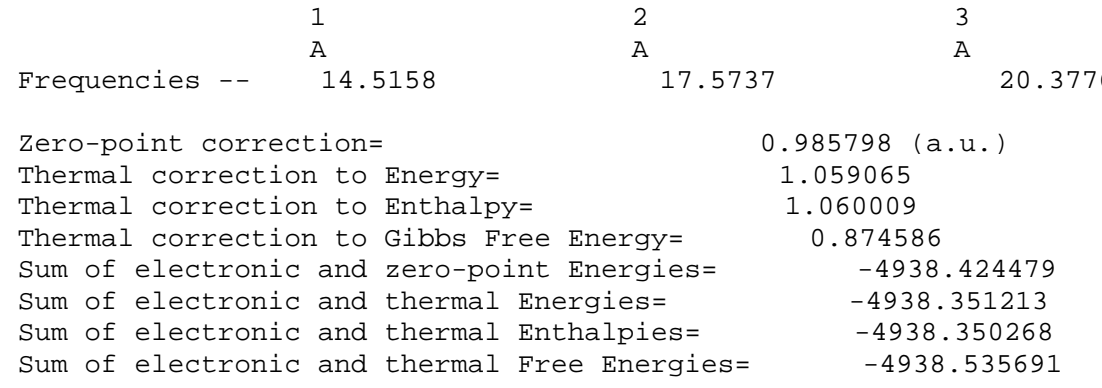

$$
\mathrm{G}(\mathrm{high})=-4940.244858 \mathrm{a} \cdot \mathrm{u} \text {. }
$$

\begin{tabular}{|c|c|c|c|}
\hline $\begin{array}{l}\mathrm{E}(\mathrm{T} \\
\mathrm{KCa}\end{array}$ & $\begin{array}{l}\text { Thermal) } \\
\text { al/Mol }\end{array}$ & $\frac{\mathrm{CV}}{\mathrm{Cal} / \mathrm{Mol}-\mathrm{Kel} \text { in }}$ & $\stackrel{\mathrm{S}}{\mathrm{Cal} / \mathrm{Mol}-\mathrm{Kel} \text { ivin }}$ \\
\hline Total & 664.573 & 279.434 & 390.254 \\
\hline Electronic & 0.000 & 0.000 & 1.377 \\
\hline Translational & $\odot .889$ & 2.981 & 47.058 \\
\hline Rotational & $\odot .889$ & 2.981 & 41.866 \\
\hline Vibrational & 662.796 & 273.473 & 299.953 \\
\hline Item & Value & \multicolumn{2}{|c|}{ Threshold Converged? } \\
\hline Maximum Force & 0.000016 & $\odot .000450$ & $\begin{array}{l}\text { erged? } \\
\text { YES }\end{array}$ \\
\hline RMS Force & 0.000002 & 0.000300 & YES \\
\hline Maximum Displacement & 0.002789 & 0.001800 & NO \\
\hline Displacement & $\odot .000354$ & 0.001200 & YES \\
\hline
\end{tabular}

Normal termination of Gaussian $\odot 9$ at Thu Jan 31 ๑2:10: 08 2019.

Figure S12-5, TS1(C8)(d)

dna@4d1-ohts. $\log$

Stoichiometry C38H49N16Na2021P2(2)

Standard orientation:

\begin{tabular}{|c|c|c|c|c|c|}
\hline \multirow{2}{*}{$\begin{array}{l}\text { Center } \\
\text { Number }\end{array}$} & \multirow{2}{*}{$\begin{array}{l}\text { Atomic } \\
\text { Number }\end{array}$} & \multirow{2}{*}{$\begin{array}{c}\text { Atomic } \\
\text { Type }\end{array}$} & \multicolumn{3}{|c|}{ Coordinates (Angstroms) } \\
\hline & & & $x$ & $\mathrm{Y}$ & Z \\
\hline & & & & 10 & 40 \\
\hline 1 & 7 & $\odot$ & -4.378089 & -2.555323 & 0.423660 \\
\hline 2 & 6 & $\odot$ & -4.566591 & -3.844900 & $\odot .017617$ \\
\hline 3 & 6 & $\odot$ & -3.519592 & -4.675377 & -0.178557 \\
\hline 4 & 6 & $\odot$ & -2.220218 & -4.168709 & 0.138042 \\
\hline 5 & 7 & $\odot$ & -2.038166 & -2.907038 & 0.530530 \\
\hline 6 & 8 & $\odot$ & -8.276411 & -0.281374 & 0.963635 \\
\hline 7 & 15 & $\odot$ & -8.211589 & 1.182968 & 0.263912 \\
\hline 8 & 8 & $\odot$ & -6.682675 & 1.652191 & 0.579637 \\
\hline 9 & 8 & $\odot$ & - 9.191233 & 2.087945 & ๑. 973961 \\
\hline 10 & 8 & $\odot$ & -8.338962 & 1.080019 & -1.244183 \\
\hline 11 & 8 & $\odot$ & -6.558221 & 5.981630 & ๑. 440374 \\
\hline 12 & 6 & $\odot$ & -5.824976 & 3.848379 & 1.196675 \\
\hline 13 & 6 & $\odot$ & -6.773187 & 4.563151 & 0.232769 \\
\hline 14 & 6 & $\odot$ & 7.465729 & -1.850541 & -3.462264 \\
\hline 15 & 6 & $\odot$ & 7.142625 & -0.436236 & -3.026657 \\
\hline 16 & 8 & $\odot$ & 6.953007 & -2.775509 & -2.472016 \\
\hline 17 & 8 & $\odot$ & 7.955856 & 1.272522 & -1.525783 \\
\hline 18 & 15 & 0 & 8.834333 & 1.830382 & -0.268661 \\
\hline 19 & 8 & $\odot$ & 7.746084 & 1.870672 & ๑. 952567 \\
\hline 20 & 8 & $\odot$ & 9.284077 & 3.226372 & -0.630168 \\
\hline 21 & 8 & $\odot$ & 9.899229 & 0.836525 & 0.132464 \\
\hline 22 & 8 & $\odot$ & 5.671189 & 2.817363 & 4.440044 \\
\hline 23 & 6 & $\odot$ & 5.700426 & 2.509660 & 2.068163 \\
\hline 24 & 6 & $\odot$ & 6.347354 & 2.069918 & 3.398228 \\
\hline 25 & 1 & $\odot$ & 6.082387 & 2.644860 & 5.304142 \\
\hline 26 & 6 & $\odot$ & -8.154793 & -3.957341 & 1.218005 \\
\hline 27 & 6 & $\odot$ & -7.716913 & -2.536288 & 1.504777 \\
\hline 28 & 6 & $\odot$ & -7.913524 & -1.557469 & 0.341210 \\
\hline 29 & 6 & $\odot$ & -6.552931 & -1.538845 & -0.350863 \\
\hline 30 & 6 & $\odot$ & -5.551576 & -1.757772 & 0.784949 \\
\hline 31 & 8 & $\odot$ & -6.284576 & -2.484410 & 1.815762 \\
\hline 32 & 1 & $\odot$ & -7.779716 & -4.613160 & 2.010308 \\
\hline
\end{tabular}




\begin{tabular}{|c|c|c|c|c|c|}
\hline 33 & 1 & 0 & -9.249939 & -3.996242 & 1.214728 \\
\hline 34 & 1 & $\odot$ & -8.252695 & -2.175867 & 2.385339 \\
\hline 35 & 1 & $\odot$ & -8.719554 & -1.850948 & -0.332318 \\
\hline 36 & 1 & $\odot$ & -6.350407 & -0.623642 & -0.899908 \\
\hline 37 & 1 & $\odot$ & -6.524370 & -2.373507 & -1.054796 \\
\hline 38 & 1 & $\odot$ & -5.178862 & -0.820763 & 1.193843 \\
\hline 39 & 8 & $\odot$ & -7.636216 & -4.363886 & -0.073431 \\
\hline 40 & 1 & $\odot$ & -7.915810 & -5.267475 & -0.293115 \\
\hline 41 & 6 & $\odot$ & -3.087723 & -2.054378 & 0.655314 \\
\hline 42 & 7 & $\odot$ & -1.143400 & -4.960574 & 0.049114 \\
\hline 43 & 8 & $\odot$ & -2.941209 & $-\odot .868762$ & $\odot .979227$ \\
\hline 44 & 1 & $\odot$ & -5.603640 & -4.152662 & -0.109936 \\
\hline 45 & 1 & $\odot$ & -3.667252 & -5.696994 & -0.500788 \\
\hline 46 & 1 & $\odot$ & -1.274514 & -5.943200 & -0.128467 \\
\hline 47 & 1 & $\odot$ & $-\odot .264471$ & -4.664132 & 0.484613 \\
\hline 48 & 6 & $\odot$ & -6.310363 & 2.503696 & 1.700611 \\
\hline 49 & 1 & $\odot$ & -7.127570 & 6.499729 & $-\odot .153563$ \\
\hline 50 & 6 & $\odot$ & -6.308597 & 4.111013 & -1.161534 \\
\hline 51 & 6 & $\odot$ & -4.826480 & 3.738548 & $-\odot .979470$ \\
\hline 52 & 8 & $\odot$ & -4.578700 & 3.701855 & 0.451797 \\
\hline 53 & 1 & $\odot$ & -5.504589 & 1.997578 & 2.233552 \\
\hline 54 & 1 & $\odot$ & -7.166090 & 2.642145 & 2.366257 \\
\hline 55 & 1 & $\odot$ & -5.609748 & 4.490818 & 2.053777 \\
\hline 56 & 1 & $\odot$ & -7.818820 & 4.298622 & 0.420624 \\
\hline 57 & 1 & $\odot$ & -6.431849 & 4.906030 & -1.899636 \\
\hline 58 & 1 & $\odot$ & -6.905815 & 3.256705 & -1.477362 \\
\hline 59 & 1 & $\odot$ & -4.148969 & 4.490065 & -1.386021 \\
\hline 60 & 7 & $\odot$ & -4.451759 & 2.462248 & -1.574553 \\
\hline 61 & 6 & $\odot$ & -5.238851 & 1.419817 & -2.025467 \\
\hline 62 & 7 & 0 & -4.552762 & $\odot .350693$ & -2.326810 \\
\hline 63 & 6 & $\odot$ & -3.243827 & $\odot .689159$ & -2.043475 \\
\hline 64 & 6 & $\odot$ & -3.162352 & 1.991734 & -1.580453 \\
\hline 65 & 7 & 0 & -2.068215 & 2.674422 & -1.182323 \\
\hline 66 & 6 & $\odot$ & $-\odot .973935$ & 1.941231 & -1.230565 \\
\hline 67 & 7 & $\odot$ & -0.946355 & $\odot .639016$ & -1.654554 \\
\hline 68 & 6 & 0 & -2.057767 & -0.101149 & -2.056385 \\
\hline 69 & 8 & $\odot$ & -1.941787 & -1.296838 & -2.356074 \\
\hline 70 & 1 & $\odot$ & -6.317057 & 1.484999 & -2.078889 \\
\hline 71 & 7 & 0 & $\odot .199242$ & 2.445086 & -0.775400 \\
\hline 72 & 1 & $\odot$ & $-\odot .043829$ & $\odot .123136$ & -1.621046 \\
\hline 73 & 1 & $\odot$ & 7.138169 & -3.694271 & -2.725024 \\
\hline 74 & 6 & 0 & 7.504102 & -0.126240 & -1.569628 \\
\hline 75 & 6 & $\odot$ & 6.199004 & -0.300854 & $-\odot .809717$ \\
\hline 76 & 6 & $\odot$ & 5.123306 & $\odot .067225$ & -1.827040 \\
\hline 77 & 8 & $\odot$ & 5.702894 & -0.193960 & -3.138699 \\
\hline 78 & 1 & $\odot$ & 7.008430 & -2.036141 & -4.439764 \\
\hline 79 & 1 & $\odot$ & 8.552969 & -1.955767 & -3.555069 \\
\hline 80 & 1 & $\odot$ & 7.650681 & $\odot .264081$ & -3.694186 \\
\hline 81 & 1 & $\odot$ & 8.306852 & -0.762440 & -1.193015 \\
\hline 82 & 1 & $\odot$ & 6.134540 & 0.321222 & 0.081217 \\
\hline 83 & 1 & $\odot$ & 6.105010 & -1.338269 & -0.482354 \\
\hline 84 & 1 & $\odot$ & 4.824717 & 1.111708 & -1.772447 \\
\hline 85 & 7 & $\odot$ & 3.894990 & -0.714374 & -1.700382 \\
\hline 86 & 6 & $\odot$ & 3.959058 & -2.075345 & -1.784892 \\
\hline 87 & 6 & $\odot$ & 2.837593 & -2.829620 & -1.756275 \\
\hline 88 & 6 & 0 & 1.586307 & -2.134328 & -1.705121 \\
\hline 89 & 7 & $\odot$ & 1.533137 & -0.801118 & -1.621214 \\
\hline 90 & 6 & $\odot$ & 2.662676 & -0.052681 & -1.613675 \\
\hline 91 & 1 & $\odot$ & 2.882718 & -3.907800 & -1.825322 \\
\hline 92 & 7 & $\odot$ & $\odot .431459$ & -2.796163 & -1.750909 \\
\hline 93 & 8 & $\odot$ & 2.637861 & 1.183028 & -1.538231 \\
\hline 94 & 1 & $\odot$ & 4.958661 & -2.489341 & -1.908523 \\
\hline 95 & 1 & $\odot$ & $\odot .417326$ & -3.801975 & -1.787530 \\
\hline 96 & 1 & $\odot$ & $-\odot .452 \odot \odot 4$ & -2.284034 & -1.835292 \\
\hline 97 & 1 & $\odot$ & 0.207414 & 3.443448 & -0.636114 \\
\hline 98 & 1 & $\odot$ & 1.081484 & 2.002019 & -1.055997 \\
\hline 99 & 6 & $\odot$ & 6.674322 & 2.856494 & 0.968562 \\
\hline 100 & 11 & 0 & -10.109660 & 2.768853 & -1.346650 \\
\hline 101 & 11 & 0 & 11.663799 & 2.579096 & $-\odot .225257$ \\
\hline 102 & 6 & 0 & 6.026176 & $\odot .571437$ & 3.482198 \\
\hline 103 & 6 & $\theta$ & 4.664443 & $\odot .527313$ & 2.799531 \\
\hline 104 & 8 & $\odot$ & 4.857085 & 1.385140 & 1.636333 \\
\hline 105 & 1 & $\odot$ & 6.157173 & 2.863241 & 0.004057 \\
\hline 106 & 1 & $\odot$ & 7.105198 & 3.844753 & 1.150871 \\
\hline 107 & 1 & $\odot$ & 5.057162 & 3.373028 & 2.263935 \\
\hline 108 & 1 & $\odot$ & 7.421779 & 2.262654 & 3.416465 \\
\hline 109 & 1 & $\odot$ & 6.017009 & $\odot .201740$ & 4.508650 \\
\hline
\end{tabular}




\begin{tabular}{|c|c|c|c|c|c|}
\hline 110 & 1 & $\odot$ & 6.757000 & 0.012373 & 2.890475 \\
\hline 111 & 1 & $\odot$ & 3.893017 & $\odot .975931$ & 3.434257 \\
\hline 112 & 7 & $\odot$ & 4.192201 & -0.773499 & 2.390185 \\
\hline 113 & 6 & $\odot$ & 4.778979 & -2.011869 & 2.594197 \\
\hline 114 & 7 & $\odot$ & 3.934706 & -3.012467 & 2.359955 \\
\hline 115 & 6 & $\odot$ & 2.786620 & -2.407004 & 1.943950 \\
\hline 116 & 6 & $\odot$ & 2.901608 & -1.016455 & 1.988478 \\
\hline 117 & 7 & $\odot$ & 1.957435 & -0.095909 & 1.778042 \\
\hline 118 & 6 & $\odot$ & 0.770902 & -0.635477 & 1.502272 \\
\hline 119 & 7 & $\odot$ & $\odot .559594$ & -1.985224 & 1.381418 \\
\hline 120 & 6 & $\odot$ & 1.528007 & -2.969320 & 1.553121 \\
\hline 121 & 7 & $\odot$ & $-\odot .295050$ & $\odot .153019$ & 1.359431 \\
\hline 122 & 8 & $\odot$ & 1.263240 & -4.161341 & 1.372154 \\
\hline 123 & 1 & $\odot$ & 5.711159 & -2.106913 & 3.125189 \\
\hline 124 & 1 & $\odot$ & -0.117943 & 1.141012 & 1.247979 \\
\hline 125 & 1 & $\odot$ & -1.226380 & -0.221987 & 1.150358 \\
\hline 126 & 1 & $\odot$ & -0.378919 & -2.310308 & 1.069029 \\
\hline 127 & 8 & $\odot$ & 6.081741 & -2.822723 & 1.009057 \\
\hline 128 & 1 & $\odot$ & 5.412044 & -3.446431 & ๑. 688511 \\
\hline
\end{tabular}

1122 basis functions

305 alpha electrons nuclear repulsion energy 16104.7182700115 Hartrees.

NAtoms $=128$ NActive $=128$

Harmonic frequencies $\left(\mathrm{cm}^{*}-1\right)$, IR intensities (KM/Mole), Raman

\begin{tabular}{|c|c|c|c|c|c|c|c|c|c|c|}
\hline & & & $\begin{array}{l}1 \\
\mathrm{~A}\end{array}$ & & $\begin{array}{l}2 \\
A\end{array}$ & & & $\begin{array}{l}3 \\
A\end{array}$ & & \\
\hline Freque & encies & -- & -195.13 & & & 10.591 & & & 4.4284 & \\
\hline Red. n & nasses & -- & 9.193 & & & 8.5639 & & & .5092 & \\
\hline Frc & onsts & -- & 0.206 & & & $\odot .0006$ & & & .0010 & \\
\hline IR Int & ten & -- & 252.629 & & & 4.6651 & & & .2220 & \\
\hline Atom & AN & $x$ & $\mathrm{Y}$ & Z & $x$ & Y & Z & $x$ & Z & \\
\hline 1 & 7 & 0.01 & 0.01 & $\odot .00$ & -0.03 & 0.00 & 0.02 & -0.07 & 0.03 & -0.03 \\
\hline 2 & 6 & $\odot . \odot \odot$ & 0.01 & $\odot . \odot \odot$ & -0.03 & 0.01 & 0.00 & -0.07 & 0.03 & -0.02 \\
\hline 3 & 6 & $-\odot . \odot \odot$ & $0.0 \odot$ & $-\odot . \odot \odot$ & -0.03 & $\odot . \odot \odot$ & $\odot .02$ & -0.07 & $\odot .03$ & -0.02 \\
\hline 4 & 6 & $-0.0 \odot$ & $-\odot .0 \odot$ & $-0.0 \odot$ & -0.03 & -0.01 & 0.05 & -0.07 & 0.03 & -0.03 \\
\hline 5 & 7 & 0.01 & $-\odot .0 \odot$ & $\odot . \odot \odot$ & -0.03 & -0.02 & $\odot .07$ & -0.07 & 0.03 & -0.04 \\
\hline 6 & 8 & $\odot . \odot \odot$ & 0.00 & $-\odot . \odot \odot$ & -0.03 & 0.02 & -0.07 & $-\odot .08$ & 0.01 & ๑. 01 \\
\hline 7 & 15 & $\odot .0 \odot$ & $\odot .0 \odot$ & $-\odot .0 \odot$ & -0.01 & $\odot .02$ & -0.07 & -0.02 & $\odot .01$ & 0.02 \\
\hline 8 & 8 & $\odot .0 \odot$ & 0.00 & -0.00 & -0.02 & 0.02 & -0.03 & -0.01 & -0.05 & 0.05 \\
\hline 9 & 8 & $\odot .00$ & 0.00 & 0.00 & -0.03 & 0.02 & -0.10 & 0.00 & 0.04 & 0.02 \\
\hline 10 & 8 & $\odot .00$ & $\odot .0 \odot$ & $-\odot . \odot \odot$ & 0.02 & $\odot .02$ & -0.07 & -0.01 & 0.03 & 0.02 \\
\hline 11 & 8 & $\odot .0 \odot$ & $-\odot . \odot \odot$ & $-\odot . \odot \odot$ & 0.01 & 0.02 & -0.06 & 0.13 & -0.05 & 0.04 \\
\hline 12 & 6 & $\odot .0 \odot$ & $\odot .0 \odot$ & $-\odot .0 \odot$ & -0.03 & $\odot .02$ & -0.02 & $\odot .05$ & -0.07 & $\odot .04$ \\
\hline 13 & 6 & $\odot .0 \odot$ & 0.00 & -0.00 & 0.00 & 0.02 & -0.05 & $\odot .08$ & -0.04 & $\odot .04$ \\
\hline 14 & 6 & -0.00 & $-0.0 \odot$ & 0.00 & -0.02 & 0.08 & -0.10 & 0.11 & -0.01 & 0.03 \\
\hline 15 & 6 & $-\odot . \odot \odot$ & $-\odot . \odot \odot$ & $-\odot .0 \odot$ & -0.03 & 0.07 & -0.06 & $\odot .09$ & -0.01 & 0.01 \\
\hline 16 & 8 & $-\odot . \odot \odot$ & $0.0 \odot$ & 0.00 & 0.03 & 0.05 & -0.10 & 0.10 & -0.01 & 0.02 \\
\hline 17 & 8 & 0.01 & 0.00 & -0.01 & -0.01 & 0.06 & -0.06 & 0.04 & 0.02 & 0.01 \\
\hline 18 & 15 & $\odot .0 \odot$ & $\odot .0 \odot$ & $-0.0 \odot$ & -0.01 & $\odot .07$ & -0.07 & $\odot .01$ & 0.05 & $\odot .02$ \\
\hline 19 & 8 & $\odot .0 \odot$ & $\odot . \odot \odot$ & -0.00 & 0.01 & $\odot .03$ & -0.05 & -0.02 & 0.03 & $\odot .0 \odot$ \\
\hline 20 & 8 & $\odot .0 \odot$ & 0.00 & -0.00 & -0.05 & 0.08 & -0.06 & -0.02 & 0.05 & $\odot .02$ \\
\hline 21 & 8 & 0.00 & 0.00 & -0.00 & 0.02 & 0.09 & $-\odot . \odot 9$ & $\odot .02$ & 0.07 & 0.05 \\
\hline 22 & 8 & -0.01 & -0.04 & -0.00 & 0.05 & -0.07 & -0.01 & -0.08 & 0.02 & -0.04 \\
\hline 23 & 6 & 0.00 & -0.01 & -0.00 & 0.01 & -0.05 & -0.01 & -0.05 & $\odot . \odot \odot$ & -0.03 \\
\hline 24 & 6 & -0.00 & -0.02 & -0.01 & 0.03 & -0.06 & -0.03 & -0.06 & 0.01 & -0.02 \\
\hline 25 & 1 & -0.01 & -0.04 & -0.00 & 0.07 & -0.07 & -0.02 & -0.08 & 0.02 & -0.03 \\
\hline 26 & 6 & 0.00 & 0.00 & 0.00 & -0.05 & 0.02 & $-\odot . \odot 8$ & -0.03 & 0.02 & 0.09 \\
\hline 27 & 6 & $\odot .0 \odot$ & $\odot .0 \odot$ & $\odot . \odot \odot$ & -0.05 & 0.01 & -0.06 & -0.04 & 0.03 & 0.05 \\
\hline 28 & 6 & $\odot .0 \odot$ & 0.00 & 0.00 & -0.02 & 0.02 & -0.06 & $-\odot . \odot 8$ & $-\odot . \odot \odot$ & 0.03 \\
\hline 29 & 6 & 0.00 & 0.00 & 0.00 & -0.00 & 0.02 & -0.03 & -0.10 & -0.01 & $-0.0 \odot$ \\
\hline 30 & 6 & 0.00 & 0.01 & $\odot .0 \odot$ & -0.03 & 0.01 & -0.01 & -0.07 & 0.03 & -0.02 \\
\hline 31 & 8 & $\odot .0 \odot$ & 0.00 & 0.00 & -0.06 & 0.01 & -0.03 & -0.03 & 0.06 & $\odot .02$ \\
\hline 32 & 1 & $0.0 \odot$ & $\odot .00$ & 0.00 & -0.07 & 0.01 & -0.07 & 0.01 & 0.04 & 0.10 \\
\hline 33 & 1 & $\odot .0 \odot$ & 0.00 & 0.00 & -0.05 & 0.02 & -0.10 & -0.03 & $-\odot .0 \odot$ & 0.13 \\
\hline 34 & 1 & $\odot .0 \odot$ & $0.0 \odot$ & $\odot .00$ & -0.07 & 0.01 & -0.07 & -0.02 & 0.04 & 0.05 \\
\hline 35 & 1 & 0.00 & $-\odot .00$ & $-0.0 \odot$ & -0.00 & 0.02 & -0.09 & -0.09 & -0.03 & 0.06 \\
\hline 36 & 1 & $\odot .0 \odot$ & 0.00 & 0.00 & $\odot .01$ & 0.02 & -0.02 & -0.12 & -0.02 & -0.03 \\
\hline 37 & 1 & 0.01 & 0.00 & 0.00 & 0.01 & 0.02 & -0.03 & -0.11 & -0.02 & 0.01 \\
\hline 38 & 1 & 0.00 & 0.01 & 0.00 & -0.04 & 0.00 & 0.01 & -0.07 & 0.05 & -0.06 \\
\hline 39 & 8 & $\odot .0 \odot$ & 0.00 & 0.00 & -0.03 & 0.02 & -0.07 & -0.06 & -0.01 & $\odot .09$ \\
\hline 40 & 1 & 0.00 & 0.00 & 0.00 & -0.02 & 0.02 & -0.08 & -0.06 & -0.01 & 0.12 \\
\hline 41 & 6 & $\odot .01$ & 0.00 & 0.00 & -0.03 & -0.01 & 0.06 & -0.07 & 0.03 & -0.03 \\
\hline 42 & 7 & -0.01 & -0.01 & -0.01 & -0.03 & -0.02 & 0.07 & -0.07 & 0.03 & -0.04 \\
\hline 43 & 8 & 0.02 & 0.00 & 0.01 & -0.03 & -0.01 & $\odot .07$ & -0.06 & 0.03 & -0.02 \\
\hline
\end{tabular}




\begin{tabular}{|c|c|c|c|c|c|c|c|c|c|c|}
\hline 44 & 1 & $-\odot . \odot \odot$ & $\odot .01$ & $\odot .0 \odot$ & $-\odot .02$ & 0.02 & $-\odot .02$ & -0.07 & $\odot .03$ & $-\odot . \odot \odot$ \\
\hline 45 & 1 & -0.01 & 0.01 & -0.00 & -0.03 & 0.01 & $\odot .01$ & -0.07 & $\odot .03$ & -0.01 \\
\hline 46 & 1 & -0.01 & -0.01 & -0.01 & -0.04 & -0.01 & 0.05 & -0.07 & $\odot .03$ & -0.04 \\
\hline 47 & 1 & -0.01 & $-\odot . \odot 2$ & -0.01 & -0.04 & $-\odot . \odot 2$ & 0.07 & -0.07 & $\odot .03$ & -0.04 \\
\hline 48 & 6 & $\odot .0 \odot$ & $\odot .00$ & -0.00 & -0.05 & 0.03 & -0.02 & 0.01 & -0.06 & 0.05 \\
\hline 49 & 1 & $-\odot . \odot \odot$ & 0.00 & -0.00 & 0.04 & 0.02 & -0.08 & 0.16 & -0.03 & 0.03 \\
\hline 50 & 6 & 0.00 & $-\odot . \odot \odot$ & -0.00 & 0.04 & 0.00 & -0.04 & 0.07 & -0.06 & 0.04 \\
\hline 51 & 6 & $\odot . \odot \odot$ & $\odot .0 \odot$ & $-\odot . \odot \odot$ & 0.03 & $-\odot . \odot \odot$ & $\odot .01$ & 0.06 & $-\odot . \odot 8$ & $\odot .05$ \\
\hline 52 & 8 & $\odot . \odot \odot$ & $-\odot .0 \odot$ & $-\odot . \odot \odot$ & -0.01 & $\odot .0 \odot$ & $\odot .01$ & 0.05 & -0.11 & $\odot .05$ \\
\hline 53 & 1 & $\odot .0 \odot$ & $\odot .0 \odot$ & 0.00 & -0.07 & 0.02 & $\odot . \odot \odot$ & -0.01 & -0.08 & 0.05 \\
\hline 54 & 1 & $\odot .0 \odot$ & $\odot .0 \odot$ & $-0.0 \odot$ & -0.07 & 0.04 & -0.05 & 0.01 & -0.03 & $\odot .04$ \\
\hline 55 & 1 & 0.00 & 0.00 & -0.00 & -0.05 & 0.02 & -0.02 & 0.07 & -0.08 & 0.04 \\
\hline 56 & 1 & $\odot .0 \odot$ & $-0.0 \odot$ & $-\odot . \odot \odot$ & $-\odot . \odot \odot$ & $\odot .03$ & -0.08 & 0.07 & $-\odot . \odot \odot$ & 0.04 \\
\hline 57 & 1 & $\odot .0 \odot$ & $-\odot . \odot \odot$ & -0.00 & $\odot .06$ & $-0.0 \odot$ & -0.05 & 0.08 & -0.06 & 0.04 \\
\hline 58 & 1 & $0.0 \odot$ & $-0.0 \odot$ & -0.00 & $\odot .04$ & 0.00 & -0.04 & 0.06 & -0.05 & 0.05 \\
\hline 59 & 1 & 0.00 & 0.00 & -0.00 & 0.05 & -0.01 & 0.02 & 0.07 & -0.08 & 0.07 \\
\hline 60 & 7 & $\odot .0 \odot$ & $-\odot . \odot \odot$ & $-\odot . \odot \odot$ & $\odot .04$ & -0.01 & 0.03 & 0.05 & -0.07 & 0.03 \\
\hline 61 & 6 & $\odot .0 \odot$ & $\odot .0 \odot$ & $-\odot . \odot \odot$ & 0.05 & -0.01 & $\odot .01$ & 0.05 & $-\odot .08$ & 0.05 \\
\hline 62 & 7 & $\odot . \odot \odot$ & $-0.0 \odot$ & $\odot . \odot \odot$ & 0.05 & -0.01 & $\odot .03$ & 0.05 & -0.07 & $\odot . \odot 4$ \\
\hline 63 & 6 & 0.00 & -0.00 & 0.00 & 0.04 & -0.02 & 0.07 & 0.06 & -0.06 & -0.00 \\
\hline 64 & 6 & $\odot .00$ & -0.00 & $\odot .0 \odot$ & 0.04 & -0.02 & 0.06 & 0.06 & -0.06 & -0.01 \\
\hline 65 & 7 & $\odot . \odot \odot$ & $-0.0 \odot$ & $\odot . \odot \odot$ & $\odot .03$ & -0.02 & $\odot . \odot 9$ & 0.06 & -0.05 & -0.03 \\
\hline 66 & 6 & $-0.0 \odot$ & $-0.0 \odot$ & $\odot .0 \odot$ & $\odot .03$ & -0.03 & 0.12 & 0.06 & -0.04 & -0.05 \\
\hline 67 & 7 & $-0.0 \odot$ & -0.00 & $\odot .0 \odot$ & $\odot .03$ & -0.03 & 0.12 & 0.07 & -0.04 & -0.05 \\
\hline 68 & 6 & $-0.0 \odot$ & $-0.0 \odot$ & $\odot . \odot \odot$ & $\odot .04$ & -0.02 & $\odot . \odot 9$ & 0.06 & -0.05 & -0.03 \\
\hline 69 & 8 & 0.00 & $-\odot . \odot \odot$ & 0.00 & 0.04 & -0.03 & 0.10 & 0.07 & -0.05 & -0.03 \\
\hline 70 & 1 & $\odot .0 \odot$ & $\odot . \odot \odot$ & $-0.0 \odot$ & 0.05 & $\odot .0 \odot$ & -0.03 & $\odot .05$ & -0.08 & $\odot . \odot 8$ \\
\hline 71 & 7 & $-0.0 \odot$ & -0.00 & 0.01 & 0.02 & -0.03 & 0.14 & 0.07 & -0.03 & -0.08 \\
\hline 72 & 1 & $\odot .0 \odot$ & 0.00 & -0.00 & 0.03 & -0.03 & 0.13 & 0.07 & -0.04 & -0.06 \\
\hline 73 & 1 & -0.01 & 0.00 & $\odot . \odot \odot$ & 0.03 & 0.06 & -0.14 & 0.11 & -0.01 & 0.03 \\
\hline 74 & 6 & $-\odot . \odot \odot$ & 0.00 & $-\odot .0 \odot$ & $\odot .02$ & 0.04 & -0.07 & 0.06 & 0.01 & 0.01 \\
\hline 75 & 6 & -0.00 & 0.02 & $-\odot .0 \odot$ & $\odot .06$ & -0.00 & -0.02 & $\odot .06$ & -0.01 & $-0.0 \odot$ \\
\hline 76 & 6 & 0.00 & 0.00 & -0.00 & 0.01 & 0.01 & 0.03 & 0.07 & -0.02 & -0.02 \\
\hline 77 & 8 & $-\odot .0 \odot$ & -0.00 & $-\odot . \odot \odot$ & -0.03 & 0.05 & $-0.0 \odot$ & 0.08 & -0.03 & -0.01 \\
\hline 78 & 1 & 0.00 & -0.00 & 0.00 & -0.05 & 0.10 & -0.09 & 0.13 & -0.02 & 0.02 \\
\hline 79 & 1 & $-\odot . \odot \odot$ & $-\odot . \odot \odot$ & $\odot .0 \odot$ & -0.02 & 0.10 & -0.15 & 0.11 & 0.00 & 0.05 \\
\hline 80 & 1 & $\odot . \odot \odot$ & $-\odot . \odot \odot$ & $-\odot . \odot \odot$ & -0.06 & 0.09 & -0.07 & $\odot .09$ & -0.01 & 0.01 \\
\hline 81 & 1 & $-\odot . \odot \odot$ & 0.00 & 0.00 & 0.06 & 0.06 & -0.11 & 0.07 & 0.03 & 0.03 \\
\hline 82 & 1 & 0.00 & 0.04 & -0.02 & 0.08 & -0.03 & -0.00 & 0.04 & -0.01 & $-0.0 \odot$ \\
\hline 83 & 1 & -0.01 & 0.03 & 0.06 & 0.08 & -0.01 & -0.05 & 0.06 & -0.01 & $-\odot . \odot \odot$ \\
\hline 84 & 1 & -0.00 & 0.00 & -0.01 & 0.01 & 0.00 & 0.07 & 0.06 & -0.02 & -0.03 \\
\hline 85 & 7 & $\odot .0 \odot$ & 0.00 & -0.00 & 0.03 & -0.01 & 0.06 & 0.07 & -0.03 & -0.02 \\
\hline 86 & 6 & 0.00 & 0.00 & 0.01 & 0.04 & -0.00 & 0.03 & 0.07 & -0.03 & -0.01 \\
\hline 87 & 6 & 0.00 & 0.00 & 0.01 & 0.04 & -0.01 & 0.04 & $\odot .08$ & -0.03 & -0.01 \\
\hline 88 & 6 & $\odot . \odot \odot$ & $-\odot .0 \odot$ & $\odot . \odot \odot$ & $\odot . \odot 4$ & -0.03 & $\odot . \odot 9$ & 0.07 & -0.03 & -0.01 \\
\hline 89 & 7 & 0.00 & -0.00 & 0.01 & 0.03 & -0.03 & 0.12 & 0.07 & -0.03 & -0.03 \\
\hline 90 & 6 & $\odot .0 \odot$ & 0.00 & 0.00 & 0.02 & -0.02 & 0.09 & 0.07 & -0.03 & -0.04 \\
\hline 91 & 1 & 0.00 & 0.00 & 0.01 & 0.05 & -0.01 & 0.02 & 0.08 & -0.03 & 0.00 \\
\hline 92 & 7 & $\odot .0 \odot$ & $-\odot . \odot \odot$ & 0.00 & 0.04 & -0.03 & 0.10 & 0.07 & -0.04 & -0.01 \\
\hline 93 & 8 & $-\odot . \odot \odot$ & $\odot . \odot \odot$ & $-\odot . \odot \odot$ & 0.01 & -0.02 & 0.11 & 0.06 & -0.03 & -0.06 \\
\hline 94 & 1 & 0.00 & $\odot .00$ & 0.01 & 0.04 & 0.01 & -0.01 & 0.08 & -0.02 & $\odot .0 \odot$ \\
\hline 95 & 1 & $\odot .00$ & $-\odot . \odot \odot$ & -0.03 & 0.05 & -0.03 & 0.07 & 0.08 & -0.04 & -0.01 \\
\hline 96 & 1 & $\odot .0 \odot$ & $-\odot . \odot \odot$ & -0.01 & $\odot .04$ & -0.04 & 0.11 & 0.07 & -0.04 & $-\odot .02$ \\
\hline 97 & 1 & -0.00 & -0.00 & $\odot .01$ & $\odot .02$ & -0.03 & 0.14 & 0.06 & $-\odot .03$ & -0.07 \\
\hline 98 & 1 & $-0.0 \odot$ & $\odot .0 \odot$ & -0.00 & $\odot .02$ & -0.03 & 0.14 & 0.07 & -0.03 & -0.07 \\
\hline 99 & 6 & 0.01 & 0.00 & 0.00 & -0.02 & -0.00 & -0.02 & -0.03 & 0.02 & -0.02 \\
\hline 100 & 11 & -0.00 & 0.00 & $-0.0 \odot$ & 0.02 & 0.03 & -0.08 & $\odot .02$ & 0.11 & 0.08 \\
\hline 101 & 11 & $-0.0 \odot$ & $-\odot . \odot \odot$ & $\odot .0 \odot$ & -0.02 & 0.12 & $-\odot .09$ & -0.02 & 0.12 & 0.05 \\
\hline 102 & 6 & 0.02 & -0.03 & -0.03 & 0.03 & -0.06 & -0.03 & -0.07 & 0.01 & -0.02 \\
\hline 103 & 6 & 0.04 & -0.07 & -0.07 & 0.02 & -0.05 & -0.02 & -0.06 & 0.01 & -0.04 \\
\hline 104 & 8 & 0.01 & $-\odot . \odot \odot$ & -0.02 & $\odot .02$ & -0.06 & -0.02 & -0.03 & $-0.0 \odot$ & -0.05 \\
\hline 105 & 1 & 0.01 & ๑. 01 & 0.00 & -0.03 & $-\odot .0 \odot$ & -0.01 & -0.02 & 0.01 & $-\odot .03$ \\
\hline 106 & 1 & 0.01 & 0.00 & 0.01 & -0.04 & $\odot .0 \odot$ & -0.01 & -0.05 & 0.02 & -0.01 \\
\hline 107 & 1 & -0.00 & -0.01 & 0.00 & -0.01 & -0.06 & 0.02 & -0.06 & -0.00 & -0.04 \\
\hline 108 & 1 & $-\odot .0 \odot$ & -0.02 & -0.00 & 0.03 & -0.06 & -0.05 & -0.06 & 0.01 & -0.01 \\
\hline 109 & 1 & $\odot .0 \odot$ & -0.04 & $-\odot .04$ & 0.04 & -0.07 & -0.03 & $-\odot . \odot 9$ & 0.02 & -0.02 \\
\hline 110 & 1 & 0.05 & -0.02 & -0.02 & 0.02 & -0.06 & -0.04 & -0.06 & $\odot .0 \odot$ & -0.00 \\
\hline 111 & 1 & 0.00 & -0.13 & -0.06 & 0.03 & -0.05 & -0.01 & -0.07 & 0.02 & -0.06 \\
\hline 112 & 7 & $\odot .08$ & -0.07 & -0.14 & 0.01 & -0.05 & -0.01 & -0.06 & 0.01 & -0.04 \\
\hline 113 & 6 & 0.12 & -0.11 & -0.23 & 0.01 & -0.05 & -0.04 & -0.07 & 0.01 & -0.04 \\
\hline 114 & 7 & -0.10 & -0.09 & $\odot .02$ & $-\odot . \odot \odot$ & -0.05 & $-\odot .02$ & -0.07 & 0.01 & -0.03 \\
\hline 115 & 6 & -0.01 & -0.05 & 0.04 & -0.01 & -0.04 & 0.03 & -0.07 & 0.02 & -0.03 \\
\hline 116 & 6 & 0.05 & -0.03 & -0.04 & -0.01 & -0.04 & 0.03 & -0.06 & 0.02 & -0.04 \\
\hline 117 & 7 & 0.06 & -0.04 & -0.03 & -0.01 & -0.04 & 0.07 & -0.06 & 0.02 & -0.03 \\
\hline 118 & 6 & 0.02 & -0.02 & -0.00 & $-\odot .02$ & -0.04 & 0.09 & -0.06 & 0.02 & -0.03 \\
\hline 119 & 7 & -0.01 & -0.02 & 0.01 & -0.03 & -0.04 & 0.09 & -0.07 & 0.02 & -0.03 \\
\hline 120 & 6 & -0.03 & -0.03 & $\odot .02$ & -0.03 & -0.04 & 0.07 & -0.07 & 0.02 & -0.03 \\
\hline
\end{tabular}




$\begin{array}{rrrrc}121 & 7 & 0.05 & -0.01 & -0.00 \\ 122 & 8 & -0.02 & -0.02 & 0.01 \\ 123 & 1 & -0.17 & -0.09 & 0.28 \\ 124 & 1 & 0.06 & -0.00 & 0.03 \\ 125 & 1 & 0.03 & 0.01 & 0.04 \\ 126 & 1 & -0.02 & -0.00 & -0.00 \\ 127 & 8 & -0.26 & 0.48 & 0.34 \\ 128 & 1 & -0.12 & 0.49 & 0.05\end{array}$

$\begin{array}{ccc}-0.02 & -0.03 & 0.12 \\ -0.04 & -0.04 & 0.07 \\ 0.03 & -0.06 & -0.08 \\ -0.02 & -0.03 & 0.11 \\ -0.02 & -0.03 & 0.12 \\ -0.03 & -0.03 & 0.10 \\ -0.07 & -0.05 & -0.11 \\ -0.09 & -0.05 & -0.08\end{array}$

$\begin{array}{ccc}-0.06 & 0.03 & -0.02 \\ -0.08 & 0.02 & -0.03 \\ -0.07 & 0.00 & -0.04 \\ -0.06 & 0.03 & -0.00 \\ -0.07 & 0.03 & -0.02 \\ -0.07 & 0.03 & -0.03 \\ -0.08 & -0.01 & -0.03 \\ -0.09 & -0.00 & -0.03\end{array}$

Zero-point correction=

Thermal correction to Energy=

$$
\begin{aligned}
0.983753 \text { (a.u. ) } \\
1.057224 \\
1.058169 \\
0.870294 \\
-4938.392509 \\
-4938.319038 \\
-4938.318094 \\
-4938.505968
\end{aligned}
$$

Sum of electronic and thermal Free Energies=

$G($ high $)=-4940.225331 \mathrm{a} \cdot \mathrm{u}$.

Total

Electronic

Translational

Rotational

Vibrational

Item

Maximum Force

RMS Force

Maximum Displacement

RMS Displacement
CV

Cal/Mol-Kelvin 278.943

0.000

2.981

2.981

272.982
$\mathrm{S}$

Cal/Mol-Kelvin

395.415

1.377

47.058

\begin{tabular}{|c|c|c|c|c|c|}
\hline \multirow{3}{*}{$\begin{array}{l}\text { Center } \\
\text { Number }\end{array}$} & \multirow{2}{*}{$\begin{array}{l}\text { Atomic } \\
\text { Number }\end{array}$} & \multirow{2}{*}{$\begin{array}{l}\text { Atomic } \\
\text { Type }\end{array}$} & \multicolumn{3}{|c|}{ Coordinates (Angstroms) } \\
\hline & & & $\mathrm{X}$ & Y & Z \\
\hline & & & - - - - - - - - & -------1 & 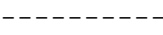 \\
\hline 1 & 7 & $\odot$ & -4.364433 & -2.544591 & 0.590066 \\
\hline 2 & 6 & $\odot$ & -4.518678 & -3.872777 & $\odot .315065$ \\
\hline 3 & 6 & $\odot$ & -3.448824 & -4.688664 & $\odot .192294$ \\
\hline 4 & 6 & $\odot$ & -2.161708 & -4.115179 & 0.438474 \\
\hline 5 & 7 & $\odot$ & -2.013954 & -2.813929 & ๑. 692099 \\
\hline 6 & 8 & $\odot$ & -8.283732 & -0.297851 & ๑. 992808 \\
\hline 7 & 15 & $\odot$ & -8.193621 & 1.129700 & $\odot .225020$ \\
\hline 8 & 8 & $\odot$ & -6.652569 & 1.582782 & $\odot .504690$ \\
\hline 9 & 8 & $\odot$ & -9.148628 & 2.086108 & ๑. 901725 \\
\hline 10 & 8 & 0 & -8.338506 & 0.963705 & -1.275374 \\
\hline 11 & 8 & $\odot$ & -6.485600 & 5.909777 & ๑. 218217 \\
\hline 12 & 6 & $\odot$ & -5.760178 & 3.792563 & 1.025147 \\
\hline 13 & 6 & $\odot$ & -6.721968 & 4.488094 & ๑. . 060321 \\
\hline 14 & 6 & $\odot$ & 7.454245 & -2.319250 & -3.237588 \\
\hline 15 & 6 & $\odot$ & 7.188145 & $-\odot .861595$ & -2.921469 \\
\hline 16 & 8 & $\odot$ & 6.893639 & -3.135415 & -2.177964 \\
\hline 17 & 8 & $\odot$ & 8. 029912 & $\odot .900427$ & -1.502534 \\
\hline 18 & 15 & $\odot$ & 8.898214 & 1.501635 & -0.253063 \\
\hline 19 & 8 & $\odot$ & 7.740254 & 1.889073 & ๑. 831461 \\
\hline 20 & 8 & $\odot$ & 9.597205 & 2.741872 & -0.756171 \\
\hline 21 & 8 & $\odot$ & 9.752809 & $\odot .425599$ & $\odot .374478$ \\
\hline 22 & 8 & $\odot$ & 5.323456 & 3.787479 & 3.736696 \\
\hline 23 & 6 & $\odot$ & 5.667641 & 2.908044 & 1.540958 \\
\hline 24 & 6 & $\odot$ & 6.104717 & 2.798880 & 3.015717 \\
\hline 25 & 1 & $\odot$ & 5.576211 & 3.798965 & 4.675538 \\
\hline 26 & 6 & $\odot$ & -8.119812 & -3.949716 & 1.493305 \\
\hline 27 & 6 & $\odot$ & -7.703038 & -2.505712 & 1.677735 \\
\hline 28 & 6 & $\odot$ & -7.917331 & -1.610171 & ○. 451686 \\
\hline 29 & 6 & 0 & -6.562051 & -1.630789 & -0.251351 \\
\hline 30 & 6 & $\odot$ & -5.554327 & -1.747037 & $\odot .893509$ \\
\hline
\end{tabular}

42.126

304.853

Normal termination of Gaussian 16 at Thu Jan 17 12:23:01 2019.

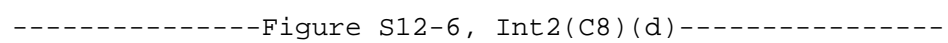

dna@4d1-ohrev. $\log$

Stoichiometry C38H49N16Na2021P2(2)

Standard orientation: 


\begin{tabular}{|c|c|c|c|c|c|}
\hline 31 & 8 & 0 & -6.270415 & -2.410629 & 1.977128 \\
\hline 32 & 1 & $\odot$ & -7.743735 & -4.539534 & 2.335527 \\
\hline 33 & 1 & $\odot$ & -9.214283 & -4.003563 & 1.484187 \\
\hline 34 & 1 & $\odot$ & -8.241190 & -2.093574 & 2.533740 \\
\hline 35 & 1 & $\odot$ & -8.728108 & -1.952376 & -0.192485 \\
\hline 36 & 1 & $\odot$ & -6.373468 & $-\odot .759619$ & -0.873066 \\
\hline 37 & 1 & $\odot$ & -6.528077 & -2.520321 & -0.884527 \\
\hline 38 & 1 & $\odot$ & -5.201277 & $-\odot .774246$ & 1.229987 \\
\hline 39 & 8 & $\odot$ & -7.583393 & -4.444262 & 0.240764 \\
\hline $4 \odot$ & 1 & $\odot$ & -7.852612 & -5.364084 & 0.084785 \\
\hline 41 & 6 & $\odot$ & -3.087994 & -1.985715 & 0.748066 \\
\hline 42 & 7 & $\odot$ & -1.062467 & -4.880171 & 0.420685 \\
\hline 43 & 8 & $\odot$ & -2.976260 & -0.768705 & 0.947692 \\
\hline 44 & 1 & $\odot$ & -5.547766 & -4.218266 & $\odot .225279$ \\
\hline 45 & 1 & $\odot$ & -3.568212 & -5.741250 & -0.024780 \\
\hline 46 & 1 & $\odot$ & -1.164052 & -5.879045 & 0.342582 \\
\hline 47 & 1 & $\odot$ & $-\odot .180520$ & -4.511877 & 0.792582 \\
\hline 48 & 6 & $\odot$ & -6.249101 & 2.471333 & 1.585501 \\
\hline 49 & 1 & $\odot$ & -7.058916 & 6.415860 & -0.382252 \\
\hline 50 & 6 & $\odot$ & -6.293818 & 3.987650 & -1.328454 \\
\hline 51 & 6 & $\odot$ & -4.809785 & 3.614325 & -1.169259 \\
\hline 52 & 8 & $\odot$ & -4.532781 & 3.606412 & 0.258047 \\
\hline 53 & 1 & $\odot$ & -5.437482 & 1.975810 & 2.119565 \\
\hline 54 & 1 & $\odot$ & -7.089038 & 2.642671 & 2.263545 \\
\hline 55 & 1 & $\odot$ & -5.519005 & 4.459924 & 1.855988 \\
\hline 56 & 1 & $\odot$ & -7.765758 & 4.241825 & 0.279290 \\
\hline 57 & 1 & $\odot$ & -6.432142 & 4.757314 & -2.090346 \\
\hline 58 & 1 & $\odot$ & -6.900947 & 3.125206 & -1.600917 \\
\hline 59 & 1 & $\odot$ & -4.138360 & 4.354002 & -1.606368 \\
\hline 60 & 7 & $\odot$ & -4.451809 & 2.324481 & -1.741407 \\
\hline 61 & 6 & $\odot$ & -5.252639 & 1.256230 & -2.100634 \\
\hline 62 & 7 & $\odot$ & -4.574754 & $\odot .174639$ & -2.372140 \\
\hline 63 & 6 & $\odot$ & -3.256503 & $\odot .53202 \odot$ & -2.163501 \\
\hline 64 & 6 & $\odot$ & -3.160870 & 1.858184 & -1.777234 \\
\hline 65 & 7 & $\odot$ & -2.053972 & 2.564764 & -1.466638 \\
\hline 66 & 6 & $\odot$ & $-\odot .957993$ & 1.832045 & -1.521279 \\
\hline 67 & 7 & $\odot$ & $-\odot .945806$ & $\odot .503904$ & -1.858714 \\
\hline 68 & 6 & $\odot$ & -2.072120 & -0.258339 & -2.165489 \\
\hline 69 & 8 & $\odot$ & -1.970254 & -1.473523 & -2.383128 \\
\hline 70 & 1 & $\odot$ & -6.332554 & 1.316467 & -2.115959 \\
\hline 71 & 7 & 0 & $\odot .231815$ & 2.368944 & -1.169263 \\
\hline 72 & 1 & $\odot$ & $-\odot .043915$ & -0.011339 & -1.805966 \\
\hline 73 & 1 & $\odot$ & 7.052881 & -4.078902 & -2.341736 \\
\hline 74 & 6 & $\odot$ & 7.517939 & -0.469659 & -1.476657 \\
\hline 75 & 6 & $\odot$ & 6.176020 & $-\odot .550851$ & -0.756817 \\
\hline 76 & 6 & $\odot$ & 5.146680 & -0.202252 & -1.832360 \\
\hline 77 & 8 & $\odot$ & 5.766410 & $-\odot .557058$ & -3.102119 \\
\hline 78 & 1 & $\odot$ & 6.997542 & -2.567097 & -4.201010 \\
\hline 79 & 1 & $\odot$ & 8.536355 & -2.478452 & -3.305742 \\
\hline 80 & 1 & $\odot$ & 7.751457 & -0.236093 & -3.617467 \\
\hline 81 & 1 & $\odot$ & 8.273490 & -1.117011 & -1.028920 \\
\hline 82 & 1 & $\odot$ & 6.098053 & $\odot .111591$ & $\odot .105927$ \\
\hline 83 & 1 & $\odot$ & 6.037073 & -1.589269 & -0.448805 \\
\hline 84 & 1 & $\odot$ & 4.889897 & $\odot .854381$ & -1.838283 \\
\hline 85 & 7 & $\odot$ & 3.888316 & $-\odot .932420$ & -1.707201 \\
\hline 86 & 6 & $\Theta$ & 3.909432 & -2.299277 & -1.698202 \\
\hline 87 & 6 & $\odot$ & 2.767060 & -3.015725 & -1.641856 \\
\hline 88 & 6 & $\odot$ & 1.536595 & -2.282915 & -1.660863 \\
\hline 89 & 7 & $\odot$ & 1.522369 & -0.946284 & -1.698615 \\
\hline 90 & 6 & $\odot$ & 2.672990 & $-\odot .230656$ & -1.729519 \\
\hline 91 & 1 & $\odot$ & 2.781093 & -4.096703 & -1.630570 \\
\hline 92 & 7 & $\odot$ & 0.367424 & -2.917358 & -1.659205 \\
\hline 93 & 8 & $\odot$ & 2.682100 & 1.005933 & -1.778458 \\
\hline 94 & 1 & $\odot$ & 4.895367 & -2.755470 & -1.770941 \\
\hline 95 & 1 & $\odot$ & $\odot .331817$ & -3.921325 & -1.593209 \\
\hline 96 & 1 & $\odot$ & $-\odot .506621$ & -2.398107 & -1.794622 \\
\hline 97 & 1 & $\odot$ & $\odot .248530$ & 3.372115 & -1.079598 \\
\hline 98 & 1 & $\odot$ & 1.107473 & 1.890980 & -1.404130 \\
\hline 99 & 6 & $\odot$ & 6.791253 & 2.955270 & 0.536963 \\
\hline 100 & 11 & $\odot$ & -10.108559 & 2.701612 & -1.370332 \\
\hline 101 & 11 & $\odot$ & 11.800262 & 1.770345 & 0.014398 \\
\hline 102 & 6 & 0 & 5.718246 & 1.370697 & 3.413608 \\
\hline 103 & 6 & 0 & 4.477419 & 1.128152 & 2.557802 \\
\hline 104 & 8 & 0 & 4.841349 & 1.732851 & 1.269924 \\
\hline 105 & 1 & 0 & 6.388918 & 2.821393 & -0.471852 \\
\hline 106 & 1 & 0 & 7.307420 & 3.917795 & $\odot .593765$ \\
\hline 107 & 1 & $\odot$ & 5.057056 & 3.810397 & 1.430553 \\
\hline
\end{tabular}




$\begin{array}{rrrrrr}108 & 1 & 0 & 7.173564 & 2.987969 & 3.143619 \\ 109 & 1 & 0 & 5.527755 & 1.273591 & 4.484380 \\ 110 & 1 & 0 & 6.509435 & 0.679235 & 3.117171 \\ 111 & 1 & 0 & 3.612867 & 1.675695 & 2.948919 \\ 112 & 7 & 0 & 4.104229 & -0.236651 & 2.350932 \\ 113 & 6 & 0 & 4.813572 & -1.451018 & 2.832840 \\ 114 & 7 & 0 & 3.955667 & -2.564632 & 2.491878 \\ 115 & 6 & 0 & 2.807544 & -2.024599 & 2.062452 \\ 116 & 6 & 0 & 2.853056 & -0.605706 & 1.993972 \\ 117 & 7 & 0 & 1.868777 & 0.224762 & 1.677570 \\ 118 & 6 & 0 & 0.705164 & -0.392517 & 1.432531 \\ 119 & 7 & 0 & 0.563538 & -1.746940 & 1.408817 \\ 120 & 6 & 0 & 1.584990 & -2.662990 & 1.670337 \\ 121 & 7 & 0 & -0.384141 & 0.343195 & 1.226219 \\ 122 & 8 & 0 & 1.375956 & -3.879705 & 1.565118 \\ 123 & 1 & 0 & 4.934985 & -1.369354 & 3.922768 \\ 124 & 1 & 0 & -0.244016 & 1.333781 & 1.098201 \\ 125 & 1 & 0 & -1.307830 & -0.072565 & 1.057044 \\ 126 & 1 & 0 & -0.358713 & -2.139950 & 1.130712 \\ 127 & 8 & 0 & 6.104773 & -1.585308 & 2.333579 \\ 128 & 1 & 0 & 6.026501 & -1.939888 & 1.441096 \\ -\ldots-\ldots \ldots \ldots-\ldots & -\ldots-\ldots\end{array}$

1122 basis functions

305 alpha electrons 304 beta electrons

nuclear repulsion energy 16148.7032771981 Hartrees.

NAtoms $=128$ NActive $=128$

Harmonic frequencies $\left(\mathrm{cm}^{* *}-1\right)$, IR intensities (KM/Mole), Raman

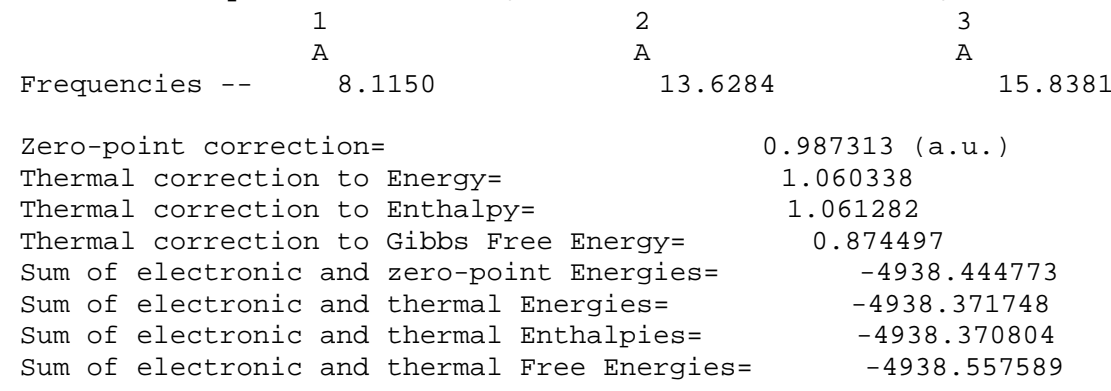

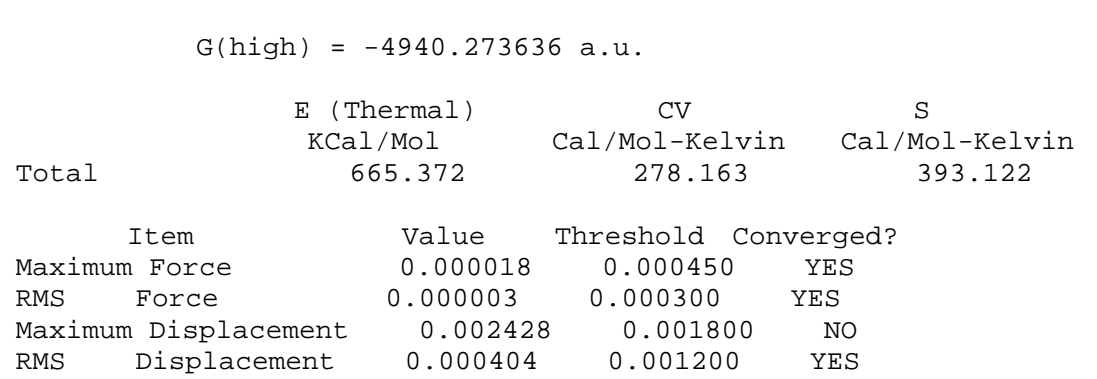

Normal termination of Gaussian 16 at Tue Jan 22 05:28:50 2019.

$============$ Figure $\mathrm{S} 13$ for $(\mathrm{b}) 5^{\prime}-\mathrm{GG}-3^{\prime}$ ======================

dna.for1bw2x. $\log$

Stoichiometry C38H53N16Na2023P2(2)

Standard orientation:

\begin{tabular}{|c|c|c|c|c|c|}
\hline \multirow{2}{*}{$\begin{array}{l}\text { Center } \\
\text { Number }\end{array}$} & \multirow{2}{*}{$\begin{array}{l}\text { Atomic } \\
\text { Number }\end{array}$} & \multirow{2}{*}{$\begin{array}{l}\text { Atomic } \\
\text { Type }\end{array}$} & \multicolumn{3}{|c|}{ Coordinates (Angstroms) } \\
\hline & & & $x$ & $Y$ & Z \\
\hline 1 & 7 & $\odot$ & 4.887174 & -1.909495 & -1.412182 \\
\hline 2 & 6 & $\odot$ & 4.790257 & -3.190169 & -0.941737 \\
\hline 3 & 6 & $\odot$ & 3.588956 & -3.795800 & -0.803026 \\
\hline 4 & 6 & 0 & 2.428486 & -3.060631 & -1.231230 \\
\hline 5 & 7 & $\odot$ & 2.549750 & -1.841230 & -1.766274 \\
\hline 6 & 8 & 0 & 8.505159 & 0.450294 & -0.772133 \\
\hline 7 & 15 & 0 & 8.058536 & 1.707001 & 0.184194 \\
\hline 8 & 8 & 0 & 6.463550 & 1.887925 & $-\odot .185065$ \\
\hline
\end{tabular}




\begin{tabular}{|c|c|c|c|c|c|}
\hline 9 & 8 & $\odot$ & 8.820354 & 2.914806 & -0.269682 \\
\hline 10 & 8 & $\odot$ & 8.061347 & 1.246113 & 1.624809 \\
\hline 11 & 8 & $\odot$ & 4.733599 & 5.695298 & $\odot .775157$ \\
\hline 12 & 6 & $\odot$ & 4.923173 & 3.746105 & -0.575354 \\
\hline 13 & 6 & $\odot$ & 5.351252 & 4.384605 & $\odot .748174$ \\
\hline 14 & 6 & $\odot$ & -8.001510 & -3.704852 & $-\odot .736 \odot 49$ \\
\hline 15 & 6 & $\odot$ & -7.938877 & -2.459393 & 0.121156 \\
\hline 16 & 8 & $\odot$ & -6.734143 & -3.899682 & -1.420391 \\
\hline 17 & 8 & $\odot$ & -8.030454 & $-\odot .053748$ & -0.093434 \\
\hline 18 & 15 & $\odot$ & -7.787327 & 1.410104 & -0.794473 \\
\hline 19 & 8 & $\odot$ & -6.303908 & 1.841802 & -0.263826 \\
\hline 20 & 8 & $\odot$ & -8.815669 & 2.325250 & -0.173985 \\
\hline 21 & 8 & $\odot$ & -7.642726 & 1.252521 & -2.282006 \\
\hline 22 & 8 & $\odot$ & -4.574310 & 5.715568 & 0.099419 \\
\hline 23 & 6 & $\odot$ & -4.911540 & 3.473093 & $\odot .816731$ \\
\hline 24 & 6 & $\odot$ & -5.227643 & 4.482012 & -0.289734 \\
\hline 25 & 1 & $\odot$ & -4.695750 & 6.395247 & -0.585265 \\
\hline 26 & 6 & $\odot$ & 8.924561 & -3.197503 & -1.499672 \\
\hline 27 & 6 & $\odot$ & 8.445085 & -1.773813 & -1.700961 \\
\hline 28 & 6 & $\odot$ & 8.301162 & $-\odot .956003$ & -0.409639 \\
\hline 29 & 6 & $\odot$ & 6.856240 & -1.216927 & -0.012529 \\
\hline 30 & 6 & $\odot$ & 6.151482 & -1.195946 & -1.366879 \\
\hline 31 & 8 & $\odot$ & 7.101534 & -1.795270 & -2.294746 \\
\hline 32 & 1 & $\odot$ & 8.859675 & -3.726332 & -2.456044 \\
\hline 33 & 1 & $\odot$ & 9.971460 & -3.183765 & -1.174818 \\
\hline 34 & 1 & $\odot$ & 9.114761 & -1.264422 & -2.397419 \\
\hline 35 & 1 & $\odot$ & 9.022541 & -1.234184 & 0.359696 \\
\hline 36 & 1 & $\odot$ & 6.478521 & -0.463390 & 0.669433 \\
\hline 37 & 1 & $\odot$ & 6.799501 & -2.200848 & 0.455028 \\
\hline 38 & 1 & $\odot$ & 5.934155 & $-\odot .177762$ & -1.680928 \\
\hline 39 & 8 & $\odot$ & 8.092216 & -3.842184 & -0.502971 \\
\hline 40 & 1 & $\odot$ & 8.318807 & -4.782526 & -0.420551 \\
\hline 41 & 6 & $\odot$ & 3.745232 & -1.216585 & -1.848323 \\
\hline 42 & 7 & $\odot$ & 1.205042 & -3.574151 & -1.146143 \\
\hline 43 & 8 & $\odot$ & 3.851602 & $-\odot .056668$ & -2.267242 \\
\hline 44 & 1 & $\odot$ & 5.736501 & -3.669149 & -0.701974 \\
\hline 45 & 1 & $\odot$ & 3.509399 & -4.810162 & -0.436911 \\
\hline 46 & 1 & $\odot$ & $\odot .960479$ & -4.341973 & -0.519613 \\
\hline 47 & 1 & 0 & $\odot .424358$ & -3.038668 & -1.530561 \\
\hline 48 & 6 & 0 & 5.939307 & 2.800199 & -1.190773 \\
\hline 49 & 1 & $\odot$ & 4.961116 & 6.160495 & 1.598166 \\
\hline 50 & 6 & $\odot$ & 4.755884 & 3.458472 & 1.814875 \\
\hline 51 & 6 & $\odot$ & 3.465518 & 2.930709 & 1.168890 \\
\hline 52 & 8 & $\odot$ & 3.672919 & 3.043201 & $-\odot .270016$ \\
\hline 53 & 1 & $\odot$ & 5.458126 & 2.199660 & -1.965332 \\
\hline 54 & 1 & $\odot$ & 6.763818 & 3.376238 & -1.618625 \\
\hline 55 & 1 & $\odot$ & 4.682946 & 4.524923 & -1.302653 \\
\hline 56 & 1 & 0 & 6.439918 & 4.465698 & 0.828235 \\
\hline 57 & 1 & $\odot$ & 4.551173 & 3.984556 & 2.749249 \\
\hline 58 & 1 & $\odot$ & 5.461307 & 2.650403 & 2. 019788 \\
\hline 59 & 1 & 0 & 2.590165 & 3.515682 & 1.439927 \\
\hline 60 & 7 & $\odot$ & 3.147890 & 1.540970 & 1.487796 \\
\hline 61 & 6 & $\odot$ & 4.084455 & $\odot .582526$ & 1.242641 \\
\hline 62 & 6 & $\odot$ & 3.823785 & -0.723046 & 1.459812 \\
\hline 63 & 6 & $\odot$ & 2.505281 & -1.063294 & 1.894845 \\
\hline 64 & 7 & $\odot$ & 1.567315 & -0.130282 & $2.07 \odot 726$ \\
\hline 65 & 6 & $\odot$ & 1.836687 & 1.183885 & 1.851611 \\
\hline 66 & 1 & $\odot$ & 4.567738 & -1.488210 & 1.297425 \\
\hline 67 & 7 & $\odot$ & 2.192982 & -2.337611 & 2.126163 \\
\hline 68 & 8 & $\odot$ & 0.976969 & 2.062398 & 1.976844 \\
\hline 69 & 1 & $\odot$ & 5.030487 & $\odot .945066$ & $\odot .849630$ \\
\hline 70 & 1 & $\odot$ & 2.879548 & -3.056522 & 1.965812 \\
\hline 71 & 1 & $\odot$ & 1.253150 & -2.606184 & 2.432065 \\
\hline 72 & 1 & $\odot$ & -0.177882 & $-\odot .676969$ & 2.310358 \\
\hline 73 & 1 & 0 & -6.854924 & -4.344157 & -2.273827 \\
\hline 74 & 6 & $\odot$ & -7.317457 & -1.239959 & -0.574679 \\
\hline 75 & 6 & $\odot$ & -5.880438 & -1.227701 & $-\odot .070523$ \\
\hline 76 & 6 & $\odot$ & -6.036155 & -1.740543 & 1.356936 \\
\hline 77 & 8 & $\odot$ & -7.090216 & -2.721756 & 1.299275 \\
\hline 78 & 1 & $\odot$ & -8.220317 & -4.571607 & -0.106263 \\
\hline 79 & 1 & 0 & -8.802394 & -3.587825 & -1.471724 \\
\hline 80 & 1 & 0 & -8.941258 & -2.218376 & 0.481964 \\
\hline 81 & 1 & $\odot$ & -7.392392 & -1.287094 & -1.661702 \\
\hline 82 & 1 & 0 & -5.419267 & -0.245340 & -0.113562 \\
\hline 83 & 1 & $\odot$ & -5.275297 & -1.932345 & -0.643374 \\
\hline 84 & 1 & $\odot$ & -6.308639 & -0.942752 & 2.054715 \\
\hline 85 & 7 & 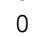 & -4.827022 & -2.388513 & 1.838328 \\
\hline
\end{tabular}




\begin{tabular}{|c|c|c|c|c|c|}
\hline 86 & 6 & 0 & -4.616290 & -3.702655 & 2.192049 \\
\hline 87 & 7 & 0 & -3.367093 & -3.968028 & 2.474596 \\
\hline 88 & 6 & 0 & -2.712114 & -2.768756 & 2.267858 \\
\hline 89 & 6 & 0 & -3.619733 & -1.763265 & 1.902331 \\
\hline 90 & 7 & 0 & -3.399562 & $-\odot .468593$ & 1.677781 \\
\hline 91 & 6 & 0 & -2.122827 & $-\odot .126747$ & 1.851385 \\
\hline 92 & 7 & $\odot$ & -1.159800 & -1.015861 & 2.271541 \\
\hline 93 & 6 & 0 & -1.359178 & -2.368381 & 2.506219 \\
\hline 94 & 8 & $\odot$ & $-\odot .420115$ & -3.090619 & 2.877303 \\
\hline 95 & 1 & 0 & -5.439404 & -4.400446 & 2.221353 \\
\hline 96 & 7 & 0 & -1.750193 & 1.132670 & 1.647851 \\
\hline 97 & 1 & 0 & -2.449577 & 1.772521 & 1.263426 \\
\hline 98 & 1 & 0 & $-\odot .779554$ & 1.435453 & 1.753061 \\
\hline 99 & 6 & 0 & -5.977216 & 2.414992 & 1.023561 \\
\hline 100 & 11 & 0 & 7.119707 & 0.281943 & 3.367002 \\
\hline 101 & 11 & 0 & -9.414613 & 4.393867 & 0.254778 \\
\hline 102 & 6 & 0 & -4.595669 & 3.873157 & -1.547229 \\
\hline 103 & 6 & $\odot$ & -3.381931 & 3.100326 & -1.019627 \\
\hline 104 & 8 & 0 & -3.649272 & 2.858040 & $\odot .399940$ \\
\hline 105 & 1 & $\odot$ & -5.591028 & 1.624570 & 1.672976 \\
\hline 106 & 1 & $\odot$ & -6.870418 & 2.862512 & 1.471040 \\
\hline 107 & 1 & 0 & -4.721746 & 3.991083 & 1.758902 \\
\hline 108 & 1 & $\odot$ & -6.304617 & 4.634882 & $-\odot .407443$ \\
\hline 109 & 1 & $\odot$ & -4.299472 & 4.637753 & -2.268021 \\
\hline 110 & 1 & 0 & -5.313758 & 3.203740 & -2.020594 \\
\hline 111 & 1 & $\odot$ & -2.459344 & 3.678695 & -1.073680 \\
\hline 112 & 7 & $\odot$ & $-3.120 \odot 26$ & 1.841360 & -1.675703 \\
\hline 113 & 6 & 0 & -4.019189 & 0.871863 & -2.091586 \\
\hline 114 & 7 & 0 & -3.442440 & -0.250805 & -2.417141 \\
\hline 115 & 6 & $\odot$ & -2.094296 & $-\odot .028915$ & -2.195270 \\
\hline 116 & 6 & 0 & -1.874600 & 1.260708 & -1.736672 \\
\hline 117 & 7 & $\odot$ & -0.712235 & 1.866564 & -1.422402 \\
\hline 118 & 6 & 0 & $\odot .326616$ & 1.060593 & -1.573573 \\
\hline 119 & 7 & 0 & 0.213782 & -0.244174 & -1.978775 \\
\hline 120 & 6 & $\odot$ & $-\odot .970670$ & -0.903454 & -2.305606 \\
\hline 121 & 7 & $\odot$ & 1.574496 & 1.482008 & -1.318518 \\
\hline 122 & 8 & 0 & -0.941303 & -2.097994 & -2.624930 \\
\hline 123 & 1 & $\odot$ & -5.083056 & 1.061924 & -2.120056 \\
\hline 124 & 1 & 0 & 1.738757 & 2.438287 & -1.047076 \\
\hline 125 & 1 & 0 & 2.387656 & 0.952337 & -1.632861 \\
\hline 126 & 1 & $\odot$ & 1.076054 & $-\odot .823756$ & -1.994979 \\
\hline 127 & 8 & 0 & -1.960537 & -2.831223 & -0.007707 \\
\hline 128 & 1 & 0 & -2.807074 & -3.207385 & -0.341387 \\
\hline 129 & 8 & $\odot$ & -4.154972 & -4.351293 & -0.779656 \\
\hline 130 & 1 & $\odot$ & -4.098665 & -4.939671 & -0.020866 \\
\hline 131 & 1 & 0 & -5.111984 & -4.166002 & -0.893779 \\
\hline 132 & 1 & $\odot$ & -1.031251 & -4.333208 & 0.365570 \\
\hline 133 & 8 & $\odot$ & $-\odot .405752$ & -5.010322 & 0.704488 \\
\hline 134 & 1 & 0 & -0.231131 & -4.658112 & 1.589187 \\
\hline
\end{tabular}

General basis read from cards: (6D, 7F)

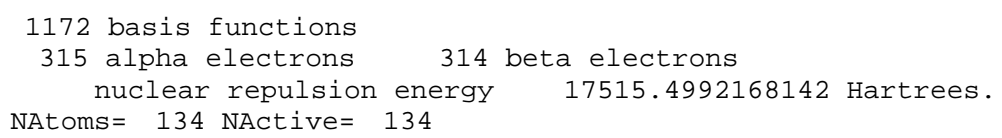

Force inversion solution in PCM.

Polarizable Continuum Model (PCM)

=ニニ=ニニニニニニニニニニニニニニニニニニニニニニニニニニニニ=

Model : PCM.

Solvent : Water, Eps $=78.355300$ Eps $($ inf $)=1.777849$

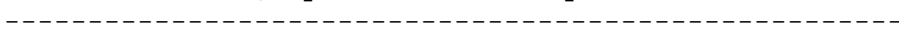

SCF Done: $E($ UwB97XD $)=-5092.25616659$ A.U. after 1 cycles NFock $=1$ Conv $=0.84 \mathrm{D}-09 \quad-\mathrm{V} / \mathrm{T}=2.0066$

$\langle\mathrm{S} x\rangle=0.0000<\mathrm{Sy}\rangle=0.0000<\mathrm{Sz}>=0.5000$

$<S^{* *} 2>=0.7567 \mathrm{~S}=0.5034$

$<$ L.S $>=0.000000000000 \mathrm{E}+00$

Annihilation of the first spin contaminant:

$\mathrm{S}^{* *} 2$ before annihilation 0.7567 , after 0.7500

Harmonic frequencies $\left(\mathrm{cm}^{*}-1\right)$, IR intensities (KM/Mole), Raman

$1 \quad 2 \quad 3$ 


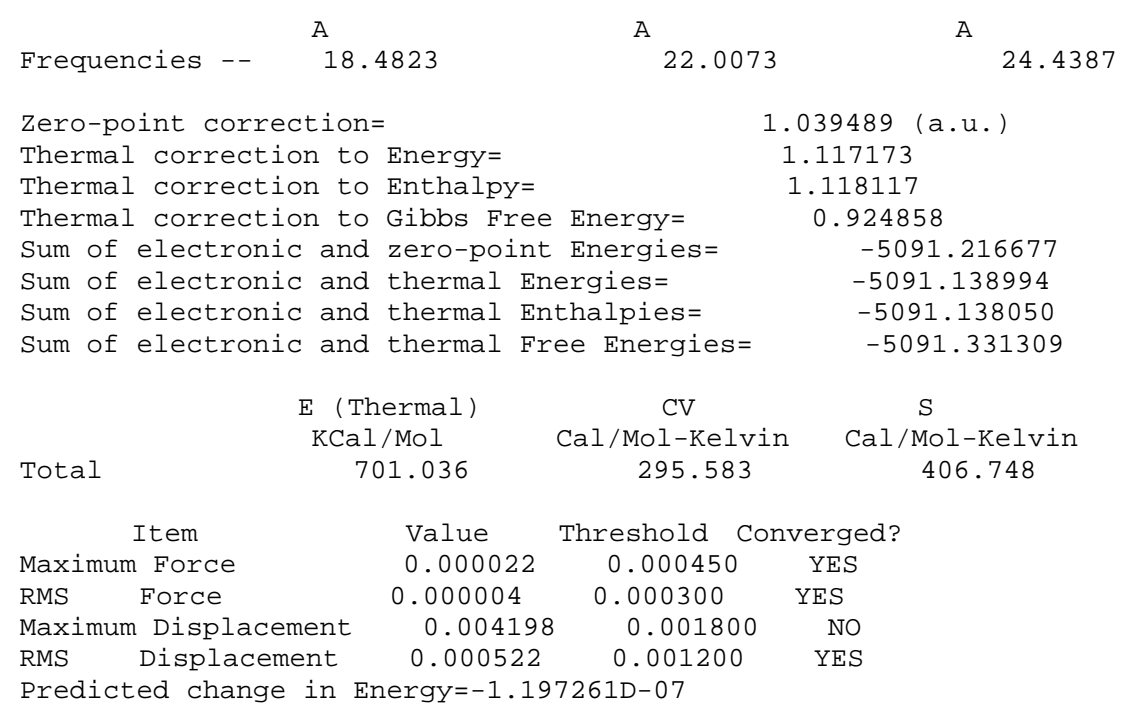

Normal termination of Gaussian 16 at Wed Aug 21 15:25:26 2019.

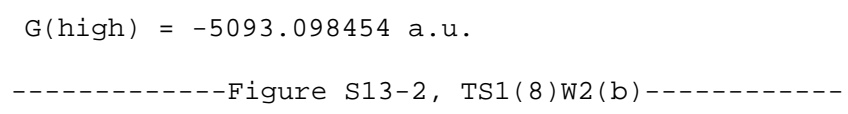

dna.ts1bw2x. log

\begin{tabular}{|c|c|c|c|c|c|}
\hline \multicolumn{2}{|c|}{ Stoichiometry } & \multicolumn{4}{|c|}{ C38H53N16Na2023P2(2) } \\
\hline Center & Atomic & Atomic & \multicolumn{3}{|c|}{ Coordinates (Angstroms) } \\
\hline Number & Number & Type & $\mathrm{X}$ & Y & Z \\
\hline- & -- & - & --------- & $----\cdots$ & $-\ldots-\ldots$ \\
\hline 1 & 7 & $\odot$ & 5.694261 & -1.858983 & -1.578252 \\
\hline 2 & 6 & $\odot$ & 5.823217 & -3.218287 & -1.541163 \\
\hline 3 & 6 & $\odot$ & 4.753306 & -4.021026 & -1.730605 \\
\hline 4 & 6 & $\odot$ & 3.489997 & -3.380436 & -1.955667 \\
\hline 5 & 7 & $\odot$ & 3.382624 & -2.049841 & -2.022531 \\
\hline 6 & 8 & $\odot$ & 8.984942 & $\odot .630647$ & -0.030031 \\
\hline 7 & 15 & $\odot$ & 8.237798 & 1.697396 & $\odot .960974$ \\
\hline 8 & 8 & $\odot$ & 6.737630 & 1.792922 & ○. 294619 \\
\hline 9 & 8 & $\odot$ & 8.942025 & 3.013450 & ๑. 819838 \\
\hline 10 & 8 & $\odot$ & 8. 015087 & 1.050853 & 2.310203 \\
\hline 11 & 8 & $\odot$ & 4.858343 & 5.443165 & 1.512271 \\
\hline 12 & 6 & $\odot$ & 5.227205 & 3.680254 & -0.046213 \\
\hline 13 & 6 & $\odot$ & 5.521325 & 4.160961 & 1.377589 \\
\hline 14 & 6 & $\odot$ & -9.182884 & -2.710829 & 2.581168 \\
\hline 15 & 6 & $\odot$ & -8.566033 & -1.340250 & 2.387323 \\
\hline 16 & 8 & $\odot$ & -8.487202 & -3.670188 & 1.750457 \\
\hline 17 & 8 & $\odot$ & -8.480448 & ๑. 591860 & $\odot .969924$ \\
\hline 18 & 15 & $\odot$ & -7.958536 & 1.576542 & -0.212263 \\
\hline 19 & 8 & $\odot$ & -6.339061 & 1.583482 & -0.055969 \\
\hline 20 & 8 & $\odot$ & -8.529707 & 2.948609 & 0.023164 \\
\hline 21 & 8 & $\odot$ & -8.228823 & 0.943087 & -1.572457 \\
\hline 22 & 8 & $\odot$ & -4.432956 & 5. 015652 & -1.712780 \\
\hline 23 & 6 & $\odot$ & -4.567264 & 3.262863 & -0.102226 \\
\hline 24 & 6 & $\odot$ & -5.080814 & 3.743138 & -1.465307 \\
\hline 25 & 1 & $\odot$ & -4.705985 & 5.374721 & -2.574271 \\
\hline 26 & 6 & $\odot$ & 9.792646 & -2.748688 & -1.380211 \\
\hline 27 & 6 & $\odot$ & 9.202248 & -1.353915 & -1.357653 \\
\hline 28 & 6 & $\odot$ & 8.827267 & -0.824646 & 0.034537 \\
\hline 29 & 6 & $\odot$ & 7.368404 & -1.241919 & 0.169432 \\
\hline 30 & 6 & $\odot$ & 6.834611 & -1.020044 & -1.244200 \\
\hline 31 & 8 & $\odot$ & 7.949485 & -1.334701 & -2.125307 \\
\hline 32 & 1 & $\odot$ & 9.898167 & -3.069088 & -2.421744 \\
\hline 33 & 1 & $\odot$ & 10.785268 & -2.725494 & -0.915914 \\
\hline 34 & 1 & $\odot$ & 9.899598 & $-\odot .665814$ & -1.840178 \\
\hline 35 & 1 & $\odot$ & 9.464923 & -1.213779 & 0. 829494 \\
\hline 36 & 1 & $\odot$ & 6.828053 & -0.668720 & ๑. 916959 \\
\hline 37 & 1 & $\odot$ & 7.339291 & -2.300849 & 0.433612 \\
\hline 38 & 1 & $\odot$ & 6.520377 & ๑.๑๑8596 & -1.399170 \\
\hline 39 & 8 & $\odot$ & 8.915059 & -3.647634 & -0.657206 \\
\hline 40 & 1 & $\odot$ & 9.252504 & -4.557676 & -0.683513 \\
\hline
\end{tabular}




\begin{tabular}{|c|c|c|c|c|c|}
\hline 41 & 6 & 0 & 4.464601 & -1.251279 & -1.871754 \\
\hline 42 & 7 & $\odot$ & 2.376033 & -4.094572 & -2.105462 \\
\hline 43 & 8 & $\odot$ & 4.394229 & -0.018954 & -1.959281 \\
\hline 44 & 1 & 0 & 6.831531 & -3.582751 & -1.351653 \\
\hline 45 & 1 & $\odot$ & 4.845770 & -5.098099 & -1.709219 \\
\hline 46 & 1 & $\odot$ & 2.408755 & -5.099994 & -2.079957 \\
\hline 47 & 1 & 0 & 1.496908 & -3.618327 & -2.329457 \\
\hline 48 & 6 & 0 & 6.315221 & 2.813517 & -0.652773 \\
\hline 49 & 1 & $\odot$ & 5.009545 & 5.810529 & 2.399801 \\
\hline 50 & 6 & 0 & 4.867037 & 3.094181 & 2.263320 \\
\hline 51 & 6 & $\odot$ & 3.642171 & 2.661035 & 1.449392 \\
\hline 52 & 8 & 0 & 3.970714 & 2.932331 & ๑.054593 \\
\hline 53 & 1 & 0 & 5.925672 & 2.297436 & -1.531699 \\
\hline 54 & 1 & $\odot$ & 7.172502 & 3.433983 & -0.925797 \\
\hline 55 & 1 & $\odot$ & 5.044658 & 4.535904 & -0.700466 \\
\hline 56 & 1 & 0 & 6.596288 & 4.256947 & 1.559731 \\
\hline 57 & 1 & $\odot$ & 4.580627 & 3.487418 & 3.240881 \\
\hline 58 & 1 & 0 & 5.567121 & 2.269545 & 2.410315 \\
\hline 59 & 1 & 0 & 2.753806 & 3.229816 & 1.710711 \\
\hline $6 \odot$ & 7 & $\odot$ & 3.274011 & 1.254767 & 1.572271 \\
\hline 61 & 6 & $\odot$ & 4.189912 & 0.286789 & 1.283640 \\
\hline 62 & 6 & 0 & 3.845884 & -1.020037 & 1.265518 \\
\hline 63 & 6 & $\odot$ & 2.467049 & -1.336696 & 1.495670 \\
\hline 64 & 7 & 0 & 1.563155 & -0.381133 & 1.723407 \\
\hline 65 & 6 & 0 & 1.917148 & 0.926871 & 1.742100 \\
\hline 66 & 1 & $\odot$ & 4.573434 & -1.791583 & 1.051194 \\
\hline 67 & 7 & $\odot$ & 2.039643 & -2.599547 & 1.475308 \\
\hline 68 & 8 & 0 & 1.093828 & 1.833192 & 1.908660 \\
\hline 69 & 1 & $\odot$ & 5.187050 & $\odot .646722$ & 1.046312 \\
\hline 70 & 1 & 0 & 2.689578 & -3.352723 & 1.323006 \\
\hline 71 & 1 & 0 & 1.064832 & -2.815953 & 1.715200 \\
\hline 72 & 1 & $\odot$ & $-\odot .255418$ & -0.783586 & 1.832456 \\
\hline 73 & 1 & 0 & -8.798206 & -4.571684 & 1.932518 \\
\hline 74 & 6 & $\odot$ & -8.437451 & -0.883833 & $\odot .928499$ \\
\hline 75 & 6 & 0 & -7.056267 & -1.382296 & 0.537288 \\
\hline 76 & 6 & 0 & -6.260020 & -1.113449 & 1.808567 \\
\hline 77 & 8 & 0 & -7.193294 & -1.337056 & 2.905223 \\
\hline 78 & 1 & $\odot$ & -9.099036 & -2.981003 & 3.639196 \\
\hline 79 & 1 & 0 & -10.245901 & -2.666583 & 2.314423 \\
\hline 80 & 1 & 0 & -9.141881 & -0.608746 & 2.958982 \\
\hline 81 & 1 & 0 & -9.242119 & -1.237183 & 0.283491 \\
\hline 82 & 1 & 0 & -6.634142 & -0.860500 & $-\odot .315454$ \\
\hline 83 & 1 & 0 & -7.093708 & -2.452791 & 0.331615 \\
\hline 84 & 1 & 0 & -5.911120 & -0.080860 & 1.839082 \\
\hline 85 & 7 & 0 & -5.090764 & -1.933747 & 1.981063 \\
\hline 86 & 6 & 0 & -4.995138 & -3.300460 & 2.130980 \\
\hline 87 & 7 & 0 & -3.760277 & -3.719067 & 2.210130 \\
\hline 88 & 6 & 0 & -2.996445 & -2.565713 & 2.091320 \\
\hline 89 & 6 & 0 & -3.812598 & -1.453073 & 1.945914 \\
\hline 90 & 7 & 0 & -3.474722 & -0.163509 & 1.792417 \\
\hline 91 & 6 & 0 & -2.167932 & ๑. 019268 & 1.741473 \\
\hline 92 & 7 & 0 & -1.265876 & -1.006754 & 1.894132 \\
\hline 93 & 6 & 0 & -1.584792 & -2.357274 & 2.061369 \\
\hline 94 & 8 & 0 & $-\odot .681758$ & -3.203692 & 2.145019 \\
\hline 95 & 1 & 0 & $-5.89 \odot \odot 44$ & -3.906271 & 2.172256 \\
\hline 96 & 7 & 0 & -1.682210 & 1.259368 & 1.550266 \\
\hline 97 & 1 & 0 & -2.278164 & 1.847836 & 0.968294 \\
\hline 98 & 1 & 0 & -0.673795 & 1.404329 & 1.507885 \\
\hline 99 & 6 & 0 & -5.584831 & 2.535940 & 0.751778 \\
\hline 100 & 11 & 0 & 6.979388 & -0.127703 & 3.833404 \\
\hline 101 & 11 & $\odot$ & -9.374509 & 2.824071 & -2.526325 \\
\hline 102 & 6 & 0 & -4.588238 & 2.663406 & -2.433238 \\
\hline 103 & 6 & 0 & -3.222944 & 2.304569 & -1.847541 \\
\hline 104 & 8 & 0 & -3.442834 & 2.366372 & $-\odot .404185$ \\
\hline 105 & 1 & 0 & -5.065481 & 1.969655 & 1.527021 \\
\hline 106 & 1 & 0 & -6.270811 & 3.260234 & 1.198440 \\
\hline 107 & 1 & 0 & -4.175695 & 4.115000 & 0.459703 \\
\hline 108 & 1 & 0 & -6.169347 & 3.851797 & -1.477394 \\
\hline 109 & 1 & 0 & -4.529542 & 3.011100 & -3.465867 \\
\hline 110 & 1 & 0 & -5.270336 & 1.812025 & -2.369647 \\
\hline 111 & 1 & 0 & -2.461026 & 3.042701 & -2.108673 \\
\hline 112 & 7 & 0 & -2.716622 & 1.002764 & -2.211427 \\
\hline 113 & 6 & 0 & -3.400883 & $-\odot .049610$ & -2.793721 \\
\hline 114 & 7 & 0 & -2.632797 & -1.098936 & -3.016401 \\
\hline 115 & 6 & 0 & -1.411885 & -0.737653 & -2.518598 \\
\hline 116 & 6 & 0 & -1.422939 & $\odot .570935$ & -2.035707 \\
\hline 117 & 7 & 0 & -0.392801 & 1.294901 & -1.571634 \\
\hline
\end{tabular}




\begin{tabular}{|c|c|c|c|c|c|}
\hline 118 & 6 & $\odot$ & 0.757897 & $\odot .628405$ & -1.611874 \\
\hline 119 & 7 & 0 & ๑. 861141 & -0.685637 & -2.000013 \\
\hline 120 & 6 & 0 & -0.185686 & -1.470663 & -2.472277 \\
\hline 121 & 7 & $\odot$ & 1.903100 & 1. 224614 & -1.264920 \\
\hline 122 & 8 & 0 & -0.001614 & -2.648857 & -2.795784 \\
\hline 123 & 1 & $\odot$ & -4.401882 & 0.067859 & -3.182643 \\
\hline 124 & 1 & 0 & 1.889229 & 2.188870 & -0.974433 \\
\hline 125 & 1 & $\Theta$ & 2.812145 & ๑. 792057 & -1.448520 \\
\hline 126 & 1 & 0 & 1.793835 & -1.146838 & -1.992987 \\
\hline 127 & 8 & 0 & -4.476363 & -1.259039 & -1.149393 \\
\hline 128 & 1 & 0 & -5.247301 & -1.035600 & -1.723567 \\
\hline 129 & 8 & 0 & -6.509816 & -0.513682 & -2.893686 \\
\hline 130 & 1 & 0 & -6.978412 & -1.277525 & -3.240333 \\
\hline 131 & 1 & 0 & -7.188962 & ०. 029562 & -2.414622 \\
\hline 132 & 1 & $\odot$ & -4.905821 & -3.041326 & -1.130166 \\
\hline 133 & $\overline{8}$ & 0 & -5.374169 & -3.898038 & -1.107580 \\
\hline 134 & 1 & $\odot$ & -6.144212 & -3.745808 & -1.661644 \\
\hline
\end{tabular}

General basis read from cards: (6D, 7F)

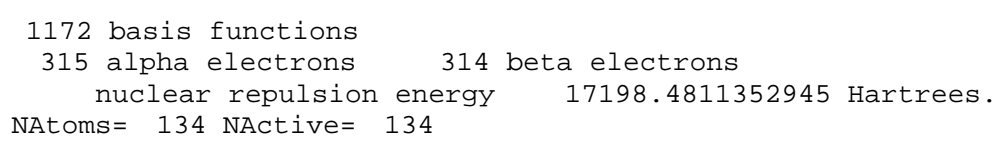

Force inversion solution in PCM.

Polarizable Continuum Model (PCM)

=ニニニニニニニニニニニニニニニニニニニニニニニニニニニニニニニニ

Model : PCM.

Atomic radii : UFF (Universal Force Field).

Polarization charges : Total charges.

Solvent : Water, Eps $=78.355300$ Eps $($ inf $)=1.777849$

SCF Done: $E($ UWB97XD) $=-5092.23508197$ A.U. after 1 cycles NFock $=1$ Conv $=0.36 \mathrm{D}-09 \quad-\mathrm{V} / \mathrm{T}=2.0066$

$\langle\mathrm{S} x\rangle=0.0000<\mathrm{Sy}\rangle=0.0000<\mathrm{Sz}>=0.5000$

$\left\langle S^{* *} 2>=0.7626 \mathrm{~S}=0.5063\right.$

$<$ L.S $>=0.000000000000 \mathrm{E}+0 \odot$

Annihilation of the first spin contaminant:

$\mathrm{S}^{* * 2}$ before annihilation 0.7626 , after 0.7501

Harmonic frequencies $\left(\mathrm{cm}^{*}-1\right)$, IR intensities (KM/Mole), Raman

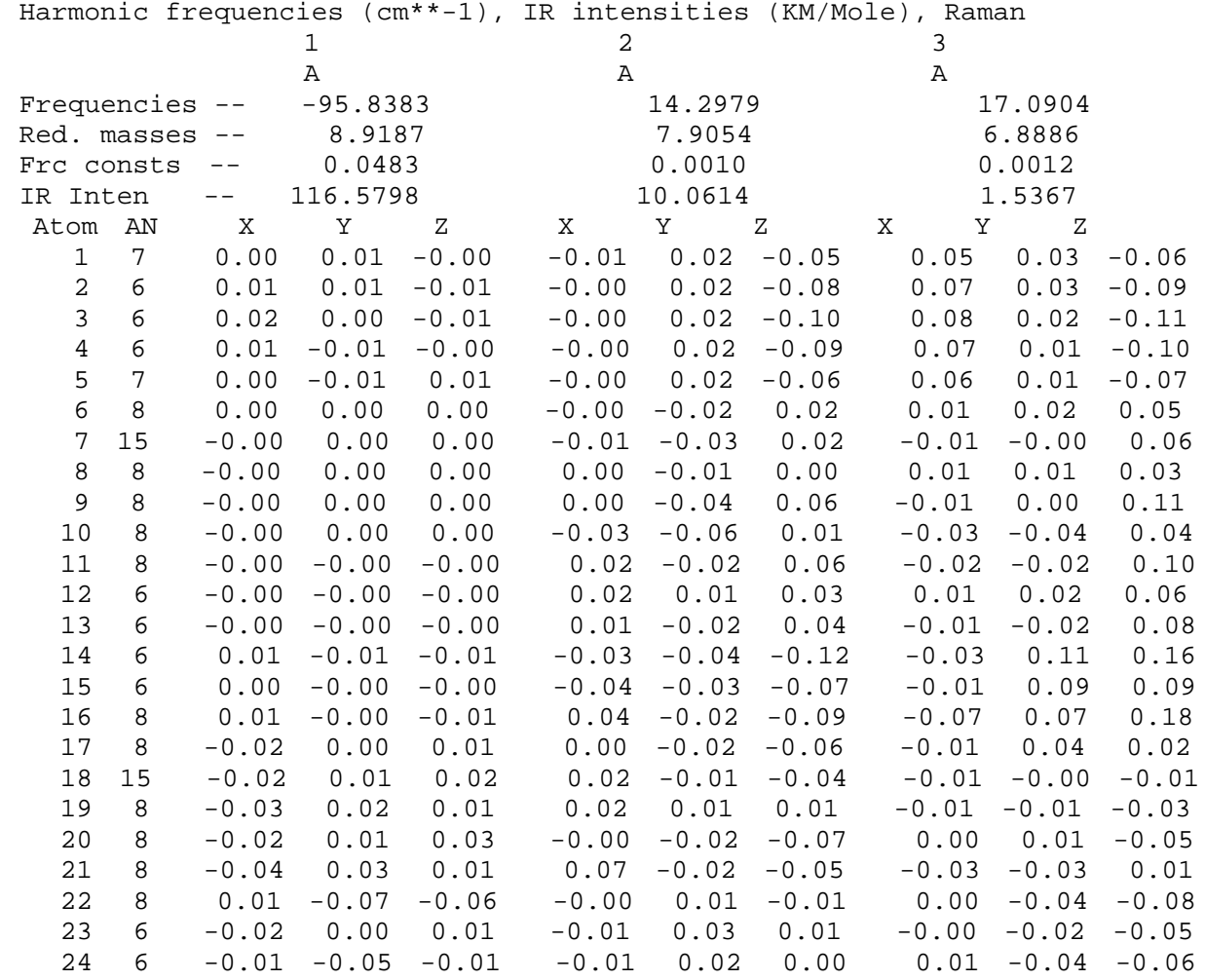




\begin{tabular}{|c|c|c|c|c|c|c|c|c|c|c|}
\hline 25 & 1 & $\odot .02$ & -0.10 & -0.08 & -0.01 & 0.01 & -0.01 & 0.01 & -0.06 & -0.09 \\
\hline 26 & 6 & -0.00 & $\odot .01$ & -0.00 & -0.01 & 0.00 & -0.05 & $\odot .06$ & 0.06 & -0.01 \\
\hline 27 & 6 & $-\odot . \odot \odot$ & $\odot .01$ & $-\odot .0 \odot$ & -0.01 & 0.00 & -0.02 & $\odot .05$ & 0.05 & 0.01 \\
\hline 28 & 6 & $\odot . \odot \odot$ & $\odot .0 \odot$ & $-\odot . \odot \odot$ & -0.01 & $-\odot .02$ & -0.02 & $\odot .02$ & 0.02 & 0.02 \\
\hline 29 & 6 & -0.00 & $\odot .01$ & $-\odot .0 \odot$ & -0.02 & -0.02 & -0.03 & 0.01 & 0.01 & -0.02 \\
\hline 30 & 6 & $-\odot . \odot \odot$ & 0.01 & $\odot . \odot \odot$ & -0.01 & 0.01 & -0.03 & 0.04 & 0.03 & -0.03 \\
\hline 31 & 8 & $-0.0 \odot$ & 0.01 & -0.00 & -0.00 & 0.03 & -0.03 & 0.06 & 0.06 & -0.01 \\
\hline 32 & 1 & -0.00 & $\odot .01$ & -0.01 & -0.01 & 0.02 & -0.05 & $\odot . \odot 8$ & 0.08 & -0.01 \\
\hline 33 & 1 & -0.00 & $\odot .01$ & -0.01 & -0.01 & -0.01 & -0.04 & 0.05 & 0.06 & 0.01 \\
\hline 34 & 1 & $-0.0 \odot$ & $\odot .01$ & $-0.0 \odot$ & $-\odot . \odot \odot$ & 0.01 & -0.01 & $\odot .05$ & 0.07 & 0.04 \\
\hline 35 & 1 & $\odot .0 \odot$ & 0.00 & -0.00 & -0.02 & -0.04 & -0.02 & $\odot . \odot \odot$ & 0.00 & 0.02 \\
\hline 36 & 1 & 0.00 & $\odot .00$ & 0.00 & -0.02 & -0.03 & -0.03 & $-\odot . \odot \odot$ & -0.00 & -0.02 \\
\hline 37 & 1 & $-\odot .0 \odot$ & 0.01 & -0.00 & $-\odot .02$ & $-\odot .02$ & -0.05 & 0.01 & 0.01 & -0.04 \\
\hline 38 & 1 & -0.00 & 0.01 & 0.00 & -0.00 & $\odot .01$ & -0.02 & 0.03 & 0.03 & -0.02 \\
\hline 39 & 8 & $\odot .0 \odot$ & 0.01 & -0.00 & -0.02 & -0.01 & -0.07 & 0.05 & 0.04 & -0.04 \\
\hline $4 \odot$ & 1 & $\odot .0 \odot$ & 0.01 & $-0.0 \odot$ & -0.02 & -0.01 & -0.08 & $\odot .06$ & 0.04 & -0.05 \\
\hline 41 & 6 & -0.00 & 0.00 & 0.01 & $-\odot .01$ & 0.02 & -0.05 & 0.05 & 0.02 & -0.06 \\
\hline 42 & 7 & $\odot .02$ & -0.02 & $-\odot . \odot \odot$ & $-\odot . \odot \odot$ & 0.02 & -0.10 & 0.08 & $-\odot . \odot \odot$ & -0.10 \\
\hline 43 & 8 & -0.02 & 0.00 & 0.02 & -0.01 & 0.02 & -0.02 & 0.03 & 0.02 & -0.04 \\
\hline 44 & 1 & 0.02 & 0.02 & -0.01 & $-0.0 \odot$ & 0.01 & -0.09 & 0.08 & 0.04 & -0.09 \\
\hline 45 & 1 & $\odot .03$ & $\odot .00$ & -0.01 & $-\odot . \odot \odot$ & $\odot .02$ & -0.12 & 0.10 & 0.02 & -0.14 \\
\hline 46 & 1 & 0.04 & -0.02 & -0.01 & $-\odot . \odot \odot$ & 0.02 & -0.12 & 0.10 & $-0.0 \odot$ & -0.11 \\
\hline 47 & 1 & $\odot .02$ & -0.03 & -0.01 & -0.00 & 0.03 & -0.09 & $\odot .07$ & -0.01 & -0.07 \\
\hline 48 & 6 & $-0.0 \odot$ & $\odot . \odot \odot$ & -0.00 & $\odot .02$ & 0.01 & 0.02 & 0.03 & 0.04 & 0.06 \\
\hline 49 & 1 & $-\odot . \odot \odot$ & $-0.0 \odot$ & $-\odot . \odot \odot$ & 0.01 & $-\odot .04$ & 0.06 & -0.03 & -0.04 & 0.11 \\
\hline 50 & 6 & $-\odot .0 \odot$ & $-\odot . \odot \odot$ & $-\odot . \odot \odot$ & $\odot . \odot \odot$ & -0.03 & 0.02 & -0.02 & $-\odot . \odot 4$ & 0.04 \\
\hline 51 & 6 & $\odot .0 \odot$ & -0.00 & -0.00 & $\odot .0 \odot$ & -0.01 & $\odot .0 \odot$ & -0.01 & -0.02 & 0.01 \\
\hline 52 & 8 & -0.00 & $-0.0 \odot$ & -0.00 & $\odot .02$ & 0.01 & 0.01 & 0.01 & 0.01 & $\odot .02$ \\
\hline 53 & 1 & $\odot .0 \odot$ & $-\odot . \odot \odot$ & -0.00 & $\odot .03$ & 0.03 & 0.00 & 0.04 & 0.06 & 0.03 \\
\hline 54 & 1 & $-0.0 \odot$ & $\odot .0 \odot$ & -0.00 & $\odot .03$ & 0.01 & 0.04 & 0.03 & 0.05 & 0.09 \\
\hline 55 & 1 & $-\odot . \odot \odot$ & $-\odot . \odot \odot$ & $-\odot . \odot \odot$ & $\odot .03$ & 0.02 & 0.04 & 0.02 & 0.03 & $\odot . \odot 8$ \\
\hline 56 & 1 & $-\odot . \odot \odot$ & $\odot . \odot \odot$ & -0.00 & $\odot .01$ & -0.03 & 0.05 & -0.01 & -0.02 & 0.09 \\
\hline 57 & 1 & $-0.0 \odot$ & $-0.0 \odot$ & -0.00 & 0.00 & -0.05 & $\odot .02$ & -0.03 & -0.07 & 0.05 \\
\hline 58 & 1 & $\odot .0 \odot$ & $-\odot . \odot \odot$ & $-\odot .0 \odot$ & $-\odot . \odot \odot$ & $-\odot .04$ & $-\odot . \odot \odot$ & -0.02 & -0.05 & $\odot .02$ \\
\hline 59 & 1 & $\odot .00$ & $-\odot .0 \odot$ & $-\odot .0 \odot$ & 0.01 & $-\odot . \odot \odot$ & $\odot .0 \odot$ & -0.01 & -0.02 & 0.01 \\
\hline 60 & 7 & $\odot .0 \odot$ & -0.00 & -0.00 & -0.01 & -0.01 & -0.02 & -0.02 & -0.02 & -0.02 \\
\hline 61 & 6 & $\odot .0 \odot$ & $-\odot . \odot \odot$ & $\odot . \odot \odot$ & -0.02 & -0.01 & -0.04 & -0.02 & -0.02 & -0.04 \\
\hline 62 & 6 & $\odot .0 \odot$ & $-\odot .0 \odot$ & 0.01 & -0.03 & -0.01 & -0.05 & -0.03 & -0.02 & -0.07 \\
\hline 63 & 6 & $\odot .0 \odot$ & $-\odot . \odot \odot$ & 0.00 & -0.03 & $\odot .0 \odot$ & -0 & -0.03 & -0.01 & $-\odot . \odot 8$ \\
\hline 64 & 7 & $\odot .0 \odot$ & $-\odot . \odot \odot$ & -0.01 & -0.02 & 0.01 & $\odot . \odot \odot$ & -0.02 & -0.02 & -0.06 \\
\hline 65 & 6 & 0.00 & $-0.0 \odot$ & $-\odot .0 \odot$ & -0.01 & 0.00 & $-0.0 \odot$ & -0.02 & -0.02 & -0.03 \\
\hline 66 & 1 & & & & & -0.01 & & -0.03 & $-\odot .01$ & -0.09 \\
\hline 67 & 7 & 0.00 & $-0.0 \odot$ & 0.00 & -0.04 & $\odot . \odot \odot$ & -0.01 & -0.04 & -0.01 & -0.11 \\
\hline 68 & 8 & 0.00 & $-\odot .0 \odot$ & -0.01 & $-0.0 \odot$ & 0.01 & 0.01 & -0.01 & -0.02 & -0.01 \\
\hline 69 & 1 & 0.00 & -0.00 & $\odot .0 \odot$ & -0.02 & -0.02 & -0.05 & -0.02 & -0.02 & -0.03 \\
\hline 70 & 1 & 0.00 & -0.00 & 0.01 & -0.04 & -0.00 & -0.02 & -0.04 & -0.01 & -0.12 \\
\hline 71 & 1 & $-\odot . \odot \odot$ & $-\odot . \odot \odot$ & $-\odot . \odot \odot$ & -0.03 & 0.01 & $\odot .04$ & -0.03 & -0.01 & -0.10 \\
\hline 72 & 1 & $\odot .0 \odot$ & $-\odot . \odot \odot$ & $-\odot .0 \odot$ & -0.01 & 0.03 & 0.06 & $-\odot . \odot 2$ & $-0.0 \odot$ & -0.04 \\
\hline 73 & 1 & 0.01 & -0.00 & -0.01 & 0.04 & -0.03 & -0.12 & -0.08 & 0.08 & 0.22 \\
\hline 74 & 6 & $\odot . \odot \odot$ & $\odot .00$ & $-\odot . \odot \odot$ & 0.04 & -0.01 & $-\odot . \odot 6$ & -0.04 & $\odot .04$ & $\odot .07$ \\
\hline 75 & 6 & $0.0 \odot$ & $\odot .02$ & -0.01 & 0.08 & 0.02 & 0.02 & -0.06 & $-\odot .0 \odot$ & 0.05 \\
\hline 76 & 6 & $\odot .0 \odot$ & 0.01 & -0.01 & -0.01 & 0.03 & $\odot .08$ & -0.02 & $\odot .03$ & $\odot .02$ \\
\hline 77 & 8 & $\odot . \odot \odot$ & $-\odot . \odot \odot$ & -0.01 & $-\odot . \odot 8$ & $-\odot . \odot \odot$ & $\odot .01$ & $\odot .01$ & $\odot .09$ & 0.05 \\
\hline 78 & 1 & 0.01 & -0.01 & -0.01 & -0.09 & -0.05 & -0.12 & -0.00 & 0.15 & 0.17 \\
\hline 79 & 1 & 0.01 & -0.01 & -0.00 & -0.01 & -0.06 & -0.19 & -0.03 & 0.12 & 0.19 \\
\hline 80 & 1 & 0.00 & -0.01 & -0.00 & -0.09 & -0.04 & -0.10 & 0.02 & 0.13 & 0.08 \\
\hline 81 & 1 & $\odot .00$ & $-\odot . \odot \odot$ & $-\odot .0 \odot$ & $\odot .09$ & -0.03 & -0.12 & -0.07 & 0.03 & 0.11 \\
\hline 82 & 1 & 0.01 & 0.02 & -0.00 & 0.13 & 0.05 & 0.06 & -0.08 & -0.05 & $\odot .01$ \\
\hline 83 & 1 & 0.01 & 0.02 & -0.01 & 0.12 & 0.03 & 0.01 & -0.09 & -0.01 & $\odot .09$ \\
\hline 84 & 1 & $\odot .0 \odot$ & 0.01 & 0.00 & -0.03 & 0.03 & 0.11 & $-\odot . \odot \odot$ & 0.03 & -0.03 \\
\hline 85 & 7 & $\odot .0 \odot$ & 0.01 & -0.01 & -0.01 & $\odot .04$ & 0.14 & -0.03 & 0.02 & 0.01 \\
\hline 86 & 6 & $-\odot . \odot \odot$ & 0.01 & -0.00 & -0.01 & 0.05 & 0.20 & -0.03 & 0.02 & 0.01 \\
\hline 87 & 7 & $-\odot . \odot \odot$ & $\odot . \odot \odot$ & $-\odot . \odot \odot$ & -0.01 & 0.05 & 0.21 & -0.03 & 0.02 & $-\odot . \odot \odot$ \\
\hline 88 & 6 & 0.00 & 0.00 & -0.01 & -0.01 & 0.04 & 0.16 & -0.03 & 0.01 & $-\odot . \odot \odot$ \\
\hline 89 & 6 & $\odot .0 \odot$ & $\odot .0 \odot$ & -0.01 & -0.01 & & 0.11 & -0.02 & 0.02 & $\odot .01$ \\
\hline 90 & 7 & 0.01 & $\odot . \odot \odot$ & $-\odot . \odot \odot$ & -0.01 & 0.03 & 0.05 & -0.02 & 0.02 & $\odot .01$ \\
\hline 91 & 6 & 0.00 & $\odot .00$ & 0.00 & -0.01 & $\odot .03$ & $\odot .04$ & -0.02 & 0.01 & $-\odot . \odot \odot$ \\
\hline 92 & 7 & $\odot .0 \odot$ & $-\odot . \odot \odot$ & -0.01 & -0.01 & 0.03 & 0.08 & -0.02 & 0.00 & -0.02 \\
\hline 93 & 6 & $\odot .0 \odot$ & $-0.0 \odot$ & -0.01 & -0.01 & 0.04 & 0.14 & -0.03 & 0.00 & -0.02 \\
\hline 94 & 8 & $\odot .0 \odot$ & $-\odot . \odot \odot$ & -0.01 & -0.01 & 0.04 & 0.17 & -0.03 & $-0.0 \odot$ & -0.05 \\
\hline 95 & 1 & $-\odot .0 \odot$ & 0.01 & $\odot . \odot \odot$ & -0.01 & 0.05 & 0.22 & -0.03 & 0.03 & 0.01 \\
\hline 96 & 7 & 0.01 & 0.00 & 0.02 & -0.01 & 0.02 & $-\odot . \odot \odot$ & -0.01 & 0.01 & -0.01 \\
\hline 97 & 1 & $\odot .00$ & 0.01 & 0.03 & -0.01 & 0.01 & -0.01 & -0.01 & 0.01 & -0.01 \\
\hline 98 & 1 & 0.00 & 0.00 & 0.01 & -0.01 & 0.02 & $-\odot . \odot \odot$ & -0.01 & 0.00 & -0.01 \\
\hline 99 & 6 & -0.02 & 0.01 & 0.01 & -0.01 & 0.03 & 0.01 & -0.01 & -0.00 & -0.04 \\
\hline 100 & 11 & $\odot . \odot \odot$ & $-\odot . \odot \odot$ & $\odot . \odot \odot$ & -0.05 & -0.06 & -0.01 & -0.05 & -0.07 & $\odot .01$ \\
\hline ७ & 11 & -0 & 0.01 & 0.00 & 0.05 & 08 & -0.10 & -0.02 & -0.01 & $-\odot$ \\
\hline
\end{tabular}




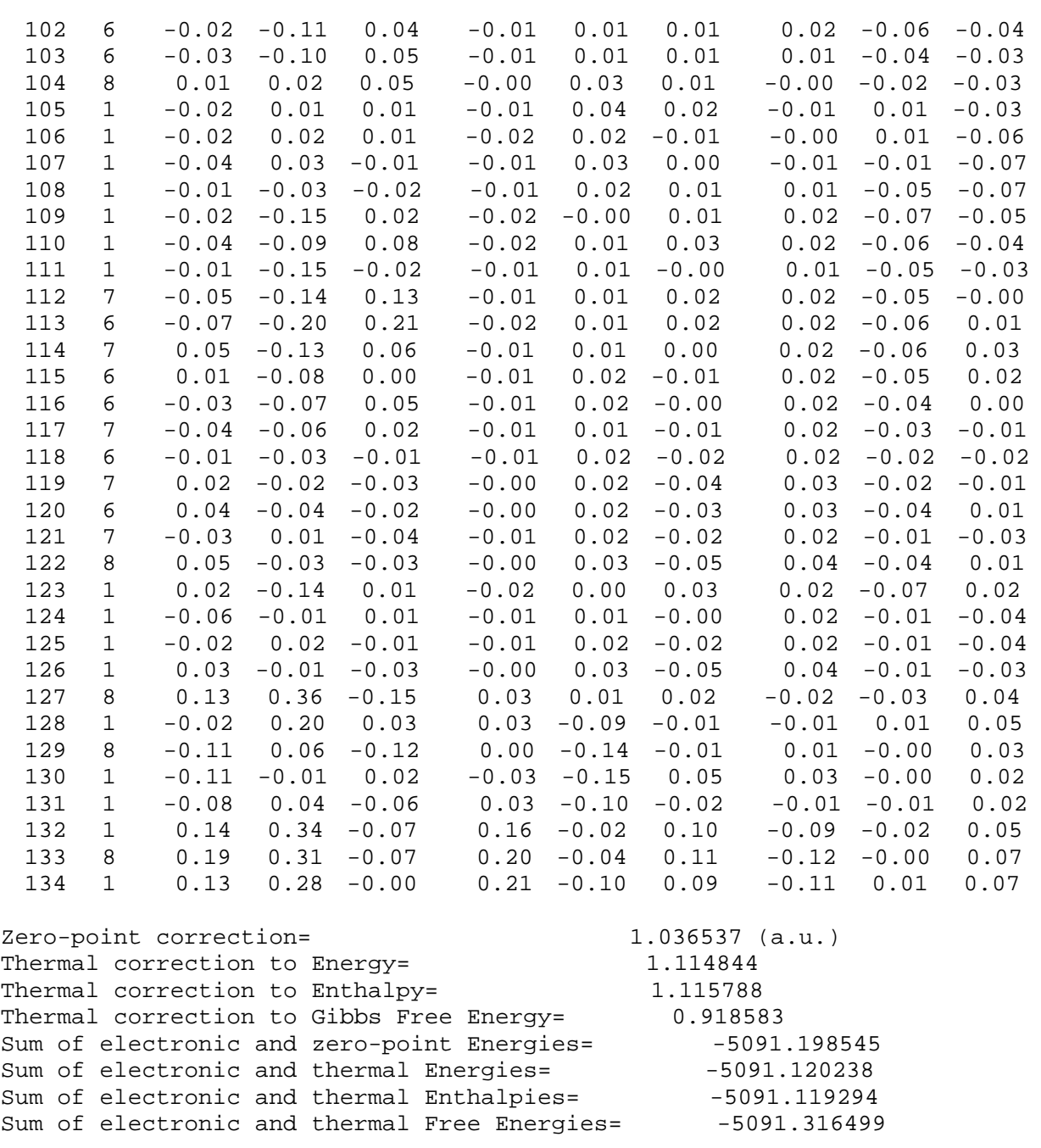

$\begin{array}{cccc} & \text { E (Thermal) } & \text { CV } & \text { S } \\ \text { KCal/Mol } & \text { Cal/Mol-Kelvin } & \text { Cal/Mol-Kelvin } \\ \text { Total } & 699.575 & 295.606 & 415.052\end{array}$

G(high) - 5093.0896313 a.u.

.

dna. rev2bw2.log

Stoichiometry C38H53N16Na2023P2(2)

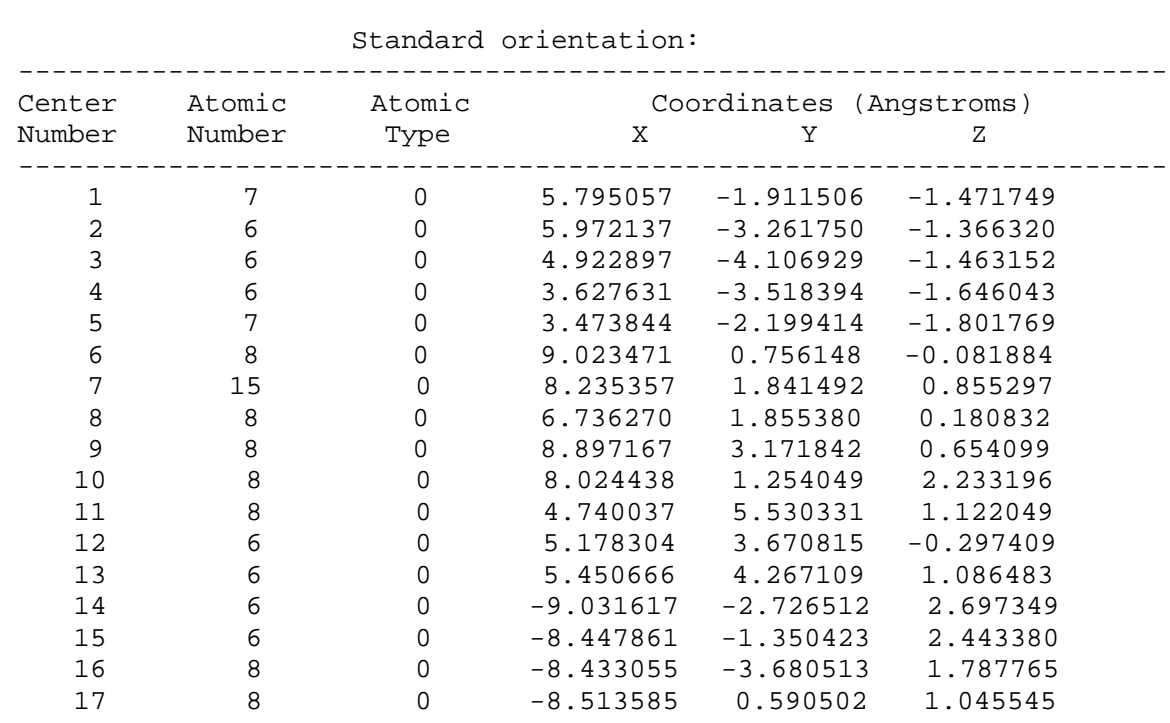




\begin{tabular}{|c|c|c|c|c|c|}
\hline 18 & 15 & $\odot$ & -8.165017 & 1.590132 & $-\odot .185087$ \\
\hline 19 & 8 & $\odot$ & -6.541680 & 1.708310 & -0.178845 \\
\hline 20 & 8 & $\odot$ & -8.802985 & 2.926547 & ๑. 094895 \\
\hline 21 & 8 & $\odot$ & -8.521295 & $\odot .931739$ & -1.510966 \\
\hline 22 & 8 & $\odot$ & -4.263121 & 4.847194 & -1.930528 \\
\hline 23 & 6 & $\odot$ & -4.662290 & 3.293706 & -0.167877 \\
\hline 24 & 6 & $\odot$ & -4.982051 & 3.623018 & -1.629728 \\
\hline 25 & 1 & $\odot$ & -4.395419 & 5.095832 & -2.861347 \\
\hline 26 & $\overline{6}$ & $\odot$ & 9.926892 & -2.640107 & -1.324223 \\
\hline 27 & 6 & $\odot$ & 9.285161 & -1.268053 & -1.341234 \\
\hline 28 & 6 & $\odot$ & 8.915585 & -0.700233 & ๑. . 037568 \\
\hline 29 & 6 & $\odot$ & 7.475340 & -1.163113 & 0. 215398 \\
\hline 30 & 6 & $\odot$ & 6.909102 & -1.017613 & -1.195975 \\
\hline 31 & 8 & $\odot$ & 8.019387 & -1.324267 & -2.085118 \\
\hline 32 & 1 & $\odot$ & 10.025618 & -2.994339 & -2.355459 \\
\hline 33 & 1 & $\odot$ & 10.926127 & -2.563709 & -0.880288 \\
\hline 34 & 1 & $\odot$ & 9.947620 & -0.573334 & -1.861997 \\
\hline 35 & 1 & $\odot$ & 9.580258 & -1.037747 & 0.833936 \\
\hline 36 & 1 & $\odot$ & 6.929416 & -0.581986 & 0.953239 \\
\hline 37 & 1 & $\odot$ & 7.490286 & -2.211377 & 0.520292 \\
\hline 38 & 1 & $\odot$ & 6.553312 & -0.008772 & -1.385047 \\
\hline 39 & 8 & $\odot$ & 9.096656 & -3.543548 & -0.552726 \\
\hline 40 & 1 & 0 & 9.467176 & -4.441003 & -0.553814 \\
\hline 41 & $\overline{6}$ & 0 & 4.536883 & -1.362415 & -1.759649 \\
\hline 42 & 7 & $\odot$ & 2.529775 & -4.271695 & -1.656376 \\
\hline 43 & 8 & $\Theta$ & 4.427175 & -0.142137 & -1.933943 \\
\hline 44 & 1 & 0 & 6.998927 & -3.582409 & -1.197686 \\
\hline 45 & 1 & $\odot$ & 5.051998 & -5.177281 & -1.381379 \\
\hline 46 & 1 & 0 & 2.598113 & -5.269809 & -1.547366 \\
\hline 47 & 1 & 0 & 1.611383 & -3.834681 & -1.793688 \\
\hline 48 & 6 & $\odot$ & 6.294930 & 2.796629 & -0.837045 \\
\hline 49 & 1 & $\odot$ & 4.863442 & 5.964600 & 1.983127 \\
\hline 50 & 6 & 0 & 4.831478 & 3.246912 & 2.049209 \\
\hline 51 & 6 & 0 & 3.623068 & 2.715102 & 1. 270449 \\
\hline 52 & 8 & $\odot$ & 3.947323 & 2.891232 & -0.141441 \\
\hline 53 & 1 & $\odot$ & 5.926227 & 2. 211809 & -1.681346 \\
\hline 54 & 1 & $\odot$ & 7.136511 & 3.421235 & -1.147093 \\
\hline 55 & 1 & $\odot$ & 4.969985 & 4.468591 & -1.014114 \\
\hline 56 & 1 & 0 & 6.520830 & 4.415076 & 1.262118 \\
\hline 57 & 1 & 0 & 4.531203 & 3.700343 & 2.995997 \\
\hline 58 & 1 & $\odot$ & 5.560561 & 2.461123 & 2.253801 \\
\hline 59 & 1 & 0 & 2.718520 & 3.276666 & 1.487857 \\
\hline 60 & 7 & $\odot$ & 3. 291642 & 1.311873 & 1.494635 \\
\hline 61 & 6 & 0 & 4.236347 & $\odot .349500$ & 1. 292799 \\
\hline 62 & 6 & $\odot$ & 3.917880 & -0.963097 & 1.327908 \\
\hline 63 & 6 & $\Theta$ & 2.532334 & -1.296246 & 1.484380 \\
\hline 64 & 7 & $\odot$ & 1.604199 & -0.351600 & 1.657675 \\
\hline 65 & 6 & $\odot$ & 1.938306 & $\odot .962111$ & 1.660346 \\
\hline 66 & 1 & $\odot$ & 4.668160 & -1.728421 & 1.179533 \\
\hline 67 & 7 & 0 & 2.128019 & -2.565176 & 1.445880 \\
\hline 68 & 8 & $\odot$ & 1.100162 & 1.859179 & 1.799242 \\
\hline 69 & 1 & $\odot$ & 5.234852 & ๑.715555 & 1.072058 \\
\hline 70 & 1 & $\odot$ & 2.792398 & -3.303234 & 1.282774 \\
\hline 71 & 1 & $\odot$ & 1.131264 & -2.798758 & 1.525696 \\
\hline 72 & 1 & $\odot$ & $-0.230 \odot 48$ & -0.788895 & 1.648399 \\
\hline 73 & 1 & $\odot$ & -8.711235 & -4.584503 & 2.006572 \\
\hline 74 & 6 & $\odot$ & -8.489521 & -0.883756 & 0.984223 \\
\hline 75 & 6 & $\odot$ & -7.170885 & -1.395641 & 0.426161 \\
\hline 76 & 6 & 0 & -6.221377 & -1.143685 & 1.592544 \\
\hline 77 & 8 & $\odot$ & -7.026354 & -1.335982 & 2.796984 \\
\hline 78 & 1 & $\odot$ & -8.825724 & -3.001493 & 3.737279 \\
\hline 79 & 1 & $\odot$ & -10.118362 & -2.686559 & 2.554410 \\
\hline 80 & 1 & $\odot$ & -8.960011 & -0.627798 & 3.082983 \\
\hline 81 & 1 & $\odot$ & -9.368817 & -1.224227 & 0.437081 \\
\hline 82 & 1 & $\odot$ & -6.857551 & -0.880040 & -0.475531 \\
\hline 83 & 1 & $\odot$ & -7.257149 & -2.463872 & ○. 225881 \\
\hline 84 & 1 & $\odot$ & -5.846652 & -0.119781 & 1.576593 \\
\hline 85 & 7 & $\odot$ & -5.056848 & -1.984976 & 1.644306 \\
\hline 86 & 6 & $\odot$ & -4.953640 & -3.360331 & 1.653491 \\
\hline 87 & 7 & $\odot$ & -3.716796 & -3.779186 & 1.677624 \\
\hline 88 & 6 & $\odot$ & -2.956890 & -2.615768 & 1.679164 \\
\hline 89 & 6 & $\odot$ & -3.779912 & -1.499819 & 1.667616 \\
\hline 90 & 7 & $\odot$ & -3.453614 & -0.201504 & 1.687073 \\
\hline 91 & 6 & $\odot$ & -2.150387 & $-0.00 \odot 453$ & 1.658408 \\
\hline 92 & 7 & $\odot$ & -1.237529 & -1.029195 & 1.666175 \\
\hline 93 & 6 & $\odot$ & -1.547202 & -2.393407 & 1.679644 \\
\hline 94 & 8 & 0 & -0.638156 & -3.237481 & 1.679916 \\
\hline
\end{tabular}




\begin{tabular}{|c|c|c|c|c|c|}
\hline 95 & 1 & 0 & -5.844217 & -3.973441 & 1.647904 \\
\hline 96 & 7 & $\odot$ & -1.685027 & 1.262152 & 1.640684 \\
\hline 97 & 1 & 0 & -2.319869 & 1.900674 & 1.160071 \\
\hline 98 & 1 & 0 & -0.682113 & 1.426223 & 1.548242 \\
\hline 99 & 6 & 0 & -5.800925 & 2.677832 & $\odot .625069$ \\
\hline 100 & 11 & $\odot$ & 6.938101 & $\odot .301362$ & 3.882925 \\
\hline 101 & 11 & $\odot$ & -9.854609 & 2.990790 & -2.179088 \\
\hline 102 & 6 & 0 & -4.414332 & 2.429856 & -2.400373 \\
\hline 103 & 6 & 0 & -3.155620 & 2.065224 & -1.608687 \\
\hline 104 & 8 & 0 & -3.508157 & 2.396823 & -0.215987 \\
\hline 105 & 1 & 0 & -5.392219 & 2.160115 & 1.495492 \\
\hline 106 & 1 & 0 & -6.487294 & 3.466131 & 0.945527 \\
\hline 107 & 1 & 0 & -4.347126 & 4.206252 & $\odot .346029$ \\
\hline 108 & 1 & 0 & -6.054921 & 3.754477 & -1.798763 \\
\hline 109 & 1 & 0 & -4.191000 & 2.670325 & -3.441941 \\
\hline 110 & 1 & $\odot$ & -5.142932 & 1.619531 & -2.369384 \\
\hline 111 & 1 & 0 & -2.310598 & 2.703262 & -1.879814 \\
\hline 112 & 7 & 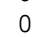 & -2.727420 & 0.702497 & -1.719402 \\
\hline 113 & 6 & 0 & -3.536939 & -0.457012 & -2.193794 \\
\hline 114 & 7 & 0 & -2.639240 & -1.587815 & -2.198416 \\
\hline 115 & 6 & 0 & -1.428600 & -1.094974 & -1.924187 \\
\hline 116 & 6 & 0 & -1.435242 & 0.299703 & -1.652029 \\
\hline 117 & 7 & 0 & $-\odot .379756$ & 1.075907 & -1.417319 \\
\hline 118 & 6 & 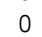 & $\odot .791770$ & 0.430764 & -1.481312 \\
\hline 119 & 7 & 0 & $\odot .900394$ & $-\odot .916987$ & -1.671121 \\
\hline 120 & 6 & 0 & -0.173295 & -1.779270 & -1.887977 \\
\hline 121 & 7 & 0 & 1.932044 & 1.111159 & -1.365110 \\
\hline 122 & 8 & 0 & 0.014285 & -2.994627 & -2.046157 \\
\hline 123 & 1 & 0 & -3.856791 & -0.237113 & -3.226770 \\
\hline 124 & 1 & $\odot$ & 1.904681 & 2.096105 & -1.156033 \\
\hline 125 & 1 & 0 & 2.846838 & $\odot .671248$ & -1.503097 \\
\hline 126 & 1 & 0 & 1.843116 & -1.353164 & -1.708042 \\
\hline 127 & 8 & 0 & -4.655799 & $-\odot .704449$ & -1.417340 \\
\hline 128 & 1 & 0 & -5.448325 & -0.471013 & -1.961600 \\
\hline 129 & 8 & 0 & -6.684043 & -0.116837 & -3.022990 \\
\hline 130 & 1 & $\odot$ & -7.048975 & $-\odot .903340$ & -3.437654 \\
\hline 131 & 1 & 0 & -7.421942 & 0.277140 & -2.482834 \\
\hline 132 & 1 & 0 & -4.980319 & -2.625660 & -1.355641 \\
\hline 133 & 8 & 0 & -5.430809 & -3.478423 & -1.474925 \\
\hline 134 & 1 & 0 & -6.241334 & -3.238108 & -1.930917 \\
\hline
\end{tabular}

General basis read from cards: (6D, 7F)

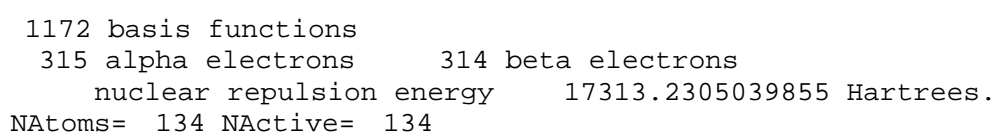

NAtoms $=134$ NActive $=134$

Force inversion solution in PCM.

Polarizable Continuum Model (PCM)

=ニニニニニニニニニニニニニニニニニニニニニニニニニニニニニニニ=

Model : PCM.

Solvent : Water, Eps $=78.355300$ Eps $($ inf $)=1.777849$

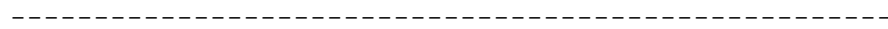

SCF Done: $E($ UWB97XD $)=-5092.29440247$ A.U. after 1 cycles

$$
\text { NFock }=1 \text { Conv }=0.19 \mathrm{D}-08 \quad-\mathrm{V} / \mathrm{T}=2.0066
$$

$\langle\mathrm{S} x\rangle=0.0000\langle\mathrm{Sy}\rangle=0.0000\langle\mathrm{Sz}\rangle=0.5000$

$\left\langle S^{* *} 2>=0.7621 \mathrm{~S}=0.5060\right.$

$<$ L.S $>=0.000000000000 \mathrm{E}+00$

Annihilation of the first spin contaminant:

$\mathrm{S}^{* * 2}$ before annihilation 0.7621 , after 0.7501

Harmonic frequencies $\left(\mathrm{cm}^{*}-1\right)$, IR intensities (KM/Mole), Raman

\begin{tabular}{|c|c|c|}
\hline $\begin{array}{l}1 \\
A\end{array}$ & $\begin{array}{l}2 \\
A\end{array}$ & $\begin{array}{l}3 \\
A\end{array}$ \\
\hline Frequencies - - & 19.5999 & 21.1322 \\
\hline Zero-point correction= & & 1.039465 (a.u.) \\
\hline Thermal correction to Energy= & & 1.117457 \\
\hline Thermal correction to Enthalpy= & & 1.118401 \\
\hline Thermal correction to Gibbs Free & Energy $=$ & 0.922931 \\
\hline Sum of electronic and zero-point & Energies= & -5091.254938 \\
\hline Sum of electronic and thermal Ene & ergies= & -5091.176945 \\
\hline
\end{tabular}


Sum of electronic and thermal Enthalpies= Sum of electronic and thermal Free Energies=

$$
\begin{gathered}
\text { E (Thermal) } \\
\text { KCal/Mol }
\end{gathered}
$$

Total
CV

Cal/Mol-Kelvin 295.387
$-5091.176001$

$-5091.371471$

$\mathrm{S}$

Cal/Mol-Kelvin 411.402

\begin{tabular}{lccc}
\multicolumn{1}{c}{ Item } & Value & Threshold Converged? \\
Maximum Force & 0.000070 & $0.00 \odot 450$ & YES \\
RMS Force & $0.000 \odot \odot 7$ & $0.00 \odot 30 \odot$ & YES \\
Maximum Displacement & 0.001817 & 0.001800 & NO \\
RMS Displacement & 0.000351 & 0.001200 & YES
\end{tabular}

Predicted change in Energy $=-2.886008 \mathrm{D}-\odot 8$

Normal termination of Gaussian 16 at Wed Jul 24 ๑๑:59:45 2019.

$\mathrm{G}(\mathrm{high})=-5093 \cdot 1412904 \mathrm{a} \cdot \mathrm{u}$.

-Figure S13-4, TS2(C8)W2(b)

dna.ts2bw2. log

Stoichiometry C38H53N16Na2023P2(2)

\begin{tabular}{|c|c|c|c|c|c|}
\hline \multirow{2}{*}{$\begin{array}{l}\text { Center } \\
\text { Number }\end{array}$} & \multirow{2}{*}{$\begin{array}{l}\text { Atomic } \\
\text { Number }\end{array}$} & \multirow{2}{*}{$\begin{array}{c}\text { Atomic } \\
\text { Type }\end{array}$} & \multicolumn{3}{|c|}{ Coordinates (Angstroms) } \\
\hline & & & $x$ & $\mathrm{Y}$ & Z \\
\hline & & 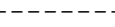 & - & & --- \\
\hline 1 & 7 & $\odot$ & 5.788049 & -1.942031 & -1.472253 \\
\hline 2 & 6 & $\odot$ & 5.961636 & -3.291097 & -1.346927 \\
\hline 3 & 6 & $\odot$ & 4.909493 & -4.134861 & -1.425238 \\
\hline 4 & 6 & $\odot$ & 3.614717 & -3.543610 & -1.602830 \\
\hline 5 & 7 & $\odot$ & 3.464376 & -2.227884 & -1.785134 \\
\hline 6 & 8 & $\odot$ & 9.009243 & 0.737860 & -0.080255 \\
\hline 7 & 15 & $\odot$ & 8.215650 & 1.832581 & $\odot .841642$ \\
\hline 8 & 8 & $\odot$ & 6.722061 & 1.842799 & $\odot .154517$ \\
\hline 9 & 8 & $\odot$ & 8.881068 & 3.160078 & 0.633631 \\
\hline 10 & 8 & $\odot$ & 7.994062 & 1.257696 & 2.223250 \\
\hline 11 & 8 & $\odot$ & 4.712062 & 5.527192 & 1.018444 \\
\hline 12 & 6 & $\odot$ & 5.170648 & 3.649275 & -0.370039 \\
\hline 13 & 6 & $\odot$ & 5.422174 & 4.263120 & 1. 010196 \\
\hline 14 & 6 & $\odot$ & -9.213822 & -2.651709 & 2.439324 \\
\hline 15 & 6 & $\odot$ & -8.564696 & -1.292256 & 2.273708 \\
\hline 16 & 8 & $\odot$ & -8.580062 & -3.595624 & 1.544237 \\
\hline 17 & 8 & $\odot$ & -8.488888 & 0.687622 & $\odot .916393$ \\
\hline 18 & 15 & 0 & -7.906213 & 1.704927 & -0.205391 \\
\hline 19 & 8 & $\odot$ & -6.297004 & 1.688432 & 0.028527 \\
\hline 20 & 8 & $\odot$ & -8.475225 & 3.075952 & $\odot .056469$ \\
\hline 21 & 8 & $\odot$ & -8.141079 & 1.140471 & -1.599911 \\
\hline 22 & 8 & $\odot$ & -4.315340 & 4.896313 & -1.935569 \\
\hline 23 & 6 & $\odot$ & -4.525535 & 3.403998 & -0.088799 \\
\hline 24 & 6 & $\odot$ & -4.957953 & 3.657769 & -1.534267 \\
\hline 25 & 1 & $\odot$ & -4.468032 & 5.061978 & -2.881447 \\
\hline 26 & 6 & $\odot$ & 9.920755 & -2.671273 & -1.278433 \\
\hline 27 & 6 & $\odot$ & 9.278638 & -1.299944 & -1.317191 \\
\hline 28 & 6 & $\odot$ & 8.896235 & -0.716919 & 0.051942 \\
\hline 29 & 6 & $\odot$ & 7.453273 & -1.174883 & ๑. 219595 \\
\hline 30 & 6 & $\odot$ & 6.901639 & -1.046617 & -1.199338 \\
\hline 31 & 8 & $\odot$ & 8.020235 & -1.366166 & -2.072859 \\
\hline 32 & 1 & 0 & 10.029538 & -3.037211 & -2.304565 \\
\hline 33 & 1 & $\odot$ & 10.915633 & -2.589468 & -0.825727 \\
\hline 34 & 1 & $\odot$ & 9.945674 & -0.610653 & -1.839344 \\
\hline 35 & 1 & 0 & 9.551878 & -1.048200 & 0.858391 \\
\hline 36 & 1 & $\odot$ & 6.899526 & -0.584069 & $\odot .943782$ \\
\hline 37 & 1 & $\odot$ & 7.462964 & -2.219406 & 0.537378 \\
\hline 38 & 1 & 0 & 6.549129 & -0.040023 & -1.405670 \\
\hline 39 & 8 & $\odot$ & 9.083101 & -3.565839 & -0.504742 \\
\hline 40 & 1 & 0 & 9.452928 & -4.463505 & -0.493140 \\
\hline 41 & 6 & $\odot$ & 4.530032 & -1.393419 & -1.766279 \\
\hline 42 & 7 & $\odot$ & 2.510367 & -4.287686 & -1.581117 \\
\hline 43 & 8 & 0 & 4.423729 & -0.176702 & -1.963106 \\
\hline 44 & 1 & 0 & 6.987970 & -3.611605 & -1.175528 \\
\hline 45 & 1 & 0 & 5.035790 & -5.204025 & -1.325585 \\
\hline 46 & 1 & $\odot$ & 2.566954 & -5.281789 & -1.435550 \\
\hline 47 & 1 & $\odot$ & 1.594561 & -3.832820 & -1.674751 \\
\hline 48 & 6 & $\odot$ & 6.296112 & 2.770536 & -0.882402 \\
\hline
\end{tabular}

Standard orientation: 


\begin{tabular}{|c|c|c|c|c|c|}
\hline 49 & 1 & 0 & 4.817459 & 5.969770 & 1.877670 \\
\hline 50 & 6 & 0 & 4.786622 & 3.255764 & 1.975065 \\
\hline 51 & 6 & 0 & 3.588531 & 2.717736 & 1.184704 \\
\hline 52 & 8 & 0 & 3.939563 & 2.868003 & -0.222993 \\
\hline 53 & 1 & $\odot$ & 5.940964 & 2.175198 & -1.725214 \\
\hline 54 & 1 & 0 & 7.141875 & 3.392223 & -1.186838 \\
\hline 55 & 1 & 0 & 4.971289 & 4.437954 & -1.099281 \\
\hline 56 & 1 & $\odot$ & 6.489614 & 4.412601 & 1.200506 \\
\hline 57 & 1 & 0 & 4.475123 & 3.719011 & 2.913414 \\
\hline 58 & 1 & 0 & 5.508676 & 2.467696 & 2.195866 \\
\hline 59 & 1 & $\odot$ & 2.682579 & 3.285832 & 1.378355 \\
\hline 60 & 7 & 0 & 3.250204 & 1.319544 & 1.429572 \\
\hline 61 & 6 & 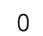 & 4.185428 & $\odot .349656$ & 1.223600 \\
\hline 62 & 6 & 0 & 3.860855 & $-\odot .960498$ & 1.291234 \\
\hline 63 & 6 & 0 & 2.477556 & -1.282765 & 1.486073 \\
\hline 64 & 7 & 0 & 1.559606 & $-\odot .330582$ & 1.670098 \\
\hline 65 & 6 & $\odot$ & 1.901938 & 0.981205 & 1.646264 \\
\hline 66 & 1 & 0 & 4.604146 & -1.732322 & 1.141280 \\
\hline 67 & 7 & 0 & 2.064828 & -2.550275 & 1.476439 \\
\hline 68 & 8 & 0 & 1.075520 & 1.885275 & 1.808079 \\
\hline 69 & 1 & 0 & 5.181079 & 0.707452 & $\odot .976390$ \\
\hline 70 & 1 & $\odot$ & 2.720059 & -3.295324 & 1.308320 \\
\hline 71 & 1 & $\odot$ & 1.071798 & -2.778788 & 1.598746 \\
\hline 72 & 1 & 0 & -0.280743 & -0.779331 & 1.727042 \\
\hline 73 & 1 & 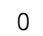 & -8.903915 & -4.495853 & 1.708903 \\
\hline 74 & 6 & 0 & -8.477138 & $-\odot .786669$ & $\odot .827450$ \\
\hline 75 & 6 & 0 & -7.123881 & -1.302022 & 0.369467 \\
\hline 76 & 6 & $\odot$ & -6.275978 & -1.097906 & 1.619114 \\
\hline 77 & 8 & 0 & -7.174922 & -1.347779 & 2.740032 \\
\hline 78 & 1 & 0 & -9.096162 & -2.965735 & 3.481927 \\
\hline 79 & 1 & 0 & -10.284866 & -2.567223 & 2.218052 \\
\hline 80 & 1 & 0 & -9.098777 & $-\odot .566989$ & 2.892120 \\
\hline 81 & 1 & 0 & -9.312606 & -1.100272 & 0.201426 \\
\hline 82 & 1 & $\odot$ & -6.720300 & -0.764989 & -0.482069 \\
\hline 83 & 1 & 0 & -7.199115 & -2.363483 & 0.129058 \\
\hline 84 & 1 & 0 & -5.908048 & $-\odot .072589$ & 1.674977 \\
\hline 85 & 7 & 0 & -5.114597 & -1.941375 & 1.714345 \\
\hline 86 & 6 & 0 & -5.021491 & -3.317050 & 1.750784 \\
\hline 87 & 7 & $\odot$ & -3.786990 & -3.742295 & 1.797977 \\
\hline 88 & 6 & $\odot$ & -3.019977 & -2.584534 & 1.785583 \\
\hline 89 & 6 & 0 & -3.834114 & -1.462928 & 1.733886 \\
\hline 90 & 7 & 0 & -3.498695 & -0.165278 & 1.706215 \\
\hline 91 & 6 & 0 & -2.190630 & 0.023496 & 1.700191 \\
\hline 92 & 7 & 0 & -1.288900 & -1.013349 & 1.755776 \\
\hline 93 & 6 & 0 & -1.608014 & -2.373689 & 1.795367 \\
\hline 94 & 8 & 0 & -0.704903 & -3.223037 & 1.827762 \\
\hline 95 & 1 & 0 & -5.916212 & -3.923708 & 1.737391 \\
\hline 96 & 7 & 0 & -1.706445 & 1.276461 & 1.666449 \\
\hline 97 & 1 & 0 & -2.308919 & 1.920973 & 1.146746 \\
\hline 98 & 1 & 0 & -0.698763 & 1.418476 & 1.587051 \\
\hline 99 & 6 & 0 & -5.558406 & 2.703339 & 0.780380 \\
\hline 100 & 11 & $\odot$ & 6.849206 & $\odot .326713$ & 3.845729 \\
\hline 101 & 11 & $\odot$ & -9.234693 & 3.411337 & -2.242286 \\
\hline 102 & 6 & 0 & -4.392063 & 2.453500 & -2.287347 \\
\hline 103 & 6 & 0 & -3.066245 & 2.146661 & -1.580032 \\
\hline 104 & 8 & 0 & -3.300369 & 2.617348 & -0.191788 \\
\hline 105 & 1 & 0 & -5.050906 & 2.188011 & 1.597347 \\
\hline 106 & 1 & 0 & -6.267111 & 3.437484 & 1.170814 \\
\hline 107 & 1 & 0 & -4.262081 & 4.351652 & 0.389468 \\
\hline 108 & 1 & 0 & -6.044646 & 3.744921 & -1.630558 \\
\hline 109 & 1 & 0 & -4.257433 & 2.649560 & -3.353833 \\
\hline 110 & 1 & 0 & -5.070170 & 1.606423 & -2.163315 \\
\hline 111 & 1 & 0 & -2.237128 & 2.746740 & -1.968437 \\
\hline 112 & 7 & 0 & -2.758108 & 0.753117 & -1.593717 \\
\hline 113 & 6 & 0 & -3.648432 & -1.026467 & -2.265756 \\
\hline 114 & 7 & 0 & -2.635003 & -1.795160 & -1.867652 \\
\hline 115 & 6 & 0 & -1.470913 & -1.140110 & -1.768451 \\
\hline 116 & 6 & 0 & -1.524256 & 0.302622 & -1.590384 \\
\hline 117 & 7 & 0 & -0.403848 & 1.058291 & -1.449702 \\
\hline 118 & 6 & 0 & $\odot .753698$ & $\odot .417926$ & -1.507825 \\
\hline 119 & 7 & 0 & $\odot .877541$ & -0.941770 & -1.630422 \\
\hline 120 & 6 & 0 & -0.200881 & -1.803866 & -1.742658 \\
\hline 121 & 7 & 0 & 1.903507 & 1.096196 & -1.446822 \\
\hline 122 & 8 & 0 & $-\odot .025062$ & -3.028408 & -1.840234 \\
\hline 123 & 1 & 0 & -3.626030 & $-\odot .547133$ & -3.247300 \\
\hline 124 & 1 & 0 & 1.885332 & 2.087331 & -1.270966 \\
\hline 125 & 1 & 0 & 2.813459 & $\odot .642375$ & -1.558211 \\
\hline
\end{tabular}




$\begin{array}{rrrrrr}126 & 1 & 0 & 1.819090 & -1.373799 & -1.675735 \\ 127 & 8 & 0 & -4.840542 & -1.272676 & -1.779967 \\ 128 & 1 & 0 & -5.565096 & -0.850321 & -2.358589 \\ 129 & 8 & 0 & -6.657734 & -0.208822 & -3.197668 \\ 130 & 1 & 0 & -7.207415 & -0.895003 & -3.588722 \\ 131 & 1 & 0 & -7.257501 & 0.312278 & -2.585220 \\ 132 & 1 & \odot & -5.309377 & -3.127253 & -1.284410 \\ 133 & 8 & 0 & -5.811500 & -3.954575 & -1.280694 \\ 134 & 1 & 0 & -6.543622 & -3.768009 & -1.875248 \\ -\end{array}$

General basis read from cards: (6D, 7F)

1172 basis functions

315 alpha electrons $\quad 314$ beta electrons

nuclear repulsion energy 17305.2816625314 Hartrees.

NAtoms $=134$ NActive $=134$

Force inversion solution in PCM.

Polarizable Continuum Model (PCM)

$\begin{array}{ll}\text { Model } & \text { : PCM. }\end{array}$

Atomic radii : UFF (Universal Force Field).

Solvent : Water, Eps $=78.355300$ Eps $($ inf $)=1.777849$

SCF Done: $E($ UWB97XD $)=-5092.26585298$ A.U. after 1 cycles NFock $=1$ Conv $=0.13 \mathrm{D}-08 \quad-\mathrm{V} / \mathrm{T}=2.0066$

$<\mathrm{S} x>=0.0000<\mathrm{Sy}\rangle=0.0000<\mathrm{Sz}>=0.5000$

$<S^{*} 2>=0.7602 \mathrm{~S}=0.5051$

$<$ L.S $>=0.000000000000 \mathrm{E}+00$

Annihilation of the first spin contaminant:

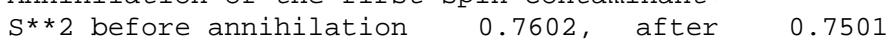

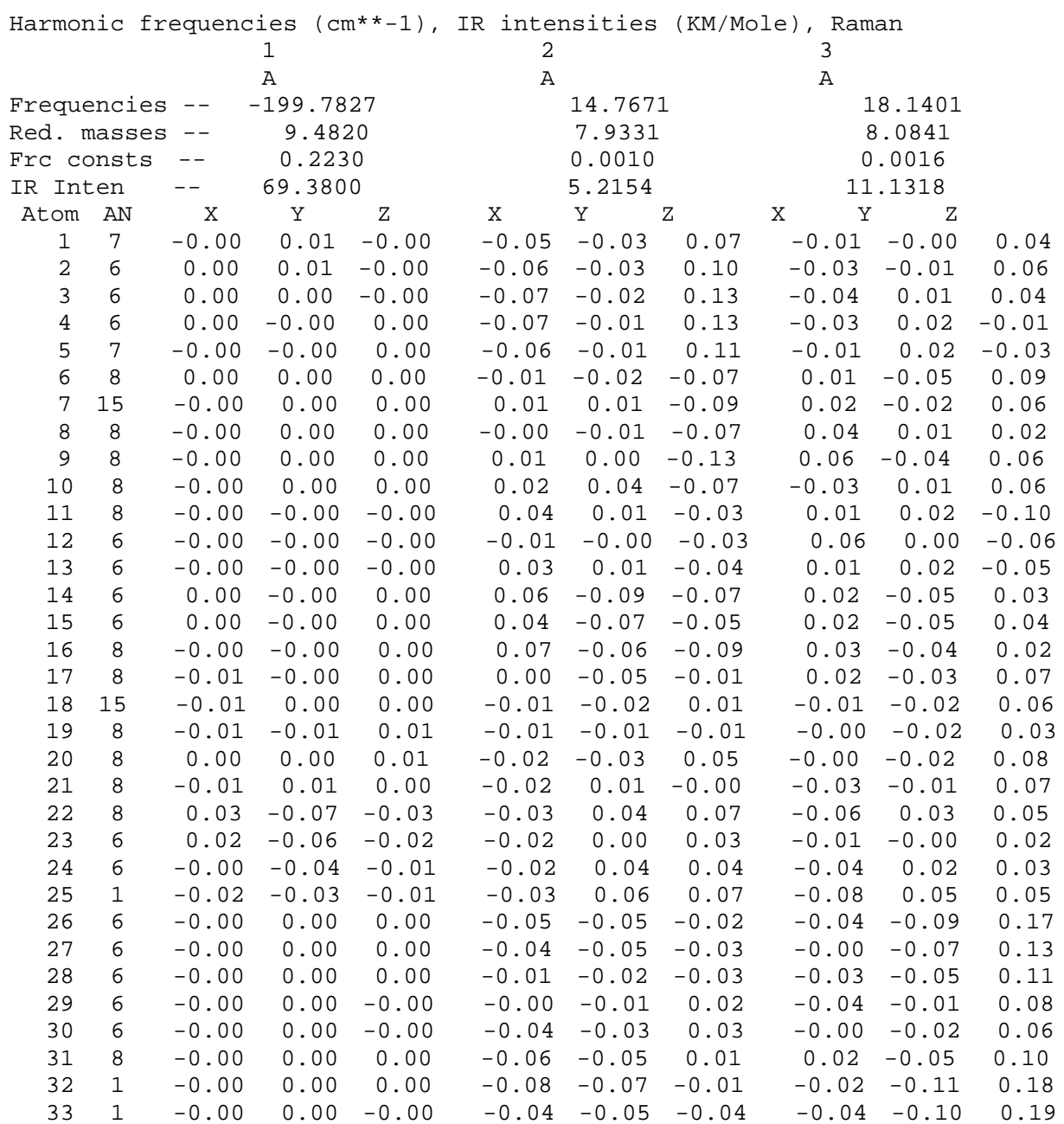




\begin{tabular}{|c|c|c|c|c|c|c|c|c|c|c|}
\hline 34 & 1 & $-\odot . \odot \odot$ & $\odot . \odot \odot$ & $\odot . \odot \odot$ & -0.05 & -0.06 & -0.06 & 0.03 & -0.10 & 0.14 \\
\hline 35 & 1 & $-\odot . \odot \odot$ & $\odot . \odot \odot$ & $\odot . \odot \odot$ & 0.02 & $\odot .0 \odot$ & $-\odot .04$ & -0.06 & -0.05 & 0.13 \\
\hline 36 & 1 & $-\odot . \odot \odot$ & $\odot . \odot \odot$ & 0.00 & 0.02 & 0.01 & $\odot .02$ & -0.05 & 0.01 & 0.05 \\
\hline 37 & 1 & $-\odot . \odot \odot$ & $0.0 \odot$ & $-\odot . \odot \odot$ & $\odot .0 \odot$ & -0.00 & 0.04 & -0.07 & -0.01 & 0.09 \\
\hline 38 & 1 & $\odot .0 \odot$ & $\odot .0 \odot$ & -0.00 & -0.04 & -0.03 & $\odot .02$ & 0.02 & -0.02 & 0.03 \\
\hline 39 & 8 & $\odot . \odot \odot$ & $\odot . \odot \odot$ & $\odot . \odot \odot$ & -0.04 & -0.03 & 0.03 & -0.07 & -0.06 & 0.16 \\
\hline 40 & 1 & $-\odot . \odot \odot$ & $\odot . \odot \odot$ & $-\odot . \odot \odot$ & -0.05 & -0.03 & $\odot .03$ & -0.10 & -0.06 & 0.19 \\
\hline 41 & 6 & $-\odot . \odot \odot$ & 0.00 & $\odot .0 \odot$ & -0.05 & -0.02 & $\odot .09$ & 0.00 & 0.01 & -0.01 \\
\hline 42 & 7 & $\odot .01$ & $-\odot . \odot \odot$ & 0.00 & -0.07 & $-\odot . \odot \odot$ & 0.15 & -0.03 & 0.03 & -0.05 \\
\hline 43 & 8 & -0.01 & $\odot . \odot \odot$ & $\odot .01$ & -0.05 & -0.02 & 0.07 & 0.02 & 0.01 & -0.02 \\
\hline 44 & 1 & $\odot .0 \odot$ & 0.01 & -0.00 & -0.06 & -0.03 & 0.09 & -0.04 & -0.02 & 0.10 \\
\hline 45 & 1 & 0.01 & 0.00 & -0.00 & -0.08 & -0.02 & 0.15 & -0.06 & 0.01 & 0.05 \\
\hline 46 & 1 & 0.01 & $-\odot . \odot \odot$ & $-\odot . \odot \odot$ & $-\odot .08$ & -0.00 & 0.16 & -0.05 & 0.03 & -0.02 \\
\hline 47 & 1 & $\odot .0 \odot$ & $-\odot . \odot \odot$ & $-\odot . \odot \odot$ & -0.07 & $-\odot . \odot \odot$ & 0.13 & -0.03 & 0.04 & $-\odot .07$ \\
\hline 48 & 6 & -0.00 & $-\odot .0 \odot$ & -0.00 & -0.03 & -0.01 & -0.06 & $\odot .09$ & 0.00 & -0.00 \\
\hline 49 & 1 & $\odot .0 \odot$ & 0.00 & -0.00 & 0.07 & 0.02 & -0.04 & -0.02 & 0.03 & -0.10 \\
\hline 50 & 6 & -0.00 & $-0.0 \odot$ & $-\odot .0 \odot$ & $\odot .06$ & 0.02 & -0.01 & -0.03 & $\odot . \odot 4$ & -0.07 \\
\hline 51 & 6 & -0.00 & $-0.0 \odot$ & $-0.0 \odot$ & 0.03 & 0.01 & 0.03 & $0.0 \odot$ & 0.02 & -0.11 \\
\hline 52 & 8 & $-\odot . \odot \odot$ & $-\odot . \odot \odot$ & $-0.0 \odot$ & -0.01 & $\odot .0 \odot$ & 0.02 & 0.06 & 0.00 & -0.09 \\
\hline 53 & 1 & -0.00 & $-\odot .0 \odot$ & -0.00 & -0.06 & -0.02 & -0.04 & 0.12 & -0.00 & -0.02 \\
\hline 54 & 1 & $-\odot . \odot \odot$ & $-\odot . \odot \odot$ & $-0.0 \odot$ & -0.04 & -0.02 & -0.09 & 0.10 & $-0.0 \odot$ & 0.03 \\
\hline 55 & 1 & $-\odot .0 \odot$ & -0.00 & -0.00 & -0.03 & -0.01 & -0.03 & 0.09 & -0.00 & -0.07 \\
\hline 56 & 1 & $-\odot . \odot \odot$ & $-\odot . \odot \odot$ & $-\odot . \odot \odot$ & $\odot . \odot 4$ & $\odot .0 \odot$ & $-\odot .08$ & $\odot . \odot \odot$ & 0.02 & -0.01 \\
\hline 57 & 1 & -0.00 & 0.00 & -0.00 & 0.09 & 0.03 & -0.01 & -0.07 & 0.05 & $-\odot .09$ \\
\hline 58 & 1 & -0.00 & $\odot .0 \odot$ & $-\odot . \odot \odot$ & 0.07 & 0.02 & -0.02 & -0.04 & 0.04 & -0.03 \\
\hline 59 & 1 & $-\odot . \odot \odot$ & $-\odot . \odot \odot$ & $\odot . \odot \odot$ & 0.04 & 0.02 & 0.06 & $-\odot .0 \odot$ & 0.02 & -0.15 \\
\hline 60 & 7 & -0.00 & $-\odot . \odot \odot$ & $-0.0 \odot$ & $\odot .04$ & $\odot .02$ & 0.05 & 0.00 & 0.03 & -0.10 \\
\hline 61 & 6 & $\odot .0 \odot$ & $-\odot . \odot \odot$ & $\odot . \odot \odot$ & $\odot .04$ & 0.02 & $\odot .08$ & 0.01 & 0.03 & -0.05 \\
\hline 62 & 6 & 0.00 & $-\odot .0 \odot$ & $\odot .0 \odot$ & 0.04 & 0.02 & 0.10 & 0.02 & 0.03 & -0.02 \\
\hline 63 & 6 & $\odot .0 \odot$ & $-\odot . \odot \odot$ & $\odot . \odot \odot$ & $\odot .04$ & 0.02 & 0.09 & $\odot .02$ & 0.02 & -0.05 \\
\hline 64 & 7 & $\odot . \odot \odot$ & $\odot .0 \odot$ & -0.00 & 0.04 & $\odot .02$ & 0.06 & 0.01 & 0.02 & -0.10 \\
\hline 65 & 6 & 0.00 & $-\odot .0 \odot$ & $\odot .0 \odot$ & 0.03 & $\odot .02$ & 0.04 & $-\odot .00$ & 0.02 & -0.12 \\
\hline 66 & 1 & $\odot .0 \odot$ & $-\odot . \odot \odot$ & $\odot .0 \odot$ & 0.05 & 0.02 & 0.12 & 0.03 & 0.03 & 0.01 \\
\hline 67 & 7 & $\odot .0 \odot$ & $-\odot . \odot \odot$ & $\odot .0 \odot$ & 0.05 & 0.01 & 0.11 & 0.03 & 0.02 & -0.03 \\
\hline 68 & 8 & -0.00 & $\odot .0 \odot$ & $-\odot . \odot \odot$ & 0.03 & 0.02 & 0.01 & -0.01 & 0.02 & -0.16 \\
\hline 69 & 1 & $\odot .0 \odot$ & $-\odot . \odot \odot$ & $-\odot . \odot \odot$ & 0.04 & 0.01 & $\odot .08$ & 0.01 & 0.03 & -0.04 \\
\hline 70 & 1 & $-\odot . \odot \odot$ & $-\odot . \odot \odot$ & $-\odot . \odot \odot$ & 0.05 & 0.01 & 0.13 & 0.04 & $\odot .02$ & 0.01 \\
\hline 71 & 1 & $-\odot . \odot \odot$ & $-\odot . \odot \odot$ & $\odot . \odot \odot$ & $\odot . \odot 4$ & 0.01 & 0.09 & 0.03 & 0.01 & -0.05 \\
\hline 72 & 1 & 0.00 & $-\odot .0 \odot$ & 0.00 & 0.04 & $\odot .02$ & 0.01 & 0.02 & -0.00 & -0.09 \\
\hline 73 & 1 & $-\odot . \odot \odot$ & $-\odot . \odot \odot$ & $\odot .0 \odot$ & $\odot .09$ & -0.07 & -0.11 & 0.03 & -0.04 & $\odot .0 \odot$ \\
\hline 74 & 6 & -0.00 & $-0.0 \odot$ & $\odot . \odot \odot$ & 0.03 & -0.04 & -0.04 & 0.02 & -0.03 & 0.05 \\
\hline 75 & 6 & $-\odot . \odot \odot$ & $\odot . \odot \odot$ & $\odot . \odot \odot$ & 0.03 & -0.01 & -0.05 & $\odot .02$ & -0.02 & $\odot .04$ \\
\hline 76 & 6 & $-\odot . \odot \odot$ & $\odot .0 \odot$ & $-\odot . \odot \odot$ & 0.03 & -0.03 & -0.05 & 0.02 & -0.03 & 0.04 \\
\hline 77 & 8 & 0.00 & $\odot .0 \odot$ & 0.00 & 0.04 & -0.06 & -0.05 & $\odot .02$ & -0.05 & $\odot .04$ \\
\hline 78 & 1 & $\odot .0 \odot$ & $-\odot .0 \odot$ & $\odot . \odot \odot$ & 0.07 & -0.11 & -0.08 & 0.03 & -0.06 & $\odot .02$ \\
\hline 79 & 1 & 0.00 & $-0.0 \odot$ & 0.00 & 0.06 & -0.10 & -0.07 & 0.02 & -0.05 & 0.03 \\
\hline 80 & 1 & 0.00 & $-0.0 \odot$ & 0.00 & $\odot .03$ & -0.10 & -0.03 & $\odot .02$ & -0.05 & 0.06 \\
\hline 81 & 1 & $-\odot . \odot \odot$ & $-\odot . \odot \odot$ & $\odot . \odot \odot$ & 0.03 & -0.04 & -0.04 & 0.02 & -0.02 & 0.05 \\
\hline 82 & 1 & -0.00 & $-0.0 \odot$ & $\odot .0 \odot$ & 0.02 & $\odot .01$ & -0.04 & $\odot .02$ & -0.01 & $\odot .05$ \\
\hline 83 & 1 & $-\odot . \odot \odot$ & $\odot .0 \odot$ & $\odot .0 \odot$ & 0.05 & -0.01 & -0.08 & $\odot .02$ & -0.01 & $\odot .03$ \\
\hline 84 & 1 & -0.00 & 0.00 & $-\odot . \odot \odot$ & 0.02 & -0.03 & -0.04 & 0.01 & -0.03 & 0.06 \\
\hline 85 & 7 & 0.00 & $\odot .00$ & -0.00 & 0.04 & -0.02 & -0.07 & 0.02 & -0.03 & 0.02 \\
\hline 86 & 6 & $\odot .0 \odot$ & $\odot . \odot \odot$ & $\odot . \odot \odot$ & 0.05 & $-\odot .02$ & -0.08 & $\odot .03$ & -0.03 & 0.01 \\
\hline 87 & 7 & -0.00 & 0.00 & $-\odot . \odot \odot$ & 0.06 & -0.01 & -0.06 & 0.03 & -0.02 & -0.02 \\
\hline 88 & 6 & $\odot .0 \odot$ & $-\odot . \odot \odot$ & -0.01 & 0.05 & $-\odot . \odot \odot$ & -0.05 & 0.03 & -0.02 & -0.03 \\
\hline 89 & 6 & & 0.00 & -0.01 & & -0.01 & -0.05 & & -0.02 & -0.00 \\
\hline 90 & 7 & $\odot .0 \odot$ & $-0.0 \odot$ & $-\odot . \odot \odot$ & 0.03 & -0.01 & -0.04 & 0.02 & -0.02 & -0.01 \\
\hline 91 & 6 & 0.00 & -0.00 & 0.00 & 0.03 & 0.00 & -0.02 & 0.02 & -0.02 & -0.04 \\
\hline 92 & 7 & $0.0 \odot$ & $-\odot .0 \odot$ & $\odot .0 \odot$ & 0.04 & 0.01 & -0.01 & 0.02 & -0.01 & -0.06 \\
\hline 93 & 6 & $\odot . \odot \odot$ & $-\odot . \odot \odot$ & $-\odot . \odot \odot$ & 0.04 & 0.01 & -0.02 & 0.03 & -0.01 & -0.06 \\
\hline 94 & 8 & 0.00 & -0.00 & $\odot . \odot \odot$ & 0.05 & $\odot .01$ & 0.01 & 0.03 & -0.01 & -0.08 \\
\hline 95 & 1 & $-\odot . \odot \odot$ & $\odot . \odot \odot$ & 0.01 & 0.06 & -0.02 & -0.09 & 0.03 & -0.03 & $\odot .03$ \\
\hline 96 & 7 & 0.01 & $-0.0 \odot$ & 0.00 & 0.02 & 0.01 & -0.01 & 0.01 & -0.01 & -0.05 \\
\hline 97 & 1 & 0.01 & -0.01 & -0.01 & 0.02 & 0.01 & -0.00 & -0.00 & -0.01 & -0.03 \\
\hline 98 & 1 & 0.01 & $-\odot . \odot \odot$ & $\odot .01$ & 0.02 & 0.02 & $-\odot . \odot \odot$ & 0.01 & -0.01 & -0.08 \\
\hline 99 & 6 & $\odot .0 \odot$ & -0.01 & -0.01 & -0.01 & -0.02 & 0.02 & 0.01 & -0.03 & 0.03 \\
\hline 100 & 11 & $-\odot . \odot \odot$ & 0.00 & 0.00 & 0.13 & 0.06 & 0.01 & $-0 . \odot 9$ & 0.04 & 0.03 \\
\hline 101 & 11 & $-0.0 \odot$ & -0.00 & $\odot .00$ & -0.02 & 0.02 & 0.05 & -0.03 & -0.01 & 0.08 \\
\hline 102 & 6 & -0.09 & $-\odot .09$ & -0.03 & -0.03 & 0.05 & 0.01 & -0.04 & 0.04 & 0.00 \\
\hline 103 & 6 & -0.12 & -0.20 & -0.05 & -0.02 & 0.03 & $-\odot . \odot \odot$ & -0.02 & $\odot .04$ & -0.02 \\
\hline 104 & 8 & -0.02 & -0.13 & -0.06 & -0.01 & 0.01 & 0.01 & -0.01 & 0.01 & -0.01 \\
\hline 105 & 1 & -0.01 & 0.00 & 0.01 & -0.01 & -0.04 & 0.01 & 0.03 & -0.04 & 0.01 \\
\hline 106 & 1 & 0.02 & 0.02 & -0.03 & -0.02 & -0.03 & 0.04 & 0.01 & -0.04 & 0.06 \\
\hline 107 & 1 & 0.09 & -0.07 & -0.03 & -0.02 & -0.01 & 0.06 & -0.01 & -0.01 & $\odot .03$ \\
\hline 108 & 1 & 0.00 & -0.00 & 0.01 & -0.02 & 0.04 & 0.05 & $-\odot . \odot 4$ & 0.01 & 0.05 \\
\hline 109 & 1 & -0.08 & -0.09 & -0.02 & -0.03 & 0.08 & 0.02 & -0.06 & 0.06 & 0.00 \\
\hline 110 & 1 & -0.19 & -0.02 & -0.04 & -0.03 & 0.05 & -0.01 & -0.02 & 0.03 & 0.00 \\
\hline
\end{tabular}




\begin{tabular}{|c|c|c|c|c|c|c|c|c|c|c|}
\hline 111 & 1 & -0.08 & -0.28 & -0.08 & -0.02 & 0.04 & 0.01 & -0.03 & 0.06 & -0.02 \\
\hline 112 & 7 & -0.07 & -0.22 & -0.07 & -0.03 & $\odot .03$ & $-\odot .03$ & -0.01 & 0.04 & -0.06 \\
\hline 113 & 6 & 0.10 & 0.48 & 0.13 & -0.03 & 0.04 & -0.07 & $-\odot .02$ & 0.04 & -0.03 \\
\hline 114 & 7 & 0.02 & 0.03 & -0.15 & -0.04 & 0.03 & -0.06 & -0.01 & 0.04 & -0.05 \\
\hline 115 & 6 & 0.04 & -0.07 & 0.01 & -0.04 & 0.03 & -0.02 & -0.01 & 0.05 & -0.07 \\
\hline 116 & 6 & 0.02 & -0.11 & 0.00 & -0.03 & 0.02 & -0.01 & -0.01 & 0.05 & $-\odot .07$ \\
\hline 117 & 7 & $-\odot .07$ & -0.04 & 0.04 & -0.03 & $\odot .02$ & 0.02 & $-\odot . \odot \odot$ & 0.05 & -0.09 \\
\hline 118 & 6 & -0.02 & -0.02 & 0.02 & -0.04 & 0.01 & 0.05 & $-\odot . \odot \odot$ & 0.04 & -0.10 \\
\hline 119 & 7 & $\odot .01$ & 0.00 & 0.02 & -0.04 & 0.01 & 0.06 & -0.01 & 0.04 & -0.09 \\
\hline 120 & 6 & $\odot .03$ & $-\odot .04$ & 0.01 & -0.05 & 0.02 & 0.02 & -0.01 & 0.05 & -0.08 \\
\hline 121 & 7 & -0.05 & $\odot .03$ & $\odot .0 \odot$ & -0.03 & $\odot . \odot \odot$ & 0.07 & $-\odot . \odot \odot$ & 0.04 & -0.11 \\
\hline 122 & 8 & $\odot .06$ & -0.04 & $\odot .00$ & -0.05 & $\odot .01$ & 0.03 & -0.01 & 0.04 & -0.08 \\
\hline 123 & 1 & -0.05 & 0.06 & -0.09 & -0.01 & 0.06 & -0.06 & -0.04 & 0.03 & -0.03 \\
\hline 124 & 1 & -0.07 & $\odot .03$ & 0.01 & -0.03 & $\odot . \odot \odot$ & 0.07 & $\odot . \odot \odot$ & $\odot .04$ & -0.10 \\
\hline 125 & 1 & $-\odot . \odot 4$ & 0.05 & 0.01 & -0.03 & -0.00 & 0.08 & $-\odot . \odot \odot$ & 0.04 & -0.09 \\
\hline 126 & 1 & 0.02 & 0.02 & 0.01 & $-\odot .04$ & $-\odot . \odot \odot$ & 0.08 & -0.01 & 0.04 & $-\odot .09$ \\
\hline 127 & 8 & $\odot .09$ & 0.36 & 0.18 & $-\odot .04$ & 0.04 & -0.10 & -0.01 & 0.03 & 0.00 \\
\hline 128 & 1 & $\odot .08$ & 0.24 & 0.15 & -0.03 & 0.06 & -0.09 & -0.02 & 0.03 & 0.02 \\
\hline 129 & 8 & -0.03 & 0.03 & -0.02 & -0.01 & 0.11 & -0.07 & -0.04 & 0.01 & 0.04 \\
\hline 130 & 1 & $\odot .04$ & -0.09 & $\odot .09$ & -0.01 & 0.13 & -0.12 & $-\odot .04$ & 0.01 & 0.04 \\
\hline 131 & 1 & -0.05 & $\odot .0 \odot$ & -0.03 & $-\odot .02$ & 0.07 & -0.05 & -0.04 & $\odot . \odot \odot$ & 0.05 \\
\hline 132 & 1 & 0.07 & $\odot .09$ & -0.03 & 0.02 & 0.03 & -0.10 & $\odot .01$ & $\odot .02$ & -0.01 \\
\hline 133 & 8 & $\odot .05$ & 0.10 & -0.03 & 0.05 & 0.01 & -0.09 & $\odot .02$ & $\odot .02$ & -0.01 \\
\hline 134 & 1 & $\odot .04$ & 0.12 & -0.01 & 0.02 & -0.02 & -0.07 & 0.01 & 0.02 & 0.00 \\
\hline
\end{tabular}

Thermochemistry

Temperature 298.150 Kelvin. Pressure 1.00000 Atm.

Zero-point correction=

Thermal correction to Energy=

Thermal correction to Enthalpy=

Thermal correction to Gibbs Free Energy=

Sum of electronic and zero-point Energies=

Sum of electronic and thermal Energies=

Sum of electronic and thermal Enthalpies=

Sum of electronic and thermal Free Energies=

$$
\begin{aligned}
1.036270(\mathrm{a} . \mathrm{u} .) \\
1.114282 \\
1.115227 \\
0.919594 \\
-5091.229583 \\
-5091.151571 \\
-5091.150626 \\
-5091.346259
\end{aligned}
$$

\begin{tabular}{|c|c|c|c|}
\hline E & $\begin{array}{l}\text { ermal) } \\
\text { /Mol } \\
99.223\end{array}$ & $\begin{array}{c}\text { CV } \\
\text { Cal/Mol-Kelvin } \\
295.040\end{array}$ & $\begin{array}{c}\mathrm{S} \\
\mathrm{Cal} / \mathrm{Mol}-\mathrm{Kel} \text { in } \\
411.744\end{array}$ \\
\hline Item & Value & \multicolumn{2}{|c|}{ Threshold Converged? } \\
\hline Maximum Force & 0.000296 & $\odot .00 \odot 450$ & $\odot$ YES \\
\hline Force & 0.000045 & 0.000300 & YES \\
\hline Maximum Displacement & 0.000235 & 0.001800 & YES \\
\hline RMS Displacement & 0.000055 & $\odot .001200$ & YES \\
\hline \multicolumn{4}{|c|}{$\begin{array}{l}\text { Predicted change in Energy }=-7.902643 \mathrm{D}-06 \\
\text { Optimization completed. }\end{array}$} \\
\hline
\end{tabular}

\begin{tabular}{|c|c|c|c|c|c|}
\hline \multirow{2}{*}{$\begin{array}{l}\text { Center } \\
\text { Number }\end{array}$} & \multirow{2}{*}{$\begin{array}{l}\text { Atomic } \\
\text { Number }\end{array}$} & \multirow{2}{*}{$\begin{array}{c}\text { Atomic } \\
\text { Type }\end{array}$} & \multicolumn{3}{|c|}{ Coordinates (Angstroms) } \\
\hline & & & $\mathrm{X}$ & $\mathrm{Y}$ & Z \\
\hline & & & -- & -1 & ------- \\
\hline 1 & 7 & $\odot$ & 5.397579 & -2.135653 & -1.371532 \\
\hline 2 & 6 & $\odot$ & 5.532146 & -3.470527 & -1.118115 \\
\hline 3 & 6 & $\odot$ & 4.446090 & -4.267296 & -1.003304 \\
\hline 4 & 6 & $\odot$ & 3.161992 & -3.639927 & -1.129480 \\
\hline 5 & 7 & $\odot$ & 3.044362 & -2.341857 & -1.421379 \\
\hline 6 & 8 & $\odot$ & 8.824847 & 0.542040 & -0.675015 \\
\hline 7 & 15 & $\odot$ & 8.237906 & 1.746162 & 0.268181 \\
\hline 8 & 8 & $\odot$ & 6.656362 & 1.798649 & -0.179922 \\
\hline 9 & 8 & $\odot$ & 8.923587 & 3.014396 & -0.142883 \\
\hline 10 & 8 & $\odot$ & 8.208289 & 1.280559 & 1.706722 \\
\hline
\end{tabular}

Normal termination of Gaussian 16 at Tue Jul 9 04:49:52 2019.

$G($ high $)=-5093 \cdot 11378025 \mathrm{a} \cdot \mathrm{u}$.

-...-Figure S13-5, Int3a(c8)W2(b)

dna. for $2 b w 2 . \log$

Stoichiometry C38H53N16Na2023P2(2)

Standard orientation: 


\begin{tabular}{|c|c|c|c|c|c|}
\hline 11 & 8 & $\odot$ & 4.834292 & 5.604985 & 0.576781 \\
\hline 12 & 6 & 0 & 5.082773 & 3.597396 & -0.675708 \\
\hline 13 & 6 & $\odot$ & 5.498044 & 4.316604 & 0.610430 \\
\hline 14 & 6 & $\odot$ & -8.986586 & -2.952975 & 1.430029 \\
\hline 15 & 6 & 0 & -8.427177 & -1.553250 & 1.582256 \\
\hline 16 & 8 & $\odot$ & -8.067082 & -3.799110 & 0.689243 \\
\hline 17 & 8 & $\odot$ & -8.278133 & $\odot .597480$ & $\odot .527257$ \\
\hline 18 & 15 & $\theta$ & -7.610771 & 1.780301 & -0.369065 \\
\hline 19 & 8 & $\odot$ & -6.086142 & 1.849222 & 0.170049 \\
\hline 20 & 8 & $\odot$ & -8.344195 & 3.068611 & -0.100429 \\
\hline 21 & 8 & 0 & -7.546644 & 1.336839 & -1.823427 \\
\hline 22 & 8 & $\odot$ & -4.102233 & 5.433219 & -1.085659 \\
\hline 23 & 6 & $\odot$ & -4.458065 & 3.659462 & $\odot .465795$ \\
\hline 24 & 6 & 0 & -4.792665 & 4.164667 & -0.937536 \\
\hline 25 & 1 & $\odot$ & -4.190703 & 5.758144 & -1.997829 \\
\hline 26 & 6 & $\odot$ & 9.505616 & -3.010728 & -1.585773 \\
\hline 27 & 6 & 0 & 8.900868 & -1.626769 & -1.703107 \\
\hline 28 & 6 & $\odot$ & $8.7 \odot 4156$ & -0.886207 & -0.371769 \\
\hline 29 & 6 & 0 & 7.282988 & -1.270097 & 0.017248 \\
\hline 30 & 6 & 0 & 6.565429 & -1.268883 & -1.330364 \\
\hline 31 & 8 & 0 & 7.557346 & -1.726976 & -2.290485 \\
\hline 32 & 1 & $\odot$ & 9.479530 & -3.490000 & -2.569758 \\
\hline 33 & 1 & $\odot$ & 10.549771 & -2.920943 & -1.264695 \\
\hline 34 & 1 & 0 & 9.515935 & -1.025268 & -2.375876 \\
\hline 35 & 1 & $\theta$ & 9.446870 & -1.152051 & 0.381624 \\
\hline 36 & 1 & $\Theta$ & 6.838394 & -0.580715 & 0.727764 \\
\hline 37 & 1 & 0 & 7.303597 & -2.273607 & 0.446709 \\
\hline 38 & 1 & $\Theta$ & 6.221879 & -0.274442 & -1.602809 \\
\hline 39 & 8 & 0 & 8.742873 & -3.781206 & $-\odot .623741$ \\
\hline 40 & 1 & 0 & 9.083255 & -4.688203 & $-\odot .558769$ \\
\hline 41 & 6 & $\Theta$ & 4.136658 & -1.560359 & -1.589117 \\
\hline 42 & 7 & $\Theta$ & 2.037682 & -4.331841 & -0.947678 \\
\hline 43 & 8 & 0 & 4.055146 & -0.365316 & -1.896440 \\
\hline 44 & 1 & $\Theta$ & 6.557845 & -3.821624 & -1.018209 \\
\hline 45 & 1 & 0 & 4.543126 & -5.326207 & $-\odot .807199$ \\
\hline 46 & 1 & 0 & 2.071446 & -5.313785 & -0.730184 \\
\hline 47 & 1 & $\Theta$ & 1.131622 & -3.868764 & -1.061005 \\
\hline 48 & 6 & $\Theta$ & 6.126311 & 2.648719 & -1.235538 \\
\hline 49 & 1 & $\theta$ & 5.044207 & 6.114705 & 1.377662 \\
\hline 50 & 6 & $\Theta$ & 4.940186 & 3.425802 & 1.726606 \\
\hline 51 & 6 & 0 & 3.662649 & 2.835424 & 1.114645 \\
\hline 52 & 8 & 0 & 3.857377 & 2.872507 & -0.330653 \\
\hline 53 & 1 & $\Theta$ & 5.667254 & 1.998516 & -1.982413 \\
\hline 54 & 1 & $\Theta$ & 6.941668 & 3.221952 & -1.684196 \\
\hline 55 & 1 & $\theta$ & 4.820815 & 4.329796 & -1.442923 \\
\hline 56 & 1 & $\odot$ & 6.583802 & 4.438954 & 0.675580 \\
\hline 57 & 1 & 0 & 4.723997 & 3.992097 & 2.634843 \\
\hline 58 & 1 & $\Theta$ & 5.670063 & 2.650843 & 1.967638 \\
\hline 59 & 1 & $\odot$ & 2.780075 & 3.421122 & 1.357892 \\
\hline 60 & 7 & 0 & 3.358086 & 1.462344 & 1.505148 \\
\hline 61 & 6 & 0 & 4.289746 & 0.486542 & 1.312677 \\
\hline 62 & 6 & $\odot$ & 3.993330 & -0.815316 & 1.515676 \\
\hline 63 & 6 & 0 & 2.632877 & -1.133319 & 1.833504 \\
\hline 64 & 7 & $\odot$ & 1.712918 & -0.179224 & 1.995432 \\
\hline 65 & 6 & $\odot$ & 2.031613 & 1.130513 & 1.841137 \\
\hline 66 & 1 & 0 & 4.734188 & -1.591068 & 1.375957 \\
\hline 67 & 7 & $\odot$ & 2.249593 & -2.402677 & 1.969360 \\
\hline 68 & 8 & $\odot$ & 1.204617 & 2.036108 & 1.989618 \\
\hline 69 & 1 & 0 & 5.257927 & $\odot .828584$ & ๑. 958218 \\
\hline 70 & 1 & 0 & 2.906230 & -3.144948 & 1.793717 \\
\hline 71 & 1 & 0 & 1.255979 & -2.638675 & 2.079843 \\
\hline 72 & 1 & 0 & -0.115329 & -0.633078 & 2.093175 \\
\hline 73 & 1 & 0 & -8.245205 & -4.735163 & 0.877205 \\
\hline 74 & 6 & $\odot$ & -8.048331 & -0.836209 & $\odot .278989$ \\
\hline 75 & 6 & 0 & -6.568480 & -1.148916 & 0.136780 \\
\hline 76 & 6 & $\odot$ & -6.080471 & -1.109534 & 1.578847 \\
\hline 77 & 8 & 0 & -7.193912 & -1.595818 & 2.375828 \\
\hline 78 & 1 & $\odot$ & -9.146113 & -3.368609 & 2.428838 \\
\hline 79 & 1 & $\odot$ & -9.950221 & -2.901752 & 0.911296 \\
\hline 80 & 1 & $\odot$ & -9.158519 & -0.951703 & 2.127710 \\
\hline 81 & 1 & 0 & -8.648513 & -1.140547 & -0.579342 \\
\hline 82 & 1 & 0 & -6.021273 & -0.455467 & -0.495852 \\
\hline 83 & 1 & 0 & -6.449537 & -2.155407 & $-\odot .257167$ \\
\hline 84 & 1 & 0 & -5.823417 & -0.093955 & 1.884954 \\
\hline 85 & 7 & 0 & -4.908976 & -1.919188 & 1.808860 \\
\hline 86 & 6 & 0 & -4.771456 & -3.291152 & 1.748602 \\
\hline 87 & 7 & $\odot$ & -3.531071 & -3.682918 & 1.867674 \\
\hline
\end{tabular}




\begin{tabular}{|c|c|c|c|c|c|}
\hline 88 & 6 & 0 & -2.806389 & -2.506138 & 1.987510 \\
\hline 89 & 6 & 0 & -3.649423 & -1.405708 & 1.944075 \\
\hline 90 & 7 & 0 & -3.349962 & -0.099792 & 1.992873 \\
\hline 91 & 6 & 0 & -2.047756 & 0.124145 & 2.036140 \\
\hline 92 & 7 & $\odot$ & -1.119184 & -0.890621 & 2.088533 \\
\hline 93 & 6 & 0 & -1.405345 & -2.259767 & 2.086219 \\
\hline 94 & 8 & 0 & -0.488611 & -3.091274 & 2.152529 \\
\hline 95 & 1 & $\odot$ & -5.636521 & -3.920782 & 1.598829 \\
\hline 96 & 7 & $\odot$ & -1.595911 & 1.389714 & 2.051546 \\
\hline 97 & 1 & 0 & -2.220775 & 2.047110 & 1.574925 \\
\hline 98 & 1 & $\odot$ & $-\odot .5930 \odot 9$ & 1.557980 & 1.955205 \\
\hline 99 & 6 & 0 & -5.545903 & 2.820355 & 1.113410 \\
\hline 100 & 11 & $\odot$ & 7.295714 & $\odot .38200 \odot$ & 3.492269 \\
\hline 101 & 11 & $\odot$ & -8.693039 & 3.528414 & -2.517328 \\
\hline 102 & 6 & $\odot$ & -4.218356 & 3.082527 & -1.856500 \\
\hline 103 & 6 & 0 & -3.007596 & 2.507353 & -1.105197 \\
\hline 104 & 8 & $\odot$ & -3.221499 & 2.905986 & $\odot .306579$ \\
\hline 105 & 1 & $\odot$ & -5.119963 & 2.252881 & 1.942113 \\
\hline 106 & 1 & $\odot$ & -6.351751 & 3.468220 & 1.468162 \\
\hline 107 & 1 & $\odot$ & -4.235461 & 4.502100 & 1.126244 \\
\hline 108 & 1 & $\odot$ & -5.870825 & 4.302148 & -1.075334 \\
\hline 109 & 1 & $\odot$ & -3.941121 & 3.482795 & -2.835578 \\
\hline 110 & 1 & $\odot$ & -4.958635 & 2.291589 & -1.991837 \\
\hline 111 & 1 & $\odot$ & -2.064739 & 2.973580 & -1.399755 \\
\hline 112 & 7 & $\odot$ & -2.976412 & 1.071643 & -1.205397 \\
\hline 113 & 6 & $\odot$ & -3.935100 & -1.298756 & -2.306982 \\
\hline 114 & 7 & $\odot$ & -2.932799 & -1.746362 & -1.637859 \\
\hline 115 & 6 & $\odot$ & -1.832671 & $-\odot .983199$ & -1.411202 \\
\hline 116 & 6 & $\odot$ & -1.815864 & $\odot .472500$ & -1.237493 \\
\hline 117 & 7 & $\odot$ & -0.614737 & 1.138752 & -1.140389 \\
\hline 118 & 6 & $\odot$ & $\odot .498359$ & $\odot .450312$ & -1.180164 \\
\hline 119 & 7 & $\odot$ & $\odot .549559$ & -0.925253 & -1.217838 \\
\hline 120 & 6 & $\odot$ & -0.571139 & -1.710982 & -1.316784 \\
\hline 121 & 7 & $\odot$ & 1.689429 & 1.059813 & -1.170819 \\
\hline 122 & 8 & $\odot$ & -0.476779 & -2.942431 & -1.351378 \\
\hline 123 & 1 & $\odot$ & -3.934024 & -0.342460 & -2.831499 \\
\hline 124 & 1 & $\odot$ & 1.731030 & 2.063135 & -1.098322 \\
\hline 125 & 1 & $\odot$ & 2.560523 & $\odot .556470$ & -1.351529 \\
\hline 126 & 1 & $\odot$ & 1.463605 & -1.414823 & -1.257779 \\
\hline 127 & 8 & $\odot$ & -5.023742 & -2.027411 & -2.418512 \\
\hline 128 & 1 & $\odot$ & -5.769173 & -1.486417 & -2.854045 \\
\hline 129 & 8 & $\odot$ & -6.877806 & -0.591033 & -3.392753 \\
\hline 130 & 1 & $\odot$ & -7.683380 & -1.100257 & -3.523298 \\
\hline 131 & 1 & $\odot$ & -7.123426 & 0.146551 & -2.763989 \\
\hline 132 & 1 & $\odot$ & -5.562395 & -3.698621 & -1.515549 \\
\hline 133 & 8 & $\odot$ & -5.977762 & -4.454739 & -1.080603 \\
\hline 134 & 1 & $\odot$ & -6.758059 & -4.089749 & $-\odot .636516$ \\
\hline
\end{tabular}

General basis read from cards: (6D, 7F)

1172 basis functions

315 alpha electrons 314 beta electrons

nuclear repulsion energy 17371.6414966613 Hartrees.

NAtoms $=134$ NActive $=134$

Force inversion solution in PCM.

Polarizable Continuum Model (PCM)

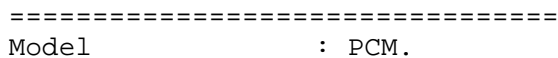

Solvent : Water, Eps $=78.355300$ Eps $($ inf $)=1.777849$

SCF Done: $E($ UWB97XD $)=-5092.28156310$ A.U. after 1 cycles NFock $=1$ Conv $=0.79 \mathrm{D}-09 \quad-\mathrm{V} / \mathrm{T}=2.0066$

$\langle$ SX $>=0.0000<$ Sy $>=0.0000\langle$ Sz $>=0.5000$

$\left\langle S^{* *} 2>=0.7709 \mathrm{~S}=0.5104\right.$

$<$ L.S $>=0.000000000000 \mathrm{E}+00$

Annihilation of the first spin contaminant:

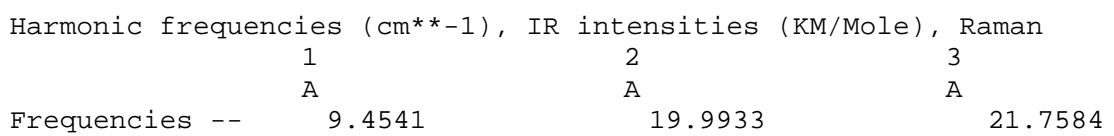


Zero-point correction=

Thermal correction to Energy=

Thermal correction to Enthalpy=

Thermal correction to Gibbs Free Energy=

Sum of electronic and zero-point Energies=

Sum of electronic and thermal Energies=

Sum of electronic and thermal Enthalpies=

Sum of electronic and thermal Free Energies=

$$
\text { E (Thermal) }
$$

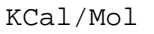

Total

$$
700.167
$$

CV
Cal/Mol-Kelvin
296.584

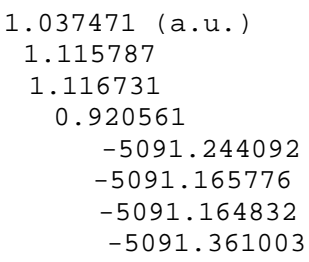

$\mathrm{S}$

Cal/Mol-Kelvin 412.875

\begin{tabular}{lccc}
\multicolumn{1}{c}{ Item } & Value & Threshold & \multicolumn{2}{c}{ Converged? } \\
Maximum Force & $0.000 \odot 43$ & $0.00 \odot 45 \odot$ & YES \\
RMS Force & $0.00 \odot \odot \odot 5$ & $0.00 \odot 3 \odot \odot$ & YES \\
Maximum Displacement & 0.005057 & $0.00180 \odot$ & NO \\
RMS Displacement & 0.001178 & $0.00120 \odot$ & YES \\
Predicted change in Energy $=-5.861650 D-08$ &
\end{tabular}

Normal termination of Gaussian 16 at Wed Jul 24 10:53:08 2019.

$\mathrm{G}(\mathrm{high})=-5093 \cdot 1258378 \mathrm{a} \cdot \mathrm{u}$.

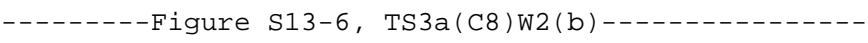

dna.ts3bw2x. $\log$

Stoichiometry C38H53N16Na2023P2(2)

\begin{tabular}{|c|c|c|c|c|c|}
\hline \multirow{2}{*}{$\begin{array}{l}\text { Center } \\
\text { Number }\end{array}$} & \multirow{2}{*}{$\begin{array}{l}\text { Atomic } \\
\text { Number }\end{array}$} & \multirow{2}{*}{$\begin{array}{l}\text { Atomic } \\
\text { Type }\end{array}$} & \multicolumn{3}{|c|}{ Coordinates (Angstroms) } \\
\hline & & & $\mathrm{X}$ & $\mathrm{Y}$ & Z \\
\hline & & & & 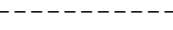 & - - - - - \\
\hline 1 & 7 & $\odot$ & 5.392118 & -2.077769 & -1.184930 \\
\hline 2 & 6 & $\odot$ & 5.523457 & -3.392755 & -0.842730 \\
\hline 3 & 6 & $\odot$ & 4.437683 & -4.188313 & -0.715199 \\
\hline 4 & 6 & $\odot$ & 3.155835 & -3.581791 & $-\odot .934592$ \\
\hline 5 & 7 & $\odot$ & 3.042637 & -2.305618 & -1.311784 \\
\hline 6 & 8 & $\odot$ & 8.785502 & 0.662026 & -0.618920 \\
\hline 7 & 15 & $\odot$ & 8.162899 & 1.923945 & ๑. 219931 \\
\hline 8 & 8 & $\odot$ & 6.587178 & 1.913424 & -0.250658 \\
\hline 9 & 8 & $\odot$ & 8.830962 & 3.169315 & -0.279770 \\
\hline 10 & 8 & $\odot$ & 8.121141 & 1.570157 & 1.689685 \\
\hline 11 & 8 & $\odot$ & 4.631266 & 5.692762 & 0.247039 \\
\hline 12 & 6 & $\odot$ & 4.973533 & 3.629908 & -0.887919 \\
\hline 13 & 6 & $\odot$ & 5.334550 & 4.430852 & $\odot .365636$ \\
\hline 14 & 6 & $\odot$ & -8.869748 & -2.937196 & ๑ . 234810 \\
\hline 15 & 6 & $\odot$ & -8.340436 & -1.746876 & 1.013791 \\
\hline 16 & 8 & $\odot$ & -7.983456 & -3.347158 & -0.825893 \\
\hline 17 & 8 & $\odot$ & -8.138530 & $\odot .650607$ & ๑.721415 \\
\hline 18 & 15 & $\odot$ & -8.088503 & 2.033322 & -0.150544 \\
\hline 19 & 8 & $\odot$ & -6.496951 & 2.383124 & -0.229106 \\
\hline 20 & 8 & $\odot$ & -8.824612 & 3.086355 & 0.644615 \\
\hline 21 & 8 & $\odot$ & -8.558751 & 1.774808 & -1.564015 \\
\hline 22 & 8 & $\odot$ & -3.771819 & 5.433583 & -1.290219 \\
\hline 23 & 6 & 0 & -4.532208 & 3.758300 & 0.226035 \\
\hline 24 & 6 & $\odot$ & -4.661784 & 4.288370 & -1.203914 \\
\hline 25 & 1 & $\odot$ & -3.750428 & 5.772562 & -2.201244 \\
\hline 26 & 6 & 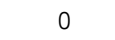 & 9.509337 & -2.940662 & -1.270111 \\
\hline 27 & 6 & $\odot$ & 8.899083 & -1.571743 & -1.491078 \\
\hline 28 & 6 & $\odot$ & 8.677487 & -0.743106 & -0.217131 \\
\hline 29 & 6 & $\odot$ & 7.255229 & -1.115166 & ๑.179895 \\
\hline 30 & 6 & $\odot$ & 6.556159 & -1.205278 & -1.175069 \\
\hline 31 & 8 & $\odot$ & 7.565080 & -1.719615 & -2.089186 \\
\hline 32 & 1 & $\odot$ & 9.500527 & -3.486236 & -2.219288 \\
\hline 33 & 1 & $\odot$ & 10.548088 & -2.822202 & -0.940868 \\
\hline 34 & 1 & $\odot$ & 9.521249 & -1.014028 & -2.194353 \\
\hline 35 & 1 & $\odot$ & 9.413066 & -0.945970 & $\odot .562352$ \\
\hline 36 & 1 & $\odot$ & 6.801067 & -0.384843 & $\odot .842427$ \\
\hline 37 & 1 & $\odot$ & 7.280162 & -2.089460 & ๑. 671901 \\
\hline 38 & 1 & $\odot$ & 6.214704 & -0.231486 & -1.515415 \\
\hline 39 & 8 & $\odot$ & 8.736837 & -3.649046 & -0.268855 \\
\hline 40 & 1 & $\odot$ & 9.085833 & -4.544751 & -0.132327 \\
\hline 41 & 6 & $\odot$ & 4.136367 & -1.526202 & -1.482626 \\
\hline 42 & 7 & $\odot$ & 2.029785 & -4.273635 & -0.763134 \\
\hline
\end{tabular}

Standard orientation: 


\begin{tabular}{|c|c|c|c|c|c|}
\hline 43 & 8 & 0 & 4.059578 & $-\odot .352221$ & -1.862933 \\
\hline 44 & 1 & $\odot$ & 6.546469 & -3.731043 & $-\odot .687427$ \\
\hline 45 & 1 & $\odot$ & 4.533244 & -5.231633 & $-\odot .447781$ \\
\hline 46 & 1 & $\theta$ & 2.060892 & -5.238172 & -0.478031 \\
\hline 47 & 1 & $\odot$ & 1.125496 & -3.827624 & -0.938322 \\
\hline 48 & 6 & $\odot$ & 6.055844 & 2.680847 & -1.367311 \\
\hline 49 & 1 & 0 & 4.803914 & 6.251260 & 1.023923 \\
\hline 50 & 6 & $\odot$ & 4.778502 & 3.581792 & 1.514651 \\
\hline 51 & 6 & $\odot$ & 3.532067 & 2.922942 & $\odot .907966$ \\
\hline 52 & 8 & 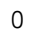 & 3.759405 & 2.891364 & -0.532624 \\
\hline 53 & 1 & $\odot$ & 5.630066 & 1.973117 & -2.080786 \\
\hline 54 & 1 & 0 & 6.864673 & 3.248942 & -1.833929 \\
\hline 55 & 1 & $\odot$ & 4.711572 & 4.310043 & -1.701753 \\
\hline 56 & 1 & $\odot$ & 6.414147 & 4.591934 & 0.448943 \\
\hline 57 & 1 & $\odot$ & 4.528095 & 4.184760 & 2.389788 \\
\hline 58 & 1 & 0 & 5.526407 & 2.841729 & 1.804632 \\
\hline 59 & 1 & $\odot$ & 2.627921 & 3.494607 & 1.100057 \\
\hline 60 & 7 & 0 & 3.257859 & 1.564604 & 1.367142 \\
\hline 61 & 6 & 0 & 4.231468 & $\odot .614959$ & 1.282720 \\
\hline 62 & 6 & $\odot$ & 3.977703 & -0.677692 & 1.579267 \\
\hline 63 & 6 & $\odot$ & 2.620040 & -1.025348 & 1.874213 \\
\hline 64 & 7 & $\odot$ & 1.657155 & -0.101459 & 1.919068 \\
\hline 65 & 6 & $\odot$ & 1.932024 & 1.205405 & 1.677549 \\
\hline 66 & 1 & 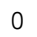 & 4.750985 & -1.432011 & 1.527151 \\
\hline 67 & 7 & $\odot$ & 2.285285 & -2.294591 & 2.105272 \\
\hline 68 & 8 & $\odot$ & 1.065910 & 2.084738 & 1.726115 \\
\hline 69 & 1 & $\odot$ & 5.197433 & $\odot .967442$ & $\odot .933843$ \\
\hline 70 & 1 & $\odot$ & 2.981513 & -3.016621 & 2.020769 \\
\hline 71 & 1 & $\odot$ & 1.300193 & -2.567572 & 2.206326 \\
\hline 72 & 1 & 0 & -0.146169 & $-\odot .638997$ & 2.008699 \\
\hline 73 & 1 & $\odot$ & -7.135757 & -3.690621 & $-\odot .485157$ \\
\hline 74 & 6 & $\odot$ & -7.749622 & $-\odot .633200$ & 0.132134 \\
\hline 75 & 6 & $\odot$ & -6.242796 & -0.835177 & 0.243114 \\
\hline 76 & 6 & $\odot$ & -6.091684 & -1.349389 & 1.670760 \\
\hline 77 & 8 & 0 & -7.229050 & -2.217751 & 1.864332 \\
\hline 78 & 1 & 0 & -9.066841 & -3.761240 & 0.929894 \\
\hline 79 & 1 & $\odot$ & -9.806887 & -2.661146 & $-\odot .252048$ \\
\hline 80 & 1 & $\odot$ & -9.119401 & -1.338683 & 1.662295 \\
\hline 81 & 1 & $\odot$ & -8.113208 & -0.679612 & -0.894986 \\
\hline 82 & 1 & $\odot$ & -5.667043 & $\odot .066443$ & $\odot .039839$ \\
\hline 83 & 1 & 0 & -5.932460 & -1.615630 & $-\odot .452896$ \\
\hline 84 & 1 & $\odot$ & -6.110309 & -0.530114 & 2.396667 \\
\hline 85 & 7 & $\odot$ & -4.885081 & -2.122151 & 1.890896 \\
\hline 86 & 6 & $\odot$ & -4.705764 & -3.482321 & 2.028882 \\
\hline 87 & 7 & $\odot$ & -3.450675 & -3.816886 & 2.174163 \\
\hline 88 & 6 & $\odot$ & -2.764118 & -2.616188 & 2.112909 \\
\hline 89 & 6 & 0 & -3.645463 & -1.557340 & 1.945981 \\
\hline 90 & 7 & $\odot$ & -3.398347 & $-\odot .241766$ & 1.860894 \\
\hline 91 & 6 & $\odot$ & -2.105067 & $\odot .035119$ & 1.890306 \\
\hline 92 & 7 & 0 & -1.138427 & $-\odot .937683$ & 2.019931 \\
\hline 93 & 6 & $\odot$ & -1.371762 & -2.309943 & 2.154829 \\
\hline 94 & 8 & $\odot$ & -0.423024 & -3.097038 & 2.284729 \\
\hline 95 & 1 & 0 & -5.550502 & -4.153659 & 2.024791 \\
\hline 96 & 7 & $\odot$ & -1.700031 & 1.311666 & 1.820365 \\
\hline 97 & 1 & $\odot$ & -2.372155 & 1.941914 & 1.373010 \\
\hline 98 & 1 & $\odot$ & -0.706506 & 1.524672 & 1.715662 \\
\hline 99 & 6 & $\odot$ & -5.802270 & 3.153454 & $\odot .795544$ \\
\hline 100 & 11 & 0 & 7.205213 & $\odot .840950$ & 3.545730 \\
\hline 101 & 11 & 0 & -10.329174 & 3.518504 & -1.299022 \\
\hline 102 & 6 & $\odot$ & -4.170778 & 3.120461 & -2.061756 \\
\hline 103 & 6 & $\odot$ & -3.061022 & 2.474940 & -1.227874 \\
\hline 104 & 8 & $\odot$ & -3.454135 & 2.776540 & 0.172477 \\
\hline 105 & 1 & $\odot$ & -5.551817 & 2.494921 & 1.631933 \\
\hline 106 & 1 & $\odot$ & -6.467480 & 3.948118 & 1.144444 \\
\hline 107 & 1 & $\odot$ & -4.207746 & 4.563586 & 0.891181 \\
\hline 108 & 1 & $\odot$ & -5.688203 & 4.582081 & -1.443455 \\
\hline 109 & 1 & $\odot$ & -3.816254 & 3.444245 & -3.043763 \\
\hline 110 & 1 & $\odot$ & -4.981849 & 2.400294 & -2.189158 \\
\hline 111 & 1 & $\odot$ & -2.092504 & 2.954838 & -1.384300 \\
\hline 112 & 7 & $\odot$ & -3.022482 & 1.048211 & -1.383927 \\
\hline 113 & 6 & $\odot$ & -3.964189 & -1.400244 & -2.445096 \\
\hline 114 & 7 & $\odot$ & -2.940566 & -1.797957 & -1.703056 \\
\hline 115 & 6 & $\odot$ & -1.852888 & -0.999718 & -1.525681 \\
\hline 116 & 6 & 0 & -1.855521 & 0.459118 & -1.420339 \\
\hline 117 & 7 & $\odot$ & -0.659467 & 1.141565 & -1.363395 \\
\hline 118 & 6 & $\odot$ & $\odot .460969$ & $\odot .466526$ & -1.343073 \\
\hline 119 & 7 & $\odot$ & $\odot .526105$ & -0.909003 & -1.297495 \\
\hline
\end{tabular}




$\begin{array}{rrrrrr}120 & 6 & 0 & -0.586974 & -1.702230 & -1.376398 \\ 121 & 7 & 0 & 1.645945 & 1.086822 & -1.359298 \\ 122 & 8 & 0 & -0.485848 & -2.937979 & -1.349195 \\ 123 & 1 & 0 & -3.873187 & -0.472242 & -3.016083 \\ 124 & 1 & 0 & 1.682394 & 2.091474 & -1.308562 \\ 125 & 1 & 0 & 2.528541 & 0.581867 & -1.460876 \\ 126 & 1 & 0 & 1.444842 & -1.391352 & -1.283167 \\ 127 & 8 & 0 & -5.039199 & -2.052693 & -2.580274 \\ 128 & 1 & 0 & -5.212328 & -3.206212 & -1.927724 \\ 129 & 8 & 0 & -5.313094 & -4.149408 & -1.369134 \\ 130 & 1 & 0 & -4.174189 & -4.381123 & -1.118871 \\ 131 & 1 & 0 & -5.668291 & -4.818064 & -1.967610 \\ 132 & 8 & 0 & -2.980109 & -4.324639 & -0.915668 \\ 133 & 1 & 0 & -2.792946 & -3.349260 & -1.160588 \\ 134 & 1 & 0 & -2.457791 & -4.853427 & -1.529555 \\ ---\ldots-----\ldots-\ldots-\ldots\end{array}$

General basis read from cards: (6D, 7F)

1172 basis functions

315 alpha electrons $\quad 314$ beta electrons

nuclear repulsion energy 17333.7440208701 Hartrees.

NAtoms $=134$

Force inversion solution in PCM.

Polarizable Continuum Model (PCM)

Model
: PCM.

Solvent : Water, Eps $=78.355300$ Eps $($ inf $)=1.777849$

SCF Done: $E($ UwB97XD) $=-5092.26620287$ A.U. after 1 cycles $\mathrm{NFock}=1$ Conv $=0.18 \mathrm{D}-08 \quad-\mathrm{V} / \mathrm{T}=2.0066$

$\langle\mathrm{S} x\rangle=0.0000<\mathrm{S} y>=0.0000<\mathrm{S} z>=0.5000$

$<S^{* *} 2>=0.7681 \mathrm{~S}=0.5090$

$<$ L.S $>=0.000000000000 \mathrm{E}+00$

Annihilation of the first spin contaminant:

$\mathrm{S}^{* *} 2$ before annihilation 0.7681 , after 0.7502

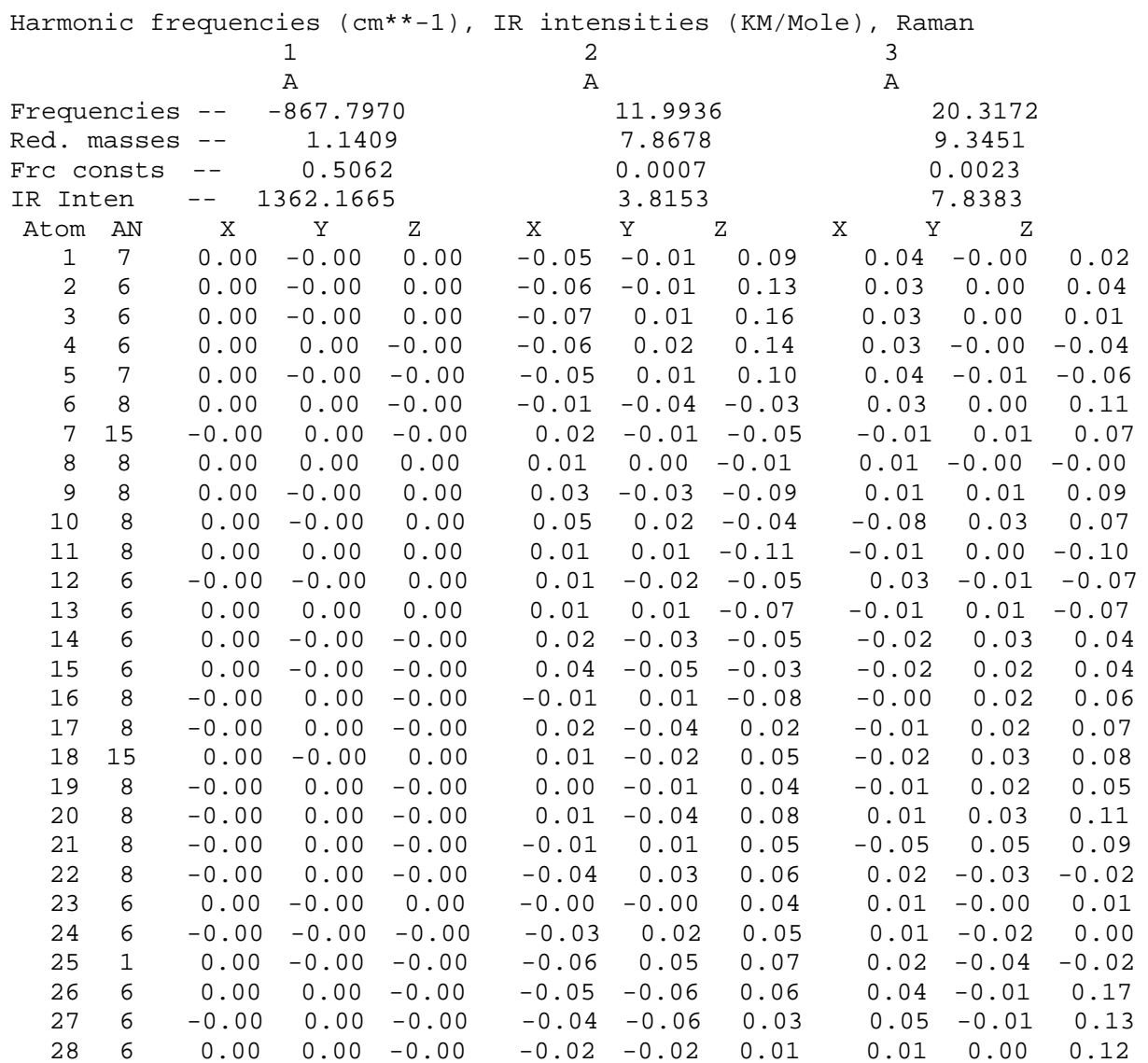




\begin{tabular}{|c|c|c|c|c|c|c|c|c|c|c|}
\hline 29 & 6 & $\odot . \odot \odot$ & $\odot . \odot \odot$ & $-\odot . \odot \odot$ & $-\odot .01$ & $-\odot . \odot \odot$ & $\odot .04$ & $-\odot . \odot \odot$ & $\odot .01$ & $\odot .07$ \\
\hline 30 & 6 & -0.00 & $-\odot . \odot \odot$ & $\odot .0 \odot$ & -0.04 & -0.03 & 0.05 & 0.04 & -0.00 & 0.05 \\
\hline 31 & 8 & 0.00 & 0.00 & -0.00 & -0.05 & -0.07 & 0.05 & 0.07 & -0.01 & $\odot .09$ \\
\hline 32 & 1 & $\odot . \odot \odot$ & $\odot . \odot \odot$ & $-\odot . \odot \odot$ & $-\odot .08$ & -0.09 & $\odot . \odot 7$ & 0.07 & -0.02 & 0.17 \\
\hline 33 & 1 & $\odot .0 \odot$ & -0.00 & $-\odot .0 \odot$ & -0.05 & -0.06 & 0.04 & 0.03 & -0.00 & 0.20 \\
\hline 34 & 1 & $\odot .0 \odot$ & $\odot .0 \odot$ & $\odot .0 \odot$ & -0.05 & -0.09 & $0.0 \odot$ & 0.07 & -0.01 & 0.15 \\
\hline 35 & 1 & $\odot .00$ & 0.00 & -0.00 & -0.01 & -0.01 & 0.00 & -0.02 & 0.01 & 0.15 \\
\hline 36 & 1 & $-\odot . \odot \odot$ & $\odot . \odot \odot$ & $-\odot . \odot \odot$ & $\odot . \odot \odot$ & $\odot . \odot 2$ & $\odot .02$ & -0.02 & $\odot .02$ & $\odot .05$ \\
\hline 37 & 1 & $-\odot . \odot \odot$ & $\odot . \odot \odot$ & $-\odot . \odot \odot$ & $-\odot . \odot 2$ & 0.01 & $\odot . \odot 6$ & $-\odot .02$ & $\odot . \odot 2$ & $\odot .08$ \\
\hline 38 & 1 & $-0.0 \odot$ & $-0.0 \odot$ & $\odot . \odot \odot$ & -0.03 & -0.03 & 0.03 & 0.05 & $-0.0 \odot$ & 0.03 \\
\hline 39 & 8 & $\odot . \odot \odot$ & $-\odot .0 \odot$ & -0.00 & -0.05 & -0.02 & 0.09 & 0.01 & 0.01 & 0.15 \\
\hline 40 & 1 & 0.00 & 0.00 & -0.00 & -0.05 & -0.02 & 0.11 & 0.01 & $\odot .01$ & 0.18 \\
\hline 41 & 6 & $\odot .00$ & $\odot .0 \odot$ & $-\odot .0 \odot$ & -0.04 & -0.01 & $\odot . \odot 8$ & 0.05 & -0.01 & -0.03 \\
\hline 42 & 7 & -0.00 & $\odot .0 \odot$ & -0.00 & -0.07 & 0.03 & 0.15 & 0.03 & $-0.0 \odot$ & -0.08 \\
\hline 43 & 8 & 0.00 & $-\odot . \odot \odot$ & -0.00 & -0.03 & -0.01 & 0.06 & 0.06 & -0.01 & -0.05 \\
\hline 44 & 1 & $\odot .0 \odot$ & $-\odot . \odot \odot$ & $\odot .00$ & $-\odot .06$ & -0.01 & 0.13 & 0.02 & 0.01 & $\odot .08$ \\
\hline 45 & 1 & $\odot .0 \odot$ & $-\odot . \odot \odot$ & 0.00 & $-\odot .08$ & 0.02 & 0.19 & 0.02 & 0.01 & 0.03 \\
\hline 46 & 1 & $-\odot . \odot \odot$ & 0.00 & $-\odot .0 \odot$ & -0.08 & $\odot .04$ & 0.17 & 0.02 & 0.00 & -0.06 \\
\hline 47 & 1 & $-0 . \odot \odot$ & $\odot . \odot \odot$ & $-\odot . \odot \odot$ & -0.06 & 0.03 & 0.12 & 0.03 & $-\odot . \odot \odot$ & -0.10 \\
\hline 48 & 6 & 0.00 & 0.00 & 0.00 & 0.00 & -0.03 & -0.03 & 0.05 & -0.01 & -0.03 \\
\hline 49 & 1 & $-\odot .0 \odot$ & -0.00 & $\odot .0 \odot$ & 0.02 & 0.04 & -0.13 & -0.03 & 0.01 & -0.11 \\
\hline 50 & 6 & $-\odot . \odot \odot$ & $\odot . \odot \odot$ & $-\odot .0 \odot$ & $\odot .01$ & $\odot .05$ & -0.05 & -0.04 & $\odot . \odot 2$ & -0.07 \\
\hline 51 & 6 & $-0.0 \odot$ & $-0.0 \odot$ & -0.00 & 0.01 & 0.02 & -0.03 & -0.02 & 0.00 & -0.10 \\
\hline 52 & 8 & $\odot . \odot \odot$ & $\odot .0 \odot$ & $-\odot .0 \odot$ & 0.00 & -0.01 & -0.03 & 0.02 & -0.01 & -0.10 \\
\hline 53 & 1 & $-0.0 \odot$ & $-\odot . \odot \odot$ & $\odot .0 \odot$ & $\odot .0 \odot$ & -0.05 & -0.01 & $\odot . \odot 8$ & -0.02 & -0.04 \\
\hline 54 & 1 & 0.00 & $-\odot . \odot \odot$ & -0.00 & 0.00 & -0.05 & -0.05 & 0.06 & -0.01 & $-0.0 \odot$ \\
\hline 55 & 1 & $\odot .0 \odot$ & $\odot . \odot \odot$ & $0.0 \odot$ & $0.0 \odot$ & -0.04 & -0.07 & 0.05 & -0.02 & -0.08 \\
\hline 56 & 1 & $\odot . \odot \odot$ & $\odot . \odot \odot$ & $\odot .0 \odot$ & 0.01 & 0.01 & -0.08 & -0.01 & ๑. 01 & $-\odot .04$ \\
\hline 57 & 1 & $-\odot . \odot \odot$ & $0.0 \odot$ & $\odot .00$ & 0.00 & $\odot .08$ & -0.07 & -0.07 & 0.03 & -0.09 \\
\hline 58 & 1 & $-0.0 \odot$ & $-0.0 \odot$ & -0.00 & $\odot .01$ & 0.06 & -0.02 & -0.05 & 0.03 & -0.04 \\
\hline 59 & 1 & $-\odot . \odot \odot$ & $-\odot . \odot \odot$ & $-\odot . \odot \odot$ & $\odot . \odot \odot$ & 0.02 & $-\odot .04$ & -0.03 & -0.00 & -0.14 \\
\hline 60 & 7 & $\odot . \odot \odot$ & $-0.0 \odot$ & $\odot .0 \odot$ & $\odot .02$ & $\odot .03$ & $\odot . \odot \odot$ & -0.02 & $\odot . \odot \odot$ & -0.10 \\
\hline 61 & 6 & $-0.0 \odot$ & 0.00 & -0.00 & 0.03 & 0.04 & 0.04 & -0.01 & 0.01 & -0.05 \\
\hline 62 & 6 & $-\odot .0 \odot$ & $-0.0 \odot$ & $-\odot . \odot \odot$ & 0.04 & 0.05 & 0.07 & -0.01 & $\odot .01$ & -0.04 \\
\hline 63 & 6 & $-0.0 \odot$ & $\odot .0 \odot$ & $-\odot .0 \odot$ & 0.04 & $\odot .04$ & 0.07 & -0.02 & $\odot .0 \odot$ & -0.08 \\
\hline 64 & 7 & 0.00 & -0.00 & 0.00 & 0.03 & 0.03 & 0.03 & -0.03 & -0.00 & -0.12 \\
\hline 65 & 6 & $-\odot . \odot \odot$ & $\odot . \odot \odot$ & $-\odot . \odot \odot$ & 0.02 & 0.03 & $\odot .0 \odot$ & -0.03 & $-0.0 \odot$ & -0.13 \\
\hline 66 & 1 & $-\odot . \odot \odot$ & $-0.0 \odot$ & $-\odot . \odot \odot$ & $\odot .05$ & 0.06 & 0.10 & $-0.0 \odot$ & $\odot .02$ & -0.01 \\
\hline 67 & 7 & 0.00 & -0.00 & 0.00 & 0.06 & 0.04 & 0.10 & -0.01 & 0.00 & -0.08 \\
\hline 68 & 8 & $\odot . \odot \odot$ & $\odot .0 \odot$ & $\odot . \odot \odot$ & 0.01 & 0.02 & -0.02 & -0.03 & -0.01 & -0.16 \\
\hline 69 & 1 & -0.00 & -0.00 & -0.00 & 0.03 & 0.04 & 0.03 & -0.00 & 0.01 & -0.03 \\
\hline 70 & 1 & $\odot .0 \odot$ & 0.00 & -0.00 & 0.06 & 0.05 & 0.11 & $-0.0 \odot$ & 0.01 & -0.05 \\
\hline 71 & 1 & 0.00 & 0.00 & $-0.0 \odot$ & 0.05 & 0.03 & 0.06 & -0.01 & -0.01 & -0.11 \\
\hline 72 & 1 & 0.00 & $-0.0 \odot$ & -0.00 & 0.04 & 0.01 & -0.02 & -0.01 & -0.03 & -0.10 \\
\hline 73 & 1 & 0.01 & 0.01 & -0.03 & $\odot . \odot \odot$ & $-\odot . \odot \odot$ & -0.12 & -0.01 & $\odot . \odot 2$ & $\odot . \odot 8$ \\
\hline 74 & 6 & 0.00 & $-\odot . \odot \odot$ & -0.00 & $\odot .02$ & -0.02 & -0.01 & -0.02 & 0.03 & 0.05 \\
\hline 75 & 6 & $\odot .0 \odot$ & $-\odot . \odot \odot$ & -0.00 & 0.02 & -0.02 & -0.04 & -0.03 & $\odot .02$ & 0.04 \\
\hline 76 & 6 & $-0.0 \odot$ & $-0.0 \odot$ & $-0.0 \odot$ & 0.04 & -0.05 & -0.05 & -0.01 & 0.00 & 0.03 \\
\hline 77 & 8 & 0.00 & 0.00 & 0.00 & 0.05 & -0.07 & -0.06 & -0.02 & $\odot .01$ & 0.04 \\
\hline 78 & 1 & $\odot .0 \odot$ & $\odot .0 \odot$ & $\odot .0 \odot$ & 0.04 & -0.05 & -0.07 & -0.04 & 0.03 & $\odot .03$ \\
\hline 79 & 1 & $0.0 \odot$ & -0.00 & 0.00 & 0.00 & -0.01 & -0.01 & -0.01 & 0.03 & 0.02 \\
\hline 80 & 1 & $\odot .00$ & $-\odot . \odot \odot$ & 0.00 & 0.05 & -0.06 & -0.00 & -0.02 & 0.01 & 0.05 \\
\hline 81 & 1 & $\odot .0 \odot$ & $-\odot . \odot \odot$ & 0.00 & $\odot .0 \odot$ & $-\odot . \odot \odot$ & -0.01 & -0.04 & 0.04 & 0.06 \\
\hline 82 & 1 & -0.00 & -0.00 & 0.00 & 0.01 & -0.01 & -0.03 & -0.02 & 0.02 & 0.04 \\
\hline 83 & 1 & 0.01 & 0.00 & -0.01 & 0.01 & $-0.0 \odot$ & -0.06 & -0.04 & 0.03 & 0.02 \\
\hline 84 & 1 & 0.00 & $-0.0 \odot$ & $0.0 \odot$ & 0.04 & -0.07 & -0.03 & $\odot .0 \odot$ & -0.01 & 0.04 \\
\hline 85 & 7 & 0.00 & -0.00 & $\odot .0 \odot$ & 0.05 & -0.04 & -0.08 & $-\odot .02$ & -0.01 & $\odot . \odot \odot$ \\
\hline 86 & 6 & $-\odot . \odot \odot$ & $\odot . \odot \odot$ & $-\odot . \odot \odot$ & 0.07 & -0.04 & -0.10 & -0.02 & -0.02 & -0.04 \\
\hline 87 & 7 & $\odot .0 \odot$ & -0.00 & $-\odot .0 \odot$ & 0.07 & -0.03 & -0.10 & -0.02 & -0.03 & -0.08 \\
\hline 88 & 6 & $\odot .0 \odot$ & $\odot .0 \odot$ & $-0.0 \odot$ & 0.06 & -0.02 & -0.08 & -0.01 & -0.03 & -0.07 \\
\hline 89 & 6 & $-\odot . \odot \odot$ & $\odot .00$ & $\odot . \odot \odot$ & 0.05 & -0.03 & -0.07 & -0.01 & -0.02 & -0.01 \\
\hline 90 & 7 & $-\odot . \odot \odot$ & $-0.0 \odot$ & $0.0 \odot$ & 0.03 & -0.03 & -0.05 & -0.01 & -0.02 & 0.01 \\
\hline 91 & 6 & $\odot .0 \odot$ & $\odot .0 \odot$ & $-\odot . \odot \odot$ & 0.03 & -0.01 & -0.03 & -0.01 & -0.02 & -0.02 \\
\hline 92 & 7 & 0.00 & -0.00 & $\odot . \odot \odot$ & 0.04 & $-0.0 \odot$ & -0.03 & -0.01 & -0.03 & -0.08 \\
\hline 93 & 6 & $\odot .0 \odot$ & $\odot .0 \odot$ & $-0.0 \odot$ & 0.05 & -0.01 & -0.06 & -0.01 & -0.03 & -0.10 \\
\hline 94 & 8 & $-\odot . \odot \odot$ & $-0.0 \odot$ & 0.00 & 0.06 & $-0.0 \odot$ & -0.05 & -0.01 & -0.04 & -0.15 \\
\hline 95 & 1 & $-\odot . \odot \odot$ & $\odot . \odot \odot$ & $-\odot . \odot \odot$ & 0.07 & -0.05 & -0.11 & -0.02 & -0.01 & -0.03 \\
\hline 96 & 7 & $-\odot . \odot \odot$ & $-0.0 \odot$ & $\odot .0 \odot$ & 0.02 & -0.01 & -0.02 & -0.01 & -0.02 & -0.01 \\
\hline 97 & 1 & -0.00 & -0.00 & $-\odot . \odot \odot$ & 0.01 & -0.01 & -0.01 & -0.02 & -0.02 & 0.02 \\
\hline 98 & 1 & $-0.0 \odot$ & -0.00 & $-0 . \odot \odot$ & 0.01 & 0.00 & -0.01 & -0.01 & -0.03 & -0.06 \\
\hline 99 & 6 & -0.00 & 0.00 & $-\odot . \odot \odot$ & $\odot .01$ & -0.03 & 0.05 & 0.01 & 0.03 & $\odot .03$ \\
\hline 100 & 11 & -0.00 & 0.00 & -0.00 & 0.05 & 0.06 & -0.02 & -0.12 & 0.05 & 0.06 \\
\hline 101 & 11 & $\odot . \odot \odot$ & $-0.0 \odot$ & $\odot .0 \odot$ & -0.01 & $\odot .0 \odot$ & 0.10 & -0.04 & 0.06 & 0.16 \\
\hline 102 & 6 & $\odot .0 \odot$ & 0.00 & 0.00 & -0.04 & 0.04 & 0.02 & 0.01 & -0.04 & 0.02 \\
\hline 103 & 6 & $\odot .0 \odot$ & $-0.0 \odot$ & $-0.0 \odot$ & -0.02 & 0.03 & -0.01 & 0.01 & -0.03 & 0.03 \\
\hline 104 & 8 & $-0.0 \odot$ & 0.00 & 0.00 & 0.01 & 0.01 & 0.00 & -0.01 & -0.02 & 0.02 \\
\hline 105 & 1 & -0.00 & -0.00 & -0.00 & 0.03 & -0.04 & 0.03 & 0.01 & 0.04 & 0.04 \\
\hline
\end{tabular}




\begin{tabular}{|c|c|c|c|c|}
\hline 106 & 1 & -0.00 & -0.00 & 0.00 \\
\hline 107 & 1 & -0.00 & $\odot .00$ & -0.00 \\
\hline 108 & 1 & -0.00 & $\odot . \odot \odot$ & $\odot . \odot \odot$ \\
\hline 109 & 1 & 0.00 & 0.00 & $\odot .00$ \\
\hline 110 & 1 & $\odot .0 \odot$ & $-\odot . \odot \odot$ & 0.00 \\
\hline 111 & 1 & -0.00 & $\odot .0 \odot$ & $\odot . \odot \odot$ \\
\hline 112 & 7 & $\odot . \odot \odot$ & $\odot .00$ & $\odot . \odot \odot$ \\
\hline 113 & 6 & -0.02 & 0.00 & -0.01 \\
\hline 114 & 7 & 0.01 & -0.03 & 0.01 \\
\hline 115 & 6 & 0.00 & -0.01 & $\odot .00$ \\
\hline 116 & 6 & -0.00 & $-\odot . \odot \odot$ & $-0.0 \odot$ \\
\hline 117 & 7 & -0.00 & $-\odot . \odot \odot$ & $-\odot .0 \odot$ \\
\hline 118 & 6 & -0.00 & -0.00 & $-0.0 \odot$ \\
\hline 119 & 7 & $\odot .0 \odot$ & $-\odot . \odot \odot$ & 0.00 \\
\hline 120 & 6 & $-0.0 \odot$ & $\odot . \odot \odot$ & $\odot . \odot \odot$ \\
\hline 121 & 7 & $\odot . \odot \odot$ & $\odot . \odot \odot$ & $\odot . \odot \odot$ \\
\hline 122 & 8 & -0.00 & $-\odot .0 \odot$ & 0.00 \\
\hline 123 & 1 & $\odot .08$ & 0.02 & $\odot .02$ \\
\hline 124 & 1 & -0.00 & -0.00 & 0.00 \\
\hline 125 & 1 & $\odot .0 \odot$ & $\odot .00$ & $\odot . \odot \odot$ \\
\hline 126 & 1 & $\odot .0 \odot$ & $\odot .0 \odot$ & $-\odot . \odot \odot$ \\
\hline 127 & 8 & 0.02 & 0.05 & -0.02 \\
\hline 128 & 1 & -0.16 & -0.46 & 0.22 \\
\hline 129 & 8 & -0.02 & -0.03 & 0.02 \\
\hline 130 & 1 & 0.79 & -0.07 & 0.13 \\
\hline 131 & 1 & -0.06 & 0.01 & -0.00 \\
\hline 132 & 8 & -0.02 & 0.04 & -0.01 \\
\hline 133 & 1 & -0.06 & 0.17 & -0.09 \\
\hline 134 & 1 & -0.07 & -0.03 & -0.01 \\
\hline
\end{tabular}

0.01
0.00
-0.04
-0.06
-0.04
-0.03
-0.03
-0.02
-0.03
-0.03
-0.03
-0.03
-0.03
-0.04
-0.04
-0.03
-0.04
-0.01
-0.02
-0.03
-0.04
-0.01
-0.02
-0.03
-0.03
-0.02
-0.03
-0.03
-0.02

$\begin{array}{cc}-0.04 & 0.08 \\ -0.01 & 0.05 \\ 0.02 & 0.08 \\ 0.06 & 0.02 \\ 0.04 & 0.02 \\ 0.03 & -0.02 \\ 0.03 & -0.03 \\ 0.04 & -0.06 \\ 0.03 & -0.05 \\ 0.03 & -0.03 \\ 0.03 & -0.02 \\ 0.03 & -0.01 \\ 0.02 & 0.01 \\ 0.02 & 0.03 \\ 0.03 & 0.01 \\ 0.02 & 0.01 \\ 0.03 & 0.02 \\ 0.05 & -0.05 \\ 0.02 & -0.00 \\ 0.01 & 0.03 \\ 0.02 & 0.05 \\ 0.04 & -0.09 \\ 0.03 & -0.12 \\ 0.02 & -0.14 \\ 0.01 & -0.13 \\ 0.03 & -0.16 \\ 0.01 & -0.10 \\ 0.01 & -0.07 \\ 0.02 & -0.10\end{array}$

$0.02 \quad 0.04 \quad 0.02$

$\begin{array}{llll}0.03 & 0.01 & -0.01\end{array}$

$\begin{array}{llll}0.01 & -0.02 & -0.00\end{array}$

$0.00 \quad-0.06 \quad 0.01$

$\begin{array}{lll}0.01 & -0.04 & 0.03\end{array}$

$\begin{array}{llll}0.01 & -0.02 & 0.03\end{array}$

$0.02 \quad-0.03 \quad 0.03$

$\begin{array}{llll}-0.00 & -0.01 & 0.02\end{array}$

$0.02-0.02-0.01$

$0.02-0.02-0.03$

$\begin{array}{llll}0.02 & -0.02 & -0.02\end{array}$

$0.02-0.02 \quad-0.06$

$0.02-0.01 \quad-0.09$

$0.03-0.01-0.10$

$0.03-0.02-0.08$

$0.02 \quad-0.01 \quad-0.14$

$\begin{array}{llll}0.03 & -0.02 & -0.09\end{array}$

$\begin{array}{lll}-0.02 & -0.00 & 0.03\end{array}$

$\begin{array}{llll}0.02 & -0.01 & -0.11\end{array}$

$0.02 \quad-0.01 \quad-0.12$

$0.03-0.01-0.11$

$\begin{array}{lll}-0.01 & 0.00 & 0.05\end{array}$

$\begin{array}{llll}-0.00 & -0.00 & 0.06\end{array}$

$0.01 \quad-0.00 \quad 0.06$

$0.02-0.02-0.00$

$\begin{array}{rrr}-0.03 & 0.01 & 0.07\end{array}$

$\begin{array}{llll}0.03 & -0.03 & -0.06\end{array}$

$0.03-0.03-0.05$

Zero-point correction=

Thermal correction to Energy=

Thermal correction to Enthalpy=

Thermal correction to Gibbs Free Energy=

Sum of electronic and zero-point Energies=

Sum of electronic and thermal Energies=

Sum of electronic and thermal Enthalpies=

Sum of electronic and thermal Free Energies=

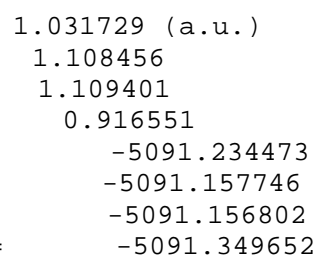

$\begin{array}{ll}\text { Total } & \\ & \text { Item }\end{array}$

Maximum Force

RMS Force

Maximum Displacement

RMS Displacement

Value Threshold Converged?

$0.000027 \quad 0.000450$ YES

$0.000003 \quad 0.000300$ YES

$\begin{array}{lll}0.000348 & 0.001200 & \text { YES }\end{array}$

Normal termination of Gaussian 16 at Sat Aug 17 09:18:39 2019.

$\mathrm{G}(\mathrm{high})=-5093 \cdot 1125927 \mathrm{a} \cdot \mathrm{u}$.

............. Figure S13-7, Int4(C8)W2(a)

dna. for $3 b w 2 x \cdot \log$

Stoichiometry C38H53N16Na2023P2(2)

Standard orientation:

\begin{tabular}{|c|c|c|c|c|c|}
\hline \multirow{2}{*}{$\begin{array}{l}\text { Center } \\
\text { Number }\end{array}$} & \multirow{2}{*}{$\begin{array}{l}\text { Atomic } \\
\text { Number }\end{array}$} & \multirow{2}{*}{$\begin{array}{c}\text { Atomic } \\
\text { Type }\end{array}$} & \multicolumn{3}{|c|}{ Coordinates (Angstroms) } \\
\hline & & & $\mathrm{x}$ & $\mathrm{Y}$ & Z \\
\hline & & & 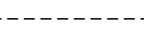 & 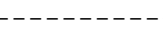 & \\
\hline 1 & 7 & $\odot$ & 5.463422 & -2.077529 & -1.186414 \\
\hline 2 & 6 & $\odot$ & 5.611597 & -3.394463 & -0.858969 \\
\hline 3 & 6 & $\odot$ & 4.536143 & -4.205537 & $-\odot .744989$ \\
\hline 4 & 6 & $\odot$ & 3.247269 & -3.612671 & -0.957000 \\
\hline 5 & 7 & $\odot$ & 3.117758 & -2.334215 & -1.322831 \\
\hline 6 & 8 & $\odot$ & 8.827289 & 0.691573 & -0.555406 \\
\hline 7 & 15 & $\odot$ & 8.172024 & 1.940052 & $\odot .277981$ \\
\hline 8 & 8 & $\odot$ & 6.606911 & 1.911700 & -0.226125 \\
\hline 9 & 8 & $\odot$ & 8.834149 & 3.197191 & -0.199820 \\
\hline 10 & 8 & $\odot$ & 8.104250 & 1.576544 & 1.744397 \\
\hline 11 & 8 & $\odot$ & 4.641987 & 5.688091 & 0.263828 \\
\hline 12 & 6 & $\odot$ & 5.000102 & 3.629719 & -0.874690 \\
\hline
\end{tabular}




\begin{tabular}{|c|c|c|c|c|c|}
\hline 13 & 6 & $\odot$ & 5.348631 & 4.428201 & ๑. 384086 \\
\hline 14 & 6 & $\Theta$ & -9.063959 & -2.802279 & 0.474177 \\
\hline 15 & 6 & $\odot$ & -8.426214 & -1.609357 & 1.166130 \\
\hline 16 & 8 & $\odot$ & -8.337894 & -3.231005 & -0.691067 \\
\hline 17 & 8 & $\odot$ & -8.180203 & $\odot .771138$ & $\odot .760415$ \\
\hline 18 & 15 & 0 & -8.105615 & 2.118451 & -0.163774 \\
\hline 19 & 8 & $\odot$ & -6.504484 & 2.421245 & -0.272360 \\
\hline 20 & 8 & $\odot$ & -8.801456 & 3.222251 & $\odot .597509$ \\
\hline 21 & 8 & $\Theta$ & -8.600558 & 1.818288 & -1.560713 \\
\hline 22 & 8 & $\odot$ & -3.707662 & 5.343650 & -1.489208 \\
\hline 23 & 6 & 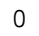 & -4.504518 & 3.765687 & $\odot .108761$ \\
\hline 24 & 6 & $\odot$ & -4.613512 & 4.216732 & -1.350086 \\
\hline 25 & 1 & $\odot$ & -3.676652 & 5.637168 & -2.415556 \\
\hline 26 & 6 & $\odot$ & 9.585470 & -2.897635 & -1.236409 \\
\hline 27 & 6 & $\odot$ & 8.966000 & -1.531634 & -1.449335 \\
\hline 28 & 6 & $\odot$ & 8.722590 & -0.718259 & -0.169456 \\
\hline 29 & 6 & $\Theta$ & 7.298273 & -1.104905 & 0.206618 \\
\hline 30 & 6 & $\odot$ & 6.616055 & -1.189797 & -1.157339 \\
\hline 31 & 8 & $\odot$ & 7.640395 & -1.683633 & -2.064949 \\
\hline 32 & 1 & $\Theta$ & 9.593056 & -3.432507 & -2.191670 \\
\hline 33 & 1 & $\odot$ & 10.618979 & -2.773911 & -0.893028 \\
\hline 34 & 1 & $\Theta$ & 9.591530 & -0.961070 & -2.139155 \\
\hline 35 & 1 & $\Theta$ & 9.449988 & -0.924330 & 0.616829 \\
\hline 36 & 1 & $\odot$ & 6.828910 & -0.384811 & $\odot .870093$ \\
\hline 37 & 1 & $\odot$ & 7.325393 & -2.083141 & 0.690592 \\
\hline 38 & 1 & $\Theta$ & 6.265996 & -0.217136 & -1.491989 \\
\hline 39 & 8 & $\odot$ & 8.807020 & -3.623943 & $-\odot .252769$ \\
\hline 40 & 1 & $\odot$ & 9.164142 & -4.516878 & -0.119141 \\
\hline 41 & 6 & $\odot$ & 4.202839 & -1.540235 & -1.485325 \\
\hline 42 & 7 & $\odot$ & 2.131303 & -4.320925 & -0.787201 \\
\hline 43 & 8 & $\Theta$ & 4.112569 & -0.364617 & -1.858176 \\
\hline 44 & 1 & $\Theta$ & 6.638602 & -3.720160 & -0.702203 \\
\hline 45 & 1 & $\Theta$ & 4.644146 & -5.250304 & -0.488498 \\
\hline 46 & 1 & $\Theta$ & 2.175832 & -5.287463 & -0.510557 \\
\hline 47 & 1 & $\odot$ & 1.221041 & -3.885649 & -0.949207 \\
\hline 48 & 6 & $\odot$ & 6.088491 & 2.683616 & -1.345644 \\
\hline 49 & 1 & $\odot$ & 4.808315 & 6.245483 & 1.042887 \\
\hline 50 & 6 & $\odot$ & 4.785779 & 3.574204 & 1.526129 \\
\hline 51 & 6 & 0 & 3.544758 & 2.917592 & 0.907179 \\
\hline 52 & 8 & 0 & 3.785155 & 2.886503 & -0.531438 \\
\hline 53 & 1 & $\odot$ & 5.671662 & 1.978075 & -2.066468 \\
\hline 54 & 1 & $\odot$ & 6.902084 & 3.253952 & -1.801089 \\
\hline 55 & 1 & $\odot$ & 4.743662 & 4.311209 & -1.689112 \\
\hline 56 & 1 & $\odot$ & 6.427029 & 4.592325 & 0.476195 \\
\hline 57 & 1 & 0 & 4.528170 & 4.173804 & 2.401482 \\
\hline 58 & 1 & $\odot$ & 5.532033 & 2.833665 & 1.818991 \\
\hline 59 & 1 & $\odot$ & 2.640154 & 3.490997 & 1.091515 \\
\hline 60 & 7 & 0 & 3.262830 & 1.559076 & 1.360943 \\
\hline 61 & 6 & $\odot$ & 4.233609 & 0.605603 & 1.284817 \\
\hline 62 & 6 & $\odot$ & 3.969963 & -0.688027 & 1.569419 \\
\hline 63 & 6 & 0 & 2.606026 & -1.031687 & 1.841003 \\
\hline 64 & 7 & $\odot$ & 1.647286 & -0.102809 & 1.880450 \\
\hline 65 & 6 & $\odot$ & 1.931990 & 1.203971 & 1.652400 \\
\hline 66 & 1 & 0 & 4.741295 & -1.444932 & 1.521781 \\
\hline 67 & 7 & $\odot$ & 2.258369 & -2.300718 & 2.052598 \\
\hline 68 & 8 & $\odot$ & 1.069493 & 2.087379 & 1.694282 \\
\hline 69 & 1 & $\odot$ & 5.205873 & $\odot .955888$ & $\odot .951535$ \\
\hline 70 & 1 & $\odot$ & 2.950291 & -3.027566 & 1.975700 \\
\hline 71 & 1 & 0 & 1.269352 & -2.567231 & 2.128156 \\
\hline 72 & 1 & 0 & -0.161156 & -0.622992 & 1.954268 \\
\hline 73 & 1 & $\odot$ & -7.478377 & -3.655758 & -0.474623 \\
\hline 74 & 6 & $\odot$ & -7.883891 & -0.547787 & $\odot .194285$ \\
\hline 75 & 6 & $\odot$ & -6.379655 & $-\odot .79814 \odot$ & 0.180684 \\
\hline 76 & 6 & $\odot$ & -6.119848 & -1.261715 & 1.610045 \\
\hline 77 & 8 & 0 & -7.258987 & -2.087200 & 1.933565 \\
\hline 78 & 1 & 0 & -9.176875 & -3.615352 & 1.201083 \\
\hline 79 & 1 & $\odot$ & -10.056910 & -2.515846 & 0.120426 \\
\hline 80 & 1 & $\odot$ & -9.129987 & -1.152965 & 1.866870 \\
\hline 81 & 1 & $\odot$ & -8.337623 & -0.620802 & -0.794204 \\
\hline 82 & 1 & $\odot$ & -5.798135 & 0.079813 & -0.099413 \\
\hline 83 & 1 & 0 & -6.158860 & -1.615592 & -0.507636 \\
\hline 84 & 1 & $\odot$ & -6.055569 & -0.411660 & 2.296946 \\
\hline 85 & 7 & $\odot$ & -4.916860 & -2.052474 & 1.773182 \\
\hline 86 & 6 & 0 & -4.751709 & -3.418173 & 1.853406 \\
\hline 87 & 7 & $\odot$ & -3.499082 & -3.772632 & 1.972394 \\
\hline 88 & 6 & $\odot$ & -2.798664 & -2.577326 & 1.955673 \\
\hline 89 & 6 & $\odot$ & -3.669895 & -1.503557 & 1.838270 \\
\hline
\end{tabular}




\begin{tabular}{|c|c|c|c|c|c|}
\hline 90 & 7 & 0 & -3.408462 & -0.188969 & 1.799335 \\
\hline 91 & 6 & 0 & -2.113354 & 0.073893 & 1.844821 \\
\hline 92 & 7 & 0 & -1.156612 & -0.911844 & 1.944528 \\
\hline 93 & 6 & 0 & -1.404271 & -2.286811 & 2.029472 \\
\hline 94 & 8 & 0 & -0.463873 & -3.085108 & 2.146757 \\
\hline 95 & 1 & 0 & -5.602371 & -4.079581 & 1.815022 \\
\hline 96 & 7 & 0 & -1.696048 & 1.348859 & 1.814265 \\
\hline 97 & 1 & 0 & -2.361912 & 1.989508 & 1.374309 \\
\hline 98 & 1 & 0 & -0.700369 & 1.554218 & 1.710342 \\
\hline 99 & 6 & 0 & -5.789645 & 3.228925 & $\odot .708682$ \\
\hline 100 & 11 & 0 & 7.175090 & 0.842442 & 3.590684 \\
\hline 101 & 11 & 0 & -10.346552 & 3.580120 & -1.345656 \\
\hline 102 & 6 & 0 & -4.126947 & 2.997860 & -2.137472 \\
\hline 103 & 6 & 0 & -3.027795 & 2.401232 & -1.255267 \\
\hline 104 & 8 & 0 & -3.456544 & 2.747656 & $\odot .118012$ \\
\hline 105 & 1 & 0 & -5.557766 & 2.612831 & 1.581861 \\
\hline 106 & 1 & 0 & -6.432836 & 4.059727 & 1. 011171 \\
\hline 107 & 1 & 0 & -4.157265 & 4.598604 & 0.726511 \\
\hline 108 & 1 & 0 & -5.633559 & 4.507939 & -1.617179 \\
\hline 109 & 1 & 0 & -3.763967 & 3.261164 & -3.134093 \\
\hline 110 & 1 & 0 & -4.942401 & 2.276600 & -2.227753 \\
\hline 111 & 1 & 0 & -2.061482 & 2.883553 & -1.415582 \\
\hline 112 & 7 & 0 & -2.969351 & 0.967065 & -1.355307 \\
\hline 113 & 6 & 0 & -4.002305 & -1.536446 & -2.291579 \\
\hline 114 & 7 & 0 & -2.870241 & -1.862785 & -1.605927 \\
\hline 115 & 6 & 0 & -1.766938 & -1.055819 & -1.462201 \\
\hline 116 & 6 & 0 & -1.792505 & 0.396445 & -1.396322 \\
\hline 117 & 7 & 0 & -0.604904 & 1.088556 & -1.372554 \\
\hline 118 & 6 & 0 & $\odot .525267$ & $\odot .427765$ & -1.357981 \\
\hline 119 & 7 & 0 & 0.607382 & -0.948856 & -1.303839 \\
\hline 120 & 6 & 0 & -0.494497 & -1.754046 & -1.351290 \\
\hline 121 & 7 & 0 & 1.699898 & 1.060824 & -1.391437 \\
\hline 122 & 8 & $\odot$ & $-\odot .403777$ & -2.985242 & -1.323199 \\
\hline 123 & 1 & 0 & -3.998713 & -0.537849 & -2.733344 \\
\hline 124 & 1 & 0 & 1.725683 & 2.066282 & -1.342395 \\
\hline 125 & 1 & 0 & 2.588692 & $\odot .562886$ & -1.483864 \\
\hline 126 & 1 & 0 & 1.532124 & -1.426253 & -1.299549 \\
\hline 127 & 8 & $\odot$ & -4.952037 & -2.305275 & -2.416666 \\
\hline 128 & 1 & 0 & -5.486906 & -3.677737 & -1.488828 \\
\hline 129 & 8 & 0 & -5.807498 & -4.398137 & -0.903010 \\
\hline 130 & 1 & $\odot$ & -3.917191 & -4.716327 & -0.697805 \\
\hline 131 & 1 & 0 & -6.099492 & -5.094382 & -1.501783 \\
\hline 132 & 8 & 0 & -3.000803 & -4.636476 & -1.012450 \\
\hline 133 & 1 & 0 & -2.774843 & -2.862597 & -1.333494 \\
\hline 134 & 1 & $\odot$ & -3.011573 & -5.034380 & -1.888553 \\
\hline
\end{tabular}

General basis read from cards: (6D, 7F)

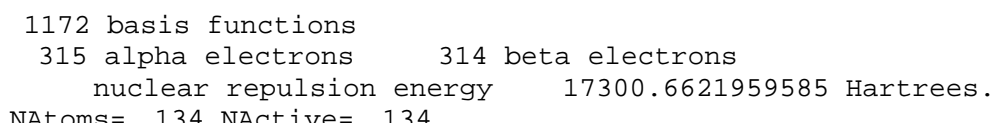

NAtoms $=134$ NActive $=134$

Force inversion solution in PCM.

Polarizable Continuum Model (PCM)

=ニニニニニニニニニニニニニニニニニニニニニニニニニニニニニニニニ

Model : PCM.

Solvent : Water, Eps $=78.355300$ Eps $($ inf $)=1.777849$

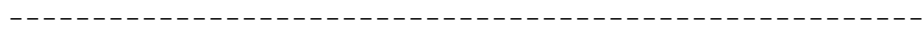

SCF Done: $E($ UWB97XD) $=-5092.29370920$ A.U. after 1 cycles NFock $=1$ Conv $=0.31 \mathrm{D}-08 \quad-\mathrm{V} / \mathrm{T}=2.0066$

$\langle\mathrm{S} X>=0.0000<\mathrm{Sy}\rangle=0.0000<\mathrm{S} z>=0.5000$

$\left\langle S^{*} * 2>=0.7721 \mathrm{~S}=0.5110\right.$

$<$ L.S $>=0.0000 \odot \odot \odot \odot \odot \odot \odot \odot E+\odot \odot$

Annihilation of the first spin contaminant:

$\mathrm{S}^{* * 2}$ before annihilation 0.7721 , after 0.7502

Harmonic frequencies $\left(\mathrm{cm}^{*}-1\right)$, IR intensities (KM/Mole), Raman

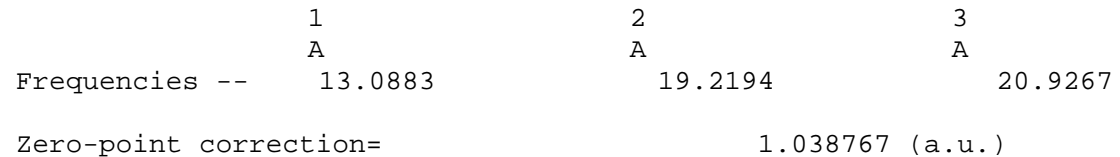


Thermal correction to Energy=

Thermal correction to Enthalpy=

Thermal correction to Gibbs Free Energy=

Sum of electronic and zero-point Energies=

Sum of electronic and thermal Energies=

Sum of electronic and thermal Enthalpies=

Sum of electronic and thermal Free Energies=

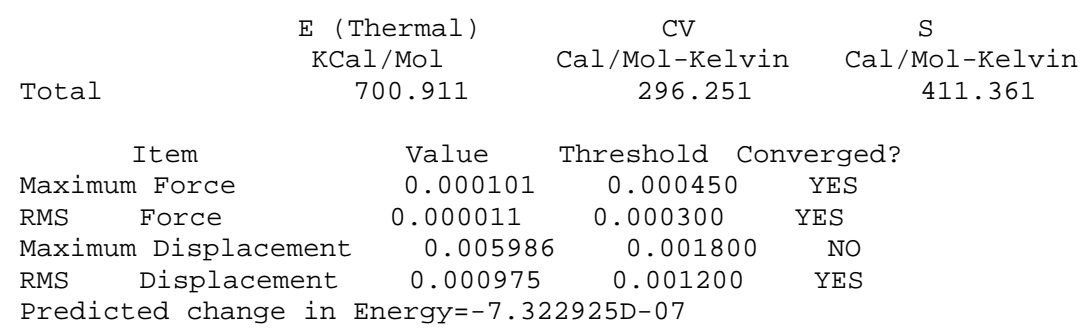

Normal termination of Gaussian 16 at Fri Aug 16 19:06:29 2019.

$\mathrm{G}(\mathrm{high})=-5093.13786015 \mathrm{a} \cdot \mathrm{u}$.

=================Figure S14 for (c) $5^{\prime}$ - GT - 3 ' ==================

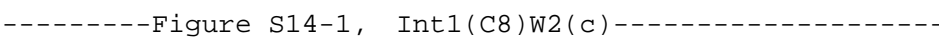

dna. for1cw2B. $\log$

Stoichiometry C39H54N15Na2023P2(2)

Standard orientation

\begin{tabular}{|c|c|c|c|c|c|}
\hline \multirow{2}{*}{$\begin{array}{l}\text { Center } \\
\text { Number }\end{array}$} & \multirow{2}{*}{$\begin{array}{l}\text { Atomic } \\
\text { Number }\end{array}$} & \multirow{2}{*}{$\begin{array}{l}\text { Atomic } \\
\text { Type }\end{array}$} & \multicolumn{3}{|c|}{ Coordinates (Angstroms) } \\
\hline & & & $\mathrm{x}$ & $\mathrm{Y}$ & $\mathrm{Z}$ \\
\hline 1 & 7 & 0 & 5.817438 & -2.157287 & -1.174570 \\
\hline 2 & 6 & 0 & 5.831792 & -3.424277 & -0.667482 \\
\hline 3 & 6 & 0 & 4.772890 & -4.245417 & -0.836244 \\
\hline 4 & 6 & 0 & 3.634251 & -3.697072 & -1.514778 \\
\hline 5 & 7 & 0 & 3.679321 & -2.511526 & -2.122718 \\
\hline 6 & 8 & 0 & 8.535846 & 0.851366 & $\odot .453439$ \\
\hline 7 & 15 & 0 & 7.591587 & 2.073322 & $\odot .994188$ \\
\hline 8 & 8 & 0 & 6.272715 & 1.949839 & $\odot .011190$ \\
\hline 9 & 8 & 0 & 8.300681 & 3.364685 & $\odot .715170$ \\
\hline 10 & 8 & 0 & 7.097050 & 1.722786 & 2.380991 \\
\hline 11 & 8 & 0 & 4.081700 & 5.625062 & 0.608423 \\
\hline 12 & 6 & 0 & 4.768907 & 3.764119 & -0.702036 \\
\hline 13 & 6 & 0 & 4.811912 & 4.376639 & $\odot .698330$ \\
\hline 14 & 6 & 0 & -9.407808 & -2.181863 & 1.610420 \\
\hline 15 & 6 & 0 & -8.611714 & -0.893620 & 1.657936 \\
\hline 16 & 8 & 0 & -8.676668 & -3.159654 & 0.831011 \\
\hline 17 & 8 & 0 & -8.098511 & 1.110823 & $\odot .413442$ \\
\hline 18 & 15 & 0 & -7.221605 & 2.033230 & -0.615469 \\
\hline 19 & 8 & $\Theta$ & -5.696545 & 1.879553 & -0.035804 \\
\hline 20 & 8 & $\odot$ & -7.686460 & 3.452145 & -0.405867 \\
\hline 21 & 8 & 0 & -7.214487 & 1.409969 & -1.988050 \\
\hline 22 & 8 & $\odot$ & -3.375938 & 5.262752 & -1.123609 \\
\hline 23 & 6 & $\theta$ & -3.926874 & 3.509997 & 0.395222 \\
\hline 24 & 6 & $\odot$ & -4.129968 & 4.025980 & -1.032898 \\
\hline 25 & 1 & 0 & -3.406752 & 5.612429 & -2.030396 \\
\hline 26 & 6 & 0 & 9.707343 & -2.695357 & 0.387506 \\
\hline 27 & 6 & 0 & 9.140748 & -1.394909 & -0.144654 \\
\hline 28 & 6 & 0 & 8.388418 & -0.542538 & 0.887346 \\
\hline 29 & 6 & $\theta$ & 6.952012 & -1.019519 & 0.734859 \\
\hline 30 & 6 & 0 & 6.843708 & -1.208964 & -0.777101 \\
\hline 31 & 8 & $\odot$ & 8.155001 & -1.684209 & -1.194720 \\
\hline 32 & 1 & $\odot$ & 10.100488 & -3.275868 & -0.453437 \\
\hline 33 & 1 & 0 & 10.529517 & -2.474081 & 1.077975 \\
\hline 34 & 1 & $\Theta$ & 9.948361 & -0.811622 & -0.592579 \\
\hline 35 & 1 & 0 & 8.779889 & -0.643666 & 1.900394 \\
\hline 36 & 1 & 0 & 6.224134 & -0.312581 & 1.121780 \\
\hline 37 & 1 & 0 & 6.844642 & -1.971563 & 1.256962 \\
\hline 38 & 1 & 0 & 6.617392 & -0.273587 & -1.284317 \\
\hline 39 & 8 & $\theta$ & 8.657499 & -3.428743 & 1.066287 \\
\hline 40 & 1 & $\odot$ & 8.976069 & -4.298706 & 1.356605 \\
\hline 41 & 6 & $\theta$ & 4.761595 & -1.711422 & -1.981559 \\
\hline
\end{tabular}

116973

$-5091.254942$

$-5091.176736$

$-5091.175792$

$-5091.371242$

Mol-Kelvin 


\begin{tabular}{|c|c|c|c|c|c|}
\hline 42 & 7 & 0 & 2.477343 & -4.366315 & -1.548687 \\
\hline 43 & 8 & $\odot$ & 4.840359 & $-\odot .598430$ & -2.519998 \\
\hline 44 & 1 & $\odot$ & 6.736087 & -3.698052 & -0.127830 \\
\hline 45 & 1 & $\odot$ & 4.763184 & -5.246777 & -0.428284 \\
\hline 46 & 1 & $\odot$ & 2.400905 & -5.272293 & -1.116675 \\
\hline 47 & 1 & $\odot$ & 1.640610 & -3.914685 & -1.930739 \\
\hline 48 & 6 & $\odot$ & 5.955546 & 2.883386 & -1.059206 \\
\hline 49 & 1 & $\odot$ & 4.061696 & 6.068764 & 1.473283 \\
\hline 50 & 6 & $\odot$ & 4.070773 & 3.350258 & 1.561377 \\
\hline 51 & 6 & $\odot$ & 3.004829 & 2.776958 & $\odot .618100$ \\
\hline 52 & 8 & $\odot$ & 3.514555 & 2.998322 & $-\odot .727573$ \\
\hline 53 & 1 & $\odot$ & 5.717802 & 2.284053 & -1.939730 \\
\hline 54 & 1 & $\odot$ & 6.825593 & 3.513419 & -1.260432 \\
\hline 55 & 1 & $\odot$ & 4.671842 & 4.548952 & -1.454802 \\
\hline 56 & 1 & $\odot$ & 5.838314 & 4.548796 & 1.036737 \\
\hline 57 & 1 & $\odot$ & 3.627105 & 3.798411 & 2.452497 \\
\hline 58 & 1 & $\odot$ & 4.767863 & 2.567841 & 1.867297 \\
\hline 59 & 1 & $\odot$ & 2.040125 & 3.276710 & $\odot .714065$ \\
\hline 60 & 7 & $\odot$ & 2.759078 & 1.356297 & $\odot .786105$ \\
\hline 61 & 6 & $\odot$ & 3.629083 & 0.339236 & $\odot .469770$ \\
\hline 62 & 7 & $\odot$ & 3.105754 & -0.853623 & $\odot .587257$ \\
\hline 63 & 6 & $\odot$ & 1.805632 & $-\odot .608870$ & $\odot .99 \odot 420$ \\
\hline 64 & 6 & $\odot$ & 1.569399 & 0.757229 & 1.117052 \\
\hline 65 & 7 & $\odot$ & $\odot .423873$ & 1.348550 & 1.479639 \\
\hline 66 & 6 & $\odot$ & -0.530485 & $\odot .45717 \odot$ & 1.712764 \\
\hline 67 & 7 & $\odot$ & -0.447185 & -0.880018 & 1.615902 \\
\hline 68 & 6 & $\odot$ & $\odot .7 \odot 4358$ & -1.456112 & 1.221864 \\
\hline 69 & 7 & $\odot$ & $\odot .747050$ & -2.794168 & 1.081297 \\
\hline 70 & 1 & $\odot$ & 4.631399 & $\odot .577942$ & $\odot .138923$ \\
\hline 71 & 1 & $\odot$ & -1.511049 & $\odot .833339$ & 1.988788 \\
\hline 72 & 1 & $\odot$ & 1.519569 & -3.190045 & 0.565840 \\
\hline 73 & 1 & $\odot$ & -0.137519 & -3.298736 & 1.051797 \\
\hline 74 & 1 & 0 & -9.137228 & -4.014260 & 0.828030 \\
\hline 75 & 6 & $\odot$ & -8.174604 & -0.351711 & $\odot .286912$ \\
\hline 76 & 6 & $\odot$ & -6.796907 & -0.966547 & 0.110994 \\
\hline 77 & 6 & $\odot$ & -6.232551 & -0.884615 & 1.523487 \\
\hline 78 & 8 & $\odot$ & -7.364641 & -1.125170 & 2.402308 \\
\hline 79 & 1 & $\odot$ & -9.557534 & -2.538641 & 2.634796 \\
\hline 80 & 1 & $\odot$ & -10.387484 & -1.983683 & 1.160055 \\
\hline 81 & 1 & $\odot$ & -9.188709 & -0.135855 & 2.193110 \\
\hline 82 & 1 & 0 & -8.871270 & -0.598465 & $-\odot .514867$ \\
\hline 83 & 1 & $\odot$ & -6.169809 & -0.459297 & -0.616415 \\
\hline 84 & 1 & $\odot$ & -6.926293 & -2.005389 & -0.197023 \\
\hline 85 & 1 & $\odot$ & -5.814577 & $\odot .096473$ & 1.733653 \\
\hline 86 & 7 & 0 & -5.170459 & -1.846014 & 1.761372 \\
\hline 87 & 6 & $\odot$ & -5.378095 & -3.176500 & 1.463378 \\
\hline 88 & 6 & 0 & -4.368406 & -4.055460 & 1.298801 \\
\hline 89 & 6 & $\odot$ & -3.009314 & -3.538709 & 1.381089 \\
\hline 90 & 7 & $\odot$ & -2.885146 & -2.245609 & 1.827984 \\
\hline 91 & 6 & $\odot$ & -3.903091 & -1.345341 & 2.017382 \\
\hline 92 & 6 & $\odot$ & -4.582953 & -5.496872 & 0.951342 \\
\hline 93 & 8 & $\odot$ & -2.002330 & -4.188358 & 1.065850 \\
\hline 94 & 8 & $\odot$ & -3.693594 & -0.188927 & 2.357348 \\
\hline 95 & 1 & $\odot$ & -6.426568 & -3.450213 & 1.362131 \\
\hline 96 & 1 & $\odot$ & -4.227832 & -5.710662 & -0.061447 \\
\hline 97 & 1 & $\Theta$ & -4.036833 & -6.151974 & 1.635480 \\
\hline 98 & 1 & $\odot$ & -5.643701 & -5.750685 & $\odot .998510$ \\
\hline 99 & 1 & $\odot$ & -1.933416 & -1.808916 & 1.819512 \\
\hline 100 & 6 & 0 & -5.115387 & 2.762384 & 0.967776 \\
\hline 101 & 11 & $\odot$ & 5.907882 & $\odot .698959$ & 3.906226 \\
\hline 102 & 11 & $\odot$ & -7.695101 & 5.615817 & $-\odot .067530$ \\
\hline 103 & 6 & 0 & -3.516026 & 2.924566 & -1.899313 \\
\hline 104 & 6 & $\odot$ & -2.343689 & 2.425690 & -1.045395 \\
\hline 105 & 8 & $\odot$ & -2.756944 & 2.627946 & 0.331485 \\
\hline 106 & 1 & $\odot$ & -4.785925 & 2.137835 & 1.799497 \\
\hline 107 & 1 & $\odot$ & -5.869668 & 3.475888 & 1.308902 \\
\hline 108 & 1 & $\odot$ & -3.672253 & 4.339849 & 1.059341 \\
\hline 109 & 1 & 0 & -5.185746 & 4.198373 & -1.259326 \\
\hline 110 & 1 & 0 & -3.186860 & 3.288712 & -2.874714 \\
\hline 111 & 1 & $\odot$ & -4.245162 & 2.122876 & -2.046884 \\
\hline 112 & 1 & 0 & -1.424297 & 2.983816 & -1.232778 \\
\hline 113 & 7 & 0 & -2.024653 & 1.028213 & -1.244395 \\
\hline 114 & 6 & 0 & -2.870864 & $-\odot .041712$ & -1.075371 \\
\hline 115 & 7 & 0 & -2.291551 & -1.181308 & -1.341041 \\
\hline 116 & 6 & 0 & -0.999793 & $-\odot .850799$ & -1.702095 \\
\hline 117 & 6 & 0 & -0.810644 & 0.519253 & -1.635146 \\
\hline 118 & 7 & 0 & $\odot .304596$ & 1.223279 & -1.899507 \\
\hline
\end{tabular}




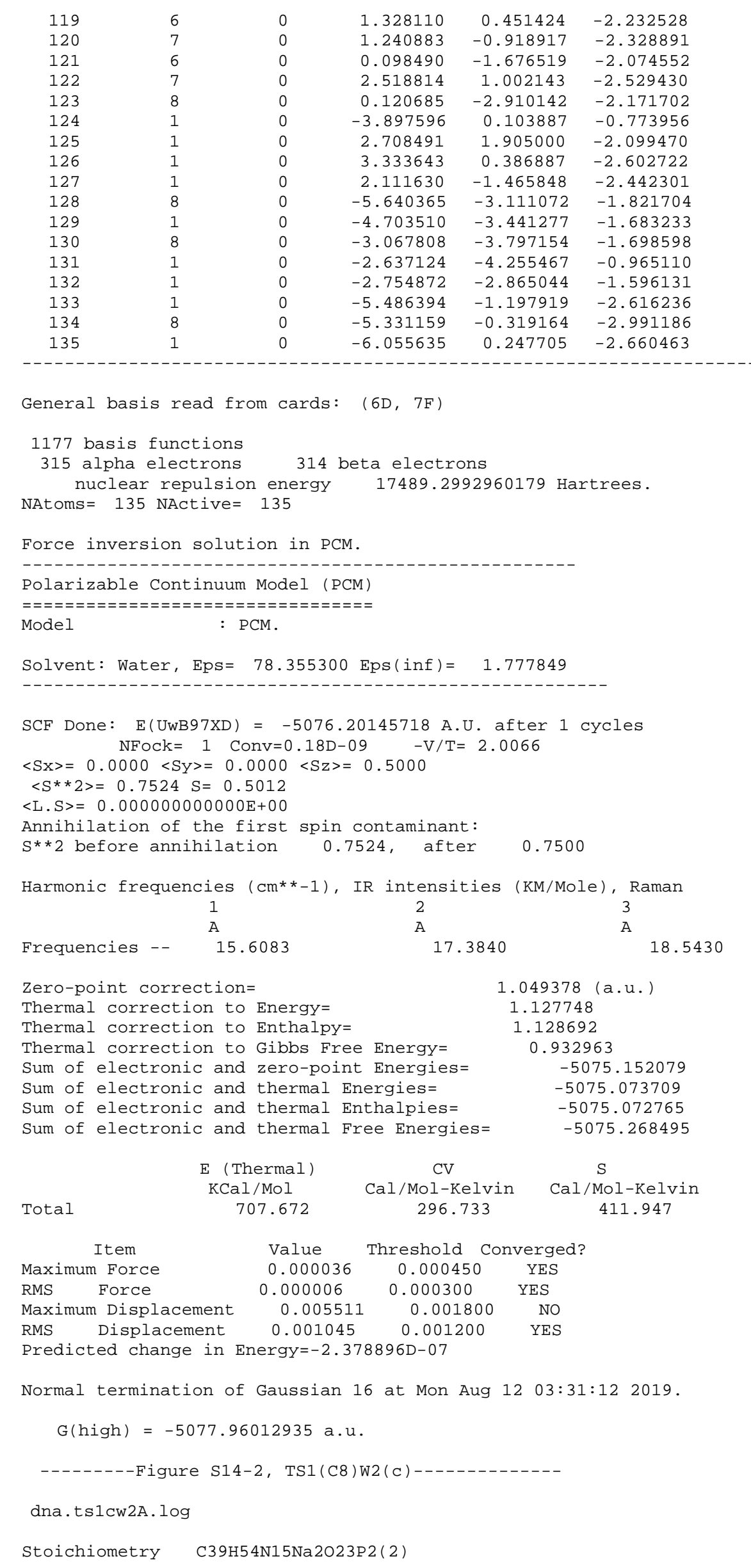


Standard orientation:

\begin{tabular}{|c|c|c|c|c|c|}
\hline \multirow{2}{*}{$\begin{array}{l}\text { Center } \\
\text { Number }\end{array}$} & \multirow{2}{*}{$\begin{array}{l}\text { Atomic } \\
\text { Number }\end{array}$} & \multirow{2}{*}{$\begin{array}{l}\text { Atomic } \\
\text { Type }\end{array}$} & \multicolumn{3}{|c|}{ Coordinates (Angstroms) } \\
\hline & & & $\mathrm{x}$ & $\mathrm{Y}$ & Z \\
\hline 1 & 7 & 0 & 5.889719 & -1.904895 & -1.091339 \\
\hline 2 & 6 & 0 & 6.052973 & -3.231280 & $-\odot .816898$ \\
\hline 3 & 6 & 0 & 5.019150 & -4.094066 & -0.929624 \\
\hline 4 & 6 & 0 & 3.756190 & -3.544509 & -1.325236 \\
\hline 5 & 7 & $\odot$ & 3.623887 & -2.259018 & -1.657065 \\
\hline 6 & 8 & 0 & 8.937542 & 0.964684 & 0.223380 \\
\hline 7 & 15 & $\theta$ & 8.079127 & 2.119727 & 1.002277 \\
\hline 8 & 8 & $\odot$ & 6.622424 & 2.047643 & 0.242149 \\
\hline 9 & 8 & 0 & 8.736627 & 3.436475 & 0.713982 \\
\hline 10 & 8 & 0 & 7.794616 & 1.661246 & 2.416083 \\
\hline 11 & 8 & $\odot$ & 4.586595 & 5.781841 & 0.822112 \\
\hline 12 & 6 & 0 & 5.093611 & 3.838439 & -0.454567 \\
\hline 13 & 6 & 0 & 5.294000 & 4.519201 & 0.900232 \\
\hline 14 & 6 & $\odot$ & -8.946322 & -2.527028 & 2.326371 \\
\hline 15 & 6 & 0 & -8.281427 & -1.170141 & 2.423795 \\
\hline 16 & 8 & 0 & -8.212071 & -3.324735 & 1.365654 \\
\hline 17 & 8 & $\odot$ & -8.228661 & 0.957011 & 1.231694 \\
\hline 18 & 15 & 0 & -8.360665 & 1.927263 & $-\odot .085907$ \\
\hline 19 & 8 & $\odot$ & -6.807263 & 1.987547 & -0.659963 \\
\hline 20 & 8 & $\odot$ & -8.710162 & 3.290852 & $\odot .458413$ \\
\hline 21 & 8 & 0 & -9.169607 & 1.257034 & -1.154682 \\
\hline 22 & 8 & 0 & -4.191979 & 4.688824 & -2.484207 \\
\hline 23 & 6 & 0 & -4.591185 & 3.028974 & -0.810415 \\
\hline 24 & 6 & 0 & -4.872577 & 3.424842 & -2.277384 \\
\hline 25 & 1 & 0 & -4.397151 & 5.042837 & -3.366179 \\
\hline 26 & 6 & $\odot$ & 10.023430 & -2.516467 & -0.570344 \\
\hline 27 & 6 & 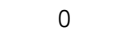 & 9.355617 & -1.172730 & $-0.78007 \odot$ \\
\hline 28 & 6 & 0 & 8.874580 & -0.473503 & 0.500329 \\
\hline 29 & 6 & 0 & 7.441844 & -0.970851 & 0.631541 \\
\hline 30 & 6 & 0 & 6.971938 & -0.973840 & -0.820965 \\
\hline 31 & 8 & $\odot$ & 8.147288 & -1.344836 & -1.597735 \\
\hline 32 & 1 & $\odot$ & 10.204045 & -2.974145 & -1.548435 \\
\hline 33 & 1 & $\odot$ & 10.986393 & -2.364542 & $-\odot .069103$ \\
\hline 34 & 1 & 0 & 10.039182 & -0.518760 & -1.325958 \\
\hline 35 & 1 & 0 & 9.494075 & -0.692770 & 1.370954 \\
\hline 36 & 1 & 0 & 6.829250 & -0.345680 & 1.272366 \\
\hline 37 & 1 & 0 & 7.466402 & -1.983960 & 1.036370 \\
\hline 38 & 1 & $\odot$ & 6.622041 & $\odot .007309$ & -1.130722 \\
\hline 39 & 8 & 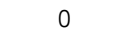 & 9.160331 & -3.358548 & 0.233895 \\
\hline 40 & 1 & $\Theta$ & 9.542963 & -4.244610 & 0.339767 \\
\hline 41 & 6 & 0 & 4.671769 & -1.406457 & -1.571139 \\
\hline 42 & 7 & $\odot$ & 2.658438 & -4.304709 & -1.361894 \\
\hline 43 & 8 & 0 & 4.582384 & -0.214531 & -1.893410 \\
\hline 44 & 1 & 0 & 7.057025 & -3.522081 & -0.513627 \\
\hline 45 & 1 & $\odot$ & 5.136682 & -5.146884 & -0.713527 \\
\hline 46 & 1 & $\odot$ & 2.719375 & -5.292682 & -1.179528 \\
\hline 47 & 1 & 0 & 1.785880 & -3.910902 & -1.726010 \\
\hline 48 & 6 & 0 & 6.218200 & 2.901490 & -0.865103 \\
\hline 49 & 1 & $\odot$ & 4.667184 & 6.266832 & 1.660923 \\
\hline 50 & 6 & 0 & 4.621778 & 3.558208 & 1.888118 \\
\hline 51 & 6 & $\odot$ & 3.466304 & 2.951704 & 1.079714 \\
\hline 52 & 8 & 0 & 3.819478 & 3.121533 & -0.324174 \\
\hline 53 & 1 & 0 & 5.873784 & 2.244979 & -1.665951 \\
\hline 54 & 1 & 0 & 7.075595 & 3.490073 & -1.201464 \\
\hline 55 & 1 & $\Theta$ & 4.953578 & 4.586484 & -1.237788 \\
\hline 56 & 1 & $\theta$ & 6.353441 & 4.677243 & 1.124158 \\
\hline 57 & 1 & $\odot$ & 4.262473 & 4.071048 & 2.782318 \\
\hline 58 & 1 & 0 & 5.338118 & 2.791527 & 2.187589 \\
\hline 59 & 1 & 0 & 2.519804 & 3.464714 & 1.253422 \\
\hline 60 & 7 & 0 & 3.221791 & 1.545513 & 1.330323 \\
\hline 61 & 6 & 0 & 4.129494 & 0.517798 & 1.213276 \\
\hline 62 & 7 & $\Theta$ & 3.584508 & -0.670325 & 1.227824 \\
\hline 63 & 6 & $\odot$ & 2.227760 & -0.413887 & 1.321956 \\
\hline 64 & 6 & 0 & 1.984236 & 0.954581 & 1.379794 \\
\hline 65 & 7 & 0 & 0.795368 & 1.569042 & 1.442671 \\
\hline 66 & 6 & 0 & -0.198147 & $\odot .692416$ & 1.431263 \\
\hline 67 & 7 & 0 & -0.112935 & -0.642818 & 1.348461 \\
\hline 68 & 6 & 0 & 1. 090918 & -1.245022 & 1.274143 \\
\hline 69 & 7 & $\Theta$ & 1.128668 & -2.579593 & 1.155364 \\
\hline 70 & 1 & $\odot$ & 5.177134 & 0.747115 & 1.077004 \\
\hline 71 & 1 & $\odot$ & -1.212933 & 1.077141 & 1.489513 \\
\hline
\end{tabular}




\begin{tabular}{|c|c|c|c|c|c|}
\hline 72 & 1 & 0 & 2.001050 & -3.038966 & 0.951174 \\
\hline 73 & 1 & 0 & $\odot .255153$ & -3.092585 & 1.041638 \\
\hline 74 & 1 & 0 & -8.587124 & -4.217639 & 1.296801 \\
\hline 75 & 6 & 0 & -8.053070 & -0.489012 & 1.064370 \\
\hline 76 & 6 & $\odot$ & -6.603325 & -0.803068 & 0.745238 \\
\hline 77 & 6 & 0 & -5.934028 & -0.863133 & 2.113532 \\
\hline 78 & 8 & 0 & -6.952133 & -1.338729 & 3.027645 \\
\hline 79 & 1 & 0 & -8.936538 & -3.001609 & 3.313020 \\
\hline 80 & 1 & 0 & -9.986854 & -2.397334 & 2.006633 \\
\hline 81 & 1 & 0 & -8.870526 & $-\odot .520495$ & 3.075699 \\
\hline 82 & 1 & $\odot$ & -8.747006 & -0.840098 & $\odot .299292$ \\
\hline 83 & 1 & $\odot$ & -6.133192 & $-\odot .05759 \odot$ & 0.113011 \\
\hline 84 & 1 & 0 & -6.544508 & -1.775602 & $\odot .254420$ \\
\hline 85 & 1 & 0 & -5.574491 & $\odot .108811$ & 2.447352 \\
\hline 86 & 7 & 0 & -4.780167 & -1.753941 & 2.118178 \\
\hline 87 & 6 & 0 & -4.954479 & -3.084653 & 1.793536 \\
\hline 88 & 6 & $\odot$ & -3.934965 & -3.902549 & 1.458826 \\
\hline 89 & 6 & 0 & -2.595780 & -3.330220 & $1.4 \odot 2533$ \\
\hline 90 & 7 & 0 & -2.501115 & $-2.009 \odot 44$ & 1.782264 \\
\hline 91 & 6 & 0 & -3.530711 & -1.158762 & 2.083270 \\
\hline 92 & 6 & $\odot$ & -4.119237 & -5.339571 & 1. 079012 \\
\hline 93 & 8 & 0 & -1.584321 & -3.947726 & 1.055844 \\
\hline 94 & 8 & $\odot$ & -3.347656 & $\odot .032385$ & 2.307643 \\
\hline 95 & 1 & 0 & -5.992648 & -3.410889 & 1.817737 \\
\hline 96 & 1 & $\odot$ & -3.832975 & -5.485446 & $\odot .033313$ \\
\hline 97 & 1 & 0 & -3.492571 & -5.993238 & 1.693014 \\
\hline 98 & 1 & $\odot$ & -5.161484 & -5.642732 & 1.201621 \\
\hline 99 & 1 & $\odot$ & -1.578816 & -1.538425 & 1.646697 \\
\hline 100 & 6 & $\odot$ & -5.795640 & 2.756954 & $\odot .05900 \odot$ \\
\hline 101 & 11 & $\odot$ & 6.596796 & 0.736797 & 4.008863 \\
\hline 102 & 11 & $\odot$ & -10.250231 & 4.864947 & $\odot .458113$ \\
\hline 103 & 6 & $\odot$ & -4.240395 & 2.291220 & -3.098573 \\
\hline 104 & 6 & $\odot$ & -3.110939 & 1.829093 & -2.179871 \\
\hline 105 & 8 & $\odot$ & -3.740131 & 1.839000 & $-\odot .864211$ \\
\hline 106 & 1 & $\odot$ & -5.482791 & 2.199369 & 0.947524 \\
\hline 107 & 1 & $\odot$ & -6.244894 & 3.703972 & 0.368034 \\
\hline 108 & 1 & $\odot$ & -4.018948 & 3.844369 & $-\odot .353685$ \\
\hline 109 & 1 & $\odot$ & -5.942015 & 3.527291 & -2.475784 \\
\hline 110 & 1 & $\odot$ & -3.889990 & 2.627400 & -4.075507 \\
\hline 111 & 1 & $\odot$ & -4.969937 & 1.486776 & -3.225339 \\
\hline 112 & 1 & $\odot$ & -2.274849 & 2.535128 & -2.190959 \\
\hline 113 & 7 & $\odot$ & -2.555802 & $\odot .524088$ & -2.427263 \\
\hline 114 & 6 & $\odot$ & -3.222034 & -0.628260 & -2.785622 \\
\hline 115 & 7 & $\odot$ & -2.413032 & -1.672615 & -2.846340 \\
\hline 116 & 6 & $\odot$ & -1.189687 & -1.195589 & -2.471801 \\
\hline 117 & 6 & $\odot$ & -1.241056 & 0.173944 & -2.224569 \\
\hline 118 & 7 & $\odot$ & -0.232843 & 1.001691 & -1.925219 \\
\hline 119 & 6 & $\odot$ & 0.943579 & 0.378596 & -1.878225 \\
\hline 120 & 7 & $\odot$ & 1.086875 & -0.981951 & -2.025903 \\
\hline 121 & 6 & $\odot$ & $\odot .058270$ & -1.873770 & -2.309048 \\
\hline 122 & 7 & $\odot$ & 2.068427 & 1.069215 & -1.689484 \\
\hline 123 & 8 & $\odot$ & 0.265575 & -3.087661 & -2.396849 \\
\hline 124 & 1 & $\odot$ & -4.242705 & $-\odot .601816$ & -3.133230 \\
\hline 125 & 1 & $\odot$ & 2.024945 & 2.054146 & -1.479832 \\
\hline 126 & 1 & $\odot$ & 2.987441 & 0.616338 & -1.704077 \\
\hline 127 & 1 & $\odot$ & 2.029098 & -1.410853 & -1.910251 \\
\hline 128 & 8 & $\odot$ & -4.278277 & -1.566639 & -0.957519 \\
\hline 129 & 1 & $\odot$ & -4.152275 & -2.506120 & -1.225300 \\
\hline 130 & 8 & $\odot$ & -3.633028 & -4.077796 & -2.101390 \\
\hline 131 & 1 & $\odot$ & -3.050252 & -3.436465 & -2.555184 \\
\hline 132 & 1 & $\odot$ & -4.335232 & -4.257366 & -2.733795 \\
\hline 133 & 1 & $\odot$ & -5.757093 & -0.801729 & -1.860137 \\
\hline 134 & 8 & $\Theta$ & -6.367035 & -0.298781 & -2.429852 \\
\hline 135 & 1 & $\odot$ & -6.542110 & 0.511280 & -1.924680 \\
\hline
\end{tabular}

General basis read from cards: (6D, 7F)

1177 basis functions

315 alpha electrons 314 beta electrons

nuclear repulsion energy 17094.2735222183 Hartrees.

NAtoms $=135$ NActive $=135$

Force inversion solution in PCM.

Polarizable Continuum Model (PCM)

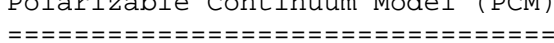


Solvent : Water, Eps $=78.355300$ Eps $($ inf $)=1.777849$

SCF Done: $E($ UWB97XD $)=-5076.18231150$ A.U. after 1 cycles NFock $=1$ Conv $=0.12 \mathrm{D}-09$ $<\mathrm{S} x>=0.0000<\mathrm{S} y>=0.0000<\mathrm{S} z>=0.5000$ $<S^{*} * 2>=0.7641 \mathrm{~S}=0.5070$

$<$ L.S $>=0.000000000000 \mathrm{E}+0 \odot$

Annihilation of the first spin contaminant:

$\mathrm{S}^{* *} 2$ before annihilation 0.7641 , after 0.7501

Harmonic frequencies $\left(\mathrm{cm}^{*}-1\right)$, IR intensities (KM/Mole), Raman

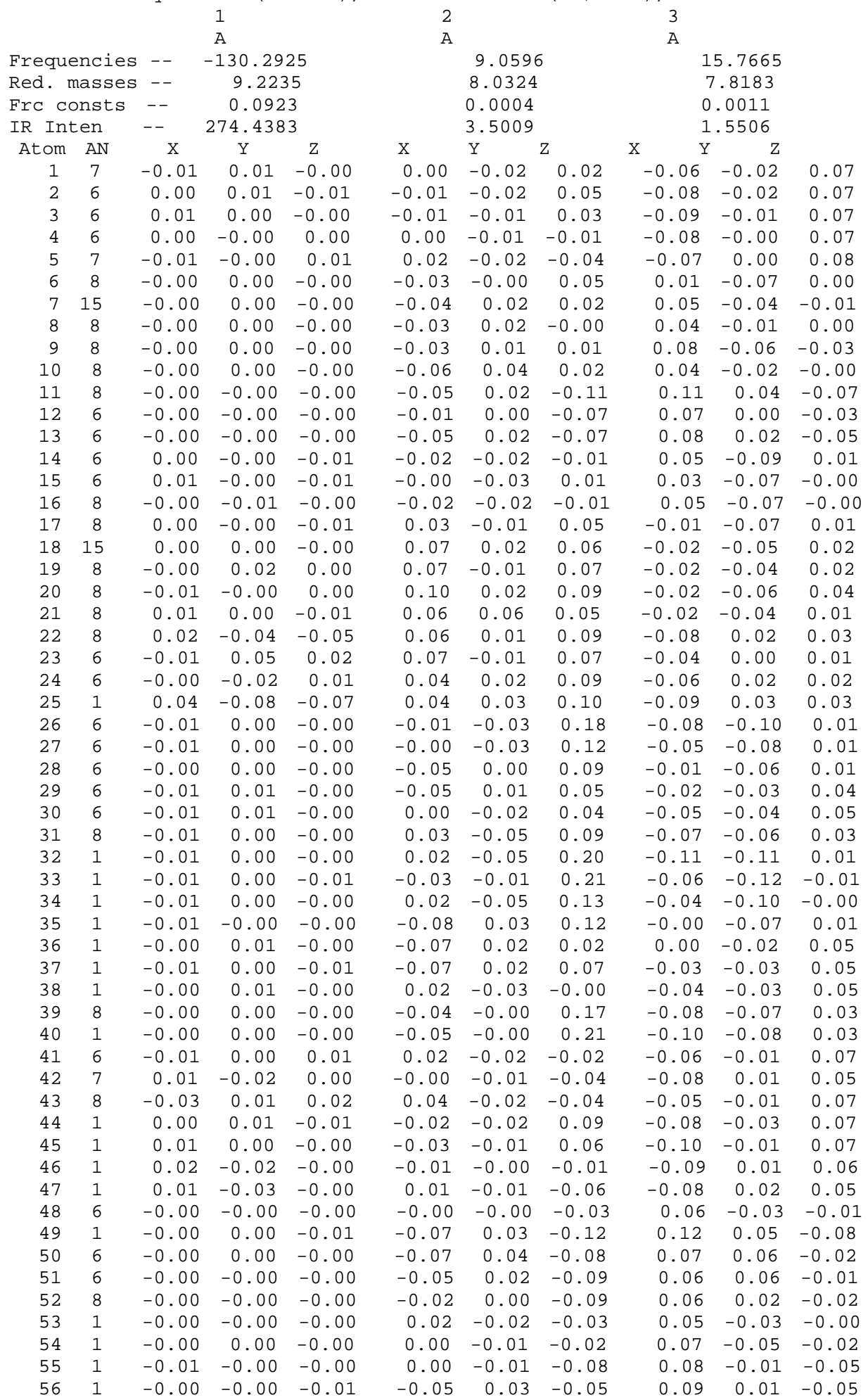




\begin{tabular}{|c|c|c|c|c|c|c|c|c|c|c|}
\hline 57 & 1 & $-\odot . \odot \odot$ & $\odot .0 \odot$ & $-\odot .0 \odot$ & -0.09 & 0.05 & -0.09 & $\odot .07$ & $\odot . \odot 8$ & -0.04 \\
\hline 58 & 1 & -0.00 & 0.00 & -0.00 & -0.07 & 0.04 & -0.05 & $\odot .06$ & 0.05 & -0.00 \\
\hline 59 & 1 & -0.00 & $-0.0 \odot$ & $-0.0 \odot$ & -0.05 & 0.03 & -0.12 & 0.06 & 0.07 & -0.02 \\
\hline 60 & 7 & $-0.0 \odot$ & -0.00 & -0.00 & -0.05 & $\odot .03$ & -0.08 & 0.04 & 0.06 & 0.02 \\
\hline 61 & 6 & $-\odot . \odot \odot$ & $\odot .0 \odot$ & $\odot . \odot \odot$ & -0.05 & 0.03 & -0.05 & $\odot .04$ & $\odot .05$ & 0.06 \\
\hline 62 & 7 & $\odot .0 \odot$ & -0.00 & 0.00 & -0.05 & 0.03 & -0.05 & 0.03 & $\odot .06$ & 0.07 \\
\hline 63 & 6 & $\odot .0 \odot$ & $-\odot . \odot \odot$ & 0.00 & -0.05 & 0.03 & -0.07 & 0.03 & 0.07 & 0.04 \\
\hline 64 & 6 & -0.00 & $-0.0 \odot$ & $\odot .0 \odot$ & -0.05 & 0.03 & -0.09 & 0.04 & 0.07 & $\odot . \odot \odot$ \\
\hline 65 & 7 & -0.00 & -0.00 & $-0.0 \odot$ & -0.06 & $\odot .02$ & -0.11 & 0.04 & 0.08 & -0.04 \\
\hline 66 & 6 & $-\odot .0 \odot$ & -0.00 & -0.01 & -0.05 & 0.02 & -0.12 & 0.03 & 0.08 & -0.05 \\
\hline 67 & 7 & -0.00 & 0.00 & -0.01 & -0.05 & 0.02 & -0.10 & 0.03 & $\odot .08$ & -0.03 \\
\hline 68 & 6 & -0.00 & -0.00 & -0.00 & -0.04 & $\odot .02$ & -0.07 & 0.03 & $\odot .07$ & $\odot .02$ \\
\hline 69 & 7 & $-\odot . \odot \odot$ & $\odot .0 \odot$ & -0.01 & -0.03 & 0.02 & -0.06 & $\odot .03$ & 0.07 & 0.04 \\
\hline 70 & 1 & $-\odot .0 \odot$ & 0.00 & 0.00 & -0.05 & 0.03 & -0.04 & 0.04 & 0.04 & $\odot .07$ \\
\hline 71 & 1 & $-\odot . \odot \odot$ & $-0.0 \odot$ & -0.01 & -0.06 & 0.01 & -0.13 & 0.04 & 0.09 & -0.09 \\
\hline 72 & 1 & $\odot .0 \odot$ & -0.01 & 0.04 & -0.02 & 0.02 & -0.01 & 0.04 & 0.07 & $\odot .09$ \\
\hline 73 & 1 & $-0.0 \odot$ & -0.01 & 0.01 & -0.03 & 0.01 & -0.05 & 0.04 & 0.07 & $\odot .01$ \\
\hline 74 & 1 & -0.00 & -0.01 & 0.00 & -0.02 & -0.02 & -0.02 & 0.07 & -0.08 & 0.01 \\
\hline 75 & 6 & 0.01 & -0.01 & -0.02 & 0.01 & -0.01 & 0.02 & -0.01 & -0.07 & -0.00 \\
\hline 76 & 6 & 0.01 & $-\odot . \odot \odot$ & -0.02 & $\odot .0 \odot$ & -0.03 & 0.02 & -0.01 & -0.05 & -0.03 \\
\hline 77 & 6 & $0.0 \odot$ & -0.00 & -0.02 & $-\odot . \odot \odot$ & -0.04 & $\odot .02$ & 0.01 & -0.03 & -0.04 \\
\hline 78 & 8 & 0.01 & -0.00 & -0.01 & -0.01 & -0.05 & $\odot .01$ & 0.04 & -0.05 & -0.02 \\
\hline 79 & 1 & $\odot .0 \odot$ & $0.0 \odot$ & $-0.0 \odot$ & -0.03 & -0.03 & -0.01 & 0.07 & -0.08 & 0.01 \\
\hline 80 & 1 & $\odot .0 \odot$ & $\odot .0 \odot$ & -0.01 & -0.01 & -0.01 & -0.01 & $\odot .04$ & -0.10 & $\odot .02$ \\
\hline 81 & 1 & 0.01 & $\odot .0 \odot$ & -0.02 & 0.00 & -0.03 & 0.02 & $\odot .02$ & -0.09 & $\odot .01$ \\
\hline 82 & 1 & 0.01 & $-0.0 \odot$ & -0.02 & $\odot .0 \odot$ & 0.01 & 0.02 & -0.02 & -0.07 & 0.01 \\
\hline 83 & 1 & $\odot .02$ & -0.02 & -0.02 & $\odot .01$ & -0.03 & 0.02 & -0.03 & -0.05 & -0.04 \\
\hline 84 & 1 & 0.02 & 0.00 & -0.02 & -0.01 & -0.03 & 0.02 & -0.01 & -0.06 & -0.02 \\
\hline 85 & 1 & $\odot .0 \odot$ & -0.00 & -0.01 & -0.00 & -0.04 & 0.03 & $-0.0 \odot$ & -0.02 & -0.05 \\
\hline 86 & 7 & $\odot . \odot \odot$ & $-\odot . \odot \odot$ & -0.02 & $-\odot . \odot \odot$ & -0.04 & 0.02 & 0.03 & $-\odot . \odot \odot$ & -0.05 \\
\hline 87 & 6 & $\odot .0 \odot$ & $0.0 \odot$ & -0.02 & $-\odot . \odot \odot$ & -0.04 & 0.00 & 0.06 & -0.01 & -0.05 \\
\hline 88 & 6 & $\odot .0 \odot$ & -0.00 & -0.01 & -0.00 & -0.03 & -0.01 & $\odot .08$ & 0.01 & -0.05 \\
\hline 89 & 6 & $\odot .0 \odot$ & $-0.0 \odot$ & -0.01 & $-\odot . \odot \odot$ & -0.02 & -0.02 & 0.06 & $\odot .04$ & -0.07 \\
\hline 90 & 7 & $-\odot .0 \odot$ & $-0.0 \odot$ & -0.02 & $-\odot . \odot \odot$ & -0.03 & $-\odot .0 \odot$ & 0.04 & 0.05 & -0.07 \\
\hline 91 & 6 & $-\odot . \odot \odot$ & $-0.0 \odot$ & -0.02 & $-\odot . \odot \odot$ & -0.03 & 0.01 & 0.02 & $\odot .02$ & -0.06 \\
\hline 92 & 6 & 0.00 & $-0.0 \odot$ & -0.01 & 0.00 & -0.03 & -0.03 & 0.11 & 0.01 & -0.04 \\
\hline 93 & 8 & 0.01 & $-\odot .0 \odot$ & $-0.0 \odot$ & $-\odot . \odot \odot$ & -0.02 & -0.03 & 0.07 & 0.06 & -0.07 \\
\hline 94 & 8 & $\odot .0 \odot$ & $-\odot . \odot \odot$ & -0.01 & -0.01 & -0.03 & 0.01 & $-\odot . \odot \odot$ & 0.03 & -0.06 \\
\hline 95 & 1 & $\odot .0 \odot$ & 0.00 & -0.02 & 0.00 & -0.04 & 0.01 & 0.07 & -0.03 & -0.03 \\
\hline 96 & 1 & -0.02 & -0.00 & -0.01 & $-\odot .01$ & -0.01 & -0.03 & $\odot .09$ & 0.01 & -0.05 \\
\hline 97 & 1 & 0.01 & $-0.0 \odot$ & -0.01 & 0.01 & -0.03 & -0.04 & 0.14 & $\odot .03$ & -0.06 \\
\hline 98 & 1 & $\odot .0 \odot$ & -0.01 & 0.01 & $\odot .0 \odot$ & -0.03 & -0.02 & 0.12 & -0.02 & -0.02 \\
\hline 99 & 1 & $-\odot .0 \odot$ & -0.00 & -0.02 & $-\odot .01$ & -0.02 & -0.03 & 0.03 & 0.07 & -0.07 \\
\hline 100 & 6 & -0.02 & 0.03 & 0.01 & 0.09 & -0.05 & 0.09 & -0.02 & -0.05 & 0.02 \\
\hline 101 & 11 & $0 . \odot \odot$ & $\odot . \odot \odot$ & 0.00 & -0.11 & 0.05 & -0.01 & 0.05 & 0.04 & 0.04 \\
\hline 102 & 11 & $-\odot . \odot \odot$ & $\odot .0 \odot$ & $-\odot . \odot \odot$ & 0.10 & 0.02 & 0.09 & -0.03 & -0.06 & 0.05 \\
\hline 103 & 6 & -0.03 & -0.07 & 0.06 & 0.01 & 0.03 & 0.05 & -0.07 & 0.03 & $-0.0 \odot$ \\
\hline 104 & 6 & -0.02 & -0.04 & 0.07 & 0.04 & 0.01 & 0.01 & -0.04 & 0.04 & -0.03 \\
\hline 105 & 8 & $\odot .04$ & 0.09 & 0.09 & 0.08 & $-\odot .0 \odot$ & 0.03 & -0.01 & 0.02 & -0.02 \\
\hline 106 & 1 & -0.01 & & & & $-\odot . \odot 9$ & & & -0.07 & $\odot .0 \odot$ \\
\hline 107 & 1 & -0.03 & $\odot .02$ & $\odot .02$ & 0.10 & -0.06 & 0.14 & -0.03 & -0.06 & 0.06 \\
\hline 108 & 1 & -0.05 & $\odot .09$ & $\odot .01$ & 0.07 & -0.02 & 0.08 & -0.05 & $\odot .01$ & 0.02 \\
\hline 109 & 1 & $\odot . \odot \odot$ & $-0.0 \odot$ & $\odot .0 \odot$ & $\odot .04$ & $\odot .04$ & 0.12 & -0.07 & 0.01 & 0.04 \\
\hline 110 & 1 & -0.03 & -0.13 & $\odot .04$ & -0.02 & 0.04 & 0.04 & -0.09 & 0.05 & -0.01 \\
\hline 111 & 1 & -0.05 & -0.07 & 0.12 & 0.00 & 0.03 & 0.07 & -0.06 & $\odot .02$ & 0.01 \\
\hline 112 & 1 & $\odot .01$ & -0.08 & -0.02 & $\odot .04$ & $\odot .01$ & -0.01 & -0.05 & 0.05 & -0.05 \\
\hline 113 & 7 & -0.06 & -0.08 & 0.16 & $\odot .03$ & 0.01 & -0.02 & -0.03 & $\odot .04$ & -0.05 \\
\hline 114 & 6 & -0.08 & -0.12 & 0.24 & 0.01 & 0.01 & -0.01 & -0.03 & 0.04 & -0.05 \\
\hline 115 & 7 & 0.05 & -0.08 & 0.03 & 0.01 & 0.01 & -0.02 & -0.03 & 0.04 & -0.04 \\
\hline 116 & 6 & $-\odot . \odot \odot$ & -0.05 & $-\odot . \odot \odot$ & & 0.00 & -0.05 & -0.04 & $\odot .04$ & -0.03 \\
\hline 117 & 6 & -0.04 & -0.03 & 0.06 & 0.03 & $0.0 \odot$ & -0.05 & -0.03 & $\odot . \odot 4$ & -0.03 \\
\hline 118 & 7 & -0.05 & -0.04 & 0.04 & 0.04 & -0.00 & -0.07 & -0.03 & 0.04 & -0.02 \\
\hline 119 & 6 & -0.02 & -0.03 & $-\odot .0 \odot$ & $\odot .03$ & -0.01 & -0.08 & -0.04 & 0.03 & -0.00 \\
\hline 120 & 7 & $\odot .01$ & -0.02 & -0.02 & 0.02 & -0.01 & -0.08 & -0.04 & 0.03 & $\odot .01$ \\
\hline 121 & 6 & 0.02 & -0.03 & -0.02 & 0.02 & -0.01 & -0.07 & $-\odot .04$ & 0.03 & $\odot .0 \odot$ \\
\hline 122 & 7 & -0.04 & -0.01 & -0.01 & $\odot .04$ & -0.01 & -0.10 & -0.04 & 0.03 & 0.01 \\
\hline 123 & 8 & 0.02 & -0.02 & -0.02 & 0.01 & -0.01 & -0.07 & -0.06 & 0.03 & 0.03 \\
\hline 124 & 1 & $\odot .05$ & -0.07 & -0.11 & 0.01 & 0.02 & 0.01 & -0.03 & $\odot . \odot 4$ & -0.06 \\
\hline 125 & 1 & -0.06 & -0.01 & -0.02 & 0.05 & -0.02 & -0.09 & -0.04 & 0.03 & $-\odot .0 \odot$ \\
\hline 126 & 1 & -0.03 & 0.01 & -0.03 & 0.04 & -0.02 & -0.09 & -0.04 & $\odot .02$ & 0.03 \\
\hline 127 & 1 & $\odot .02$ & -0.01 & -0.01 & 0.02 & -0.02 & -0.08 & -0.05 & 0.02 & 0.04 \\
\hline 128 & 8 & 0.21 & 0.50 & -0.30 & $\odot .02$ & 0.02 & $\odot . \odot \odot$ & -0.01 & $\odot .04$ & -0.05 \\
\hline 129 & 1 & 0.11 & 0.45 & -0.08 & 0.01 & 0.02 & $-0.0 \odot$ & -0.00 & 0.04 & -0.04 \\
\hline 130 & 8 & -0.03 & -0.09 & $-\odot .0 \odot$ & -0.01 & 0.02 & -0.02 & 0.02 & 0.02 & -0.03 \\
\hline 131 & 1 & $\odot .01$ & -0.10 & 0.05 & -0.01 & 0.01 & -0.02 & 0.00 & 0.03 & -0.04 \\
\hline 132 & 1 & -0.09 & 0.10 & $\odot .01$ & -0.02 & 0.03 & -0.01 & 0.02 & 0.01 & -0.02 \\
\hline 133 & 1 & 0.18 & 0.12 & 0.02 & 0.02 & 0.01 & 0.02 & -0.02 & -0.00 & -0.05 \\
\hline
\end{tabular}




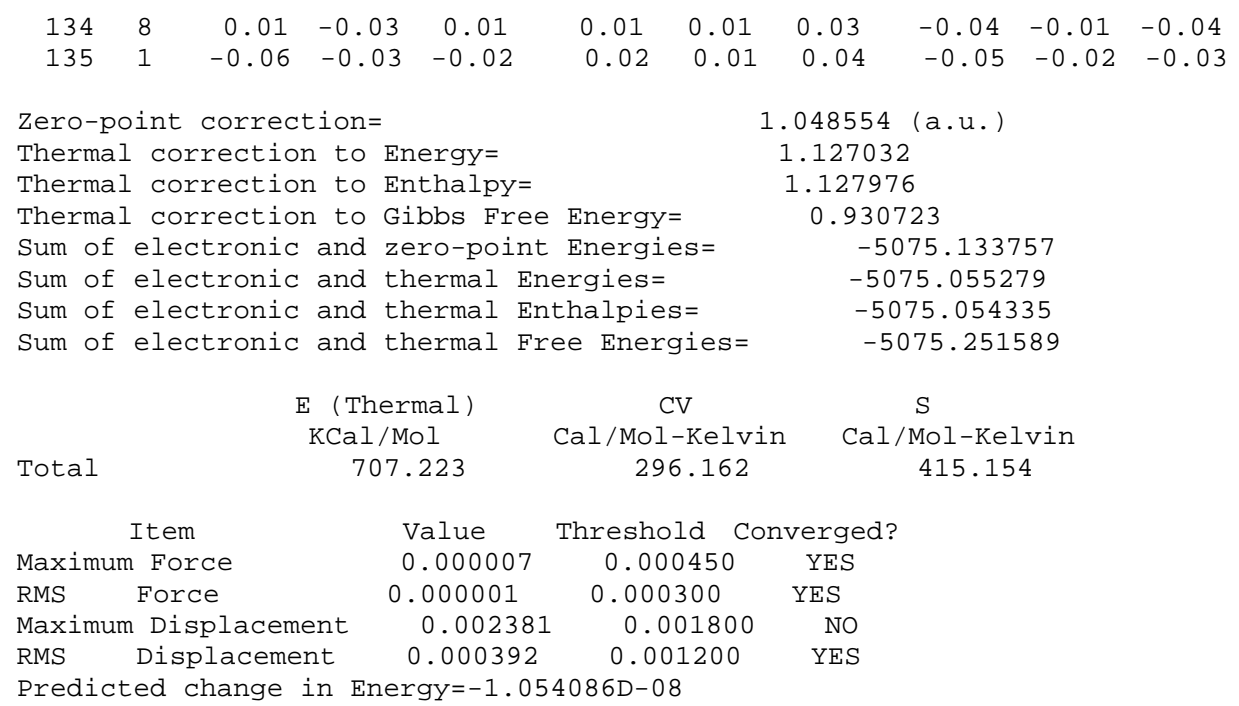

Normal termination of Gaussian 16 at Sat Jul 27 11:58:29 2019.

$G($ high $)=-5077.0160424 \mathrm{a} \cdot \mathrm{u}$.

-............... Int2(C8)W2(c)

dna. rev1cw2B. $\log$

Stoichiometry C39H54N15Na2023P2(2)

Standard orientation:

\begin{tabular}{|c|c|c|c|c|c|}
\hline \multirow{2}{*}{$\begin{array}{l}\text { Center } \\
\text { Number }\end{array}$} & \multirow{2}{*}{$\begin{array}{l}\text { Atomic } \\
\text { Number }\end{array}$} & \multirow{2}{*}{$\begin{array}{c}\text { Atomic } \\
\text { Type }\end{array}$} & \multicolumn{3}{|c|}{ Coordinates (Angstroms) } \\
\hline & & & $\mathrm{x}$ & $\mathrm{Y}$ & Z \\
\hline & & -- & ------ & ------- & ------- \\
\hline 1 & 7 & $\odot$ & 5.932678 & -2.011219 & -1.078370 \\
\hline 2 & 6 & $\odot$ & 6.011603 & -3.315350 & -0.683935 \\
\hline 3 & 6 & $\odot$ & 4.988903 & -4.169168 & -0.908815 \\
\hline 4 & 6 & $\odot$ & 3.825946 & -3.630840 & -1.551301 \\
\hline 5 & 7 & $\odot$ & 3.800728 & -2.383768 & -2.024286 \\
\hline 6 & 8 & $\odot$ & 8.686571 & 0.942068 & $\odot .569595$ \\
\hline 7 & 15 & $\odot$ & 7.725895 & 2.122099 & 1.170806 \\
\hline 8 & 8 & $\odot$ & 6.373809 & 1.981788 & ๑. 239199 \\
\hline 9 & 8 & 0 & 8.391825 & 3.436796 & ๑. 894287 \\
\hline 10 & 8 & $\odot$ & 7.285583 & 1.728032 & 2.564030 \\
\hline 11 & 8 & 0 & 4.220000 & 5.663753 & $\odot .791874$ \\
\hline 12 & 6 & $\odot$ & 4.862142 & 3.777778 & -0.508731 \\
\hline 13 & 6 & $\odot$ & 4.945816 & 4.412555 & $\odot .880023$ \\
\hline 14 & 6 & $\odot$ & -9.345695 & -2.251456 & 2.268981 \\
\hline 15 & 6 & $\odot$ & -8.589047 & -0.939326 & 2.236481 \\
\hline 16 & 8 & $\odot$ & -8.666815 & -3.209660 & 1.421196 \\
\hline 17 & 8 & $\odot$ & -8.243431 & 1.082045 & $\odot .974932$ \\
\hline 18 & 15 & 0 & -7.646771 & 2.036680 & -0.213381 \\
\hline 19 & 8 & $\odot$ & -6.032392 & 1.846191 & -0.079513 \\
\hline 20 & 8 & $\odot$ & - 8.019608 & 3.450843 & ๑. 157928 \\
\hline 21 & 8 & $\odot$ & -8.014951 & 1.453923 & -1.556223 \\
\hline 22 & 8 & $\odot$ & -3.631552 & 4.727698 & -1.981579 \\
\hline 23 & 6 & $\odot$ & -3.986949 & 3. 151038 & -0.217609 \\
\hline 24 & 6 & $\odot$ & -4.410423 & 3.549168 & -1.641307 \\
\hline 25 & 1 & $\odot$ & -3.822799 & 5.004689 & -2.894029 \\
\hline 26 & 6 & $\odot$ & 9.902764 & -2.576035 & 0. 240419 \\
\hline 27 & 6 & $\odot$ & 9.292472 & -1.254261 & -0.179390 \\
\hline 28 & 6 & $\odot$ & 8.586719 & -0.474212 & ๑. 939132 \\
\hline 29 & 6 & $\odot$ & 7.153172 & -0.975701 & 0.842200 \\
\hline 30 & 6 & $\odot$ & 6.960410 & -1.069493 & -0.669703 \\
\hline 31 & 8 & $\odot$ & 8.253433 & -1.492167 & -1.190409 \\
\hline 32 & 1 & $\odot$ & 10.260356 & -3.095391 & -0.654620 \\
\hline 33 & 1 & $\odot$ & 10.756209 & -2.384476 & ๑. 901262 \\
\hline 34 & 1 & $\odot$ & 10.067991 & -0.633911 & -0.634044 \\
\hline 35 & 1 & $\odot$ & 9.038243 & -0.618904 & 1.921397 \\
\hline 36 & 1 & $\odot$ & 6.437021 & -0.313644 & 1.318883 \\
\hline 37 & 1 & $\odot$ & 7.098862 & -1.961593 & 1.306465 \\
\hline 38 & 1 & $\odot$ & 6.690039 & -0.107634 & -1.098453 \\
\hline 39 & 8 & $\odot$ & 8.900109 & -3.369626 & 0.922850 \\
\hline
\end{tabular}




\begin{tabular}{|c|c|c|c|c|c|}
\hline 40 & 1 & 0 & 9.246268 & -4.250006 & 1.141423 \\
\hline 41 & 6 & $\odot$ & 4.834290 & -1.538629 & -1.806496 \\
\hline 42 & 7 & $\odot$ & 2.718414 & -4.364696 & -1.690128 \\
\hline 43 & 8 & $\odot$ & 4.830384 & -0.369142 & -2.216977 \\
\hline 44 & 1 & $\odot$ & 6.939296 & -3.594548 & -0.188382 \\
\hline 45 & 1 & $\odot$ & 5.036281 & -5.202137 & -0.592715 \\
\hline 46 & 1 & $\odot$ & 2.704050 & -5.325753 & -1.391578 \\
\hline 47 & 1 & $\odot$ & 1.901434 & -3.971784 & -2.166753 \\
\hline 48 & 6 & $\odot$ & 6.027806 & 2.866753 & $-\odot .863936$ \\
\hline 49 & 1 & $\odot$ & 4.229170 & 6.121269 & 1.649693 \\
\hline 50 & 6 & $\odot$ & 4.219967 & 3.403394 & 1.777320 \\
\hline 51 & 6 & $\odot$ & 3.124848 & 2.831914 & $\odot .869032$ \\
\hline 52 & 8 & $\odot$ & 3.589294 & 3.043249 & $-\odot .497636$ \\
\hline 53 & 1 & $\odot$ & 5.753440 & 2.227374 & -1.704581 \\
\hline 54 & 1 & $\odot$ & 6.896123 & 3.477694 & -1.123260 \\
\hline 55 & 1 & $\odot$ & 4.770448 & 4.549780 & -1.275317 \\
\hline 56 & 1 & $\odot$ & 5.981828 & 4.582690 & 1.188329 \\
\hline 57 & 1 & $\odot$ & 3.802194 & 3.869102 & 2.671902 \\
\hline 58 & 1 & $\odot$ & 4.919335 & 2.621943 & 2.079183 \\
\hline 59 & 1 & $\odot$ & 2.168797 & 3.342361 & $\odot .989852$ \\
\hline 60 & 7 & $\odot$ & 2.864595 & 1.417164 & 1.043147 \\
\hline 61 & 6 & $\odot$ & 3.758079 & $\odot .394263$ & ๑. 820108 \\
\hline 62 & 7 & $\odot$ & 3.202893 & $-\odot .789142$ & $\odot .784398$ \\
\hline 63 & 6 & $\odot$ & 1.855097 & -0.530868 & 0.960539 \\
\hline 64 & 6 & $\odot$ & 1.624381 & 0.832718 & 1.113511 \\
\hline 65 & 7 & $\odot$ & 0.445535 & 1.445743 & 1.280484 \\
\hline 66 & 6 & $\odot$ & -0.554949 & $\odot .576580$ & 1.279488 \\
\hline 67 & 7 & $\odot$ & -0.480459 & $-0.75664 \odot$ & 1.132644 \\
\hline 68 & 6 & $\odot$ & $\odot .712226$ & -1.356328 & $\odot .95 \odot 272$ \\
\hline 69 & 7 & $\odot$ & 0.740202 & -2.683724 & $\odot .765848$ \\
\hline 70 & 1 & $\odot$ & 4.801891 & 0.626253 & $\odot .653405$ \\
\hline 71 & 1 & $\odot$ & -1.563210 & $\odot .966074$ & 1.384922 \\
\hline 72 & 1 & 0 & 1.601076 & -3.142014 & 0.514521 \\
\hline 73 & 1 & $\odot$ & -0.133845 & -3.204271 & 0.744946 \\
\hline 74 & 1 & $\odot$ & -9.099025 & -4.077843 & 1.466176 \\
\hline 75 & 6 & 0 & -8.314808 & -0.379905 & 0.831456 \\
\hline 76 & 6 & $\odot$ & -6.947995 & -0.957945 & 0.500606 \\
\hline 77 & 6 & $\odot$ & -6.241706 & -0.850242 & 1.847640 \\
\hline 78 & 8 & $\odot$ & -7.262917 & -1.131744 & 2.842212 \\
\hline 79 & 1 & $\odot$ & -9.381239 & -2.609123 & 3.303352 \\
\hline 80 & 1 & 0 & -10.371251 & -2.084459 & 1.919311 \\
\hline 81 & 1 & $\odot$ & -9.129020 & -0.200638 & 2.833384 \\
\hline 82 & 1 & $\odot$ & -9.089710 & -0.629444 & 0.106003 \\
\hline 83 & 1 & $\odot$ & -6.406763 & -0.421406 & -0.271869 \\
\hline 84 & 1 & $\odot$ & -7.073356 & -2.002524 & 0.210353 \\
\hline 85 & 1 & $\odot$ & -5.843767 & $\odot .148611$ & 2.011396 \\
\hline 86 & 7 & $\odot$ & -5.124999 & -1.768530 & 1.978458 \\
\hline 87 & 6 & $\odot$ & -5.325596 & -3.118912 & 1.786944 \\
\hline 88 & 6 & $\odot$ & -4.316161 & -3.983205 & 1.553066 \\
\hline 89 & 6 & 0 & -2.978677 & -3.427425 & 1.417837 \\
\hline 90 & 7 & $\odot$ & -2.843573 & -2.098630 & 1.731906 \\
\hline 91 & 6 & $\odot$ & -3.853872 & -1.217569 & 2.027514 \\
\hline 92 & 6 & $\odot$ & -4.519132 & -5.448660 & 1.316992 \\
\hline 93 & 8 & $\odot$ & -1.997009 & -4.072479 & 1.017188 \\
\hline 94 & 8 & $\odot$ & -3.637886 & -0.043483 & 2.290055 \\
\hline 95 & 1 & $\odot$ & -6.370964 & -3.419694 & 1.825835 \\
\hline 96 & 1 & $\odot$ & -4.245834 & -5.712898 & 0.290841 \\
\hline 97 & 1 & $\odot$ & -3.894953 & -6.045851 & 1.987718 \\
\hline 98 & 1 & 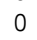 & -5.563267 & -5.725227 & 1.476372 \\
\hline 99 & 1 & $\odot$ & -1.915438 & -1.647472 & 1.549575 \\
\hline 100 & 6 & 0 & -5.127061 & 2.698510 & 0.672520 \\
\hline 101 & 11 & $\Theta$ & 6.075496 & 0.726750 & 4.088617 \\
\hline 102 & 11 & 0 & -7.644931 & 5.590948 & $-\odot .213720$ \\
\hline 103 & 6 & 0 & -4.008732 & 2.340390 & -2.490549 \\
\hline 104 & 6 & $\odot$ & -2.727292 & 1.877111 & -1.796711 \\
\hline 105 & 8 & $\odot$ & -3.021633 & 2.061478 & -0.372592 \\
\hline 106 & 1 & $\odot$ & -4.728194 & 2.117916 & 1.506712 \\
\hline 107 & 1 & 0 & -5.674364 & 3.567379 & 1.047770 \\
\hline 108 & 1 & 0 & -3.479197 & 3.997637 & $\odot .255077$ \\
\hline 109 & 1 & 0 & -5.479481 & 3.767452 & -1.708432 \\
\hline 110 & 1 & 0 & -3.845138 & 2.599664 & -3.539090 \\
\hline 111 & 1 & 0 & -4.791343 & 1.578557 & -2.429121 \\
\hline 112 & 1 & $\theta$ & -1.885530 & 2.525420 & -2.057412 \\
\hline 113 & 7 & 0 & -2.313409 & $\odot .530227$ & -2.050735 \\
\hline 114 & 6 & 0 & -3.168444 & -0.617374 & -2.401371 \\
\hline 115 & 7 & 0 & -2.262892 & -1.693081 & -2.737584 \\
\hline 116 & 6 & 0 & -1.029785 & -1.219921 & -2.547715 \\
\hline
\end{tabular}




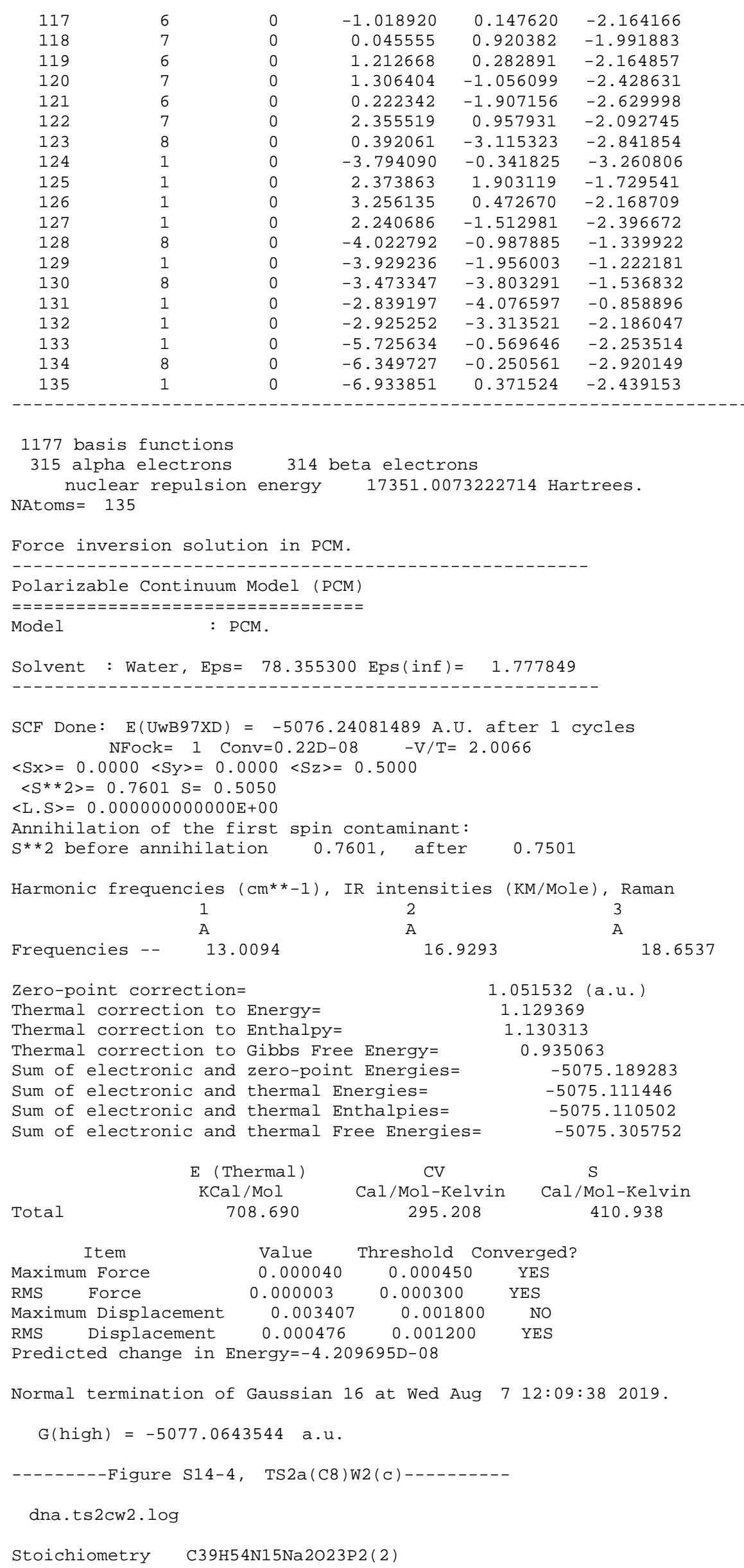


Standard orientation:

\begin{tabular}{|c|c|c|c|c|c|}
\hline \multirow{2}{*}{$\begin{array}{l}\text { Center } \\
\text { Number }\end{array}$} & \multirow{2}{*}{$\begin{array}{l}\text { Atomic } \\
\text { Number }\end{array}$} & \multirow{2}{*}{$\begin{array}{c}\text { Atomic } \\
\text { Type }\end{array}$} & \multicolumn{3}{|c|}{ Coordinates (Angstroms) } \\
\hline & & & $x$ & $\mathrm{Y}$ & z \\
\hline 1 & 7 & $\odot$ & 4.966673 & -2.155798 & -1.002669 \\
\hline 2 & 6 & $\odot$ & 5.109132 & -3.505680 & $-\odot .863080$ \\
\hline 3 & 6 & 0 & 4.034007 & -4.307467 & -0.699193 \\
\hline 4 & 6 & 0 & 2.746383 & -3.680653 & -0.713265 \\
\hline 5 & 7 & 0 & 2.616144 & -2.358671 & $-\odot .858860$ \\
\hline 6 & 8 & 0 & 8.762781 & 0.227812 & -1.089116 \\
\hline 7 & 15 & $\theta$ & 8.430465 & 1.539001 & -0.177156 \\
\hline 8 & 8 & 0 & 6.843647 & 1.786999 & -0.492659 \\
\hline 9 & 8 & 0 & 9.248155 & 2.678567 & -0.709859 \\
\hline 10 & 8 & 0 & 8.468042 & 1.161059 & 1.290576 \\
\hline 11 & 8 & 0 & 5.878706 & 5.939333 & $\odot .228128$ \\
\hline 12 & 6 & 0 & 5.582676 & 3.837158 & -0.845688 \\
\hline 13 & 6 & $\theta$ & 6.359860 & 4.571635 & 0.249323 \\
\hline 14 & 6 & 0 & -7.585240 & -2.197954 & 2.994712 \\
\hline 15 & 6 & 0 & -7.262180 & -0.738922 & 2.744773 \\
\hline 16 & 8 & 0 & -7.062815 & -2.983223 & 1.895248 \\
\hline 17 & 8 & 0 & -8.063049 & 1.074984 & 1.387981 \\
\hline 18 & 15 & 0 & -8.675555 & 1.843265 & $\odot .082156$ \\
\hline 19 & 8 & $\Theta$ & -7.341247 & 2.493930 & -0.600574 \\
\hline 20 & 8 & $\odot$ & -9.603748 & 2.909985 & $\odot .577902$ \\
\hline 21 & 8 & 0 & -9.131865 & 0.811142 & -0.933851 \\
\hline 22 & 8 & 0 & -4.372290 & 4.840821 & -2.457662 \\
\hline 23 & 6 & $\theta$ & -5.212378 & 3.625368 & -0.575209 \\
\hline 24 & 6 & 0 & -5.294344 & 3.773913 & -2.105734 \\
\hline 25 & 1 & 0 & -4.378124 & 4.984787 & -3.419288 \\
\hline 26 & 6 & 0 & 8.787492 & -3.331814 & -2.125440 \\
\hline 27 & 6 & $\theta$ & 8.312993 & -1.893772 & -2.095818 \\
\hline 28 & 6 & 0 & 8.499554 & -1.172814 & -0.753018 \\
\hline 29 & 6 & $\theta$ & 7.161584 & -1.395267 & -0.055101 \\
\hline 30 & 6 & 0 & 6.153970 & -1.333218 & -1.200915 \\
\hline 31 & 8 & 0 & 6.872824 & -1.826718 & -2.370440 \\
\hline 32 & 1 & $\odot$ & 8.458475 & -3.793340 & -3.062106 \\
\hline 33 & 1 & $\Theta$ & 9.882863 & -3.346144 & -2.090151 \\
\hline 34 & 1 & 0 & 8.824854 & -1.339665 & -2.885510 \\
\hline 35 & 1 & 0 & 9.346053 & -1.552305 & -0.178662 \\
\hline 36 & 1 & 0 & 6.949026 & -0.666014 & 0.718497 \\
\hline 37 & 1 & 0 & 7.177770 & -2.390987 & $\odot .392500$ \\
\hline 38 & 1 & 0 & 5.807934 & -0.318315 & -1.380505 \\
\hline 39 & 8 & $\theta$ & 8.237969 & -4.040624 & $-\odot .986378$ \\
\hline 40 & 1 & 0 & 8.508460 & -4.973156 & -0.994084 \\
\hline 41 & 6 & $\theta$ & 3.699520 & -1.557132 & -1.013332 \\
\hline 42 & 7 & 0 & 1.634263 & -4.404583 & $-\odot .576752$ \\
\hline 43 & 8 & 0 & 3.600347 & -0.332270 & -1.162300 \\
\hline 44 & 1 & 0 & 6.133154 & -3.873448 & -0.907350 \\
\hline 45 & 1 & $\Theta$ & 4.142497 & -5.378126 & -0.593793 \\
\hline 46 & 1 & 0 & 1.694827 & -5.407697 & -0.520739 \\
\hline 47 & 1 & 0 & $\odot .718957$ & -3.977329 & -0.737409 \\
\hline 48 & 6 & 0 & 6.334331 & 2.696022 & -1.503684 \\
\hline 49 & 1 & 0 & 6.335898 & 6.468972 & $\odot .903198$ \\
\hline 50 & 6 & 0 & 5.957734 & 3.850070 & 1.542829 \\
\hline 51 & 6 & 0 & 4.540160 & 3.332536 & 1.258453 \\
\hline 52 & 8 & $\theta$ & 4.373416 & 3.343221 & -0.187299 \\
\hline 53 & 1 & 0 & 5.654750 & 2.125812 & -2.139345 \\
\hline 54 & 1 & 0 & 7.159234 & 3.091187 & -2.102603 \\
\hline 55 & 1 & 0 & 5.265156 & 4.545280 & -1.614772 \\
\hline 56 & 1 & $\theta$ & 7.440088 & 4.535102 & 0.076148 \\
\hline 57 & 1 & 0 & 5.972683 & 4.522245 & 2.402988 \\
\hline 58 & 1 & $\theta$ & 6.654907 & 3.033231 & 1.733823 \\
\hline 59 & 1 & 0 & 3.766582 & 3.977158 & 1.677292 \\
\hline 60 & 7 & 0 & 4.272743 & 1.995524 & 1.756095 \\
\hline 61 & 6 & 0 & 5.115926 & $\odot .907119$ & 1.791690 \\
\hline 62 & 7 & $\Theta$ & 4.504833 & -0.224951 & 2.025521 \\
\hline 63 & 6 & $\odot$ & 3.172664 & $\odot .129370$ & 2.126826 \\
\hline 64 & 6 & $\theta$ & 3.007996 & 1.499047 & 1.966873 \\
\hline 65 & 7 & 0 & 1.855871 & 2.184843 & 2.007238 \\
\hline 66 & 6 & 0 & ๑.823201 & 1.378076 & 2.205898 \\
\hline 67 & 7 & 0 & 0.832637 & $\odot .043396$ & 2.336711 \\
\hline 68 & 6 & 0 & 1.997524 & -0.629794 & 2.278354 \\
\hline 69 & 7 & 0 & 1.981315 & -1.970710 & 2.381522 \\
\hline 70 & 1 & $\theta$ & 6.177607 & 1.022348 & 1.629503 \\
\hline 71 & 1 & $\odot$ & $-\odot .165686$ & 1.828441 & 2.248846 \\
\hline
\end{tabular}




\begin{tabular}{|c|c|c|c|c|c|}
\hline 72 & 1 & 0 & 2.829138 & -2.457478 & 2.136628 \\
\hline 73 & 1 & 0 & 1.095683 & -2.448239 & 2.198500 \\
\hline 74 & 1 & 0 & -7.167963 & -3.934015 & 2.059524 \\
\hline 75 & 6 & 0 & -7.524425 & -0.282793 & 1.305666 \\
\hline 76 & 6 & $\odot$ & -6.155237 & -0.328755 & 0.626373 \\
\hline 77 & 6 & 0 & -5.150915 & -0.119095 & 1.763964 \\
\hline 78 & 8 & 0 & -5.846825 & -0.475852 & 2.995369 \\
\hline 79 & 1 & 0 & -7.135693 & -2.505909 & 3.944206 \\
\hline 80 & 1 & 0 & -8.672880 & -2.317070 & 3.061638 \\
\hline 81 & 1 & 0 & -7.839250 & $-\odot .123818$ & 3.439076 \\
\hline 82 & 1 & $\odot$ & -8.256191 & -0.912824 & 0.799788 \\
\hline 83 & 1 & $\odot$ & -6.031126 & 0.437509 & -0.138976 \\
\hline 84 & 1 & 0 & -6.031178 & -1.310790 & 0.169831 \\
\hline 85 & 1 & 0 & -4.820952 & $\odot .915379$ & 1.823409 \\
\hline 86 & 7 & 0 & -3.939641 & -0.927300 & 1.667790 \\
\hline 87 & 6 & 0 & -4.031800 & $-2.28607 \odot$ & 1.452171 \\
\hline 88 & 6 & $\odot$ & -2.962676 & -3.109401 & 1.417657 \\
\hline 89 & 6 & 0 & -1.656528 & -2.537269 & 1.683586 \\
\hline 90 & 7 & 0 & -1.645663 & -1.176994 & 1.911687 \\
\hline 91 & 6 & 0 & -2.724984 & -0.330997 & 1.976535 \\
\hline 92 & 6 & $\odot$ & -3.058648 & -4.574668 & 1.122171 \\
\hline 93 & 8 & 0 & $-\odot .606037$ & -3.185443 & 1.729949 \\
\hline 94 & 8 & $\odot$ & -2.604539 & $\odot .846532$ & 2.286069 \\
\hline 95 & 1 & 0 & -5.045636 & -2.659696 & 1.333527 \\
\hline 96 & 1 & $\odot$ & -2.460642 & -4.811022 & $\odot .236159$ \\
\hline 97 & 1 & 0 & -2.670063 & -5.175207 & 1.950004 \\
\hline 98 & 1 & $\odot$ & -4.093919 & -4.862796 & 0.930093 \\
\hline 99 & 1 & $\odot$ & $-\odot .72336 \odot$ & -0.736731 & 2.124162 \\
\hline 100 & 6 & $\odot$ & -6.545229 & 3.478116 & 0.116485 \\
\hline 101 & 11 & $\odot$ & 8.563016 & 0.050898 & 3.170419 \\
\hline 102 & 11 & $\odot$ & -9.482299 & -1.107775 & -2.035873 \\
\hline 103 & 6 & $\odot$ & -4.816354 & 2.417992 & -2.627219 \\
\hline 104 & 6 & $\odot$ & -3.785988 & 1.997058 & -1.578072 \\
\hline 105 & 8 & $\odot$ & -4.412366 & 2.431100 & -0.317370 \\
\hline 106 & 1 & $\odot$ & -6.390363 & 3.144746 & 1.146959 \\
\hline 107 & 1 & $\odot$ & -7.077269 & 4.434325 & 0.121234 \\
\hline 108 & 1 & $\odot$ & -4.695112 & 4.500247 & -0.167047 \\
\hline 109 & 1 & $\odot$ & -6.305650 & 4.018773 & -2.441529 \\
\hline 110 & 1 & $\odot$ & -4.395888 & 2.480016 & -3.633942 \\
\hline 111 & 1 & $\odot$ & -5.649953 & 1.710459 & -2.626879 \\
\hline 112 & 1 & $\odot$ & -2.850600 & 2.556068 & -1.697441 \\
\hline 113 & 7 & $\odot$ & -3.545901 & 0.591439 & -1.539641 \\
\hline 114 & 6 & $\odot$ & -4.258507 & -1.057680 & -2.605569 \\
\hline 115 & 7 & $\odot$ & -3.377906 & -1.906014 & -2.063772 \\
\hline 116 & 6 & $\odot$ & -2.244513 & -1.297369 & -1.669648 \\
\hline 117 & 6 & $\odot$ & -2.322126 & 0.124163 & -1.400052 \\
\hline 118 & 7 & $\odot$ & -1.230899 & $\odot .862886$ & -1.083381 \\
\hline 119 & 6 & $\odot$ & -0.078853 & 0.212009 & -1.016065 \\
\hline 120 & 7 & $\odot$ & $\odot .039657$ & -1.150314 & -1.094650 \\
\hline 121 & 6 & $\odot$ & -1.012026 & -1.992405 & -1.419003 \\
\hline 122 & 7 & $\Theta$ & 1.058863 & $\odot .890276$ & -0.857963 \\
\hline 123 & 8 & $\odot$ & -0.846537 & -3.216407 & -1.492427 \\
\hline 124 & 1 & $\odot$ & -4.010668 & $-\odot .45 \odot \odot 32$ & -3.473393 \\
\hline 125 & 1 & $\odot$ & 0.995126 & 1.888221 & -0.744642 \\
\hline 126 & 1 & $\odot$ & 1.979961 & 0.439230 & $-\odot .890687$ \\
\hline 127 & 1 & $\odot$ & $\odot .977571$ & -1.586685 & $-\odot .977417$ \\
\hline 128 & 8 & $\odot$ & -5.549511 & -1.320519 & -2.526642 \\
\hline 129 & 1 & $\odot$ & -5.690816 & -2.101470 & -1.920225 \\
\hline 130 & 8 & $\odot$ & -5.409780 & -3.526110 & -1.094590 \\
\hline 131 & 1 & $\odot$ & -4.455944 & -3.362895 & -1.231761 \\
\hline 132 & 1 & $\odot$ & -5.621574 & -4.291113 & -1.640359 \\
\hline 133 & 1 & $\odot$ & -6.997567 & -0.171160 & -3.011832 \\
\hline 134 & 8 & $\odot$ & -7.798516 & 0.314957 & -3.253911 \\
\hline 135 & 1 & $\odot$ & -8.064298 & 0.778235 & -2.436753 \\
\hline
\end{tabular}

General basis read from cards: (6D, 7F)

1177 basis functions

315 alpha electrons 314 beta electrons

nuclear repulsion energy 17224.3981561936 Hartrees.

NAtoms $=135$ NActive $=135$

Force inversion solution in PCM.

Polarizable Continuum Model (PCM)

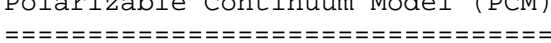


Solvent : Water, Eps $=78.355300$ Eps $($ inf $)=1.777849$

SCF Done: $E($ UWB97XD) $=-5076.21415353$ A.U. after 1 cycles NFock $=1$ Conv $=0.46 \mathrm{D}-08$

$\langle\mathrm{S} x\rangle=0.0000<\mathrm{S} y>=0.0000<\mathrm{Sz}>=0.5000$

$<S^{* *} 2>=0.7619 \mathrm{~S}=0.5059$

$<$ L.S $>=0.0000 \odot \odot \odot \odot \odot \odot \odot \odot E+\odot \odot$

Annihilation of the first spin contaminant:

$\mathrm{S}^{* * 2}$ before annihilation 0.7619 , after 0.7501

Harmonic frequencies $\left(\mathrm{cm}^{*}-1\right)$, IR intensities (KM/Mole), Raman

\begin{tabular}{|c|c|c|c|c|c|c|c|c|c|c|}
\hline & \multicolumn{3}{|c|}{1} & \multicolumn{3}{|l|}{2} & \multicolumn{3}{|l|}{3} \\
\hline & & \multicolumn{3}{|c|}{ A } & \multicolumn{3}{|l|}{ A } & \multicolumn{2}{|l|}{ A } & \\
\hline \multicolumn{2}{|c|}{ Frequencies } & - & \multicolumn{2}{|c|}{-228.5625} & \multicolumn{3}{|c|}{11.8502} & \multicolumn{3}{|c|}{13.7791} \\
\hline \multicolumn{2}{|c|}{ Red. masses } & - & \multicolumn{2}{|c|}{8.6006} & & 6.8180 & & & .6338 & \\
\hline FrC $\mathrm{CC}$ & onsts & - & 0.264 & & & 0.0006 & & & .0010 & \\
\hline IR Int & Een & -- & 108.867 & & & 2.0529 & & & .2391 & \\
\hline Atom & AN & $x$ & $Y$ & Z & $x$ & $Y$ & Z & $x$ & $Z$ & \\
\hline 1 & 7 & $-\odot .00$ & 0.01 & -0.00 & $\odot .02$ & -0.03 & $\odot . \odot 3$ & $\odot .01$ & -0.02 & -0.05 \\
\hline 2 & 6 & $-\odot . \odot \odot$ & $\odot . \odot \odot$ & $-0.0 \odot$ & 0.01 & -0.03 & $\odot .07$ & 0.02 & -0.02 & -0.06 \\
\hline 3 & 6 & $\odot .00$ & $\odot .0 \odot$ & 0.00 & $\odot .0 \odot$ & -0.02 & 0.08 & 0.03 & -0.03 & -0.02 \\
\hline 4 & 6 & $\odot . \odot \odot$ & -0.00 & $\odot . \odot \odot$ & $\odot . \odot \odot$ & -0.02 & 0.03 & $\odot .03$ & -0.03 & 0.03 \\
\hline 5 & 7 & -0.00 & 0.00 & $\odot .00$ & 0.01 & -0.02 & -0.01 & 0.02 & -0.03 & 0.05 \\
\hline 6 & 8 & $\odot .0 \odot$ & $\odot .0 \odot$ & $\odot .0 \odot$ & -0.02 & -0.02 & $\odot .0 \odot$ & -0.05 & 0.05 & -0.09 \\
\hline 7 & 15 & $\odot . \odot \odot$ & $\odot . \odot \odot$ & $\odot . \odot \odot$ & -0.03 & $\odot .01$ & $-\odot .04$ & -0.06 & $\odot .03$ & -0.07 \\
\hline 8 & 8 & $-\odot . \odot \odot$ & $\odot . \odot \odot$ & $\odot . \odot \odot$ & -0.03 & -0.01 & -0.05 & -0.07 & 0.01 & -0.04 \\
\hline 9 & 8 & $-\odot . \odot \odot$ & $\odot . \odot \odot$ & $\odot . \odot \odot$ & -0.03 & $\odot . \odot \odot$ & -0.07 & -0.09 & $\odot .05$ & -0.08 \\
\hline 10 & 8 & -0.00 & 0.00 & 0.00 & -0.03 & 0.05 & -0.03 & -0.02 & 0.02 & -0.08 \\
\hline 11 & 8 & $-\odot . \odot \odot$ & $\odot . \odot \odot$ & $\odot . \odot \odot$ & -0.07 & $\odot . \odot \odot$ & -0.13 & -0.11 & $-\odot .01$ & 0.03 \\
\hline 12 & 6 & $\odot .0 \odot$ & $\odot . \odot \odot$ & $-0.0 \odot$ & -0.04 & -0.02 & -0.09 & -0.10 & $-0.0 \odot$ & 0.01 \\
\hline 13 & 6 & $\odot . \odot \odot$ & $\odot . \odot \odot$ & $-\odot . \odot \odot$ & -0.05 & $\odot .01$ & -0.10 & -0.09 & $-\odot . \odot \odot$ & $\odot . \odot \odot$ \\
\hline 14 & 6 & $-0.0 \odot$ & $-\odot .0 \odot$ & $\odot .0 \odot$ & -0.04 & -0.08 & -0.07 & -0.05 & 0.03 & -0.04 \\
\hline 15 & 6 & $-0.0 \odot$ & $-0.0 \odot$ & 0.01 & -0.03 & -0.07 & -0.03 & -0.03 & $\odot .03$ & -0.04 \\
\hline 16 & 8 & $-\odot . \odot \odot$ & $\odot .00$ & $\odot . \odot \odot$ & -0.02 & -0.05 & $-\odot . \odot 8$ & -0.03 & 0.03 & -0.03 \\
\hline 17 & 8 & $-\odot . \odot \odot$ & $\odot . \odot \odot$ & $\odot .0 \odot$ & -0.01 & -0.03 & ๑. 01 & $\odot . \odot \odot$ & 0.03 & -0.06 \\
\hline 18 & 15 & -0.00 & 0.00 & 0.00 & 0.01 & 0.01 & 0.03 & 0.03 & 0.02 & -0.08 \\
\hline 19 & 8 & $\odot .0 \odot$ & -0.00 & -0.00 & $\odot .02$ & $\odot .02$ & $\odot .06$ & 0.04 & 0.02 & -0.05 \\
\hline 20 & 8 & $\odot . \odot \odot$ & $\odot . \odot \odot$ & $\odot . \odot \odot$ & 0.01 & $-\odot . \odot \odot$ & 0.05 & 0.02 & 0.03 & -0.10 \\
\hline 21 & 8 & -0.00 & 0.00 & 0.00 & 0.01 & 0.03 & -0.00 & 0.05 & 0.02 & -0.09 \\
\hline 22 & 8 & 0.02 & -0.05 & $-\odot . \odot \odot$ & 0.05 & 0.05 & 0.14 & 0.11 & $-0.0 \odot$ & 0.02 \\
\hline 23 & 6 & $\odot .03$ & -0.07 & -0.02 & 0.03 & $\odot . \odot \odot$ & 0.10 & 0.05 & 0.01 & $-\odot . \odot \odot$ \\
\hline 24 & 6 & $\odot . \odot \odot$ & -0.03 & -0.01 & 0.04 & 0.04 & 0.11 & 0.09 & 0.01 & $-0.0 \odot$ \\
\hline 25 & 1 & -0.02 & -0.01 & 0.00 & 0.06 & 0.07 & 0.15 & 0.13 & -0.00 & 0.02 \\
\hline 26 & 6 & -0.00 & 0.00 & -0.00 & 0.05 & -0.07 & 0.16 & 0.03 & 0.05 & -0.10 \\
\hline 27 & 6 & $-0.0 \odot$ & $\odot .0 \odot$ & $0.0 \odot$ & $\odot .04$ & -0.07 & 0.09 & -0.01 & $\odot .04$ & -0.09 \\
\hline 28 & 6 & $-0.0 \odot$ & $\odot .0 \odot$ & $\odot .0 \odot$ & 0.01 & -0.01 & 0.06 & $-0.0 \odot$ & 0.04 & -0.09 \\
\hline 29 & 6 & $-\odot . \odot \odot$ & $\odot .00$ & -0.00 & -0.00 & $\odot .00$ & 0.04 & 0.01 & $\odot .0 \odot$ & -0.08 \\
\hline 30 & 6 & $-\odot . \odot \odot$ & $\odot . \odot \odot$ & -0.00 & 0.02 & -0.04 & $\odot .02$ & -0.00 & -0.01 & -0.06 \\
\hline 31 & 8 & $-0.0 \odot$ & 0.00 & 0.00 & $\odot .05$ & $-\odot .09$ & 0.06 & -0.01 & 0.00 & -0.07 \\
\hline 32 & 1 & $-\odot . \odot \odot$ & 0.00 & 0.00 & 0.08 & -0.11 & 0.17 & 0.03 & 0.05 & -0.10 \\
\hline 33 & 1 & -0.00 & 0.00 & -0.00 & 0.05 & -0.06 & 0.19 & 0.03 & 0.07 & -0.10 \\
\hline 34 & 1 & -0.00 & 0.00 & 0.00 & 0.05 & -0.10 & 0.07 & -0.03 & 0.05 & -0.10 \\
\hline 35 & 1 & $-0.0 \odot$ & -0.00 & $\odot .0 \odot$ & $\odot .0 \odot$ & 0.03 & 0.09 & 0.01 & 0.06 & -0.10 \\
\hline 36 & 1 & -0.00 & 0.00 & 0.00 & -0.03 & 0.03 & 0.01 & 0.00 & -0.01 & -0.07 \\
\hline 37 & 1 & $-0.0 \odot$ & $\odot .0 \odot$ & -0.00 & $-\odot .0 \odot$ & 0.02 & 0.08 & 0.04 & 0.00 & -0.08 \\
\hline 38 & 1 & -0.00 & $\odot .0 \odot$ & -0.00 & 0.03 & -0.05 & -0.03 & -0.02 & -0.01 & -0.06 \\
\hline 39 & 8 & -0.00 & 0.00 & 0.00 & $\odot .02$ & -0.02 & 0.17 & 0.04 & 0.03 & -0.11 \\
\hline 40 & 1 & $-0.0 \odot$ & $\odot .0 \odot$ & -0.00 & 0.03 & -0.02 & 0.22 & 0.06 & 0.03 & -0.12 \\
\hline 41 & 6 & $-0.0 \odot$ & 0.00 & 0.00 & 0.02 & -0.03 & -0.01 & 0.01 & -0.03 & 0.00 \\
\hline 42 & 7 & 0.00 & -0.00 & $\odot .0 \odot$ & -0.00 & -0.01 & 0.02 & 0.03 & -0.04 & 0.07 \\
\hline 43 & 8 & -0.01 & $\odot .0 \odot$ & $\odot . \odot \odot$ & 0.03 & -0.03 & $-\odot .04$ & -0.01 & $-\odot .03$ & $\odot . \odot \odot$ \\
\hline 44 & 1 & 0.00 & 0.01 & -0.00 & 0.01 & -0.03 & 0.10 & 0.02 & -0.01 & -0.09 \\
\hline 45 & 1 & $\odot . \odot \odot$ & $\odot .0 \odot$ & $\odot . \odot \odot$ & -0.00 & -0.02 & 0.11 & 0.04 & -0.03 & -0.03 \\
\hline 46 & 1 & 0.01 & -0.00 & 0.00 & -0.01 & -0.00 & 0.05 & 0.04 & -0.04 & 0.06 \\
\hline 47 & 1 & $\odot . \odot \odot$ & $-0.0 \odot$ & -0.01 & $\odot .0 \odot$ & $-0.0 \odot$ & $-\odot . \odot \odot$ & 0.03 & -0.04 & 0.09 \\
\hline 48 & 6 & $-\odot . \odot \odot$ & 0.00 & 0.00 & -0.03 & -0.03 & -0.06 & -0.10 & 0.01 & -0.02 \\
\hline 49 & 1 & $\odot . \odot \odot$ & 0.00 & -0.00 & -0.07 & $\odot .02$ & -0.14 & -0.10 & -0.01 & $\odot .02$ \\
\hline 50 & 6 & 0.00 & 0.00 & $\odot .0 \odot$ & -0.05 & 0.03 & -0.08 & -0.05 & -0.02 & 0.00 \\
\hline 51 & 6 & $\odot . \odot \odot$ & $\odot .0 \odot$ & $-0.0 \odot$ & -0.04 & 0.01 & -0.08 & -0.05 & -0.03 & $\odot .03$ \\
\hline 52 & 8 & $-\odot . \odot \odot$ & 0.00 & 0.00 & -0.04 & -0.02 & -0.08 & -0.08 & -0.03 & 0.03 \\
\hline 53 & 1 & $-\odot . \odot \odot$ & 0.00 & $\odot . \odot \odot$ & -0.02 & -0.04 & -0.06 & -0.11 & 0.01 & -0.01 \\
\hline 54 & 1 & $-0.0 \odot$ & $-0.0 \odot$ & -0.00 & -0.03 & -0.03 & -0.07 & -0.12 & 0.03 & -0.03 \\
\hline 55 & 1 & $-\odot . \odot \odot$ & 0.00 & 0.00 & -0.05 & -0.04 & -0.10 & -0.13 & $0.0 \odot$ & 0.03 \\
\hline 56 & 1 & $0.0 \odot$ & $\odot . \odot \odot$ & $-0.0 \odot$ & -0.05 & 0.01 & -0.10 & $-\odot .09$ & 0.01 & -0.02 \\
\hline
\end{tabular}




\begin{tabular}{|c|c|c|c|c|c|c|c|c|c|c|}
\hline 57 & 1 & $\odot . \odot \odot$ & $\odot . \odot \odot$ & $-\odot . \odot \odot$ & -0.05 & $\odot .04$ & -0.10 & -0.03 & -0.03 & 0.01 \\
\hline 58 & 1 & $\odot . \odot \odot$ & $\odot .0 \odot$ & $\odot .0 \odot$ & -0.04 & 0.04 & -0.07 & -0.04 & -0.02 & $-\odot .02$ \\
\hline 59 & 1 & 0.00 & $0.0 \odot$ & $\odot .0 \odot$ & -0.05 & $\odot .02$ & -0.09 & -0.05 & -0.03 & 0.05 \\
\hline 60 & 7 & $0.0 \odot$ & $-\odot .0 \odot$ & -0.00 & -0.04 & 0.02 & -0.05 & -0.03 & -0.03 & 0.03 \\
\hline 61 & 6 & $\odot . \odot \odot$ & $\odot . \odot \odot$ & $\odot . \odot \odot$ & -0.03 & $\odot .03$ & -0.04 & -0.03 & -0.03 & -0.02 \\
\hline 62 & 7 & $\odot . \odot \odot$ & $\odot . \odot \odot$ & $\odot .0 \odot$ & -0.02 & 0.03 & -0.02 & -0.02 & -0.04 & -0.02 \\
\hline 63 & 6 & $\odot .0 \odot$ & $-\odot . \odot \odot$ & $\odot .0 \odot$ & -0.02 & 0.02 & -0.02 & -0.02 & -0.04 & 0.04 \\
\hline 64 & 6 & $\odot . \odot \odot$ & $\odot .0 \odot$ & $-\odot .0 \odot$ & -0.03 & $\odot . \odot 2$ & -0.04 & $-\odot .02$ & -0.03 & $\odot .08$ \\
\hline 65 & 7 & $-0.0 \odot$ & $\odot .0 \odot$ & $-\odot .0 \odot$ & -0.04 & 0.01 & -0.04 & -0.02 & -0.03 & 0.14 \\
\hline 66 & 6 & $\odot .0 \odot$ & 0.00 & $\odot . \odot \odot$ & -0.03 & 0.01 & -0.03 & -0.01 & -0.04 & 0.16 \\
\hline 67 & 7 & $\odot .0 \odot$ & $\odot .0 \odot$ & 0.00 & -0.02 & 0.01 & -0.02 & -0.01 & -0.04 & 0.13 \\
\hline 68 & 6 & $\odot .0 \odot$ & -0.00 & $\odot . \odot \odot$ & -0.02 & 0.01 & -0.02 & -0.01 & -0.04 & 0.07 \\
\hline 69 & 7 & $-\odot . \odot \odot$ & $\odot .0 \odot$ & $-\odot . \odot \odot$ & -0.01 & 0.02 & -0.01 & -0.01 & -0.04 & 0.03 \\
\hline 70 & 1 & 0.00 & $\odot .0 \odot$ & $\odot .0 \odot$ & -0.03 & 0.03 & -0.04 & -0.04 & -0.03 & -0.07 \\
\hline 71 & 1 & $\odot . \odot \odot$ & $\odot . \odot \odot$ & $\odot . \odot \odot$ & -0.03 & $\odot . \odot \odot$ & -0.03 & -0.01 & -0.04 & 0.21 \\
\hline 72 & 1 & 0.00 & $-\odot . \odot \odot$ & $\odot .0 \odot$ & -0.01 & 0.02 & $-0.0 \odot$ & -0.02 & -0.04 & -0.03 \\
\hline 73 & 1 & $-\odot .0 \odot$ & -0.00 & $\odot .00$ & -0.01 & 0.01 & -0.02 & -0.02 & -0.03 & 0.06 \\
\hline 74 & 1 & 0.00 & 0.00 & -0.00 & -0.02 & -0.05 & -0.11 & -0.04 & 0.03 & -0.03 \\
\hline 75 & 6 & $-0.0 \odot$ & 0.00 & 0.01 & -0.01 & -0.03 & -0.02 & $0.0 \odot$ & $\odot .03$ & -0.05 \\
\hline 76 & 6 & $-\odot . \odot \odot$ & $-0.0 \odot$ & 0.01 & $-\odot . \odot \odot$ & -0.01 & $\odot . \odot \odot$ & 0.02 & $\odot .02$ & -0.02 \\
\hline 77 & 6 & -0.00 & $-0.0 \odot$ & 0.01 & -0.02 & -0.04 & $\odot .02$ & -0.00 & $\odot .02$ & $\odot .00$ \\
\hline 78 & 8 & -0.00 & $-0.0 \odot$ & $\odot .0 \odot$ & -0.03 & -0.08 & $\odot . \odot \odot$ & -0.03 & $\odot .02$ & -0.01 \\
\hline 79 & 1 & $-\odot . \odot \odot$ & $-0.0 \odot$ & $\odot .0 \odot$ & -0.06 & -0.10 & -0.07 & -0.07 & 0.03 & -0.03 \\
\hline 80 & 1 & $-0.0 \odot$ & -0.00 & $0.0 \odot$ & -0.04 & -0.08 & -0.10 & -0.05 & $\odot .04$ & -0.06 \\
\hline 81 & 1 & -0.00 & -0.00 & 0.01 & -0.04 & -0.09 & -0.02 & -0.04 & 0.04 & -0.05 \\
\hline 82 & 1 & -0.00 & $\odot .0 \odot$ & 0.01 & $-\odot .0 \odot$ & -0.01 & -0.04 & 0.01 & 0.03 & -0.06 \\
\hline 83 & 1 & -0.01 & -0.01 & $\odot .01$ & $\odot .01$ & 0.01 & 0.02 & 0.04 & 0.03 & -0.01 \\
\hline 84 & 1 & -0.01 & -0.01 & 0.02 & 0.01 & 0.00 & -0.02 & 0.02 & 0.02 & -0.02 \\
\hline 85 & 1 & $-0.0 \odot$ & -0.00 & 0.01 & -0.02 & -0.04 & 0.06 & 0.00 & 0.01 & 0.01 \\
\hline 86 & 7 & $-\odot . \odot \odot$ & & $-\odot . \odot \odot$ & & -0.03 & $\odot .01$ & -0.01 & $\odot . \odot \odot$ & $\odot .03$ \\
\hline 87 & 6 & $0.0 \odot$ & $-\odot .0 \odot$ & -0.00 & -0.00 & -0.03 & -0.02 & -0.02 & $\odot .01$ & 0.03 \\
\hline 88 & 6 & $\odot . \odot \odot$ & $\odot .0 \odot$ & -0.00 & $\odot .00$ & -0.02 & -0.04 & -0.03 & -0.01 & 0.05 \\
\hline 89 & 6 & $-\odot . \odot \odot$ & $\odot .0 \odot$ & $\odot . \odot \odot$ & $-\odot .0 \odot$ & -0.01 & -0.03 & -0.02 & -0.02 & 0.08 \\
\hline 90 & 7 & $-\odot .0 \odot$ & $\odot .0 \odot$ & $\odot .0 \odot$ & -0.01 & -0.02 & $\odot . \odot \odot$ & -0.02 & -0.02 & $\odot .08$ \\
\hline 91 & 6 & $-\odot . \odot \odot$ & $0.0 \odot$ & $\odot .0 \odot$ & -0.02 & -0.03 & 0.03 & -0.01 & -0.01 & 0.05 \\
\hline 92 & 6 & $\odot .0 \odot$ & $\odot .0 \odot$ & -0.01 & 0.01 & -0.01 & -0.07 & -0.03 & $-0.0 \odot$ & $\odot .05$ \\
\hline 93 & 8 & $-\odot .0 \odot$ & $\odot .0 \odot$ & $0.0 \odot$ & $\odot . \odot \odot$ & -0.00 & -0.05 & -0.03 & -0.03 & 0.10 \\
\hline 94 & 8 & $-\odot . \odot \odot$ & $\odot .0 \odot$ & $-\odot . \odot \odot$ & -0.02 & -0.03 & 0.05 & $-0.0 \odot$ & -0.01 & 0.05 \\
\hline 95 & 1 & $\odot .0 \odot$ & $-\odot . \odot \odot$ & -0.01 & $-0.0 \odot$ & -0.03 & -0.03 & -0.02 & 0.01 & 0.01 \\
\hline 96 & 1 & 0.01 & $0.0 \odot$ & 0.00 & 0.02 & 0.01 & -0.08 & -0.01 & -0.01 & 0.07 \\
\hline 97 & 1 & -0.01 & -0.00 & $-\odot . \odot \odot$ & 0.01 & -0.03 & -0.08 & -0.06 & -0.01 & 0.06 \\
\hline 98 & 1 & $\odot . \odot \odot$ & 0.01 & -0.03 & 0.01 & -0.01 & -0.08 & -0.03 & $\odot .01$ & 0.03 \\
\hline 99 & 1 & $-\odot .0 \odot$ & -0.00 & 0.01 & -0.01 & -0.01 & -0.00 & -0.02 & -0.03 & 0.10 \\
\hline 100 & 6 & 0.03 & -0.03 & -0.00 & $\odot .02$ & -0.01 & $\odot .09$ & 0.03 & $\odot .02$ & -0.03 \\
\hline 101 & 11 & $\odot .0 \odot$ & 0.00 & $\odot .0 \odot$ & -0.03 & 0.07 & -0.02 & -0.02 & 0.02 & -0.08 \\
\hline 102 & 11 & $0.0 \odot$ & $-\odot . \odot \odot$ & $\odot . \odot \odot$ & $\odot .02$ & 0.06 & -0.06 & 0.07 & 0.02 & -0.09 \\
\hline 103 & 6 & -0.08 & -0.05 & -0.06 & 0.05 & 0.05 & 0.08 & 0.08 & $\odot .0 \odot$ & 0.01 \\
\hline 104 & 6 & -0.11 & -0.17 & -0.11 & 0.03 & 0.02 & 0.08 & 0.05 & -0.01 & 0.03 \\
\hline 105 & 8 & -0.01 & -0.12 & -0.06 & 0.01 & -0.01 & $\odot .08$ & 0.03 & $\odot . \odot \odot$ & $\odot .02$ \\
\hline 106 & 1 & 0.02 & -0.03 & $-\odot . \odot \odot$ & & -0.03 & $\odot .08$ & $\odot . \odot \odot$ & $\odot .02$ & -0.03 \\
\hline 107 & 1 & 0.06 & -0.01 & $-\odot .00$ & 0.02 & -0.00 & 0.10 & 0.04 & 0.02 & -0.05 \\
\hline 108 & 1 & $\odot .08$ & -0.11 & -0.00 & 0.03 & -0.01 & 0.13 & 0.05 & 0.01 & 0.01 \\
\hline 109 & 1 & $\odot .0 \odot$ & 0.01 & 0.02 & 0.05 & $\odot .05$ & 0.10 & 0.10 & 0.02 & -0.03 \\
\hline 110 & 1 & -0.08 & -0.03 & -0.06 & 0.06 & 0.08 & 0.08 & 0.10 & -0.00 & 0.02 \\
\hline 111 & 1 & -0.15 & $\odot .02$ & -0.10 & 0.04 & 0.06 & $\odot .05$ & 0.07 & 0.01 & -0.01 \\
\hline 112 & 1 & -0.07 & -0.24 & -0.12 & $\odot .03$ & $\odot .03$ & 0.11 & 0.06 & -0.02 & 0.05 \\
\hline 113 & 7 & -0.05 & -0.19 & -0.14 & $\odot .03$ & $\odot .02$ & 0.05 & $\odot .04$ & -0.01 & 0.04 \\
\hline 114 & 6 & $\odot . \odot 8$ & 0.44 & 0.19 & 0.02 & 0.05 & $\odot . \odot \odot$ & 0.06 & $-0.0 \odot$ & $\odot .02$ \\
\hline 115 & 7 & 0.05 & 0.04 & -0.10 & $\odot .02$ & 0.04 & -0.02 & 0.04 & -0.01 & 0.05 \\
\hline 116 & 6 & 0.04 & -0.06 & 0.01 & 0.03 & $\odot .02$ & -0.01 & 0.03 & -0.02 & $\odot .07$ \\
\hline 117 & 6 & 0.02 & -0.10 & -0.02 & 0.03 & 0.02 & $\odot .02$ & $\odot .03$ & -0.01 & 0.07 \\
\hline 118 & 7 & -0.07 & -0.04 & $\odot .04$ & 0.04 & 0.01 & 0.03 & 0.03 & -0.02 & 0.09 \\
\hline 119 & 6 & -0.02 & -0.02 & 0.03 & 0.04 & $0.0 \odot$ & $\odot .0 \odot$ & 0.03 & -0.02 & 0.11 \\
\hline 120 & 7 & 0.01 & 0.00 & 0.02 & 0.03 & $\odot .0 \odot$ & -0.02 & 0.02 & -0.02 & 0.11 \\
\hline 121 & 6 & $\odot .02$ & -0.03 & 0.02 & 0.02 & 0.01 & -0.03 & 0.03 & -0.02 & 0.09 \\
\hline 122 & 7 & -0.05 & 0.02 & -0.00 & $\odot .04$ & -0.00 & -0.01 & 0.02 & -0.02 & 0.14 \\
\hline 123 & 8 & 0.05 & -0.03 & $\odot .01$ & 0.02 & 0.01 & -0.05 & 0.03 & -0.02 & 0.09 \\
\hline 124 & 1 & $\odot .02$ & $\odot .01$ & -0.15 & $\odot .03$ & 0.08 & $\odot .02$ & $\odot .08$ & -0.01 & 0.03 \\
\hline 125 & 1 & -0.07 & $\odot .02$ & $\odot .02$ & 0.05 & -0.01 & 0.03 & 0.02 & -0.02 & 0.10 \\
\hline 126 & 1 & -0.04 & $\odot .04$ & $-\odot . \odot \odot$ & 0.04 & -0.01 & -0.02 & 0.02 & $-\odot .02$ & 0.10 \\
\hline 127 & 1 & $\odot .02$ & 0.02 & 0.01 & 0.03 & -0.00 & -0.03 & 0.02 & -0.02 & 0.11 \\
\hline 128 & 8 & 0.05 & 0.34 & 0.20 & 0.02 & $\odot .06$ & -0.00 & 0.05 & 0.00 & -0.01 \\
\hline 129 & 1 & 0.10 & 0.32 & 0.16 & 0.02 & 0.04 & -0.02 & 0.03 & 0.01 & -0.01 \\
\hline 130 & 8 & 0.03 & 0.05 & -0.02 & 0.02 & 0.02 & -0.06 & 0.01 & 0.01 & 0.01 \\
\hline 131 & 1 & 0.01 & 0.05 & -0.15 & $\odot .02$ & $\odot .02$ & -0.05 & 0.01 & $\odot .0 \odot$ & 0.02 \\
\hline 132 & 1 & -0.07 & 0.16 & -0.14 & 0.02 & 0.04 & -0.08 & 0.01 & $\odot .01$ & 0.01 \\
\hline 133 & 1 & -0.00 & 0.02 & 0.02 & 0.03 & 0.07 & 0.00 & 0.08 & 0.02 & -0.05 \\
\hline
\end{tabular}




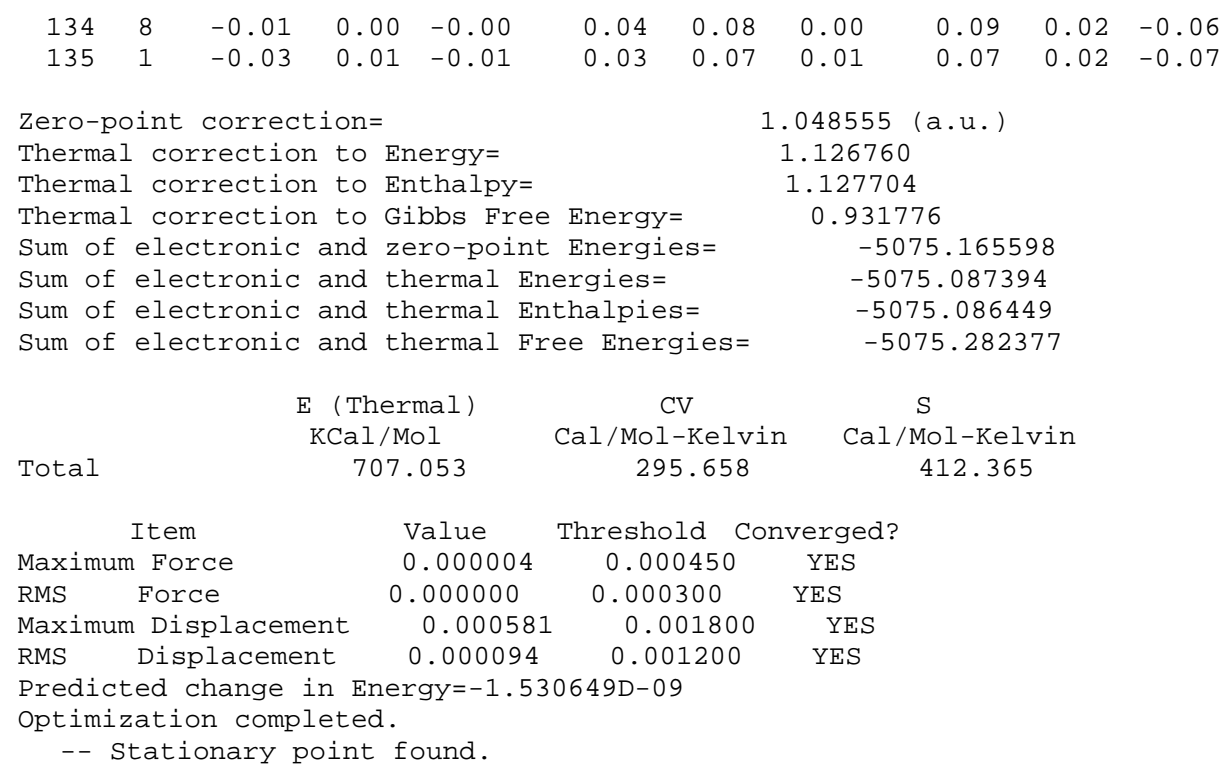

Normal termination of Gaussian 16 at Mon Jul 15 17:34:06 2019.

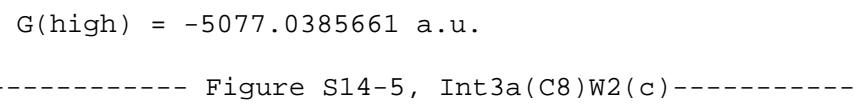

\begin{tabular}{|c|c|c|c|c|c|}
\hline \multirow{2}{*}{$\begin{array}{l}\text { Center } \\
\text { Number }\end{array}$} & \multirow{2}{*}{$\begin{array}{l}\text { Atomic } \\
\text { Number }\end{array}$} & \multirow{2}{*}{$\begin{array}{c}\text { Atomic } \\
\text { Type }\end{array}$} & \multicolumn{3}{|c|}{ Coordinates (Angstroms) } \\
\hline & & & $\mathrm{x}$ & $\mathrm{Y}$ & Z \\
\hline 1 & 7 & $\odot$ & -5.522579 & 2.177714 & -1.044369 \\
\hline 2 & 6 & $\odot$ & -5.721910 & 3.428919 & -0.539246 \\
\hline 3 & 6 & $\odot$ & -4.687264 & 4.283715 & -0.377764 \\
\hline 4 & 6 & $\Theta$ & -3.389904 & 3.804171 & -0.748161 \\
\hline 5 & 7 & $\odot$ & -3.219852 & 2.618169 & -1.330436 \\
\hline 6 & 8 & $\odot$ & -8.658366 & $-\odot .833269$ & -0.732530 \\
\hline 7 & 15 & $\odot$ & -7.931061 & -2.134587 & -0.064311 \\
\hline 8 & 8 & $\odot$ & -6.349141 & -1.899568 & -0.465838 \\
\hline 9 & 8 & $\odot$ & -8.447396 & -3.354581 & -0.769616 \\
\hline 10 & 8 & $\odot$ & -7.988485 & -2.011990 & 1.444494 \\
\hline 11 & 8 & $\odot$ & -4.444196 & -5.654645 & 0.282930 \\
\hline 12 & 6 & $\Theta$ & -4.652755 & -3.624806 & -0.940718 \\
\hline 13 & 6 & $\odot$ & -5.141778 & -4.384548 & 0.293502 \\
\hline 14 & 6 & $\odot$ & 8.884447 & 1.826769 & 1.404648 \\
\hline 15 & 6 & $\odot$ & 8.259783 & $\odot .451771$ & 1.525465 \\
\hline 16 & 8 & $\odot$ & 8.047389 & 2.647608 & 0.552317 \\
\hline 17 & 8 & 0 & 8.128855 & -1.592633 & 0.253493 \\
\hline 18 & 15 & $\odot$ & 7.999103 & -2.557378 & -1.059564 \\
\hline 19 & 8 & $\odot$ & 6.432505 & -3.015107 & -1.017750 \\
\hline 20 & 8 & $\odot$ & 8.908174 & -3.744196 & -0.833584 \\
\hline 21 & 8 & $\odot$ & 8.198811 & -1.768039 & -2.333487 \\
\hline 22 & 8 & $\odot$ & 2.663895 & -4.972509 & -0.748229 \\
\hline 23 & 6 & $\odot$ & 4. $4 \odot 7127$ & -3.612901 & 0.169357 \\
\hline 24 & 6 & $\odot$ & 3.668145 & -3.993599 & -1.130408 \\
\hline 25 & 1 & $\odot$ & 2.168635 & -5.266223 & -1.531712 \\
\hline 26 & 6 & $\odot$ & -9.691490 & 2.750967 & -0.936940 \\
\hline 27 & 6 & $\odot$ & -8.993239 & 1.461707 & -1.318980 \\
\hline 28 & 6 & $\odot$ & -8.673538 & 0.514842 & -0.154057 \\
\hline 29 & 6 & $\odot$ & -7.278861 & 0.957483 & 0.266808 \\
\hline 30 & 6 & $\odot$ & -6.623902 & 1.231187 & -1.086585 \\
\hline 31 & 8 & $\odot$ & -7.690726 & 1.765956 & -1.924146 \\
\hline 32 & 1 & $\odot$ & -9.753802 & 3.390131 & -1.823767 \\
\hline 33 & 1 & 0 & -10.707978 & 2.521954 & $\begin{array}{r}-0.596273 \\
\end{array}$ \\
\hline 34 & 1 & 0 & -9.597005 & 0.940970 & -2.065581 \\
\hline 35 & 1 & 0 & -9.408205 & 0.549320 & 0.651223 \\
\hline 36 & 1 & 0 & -6.756498 & 0.207078 & 0.854483 \\
\hline 37 & 1 & 0 & -7.369932 & 1.874043 & 0.851968 \\
\hline
\end{tabular}




\begin{tabular}{|c|c|c|c|c|c|}
\hline 38 & 1 & 0 & -6.233117 & $\odot .319480$ & -1.531014 \\
\hline 39 & 8 & $\odot$ & -8.940878 & 3.409851 & 0.113054 \\
\hline 40 & 1 & $\odot$ & -9.339160 & 4.266556 & $\odot .337023$ \\
\hline 41 & 6 & 0 & -4.261410 & 1.769084 & -1.496311 \\
\hline 42 & 7 & $\odot$ & -2.291751 & 4.530456 & -0.503312 \\
\hline 43 & 8 & $\odot$ & -4.127167 & $\odot .654594$ & -2.018584 \\
\hline 44 & 1 & 0 & -6.753498 & 3.670435 & $-\odot .290190$ \\
\hline 45 & 1 & $\odot$ & -4.832519 & 5.274264 & 0.030372 \\
\hline 46 & 1 & $\odot$ & -2.370567 & 5.465384 & -0.138392 \\
\hline 47 & 1 & 0 & -1.387213 & 4.202674 & $-\odot .848060$ \\
\hline 48 & 6 & $\odot$ & -5.654536 & -2.634290 & -1.515575 \\
\hline 49 & 1 & 0 & -4.688967 & -6.182247 & 1.061993 \\
\hline 50 & 6 & 0 & -4.685271 & -3.500603 & 1.459793 \\
\hline 51 & 6 & $\odot$ & -3.368734 & -2.892165 & 0.960860 \\
\hline 52 & 8 & $\odot$ & -3.425756 & -2.951242 & -0.494729 \\
\hline 53 & 1 & 0 & -5.131826 & -1.894485 & -2.123858 \\
\hline 54 & 1 & $\odot$ & -6.387569 & -3.171785 & -2.121792 \\
\hline 55 & 1 & 0 & -4.355745 & -4.324435 & -1.724630 \\
\hline 56 & 1 & 0 & -6.225371 & -4.535937 & 0.277207 \\
\hline 57 & 1 & $\odot$ & -4.545310 & -4.069032 & 2.381525 \\
\hline 58 & 1 & 0 & -5.429143 & -2.720661 & 1.635152 \\
\hline 59 & 1 & $\odot$ & -2.493214 & -3.450941 & 1.293094 \\
\hline $6 \odot$ & 7 & $\odot$ & -3.155238 & -1.513847 & 1.350568 \\
\hline 61 & 6 & 0 & -3.959686 & -0.445809 & 1.025189 \\
\hline 62 & 7 & 0 & -3.407218 & $\odot .716494$ & 1.253356 \\
\hline 63 & 6 & 0 & -2.146313 & $\odot .400031$ & 1.725644 \\
\hline 64 & 6 & 0 & -1.967498 & $-\odot .978724$ & 1.782283 \\
\hline 65 & 7 & 0 & -0.863528 & -1.641459 & 2.151667 \\
\hline 66 & 6 & 0 & 0.114037 & -0.803857 & 2.467513 \\
\hline 67 & 7 & 0 & $\odot .09 \odot 40 \odot$ & 0.536368 & 2.439084 \\
\hline 68 & 6 & 0 & -1.019381 & 1.186427 & 2.037488 \\
\hline 69 & 7 & 0 & -0.984539 & 2.526729 & 1.954179 \\
\hline 70 & 1 & 0 & -4.931305 & -0.623084 & 0.582634 \\
\hline 71 & 1 & 0 & 1.066307 & -1.231448 & 2.771500 \\
\hline 72 & 1 & 0 & -1.736154 & 3.006281 & 1.482120 \\
\hline 73 & 1 & 0 & -0.077728 & 2.992933 & 2.014551 \\
\hline 74 & 1 & 0 & 8.406641 & 3.546076 & $\odot .472075$ \\
\hline 75 & 6 & 0 & 7.826581 & -0.158748 & $\odot .186407$ \\
\hline 76 & 6 & 0 & 6.328616 & 0.113496 & 0.112490 \\
\hline 77 & 6 & 0 & 5.871249 & 0.165292 & 1.572438 \\
\hline 78 & 8 & 0 & 7.053524 & $\odot .51740 \odot$ & 2.350568 \\
\hline 79 & 1 & 0 & 8.973170 & 2.268299 & $2.4 \odot 2668$ \\
\hline 80 & 1 & 0 & 9.886336 & 1.727545 & 0.970821 \\
\hline 81 & 1 & 0 & 8.968003 & -0.214892 & 2.023774 \\
\hline 82 & 1 & 0 & 8.368032 & 0.263794 & -0.660795 \\
\hline 83 & 1 & 0 & 5.773820 & -0.654305 & -0.426413 \\
\hline 84 & 1 & 0 & 6.178987 & 1.079990 & -0.371295 \\
\hline 85 & 1 & $\odot$ & 5.477180 & $-\odot .791826$ & 1.906092 \\
\hline 86 & 7 & 0 & 4.824683 & 1.148965 & 1.822610 \\
\hline 87 & 6 & 0 & 5.042644 & 2.477005 & 1.523011 \\
\hline 88 & 6 & 0 & 4.086710 & 3.426056 & 1.617213 \\
\hline 89 & 6 & 0 & 2.747836 & 3.000337 & 1.989016 \\
\hline $9 \odot$ & 7 & 0 & 2.633089 & 1.677118 & 2.347771 \\
\hline 91 & 6 & 0 & 3.618306 & 0.719503 & 2.351115 \\
\hline 92 & 6 & 0 & 4.328106 & 4.875843 & 1.327915 \\
\hline 93 & 8 & 0 & 1.754009 & 3.736272 & 2.011507 \\
\hline 94 & 8 & 0 & 3.424303 & -0.401342 & 2.797880 \\
\hline 95 & 1 & 0 & 6.060370 & 2.700152 & 1.206217 \\
\hline 96 & 1 & 0 & 3.559326 & 5.264793 & 0.656283 \\
\hline 97 & 1 & 0 & 4.290118 & 5.470525 & 2.247338 \\
\hline 98 & 1 & 0 & 5.303992 & 5.024002 & 0.861269 \\
\hline 99 & 1 & 0 & 1.677290 & 1.315121 & 2.540573 \\
\hline 100 & 6 & $\odot$ & 5.909805 & -3.736292 & $\odot .136211$ \\
\hline 101 & 11 & 0 & -8.047350 & -3.578046 & 3.022024 \\
\hline 102 & 11 & 0 & 10.029892 & -3.261278 & -3.050569 \\
\hline 103 & 6 & 0 & 3.042472 & -2.678568 & -1.597894 \\
\hline 104 & 6 & 0 & 2.862863 & -1.878950 & -0.296536 \\
\hline 105 & 8 & $\odot$ & 4.065952 & -2.230178 & 0.455339 \\
\hline 106 & 1 & $\odot$ & 6.328467 & -3.309864 & 1.052506 \\
\hline 107 & 1 & $\odot$ & 6.201014 & -4.788051 & 0.060691 \\
\hline 108 & 1 & 0 & 4.026066 & -4.261497 & $\odot .968009$ \\
\hline 109 & 1 & $\odot$ & 4.344392 & -4.420409 & -1.876860 \\
\hline 110 & 1 & $\odot$ & 2.102294 & -2.818308 & -2.134260 \\
\hline 111 & 1 & 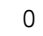 & 3.745944 & -2.137619 & -2.238489 \\
\hline 112 & 1 & $\odot$ & 1.983806 & -2.234201 & 0.257613 \\
\hline 113 & 7 & $\odot$ & 2.848017 & -0.463025 & -0.488369 \\
\hline 114 & 6 & 0 & 3.920482 & 1.858181 & -1.665386 \\
\hline
\end{tabular}




\begin{tabular}{|c|c|c|c|c|c|}
\hline 115 & 7 & 0 & 2.764550 & 2.325052 & -1.315973 \\
\hline 116 & 6 & 0 & 1.696469 & $1.5 \odot 4949$ & -1.134805 \\
\hline 117 & 6 & 0 & 1.711496 & $\odot .072657$ & $-\odot .836158$ \\
\hline 118 & 7 & 0 & $\odot .556013$ & -0.672679 & $-\odot .971291$ \\
\hline 119 & 6 & 0 & -0.569603 & -0.055930 & -1.222766 \\
\hline 120 & 7 & 0 & $-\odot .681755$ & 1.318198 & -1.305407 \\
\hline 121 & 6 & 0 & ๑.398814 & 2.150111 & -1.266652 \\
\hline 122 & 7 & 0 & -1.707571 & -0.726810 & -1.432267 \\
\hline 123 & 8 & 0 & $\odot .235064$ & 3.379752 & -1.362452 \\
\hline 124 & 1 & 0 & 4.110024 & $\odot .808283$ & -1.878222 \\
\hline 125 & 1 & 0 & -1.761459 & -1.714343 & -1.229098 \\
\hline 126 & 1 & 0 & -2.585081 & -0.235140 & -1.615831 \\
\hline 127 & 1 & 0 & -1.615579 & 1.769827 & -1.387814 \\
\hline 128 & 8 & 0 & 4.967789 & 2.623853 & -1.811741 \\
\hline 129 & 1 & 0 & 4.740145 & 3.609821 & -1.733877 \\
\hline 130 & 8 & 0 & 4.503310 & 5.149220 & -1.916118 \\
\hline 131 & 1 & 0 & 3.551731 & 5.306339 & -1.684940 \\
\hline 132 & 1 & 0 & 4.570324 & 5.336141 & -2.857846 \\
\hline 133 & 8 & 0 & 1.960901 & 5.500452 & -1.232389 \\
\hline 134 & 1 & 0 & 1.533620 & 4.618522 & -1.236288 \\
\hline 135 & 1 & 0 & 1.457826 & 6.020408 & -1.865697 \\
\hline
\end{tabular}

General basis read from cards: (6D, 7F)

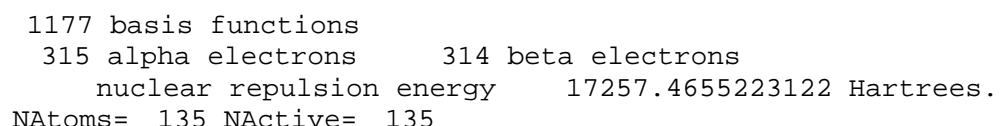

NAtoms $=135$ NActive $=135$

Force inversion solution in PCM.

Polarizable Continuum Model (PCM)

=ニニニニニニニニニニニニニニニニニニニニニニニニニニニニニニニ=

Model : PCM.

Atomic radii : UFF (Universal Force Field).

Solvent : Water, Eps $=78.355300$ Eps $($ inf $)=1.777849$

SCF Done: $E($ UWB97XD) $=-5076.22242581$ A.U. after 1 cycles NFock $=1$ Conv $=0.19 \mathrm{D}-08 \quad-\mathrm{V} / \mathrm{T}=2.0066$

$\langle\mathrm{S} X>=0.0000\langle\mathrm{~S} y\rangle=0.0000\langle\mathrm{~S} z\rangle=0.5000\langle\mathrm{~S} * * 2\rangle=0.7735 \mathrm{~S}=0.5117$

$<$ L.S $>=0.000000000000 \mathrm{E}+00$

Annihilation of the first spin contaminant:

$\mathrm{S}^{* *} 2$ before annihilation 0.7735 , after 0.7503

Harmonic frequencies $\left(\mathrm{cm}^{*}-1\right)$, IR intensities (KM/Mole), Raman

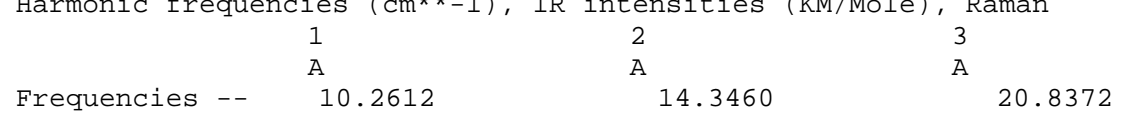

Zero-point correction=

Thermal correction to Energy=

Thermal correction to Enthalpy=

Thermal correction to Gibbs Free Energy=

Sum of electronic and zero-point Energies=

Sum of electronic and thermal Energies=

Sum of electronic and thermal Enthalpies=

1.047346 (a.u.)

1.126488

1.127432

0.928388

$-5075.175080$

$-5075.095938$

$-5075.094994$

Sum of electronic and thermal Free Energies=

$-5075.294038$

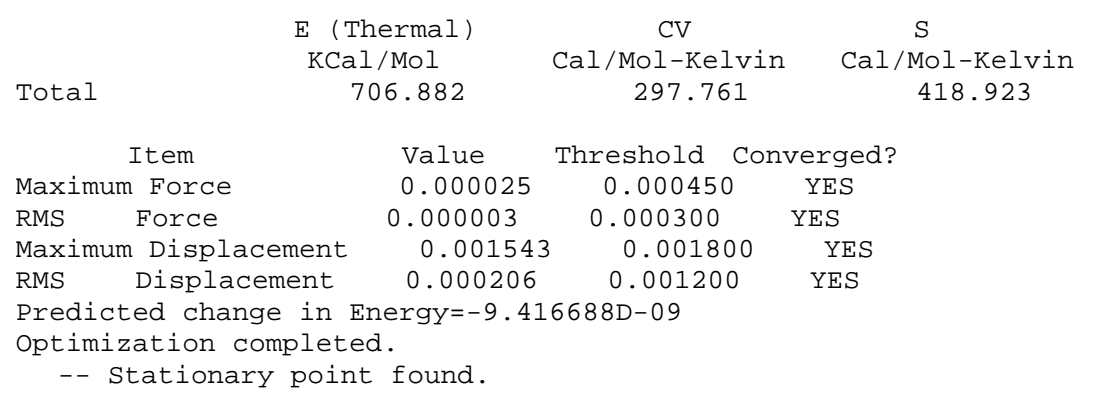

Normal termination of Gaussian 16 at Tue Aug 20 13:38:16 2019.

$\mathrm{G}(\mathrm{high})=-5077.0540578 \mathrm{a} \cdot \mathrm{u}$. 


\begin{tabular}{|c|c|c|c|c|c|}
\hline \multicolumn{2}{|c|}{ Stoichiometry } & \multicolumn{4}{|c|}{ C39H54N15Na2023P2(2) } \\
\hline \multicolumn{6}{|c|}{ Standard orientation: } \\
\hline Center & Atomic & Atomic & $\mathrm{Coc}$ & rdinates $(A$ & igstroms) \\
\hline Number & Number & Type & $x$ & $\mathrm{Y}$ & Z \\
\hline 1 & 7 & 0 & -5.563736 & 2.174199 & -1.097736 \\
\hline 2 & 6 & $\odot$ & -5.760422 & 3.429684 & -0.601684 \\
\hline 3 & 6 & $\odot$ & -4.726646 & 4.289188 & $-\odot .462790$ \\
\hline 4 & 6 & $\odot$ & -3.433115 & 3.810011 & $-\odot .848372$ \\
\hline 5 & 7 & 0 & -3.268982 & 2.623522 & -1.430876 \\
\hline 6 & 8 & $\odot$ & -8.676804 & $-\odot .848357$ & -0.694311 \\
\hline 7 & 15 & $\odot$ & -7.933667 & -2.135788 & $-\odot .016958$ \\
\hline 8 & 8 & 0 & -6.358018 & -1.895197 & -0.440170 \\
\hline 9 & 8 & $\odot$ & -8.449514 & -3.368921 & -0.699586 \\
\hline 10 & 8 & $\odot$ & -7.977614 & -1.994100 & 1.490864 \\
\hline 11 & 8 & $\odot$ & -4.426980 & -5.609868 & 0.427370 \\
\hline 12 & 6 & $\odot$ & -4.653673 & -3.625280 & $-\odot .866611$ \\
\hline 13 & 6 & $\odot$ & -5.129955 & -4.342967 & 0.397671 \\
\hline 14 & 6 & 0 & 8.932650 & 1.713511 & 1.438318 \\
\hline 15 & 6 & $\odot$ & 8.270397 & $\odot .354069$ & 1.530266 \\
\hline 16 & 8 & $\odot$ & 8.122453 & 2.572719 & $\odot .598148$ \\
\hline 17 & 8 & $\odot$ & 8.096455 & -1.659067 & 0.218019 \\
\hline 18 & 15 & $\odot$ & 7.937186 & -2.601046 & -1.107576 \\
\hline 19 & 8 & 0 & 6.365007 & -3.036718 & -1.053242 \\
\hline 20 & 8 & $\odot$ & 8.831770 & -3.804311 & -0.910543 \\
\hline 21 & 8 & 0 & 8.134845 & -1.796892 & -2.372547 \\
\hline 22 & 8 & $\odot$ & 2.574323 & -4.937812 & -0.763510 \\
\hline 23 & 6 & $\odot$ & 4.340651 & -3.604105 & 0.150336 \\
\hline 24 & 6 & $\odot$ & 3.588168 & -3.968448 & -1.146451 \\
\hline 25 & 1 & $\odot$ & 2.066841 & -5.215629 & -1.544968 \\
\hline 26 & 6 & $\odot$ & -9.735883 & 2.726263 & -0.925843 \\
\hline 27 & 6 & 0 & -9.035273 & 1.437203 & -1.304194 \\
\hline 28 & 6 & $\odot$ & -8.690180 & $\odot .506994$ & -0.133089 \\
\hline 29 & 6 & 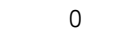 & -7.290851 & $\odot .961943$ & 0.257773 \\
\hline 30 & 6 & 0 & -6.660879 & 1.222579 & -1.110108 \\
\hline 31 & 8 & $\odot$ & -7.745220 & 1.742713 & -1.934600 \\
\hline 32 & 1 & $\odot$ & -9.815969 & 3.355131 & -1.818599 \\
\hline 33 & 1 & 0 & -10.745552 & 2.494626 & $-\odot .567056$ \\
\hline 34 & 1 & $\odot$ & -9.647928 & 0.903310 & -2.034138 \\
\hline 35 & 1 & $\odot$ & -9.411060 & $\odot .547714$ & 0.684259 \\
\hline 36 & 1 & 0 & -6.753974 & $\odot .221256$ & 0.844846 \\
\hline 37 & 1 & 0 & -7.376429 & 1.885202 & 0.833137 \\
\hline 38 & 1 & $\odot$ & -6.273430 & 0.307610 & -1.551052 \\
\hline 39 & 8 & 0 & -8.973489 & 3.401290 & 0.105236 \\
\hline 40 & 1 & $\odot$ & -9.373122 & 4.258412 & 0.325129 \\
\hline 41 & 6 & $\Theta$ & -4.307643 & 1.766357 & -1.566759 \\
\hline 42 & 7 & 0 & -2.332689 & 4.537813 & -0.619873 \\
\hline 43 & 8 & $\odot$ & -4.176231 & 0.644884 & -2.074619 \\
\hline 44 & 1 & 0 & -6.788921 & 3.669658 & -0.339094 \\
\hline 45 & 1 & 0 & -4.869037 & 5.282390 & -0.060016 \\
\hline 46 & 1 & $\odot$ & -2.407856 & 5.472814 & -0.254380 \\
\hline 47 & 1 & 0 & -1.428624 & 4.202503 & -0.964465 \\
\hline 48 & 6 & 0 & -5.664652 & -2.660610 & -1.468574 \\
\hline 49 & 1 & $\odot$ & -4.658335 & -6.106767 & 1.230406 \\
\hline 50 & 6 & 0 & -4.668188 & -3.415896 & 1.527587 \\
\hline 51 & 6 & 0 & -3.355673 & -2.826234 & 0.998262 \\
\hline 52 & 8 & $\odot$ & -3.427655 & -2.928868 & $-\odot .454007$ \\
\hline 53 & 1 & 0 & -5.150029 & -1.938368 & -2.104203 \\
\hline 54 & 1 & 0 & -6.397876 & -3.222162 & -2.052278 \\
\hline 55 & 1 & $\odot$ & -4.358438 & -4.350902 & -1.627256 \\
\hline 56 & 1 & $\odot$ & -6.212913 & -4.499847 & 0.396169 \\
\hline 57 & 1 & $\odot$ & -4.522539 & -3.949317 & 2.469183 \\
\hline 58 & 1 & $\odot$ & -5.412317 & -2.630892 & 1.677432 \\
\hline 59 & 1 & $\odot$ & -2.478751 & -3.377548 & 1.339418 \\
\hline 60 & 7 & $\odot$ & -3.133969 & -1.437904 & 1.344491 \\
\hline 61 & 6 & $\odot$ & -3.928525 & -0.373328 & $\odot .986201$ \\
\hline 62 & 7 & $\odot$ & -3.369044 & 0.790614 & 1.188063 \\
\hline 63 & 6 & $\odot$ & -2.113717 & 0.478114 & 1.677769 \\
\hline 64 & 6 & 0 & -1.946208 & $-\odot .900125$ & 1.772317 \\
\hline 65 & 7 & 0 & -0.853014 & -1.561926 & 2.173368 \\
\hline
\end{tabular}




\begin{tabular}{|c|c|c|c|c|c|}
\hline 66 & 6 & 0 & 0.126808 & -0.724425 & 2.481724 \\
\hline 67 & 7 & 0 & 0.113948 & 0.614721 & 2.418931 \\
\hline 68 & 6 & 0 & -0.983759 & 1.263123 & 1.984450 \\
\hline 69 & 7 & 0 & -0.934397 & 2.601131 & 1.869138 \\
\hline 70 & 1 & 0 & -4.899125 & -0.554103 & 0.542930 \\
\hline 71 & 1 & 0 & 1.071738 & -1.150713 & 2.809136 \\
\hline 72 & 1 & $\odot$ & -1.669657 & 3.076861 & 1.367649 \\
\hline 73 & 1 & $\odot$ & -0.024834 & 3.059008 & 1.940447 \\
\hline 74 & 1 & 0 & 8.507471 & 3.461876 & 0.535409 \\
\hline 75 & 6 & 0 & 7.823631 & -0.217719 & 0.178605 \\
\hline 76 & 6 & 0 & 6.332212 & 0.089931 & 0.109108 \\
\hline 77 & 6 & 0 & 5.874950 & $\odot .127283$ & 1.569815 \\
\hline 78 & 8 & 0 & 7.065395 & $\odot .434061$ & 2.355412 \\
\hline 79 & 1 & $\odot$ & 9.028962 & 2.133559 & 2.444917 \\
\hline 80 & 1 & 0 & 9.933509 & 1.594918 & 1.006869 \\
\hline 81 & 1 & 0 & 8.959938 & -0.341413 & 2.015227 \\
\hline 82 & 1 & 0 & 8.375397 & 0.209578 & -0.659463 \\
\hline 83 & 1 & 0 & 5.760423 & -0.657902 & -0.440073 \\
\hline 84 & 1 & 0 & 6.199324 & 1.065823 & -0.362214 \\
\hline 85 & 1 & 0 & 5.454872 & -0.825159 & 1.885203 \\
\hline 86 & 7 & 0 & 4.855943 & 1.134967 & 1.836593 \\
\hline 87 & 6 & $\odot$ & 5.115604 & 2.461466 & 1.570625 \\
\hline 88 & 6 & 0 & 4.187750 & 3.436636 & 1.677910 \\
\hline 89 & 6 & 0 & 2.829570 & 3.040409 & 2.010826 \\
\hline 90 & 7 & 0 & 2.676884 & 1.716080 & 2.353927 \\
\hline 91 & 6 & 0 & 3.636280 & 0.733078 & 2.356468 \\
\hline 92 & 6 & 0 & 4.490119 & 4.883906 & 1.434826 \\
\hline 93 & 8 & 0 & 1.850935 & 3.796566 & 2.017073 \\
\hline 94 & 8 & 0 & 3.413040 & -0.384797 & 2.797046 \\
\hline 95 & 1 & 0 & 6.141652 & 2.661070 & 1.266353 \\
\hline 96 & 1 & 0 & 3.751547 & 5.322797 & $\odot .761310$ \\
\hline 97 & 1 & 0 & 4.458272 & 5.454904 & 2.369288 \\
\hline 98 & 1 & 0 & 5.482601 & 5.004617 & $\odot .994750$ \\
\hline 99 & 1 & 0 & 1.710165 & 1.375926 & 2.528886 \\
\hline 100 & 6 & $\odot$ & 5.840863 & -3.751148 & 0.104496 \\
\hline 101 & 11 & $\odot$ & -8.018315 & -3.570801 & 3.063774 \\
\hline 102 & 11 & $\odot$ & 9.944985 & -3.306071 & -3.119633 \\
\hline 103 & 6 & 0 & 2.976058 & -2.643167 & -1.601126 \\
\hline 104 & 6 & 0 & 2.813766 & -1.849233 & -0.294043 \\
\hline 105 & 8 & 0 & 4.022352 & -2.218241 & 0.444565 \\
\hline 106 & 1 & $\odot$ & 6.274692 & -3.332716 & 1.017365 \\
\hline 107 & 1 & 0 & 6.114452 & -4.807404 & 0.024705 \\
\hline 108 & 1 & 0 & 3.955824 & -4.251306 & $\odot .948572$ \\
\hline 109 & 1 & 0 & 4.254187 & -4.399815 & -1.899543 \\
\hline 110 & 1 & 0 & 2.031039 & -2.767307 & -2.132582 \\
\hline 111 & 1 & 0 & 3.681866 & -2.106113 & -2.242504 \\
\hline 112 & 1 & $\odot$ & 1.938432 & -2.205216 & 0.265995 \\
\hline 113 & 7 & 0 & 2.807283 & $-\odot .431764$ & $-\odot .47 \odot 215$ \\
\hline 114 & 6 & 0 & 3.932939 & 1.947265 & -1.554746 \\
\hline 115 & 7 & 0 & 2.721014 & 2.388235 & -1.221446 \\
\hline 116 & 6 & $\odot$ & 1.655938 & 1.545869 & -1.111862 \\
\hline 117 & 6 & 0 & 1.677994 & 0.108939 & -0.835694 \\
\hline 118 & 7 & $\odot$ & 0.528985 & -0.645505 & -0.998508 \\
\hline 119 & 6 & 0 & $-\odot .598052$ & -0.038939 & -1.263669 \\
\hline 120 & 7 & 0 & $-\odot .718180$ & 1.329821 & -1.358785 \\
\hline 121 & 6 & 0 & 0.356943 & 2.175650 & -1.300895 \\
\hline 122 & 7 & 0 & -1.729489 & -0.723563 & -1.485054 \\
\hline 123 & 8 & 0 & $\odot .186834$ & 3.400338 & -1.428083 \\
\hline 124 & 1 & 0 & 4.067565 & $\odot .879674$ & -1.745508 \\
\hline 125 & 1 & 0 & -1.783505 & -1.702989 & -1.246001 \\
\hline 126 & 1 & 0 & -2.610261 & $-\odot .237535$ & -1.662792 \\
\hline 127 & 1 & 0 & -1.650542 & 1.772817 & -1.469758 \\
\hline 128 & 8 & 0 & 4.939073 & 2.695705 & -1.678925 \\
\hline 129 & 1 & 0 & 4.824809 & 4.096062 & -1.811994 \\
\hline 130 & 8 & 0 & 4.656179 & 5.132217 & -1.962581 \\
\hline 131 & 1 & 0 & 3.529853 & 5.208844 & -1.666455 \\
\hline 132 & 1 & 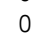 & 4.776871 & 5.317252 & -2.901299 \\
\hline 133 & 8 & 0 & 2.417000 & 5.002141 & -1.172834 \\
\hline 134 & 1 & 0 & 2.434491 & 3.974340 & -1.088290 \\
\hline 135 & 1 & 0 & 1.651099 & 5.162620 & -1.737142 \\
\hline
\end{tabular}

General basis read from cards: (6D, 7F)

1177 basis functions

315 alpha electrons

314 beta electrons

nuclear repulsion energy 17275.4813435240 Hartrees. 
NAtoms $=135$ NActive $=135$

Force inversion solution in PCM.

Polarizable Continuum Model (PCM)

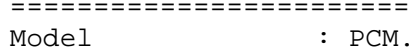

Atomic radii : UFF (Universal Force Field).

Solvent : Water, Eps $=78.355300$ Eps $($ inf $)=1.777849$

SCF Done: $E($ UWB97XD) $=-5076.20938043$ A.U. after 1 cycles NFock $=1$ Conv $=0.52 \mathrm{D}-09 \quad-\mathrm{V} / \mathrm{T}=2.0066$

$\langle\mathrm{S} x\rangle=0.0000<\mathrm{Sy}\rangle=0.0000<\mathrm{Sz}>=0.5000$

$<S^{*} 2>=0.7701 \mathrm{~S}=0.5100$

$<$ L.S $>=0.000000000000 \mathrm{E}+00$

Annihilation of the first spin contaminant:

$\mathrm{S}^{* * 2}$ before annihilation 0.7701 , after 0.7502

Harmonic frequencies $\left(\mathrm{cm}^{*}-1\right)$, IR intensities (KM/Mole), Raman

\begin{tabular}{|c|c|c|c|c|c|c|c|c|c|c|}
\hline & & & $\begin{array}{l}1 \\
A\end{array}$ & & A & & & $\begin{array}{l}3 \\
A\end{array}$ & & \\
\hline Frequ & encies & -- & -621.61 & & & 9.711 & & & 6.4940 & \\
\hline Red. & nasses & -- & 1.33 & & & 8.3997 & & & .9558 & \\
\hline Frc C & onsts & - - & 0.303 & & & 0.0005 & & & .0016 & \\
\hline IR In & Een & -- & 794.739 & & & 3.8432 & & & .7463 & \\
\hline Atom & AN & $x$ & $Y$ & Z & $x$ & $\mathrm{Y}$ & Z & $x$ & Z & \\
\hline 1 & 7 & $\odot . \odot \odot$ & $-0.0 \odot$ & $-\odot . \odot \odot$ & 0.01 & -0.02 & $-\odot .02$ & -0.01 & 0.01 & 0.06 \\
\hline 2 & 6 & -0.00 & 0.00 & $\odot . \odot \odot$ & 0.01 & -0.01 & -0.06 & $\odot .01$ & 0.01 & 0.06 \\
\hline 3 & 6 & -0.00 & -0.00 & 0.00 & 0.01 & -0.01 & -0.06 & 0.01 & 0.00 & 0.06 \\
\hline 4 & 6 & 0.00 & 0.00 & $0.0 \odot$ & 0.02 & -0.03 & $-\odot .02$ & 0.01 & -0.00 & 0.05 \\
\hline 5 & 7 & $-\odot . \odot \odot$ & $-0.0 \odot$ & $\odot .0 \odot$ & 0.02 & -0.05 & $\odot .02$ & -0.01 & $-0 . \odot \odot$ & 0.04 \\
\hline 6 & 8 & -0.00 & -0.00 & 0.00 & -0.04 & 0.02 & -0.02 & -0.04 & $\odot .04$ & 0.06 \\
\hline 7 & 15 & -0.00 & $0.0 \odot$ & -0.00 & -0.06 & 0.04 & 0.03 & -0.05 & $\odot .03$ & 0.03 \\
\hline 8 & 8 & $\odot .0 \odot$ & -0.00 & $\odot .0 \odot$ & -0.06 & 0.01 & $\odot .04$ & -0.05 & 0.01 & 0.02 \\
\hline 9 & 8 & $\odot . \odot \odot$ & 0.00 & $-\odot .0 \odot$ & -0.07 & $\odot .02$ & 0.05 & -0.07 & 0.04 & 0.03 \\
\hline 10 & 8 & $\odot .0 \odot$ & $-\odot . \odot \odot$ & -0.00 & -0.08 & 0.08 & 0.02 & -0.02 & 0.01 & 0.04 \\
\hline 11 & 8 & -0.00 & -0.00 & -0.00 & -0.09 & 0.01 & 0.12 & -0.06 & -0.02 & -0.10 \\
\hline 12 & 6 & -0.00 & 0.00 & $-0.0 \odot$ & -0.07 & -0.01 & 0.08 & -0.07 & 0.01 & -0.05 \\
\hline 13 & 6 & 0.00 & $\odot . \odot \odot$ & -0.00 & $-\odot . \odot 8$ & $\odot .02$ & $\odot .09$ & -0.05 & -0.01 & -0.06 \\
\hline 14 & 6 & -0.00 & 0.00 & -0.00 & -0.01 & 0.01 & 0.07 & -0.02 & 0.08 & -0.01 \\
\hline 15 & 6 & $\odot .00$ & -0.00 & $\odot . \odot \odot$ & $-\odot . \odot \odot$ & $-0.0 \odot$ & 0.03 & $\odot . \odot \odot$ & 0.07 & $\odot .02$ \\
\hline 16 & 8 & $-\odot . \odot \odot$ & $-0.0 \odot$ & $-0.0 \odot$ & 0.00 & 0.02 & 0.07 & -0.03 & 0.05 & -0.04 \\
\hline 17 & 8 & -0.00 & 0.00 & 0.00 & $\odot . \odot 4$ & 0.03 & -0.03 & 0.03 & 0.03 & 0.09 \\
\hline 18 & 15 & $0.0 \odot$ & -0.00 & -0.00 & 0.07 & 0.06 & -0.05 & 0.10 & -0.02 & 0.11 \\
\hline 19 & 8 & $-0.0 \odot$ & 0.00 & 0.00 & 0.08 & 0.03 & -0.07 & 0.09 & -0.00 & 0.03 \\
\hline 20 & 8 & -0.00 & $\odot . \odot \odot$ & $\odot . \odot \odot$ & $\odot . \odot 9$ & $\odot . \odot 8$ & -0.08 & 0.08 & -0.02 & 0.21 \\
\hline 21 & 8 & $-0.0 \odot$ & $\odot .0 \odot$ & $\odot . \odot \odot$ & $\odot .06$ & 0.10 & -0.03 & 0.18 & -0.06 & 0.10 \\
\hline 22 & 8 & 0.00 & $\odot . \odot \odot$ & $\odot . \odot \odot$ & 0.11 & -0.04 & -0.09 & 0.10 & $-\odot .02$ & -0.16 \\
\hline 23 & 6 & $\odot .0 \odot$ & -0.00 & -0.00 & 0.09 & -0.02 & -0.08 & 0.03 & $\odot .01$ & -0.06 \\
\hline 24 & 6 & 0.00 & -0.00 & -0.00 & 0.08 & -0.01 & -0.08 & 0.08 & $\odot .02$ & -0.10 \\
\hline 25 & 1 & $\odot .00$ & $-\odot .0 \odot$ & $\odot .00$ & 0.11 & -0.04 & -0.09 & 0.15 & -0.03 & -0.18 \\
\hline 26 & 6 & -0.00 & $0.0 \odot$ & 0.00 & 0.02 & 0.03 & -0.15 & $\odot .00$ & 0.06 & 0.10 \\
\hline 27 & 6 & $\odot .00$ & 0.00 & $\odot .00$ & 0.01 & 0.01 & -0.10 & -0.02 & 0.05 & 0.08 \\
\hline 28 & 6 & $\odot . \odot \odot$ & $\odot . \odot \odot$ & $\odot . \odot \odot$ & -0.03 & $\odot . \odot 4$ & -0.06 & -0.02 & 0.04 & 0.07 \\
\hline 29 & 6 & -0.00 & 0.00 & 0.00 & -0.04 & 0.03 & -0.03 & -0.01 & $\odot .02$ & 0.06 \\
\hline 30 & 6 & 0.00 & $\odot .0 \odot$ & $\odot .0 \odot$ & $\odot . \odot \odot$ & -0.01 & $-\odot .02$ & -0.02 & 0.02 & 0.06 \\
\hline 31 & 8 & -0.00 & 0.00 & $\odot . \odot \odot$ & $\odot .03$ & -0.03 & -0.07 & -0.02 & 0.04 & 0.07 \\
\hline 32 & 1 & $\odot .00$ & 0.00 & $\odot .0 \odot$ & 0.06 & 0.01 & -0.17 & 0.00 & 0.07 & 0.10 \\
\hline 33 & 1 & -0.00 & $\odot .0 \odot$ & $\odot . \odot \odot$ & 0.01 & 0.06 & -0.17 & $\odot . \odot \odot$ & 0.07 & 0.10 \\
\hline 34 & 1 & -0.00 & $\odot .0 \odot$ & 0.00 & $\odot .02$ & -0.00 & -0.10 & -0.03 & 0.07 & 0.08 \\
\hline 35 & 1 & $-\odot .0 \odot$ & $-\odot . \odot \odot$ & $\odot .0 \odot$ & -0.06 & 0.08 & -0.08 & -0.01 & 0.04 & 0.08 \\
\hline 36 & 1 & 0.00 & 0.00 & $\odot .00$ & -0.06 & 0.04 & $-\odot . \odot \odot$ & -0.01 & 0.01 & 0.05 \\
\hline 37 & 1 & 0.00 & 0.00 & $\odot .00$ & -0.04 & 0.05 & -0.06 & 0.01 & 0.01 & 0.07 \\
\hline 38 & 1 & $\odot .0 \odot$ & 0.00 & $\odot . \odot \odot$ & 0.01 & -0.03 & 0.01 & -0.03 & 0.02 & 0.05 \\
\hline 39 & 8 & -0.00 & $-0.0 \odot$ & $\odot . \odot \odot$ & 0.00 & 0.05 & -0.15 & $\odot .02$ & 0.04 & 0.10 \\
\hline 40 & 1 & $\odot .0 \odot$ & $\odot .0 \odot$ & $-0.0 \odot$ & $\odot .01$ & 0.06 & -0.18 & 0.03 & 0.04 & 0.11 \\
\hline 41 & 6 & $\odot . \odot \odot$ & $\odot . \odot \odot$ & $\odot . \odot \odot$ & $\odot . \odot 2$ & $-\odot . \odot 4$ & 0.02 & $-\odot .01$ & $\odot . \odot \odot$ & $\odot .05$ \\
\hline 42 & 7 & -0.00 & $-0.0 \odot$ & $-0.0 \odot$ & $\odot .02$ & -0.03 & -0.02 & 0.01 & -0.01 & 0.04 \\
\hline 43 & 8 & $\odot . \odot \odot$ & $-0.0 \odot$ & $\odot .0 \odot$ & $\odot .02$ & -0.06 & 0.05 & -0.02 & $\odot . \odot \odot$ & 0.05 \\
\hline 44 & 1 & $-\odot . \odot \odot$ & $\odot . \odot \odot$ & $\odot . \odot \odot$ & $\odot . \odot \odot$ & $\odot .01$ & $-\odot . \odot 9$ & 0.01 & 0.02 & $\odot .07$ \\
\hline 45 & 1 & $-\odot .0 \odot$ & $\odot .0 \odot$ & 0.00 & 0.01 & $\odot .00$ & $-\odot . \odot 9$ & 0.02 & $\odot . \odot \odot$ & 0.06 \\
\hline 46 & 1 & $\odot . \odot \odot$ & $-\odot . \odot \odot$ & $-\odot . \odot \odot$ & 0.01 & $-\odot . \odot 2$ & -0.05 & $\odot .02$ & $-\odot .01$ & 0.04 \\
\hline 47 & 1 & -0.00 & -0.00 & -0.00 & 0.02 & -0.04 & 0.00 & 0.01 & -0.01 & 0.02 \\
\hline 48 & 6 & -0.00 & $\odot . \odot \odot$ & -0.00 & -0.06 & -0.01 & 0.06 & -0.07 & 0.03 & -0.01 \\
\hline 49 & 1 & -0.00 & $\odot . \odot \odot$ & $-\odot . \odot \odot$ & -0.10 & 0.03 & 0.12 & -0.05 & -0.04 & -0.11 \\
\hline
\end{tabular}




\begin{tabular}{|c|c|c|c|c|c|c|c|c|c|c|}
\hline 50 & 6 & $\odot . \odot \odot$ & $-\odot . \odot \odot$ & $-\odot . \odot \odot$ & $-\odot .08$ & 0.03 & $\odot . \odot 8$ & $-\odot .03$ & $-\odot . \odot 4$ & -0.04 \\
\hline 51 & 6 & 0.00 & 0.00 & 0.00 & -0.07 & 0.01 & 0.07 & -0.03 & -0.03 & -0.05 \\
\hline 52 & 8 & $\odot .0 \odot$ & $\odot .0 \odot$ & $-0.0 \odot$ & -0.06 & -0.01 & 0.07 & -0.06 & $-\odot .0 \odot$ & -0.05 \\
\hline 53 & 1 & $-\odot . \odot \odot$ & $\odot .0 \odot$ & $\odot .0 \odot$ & -0.05 & -0.03 & $\odot .05$ & $-\odot . \odot 8$ & 0.04 & -0.01 \\
\hline 54 & 1 & 0.00 & $\odot .0 \odot$ & -0.00 & -0.06 & -0.02 & 0.06 & $-\odot .09$ & 0.05 & -0.01 \\
\hline 55 & 1 & $\odot .0 \odot$ & $\odot .0 \odot$ & -0.00 & -0.07 & -0.03 & $\odot .09$ & $-\odot .08$ & 0.03 & -0.07 \\
\hline 56 & 1 & $\odot .00$ & 0.00 & -0.00 & -0.08 & 0.03 & 0.09 & -0.05 & -0.01 & -0.04 \\
\hline 57 & 1 & $-\odot .0 \odot$ & $-0.0 \odot$ & $-\odot . \odot \odot$ & $-\odot .09$ & $\odot .04$ & 0.09 & -0.01 & -0.07 & -0.06 \\
\hline 58 & 1 & $\odot . \odot \odot$ & $-\odot . \odot \odot$ & $-\odot . \odot \odot$ & -0.07 & 0.04 & $\odot .06$ & -0.02 & -0.04 & -0.01 \\
\hline 59 & 1 & $\odot .0 \odot$ & $-\odot . \odot \odot$ & $-0.0 \odot$ & -0.08 & $\odot .0 \odot$ & $\odot .08$ & -0.03 & -0.04 & -0.08 \\
\hline 60 & 7 & $\odot .0 \odot$ & $-\odot . \odot \odot$ & -0.00 & -0.05 & 0.01 & $\odot .06$ & -0.03 & -0.04 & -0.03 \\
\hline 61 & 6 & -0.00 & 0.00 & -0.00 & -0.05 & 0.01 & 0.06 & -0.04 & -0.03 & 0.01 \\
\hline 62 & 7 & $-\odot .0 \odot$ & $-0.0 \odot$ & $\odot .0 \odot$ & -0.04 & 0.01 & $\odot .05$ & -0.04 & $-\odot . \odot 4$ & 0.03 \\
\hline 63 & 6 & $-\odot . \odot \odot$ & -0.00 & $0.0 \odot$ & -0.03 & 0.00 & 0.03 & -0.03 & -0.04 & -0.01 \\
\hline 64 & 6 & $-0.0 \odot$ & $-0.0 \odot$ & 0.00 & -0.04 & 0.00 & 0.04 & -0.02 & -0.05 & -0.04 \\
\hline 65 & 7 & $-0.0 \odot$ & $-0.0 \odot$ & $\odot .0 \odot$ & -0.04 & -0.01 & 0.03 & -0.01 & -0.05 & -0.08 \\
\hline 66 & 6 & $-\odot . \odot \odot$ & $-0.0 \odot$ & $-\odot . \odot \odot$ & -0.03 & -0.01 & 0.01 & -0.01 & -0.05 & -0.08 \\
\hline 67 & 7 & $-\odot . \odot \odot$ & $-\odot . \odot \odot$ & $-\odot . \odot \odot$ & $-\odot .02$ & -0.01 & $-0.0 \odot$ & -0.01 & -0.05 & -0.05 \\
\hline 68 & 6 & $-0 . \odot \odot$ & $\odot . \odot \odot$ & $\odot . \odot \odot$ & -0.02 & -0.01 & $\odot .01$ & -0.02 & -0.05 & -0.02 \\
\hline 69 & 7 & 0.00 & -0.00 & 0.00 & -0.01 & -0.01 & 0.01 & -0.03 & -0.04 & 0.01 \\
\hline 70 & 1 & $-\odot . \odot \odot$ & 0.00 & $-0.0 \odot$ & -0.05 & $\odot .02$ & $\odot .07$ & -0.05 & -0.03 & 0.03 \\
\hline 71 & 1 & $\odot . \odot \odot$ & $-0.0 \odot$ & $-\odot . \odot \odot$ & $-\odot .03$ & -0.02 & $-\odot .01$ & $\odot .0 \odot$ & $-\odot .06$ & -0.11 \\
\hline 72 & 1 & $\odot .0 \odot$ & $\odot .0 \odot$ & $-0.0 \odot$ & -0.02 & $-\odot .0 \odot$ & 0.02 & -0.04 & -0.04 & 0.03 \\
\hline 73 & 1 & $\odot .0 \odot$ & $-\odot .0 \odot$ & $\odot .0 \odot$ & -0.01 & -0.01 & $\odot .0 \odot$ & -0.03 & -0.04 & -0.00 \\
\hline 74 & 1 & $-0.0 \odot$ & 0.00 & 0.00 & $-\odot .0 \odot$ & 0.02 & $\odot .09$ & -0.05 & 0.05 & -0.05 \\
\hline 75 & 6 & 0.00 & $-0.0 \odot$ & -0.00 & 0.02 & 0.03 & 0.01 & 0.03 & 0.02 & 0.03 \\
\hline 76 & 6 & $\odot .0 \odot$ & $\odot . \odot \odot$ & $0.0 \odot$ & 0.02 & 0.01 & $-\odot .01$ & 0.03 & 0.01 & $-0.0 \odot$ \\
\hline 77 & 6 & $-0.0 \odot$ & 0.00 & 0.00 & -0.00 & -0.02 & -0.02 & 0.01 & 0.03 & -0.01 \\
\hline 78 & 8 & $\odot . \odot \odot$ & $-\odot .0 \odot$ & -0.00 & -0.02 & -0.03 & 0.01 & -0.01 & 0.07 & -0.00 \\
\hline 79 & 1 & $-0.0 \odot$ & $\odot .0 \odot$ & -0.00 & -0.03 & -0.01 & 0.08 & -0.04 & 0.10 & -0.02 \\
\hline 80 & 1 & -0.00 & 0.00 & 0.00 & $-\odot .0 \odot$ & 0.02 & $\odot . \odot 9$ & -0.02 & $\odot .09$ & $0.0 \odot$ \\
\hline 81 & 1 & $\odot . \odot \odot$ & $-0.0 \odot$ & $\odot . \odot \odot$ & -0.01 & -0.01 & $\odot . \odot 2$ & $\odot .01$ & 0.10 & 0.04 \\
\hline 82 & 1 & 0.00 & $-\odot .0 \odot$ & -0.00 & 0.03 & 0.06 & 0.03 & 0.04 & -0.00 & 0.02 \\
\hline 83 & 1 & $-\odot .0 \odot$ & $\odot .0 \odot$ & -0.00 & $\odot .04$ & 0.01 & -0.03 & 0.04 & -0.01 & 0.00 \\
\hline 84 & 1 & 0.01 & 0.00 & 0.00 & 0.02 & 0.02 & 0.00 & 0.02 & 0.00 & -0.02 \\
\hline 85 & 1 & $\odot .0 \odot$ & $-\odot . \odot \odot$ & $-\odot . \odot \odot$ & -0.01 & -0.02 & -0.04 & 0.02 & 0.02 & $\odot .0 \odot$ \\
\hline 86 & 7 & $-\odot . \odot \odot$ & $-0.0 \odot$ & $-\odot . \odot \odot$ & -0.01 & -0.02 & -0.02 & -0.02 & 0.01 & -0.04 \\
\hline 87 & 6 & $\odot .0 \odot$ & $\odot .0 \odot$ & 0.00 & -0.01 & -0.02 & 0.01 & -0.03 & 0.01 & -0.04 \\
\hline 88 & 6 & $\odot .0 \odot$ & 0.00 & -0.00 & -0.01 & -0.02 & 0.02 & -0.04 & -0.00 & -0.04 \\
\hline 89 & 6 & $-\odot . \odot \odot$ & $\odot .0 \odot$ & $-\odot . \odot \odot$ & -0.01 & -0.02 & 0.00 & -0.04 & -0.02 & -0.04 \\
\hline 90 & 7 & 0.00 & -0.00 & -0.00 & -0.01 & -0.03 & -0.03 & -0.03 & -0.02 & -0.05 \\
\hline 91 & 6 & $\odot .0 \odot$ & $-0.0 \odot$ & -0.00 & -0.02 & -0.03 & -0.04 & -0.01 & -0.01 & -0.05 \\
\hline 92 & 6 & $-0.0 \odot$ & 0.00 & -0.00 & -0.00 & -0.01 & 0.06 & -0.06 & $\odot .0 \odot$ & -0.05 \\
\hline 93 & 8 & 0.00 & $-0.0 \odot$ & 0.00 & -0.01 & -0.02 & 0.01 & -0.05 & -0.03 & -0.03 \\
\hline 94 & 8 & $-\odot . \odot \odot$ & $-\odot . \odot \odot$ & $-\odot . \odot \odot$ & -0.02 & -0.04 & -0.06 & $-\odot . \odot \odot$ & -0.01 & -0.06 \\
\hline 95 & 1 & $\odot .0 \odot$ & $\odot .0 \odot$ & $\odot . \odot \odot$ & $-0.0 \odot$ & -0.01 & 0.03 & -0.03 & 0.02 & -0.04 \\
\hline 96 & 1 & -0.01 & -0.02 & -0.01 & 0.01 & 0.00 & 0.05 & -0.06 & -0.01 & -0.06 \\
\hline 97 & 1 & -0.01 & 0.00 & -0.01 & -0.02 & -0.03 & 0.07 & -0.09 & 0.01 & -0.05 \\
\hline 98 & 1 & 0.00 & $\odot .0 \odot$ & 0.01 & 0.01 & $-\odot . \odot \odot$ & $\odot .08$ & -0.06 & 0.01 & -0.03 \\
\hline 99 & 1 & $\odot .0 \odot$ & $-\odot . \odot \odot$ & 0.00 & $-\odot .02$ & -0.03 & -0.03 & -0.02 & -0.03 & -0.05 \\
\hline 100 & 6 & $\odot . \odot \odot$ & $-0.0 \odot$ & $\odot .0 \odot$ & 0.09 & -0.01 & -0.08 & $\odot .03$ & $-\odot . \odot \odot$ & 0.01 \\
\hline 101 & 11 & $\odot .0 \odot$ & $-\odot . \odot \odot$ & -0.00 & -0.10 & 0.09 & 0.03 & -0.02 & $\odot . \odot \odot$ & 0.03 \\
\hline 102 & 11 & $\odot . \odot \odot$ & $-\odot . \odot \odot$ & $-\odot . \odot \odot$ & 0.07 & 0.14 & -0.07 & 0.17 & -0.10 & 0.20 \\
\hline 103 & 6 & $\odot .0 \odot$ & -0.00 & $-\odot . \odot \odot$ & 0.05 & -0.02 & -0.04 & 0.06 & 0.02 & -0.08 \\
\hline 104 & 6 & 0.00 & $-0.0 \odot$ & $-\odot . \odot \odot$ & 0.06 & -0.04 & -0.03 & 0.03 & $-0.0 \odot$ & -0.08 \\
\hline 105 & 8 & & -0.00 & -0.00 & 0.07 & -0.03 & -0.05 & 0.03 & 0.01 & -0.07 \\
\hline 106 & 1 & $\odot . \odot \odot$ & $-0.0 \odot$ & 0.00 & $\odot .09$ & -0.02 & -0.08 & -0.01 & -0.01 & 0.03 \\
\hline 107 & 1 & $\odot . \odot \odot$ & $-\odot . \odot \odot$ & $-\odot . \odot \odot$ & 0.10 & -0.00 & -0.11 & $\odot .03$ & -0.00 & $\odot . \odot 2$ \\
\hline 108 & 1 & 0.00 & 0.00 & $\odot . \odot \odot$ & 0.10 & -0.04 & -0.08 & -0.01 & 0.01 & -0.08 \\
\hline 109 & 1 & $0.0 \odot$ & $-0.0 \odot$ & $-\odot .0 \odot$ & 0.08 & 0.02 & -0.09 & 0.12 & 0.06 & -0.09 \\
\hline 110 & 1 & $-\odot . \odot \odot$ & $\odot . \odot \odot$ & $\odot .0 \odot$ & 0.04 & -0.03 & -0.03 & 0.06 & $-\odot . \odot \odot$ & -0.09 \\
\hline 11 & 1 & $0.0 \odot$ & $-0.0 \odot$ & $-\odot . \odot \odot$ & 0.02 & 0.01 & -0.05 & 0.05 & 0.04 & -0.07 \\
\hline 112 & 1 & 0.00 & 0.00 & $-\odot .0 \odot$ & 0.07 & -0.05 & -0.02 & 0.03 & $-\odot .02$ & -0.09 \\
\hline 113 & 7 & $\odot .0 \odot$ & -0.00 & -0.00 & 0.04 & -0.04 & -0.01 & 0.01 & $\odot .00$ & -0.07 \\
\hline 114 & 6 & -0.01 & 0.01 & $\odot .0 \odot$ & 0.01 & -0.02 & $-0.0 \odot$ & 0.00 & 0.01 & -0.05 \\
\hline 115 & 7 & 0.02 & -0.04 & -0.01 & 0.02 & -0.03 & 0.02 & $-\odot . \odot \odot$ & 0.00 & -0.06 \\
\hline 116 & 6 & $\odot .01$ & -0.01 & $-\odot . \odot \odot$ & 0.02 & $-\odot .04$ & 0.02 & $\odot . \odot \odot$ & $-\odot . \odot \odot$ & -0.05 \\
\hline 117 & 6 & $-\odot . \odot \odot$ & -0.01 & $\odot .0 \odot$ & 0.03 & -0.04 & 0.01 & 0.01 & $-\odot . \odot \odot$ & -0.06 \\
\hline 118 & 7 & -0.00 & -0.00 & $\odot .00$ & 0.03 & -0.04 & 0.03 & 0.01 & -0.01 & -0.05 \\
\hline 119 & 6 & $-\odot . \odot \odot$ & -0.00 & $\odot . \odot \odot$ & 0.02 & -0.05 & 0.04 & $\odot .0 \odot$ & -0.01 & -0.03 \\
\hline 120 & 7 & 0.00 & -0.00 & -0.00 & 0.02 & -0.05 & 0.04 & -0.00 & -0.01 & -0.01 \\
\hline 121 & 6 & $\odot . \odot \odot$ & 0.00 & -0.00 & $\odot .02$ & -0.05 & $\odot .02$ & -0.00 & -0.00 & -0.03 \\
\hline 122 & 7 & $-\odot . \odot \odot$ & $-\odot . \odot \odot$ & $\odot . \odot \odot$ & 0.02 & -0.05 & 0.07 & $-\odot . \odot \odot$ & -0.01 & -0.01 \\
\hline 123 & 8 & -0.01 & -0.01 & -0.00 & 0.01 & -0.05 & 0.02 & -0.01 & -0.00 & -0.03 \\
\hline 124 & 1 & $\odot .09$ & $\odot .02$ & 0.01 & $\odot .02$ & -0.02 & $-\odot .02$ & 0.01 & 0.01 & -0.03 \\
\hline 125 & 1 & -0.00 & -0.00 & -0.00 & 0.03 & -0.05 & 0.07 & 0.01 & -0.01 & -0.03 \\
\hline 126 & 1 & $\odot .0 \odot$ & 0.00 & -0.00 & $\odot .02$ & -0.06 & 0.07 & -0.01 & -0.01 & 0.01 \\
\hline
\end{tabular}




$\begin{array}{rrrrrrrrrrr}127 & 1 & 0.00 & -0.00 & 0.00 & 0.02 & -0.05 & 0.04 & -0.01 & -0.01 & 0.01 \\ 128 & 8 & -0.00 & 0.07 & -0.02 & 0.01 & -0.01 & 0.01 & -0.0 \odot & 0.01 & -0.05 \\ 129 & 1 & -0.07 & -0.4 \odot & 0.06 & 0.00 & -0.01 & 0.04 & -0.0 \odot & 0.01 & -0.06 \\ 130 & 8 & 0.01 & -0.08 & 0.01 & -0.00 & -0.01 & 0.06 & -0.01 & 0.01 & -0.07 \\ 131 & 1 & 0.79 & 0.08 & -0.27 & -0.0 \odot & -0.02 & 0.06 & -0.01 & 0.01 & -0.08 \\ 132 & 1 & -0.00 & -0.00 & 0.02 & -0.01 & 0.01 & 0.06 & 0.0 \odot & 0.01 & -0.07 \\ 133 & 8 & -0.05 & 0.07 & 0.01 & 0.0 \odot & -0.04 & 0.06 & -0.01 & 0.00 & -0.09 \\ 134 & 1 & -0.08 & 0.25 & 0.08 & 0.01 & -0.04 & 0.04 & -0.01 & 0.00 & -0.08 \\ 135 & 1 & -0.11 & -0.02 & 0.09 & -0.0 \odot & -0.03 & 0.06 & -0.01 & 0.01 & -0.09\end{array}$

Zero-point correction=

Thermal correction to Energy=

Thermal correction to Enthalpy=

1.041978 (a.u.)

1.119776

1.120720

$\odot .924355$

$-5075.167402$

$-5075.089605$

$-5075.088660$

Sum of electronic and thermal Energies=

Sum of electronic and thermal Enthalpies=

$-5075.285026$

Sum of electronic and thermal Free Energies=

$\mathrm{S}$

Total

$$
\begin{gathered}
\text { E (Thermal) } \\
\text { KCal/Mol }
\end{gathered}
$$

$\mathrm{CV}$

Cal/Mol-Kelvin Cal/Mol-Kelvin 293. 857

413.286

\begin{tabular}{lccc}
\multicolumn{1}{c}{ Item } & Value & Threshold Converged? \\
Maximum Force & $0.00 \odot \odot 18$ & $0.00 \odot 450$ & YES \\
RMS Force & $0.000 \odot \odot 2$ & $0.00 \odot 30 \odot$ & YES \\
Maximum Displacement & 0.001887 & 0.001800 & NO \\
RMS Displacement & $0.00 \odot 247$ & $0.00120 \odot$ & YES
\end{tabular}

Predicted change in Energy $=-2.360386 \mathrm{D}-\odot 8$

Normal termination of Gaussian 16 at Thu Aug 15 11:11:12 2019.

$\mathrm{G}(\mathrm{high})=-5077.0405625 \mathrm{a} \cdot \mathrm{u}$.

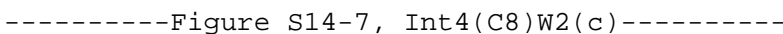

dna. for $3 c w 2 x \cdot \log$

Stoichiometry C39H54N15Na2023P2(2)

\begin{tabular}{|c|c|c|c|c|c|}
\hline \multirow{2}{*}{$\begin{array}{l}\text { Center } \\
\text { Number }\end{array}$} & \multirow{2}{*}{$\begin{array}{l}\text { Atomic } \\
\text { Number }\end{array}$} & \multirow{2}{*}{$\begin{array}{c}\text { Atomic } \\
\text { Type }\end{array}$} & \multicolumn{3}{|c|}{ Coordinates (Angstroms) } \\
\hline & & & $x$ & $\mathrm{Y}$ & Z \\
\hline & & & $---\cdots$ & - & . \\
\hline 1 & 7 & $\odot$ & -5.588880 & 2.210600 & -0.945532 \\
\hline 2 & 6 & $\odot$ & -5.793887 & 3.427793 & $-\odot .365095$ \\
\hline 3 & 6 & $\odot$ & -4.772415 & 4.297757 & -0.201277 \\
\hline 4 & 6 & $\odot$ & -3.481726 & 3.869061 & -0.648821 \\
\hline 5 & 7 & $\odot$ & -3.311465 & 2.722687 & -1.306210 \\
\hline 6 & 8 & $\odot$ & -8.619411 & -0.902743 & -0.628108 \\
\hline 7 & 15 & $\odot$ & -7.824404 & -2.211305 & -0.058480 \\
\hline 8 & 8 & $\odot$ & -6.272870 & -1.912004 & -0.531389 \\
\hline 9 & 8 & $\odot$ & -8.343862 & -3.413453 & -0.791626 \\
\hline 10 & 8 & $\odot$ & -7.805788 & -2.155839 & 1.455202 \\
\hline 11 & 8 & $\odot$ & -4.281706 & -5.664786 & ๑. . 031876 \\
\hline 12 & 6 & $\odot$ & -4.564809 & -3.596172 & -1.108446 \\
\hline 13 & 6 & $\odot$ & -5.007384 & -4.412604 & 0.106824 \\
\hline 14 & 6 & $\odot$ & 8.952272 & 1.774154 & 1.371449 \\
\hline 15 & 6 & 0 & 8.316632 & $\odot .407271$ & 1.521792 \\
\hline 16 & 8 & $\odot$ & 8.094348 & 2.600596 & ๑. 545888 \\
\hline 17 & 8 & $\odot$ & 8.093297 & -1.651993 & 0.290669 \\
\hline 18 & 15 & 0 & 7.920794 & -2.635405 & -1.003640 \\
\hline 19 & 8 & $\odot$ & 6.328477 & -2.996419 & -0.972902 \\
\hline 20 & 8 & $\odot$ & 8.753726 & -3.868498 & -0.737276 \\
\hline 21 & 8 & $\odot$ & 8.183349 & -1.888845 & -2.290985 \\
\hline 22 & 8 & $\odot$ & 2.514342 & -4.830950 & -0.791190 \\
\hline 23 & 6 & $\odot$ & 4.256643 & -3.503278 & 0.173688 \\
\hline 24 & 6 & $\odot$ & 3.538280 & -3.862667 & -1.144595 \\
\hline 25 & 1 & $\odot$ & 2.040754 & -5.123496 & -1.588339 \\
\hline 26 & 6 & $\odot$ & -9.764778 & 2.652951 & -0.602945 \\
\hline 27 & 6 & $\odot$ & -9.049505 & 1.406501 & -1.082691 \\
\hline 28 & 6 & $\odot$ & -8.643871 & 0.414981 & ๑ . 016492 \\
\hline 29 & 6 & 0 & -7.242405 & 0.878945 & ๑. 388147 \\
\hline 30 & 6 & $\odot$ & -6.666348 & 1.236246 & -0.981578 \\
\hline 31 & 8 & $\odot$ & -7.788638 & 1.781061 & -1.735173 \\
\hline 32 & 1 & $\odot$ & -9.890081 & 3.332143 & -1.452588 \\
\hline
\end{tabular}

Standard orientation: 


\begin{tabular}{|c|c|c|c|c|c|}
\hline 33 & 1 & 0 & -10.755769 & 2.375260 & -0.225313 \\
\hline 34 & 1 & $\odot$ & -9.674671 & $\odot .903744$ & -1.823975 \\
\hline 35 & 1 & 0 & -9.336844 & ๑. 388164 & $\odot .858176$ \\
\hline 36 & 1 & 0 & -6.670000 & 0.118161 & 0.912285 \\
\hline 37 & 1 & $\odot$ & -7.328535 & 1.764178 & 1.020381 \\
\hline 38 & 1 & $\odot$ & -6.276031 & $\odot .358794$ & -1.490829 \\
\hline 39 & 8 & 0 & -8.982130 & 3.283410 & 0.441048 \\
\hline 40 & 1 & $\odot$ & -9.392184 & 4.116642 & 0.724541 \\
\hline 41 & 6 & 0 & -4.341011 & 1.859026 & -1.473933 \\
\hline 42 & 7 & $\odot$ & -2.390202 & 4.605075 & -0.404431 \\
\hline 43 & 8 & $\odot$ & -4.205437 & $\odot .778023$ & -2.062539 \\
\hline 44 & 1 & 0 & -6.818506 & 3.629586 & $-\odot .058550$ \\
\hline 45 & 1 & 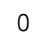 & -4.921899 & 5.261233 & $\odot .265895$ \\
\hline 46 & 1 & $\odot$ & -2.471530 & 5.514029 & 0.020480 \\
\hline 47 & 1 & 0 & -1.492936 & 4.317187 & $-\odot .795965$ \\
\hline 48 & 6 & 0 & -5.600392 & -2.606359 & -1.621645 \\
\hline 49 & 1 & $\odot$ & -4.499894 & -6.229552 & 0.792598 \\
\hline 50 & 6 & 0 & -4.543146 & -3.562466 & 1.294794 \\
\hline 51 & 6 & 0 & -3.250659 & -2.909536 & $\odot .790991$ \\
\hline 52 & 8 & $\odot$ & -3.341300 & -2.913835 & -0.664366 \\
\hline 53 & 1 & 0 & -5.110309 & -1.840354 & -2.224608 \\
\hline 54 & 1 & $\odot$ & -6.343370 & -3.139062 & -2.220022 \\
\hline 55 & 1 & $\odot$ & -4.271992 & -4.258435 & -1.925611 \\
\hline 56 & 1 & 0 & -6.087418 & -4.588292 & 0.109282 \\
\hline 57 & 1 & 0 & -4.371362 & -4.161453 & 2.191470 \\
\hline 58 & 1 & 0 & -5.297841 & -2.805167 & 1.515840 \\
\hline 59 & 1 & 0 & -2.357030 & -3.462610 & 1. 081942 \\
\hline 60 & 7 & $\odot$ & -3.053707 & -1.541801 & 1.226024 \\
\hline 61 & 6 & 0 & -3.868571 & $-\odot .473143$ & 0.929339 \\
\hline 62 & 7 & 0 & -3.329443 & 0.687680 & 1.194688 \\
\hline 63 & 6 & $\odot$ & -2.068466 & $\odot .370246$ & 1.665646 \\
\hline 64 & 6 & 0 & -1.875085 & -1.007698 & 1.683223 \\
\hline 65 & 7 & 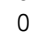 & -0.768330 & -1.669070 & 2.046753 \\
\hline 66 & 6 & 0 & $\odot .195740$ & $-\odot .830864$ & 2.400167 \\
\hline 67 & 7 & 0 & $\odot .159494$ & $\odot .509587$ & 2.406781 \\
\hline 68 & 6 & 0 & -0.951896 & 1.158884 & 2.008280 \\
\hline 69 & 7 & $\odot$ & $-\odot .927597$ & 2.500455 & 1.952979 \\
\hline 70 & 1 & 0 & -4.836755 & $-\odot .648010$ & 0.477902 \\
\hline 71 & 1 & 0 & 1.146425 & -1.257750 & 2.710596 \\
\hline 72 & 1 & $\odot$ & -1.689664 & 2.987578 & 1.506275 \\
\hline 73 & 1 & 0 & -0.026192 & 2.973925 & 2.032460 \\
\hline 74 & 1 & 0 & 8.461486 & 3.493889 & 0.446034 \\
\hline 75 & 6 & $\odot$ & 7.836605 & -0.211023 & 0.202418 \\
\hline 76 & 6 & 0 & 6.345484 & $\odot .101830$ & $\odot .162606$ \\
\hline 77 & 6 & 0 & 5.929186 & $\odot .148363$ & 1.635957 \\
\hline 78 & 8 & 0 & 7.133720 & 0.494533 & 2.379577 \\
\hline 79 & 1 & 0 & 9.081628 & 2.218998 & 2.363515 \\
\hline 80 & 1 & 0 & 9.936874 & 1.661051 & $\odot .9 \odot 2906$ \\
\hline 81 & 1 & $\odot$ & 9.030509 & -0.263108 & 2.006523 \\
\hline 82 & 1 & 0 & 8.370304 & $\odot .180802$ & -0.664323 \\
\hline 83 & 1 & $\odot$ & 5.757846 & -0.644199 & -0.372355 \\
\hline 84 & 1 & 0 & 6.215628 & 1.080145 & $-\odot .305156$ \\
\hline 85 & 1 & 0 & 5.541974 & -0.808501 & 1.978956 \\
\hline 86 & 7 & 0 & 4.893044 & 1.134048 & 1.916126 \\
\hline 87 & 6 & $\odot$ & 5.108253 & 2.458387 & 1.600416 \\
\hline 88 & 6 & $\odot$ & 4.148090 & 3.404780 & 1.669291 \\
\hline 89 & 6 & $\odot$ & 2.808413 & 2.977383 & 2.037546 \\
\hline 90 & 7 & 0 & 2.699919 & 1.663635 & 2.434578 \\
\hline 91 & 6 & $\odot$ & 3.686074 & 0.705773 & 2.445203 \\
\hline 92 & 6 & $\odot$ & 4.381381 & 4.836604 & 1.295743 \\
\hline 93 & 8 & 0 & 1.806540 & 3.702115 & 2.017070 \\
\hline 94 & 8 & $\theta$ & 3.493823 & -0.414025 & 2.893557 \\
\hline 95 & 1 & $\odot$ & 6.124314 & 2.678823 & 1.277112 \\
\hline 96 & 1 & 0 & 3.728579 & 5.126699 & $\odot .466776$ \\
\hline 97 & 1 & $\odot$ & 4.163673 & 5.503040 & 2.136842 \\
\hline 98 & 1 & $\odot$ & 5.420794 & 4.989772 & $\odot .996229$ \\
\hline 99 & 1 & 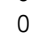 & $1.7407 \odot 2$ & 1.297230 & 2.599370 \\
\hline 100 & 6 & 0 & 5.753849 & -3.680249 & 0.179123 \\
\hline 101 & 11 & $\odot$ & -7.823987 & -3.720721 & 3.036197 \\
\hline 102 & 11 & $\odot$ & 9.934273 & -3.540301 & -2.924039 \\
\hline 103 & 6 & 0 & 2.938953 & -2.534534 & -1.612909 \\
\hline 104 & 6 & 0 & 2.755149 & -1.746992 & -0.304435 \\
\hline 105 & 8 & 0 & 3.953532 & -2.107811 & 0.446046 \\
\hline 106 & 1 & 0 & 6.168113 & -3.256487 & 1.098784 \\
\hline 107 & 1 & 0 & 6.007221 & -4.743053 & $\odot .125531$ \\
\hline 108 & 1 & 0 & 3.831026 & -4.134365 & 0.963744 \\
\hline 109 & 1 & 0 & 4.223825 & -4.290446 & -1.881629 \\
\hline
\end{tabular}




$\begin{array}{rrrrrr}110 & 1 & 0 & 2.004368 & -2.656746 & -2.162267 \\ 111 & 1 & 0 & 3.659125 & -1.998167 & -2.238531 \\ 112 & 1 & 0 & 1.871360 & -2.106336 & 0.240147 \\ 113 & 7 & 0 & 2.742988 & -0.327884 & -0.471968 \\ 114 & 6 & 0 & 3.964660 & 2.084346 & -1.460055 \\ 115 & 7 & 0 & 2.686340 & 2.458866 & -1.147177 \\ 116 & 6 & 0 & 1.583766 & 1.640345 & -1.076123 \\ 117 & 6 & 0 & 1.612075 & 0.207622 & -0.846141 \\ 118 & 7 & 0 & 0.472073 & -0.538576 & -1.050888 \\ 119 & 6 & 0 & -0.652577 & 0.075476 & -1.316116 \\ 120 & 7 & 0 & -0.770896 & 1.452098 & -1.363667 \\ 121 & 6 & 0 & 0.298624 & 2.290061 & -1.268738 \\ 122 & 7 & 0 & -1.775801 & -0.596687 & -1.581028 \\ 123 & 8 & 0 & 0.151695 & 3.520055 & -1.355344 \\ 124 & 1 & 0 & 4.118436 & 1.004419 & -1.516219 \\ 125 & 1 & 0 & -1.834018 & -1.588290 & -1.391744 \\ 126 & 1 & 0 & -2.654987 & -0.102083 & -1.752886 \\ 127 & 1 & 0 & -1.707620 & 1.902882 & -1.446412 \\ 128 & 8 & 0 & 4.857533 & 2.904125 & -1.642228 \\ 129 & 1 & 0 & 4.581555 & 4.443499 & -2.607878 \\ 130 & 8 & 0 & 4.245578 & 5.173442 & -3.159621 \\ 131 & 1 & 0 & 2.812435 & 5.415545 & -2.100653 \\ 132 & 1 & 0 & 3.981764 & 4.746989 & -3.980553 \\ 133 & 8 & 0 & 2.247638 & 5.363631 & -1.304595 \\ 134 & 1 & 0 & 2.520868 & 3.478328 & -1.119013 \\ 135 & 1 & 0 & 1.395260 & 4.998585 & -1.578591 \\ -\ldots-\ldots-\ldots & -\ldots-\ldots\end{array}$

General basis read from cards: (6D, 7F)

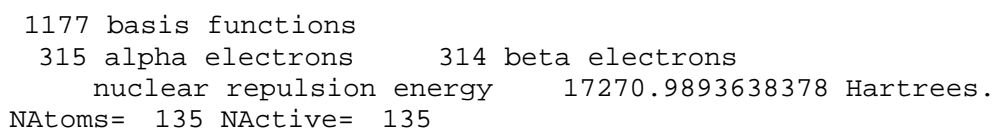

Force inversion solution in PCM.

Polarizable Continuum Model (PCM)

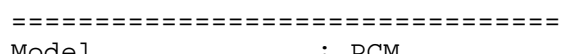

Atomic radii : UFF (Universal Force Field).

Solvent : Water, Eps $=78.355300$ Eps $($ inf $)=1.777849$

Solvent : -

SCF Done: $E($ UWB97XD $)=-5076.23597276$ A.U. after 1 cycles NFock $=1$ Conv $=0.32 \mathrm{D}-08 \quad-\mathrm{V} / \mathrm{T}=2.0066$

$\langle\mathrm{S} x\rangle=0.0000<\mathrm{Sy}\rangle=0.0000<\mathrm{S} z>=0.5000$

$<\mathrm{S} * * 2>=0.7744 \mathrm{~S}=0.5121$

$<$ L.S $>=0.000000000000 \mathrm{E}+00$

Annihilation of the first spin contaminant:

$\mathrm{S}^{* * 2}$ before annihilation 0.7744 , after 0.7502

Harmonic frequencies $\left(\mathrm{cm}^{*}-1\right)$, IR intensities (KM/Mole), Raman

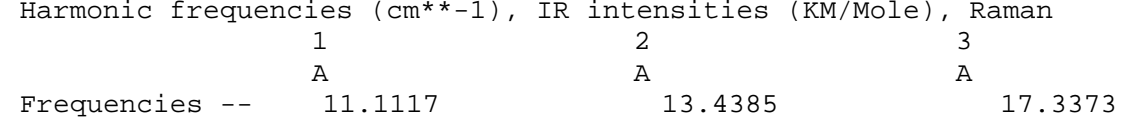

Zero-point correction=

Thermal correction to Energy=

Thermal correction to Enthalpy=
1.048383 (a.u.)
1.127758
1.128702
0.928774
$-5075.187590$
$-5075.108215$
$-5075.107271$
$-5075.307198$

Sum of electronic and thermal Energies=

Sum of electronic and thermal Enthalpies=

Sum of electronic and thermal Free Energies=

\section{$\mathrm{S}$}

$$
\begin{gathered}
\text { E (Thermal) } \\
\text { KCal/Mol }
\end{gathered}
$$

CV

Cal/Mol-Kelvin Cal/Mol-Kelvin

Total

707.679

298. 099

420.783

\begin{tabular}{lccc}
\multicolumn{1}{c}{ Item } & Value & Threshold Converged? \\
Maximum Force & $0.00 \odot \odot 22$ & $0.00 \odot 450$ & YES \\
RMS Force & $0.00 \odot \odot \odot 3$ & $\odot .0 \odot \odot 3 \odot \odot$ & YES \\
Maximum Displacement & 0.001464 & $0.00180 \odot$ & YES \\
RMS Displacement & 0.000198 & 0.001200 & YES \\
Predicted change in Energy $=-1.477774 \mathrm{D}-08$ &
\end{tabular}


optimization completed.

- - Stationary point found.

Normal termination of Gaussian 16 at Tue Aug 20 10:39:52 2019.

$G($ high $)=-5077.0680527 \mathrm{a} \cdot \mathrm{u}$.

ニニニニニニニニニニニニニニ=ニ= Figure S15 for (d) $5^{\prime}-\mathrm{GC}-3^{\prime}$ =ニ=ニ=ニ=ニニ=ニ=ニ=ニ=ニ

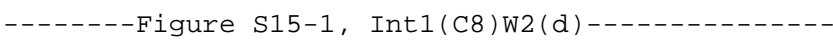

dna. for $1 d w 2 . \log$

Stoichiometry C38H53N16Na2023P2(2)

Standard orientation:

\begin{tabular}{|c|c|c|c|c|c|}
\hline Center & Atomic & Atomic & \multirow{2}{*}{\multicolumn{3}{|c|}{ 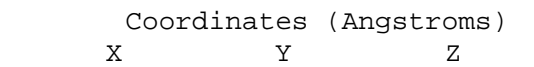 }} \\
\hline Number & Number & Type & & & \\
\hline & & & & & \\
\hline 1 & 7 & $\odot$ & 4.815367 & 2.324506 & ๑. 720241 \\
\hline 2 & 6 & $\odot$ & 4.890460 & 3.669017 & ๑. 501311 \\
\hline 3 & 6 & $\odot$ & 3.773533 & 4.414350 & ๑. 357395 \\
\hline 4 & 6 & $\odot$ & 2.518399 & 3.739927 & ๑. 494199 \\
\hline 5 & 7 & $\odot$ & 2.449557 & 2.428583 & ๑. 735844 \\
\hline 6 & 8 & $\odot$ & 8.818010 & ๑. 210408 & ๑. 863113 \\
\hline 7 & 15 & $\odot$ & 8.487108 & -1.177586 & ०. . 090208 \\
\hline 8 & 8 & $\odot$ & 6.948833 & -1.480817 & 0.537281 \\
\hline 9 & 8 & $\odot$ & 9.411376 & -2.242653 & 0.631441 \\
\hline 10 & 8 & $\odot$ & 8.459764 & $-\odot .989643$ & -1.414805 \\
\hline 11 & 8 & 0 & 6.169271 & -5.734151 & 0.118462 \\
\hline 12 & 6 & $\odot$ & 5.826423 & -3.577320 & 1.061378 \\
\hline 13 & 6 & $\odot$ & 6.593199 & -4.353458 & -0.012780 \\
\hline 14 & 6 & 0 & -7.743852 & 2.424441 & -2.891622 \\
\hline 15 & 6 & $\odot$ & -7.426635 & $\odot .962934$ & -2.653904 \\
\hline 16 & 8 & $\odot$ & -7.125235 & 3.206413 & -1.842604 \\
\hline 17 & 8 & $\odot$ & -8.213204 & -0.842694 & -1.266815 \\
\hline 18 & 15 & $\odot$ & -8.818994 & -1.575663 & ๑. . 061432 \\
\hline 19 & 8 & $\odot$ & -7.480875 & -2.212009 & $\odot .755040$ \\
\hline 20 & 8 & $\odot$ & -9.744034 & -2.670742 & -0.413004 \\
\hline 21 & 8 & $\odot$ & -9.376955 & -0.564230 & 1.035331 \\
\hline 22 & 8 & $\odot$ & -4.740491 & -4.760337 & 2.728264 \\
\hline 23 & 6 & $\odot$ & -5.442014 & -3.504984 & 0.821789 \\
\hline 24 & 6 & $\odot$ & -5.604892 & -3.660428 & 2.345148 \\
\hline 25 & 1 & $\odot$ & -4.812622 & -4.931931 & 3.682680 \\
\hline 26 & 6 & $\odot$ & 8.521350 & 3.777111 & 1.810817 \\
\hline 27 & 6 & $\odot$ & 8.158773 & 2.306232 & 1.821954 \\
\hline 28 & 6 & $\odot$ & 8.407084 & 1.571904 & $\odot .497574$ \\
\hline 29 & 6 & $\odot$ & 7.055835 & 1.651621 & -0.207983 \\
\hline 30 & 6 & $\odot$ & 6.044549 & 1.570149 & $\odot .936052$ \\
\hline 31 & 8 & $\odot$ & 6.730383 & 2.128971 & 2.098611 \\
\hline 32 & 1 & $\odot$ & 8.132616 & 4.244821 & 2.721156 \\
\hline 33 & 1 & $\odot$ & 9.612976 & 3.873653 & 1.801845 \\
\hline 34 & 1 & $\odot$ & 8.711897 & 1.816024 & 2.625947 \\
\hline 35 & 1 & $\odot$ & 9.214138 & 2. 012517 & -0.088334 \\
\hline 36 & 1 & $\odot$ & 6.908156 & 0.871495 & -0.948062 \\
\hline 37 & 1 & $\odot$ & 7.001506 & 2.620254 & -0.708655 \\
\hline 38 & 1 & $\odot$ & 5.750789 & $\odot .544290$ & 1.141994 \\
\hline 39 & 8 & $\odot$ & 7.953207 & 4.400070 & $\odot .631595$ \\
\hline 40 & 1 & $\odot$ & 8.170067 & 5.345885 & $\odot .598400$ \\
\hline 41 & 6 & $\odot$ & 3.576214 & 1.681261 & $\odot .858604$ \\
\hline 42 & 7 & $\odot$ & 1.373078 & 4.420315 & $\odot .396490$ \\
\hline 43 & 8 & $\odot$ & 3.542114 & $\odot .467770$ & 1.098573 \\
\hline 44 & 1 & $\odot$ & 5.896563 & 4.084871 & $\odot .475504$ \\
\hline 45 & 1 & $\odot$ & 3.827527 & 5.480485 & ๑. 184506 \\
\hline 46 & 1 & $\odot$ & 1.397136 & 5.422293 & $\odot .303690$ \\
\hline 47 & 1 & $\odot$ & ๑. 488791 & 3.971323 & ๑. 648210 \\
\hline 48 & 6 & $\odot$ & 6.548686 & -2.365847 & 1. 619101 \\
\hline 49 & 1 & $\odot$ & 6.598474 & -6.285097 & -0.558033 \\
\hline 50 & 6 & $\odot$ & 6.114737 & -3.736280 & -1.332557 \\
\hline 51 & 6 & $\odot$ & 4.686358 & -3.260871 & -1.030542 \\
\hline 52 & 8 & $\odot$ & 4.576632 & -3.175200 & $\odot .421405$ \\
\hline 53 & 1 & $\odot$ & 5.873019 & -1.798859 & 2.261184 \\
\hline 54 & 1 & $\odot$ & 7.425506 & -2.681799 & 2.190350 \\
\hline 55 & 1 & $\odot$ & 5.570103 & -4.245985 & 1.886998 \\
\hline 56 & 1 & $\odot$ & 7.677048 & -4.267236 & 0.115474 \\
\hline 57 & 1 & $\odot$ & 6.132119 & -4.460306 & -2.149818 \\
\hline
\end{tabular}




\begin{tabular}{|c|c|c|c|c|c|}
\hline 58 & 1 & $\odot$ & 6.765115 & -2.902621 & -1.598964 \\
\hline 59 & 1 & $\odot$ & 3.929601 & -3.972126 & -1.363597 \\
\hline 60 & 7 & $\odot$ & 4.340646 & -1.983201 & -1.618188 \\
\hline 61 & 6 & 0 & 5.145242 & -0.880291 & -1.845925 \\
\hline 62 & 7 & $\odot$ & 4.471775 & $\odot .190997$ & -2.159567 \\
\hline 63 & 6 & $\odot$ & 3.147435 & -0.210767 & -2.120310 \\
\hline 64 & 6 & $\odot$ & 3.048560 & -1.551931 & -1.786557 \\
\hline 65 & 7 & $\odot$ & 1.943338 & -2.309347 & -1.652583 \\
\hline 66 & 6 & $\odot$ & 0.832163 & -1.616318 & -1.844670 \\
\hline 67 & 7 & 0 & $\odot .814942$ & -0.275677 & -2.137707 \\
\hline 68 & 6 & $\odot$ & 1.943640 & $\odot .532349$ & -2.283243 \\
\hline 69 & 8 & 0 & 1.822679 & 1.742854 & -2.518185 \\
\hline 70 & 1 & $\odot$ & 6.222376 & -0.944515 & -1.774483 \\
\hline 71 & 7 & $\odot$ & $-\odot .359721$ & -2.226811 & -1.764640 \\
\hline 72 & 1 & $\odot$ & -0.101841 & $\odot .213565$ & -2.154499 \\
\hline 73 & 1 & $\odot$ & -7.285405 & 4.155178 & -1.970066 \\
\hline 74 & 6 & $\odot$ & -7.637866 & $\odot .505508$ & -1.206130 \\
\hline 75 & 6 & 0 & -6.240968 & 0.498427 & $-\odot .590815$ \\
\hline 76 & 6 & $\odot$ & -5.293033 & $\odot .274231$ & -1.772278 \\
\hline 77 & 8 & $\odot$ & -6.021685 & $\odot .694882$ & -2.961609 \\
\hline 78 & 1 & $\odot$ & -7.363501 & 2.718220 & -3.875534 \\
\hline 79 & 1 & $\odot$ & -8.831658 & 2.560308 & -2.878081 \\
\hline 80 & 1 & $\odot$ & -8.035079 & $\odot .353175$ & -3.326176 \\
\hline 81 & 1 & 0 & -8.328030 & 1.148050 & $-\odot .658539$ \\
\hline 82 & 1 & $\odot$ & -6.128521 & -0.296768 & 0.145971 \\
\hline 83 & 1 & $\odot$ & -6.050884 & 1.460007 & -0.109884 \\
\hline 84 & 1 & $\odot$ & -5.002866 & -0.768273 & -1.879313 \\
\hline 85 & 7 & $\odot$ & -4.046776 & 1.035091 & -1.701227 \\
\hline 86 & 6 & $\odot$ & -4.097572 & 2.393788 & -1.573808 \\
\hline 87 & 6 & $\odot$ & -2.971414 & 3.139482 & -1.607521 \\
\hline 88 & 6 & $\odot$ & -1.736270 & 2.451936 & -1.819971 \\
\hline 89 & 7 & $\odot$ & -1.693303 & 1.121762 & -1.955641 \\
\hline 90 & 6 & $\odot$ & -2.825595 & $\odot .379290$ & -1.915579 \\
\hline 91 & 1 & $\odot$ & -3.008046 & 4.214035 & -1.493438 \\
\hline 92 & 7 & $\odot$ & $-\odot .586599$ & 3.119453 & -1.907995 \\
\hline 93 & 8 & $\odot$ & -2.811133 & $-\odot .850448$ & -2.063773 \\
\hline 94 & 1 & $\odot$ & -5.091176 & 2.819813 & -1.464974 \\
\hline 95 & 1 & $\odot$ & $-\odot .570006$ & 4.114614 & -1.762550 \\
\hline 96 & 1 & $\odot$ & $\odot .292827$ & 2.625686 & -2.094950 \\
\hline 97 & 1 & $\odot$ & $-\odot .362131$ & -3.178393 & -1.438674 \\
\hline 98 & 1 & 0 & -1.243805 & -1.714219 & -1.840272 \\
\hline 99 & 6 & 0 & -6.721333 & -3.246772 & 0.066268 \\
\hline 100 & 11 & 0 & 9.962918 & -2.953604 & -1.750140 \\
\hline 101 & 11 & 0 & -11.570532 & -1.632426 & 1.071632 \\
\hline 102 & 6 & 0 & -5.095729 & -2.327798 & 2.902334 \\
\hline 103 & 6 & 0 & -3.979442 & -1.988387 & 1.918337 \\
\hline 104 & 8 & 0 & -4.534141 & -2.371349 & 0.623701 \\
\hline 105 & 1 & $\odot$ & -6.489765 & -2.915855 & -0.950584 \\
\hline 106 & 1 & 0 & -7.320844 & -4.160555 & 0.021058 \\
\hline 107 & 1 & 0 & -4.968477 & -4.407819 & $\odot .425084$ \\
\hline 108 & 1 & 0 & -6.640040 & -3.862968 & 2.630668 \\
\hline 109 & 1 & 0 & -4.746250 & -2.402752 & 3.933131 \\
\hline 110 & 1 & 0 & -5.899002 & -1.589783 & 2.841004 \\
\hline 111 & 1 & 0 & -3.081980 & -2.581269 & 2.113313 \\
\hline 112 & 7 & 0 & -3.571102 & $-\odot .610639$ & 1.866946 \\
\hline 113 & 6 & 0 & -4.340997 & 0.524189 & 2.026274 \\
\hline 114 & 7 & 0 & -3.662968 & 1.623754 & 1.859765 \\
\hline 115 & 6 & 0 & -2.377788 & 1.206399 & 1.564328 \\
\hline 116 & 6 & 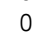 & -2.300776 & $-\odot .179351$ & 1.568883 \\
\hline 117 & 7 & 0 & -1.222901 & $-\odot .958493$ & 1.391986 \\
\hline 118 & 6 & 0 & -0.112336 & $-\odot .258456$ & 1.206367 \\
\hline 119 & 7 & 0 & $-\odot .085563$ & 1.115390 & 1.149903 \\
\hline 120 & 6 & 0 & -1.183641 & 1.938070 & 1.335944 \\
\hline 121 & 7 & 0 & 1.064222 & $-\odot .880060$ & 1.079018 \\
\hline 122 & 8 & 0 & -1.045129 & 3.177266 & 1.294313 \\
\hline 123 & 1 & 0 & -5.394986 & $\odot .496338$ & 2.269312 \\
\hline 124 & 1 & 0 & 1.059045 & -1.885812 & 1.052925 \\
\hline 125 & 1 & 0 & 1.955966 & -0.376112 & 1.015095 \\
\hline 126 & 1 & 0 & $\odot .828136$ & 1.587193 & $\odot .982479$ \\
\hline 127 & 8 & 0 & -5.311310 & 3.341336 & 1.130700 \\
\hline 128 & 1 & 0 & -4.523056 & 3.856846 & 1.448634 \\
\hline 129 & 8 & 0 & -3.151617 & 4.823272 & 1.874905 \\
\hline 130 & 1 & 0 & -3.142417 & 4.821872 & 2.836791 \\
\hline 131 & 1 & 0 & -2.419055 & $4.217 \odot 23$ & 1.630286 \\
\hline 132 & 1 & 0 & -6.449286 & 2.529152 & 2.248478 \\
\hline 133 & 8 & 0 & -7.038002 & 1.937048 & 2.758083 \\
\hline 134 & 1 & $\odot$ & -6.707283 & 1.999504 & 3.658468 \\
\hline
\end{tabular}


General basis read from cards: (6D, 7F)

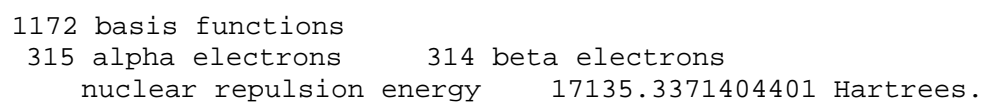

\begin{tabular}{|c|c|c|c|c|c|}
\hline \multirow{3}{*}{$\begin{array}{l}\text { Center } \\
\text { Number }\end{array}$} & \multirow{2}{*}{$\begin{array}{l}\text { Atomic } \\
\text { Number }\end{array}$} & \multirow{2}{*}{$\begin{array}{c}\text { Atomic } \\
\text { Type }\end{array}$} & \multicolumn{3}{|c|}{ Coordinates (Angstroms) } \\
\hline & & & $x$ & $\mathrm{Y}$ & Z \\
\hline & & 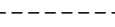 & -1 & - & ------ \\
\hline 1 & 7 & $\odot$ & 4.540269 & 2.547064 & 0.311219 \\
\hline 2 & 6 & $\odot$ & 4.705011 & 3.812656 & -0.172723 \\
\hline 3 & 6 & $\odot$ & 3.643755 & 4.616228 & -0.400632 \\
\hline 4 & 6 & $\odot$ & 2.355828 & 4.110167 & -0.039883 \\
\hline 5 & 7 & $\odot$ & 2.196976 & 2.870946 & 0.428926 \\
\hline 6 & 8 & $\odot$ & 8.477010 & $\odot .341518$ & $\odot .870672$ \\
\hline 7 & 15 & $\odot$ & 8.425616 & -1.158155 & 0.247776 \\
\hline 8 & 8 & $\odot$ & 6.917132 & -1.642226 & 0.629548 \\
\hline 9 & 8 & $\odot$ & 9.444465 & -2.005644 & 0.973198 \\
\hline 10 & 8 & 0 & 8.508854 & -1.128428 & -1.266683 \\
\hline 11 & 8 & $\odot$ & 6.817337 & -5.974970 & 0.700432 \\
\hline
\end{tabular}




\begin{tabular}{|c|c|c|c|c|c|}
\hline 12 & 6 & $\odot$ & 6.098139 & -3.810421 & 1.376808 \\
\hline 13 & 6 & $\Theta$ & 7.018651 & -4.567590 & 0.417975 \\
\hline 14 & 6 & $\odot$ & -7.225994 & 1.301696 & -3.847425 \\
\hline 15 & 6 & $\odot$ & -6.896884 & -0.054492 & -3.257521 \\
\hline 16 & 8 & $\odot$ & -6.821320 & 2.333365 & -2.913342 \\
\hline 17 & 8 & $\odot$ & -7.737026 & -1.643345 & -1.644864 \\
\hline 18 & 15 & $\odot$ & -8.628755 & -2.135368 & -0.368896 \\
\hline 19 & 8 & $\odot$ & -7.570899 & -2.047579 & 0.875295 \\
\hline 20 & 8 & $\Theta$ & -9.023697 & -3.567872 & -0.636897 \\
\hline 21 & 8 & $\odot$ & -9.735337 & -1.151053 & -0.067300 \\
\hline 22 & 8 & 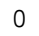 & -5.625116 & -2.850168 & 4.445530 \\
\hline 23 & 6 & $\Theta$ & -5.527351 & -2.528151 & 2.076864 \\
\hline 24 & 6 & $\odot$ & -6.221348 & -2.068256 & 3.379160 \\
\hline 25 & 1 & $\odot$ & -6.081738 & -2.678909 & 5.286678 \\
\hline 26 & 6 & $\Theta$ & 8.329242 & 4.024400 & 0.906354 \\
\hline 27 & 6 & $\odot$ & 7.910387 & 2.621206 & 1.292669 \\
\hline 28 & 6 & $\Theta$ & 8.081030 & 1.574751 & 0.185288 \\
\hline 29 & 6 & $\odot$ & 6.700012 & 1.499844 & -0.460536 \\
\hline 30 & 6 & $\odot$ & 5.731600 & 1.783831 & 0.689403 \\
\hline 31 & 8 & $\Theta$ & 6.487698 & 2.581732 & 1.647574 \\
\hline 32 & 1 & $\odot$ & 7.976154 & 4.725579 & 1.669338 \\
\hline 33 & 1 & $\Theta$ & 9.423364 & 4.068508 & $\odot .863589$ \\
\hline 34 & 1 & $\Theta$ & 8.473725 & 2.318258 & 2.177650 \\
\hline 35 & 1 & $\odot$ & 8.861766 & 1.834963 & -0.530391 \\
\hline 36 & 1 & $\odot$ & 6.490607 & $\odot .548946$ & -0.942049 \\
\hline 37 & 1 & $\Theta$ & 6.639259 & 2.286265 & -1.216096 \\
\hline 38 & 1 & $\odot$ & 5.379707 & $\odot .872880$ & 1.169466 \\
\hline 39 & 8 & $\odot$ & 7.764257 & 4.347315 & -0.389118 \\
\hline 40 & 1 & $\odot$ & 8.023483 & 5.239407 & $-\odot .671275$ \\
\hline 41 & 6 & $\odot$ & 3.261400 & 2.042856 & ๑.589894 \\
\hline 42 & 7 & $\Theta$ & 1.267809 & 4.879840 & -0.164310 \\
\hline 43 & 8 & $\Theta$ & 3.137353 & 0.876264 & 0.985472 \\
\hline 44 & 1 & $\Theta$ & 5.735867 & 4.123865 & -0.336699 \\
\hline 45 & 1 & $\Theta$ & 3.771941 & 5.618854 & -0.784812 \\
\hline 46 & 1 & $\odot$ & 1.378364 & 5.850657 & -0.407632 \\
\hline 47 & 1 & $\odot$ & ๑.391033 & 4.588829 & $\odot .275130$ \\
\hline 48 & 6 & $\odot$ & 6.593018 & -2.442428 & 1.801855 \\
\hline 49 & 1 & $\odot$ & 7.369565 & -6.519588 & 0.114108 \\
\hline 50 & 6 & 0 & 6.508020 & -4.183707 & -0.980240 \\
\hline 51 & 6 & 0 & 5.029674 & -3.812205 & -0.769337 \\
\hline 52 & 8 & $\odot$ & 4.828664 & -3.700572 & 0.665477 \\
\hline 53 & 1 & $\odot$ & 5.804474 & -1.916861 & 2.341803 \\
\hline 54 & 1 & $\odot$ & 7.474348 & -2.546012 & 2.439903 \\
\hline 55 & 1 & $\odot$ & 5.912596 & -4.412820 & 2. 269171 \\
\hline 56 & 1 & 0 & 8.068863 & -4.291331 & 0.557759 \\
\hline 57 & 1 & $\odot$ & 6.614871 & -5.010498 & -1.685151 \\
\hline 58 & 1 & $\odot$ & 7.089596 & -3.341001 & -1.351557 \\
\hline 59 & 1 & 0 & 4.346113 & -4.589205 & -1.113014 \\
\hline 60 & 7 & $\odot$ & 4.624928 & -2.572828 & -1.419554 \\
\hline 61 & 6 & $\odot$ & 5.389664 & -1.548054 & -1.943457 \\
\hline 62 & 7 & 0 & 4.684677 & -0.504016 & -2.286097 \\
\hline 63 & 6 & $\odot$ & 3.386514 & -0.840610 & -1.956091 \\
\hline 64 & 6 & $\odot$ & 3.330049 & -2.117052 & -1.421911 \\
\hline 65 & 7 & 0 & 2.251844 & -2.788614 & -0.964888 \\
\hline 66 & 6 & $\odot$ & 1.148187 & -2.071257 & -1.031953 \\
\hline 67 & 7 & $\odot$ & 1.096759 & -0.794354 & -1.524678 \\
\hline 68 & 6 & $\odot$ & 2.191204 & -0.064763 & -1.987777 \\
\hline 69 & 8 & $\odot$ & 2.055264 & 1.111449 & -2.348806 \\
\hline 70 & 1 & 0 & 6.467040 & -1.604992 & -2.018503 \\
\hline 71 & 7 & 0 & -0.010987 & -2.561594 & -0.526813 \\
\hline 72 & 1 & $\odot$ & 0.188288 & -0.287692 & -1.504711 \\
\hline 73 & 1 & $\odot$ & -6.992853 & 3.217139 & -3.276809 \\
\hline 74 & 6 & $\odot$ & -7.352568 & -0.235268 & -1.805481 \\
\hline 75 & 6 & $\odot$ & -6.115298 & $\odot .092307$ & -0.987218 \\
\hline 76 & 6 & 0 & -4.955865 & -0.354684 & -1.873987 \\
\hline 77 & 8 & 0 & -5.446232 & -0.257787 & -3.244820 \\
\hline 78 & 1 & $\odot$ & -6.698259 & 1.412941 & -4.800322 \\
\hline 79 & 1 & $\odot$ & -8.305135 & 1.358373 & -4.032214 \\
\hline 80 & 1 & $\odot$ & -7.335633 & -0.831378 & -3.888238 \\
\hline 81 & 1 & 0 & -8.208341 & 0.391973 & -1.550450 \\
\hline 82 & 1 & 0 & -6.091314 & -0.395718 & -0.014686 \\
\hline 83 & 1 & 0 & -6.076211 & 1.167343 & -0.819118 \\
\hline 84 & 1 & $\odot$ & -4.644763 & -1.378801 & -1.680335 \\
\hline 85 & 7 & 0 & -3.751389 & 0.464335 & -1.752784 \\
\hline 86 & 6 & $\odot$ & -3.833671 & 1.811250 & -1.953424 \\
\hline 87 & 6 & $\odot$ & -2.729680 & 2.591844 & -1.930887 \\
\hline 88 & 6 & $\odot$ & -1.472444 & 1.934655 & -1.752339 \\
\hline
\end{tabular}




\begin{tabular}{|c|c|c|c|c|c|}
\hline 89 & 7 & $\Theta$ & -1.397887 & 0.614381 & -1.554183 \\
\hline 90 & 6 & $\odot$ & -2.510354 & $-\odot .159409$ & -1.556315 \\
\hline 91 & 1 & $\odot$ & -2.794379 & 3.658690 & -2.093968 \\
\hline 92 & 7 & $\odot$ & -0.327996 & 2.618027 & -1.786276 \\
\hline 93 & 8 & $\odot$ & -2.460299 & -1.386710 & -1.398915 \\
\hline 94 & 1 & $\odot$ & -4.829270 & 2.192272 & -2.166799 \\
\hline 95 & 1 & $\odot$ & -0.333929 & 3.611632 & -1.946487 \\
\hline 96 & 1 & $\odot$ & $\odot .564048$ & 2.117092 & -1.834565 \\
\hline 97 & 1 & $\odot$ & -0.004321 & -3.551938 & -0.337895 \\
\hline 98 & 1 & $\odot$ & -0.901221 & -2.149476 & $-\odot .828891$ \\
\hline 99 & 6 & $\odot$ & -6.450025 & -2.971111 & 0.969073 \\
\hline 100 & 11 & $\odot$ & 10.298787 & -2.779067 & -1.344397 \\
\hline 101 & 11 & $\odot$ & -11.459949 & -2.885313 & $-\odot .322896$ \\
\hline 102 & 6 & $\odot$ & -5.856397 & $-\odot .580823$ & 3.489195 \\
\hline 103 & 6 & $\odot$ & -4.495912 & -0.561694 & 2.800791 \\
\hline 104 & 8 & $\odot$ & -4.723047 & -1.386822 & 1.621613 \\
\hline 105 & 1 & $\odot$ & -5.905152 & -2.994158 & ๑. 020184 \\
\hline 106 & 1 & $\odot$ & -6.833189 & -3.972149 & 1.185735 \\
\hline 107 & 1 & $\odot$ & -4.851432 & -3.352795 & 2.327384 \\
\hline 108 & 1 & $\odot$ & -7.301386 & -2.223545 & 3.344273 \\
\hline 109 & 1 & $\odot$ & -5.825485 & $-\odot .237288$ & 4.524703 \\
\hline 110 & 1 & $\odot$ & -6.572302 & $\odot .021712$ & 2.918940 \\
\hline 111 & 1 & $\odot$ & -3.733423 & -1.046034 & 3.420768 \\
\hline 112 & 7 & $\odot$ & -3.982312 & 0.731736 & 2.422412 \\
\hline 113 & 6 & $\odot$ & -4.588628 & 1.964597 & 2.532514 \\
\hline 114 & 7 & $\odot$ & -3.734612 & 2.966093 & 2.343522 \\
\hline 115 & 6 & $\odot$ & -2.565628 & $2.36 \odot 495$ & 1.996810 \\
\hline 116 & 6 & $\odot$ & -2.675241 & $\odot .971071$ & 2.069715 \\
\hline 117 & 7 & $\odot$ & -1.720488 & 0.059010 & 1.892380 \\
\hline 118 & 6 & $\odot$ & -0.541205 & $\odot .604258$ & 1.588462 \\
\hline 119 & 7 & $\odot$ & -0.354078 & $1.95 \odot 421$ & 1.387550 \\
\hline 120 & 6 & $\odot$ & -1.341636 & 2.913439 & 1.514027 \\
\hline 121 & 7 & $\odot$ & $\odot .535720$ & $-\odot .171541$ & 1.483293 \\
\hline 122 & 8 & $\odot$ & -1.132497 & 4.095496 & 1.200102 \\
\hline 123 & 1 & $\odot$ & -5.590447 & 2.066450 & 2.918367 \\
\hline 124 & 1 & $\odot$ & $\odot .372922$ & -1.166991 & 1.427799 \\
\hline 125 & 1 & $\odot$ & 1.458655 & $\odot .207445$ & 1.241230 \\
\hline 126 & 1 & $\odot$ & 0.568848 & 2.273274 & 1.023188 \\
\hline 127 & 8 & $\odot$ & -5.369662 & 2.555858 & $\odot .555472$ \\
\hline 128 & 1 & $\odot$ & -4.915960 & $3.4104 \odot 2$ & $\odot .413206$ \\
\hline 129 & 8 & $\odot$ & -3.816296 & 5.113617 & $\odot .518777$ \\
\hline 130 & 1 & $\odot$ & -3.980871 & 4.833328 & 1.431509 \\
\hline 131 & 1 & $\odot$ & -2.883815 & 4.861378 & 0.429964 \\
\hline 132 & 1 & $\odot$ & -6.975309 & 2.145936 & 1.203073 \\
\hline 133 & 8 & $\odot$ & -7.723355 & 1.716436 & 1.663168 \\
\hline 134 & 1 & $\odot$ & -8.151682 & 2.427039 & 2.147709 \\
\hline
\end{tabular}

General basis read from cards: (6D, 7F)

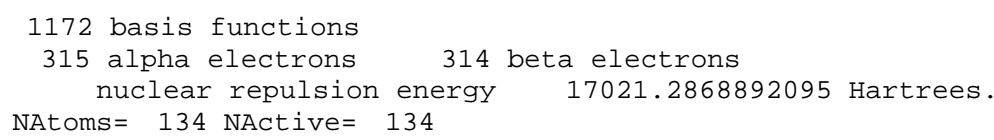

NAtoms $=134$ NActive $=134$

Force inversion solution in PCM.

Polarizable Continuum Model (PCM)

$\begin{array}{ll}\text { Model } & \text { : PCM. }\end{array}$

Solvent : Water, Eps $=78.355300$ Eps $($ inf $)=1.777849$

SCF Done: $\mathrm{E}($ UwB97XD) $=-5092.22368129$ A.U. after 1 cycles

SCF Done: $E(U W B 97 X D)=-5092.22368129$ A.U. after 1 cycles
NFock $=1$ Conv $=0.30 \mathrm{D}-09 \quad-\mathrm{V} / \mathrm{T}=2.0066$

$\langle\mathrm{S} x\rangle=0.0000<\mathrm{S} y>=0.0000<\mathrm{S} z>=0.5000$

$<S^{* *} 2>=0.7670 \mathrm{~S}=0.5084$

$<$ L.S $>=0.000000000000 \mathrm{E}+00$

Annihilation of the first spin contaminant:

$\mathrm{S}^{* * 2}$ before annihilation 0.7670 , after 0.7501

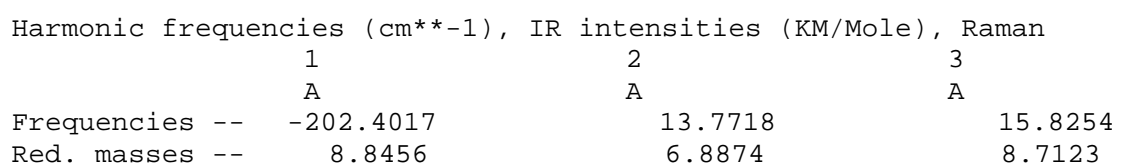

$\begin{array}{llll}\text { Red. masses - } & 8.8456 & 6.8874 & 8.7123\end{array}$ 


\begin{tabular}{|c|c|c|c|c|c|c|c|c|c|c|}
\hline \multirow{2}{*}{\multicolumn{2}{|c|}{$\begin{array}{l}\text { Frc consts } \\
\text { IR Inten }\end{array}$}} & \multirow{2}{*}{\multicolumn{3}{|c|}{$\begin{array}{r}0.2135 \\
376.8979\end{array}$}} & \multirow{2}{*}{\multicolumn{3}{|c|}{$\begin{array}{l}\odot .0008 \\
\odot .3651\end{array}$}} & \multicolumn{3}{|c|}{0.0013} \\
\hline & & & & & & & & & .8966 & \\
\hline & & $x$ & $Y$ & Z & $x$ & $Y$ & Z & $x$ & Z & \\
\hline 1 & 7 & $\odot . \odot \odot$ & $\odot .01$ & $-\odot .0 \odot$ & $-\odot . \odot \odot$ & -0.01 & $\odot .04$ & $-\odot .01$ & $\odot .01$ & 0.02 \\
\hline 2 & 6 & $-\odot . \odot \odot$ & 0.01 & $-\odot . \odot \odot$ & $\odot .01$ & $-\odot . \odot \odot$ & 0.06 & -0.02 & $\odot .0 \odot$ & $\odot .0 \odot$ \\
\hline 3 & 6 & $-\odot . \odot \odot$ & $\odot . \odot \odot$ & $\odot . \odot \odot$ & $\odot .02$ & 0.01 & $\odot .05$ & $-\odot . \odot 2$ & $\odot .01$ & $\odot .03$ \\
\hline 4 & 6 & $-\odot . \odot \odot$ & -0.00 & $\odot .0 \odot$ & 0.01 & 0.01 & $\odot .02$ & $-\odot .01$ & 0.02 & 0.07 \\
\hline 5 & 7 & $\odot . \odot \odot$ & $-\odot .0 \odot$ & $-\odot .0 \odot$ & $-\odot . \odot \odot$ & $\odot .01$ & $\odot . \odot \odot$ & -0.00 & 0.02 & ๑.๑8 \\
\hline 6 & 8 & $\odot . \odot \odot$ & $\odot . \odot \odot$ & $\odot . \odot \odot$ & $-\odot .01$ & $-\odot . \odot 4$ & $\odot .04$ & $-\odot . \odot \odot$ & $-\odot .01$ & -0.10 \\
\hline 7 & 15 & $\odot . \odot \odot$ & $\odot . \odot \odot$ & $\odot . \odot \odot$ & $\odot . \odot \odot$ & $-\odot . \odot 2$ & -0.01 & $-\odot . \odot \odot$ & $-\odot .01$ & $-\odot .09$ \\
\hline 8 & 8 & $\odot . \odot \odot$ & $\odot .0 \odot$ & $\odot . \odot \odot$ & $\odot . \odot \odot$ & $-\odot . \odot 4$ & -0.03 & 0.01 & -0.03 & -0.05 \\
\hline 9 & 8 & $\odot . \odot \odot$ & $\odot .00$ & $\odot .0 \odot$ & $\odot . \odot \odot$ & $-\odot . \odot 4$ & -0.04 & $\odot .03$ & $\odot .0 \odot$ & -0.11 \\
\hline 10 & 8 & $\odot . \odot \odot$ & $\odot . \odot \odot$ & $\odot . \odot \odot$ & $\odot .0 \odot$ & 0.03 & -0.01 & -0.04 & -0.02 & $-\odot .09$ \\
\hline 11 & 8 & $\odot .0 \odot$ & $\odot . \odot \odot$ & $\odot . \odot \odot$ & 0.05 & -0.04 & -0.16 & $\odot .01$ & $-\odot .02$ & 0.01 \\
\hline 12 & 6 & $\odot .0 \odot$ & $\odot . \odot \odot$ & $\odot . \odot \odot$ & 0.02 & $-\odot . \odot 7$ & $-\odot . \odot 9$ & 0.03 & $-\odot .01$ & $\odot .0 \odot$ \\
\hline 13 & 6 & $\odot . \odot \odot$ & $\odot . \odot \odot$ & $\odot . \odot \odot$ & 0.03 & $-\odot .03$ & -0.11 & $\odot . \odot \odot$ & -0.03 & -0.02 \\
\hline 14 & 6 & $-\odot .0 \odot$ & $-\odot . \odot \odot$ & $-\odot . \odot \odot$ & $-\odot . \odot \odot$ & $-\odot .09$ & -0.03 & 0.04 & $-\odot .01$ & $-\odot .06$ \\
\hline 15 & 6 & $-\odot .0 \odot$ & $-\odot . \odot \odot$ & 0.00 & 0.01 & $-\odot . \odot 7$ & $\odot . \odot \odot$ & 0.05 & $-\odot .01$ & -0.05 \\
\hline 16 & 8 & $-\odot .0 \odot$ & $-\odot . \odot \odot$ & $-\odot . \odot \odot$ & $-\odot .01$ & -0.06 & -0.05 & 0.02 & -0.01 & -0.05 \\
\hline 17 & 8 & $\odot .01$ & $\odot . \odot \odot$ & 0.01 & $\odot .02$ & $-\odot .05$ & ๑.०3 & $\odot .05$ & $-\odot . \odot 2$ & $-\odot .06$ \\
\hline 18 & 15 & $\odot . \odot \odot$ & $\odot . \odot \odot$ & $\odot . \odot \odot$ & 0.01 & -0.03 & 0.03 & 0.04 & $-\odot . \odot 4$ & $-\odot .08$ \\
\hline 19 & 8 & $\odot .0 \odot$ & $\odot . \odot \odot$ & $\odot . \odot \odot$ & $-\odot . \odot \odot$ & 0.01 & $\odot .04$ & $\odot .01$ & $-\odot . \odot \odot$ & -0.06 \\
\hline 20 & 8 & $\odot . \odot \odot$ & $\odot . \odot \odot$ & $\odot . \odot \odot$ & 0.03 & $-\odot .04$ & 0.06 & 0.09 & -0.05 & $-\odot . \odot 8$ \\
\hline 21 & 8 & $\odot . \odot \odot$ & $\odot . \odot \odot$ & $-\odot .0 \odot$ & $-\odot . \odot \odot$ & $-\odot .04$ & $-\odot . \odot \odot$ & 0.01 & $-\odot .07$ & -0.11 \\
\hline 22 & 8 & $-\odot .01$ & $-\odot .04$ & 0.00 & -0.05 & 0.13 & 0.09 & -0.10 & 0.03 & 0.01 \\
\hline 23 & 6 & $\odot . \odot \odot$ & $-\odot .01$ & $\odot . \odot \odot$ & $-\odot . \odot 2$ & 0.05 & $\odot . \odot 8$ & $-\odot . \odot 2$ & $\odot . \odot 3$ & 0.01 \\
\hline 24 & 6 & $-\odot . \odot \odot$ & -0.03 & 0.01 & -0.04 & 0.09 & $\odot .05$ & -0.06 & 0.03 & $-\odot .01$ \\
\hline 25 & 1 & $-\odot .0 \odot$ & -0.04 & $\odot .0 \odot$ & -0.05 & 0.16 & $\odot .08$ & -0.13 & $\odot .03$ & $-\odot .01$ \\
\hline 26 & 6 & $\odot . \odot \odot$ & 0.00 & $-\odot .0 \odot$ & $-\odot .01$ & $-\odot . \odot 4$ & 0.17 & 0.03 & -0.01 & -0.15 \\
\hline 27 & 6 & $\odot . \odot \odot$ & $\odot . \odot \odot$ & $-\odot .0 \odot$ & $-\odot .02$ & $-\odot .06$ & 0.11 & 0.03 & $\odot .0 \odot$ & -0.11 \\
\hline 28 & 6 & $\odot . \odot \odot$ & $\odot . \odot \odot$ & $-\odot .00$ & $-\odot . \odot \odot$ & -0.02 & $\odot .08$ & $-\odot .02$ & -0.02 & -0.10 \\
\hline 29 & 6 & $\odot . \odot \odot$ & $\odot . \odot \odot$ & $-\odot . \odot \odot$ & 0.01 & $\odot . \odot \odot$ & $\odot .05$ & -0.05 & $-\odot .02$ & -0.05 \\
\hline 30 & 6 & $\odot .0 \odot$ & 0.01 & -0.00 & $-\odot .02$ & $-\odot .03$ & $\odot .04$ & $-\odot . \odot \odot$ & 0.01 & $-\odot .01$ \\
\hline 31 & 8 & $\odot . \odot \odot$ & $\odot .0 \odot$ & $-\odot . \odot \odot$ & -0.03 & -0.06 & $\odot .08$ & 0.04 & $\odot .02$ & -0.06 \\
\hline 32 & 1 & $\odot . \odot \odot$ & $\odot . \odot \odot$ & $-\odot .0 \odot$ & $-\odot .03$ & $-\odot .07$ & 0.18 & $\odot .06$ & 0.01 & -0.15 \\
\hline 33 & 1 & $\odot .0 \odot$ & $\odot . \odot \odot$ & $-\odot .0 \odot$ & $-\odot .01$ & $-\odot . \odot 4$ & 0.20 & $\odot .02$ & $-\odot .02$ & -0.20 \\
\hline 34 & 1 & $\odot . \odot \odot$ & $\odot . \odot \odot$ & $-\odot . \odot \odot$ & $-\odot .04$ & $-\odot .09$ & 0.11 & $\odot .06$ & $\odot .01$ & -0.13 \\
\hline 35 & 1 & $\odot . \odot \odot$ & $-\odot . \odot \odot$ & $\odot . \odot \odot$ & 0.01 & 0.01 & 0.10 & -0.05 & -0.03 & -0.14 \\
\hline 36 & 1 & $\odot .0 \odot$ & $\odot .0 \odot$ & $-\odot . \odot \odot$ & $\odot .01$ & 0.01 & 0.02 & -0.07 & -0.03 & -0.02 \\
\hline 37 & 1 & $\odot . \odot \odot$ & $\odot .0 \odot$ & $-\odot .0 \odot$ & 0.02 & $\odot .02$ & $\odot .07$ & $-\odot . \odot 7$ & $-\odot .03$ & -0.06 \\
\hline 38 & 1 & $-\odot . \odot \odot$ & $\odot .01$ & $-\odot . \odot \odot$ & -0.03 & -0.04 & 0.01 & 0.01 & $\odot .02$ & 0.02 \\
\hline 39 & 8 & $\odot . \odot \odot$ & $\odot . \odot \odot$ & $-\odot .00$ & 0.02 & $-\odot . \odot \odot$ & 0.17 & $-\odot .02$ & -0.03 & -0.14 \\
\hline 40 & 1 & $\odot .0 \odot$ & $\odot . \odot \odot$ & $-\odot . \odot \odot$ & $\odot .03$ & $\odot .01$ & 0.20 & $-\odot .03$ & $-\odot .03$ & -0.16 \\
\hline 41 & 6 & $\odot .01$ & $\odot . \odot \odot$ & $-\odot .0 \odot$ & $-\odot .01$ & $-\odot . \odot \odot$ & $\odot .02$ & $-\odot . \odot \odot$ & $\odot .02$ & $\odot .06$ \\
\hline 42 & 7 & $-\odot .01$ & $-\odot .01$ & 0.01 & 0.02 & $\odot .01$ & $-\odot .01$ & -0.01 & 0.03 & $\odot .09$ \\
\hline 43 & 8 & 0.02 & $\odot . \odot \odot$ & -0.01 & -0.03 & $-\odot .0 \odot$ & $\odot .01$ & $-\odot . \odot \odot$ & 0.02 & $\odot .07$ \\
\hline 44 & 1 & $-\odot . \odot \odot$ & $\odot .01$ & $-\odot . \odot \odot$ & $\odot .02$ & $-\odot .01$ & $\odot .09$ & -0.02 & $-\odot .01$ & -0.04 \\
\hline 45 & 1 & $-\odot .01$ & $\odot . \odot \odot$ & $\odot . \odot \odot$ & $\odot .04$ & 0.01 & 0.07 & $-\odot .02$ & 0.01 & $\odot .01$ \\
\hline 46 & 1 & $-\odot .02$ & -0.01 & 0.01 & 0.03 & $\odot .01$ & $-\odot . \odot \odot$ & $-\odot .01$ & $\odot .02$ & $\odot .08$ \\
\hline 47 & 1 & $-\odot .01$ & $-\odot .02$ & 0.01 & 0.01 & 0.01 & -0.03 & -0.01 & $\odot .03$ & 0.10 \\
\hline 48 & 6 & $\odot .0 \odot$ & $\odot .0 \odot$ & $\odot .0 \odot$ & 0.01 & $-\odot . \odot 8$ & -0.05 & 0.05 & -0.01 & -0.03 \\
\hline 49 & 1 & $-\odot . \odot \odot$ & $\odot .0 \odot$ & $\odot . \odot \odot$ & 0.07 & -0.01 & -0.17 & -0.01 & -0.03 & $-0.0 \odot$ \\
\hline 50 & 6 & $\odot . \odot \odot$ & $-\odot . \odot \odot$ & $\odot . \odot \odot$ & 0.03 & 0.01 & -0.10 & -0.03 & -0.04 & $-\odot .01$ \\
\hline 51 & 6 & $\odot . \odot \odot$ & $\odot .0 \odot$ & $\odot .0 \odot$ & 0.02 & -0.01 & $-\odot . \odot 9$ & -0.02 & $-\odot .03$ & 0.03 \\
\hline 52 & 8 & $\odot . \odot \odot$ & $-\odot . \odot \odot$ & $\odot . \odot \odot$ & $\odot .02$ & -0.06 & $-\odot .08$ & $\odot .01$ & $-\odot .02$ & 0.03 \\
\hline 53 & 1 & $-\odot .0 \odot$ & $\odot . \odot \odot$ & $\odot . \odot \odot$ & $\odot . \odot \odot$ & -0.10 & -0.04 & $\odot .07$ & $-\odot . \odot \odot$ & $-\odot .01$ \\
\hline 54 & 1 & $-\odot . \odot \odot$ & $\odot .0 \odot$ & $\odot . \odot \odot$ & 0.01 & $-\odot . \odot 9$ & -0.05 & $\odot .07$ & -0.01 & $-\odot .06$ \\
\hline 55 & 1 & $\odot . \odot \odot$ & $\odot . \odot \odot$ & $\odot . \odot \odot$ & 0.03 & -0.10 & -0.11 & 0.06 & $-\odot .0 \odot$ & $\odot .01$ \\
\hline 56 & 1 & $\odot .0 \odot$ & $\odot .0 \odot$ & $\odot .0 \odot$ & 0.03 & $-\odot . \odot 2$ & -0.11 & $\odot .01$ & -0.03 & -0.05 \\
\hline 57 & 1 & $\odot . \odot \odot$ & $-\odot .0 \odot$ & $\odot . \odot \odot$ & 0.03 & 0.03 & -0.12 & -0.06 & -0.05 & $\odot . \odot \odot$ \\
\hline 58 & 1 & $\odot . \odot \odot$ & $-\odot . \odot \odot$ & $\odot . \odot \odot$ & $\odot .02$ & 0.02 & $-\odot . \odot 8$ & -0.04 & -0.05 & $-\odot .03$ \\
\hline 59 & 1 & $\odot . \odot \odot$ & -0.00 & $\odot . \odot \odot$ & 0,03 & $\odot . \odot \odot$ & -0.12 & -0.04 & -0.03 & $\odot .05$ \\
\hline 60 & 7 & $\odot .0 \odot$ & $-\odot . \odot \odot$ & $\odot . \odot \odot$ & 0.02 & 0.02 & $-\odot .04$ & -0.03 & -0.03 & 0.03 \\
\hline 61 & 6 & $\odot . \odot \odot$ & $-\odot . \odot \odot$ & $\odot . \odot \odot$ & $\odot .01$ & $\odot .03$ & -0.03 & -0.03 & -0.05 & $-\odot . \odot \odot$ \\
\hline 62 & 7 & $\odot . \odot \odot$ & $-\odot . \odot \odot$ & $-\odot .0 \odot$ & 0.01 & $\odot .03$ & -0.01 & -0.03 & -0.04 & 0.01 \\
\hline 63 & 6 & $\odot . \odot \odot$ & $-\odot .0 \odot$ & $-\odot . \odot \odot$ & 0.01 & 0.03 & $\odot .0 \odot$ & -0.02 & $-\odot .02$ & 0.06 \\
\hline 64 & 6 & $\odot . \odot \odot$ & $-\odot . \odot \odot$ & $-\odot . \odot \odot$ & $\odot .02$ & $\odot .02$ & $-\odot .02$ & -0.03 & $-\odot .02$ & 0.07 \\
\hline 65 & 7 & $\odot . \odot \odot$ & -0.00 & $-\odot . \odot \odot$ & 0.02 & $\odot .01$ & -0.02 & $-\odot .02$ & $\odot . \odot \odot$ & 0.10 \\
\hline 66 & 6 & $-\odot . \odot \odot$ & $-\odot . \odot \odot$ & $-\odot . \odot \odot$ & 0.02 & 0.01 & 0.01 & -0.01 & $\odot .01$ & 0.13 \\
\hline 67 & 7 & $-\odot . \odot \odot$ & $-\odot . \odot \odot$ & $-\odot . \odot \odot$ & $\odot .02$ & $\odot .02$ & $\odot . \odot 2$ & $-\odot .01$ & $\odot .01$ & 0.12 \\
\hline 68 & 6 & $-\odot . \odot \odot$ & $-\odot . \odot \odot$ & $-\odot . \odot \odot$ & 0.01 & $\odot .02$ & $\odot .01$ & $-\odot .01$ & $-\odot . \odot \odot$ & $\odot . \odot 9$ \\
\hline 69 & 8 & $\odot . \odot \odot$ & $-\odot . \odot \odot$ & $\odot . \odot \odot$ & 0.01 & 0.03 & 0.02 & $-\odot .01$ & $-\odot . \odot \odot$ & $\odot .09$ \\
\hline 70 & 1 & $\odot . \odot \odot$ & $\odot . \odot \odot$ & $\odot .0 \odot$ & 0.01 & 0.03 & -0.04 & -0.04 & -0.06 & -0.04 \\
\hline 71 & 7 & $\odot . \odot \odot$ & $-\odot . \odot \odot$ & $-\odot .01$ & 0.03 & $\odot .01$ & $\odot .02$ & -0.01 & 0.03 & 0.16 \\
\hline 72 & 1 & $\odot . \odot \odot$ & $-\odot . \odot \odot$ & $\odot .01$ & $\odot .02$ & $\odot .01$ & 0.03 & $-\odot .01$ & 0.02 & 0.13 \\
\hline 73 & 1 & $-\odot .0 \odot$ & $-\odot . \odot \odot$ & $\odot .0 \odot$ & $-\odot .01$ & -0.07 & -0.06 & 0.02 & $-\odot .01$ & -0.06 \\
\hline 74 & 6 & $-\odot . \odot \odot$ & $-\odot . \odot \odot$ & $\odot . \odot \odot$ & 0.01 & $-\odot .05$ & $\odot . \odot \odot$ & $\odot .03$ & $-\odot .02$ & $-\odot .06$ \\
\hline
\end{tabular}




\begin{tabular}{|c|c|c|c|c|}
\hline 75 & 6 & $\odot .0 \odot$ & 0.02 & $-0.0 \odot$ \\
\hline 76 & 6 & $\odot . \odot \odot$ & $\odot . \odot \odot$ & $-\odot . \odot \odot$ \\
\hline 77 & 8 & $-0.0 \odot$ & -0.00 & 0.00 \\
\hline 78 & 1 & $-\odot . \odot \odot$ & $-\odot . \odot \odot$ & $-0.0 \odot$ \\
\hline 79 & 1 & -0.00 & 0.00 & -0.00 \\
\hline 80 & 1 & -0.00 & $-0.0 \odot$ & $\odot .0 \odot$ \\
\hline 81 & 1 & $-0.0 \odot$ & $-\odot . \odot \odot$ & $\odot . \odot \odot$ \\
\hline 82 & 1 & 0.00 & 0.04 & 0.01 \\
\hline 83 & 1 & -0.02 & 0.02 & -0.06 \\
\hline 84 & 1 & -0.01 & $\odot .0 \odot$ & 0.01 \\
\hline 85 & 7 & $\odot .0 \odot$ & 0.00 & -0.01 \\
\hline 86 & 6 & 0.01 & $-\odot . \odot \odot$ & -0.04 \\
\hline 87 & 6 & 0.01 & $-\odot .0 \odot$ & -0.02 \\
\hline 88 & 6 & 0.00 & -0.00 & $-\odot .0 \odot$ \\
\hline 89 & 7 & $\odot . \odot \odot$ & $-\odot . \odot \odot$ & $-\odot . \odot \odot$ \\
\hline 90 & 6 & 0.00 & 0.00 & -0.00 \\
\hline 91 & 1 & 0.01 & -0.00 & -0.04 \\
\hline 92 & 7 & 0.00 & -0.00 & -0.00 \\
\hline 93 & 8 & $-\odot . \odot \odot$ & 0.00 & $\odot .01$ \\
\hline 94 & 1 & 0.01 & $-0.0 \odot$ & -0.07 \\
\hline 95 & 1 & 0.00 & 0.00 & 0.02 \\
\hline 96 & 1 & $\odot .00$ & 0.00 & $\odot .02$ \\
\hline 97 & 1 & $\odot .0 \odot$ & -0.00 & $-\odot . \odot \odot$ \\
\hline 98 & 1 & $-\odot . \odot \odot$ & 0.00 & 0.00 \\
\hline 99 & 6 & 0.01 & 0.01 & -0.00 \\
\hline 100 & 11 & -0.00 & 0.00 & 0.00 \\
\hline 101 & 11 & $-\odot . \odot \odot$ & $-\odot .0 \odot$ & $-\odot . \odot \odot$ \\
\hline 102 & 6 & $\odot .01$ & -0.04 & $\odot .04$ \\
\hline 103 & 6 & 0.03 & -0.06 & 0.07 \\
\hline 104 & 8 & 0.01 & $\odot .01$ & $\odot .03$ \\
\hline 105 & 1 & 0.01 & 0.02 & -0.00 \\
\hline 106 & 1 & 0.01 & 0.00 & -0.02 \\
\hline 107 & 1 & $\odot .0 \odot$ & -0.01 & -0.00 \\
\hline 108 & 1 & -0.00 & -0.02 & 0.00 \\
\hline 109 & 1 & -0.02 & -0.04 & 0.04 \\
\hline 110 & 1 & $\odot .04$ & -0.03 & 0.02 \\
\hline 111 & 1 & $-\odot . \odot \odot$ & -0.12 & 0.06 \\
\hline 112 & 7 & 0.04 & -0.05 & 0.15 \\
\hline 113 & 6 & $\odot .08$ & -0.09 & 0.35 \\
\hline 114 & 7 & -0.13 & $-\odot . \odot 8$ & -0.01 \\
\hline 115 & 6 & -0.04 & -0.05 & -0.06 \\
\hline 116 & 6 & 0.01 & -0.01 & 0.02 \\
\hline 117 & 7 & $\odot . \odot 3$ & -0.03 & $\odot .02$ \\
\hline 118 & 6 & -0.01 & -0.02 & -0.00 \\
\hline 119 & 7 & -0.04 & -0.01 & -0.01 \\
\hline 120 & 6 & -0.06 & -0.03 & -0.03 \\
\hline 121 & 7 & 0.03 & -0.00 & 0.01 \\
\hline 122 & 8 & -0.04 & -0.01 & -0.02 \\
\hline 123 & 1 & -0.06 & -0.11 & $\odot . \odot \odot$ \\
\hline 124 & 1 & 0.04 & 0.00 & $-\odot . \odot 4$ \\
\hline 125 & 1 & 0.01 & 0.01 & -0.04 \\
\hline 126 & 1 & -0.05 & 0.00 & 0.01 \\
\hline 127 & 8 & -0.01 & 0.44 & -0.39 \\
\hline 128 & 1 & 0.13 & 0.40 & -0.35 \\
\hline 129 & 8 & $\odot .00$ & -0.06 & $\odot .02$ \\
\hline 130 & 1 & $-\odot . \odot \odot$ & 0.04 & 0.05 \\
\hline 131 & 1 & 0.01 & -0.06 & 0.05 \\
\hline 132 & 1 & -0.11 & 0.14 & -0.06 \\
\hline 133 & 8 & 0.03 & 0.01 & 0.01 \\
\hline 134 & 1 & -0.09 & -0.05 & -0.01 \\
\hline
\end{tabular}

\begin{tabular}{|c|c|c|}
\hline 0.01 & -0.03 & -0.00 \\
\hline 0.01 & -0.03 & 0.01 \\
\hline 0.01 & -0.06 & 0.00 \\
\hline-0.00 & -0.10 & -0.03 \\
\hline$-\odot . \odot \odot$ & -0.10 & -0.03 \\
\hline 0.02 & -0.09 & $\odot .02$ \\
\hline$\odot .01$ & -0.05 & -0.01 \\
\hline 0.01 & -0.01 & $\odot .01$ \\
\hline$\odot .0 \odot$ & -0.02 & -0.02 \\
\hline$\odot .02$ & -0.03 & 0.02 \\
\hline 0.01 & -0.02 & -0.01 \\
\hline$-0.0 \odot$ & -0.03 & -0.04 \\
\hline-0.01 & -0.02 & -0.04 \\
\hline-0.01 & -0.00 & -0.01 \\
\hline$\odot .0 \odot$ & $\odot . \odot \odot$ & 0.01 \\
\hline$\odot .01$ & -0.01 & $\odot .02$ \\
\hline-0.02 & -0.02 & -0.07 \\
\hline-0.01 & 0.01 & -0.01 \\
\hline 0.02 & $-\odot . \odot \odot$ & 0.05 \\
\hline$-\odot . \odot \odot$ & -0.04 & -0.05 \\
\hline-0.02 & $0.0 \odot$ & -0.04 \\
\hline-0.01 & 0.01 & $\odot . \odot \odot$ \\
\hline 0.03 & $\odot .01$ & 0.01 \\
\hline 0.03 & 0.01 & 0.03 \\
\hline$-\odot . \odot \odot$ & 0.01 & 0.08 \\
\hline 0.01 & 0.05 & -0.08 \\
\hline 0.02 & -0.07 & 0.03 \\
\hline$-\odot .04$ & 0.10 & 0.01 \\
\hline$-\odot .03$ & $\odot . \odot 8$ & 0.03 \\
\hline-0.01 & 0.03 & 0.05 \\
\hline 0.01 & -0.01 & 0.09 \\
\hline-0.01 & 0.02 & 0.11 \\
\hline-0.02 & 0.06 & 0.11 \\
\hline-0.03 & $\odot .09$ & 0.05 \\
\hline-0.06 & 0.13 & -0.00 \\
\hline-0.04 & $\odot .07$ & -0.02 \\
\hline-0.04 & 0.10 & 0.05 \\
\hline-0.03 & 0.06 & -0.01 \\
\hline-0.03 & $\odot .07$ & -0.04 \\
\hline-0.02 & 0.06 & -0.06 \\
\hline$-\odot . \odot 2$ & 0.05 & -0.04 \\
\hline-0.03 & 0.05 & -0.01 \\
\hline-0.03 & 0.05 & 0.01 \\
\hline$-\odot .03$ & $\odot .03$ & $\odot . \odot \odot$ \\
\hline-0.02 & 0.03 & -0.02 \\
\hline$-\odot .02$ & 0.04 & -0.04 \\
\hline-0.03 & 0.03 & 0.01 \\
\hline-0.01 & 0.03 & -0.06 \\
\hline-0.03 & 0.08 & -0.05 \\
\hline-0.04 & 0.03 & 0.02 \\
\hline-0.03 & 0.02 & 0.01 \\
\hline-0.02 & 0.02 & -0.02 \\
\hline-0.01 & 0.03 & -0.06 \\
\hline-0.01 & 0.03 & -0.08 \\
\hline-0.01 & $\odot .02$ & -0.10 \\
\hline-0.01 & 0.04 & -0.10 \\
\hline-0.01 & 0.02 & -0.09 \\
\hline-0.02 & 0.03 & -0.08 \\
\hline-0.02 & 0.03 & -0.09 \\
\hline-0.03 & 0.03 & -0.09 \\
\hline
\end{tabular}

$0.01 \quad 0.01 \quad-0.04$

$0.03 \quad 0.01 \quad-0.01$

$0.05 \quad 0.01 \quad-0.02$

$0.06-0.01-0.05$

$0.05-0.02 \quad-0.07$

$0.07-0.01 \quad-0.06$

$0.01 \quad-0.03 \quad-0.07$

$\begin{array}{llll}-0.00 & 0.01 & -0.03\end{array}$

$\begin{array}{llll}-0.01 & 0.01 & -0.05\end{array}$

$\begin{array}{llll}0.03 & 0.01 & -0.01\end{array}$

$0.02 \quad 0.02 \quad 0.01$

$\begin{array}{llll}0.02 & 0.02 & -0.00\end{array}$

$0.01 \quad 0.02 \quad 0.02$

$\begin{array}{lll}0.01 & 0.03 & 0.07\end{array}$

$\begin{array}{llll}0.01 & 0.03 & 0.09\end{array}$

$0.01 \quad 0.03 \quad 0.06$

$0.02 \quad 0.02 \quad 0.01$

$\begin{array}{lll}0.01 & 0.03 & 0.10\end{array}$

$\begin{array}{llll}0.02 & 0.03 & 0.08\end{array}$

$\begin{array}{llll}0.02 & 0.01 & -0.04\end{array}$

$\begin{array}{lll}0.01 & 0.03 & 0.08\end{array}$

$0.01 \quad 0.03 \quad 0.11$

$\begin{array}{lll}-0.01 & 0.03 & 0.15\end{array}$

$\begin{array}{lll}-0.01 & 0.03 & 0.14\end{array}$

$\begin{array}{llll}0.02 & 0.01 & -0.02\end{array}$

$\begin{array}{lll}-0.02 & -0.03 & -0.09\end{array}$

$0.06-0.11-0.10$

$\begin{array}{lll}-0.05 & 0.03 & 0.00\end{array}$

$\begin{array}{lll}-0.04 & 0.03 & 0.03\end{array}$

$\begin{array}{lll}-0.01 & 0.04 & 0.03\end{array}$

$0.05 \quad 0.01 \quad 0.00$

$0.03 \quad 0.01 \quad-0.02$

$\begin{array}{lll}-0.01 & 0.04 & 0.04\end{array}$

$\begin{array}{llll}-0.06 & 0.04 & -0.05\end{array}$

$\begin{array}{lll}-0.08 & 0.03 & 0.00\end{array}$

$\begin{array}{lll}-0.04 & 0.04 & -0.01\end{array}$

$\begin{array}{lll}-0.05 & 0.03 & 0.05\end{array}$

$\begin{array}{lll}-0.03 & 0.03 & 0.05\end{array}$

$\begin{array}{lll}-0.03 & 0.03 & 0.02\end{array}$

$\begin{array}{lll}-0.03 & 0.03 & 0.03\end{array}$

$\begin{array}{lll}-0.02 & 0.03 & 0.06\end{array}$

$\begin{array}{lll}-0.02 & 0.03 & 0.07\end{array}$

$\begin{array}{lll}-0.01 & 0.04 & 0.09\end{array}$

$\begin{array}{lll}-0.01 & 0.04 & 0.10\end{array}$

$\begin{array}{lll}-0.01 & 0.04 & 0.10\end{array}$

$\begin{array}{lll}-0.02 & 0.03 & 0.08\end{array}$

$\begin{array}{lll}-0.01 & 0.04 & 0.12\end{array}$

$\begin{array}{lll}-0.02 & 0.03 & 0.08\end{array}$

$\begin{array}{lll}-0.04 & 0.03 & -0.00\end{array}$

$\begin{array}{lll}-0.01 & 0.04 & 0.11\end{array}$

$\begin{array}{lll}-0.01 & 0.04 & 0.11\end{array}$

$\begin{array}{lll}-0.01 & 0.04 & 0.10\end{array}$

$0.03-0.01-0.02$

$\begin{array}{llll}0.02 & -0.00 & -0.02\end{array}$

$\begin{array}{lll}-0.01 & 0.00 & -0.00\end{array}$

$\begin{array}{lll}-0.03 & 0.02 & -0.00\end{array}$

$\begin{array}{lll}-0.01 & 0.01 & 0.02\end{array}$

$0.01-0.01 \quad-0.07$

$-0.01-0.00 \quad-0.09$

Zero-point correction=

Thermal correction to Energy=

Thermal correction to Enthalpy=

Thermal correction to Gibbs Free Energy=

Sum of electronic and zero-point Energies=

Sum of electronic and thermal Energies=

Sum of electronic and thermal Enthalpies=

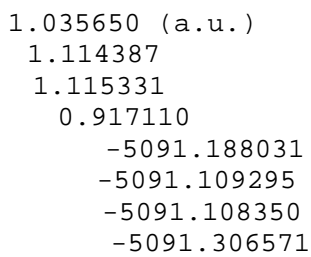

Total Maximum Force RMS Force

Value $\odot .000013$ Threshold Converged? $\odot .000002$ 0.000450 YES 0.000300 YES 
$\begin{array}{lccr}\text { Maximum Displacement } & 0.005264 & 0.001800 & \text { NO } \\ \text { RMS Displacement } & 0.000600 & 0.001200 & \text { YES }\end{array}$

Predicted change in Energy $=-1.345425 D-07$

Normal termination of Gaussian 16 at Sat Jul 13 07:24:21 2019.

$G($ high $)=-5093.0840802 \mathrm{a} \cdot \mathrm{u}$.

- Figure S15-3, Int2(C8)W2(d) - . . . .

dna. rev1dw2. log

Stoichiometry C38H53N16Na2023P2(2)

Standard orientation

\begin{tabular}{|c|c|c|c|c|c|}
\hline \multirow{2}{*}{$\begin{array}{l}\text { Center } \\
\text { Number }\end{array}$} & \multirow{2}{*}{$\begin{array}{l}\text { Atomic } \\
\text { Number }\end{array}$} & \multirow{2}{*}{$\begin{array}{c}\text { Atomic } \\
\text { Type }\end{array}$} & \multicolumn{3}{|c|}{ Coordinates (Angstroms) } \\
\hline & & & $\mathrm{x}$ & $\mathrm{Y}$ & Z \\
\hline 1 & 7 & 0 & 4.344739 & 2.536426 & 0.840621 \\
\hline 2 & 6 & 0 & 4.429552 & 3.896888 & $\odot .795716$ \\
\hline 3 & 6 & $\odot$ & 3.317572 & 4.661071 & 0.695745 \\
\hline 4 & 6 & $\odot$ & 2.060709 & 3.982034 & $\odot .713223$ \\
\hline 5 & 7 & 0 & 1.985993 & 2.651623 & 0.711513 \\
\hline 6 & 8 & $\odot$ & 8.264777 & 0.365762 & 1.309734 \\
\hline 7 & 15 & $\odot$ & 8.256792 & $-\odot .979486$ & 0.387150 \\
\hline 8 & 8 & 0 & 6.689425 & -1.437895 & $\odot .459968$ \\
\hline 9 & 8 & $\odot$ & 9.091386 & -1.992252 & 1.137978 \\
\hline 10 & 8 & 0 & 8.536776 & -0.650072 & -1.055080 \\
\hline 11 & 8 & $\odot$ & 6.389395 & -5.722182 & -0.090581 \\
\hline 12 & 6 & $\odot$ & 5.657844 & -3.620410 & 0.755924 \\
\hline 13 & 6 & 0 & 6.690195 & -4.304347 & -0.142107 \\
\hline 14 & 6 & 0 & -7.345470 & 3.052121 & -2.070576 \\
\hline 15 & 6 & $\odot$ & -7.028119 & 1.600796 & -2.359555 \\
\hline 16 & 8 & 0 & -6.458259 & 3.568328 & -1.035448 \\
\hline 17 & 8 & $\odot$ & -7.638887 & -0.584912 & -1.566984 \\
\hline 18 & 15 & $\Theta$ & -8.538675 & -1.513935 & -0.560976 \\
\hline 19 & 8 & $\odot$ & -7.399804 & -2.354956 & 0.265738 \\
\hline 20 & 8 & $\odot$ & -9.339824 & -2.438398 & -1.430160 \\
\hline 21 & 8 & $\odot$ & -9.194776 & -0.626768 & 0.475024 \\
\hline 22 & 8 & 0 & -4.745025 & -5.000662 & 2.275421 \\
\hline 23 & 6 & $\odot$ & -5.343923 & -3.613274 & 0.426906 \\
\hline 24 & 6 & 0 & -5.635739 & -3.915919 & 1.906119 \\
\hline 25 & 1 & 0 & -4.884697 & -5.251612 & 3.204490 \\
\hline 26 & 6 & 0 & 7.982144 & 3.953192 & 2.152193 \\
\hline 27 & 6 & 0 & 7.604580 & 2.487079 & 2.156406 \\
\hline 28 & 6 & 0 & 7.932122 & 1.720539 & $\odot .868871$ \\
\hline 29 & 6 & $\odot$ & 6.634866 & 1.803636 & $\odot .066373$ \\
\hline 30 & 6 & 0 & 5.548950 & 1.757192 & 1.140530 \\
\hline 31 & 8 & 0 & 6.156921 & 2.325392 & 2.336367 \\
\hline 32 & 1 & $\odot$ & 7.554007 & 4.433177 & 3.038329 \\
\hline 33 & 1 & 0 & 9.073810 & 4.037919 & 2.197280 \\
\hline 34 & 1 & 0 & 8.092817 & 2.004030 & 3.005264 \\
\hline 35 & 1 & $\odot$ & 8.786632 & 2.131821 & $\odot .329393$ \\
\hline 36 & 1 & $\Theta$ & 6.519571 & 1.011060 & -0.668717 \\
\hline 37 & 1 & 0 & 6.619720 & 2.766918 & -0.448875 \\
\hline 38 & 1 & 0 & 5.219645 & $\odot .739936$ & 1.342311 \\
\hline 39 & 8 & $\odot$ & 7.478732 & 4.572237 & $\odot .942324$ \\
\hline 40 & 1 & 0 & 7.724273 & 5.510825 & 0.902059 \\
\hline 41 & 6 & $\odot$ & 3.108162 & 1.887691 & $\odot .744554$ \\
\hline 42 & 7 & 0 & 0.905723 & 4.671092 & 0.717547 \\
\hline 43 & 8 & 0 & 3.070095 & 0.650802 & 0.706966 \\
\hline 44 & 1 & 0 & 5.437525 & 4.304912 & $\odot .859092$ \\
\hline 45 & 1 & 0 & 3.379394 & 5.740114 & $\odot .663898$ \\
\hline 46 & 1 & 0 & 0.941873 & 5.667219 & $\odot .866006$ \\
\hline 47 & 1 & $\odot$ & $\odot .047288$ & 4.185923 & $\odot .997813$ \\
\hline 48 & 6 & $\odot$ & 6.142727 & -2.357467 & 1.441166 \\
\hline 49 & 1 & 0 & 6.990058 & -6.216331 & -0.674260 \\
\hline 50 & 6 & $\odot$ & 6.432727 & -3.712242 & -1.535752 \\
\hline 51 & 6 & $\odot$ & 4.952522 & -3.297389 & -1.515764 \\
\hline 52 & 8 & 0 & 4.529020 & -3.330372 & -0.123405 \\
\hline 53 & 1 & $\odot$ & 5.301874 & -1.856765 & 1.924128 \\
\hline 54 & 1 & $\odot$ & 6.901958 & -2.607802 & 2.186996 \\
\hline 55 & 1 & $\Theta$ & 5.300779 & -4.321408 & 1.514244 \\
\hline 56 & 1 & 0 & 7.712910 & -4.114396 & 0.197603 \\
\hline 57 & 1 & 0 & 6.632600 & -4.439797 & -2.324929 \\
\hline 58 & 1 & $\odot$ & 7.089615 & -2.856400 & -1.687079 \\
\hline
\end{tabular}




\begin{tabular}{|c|c|c|c|c|c|}
\hline 59 & 1 & $\odot$ & 4.310087 & -3.999299 & -2.048446 \\
\hline 60 & 7 & $\odot$ & 4.691645 & -1.981950 & -2.075640 \\
\hline 61 & 6 & $\odot$ & 5.536729 & $-\odot .894137$ & -2.214857 \\
\hline 62 & 7 & 0 & 4.910258 & $\odot .203218$ & -2.538104 \\
\hline 63 & 6 & $\odot$ & 3.578685 & $-\odot .164598$ & -2.599849 \\
\hline 64 & 6 & $\odot$ & 3.424550 & -1.512142 & -2.320195 \\
\hline 65 & 7 & $\odot$ & 2.287460 & -2.236038 & -2.274789 \\
\hline 66 & 6 & $\odot$ & 1.211519 & -1.500609 & -2.500233 \\
\hline 67 & 7 & $\odot$ & 1.250412 & $-\odot .147487$ & -2.723653 \\
\hline 68 & 6 & $\odot$ & 2.408117 & $\odot .627758$ & -2.756511 \\
\hline 69 & 8 & $\odot$ & 2.337782 & 1.858650 & -2.888294 \\
\hline 70 & 1 & $\odot$ & 6.603805 & -0.959328 & -2.047015 \\
\hline 71 & 7 & $\odot$ & -0.004126 & -2.073069 & -2.515900 \\
\hline 72 & 1 & $\odot$ & $\odot .351896$ & $\odot .373719$ & -2.713647 \\
\hline 73 & 1 & $\odot$ & -6.393850 & 4.536651 & -1.079401 \\
\hline 74 & 6 & 0 & -7.012599 & $\odot .662405$ & -1.141998 \\
\hline 75 & 6 & $\odot$ & -5.534415 & $\odot .455876$ & -0.836393 \\
\hline 76 & 6 & $\odot$ & -4.831625 & 0.639546 & -2.181022 \\
\hline 77 & 8 & $\odot$ & -5.705325 & 1.491825 & -2.973430 \\
\hline 78 & 1 & $\odot$ & -7.211055 & 3.636218 & -2.983876 \\
\hline 79 & 1 & $\odot$ & -8.384335 & 3.138669 & -1.737774 \\
\hline 80 & 1 & $\odot$ & -7.765011 & 1.233338 & -3.078157 \\
\hline 81 & 1 & $\odot$ & -7.568440 & 1.063104 & -0.291602 \\
\hline 82 & 1 & $\odot$ & -5.328847 & -0.516622 & $-\odot .386401$ \\
\hline 83 & 1 & $\odot$ & -5.198849 & 1.229028 & -0.141133 \\
\hline 84 & 1 & $\odot$ & -4.659628 & $-\odot .298408$ & -2.704742 \\
\hline 85 & 7 & $\odot$ & -3.534227 & 1.303822 & -2.067098 \\
\hline 86 & 6 & $\odot$ & -3.493830 & 2.592812 & -1.618993 \\
\hline 87 & 6 & $\odot$ & -2.326741 & 3.261569 & -1.520047 \\
\hline 88 & 6 & $\odot$ & -1.144928 & 2.570717 & -1.941533 \\
\hline 89 & 7 & $\odot$ & -1.192940 & 1.306026 & -2.375234 \\
\hline $9 \odot$ & 6 & $\odot$ & -2.363625 & $\odot .625322$ & -2.424959 \\
\hline 91 & 1 & $\odot$ & -2.288930 & 4.278356 & -1.155715 \\
\hline 92 & 7 & $\odot$ & 0.035184 & 3.184744 & -1.931303 \\
\hline 93 & 8 & $\odot$ & -2.428120 & $-\odot .563626$ & -2.767742 \\
\hline 94 & 1 & $\odot$ & -4.456711 & 3.031349 & -1.373153 \\
\hline 95 & 1 & $\odot$ & 0.131713 & 4.086225 & -1.489123 \\
\hline 96 & 1 & $\odot$ & 0.880366 & 2.694206 & -2.244322 \\
\hline 97 & 1 & $\odot$ & -0.054713 & -3.050426 & -2.283433 \\
\hline 98 & 1 & $\odot$ & -0.865532 & -1.529983 & -2.618641 \\
\hline 99 & 6 & 0 & -6.557062 & -3.318162 & $-\odot .422592$ \\
\hline 100 & 11 & $\odot$ & 10.655976 & -3.535206 & 1.144061 \\
\hline 101 & 11 & $\odot$ & -9.495935 & -0.965817 & 2.638540 \\
\hline 102 & 6 & $\odot$ & -5.270162 & -2.615810 & 2.626818 \\
\hline 103 & 6 & $\odot$ & -4.094103 & -2.104896 & 1.797416 \\
\hline 104 & 8 & $\odot$ & -4.463776 & -2.443847 & ๑.419046 \\
\hline 105 & 1 & $\odot$ & -6.232731 & -2.908358 & -1.384237 \\
\hline 106 & 1 & $\odot$ & -7.126972 & -4.235590 & $-\odot .597851$ \\
\hline 107 & 1 & $\odot$ & -4.805665 & -4.462253 & $-\odot .005294$ \\
\hline 108 & 1 & $\odot$ & -6.678353 & -4.200800 & 2.071055 \\
\hline 109 & 1 & $\odot$ & -5.012269 & -2.774457 & 3.676081 \\
\hline 110 & 1 & $\odot$ & -6.108971 & -1.923307 & 2.550115 \\
\hline 111 & 1 & $\odot$ & -3.172708 & -2.646163 & 2.034391 \\
\hline 112 & 7 & $\odot$ & -3.832264 & $-\odot .6967 \odot 7$ & 1.878097 \\
\hline 113 & 6 & $\odot$ & -4.588410 & 0.330133 & 2.634719 \\
\hline 114 & 7 & $\odot$ & -3.873061 & 1.573300 & 2.424574 \\
\hline 115 & 6 & $\odot$ & -2.718063 & 1.233255 & 1.833857 \\
\hline 116 & 6 & $\odot$ & -2.654100 & -0.149988 & 1.509871 \\
\hline 117 & 7 & $\odot$ & -1.633575 & -0.811454 & 0.978372 \\
\hline 118 & 6 & $\odot$ & $-\odot .542383$ & $-\odot .056836$ & ๑.795911 \\
\hline 119 & 7 & $\odot$ & $-\odot .515422$ & 1.288754 & $1.0 \odot 7440$ \\
\hline 120 & 6 & $\odot$ & -1.577872 & 2.042446 & 1.513960 \\
\hline 121 & 7 & 0 & 0.586192 & -0.635077 & $\odot .391769$ \\
\hline 122 & 8 & $\odot$ & -1.461046 & 3.266034 & 1.658723 \\
\hline 123 & 1 & $\odot$ & -4.545201 & $\odot .051701$ & 3.700971 \\
\hline 124 & 1 & $\odot$ & $\odot .566609$ & -1.634181 & $\odot .266120$ \\
\hline 125 & 1 & $\odot$ & 1.489724 & -0.145880 & $\odot .419586$ \\
\hline 126 & 1 & $\odot$ & $\odot .377512$ & 1.797633 & $\odot .840027$ \\
\hline 127 & 8 & $\odot$ & -5.932960 & $\odot .396930$ & 2.273018 \\
\hline 128 & 1 & $\odot$ & -6.134671 & 1.307153 & 1.924078 \\
\hline 129 & 8 & $\odot$ & -6.235222 & 2.903348 & 1.565980 \\
\hline 130 & 1 & $\odot$ & -5.287982 & 2.977525 & 1.769935 \\
\hline 131 & 1 & $\odot$ & -6.336028 & 3.117526 & $\odot .613390$ \\
\hline 132 & 1 & $\odot$ & -7.087111 & $-\odot .066146$ & 3.517264 \\
\hline 133 & 8 & $\odot$ & -7.793089 & $-\odot .404731$ & 4.104940 \\
\hline 134 & 1 & $\odot$ & -7.397133 & -1.142719 & 4.576349 \\
\hline
\end{tabular}


General basis read from cards: (6D, 7F)

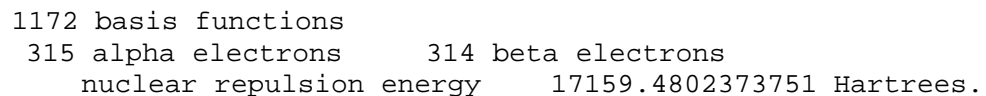

Force inversion solution in PCM.

Polarizable Continuum Model (PCM)

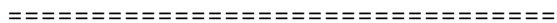

Model : PCM.

Atomic radii : UFF (Universal Force Field).

Solvent : Water, Eps $=78.355300$ Eps $($ inf $)=1.777849$

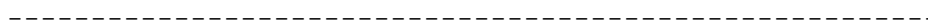

SCF Done: $E($ UwB97XD) $=-5092.30024686$ A.U. after 1 cycles NFock $=1$ Conv $=0.15 \mathrm{D}-08 \quad-\mathrm{V} / \mathrm{T}=2.0066$

$\langle\mathrm{S} X>=0.0000<\mathrm{Sy}\rangle=0.0000<\mathrm{S} z>=0.5000$

$<S^{*} * 2>=0.7604 \mathrm{~S}=0.5052$

$<$ L.S $>=0.000000000 \odot \odot \odot E+\odot \odot$

Annihilation of the first spin contaminant:

$\mathrm{S}^{* * 2}$ before annihilation 0.7604 , after 0.7501

Harmonic frequencies $\left(\mathrm{cm}^{*}-1\right)$, IR intensities (KM/Mole), Raman

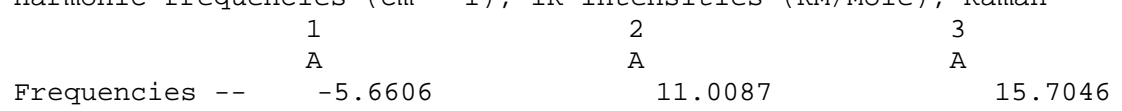

Zero-point correction=

Thermal correction to Energy=

1.040671 (a.u.)

1.117012

1.117956

0.926291

$-5091.259575$

$-5091.183235$

$-5091.182291$

Sum of electronic and zero-point Energies=

Sum of electronic and thermal Energies=

$-5091.373956$

Sum of electronic and thermal Free Energies=

$\mathrm{S}$
Total $\mathrm{KCal} / \mathrm{Mol}$
Cal/Mol-Kelvin Cal/Mol-Kelvin 700.936 291.957 403.394

E (Thermal)

CV

\begin{tabular}{lccc}
\multicolumn{1}{c}{ Item } & Value & Threshold Converged? \\
Maximum Force & 0.000184 & $0.00 \odot 450$ & YES \\
RMS Force & 0.000023 & 0.000300 & YES \\
Maximum Displacement & 0.029448 & $0.00180 \odot$ & NO \\
RMS Displacement & 0.005898 & 0.001200 & NO
\end{tabular}

Predicted change in Energy $=-3.029262 \mathrm{D}-06$

Normal termination of Gaussian 16 at Fri Aug 16 04:28:14 2019.

$\mathrm{G}(\mathrm{high})=-5093.1388678 \mathrm{a} \cdot \mathrm{u}$.

.............. Figure S15-4, TS2a(C8)W2(d)

dna.ts2dw2. $\log$

Stoichiometry C38H53N16Na2023P2(2)

Standard orientation:

\begin{tabular}{|c|c|c|c|c|c|}
\hline \multirow{2}{*}{$\begin{array}{l}\text { Center } \\
\text { Number }\end{array}$} & \multirow{2}{*}{$\begin{array}{l}\text { Atomic } \\
\text { Number }\end{array}$} & \multirow{2}{*}{$\begin{array}{c}\text { Atomic } \\
\text { Type }\end{array}$} & \multicolumn{3}{|c|}{ Coordinates (Angstroms) } \\
\hline & & & $x$ & $\mathrm{Y}$ & Z \\
\hline.---- & 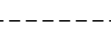 & & ----- & -------- & 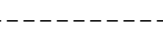 \\
\hline 1 & 7 & $\odot$ & 4.529480 & -2.575037 & -0.780886 \\
\hline 2 & 6 & $\odot$ & 4.607821 & -3.921377 & -0.578930 \\
\hline 3 & 6 & $\odot$ & 3.489800 & -4.667744 & -0.422792 \\
\hline 4 & 6 & $\odot$ & 2.236561 & -3.989895 & -0.536258 \\
\hline 5 & 7 & $\odot$ & 2.169710 & -2.667844 & $-\odot .687948$ \\
\hline 6 & 8 & $\odot$ & 8.419810 & -0.378425 & -1.241880 \\
\hline 7 & 15 & $\odot$ & 8.252126 & 1.064015 & -0.514243 \\
\hline 8 & 8 & $\odot$ & 6.669076 & 1.391201 & -0.722684 \\
\hline 9 & 8 & $\odot$ & 9.100143 & 2.063386 & -1.263387 \\
\hline 10 & 8 & $\odot$ & 8.471719 & 0.953139 & ๑. 982854 \\
\hline 11 & 8 & $\odot$ & 6.160577 & 5.705723 & -0.640134 \\
\hline
\end{tabular}




\begin{tabular}{|c|c|c|c|c|c|}
\hline 12 & 6 & $\odot$ & 5.562563 & 3.492065 & -1.272573 \\
\hline 13 & 6 & 0 & 6.532023 & 4.320437 & -0.425962 \\
\hline 14 & 6 & $\odot$ & -7.339702 & -2.982884 & 2.270177 \\
\hline 15 & 6 & 0 & -7.075571 & -1.504314 & 2.459298 \\
\hline 16 & 8 & 0 & -6.541088 & -3.500179 & 1.162358 \\
\hline 17 & 8 & 0 & -7.796405 & 0.610695 & 1.585387 \\
\hline 18 & 15 & 0 & -8.564944 & 1.563871 & 0.493963 \\
\hline 19 & 8 & 0 & -7.313681 & 2.307554 & -0.257916 \\
\hline 20 & 8 & $\odot$ & -9.373025 & 2.555986 & 1.275875 \\
\hline 21 & 8 & 0 & -9.194672 & ๑.680964 & -0.559024 \\
\hline 22 & 8 & 0 & -4.636299 & 4.944066 & -2.210429 \\
\hline 23 & 6 & 0 & -5.269194 & 3.599202 & -0.338315 \\
\hline 24 & 6 & 0 & -5.514298 & 3.849143 & -1.834317 \\
\hline 25 & 1 & 0 & -4.730253 & 5.139103 & -3.158446 \\
\hline 26 & 6 & $\odot$ & 8.280650 & -4.051270 & -1.692218 \\
\hline 27 & 6 & $\odot$ & 7.873839 & -2.605974 & -1.886030 \\
\hline 28 & 6 & 0 & 8.098029 & -1.692622 & -0.674041 \\
\hline 29 & 6 & $\odot$ & 6.755244 & -1.731791 & 0.050836 \\
\hline 30 & 6 & 0 & 5.744905 & -1.822432 & -1.091533 \\
\hline 31 & 8 & $\odot$ & 6.437753 & -2.510493 & -2.172038 \\
\hline 32 & 1 & $\odot$ & 7.935672 & -4.634834 & -2.552029 \\
\hline 33 & 1 & 0 & 9.373879 & -4.108989 & -1.642017 \\
\hline 34 & 1 & 0 & 8.406550 & -2.205765 & -2.751058 \\
\hline 35 & 1 & $\odot$ & 8.930846 & -2.007270 & -0.044162 \\
\hline 36 & 1 & 0 & 6.570489 & -0.874811 & 0.693538 \\
\hline 37 & 1 & 0 & 6.728791 & -2.640800 & 0.656436 \\
\hline 38 & 1 & $\odot$ & 5.423310 & -0.838522 & -1.426327 \\
\hline 39 & 8 & 0 & 7.691238 & -4.550942 & -0.466426 \\
\hline 40 & 1 & 0 & 7.948318 & -5.473694 & -0.306912 \\
\hline 41 & 6 & $\odot$ & 3.294411 & -1.916074 & -0.795753 \\
\hline 42 & 7 & $\odot$ & 1.076307 & -4.666779 & -0.481344 \\
\hline 43 & 8 & 0 & 3.260873 & -0.684837 & -0.915217 \\
\hline 44 & 1 & 0 & 5.616146 & -4.332460 & -0.564842 \\
\hline 45 & 1 & 0 & 3.545474 & -5.736603 & -0.269169 \\
\hline 46 & 1 & 0 & 1.103435 & -5.673603 & -0.512572 \\
\hline 47 & 1 & $\odot$ & $\odot .225068$ & -4.203792 & -0.818245 \\
\hline 48 & 6 & $\odot$ & 6.124922 & 2.190386 & -1.810004 \\
\hline 49 & 1 & 0 & 6.730820 & 6.293362 & -0.115888 \\
\hline 50 & 6 & 0 & 6.252903 & 3.872180 & 1.015454 \\
\hline 51 & 6 & $\odot$ & 4.786416 & 3.413177 & $\odot .999301$ \\
\hline 52 & 8 & $\odot$ & 4.420935 & 3.235158 & $-\odot .399602$ \\
\hline 53 & 1 & $\odot$ & 5.325227 & 1.604912 & -2.265502 \\
\hline 54 & 1 & 0 & 6.901672 & 2.396868 & -2.550936 \\
\hline 55 & 1 & 0 & 5.200000 & 4.091658 & -2.111148 \\
\hline 56 & 1 & 0 & 7.573952 & 4.147914 & -0.713831 \\
\hline 57 & 1 & 0 & 6.409625 & 4.685190 & 1.727173 \\
\hline 58 & 1 & $\odot$ & 6.927011 & 3.056301 & 1.276354 \\
\hline 59 & 1 & 0 & 4.106170 & 4.162048 & 1.406330 \\
\hline 60 & 7 & 0 & 4.535748 & 2.181569 & 1.724573 \\
\hline 61 & 6 & $\odot$ & 5.389234 & 1.119503 & 1.971783 \\
\hline 62 & 7 & 0 & 4.774267 & ๑.061858 & 2.423103 \\
\hline 63 & 6 & 0 & 3.441117 & 0.428530 & 2.462411 \\
\hline 64 & 6 & $\odot$ & 3.274131 & 1.735975 & 2.035451 \\
\hline 65 & 7 & $\odot$ & 2.132307 & 2.444738 & 1.923736 \\
\hline 66 & 6 & $\odot$ & 1.064234 & 1.734813 & 2.250671 \\
\hline 67 & 7 & $\odot$ & 1.115825 & $\odot .420135$ & 2.637957 \\
\hline 68 & 6 & $\odot$ & 2.277638 & -0.343490 & 2.733638 \\
\hline 69 & 8 & 0 & 2.213646 & -1.551742 & 3.004478 \\
\hline 70 & 1 & 0 & 6.454730 & 1.179921 & 1.792052 \\
\hline 71 & 7 & 0 & -0.154928 & 2.298566 & 2.218290 \\
\hline 72 & 1 & 0 & 0.222950 & -0.110096 & 2.670597 \\
\hline 73 & 1 & $\odot$ & -6.386383 & -4.455109 & 1.250572 \\
\hline 74 & 6 & $\odot$ & -7.172901 & -0.647895 & 1.189800 \\
\hline 75 & 6 & $\odot$ & -5.726395 & -0.439053 & 0.754043 \\
\hline 76 & 6 & 0 & -4.931035 & -0.491101 & 2.059405 \\
\hline 77 & 8 & $\odot$ & -5.725627 & -1.298647 & 2.978269 \\
\hline 78 & 1 & 0 & -7.073116 & -3.515621 & 3.185323 \\
\hline 79 & 1 & $\odot$ & -8.400415 & -3.142630 & 2.054588 \\
\hline 80 & 1 & $\odot$ & -7.784026 & -1.127418 & 3.201093 \\
\hline 81 & 1 & $\odot$ & -7.781077 & -1.107492 & 0.409274 \\
\hline 82 & 1 & 0 & -5.570320 & 0.501989 & 0.226221 \\
\hline 83 & 1 & 0 & -5.415511 & -1.251272 & 0.092043 \\
\hline 84 & 1 & 0 & -4.766099 & 0.494204 & 2.489963 \\
\hline 85 & 7 & 0 & -3.619317 & -1.119856 & 1.939756 \\
\hline 86 & 6 & $\odot$ & -3.552013 & -2.430235 & 1.561799 \\
\hline 87 & 6 & $\odot$ & -2.376429 & -3.089974 & 1.525886 \\
\hline 88 & 6 & $\odot$ & -1.212407 & -2.364809 & 1.938249 \\
\hline
\end{tabular}




\begin{tabular}{|c|c|c|c|c|c|}
\hline 89 & 7 & $\odot$ & -1.288023 & -1.085843 & 2.321536 \\
\hline 90 & 6 & $\odot$ & -2.467852 & -0.419258 & 2.321717 \\
\hline 91 & 1 & $\odot$ & -2.317974 & -4.121210 & 1. 208443 \\
\hline 92 & 7 & $\odot$ & -0.021372 & -2.957888 & 1.971263 \\
\hline 93 & 8 & $\odot$ & -2.554601 & 0.773779 & 2.643135 \\
\hline 94 & 1 & $\odot$ & -4.502214 & -2.893590 & 1.316389 \\
\hline 95 & 1 & $\odot$ & $\odot .100600$ & -3.881926 & 1.585861 \\
\hline 96 & 1 & $\odot$ & $\odot .801737$ & -2.449869 & 2. 310798 \\
\hline 97 & 1 & $\odot$ & -0.220052 & 3. 225999 & 1.833892 \\
\hline 98 & 1 & $\odot$ & -1.009174 & 1.757150 & 2.374485 \\
\hline 99 & 6 & $\odot$ & -6.513687 & 3.289232 & $\odot .460398$ \\
\hline 100 & 11 & $\odot$ & 10.134480 & 2.728964 & 1.037674 \\
\hline 101 & 11 & $\odot$ & -9.315740 & -0.129767 & -2.572284 \\
\hline 102 & 6 & $\odot$ & -5.089470 & 2.536556 & -2.490986 \\
\hline 103 & 6 & 0 & -3.930207 & 2.063011 & -1.614576 \\
\hline 104 & 8 & $\odot$ & -4.347673 & 2.467511 & -0.256249 \\
\hline 105 & 1 & 0 & -6.228113 & 2.889023 & 1.438315 \\
\hline 106 & 1 & $\odot$ & -7.106988 & 4.197383 & 0.603480 \\
\hline 107 & 1 & 0 & -4.784060 & 4.480513 & ๑. . 093399 \\
\hline 108 & 1 & 0 & -6.556535 & 4.106417 & -2.043308 \\
\hline 109 & 1 & $\odot$ & -4.805435 & 2.659438 & -3.539037 \\
\hline 110 & 1 & 0 & -5.903701 & 1.811044 & -2.413393 \\
\hline 111 & 1 & 0 & -3.000159 & 2.595765 & -1.844089 \\
\hline 112 & 7 & 0 & -3.752603 & 0.649417 & -1.655303 \\
\hline 113 & 6 & 0 & -4.515792 & -0.958411 & -2.737188 \\
\hline 114 & 7 & $\odot$ & -3.748043 & -1.861348 & -2.123671 \\
\hline 115 & 6 & 0 & -2.589484 & -1.329322 & -1.689773 \\
\hline 116 & 6 & $\odot$ & -2.583617 & 0.100015 & -1.422724 \\
\hline 117 & 7 & $\odot$ & -1.479124 & 0.768112 & -1.006858 \\
\hline 118 & 6 & 0 & -0.375697 & 0.050200 & -0.876436 \\
\hline 119 & 7 & $\odot$ & -0.324005 & -1.309511 & -1.019745 \\
\hline 120 & 6 & 0 & -1.402650 & -2.089317 & -1.410688 \\
\hline 121 & 7 & 0 & 0.779375 & 0.653860 & -0.580963 \\
\hline 122 & 8 & $\odot$ & -1.280918 & -3.318772 & -1.511897 \\
\hline 123 & 1 & 0 & -4.146259 & -0.410245 & -3.603597 \\
\hline 124 & 1 & $\odot$ & 0.765954 & 1.657581 & -0.503896 \\
\hline 125 & 1 & 0 & 1.679015 & 0.165097 & -0.635151 \\
\hline 126 & 1 & 0 & 0.578056 & -1.801474 & -0.864297 \\
\hline 127 & 8 & 0 & -5.826407 & -1.056613 & -2.744048 \\
\hline 128 & 1 & 0 & -6.201010 & -1.737732 & -2.099597 \\
\hline 129 & 8 & $\odot$ & -7.017582 & -2.859758 & -1.363644 \\
\hline 130 & 1 & $\odot$ & -6.841658 & -3.093231 & -0.420475 \\
\hline 131 & 1 & 0 & -7.972817 & -2.794194 & -1.451845 \\
\hline 132 & 1 & $\odot$ & -6.934776 & -0.013370 & -3.807436 \\
\hline 133 & 8 & $\odot$ & -7.708981 & 0.455727 & -4.159861 \\
\hline 134 & 1 & 0 & -7.459181 & 1.383297 & -4.185684 \\
\hline
\end{tabular}

General basis read from cards: (6D, 7F)

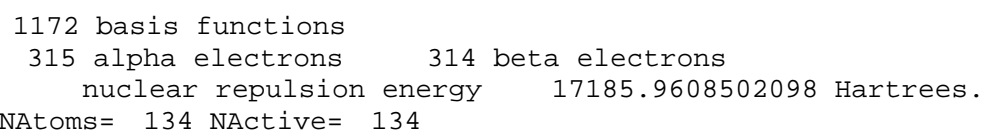

NAtoms $=134$ NActive $=134$

Force inversion solution in PCM.

Polarizable Continuum Model (PCM)

$\begin{array}{ll}\text { Model } & \text { : PCM. }\end{array}$

Solvent : Water, Eps $=78.355300$ Eps $($ inf $)=1.777849$

-

SCF Done: $E(U W B 97 X D)=-5092.26796890$ A.U. after 1 cycles $\mathrm{NFock}=1$ Conv $=0.64 \mathrm{D}-09 \quad-\mathrm{V} / \mathrm{T}=2.0066$

$\langle\mathrm{S} x\rangle=0.0000<\mathrm{Sy}\rangle=0.0000<\mathrm{S} z>=0.5000$

$<S^{* *} 2>=0.7609 \mathrm{~S}=0.5054$

$<$ L.S $>=0.000000000000 \mathrm{E}+00$

Annihilation of the first spin contaminant:

$\mathrm{S}^{* * 2}$ before annihilation 0.7609 , after 0.7501

Harmonic frequencies $\left(\mathrm{cm}^{*}-1\right)$, IR intensities (KM/Mole), Raman

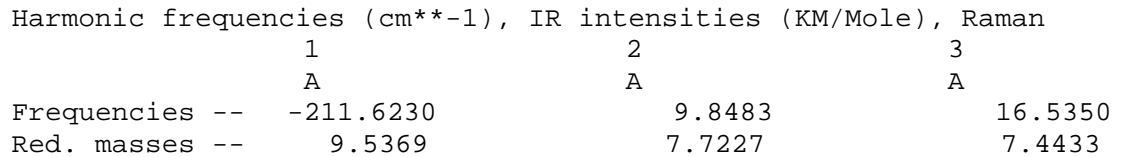




\begin{tabular}{|c|c|c|c|c|c|c|c|c|c|c|}
\hline \multirow{2}{*}{\multicolumn{2}{|c|}{$\begin{array}{l}\text { Frc consts } \\
\text { IR Inten }\end{array}$}} & \multirow{2}{*}{\multicolumn{3}{|c|}{$\begin{array}{r}0.2516 \\
116.6449\end{array}$}} & \multicolumn{3}{|c|}{$\odot .0004$} & \multicolumn{3}{|c|}{0.0012} \\
\hline & & & & & & 2.3073 & & & .3954 & \\
\hline & & $x$ & $Y$ & Z & $x$ & $Y$ & Z & $x$ & Z & \\
\hline 1 & 7 & $-\odot . \odot \odot$ & $\odot .01$ & $-\odot . \odot \odot$ & $-\odot .01$ & $\odot .03$ & $-\odot .01$ & -0.05 & $-\odot . \odot 2$ & $\odot .02$ \\
\hline 2 & 6 & $-\odot . \odot \odot$ & $\odot . \odot \odot$ & $-\odot . \odot \odot$ & $-\odot .01$ & 0.03 & $-\odot .02$ & $-\odot .06$ & $-\odot . \odot 2$ & $\odot .02$ \\
\hline 3 & 6 & $\odot . \odot \odot$ & $\odot . \odot \odot$ & $\odot . \odot \odot$ & $-\odot .01$ & $\odot . \odot 2$ & -0.04 & $-\odot .07$ & $-\odot .01$ & $\odot . \odot \odot$ \\
\hline 4 & 6 & $-\odot . \odot \odot$ & $\odot . \odot \odot$ & $\odot . \odot \odot$ & -0.01 & 0.02 & -0.04 & -0.06 & $0.0 \odot$ & $-\odot . \odot 2$ \\
\hline 5 & 7 & $-\odot . \odot \odot$ & $\odot . \odot \odot$ & $\odot . \odot \odot$ & $-\odot . \odot 2$ & $\odot . \odot 2$ & $-\odot .03$ & -0.05 & $\odot . \odot \odot$ & $-\odot . \odot 2$ \\
\hline 6 & 8 & $\odot . \odot \odot$ & $\odot . \odot \odot$ & $\odot . \odot \odot$ & $\odot .02$ & $\odot .0 \odot$ & 0.02 & $-\odot .03$ & -0.05 & 0.05 \\
\hline 7 & 15 & $\odot . \odot \odot$ & $\odot . \odot \odot$ & $\odot . \odot \odot$ & $\odot .04$ & -0.01 & 0.05 & $-\odot . \odot \odot$ & -0.03 & 0.03 \\
\hline 8 & 8 & $-\odot . \odot \odot$ & $\odot . \odot \odot$ & $\odot . \odot \odot$ & 0.05 & $\odot . \odot 2$ & $\odot .05$ & $-\odot . \odot \odot$ & $-\odot .01$ & $\odot . \odot 3$ \\
\hline 9 & 8 & $\odot . \odot \odot$ & $\odot . \odot \odot$ & $\odot . \odot \odot$ & $\odot .07$ & $-\odot .01$ & $\odot . \odot 8$ & 0.01 & -0.06 & $\odot .0 \odot$ \\
\hline 10 & 8 & $-\odot . \odot \odot$ & $\odot . \odot \odot$ & $\odot . \odot \odot$ & 0.03 & -0.05 & 0.05 & $\odot . \odot \odot$ & -0.01 & $\odot .03$ \\
\hline 11 & 8 & $-\odot . \odot \odot$ & $\odot . \odot \odot$ & $\odot . \odot \odot$ & 0.14 & 0.03 & 0.12 & $\odot .05$ & $-\odot . \odot \odot$ & $-\odot . \odot 9$ \\
\hline 12 & 6 & $-\odot . \odot \odot$ & $\odot . \odot \odot$ & $-\odot . \odot \odot$ & 0.10 & 0.05 & $\odot . \odot 8$ & $\odot .02$ & $-\odot .01$ & $-\odot .03$ \\
\hline 13 & 6 & $-\odot . \odot \odot$ & $\odot . \odot \odot$ & $-\odot . \odot \odot$ & 0.11 & 0.02 & 0.10 & 0.03 & $\odot . \odot \odot$ & -0.05 \\
\hline 14 & 6 & $-\odot . \odot \odot$ & $-\odot . \odot \odot$ & $\odot . \odot \odot$ & $\odot .02$ & 0.05 & 0.10 & 0.10 & -0.08 & $-\odot .05$ \\
\hline 15 & 6 & $-\odot . \odot \odot$ & $-\odot . \odot \odot$ & $\odot .0 \odot$ & 0.02 & 0.05 & 0.06 & 0.07 & -0.08 & -0.03 \\
\hline 16 & 8 & $\odot . \odot \odot$ & $-\odot . \odot \odot$ & $\odot .01$ & $-\odot .01$ & $\odot . \odot 2$ & $\odot . \odot 9$ & 0.10 & $-\odot . \odot 4$ & $-\odot .07$ \\
\hline 17 & 8 & $\odot . \odot \odot$ & $-\odot . \odot \odot$ & $-\odot . \odot \odot$ & $-\odot .01$ & 0.03 & 0.02 & $\odot .03$ & -0.07 & $\odot .03$ \\
\hline 18 & 15 & $-\odot . \odot \odot$ & $-\odot . \odot \odot$ & $\odot . \odot \odot$ & -0.03 & -0.01 & 0.01 & 0.00 & -0.06 & 0.06 \\
\hline 19 & 8 & $-\odot . \odot \odot$ & $-\odot . \odot \odot$ & $-\odot . \odot \odot$ & $-\odot .05$ & $-\odot . \odot 2$ & -0.03 & $-\odot . \odot 2$ & -0.03 & 0.05 \\
\hline 20 & 8 & $\odot . \odot \odot$ & $\odot . \odot \odot$ & $\odot . \odot \odot$ & $-\odot .03$ & $\odot . \odot \odot$ & $-\odot . \odot \odot$ & $\odot .01$ & $-\odot . \odot 8$ & $\odot . \odot 9$ \\
\hline 21 & 8 & $-0.0 \odot$ & $-\odot . \odot \odot$ & $\odot . \odot \odot$ & $-\odot . \odot 4$ & -0.04 & 0.04 & $-\odot . \odot \odot$ & -0.04 & 0.05 \\
\hline 22 & 8 & $\odot . \odot 2$ & $-\odot .07$ & $-\odot . \odot 2$ & $-\odot . \odot 8$ & -0.05 & -0.11 & $-\odot . \odot 7$ & $\odot . \odot 4$ & 0.07 \\
\hline 23 & 6 & 0.03 & $-\odot .07$ & $-\odot .03$ & $-\odot .06$ & -0.01 & $-\odot .08$ & -0.03 & $-\odot .01$ & 0.05 \\
\hline 24 & 6 & $\odot .0 \odot$ & -0.04 & -0.02 & $-\odot .07$ & -0.04 & -0.08 & -0.05 & 0.02 & 0.05 \\
\hline 25 & 1 & $-\odot . \odot 2$ & $-\odot . \odot 2$ & $-\odot . \odot \odot$ & $-\odot . \odot 9$ & $-\odot .07$ & -0.11 & $-\odot . \odot 8$ & $\odot . \odot 6$ & $\odot .07$ \\
\hline 26 & 6 & $-\odot . \odot \odot$ & $\odot . \odot \odot$ & $-\odot . \odot \odot$ & $-\odot .04$ & 0.02 & $-\odot .08$ & $-\odot .04$ & $-\odot .06$ & 0.15 \\
\hline 27 & 6 & $-\odot . \odot \odot$ & $\odot . \odot \odot$ & $\odot . \odot \odot$ & -0.03 & 0.03 & -0.04 & $-\odot . \odot 2$ & $-\odot .06$ & 0.10 \\
\hline 28 & 6 & $-\odot . \odot \odot$ & $\odot . \odot \odot$ & $\odot . \odot \odot$ & $-\odot . \odot \odot$ & $-\odot .01$ & $-\odot .01$ & -0.05 & $-\odot .03$ & $\odot .08$ \\
\hline 29 & 6 & $-\odot . \odot \odot$ & $\odot . \odot \odot$ & $-\odot . \odot \odot$ & $-\odot . \odot \odot$ & $-\odot .01$ & $-\odot .01$ & $-\odot . \odot 6$ & $-\odot . \odot \odot$ & 0.05 \\
\hline 30 & 6 & $-\odot . \odot \odot$ & $\odot . \odot \odot$ & $-\odot . \odot \odot$ & $-\odot .01$ & 0.03 & $\odot . \odot \odot$ & $-\odot . \odot 4$ & -0.03 & 0.03 \\
\hline 31 & 8 & $-\odot . \odot \odot$ & $\odot . \odot \odot$ & $\odot . \odot \odot$ & $-\odot .03$ & $\odot .06$ & $-\odot .02$ & $-\odot . \odot 2$ & $-\odot . \odot 6$ & $\odot .07$ \\
\hline 32 & 1 & $-\odot . \odot \odot$ & $\odot . \odot \odot$ & $-\odot . \odot \odot$ & $-\odot .07$ & 0.04 & $-\odot .09$ & $-\odot .02$ & $-\odot .08$ & 0.15 \\
\hline 33 & 1 & $-\odot . \odot \odot$ & $\odot . \odot \odot$ & $-\odot . \odot \odot$ & $-\odot .04$ & $\odot . \odot \odot$ & -0.10 & -0.04 & $-\odot .07$ & 0.18 \\
\hline 34 & 1 & $-\odot . \odot \odot$ & $\odot . \odot \odot$ & $\odot . \odot \odot$ & $-\odot .03$ & $\odot .05$ & -0.03 & $\odot . \odot \odot$ & $-\odot . \odot 9$ & 0.10 \\
\hline 35 & 1 & $-0.0 \odot$ & $-\odot . \odot \odot$ & $\odot . \odot \odot$ & -0.01 & -0.04 & -0.03 & -0.06 & -0.02 & 0.11 \\
\hline 36 & 1 & $-\odot . \odot \odot$ & $\odot . \odot \odot$ & $\odot . \odot \odot$ & 0.02 & -0.02 & 0.01 & -0.07 & 0.01 & 0.03 \\
\hline 37 & 1 & $-\odot . \odot \odot$ & $\odot . \odot \odot$ & $-\odot . \odot \odot$ & -0.01 & -0.02 & -0.02 & $-\odot . \odot 8$ & 0.01 & ๑.๑8 \\
\hline 38 & 1 & $-\odot . \odot \odot$ & $\odot . \odot \odot$ & $-\odot . \odot \odot$ & -0.01 & 0.04 & 0.03 & -0.02 & $-\odot .04$ & $-0.0 \odot$ \\
\hline 39 & 8 & $-\odot . \odot \odot$ & $\odot . \odot \odot$ & $\odot . \odot \odot$ & $-\odot .03$ & $-\odot . \odot \odot$ & -0.08 & -0.07 & -0.03 & 0.14 \\
\hline 40 & 1 & $-\odot . \odot \odot$ & $\odot . \odot \odot$ & $-\odot . \odot \odot$ & $-\odot .03$ & $-\odot .01$ & -0.11 & $-\odot . \odot 8$ & $-\odot . \odot 2$ & 0.17 \\
\hline 41 & 6 & $-\odot . \odot \odot$ & $\odot . \odot \odot$ & $\odot . \odot \odot$ & $-\odot .02$ & $\odot . \odot 2$ & -0.02 & $-\odot .04$ & $-\odot . \odot 1$ & $\odot .01$ \\
\hline 42 & 7 & $\odot . \odot \odot$ & $-\odot . \odot \odot$ & $\odot . \odot \odot$ & $-\odot . \odot \odot$ & $\odot .01$ & -0.04 & $-\odot .07$ & 0.01 & $-\odot . \odot 4$ \\
\hline 43 & 8 & $-\odot .01$ & $\odot . \odot \odot$ & $\odot . \odot \odot$ & $-\odot .02$ & $\odot .02$ & -0.01 & $-\odot .03$ & $-\odot . \odot \odot$ & $\odot .03$ \\
\hline 44 & 1 & $\odot . \odot \odot$ & 0.01 & $-\odot . \odot \odot$ & -0.01 & 0.03 & -0.02 & -0.06 & -0.03 & 0.05 \\
\hline 45 & 1 & $\odot . \odot \odot$ & $\odot . \odot \odot$ & $\odot . \odot \odot$ & $-\odot . \odot \odot$ & $\odot . \odot 2$ & -0.05 & $-\odot . \odot 8$ & $-\odot .01$ & $\odot .01$ \\
\hline 46 & 1 & $\odot .01$ & $-\odot . \odot \odot$ & $\odot .01$ & $\odot . \odot \odot$ & $\odot .01$ & $-\odot . \odot 4$ & $-\odot . \odot 8$ & $\odot .01$ & $-\odot .05$ \\
\hline 47 & 1 & $-0.0 \odot$ & $-\odot . \odot \odot$ & $-0 . \odot \odot$ & -0.01 & $0.0 \odot$ & -0.03 & -0.06 & 0.02 & -0.07 \\
\hline 48 & 6 & $-\odot . \odot \odot$ & $\odot . \odot \odot$ & $\odot . \odot \odot$ & $\odot . \odot 8$ & $\odot .05$ & $\odot . \odot 6$ & $\odot .01$ & $-\odot .03$ & $\odot . \odot \odot$ \\
\hline 49 & 1 & 0.00 & $\odot .0 \odot$ & $-\odot . \odot \odot$ & 0.15 & 0.01 & 0.14 & 0.06 & 0.01 & -0.12 \\
\hline 50 & 6 & $-\odot . \odot \odot$ & $\odot . \odot \odot$ & $\odot . \odot \odot$ & 0.09 & $-\odot . \odot \odot$ & $\odot . \odot 9$ & 0.03 & 0.05 & -0.04 \\
\hline 51 & 6 & $-\odot . \odot \odot$ & $\odot . \odot \odot$ & $-\odot . \odot \odot$ & $\odot .08$ & 0.02 & $\odot . \odot 7$ & 0.03 & $\odot .05$ & $-\odot . \odot 3$ \\
\hline 52 & 8 & $-\odot . \odot \odot$ & $\odot . \odot \odot$ & $\odot . \odot \odot$ & $\odot . \odot 9$ & $\odot .06$ & $\odot . \odot 7$ & 0.02 & 0.03 & $-\odot . \odot 2$ \\
\hline 53 & 1 & $-0.0 \odot$ & $\odot . \odot \odot$ & $\odot . \odot \odot$ & 0.07 & $\odot .08$ & 0.04 & -0.00 & $-\odot .04$ & 0.02 \\
\hline 54 & 1 & $-\odot . \odot \odot$ & $\odot . \odot \odot$ & $-\odot . \odot \odot$ & $\odot . \odot 9$ & $\odot .05$ & $\odot .07$ & $\odot .01$ & $-\odot . \odot 6$ & $-\odot . \odot \odot$ \\
\hline 55 & 1 & $-\odot . \odot \odot$ & $\odot . \odot \odot$ & $\odot . \odot \odot$ & 0.12 & $\odot . \odot 8$ & $\odot .09$ & 0.03 & $-\odot .03$ & $-\odot .05$ \\
\hline 56 & 1 & $-\odot . \odot \odot$ & $\odot . \odot \odot$ & $-\odot . \odot \odot$ & 0.11 & $-\odot . \odot \odot$ & 0.11 & $\odot .03$ & -0.02 & $-\odot .05$ \\
\hline 57 & 1 & $-\odot . \odot \odot$ & $\odot . \odot \odot$ & $-\odot . \odot \odot$ & $\odot . \odot 9$ & $-\odot . \odot 2$ & 0.11 & 0.03 & $\odot .07$ & $-\odot .07$ \\
\hline 58 & 1 & $-\odot . \odot \odot$ & $\odot . \odot \odot$ & $-\odot . \odot \odot$ & $\odot . \odot 8$ & $-\odot .02$ & $\odot . \odot 9$ & $\odot .03$ & $\odot .05$ & $-\odot . \odot 2$ \\
\hline 59 & 1 & $0.0 \odot$ & $\odot . \odot \odot$ & $\odot . \odot \odot$ & 0.09 & 0.02 & 0.09 & 0.03 & 0.06 & -0.04 \\
\hline 60 & 7 & $-\odot . \odot \odot$ & $\odot . \odot \odot$ & $-\odot . \odot \odot$ & $\odot .06$ & $\odot . \odot \odot$ & $\odot .04$ & $\odot .03$ & $\odot . \odot 7$ & $-\odot . \odot \odot$ \\
\hline 61 & 6 & $-\odot . \odot \odot$ & $\odot . \odot \odot$ & $\odot . \odot \odot$ & 0.05 & $-\odot .01$ & $\odot . \odot 4$ & 0.03 & 0.08 & 0.03 \\
\hline 62 & 7 & $\odot . \odot \odot$ & $\odot . \odot \odot$ & $\odot . \odot \odot$ & 0.03 & $-\odot .01$ & 0.01 & $\odot .04$ & 0.08 & 0.04 \\
\hline 63 & 6 & $\odot . \odot \odot$ & $\odot .0 \odot$ & $\odot . \odot \odot$ & 0.03 & $-\odot . \odot \odot$ & $-\odot .01$ & $\odot .03$ & 0.07 & 0.02 \\
\hline 64 & 6 & $\odot . \odot \odot$ & $-\odot . \odot \odot$ & $0.0 \odot$ & 0.05 & $\odot .0 \odot$ & 0.01 & 0.03 & 0.06 & $-\odot . \odot \odot$ \\
\hline 65 & 7 & $-\odot . \odot \odot$ & $-\odot . \odot \odot$ & $-\odot . \odot \odot$ & $\odot .06$ & 0.01 & $-\odot . \odot \odot$ & $\odot . \odot 2$ & 0.05 & $-\odot .03$ \\
\hline 66 & 6 & $-\odot . \odot \odot$ & $\odot . \odot \odot$ & $\odot . \odot \odot$ & $\odot .05$ & $\odot .01$ & $-\odot . \odot 4$ & $\odot . \odot 2$ & $\odot .05$ & $-\odot . \odot 3$ \\
\hline 67 & 7 & $-\odot . \odot \odot$ & $-\odot . \odot \odot$ & $-\odot . \odot \odot$ & $\odot .03$ & 0.01 & $-\odot .06$ & $\odot .03$ & $\odot .05$ & $-\odot .01$ \\
\hline 68 & 6 & $\odot . \odot \odot$ & $-\odot . \odot \odot$ & $-\odot . \odot \odot$ & 0.02 & -0.00 & -0.05 & 0.04 & $\odot .07$ & $\odot .01$ \\
\hline 69 & 8 & $\odot . \odot \odot$ & $-\odot . \odot \odot$ & $\odot . \odot \odot$ & $\odot .01$ & $-\odot .01$ & $-\odot .07$ & $\odot .04$ & 0.07 & $\odot .02$ \\
\hline 70 & 1 & $-\odot .0 \odot$ & $\odot . \odot \odot$ & $\odot . \odot \odot$ & 0.05 & -0.01 & 0.06 & 0.03 & 0.08 & $\odot .04$ \\
\hline 71 & 7 & -0.00 & $-0.0 \odot$ & -0.00 & 0.05 & 0.02 & -0.05 & 0.02 & 0.04 & -0.05 \\
\hline 72 & 1 & $-\odot . \odot \odot$ & $-\odot . \odot \odot$ & $\odot . \odot \odot$ & $\odot .03$ & $\odot .01$ & $-\odot .08$ & $\odot .04$ & $\odot .05$ & $-\odot . \odot 2$ \\
\hline 73 & 1 & -0.01 & $-\odot . \odot \odot$ & $\odot .0 \odot$ & -0.01 & 0.03 & 0.11 & 0.12 & -0.04 & $-\odot .09$ \\
\hline 74 & 6 & -0.00 & $-0.0 \odot$ & 0.00 & -0.01 & 0.02 & 0.04 & 0.04 & -0.05 & -0.01 \\
\hline
\end{tabular}




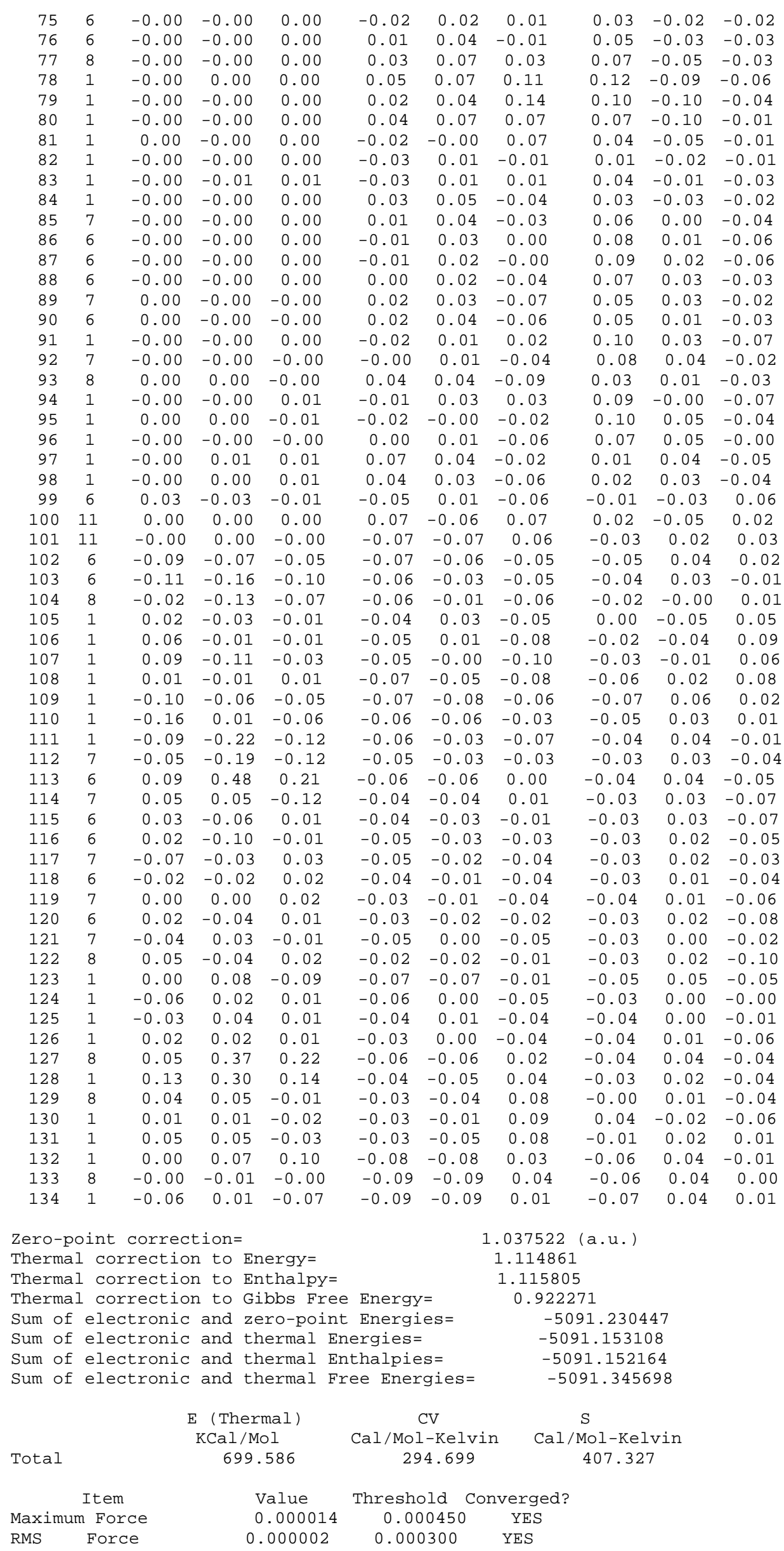


$\begin{array}{lccc}\text { Maximum Displacement } & 0.001386 & 0.001800 & \text { YES } \\ \text { RMS Displacement } & 0.000250 & 0.001200 & \text { YES }\end{array}$

Predicted change in Energy $=-2.406887 \mathrm{D}-08$

optimization completed.

- - Stationary point found.

Normal termination of Gaussian 16 at Mon Jul 15 13:25:33 2019.

$G($ high $)=-5093.1112105 \mathrm{a} \cdot \mathrm{u}$.

-Figure S15-5, Int3a(C8)W2(d)

dna. rev3dw2. $\log$

Stoichiometry C38H53N16Na2023P2(2)

Standard orientation:

\begin{tabular}{|c|c|c|c|c|c|}
\hline \multirow{2}{*}{$\begin{array}{l}\text { Center } \\
\text { Number }\end{array}$} & \multirow{2}{*}{$\begin{array}{l}\text { Atomic } \\
\text { Number }\end{array}$} & \multirow{2}{*}{$\begin{array}{c}\text { Atomic } \\
\text { Type }\end{array}$} & \multicolumn{3}{|c|}{ Coordinates (Angstroms) } \\
\hline & & & $\mathrm{x}$ & $\mathrm{Y}$ & Z \\
\hline 1 & 7 & $\odot$ & -2.966231 & 2.653690 & -0.753669 \\
\hline 2 & 6 & 0 & -3.216768 & 3.553419 & 0.236110 \\
\hline 3 & 6 & 0 & -2.203087 & 4.248011 & $\odot .8 \odot 4358$ \\
\hline 4 & 6 & 0 & -0.880887 & 3.974549 & $\odot .340763$ \\
\hline 5 & 7 & $\odot$ & -0.638066 & 3.031548 & -0.573478 \\
\hline 6 & 8 & $\odot$ & -6.328305 & $\odot .084703$ & -2.292645 \\
\hline 7 & 15 & $\odot$ & -7.842201 & -0.460116 & -2.636196 \\
\hline 8 & 8 & $\odot$ & -7.923539 & -1.810017 & -1.709255 \\
\hline 9 & 8 & 0 & -7.859926 & $-\odot .809987$ & -4.093806 \\
\hline 10 & 8 & $\odot$ & -8.853476 & 0.483393 & -2.026793 \\
\hline 11 & 8 & $\odot$ & -8.303685 & -5.539716 & 0.351965 \\
\hline 12 & 6 & 0 & -7.078279 & -3.929495 & -0.905776 \\
\hline 13 & 6 & 0 & -8.373631 & -4.171076 & $-\odot .120786$ \\
\hline 14 & 6 & $\odot$ & 6.477777 & 0.493153 & 2.390414 \\
\hline 15 & 6 & $\odot$ & 5.888641 & $-\odot .889180$ & 2.219145 \\
\hline 16 & 8 & $\odot$ & 5.685556 & 1.437912 & 1.650619 \\
\hline 17 & 8 & $\odot$ & 5.829827 & -2.771545 & $\odot .678755$ \\
\hline 18 & 15 & $\odot$ & 7.302662 & -3.390786 & $\odot .345820$ \\
\hline 19 & 8 & $\odot$ & 7.437319 & -3.121760 & -1.256869 \\
\hline 20 & 8 & $\odot$ & 7.240600 & -4.869178 & $\odot .655918$ \\
\hline 21 & 8 & 0 & 8.398124 & -2.594908 & 1.021253 \\
\hline 22 & 8 & $\odot$ & 7.040789 & -2.038654 & -5.349697 \\
\hline 23 & 6 & $\odot$ & 6.108564 & -2.561551 & -3.206450 \\
\hline 24 & 6 & 0 & 7.329671 & -1.954409 & -3.926717 \\
\hline 25 & 1 & $\odot$ & 7.787040 & -1.685186 & -5.863103 \\
\hline 26 & 6 & $\odot$ & -6.958865 & 2.844152 & $\odot .194614$ \\
\hline 27 & 6 & 0 & -6.436987 & 2.216030 & -1.090651 \\
\hline 28 & 6 & 0 & -6.076263 & $\odot .725672$ & -1.004628 \\
\hline 29 & 6 & $\odot$ & -4.578839 & $\odot .725845$ & -0.736385 \\
\hline 30 & 6 & $\odot$ & -4.073765 & 1.980616 & -1.455179 \\
\hline 31 & 8 & $\odot$ & -5.198312 & 2.884404 & -1.515295 \\
\hline 32 & 1 & $\odot$ & -7.170924 & 3.898368 & $\odot .0 \odot 4418$ \\
\hline 33 & 1 & $\odot$ & -7.899209 & 2.348810 & $\odot .468469$ \\
\hline 34 & 1 & 0 & -7.173541 & 2.368906 & -1.880476 \\
\hline 35 & 1 & 0 & -6.639099 & $\odot .203505$ & -0.230209 \\
\hline 36 & 1 & $\odot$ & -4.090868 & -0.186290 & -1.077874 \\
\hline 37 & 1 & $\odot$ & -4.411367 & $\odot .844785$ & $\odot .335735$ \\
\hline 38 & 1 & 0 & -3.718956 & 1.769791 & -2.462896 \\
\hline 39 & 8 & $\odot$ & -6.015690 & 2.808346 & 1.281375 \\
\hline 40 & 1 & 0 & -6.064504 & 1.961324 & 1.790345 \\
\hline 41 & 6 & 0 & -1.660297 & 2.338586 & -1.134499 \\
\hline 42 & 7 & 0 & 0.168996 & 4.635319 & $\odot .847147$ \\
\hline 43 & 8 & 0 & -1.485449 & 1.440308 & -1.970796 \\
\hline 44 & 1 & 0 & -4.256694 & 3.634506 & $\odot .540468$ \\
\hline 45 & 1 & $\odot$ & -2.389498 & 4.966245 & 1.591101 \\
\hline 46 & 1 & $\Theta$ & 0.022733 & 5.432585 & 1.444410 \\
\hline 47 & 1 & $\odot$ & 1.109248 & 4.443550 & $\odot .497392$ \\
\hline 48 & 6 & $\odot$ & -7.197865 & -3.008977 & -2.097788 \\
\hline 49 & 1 & 0 & -9.100338 & -5.764456 & 0.862289 \\
\hline 50 & 6 & 0 & -8.284594 & -3.171689 & 1.036771 \\
\hline 51 & 6 & $\odot$ & -6.788249 & -3.165437 & 1.343967 \\
\hline 52 & 8 & $\Theta$ & -6.139708 & -3.343953 & 0.054770 \\
\hline 53 & 1 & 0 & -6.195081 & -2.744171 & -2.445616 \\
\hline 54 & 1 & $\odot$ & -7.736795 & -3.507995 & -2.908142 \\
\hline 55 & 1 & $\odot$ & -6.674300 & -4.889709 & -1.237547 \\
\hline 56 & 1 & 0 & -9.266094 & -4.015391 & -0.732717 \\
\hline
\end{tabular}




\begin{tabular}{|c|c|c|c|c|c|}
\hline 57 & 1 & 0 & -8.896033 & -3.455885 & 1.894359 \\
\hline 58 & 1 & $\odot$ & -8.603482 & -2.198310 & $\odot .659709$ \\
\hline 59 & 1 & $\odot$ & -6.498918 & -3.991435 & 1.997362 \\
\hline 60 & 7 & $\odot$ & -6.281452 & -1.937398 & 1.925949 \\
\hline 61 & 6 & $\odot$ & -6.900555 & -0.716979 & 2.067118 \\
\hline 62 & 7 & $\odot$ & -6.081146 & $\odot .228600$ & 2.447748 \\
\hline 63 & 6 & $\odot$ & -4.852912 & -0.396715 & 2.560156 \\
\hline 64 & 6 & $\odot$ & -4.957542 & -1.739117 & 2.240003 \\
\hline 65 & 7 & $\odot$ & -3.987507 & -2.667874 & 2.201504 \\
\hline 66 & 6 & $\odot$ & -2.789749 & -2.161480 & 2.451892 \\
\hline 67 & 7 & $\odot$ & -2.567598 & -0.832387 & 2.726216 \\
\hline 68 & 6 & $\odot$ & -3.555398 & $\odot .146276$ & 2.802851 \\
\hline 69 & 8 & $\odot$ & -3.269740 & 1.328070 & 3.026029 \\
\hline 70 & 1 & $\odot$ & -7.956280 & -0.580987 & 1.889625 \\
\hline 71 & 7 & $\odot$ & -1.713551 & -2.962225 & 2.464067 \\
\hline 72 & 1 & $\odot$ & -1.584556 & -0.499579 & 2.796871 \\
\hline 73 & 1 & $\odot$ & 6.078704 & 2.339528 & 1.683772 \\
\hline 74 & 6 & $\odot$ & 5.645611 & -1.317683 & 0.763701 \\
\hline 75 & 6 & $\odot$ & 4.180036 & -1.012884 & $\odot .516115$ \\
\hline 76 & 6 & $\odot$ & 3.523139 & -1.196299 & 1.886952 \\
\hline 77 & 8 & $\odot$ & 4.571435 & -0.963098 & 2.866485 \\
\hline 78 & 1 & $\odot$ & 6.495020 & 0.743598 & 3.458195 \\
\hline 79 & 1 & $\odot$ & 7.511398 & $\odot .475870$ & 2.021994 \\
\hline 80 & 1 & $\odot$ & 6.540667 & -1.612585 & 2.712639 \\
\hline 81 & 1 & $\odot$ & 6.290765 & -0.816346 & $\odot .041749$ \\
\hline 82 & 1 & $\odot$ & 3.756655 & -1.655505 & -0.253055 \\
\hline 83 & 1 & $\odot$ & 4.096831 & 0.026200 & 0.192768 \\
\hline 84 & 1 & $\odot$ & 3.112847 & -2.193547 & 2.028592 \\
\hline 85 & 7 & $\odot$ & 2.428281 & -0.258114 & 2.142014 \\
\hline 86 & 6 & 0 & 2.700449 & 1.078905 & 2.183499 \\
\hline 87 & 6 & $\odot$ & 1.726494 & 1.973746 & 2.458256 \\
\hline 88 & 6 & $\odot$ & $\odot .416975$ & 1.458225 & 2.715106 \\
\hline 89 & 7 & $\odot$ & $\odot .156303$ & $\odot .147576$ & 2.665764 \\
\hline 90 & 6 & $\odot$ & 1.137553 & -0.744278 & 2.380395 \\
\hline 91 & 1 & $\odot$ & 1.930928 & 3.034560 & 2.476215 \\
\hline 92 & 7 & $\odot$ & -0.581843 & 2.282193 & 3.036856 \\
\hline 93 & 8 & $\odot$ & $\odot .927730$ & -1.964636 & 2.320513 \\
\hline 94 & 1 & $\odot$ & 3.740976 & 1.353286 & 1.994221 \\
\hline 95 & 1 & $\odot$ & -0.424738 & 3.275365 & 2.988954 \\
\hline 96 & 1 & $\odot$ & -1.544544 & 1.936144 & 3.089636 \\
\hline 97 & 1 & $\odot$ & -1.858863 & -3.902165 & 2.133101 \\
\hline 98 & 1 & $\odot$ & $-\odot .759956$ & -2.581880 & 2.457725 \\
\hline 99 & 6 & $\odot$ & 6.428641 & -3.623920 & -2.183759 \\
\hline 100 & 11 & 0 & -10.227287 & 2.136627 & -1.681939 \\
\hline 101 & 11 & 0 & 9.214506 & -4.599278 & 2.196815 \\
\hline 102 & 6 & 0 & 7.362614 & $-\odot .508990$ & -3.431347 \\
\hline 103 & 6 & $\odot$ & 5.895775 & $-\odot .197739$ & -3.138188 \\
\hline 104 & 8 & $\odot$ & 5.421542 & -1.461306 & -2.542171 \\
\hline 105 & 1 & $\odot$ & 5.518986 & -3.893056 & -1.641143 \\
\hline 106 & 1 & 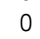 & 6.830311 & -4.512439 & -2.678061 \\
\hline 107 & 1 & 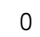 & 5.451539 & -2.997575 & -3.969053 \\
\hline 108 & 1 & 0 & 8.251804 & -2.496453 & -3.698526 \\
\hline 109 & 1 & 0 & 7.809827 & $\odot .174754$ & -4.157212 \\
\hline 110 & 1 & $\odot$ & 7.920843 & $-\odot .448656$ & -2.492387 \\
\hline 111 & 1 & $\odot$ & 5.329627 & -0.036928 & -4.066732 \\
\hline 112 & 7 & 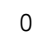 & 5.741161 & 0.902759 & -2.231068 \\
\hline 113 & 6 & $\odot$ & 6.571106 & 3.362855 & -1.286810 \\
\hline 114 & 7 & 0 & 5.421040 & 3.274995 & $-\odot .705678$ \\
\hline 115 & 6 & $\theta$ & 4.397829 & 2.558829 & -1.213212 \\
\hline 116 & 6 & $\odot$ & 4.533820 & 1.360772 & -2.043702 \\
\hline 117 & 7 & $\odot$ & 3.415035 & $\odot .780272$ & -2.587640 \\
\hline 118 & 6 & $\odot$ & 2.235062 & 1.214060 & -2.223236 \\
\hline 119 & 7 & $\odot$ & 2.033587 & 2.234319 & -1.315647 \\
\hline 120 & 6 & 0 & 3.066593 & 2.967572 & -0.803492 \\
\hline 121 & 7 & 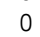 & 1.124788 & $\odot .663397$ & -2.724477 \\
\hline 122 & 8 & $\odot$ & 2.836772 & 3.925265 & $-\odot .042075$ \\
\hline 123 & 1 & 0 & 6.745668 & 3.121554 & -2.333312 \\
\hline 124 & 1 & $\odot$ & 1.245376 & $-\odot .110504$ & -3.356343 \\
\hline 125 & 1 & $\odot$ & $\odot .182526$ & $\odot .962515$ & -2.451213 \\
\hline 126 & 1 & 0 & 1.067809 & 2.514053 & -1.047909 \\
\hline 127 & 8 & 0 & 7.624914 & 3.818639 & -0.662373 \\
\hline 128 & 1 & $\odot$ & 7.401875 & 3.945639 & 0.307061 \\
\hline 129 & 8 & $\odot$ & 6.820918 & 3.961122 & 1.819435 \\
\hline 130 & 1 & $\odot$ & 5.991805 & 4.517554 & 1.810734 \\
\hline 131 & 1 & $\odot$ & 7.355216 & 4.230965 & 2.571930 \\
\hline 132 & 8 & $\odot$ & 4.566271 & 5.285814 & 1.619762 \\
\hline 133 & 1 & $\odot$ & 4.081438 & 4.766508 & 0.943728 \\
\hline
\end{tabular}




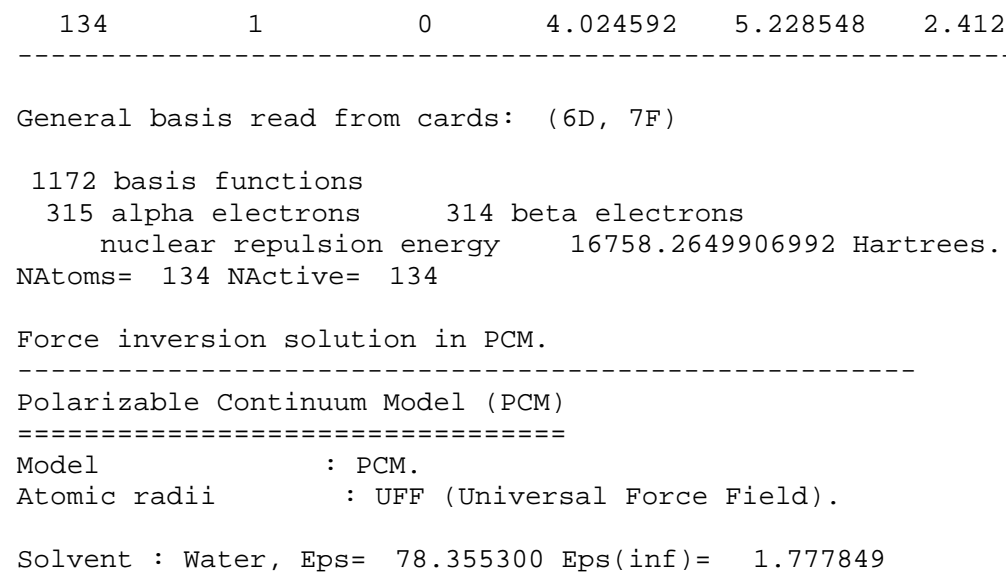

General basis read from cards: (6D, 7F)

\begin{tabular}{|c|c|c|c|c|c|}
\hline Center & Atomic & Atomic & \multicolumn{3}{|c|}{ Coordinates (Angstroms) } \\
\hline Number & Number & Type & $\mathrm{X}$ & Y & Z \\
\hline--- & - & & & - & ----- \\
\hline 1 & 7 & $\odot$ & -3.045696 & 2.674148 & -0.719579 \\
\hline 2 & 6 & $\odot$ & -3.284229 & 3.561189 & 0.284436 \\
\hline 3 & 6 & $\odot$ & -2.263419 & 4.244796 & 0.853650 \\
\hline 4 & 6 & $\odot$ & -0.946178 & 3.974368 & 0.374182 \\
\hline 5 & 7 & $\odot$ & -0.715655 & 3.043258 & -0.555095 \\
\hline 6 & 8 & $\odot$ & -6.416958 & 0.117213 & -2.259402 \\
\hline 7 & 15 & $\odot$ & -7.931153 & -0.437520 & -2.585192 \\
\hline 8 & 8 & 0 & -7.977118 & -1.809142 & -1.688354 \\
\hline 9 & 8 & 0 & -7.973923 & $-\odot .753676$ & -4.050007 \\
\hline
\end{tabular}




\begin{tabular}{|c|c|c|c|c|c|}
\hline 10 & 8 & $\odot$ & -8.941197 & 0.479263 & -1.934253 \\
\hline 11 & 8 & $\odot$ & -8.209469 & -5.580760 & ๑. 318126 \\
\hline 12 & 6 & $\odot$ & -7.056684 & -3.917012 & -0.938166 \\
\hline 13 & 6 & $\odot$ & -8.323317 & -4.203838 & -0.121131 \\
\hline 14 & 6 & $\odot$ & 6.564662 & 0.457704 & 2.489403 \\
\hline 15 & 6 & $\odot$ & 5.950733 & -0.904195 & 2.257673 \\
\hline 16 & 8 & $\odot$ & 5.805527 & 1.448566 & 1.769746 \\
\hline 17 & 8 & $\odot$ & 5.888346 & -2.734508 & ๑. 661806 \\
\hline 18 & 15 & 0 & 7.344271 & -3.371507 & ๑. 292387 \\
\hline 19 & 8 & $\odot$ & 7.434364 & -3.118883 & -1.316043 \\
\hline 20 & 8 & $\odot$ & 7.278566 & -4.847170 & ๑. 615432 \\
\hline 21 & 8 & $\odot$ & 8.463146 & -2.579353 & ๑. 932591 \\
\hline 22 & 8 & $\odot$ & 6.888130 & -1.997012 & -5.375142 \\
\hline 23 & 6 & $\odot$ & 6.026224 & -2.560844 & -3.211962 \\
\hline 24 & 6 & $\odot$ & 7.220121 & -1.934375 & -3.960168 \\
\hline 25 & 1 & $\odot$ & 7.612448 & -1.621519 & -5.904151 \\
\hline 26 & 6 & $\odot$ & -7.019872 & 2.816736 & $\odot .300269$ \\
\hline 27 & 6 & $\odot$ & -6.517991 & 2.219259 & -1.007610 \\
\hline 28 & 6 & $\odot$ & -6.145813 & $\odot .730048$ & -0.961696 \\
\hline 29 & 6 & $\odot$ & -4.643825 & 0.734369 & -0.720734 \\
\hline 30 & 6 & $\odot$ & -4.160083 & 2.009465 & -1.418524 \\
\hline 31 & 8 & $\odot$ & -5.292057 & 2.905349 & -1.440581 \\
\hline 32 & 1 & $\odot$ & -7.247859 & 3.871715 & $\odot .134231$ \\
\hline 33 & 1 & $\odot$ & -7.948669 & 2.305900 & $\odot .584851$ \\
\hline 34 & 1 & $\odot$ & -7.269747 & 2.383615 & -1.780683 \\
\hline 35 & 1 & $\odot$ & -6.691315 & $\odot .186494$ & -0.189638 \\
\hline 36 & 1 & $\odot$ & -4.156457 & -0.165915 & -1.093040 \\
\hline 37 & 1 & $\odot$ & -4.457101 & 0.828142 & $\odot .350772$ \\
\hline 38 & 1 & $\odot$ & -3.820478 & 1.824147 & -2.436523 \\
\hline 39 & 8 & $\odot$ & -6.053191 & 2.773078 & 1.365605 \\
\hline 40 & 1 & $\odot$ & -6.075323 & 1.915148 & 1.858123 \\
\hline 41 & 6 & $\odot$ & -1.743808 & 2. 359861 & -1.115953 \\
\hline 42 & 7 & $\odot$ & ๑. 111293 & 4.624520 & ๑. 878580 \\
\hline 43 & 8 & $\odot$ & -1.578595 & 1.471635 & -1.964847 \\
\hline 44 & 1 & $\odot$ & -4.320769 & 3.640442 & ๑. 599663 \\
\hline 45 & 1 & $\odot$ & -2.440995 & 4.951980 & 1.652354 \\
\hline 46 & 1 & $\odot$ & -0.027866 & 5.415264 & 1. 486192 \\
\hline 47 & 1 & $\odot$ & 1.049839 & 4.431667 & ๑. 519529 \\
\hline 48 & 6 & $\odot$ & -7.233561 & -2.984601 & -2.113409 \\
\hline 49 & 1 & $\odot$ & -8.984453 & -5.834715 & 0.847719 \\
\hline 50 & 6 & $\odot$ & -8.221704 & -3.228921 & 1.056144 \\
\hline 51 & 6 & $\odot$ & -6.717413 & -3.197696 & 1. 318185 \\
\hline 52 & 8 & $\odot$ & -6.107008 & -3.318386 & $\odot .003358$ \\
\hline 53 & 1 & $\odot$ & -6.248353 & -2.690383 & -2.486761 \\
\hline 54 & 1 & $\odot$ & -7.783662 & -3.486610 & -2.914339 \\
\hline 55 & 1 & $\odot$ & -6.638278 & -4.862300 & -1.294334 \\
\hline 56 & 1 & $\odot$ & -9.236626 & -4.054885 & -0.703198 \\
\hline 57 & 1 & $\odot$ & -8.800666 & -3.546261 & 1.924579 \\
\hline 58 & 1 & $\odot$ & -8.573061 & -2.254265 & 0.712528 \\
\hline 59 & 1 & $\odot$ & -6.390642 & -4.038403 & 1.934145 \\
\hline 60 & 7 & $\odot$ & -6.217414 & -1.980140 & 1.925248 \\
\hline 61 & 6 & $\odot$ & -6.846927 & -0.769851 & 2.102026 \\
\hline 62 & 7 & $\odot$ & -6.031735 & ๑. 176124 & 2.490276 \\
\hline 63 & 6 & $\odot$ & -4.794894 & -0.437834 & 2.570337 \\
\hline 64 & 6 & $\odot$ & -4.890862 & -1.774125 & 2.222832 \\
\hline 65 & 7 & $\odot$ & -3.913310 & -2.692530 & 2.150027 \\
\hline 66 & 6 & $\odot$ & -2.716836 & -2.180783 & 2.396471 \\
\hline 67 & 7 & $\odot$ & -2.503452 & -0.855784 & 2.696102 \\
\hline 68 & 6 & $\odot$ & -3.498855 & 0.112351 & 2.806258 \\
\hline 69 & 8 & $\odot$ & -3.219728 & 1.291320 & 3.050970 \\
\hline 70 & 1 & $\odot$ & -7.906778 & -0.641478 & 1.944205 \\
\hline 71 & 7 & $\odot$ & -1.634115 & -2.971676 & 2.377619 \\
\hline 72 & 1 & $\odot$ & -1.522610 & -0.516449 & 2.765023 \\
\hline 73 & 1 & $\odot$ & 6.233561 & 2.327311 & 1.826557 \\
\hline 74 & 6 & $\odot$ & 5.732611 & -1.280770 & $\odot .784058$ \\
\hline 75 & 6 & $\odot$ & 4.280798 & -0.936566 & 0.514909 \\
\hline 76 & 6 & $\odot$ & 3.591666 & -1.168941 & 1.860637 \\
\hline 77 & 8 & $\odot$ & 4.618603 & -0.971301 & 2.872589 \\
\hline 78 & 1 & $\odot$ & 6.565518 & 0.671153 & 3.564849 \\
\hline 79 & 1 & $\odot$ & 7.603634 & 0.432651 & 2.138115 \\
\hline 80 & 1 & $\odot$ & 6.578041 & -1.656057 & 2.741342 \\
\hline 81 & 1 & $\odot$ & 6.406516 & -0.769533 & ๑. 096304 \\
\hline 82 & 1 & $\odot$ & 3.869586 & -1.532359 & -0.297036 \\
\hline 83 & 1 & $\odot$ & 4.227782 & 0.117512 & ๑. 236833 \\
\hline 84 & 1 & $\odot$ & 3.179217 & -2.170623 & 1.956837 \\
\hline 85 & 7 & $\odot$ & 2.492700 & -0.240614 & 2.126178 \\
\hline 86 & 6 & $\odot$ & 2.757959 & 1.096978 & 2.19568 \\
\hline
\end{tabular}




\begin{tabular}{|c|c|c|c|c|c|}
\hline 87 & 6 & 0 & 1.776489 & 1.981672 & 2.476277 \\
\hline 88 & 6 & 0 & $\odot .467737$ & 1.454347 & 2.714761 \\
\hline 89 & 7 & 0 & $\odot .215107$ & $\odot .143312$ & 2.643503 \\
\hline 90 & 6 & 0 & 1.203031 & -0.737813 & 2.349073 \\
\hline 91 & 1 & 0 & 1.972965 & 3.043506 & 2.513945 \\
\hline 92 & 7 & 0 & -0.537363 & 2.267445 & 3.043948 \\
\hline 93 & 8 & $\odot$ & 1.000435 & -1.958039 & 2.267675 \\
\hline 94 & 1 & $\odot$ & 3.798900 & 1.379826 & 2.027444 \\
\hline 95 & 1 & $\odot$ & -0.388352 & 3.262229 & 2.999948 \\
\hline 96 & 1 & 0 & -1.497210 & 1.913204 & 3.094534 \\
\hline 97 & 1 & 0 & -1.774993 & -3.907549 & 2.033715 \\
\hline 98 & 1 & 0 & -0.682912 & -2.585420 & 2.379817 \\
\hline 99 & 6 & $\odot$ & 6.392643 & -3.623809 & -2.205333 \\
\hline 100 & 11 & 0 & -10.295242 & 2.138841 & -1.543700 \\
\hline 101 & 11 & 0 & 9.273175 & -4.586313 & 2.120005 \\
\hline 102 & 6 & $\odot$ & 7.257813 & $-\odot .497546$ & -3.442118 \\
\hline 103 & 6 & $\odot$ & 5.795383 & $-\odot .197744$ & -3.117028 \\
\hline 104 & 8 & $\odot$ & 5.341776 & -1.473163 & -2.524351 \\
\hline 105 & 1 & $\odot$ & 5.506655 & -3.901641 & -1.629214 \\
\hline 106 & 1 & $\odot$ & 6.782091 & -4.507728 & -2.717279 \\
\hline 107 & 1 & 0 & 5.355141 & -3.003328 & -3.958444 \\
\hline 108 & 1 & $\odot$ & 8.152239 & -2.474016 & -3.769742 \\
\hline 109 & 1 & $\odot$ & 7.687867 & $\odot .200182$ & -4.165234 \\
\hline 110 & 1 & $\Theta$ & 7.834020 & $-\odot .450967$ & -2.513224 \\
\hline 111 & 1 & $\odot$ & 5.211390 & $-\odot .037684$ & -4.034314 \\
\hline 112 & 7 & $\odot$ & 5.651409 & 0.896946 & -2.203074 \\
\hline 113 & 6 & $\Theta$ & 6.559900 & 3.312392 & -1.238353 \\
\hline 114 & 7 & $\odot$ & 5.332005 & 3.331691 & -0.721625 \\
\hline 115 & 6 & 0 & 4.317431 & 2.592580 & -1.221878 \\
\hline 116 & 6 & $\odot$ & 4.451523 & 1.385601 & -2.038341 \\
\hline 117 & 7 & $\odot$ & 3.332279 & $\odot .816135$ & -2.596353 \\
\hline 118 & 6 & $\odot$ & 2.151653 & 1.249321 & -2.233318 \\
\hline 119 & 7 & $\odot$ & 1.950334 & 2.256985 & -1.316751 \\
\hline 120 & 6 & $\odot$ & 2.985378 & 2.994489 & -0.803478 \\
\hline 121 & 7 & $\odot$ & 1.041312 & $\odot .706537$ & -2.748568 \\
\hline 122 & 8 & $\odot$ & 2.764792 & 3.942730 & -0.031332 \\
\hline 123 & 1 & $\odot$ & 6.706687 & 2.978623 & -2.267606 \\
\hline 124 & 1 & $\odot$ & 1.162590 & $-\odot .069224$ & -3.377743 \\
\hline 125 & 1 & $\Theta$ & $\odot . \odot 9987 \odot$ & $\odot .997079$ & -2.466539 \\
\hline 126 & 1 & 0 & $\odot .986014$ & 2.532310 & -1.044608 \\
\hline 127 & 8 & $\odot$ & 7.571172 & 3.721985 & -0.604202 \\
\hline 128 & 1 & $\odot$ & 7.356868 & 3.897068 & $\odot .692280$ \\
\hline 129 & 8 & $\odot$ & 7.028170 & 3.995635 & 1.745408 \\
\hline 130 & 1 & $\odot$ & 6.009091 & 4.655214 & 1.638485 \\
\hline 131 & 1 & $\odot$ & 7.715412 & 4.372332 & 2.305427 \\
\hline 132 & 8 & 0 & 5.018284 & 5.118782 & 1.186998 \\
\hline 133 & 1 & $\odot$ & 4.943965 & 4.500711 & 0.371629 \\
\hline 134 & 1 & $\odot$ & 4.227892 & 4.932098 & 1.708659 \\
\hline
\end{tabular}

General basis read from cards: (6D, 7F)

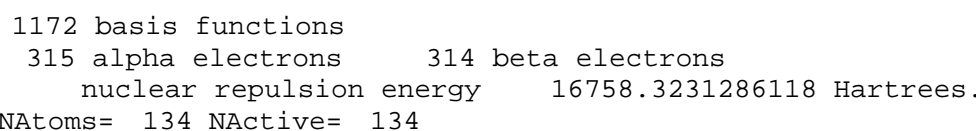

NAtoms $=134$ NActive $=134$

Force inversion solution in PCM.

Polarizable Continuum Model (PCM)

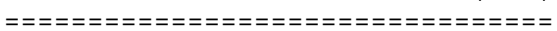

Model : PCM.

Atomic radii : UFF (Universal Force Field).

Solvent : Water, Eps= 78.355300 Eps (inf) $=1.777849$

-

SCF Done: $E($ UWB97XD $)=-5092.25833219$ A.U. after 1 cycles NFock $=1$ Conv $=0.30 \mathrm{D}-08 \quad-\mathrm{V} / \mathrm{T}=2.0066$

$\langle$ Sx $>=0.0000\langle$ Sy $\rangle=0.0000\langle$ Sz $>=0.5000$

$<S^{* *} 2>=0.7649 \mathrm{~S}=0.5074$

$<$ L.S $>=0.000000000000 \mathrm{E}+00$

Annihilation of the first spin contaminant:

$\mathrm{S}^{* * 2}$ before annihilation 0.7649 , after 0.7501

Harmonic frequencies $\left(\mathrm{cm}^{* *}-1\right)$, IR intensities (KM/Mole), Raman 


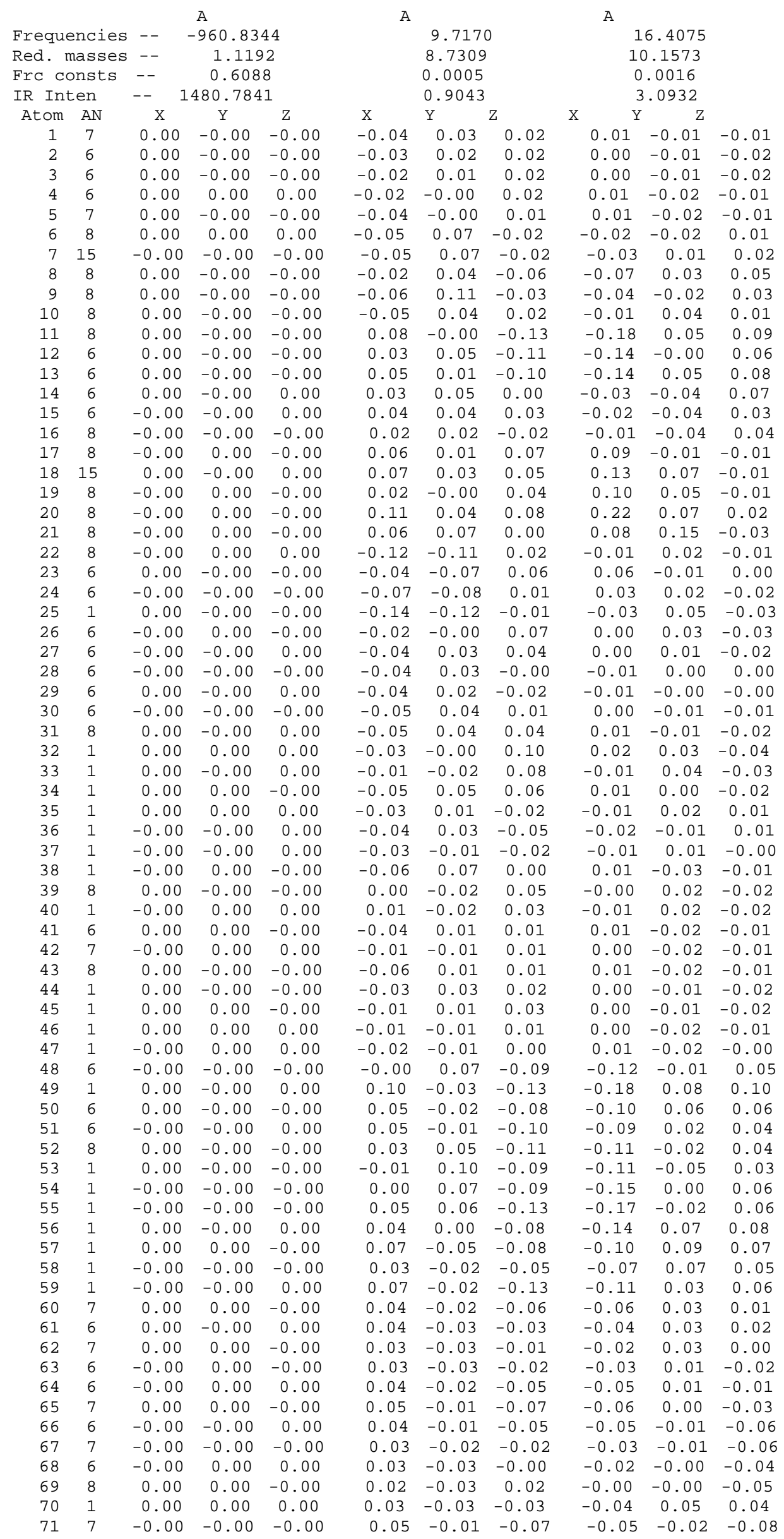




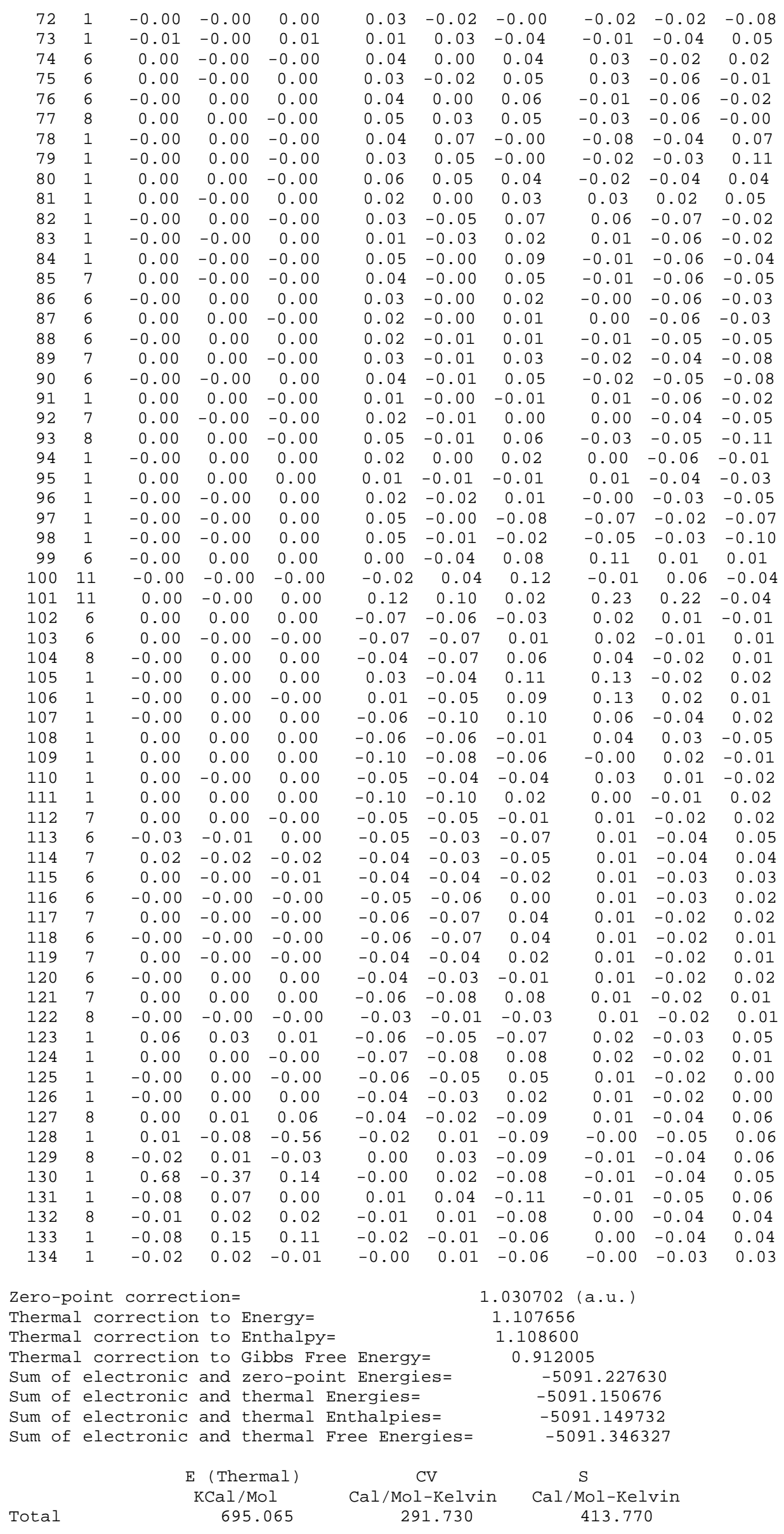




\begin{tabular}{lccc}
\multicolumn{1}{c}{ Item } & Value & Threshold Converged? \\
Maximum Force & 0.000009 & 0.000450 & YES \\
RMS Force & 0.000002 & 0.000300 & YES \\
Maximum Displacement & 0.001958 & $0.00180 \odot$ & NO \\
RMS Displacement & 0.000397 & 0.001200 & YES \\
Predicted change in Energy $=-2.206142 D-08$ &
\end{tabular}

Normal termination of Gaussian 16 at Sat Jul 20 10:45:17 2019.

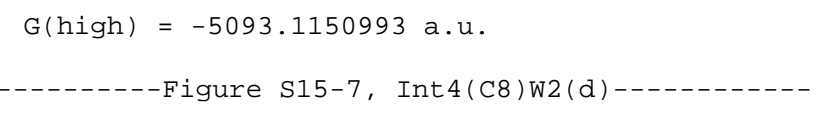

dna.for3dw2. $\log$

Stoichiometry C38H53N16Na2023P2(2)

Standard orientation:

\begin{tabular}{|c|c|c|c|c|c|}
\hline \multirow{2}{*}{$\begin{array}{l}\text { Center } \\
\text { Number }\end{array}$} & \multirow{2}{*}{$\begin{array}{l}\text { Atomic } \\
\text { Number }\end{array}$} & \multirow{2}{*}{$\begin{array}{l}\text { Atomic } \\
\text { Type }\end{array}$} & \multicolumn{3}{|c|}{ Coordinates (Angstroms) } \\
\hline & & & $\mathrm{x}$ & $Y$ & Z \\
\hline 1 & 7 & $\odot$ & -2.890985 & 2.651393 & $-\odot .836797$ \\
\hline 2 & 6 & $\odot$ & -3.150471 & 3.576482 & $\odot .126975$ \\
\hline 3 & 6 & $\odot$ & -2.140752 & 4.281079 & $\odot .689946$ \\
\hline 4 & 6 & $\odot$ & -0.814008 & 3.988525 & $\odot .252024$ \\
\hline 5 & 7 & $\odot$ & -0.563175 & 3.021945 & $-\odot .635578$ \\
\hline 6 & 8 & 0 & -6.257727 & $\odot .021193$ & -2.295469 \\
\hline 7 & 15 & $\odot$ & -7.774880 & -0.530528 & -2.611604 \\
\hline 8 & 8 & $\odot$ & -7.872829 & -1.825097 & -1.610138 \\
\hline 9 & 8 & 0 & -7.792312 & -0.963043 & -4.046813 \\
\hline 10 & 8 & $\odot$ & -8.777919 & $\odot .455561$ & -2.058070 \\
\hline 11 & 8 & $\odot$ & -8.482703 & -5.459832 & 0.568607 \\
\hline 12 & 6 & $\odot$ & -7.162347 & -3.965929 & -0.732902 \\
\hline 13 & 6 & $\odot$ & -8.482478 & -4.113746 & 0.031182 \\
\hline 14 & 6 & $\odot$ & 6.458786 & $\odot .489608$ & 2.388203 \\
\hline 15 & 6 & $\odot$ & 5.843728 & $-\odot .879018$ & 2.199100 \\
\hline 16 & 8 & $\odot$ & 5.695616 & 1.476139 & 1.664429 \\
\hline 17 & 8 & $\odot$ & 5.754974 & -2.761430 & 0.664306 \\
\hline 18 & 15 & $\odot$ & 7.218791 & -3.419298 & $\odot .363555$ \\
\hline 19 & 8 & $\odot$ & 7.374034 & -3.198824 & -1.244379 \\
\hline 20 & 8 & $\odot$ & 7.121602 & -4.887327 & $\odot .712261$ \\
\hline 21 & 8 & $\odot$ & 8.321989 & -2.626910 & 1.029163 \\
\hline 22 & 8 & $\odot$ & 7.013400 & -2.171730 & -5.359470 \\
\hline 23 & 6 & $\odot$ & 6.069433 & -2.651980 & -3.212520 \\
\hline 24 & 6 & $\odot$ & 7.307592 & -2.087979 & -3.937821 \\
\hline 25 & 1 & 0 & 7.768102 & -1.842775 & -5.876804 \\
\hline 26 & 6 & $\odot$ & $-6.9 \odot 4516$ & 2.897260 & $\odot .042746$ \\
\hline 27 & 6 & $\odot$ & -6.359932 & 2.214974 & -1.204455 \\
\hline 28 & 6 & $\odot$ & -6.006122 & 0.728782 & -1.042813 \\
\hline 29 & 6 & $\odot$ & -4.510457 & 0.734509 & -0.765670 \\
\hline 30 & 6 & $\odot$ & -3.992949 & 1.958125 & -1.527488 \\
\hline 31 & 8 & 0 & -5.112202 & 2.863396 & -1.633605 \\
\hline 32 & 1 & $\odot$ & -7.101490 & 3.945586 & -0.190996 \\
\hline 33 & 1 & $\odot$ & -7.855460 & 2.419782 & $\odot .311864$ \\
\hline 34 & 1 & $\odot$ & -7.081003 & 2.334583 & -2.014159 \\
\hline 35 & 1 & $\odot$ & -6.574677 & 0.251175 & -0.244224 \\
\hline 36 & 1 & $\odot$ & -4.023823 & -0.192437 & -1.066960 \\
\hline 37 & 1 & $\odot$ & -4.349666 & 0.895513 & 0.301734 \\
\hline 38 & 1 & $\odot$ & -3.626649 & 1.708478 & -2.522042 \\
\hline 39 & 8 & $\odot$ & -5.986269 & 2.892466 & 1.151465 \\
\hline $4 \odot$ & 1 & $\Theta$ & -6.052978 & 2. 062717 & 1.685822 \\
\hline 41 & 6 & $\odot$ & -1.582455 & 2.321431 & -1.193109 \\
\hline 42 & 7 & $\odot$ & 0.232105 & 4.656179 & 0.755923 \\
\hline 43 & 8 & $\odot$ & -1.401956 & 1.402403 & -2.005477 \\
\hline 44 & 1 & $\odot$ & -4.194000 & 3.669619 & $\odot .415956$ \\
\hline 45 & 1 & $\odot$ & -2.332985 & 5.019527 & 1.456281 \\
\hline 46 & 1 & $\odot$ & 0.084641 & 5.463958 & 1.338312 \\
\hline 47 & 1 & $\odot$ & 1.174899 & 4.448375 & 0.428025 \\
\hline 48 & 6 & $\odot$ & -7.205442 & -3.071415 & -1.950295 \\
\hline 49 & 1 & $\odot$ & -9.297565 & -5.624969 & 1.072842 \\
\hline 50 & 6 & $\odot$ & -8.373705 & -3.063551 & 1.141173 \\
\hline 51 & 6 & $\odot$ & -6.883620 & -3.096155 & 1.482748 \\
\hline 52 & 8 & 0 & -6.211177 & -3.416101 & 0.235791 \\
\hline 53 & 1 & $\odot$ & -6.182103 & -2.869960 & -2.280584 \\
\hline 54 & 1 & $\odot$ & -7.751938 & -3.562488 & -2.760458 \\
\hline 55 & 1 & $\odot$ & -6.804523 & -4.954327 & -1.032566 \\
\hline
\end{tabular}




\begin{tabular}{|c|c|c|c|c|c|}
\hline 56 & 1 & $\odot$ & -9.354187 & -3.948636 & -0.607809 \\
\hline 57 & 1 & $\odot$ & -9.011858 & -3.281179 & 1.998864 \\
\hline 58 & 1 & $\odot$ & -8.648716 & -2.099371 & 0.710488 \\
\hline 59 & 1 & $\odot$ & -6.643707 & -3.872248 & 2.212769 \\
\hline 60 & 7 & 0 & -6.343284 & -1.839063 & 1.972616 \\
\hline 61 & 6 & $\odot$ & -6.946445 & -0.606276 & 2.066834 \\
\hline 62 & 7 & $\odot$ & -6.112805 & 0.345108 & 2.400224 \\
\hline 63 & 6 & $\odot$ & -4.892427 & -0.290542 & 2.531147 \\
\hline 64 & 6 & $\odot$ & -5.014997 & -1.644257 & 2.269869 \\
\hline 65 & 7 & $\odot$ & -4.053953 & -2.583812 & 2.26659 \\
\hline 66 & 6 & 0 & -2.849625 & -2.080141 & 2.486465 \\
\hline 67 & 7 & $\odot$ & -2.611383 & -0.743728 & 2.70533 \\
\hline 68 & 6 & 0 & -3.588425 & 0.246664 & 2.748360 \\
\hline 69 & 8 & 0 & -3.290114 & 1.433529 & 2.924421 \\
\hline 70 & 1 & $\odot$ & -8.001494 & -0.464485 & 1.89094 \\
\hline 71 & 7 & $\odot$ & -1.780127 & -2.890736 & 2.52369 \\
\hline 72 & 1 & $\odot$ & -1.624847 & -0.417980 & 2.759940 \\
\hline 73 & 1 & $\odot$ & 5.939741 & 2.385705 & 1.959071 \\
\hline 74 & 6 & 0 & 5.601477 & -1.304791 & 0.742439 \\
\hline 75 & 6 & $\odot$ & 4.142239 & -0.974916 & 0.490091 \\
\hline 76 & 6 & $\odot$ & 3.476421 & -1.160782 & 1.856284 \\
\hline 77 & 8 & $\odot$ & 4.523101 & -0.935750 & 2.840034 \\
\hline 78 & 1 & $\odot$ & 6.475434 & 0.722531 & 3.458968 \\
\hline 79 & 1 & $\odot$ & 7.492620 & $\odot .459341$ & 2.022637 \\
\hline 80 & 1 & $\odot$ & 6.483381 & -1.613841 & 2.692003 \\
\hline 81 & 1 & $\odot$ & 6.261043 & -0.818826 & 0.022322 \\
\hline 82 & 1 & $\odot$ & 3.712645 & -1.599943 & -0.290183 \\
\hline 83 & 1 & 0 & 4.075106 & 0.069644 & 0.18100 \\
\hline 84 & 1 & $\odot$ & 3.059387 & -2.156136 & 1.991638 \\
\hline 85 & 7 & 0 & 2.387912 & -0.215333 & 2.110171 \\
\hline 86 & 6 & $\odot$ & 2.674731 & 1.117609 & 2.167795 \\
\hline 87 & 6 & $\odot$ & 1.711455 & 2.023244 & 2.441775 \\
\hline 88 & 6 & 0 & 0.392457 & 1.521628 & 2.678806 \\
\hline 89 & 7 & 0 & 0.116940 & 0.214619 & 2.616442 \\
\hline 90 & 6 & $\odot$ & 1.089456 & -0.688176 & 2.334691 \\
\hline 91 & 1 & 0 & 1.930370 & 3.081003 & 2.475032 \\
\hline 92 & 7 & $\odot$ & $-\odot .599789$ & 2.355707 & 2.993146 \\
\hline 93 & 8 & $\odot$ & 0.866125 & -1.905220 & 2.265958 \\
\hline 94 & 1 & 0 & 3.719078 & 1.378278 & 1.990720 \\
\hline 95 & 1 & $\odot$ & $-\odot .429067$ & 3.347231 & 2.960673 \\
\hline 96 & 1 & $\odot$ & -1.567982 & 2.021710 & 3.025937 \\
\hline 97 & 1 & 0 & -1.937509 & -3.839778 & 2.22550 \\
\hline 98 & 1 & $\odot$ & -0.825399 & -2.517042 & 2.47567 \\
\hline 99 & 6 & $\odot$ & 6.359689 & -3.699299 & -2.16595 \\
\hline 100 & 11 & 0 & -10.140261 & 2.146216 & -1.8985 \\
\hline 101 & 11 & 0 & 9.068332 & -4.655093 & 2.2648 \\
\hline 102 & 6 & 0 & 7.384588 & $-\odot .640952$ & -3.4527 \\
\hline 103 & 6 & $\odot$ & 5.926449 & $-\odot .282056$ & -3.1728 \\
\hline 104 & 8 & $\odot$ & 5.405626 & -1.522099 & -2.5727 \\
\hline 105 & 1 & 0 & 5.441039 & -3.935721 & -1.6233 \\
\hline 106 & 1 & 0 & 6.744302 & -4.606976 & -2.6383 \\
\hline 107 & 1 & 0 & 5.404655 & -3.086811 & -3.9686 \\
\hline 108 & 1 & 0 & 8.212943 & -2.656401 & -3.7069 \\
\hline 109 & 1 & $\theta$ & 7.858213 & $\odot .023003$ & -4.17998 \\
\hline 110 & 1 & $\odot$ & 7.937810 & $-\odot .592507$ & -2.5099 \\
\hline 111 & 1 & 0 & 5.372444 & -0.101457 & -4.10473 \\
\hline 112 & 7 & 0 & 5.804326 & ๑.831558 & -2.2754 \\
\hline 113 & 6 & 0 & 6.850360 & 2.996510 & -0.7313 \\
\hline 114 & 7 & 0 & 5.499839 & 3.249719 & -0.7236 \\
\hline 115 & 6 & $\theta$ & 4.476557 & 2.484887 & -1.21064 \\
\hline 116 & 6 & $\odot$ & 4.608256 & 1.301125 & -2.05798 \\
\hline 117 & 7 & 0 & 3.482198 & 0.738727 & -2.6006 \\
\hline 118 & 6 & $\odot$ & 2.302127 & 1.173932 & -2.24395 \\
\hline 119 & 7 & 0 & 2.106015 & 2.198482 & -1.34001 \\
\hline 120 & 6 & 0 & 3.140251 & 2.924444 & -0.8283 \\
\hline 121 & 7 & 0 & 1.193630 & $\odot .626690$ & -2.74685 \\
\hline 122 & 8 & 0 & 2.926651 & 3.885387 & -0.0726 \\
\hline 123 & 1 & 0 & 7.146446 & 2.124587 & -1.3170 \\
\hline 124 & 1 & 0 & 1.314614 & -0.148325 & -3.3776 \\
\hline 125 & 1 & 0 & $\odot .250190$ & $\odot .929097$ & -2.4769 \\
\hline 126 & 1 & 0 & 1.140414 & 2.491884 & -1.07804 \\
\hline 127 & 8 & 0 & 7.629340 & 3.721695 & -0.1205 \\
\hline 128 & 1 & 0 & 7.121938 & 4. 099688 & 1.57485 \\
\hline 129 & 8 & $\theta$ & 6.526417 & 4.019077 & 2.34626 \\
\hline 130 & 1 & $\theta$ & 5.308894 & 5.252828 & 1.62439 \\
\hline 131 & 1 & 0 & 7.072291 & 4.111233 & 3.13283 \\
\hline 132 & 8 & 0 & 4.883669 & 5.651644 & 0.846908 \\
\hline
\end{tabular}




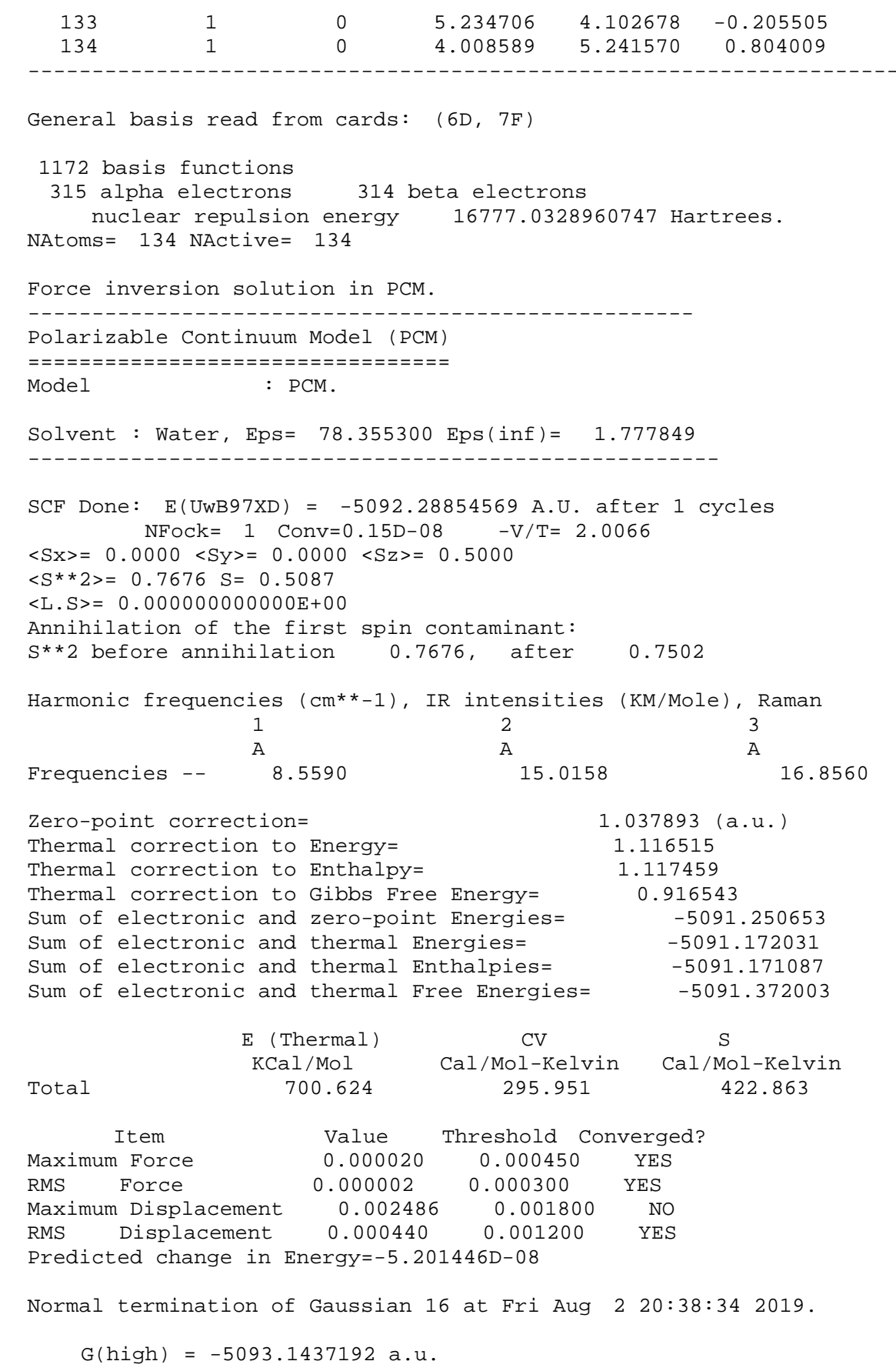

